\title{
Chapter 1 \\ Assessment of Undiscovered Oil and Gas Resources of the Anadarko Basin Province of Oklahoma, Kansas, Texas, and Colorado, 2010
}

By Debra K. Higley, Stephanie B. Gaswirth, Marvin M. Abbott, Ronald R. Charpentier, Troy A. Cook, Geoffrey S. Ellis, Nicholas J. Gianoutsos, Joseph R. Hatch, Timothy R. Klett, Philip H. Nelson, Mark J. Pawlewicz, Ofori N. Pearson, Richard M. Pollastro, and Christopher J. Schenk

Chapter 1 of 13

Petroleum Systems and Assessment of Undiscovered Oil and Gas in the Anadarko Basin Province, Colorado, Kansas, Oklahoma, and Texas-USGS Province 58

Compiled by Debra K. Higley

U.S. Geological Survey Digital Data Series DDS-69-EE 


\title{
U.S. Department of the Interior SALLY JEWELL, Secretary
}

\section{U.S. Geological Survey \\ Suzette M. Kimball, Acting Director}

\author{
U.S. Geological Survey, Reston, Virginia: 2014
}

For more information on the USGS - the Federal source for science about the Earth, its natural and living resources, natural hazards, and the environment, visit http://www.usgs.gov or call 1-888-ASK-USGS.

For an overview of USGS information products, including maps, imagery, and publications, visit http://WwW.usgs.gov/pubprod

To order this and other USGS information products, visit http://store.usgs.gov

Any use of trade, firm, or product names is for descriptive purposes only and does not imply endorsement by the U.S. Government.

Although this information product, for the most part, is in the public domain, it also may contain copyrighted materials as noted in the text. Permission to reproduce copyrighted items must be secured from the copyright owner.

Suggested citation:

Higley, D.K., Gaswirth, S.B., Abbott, M.M., Charpentier, R.R., Cook, T.A., Ellis, G.S., Gianoutsos, N.J., Hatch, J.R., Klett, T.R., Nelson, P.H., Pawlewicz, M.J., Pearson, O.N., Pollastro, R.M., and Schenk, C.J., 2014, Assessment of undiscovered oil and gas resources of the Anadarko Basin Province of Oklahoma, Kansas, Texas, and Colorado, 2010, chap. 1, in Higley, D.K., compiler, Petroleum systems and assessment of undiscovered oil and gas in the Anadarko Basin Province, Colorado, Kansas, Oklahoma, and Texas_USGS Province 58: U.S. Geological Survey Digital Data Series DDS-69-EE, 3 p. http://dx.doi.org/10.3133/ds69EE.

ISSN 2327-638X (online) 


\section{Contents}

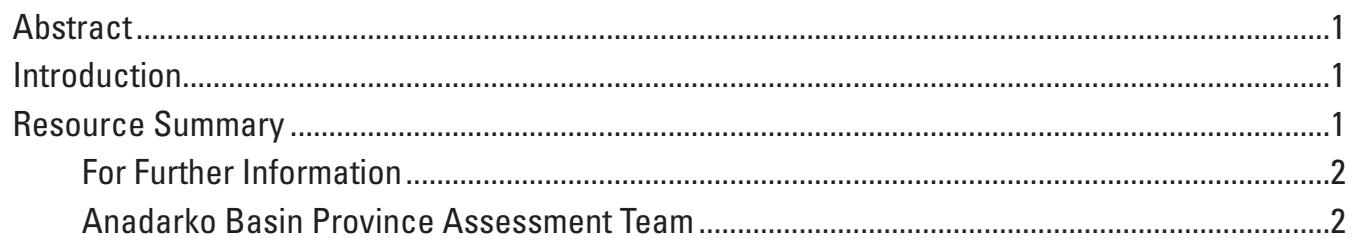

\section{Figure}

1. Map showing boundaries of the Anadarko Basin Province (red line), the Woodford Composite total petroleum system (TPS, black line), and the Pennsylvanian Composite TPS (blue line)

\section{Table}

1. Anadarko Basin Province assessment results listed by total petroleum system (TPS) and assessment unit (AU). Included are estimated volumes of undiscovered technically recoverable oil, gas, and natural gas liquids (NGL).. 



\title{
Assessment of Undiscovered Oil and Gas Resources of the Anadarko Basin Province of Oklahoma, Kansas, Texas, and Colorado, 2010
}

\author{
Compiled by Debra K. Higley
}

\begin{abstract}
The U.S. Geological Survey, using a geoscience-based assessment methodology, estimated mean technically recoverable undiscovered continuous and conventional resources that total 495 million barrels of oil, 27.5 trillion cubic feet of natural gas, and 410 million barrels of natural gas liquids in the Anadarko Basin Province; this assessment includes the Las Animas arch area of southeastern Colorado. The province is at a mature stage of exploration and development for conventional resources. Mean undiscovered continuous resources are estimated at 79 percent of oil, 90 percent of natural gas, and 81 percent of natural gas liquids in the province.
\end{abstract}

\section{Introduction}

The U.S. Geological Survey (USGS) in 2010 completed an assessment of the undiscovered oil and gas potential of the Anadarko Basin Province of western Oklahoma, western Kansas, northern Texas, and southeastern Colorado (fig. 1). The assessment is based on the geologic elements of each defined total petroleum system (TPS), including (1) hydrocarbon source rocks (source-rock richness and thermal maturation, hydrocarbon generation, adsorption, and migration); (2) reservoir rock type (conventional or continuous), distribution, and quality; and (3) types and distribution of reservoir traps and seals, including timing relative to petroleum generation and migration. Using this geologic framework, the USGS defined 2 TPSs, with 12 included assessment units (AU), and quantitatively estimated undiscovered oil, gas, and natural gas liquids resources in each $\mathrm{AU}$ (table 1).

The Anadarko Basin Province is in a mature state of exploration and development for conventional resources. Much of the production is reported as being commingled from numerous formations that were deposited over broad age ranges; this commingling influenced grouping of formations into the AUs. The Woodford Composite and Pennsylvanian Composite TPSs represent source rock contributions from numerous Ordovician through Pennsylvanian formations. The
Woodford Composite TPS source rocks primarily contribute to Cambrian through Mississippian reservoirs, and those of the Pennsylvanian Composite TPS to Pennsylvanian and Permian reservoirs. Migration and accumulation of hydrocarbons from variable sources can occur along fault systems and updip from the extent of the Woodford Shale and other source rocks. Biogenic gas from the Cretaceous Niobrara Formation is produced from western Kansas and eastern Colorado; however, that resource was evaluated in the Denver Basin Province assessment (USGS Fact Sheet 002-03).

\section{Resource Summary}

The USGS assessment of undiscovered conventional and continuous (unconventional) resources within the province resulted in mean estimates of 495 million barrels of oil (MMBO), 27 trillion cubic feet of natural gas (TCFG), and 410 million barrels of natural gas liquids (MMBNGL) within 12 AUs in the 2 TPSs (table 1). Much of the remaining conventional resources are from field growth in this mature province. Continuous resources are focused in the deep part of the Anadarko Basin in Oklahoma and Texas. Boundaries of the Woodford Shale Oil and Woodford Shale Gas AUs and locations of sweet spots within them were based mainly on (1) extent and thickness of the formation, (2) filling of underlying Hunton Formation eroded channels, (3) historical and estimated ultimate production from existing wells, and (4) levels of thermal maturation based on 1D, 2D, and 3D petroleum system models and on vitrinite reflectance maps and data. The Thirteen Finger Limestone-Atoka Shale Gas continuous AU does not have documented production and has limited published information. Boundaries for this AU were based largely on characteristics such as thickness and lateral extent of included formations from well-log examination, and it is within the boundary of wet and dry gas generation based on $1 \mathrm{D}$ and $3 \mathrm{D}$ petroleum system models. This uncertainty is reflected in a fairly broad range of F5 to F95 resource estimates (table 1); mean undiscovered resources are $6.85 \mathrm{TCFG}$ and 82 MMBNGL. 


\section{For Further Information}

Supporting geologic studies of total petroleum systems and assessment units and reports on the methodology used in the Anadarko Basin Province assessment are in preparation. Assessment results and geologic reports will be available as completed at the USGS Web site http://energy.cr.usgs.gov/ oilgas/noga/.

\section{Anadarko Basin Province Assessment Team:}

Debra K. Higley (Task Leader; higley@usgs.gov), Stephanie B. Gaswirth, Marvin M. Abbott, Ronald R. Charpentier, Troy A. Cook, Geoffrey S. Ellis, Nicholas J. Gianoutsos, Joseph R. Hatch, Timothy R. Klett, Philip H. Nelson, Mark J. Pawlewicz, Ofori N. Pearson, Richard M. Pollastro, and Christopher J. Schenk (National Assessment of Oil and Gas Project Chief).

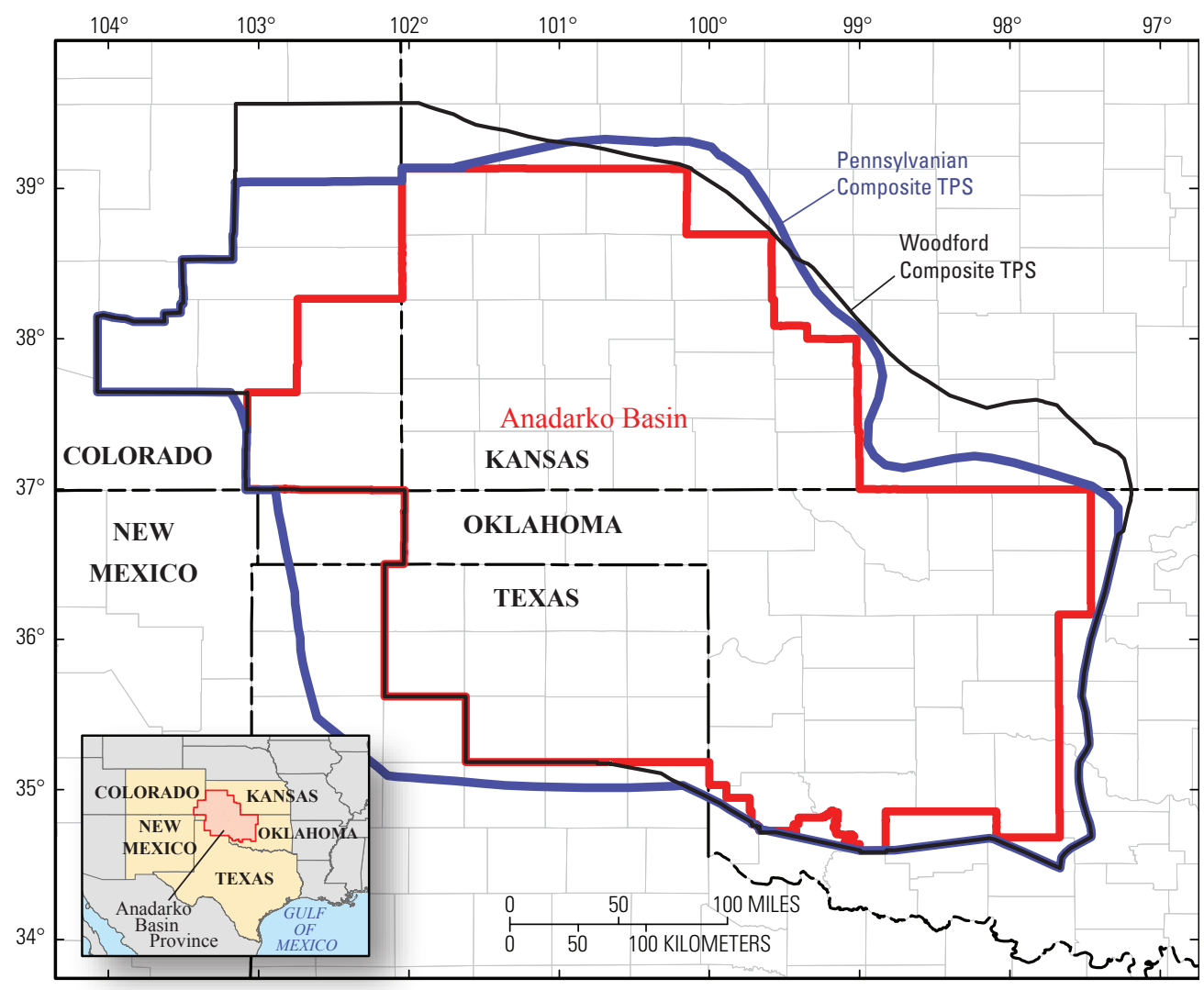

Figure 1. Map showing boundaries of the Anadarko Basin Province (red line), the Woodford Composite total petroleum system (TPS, black line), and the Pennsylvanian Composite TPS (blue line). 
Table 1. Anadarko Basin Province assessment results listed by total petroleum system (TPS) and assessment unit (AU). Included are estimated volumes of undiscovered technically recoverable oil, gas, and natural gas liquids (NGL).

[MMBO, million barrels of oil; BCFG, billion cubic feet of gas; MMBNGL, million barrels of natural gas liquids; gray shading, not applicable. Type refers to mainly oil or gas accumulations in the assessment unit. Fractiles (F95, F50, F5) are fully risked estimates. F95 denotes a 95-percent chance of at least the amount tabulated. Other fractiles are defined similarly. Fractiles are additive only under the assumption of perfect positive correlation]

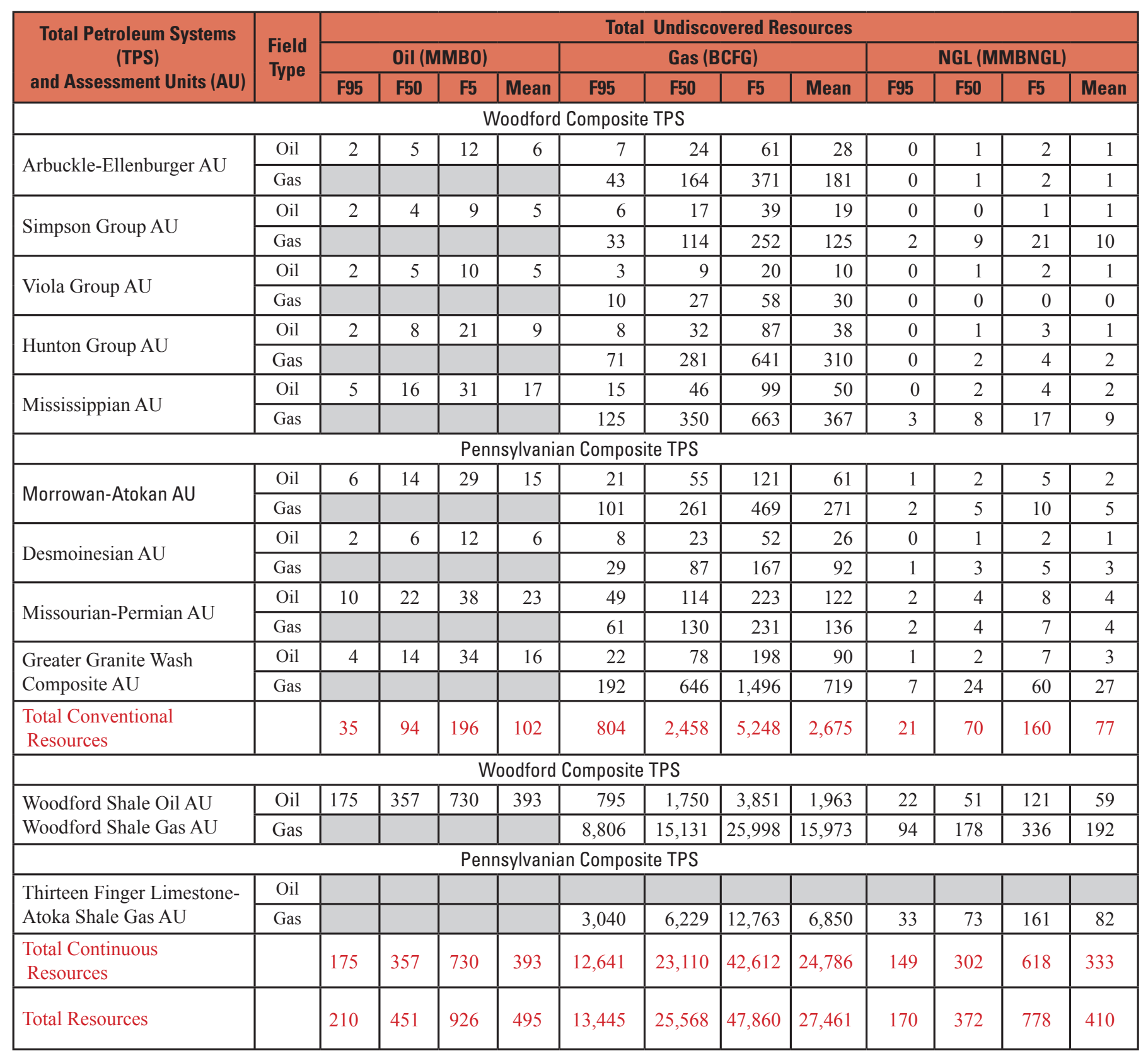





\section{Chapter 2 \\ Overview}

By Debra K. Higley and Stephanie B. Gaswirth

Click here to return to

Volume Title Page

Chapter 2 of 13

Petroleum Systems and Assessment of Undiscovered Oil and Gas in the Anadarko Basin Province, Colorado, Kansas, Oklahoma, and Texas-USGS Province 58

Compiled by Debra K. Higley

U.S. Geological Survey Digital Data Series DDS-69-EE 


\title{
U.S. Department of the Interior SALLY JEWELL, Secretary
}

\section{U.S. Geological Survey Suzette M. Kimball, Acting Director}

\author{
U.S. Geological Survey, Reston, Virginia: 2014
}

For more information on the USGS - the Federal source for science about the Earth, its natural and living resources, natural hazards, and the environment, visit http://Www.usgs.gov or call 1-888-ASK-USGS.

For an overview of USGS information products, including maps, imagery, and publications, visit http://WwW.usgs.gov/pubprod

To order this and other USGS information products, visit http://store.usgs.gov

Any use of trade, firm, or product names is for descriptive purposes only and does not imply endorsement by the U.S. Government.

Although this information product, for the most part, is in the public domain, it also may contain copyrighted materials as noted in the text. Permission to reproduce copyrighted items must be secured from the copyright owner.

Suggested citation:

Higley, D.K., and Gaswirth, S.B., 2014, Overview, chap. 2, in Higley, D.K., compiler, Petroleum systems and assessment of undiscovered oil and gas in the Anadarko Basin Province, Colorado, Kansas, Oklahoma, and TexasUSGS Province 58: U.S. Geological Survey Digital Data Series DDS-69-EE, 5 p. http://dx.doi.org/10.3133/ds69EE.

ISSN 2327-638X (online) 


\section{Contents}

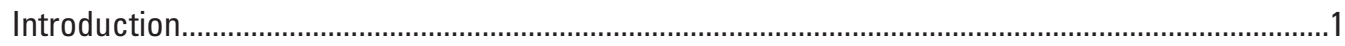

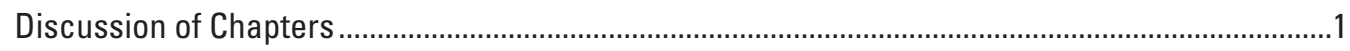

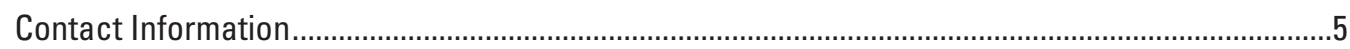

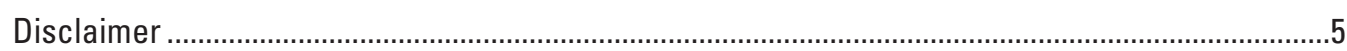

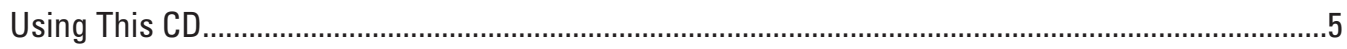

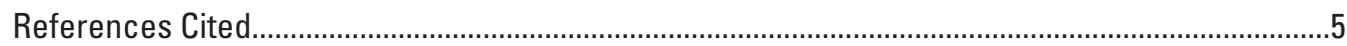

\section{Figures}

1. Map showing the Anadarko Basin Province is delineated by the maximum areal extent of the Woodford Composite and Pennsylvanian Composite Total Petroleum Systems (TPSs). The province includes the Anadarko Basin (red line), part of the Palo Duro Basin, and the Las Animas arch....

2. Generalized surface and subsurface stratigraphic columns for the Anadarko Basin and the Southern Oklahoma Fold Belt Provinces for the Precambrian to Mississippian. Assessment units (AU) are included in the Woodford Composite TPS. Blue text and lowercase descriptors indicate informal status. Wavy horizontal lines and vertical bars indicate unconformities and their duration. Modified from Bebout and others (1993) and Henry and Hester (1995). Ages in millions of years before present (Ma) from Haq and Van Eysinga (1998) and Gradstein and others (2004) (red text). Fm., Formation; Mbr., Member.

3. Generalized surface and subsurface stratigraphic columns for the Anadarko Basin and the Southern Oklahoma Fold Belt Provinces for the Precambrian to Mississippian. Assessment units (AU) are included in the Woodford Composite TPS. Italic (blue) text and lowercase descriptors indicate informal status. Wavy horizontal lines and vertical bars indicate unconformities and their duration. Modified from Bebout and others (1993) and Henry and Hester (1995). Ages in millions of years before present (Ma) from Haq and Van Eysinga (1998) and Gradstein and others (2004) (red text). Fm., Formation; Mbr., Member..... 



\section{Overview}

By Debra K. Higley and Stephanie B. Gaswirth

\section{Introduction}

This publication provides research results and related data in support of the U.S. Geological Survey (USGS) assessment of the undiscovered oil and gas resource potential of the Anadarko Basin Province of western Oklahoma and Kansas, northern Texas, and southeastern Colorado (fig. 1). This province area includes the Las Animas arch of southeastern Colorado, part of the Palo Duro Basin of Texas, and the Anadarko Basin. This is hereafter referred to as the 2011 assessment, which corresponds to the publication release date of the assessment results (Higley and others, 2011). Results of the geologic analysis and resource assessment (chapter 1) are based on the geologic elements of each defined total petroleum system (TPS), including hydrocarbon source rocks (source-rock maturation, hydrocarbon generation and migration), reservoir rocks (sequence stratigraphic and petrophysical properties), hydrocarbon traps (trapping mechanisms and timing), and seals. Using this geologic framework, the USGS defined 2 TPSs, the Woodford Composite TPS and Pennsylvanian Composite TPS (fig. 1) and 12 included assessment units (AU) (chapters 1 and 5-7), and quantitatively estimated the undiscovered oil and gas resources within these AUs. The assigned TPS and AU names and numeric codes follow.

Woodford Composite TPS 505801:

1. Arbuckle-Ellenburger, AU 50580101

2. Simpson Group, AU 50580102

3. Viola Group, AU 50580103

4. Hunton Group, AU 50580104

5. Mississippian, AU 50580105

6. Woodford Shale Gas, AU 50580161

7. Woodford Shale Oil, AU 50580162

8. Pennsylvanian Composite TPS 505802:

9. Morrowan-Atokan, AU 50580201

10. Desmoinesian, AU 50580202

11. Missourian-Permian, AU 50580203

12. Greater Granite Wash Composite, AU 50580204
13. Thirteen Finger Limestone-Atoka Shale Gas, AU 50580261

There are nine conventional and three continuous AUs. Continuous AUs are the (1) Devonian and Mississippian Woodford Shale Gas and (2) Woodford Shale Oil AUs of the Woodford Composite TPS, and (3) the Pennsylvanian Thirteen Finger Limestone-Atoka Shale of the Pennsylvanian Composite TPS. The stratigraphic charts show units within the Woodford Composite TPS (fig. 2) and Pennsylvanian Composite TPS (fig. 3).

\section{Discussion of Chapters}

The 13 chapters included in DDS-69-EE cover topics that range from the oil and gas resource assessment results (chapter 1) of the Anadarko Basin Province, to background geological and geochemical research (chapters 3-11), tabular data and graphs in support of the assessment (chapter 12), and data releases of geographic information systems (GIS) shape files, zmap-format grid files that were used to build petroleum system models, and a standalone three-dimensional (3D) geologic model (chapter 13). Information on individual chapters is below.

Chapter 3. Thermal maturation history is based on one-dimensional (1D) and four-dimensional (4D) petroleum system models created as part of this study and on published research. This information was used to model hydrocarbon generation, migration, and accumulation through time for three assigned source intervals, the Ordovician Oil Creek Formation, Devonian-Mississippian Woodford Shale, and the informal Atokan Thirteen Finger limestone. Thermal maturation boundaries were also used to delineate continuous assessment units.

Chapter 4. The geochemistry of produced gases from the Anadarko Basin was analyzed with particular regard for their source and timing of generation, and this information helped to define the assessment units used in the assessment of undiscovered resources.

Chapter 5. The geology and assessment of Cambrian through Devonian stratigraphy of the Anadarko Basin Province is discussed. Included are descriptions of the units that compose the AUs: the Reagan Sandstone, the Arbuckle Group, the Simpson Group, the Viola Group, the Sylvan Shale, the Hunton Group, and the Misener sand (fig. 2). 


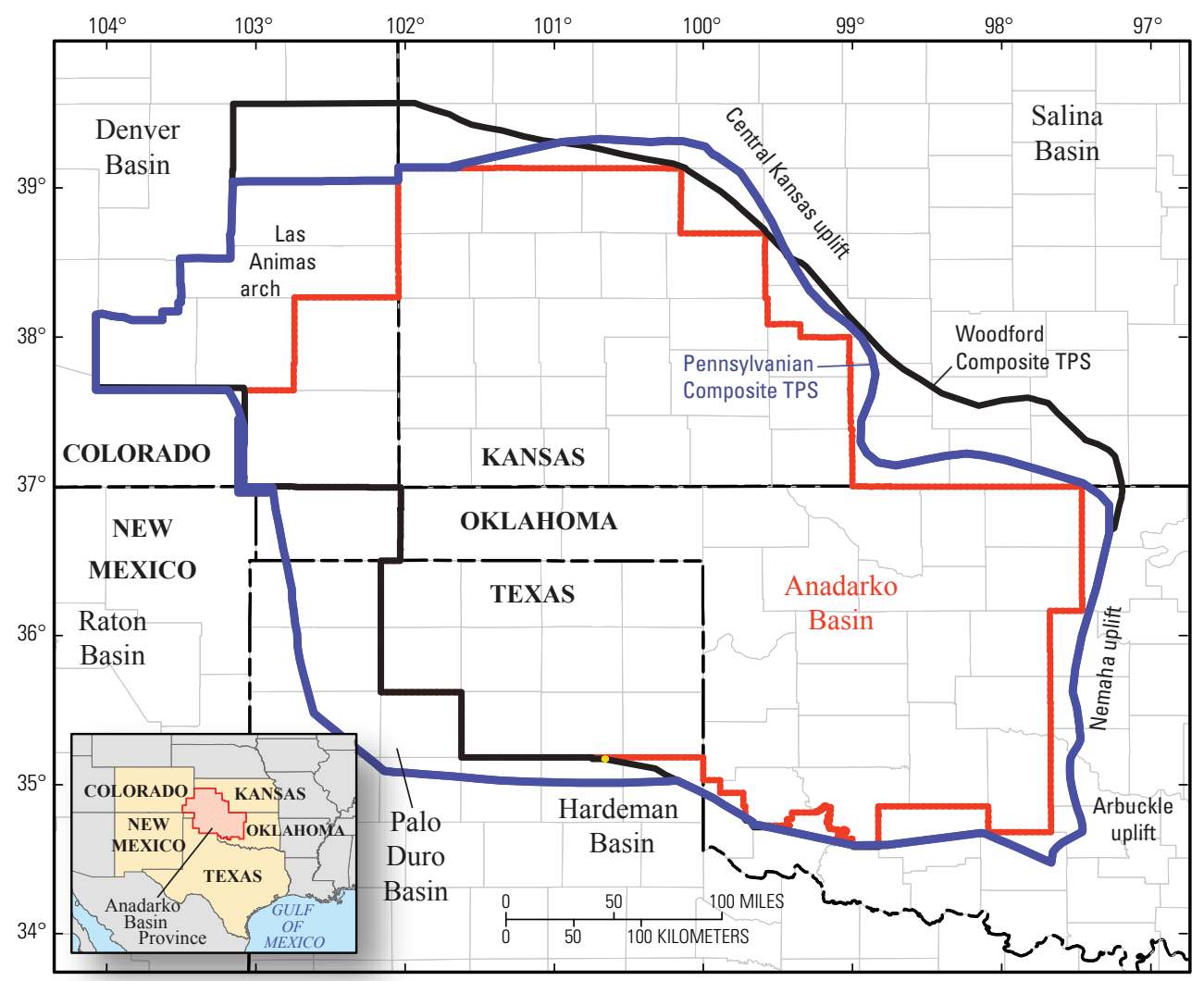

Figure 1. Map showing the Anadarko Basin Province is delineated by the maximum areal extent of the Woodford Composite and Pennsylvanian Composite Total Petroleum Systems (TPSs). The province includes the Anadarko Basin (red line), part of the Palo Duro Basin, and the Las Animas arch.

Chapter 6. Contained are the geology, thermal maturation history, and assessment of continuous resources of the Devonian-Mississippian Woodford Shale Gas and Woodford Shale Oil AUs (fig. 2).

Chapter 7. Conventional undiscovered resources of Mississippian through Permian AUs are discussed, as well as continuous gas resources from the Atokan Thirteen Finger limestone AU (figs. 2 and 3). Background information on these AUs includes the geologic characteristics and thermal maturation and petroleum production histories of the source and reservoir rocks.

Chapters 8 and 9. Pore pressures in Pennsylvanian strata of the greater Anadarko Basin range from overpressure in the deep basin, to normal pressure in the northeastern flank, to underpressure in the northwestern flank. The characteristics and evolution of the overpressured system are discussed in chapter 8, along with the finding of a paleo-overpressure zone more extensive than the present-day area of overpressure. The characteristics and causes of the underpressure, which grades into normal pressure along the northern flank of the basin, are discussed in chapter 9 .

Chapter 10. Lithologies derived from mud logs, sample logs, and geophysical logs are presented on eight structural cross sections that cover the Oklahoma portion of the Anadarko Basin. There are three major lithologic groups: (1) the carbonate-dominated units of Mississippian age and older, (2) mostly siliciclastic units of Pennsylvanian age, and (3) evaporites and red shales of Permian age.

Chapter 11. This discussion of the tectonic and structural evolution of the Anadarko Basin is accompanied by a structural interpretation of a key two-dimensional (2D) seismic line and associated structural restoration.

Chapter 12. Contained are tabular data and graphs used in support of assessment of undiscovered oil and gas resources of the Anadarko Basin Province.

Chapter 13. Included are grid files and associated readme and metadata files used to build and document a 4D petroleum system model of the province. The $2 \mathrm{D}$ grid files comprise (1) 26 structural surfaces across the province, (2) estimated eroded thickness of strata in the Cenozoic, (3) total organic carbon content of the Woodford Shale, and (4) basement heat flow. Also included with this chapter is a 3D standalone geologic model of the Anadarko Basin Province that incorporates the $2 \mathrm{D}$ grid files. 


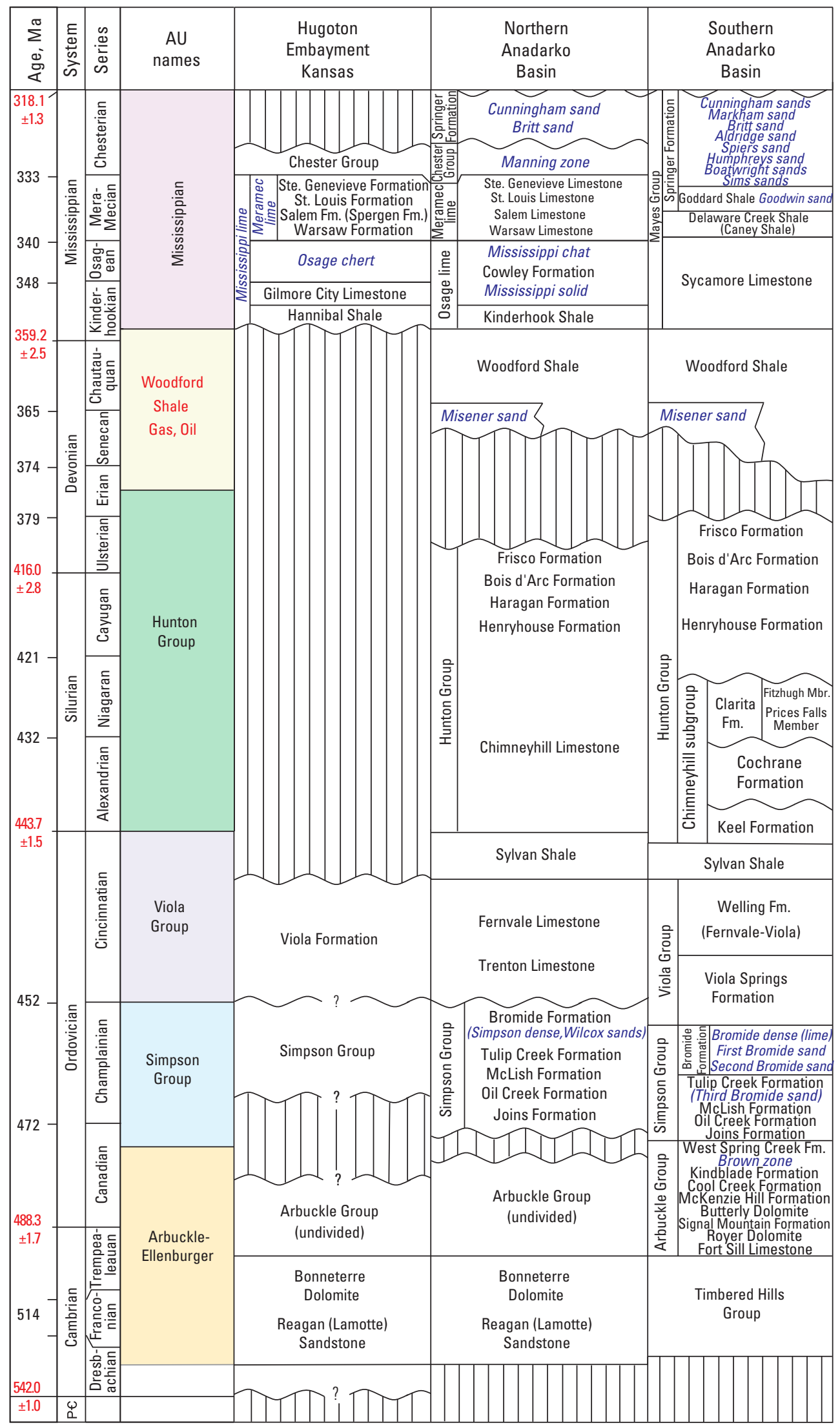

Figure 2. Generalized surface and subsurface stratigraphic columns for the Anadarko Basin and the Southern Oklahoma Fold Belt Provinces for the Precambrian to Mississippian. Assessment units (AU) are included in the Woodford Composite TPS. Blue text and lowercase descriptors indicate informal status. Wavy horizontal lines and vertical bars indicate unconformities and their duration. Modified from Bebout and others (1993) and Henry and Hester (1995). Ages in millions of years before present (Ma) from Haq and Van Eysinga (1998) and Gradstein and others (2004) (red text). Fm., Formation; Mbr., Member. 


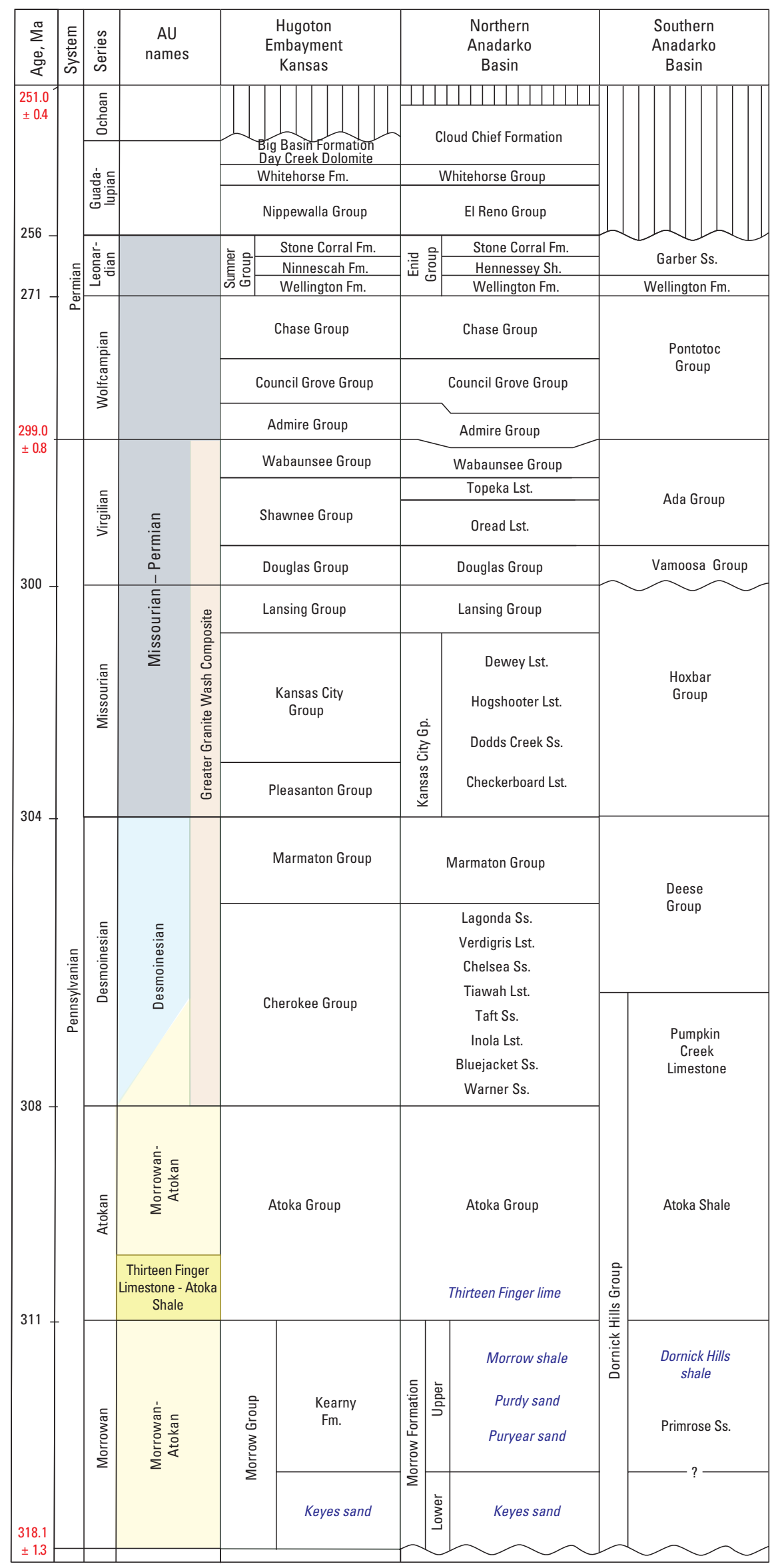

Figure 3. Generalized surface and subsurface stratigraphic columns for the Anadarko Basin and the Southern Oklahoma Fold Belt Provinces for the Precambrian to Mississippian. Assessment units (AU) are included in the Woodford Composite TPS. Italic (blue) text and lowercase descriptors indicate informal status. Wavy horizontal lines and vertical bars indicate unconformities and their duration. Modified from Bebout and others (1993) and Henry and Hester (1995). Ages in millions of years before present (Ma) from Haq and Van Eysinga (1998) and Gradstein and others (2004) (red text). Fm., Formation; Mbr., Member. 


\section{Contact Information}

This volume is one of a series of products resulting from the National Oil and Gas Assessment project of the U.S. Geological Survey. Inquiries about this CD-ROM or the project should be addressed to:

Christopher J. Schenk, Project Chief

U.S. Geological Survey

Box 25046, Mail Stop 939

Denver Federal Center

Denver, CO 80225-0046

Telephone: (303) 236-5796

E-mail: schenk@usgs.gov

\section{Disclaimer}

This Compact Disc-Read Only Memory (CD-ROM) publication was prepared by an agency of the United States Government. Neither the United States Government nor any agency thereof, nor any of its employees, make any warranty, expressed or implied, or assume any legal liability or responsibility for the accuracy, completeness, or usefulness of any information, apparatus, product, or process disclosed in this report, or represent that its use would not infringe privately owned rights. Reference therein to any specific commercial product, process, or service by trade name, trademark, manufacturer, or otherwise does not necessarily constitute or imply its endorsement, recommendation, or favoring by the United States Government or any agency thereof. Although all data and software published on this CD-ROM have been used by the U.S. Geological Survey, no warranty, expressed or implied, is made by the U.S. Geological Survey as to the accuracy of the data and related materials and(or) the functioning of the software. The act of distribution shall not constitute any such warranty, and no responsibility is assumed by the U.S. Geological Survey in the use of these data, the software, or related materials.

\section{Using This CD}

The descriptive and interpretive text chapters of this volume are in PDF format. Use Acrobat Reader Version 7.0 or later to access and view these chapters.

Data-table files are presented as tab-delimited text files (.tab files), usable in spreadsheet and database software. Graphical and summary-table files are presented as portable document format files (.pdf files). Zmap-format grids (.DAT) and the associated readme file include geographic coordinate data. Geographic Information System (GIS) data associated with the Anadarko Basin resource assessment can be accessed from the following website: http://energy.usgs.gov/OilGas/ AssessmentsData/NationalOilGasAssessment/USBasinSummaries.aspx? provcode $=5058$.

\section{References Cited}

Bebout, D.G., White, W.A., Hentz, T.F., and Grasmick, M.K., eds., 1993, Atlas of major mid-continent gas reservoirs: Bureau of Economic Geology, 85 p.

Gradstein, F.M., Ogg, J.G., Smith, A.G., Bleeker, Wouter, Lourens, L.J., 2004, A new geologic time scale with special reference to Precambrian and Neogene: Episodes, June 2004 , v. 27 , no. 2 , p. $83-100$.

Haq, B.U., and Van Eysinga, F.W.B., 1998, Geological time table: Elsevier Science, Amsterdam, New York, 1 chart.

Henry, M.E., and Hester, T.C., 1995, Anadarko Basin Province (058), in Gautier, D.L., Dolton, G.L., Takahashi, K.I., and Varnes, K.L., eds., 1995 National assessment of United States oil and gas resources on CD-ROM: U.S. Geological Survey Digital Data Series 30, 51 p., accessed April 20, 2011, at http://energy.cr.usgs.gov/oilgas/noga/1995.html.

Higley, D.K., Gaswirth, S.B., Abbott, M.M., Charpentier, R.R., Cook, T.A., Ellis, G.S., Gianoutsos, N.J., Hatch, J.R., Klett, T.R., Nelson, Philip, Pawlewicz, M.J., Pearson, O.N., Pollastro, R.M., and Schenk, C.J., 2011, Assessment of undiscovered oil and gas resources of the Anadarko Basin province of Oklahoma, Kansas, Texas, and Colorado, 2010: U.S. Geological Survey Fact Sheet 2011-3003, 2 p., accessed February 2011, at http://pubs.usgs.gov/ $f_{S} / 2011 / 3003 /$. 



\section{Chapter 3 \\ Thermal Maturation of Petroleum Source Rocks in the Anadarko Basin Province, Colorado, Kansas, Oklahoma, and Texas}

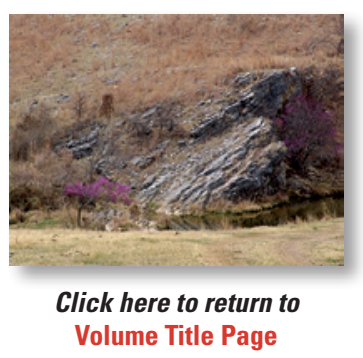

By Debra K. Higley

Chapter 3 of 13

Petroleum Systems and Assessment of Undiscovered Oil and Gas in the Anadarko Basin Province, Colorado, Kansas, Oklahoma, and Texas-USGS Province 58

Compiled by Debra K. Higley

U.S. Geological Survey Digital Data Series DDS-69-EE 


\title{
U.S. Department of the Interior SALLY JEWELL, Secretary
}

\section{U.S. Geological Survey \\ Suzette M. Kimball, Acting Director}

\author{
U.S. Geological Survey, Reston, Virginia: 2014
}

For more information on the USGS - the Federal source for science about the Earth, its natural and living resources, natural hazards, and the environment, visit http://Www.usgs.gov or call 1-888-ASK-USGS.

For an overview of USGS information products, including maps, imagery, and publications, visit http://WwW.usgs.gov/pubprod

To order this and other USGS information products, visit http://store.usgs.gov

Any use of trade, firm, or product names is for descriptive purposes only and does not imply endorsement by the U.S. Government.

Although this information product, for the most part, is in the public domain, it also may contain copyrighted materials as noted in the text. Permission to reproduce copyrighted items must be secured from the copyright owner.

Suggested citation:

Higley, D.K., 2014, Thermal maturation of petroleum source rocks in the Anadarko Basin Province, Colorado, Kansas, Oklahoma, and Texas, chap. 3, in Higley, D.K., compiler, Petroleum systems and assessment of undiscovered oil and gas in the Anadarko Basin Province, Colorado, Kansas, Oklahoma, and Texas-USGS Province 58: U.S. Geological Survey Digital Data Series DDS-69-EE, 53 p., http://dx.doi.org/10.3133/ds69EE. 


\section{Contents}

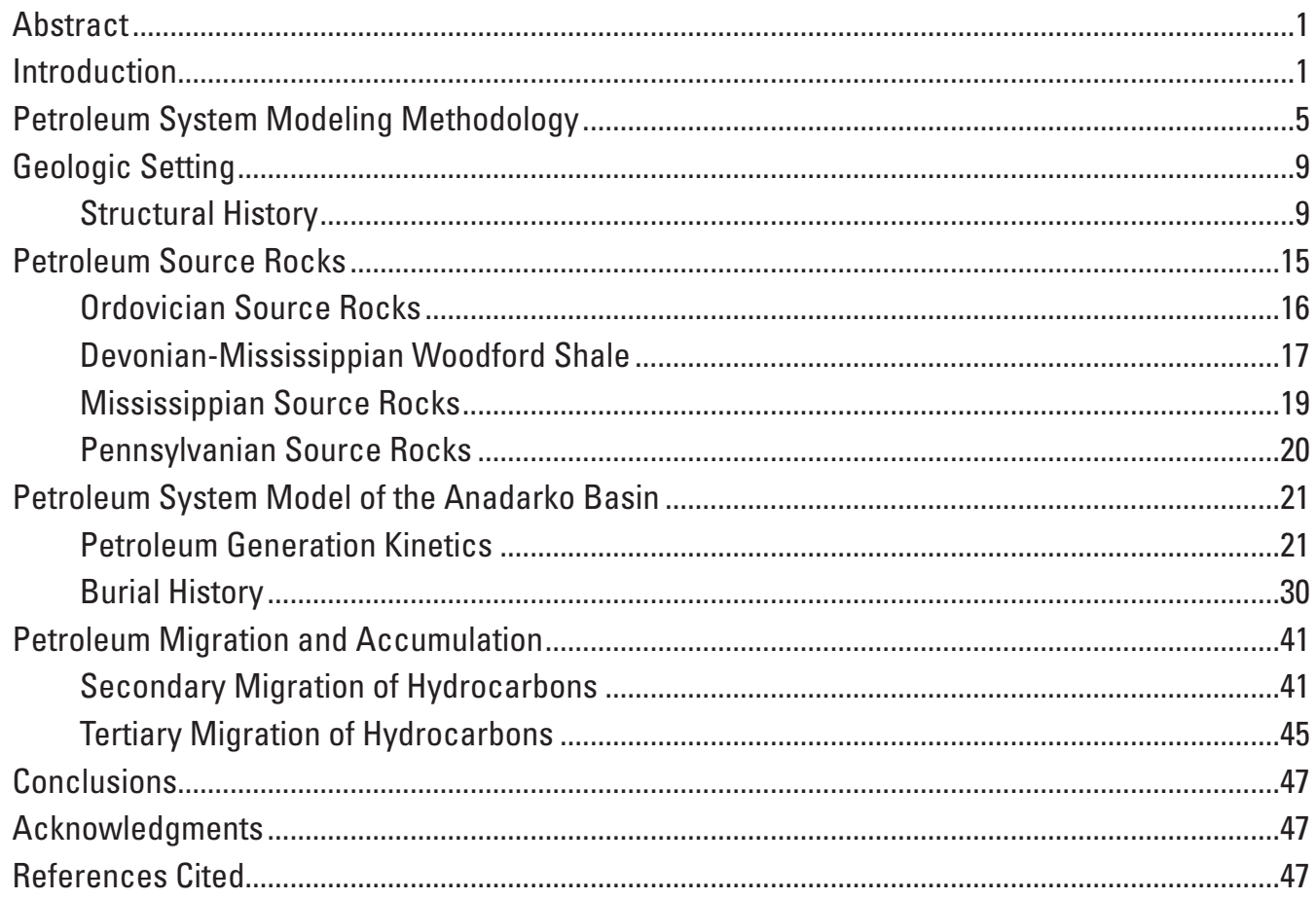

\section{Figures}

1. Map showing Anadarko Basin Province (dashed line) and boundaries of total petroleum systems (TPS) and assessment units (AU). Only AUs that contain source rocks are shown. The Woodford Shale is divided into the Woodford Shale Oil and Woodford Shale Gas AUs based mainly on levels of thermal maturation and formation thickness. The Desmoinesian and Missourian-Permian AUs share a common boundary with the Pennsylvanian Composite TPS, with the exception that the AUs exclude an area of the deep basin that is the Granite Wash Composite AU (not shown). There are common boundaries for most AUs..

2. Generalized stratigraphic column for the Anadarko Basin Province with oil and gas source rocks (brown text). Increases in source rock potential are indicated by larger numbers. The expected hydrocarbons header indicates whether the source rock is more oil or gas prone. Hatch (oral commun., 2010), and modified from Burruss and Hatch (1989). Vertical lines show a generalized time range of unconformity from Bebout and others (1993, fig. 5). Gp., Group; Fm., Formation.

3. Map showing basement heat flow contours across the Anadarko Basin based on data from Carter and others (1998), Blackwell and Richards (2004), and data downloads from the Southern Methodist University Web site ( http://smu.edu/geothermal/). Basin areas within and north of the Wichita Mountain and Amarillo uplifts exhibit generally lower heat flows than other basin areas. Highest measured heat flow is in the northwest, along the Las Animas arch. The northwest trending Central Kansas uplift (CKU) also exhibits elevated heat flow values. Contours are in milliwatts per square meter $\left(\mathrm{mW} / \mathrm{m}^{2}\right)$. Red fault lines are from Adler and others (1971). 
4. Map showing formation temperatures on the top of the Woodford Shale model layer. Cross section location is orange line on map. Black contour lines are temperature on the Woodford Shale from Gallardo and Blackwell (1999). Formation temperatures on the cross section are from ground surface to the Precambrian. The red dot is location of the Woodford Shale layer. Elevation in meters is relative to sea level. Purple fault lines are from Adler and others (1971)

5. Map showing modeled Celsius $\left({ }^{\circ} \mathrm{C}\right)$ temperatures on the top of the Wabaunsee layer. Oklahoma (black) contour lines from Gallardo and Blackwell (1999) are estimated temperatures on the top of the Pennsylvanian, which is roughly analogous to the Wabaunsee elevation. Both sets of contours show general increase in temperature with greater burial depth. Increasing depth with burial is shown by the underlying Precambrian through Morrowan layers (red arrow). 3D view is 10 times vertical exaggeration. Purple fault lines are from Adler and others (1971)

6. View to the southeast of elevation on the top of the Woodford Shale model layer is from the two-dimensional WoodfordTft.DAT grid file (Higley and others, 2014) that was created using Earthvision ${ }^{\circledR}$. Vertical exaggeration is 18 times. Woodford extent is approximated by the $-12,000$-foot elevation contour, although Woodford is also present east of the Central Kansas uplift (CKU). Major structures are labeled. Precambrian faults (red) are from Adler and others (1971). Wichita Mountain uplift bounding faults are vertical for four-dimensional model purposes only, and the Woodford Shale surface in this area is unknown because of insufficient data. Data sources for this surface include Woodford picks from well logs, Rottmann (2000a, 2000b), and edited IHS Energy (2009a, 2009b) formation tops

7. View to the southeast showing Woodford Shale layer lithofacies. Vertical exaggeration is 18 times. View orientation is similar to the 1-kilometer grid spacing Earthvision ${ }^{\circledR}$ image (fig. 6). This PetroMod® image shows underlying and lateral facies changes for the Woodford Shale layer, which is shown in white. Lateral lithofacies within the Woodford layer are primarily limestone and dolomite of the Viola Group. Because the purpose of this image is to show lateral changes in lithofacies assignments on a model layer, this information is generalized in the explanation and not all listed formations are visible. The southern half of the Kansas Woodford has almost 0 meter thickness and represents grid extrapolation between the northeast Kansas and Oklahoma extents. Vertical yellow bars are faults from Adler and others (1971). CKU, Central Kansas uplift; Ist, limestone; sh, shale; $U$, upper; L, lower...

8. View to the southeast of elevation on the top of the Desmoinesian layer (Higley and others, 2014, DesmoinesianTft.DAT grid file). Vertical exaggeration is 18 times. Major structures are labeled. CKU is Central Kansas uplift. Precambrian faults (red) are from Adler and others (1971). Wichita Mountain and Amarillo uplift faults are vertical for three-dimensional Earthvision ${ }^{\circledR}$ model purposes only, and presence of the Desmoinesian in this area is unknown because of insufficient data. Data sources for this surface include picks from well logs and edited IHS Energy (2009a, 2009b) well formation tops. Elevation is relative to sea level.

9. Map showing thickness of strata between the Hunton Group and Desmoinesian layers ranges from less than 500 meters over most of the Kansas shelf to more than 5,000 meters in the deep basin of Oklahoma. This wedge of units is oriented northwest-southeast. Precambrian faults (blue lines) and named structures are from Adler and others (1971). The north-south red line is the general location of the figure 10 image. 
10. Cube dissection of the four-dimensional petroleum system model shows present-day elevation on the Desmoinesian layer surface; color variations on the Desmoinesian correspond to lithologies from Adler and others (1971). Vertical color bands represent lithofacies assignments for the Desmoinesian through Precambrian layers. For example, the labeled Mississippian layer is about 900 meters (2,900 feet) thick at the location. Location of the slice is in figure 9. Vertical exaggeration is 15 times.

11. Map showing vitrinite reflectance $\left(R_{0}\right)$ contours for the Woodford Shale; black line contours are in increments of $0.4 \% R_{0}$. Generation stages on $R_{0}$ legend are generalized, as gas generation also occurs within the oil generation range. Black triangles and white dots include $\mathrm{R}_{\mathrm{o}}$ data from Cardott (1989; written commun., 2011), Price (1997), and Mark Pawlewicz (written commun., 2010). Labeled white dot well locations are (1) Bertha Rogers 1, (2) Petree Ranch 1, (3) West Edmond SWD 1-24, and (4) Streeter 1. Brown lines are faults in the underlying Hunton Group (Rottmann, 2000a, pl. 3)

12. Cross section showing the Woodford Shale (WDFD, blue line) and terminus of the Morrow Group (MRRW) and overlying Thirteen Finger Limestone portion of the Atoka Group (ATOKA, gold line) in the deep Anadarko Basin. Line of section is shown in figure 13. Vertical scale is in feet relative to the top of the WDFD. MRMT is Marmaton Group, CHRK is Cherokee Group, MSSP is Mississippian, HNTN is Hunton Group, SLVN is Sylvan Shale, VIOL is Viola Group, and SPRG, CSTR, and MRMC are the respective Mississippian Springer Formation, Chester Group, and Meramec lime. Labeled well log traces are gamma (0 to $200 \mathrm{API}$ ), resistivity, sonic density, neutron density, spontaneous potential, and density

13. Three-dimensional isopach image of the Woodford Shale in the Anadarko Basin. Vitrinite reflectance $\left(\% \mathrm{R}_{0}\right)$ contours (white lines) from figure 11 approximate end of oil generation at $1.2 \% R_{0}$ to overmature for petroleum generation $\left(4 \% R_{0}\right)$. White dot well locations are, from left to right, Bertha Rogers 1, Petree 1, and Streeter 1. Dashed white line is location of figure 12 cross section. Woodford thickness is derived from Rottmann (2000b, pl. 2), analysis of more than 100 well logs, and edited formation tops from IHS Energy (2009a, 2009b). Faults (red) are from Rottmann (2000a, pl. 3). Image is tilted and 1,300 times vertical exaggeration.....

14. Map showing total organic carbon (TOC) content in weight percent (wt \%) for the Woodford Shale based on TOC data from Burruss and Hatch (1989) and mean values from Hester and others (1990). TOC ranges from 0.08 to $14.05 \mathrm{wt} \%$ and averages $3.7 \mathrm{wt} \%$ for core and well log density calculations from 123 wells. There is considerable TOC variation, but almost the entire area that is thermally mature for petroleum generation contains greater than $2 \mathrm{wt} \%$ TOC. Oil and gas generation depletes TOC and HI (hydrogen index), the fuels for petroleum, so original TOC and $\mathrm{HI}$ values would have been greater. Red lines are faults from Adler and others (1971).

15. View to the southeast of elevation on the top of the Thirteen Finger limestone layer (Higley and others, 2014, ThirteenFingerTft.DAT grid file). Vertical exaggeration is 18 times. Thirteen Finger lime extent is approximated by the light blue line. Major structures are labeled. Precambrian faults (red) are from Adler and others (1971). Wichita Mountain uplift faults are vertical for four-dimensional model purposes only, and surface in this area is unknown because of insufficient data. Data sources for this surface include Thirteen Finger limestone picks from well logs and edited IHS Energy $(2009 a, 2009 b)$ formation tops. Elevation is relative to sea level. CKU, Central Kansas uplift 
16. Isopach map of the top of the stratigraphic interval from the Thirteen Finger limestone to the Morrow Formation, based on Andrews (1999a, b), examination of more than 120 well logs, and edited tops from IHS Energy (2009a, 2009b). Morrow Formation faults (green) are modified from Andrews (1999b). Color contour interval is 20 feet and (black) line contours are 10-feet intervals. Figure 17 cross sections are labeled $A-A^{\prime}$ and $B-B^{\prime}$. White polygons are Perryton (Texas) and Knowles Northwest (Oklahoma Panhandle) oil fields...

17. A, Southwest to northeast and $B$, southeast to northwest cross sections of the Thirteen Finger limestone (TRFG) of the Pennsylvanian Atoka Group (ATOKA) in the Anadarko Basin. Lines of section are shown in figure 16. Vertical scale is feet relative to the base of the Thirteen Finger. MRMT is Marmaton Group, CHRK is Cherokee Group, MRRW is Morrow Group, and MSSP is top of the Mississippian. Labeled well log traces are gamma (0 to 200 American Petroleum Institute [API] range), resistivity, sonic density, and neutron density

18. Graphic showing comparison of calculated vitrinite reflectance $\left(\% \mathrm{R}_{0}\right)$ and transformation ratios (TR) using Woodford Shale and Phosphoria kinetic algorithms from Lewan and Ruble (2002), gas generation from coals based on Pepper and Corvi (1995a, 1995b), and dry gas generation using Tsuzuki and others (1999). Calculations are for the Bertha Rogers 1 and Petree Ranch 1 wells shown on figure 11. Woodford hydrous pyrolysis (HP) TR of onset- (0.1percent) to-completion of oil generation (99 percent) correlates to a range of about 0.6 to $1.2 \% R_{0}$; Woodford Rock Eval pyrolysis (REV) TR range is much broader at approximately 0.4 to $2.6 \%$ $\mathrm{R}_{\mathrm{o}^{\prime}}$ and Phosphoria HP TR onset and completion are about 0.4 to $0.8 \% \mathrm{R}_{0}$. Woodford REV TR is at 81 percent when the HP TR has completed at 100 percent. Pepper and Corvi $(1995 a, 1995 b)$ kinetics provides a range of gas generation from 0.55 to $3.0 \%$ $\mathrm{R}_{\mathrm{o}}$ from the Type III kerogen in coals. Basin heating rate of $5^{\circ} \mathrm{C} / \mathrm{m}$.y. was based on the Bertha Rogers 1 well; present-day geothermal gradient is $21.3^{\circ} \mathrm{C} / \mathrm{km}$ to the Woodford ( $19^{\circ} \mathrm{C}$ surface and $200^{\circ} \mathrm{C}$ Woodford temperature at 8,486.5-meter depth) ...27

19. Graph showing depth distribution of wet and dry gas in the Anadarko Basin and of $1 D$ vitrinite reflectance $\left(\% \mathrm{R}_{0}\right)$ profiles for the Bertha Rogers 1 and Petree Ranch 1 wells (fig. 11). Depth range for end of oil generation to start of dry gas generation for the one-dimensional wells is about 3,200 to 5,000 meters. Increase in dry gas fraction and decrease in scatter below 5,000 meters indicates gas is becoming progressively dryer for these samples, with 1.0 being 100 percent methane. Gas data are from the Energy geochemistry data base (U.S. Geological Survey, 2010). The blue symbols are mostly Colorado and Kansas wells. Oklahoma and Texas wells were selected for detailed study because Colorado and Kansas portions of the province are thermally immature for gas generation. Light blue background is a generalized boundary for gas distribution..

20. Burial history curves for the Bertha Rogers 1 and Petree Ranch 1 wells. Modeled vitrinite reflectance (percent Ro) through time includes heat flow of about 68 milliwatts per square meter $\left(\mathrm{mW} / \mathrm{m}^{2}\right)$ to 260 million years ago (Ma) followed by 42 $\mathrm{mW} / \mathrm{m}^{2}$ for Bertha Rogers 1, and $68 \mathrm{~mW} / \mathrm{m}^{2}$ to $260 \mathrm{Ma}$ followed by $52 \mathrm{~mW} / \mathrm{m}^{2}$ for Petree Ranch 1. Pale-yellow and blue lines follow the Thirteen Finger limestone and Woodford Shale, respectively. Gp., Group; Lst., Limestone; Ord., Ordovician; Sil., Silurian, Neog., Neogene 
21. Burial history transformation ratios (TR) for the Bertha Rogers 1 well based on Woodford Shale hydrous pyrolysis (HP) kinetics (Lewan and Ruble, 2002). Depth compared to temperature and vitrinite reflectance profiles are calibrated at (1) 40 milliwatts per square meter $\left(\mathrm{mW} / \mathrm{m}^{2}\right)$ heat flow (HF) through time, and (2) 70 $\mathrm{mW} / \mathrm{m}^{2}$ to 260 million years ago (Ma) followed by $42 \mathrm{~mW} / \mathrm{m}^{2}$ to present. Woodford Shale interval is marked with the blue line and the Thirteen Finger limestone by the gold line. Vitrinite reflectance $\left(\% \mathrm{R}_{0}\right)$ calibration data are from Price (1997). Temperature dots are corrected bottom hole temperatures (BHT) from Gallardo and Blackwell $(1999$, fig. 8) and bars show range from measured to maximum estimated BHT. Gp., Group; Ord., Ordovician; Sil., Silurian, Neog., Neogene ...

22. Burial history for the Bertha Rogers 1 well based on Phosphoria Formation hydrous pyrolysis (HP) kinetics (Lewan and Ruble, 2002). Depths are shown relative to calculated porosity, lithostatic, pore, and hydrostatic pressure; light blue line is hydrostatic pressure of 9,800 Pascals/meter $(\mathrm{MPa} / \mathrm{m})(0.465 \mathrm{psi} / \mathrm{ft})$. Yellow band is probable zone of overpressure based on Al-Shaieb and others (1994). Woodford Shale interval is marked with the blue line and the Thirteen Finger limestone by the gold line. Ord., Ordovician; Sil., Silurian, Neog., Neogene.

23. Burial history for the Petree Ranch 1 well based on Woodford Shale and Phosphoria Formation hydrous pyrolysis (HP) kinetics (Lewan and Ruble, 2002). Model is calibrated using 68 milliwatts per square meter $\left(\mathrm{mW} / \mathrm{m}^{2}\right)$ heat flow to 260 million years before present (Ma), then $52 \mathrm{~mW} / \mathrm{m}^{2}$ to present. Woodford Shale interval is marked on the transformation ratio (TR) charts with the blue line, and the Thirteen Finger limestone by the gold line. Vitrinite reflectance $\left(\% R_{0}\right)$ calibration data are from Pawlewicz (1989) (brown dots) and Woodford Shale estimates from Cardott (1989) (yellow and red dots). Temperature data from Pawlewicz (1989, table 2) (blue dots and connecting line) were calculated based on $1.3^{\circ} \mathrm{F}$ increase per 100 feet depth from a $15^{\circ} \mathrm{C}\left(60^{\circ} \mathrm{F}\right)$ surface temperature. The corrected bottom hole temperature (BHT) (yellow dot) was derived using Gallardo and Blackwell (1999, fig. 7). Ord., Ordovician; Sil., Silurian, Neog., Neogene

24. Graph showing history of generation of oil from the Oil Creek Formation, Woodford Shale, and Thirteen Finger limestone sources in the four-dimensional petroleum system model based on Woodford Shale HP kinetics. Gray bars delineate periods of peak oil generation. Early oil generation from the Oil Creek layer, prior to about 70 million years before present (Ma), is in a narrow band that is proximal to the Wichita Mountain uplift. This band exceeds maximum depth of the formation and is the result of poor data control in the area bordering the fault zone. The error could have been removed by clipping the four-dimensional model extent to the basin axis and northward, but less of the model would have been visible and usable...

25. South to north cross sections of thermal maturation using Woodford transformation ratio (TR) at present $(0 \mathrm{Ma})$ and at 300 million years before present (Ma). The inset shows the location of these extracts from the four-dimensional model. Yellow and pink dots are approximate locations of the Woodford Shale and Thirteen Finger limestone, respectively. Elevation is relative to sea level, and vertical and lateral scales are equivalent for the cross sections. Map image is TR on the Woodford Shale layer at $0 \mathrm{Ma}$ and at 10 times vertical exaggeration. Red line is the approximate location of the cross section. 
26. Images showing present-day vitrinite reflectance $\left(\% R_{0}\right)$ on Ordovician to Virgilian model layers (fig. 2), using Sweeney and Burnham (1990) kinetics. Changes in thermal maturity on the shelf areas of Kansas and Colorado are minor and source rocks are mostly immature for oil generation at less than $0.6 \% \mathrm{R}_{0}$. Thermal maturity for source rocks in the deep basin of Oklahoma and Texas ranges generally from mature for oil generation to overmature for gas generation at about $4 \% R_{0}$ and greater. Red contour lines mark the 99 percent transformation ratio for each layer; it is approximately equivalent to $1.2 \% \mathrm{R}_{0}$. Precambrian faults (Adler and others, 1971) are shown as blue lines.

27. Images showing transformation ratios (TR) through time on tops of the Oil Creek, Woodford Shale, and Thirteen Finger Limestone layers. Ten times vertical exaggeration results in variable shading within contours. Onset of oil generation was about 370 million years ago (Ma) (Oil Creek), $330 \mathrm{Ma}$ (Woodford), and 300 $\mathrm{Ma}$ (Thirteen Finger) based on modeled vitrinite reflectance and 0.1 percent TR. Completion of oil generation is indicated by 99 percent TR. Source rocks are overmature for oil generation at greater than 99 percent TR (medium gray). Precambrian faults (Adler and others, 1971) are shown as blue lines

28. Map and rotated three dimensional images of modeled petroleum migration catchments on the Thirteen Finger limestone layer. Layer boundaries extend across the images but catchments represent greater than zero meter thickness for the Thirteen Finger limestone. Oil and gas flow paths are compartmentalized within each catchment until a change in lithofacies or structure results in petroleum accumulations or loss to bounding formations. The "cluttered" compartments on the west represent more complex flow and are represented on flow path maps as broken lines or groups of rays. Olive green lines and vertical bars are Precambrian faults of Adler and others (1971)

29. Two views of modeled oil (green) and gas (red) flow path lines, and oil (yellow) accumulations for the Thirteen Finger limestone. Layer shown in dark gray on three dimensional map view is the top of the Morrow Group layer. The gray space in the deep basin that is devoid of oil flow paths is the area that is mature for gas generation from the Thirteen Finger limestone. Inset image is a rotated view at 10 times vertical exaggeration

30. Images showing oil migration flow paths from 320 to 40 million years ago (Ma). Blank migration areas can indicate no migration, (invisible) Darcy flow through lithofacies, or overmaturity for petroleum generation. Hunton Group facies from Howery (1993) are delineated by orange lines for limestone (Darcy flow area) and dolomite (flow path migration). Onset of oil generation preceded migration from the Oil Creek Formation layer source rocks (light green), which started about $340 \mathrm{Ma}$. This was followed by Woodford Shale oil generation about $335 \mathrm{Ma}$, then the Thirteen Finger limestone (blue) about $300 \mathrm{Ma}$. Lst, limestone. Maroon lines are Precambrian faults of Adler and others (1971).

31. Image showing present-day oil and gas migration flow paths. Blank migration areas indicate no migration, (invisible) Darcy flow through lithofacies, or overmaturity for petroleum generation. Oil migration flow paths from mostly 0il Creek layer source rocks (light green) are overlain by mostly Woodford Shale (dark green), and then Thirteen Finger limestone (blue) flow paths. Gas (red) flow paths are not differentiated by source rocks. Maroon lines are the fault system from Adler and others (1971) 
32. Images showing modeled gas (red) and oil accumulations at 300 million years before present (Ma) and present day for above named layers. Some major oil and gas fields are outlined and labeled. Brown lines are the Precambrian fault system from Adler and others (1971); the purple lines north of this fault system are modeled locations of the Wichita Mountain uplift at $200 \mathrm{Ma}$ and at present. Accumulations preferentially form on large structures, such as the Cement field, and updip pinchout of reservoir layers against seal layers, such as for the Hunton Group accumulations in northeast Oklahoma. Stratigraphic traps are commonly not filled in this leaky four-dimensional model because of the coarse grid size and lithologic assignments.

\section{Tables}

1. Anadarko Basin Province assessment results are listed by name and code of total petroleum system (TPS) and assessment unit (AU).

2. Summary of kinetic parameters derived for expelled-oil generation based on hydrous pyrolysis (HP) in isothermal experiments, and nonisothermal open-system pyrolysis (REV) that were derived from Lewan and Ruble (2002, tables 4 and 5) and cracking of oil to gas (Tsuzuki and others, 1999). The Woodford Shale analyses were on an unweathered, thermally immature sample that contained 12.7 percent total organic carbon (TOC), from section 25, T. 2 S., R. 1 E., Carter Co., Oklahoma (Lewan, 1983).

3. Onset of oil generation in the Anadarko Basin 4D model, and onset and completion dates in millions of years before present (Ma) for the Bertha Rogers 1 and Petree Ranch 1 one-dimensional models based on Woodford Shale hydrous pyrolysis (HP) kinetics (Lewan and Ruble, 2002). The Douglas Group overlies the youngest potential petroleum source rocks on figure 2. The (HP) kinetics-based onset of oil generation is 0.1 percent transformation ratio (TR) and completion is 99 percent TR. Oil generation histories in the four-dimensional model were recorded at $10 \mathrm{Ma}$ increments, and the plus symbol indicates onset was before the listed age 



\title{
Thermal Maturation of Petroleum Source Rocks in the Anadarko Basin Province, Colorado, Kansas, Oklahoma, and Texas
}

\author{
By Debra K. Higley
}

\section{Abstract}

Petroleum source rocks are thermally mature for oil and gas generation across most of the Anadarko Basin in Oklahoma and Texas based on one-dimensional and fourdimensional petroleum system models. Three petroleum source rocks were defined in the four-dimensional model. They are the Oil Creek Formation of the Ordovician Simpson Group, the Devonian-Mississippian Woodford Shale, and the informal Thirteen Finger limestone of the Pennsylvanian Atoka Group. Modeled onset of oil generation in the basin was about 370 million years ago for the Oil Creek Formation, 330 million years ago for the Woodford Shale, and 300 million years ago for the Thirteen Finger limestone. The deep basin of Oklahoma and Texas is thermally mature for gas from Atokan and older source rocks. Deep basin areas that are overmature for gas generation are within the gas preservation window, as indicated by gas production from Woodford and older formations in this area, and on modeled levels of thermal maturation. Almost all of the Colorado and Kansas portions of the basin are thermally immature for petroleum generation. However, Mississippian and older source rocks may be marginally mature to mature for oil generation in the Las Animas arch of southeastern Colorado based on calculated vitrinite reflectance and transformation ratios from a four-dimensional petroleum system model.

Oil and gas migration pathways were mainly radially northward from the deep basin in Oklahoma and proximal Texas, and north and west from the Texas Panhandle. Petroleum was further funneled by local structures. The Nemaha uplift in central Oklahoma was a barrier that limited eastward migration. Northward flow was diverted toward the Pratt anticline and Central Kansas uplift in south-central Kansas, and to the northwest Hugoton embayment. Decrease in burial pressure associated with the Laramide uplift and erosion resulted in a probable volume increase of gas that displaced oil in reservoirs with subsequent renewed gas migration. Evidence for this includes Permian gas reservoirs of the southern Central Kansas uplift and Pratt anticline areas, and the Hugoton embayment gas fields.

\section{Introduction}

The U.S. Geological Survey (USGS) recently completed an assessment of the undiscovered oil and gas resource potential of the Anadarko Basin Province of western Oklahoma and Kansas, northern Texas, and southeastern Colorado (fig. 1). This is hereafter referred to as the 2011 assessment, which corresponds to the publication release date of the assessment results (Higley and others, 2011). This province area includes the Anadarko Basin, Las Animas arch, and a portion of the Palo Duro Basin. Stratigraphic units range in age from Precambrian to present, and petroleum is produced from Cambrian through Permian strata. Mesozoic strata are absent over most of the basin, being limited primarily to the Colorado and Kansas portions. The Cretaceous Niobrara Formation is productive near the northwestern boundary of the basin, but this biogenic methane resource was included in the Denver Basin assessment (Higley and Cox, 2007; Higley and others, 2007).

Figure 1 shows the province boundary and labeled total petroleum systems (TPS) and assessment units (AU). Total petroleum systems and included AUs are defined in Magoon and Dow (1994). The TPS concept basically incorporates hydrocarbon source rocks (source-rock maturation, hydrocarbon generation, migration, and accumulation), reservoir rocks (sequence stratigraphy and petrophysical properties), and hydrocarbon traps (trap formation, timing, and seals) whose provenance is a pod or closely related pods of active source rock. The TPS is basically a hydrocarbon fluid system that links petroleum source rocks, migration pathways, and real and (or) hypothetical oil and gas accumulations. An AU within a TPS is defined as a mappable volume of strata that incorporates petroleum accumulations (discovered and undiscovered) that have the same hydrocarbon source $\operatorname{rock}(\mathrm{s})$, and similar geologic and economic factors that control oil and (or) gas entrapment, exploration, and development. These accumulations should therefore be sufficiently homogeneous in terms of geology, exploration strategy, and risk so that the chosen method of resource assessment is applicable. A TPS might contain a single or multiple AUs, depending on whether differences are sufficient to warrant separation. Assigning AUs is 


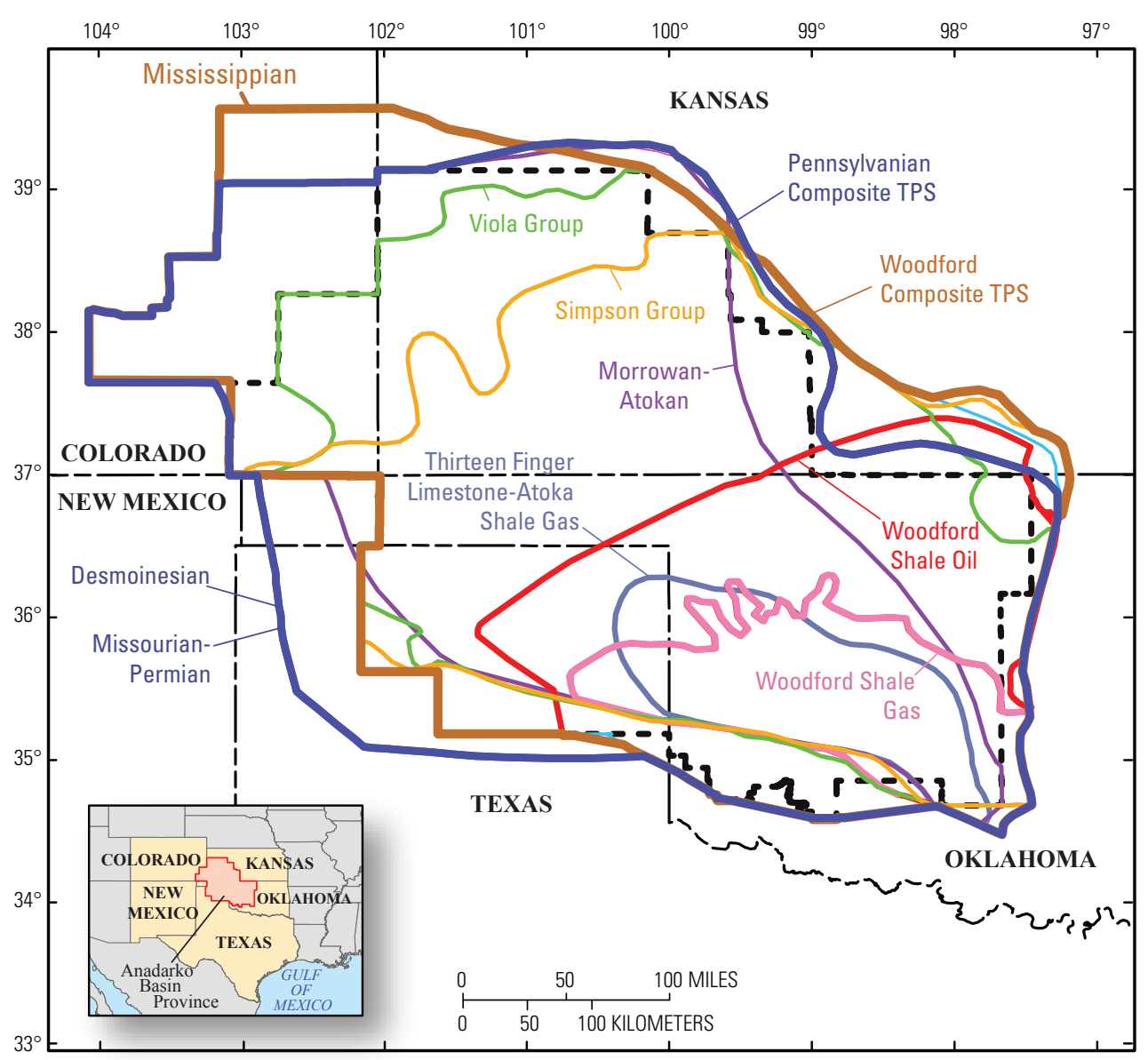

Figure 1. Map showing Anadarko Basin Province (dashed line) and boundaries of total petroleum systems (TPS) and assessment units (AU). Only AUs that contain source rocks are shown. The Woodford Shale is divided into the Woodford Shale Oil and Woodford Shale Gas AUs based mainly on levels of thermal maturation and formation thickness. The Desmoinesian and Missourian-Permian AUs share a common boundary with the Pennsylvanian Composite TPS, with the exception that the AUs exclude an area of the deep basin that is the Granite Wash Composite AU (not shown). There are common boundaries for most AUs.

also based on the quality and availability of petroleum source, reservoir, well history, and production data. Each AU can incorporate several exploration plays that are based on different reservoir formations, trap types, exploration strategies, and discovery histories.

The Anadarko Basin Province includes two TPSs that include nine conventional and three continuous AUs (Higley and others, 2011). The stratigraphic column in the basin (fig. 2) lists Ordovician through Pennsylvanian shales and limestones that are petroleum source and potential source rocks. The Woodford Composite TPS encompasses Ordovician through Mississippian petroleum source rocks and is named for the principal source rock. The Pennsylvanian Composite TPS includes the remaining petroleum source rocks. Reasoning behind this generalized TPS division is that geochemical research in the basin has been focused on the Woodford Shale, with lesser information on other possible petroleum source rocks. Continuous AUs are the Woodford Shale Gas and Woodford Shale Oil of the Woodford Composite TPS, and the Thirteen Finger Limestone-Atoka Shale of the Pennsylvanian Composite TPS. The AU assignments and associated codes are listed below; they are based primarily on (1) the lateral extent and thickness of associated reservoir and petroleum source formations, (2) timing and degree of thermal maturation of potential contributing petroleum source rocks, (3) oil and gas generation and migration pathways through time based on 4D petroleum system modeling of the basin, and (4) faults and other structures that would limit or enhance oil and gas accumulations. Results of the oil, gas, and natural gas liquids (NGL) assessment are listed in table 1. 


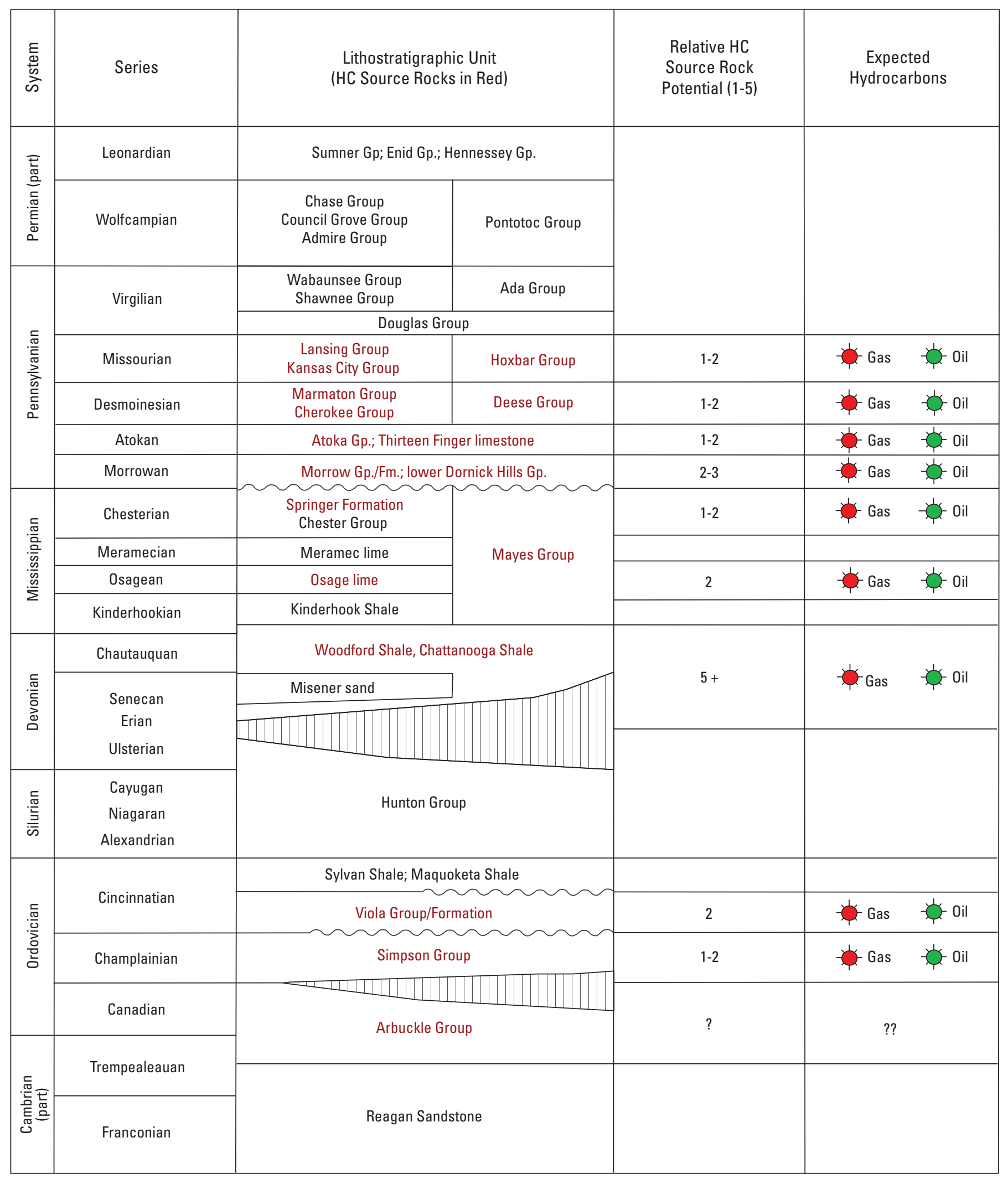

Figure 2. Generalized stratigraphic column for the Anadarko Basin Province with oil and gas source rocks (brown text). Increases in source rock potential are indicated by larger numbers. The expected hydrocarbons header indicates whether the source rock is more oil or gas prone. Hatch (oral commun., 2010), and modified from Burruss and Hatch (1989). Vertical lines show a generalized time range of unconformity from Bebout and others (1993, fig. 5). Gp., Group; Fm., Formation. 
Table 1. Anadarko Basin Province assessment results are listed by name and code of total petroleum system (TPS) and assessment unit (AU).

[Resources are undiscovered oil, gas, and (or) natural gas liquids. MMBO, million barrels of oil; BCFG, billion cubic feet of gas; MMBNGL, million barrels of natural gas liquids. Type refers to mainly oil or gas accumulations in the AU. Fractiles are fully risked estimates. F95 denotes a 95-percent chance of at least the amount tabulated. F50 and F5 fractiles are defined similarly. Fractiles are additive only under the assumption of perfect positive correlation. Gray shading indicates not applicable]

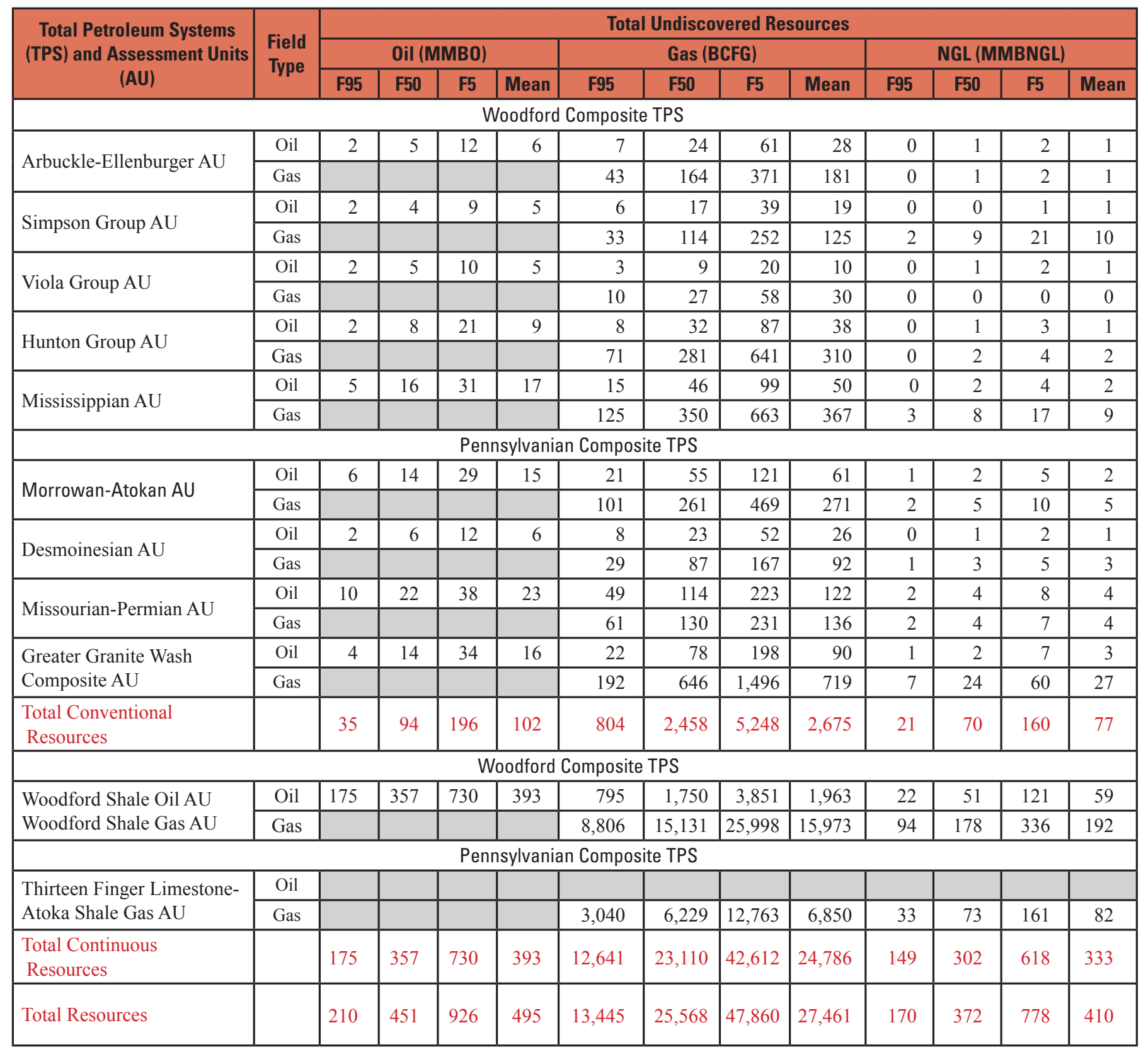


Woodford Composite TPS 505801:

1. Arbuckle-Ellenburger, AU 50580101

2. Simpson Group, AU 50580102

3. Viola Group, AU 50580103

4. Hunton Group, AU 50580104

5. Mississippian, AU 50580105

6. Woodford Shale Gas, AU 50580161

7. Woodford Shale Oil, AU 50580162

Pennsylvanian Composite TPS 505802:

1. Morrowan-Atokan, AU 50580201

2. Desmoinesian, AU 50580202

3. Missourian-Permian, AU 50580203

4. Greater Granite Wash Composite, AU 50580204

5. Thirteen Finger Limestone-Atoka Shale Gas, AU 50580261

Petroleum productive and potentially productive formations were divided into the two TPSs, with Ordovician through Mississippian source rocks mainly contributing to reservoirs of that age range, and Pennsylvanian source rocks to those of Pennsylvanian and Permian age reservoirs (fig. 2). The Hunton Group is not listed in figure 2 as containing petroleum source rocks. Also, there is only limited evidence for source potential from the ArbuckleEllenburger AU (Burruss and Hatch, 1989; J. Hatch, oral communication, 2010). The TPS and AU divisions were also influenced by the accuracy and existence of well history and production data. For example, much of the Atokan petroleum production is reported commingled with Morrowan production. The Thirteen Finger Limestone-Atokan Shale Gas continuous AU is based largely on source rock potential based on Carr and Hentz (2009) and on low permeability and source rock potential from well log analysis. Source rock research and data have been focused on the Woodford Shale in the Anadarko and adjacent basins. Most of the vitrinite reflectance data in the Anadarko Basin is from Woodford Shale cores, well cuttings, and outcrop samples. The onedimensional (1D) and four-dimensional (4D) petroleum system models are preferentially calibrated to the Woodford Shale vitrinite reflectance data of Cardott (1989), Price (1997), and two wells in the Edmond West field (Chesapeake Energy [written communication, 2008] and Mark Pawlewicz [written communication, 2010] analyses). Source rock potential for other petroleum source rocks incorporate published research, vitrinite reflectance values from Pawlewicz (1989), and results of the 1D and 4D models of this study.

\section{Petroleum System Modeling Methodology}

Petroleum system modeling is designed to recreate, through time, oil and gas generation, expulsion, migration, saturation, accumulation, and loss for included petroleum source and reservoir rocks, and at well to basin scales. The timing and extent of petroleum generation from each source rock requires kinetic parameters that relate the geologic time required for generation reactions to the burial temperatures of source rocks. Development and assignment of kinetic algorithms for the Woodford Shale and other petroleum source rocks are discussed in Lewan $(1983,1985)$ and Lewan and Ruble (2002). Created models show levels of thermal maturation and generation of oil and gas through time based on kinetic factors such as transformation ratios (TR) and levels of thermal maturity for source organofacies based on calculated vitrinite reflectance, TR, and other factors. 1D, two-dimensional (2D), and 4D models commonly integrate influences through time of (a) basal heat flow, (b) water depth, (c) surface temperature, (d) time and extent of deposition and erosion, (e) vertical and lateral lithologic properties within each layer, (f) profiles of lithofacies decompaction through time, and (g) geochemical characteristics for each source organofacies, such as total organic carbon (TOC) content and hydrogen index (HI).

Maps and models were generated using Dynamic Graphics ${ }^{\circledR}$ Earthvision ${ }^{\circledR}$ (Dynamic Graphics ${ }^{\circledR}$ and Earthvision ${ }^{\circledR}$ are registered trademarks of Dynamic Graphics, Inc.) and PetroMod ${ }^{\circledR}$ software (Schlumberger, 2011). Schlumberger Pet$\operatorname{roMod}^{\circledR}$ software was used for 1D and 4D petroleum system models. PetroMod ${ }^{\circledR}$ is pressure/volume/temperature (PVT) modeling software. The Anadarko 4D model incorporates flow path and Darcy flow migration that is referred to as hybridDarcy in PetroMod ${ }^{\circledR}$ documentation. Flow path migration of liquid and vapor components of petroleum and water is largely buoyancy-driven by hydrostatic pressure changes, whereas the Darcy flow is a multiphase process that uses gas diffusion and PVT to model migration. Structural relief on the top of the carrier (reservoir) layers is the primary control on migration pathways for oil and gas. Petroleum migration flow paths are vertical and lateral. When fluids and gases enter a reservoir or other permeable layer, the flow through the most permeable lithofacies is toward the top of the layer, and then laterally upward along the overlying seal layer. This seal layer can be breached by its thinning or lateral change to a permeable lithology, or the presence of an open fault system. Flow is then mostly vertical until the next seal is reached. These flow paths are more readily viewed than the more diffuse oil and gas Darcy flow within the reservoir layer. Oil and gas trapped as a result of accumulation or Darcy saturation in reservoirs does not further migration without a change in PVT conditions, such as uplift, erosion, open faults, or change in hydrodynamic flow. Modeling requirements, strengths, and weaknesses are discussed in Higley and others (2006). 
Faults associated with the Wichita Mountain, Amarillo, Nemaha, and Central Kansas (CKU) uplifts are modeled as closed through time (see fig. 3 for locations). Main potential effects of this are to decrease petroleum migration east of the Nemaha uplift and south of the Wichita Mountain and Amarillo uplifts, and to channel migration in areas such as the Pratt anticline and CKU. Faults along the northern boundaries of the Wichita Mountain and Amarillo uplifts are modeled as vertical, with only minimal horizontal offset from Precambrian to ground surface. Also critical to migration and accumulation is the proximity of reservoir strata to vertical and lateral seals and to thermally mature source rock.

Petroleum system models were constructed using formation tops, lithologies, and ages of deposition and erosion from numerous sources. Data sources for the models include (1) formation top picks in more than 220 wells across the province, (2) IHS Energy (2009a, 2009b) and Kansas Geological Survey (2010, http://www.kgs.ku.edu/PRS/petroDB.html) formation tops, and (3) maps and data from Fay (1964), Rascoe and Hyne (1987), Robbins and Keller (1992), Cederstrand, and Becker (1998), Andrews (1999a, 1999b, 2001), and Rottmann (2000a, 2000b). Formation ages and lithologies are commonly generalized and include Denison and others (1984), Ludvigson and others (2009), and the National Geologic Map Database (2011, http://ngmdb.usgs.gov/Geolex/). Figures within this chapter of the report may have feet or meters as vertical scales. This is because the $1 \mathrm{D}, 2 \mathrm{D}$, and $4 \mathrm{D}$ petroleum system models have meters as the vertical scale, largely because of ease in using the modeling software. Vertical scale of most contour maps and of well-log cross sections are in feet because that is traditionally used in the United States. Tilted three-dimensional (3D) images generally do not have map scales because edges would have different scales, with the associated clutter.

4D petroleum system models consist of stacked layers of formations and groups of formations. The Anadarko Basin model contains 30 layers. Below are names of modeled layers that correspond generally to associated formation, group, and period names.
1. Surface
16. Atokan
2. OgallalaBase
17. Thirteen Finger
3. Cretaceous
18. MorrowU
4. Permian
19. MorrowM
5. Blaine
20. MorrowL
6. StoneCorral
21. Springer
7. Wellington
22. Woodford
8. Chase
23. Hunton
9. CouncilGrove
24. Sylvan
10. Wabaunsee
25. Viola
11. HeebnerT
26. SimpsonT

12. HeebnerB

27. Oil Creek

13. Douglas

14. Desmoinesian

15. Cherokee

28. SimpsonB

29. Arbuckle

30. Precambrian

Formations that compose each layer were grouped based on common petroleum system elements of reservoir, source, seal, overburden, or underburden. Assigned petroleum source rocks in the model are the Oil Creek, Woodford, and Thirteen Finger layers; these correspond generally to the petroleum source rocks in figure 2. All model layers extend across the study area, even if the included formation(s) has limited lateral extent. This is because of a software requirement that all grid cells be populated with numbers representing the structural elevation relative to sea level, or isopach thickness of zero or greater. The structural surface of layers is somewhat like a geologic map in that the extent of the modeled formation is shown, as well as any lateral subcropping formations. Some layers consist of one formation, such as for the Sylvan Shale, Hunton Group, Woodford Shale, Chase Group, and Blaine Formation, and others are compound, such as the Desmoinesian and Missourian layers. Selection of layer intervals was based mainly on the quality of formation tops data and their relative importance as source, reservoir, and seal units.

$\mathrm{X}$ and $\mathrm{Y}$ axis grid spacing are 6 kilometers $(\mathrm{km})$ for the 4D basin models, primarily because of computer processing and display limitations. File sizes of these "simplified" 4D models can exceed 170 gigabytes. The coarse gridding results in somewhat pixilated graphic images. Spacing is 1 kilometer $(\mathrm{km})$ for published grids (Higley and others, 2014) and as initial "pre-filtered" input of the 4D models. Intervals with their associated lithologic properties are homogenized at this 6-km scale. A primary result of this homogenization is that most generated oil and gas is not trapped within but instead migrates outside the model; this lost petroleum is mainly along the northern extension of the model in Colorado and Kansas. Models for stratigraphic traps and those constrained by PVT history require more detailed lithologic assignments and closer grid spacing.

A heat flow map (fig. 3) was assigned as basement heat flow in the 4D model. Variation in measured heat flow is probably greater than that shown, as is indicated by "bull's eye" contours in areas of closer data control. There are areas in western Kansas that display minor change in heat flow; these result from fewer data points, and perhaps less variation than other map areas. Data sources for downhole temperature include Carter and others (1998), Gallardo and Blackwell (1999), Price (1997), and drillstem test and corrected borehole temperatures. These well data were used to calibrate 1D models, and were also compared to 4D model temperatures for further calibration. Shown on figures 4 and 5 are present-day modeled and measured temperatures on the Devonian-Mississippian Woodford and the Virgilian Wabaunsee layers. Model layers and datasets show trends of increasing temperature with burial and similar temperature values. Woodford layer contours show the 


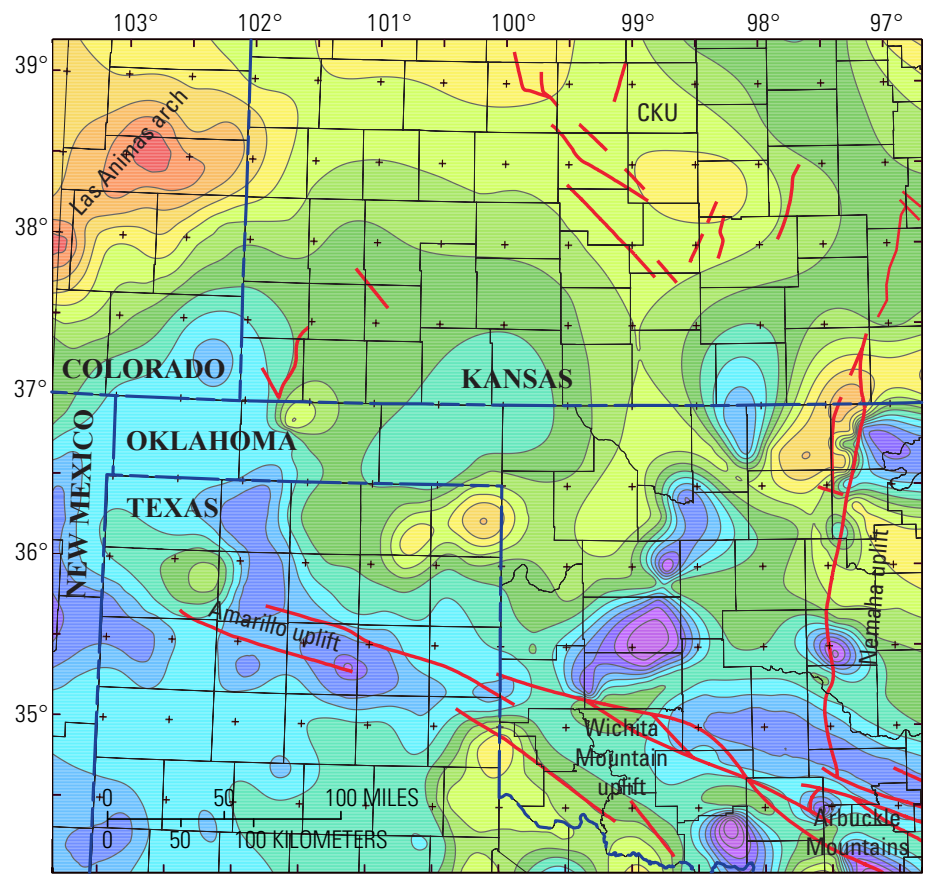

EXPLANATION

Heat Flow $\left(\mathrm{mW} / \mathrm{m}^{2}\right)$

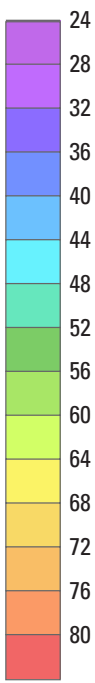

Figure 3. Map showing basement heat flow contours across the Anadarko Basin based on data from Carter and others (1998), Blackwell and Richards (2004), and data downloads from the Southern Methodist University Web site (http://smu.edu/geothermal/. Basin areas within and north of the Wichita Mountain and Amarillo uplifts exhibit generally lower heat flows than other basin areas. Highest measured heat flow is in the northwest, along the Las Animas arch. The northwest trending Central Kansas uplift (CKU) also exhibits elevated heat flow values. Contours are in milliwatts per square meter $\left(\mathrm{mW} / \mathrm{m}^{2}\right)$. Red fault lines are from Adler and others (1971).
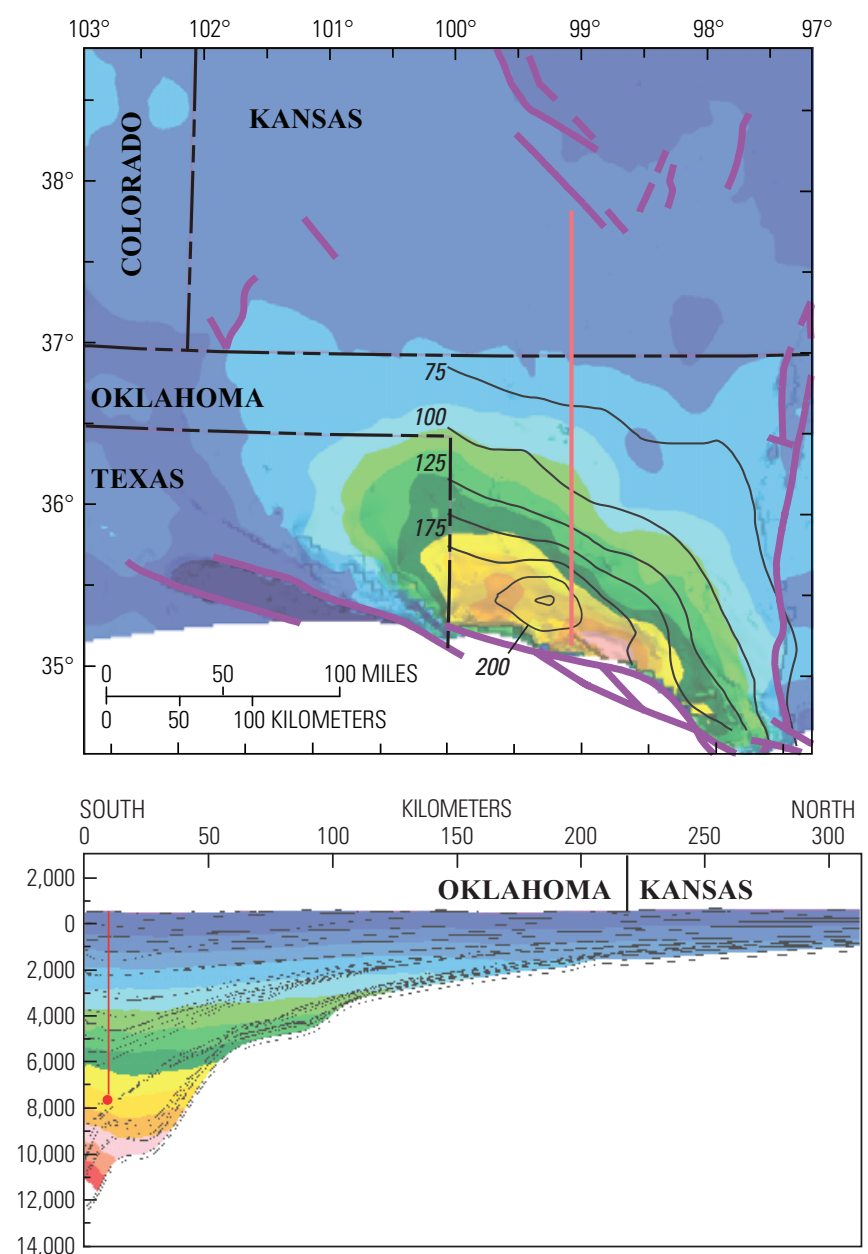

Figure 4. Map showing formation temperatures on the top of the Woodford Shale model layer. Cross section location is orange line on map. Black contour lines are temperature on the Woodford Shale from Gallardo and Blackwell (1999). Formation temperatures on the cross section are from ground surface to the Precambrian. The red dot is location of the Woodford Shale layer. Elevation in meters is relative to sea level. Purple fault lines are from Adler and others (1971).

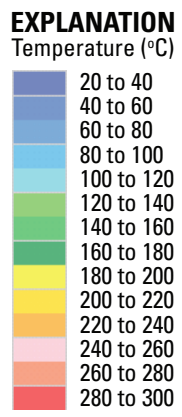




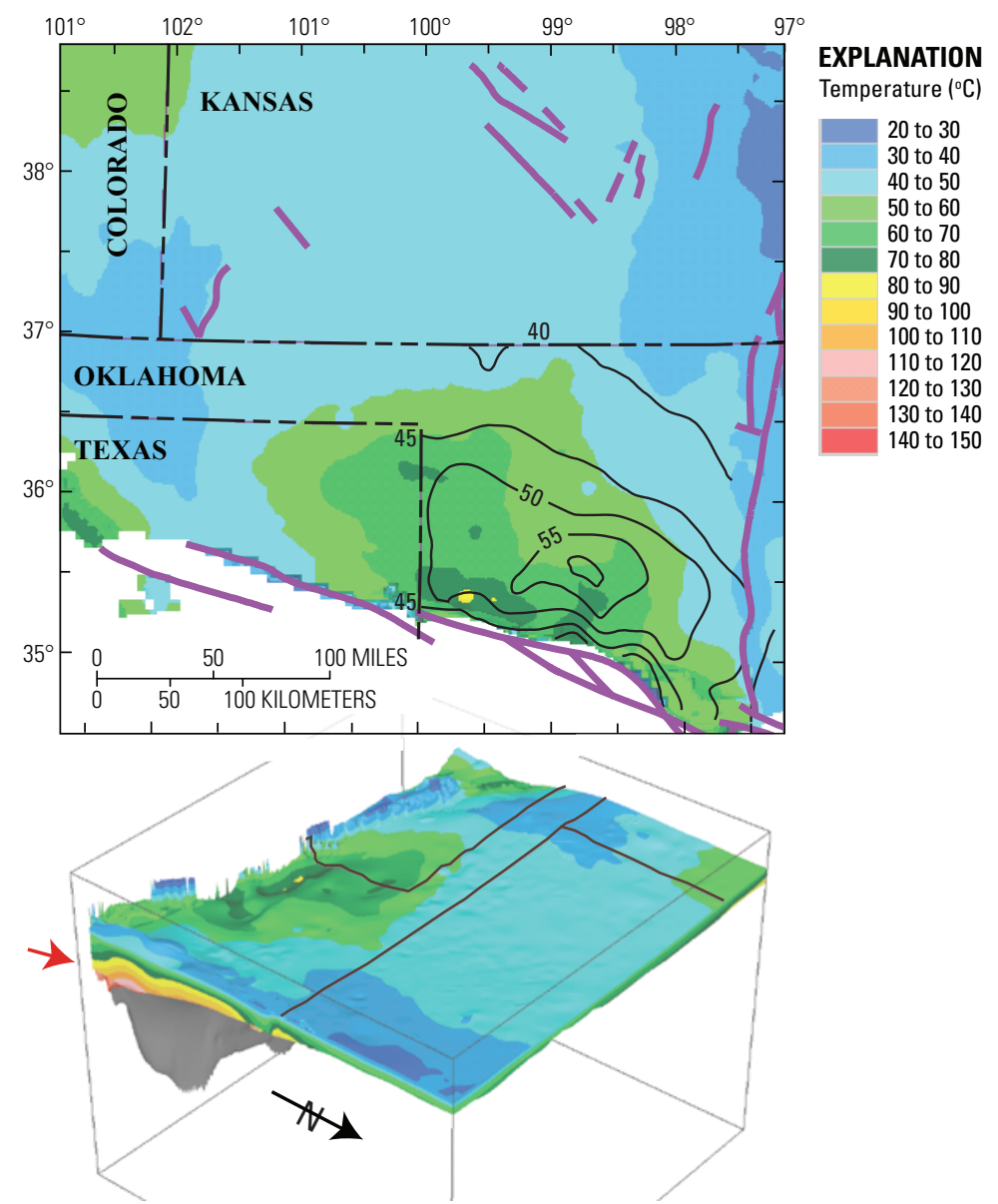

Figure 5. Map showing modeled Celsius $\left({ }^{\circ} \mathrm{C}\right)$ temperatures on the top of the Wabaunsee layer. Oklahoma (black) contour lines from Gallardo and Blackwell (1999) are estimated temperatures on the top of the Pennsylvanian, which is roughly analogous to the Wabaunsee elevation. Both sets of contours show general increase in temperature with greater burial depth. Increasing depth with burial is shown by the underlying Precambrian through Morrowan layers (red arrow). 3D view is 10 times vertical exaggeration. Purple fault lines are from Adler and others (1971). 
same northwest trend and similar values to those of Gallardo and Blackwell (1999). This indicates that temperatures for the 4D model compare generally with measured data. Subsurface temperatures are influenced by various factors. The Precambrian in the deep basin is primarily rhyolite, which is cooler than the mainly granitic rocks of the shallower basement and shelf regions. The rhyolite could influence thermal maturation by dampening thermal effects, essentially slowing onset of generation from overlying rocks. The Carter and others (1998) measured heat flow in the basin decreased from 55 to 64 milliwatts per square meter $\left(\mathrm{mW} / \mathrm{m}^{2}\right)$ in the northern part, to 39 to $54 \mathrm{~mW} / \mathrm{m}^{2}$ in the southern part. Lee and Deming (1999) incorporated this information into their mean heat flows in the northern and southern basin of $39 \mathrm{~mW} / \mathrm{m}^{2}$ and $51 \mathrm{~mW} / \mathrm{m}^{2}$, respectively. They found no evidence that basement lithology influenced the increased heat flow from the south to north. Their work was based on thermal conductivity measurements of drill cuttings from nine wells in the basin. These and other heat flow data were contoured (fig. 3) and applied to the 4D model. Basement heat flow exhibits a heterogeneous distribution (fig. 3) with somewhat lower values in the southern part of the basin and greater heat flow across parts of the Las Animas arch, Central Kansas uplift, and parts of the Nemaha uplift. Lithofacies that overlie source intervals can also influence onset of maturation. For example, evaporites have good thermal conductivity that allows heat conduction away from kitchen areas, whereas thick shale and coal beds have the opposite effect, acting as thermal blankets that insulate underlying rocks. Minor differences in assigned lithofacies have no effect on modeling results.

\section{Geologic Setting}

\section{Structural History}

The Anadarko Basin is a south-dipping asymmetric basin in which the present configuration is controlled mainly by the late Paleozoic Wichita Mountain uplift and the Ouachita thrust plate (Amsden, 1975, 1989). Southern Oklahoma was initially described by Shatski (1946) as a failed Cambrian aulacogen. The deepest part of the basin is along and north of this rift zone and is proximal to its southern margin along the Wichita Mountain uplift (fig. 6). The tectonic history of the Anadarko Basin strongly influenced rate of sediment deposition, sediment sources, depositional environments, erosion, and especially burial depth of reservoir, seal, and source rocks. Depth of burial through time is the main control on thermal maturation of petroleum source rocks, although oil and gas generation is also influenced by factors such as basement heat flow and thermal conductivity of lithofacies. The focus of this is on oil and gas generation, migration and accumulation through time from source rocks in the basin.

The Wichita Mountain and Amarillo uplifts and adjacent basins were strongly influenced by structural, stratigraphic, and thermal evolution of the southern Oklahoma aulacogen
(Rascoe and Adler, 1983) that was emplaced on the Precambrian craton (Ham and others, 1964). It is about 100 miles (mi) [160 kilometers $(\mathrm{km})]$ wide and $300 \mathrm{mi}(480 \mathrm{~km})$ long with the southern boundary approximated by the Wichita Mountain uplift (fig. 6). The Wichita Mountain and Amarillo uplifts include a number of faults, such as Mountain View, Cordell, Cement, Meers, and Duncan-Criner (Luza, 1989) that are along and proximal to the northern boundary of the uplifts,

Early and Middle Cambrian rifting and igneous activity was the initial phase in basin development (Denison, 1976; Gallardo and Blackwell, 1999). Increased subsidence in the aulacogen during the Early Cambrian resulted in a greater rate of deposition near the present-day basin axis; the subsidence rate slowed by the beginning of the Silurian (Feinstein, 1981). This is indicated by the relatively thin Silurian and Devonian rocks (Johnson and others, 1988; Johnson, 1989) that are of limited lateral extent. Figure 7 shows generalized lateral extent of the Woodford Shale within the Woodford Shale model layer. Model layers are somewhat like geologic maps through time in that they display the extent of the named formation, and "subcropping" lateral formations. All formations on the surface of figure 7 are assigned to the Woodford layer. The Viola layer, for example, would show the same assigned lithofacies of the Viola Group and any underlying formations outside its extent.

The Ouachita-Marathon orogeny and related tectonism began in Mississippian (probably Chesterian) time (Burgess, 1976; Perry, 1989). Anadarko began to form as an independent structural basin in Late Mississippian time, when the Texas promontory of the southern continental margin of Paleozoic North America first reacted to early stages of plate collision with Gondwana or an intervening microplate (Perry, 1989). During Early to Late Pennsylvanian time the principal tectonic activity in the basin occurred in several pulses: (1) the Morrowan Wichita Mountain and Amarillo uplifts were accompanied by downwarping of the basin (Gallardo and Blackwell, 1999) with greater thickness of Morrowan strata in the deep basin relative to the shelf; and (2) Late Morrowan uplift along most of the Wichita Mountain and Amarillo blocks is evidenced by the upper Morrowan fan-delta chert conglomerate (Johnson, 1989). These were derived from Mississippian cherty limestones and dolomites that mantled the uplift (Johnson, 1989).

Climax of the Wichita orogeny during the Atokan was evidenced by Wichita Mountain and Amarillo uplift of thousands of feet relative to the proximal basin axis to north (Ball and others, 1991). Overthrusting associated with the uplift resulted in $15 \pm 5 \mathrm{~km}(9 \pm 3 \mathrm{mi})$ of crustal shortening (Brewer and others, 1983). The uplift supplied sediments to the informally named Granite Wash, which was deposited from Atokan through much of the Virgilian time. Transition from arkosic granite wash to felsic quartz grains extended some 30 miles northward from the mountain front during the Atokan (Ball and others, 1991). The Wichita Mountain and Amarillo uplifts persisted as major positive tectonic features during Missourian time (Johnson, 1989). The Anadarko Basin has 


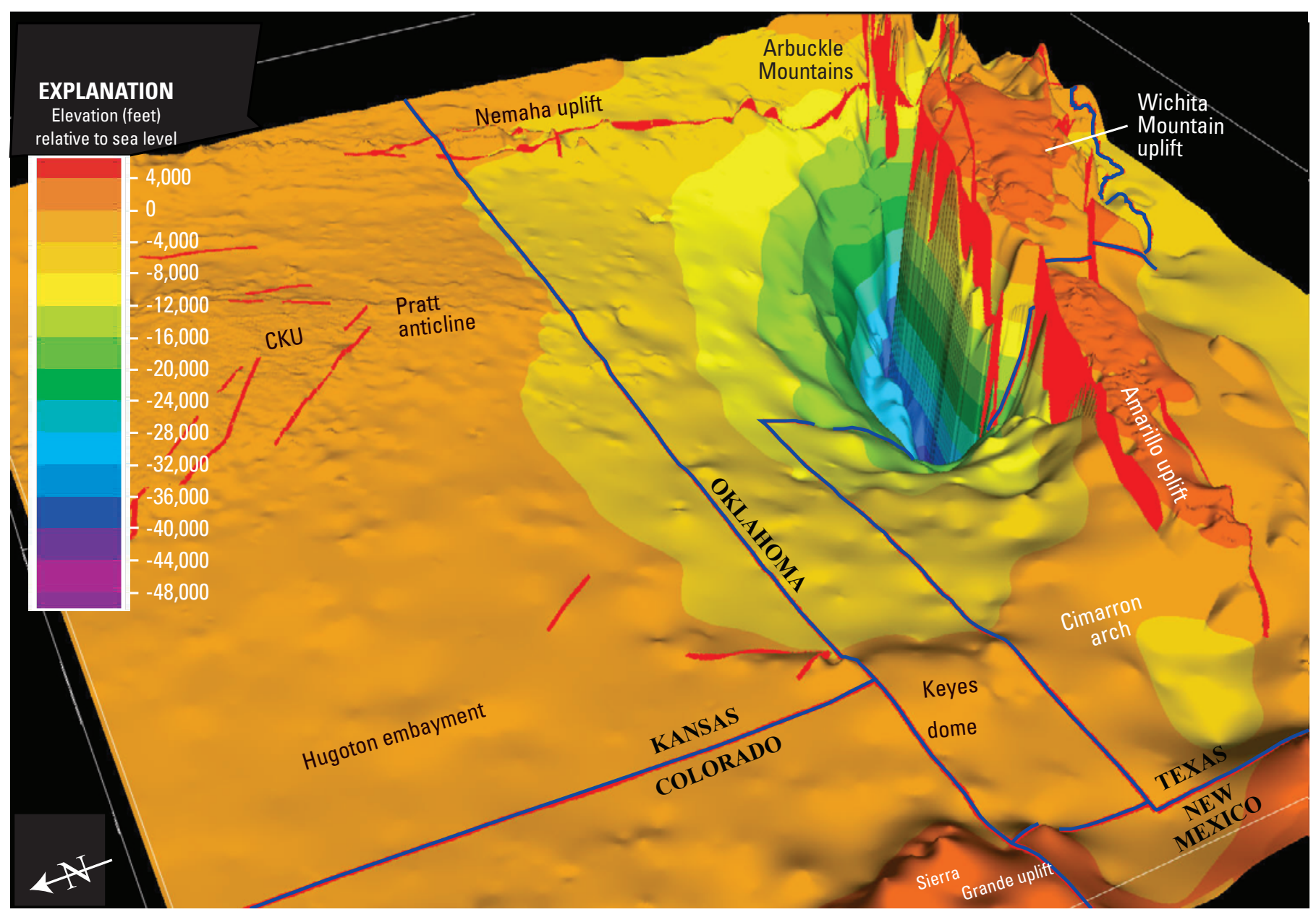

Figure 6. View to the southeast of elevation on the top of the Woodford Shale model layer is from the two-dimensional WoodfordTft.DAT grid file (Higley and others, 2014) that was created using Earthvision ${ }^{\circledR}$. Vertical exaggeration is 18 times. Woodford extent is approximated by the -12,000-foot elevation contour, although Woodford is also present east of the Central Kansas uplift (CKU). Major structures are labeled. Precambrian faults (red) are from Adler and others (1971). Wichita Mountain uplift bounding faults are vertical for fourdimensional model purposes only, and the Woodford Shale surface in this area is unknown because of insufficient data. Data sources for this surface include Woodford picks from well logs, Rottmann (2000a, 2000b), and edited IHS Energy (2009a, 2009b) formation tops. 


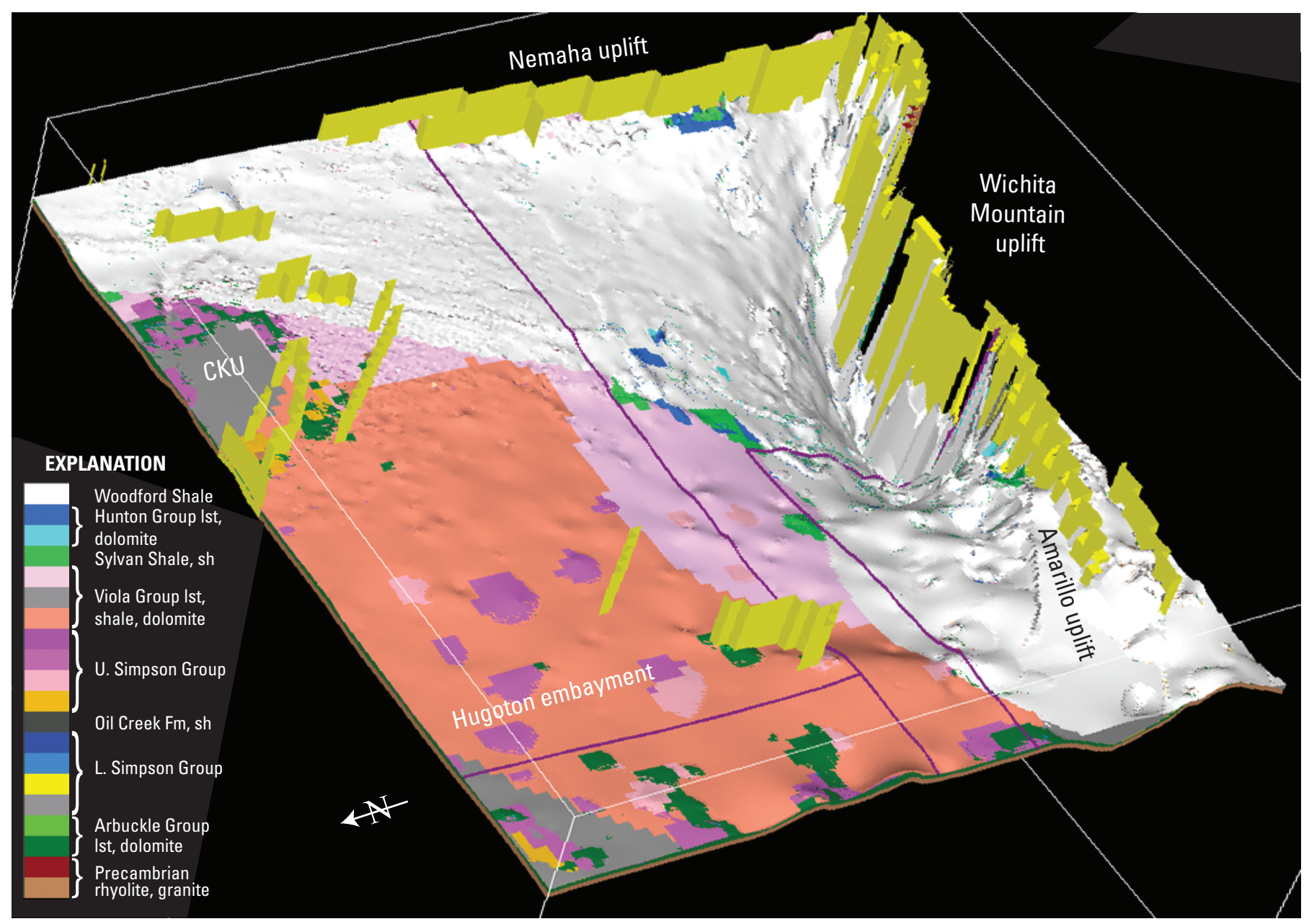

Figure 7. View to the southeast showing Woodford Shale layer lithofacies. Vertical exaggeration is 18 times. View orientation is similar to the 1-kilometer grid spacing Earthvision® image (fig. 6). This PetroMod® image shows underlying and lateral facies changes for the Woodford Shale layer, which is shown in white. Lateral lithofacies within the Woodford layer are primarily limestone and dolomite of the Viola Group. Because the purpose of this image is to show lateral changes in lithofacies assignments on a model layer, this information is generalized in the explanation and not all listed formations are visible. The southern half of the Kansas Woodford has almost 0 meter thickness and represents grid extrapolation between the northeast Kansas and Oklahoma extents. Vertical yellow bars are faults from Adler and others (1971). CKU, Central Kansas uplift; Ist, limestone; sh, shale; U, upper; L, lower. 
essentially been structurally dormant since Early Permian time (Perry, 1989), as indicated by relatively uniform thicknesses of Wolfcampian and Leonardian formations, and that Council Grove, Chase, and Hennessey Groups, and other strata can be fairly readily correlated across the basin. Guadalupian and younger units exhibit more irregular distribution because of nondeposition and uplift, tilting, and erosion associated with the Laramide orogeny. The Laramide orogeny in the southern Rocky Mountains began about 67.5 million years before present (Ma) and ended about $50 \mathrm{Ma}$ (Tweto, 1975). Johnson and others (1988) indicated that southern Oklahoma has been structurally quiescent since the Permian, except for the Laramide orogeny causing slight eastward tilting. Subdued modest reactivation of older basin faults may have occurred during the Jurassic and Cretaceous, and minor Holocene movement has occurred on the Meers fault of the Wichita Mountain uplift (Crone and Luza, 1986), and possibly other faults in the basin (Perry, 1989).

Present-day surface expression of the Anadarko Basin is broad and low-relief with elevation decrease and drainage from west to east. This is in contrast to subsurface relief of Wolfcampian and older strata, which form a broad shelf over the Kansas and eastern Colorado portions of the basin that deepens gradually southward into Oklahoma and Texas. Northern Oklahoma and northeastern Texas evidence a marked increase in slope as the basin radially deepens southward to the Wichita Mountain uplift and its bounding high-angle reverse faults. Figures 6 and 7 are views to the southeast on the Woodford Shale layer. These and other three-dimensional (3D) structural images extend to the edges of the model, rather than terminating at the limits of contained formations. The slope of the Woodford surface increases gradually from the shelf southward to the basin axis; then slopes steeply upward toward the Wichita Mountain uplift and bounding faults. This slope increase is mirrored by increased thickness of most Pennsylvanian and older formations. The deep basin gradually filled with sediments through time. Figure 8 shows elevation on the Desmoinesian layer. Desmoinesian strata covered most of the Anadarko Basin, but is absent across most of the Wichita Mountain uplift, Central Kansas uplift, the Cimarron arch of northeastern New Mexico, and the Sierra Grande uplift of northeastern New Mexico and southeastern Colorado.

The figure 9 isopach of strata between the top of the Hunton Group and the Desmoinesian layers shows southward increase in thickness. The absence of contours just north of the Wichita Mountain uplift is because of variable thicknesses of layers next to the uplift and associated terminations of layers against fault segments. Greatest thickness of strata is along the basin axis, proximal and north of the Wichita Mountain uplift. Thickness changes in southeastern Colorado are associated with the Las Animas arch, primarily a Laramide feature that also experienced movement during the late Paleozoic (Rascoe, 1978). The pronounced thickening of strata in the deep basin is also shown by the cube slice of Precambrian through Desmoinesian layers from the 4D petroleum system model in figure 10. It shows the same break in slope southward that results in the wedge of sedimentation. The Atokan through Desmoinesian strata generalized lithofacies assignments were from Adler and others (1971) and are displayed as the gray, green and blue colors on the top slice of the model (fig. 10). Each color change across the vertical cut corresponds to a unique lithofacies assignment. Some of these extend across layers, such as for the Atokan through Desmoinesian layers. The result of this homogenization is a fairly leaky petroleum system model. For example, the Mississippian layer is about 900 meters (m) thick at the marked location, when corrected to vertical. The lithologic assignment (modified from Adler and others, 1971) for the light green bands in this layer is about 40 percent limestone, 30 percent sandstone, and 30 percent shale, and the intervening dark green band is 60 percent limestone, 20 percent sandstone, and 20 percent shale. Unless these assigned lithofacies truncate against or form a structure under a low-permeability seal layer, oil and gas is not trapped.

Model layers can be modified to better represent the vertical and lateral lithofacies variation in the basin, but this was beyond the scope of this project. Lithofacies for most layers were derived from the Adler and others (1971) generalized clastic and carbonate assignments. An overpressured zone in the basin that persisted through geologic time could not be modeled, at least partly because of the coarse 6 to $9 \mathrm{~km}$ grid sizes of the 4D model and the generalized lithofacies assignments. Most generated petroleum for the Pennsylvanian through Permian strata migrated outside the model edges. The Al-Shaieb and others (1994) megacompartment complex (MCC) is composed of Devonian, Mississippian, and Pennsylvanian stratigraphic intervals and is characterized by overpressured conditions in the Anadarko Basin that exceed the normal gradient of 10.515 kilopascal per meter $(\mathrm{kPa} / \mathrm{m})[0.465$ pounds per square inch per foot $(\mathrm{psi} / \mathrm{ft})]$. The isolation of the MCC is maintained through considerably long geologic times (early Missourian to present) via encasement by an intricately complex framework of top, basal, and lateral seals (Al-Shaieb and others, 1990).

Primary effect of the wedge of overburden is that thermal maturation of source rocks increases to the south. The primary kitchen area in the Anadarko Basin is in the Oklahoma and northeastern Texas portions (fig. 11). Thermal maturation ranges from onset to completion of oil generation from about 0.6 percent vitrinite reflectance $\left(R_{o}\right)$ to 1.2 percent $R_{o}$, and overmature for gas generation at greater than 4 percent $\mathrm{R}_{\mathrm{o}}$. Increased slope of $\mathrm{R}_{\mathrm{o}}$ contours is mirrored by depth of the Woodford Shale and thickness of overburden. The general northwest trend of $\mathrm{R}_{\mathrm{o}}$ contours is similar to those of overburden thickness. The Cardott (1989) least squares regression correlation was 0.893 for 80 of the Woodford Shale $\mathrm{R}_{\mathrm{o}}$ values compared to depth. Spread in $\mathrm{R}_{\mathrm{o}}$ correlations can be influenced by (1) initial transformation of organic matter to vitrinite; (2) random experimental error; (3) interpretation of low-gray (low reflectance) vitrinite populations; (4) anisotropy of vitrinite-broadening reflectance histograms starting at 1.0 percent $R_{0}$, with a noticeable effect at greater than 2 percent $R_{0}$ (Dow, 1977; Hunt, 1979); and (5) suppression of $R_{0}$ in alginite-rich 


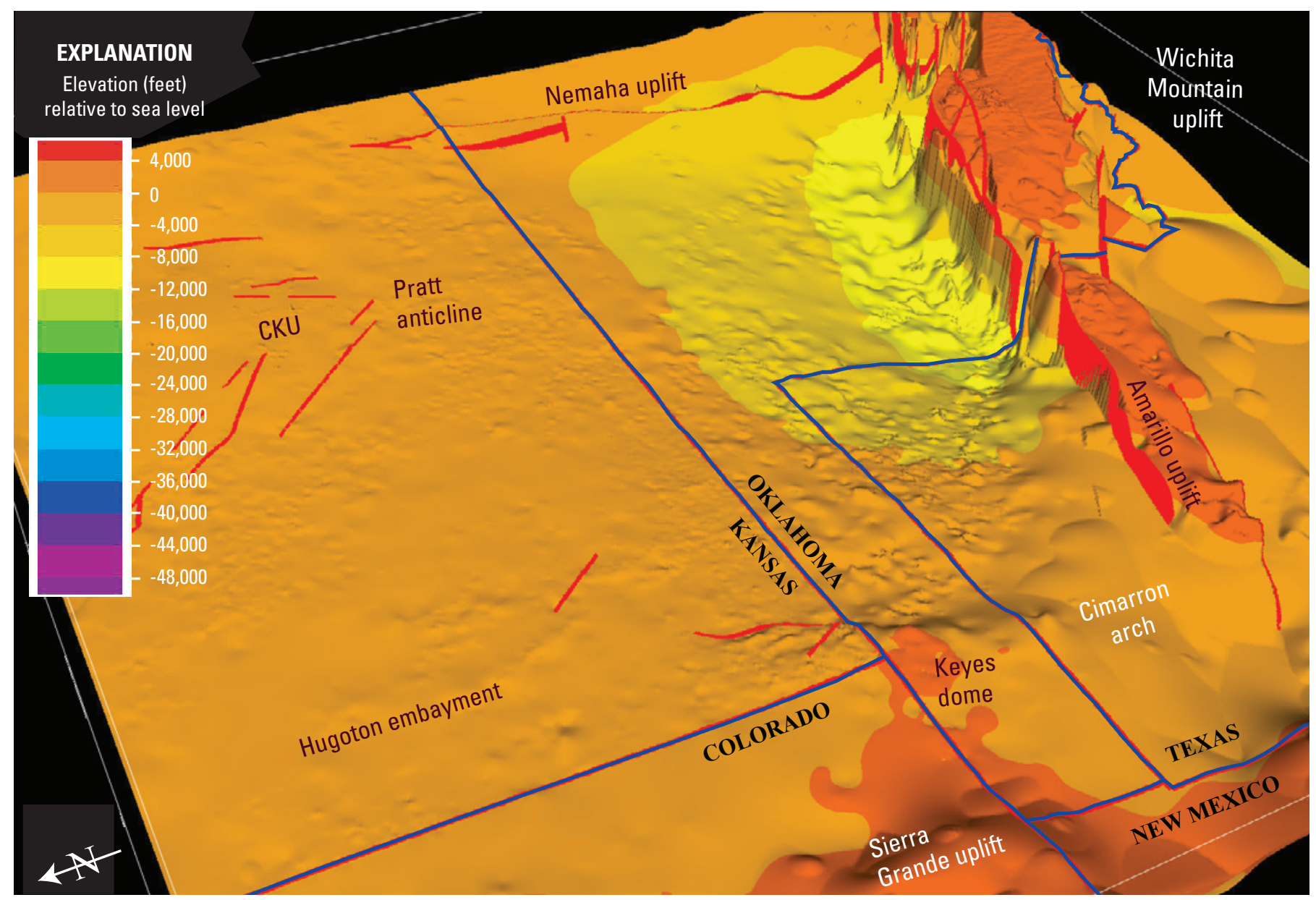

Figure 8. View to the southeast of elevation on the top of the Desmoinesian layer (Higley and others, 2014, DesmoinesianTft.DAT grid file). Vertical exaggeration is 18 times. Major structures are labeled. CKU is Central Kansas uplift. Precambrian faults (red) are from Adler and others (1971). Wichita Mountain and Amarillo uplift faults are vertical for three-dimensional Earthvision ${ }^{\circledR}$ model purposes only, and presence of the Desmoinesian in this area is unknown because of insufficient data. Data sources for this surface include picks from well logs and edited IHS Energy (2009a, 2009b) well formation tops. Elevation is relative to sea level.

rocks (Cardott, 1989; p. 41), which may be a factor in the Type II kerogen within the Woodford Shale.

Part of the area proximal to the Nemaha uplift (eastern part of fig. 11) is thermally immature for oil generation from the Woodford Shale based on Cardott (1989), but immature to mature based on $\mathrm{R}_{\mathrm{o}}$ measurements for the West Edmond SWD 1-24 (0.55 percent mean $\left.\mathrm{R}_{\mathrm{o}}\right)$ and Streeter 1 ( 0.75 percent mean $\mathrm{R}_{\mathrm{o}}$ ) wells from Brian Cardott (written commun., 2011) and Mark Pawlewicz (written commun., 2010), respectively (fig. 11). The West Edmond SWD 1-24 sample was also analyzed by Chesapeake Energy (on January 10, 2008) with an estimated mean of 0.83 percent $R_{o}$ and a range of 0.44 to 0.96 percent $\mathrm{R}_{\mathrm{o}}$ based on Rock Eval Tmax values for 19 samples of the Woodford Shale interval. Gaswirth and Higley (2014) calibrated 1D petroleum system models of these wells using the measured $\mathrm{R}_{\mathrm{o}}$ data. The Streeter 1 Woodford Shale sample was located just above the Hunton cored interval, and sample quality was marginal. It was analyzed using Rock Eval pyrolysis with associated TOC of 0.96 weight percent (wt $\%$ ), Tmax of $550{ }^{\circ} \mathrm{C}$, and low hydrocarbon and oxygen indices of 68 and 39, respectively. These wells may also record localized elevated thermal maturation. Possible increased thermal maturation along the Nemaha uplift could be associated with hydrothermal fluids along these deep faults, and increased erosion compared to wells to the west also contributed to greater thermal maturation.

Fractures are present within the Woodford Shale throughout its extent in Oklahoma and Arkansas, and province areas in which fractures are most commonly filled with bitumen include the Arbuckle Mountains and the southern part of the Anadarko Basin; these were areas of intense late Paleozoic orogenic activity (Comer and Hinch, 1987). Fractures filled with bitumen were located in Woodford samples along the Nemaha uplift in central Oklahoma, and in the southern Anadarko Basin 


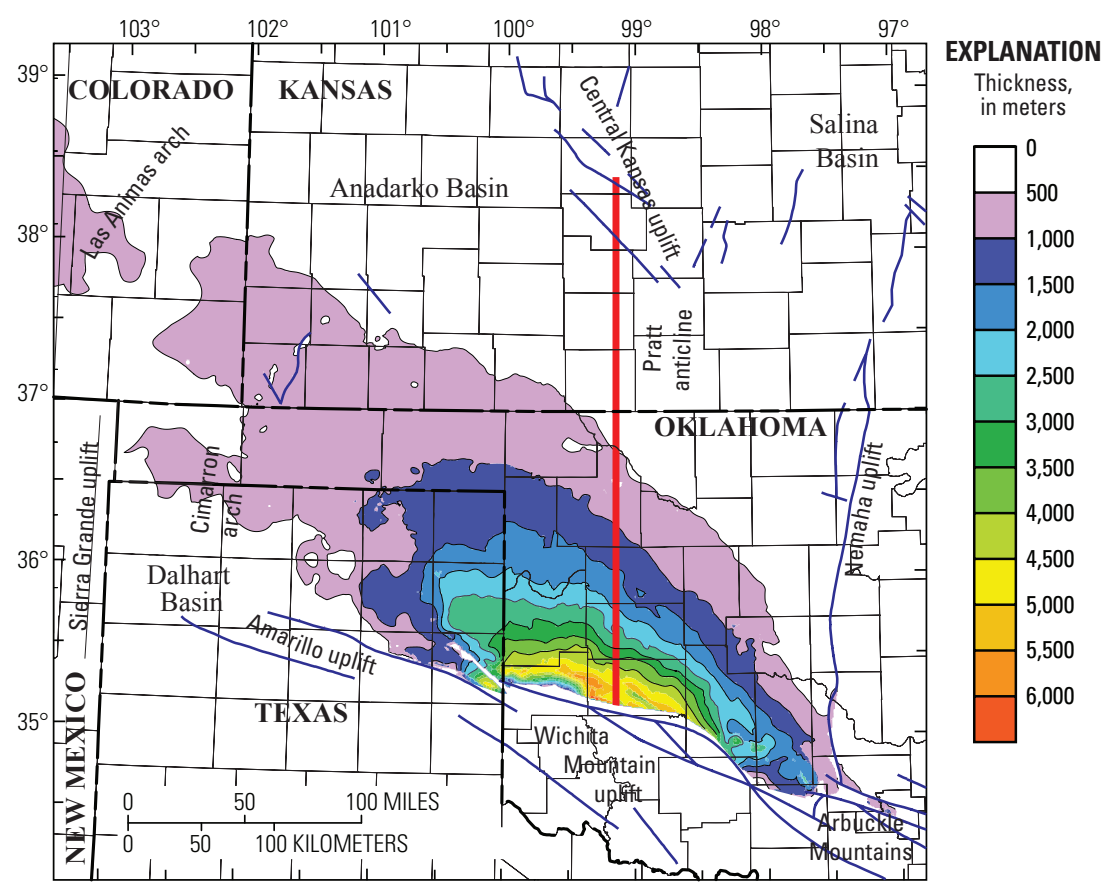

Figure 9. Map showing thickness of strata between the Hunton Group and Desmoinesian layers ranges from less than 500 meters over most of the Kansas shelf to more than 5,000 meters in the deep basin of Oklahoma. This wedge of units is oriented northwest-southeast. Precambrian faults (blue lines) and named structures are from Adler and others (1971). The north-south red line is the general location of the figure 10 image.

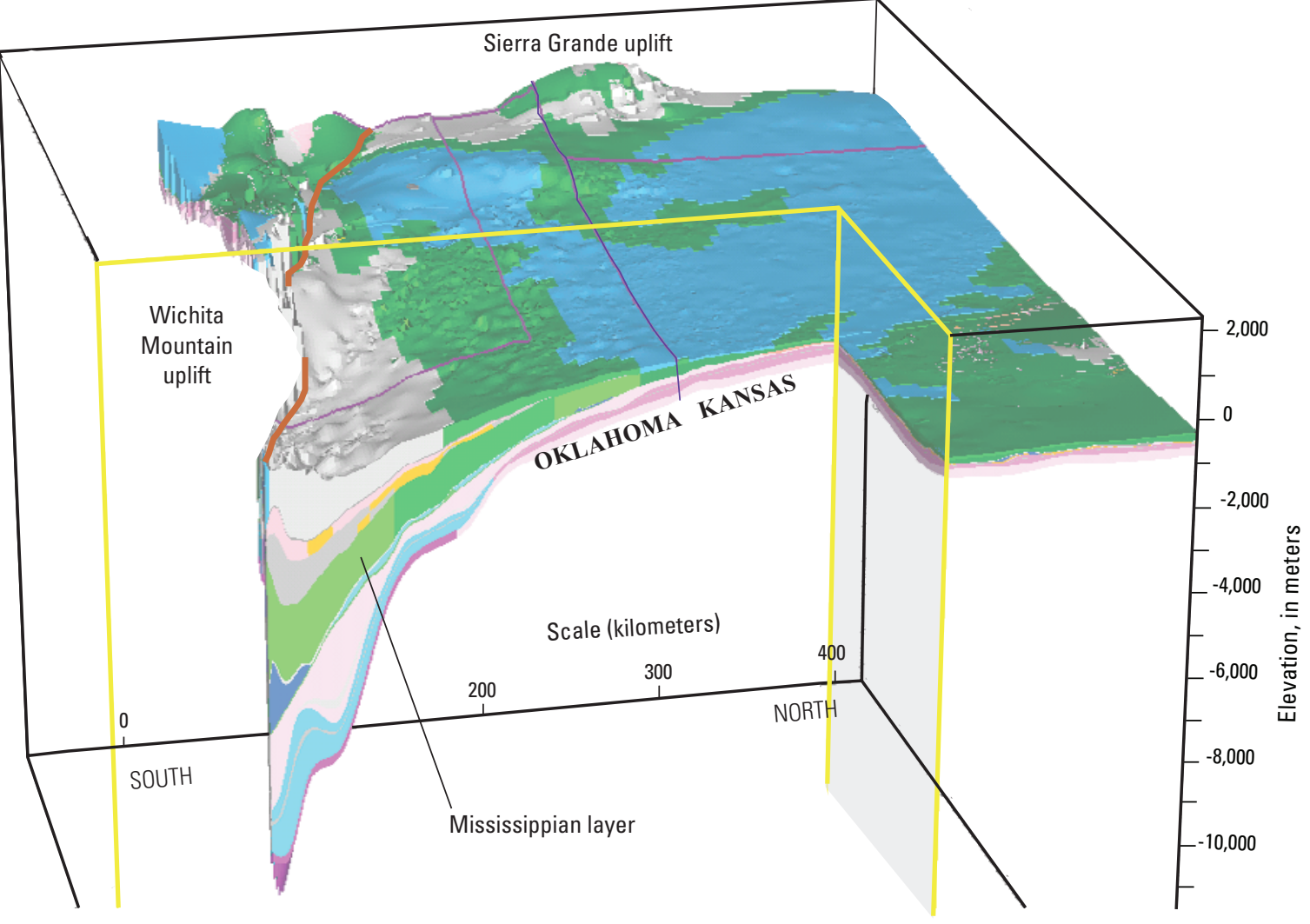

Figure 10. Cube dissection of the four-dimensional petroleum system model shows present-day elevation on the Desmoinesian layer surface; color variations on the Desmoinesian correspond to lithologies from Adler and others (1971). Vertical color bands represent lithofacies assignments for the Desmoinesian through Precambrian layers. For example, the labeled Mississippian layer is about 900 meters (2,900 feet) thick at the location. Location of the slice is in figure 9. Vertical exaggeration is 15 times. 


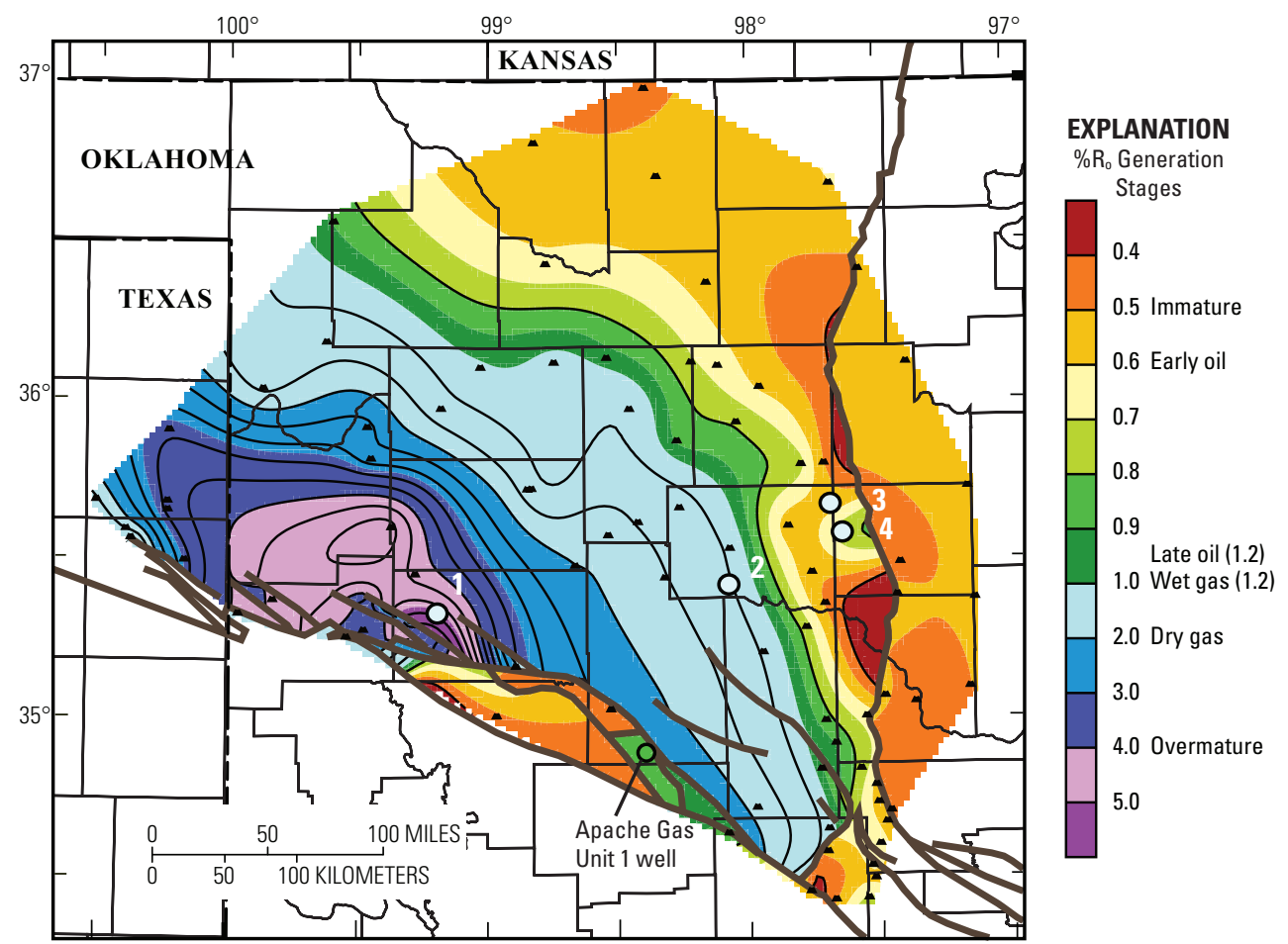

Figure 11. Map showing vitrinite reflectance $\left(R_{0}\right)$ contours for the Woodford Shale; black line contours are in increments of $0.4 \% R_{0}$. Generation stages on $R_{0}$ legend are generalized, as gas generation also occurs within the oil generation range. Black triangles and white dots include $\mathrm{R}_{0}$ data from Cardott (1989; written commun., 2011), Price (1997), and Mark Pawlewicz (written commun., 2010). Labeled white dot well locations are (1) Bertha Rogers 1, (2) Petree Ranch 1, (3) West Edmond SWD 1-24, and (4) Streeter 1. Brown lines are faults in the underlying Hunton Group (Rottmann, 2000a, pl. 3).

and Arbuckle Mountains (Comer and Hinch, 1987). Bitumen is assumed to be sourced from the Woodford as generated oil migrated laterally and then vertically along the faults. Vitrinite reflectance was contoured relative to the Hunton faults of Rottmann (2000a), which also isolates samples from the Wichita Mountain uplift relative to those in the basin. That the uplifted samples exhibit lower levels of thermal maturation than samples located north of the fault system shows that uplift occurred prior to maximum burial in the basin. Thermal maturation in the Wichita Mountain uplift is extrapolated from several outcrop samples and does not reflect the actual conditions because of the complexity of faulting, timing of uplift, and distribution of the Woodford Shale. Uplift samples should be considered point sources because of extensive faulting. For example, the Woodford Shale is present at least 4 times within the 1,930- to 19,000-ft depth interval of the Apache Gas Unit 1 well (fig. 11); this well was not sampled, but it is likely that there would be a spread of $R_{o}$ values.

\section{Petroleum Source Rocks}

Petroleum source rocks in the Anadarko Basin were depPetroleum source rocks in the Anadarko Basin were deposited in marine environments. Burruss and Hatch (1989) and Joseph Hatch (oral commun, 2010) identified shale intervals of the Simpson Group, Woodford Shale, and Middle and Upper Pennsylvanian strata as the main source rocks in the Anadarko Basin (fig. 2). Burruss and Hatch (1989, p. 57) identified the following Anadarko Basin source rocks as having moderate to good hydrocarbon potential based on oil and source rocks geochemical analyses: (1) Ordovician Simpson Group shale, (2) Devonian-Mississippian Woodford Shale, and (3) Pennsylvanian black shales. They indicated absent to moderate source potential for the Ordovician Sylvan Shale based on geochemical analysis of oil and rock samples in Kansas and Oklahoma. There is no evidence for source potential from the Sylvan (or equivalent Maquoketa) Shale in the Anadarko Basin based on studies by Hatch and others (1987) and Wang and Philp (1997), and the poor gamma response from examination of about 90 well logs across the basin. The primary and most studied petroleum source rock in the Anadarko Basin is the Devonian-Mississippian Woodford Shale; equivalent strata are termed the Chattanooga Shale in Kansas. Because of limited source rock information for many other formations, all but the Woodford will be grouped by system. The Woodford Composite TPS includes the Cambrian through Mississippian section, and the Pennsylvanian Composite TPS includes source and reservoir rocks of Pennsylvanian through Permian age.

Three layers in the 4D petroleum system model were assigned as petroleum source rocks based partly on the Burruss and Hatch (1989) determination of three oil types in the Anadarko Basin. They conducted geochemical analysis of 104 crude oils and 190 core samples of Cambrian through Pennsylvanian dark colored shales, and their sampled oils and source intervals are from (1) the Middle Ordovician Simpson Group, (2) Silurian to Mississippian reservoirs, and (3) Pennsylvanian strata. The modeled source intervals are the 
Oil Creek Formation layer of the Simpson Group, the Woodford Shale layer, and the informal Thirteen Finger limestone layer (fig. 2). The Thirteen Finger limestone was chosen to represent Pennsylvanian source rocks because I mapped the thickness and extent of this high-gamma interval formation across the Anadarko Basin Province and therefore had a layer to assign source properties.

This definition of three petroleum source rock layers serves to model which source(s) contribute to which reservoir layers, and also if this contribution varies across the basin and through time. Three assigned source intervals in a basin that contains probable other source rocks further homogenizes the model, but can also be used to determine if all potential reservoir intervals are charged. For example, does the Oil Creek layer source charge underlying Arbuckle layer reservoirs? If not, is it because of the coarseness of the model and (or) would this indicate source intervals in the Arbuckle?

Middle Ordovician Simpson Group reservoirs contain "typical" Ordovician oils that are characterized by (1) strong odd-carbon predominance in the $\mathrm{C}_{13}$ to $\mathrm{C}_{19}$ n-alkanes, (2) few or no acyclic isoprenoids, and $(3) \delta^{13} \mathrm{C}$ values of -33.9 parts per thousand (ppt) (saturates) and -33.7 ppt (aromatics) (Burruss and Hatch, 1989). Oils from Silurian to Mississippian reservoirs show little or no odd-carbon predominance in the $\mathrm{n}$-alkanes, a regular decrease in abundance of n-alkanes with increasing carbon number, pristane/phytane ratios ( $\mathrm{pr} / \mathrm{ph})$ of 1.1 to 1.5 , and delta carbon- $13\left(\delta^{13} \mathrm{C}\right)$ values of $-30.6 \mathrm{ppt}$ (saturates) and -30.1 ppt (aromatics). Oils in Pennsylvanian reservoirs have the greatest amounts of carbon $15+\left(\mathrm{C}_{15+}\right)$ hydrocarbons, are isotopically heavy (-27.5 ppt (saturates) and $-26.4 \mathrm{ppt}$ (aromatics), have methyl-cyclohexane as the most abundant hydrocarbon, and have $\mathrm{pr} / \mathrm{ph}$ values from 2.0 to 0.9 .

The Wavrek (1992) gas chromatography analysis of 385 Cambrian through Cretaceous crude oils from 83 fields across the Ardmore and Marietta Basins, south and east of the Anadarko Basin resulted in the assignment of 7 oil families. These A through $\mathrm{G}$ families were based largely on relative n-alkane distribution, cyclic isoprenoids, alkylated cyclic hydrocarbons, alkylated sulfur compounds, aryl-isoprenoids, hopanes, steranes, and carbon isotopes. Types A through E oils appear to correlate with respective source facies within the (A) Pennsylvanian (Atoka Group?), (B) Mississippian (Goddard Shale, Caney Shale, and Sycamore Limestone of the Mayes Group), (C) Devonian-Mississippian Woodford Shale, (D) upper Middle Ordovician Viola Group, and (E) Middle Ordovician Simpson Group (Wavrek, 1992). Type F oil was from Arbuckle Group reservoirs, but had uncertain parentage, and Type $\mathrm{G}$ oil had some characteristics of mixed oil types and possible effects of multiple sources because of proximity to the Ouachita thrust zone. Wavrek (1992) assigned greatest importance as a source rock to Type C, the Woodford Shale.

\section{Ordovician Source Rocks}

The Cambrian and Ordovician Arbuckle Group lithologies include limestones with algal mats and boundstones
(Donovan, 1986), bedded and disseminated anhydrite (Latham, 1970; St. John and Eby, 1978; Ragland and Donovan, 1985; Donovan, 1986), and gray to dark gray limestone and some shale (Fay, 1989). The Arbuckle and Ellenburger Groups are partially equivalent and about 7,000 feet (ft) $(2,150 \mathrm{~m})$ thick in the deep basin; petroleum production is confined to the upper 200-300 ft (Cardwell, 1977a). Pyrolysis-gas chromatographic analysis reveals that, in most of Oklahoma, the Arbuckle section is thermally mature but is deficient in organic matter (Cardwell, 1977a, 1977b). The Arbuckle Group is unlikely to contain petroleum source rocks in the Anadarko Basin. If present, source rocks are most likely proximal to the subsiding southern Oklahoma aulacogen (Johnson and Cardott, 1992), an area that is currently mostly overmature for hydrocarbon generation. This generation would have resulted in depletion of TOC, so original TOC would have been greater. TOC from 21 Arbuckle and Ellenburger carbonate samples ranged from $0.03-0.24$ wt \%, with an average of $0.1 \%$ (Cardwell, 1977a), less than good quality source rock of greater than $1.0 \mathrm{wt} \%$ TOC (Hatch and others, 1987). The Trask and Panode (1942, table 94, p. 263) Arbuckle TOC values ranged from 0.3 to 0.8 wt \% for 6 samples from 6 wells just east of the basin in Kansas and Oklahoma. Cardwell (1977a, p. 42) also observed that the composition of 9 Arbuckle oils from western Oklahoma were closely similar to those of 14 Desmoinesian oils from northeast Oklahoma and southeast Kansas.

The Ordovician Simpson and Viola Groups contain mainly oil-prone source intervals (fig. 2) of Types I and II kerogen based on Rock-Eval pyrolysis results (Rice and others, 1989; Burruss and Hatch, 1989). Wang and Philp (1997) indicated the Viola and Woodford petroleum source intervals are mostly Type II kerogen based on Rock-Eval pyrolysis. The Simpson Group reaches a maximum thickness of 2,300 ft (700 m), of which about 50 percent is shale (Schramm, 1964). Sparse sampling on the shelf indicated as much as 14 percent of these shales may contain more than the minimum $1.0 \mathrm{wt} \%$ TOC required for a petroleum source rock (Hatch and others, 1987). Burruss and Hatch (1989) state Ordovician rocks generally exhibit TOC less than $1.0 \mathrm{wt} \%$, although several Kansas samples have TOC between 1.0 and 9.0 wt \%. Joseph Hatch (oral commun., 2010) unpublished TOC data ranged from 0.02 to $18.2 \mathrm{wt} \%$ and averaged $1.54 \mathrm{wt} \%$ for 39 Ordovician core samples from 4 wells in Oklahoma and Kansas. The average was increased because of TOC values of 5.34, 8.81, and $18.2 \mathrm{wt} \%$ from a well located northeast of the province at about latitude $38.19 \mathrm{~N}$. and longitude $97.66 \mathrm{~W}$.; hydrocarbon index (HI) values for this well were 665 to 787 milligrams sulfur per gram of total organic carbon $\left(\mathrm{mg} \mathrm{S}_{2} / \mathrm{g}\right)$ TOC.

The Sylvan Shale is isolated to the deep basin in Oklahoma and Texas. It is light gray to grayish green and waxy (Johnson and others, 1988), with an average thickness of less than $250 \mathrm{ft}$ (80 m) (Wang and Philp, 1997). Well log gamma ray signatures were subdued for the Sylvan Shale in 88 wells scattered across the Oklahoma and Texas portions of the basin. Signatures were distinct enough to correlate the formation, but 
the minor gamma response is characteristic of the generally low TOC for this formation in the province.

\section{Devonian-Mississippian Woodford Shale}

The Woodford Shale in the Anadarko Basin is a carbonaceous, siliceous, pyritic, dark-gray to black shale (Cardott, 1989), the general extent of which is outlined in figure 1 by the Woodford Shale Oil AU. Equivalent strata northeast of the province in Kansas are called the Chattanooga Shale. The Woodford Shale was deposited on a regional erosional surface of the Silurian-Devonian Hunton Group (fig. 2). This unconformity developed across a broad upwarping that was one of the most widespread in the entire Midcontinent (Amsden, 1975, 1989); it was accompanied by little, if any, folding or faulting except for faulting along the Nemaha uplift (Johnson, 1989). This upwarping partially isolated the epicontinental seaway. The organic-rich Woodford black shales were deposited under anoxic conditions during the early Kaskaskia transgression of this shallow epicontinental sea (von Almen, 1970; Walper, 1977; Sullivan, 1985; Johnson and Cardott, 1992; Lambert, 1993). Evidence of burrowing in exposed organic-rich rocks in the Arbuckle Mountains (fig. 3) suggests there were also periods of oxic depositional conditions (Neil Fishman, oral commun., March 2, 2011). Egenhoff and others (2011) indicated a basinward progression in the Woodford from siliciclastic mudstones to successively increasing downslope Tasmanites and then radiolarian cherts; short-term highstands completely shut off delivery of very fine-grained sediments to distal parts of the passive margin during Woodford deposition, enabling good preservation of Milankovitch cycles in the formation.

Elevation on the top of the Woodford Shale in the Anadarko Basin ranges from about $-2,500 \mathrm{ft}(-760 \mathrm{~m})$ on the southern Kansas shelf, to $-27,000 \mathrm{ft}(-8,000 \mathrm{~m})$ in southern Oklahoma (fig. 3). The formation is mostly absent because of erosion in the Wichita Mountain and Amarillo uplifts, although the Woodford Shale Gas AU includes an area of Woodford in the Wichita Mountain uplift proximal to the fault zone. The Woodford Shale is a fairly easy pick from well logs because of the large gamma kick and low permeability of this formation (fig. 12). Gamma-ray responses are commonly greater than 160 American Petroleum Institute (API) units (Amsden, 1975; Sullivan, 1985). The figure 12 cross section is located perpendicular to a thick interval of Woodford channel fill (fig. 13). The easternmost well in the cross section exhibits lateral thinning of the Mississippian section and absence of Morrow Formation and Thirteen Finger limestone.

Thickness of the Woodford Shale varies greatly across the province. It ranges up to $375 \mathrm{ft}$ thick and averages $40 \mathrm{ft}$ based on Rottmann (2000b), well-log picks from about 100 wells across the basin, and edited formation tops from IHS Energy (2009a, 2009b). Greatest thicknesses are in the southern part of the basin in Oklahoma and locally as fill within eroded channels of the underlying Hunton Group (figs. 12 and 13).
This is also the basin area that is most thermally mature for gas generation. Areas within the Woodford Shale AUs boundaries (fig. 1) where the unit is absent or thin resulted from erosion or nondeposition. There are a few erosional remnants outside the Woodford Shale Oil AU boundary, and the unit is present northeast of the province in Kansas. Smooth contours near the southeastern corner of figure 13 reflect limited data and variable thicknesses because of extensive faulting. The Misener sand is a lower unit of the Woodford Shale that is located mainly in the northeastern basin in Oklahoma and Kansas. Petroleum resources of this unit were evaluated with the Hunton Group AU because the Misener sand reservoirs are conventional, and much of the petroleum production was reported as commingled with that of the Hunton Group.

The Woodford Shale comprises upper, middle, and lower informal members of carbonate- and silica-rich shales based on well-log signatures, palynomorphs, and geochemical differences (Urban, 1960; von Almen, 1970; Sullivan, 1983, 1985; Hester and others, 1990; Lambert, 1993). The middle shale member has the greatest areal extent and thickness, and is also the most organic rich (Lambert, 1993). The Woodford Shale exhibits two habits, fissile and nonfissile shale. Nonfissile contains phosphatic and siliceous shales, minor thin dolomite beds, phosphatic nodules, and rare chert; fissile shales are composed of laminations less than 1 centimeter $(\mathrm{cm})$ thick and are more organic-rich than nonfissile shale (Krystyniak and Paxton, 2006). The Woodford has abundant Type A amorphous Type II kerogen [partially equivalent to the liptinite (exinite) maceral group] with the remaining primarily Type III kerogen (vitrinite) and Type I kerogen (Tasmanite alginite) (Lewan, 1983; Thompson and Dembicki, 1986; Crossey and others, 1986; Comer and Hinch, 1987; Burwood and others, 1988; Krystyniack and Paxton, 2006). Applied to the Woodford Shale layer within the 4D PetroMod ${ }^{\circledR}$ model were a generalized composition of the Woodford Shale is 37 percent quartz, 26 percent illite, 15 percent kerogen, 17 percent carbonate, and 5 percent kaolinite (Abousleiman, 2008), and a HI of $527 \mathrm{mg}$ $\mathrm{S}_{2} / \mathrm{g}$ TOC based on Lewan and Ruble (2002). HI data ranged from 3 to $1,127 \mathrm{mg} \mathrm{S}_{2} / \mathrm{g}$ TOC and averaged $260 \mathrm{mg} \mathrm{S}_{2} / \mathrm{g}$ TOC based on analyses of 97 core samples from 30 wells that were scattered across Oklahoma and Kansas (Burruss and Hatch, 1989; Joseph Hatch, oral commun., 2010). Kirkland and others (1992) indicated a $\mathrm{HI}$ range of 500 to $800 \mathrm{mg} \mathrm{S}_{2} / \mathrm{g}$ TOC for their samples. The Oklahoma portion of the province is mostly mature to overmature for petroleum generation, whereas the Woodford (Chattanooga) in Kansas is immature. As such, the HI of 17 Kansas Woodford (Chattanooga) samples ranged from 89 to 600 and averaged $321 \mathrm{mg} \mathrm{S}_{2} / \mathrm{g}$ TOC.

Based on analysis of 101 Woodford core samples from 30 wells that were scattered throughout its extent in Oklahoma and Kansas, Burruss and Hatch (1989) indicated the TOC ranged from greater than $2 \mathrm{wt} \%$ to $14 \mathrm{wt} \%$. Joseph Hatch (oral commun., 2010) indicated that TOC data averaged $4 \mathrm{wt} \%$. Estimated average TOC of a somewhat conservative $3.5 \mathrm{wt} \%$ was used for 1D models and was based on data from Hester and others (1990), Comer and Hinch 
Chesapeake Energy Corporation

T. 16 N., R. 12 W., Sec. 4

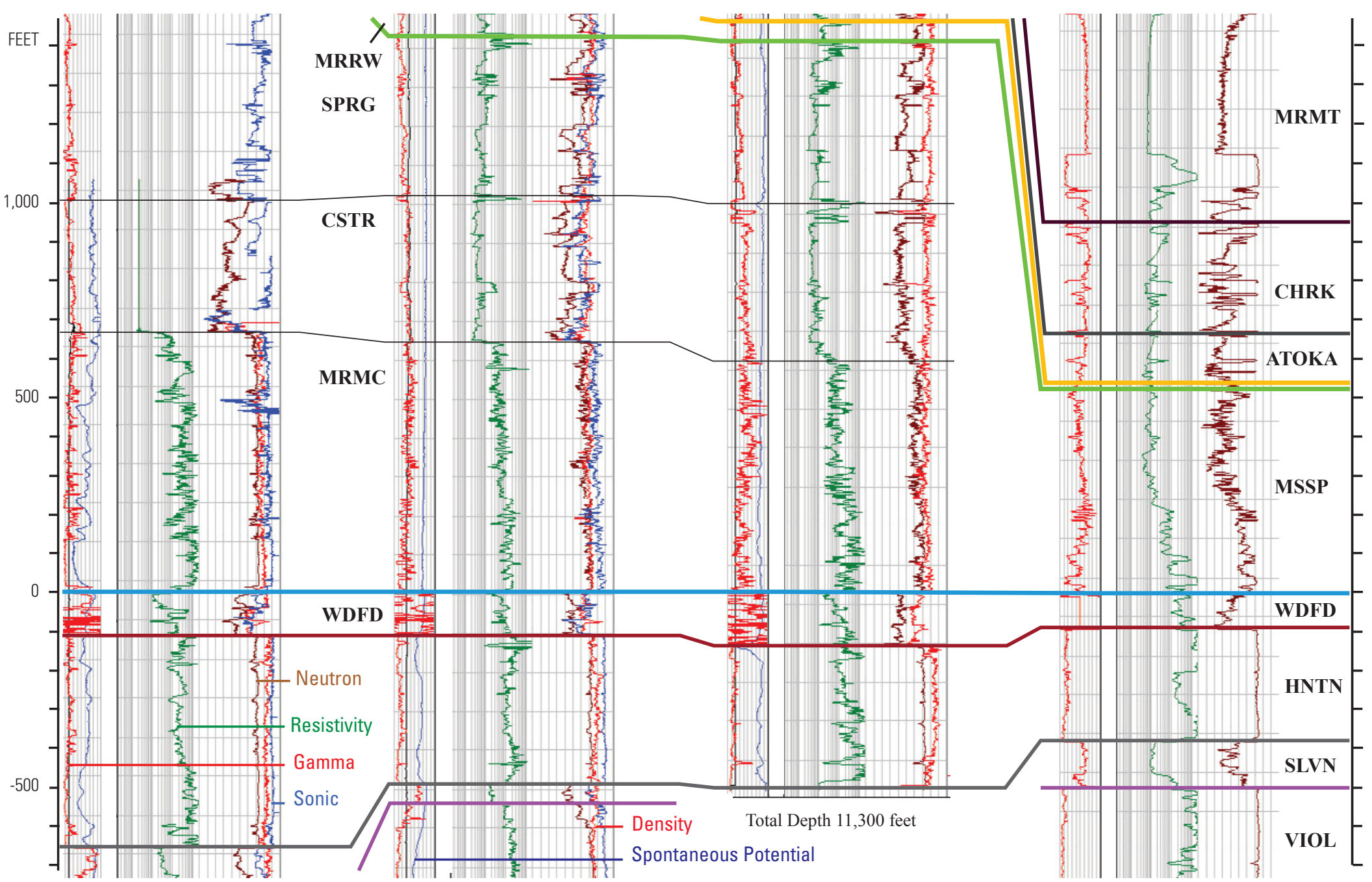

Figure 12. Cross section showing the Woodford Shale (WDFD, blue line) and terminus of the Morrow Group (MRRW) and overlying Thirteen Finger Limestone portion of the Atoka Group (ATOKA, gold line) in the deep Anadarko Basin. Line of section is shown in figure 13. Vertical scale is in feet relative to the top of the WDFD. MRMT is Marmaton Group, CHRK is Cherokee Group, MSSP is Mississippian, HNTN is Hunton Group, SLVN is Sylvan Shale, VIOL is Viola Group, and SPRG, CSTR, and MRMC are the respective Mississippian Springer Formation, Chester Group, and Meramec lime. Labeled well log traces are gamma (0 to 200 API), resistivity, sonic density, neutron density, spontaneous potential, and density.
Dragging Otter 1-29 Chesapeake Energy Corporation

Samedan Oil Corporation

Chesapeake Energy Corporation

T. 15 N., R. 9 W., Sec. 29

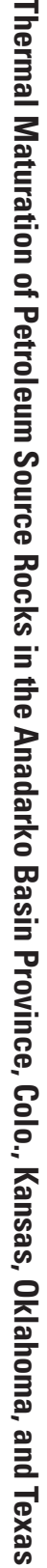




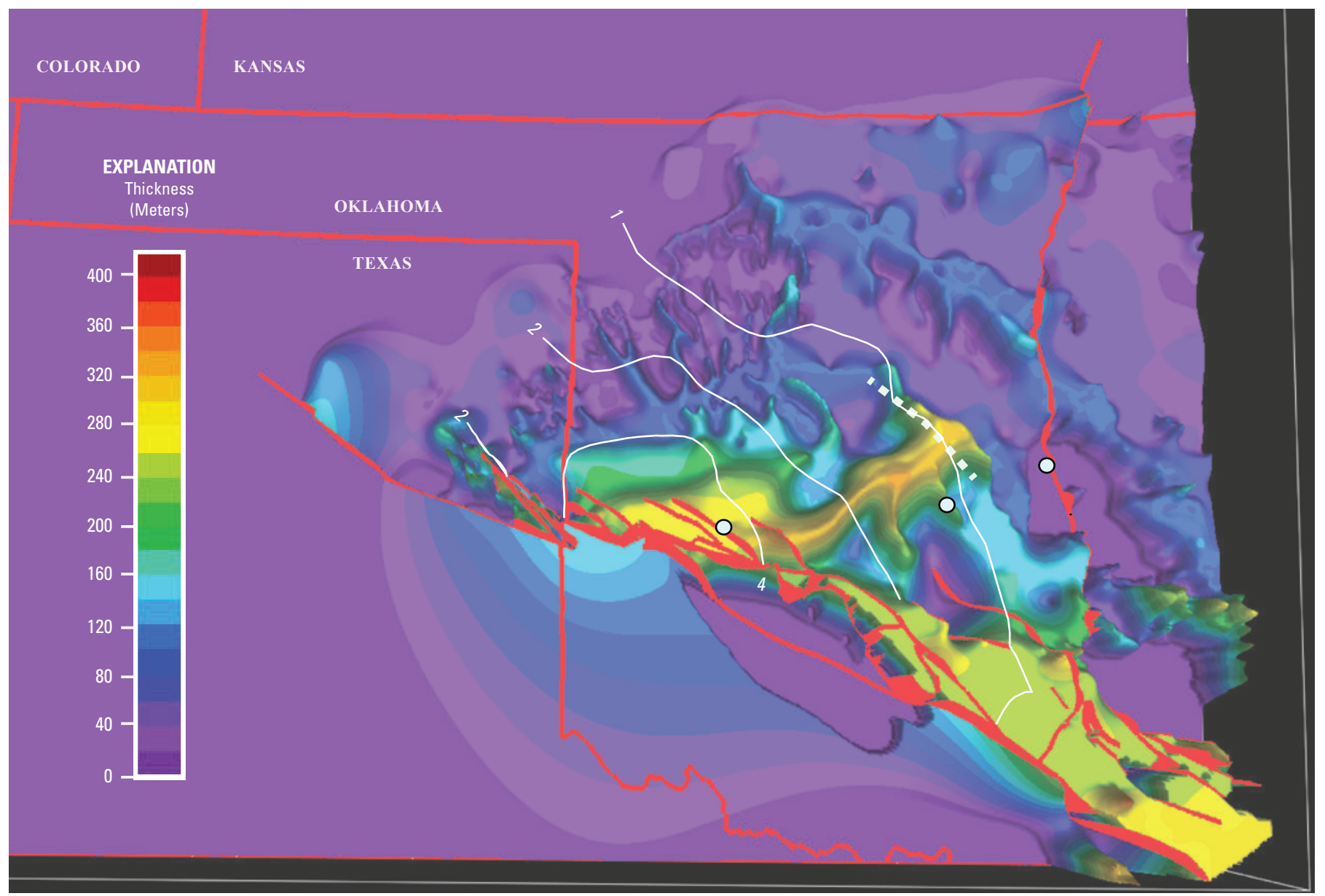

Figure 13. Three-dimensional isopach image of the Woodford Shale in the Anadarko Basin. Vitrinite reflectance $\left(\% R_{0}\right)$ contours (white lines) from figure 11 approximate end of oil generation at $1.2 \% R_{0}$ to overmature for petroleum generation $\left(4 \% R_{0}\right)$. White dot well locations are, from left to right, Bertha Rogers 1, Petree 1, and Streeter 1. Dashed white line is location of figure 12 cross section. Woodford thickness is derived from Rottmann (2000b, pl. 2), analysis of more than 100 well logs, and edited formation tops from IHS Energy (2009a, 2009b). Faults (red) are from Rottmann (2000a, pl. 3). Image is tilted and 1,300 times vertical exaggeration.

(1987), Burruss and Hatch (1989), Price (1997), Joseph Hatch (oral commun., 2010), and the Energy Geochemistry Data Base (U.S. Geological Survey, 2010). 1D models are located in areas that are mature to overmature for petroleum generation from the Woodford Shale. As such, original TOC was greater. The 4D model incorporated a map generated from these TOC data (fig. 14), instead of assigning a value for the entire layer. The Comer and Hinch (1987) Woodford mean TOC content of $5.4 \pm 6.9$ percent by wt was based on analysis of 300 samples from 16 cores and 43 outcrops that were distributed across Oklahoma and Arkansas; uncertainty in TOC resulted from a range of less than $0.1 \mathrm{wt} \%$ in some chert beds to $26 \mathrm{wt} \%$ in highly compacted black shale. Their location A21 near the southeast corner of figure 14 averaged $5.5 \pm 3.9$ percent by weight TOC and this Type II kerogen was in early stage oil generation. Krystyniack and Paxton (2006) TOC values ranged from 0.8 to $17 \mathrm{wt} \%$ for upper Woodford outcrop samples of south-central Oklahoma.
Comer and Hinch (1987) indicated that chert beds contained less TOC, but more total bitumen and hydrocarbons per gram. This may be indicative of greater fracturing and petroleum retention in chert beds.

\section{Mississippian Source Rocks}

Middle Ordovician through lowermost Mississippian sedimentary rocks in the basin are composed of fossiliferous shallow-water marine carbonates interbedded with fine-grained to moderately coarse-grained clastics that were derived from the northeast and east (Johnson and others, 1988). Probable Mississippian petroleum source rocks are within the informal Osage lime and Springer Formation (fig. 2) (Wang and Philp, 1997). Average thickness of the Springer Formation is about $500 \mathrm{ft}(150 \mathrm{~m})$ and more than 60 percent of it is shale and mudstone (Wang and Philp, 1997). Organic-carbon values for 


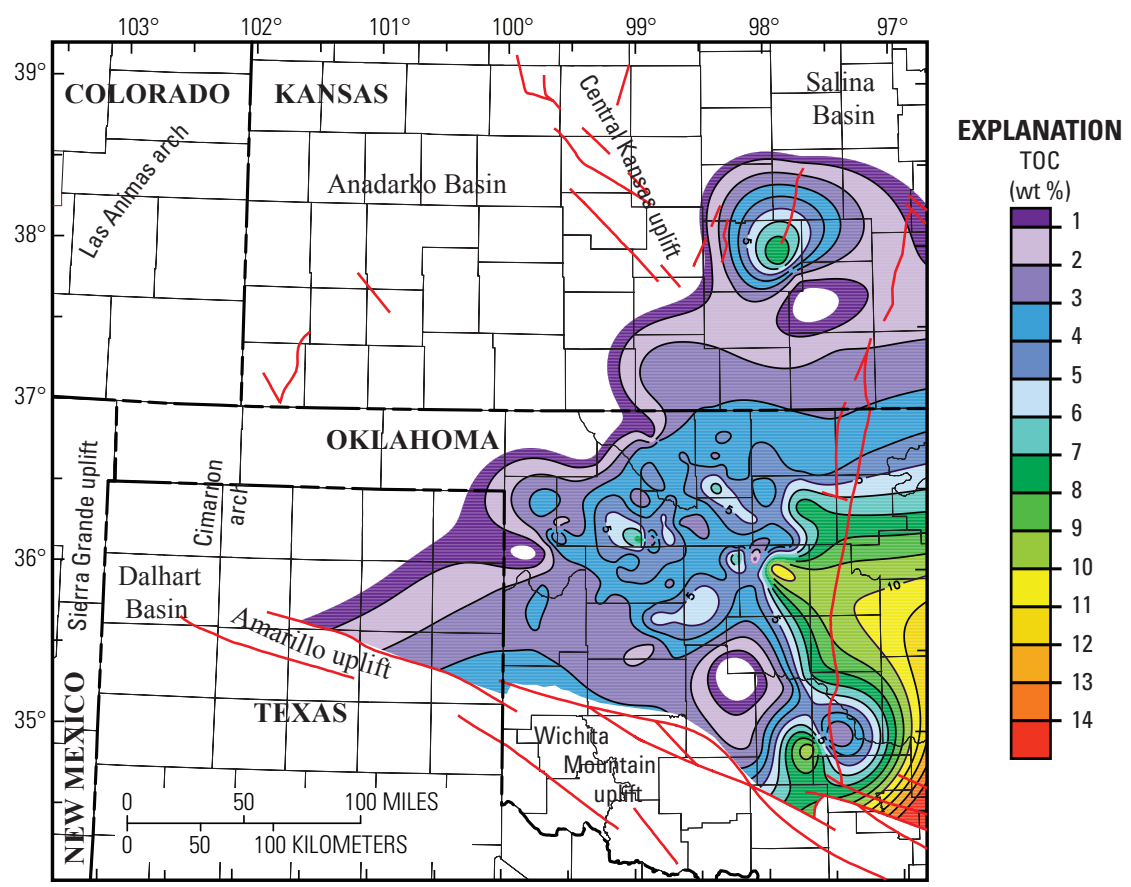

Figure 14. Map showing total organic carbon (TOC) content in weight percent (wt $\%$ ) for the Woodford Shale based on TOC data from Burruss and Hatch (1989) and mean values from Hester and others (1990). TOC ranges from 0.08 to $14.05 \mathrm{wt} \%$ and averages $3.7 \mathrm{wt} \%$ for core and well log density calculations from 123 wells. There is considerable TOC variation, but almost the entire area that is thermally mature for petroleum generation contains greater than $2 \mathrm{wt} \%$ TOC. Oil and gas generation depletes TOC and HI (hydrogen index), the fuels for petroleum, so original TOC and HI values would have been greater. Red lines are faults from Adler and others (1971).

thick shales in the Springer and Pennsylvanian (Morrowan) producing intervals range from 0.5 to $3.4 \mathrm{wt} \%$; however, the kerogen is mostly Type III, indicating that it is primarily gas-prone (Hatch and others, 1987; Rice and others, 1989; Wang and Philp, 1997) (fig. 2). Johnson and Cardott (1992, fig. 7) indicate that Mississippian shales and novaculite in the Ouachita trough, southeast of the Anadarko Basin, contain Types II and III kerogen with TOC values ranging from less than 1.0 to $1.9 \mathrm{wt} \%$. The Mississippian ended with regional uplift and erosion that marked the onset of the Wichita orogeny, and resulted in erosion of Devonian and older strata, such as the Woodford Shale on structural highs around the margin of the basin (Ball and others, 1991).

\section{Pennsylvanian Source Rocks}

Morrowan, Atokan, Desmoinesian, and Missourian organic-rich shales are important petroleum source rocks in the Anadarko Basin (fig. 2) (Hatch and others, 1987; Burruss and Hatch, 1989). Absence of uppermost Morrowan through Atokan strata over most of the Cimarron arch indicates this structure formed toward the end of tectonic activity that gave rise to the Wichita Mountain and Amarillo uplifts (fig. 14) (Johnson, 1989). These strata are also absent over the Sierra Grande uplift (fig. 9), a broad regional feature that was active during the Precambrian and a positive feature since Paleozoic time (Speer, 1976). The Morrow Group averages $1,500 \mathrm{ft}(460 \mathrm{~m})$ in thickness in the Oklahoma portion of the Anadarko Basin, and comprises more than 60 percent shale and mudstone (Wang and Philp, 1997). As is the case with most other formations in the basin, the Morrow thins northward from the basin axis and across the shelf. Deposition across the province varied from shallow marine to shoreline and deltaic environments (Tsiris, 1983). Lower Morrow Group lithofacies are mainly black shale beds with interbedded sandstone and thin limestone layers; these probably represent deposition in a shallow transgressive sea (Wang and Philp, 1997). The middle Morrow is dominantly marine shales and carbonates, and the upper Morrow contains black shale, thin coal layers, and recycled organic matter (Wang and Philp, 1997). Unpublished data for Morrowan shales from core of 14 wells in the Oklahoma portion of the basin were from Joseph Hatch (oral commun., 2010); results ranged from 0.48 to $10.71 \mathrm{wt} \%$ TOC and averaged $1.72 \mathrm{wt}$ $\%$; HI ranged from 15 to $179 \mathrm{mg} \mathrm{S}_{2} / \mathrm{g}$ TOC and averaged 46. 
As is the case for Pennsylvanian and older rocks in the deep basin, sampled areas are thermally mature for petroleum generation and original TOC and HI values would have been greater. 4D model initial TOC and HI values are calculated by PetroMod ${ }^{\circledR}$ software based on present-day thermal maturation of source rocks and their burial through time.

Atokan Series source rocks include shales and limestones of the Atoka Group and underlying Thirteen Finger limestone. The Atokan Series averages $390 \mathrm{ft}$ thick in Texas and the Oklahoma Panhandle and overlies a second-order unconformity at the top of the Morrow Group (Carr and Hentz, 2009). The Thirteen Finger limestone, also called the 13 Finger lime and Thirteen Finger lime, consists of fissile, organic-rich shale interbedded with fossiliferous wackestones that represents a third-order transgressive systems tract (Carr and Hentz, 2009; Carr and others, 2009). Depths range from about 4,000 to $18,500 \mathrm{ft}$ across the mapped extent of the unit. Elevation of the Thirteen Finger limestone, based partly on examination of 125 wells across the basin, ranges from about $-4,000 \mathrm{ft}$ on the shelf areas of Colorado and Kansas, to $-17,000 \mathrm{ft}$ in the deep basin area of Oklahoma and Texas (fig. 15). There is limited change in elevation of the formation across the Colorado and Kansas shelf, aside from uplifts associated with the Las Animas arch in southeastern Colorado, and the Keyes dome. This dome (fig. 6) forms a trap for modeled Thirteen Finger limestone oil accumulations. The Cimarron arch and Sierra Grande uplift (fig. 6) were positive features during Morrowan and Atokan time, which prevented deposition of these strata. Thickness of the Thirteen Finger limestone is variable across its extent (fig. 16) and averages $95 \mathrm{ft}$ based on examination of 125 well logs. Semicircular contours represent increased thickness along a curved axis of deposition, and data extrapolation in areas with sparse well coverage. Also shown in figure 16 is overall thinning of strata from the deep basin to shelf areas, although there is a northwest trend of increased thickness from the deep basin towards southeastern Colorado. The Perryton and Knowles Northwest oil fields (fig. 16) have cumulative production from the Thirteen Finger limestone of about 160,000 barrels of oil, 300 million cubic feet of gas, and 6,900 barrels of water (IHS Energy, 2010). These fields are considered to be conventional based on decline curves of petroleum and water production. Perryton and Knowles Northwest fields are also located in an area marginally mature to mature for oil generation from the Thirteen Finger limestone, based on modeled TR and $\mathrm{R}_{\mathrm{o}}$ values.

The thickest, most organic-rich interval in the Atokan Series is in the uppermost part of the Thirteen Finger limestone and it probably represents a third-order marine condensed section (Carr and Hentz, 2009). Carr and Hentz (2009) estimated the average TOC ranged from about 4-6 wt \% based on petrophysical examination of 28 wells. The Thirteen Finger limestone is characterized on well logs as stacked thin shale and carbonate beds with irregular high gamma kicks of 140 API and greater (fig. 17). This results in the "fingers" appearance. It is fairly readily correlated across the extent of the underlying Morrow Group in the province, except where proximal to the Wichita Mountain uplift. This is primarily because of tilting of strata and increased contribution of clastic sediments relative to organics. Gamma response for cross section $A-A^{\prime}$ is less pronounced than that of $B-B^{\prime}$. This suggests that the Atokan section in $B-B^{\prime}$ contains more organic matter. Source rocks in $A-A$ ' are also thermally mature for oil and (or) gas generation, which would deplete the TOC and HI values. Gamma response of the overlying Atoka Group is unremarkable in the $A-A^{\prime}$ ' cross section but the $B-B^{\prime}$ response is similar to that of the Thirteen Finger limestone. The Atoka Group source interval differs in that it is primarily shale, rather than thin limestone and shale beds.

Desmoinesian and Missourian source rocks (fig. 2) are primarily organic-rich shales. Sixteen of the sample analyses results from Joseph Hatch (oral commun., 2010) were loosely categorized as Desmoinesian source rocks, 13 from Oklahoma, 1 from Texas, and 2 from western Kansas-TOC values ranged from 0.08 to $11.2 \mathrm{wt} \%$, averaging $2.9 \mathrm{wt} \%$, and $\mathrm{HI}$ values ranged from 7 to $442 \mathrm{mg} \mathrm{S} / \mathrm{g}$ TOC, averaging $144 \mathrm{mg}$ $\mathrm{S}_{2} / \mathrm{g}$ TOC. Two samples from the Missourian section in Oklahoma and 4 from western Kansas ranged from 0.5 to $39.4 \mathrm{wt}$ $\%$ TOC, averaging $11.4 \mathrm{wt} \%$, and $\mathrm{HI}$ values ranged from 7 to

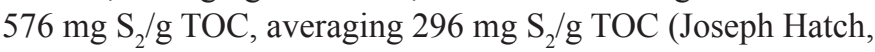
oral commun., 2010).

\section{Petroleum System Model of the Anadarko Basin}

\section{Petroleum Generation Kinetics}

The 4D model of the Anadarko Basin was constructed of 30 layers of strata from Precambrian to ground surface. Incorporated in the model are maps of basal heat flow, water depth through time, periods and thicknesses of deposition and erosion, total organic carbon (TOC) and hydrogen indices (HI) for the Woodford Shale and other source rocks, and lithofacies for each layer. Ideally, once the 4D model is constructed, it can be readily calibrated to available temperature and thermal maturation data, but in actuality, numerous modifications and iterations are necessary to achieve "final" models. Changes are designed to fit the 4D model to generated 1D models and to measured temperature and thermal maturation indicators across the basin. 1D models and 1D extractions from the 4D model were calibrated using temperature and $R_{o}$ data. $1 \mathrm{D}$ models were compared to $1 \mathrm{D}$ extractions from the $3 \mathrm{D}$ model as a further calibration step. Modeled $\mathrm{R}_{\mathrm{o}}$ (Sweeney and Burnham, 1990) was preferentially calibrated on measured $R_{0}$ from the Woodford Shale (Cardott, 1989; Price, 1997).

Onsets of petroleum generation and expulsion across the basin were determined primarily using modeled $\mathrm{R}_{\mathrm{o}}$ and Woodford Shale hydrous pyrolysis (HP) kinetics (table 2) that were applied to potential source rocks of the Ordovician Simpson Group, Devonian-Mississippian Woodford Shale, 


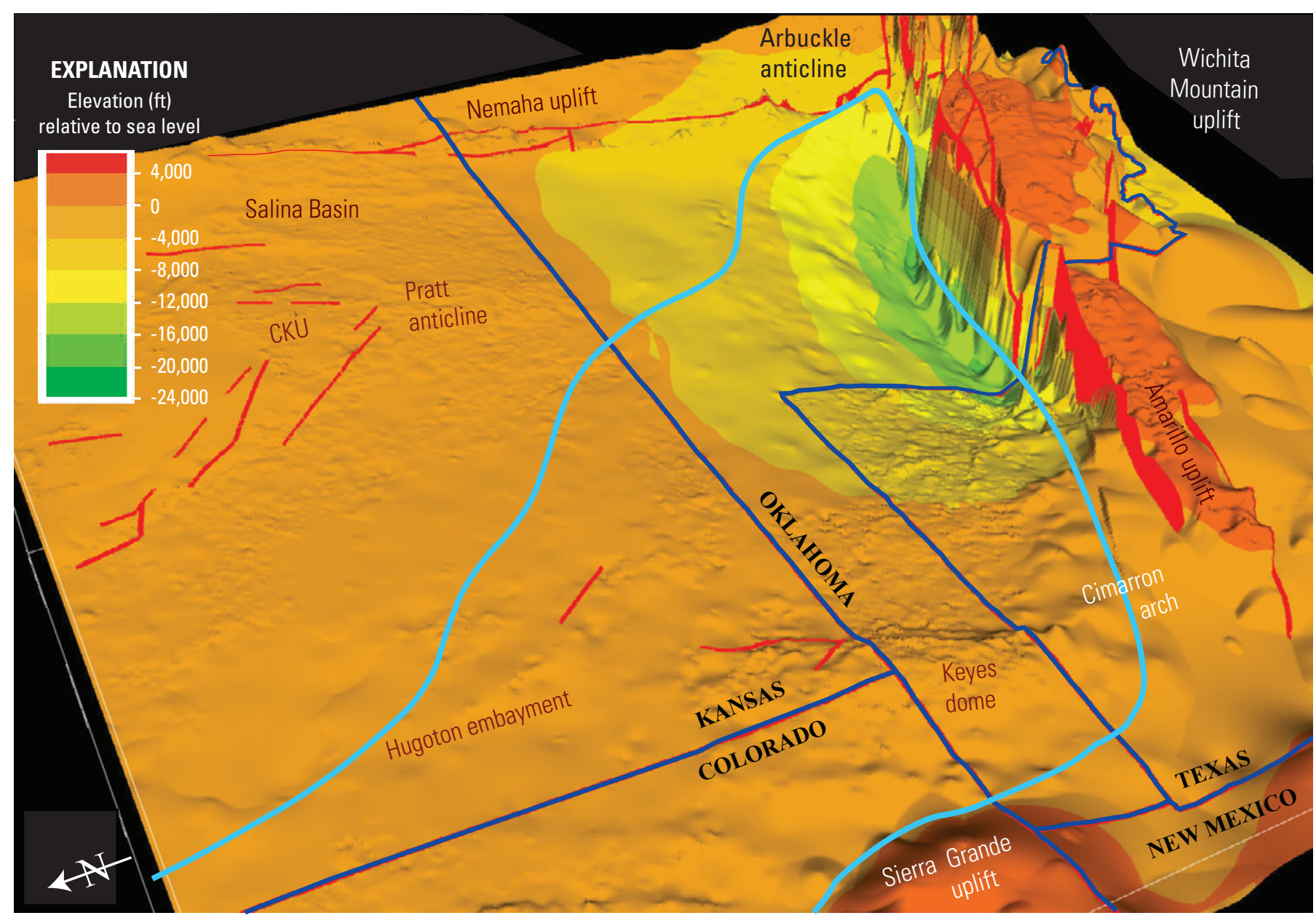

Figure 15. View to the southeast of elevation on the top of the Thirteen Finger limestone layer (Higley and others, 2014, ThirteenFingerTft.DAT grid file). Vertical exaggeration is 18 times. Thirteen Finger lime extent is approximated by the light blue line. Major structures are labeled. Precambrian faults (red) are from Adler and others (1971). Wichita Mountain uplift faults are vertical for four-dimensional model purposes only, and surface in this area is unknown because of insufficient data. Data sources for this surface include Thirteen Finger limestone picks from well logs and edited IHS Energy (2009a, 2009b) formation tops. Elevation is relative to sea level. CKU, Central Kansas uplift.

and Pennsylvanian Thirteen Finger limestone. Heat flow and erosional history, estimated depositional and thermal properties of assigned lithofacies, and choice of kinetic algorithms can significantly affect timing of generation. Petroleum modeling software can also affect results and associated conclusions (Higley and others, 2006). For these reasons I used several kinetic algorithms and heating histories. It is preferable to have kinetic algorithms calculated from and specific for each petroleum source rock, but those values were only available for the Woodford Shale.

Phosphoria Formation HP kinetics (Lewan and Ruble, 2002) was also applied to the models to investigate the effect of organic sulfur on timing of petroleum generation. Some wells in the Panhandle and Hugoton field area of the western basin produce sour oil and (or) gas from mostly Pennsylvanian reservoirs, but sour oil and (or) gas has also been reported from scattered Cambrian and Ordovician Arbuckle through Permian Chase reservoirs across the basin (Mason,
1968; Owen, 1975; Nehring and Associates, Inc., 2009). The Woodford Shale contains a fairly low ratio of organicsulfur to carbon at $0.023\left(\mathrm{~S}_{\text {org }} / \mathrm{C}\right)$; the Phosphoria Formation is fairly high at $0.045023 \mathrm{~S}_{\text {org }} / \mathrm{C}$ (Lewan and Ruble, 2002, table 1). HP kinetics depend partly on the organic-sulfur content of the original kerogen (Lewan and Ruble, 2002). Impact of the Type IIS kerogen organic sulfur on generation of oil is detailed in Lewan and Ruble (2002) and illustrated in Higley and others (2009). To simplify a complex process, essentially the organic sulfur results in sulfur-radical initiators that increase the associated reaction rates during thermal maturation of the source rock. A primary effect is that the onsetthrough-completion of oil generation and expulsion occurs at decreased levels of thermal maturation. The Tsuzuki and others' (1999) secondary and tertiary generation (cracking) of oil to gas kinetics were applied for each petroleum source rock in the model. Because of the coarse grid spacing and generalized lithologies, little secondary or tertiary gas 


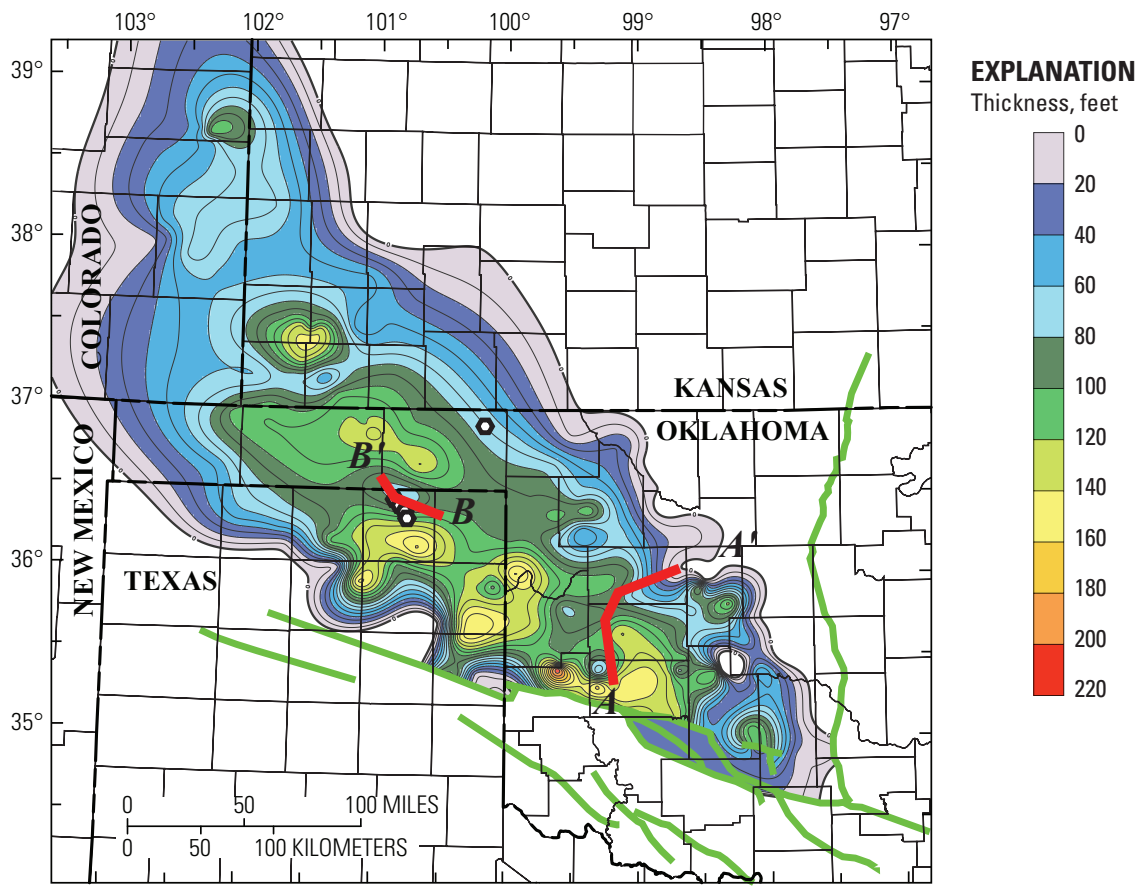

Figure 16. Isopach map of the top of the stratigraphic interval from the Thirteen Finger limestone to the Morrow Formation, based on Andrews (1999a, b), examination of more than 120 well logs, and edited tops from IHS Energy (2009a, 2009b). Morrow Formation faults (green) are modified from Andrews (1999b). Color contour interval is 20 feet and (black) line contours are 10 -feet intervals. Figure 17 cross sections are labeled $A-A^{\prime}$ and $B-B^{\prime}$. White polygons are Perryton (Texas) and Knowles Northwest (Oklahoma Panhandle) oil fields.

Table 2. Summary of kinetic parameters derived for expelled-oil generation based on hydrous pyrolysis (HP) in isothermal experiments, and nonisothermal open-system pyrolysis (REV) that were derived from Lewan and Ruble (2002, tables 4 and 5) and cracking of oil to gas (Tsuzuki and others, 1999). The Woodford Shale analyses were on an unweathered, thermally immature sample that contained 12.7 percent total organic carbon (TOC), from section 25 , $\mathrm{T}$. 2 S., R. 1 E., Carter Co., Oklahoma (Lewan, 1983).

\begin{tabular}{|c|c|c|c|c|c|}
\hline $\begin{array}{l}\text { Formation- } \\
\text { Name }\end{array}$ & SampleNumber & KerogenType & $\begin{array}{l}\text { Activation energy, } \mathrm{E}_{\mathrm{a}} \\
\quad \text { (kcal/mol) }\end{array}$ & $\begin{array}{l}\text { Frequency factor } A_{0} \\
\qquad\left(m \cdot y^{-1}\right)\end{array}$ & $\mathrm{HImgHC} / \mathrm{gTOC}$ \\
\hline Phosphoria & P-64 & Type II-S & 42.71 & $4.31 \mathrm{E} \times 10^{23}$ & 276.0 \\
\hline Woodford & WD-5 & Type II & 52.16 & $5.706 \mathrm{E} \times 10^{26}$ & 246.2 \\
\hline \multirow[t]{2}{*}{ Woodford } & WD-5 (REV) & Type II & 56.1 (Mean) & $8.111 \mathrm{E} \times 10^{26}$ & 440.0 \\
\hline & & $\left(\mathrm{C}_{15^{+}} \text {crude oil }\right)^{1}$ & 76.00 & $3.419 \mathrm{E} \times 10^{33}$ & \\
\hline
\end{tabular}

\footnotetext{
${ }^{1}$ Tsuzuki and others (1999) secondary gas generation kinetics was applied to all petroleum source rocks.
} 

一

Hohnke Leo Unit 1

Exxon Corporation

Arkla Exploratio

T. 14 N., R. 19 W., Sec. 6

T. 10 N., R. 18 W., Sec. 6

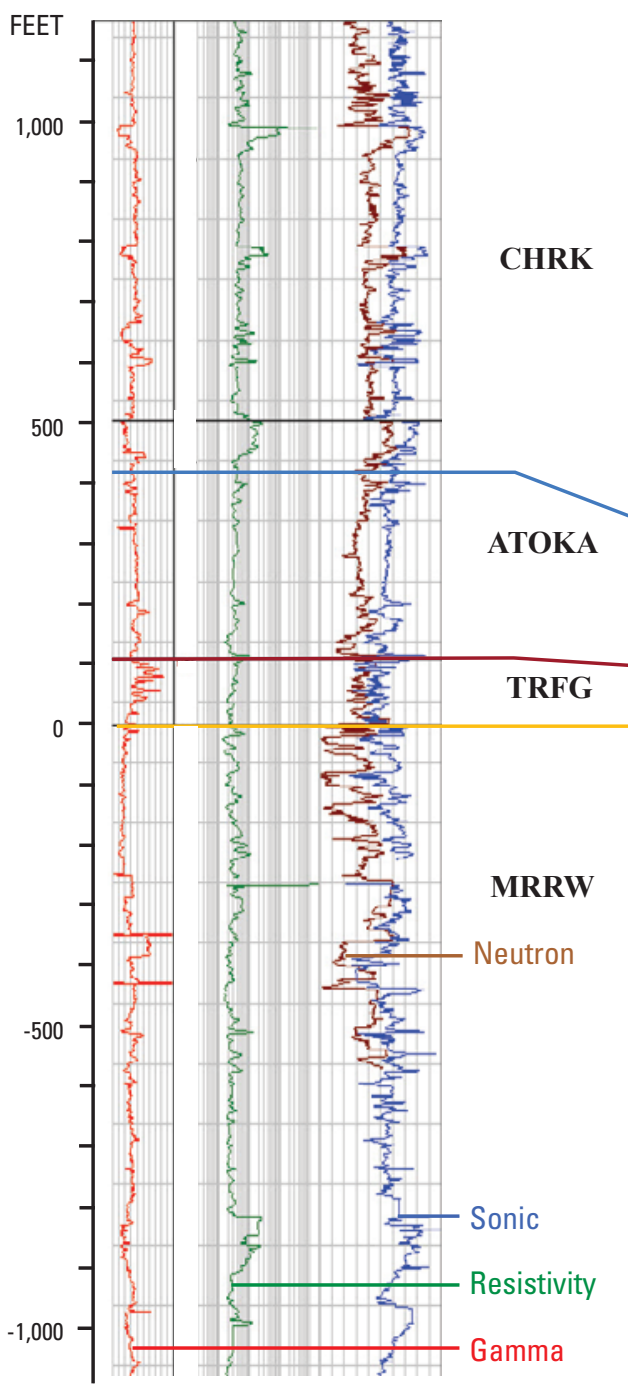

Porter Willie 1-9

Monsanto Company

add Petroleum Company

T. 16 N., R. 17 W., Sec. 18

T. 17 N., R. 14 W., Sec. 9 (ATOKA) in the Anadarko Basin. Lines of section are shown in figure 16. Vertical scale is feet relative to the base of the Thirteen Finger. MRMT is Marmaton Group, CHRK is Cherokee Group, MRRW is Morrow Group, and MSSP is top of the Mississippian. Labeled well log traces are gamma (0 to 200 American Petroleum Institute [API] range), resistivity, sonic density, and neutron density. 
Sturtz-Deeds Unit 1 Arco Oil \& Gas Corporation

FEET
McGarraugh 2-129

Anadarko Petroleum Corporation

Survey: SPRR Abs: 333, Blk. 10, S. 129
Hale 1-7

Chesapeake Energy Corporation

T. 1 N., R. 20 E., Sec. 7

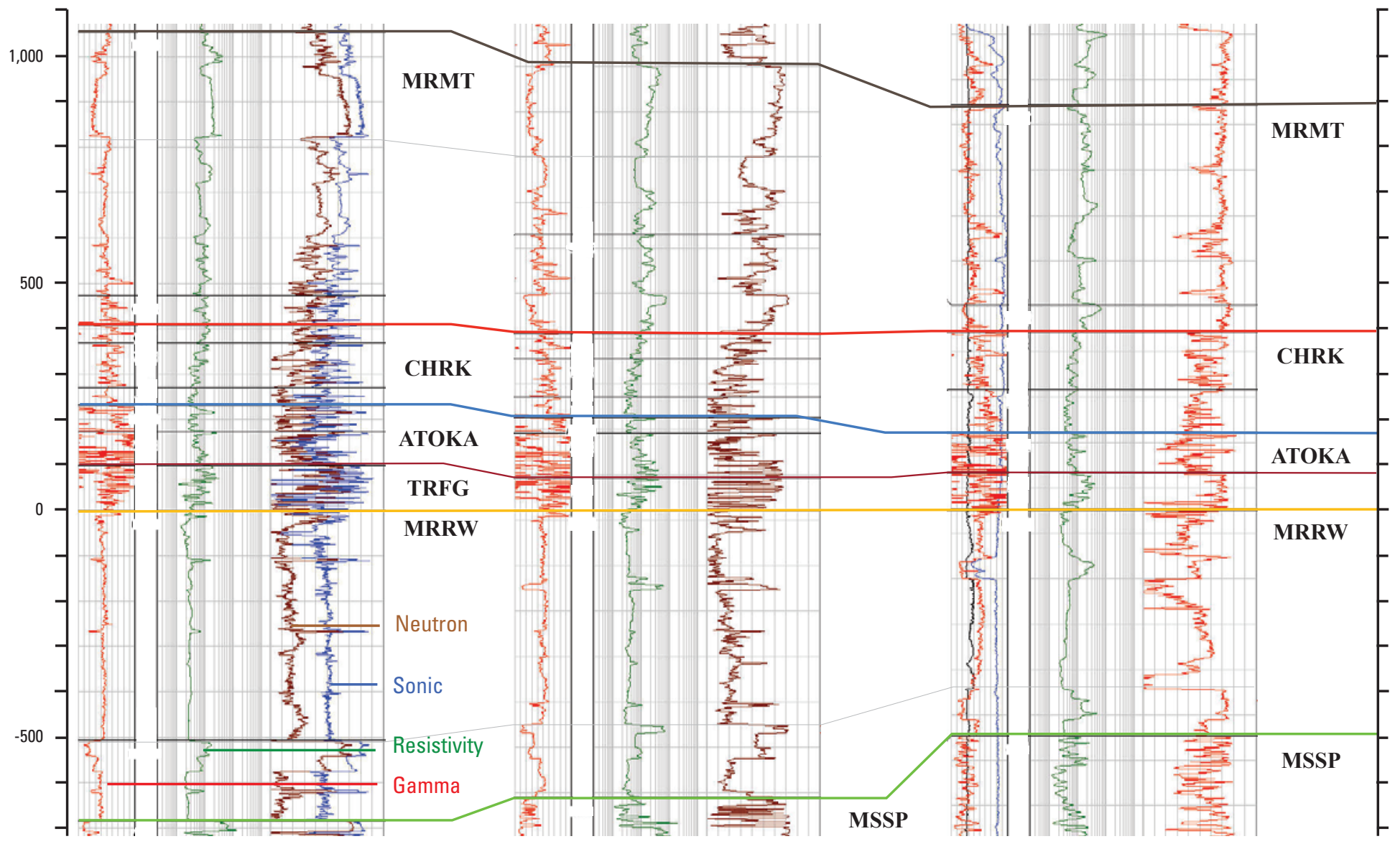

Figure 17. A, Southwest to northeast and $B$, southeast to northwest cross sections of the Thirteen Finger limestone (TRFG) of the Pennsylvanian Atoka Group (ATOKA) in the Anadarko Basin. Lines of section are shown in figure 16. Vertical scale is feet relative to the base of the Thirteen Finger. MRMT is Marmaton Group, CHRK is Cherokee Group, MRRW is Morrow Group, and MSSP is top of the Mississippian. Labeled well log traces are gamma (0 to 200 American Petroleum Institute [API] range), resistivity, sonic density, and neutron density.-Continued 
was generated in the model. Basically, most generated oil migrated out of the deep basin instead of being trapped and subject to secondary or tertiary generation of gas. Future improvements in computer processing and display, and in software design, may allow for greater complexity of the basin-scale model and results.

The timing and extent of petroleum generation from a source rock requires determining kinetic parameters that relate to the length of geologic time responsible for generation reactions to burial temperatures. This process is detailed in Lewan and Ruble (2002) and briefly summarized here. Applying kinetic parameters is typically accomplished using the Arrhenius equation, which for first-order reactions can be expressed in terms of the transformation ratio $(X)$ as

$$
X=1-\exp \left\{\mathbf{t A}_{\mathrm{o}}\left(\exp \left[-\mathrm{E}_{\mathrm{a}} / \mathrm{RT}\right]\right)\right\}
$$

The transformation ratio $(X)$ is the decimal fraction of the amount of petroleum product generated at a given time ( $\mathbf{t})$ and temperature $(\mathbf{T})$ as determined by the kinetic parameters $E_{a}$ and $A_{o}$. The activation energy $\left(E_{a}\right)$ represents the energy barrier the reactants (kerogen or bitumen) must reach or exceed for the reaction to continue. The frequency factor $\left(\mathrm{A}_{\mathrm{o}}\right)$ represents how often the reactants reach the energy barrier in a given amount of time for the reaction to proceed. $\mathrm{R}$ is the universal gas constant. HP kinetic parameters are used to determine narrow oil generation and expulsion windows that extend over a wide range of temperatures and depths. Timing of oil and gas generation is indicated on the petroleum system models by the following boundaries. Transformation ratios (TR) of oil generation and expulsion are onset (0.1 percent), peak (50 percent), and completion (99 percent). Vitrinite reflectance boundaries are presented graphically as onset of generation of oil $\left(0.6 \% \mathrm{R}_{\mathrm{o}}\right)$, end of oil and start of wet gas generation $\left(1.2 \% \mathrm{R}_{\mathrm{o}}\right)$, dry gas onset $\left(2 \% \mathrm{R}_{\mathrm{o}}\right)$, and overmature for gas generation $\left(4 \% \mathrm{R}_{\mathrm{o}}\right)$. Carter and others (1998) estimated the main intervals of oil generation at 0.7 to $0.9 \% \mathrm{R}_{\mathrm{o}}$, and at depths in the deep Anadarko Basin of 2,800 to 3,800 m (eastern) and 2,200 to 3,000 m (western). The Cardott (1989) oil window is 0.6 to $1.3 \% \mathrm{R}_{\mathrm{o}}$, and Cardott and Lambert (1985) estimated the limit of preservation of dry gas at $5 \% \mathrm{R}_{\mathrm{o}}$.

Woodford, Phosphoria, and Tsuzuki HP, Rock Eval (REV), and $\mathrm{R}_{\mathrm{o}}$ curves in figure 18 are modified from Lewan (written commun., 2011). Shown are $\mathrm{R}_{\mathrm{o}}$ signatures for onsetthrough-completion of oil generation using Woodford Shale HP and REV kinetics, and Phosphoria Formation HP kinetics (Lewan and Ruble, 2002). The Pepper and Corvi (1995a, 1995b) kinetics for Type III kerogen is only to illustrate that gas generation can occur at low levels and over a broad range of thermal maturation; in this case, a range of 0.55 to $3.0 \% \mathrm{R}_{\text {. }}$. REV kinetics was not used for the 1D and 4D models because the broad window of oil generation extends from about 0.4 to $2.6 \% \mathrm{R}_{\mathrm{o}}$, which appears to underestimate and overestimate the thermal maturity for oil generation. Phosphoria HP kinetics is specific to Type IIS kerogen, and shows the same early onset of generation at about $0.4 \% \mathrm{R}_{\mathrm{o}}$ with generation completed by $0.8 \% \mathrm{R}_{\circ}$. This would result in early generation of oil, and completion when the $\% \mathrm{R}_{\mathrm{o}}$ is in the oil generation window for source rocks that are low in Type IIS kerogen. However, source rocks that are believed to be thermally immature or marginally mature for oil may be in the oil generation window if they contain Type IIS kerogen. REV and Phosphoria TR maps of potential source rocks in the Colorado and Kansas portions of the province would be thermally mature for oil generation over almost the entire extent because of the onset at $0.4 \% \mathrm{R}_{\mathrm{o}}$ equivalent.

The Woodford TR completion of oil generation roughly outlines the $1.2 \% \mathrm{R}_{\mathrm{o}}$ contour of Cardott (1989). The HP TR onset and completion of oil generation are equivalent to about 0.6 and $1.2 \% \mathrm{R}_{\mathrm{o}}$, respectively, which approximately brackets onset-to-completion of oil generation. Oil cracking to gas starts at about $1.7 \% \mathrm{R}_{\mathrm{o}}$ based on Tsuzuki (1999) HP kinetics (fig. 18). Cardott (1989) indicated that the 1.3-2.0\% $\mathrm{R}_{\mathrm{o}}$ window for the Woodford Shale brackets generation and preservation of condensate and wet gas, with $2.0 \% \mathrm{R}_{\mathrm{o}}$ marking the onset of thermal cracking of oil. The Woodford HP kinetics records onset-through-completion of oil generation and expulsion. However, gas generation and expulsion also occurs during oil generation. Instead of the abrupt onset-throughcompletion of oil and gas generation steps that are implied by $R_{0}$ scales (fig. 20), oil and gas generation overlap. Significant volumes of thermogenic gas can be generated during oil generation, and this generated gas is not restricted to the high thermal maturities of greater than $1.2 \% \mathrm{R}_{\mathrm{o}}$ that represent postoil generation (Lewan, 2002). Timing and volumes of oil and gas generation are dependent upon the kinetics specific to each petroleum source rock.

The figure 19 PetroMod $^{\circledR} 1 \mathrm{D}$ wells use Sweeney and Burnham (1990) kinetics to model downhole $R_{0}$ curves for the Bertha Rogers 1 and Petree Ranch 1 wells (fig. 20), which are compared to gas composition from the Energy geochemistry data base (U.S. Geological Survey, 2010). The calculated surface $R_{o}$ of 0.4 percent represents depth of erosion relative to a thermally immature baseline of about $0.26 \% \mathrm{R}_{\mathrm{o}}$. Distribution of wet and dry gas across the province is illustrated by the methane/(methane+ethane+propane) composition of gas and sample depth (fig. 19). Gas becomes isotopically heavier and chemically dryer with increasing thermal maturity and depth of burial. Pure methane is the 1.0 end member with progressively wetter gases shown by smaller fractions. The gas distribution shows extreme scatter vertically and laterally, probably because of long-distance migration in the province, contributions of gases from different petroleum source rocks, and composition of the contributing source rocks. Gas samples less than 1,000-m depth are almost exclusively from Permian strata in Kansas, and in the panhandle areas of Texas and Oklahoma. Permian gas fields are sourced from petroleum source rocks that are deeper in the basin, and based on the methane gas distribution scale from about 0.7 to 1.0 , from ranges of thermal maturity. Pennsylvanian and Mississippian gases also show considerable scatter, but there is a general trend of increasing dryness with depth. Gas samples 


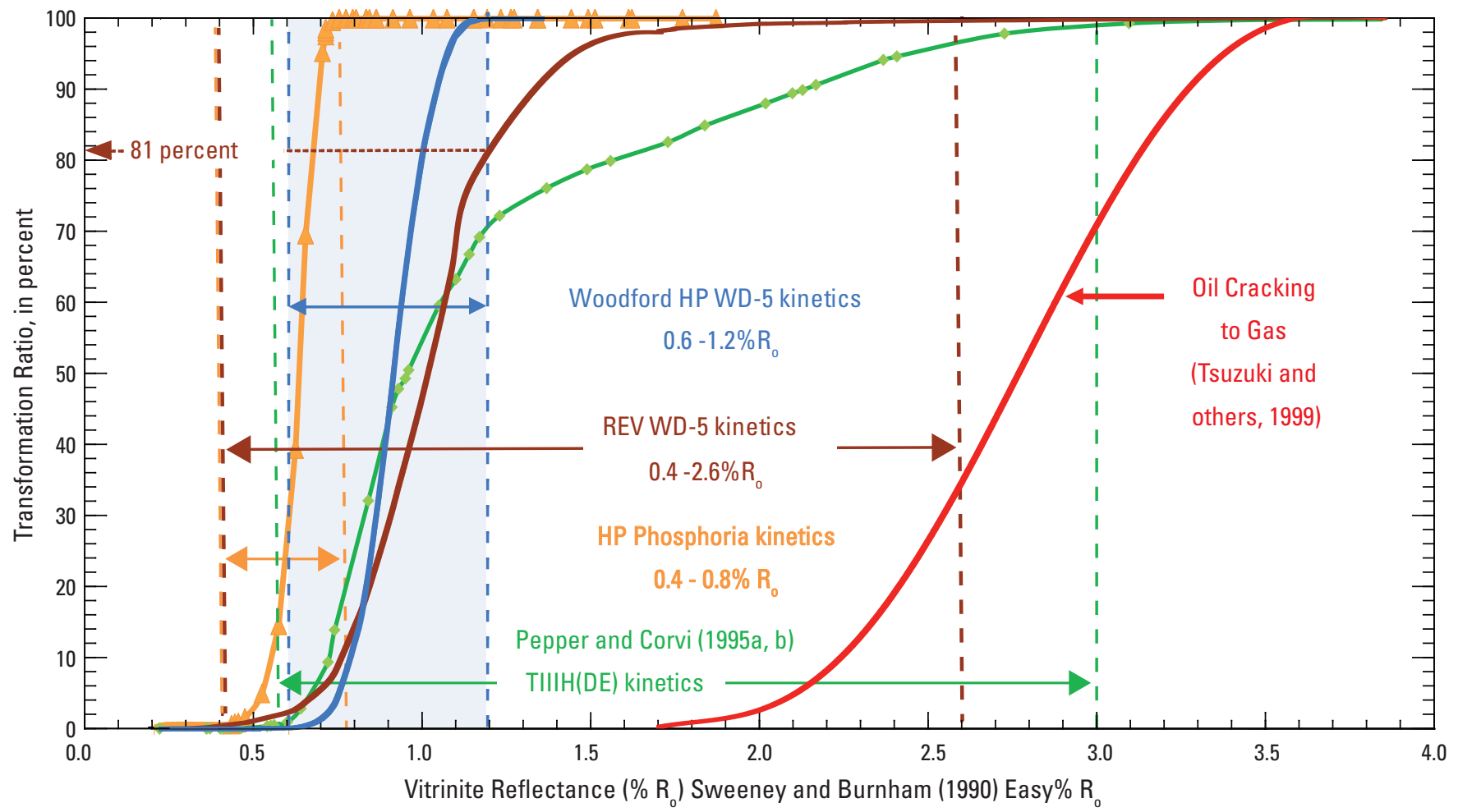

Figure 18. Graphic showing comparison of calculated vitrinite reflectance $\left(\% R_{0}\right)$ and transformation ratios (TR) using Woodford Shale and Phosphoria kinetic algorithms from Lewan and Ruble (2002), gas generation from coals based on Pepper and Corvi (1995a, 1995b), and dry gas generation using Tsuzuki and others (1999). Calculations are for the Bertha Rogers 1 and Petree Ranch 1 wells shown on figure 11. Woodford hydrous pyrolysis (HP) TR of onset- (0.1percent) to-completion of oil generation (99 percent) correlates to a range of about 0.6 to $1.2 \% \mathrm{R}_{\mathrm{o}}$; Woodford Rock Eval pyrolysis (REV) TR range is much broader at approximately 0.4 to $2.6 \% R_{0^{\prime}}$ and Phosphoria HP TR onset and completion are about 0.4 to $0.8 \% R_{0}$. Woodford REV TR is at 81 percent when the HP TR has completed at 100 percent. Pepper and Corvi $(1995 a, 1995 b)$ kinetics provides a range of gas generation from 0.55 to $3.0 \% R_{0}$ from the Type III kerogen in coals. Basin heating rate of $5^{\circ} \mathrm{C} / \mathrm{m}$.y. was based on the Bertha Rogers 1 well; present-day geothermal gradient is $21.3^{\circ} \mathrm{C} / \mathrm{km}$ to the Woodford $\left(19^{\circ} \mathrm{C}\right.$ surface and $200^{\circ} \mathrm{C}$ Woodford temperature at 8,486.5-meter depth). 


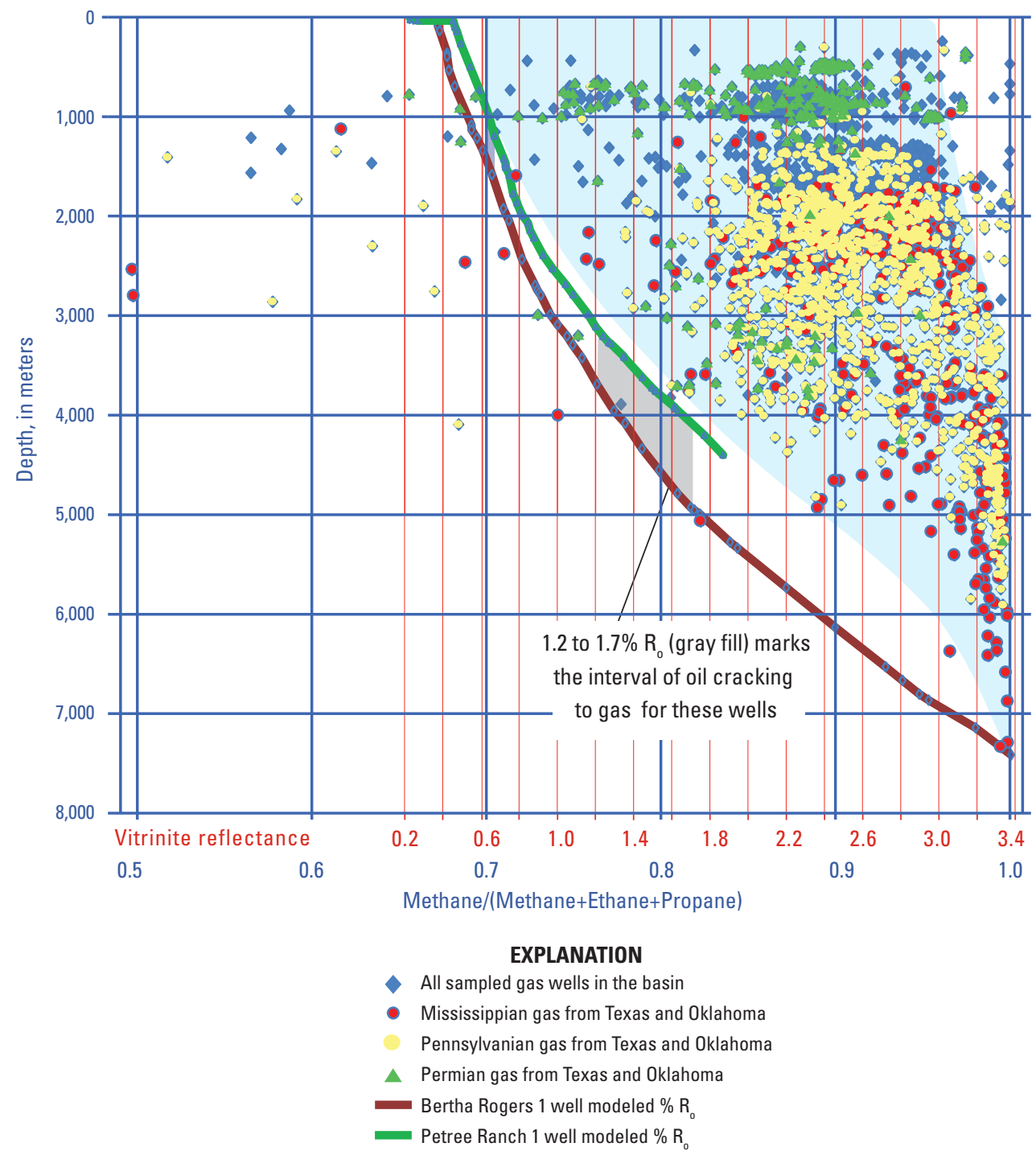

Figure 19. Graph showing depth distribution of wet and dry gas in the Anadarko Basin and of $1 \mathrm{D}$ vitrinite reflectance $\left(\% R_{0}\right)$ profiles for the Bertha Rogers 1 and Petree Ranch 1 wells (fig. 11). Depth range for end of oil generation to start of dry gas generation for the one-dimensional wells is about 3,200 to 5,000 meters. Increase in dry gas fraction and decrease in scatter below 5,000 meters indicates gas is becoming progressively dryer for these samples, with 1.0 being 100 percent methane. Gas data are from the Energy geochemistry data base (U.S. Geological Survey, 2010). The blue symbols are mostly Colorado and Kansas wells. Oklahoma and Texas wells were selected for detailed study because Colorado and Kansas portions of the province are thermally immature for gas generation. Light blue background is a generalized boundary for gas distribution. 


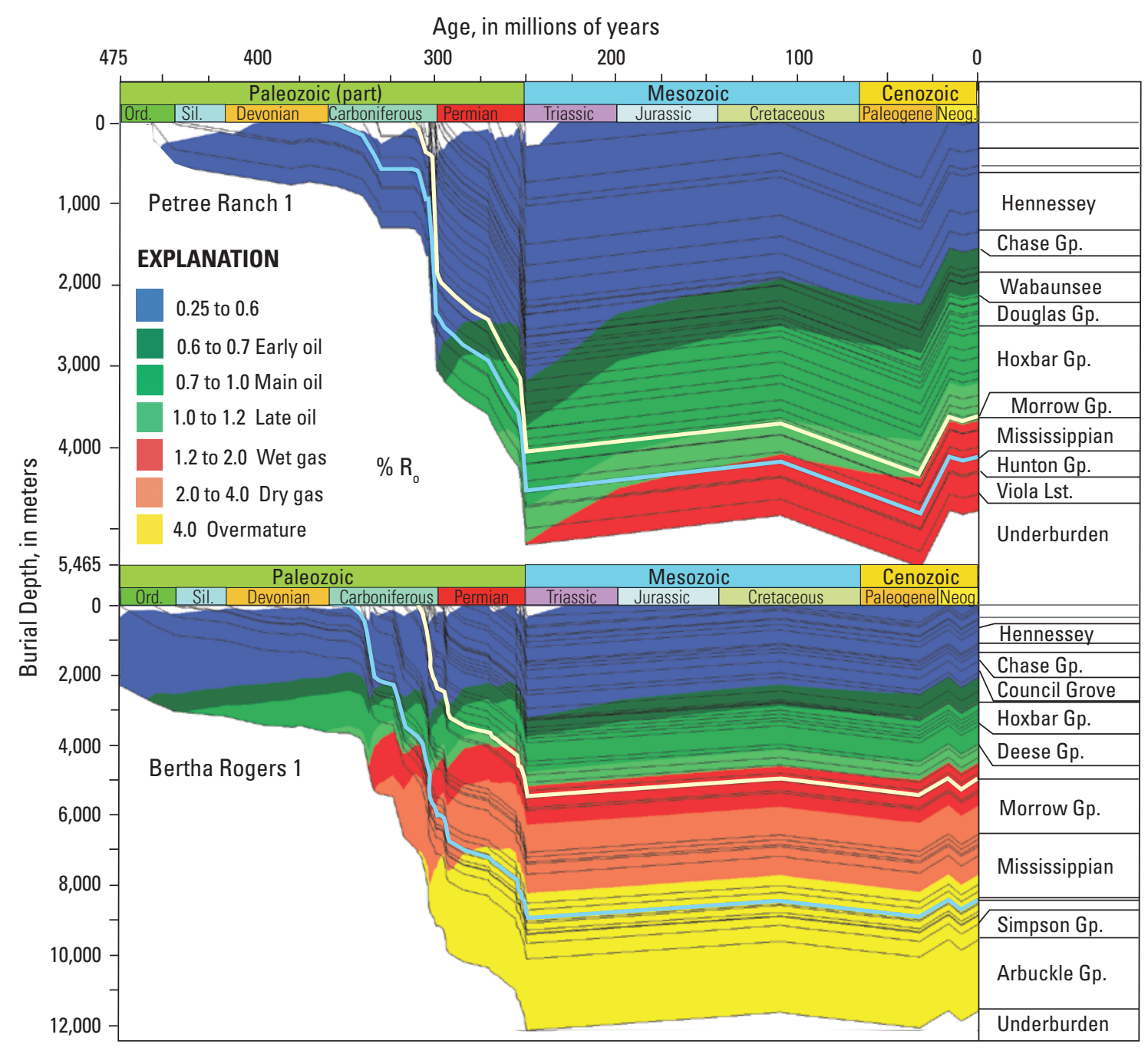

Figure 20. Burial history curves for the Bertha Rogers 1 and Petree Ranch 1 wells. Modeled vitrinite reflectance (percent Ro) through time includes heat flow of about 68 milliwatts per square meter $\left(\mathrm{mW} / \mathrm{m}^{2}\right)$ to 260 million years ago (Ma) followed by $42 \mathrm{~mW} / \mathrm{m}^{2}$ for Bertha Rogers 1, and 68 $\mathrm{mW} / \mathrm{m}^{2}$ to $260 \mathrm{Ma}$ followed by $52 \mathrm{~mW} / \mathrm{m}^{2}$ for Petree Ranch 1. Pale-yellow and blue lines follow the Thirteen Finger limestone and Woodford Shale, respectively. Gp., Group; Lst., Limestone; Ord., Ordovician; Sil., Silurian, Neog., Neogene. 
greater than 5,000 $\mathrm{m}$ approach the methane composition. Any correlation of gas with modeled $\mathrm{R}_{\mathrm{o}}$ is generalized, in that gas dryness increases with burial depth with associated increase in thermal maturation.

Vitrinite reflectance history profiles for Petree Ranch 1 and Bertha Rogers 1 wells (fig. 20) show similar trends of increased burial and maturation that start about $350 \mathrm{Ma}$. The Woodford Shale is overmature for gas generation in Bertha Rogers 1, and is in the gas generation window for Petree Ranch 1. The Thirteen Finger limestone in Bertha Rogers 1 is in the wet gas window, and for Petree Ranch 1 is on the boundary between the $1.2 \% \mathrm{R}_{\mathrm{o}}$ end of oil generation and start of wet gas generation. The actual boundaries between oil and gas generation are more gradual, with wet gas generation within the oil generation window.

\section{Burial History}

Burial history trends through time across the province record (1) relatively slow rates of deposition until about $350 \mathrm{Ma}$ that is associated with the Cambrian through Early Mississippian development of the southern Oklahoma trough, (2) more rapid rates of deposition in the deep basin from early Carboniferous through Leonardian (fig. 2) that is associated with late Paleozoic tectonism and formation of the basin, and (3) middle Permian to present periods of hiatus and (or) deposition and erosion in the deep basin, and slower rates of deposition on the shelf. Because most of the Permian (Guadalupian) through Mesozoic section is missing in the Oklahoma and Texas portions of the basin, reconstruction of this section is based largely on deposition and erosion of strata to the north and west.

Based on 4D model Woodford HP and vitrinite reflectance kinetics, thermal maturation of petroleum source rocks in the basin began as early as Devonian in the deep basin and was essentially completed by the end of the Permian. Woodford $\mathrm{HP}$ and $\mathrm{R}_{\mathrm{o}}$ kinetics resulted in similar onset of oil generation. The Phosphoria HP kinetics is designed for Type IIS source rocks and had a narrow range of oil generation that corresponded to about 0.4 to $0.8 \% \mathrm{R}_{\mathrm{o}}$; this information is provided here in case Type IIS source rocks are present in the basin. A period of Permian-Cretaceous deposition and erosion was modeled from about 250 to $110 \mathrm{Ma}$. This was included to evaluate effects on heating history, and because strata from all or most of this age range are absent across the province. Effects were minimal for this $500 \mathrm{~m}$ of eroded strata in Bertha Rogers 1 because it followed most petroleum generation in the basin. The second phase mainly followed onset of the Laramide orogeny, which began in the southern Rocky Mountains about 67.5 Ma and ended about $50 \mathrm{Ma}$ (Tweto, 1975). The Laramide orogeny influenced uplift, tilting, and erosion such that Mesozoic strata and erosional remnants are present in the Kansas and Colorado portions of the basin, but absent from all but a few scattered places in the Texas and Oklahoma portions. Laramide uplift and erosion resulted in widespread deposition of Ogallala Formation sediments on the plains
(Jorgensen, 1989), which was followed by the last phase of erosion about $10 \mathrm{Ma}$; the base of the eastward-thinning Ogallala aquifer wedge was derived from data in Cederstrand and Becker (1998). Ogallala Formation fluvial and eolian sediments were deposited upon an erosional surface of Permian strata and locally on Jurassic or Cretaceous formations; the Ogallala Formation probably mantled almost all the entire basin, but post-Tertiary erosion has removed much of it with only 200-600 ft remaining in the western part of the basin (Johnson, 1989).

1D burial histories for the Bertha Rogers 1 and Petree Ranch 1 wells (figs. 21-23) use Woodford and Phosphoria HP kinetics from Lewan and Ruble (2002). Bertha Rogers 1 is the deepest well in the onshore United States at 31,441 feet (9,583 m); it reaches total depth in the Arbuckle Group (fig. 2) and was productive of gas from the Atokan granite wash sequence (Rowland, 1974; Davis and Northcutt, 1989; IHS Energy, 2009a). A small show of high-sulfur gas was recorded at the surface, and sulfur crystals were circulated to the surface after this overpressured well was controlled [bottom hole pressure was 24,835 pounds per square inch $\left(\mathrm{lb} / \mathrm{in}^{2}\right)$ ] (Rowland, 1974; Davis and Northcutt, 1989). Arbuckle strata were the source of the sulfur, but the source of sour oil and gas in other areas of the basin is not known. Providing the Phosphoria kinetics gives timing of generation for potential Type IIS source rocks.

Burial history charts created for the Bertha Rogers 1 well were calibrated to $\mathrm{R}_{\mathrm{o}}$ data from Price (1997), and to corrected bottom-hole temperatures (BHT) from Gallardo and Blackwell (1999, p. 348) which were based primarily on their discussion and table of predicted temperatures that were approximately $21^{\circ} \mathrm{C}$ greater than measured BHT. Respective TR oil generation percentages for onset, peak, and completion are $0.1,50$, and 99, and modeled $R_{0}$ equivalent maximum values of 4.66 percent are shown on the $\mathrm{R}_{\mathrm{o}}$ versus depth chart as the vertical line segment (fig. 21). Measured $R_{o}$ from Price (1997) were about 5.3 percent, 4.9 percent and 6.0 percent for the Woodford Shale, Hunton Group, and Sylvan Shale (fig. 2), respectively. Strata older than the Hunton (Silurian) precede the existence of deciduous plants, which are the source of vitrinite, so Silurian and older strata more likely record pyrobitumen and are therefore suspect. I was not able to fit modeled Woodford $\mathrm{R}_{\mathrm{o}}$ data to measured $\mathrm{R}_{\mathrm{o}}$ or temperature using stable heat flow (HF) values through time. Lee and Deming (1999) indicated an absence of evidence that the thermal state of the Anadarko Basin has changed significantly since the late Paleozoic (250 Ma). Carter and others (1998) applied constant HF through the Paleozoic for their 1D models, although they indicated that the Arbuckle Group may have been subjected to a slightly higher HF because of decay of the Cambrian rifting. They ascribe a Late Pennsylvanian and Early Permian increase in measured thermal maturity on the models because of heating effects of shales that dominated the Pennsylvanian section. 1D models in the deep basin were best calibrated using variable heat flows (HF) through time. Modeled HF for Bertha Rogers 1 was $68 \mathrm{~mW} / \mathrm{m}^{2}$ from Cambrian to $260 \mathrm{Ma}$, then $42 \mathrm{~mW} / \mathrm{m}^{2}$ to the 

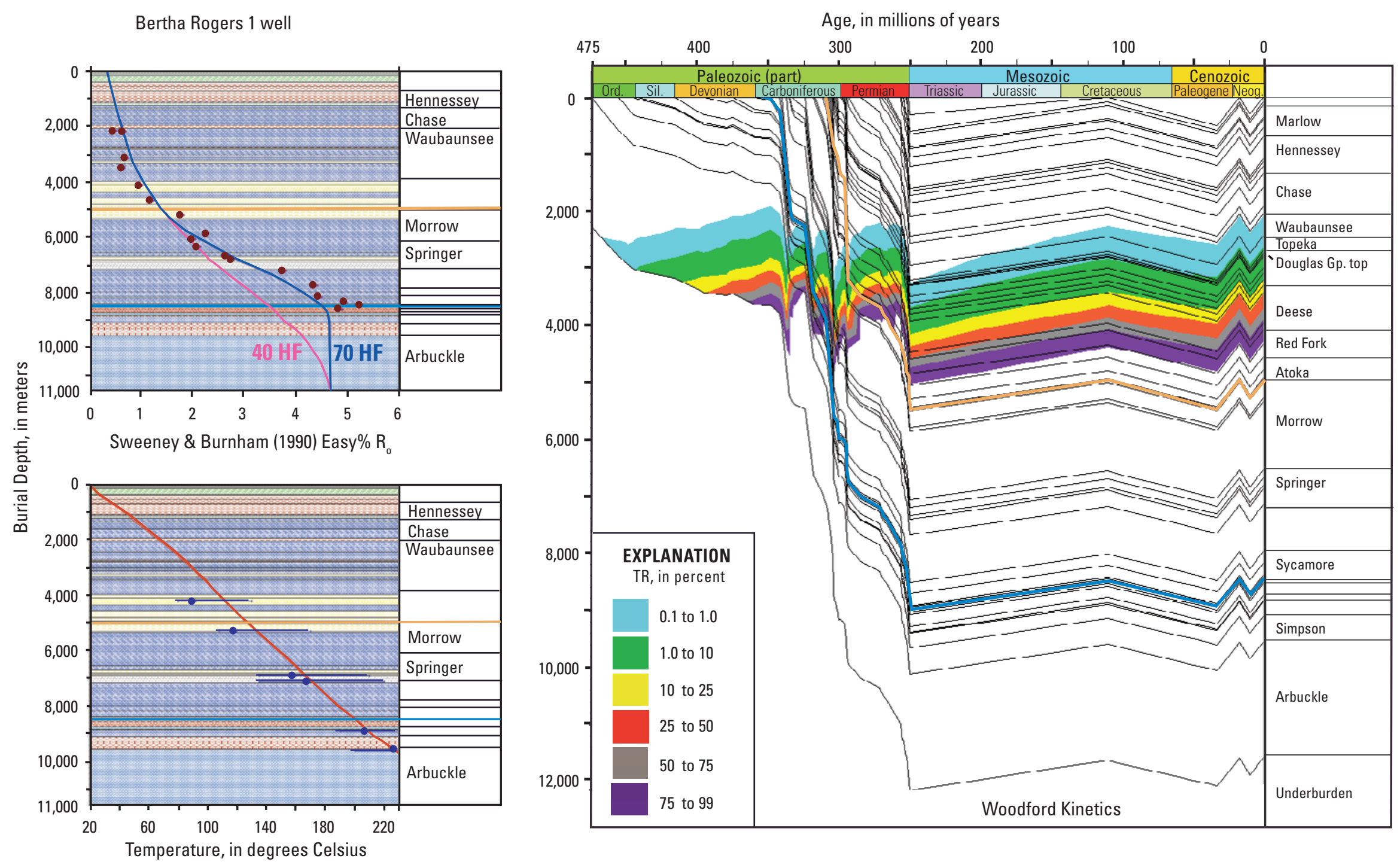

Figure 21. Burial history transformation ratios (TR) for the Bertha Rogers 1 well based on Woodford Shale hydrous pyrolysis (HP) kinetics (Lewan and Ruble, 2002). Depth compared to temperature and vitrinite reflectance profiles are calibrated at (1) 40 milliwatts per square meter $\left(\mathrm{mW} / \mathrm{m}^{2}\right)$ heat flow (HF) through time, and (2) $70 \mathrm{~mW} / \mathrm{m}^{2}$ to 260 million years ago (Ma) followed by $42 \mathrm{~mW} / \mathrm{m}^{2}$ to present. Woodford Shale interval is marked with the blue line and the Thirteen Finger limestone by the gold line. Vitrinite reflectance (\% R) calibration data are from Price (1997). Temperature dots are corrected bottom hole temperatures (BHT) from Gallardo and Blackwell (1999, fig. 8) and bars show range from measured to maximum estimated BHT. Gp., Group; Ord., Ordovician; Sil., Silurian, Neog., Neogene. 

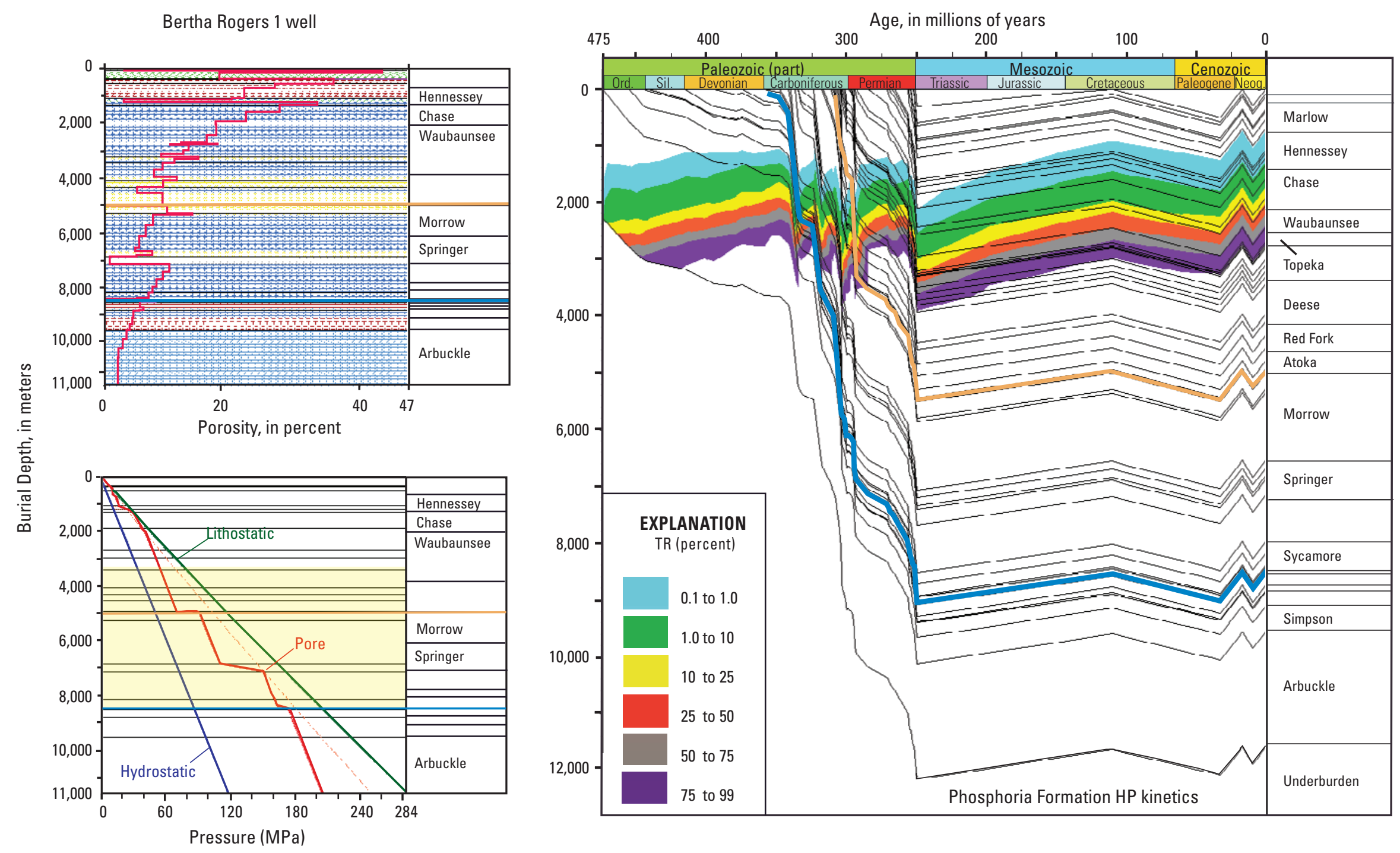

Figure 22. Burial history for the Bertha Rogers 1 well based on Phosphoria Formation hydrous pyrolysis (HP) kinetics (Lewan and Ruble, 2002). Depths are shown relative to calculated porosity, lithostatic, pore, and hydrostatic pressure; light blue line is hydrostatic pressure of $9,800 \mathrm{Pascals} / \mathrm{meter}(\mathrm{MPa} / \mathrm{m})(0.465 \mathrm{psi} / \mathrm{ft})$. Yellow band is probable zone of overpressure based on Al-Shaieb and others (1994). Woodford Shale interval is marked with the blue line and the Thirteen Finger limestone by the gold line. Ord., Ordovician; Sil., Silurian, Neog., Neogene. 
Petree Ranch 1 well

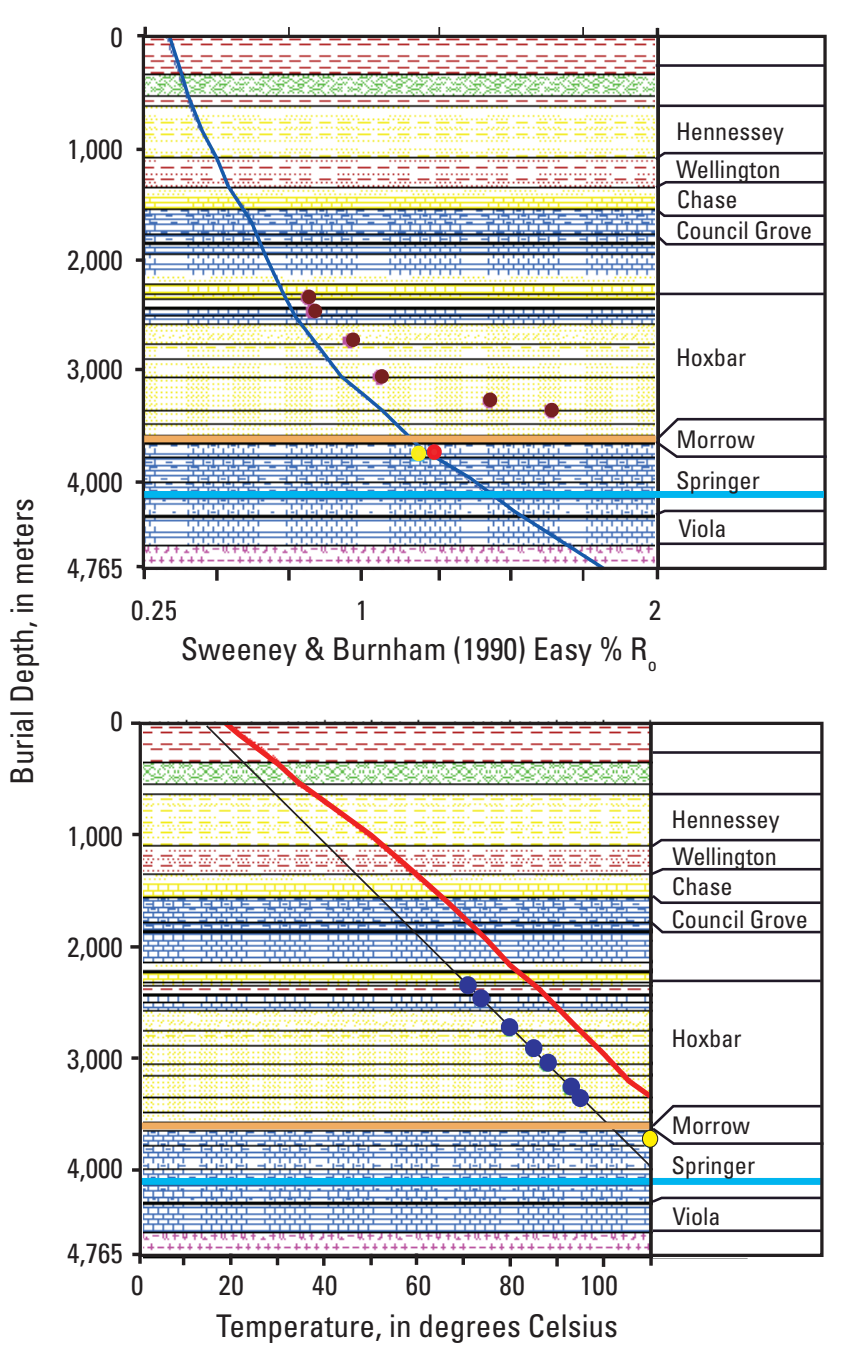

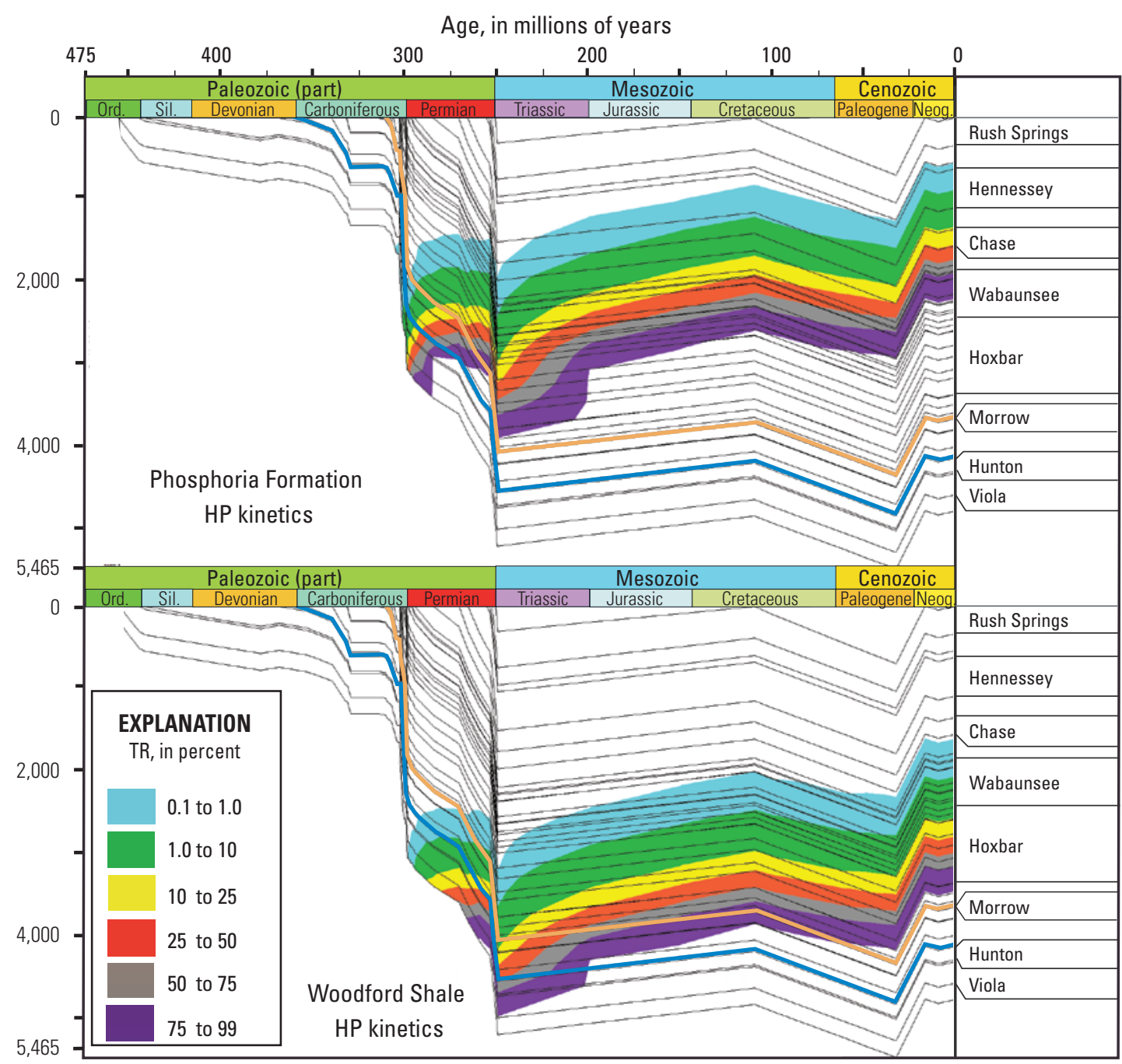

Figure 23. Burial history for the Petree Ranch 1 well based on Woodford Shale and Phosphoria Formation hydrous pyrolysis (HP) kinetics (Lewan and Ruble, 2002). Model is calibrated using 68 milliwatts per square meter $\left(\mathrm{mW} / \mathrm{m}^{2}\right)$ heat flow to 260 million years before present (Ma), then $52 \mathrm{~mW} / \mathrm{m}^{2}$ to present. Woodford Shale interval is marked on the transformation ratio (TR) charts with the blue line, and the Thirteen Finger limestone by the gold line. Vitrinite reflectance (\% $\mathrm{R}_{0}$ ) calibration data are from Pawlewicz (1989) (brown dots) and Woodford Shale estimates from Cardott (1989) (yellow and red dots). Temperature data from Pawlewicz (1989, table 2) (blue dots and connecting line) were calculated based on $1.3^{\circ} \mathrm{F}$ increase per 100 feet depth from a $15^{\circ} \mathrm{C}\left(60^{\circ} \mathrm{F}\right.$ ) surface temperature. The corrected bottom hole temperature (BHT) (yellow dot) was derived using Gallardo and Blackwell (1999, fig. 7). Ord., Ordovician; Sil., Silurian, Neog., Neogene. 
present (figs. 21 and 22). Petree Ranch 1 was assigned an HF of $68 \mathrm{~mW} / \mathrm{m}^{2}$ to $260 \mathrm{Ma}$, then $52 \mathrm{~mW} / \mathrm{m}^{2}$ to present (fig. 23). Present-day HF in the area of the wells is about $54 \pm 4 \mathrm{~mW} /$ $\mathrm{m}^{2}$ based on Carter and others (1998) and 45-49 $\mathrm{mW} / \mathrm{m}^{2}$ based on Blackwell and Richards (2004); these sources were used to generate an HF map of the basin that was applied through time for the 4D model. The 4D model did not use variable heat flow through time, a possible effect of which could be a slight underestimation of the level of thermal maturation in the deep basin.

The Bertha Rogers 1 temperature-depth profile calibrates fairly well with measured temperature (fig. 21); however, measured $\mathrm{R}_{\mathrm{o}}$ exhibits considerable spread. All $\mathrm{R}_{\mathrm{o}}$ depth curves were preferentially calibrated to the Woodford Shale. The spread in $\mathrm{R}_{\mathrm{o}}$ data for source rocks of the Anadarko Basin may have been influenced by various data quality and vitrinite suppression factors. Cardott (1989) indicated increased anisotropy of $\mathrm{R}_{\mathrm{o}}$ histograms starting at $1.0 \% \mathrm{R}_{\mathrm{o}}$, with a noticeable effect at greater than $\% \mathrm{R}_{\mathrm{o}}$ (Dow, 1977; Hunt, 1979). This suggests that the $\mathrm{R}_{\mathrm{o}}$ /depth curve deflection that begins at about $2 \% \mathrm{R}_{\mathrm{o}}$ could be influenced by the "increasing anisotropy of the vitrinite and associated need to measure maximum reflectance; which cannot be done using random reflectance measurements" according to Brian Cardott (written commun., June 2011). Mixing of macerals such as the mainly marine Type II of the Woodford Shale with Type III vitrinite from recycled woody organic matter should not have increased $\mathrm{R}_{\mathrm{o}}$ because there were few older source rocks that would have contained vitrinite (Cardott, 1989). Mixing should have minimal effect on increase in maturation in younger source rocks mainly because depositional processes predominated in the basin, and vitrinite macerals from sediment input from the north and west were probably less thermally mature. If mixing occurred, then the main effect could have been a decrease in measured maturation. However, mixing of macerals is generally detected during analysis of the vitrinite. Scatter in $\mathrm{R}_{\mathrm{o}}$ data and calibration problems can also result from suppression of vitrinite from liptinite macerals; these "oily" particles could decrease the reflectance of vitrinite macerals. Cardott (1989) suspected that Woodford Shale $\mathrm{R}_{\mathrm{o}}$ values less than $1.3 \% \mathrm{R}_{\mathrm{o}}$ were suppressed, but did not believe this continued past 1.3 percent because of the increase in reflectance of liptinite macerals above this level. In contrast, Price and Barker (1985, p. 80) believed potential suppression extended to at least $4.0 \% \mathrm{R}_{\mathrm{o}}$.

Overpressure can retard organic matter maturation and associated $\mathrm{R}_{\mathrm{o}}$ (Hao and others, 2007). That the Woodford Shale in the deep basin is overpressured (Al-Shaieb and others, 1994) could have decreased reflectance of vitrinite. The effect of suppressed vitrinite on the Petree Ranch 1 well would be to underestimate Woodford Shale $\mathrm{R}_{\mathrm{o}}$ values. This could be a factor in increased reflectance of shallower formations, although measured reflectance in other wells from Pawlewicz (1989) show considerable scatter, including one well in which vitrinite reflectance decreases downhole. Pressure and temperature data are not available for most wells in the Oklahoma portion of the basin; estimated downhole temperatures for the Petree Ranch 1 well (fig. 23) are from
Pawlewicz (1989) and Gallardo and Blackwell (1999). I was not able to fit the model to estimated downhole temperature for this well, possibly because of the quality of temperature data, and that Pawlewicz (1989) used a cooler surface temperature of $15^{\circ} \mathrm{C}$ compared to the $19^{\circ} \mathrm{C}$ assigned in this study. His geothermal gradient was 23.7 degrees Celsius per kilometer $\left({ }^{\circ} \mathrm{C} / \mathrm{km}\right)$, compared to the modeled geothermal gradient of $24.57{ }^{\circ} \mathrm{C} / \mathrm{km}$; the curved line was also influenced by thermal conductivity of downhole lithofacies.

The onset and completion of oil generation shown on table 3 are based on $0.1 \%$ TR $\left(0.6 \% \mathrm{R}_{\mathrm{o}}\right)$ and $0.99 \% \mathrm{TR}$ $\left(1.2 \% \mathrm{R}_{\mathrm{o}}\right)$, respectively. These boundaries were based on Woodford HP kinetics that was applied to petroleum system models. Onset of oil cracking to gas is at 1.7\% $\mathrm{R}_{\mathrm{o}}$. Although the burial history plots show abrupt onset and completion for thermal maturity, the time ranges of petroleum generation are more gradual. Figure 24 shows oil generation through time for the Oil Creek Formation, Woodford Shale, and Thirteen Finger limestone petroleum source rocks. These are displayed as onset to completion, rather than volumes, partly because thickness of the Oil Creek layer is generalized and does not represent the actual thickness of the source interval. Oil generation and expulsion started about $370 \mathrm{Ma}$ with the Oil Creek source rocks, reached peak generation about 290 Ma to 220 Ma with contributions from all three source rocks, and was relatively dormant until about $80 \mathrm{Ma}$ when Laramide burial caused a brief increase in generation and expulsion (table 3, fig. 24). The primary factor causing increased thermal maturation is burial depth. For example, the Woodford Shale entered the oil generation window about 30 million years (m.y.) before the Thirteen Finger limestone because of deposition of thousands of feet of intervening Mississippian and Morrowan strata. Because Anadarko has a deep basin and shallow shelf, age ranges for hydrocarbon generation vary laterally as well, which allowed for longer periods of oil and gas generation, migration, and trapping. Carter and others (1998) indicated that Upper Pennsylvanian to Lower Permian source rocks in the deep basin moved into the hydrocarbon generation window during Cretaceous burial of the region. This is somewhat more recent than the Missourian onset of generation about $260 \mathrm{Ma}$ for the 4D and 1D Bertha Rogers 1 models. The primary factor between the different onset times of Carter and others (1998) and my $1 \mathrm{D}$ models is that their maximum burial and erosion followed $110 \mathrm{Ma}$, and mine was about $250 \mathrm{Ma}$. Missourian source rocks in the Bertha Rogers 1 well are now in the oil generation window $\left(0.9 \% \mathrm{R}_{\mathrm{o}}\right)$. If Virgilian and (or) Permian source rocks exist, their onset of generation would have been about 230 Ma using values of 0.1 percent TR for the Bertha Rogers 1 and Petree Ranch 1 wells (figs. 21 and 23).

Calculations also include burial depth compared to measured and modeled downhole porosity and pressure. Because these data were not available for calibration, only the modeled values are shown in figure 22. Porosity and pressure are independent of the applied kinetics and are largely dependent upon burial depth and physical properties of assigned lithofacies. Jumps in pore pressure with depth are tied to effects of 
Table 3. Onset of oil generation in the Anadarko Basin 4D model, and onset and completion dates in millions of years before present $(\mathrm{Ma})$ for the Bertha Rogers 1 and Petree Ranch 1 one-dimensional models based on Woodford Shale hydrous pyrolysis (HP) kinetics (Lewan and Ruble, 2002). The Douglas Group overlies the youngest potential petroleum source rocks on figure 2. The (HP) kinetics-based onset of oil generation is 0.1 percent transformation ratio (TR) and completion is 99 percent TR. Oil generation histories in the four-dimensional model were recorded at $10 \mathrm{Ma}$ increments, and the plus symbol indicates onset was before the listed age.

\begin{tabular}{|c|c|c|c|c|c|}
\hline \multicolumn{2}{|c|}{ Four Dimensional Model } & \multicolumn{2}{|c|}{ Bertha Rogers 1} & \multicolumn{2}{|c|}{ Petree Ranch 1} \\
\hline & Onset & Onset & End & Onset & End \\
\hline Virgilian (Douglas Group) & 270 & 100 & & & \\
\hline Thirteen Finger limestone & +300 & 290 & 265 & 270 & \\
\hline Woodford Shale & 330 & 330 & 310 & 290 & 200 \\
\hline Oil Creek Formation & +370 & 340 & 320 & & \\
\hline
\end{tabular}

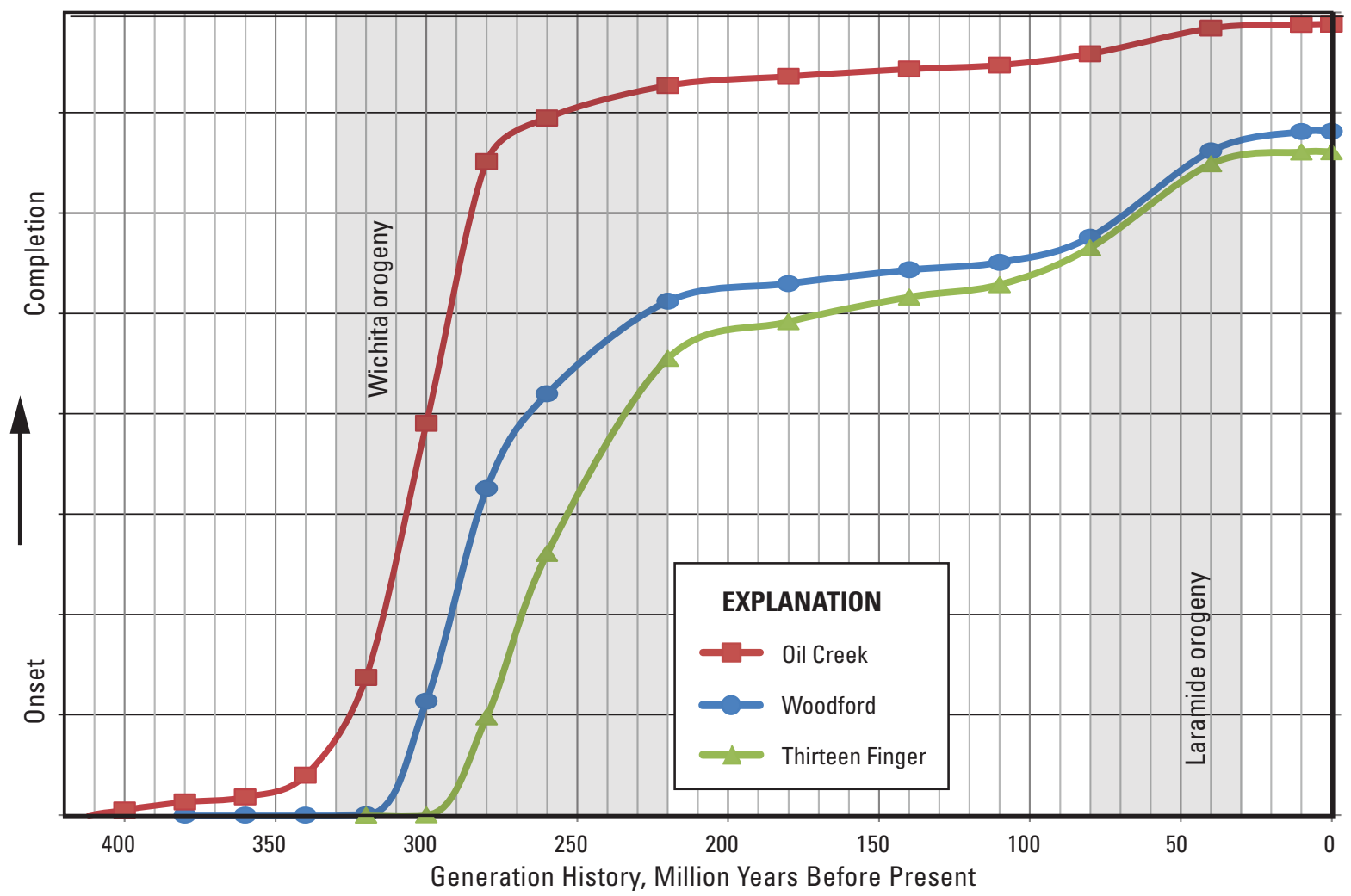

Figure 24. Graph showing history of generation of oil from the Oil Creek Formation, Woodford Shale, and Thirteen Finger limestone sources in the four-dimensional petroleum system model based on Woodford Shale HP kinetics. Gray bars delineate periods of peak oil generation. Early oil generation from the Oil Creek layer, prior to about 70 million years before present (Ma), is in a narrow band that is proximal to the Wichita Mountain uplift. This band exceeds maximum depth of the formation and is the result of poor data control in the area bordering the fault zone. The error could have been removed by clipping the four-dimensional model extent to the basin axis and northward, but less of the model would have been visible and usable. 
near-surface evaporite seals and porosity of various lithologies, such as shale and low-permeability sandstone and limestone. The pressure/depth increases for the Woodford (blue line) and Thirteen Finger (gold line) strata are because of these and bounding low-permeability strata. The entire well is overpressured in this $1 \mathrm{D}$ model. The actual zone of overpressure is probably limited to Woodford through Desmoinesian strata (yellow zone, fig. 22) based on Al-Shaieb and others (1994). Because 1D models are points and are not influenced by 3D effects of PVT, pressure increase is tied to lithofacies and rate of burial depth.

Changes in thermal maturation laterally and through time are more easily viewed on the cross section extractions from the 4D model at $300 \mathrm{Ma}$ and at present (fig. 25). The Woodford Shale yellow dot for the Bertha Rogers 1 location was mature for oil generation at $300 \mathrm{Ma}$, and is currently overmature. The 4D model and associated extractions are based on elevation relative to sea level and generated 1D models are depth from ground surface. Only the deep basin was thermally mature for oil and gas at $300 \mathrm{Ma}$ (fig. 25) compared to the present generation range of mature for oil to overmature for gas. Southern Kansas is now only marginally mature for oil generation.

Modeled map views of present-day thermal maturation are shown for Arbuckle through Douglas Groups 4D layers in figure 26. Calculated $\mathrm{R}_{\mathrm{o}}$ is displayed for each model layer. The red contours represent the $99 \%$ TR in the transformation ratio maps, and approximates the end of oil generation at $1.2 \% \mathrm{R}_{0}$. The Douglas layer overlies potential Missourian source rocks for which the deep basin is in the oil-to-gas generation window. There are no potential source rocks in the Sierra Grande uplift or Cimarron arch (fig. 8) and the lower levels of thermal maturity in those areas represent only shallower overburden and lower basement heat flow (fig. 3). The Colorado and Kansas portions of the province are almost exclusively immature to marginally mature for oil generation. Elevated thermal maturation in the Las Animas arch and CKU also have increased basement heat flow (fig. 3). Basinward increases in thermal maturation primarily reflect greater overburden thickness. This is why the Texas and Oklahoma portions of the province exhibit the most change from the Arbuckle to Douglas layers. Thermal conductivity of lithofacies also impacts the heat of underlying formations. The Oil Creek layer map image is not shown, but is only slightly less mature than that of the underlying Arbuckle layer. The Woodford Shale is located primarily in the Texas and Oklahoma portions of the Anadarko Basin Province (fig. 1), and this layer incorporates extents of underlying formations. This is why the oil generation window (yellow) in Colorado is almost identical for the Woodford, Viola, and Arbuckle; this area of the layer is primarily the top of the Arbuckle Group. Figure 16 shows the extent of the Thirteen Finger limestone. Almost the entire unit in the deep basin of Oklahoma and Texas is in the oil and gas generation window.

Map images of thermal maturation through time are displayed in figure 27 based on transformation ratios, and the trends are similar for $\mathrm{R}_{\mathrm{o}}$ through time. Maturation started in the deep basin and expanded radially northward through time for each source rock. The largest increase in maturation corresponds to that of 1D models and is tied to basin downwarping and associated increased sediment deposition from about 350 to $250 \mathrm{Ma}$. Maturation continues past $240 \mathrm{Ma}$, but at a slower rate. 


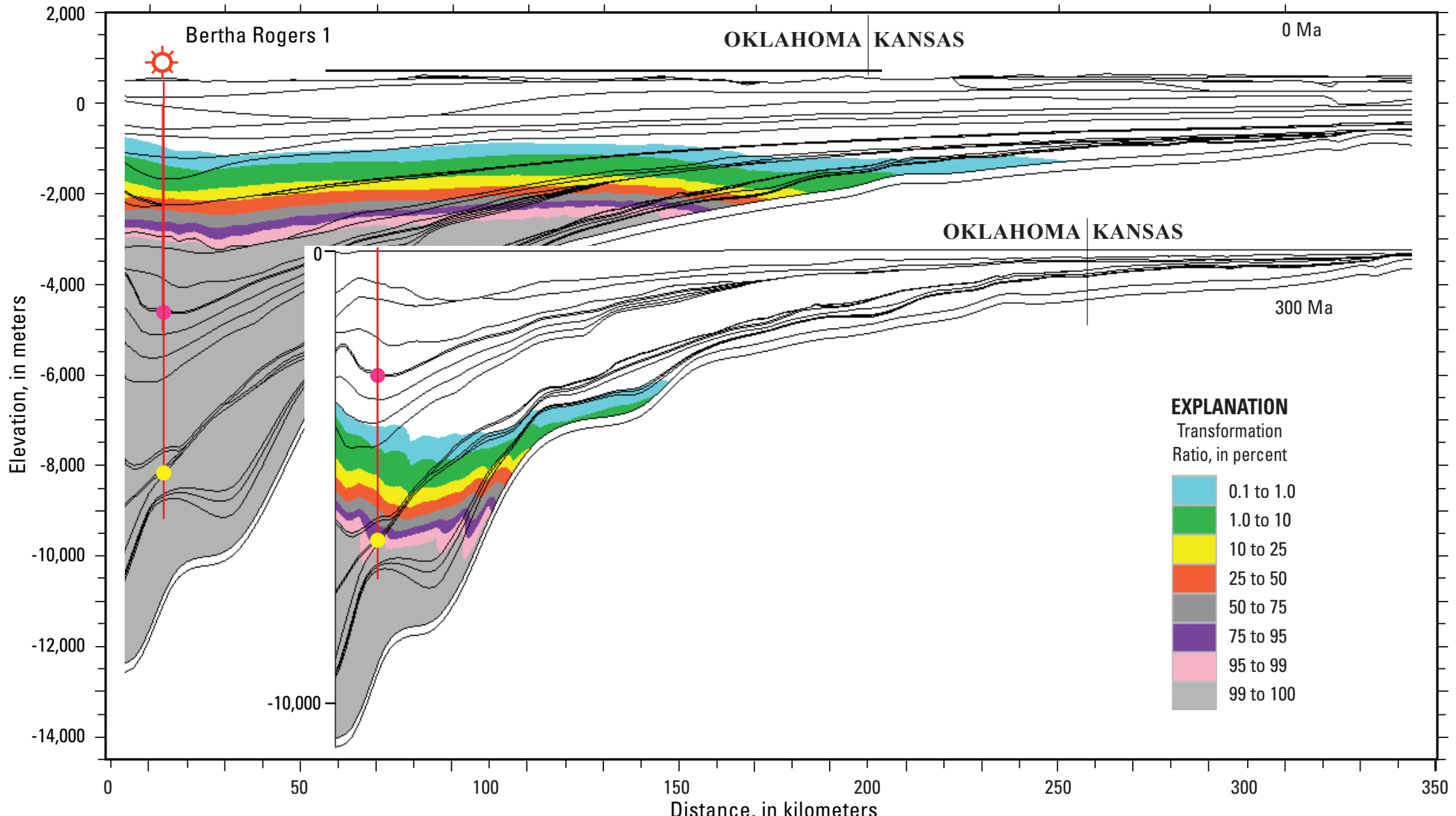

Figure 25. South to north cross sections of thermal maturation using Woodford transformation ratio (TR) at present (0 Ma) and at 300 million years before present (Ma). The inset shows the location of these extracts from the four-dimensional model. Yellow and pink dots are approximate locations of the Woodford Shale and Thirteen Finger limestone, respectively. Elevation is relative to sea level, and vertical and lateral scales are equivalent for the cross sections. Map image is TR on the Woodford Shale layer at $0 \mathrm{Ma}$ and at 10 times vertical exaggeration. Red line is the approximate location of the cross section.

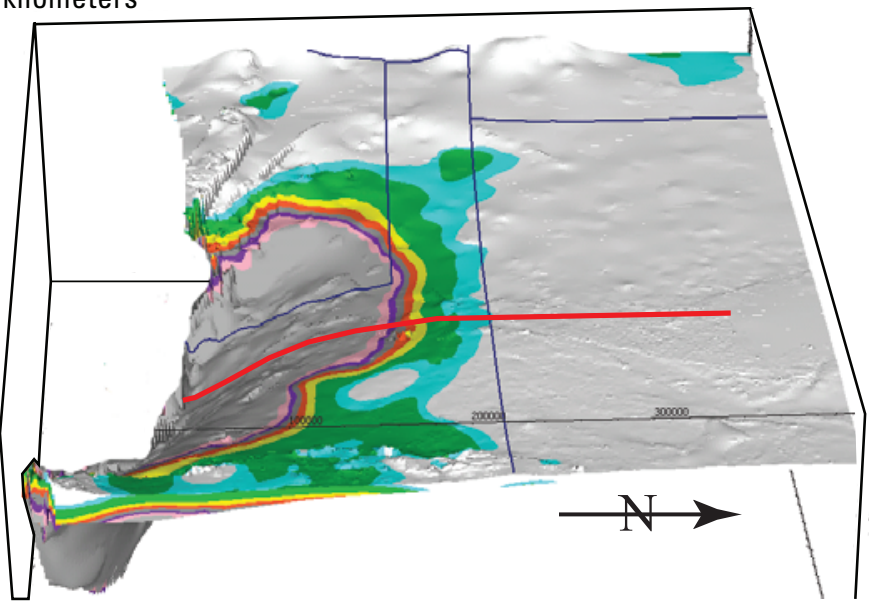



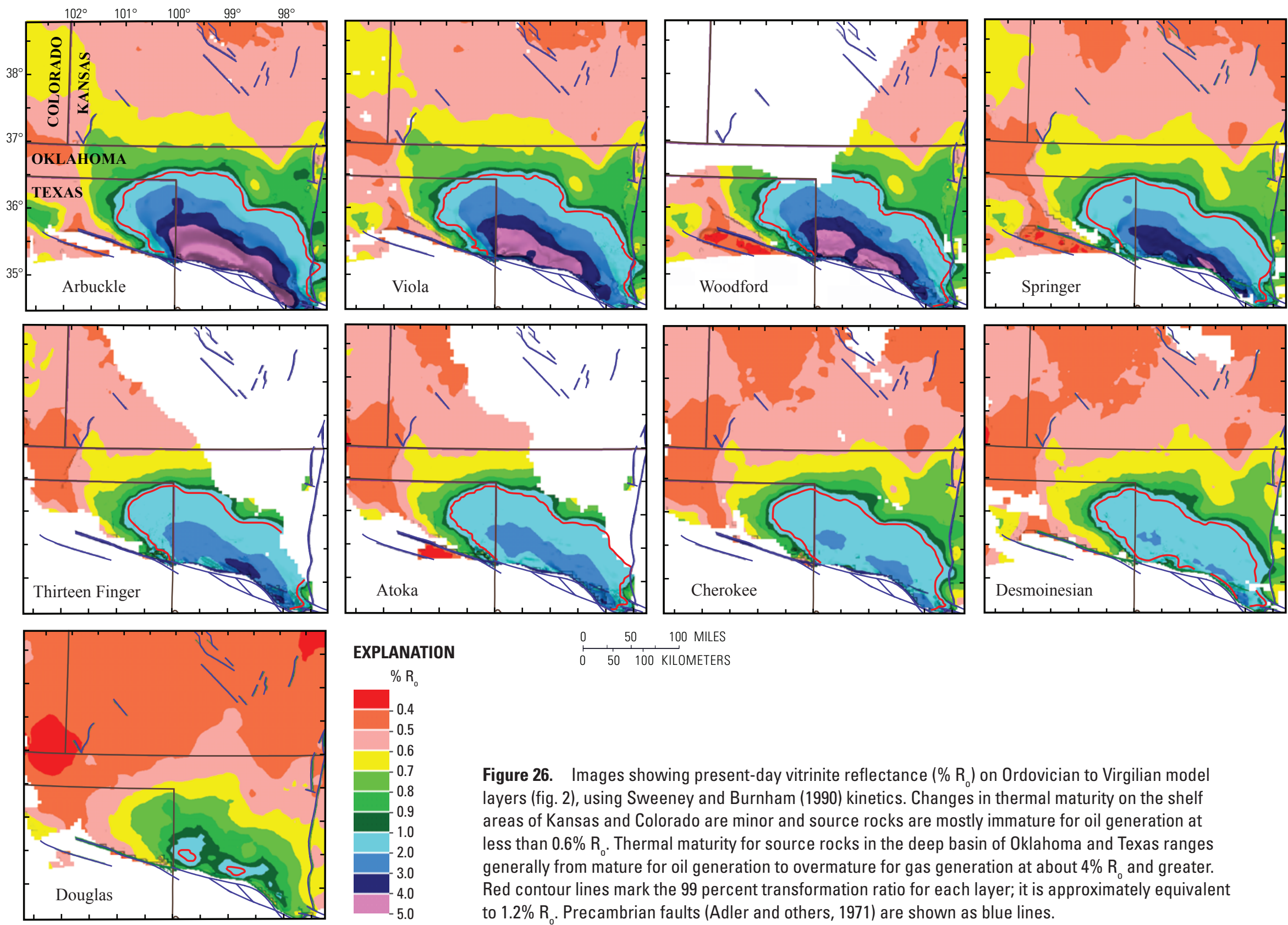

Figure 26. Images showing present-day vitrinite reflectance $\left(\% R_{0}\right)$ on Ordovician to Virgilian model layers (fig. 2), using Sweeney and Burnham (1990) kinetics. Changes in thermal maturity on the shelf areas of Kansas and Colorado are minor and source rocks are mostly immature for oil generation at less than $0.6 \% \mathrm{R}_{\mathrm{o}}$. Thermal maturity for source rocks in the deep basin of Oklahoma and Texas ranges generally from mature for oil generation to overmature for gas generation at about $4 \% R_{0}$ and greater. Red contour lines mark the 99 percent transformation ratio for each layer; it is approximately equivalent to $1.2 \% R_{0}$. Precambrian faults (Adler and others, 1971) are shown as blue lines. 

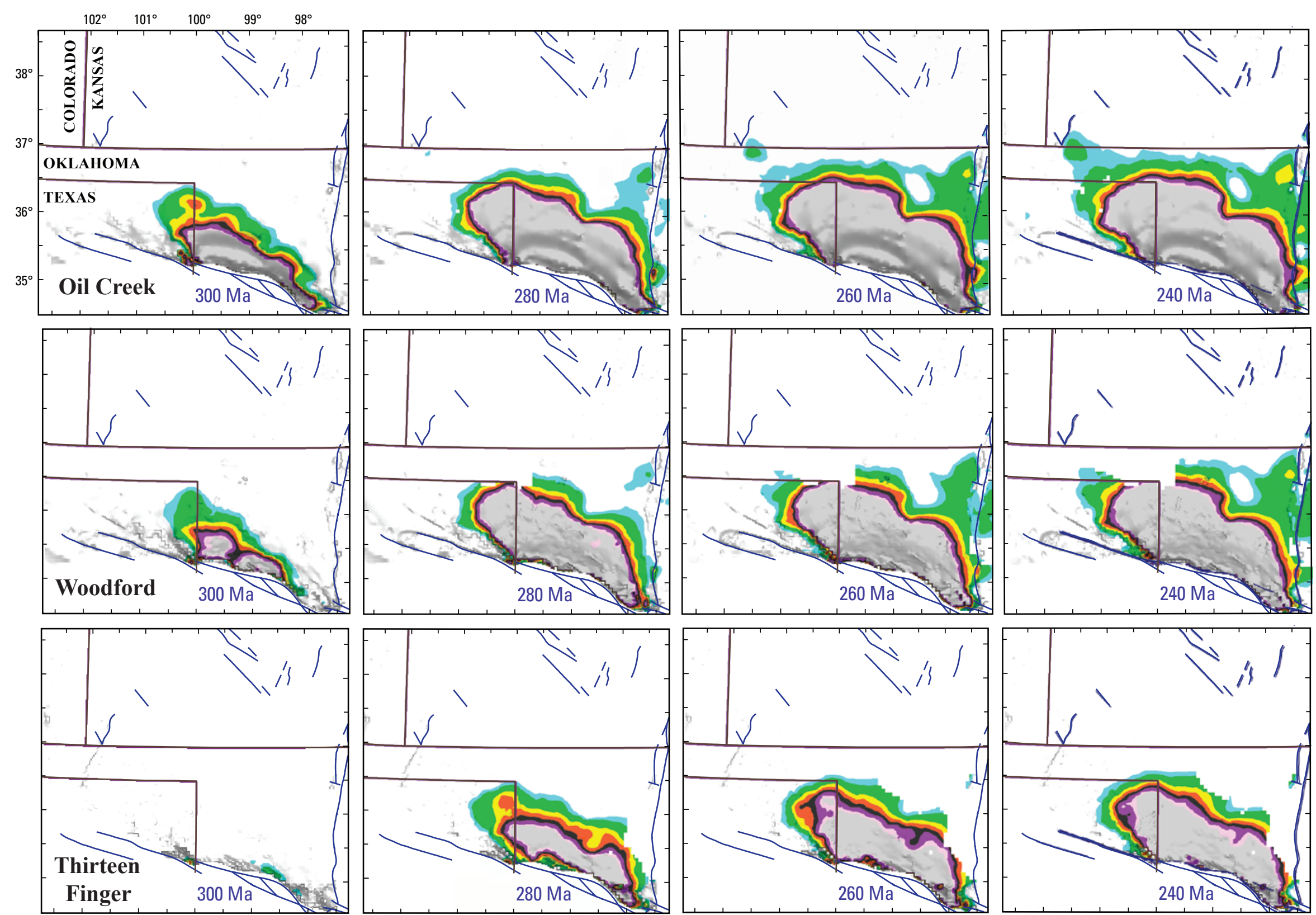

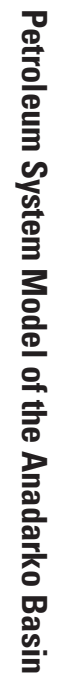




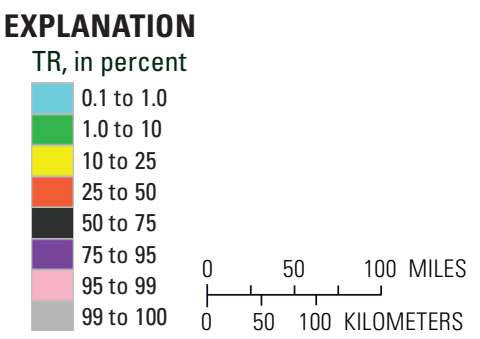

Figure 27 (previous page). Images showing transformation ratios (TR) through time on tops of the Oil Creek, Woodford Shale, and Thirteen Finger Limestone layers. Ten times vertical exaggeration results in variable shading within contours. Onset of oil generation was about 370 million years ago (Ma) (Oil Creek), $330 \mathrm{Ma}$ (Woodford), and $300 \mathrm{Ma}$ (Thirteen Finger) based on modeled vitrinite reflectance and 0.1 percent TR. Completion of oil generation is indicated by 99 percent TR. Source rocks are overmature for oil generation at greater than 99 percent TR (medium gray). Precambrian faults (Adler and others, 1971) are shown as blue lines.

\section{Petroleum Migration and Accumulation}

A general division of oil and gas migration is (1) primary, which is basically expulsion from the source $\operatorname{rock}(\mathrm{s}),(2)$ secondary, or migration from the source rock through permeable carrier beds, and (3) tertiary migration that is remobilization of oil and (or) gas. Tertiary migration can result from tilting of the basin, development of structures such as the Wichita Mountain and Amarillo uplifts, or any hydrodynamic, erosional, or other process that changes the PVT conditions across or within the basin. Some of these PVT factors are discussed in the petroleum system modeling section of this report.

\section{Secondary Migration of Hydrocarbons}

The axis of the southern Oklahoma aulacogen is south of the present southern boundary of the Anadarko Basin (Perry, 1989), and the thickest, hottest, and most deeply buried lower Paleozoic section is probably hidden beneath the thrust plates of the Wichita Mountain uplift. Hydrocarbon generation and migration from contained source rocks were essentially synchronous with those to the north because of Morrowan onset of uplift, and would have included lateral migration along carrier beds and vertical migration along faults and fractures. Because the faults were modeled through time as closed (impermeable) faults, petroleum from source rocks located south of the faults did not migrate north in the 4D model. Lateral oil and gas migration pathways were mainly radially northward from the deep basin in Oklahoma and proximal Texas, and north and west from the Texas Panhandle. Petroleum was further funneled by local structures. The Nemaha uplift forms the eastern border of the province (fig. 6). It is the axis of the broad, gently raised central Oklahoma platform, and consists of a discontinuous series of block-faulted uplifts, each of which is in the general range of 5-20 mi long (north-south) and 3-5 mi wide (Johnson, 1989). This uplift was primarily a barrier to eastward migration from underlying source rocks because of termination of reservoir against nonreservoir facies, nondeposition of some source and reservoir formations, and decreased permeability because of fault gouge. Faults along the uplift would have been open or closed through time. They were modeled as closed through time, but leakage occurred between fault segments, such as for the CKU and Nemaha uplift. Lateral flow of petroleum is terminated or diverted by faults. Leakage is shown by modeled flow paths outside the basin margins. In actuality, vertical permeability of this and other fault systems in the basin probably varied through time. Michael and others (1989) indicated that biodegraded "tar-sand" bitumen deposits located in the southern Ardmore and Anadarko Basins and proximal to major fault systems were sourced from the Woodford Shale and Oil Creek Formations.

Migration pathways are more easily visualized as catchments, also known as drainage pathways. Figures 28 and 29 show modeled petroleum migration catchments and pathways for the Thirteen Finger limestone layer. Orientation of images is based on the best angle for viewing accumulations and flow. The Thirteen Finger limestone terminates north of the Adler and others' (1971) Precambrian fault trace in the Wichita Mountain uplift in figures 28 and 29 because the layer was gridded using (Morrow Group) fault contacts modified from Andrews (1999a). Catchments and flow in east-central Oklahoma are mostly northward and are shown on the figures as wide catchments and smooth rays. Catchments are more complex in Texas, the Oklahoma Panhandle, western Kansas, and Colorado. This is reflected in figure 15 by the variable surface of the Thirteen Finger limestone layer. Catchments and flow paths could have been simplified by smoothing generated grid files, but doing so could remove subtle structures. One prominent feature is the oval maroon catchment shown at the intersection of Colorado, Kansas, and Oklahoma in figure 28 and is mirrored in figure 29 as an oil accumulation in the structural trap at Keys dome (fig. 15). Oil flow is directed to this and other accumulations in the western basin. Other accumulations are mostly updip pinchouts into low-permeability lithofacies of the overlying Atokan layer, particularly along the eastern terminus of the Thirteen Finger limestone. Whether these modeled accumulations correspond to actual Thirteen Finger limestone petroleum reservoirs is not known, but there is scattered production from Morrowan, Atokan, and Permian strata within Keyes dome (IHS Energy, 2009a, 2010). Again, the 4D model is coarse, so accumulations should be viewed more as terminations of flow rather than actual reservoirs. The gas migration pathways in figure 29 are partly obscured by overlying oil flow paths, but show radial outward flow from the deep basin. Absence of flow in the deep basin outlines the area that is thermally mature to overmature for gas generation. 


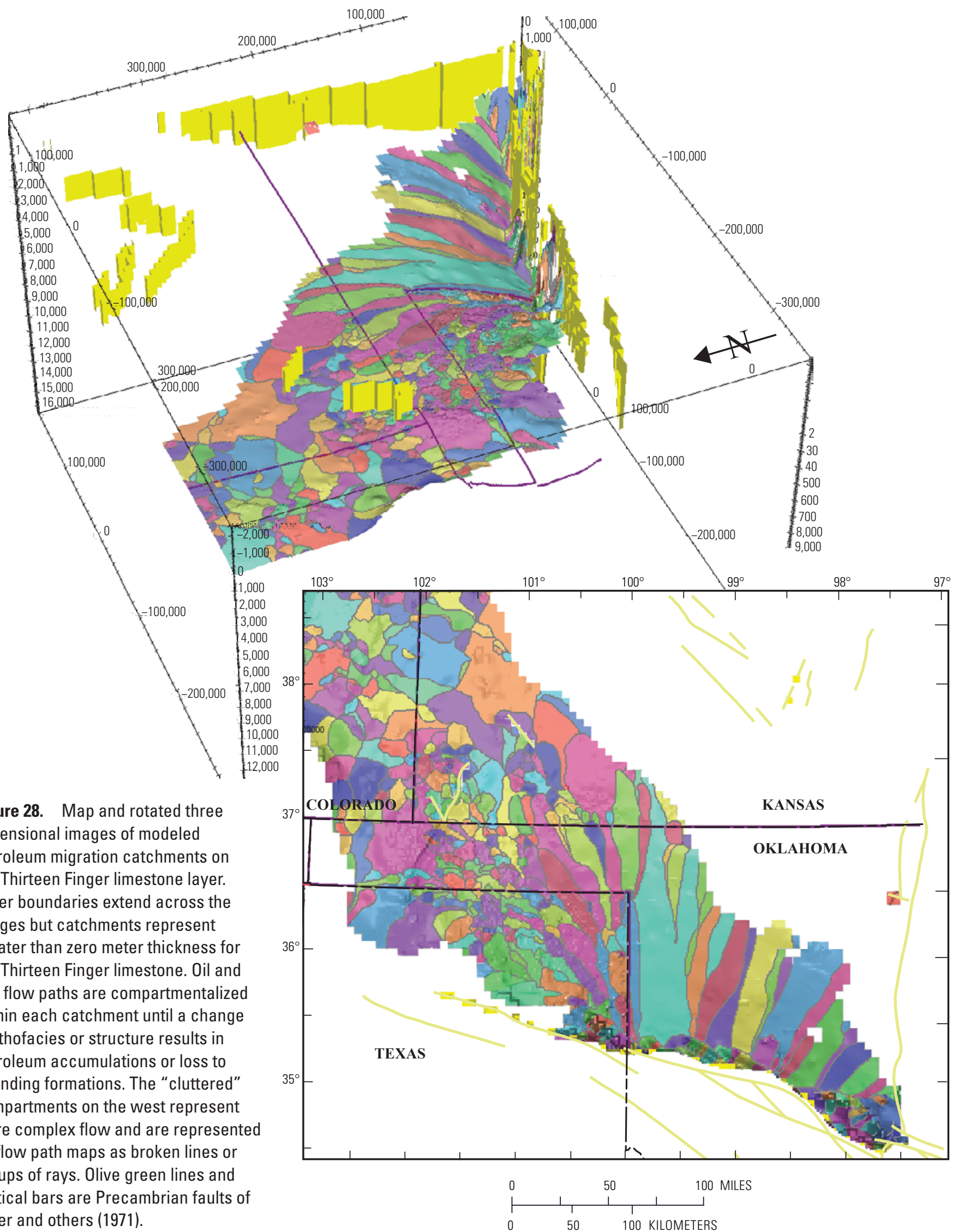



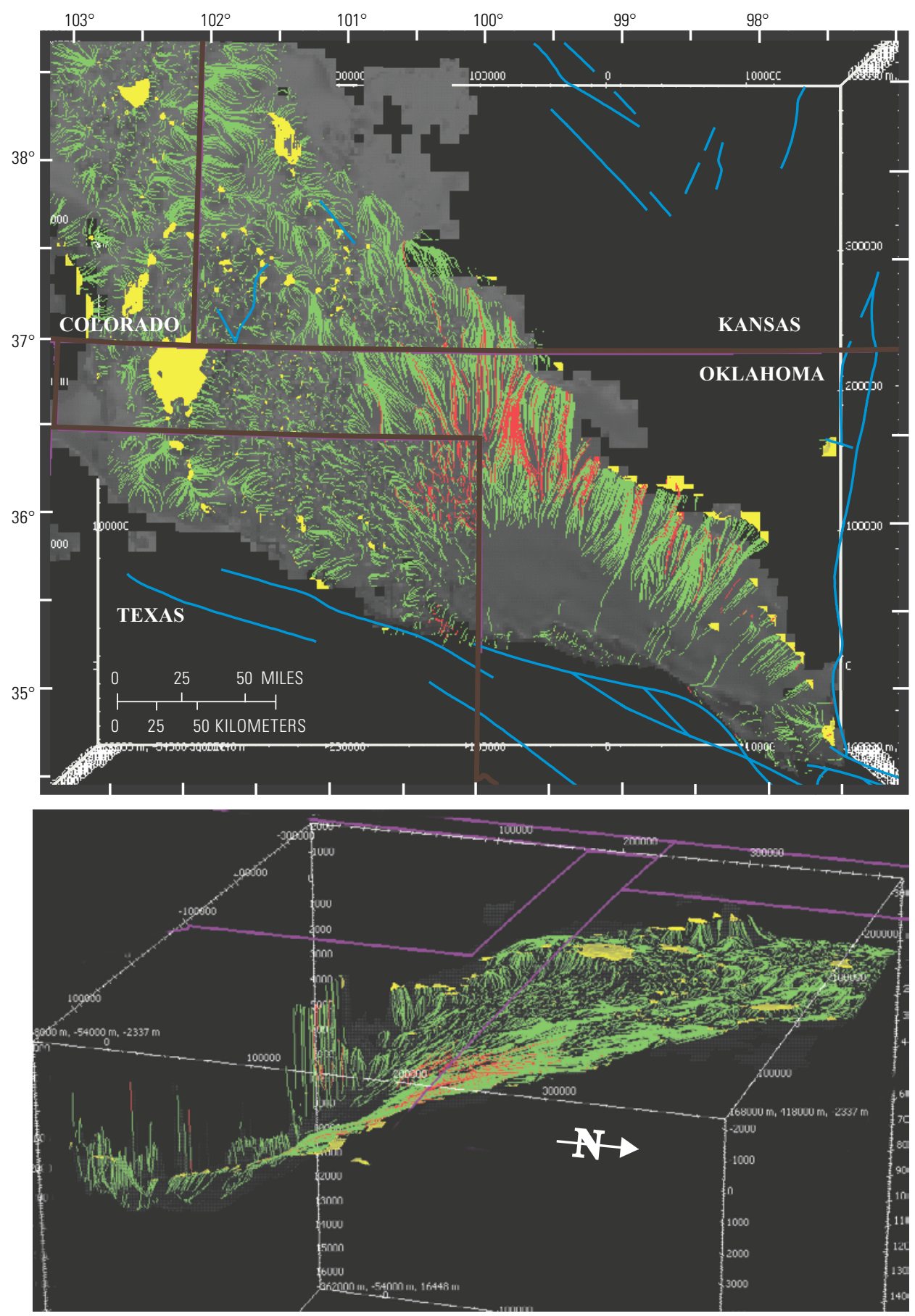

Figure 29. Two views of modeled oil (green) and gas (red) flow path lines, and oil (yellow) accumulations for the Thirteen Finger limestone. Layer shown in dark gray on three dimensional map view is the top of the Morrow Group layer. The gray space in the deep basin that is devoid of oil flow paths is the area that is mature for gas generation from the Thirteen Finger limestone. Inset image is a rotated view at 10 times vertical exaggeration. 

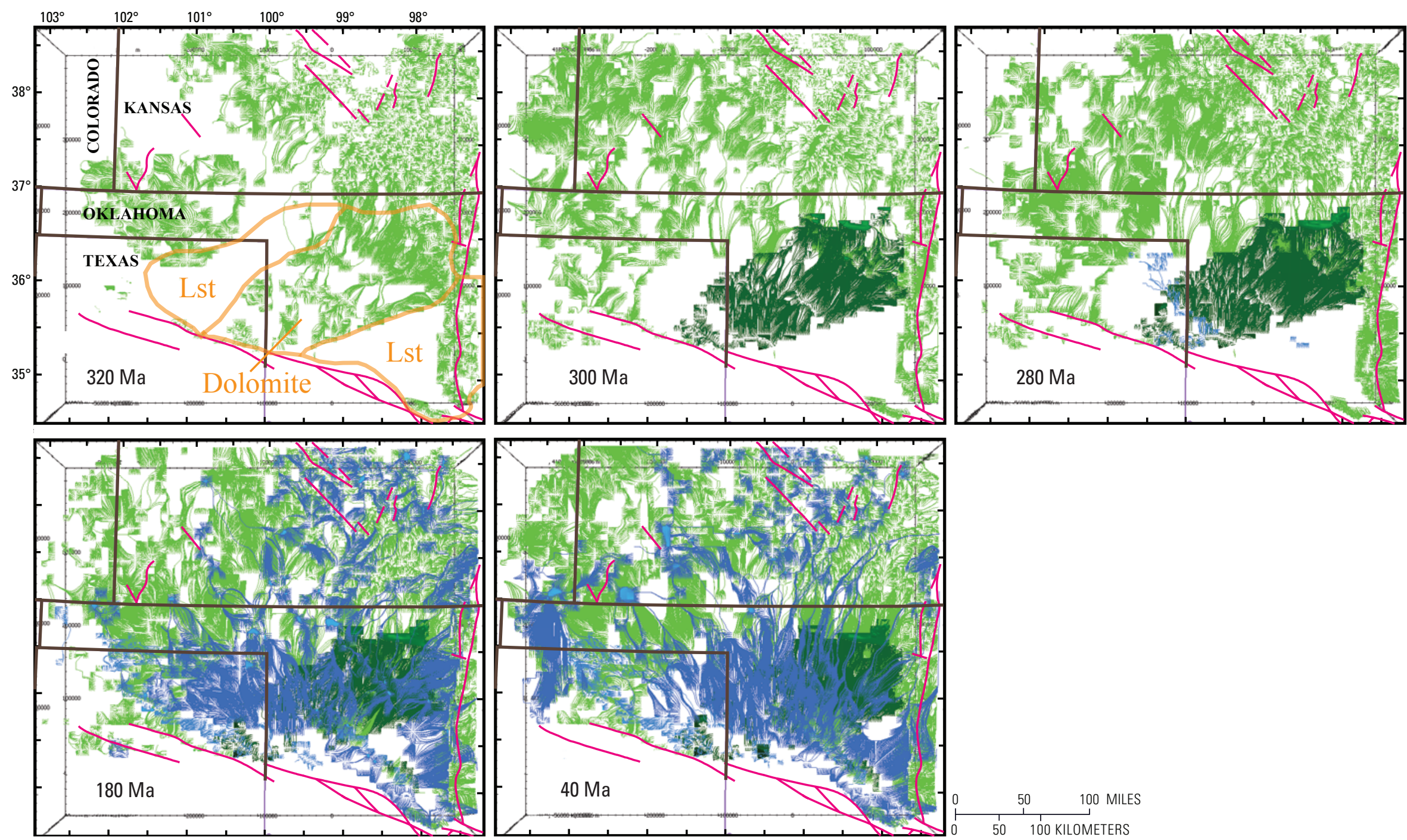

Figure 30. Images showing oil migration flow paths from 320 to 40 million years ago (Ma). Blank migration areas can indicate no migration, (invisible) Darcy flow through lithofacies, or overmaturity for petroleum generation. Hunton Group facies from Howery (1993) are delineated by orange lines for limestone (Darcy flow area) and dolomite (flow path migration). Onset of oil generation preceded migration from the Oil Creek Formation layer source rocks (light green), which started about $340 \mathrm{Ma}$. This was followed by Woodford Shale oil generation about $335 \mathrm{Ma}$, then the Thirteen Finger limestone (blue) about $300 \mathrm{Ma}$. Lst, limestone. Maroon lines are Precambrian faults of Adler and others (1971). 
The isolated square of flow and accumulation east of where the formation exists is a gridding artifact.

Flow from the deep basin in Oklahoma and eastern Texas was northward toward the Pratt anticline and Central Kansas uplift (CKU) and northwest toward the Hugoton embayment (locations are on fig. 15). Figure 30 shows oil flow paths and accumulations for petroleum source rocks through time. Light green corresponds to Oil Creek as the primary source for flow path accumulations, dark green is for a primarily Woodford Shale source, and dark blue flow paths mark the Thirteen Finger limestone as the main source for oil accumulations. Change of primary-contributing-source through time is marked by change in ray colors. These ray colors do not reflect the volumes of migrating oil because flow paths are visual rather than volumetric. Modeled Darcy flow is invisible and through low-permeability lithofacies. It is "present" as the two blank flow areas north of the Wichita Mountain uplift in the 320 Ma through 280 Ma images in figure 30 . These correspond to the Hunton Group limestone lithofacies from Howery (1993), which have lower permeability than the dolomite facies that form the (dark green) Hunton fairway of production from 300 through $40 \mathrm{Ma}$. The Woodford Shale is also the primary source rock for Hunton accumulations in the 4D model. The blue vertical flow paths in western Oklahoma and Texas begin in an area with no Woodford or Thirteen Finger limestone source rocks. These particular flow paths originate from the underlying Oil Creek source rock, and they merge updip with Oil Creek, Woodford, and Thirteen Finger flow paths from the deep basin.

Long distance migration of oil and gas in the Anadarko Basin is indicated by the progressive depletion of toluene in oils with increased distance from the kitchen area (Burruss and Hatch, 1989, p. 61), and that most of the Kansas and Colorado portions of the province are thermally immature for oil or gas generation but contain abundant oil and gas resources. Lateral migration can exceed $200 \mathrm{~km}(124 \mathrm{mi})$, which is the distance between the extent of thermally mature source rocks for the Oil Creek layer and the northern limit of the model. Maximum migration is probably considerably greater as evidenced by the abundance of oil and gas resources in the Central Kansas uplift and the Cambridge arch to the north, areas that are thermally immature for petroleum generation.

\section{Tertiary Migration of Hydrocarbons}

Impact of the Laramide orogeny included withdrawal of the Cretaceous epicontinental seaway and regional uplift that caused an eastward and southeastward tilt to the Anadarko Basin (Johnson, 1989). Primary modeled effect of this tilting is remobilization of some trapped oil and gas with subsequent remigration, perhaps resulting in the red flow paths of gas migration in the northern basin (fig. 31). Uplift and erosion can also influence pressure in formations across the basin, resulting in the release of pressure by removal of overburden, particularly if they are seals, and associated modification of hydrologic flow. The Permian layers provide an example of gas remigration and diffusion during the Laramide uplift and erosion in gas reservoirs of the southern Central Kansas uplift and Pratt anticline, and the Hugoton embayment (fig. 32). The Laramide orogeny redistributed Panhandle field fluid columns, possibly spilling gas northward into the Hugoton embayment (Sorenson, 2005). Regional uplift, erosion, and associated drop in pressure in the Hugoton embayment and CKU areas resulted in a volume increase in the gas within the Chase and underlying layers. Gas flow paths are primarily in the Douglas Group through Permian layers. The mostly northern flow of Oil Creek- and Thirteen Finger-sourced petroleum from the Panhandle field area and northwesterly curving Thirteen Finger flow in Kansas suggests these are primary sources for the Hugoton field area of southwest Kansas. Oil Creek is the primary modeled source for petroleum in the panhandle area; however, north of this field there are numerous and variable source rocks that contribute to accumulations.

The modeled source rock contributions to petroleum accumulations in the Anadarko Basin correspond generally to the Burruss and Hatch (1989) determination of three petroleum systems; divisions were based on carbon stable-isotopic compositions and gasoline-range hydrocarbon compositions of oil that correlated with extracts of Ordovician, Devonian, and Pennsylvanian source rocks. These divisions correspond generally to our Ordovician Oil Creek, Devonian-Mississippian Woodford, and Pennsylvanian Thirteen Finger limestone source rock layers.

Within the 4D model, the Oil Creek primarily sources Arbuckle through Viola petroleum accumulations, the Woodford Shale is the major source for Hunton, Springer, and lower Morrow accumulations, and the Thirteen Finger limestone is the main source for middle Morrow through Permian accumulations. Each color bar in figure 32 shows petroleum accumulations specific to the corresponding named layer, and each of these layers can have contributions from one or more source rocks. Contributions from sources can change through time as shallower source rocks enter the oil and gas generation and expulsion windows. I will only list generalized source rock contributions for modeled accumulations at $0 \mathrm{Ma}$.

The Oil Creek Formation of the Simpson Group was the only petroleum source layer that contributed to the Arbuckle layer accumulations (fig. 32). These minor accumulations are located primarily in uplifted and exposed areas of the Central Kansas uplift and Panhandle field. The Oil Creek source layer is the only contributor to lower Simpson petroleum accumulations, but minor upper (overlying) Simpson accumulations include about 25 percent contribution from Woodford sources. The Viola layer petroleum sources include Oil Creek (71 percent) and Woodford (29 percent) layers. Hunton accumulations have the Woodford Shale as the primary source at about 77 percent of the oil and wet gas; the remainder is sourced from the Oil Creek layer. Most of the modeled Hunton petroleum accumulated along the northern boundary of the Sooner trend (fig. 32), which is along the northern terminus of the formation against the sealing Woodford Shale layer. Sooner-trend oil and associated gases are produced from 


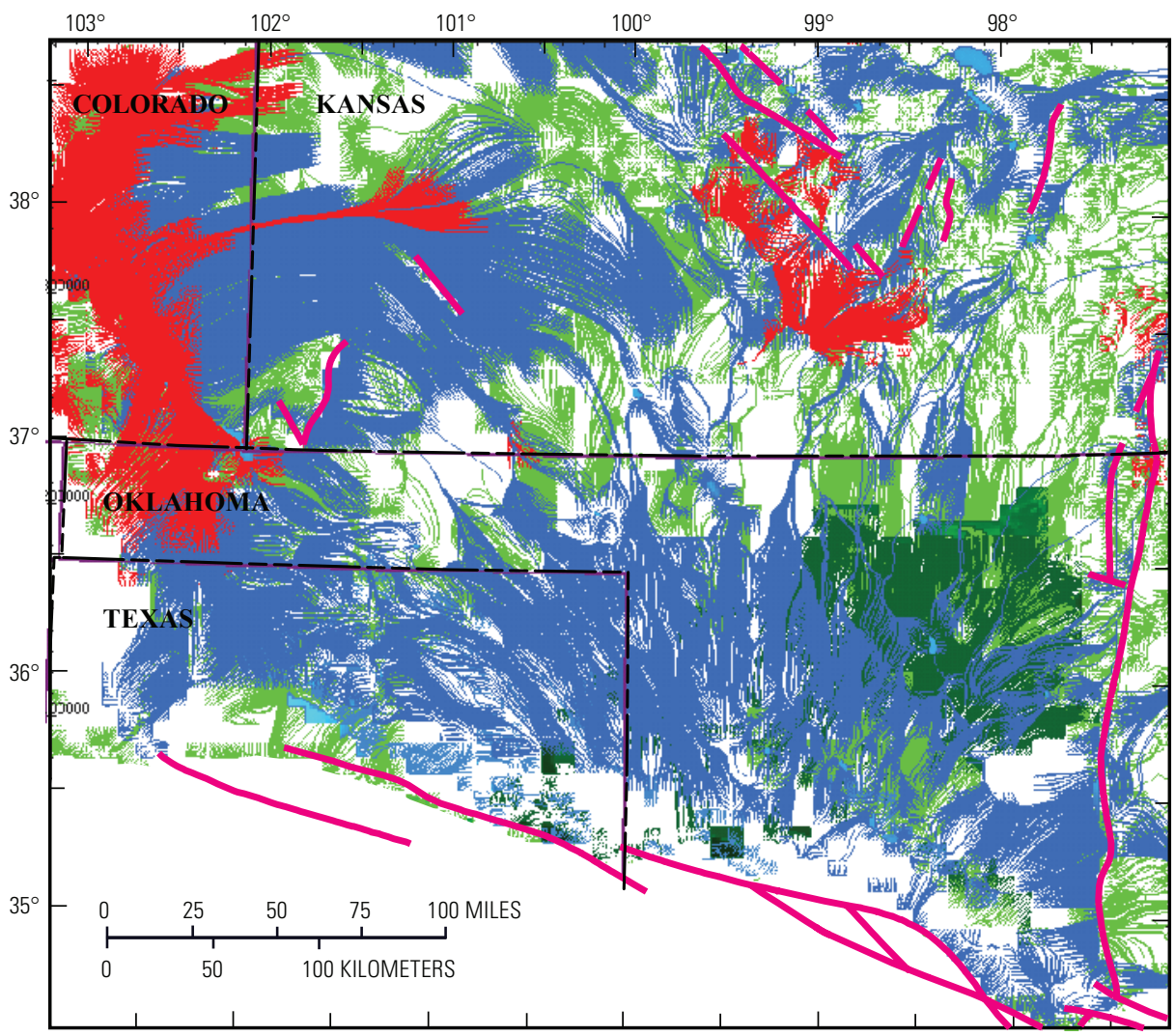

Figure 31. Image showing present-day oil and gas migration flow paths. Blank migration areas indic ate no migration, (invisible) Darcy flow through lithofacies, or overmaturity for petroleum generation. Oil migration flow paths from mostly Oil Creek layer source rocks (light green) are overlain by mostly Woodford Shale (dark green), and then Thirteen Finger limestone (blue) flow paths. Gas (red) flow paths are not differentiated by source rocks. Maroon lines are the fault system from Adler and others (1971).

Silurian, Devonian, and Mississippian carbonates at depths as great as 9,600 ft, including Hunton Group carbonates, and were generated from Type-II kerogen during the mature stage; associated oil correlates with extracts of the Woodford Shale (Rice and others, 1989). Gases are isotopically light $\left(\delta^{13} \mathrm{C}\right.$ values of -47.3 to $-40.6 \mathrm{ppt})$ and chemically wetter $\left(\mathrm{C}_{1} / \mathrm{C}_{1-5}\right.$ values of 0.67 to 0.99 ) than those derived from Type-III kerogen at an equivalent level of thermal maturity (Rice and others, 1989). Hunton petroleum in the Cement field (fig. 32) is sourced partly from the Oil Creek and primarily from the Woodford. A number of modeled source rocks contribute to Springer layer production across the study area, but the primary source is Woodford at 49 percent, followed by Oil Creek at 31 percent, and Thirteen Finger limestone at 20 percent. The Morrow was divided into three layers with assigned lithofacies boundaries based on Andrews (1999b). Lower Morrow is primarily sourced from the Woodford (76 percent) with secondary Oil Creek (23 percent). The middle Morrow layer has 98 percent Woodford and 2 percent Oil Creek layer contributions; if were there were additional petroleum source rocks, these percentages would change. The upper Morrow layer is sourced 74 percent from the Thirteen Finger and 26 percent from the Woodford layer; Woodford contributions are focused along the updip limit of the Morrow layers.

Model results include petroleum volumes remaining within source rocks. This is primarily because the low permeability of the Oil Creek, Woodford, and deep-basin Thirteen Finger layers resulted in saturation rather than discrete accumulations. Technically, these layers would be mostly or completely self-sourced. Although saturation/accumulation seems a subtle difference, the saturation results do not include petroleum migration, accumulation, or reported volumes. Unlike for the Oil Creek Formation and Woodford Shale source rocks, PetroMod ${ }^{\circledR}$ calculated oil reservoir accumulations for the Thirteen Finger limestone north of the deep basin (figs. 28 and 29). This layer is defined as a source rock with the general lithofacies assignment of shaley limestone. The area of the deep basin that is mature for Thirteen Finger limestone gas generation contains some peripheral oil and wet gas accumulations with the Woodford Shale as a contributing source, but 


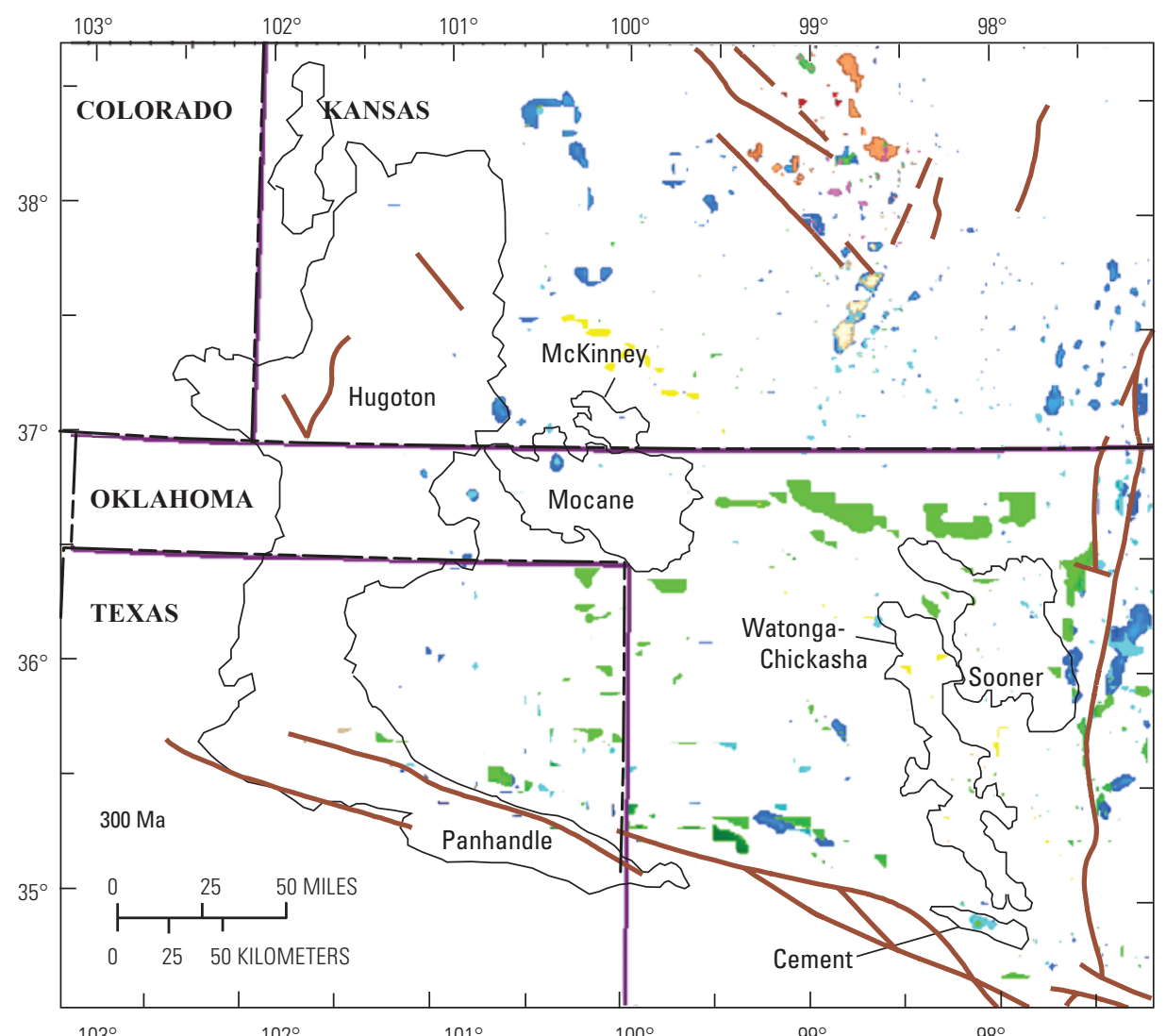

Figure 32. Images showing modeled gas (red) and oil accumulations at 300 million years before present (Ma) and present day for above named layers. Some major oil and gas fields are outlined and labeled. Brown lines are the Precambrian fault system from Adler and others (1971); the purple lines north of this fault system are modeled locations of the Wichita Mountain uplift at $200 \mathrm{Ma}$ and at present. Accumulations preferentially form on large structures, such as the Cement field, and updip pinchout of reservoir layers against seal layers, such as for the Hunton Group accumulations in northeast Oklahoma. Stratigraphic traps are commonly not filled in this leaky four-dimensional model because of the coarse grid size and lithologic assignments.

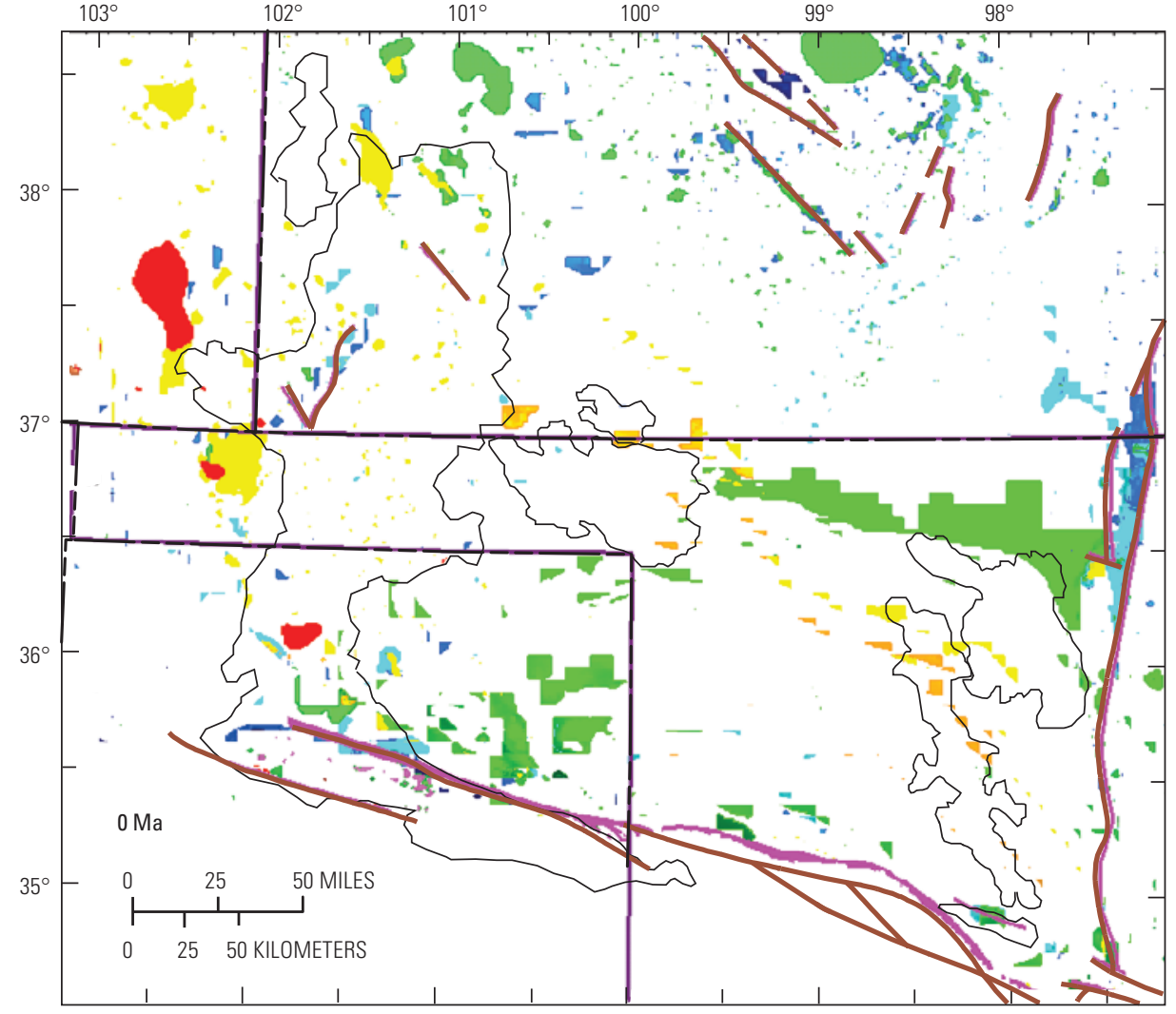

EXPLANATION

4D model layer names

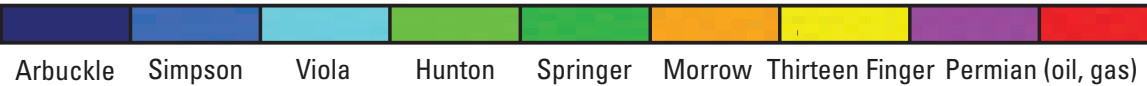


98 percent of the oil in this layer is sourced from the Thirteen Finger limestone, with about 2 percent Woodford Shale. Permian oil and gas is sourced 56 percent from the Thirteen Finger limestone, 26 percent from the Oil Creek layer, and 18 percent from the Woodford Shale; most of the Permian gas accumulations are in the panhandle through western Hugoton field areas (fig. 32). Probable reasons for this mix are (1) Woodford Shale and Thirteen Finger limestone source rocks are absent in most of the Amarillo uplift area, so the Permian blue "rectangle" southwest of Colorado in figure 30 at 40 Ma was mostly migration of Oil Creek hydrocarbons updip along the flanks of the basin; (2) the isopach of Woodford through Desmoinesian strata is thin in the Texas panhandle (fig. 9), which could allow for greater contributions from deeper source rocks, which could also be a factor in sour oil and gas in parts of the Panhandle field; and (3) this leaky 4D model allows for more vertical and lateral migration than a more complex model would. Isolating the model to smaller areas of the basin and running it with closer grid spacing and more complex vertical and lateral lithofacies would provide additional information.

\section{Conclusions}

The Anadarko Basin is an important petroleum province in the United States. The richness of oil and gas production is reflected by petroleum source rocks that range in age from Ordovician through Pennsylvanian. These source rocks are most thermally mature in the deep basin of southwestern Oklahoma and northern Texas. Oil and gas generation, migration, and accumulation were strongly influenced by the history of deposition, uplift, and erosion. A 4D petroleum system model of the Anadarko Basin Province was built using 30 layers of Precambrian to present-day stratigraphic units with assigned lithofacies and ages of deposition and erosion. 1D models were constructed and calibrated using vitrinite reflectance and temperature data. These were used to calibrate the 4D model, and 1D extractions from the model were used for further calibration.

Three source intervals were incorporated in these models, mainly to derive timing of oil and gas generation and migration, and to evaluate total petroleum systems based on source contributions to petroleum accumulations. Petroleum source rocks are the Oil Creek Formation of the Ordovician Simpson Group, the Devonian-Mississippian Woodford Shale, and the Thirteen Finger limestone of the Pennsylvanian Atoka Group. These modeled source rock contributions to petroleum accumulations correspond generally to the determination of three petroleum systems in the basin. Within the 4D model, the Oil Creek primarily sources Arbuckle through Viola petroleum accumulations; the Woodford Shale is the major source for Hunton, Springer, and lower Morrow accumulations; and the Thirteen Finger limestone is the main source for upper Morrow through Permian accumulations.

\section{Acknowledgments}

Brian Cardott of the Oklahoma Geological Survey and Neil Fishman of the U.S. Geological Survey provided excellent information and reviews that improved this report. Richard Keefer of the U.S. Geological Survey greatly improved the clarity of this report through his editorial review. Michael Lewan gave me the comparison chart of Woodford Shale hydrous pyrolysis transformation ratios and vitrinite reflectance; he has also been a valuable source of knowledge in my research. Chesapeake Energy personnel have been most generous with their time, data, and expertise, for which I am grateful. I would like to thank Kathy Winner and IHS Energy for providing information for our mapping and modeling efforts.

\section{References Cited}

Abousleiman, Younane, Tran, M.H., Hoang, S., Bobko, C., Ortega, A., and Ulm, F.J., 2008, Study characterizes Woodford Shale: The American Oil and Gas Reporter, January 2008 , v. 51, no. 1 , p. 106-115.

Adler, F.J., Caplan, W.M., Carlson, M.P., Goebel, E.D., Henslee H.T., Hick, I.C., Larson, T.G., McCracken, M.H., Parker, M.C., Rascoe, G., Jr., Schramm, M.W., and Wells, J.S.,1971, Future petroleum provinces of the mid-continent, in Cram, I.H., ed., Future petroleum provinces of the United States-Their geology and potential: American Association of Petroleum Geologist Memoir 15, v. 2, p. 985-1120.

Al-Shaieb, Zuhair, Puckett, J.O., Abdalla, A.A., and Ely, P.B., 1994, Three levels of compartmentation within the overpressured interval of the Anadarko Basin, in Ortoleva, P.J., ed., Basin compartments and seals: American Association of Petroleum Geologists Memoir 61, p. 69-83.

Amsden, T.W., 1975, Hunton Group (Late Ordovician, Silurian, and Early Devonian) in the Anadarko Basin of Oklahoma: Oklahoma Geological Survey Bulletin 129, 136 p.

Amsden, T.W., 1989, Depositional and post-depositional history of middle Paleozoic (late Ordovician through early Devonian) strata in the ancestral Anadarko Basin, in Johnson, K.S., ed., Anadarko Basin symposium, 1988: Oklahoma Geological Survey Circular 90, p. 143-146.

Andrews, R.D., 1999a, Map showing regionlal (sic) structure at the top of the Morrow Formation in the Anadarko Basin and shelf of Oklahoma: Oklahoma Geological Survey Special Publication 99-4, pl. 3.

Andrews, R.D., 1999b, Morrow gas play in the Anadarko Basin and shelf of Oklahoma: Oklahoma Geological Survey Special Publication 99-4, 133 p., 7 pls. 
Andrews, R.D., 2001, Map showing regional structure at the top of the Springer Group in the Ardmore Basin, and the Anadarko Basin and shelf of Oklahoma: Oklahoma Geological Survey Special Publication 2001-1, pl. 5.

Ball, M.M., Henry, M.E., and Frezon, S.E., 1991, Petroleum geology of the Anadarko Basin region, province (115), Kansas, Oklahoma, and Texas: U.S. Geological Survey OpenFile Report 88-450W, 36 p.

Bebout, D.G., White, W.A., Hentz, T.F., and Grasmick, M.K. eds., 1993, Atlas of major mid-continent gas reservoirs: Bureau of Economic Geology, 85 p.

Blackwell, D.D., and Richards, M., 2004, Geothermal map of North America: American Association of Petroleum Geologists, 1 sheet, scale 1:6,500,000.

Brewer, J.A., Good, R., Oliver, J.E., Brown, L.D., and Kaufman, S., 1983, COCORP profiling across southern Oklahoma aulacogen: Overthrusting of the Wichita Mountains and compression within the Anadarko Basin: Geology, v. 11, p. 109-114.

Burgess, W.J., 1976, Geological evolution of the midcontinent and Gulf Coast areas - A plate tectonics view: Transactions, Gulf Coast Association of Geological Societies, v. 26, p. 132-143.

Burruss, R.C., and Hatch, J.R., 1989, Geochemistry of oils and hydrocarbon source rocks, greater Anadarko Basin: Evidence for multiple sources of oils and long-distance oil migration, in Johnson, K.S., ed., Anadarko Basin symposium, 1988: Oklahoma Geological Survey Circular 90, p. 53-64.

Burwood, R., Drozd, R.J., Halpern, H.I., and Sedivy, R.R., 1988, Carbon isotopic variations of kerogen pyrolyzates: Organic Geochemistry, v. 12, p. 195-205.

Cardott, B.J., 1989, Thermal maturation of the Woodford Shale in the Anadarko Basin, in Johnson, K.S., ed., Anadarko Basin symposium, 1988: Oklahoma Geological Survey Circular 90, p. 32-46.

Cardott, B.J., and Lambert, M.W., 1985, Thermal maturation by vitrinite reflectance of Woodford Shale, Anadarko Basin, Oklahoma: American Association of Petroleum Geologists Bulletin, v. 69, no. 11, p. 1982-1998.

Cardwell, A.L., 1977a, Petroleum source-rock potential of Arbuckle and Ellenburger Groups, southern mid-continent, United States: Quarterly of the Colorado School of Mines, July 1977 , v. 72, no. 3, 134 p., 1 pl.

Cardwell, A.L., 1977b, Petroleum source-rock potential of the Arbuckle Group (Cambrian-Ordovician), Oklahoma: American Association of Petroleum Geologists Bulletin, v. 61 , no. 8 , p. 1374-1375.
Carr, D.L., and Hentz, T.F., 2009, Sequence stratigraphy and organic richness of the Lower Pennsylvanian Atoka Series, northwest Anadarko Basin, Texas and Oklahoma Panhandle: American Association of Petroleum Geologists Annual Convention, June 7-10, 2009, Denver, Colo., 1 p.

Carr, D.L., Hentz, T.F., Ambrose, W.A., Ambrose, W.A., Potter, E., and Clift, S.J., 2009, Sequence stratigraphy, depositional systems, and production trends in the Atoka Series and Mid-Pennsylvanian Cleveland and Marmaton Formations, western Anadarko Basin - core workshop: The University of Texas at Austin, Bureau of Economic Geology, Workshop Workbook, no. SW0019, variously paginated.

Carter, L.S., Kelly, S.A., Blackwell, D.D., and Naeser, N.D., 1998, Heat flow and thermal history of the Anadarko Basin, Oklahoma: American Association of Petroleum Geologists Bulletin, v. 82, no. 2, p. 291-316.

Cederstrand, J.R., and Becker, M.F., 1998, Digital map of base of aquifer for the High Plains aquifer in parts of Colorado, Kansas, Nebraska, New Mexico, Oklahoma, South Dakota, Texas, and Wyoming: U.S. Geological Survey OpenFile Report 98-393, digital data and metadata, accessed September 2011, at http://cohyst.dnr.ne.gov/metadata/ m001aqbs_99.html.

Comer, J.B., and Hinch, H.H., 1987, Recognizing and quantifying expulsion of oil from the Woodford Formation and age-equivalent rocks in Oklahoma and Arkansas: American Association of Petroleum Geologists Bulletin, v. 71, no. 7, p. 844-858.

Crone, A.J., and Luza, K.V., 1986, Holocene deformation associated with the Meers fault, southwestern OklahomaFragments of an aulacogen?: Geological Society of America Annual Meeting, 1986, Guidebook for Field Trip 12, Oklahoma Geological Survey Guidebook 24, p. 68-74.

Crossey, L.J., Hagen, E.S., Surdam, R.C., and Papoint, T.W., 1986, Correlation of organic parameters derived from elemental analysis and programmed pyrolysis of kerogen, in Gautier, D.L., ed., Roles of organic matter in sediment diagenesis: Society of Economic Paleontologists and Mineralogists Special Publication 38, p. 35-45.

Davis, H.G., and Northcutt, R.A., 1989, The greater Anadarko Basin: An overview of petroleum exploration and development, in Johnson, K.S., ed., Anadarko Basin symposium, 1988: Oklahoma Geological Survey Circular 90, p. 13-24.

Denison, R.E., 1976, Evolution of the Anadarko Basin: American Association of Petroleum Geologists Bulletin, v. 60, p. 325 .

Denison, R.E., Lidiak, E.G., Bickford, M.E., and Kisvarsanyi, E.B., 1984, Geology and geochronology of Precambrian rocks in the central interior region of the United States: U.S. Geological Survey Professional Paper 1241-C, 20 p. 
Donovan, R.N., 1986, The Slick Hills of southwestern Oklahoma-Fragments of an aulacogen?: Oklahoma Geological Survey Guidebook 24, 112 p.

Dow, W.G., 1977, Kerogen studies and geological interpretations: Journal of Chemical Exploration, v. 7, p. 79-99.

Egenhoff, S.O., Fishman, N.S., and Paxton, S.T., 2011, Cyclic nature of intracratonic versus passive margin mudstone systems - A reservoir perspective: American Association of Petroleum Geologists 2011 National Meeting, 1 p.

Fay, R.O., 1964, The Blaine and related formations of northwestern Oklahoma and southern Kansas: Oklahoma Geological Survey Bulletin 98, 238 p., 24 pls.

Fay, R.O., 1989, Geology of the Arbuckle Mountains along Interstate 35, Carter and Murray counties, Oklahoma: Oklahoma Geological Survey Guidebook 26, 50 p.

Feinstein, Shimon, 1981, Subsidence and thermal history of southern Oklahoma aulacogen: Implications for petroleum exploration: American Association of Petroleum Geologists Bulletin, v. 65, v. 12, p. 2521-2533.

Gallardo, Jaquidon, and Blackwell, D. D., 1999, Thermal structure of the Anadarko Basin: American Association of Petroleum Geologists Bulletin, v. 83, no. 2, p. 333-361.

Gaswirth, S.B., and Higley, D.K., 2014, Geologic assessment of undiscovered oil and gas resources in the CambrianDevonian stratigraphy of the Anadarko Basin, Oklahoma, Kansas, Texas, and Colorado, Chap. 5, in Higley, D.K., comp., Petroleum systems and assessment of undiscovered oil and gas in the Anadarko Basin Province, Colorado, Kansas, Oklahoma, and Texas-USGS Province 58: U.S. Geological Survey Digital Data Series DDS-69-EE, 42 p.

Ham, W.E., Denison, R.E., and Merritt, C.H., 1964, Basement rocks and structural evolution of southern Oklahoma: Oklahoma Geological Survey Bulletin 95, 302 p.

Hao, F., Zou, H., Gong, Z., Yang, S., and Zeng, Z., 2007, Hierarchies of overpressure retardation of organic matter maturation: Case studies from petroleum basins in China: American Association of Petroleum Geologists Bulletin, v. 91, p. 1467-1498.

Hatch, J.R., Jacobson, S.R., Witzke, B.J., Risatti, J.B., Anders, D.E., Watney, W.L., Newell, K.D., and Vuletich , A.K., 1987, Possible late Middle Ordovician organic carbon isotope excursion; evidence from Ordovician oils and hydrocarbon source rocks, mid-continent and east-central United States: American Association of Petroleum Geologists Bulletin v. 71, no. 11, p. 1342-1354.

Hester, T.C., Schmoker, J.W., and Sahl, H.L., 1990, Logderived regional source-rock characteristics of the Woodford Shale, Anadarko Basin, Oklahoma: U.S. Geological Survey Bulletin 1866-D, 38 p.
Higley, D.K., Charpentier, R.R., Cook, T.A., Klett, T.R., Pollastro, R.M., and Schmoker, J.W., 2007, Executive summary-2002 Assessment of undiscovered oil and gas in the Denver Basin Province, Colorado, Kansas, Nebraska, South Dakota, and Wyoming, Chap. 4, in Higley, D.K., compiler, Petroleum systems and assessment of undiscovered oil and gas in the Denver Basin Province, Colorado, Kansas, Nebraska, South Dakota, and Wyoming_-USGS Province 39: U.S. Geological Survey Digital Data Series 69-P, 4 p.

Higley, D.K., Lewan, M.D., Roberts, L.N.R., and Henry, M.E., 2006, Petroleum system modeling capabilities for use in oil and gas resource assessments: U.S. Geological Survey Open-File Report 2006-1024, 18 p., accessed September 2011, at http://pubs.usgs.gov/of/2006/1024/.

Higley, D.K., and Cox, D.O., 2007, Oil and gas exploration and development along the Front Range in the Denver Basin of Colorado, Nebraska, and Wyoming, Chap. 2, in Higley, D.K., compiler, Petroleum systems and assessment of undiscovered oil and gas in the Denver Basin Province, Colorado, Kansas, Nebraska, South Dakota, and Wyoming-USGS Province 39: U.S. Geological Survey Digital Data Series 69-P, 40 p., accessed September 2011, at http:// pubs.usgs.gov/dds/dds-069/dds-069-p/REPORTS/69_p_ CHAP_LIST.pdf.

Higley, D.K., Lewan, M.D., Roberts, L.N.R., and Henry, M.E., 2009, Timing and petroleum sources for the Lower Cretaceous Mannville Group oil sands of northern Alberta based on 4-D modeling: American Association of Petroleum Geologists Bulletin, v. 93 no. 2, p. 203-230.

Higley, D.K., Gaswirth, S.B., Abbott, M.M., Charpentier, R.R., Cook, T.A., Ellis, G.S., Gianoutsos, N.J., Hatch, J.R., Klett, T.R., Nelson, Philip, Pawlewicz, M.J., Pearson, O.N., Pollastro, R.M., and Schenk, C.J., 2011, Assessment of undiscovered oil and gas resources of the Anadarko Basin Province of Oklahoma, Kansas, Texas, and Colorado, 2010: U.S. Geological Survey Fact Sheet 2011-3003, 2 p., accessed February 2011, at http://pubs.usgs.gov/ $f_{s} / 2011 / 3003 /$.

Higley, D.K., Gianoutsos, N.J., Pantea, M.P., and Strickland, S.M., 2014, Precambrian to ground surface grid cell maps and 3D model of the Anadarko Basin Province, Chap. 13, in Higley, D.K., comp., Petroleum systems and assessment of undiscovered oil and gas in the Anadarko Basin Province, Colorado, Kansas, Oklahoma, and Texas-USGS Province 58: U.S. Geological Survey Digital Data Series 69-EE, 7 p.

Howery, S.D., 1993, A regional look at Hunton production in the Anadarko Basin, in Johnson, K.S., ed., Hunton Group core workshop and field trip: Oklahoma Geological Survey Special Publication 93-4, p. 77-81.

Hunt, J.M., 1979, Petroleum geochemistry and geology: San Francisco, W.H. Freeman, 617 p. 
IHS Energy, 2009a, IHS energy well database: Unpublished database available from IHS Energy, 15 Inverness Way East, Englewood, CO 80112.

IHS Energy, 2009b, GDS database: Unpublished geological data services database available from IHS Energy, 15 Inverness Way East, Englewood, CO 80112.

IHS Energy, 2010, IHS energy production data on CD-ROM: Unpublished database available from IHS Energy, 15 Inverness Way East, Englewood, CO 80112.

Johnson, K.S., 1989, Geologic evolution of the Anadarko Basin, in Johnson, K.S., ed., Anadarko Basin symposium, 1988: Oklahoma Geological Survey Circular 90, p. 3-12.

Johnson, K.S., Amsden, T.W., Denison, R.E., Dutton, S.P., Goldstein, A.G., Rascoe, B., Jr., Sutherland, P.K., and Thompson, D.M., 1988, Southern Midcontinent region, in Sloss, L.L., ed., Sedimentary cover-North American craton, U.S., The geology of North America: Boulder, Colo., Geological Society of America, v. D-2, p. 307-359.

Johnson, K.S., and Cardott, B.J., 1992, Geologic framework and hydrocarbon source rocks of Oklahoma, in Johnson, K.S., and Cardott, B.J., eds., Source rocks in the southern Midcontinent, 1990 symposium: Oklahoma Geological Survey Circular 93, p. 21-37.

Jorgensen, D.G., 1989, Paleohydrology of the Anadarko Basin, central United States, in Johnson, K.S., ed., Anadarko Basin symposium, 1988: Oklahoma Geological Survey Circular 90, p. 176-193.

Kansas Geological Survey, 2010, downloadable formations tops and LAS well data, accessed February 2011, at http://www.kgs.ku.edu/PRS/petroDB.html.

Kirkland, D.W., Denison, R.E., Summers, D.M., and Gormly, J.R., 1992, Geology and organic geochemistry of the Woodford Shale in the Criner Hills and western Arbuckle Mountains, in Johnson, K.S., and Cardott, B.J., eds., Source rocks in the southern Midcontinent, 1990 symposium: Oklahoma Geological Survey Circular 93, p. 38-69.

Krystyniak, A.M., and Paxton, S.T., 2006, Outcrop-based gamma-ray characterization of the Woodford Shale of southcentral Oklahoma: Oklahoma Geological Survey, Coalbed methane and gas shales in the southern Midcontinent conference, March 21, 2006, Oklahoma City, Okla., 16 p.

Lambert, M.W., 1993, Internal stratigraphy and organic facies of the Devonian-Mississippian Chattanooga (Woodford) Shale in Oklahoma and Kansas: American Association of Petroleum Geology Studies in Geology 37: Source rocks in a sequence stratigraphic framework, ch. 11, p. 163-176.

Latham, J.W., 1970, Healdton field, Carter County, Oklahoma: American Association of Petroleum Geologists Memoir 14, p. 255-276.
Lee, Youngmin, and Deming, David, 1999, Heat flow and thermal history of the Anadarko Basin and the western Oklahoma Platform: Tectonophysics, v. 313, no. 4, p. 399-410.

Lewan, M.D., 1983, Effect of thermal maturation on stable organic carbon isotopes as determined by hydrous pyrolysis of Woodford Shale: Geochemica et Cosmochimica Acta, v. 47, p. 1471-1479.

Lewan, M.D., 1985, Evaluation of petroleum generation by hydrous pyrolysis experimentation: Philosophical Transactions of the Royal Society, A 315, p. 123-134.

Lewan, M.D., 2002, Fundamental issues on thermogenic gas generation from source-rock maturation and reservoir-oil cracking: Rocky Mountain Association of Geologists Symposium on Innovative Gas Exploration Concepts, October 1, 2002, abstract with program, 2 p.

Lewan, M.D., and Ruble, T.E., 2002, Comparison of petroleum generation kinetics by isothermal hydrous and nonisothermal open-system pyrolysis: Organic Geochemistry, v. 33, no. 12, p. 1457-1475.

Ludvigson, G.A., Sawin, R.S., Franseen, E.K., Watney, W.L., West, R.R., and Smith, J.J., 2009, A review of the stratigraphy of the Ogallala Formation and revision of Neogene ("Tertiary") nomenclature in Kansas: Kansas Geological Survey, Current Research in Earth Sciences, Bulletin 256, part 2, 9 p., accessed February 2011, at http://www.kgs. ku.edu/Current/2009/Ludvigson/index.html.

Luza, K.V., 1989, Neotectonics and seismicity of the Anadarko Basin, in Johnson, K.S., ed., Anadarko Basin symposium, 1988: Oklahoma Geological Survey Circular 90, p. $121-132$.

Magoon, L.B., and Dow, W.G., 1994, The petroleum system, in Magoon, L.B., and Dow, W.G., eds., The petroleum system-From source to trap: American Association of Petroleum Geologists Memoir 60, p. 3-24.

Mason, J.W., 1968, Hugoton Panhandle Field, Kansas, Oklahoma and Texas, in M 9: Natural gases of North America, volume two: American Association of Petroleum Geologists Volume A008, p. 1539-1547.

Michael, G.E., Lin, L.H., Philp, R.P., Lewis, C.A., and Jones, P.J., 1989, Biodegradation of tar-sand bitumens from the Ardmore/Anadarko Basins, Oklahoma-II. Correlation of oils, tar sands and source rocks: Organic Geochemistry, v. 14 , p. 619-633.

National Geologic Map Database, 2011: U.S. Geological Survey, accessed June 2011, at http://ngmdb.usgs.gov/Geolex/.

Nehring and Associates, Inc., 2009, Significant oil and gas fields database: Colorado Springs, Colo., NRG Associates, Inc. (Database available from NRG Associates, Inc., P.O. Box 1655, Colorado Springs, CO 80901). 
Owen, E.W., 1975, Part II. Panhandle-Hugoton gas and oil field, in Owen, E.W., ed., Trek of the oil finders: A history of exploration for petroleum: American Association of Petroleum Geologists Memoir 6, p. 939-959.

Pawlewicz, M.J., 1989, Thermal maturation of the eastern Anadarko Basin, Oklahoma: U.S. Geological Survey Bulletin 1866-C, p. C1-C12., accessed September 2010, at http:// pubs.er.usgs.gov/djvu/B/bull_1866_c.djvu.

Pepper, A.S., and Corvi, P.J., 1995a, Simple kinetic models of petroleum formation: Part I. Oil and gas generation from kerogen: Marine and Petroleum Geology, v. 12, p. 291-319.

Pepper, A.S., and Corvi, P.J., 1995b, Simple kinetic models of petroleum formation: Part III. Modeling an open system: Marine and Petroleum Geology, v. 12, p. 417-452.

Perry, W.J., Jr., 1989, Tectonic evolution of the Anadarko Basin region, Oklahoma: U.S. Geological Survey Bulletin 1866-A, p. A1-A19.

Price, L.C., 1997, Minimum thermal stability levels and controlling parameters of methane, as determined by $\mathrm{C}_{15}{ }^{+}$ hydrocarbon thermal stabilities, in Dyman, T.S., Rice, D.D., and Westcott, P.A., eds., Geologic controls of deep natural gas resources in the United States: U.S. Geological Survey Bulletin 2146-K, p. 135-176.

Price L.C., and Barker, C.E., 1985, Suppression of vitrinite reflectance in amorphous rich kerogen-a major unrecognized problem: Journal of Petroleum Geology, v. 8, p. 59-84.

Ragland, D.A., and Donovan, R.N., 1985, The Cool Creek Formation (Ordovician) at Turner Falls in the Arbuckle Mountains of southern Oklahoma: Oklahoma Geology Notes, v. 45, p. 132-148.

Rascoe, B., Jr., 1978, Sedimentary cycles in the Virgilian Stage (Upper Pennsylvanian) of the Anadarko Basin, parts 1 and 2: Oklahoma City Geological Society Shale Shaker, v. 28 , p. $123-131$, p. 144-149.

Rascoe, B., Jr., and Adler, F.J., 1983, Permo-Carboniferous hydrocarbon accumulations, mid-continent USA: American Association of Petroleum Geologists Bulletin, v. 67, no. 6, p. 979-1001.

Rascoe, B., Jr., and Hyne, N.J., 1987, Petroleum geology of the Midcontinent: Tulsa Geological Society Special Publication 3, $162 \mathrm{p}$.

Rice, D.D., Threlkeld, C.N., and Vuletich, A.K., 1989, Characterization and origin of natural gases of the Anadarko Basin, in Johnson, K.S., ed., Anadarko Basin symposium, 1988: Oklahoma Geological Survey Circular 90, p. 47-52.

Robbins, S.L., and Keller, G.R., 1992, Complete Bouguer and isostatic residual gravity maps of the Anadarko Basin, Wichita Mountains, and surrounding areas, Oklahoma,
Kansas, Texas, and Colorado: U.S. Geological Survey Bulletin 1866-G, 11 p., 2 pls.

Rottmann, Kurt, 2000a, Structure map of Hunton Group in Oklahoma and Texas Panhandle: Oklahoma Geological Survey Special Publication 2000-2, pl. 3.

Rottmann, Kurt, 2000b, Isopach map of Woodford Shale in Oklahoma and Texas Panhandle: Oklahoma Geological Survey Special Publication 2000-2, pl. 2.

Rowland, T.L., 1974, Lone Star 1 Rogers unit captures world depth record: Oklahoma Geology Notes, v. 34, p. 185-189.

St. John, J.W., Jr., and Eby, E.E., 1978, Peritidal carbonates and evidence for vanished evaporites in the Lower Ordovician Cool Creek Formation, Arbuckle Mountains, Oklahoma: Gulf Coast Association of Geological Societies Transactions, v. 28, part 2, p. 589-599.

Schlumberger, 2011, PetroMod Basin and Petroleum Systems Modeling Software: IES GmbH, Ritterstrasser, 23, 52072 Aachen, Germany.

Schramm, M.W., Jr., 1964, Paleogeologic and quantitative lithofacies analysis, Simpson Group, Oklahoma: American Association of Petroleum Geologists Bulletin, v. 48, no. 7, p. 1164-1195.

Shatski, N.S., 1946, The Great Donets Basin and Wichita System-comparative tectonics of ancient platforms: USSG, Akad. Nauk Izv. Geological Serial no. 1, p. 5-62.

Sorenson, R.P., 2005, A dynamic model for the Permian Panhandle and Hugoton fields, western Anadarko Basin: American Association of Petroleum Geologists Bulletin, v. 89 , no. 7, p. 921-938.

Speer, W.R., 1976, Oil and gas exploration in the Raton Basin, in Ewing, R.C., and Kues, B.S., eds., Guidebook of Vermejo Park, northeastern New Mexico: New Mexico Geological Society 27th Field Conference Guidebook, 1976, p. 217-226.

Sullivan, K.L., 1983, Organic facies variation of the Woodford Shale in western Oklahoma: University of Oklahoma, M.S. thesis, $100 \mathrm{p}$.

Sullivan, K.L., 1985, Organic facies variation of the Woodford Shale in western Oklahoma: Oklahoma City Geological Survey Shale Shaker, v. 35, no. 4, p. 76-89.

Sweeney, J.J., and Burnham, A.K., 1990, Evaluation of a simple model of vitrinite reflectance based on chemical kinetics: American Association of Petroleum Geologists Bulletin, v. 74, p. 1559-1570.

Thompson, C.L., and Dembicki, H., Jr., 1986, Optical characteristics of amorphous kerogens and the hydrocarbongenerating potential of source rocks: International Journal of Coal Geology, v. 6, p. 229-249. 
Trask, P.D., and Patnode, H.W., 1942, Mid-continent area: Regional studies: American Association of Petroleum Geologists SP 12: Source beds of petroleum, p. 255-285.

Tsuzuki, N., Takeda, N., Suzuki, M., and Yokoi, K., 1999, The kinetic modeling of oil cracking by hydrothermal pyrolysis experiments: International Journal of Coal Geology, v. 39, no. $1-3$, p. $227-250$.

Tsiris, V.L., 1983, Organic geochemistry and thermal history of the uppermost Morrow shale (Lower Pennsylvanian) in the Anadarko Basin, Oklahoma: University of Oklahoma, unpublished M.S. thesis, Norman, Oklahoma, 163 p.

Tweto, O., 1975, Laramide (Late Cretaceous-early Tertiary) orogeny in the southern Rocky Mountains, in Curtis, B., ed., Cenozoic Geology of the Southern Rocky Mountains: Geological Society of America Memoir 144, p. 1-44.

Urban, J.B., 1960, Microfossils of the Woodford Shale (Devonian) of Oklahoma: University of Oklahoma, unpublished M.S. thesis, $77 \mathrm{p}$.

U.S. Geological Survey, 2010, Energy geochemistry data base, accessed June 2011, at http://energy.cr.usgs.gov/prov/og/.

von Almen, W.E., 1970, Palynomorphs of the Woodford Shale of south central Oklahoma with observations on their significance in zonation and paleoecology: Michigan State University, Ph. D. dissertation, East Lansing, Michigan, $179 \mathrm{p}$.

Walper, J.L., 1977, The geotectonic evolution of the Wichita aulacogen, Oklahoma, in Henry, G.E., ed., Basins of the Southwest, phase 2: North Texas Geological Society, p. 192-211.

Wang, H.D., and Philp, R.P., 1997, Geochemical study of potential source rocks and crude oils in the Anadarko Basin, Oklahoma: American Association of Petroleum Geologists Bulletin, v. 81, no. 2, p. 249-274.

Wavrek, D.A., 1992, Characterization of oil types in the Ardmore and Marietta Basins, southern Oklahoma aulacogen, in Johnson, K.S., and Cardott, B.J., eds., Source rocks in the southern Midcontinent, 1990 symposium: Oklahoma Geological Survey Circular 93, p. 185-195. 


\section{Chapter 4 Geochemistry of Natural Gases of the Anadarko Basin}

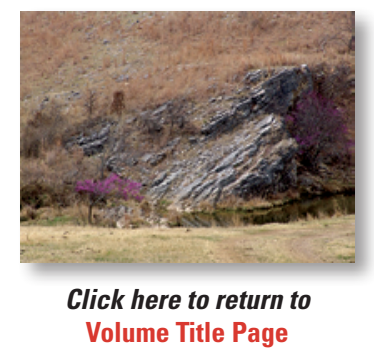

By Geoffrey S. Ellis

Chapter 4 of 13

Petroleum Systems and Assessment of Undiscovered Oil and Gas in the Anadarko Basin Province, Colorado, Kansas, Oklahoma, and Texas-USGS Province 58

Compiled by Debra K. Higley

U.S. Geological Survey Digital Data Series DDS-69-EE 


\title{
U.S. Department of the Interior SALLY JEWELL, Secretary
}

\section{U.S. Geological Survey Suzette M. Kimball, Acting Director}

\author{
U.S. Geological Survey, Reston, Virginia: 2014
}

For more information on the USGS - the Federal source for science about the Earth, its natural and living resources, natural hazards, and the environment, visit http://Www.usgs.gov or call 1-888-ASK-USGS.

For an overview of USGS information products, including maps, imagery, and publications, visit http://WwW.usgs.gov/pubprod

To order this and other USGS information products, visit http://store.usgs.gov

Any use of trade, firm, or product names is for descriptive purposes only and does not imply endorsement by the U.S. Government.

Although this information product, for the most part, is in the public domain, it also may contain copyrighted materials as noted in the text. Permission to reproduce copyrighted items must be secured from the copyright owner.

Suggested citation:

Ellis, G.S., 2014, Geochemistry of natural gases of the Anadarko Basin, chap. 4, in Higley, D.K., compiler, Petroleum systems and assessment of undiscovered oil and gas in the Anadarko Basin Province, Colorado, Kansas, Oklahoma, and Texas_-USGS Province 58: U.S. Geological Survey Digital Data Series DDS-69-EE, 31 p.,

http://dx.doi.org/10.3133/ds69EE.

ISSN 2327-638X (online) 


\section{Contents}

Abstract
Introduction.
Geologic Setting and Gas Geochemistry
Methods.
$\quad$ Analytical Techniques Sample Collection
$\quad$ Stable Isotopic Composition
$\quad$ Central Anadarko Basin
$\quad$ Sanhandle Hugoton Field
$\quad$ Central Kansas Uplift.

\section{Figures}

1. Map showing the locations of the Anadarko Basin Province (red line on inset map) and the interpretive regions used in this study: central Anadarko Basin (red line), Panhandle-Hugoton field (green line), Sooner trend (blue line), and central Kansas uplift (yellow line). The boundaries of the interpretive regions are modified from Rice and others (1988b) and Jenden and others (1988). The well locations for the gas geochemistry data used in this study are shown as black circles...

2. Generalized stratigraphic column for the Anadarko Basin Province with oil and gas source rocks highlighted (red text). Greater source rock potential is indicated by larger numbers. The expected hydrocarbons header indicates whether the source rock is more oil- or gas-prone (Burruss and Hatch, 1989; J. Hatch, oral communication, 2010). Vertical lines show a generalized time range of unconformity from Bebout and others (1993, fig. 5). Gp., Group; Fm., Formation ...............4

3. Plot of the stable carbon isotopic composition of methane, ethane, and propane versus the reciprocal of the carbon number of the hydrocarbon species. Data are average values for each of the interpretive regions of the study area. Heavy black lines indicate the expected trends for thermogenic and abiogenic sources of hydrocarbon gases. Microbial gases have isotopically depleted methane compositions as shown by the arrow ..

4. Plot of the stable carbon versus the stable hydrogen isotopic composition of methane. Note that stable hydrogen isotopic data for methane are not available for all of the gases in this study. Assoc. Gas, oil associated natural gas..

5. Plot of the gas wetness $\left(\Sigma C_{2+} / \Sigma C_{1}+C_{2+}\right)$ versus the stable carbon isotopic composition of methane 


\section{Tables}

1. Molecular compositional data (in mole percent) for natural gases from the Anadarko Basin Province by region ................................................................................

2. Molecular and stable isotopic composition of gases from the central Anadarko Basin.

3. Molecular and stable isotopic composition of gases from the Panhandle Hugoton region of the Anadarko Basin Province

4. Molecular and stable isotopic composition of gases from the Sooner trend region of the Anadarko Basin Province

5. Molecular and stable isotopic composition of gases from the central Kansas uplift region of the Anadarko Basin Province. 


\title{
Geochemistry of Natural Gases of the Anadarko Basin
}

\author{
By Geoffrey S. Ellis
}

\section{Abstract}

The sources and timing of natural gas generation as well as the migration pathways that lead to the distribution of gas accumulations throughout the Anadarko Basin Province were investigated using a geochemical dataset from more than 400 natural gas wells. The molecular and stable isotopic composition of the hydrocarbon gases indicate that there is no significant contribution of gas from abiogenic sources, and that the majority of the gases are derived from thermal maturation of organic matter. Limited contributions from microbial sources may be possible in localized areas, but this is not thought to be a significant source of gas in the province. The molecular and stable carbon and hydrogen isotopic compositions of the hydrocarbon gases indicate mature (oil window) to post mature (dry gas window) sources for gas generation, which is consistent with the findings of previous studies. Gases produced from the deepest reservoirs in the central Anadarko Basin show evidence for gas contributions from oil cracking. The geochemistry of the gases produced from the giant Panhandle Hugoton fields is remarkably homogeneous and likely reflects a single Early Mississippian or older source in the central Anadarko Basin. Uncommonly high concentrations of nitrogen are encountered in the Panhandle and Hugoton fields, and are derived from a mixture of crustal sources and thermally mature sedimentary organic matter. Gas reservoirs in the central Kansas uplift are also thought to be primarily sourced from lower Paleozoic source rocks in the central Anadarko Basin and charged via long distance migration (several hundred miles). Locally sourced, early thermogenic gas may constitute a small portion of the gas accumulations in the central Kansas uplift and Panhandle Hugoton regions. Sooner trend gas production is associated with Woodford Shale-sourced oil, and these hydrocarbons are not likely to have been generated locally but rather migrated from deeper portions of the central Anadarko Basin.

\section{Introduction}

The Anadarko Basin Province covers an area of approximately 58,000 square miles of southwestern Kansas, northwestern Oklahoma, southeastern Colorado, and northern parts of the Texas Panhandle (fig. 1). The province includes the
Anadarko Basin which is bounded by the Nemaha ridge to the east, the Amarillo-Wichita uplift to the south, and a broad shallow shelf (Hugoton embayment) to the west and northwest of the basin proper (Higley, 2014). The Anadarko Basin is one of the deepest basins on the North American craton, containing in excess of 40,000 feet of Paleozoic sedimentary rocks (Ham and Wilson, 1967); it is also the setting for the Bertha Rogers 1 well, which at a total depth of 31,441 feet $(9,583$ meters $)$ is the deepest well ever drilled in the United States (Johnson and others, 1988).

Oil and gas development in the province dates back to the early 1900s. It contains one of the largest commercial accumulations of hydrocarbons in the United States (Petroleum Information Corporation, 1982); as of mid-2011, the Anadarko Basin Province has produced approximately 5 billion barrels of oil and 150 trillion cubic feet of natural gas (IHS Energy, 2011). In 2009, the Hugoton gas field was ranked the 9th largest natural gas accumulation in the United States in terms of proved reserves (Energy Information Administration, 2010). The 2011 U.S. Geological Survey (USGS) assessment of the Anadarko Basin Province estimated the mean undiscovered technically recoverable resource potential to be 495 million barrels of oil, 27.5 trillion cubic feet of gas, and 410 million barrels of natural gas liquids (Higley and others, 2011).

The objectives of this study are to provide a better understanding of (1) the sources and timing of natural gas generation, and (2) the migration pathways that lead to the distribution of gas accumulations throughout the Anadarko Basin Province. To achieve these objectives, a review of the published literature on the geochemistry of natural gases produced from the Anadarko Basin Province was conducted and a suite of gas samples was collected and analyzed to augment existing datasets.

\section{Geologic Setting and Gas Geochemistry}

The geologic evolution of the Anadarko Basin Province is described by Higley (2014) and only a brief overview is provided herein. The Anadarko Basin is structurally asymmetric with a deep northwest-southeast trending axis at the south margin that parallels the Amarillo-Wichita Mountain front, and a broad shallow shelf region to the north and west 


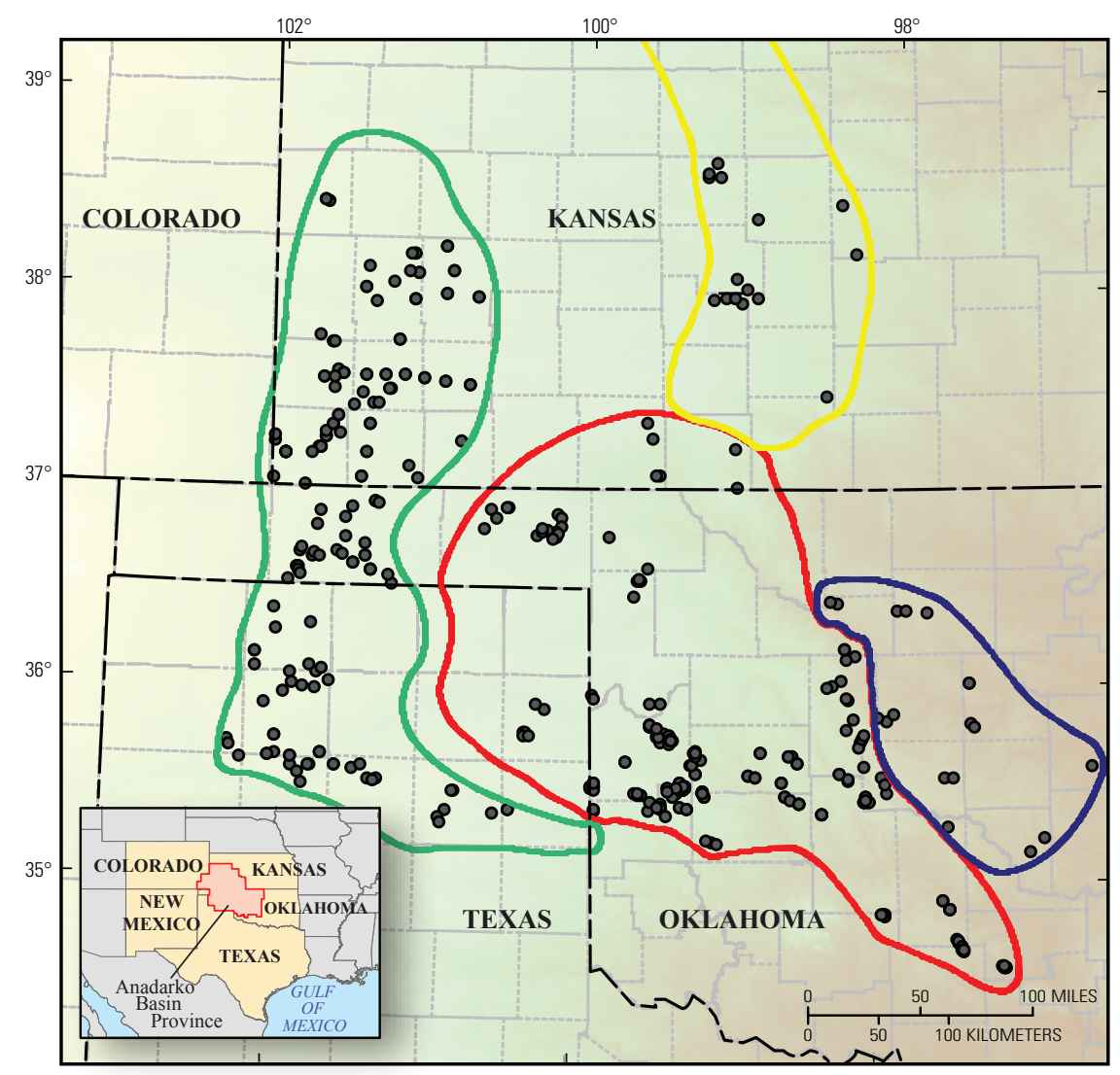

Figure 1. Map showing the locations of the Anadarko Basin Province (red line on inset map) and the interpretive regions used in this study: central Anadarko Basin (red line), Panhandle-Hugoton field (green line), Sooner trend (blue line), and central Kansas uplift (yellow line). The boundaries of the interpretive regions are modified from Rice and others (1988b) and Jenden and others (1988). The well locations for the gas geochemistry data used in this study are shown as black circles.

(Adler, 1971). From the late Precambrian through the Middle Cambrian, what is now the southern midcontinent of North America, experienced extensive igneous activity and graben formation related to thermally driven rifting (Ham and others, 1964). The present-day Anadarko Basin is thought to have developed on the southern Oklahoma aulacogen that formed during the Middle to Late Cambrian (Shatski, 1946; Burke, 1977; Hoffman and others, 1989). From the Late Cambrian through the Mississippian, this region experienced an epeirogenic phase that was characterized by shallow marine sedimentation in an epicontinental sea (Craig and Varnes, 1979; Frezon and Jordan, 1979; Johnson and others, 1988). Pennsylvanian time brought about dramatic changes to the Anadarko Basin as regional orogenic activity produced significant folding, faulting, and subsidence. Basin fill was initially provided by the uplifting Amarillo-Wichita block to the south, followed later by development of the Nemaha uplift to the east and the Cimarron arch to the northwest, resulting in the deposition of as much as 18,000 feet of clastic and carbonate sediments (Johnson, 1989). The postPennsylvanian sedimentary record within the Anadarko Basin is dominated by Permian red beds, carbonates and evaporites (Jordan and Vosburg, 1963; McKee and others, 1967; Johnson and others, 1988). Sedimentary deposition during the Mesozoic and Cenozoic produced a relatively thin sequence of strata that has been partially or completely eroded throughout extensive parts of the province (Johnson and others, 1988; Johnson, 1989). The generalized stratigraphy of the Anadarko Basin Province is shown in figure 2.

Several organic-rich lithologies have been identified as potential petroleum source rocks in the province (Bartram and others, 1950; Hatch and others, 1986; Johnson and Cardott, 1992; Wang and Philp, 1997), with the Upper Devonian and Lower Mississippian Woodford Shale widely recognized as the most significant source (Hatch and others, 1986; Comer and Hinch, 1987; Rice and others, 1988b; Johnson and Cardott, 1992; Wang and Philp, 1997). The total organic carbon (TOC) content in the Woodford Shale 
is reported to range from less than 0.1 weight percent (wt $\%$ ) to in excess of $25 \mathrm{wt} \%$ (Webb, 1976; Comer and Hinch, 1987). Hydrogen index values, determined from Rock Eval pyrolysis of outcrop samples of relatively low thermal maturity, are reported to range from approximately 250 to 850 milligrams of hydrocarbon per gram of total organic carbon (mg HC/g TOC) (Johnson and Cardott, 1992), and the kerogen is predominantly oil-prone Type II (Lewan, 1983; Cardwell, 1985). A number of Pennsylvanian marine shales are also recognized as important petroleum source rocks in the Anadarko Basin, including (1) the Lower Pennsylvanian Morrow Group that has TOC contents ranging from about 0.5 to greater than $10 \mathrm{wt} \%$ (average TOC $=1.7 \mathrm{wt} \%$ ) and hydrogen index values ranging from 15 to $179 \mathrm{mg} \mathrm{HC} / \mathrm{g}$ TOC (average HI = 46 mg HC/g TOC) (Higley, 2014), which is indicative of a gas-prone source rock; and (2) the Middle and Upper Pennsylvanian shales (Atokan, Desmoinesian, and Virgilian) that are characterized as having good hydrocarbon generation potential (Johnson and Cardott, 1992), with TOC contents ranging from 6 to $18 \mathrm{wt} \%$ and kerogen that is predominately Type II (oil prone) (Hatch and others, 1986).

The Ordovician Simpson and Viola Groups and the Sylvan Shale have all been proposed to be potential viable source rocks in the province (Johnson and Cardott, 1992). Burruss and Hatch (1989) studied the Simpson and Viola Groups and reported that, although the TOC contents are generally less than $1 \mathrm{wt} \%$, some samples from the Kansas shelf are as high as 9 wt $\%$, and that Rock-Eval pyrolysis data show the kerogen to be a mixture of Types I and II. Overall, the authors concluded that the source rock potential of the Simpson and Viola Groups is poor to moderate within the Anadarko Basin proper and a little better on the Kansas shelf. Although the Upper Ordovician Sylvan Shale contains oil-prone Type II kerogen, TOC contents are generally less than $1 \mathrm{wt} \%$ and the source rock potential is reported to be poor to moderate (Johnson and Cardott, 1992; Wang and Philp, 1997). Early studies of the occurrence of oil and gas in the Upper Cambrian and Lower Ordovician Arbuckle Group indicated a potential indigenous source for the hydrocarbons (Bartram and others, 1950; Webb, 1976); however, subsequent work has shown that these accumulations were sourced from younger strata and the Arbuckle Group is no longer thought to have contributed significant amounts of petroleum to the province (Cardwell, 1985; Jones and Philp, 1990).

Several previous studies have examined the geochemistry of produced natural gases from the Anadarko Basin Province (Moore, 1982; Hatch and others, 1986; Jenden and others, 1988; Rice and others, 1988b, 1988a, 1989; Price, 1995). From 1917 through 1980 the U.S. Bureau of Mines collected more than 10,000 samples of produced natural gases from across the United States and analyzed them for their chemical composition. Of those samples, more than 2,500 were collected from the Anadarko Basin Province (Moore, 1982). Researchers from the USGS examined the molecular and stable isotopic composition $\left(\delta^{13} \mathrm{C}\right.$ and $\left.\delta^{2} \mathrm{H}\right)$ of produced gases from the central Anadarko Basin, the Sooner trend, and the Panhandle-Hugoton fields in efforts to characterize the source, thermal maturity, and migration pathways of the gas accumulations in the Anadarko Basin Province (Hatch and others, 1986; Rice and others, 1988b, 1988a, 1989). Jenden and others (1988) studied the geochemistry of produced natural gas from Kansas, which includes the northeastern portion of the Hugoton field, to determine the mechanisms of formation for the gas accumulations in that state. A subsequent study by Price (1995) of how deep, high-thermal maturity gas accumulations may have formed focused in part on the geochemistry of natural gases from the deep portion of the central Anadarko Basin. The present report provides new geochemical data from producing gas wells in the Anadarko Basin Province and offers an interpretation of their significance in light of these previous studies.

\section{Methods}

\section{Gas Sample Collection}

A total of 96 produced natural gas samples were collected at or near the individual well heads. Well locations were selected such that only those wells that produce from a single stratigraphic interval were sampled (that is, no commingled gases), and a representative spatial distribution through the central Anadarko Basin was achieved. All gases were collected in Isotubes ${ }^{\circledR}$ following the protocol established by Isotech, Inc. (http://www.isotechlabs.com/customersupport/ samplingprocedures/WellSM.pdf). Once collected, gas samples were immediately shipped to the USGS Organic Geochemistry Laboratory in Denver, Colorado, for analysis.

\section{Analytical Techniques}

The molecular composition of the gases was determined using an Agilent 6890 gas chromatograph (GC) (Agilent Technologies) customized for the analysis of light natural gas components (Wasson ECE Instrumentation). The instrument contains eight columns and three detectors [two thermal conductivity detectors (TCD) and one flame ionization detector (FID)], allowing for the analysis of both hydrocarbon and nonhydrocarbon components in a single injection. One TCD uses nitrogen as the carrier gas, and the other TCD and the FID use helium as carrier gas. Analytes that are routinely quantitated include: methane, ethane, ethylene, propane, propylene, n-butane, i-butane, isobutene, 1-butene, cis-2-butene, trans2-butene, n-pentane, i-pentane, neopentane, n-hexane, benzene, n-heptane, carbon dioxide, carbon monoxide, nitrogen, helium, hydrogen, and hydrogen sulfide. Oxygen and argon are detected but are reported together because of coelution. The minimum reporting level for all analytes is 0.01 mole percent (mol \%). Instrument calibration is based on four separate standard reference-gas mixtures and the concentrations of all components are verified to be within $0.6 \mathrm{~mol} \%$ of the known values. For all analytes of interest in this study, the standard 


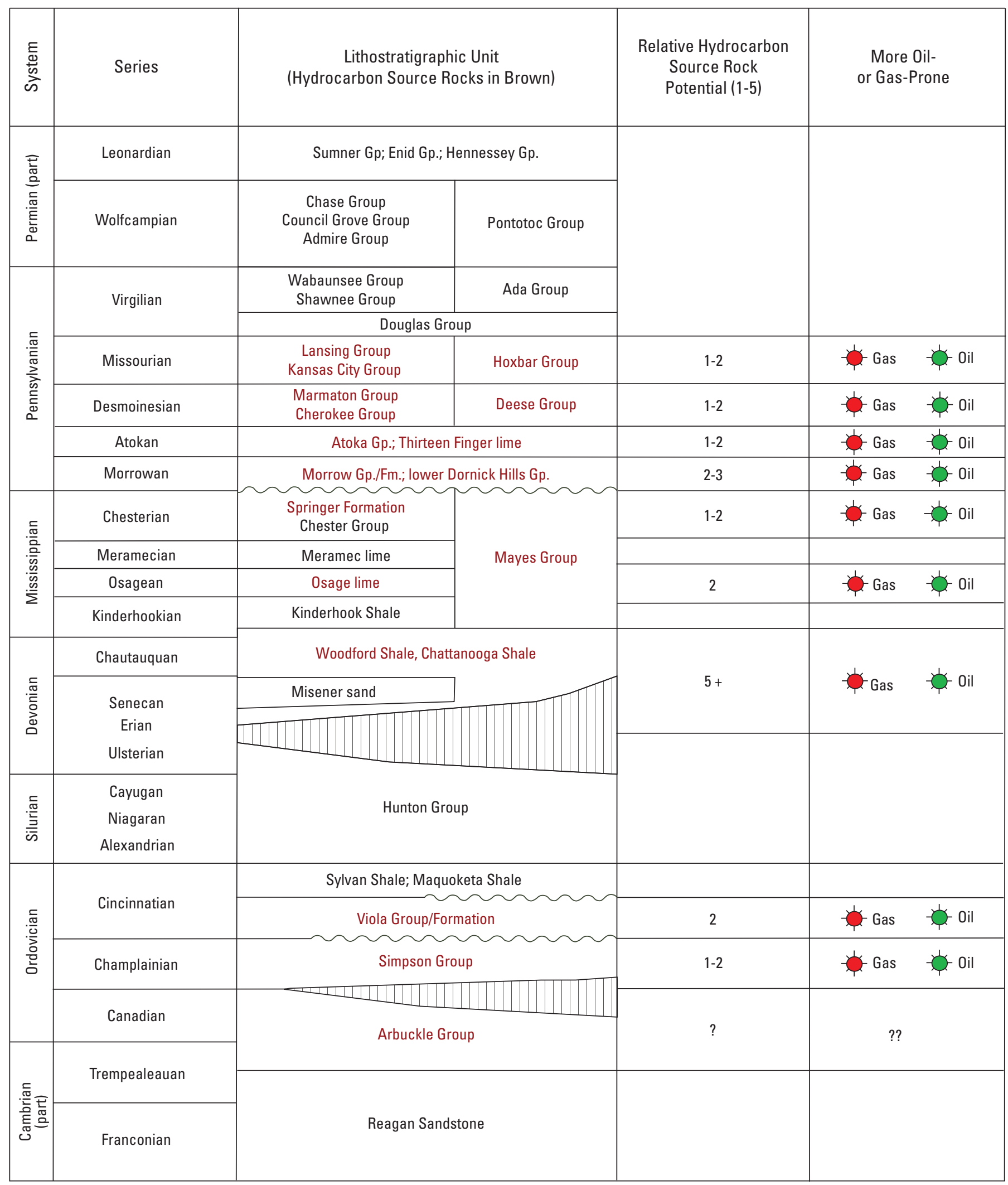

Figure 2. Generalized stratigraphic column for the Anadarko Basin Province with oil and gas source rocks highlighted (red text). Greater source rock potential is indicated by larger numbers. The expected hydrocarbons header indicates whether the source rock is more oil- or gas-prone (Burruss and Hatch, 1989; J. Hatch, oral communication, 2010). Vertical lines show a generalized time range of unconformity from Bebout and others (1993, fig. 5). Gp., Group; Fm., Formation. 
deviation of replicate analyses $(\mathrm{n}=19)$ of the gas standards ranged from 0.00 to $0.67 \mathrm{~mol} \%$ and averaged $0.15 \mathrm{~mol} \%$.

Where a sufficient quantity of gas was available, the ${ }^{13} \mathrm{C} /{ }^{12} \mathrm{C}$ ratio of the methane, ethane, and propane of the natural gas samples was determined using a method modeled after Baylis and others (1994). A natural gas sample is introduced from a custom-built autosampler into an Agilent 6890 GC (Agilent Technologies) through a sample-loop injector of variable size. Gas components are chromatographically separated on a 50 -meter $(\mathrm{m}) \times 0.32$-millimeter $(\mathrm{mm}) \times$ 5-micrometer (mm) PoraBond-Q column (Agilent Technologies). Typical GC conditions are as follows: helium carrier gas at 1.5 milliliters per minute $(\mathrm{mL} / \mathrm{min}) ; 40$ degrees Celsius $\left({ }^{\circ} \mathrm{C}\right)$ initial temperature; hold for 6 minutes; ramp to $150{ }^{\circ} \mathrm{C}$ at 15 degrees Celsius per minute $\left({ }^{\circ} \mathrm{C} / \mathrm{min}\right)$; ramp to $250^{\circ} \mathrm{C}$ at $30^{\circ} \mathrm{C} / \mathrm{min}$; hold for 5 minutes. The individual components are then combusted in the helium stream [with a small addition of $\left.\left(\mathrm{O}_{2}\right)\right]$ at $1,050{ }^{\circ} \mathrm{C}$ in an in-line Alsint-99.7 ceramic reactor [0.5-mm inner diameter (I.D.) x 6-mm outer diameter (O.D.) x 500-mm length]. The resulting combustion products [primarily $\left(\mathrm{CO}_{2}\right)$ and $\left(\mathrm{H}_{2} \mathrm{O}\right)$ ] are passed through an in-line Nafion ${ }^{\circledR}$ drier to remove $\mathrm{H}_{2} \mathrm{O}$, and the final analyte $\mathrm{CO}_{2}$ is passively drawn via open split into the source of a GV-Elementar IsoPrime isotope ratio mass spectrometer for subsequent carbon isotope analysis.

Carbon isotope values from the instrument undergo offline isotope corrections for drift from isotopic linearity because of any systematic error in the autosampler, chromatographic, and oxidation processes. Drift-corrected values are then normalized on the Vienna Pee Dee Belemnite (VPDB) scale using two well-calibrated, working laboratory methane standards. On average, for every 6 samples run in replicate ( $\mathrm{n}=3,18$ total determinations), 6 or more analyses of standards are performed to ensure proper analytical calibration. The final carbon isotope values represent the average of multiple replicate analyses (generally, $n=3$ ) with a standard deviation of generally better than 0.2 per mil (\%). All final $\delta^{13} \mathrm{C}$ values are reported relative to the international standard VPDB.

The ${ }^{2} \mathrm{H} /{ }^{1} \mathrm{H}$ ratio of methane in the gas samples was determined using a method modeled after Burgøyne and Hayes (1998). A natural gas sample is introduced into an Agilent $6890 \mathrm{GC}$ via an autosampler through a sample-loop injector. Methane is chromatographically separated on a $30-\mathrm{m} \mathrm{x}$ 0.32-mm x 1-mm Porplot-Q column (Agilent Technologies). The following GC conditions are used: helium carrier gas at $1 \mathrm{~mL} / \mathrm{min}$; $65^{\circ} \mathrm{C}$ initial temperature; hold for 12 minutes; ramp to $225^{\circ} \mathrm{C}$ at $25{ }^{\circ} \mathrm{C} / \mathrm{min}$; hold for 5 minutes. The eluent methane is then pyrolyzed in the helium stream at $1,450{ }^{\circ} \mathrm{C}$ in an in-line Alsint-99.7 ceramic reactor (0.5-mm I.D. x 6-mm O.D. $x$ 500-mm length). The resulting $\mathrm{H}_{2}$ analyte is passively drawn via open split into the source of a GV-Elementar IsoPrime isotope ratio mass spectrometer for subsequent hydrogen isotope analysis.

Raw delta values from the instrument were drift corrected for isotopic nonlinearity because of temporal variations in the autosampler, the GC, and the physical chemistry of the ceramic pyrolysis reactor, and then were normalized to the internationally accepted standard mean ocean water-standard light arctic precipitation (SMOW-SLAP) hydrogen isotope scale using two calibrated, working laboratory methane standards. On average, for every 5 replicate analyses of 5 samples $(\mathrm{n}=5,25$ total determinations), 50 analyses of standards were performed to ensure proper analytical calibration. The final hydrogen isotope values represent the average of multiple replicate analyses $(\mathrm{n}=5)$ with a standard deviation of better than $1 \%$. All final $\delta^{2} \mathrm{H}$ values are presented relative to the international standard, Vienna Standard Mean Ocean Water (VSMOW).

\section{Results}

For the purposes of this study, natural gas production from the Anadarko Basin Province is considered in four separate regions within the province. Rice and others (1988b) suggested that, on the basis of the age of the reservoir rocks, type of trap, and the composition and origin of the gases, three distinct areas of the Anadarko Basin Province can be defined as: the central Anadarko Basin, the Panhandle-Hugoton field, and the Sooner trend. In their study of the gases of Kansas, Jenden and others (1988) distinguished between the gases of the Hugoton embayment and the central Kansas uplift on the basis of the regional geology and their interpreted origin of the gases in each region. In the present study, the Hugoton embayment gases are included with the Panhandle-Hugoton field gases and the central Kansas uplift constitutes the fourth region (in addition to the 3 regions identified by Rice and others, 1988b). The spatial distribution of these four regions is shown in figure 1.

\section{Gas Molecular Composition}

The U.S. Bureau of Mines natural gas study (Moore, 1982) contains approximately 2,300 gas wells from within the four regions of the Anadarko Basin Province (the central Anadarko Basin, the Panhandle-Hugoton field, the Sooner trend, and the central Kansas uplift). Because this dataset is too extensive to include here, the results from these samples are summarized (minimum, maximum, and average values) in table 1 .

Of the 96 gas samples that were collected and sent to the laboratory for this study, 10 contained an insufficient amount of gas to allow for accurate molecular compositional analysis and no data were generated. Two additional samples contained greater than $10 \mathrm{~mol} \%$ air and were deemed too contaminated to be useful; the associated data for these two wells are not included in this report. Of the remaining 84 samples, 11 contained oxygen plus argon concentrations that indicated levels of air contamination that were less than $10 \mathrm{~mol} \%$ but were higher than the expected "background" concentration of oxygen plus argon in produced natural gases from the Anadarko 
Basin Province. The "background" concentration of oxygen plus argon in Anadarko Basin Province gases was determined by examining natural gas compositional data from 4,117 wells within the Anadarko Basin and surrounding provinces (Moore, 1982). The sum of the mean oxygen $(0.10 \pm 0.34 \mathrm{~mol}$ $\%)$ and argon $(0.02 \pm 0.10 \mathrm{~mol} \%)$ concentrations plus one standard deviation $(0.55 \mathrm{~mol} \%)$ was taken to be the maximum expected "background" oxygen-plus-argon concentration. For the samples that contained oxygen-plus-argon concentrations that exceeded the maximum "background" level, the "background" concentration $(0.55 \mathrm{~mol} \%)$ was subtracted and the remainder was assumed to be because of air contamination. The nitrogen concentration was then corrected for the assumed air contamination based on the atmospheric abundances of nitrogen (78.08 volume \%) and oxygen plus argon (21.88 volume $\%)$. The concentrations of all of the gas components were then renormalized to 100 percent using the "air-free" nitrogen value. The gas wetness was calculated on an "air-free" basis using the following equation:

$$
\text { Wetness }=\frac{C_{2}+C_{3}+n C_{4}+i C_{4}+n C_{5}+i C_{5}}{C_{1}+C_{2}+C_{3}+n C_{4}+i C_{4}+n C_{5}+i C_{5}}
$$

where $C_{1}=$ mol \% methane, $C_{2}=$ mol \% ethane, $C_{3}=\operatorname{mol} \%$ propane, $n C_{4}=\mathrm{mol} \%$ normal butane, $i C_{4}=$ mol \% isobutane, $n C_{5}=\mathrm{mol} \%$ normal pentane, and $\mathrm{i}_{5}=\mathrm{mol} \%$ isopentane. The molecular compositional data for the gas samples collected for this study are shown in table 2 .

In addition to the data generated from the samples collected for this study, published data from producing gas wells in the central Anadarko Basin are included in table 2. These include 49 wells from Jenden and Kaplan (1989a),
6 wells from Jenden and others (1988), and 89 wells from Rice and others (1988a). To fully characterize the geochemistry of produced gases from the Anadarko Basin Province additional published data from other parts of the province are included in this report. Table 3 contains molecular compositional data for gases produced from the PanhandleHugoton field in the Texas and Oklahoma panhandles and southwestern Kansas. These include 31 wells from Ballentine and Sherwood Lollar (2002), 72 wells from Jenden and Kaplan (1989a), 27 wells from Jenden and others (1988), and 36 wells from Rice and others (1988a). Table 4 contains molecular compositional data for 18 gases produced from the Sooner trend on the eastern flank of the Anadarko Basin (Rice and others, 1988a). Table 5 contains molecular compositional data for produced gases from 15 wells in the central Kansas uplift at the north end of the Anadarko Basin Province (Jenden and others, 1988). Four wells identified by Jenden and others (1988) as being part of the Hugoton field (Dutton 1-17, Embry Lease, Chalk A 1-18, and EnlowMiller \#1) are considered here to be part of the central Kansas uplift given their proximity to other wells in this region (about 5 miles to the nearest central Kansas uplift well and about 80 miles to the nearest Hugoton well).

\section{Stable Isotopic Composition}

Of the 84 samples analyzed for molecular composition, 9 did not contain a sufficient quantity of gas to allow for determination of the $\delta^{13} \mathrm{C}$ composition of methane, ethane, or propane. One of the remaining 75 samples contained concentrations of ethane and propane that were too low to allow

Table 1. Molecular compositional data (in mole percent) for natural gases from the Anadarko Basin Province by region.

$\left[\mathrm{N}_{2}\right.$, nitrogen; $\mathrm{CO}_{2}$, carbon dioxide; $\mathrm{H}_{2}$, hydrogen; He, helium; $\mathrm{C}_{1}$, methane; $\mathrm{C}_{2}$, ethane; $\mathrm{C}_{3}$, propane; n- $\mathrm{C}_{4}$, n-butane; i-C $\mathrm{C}_{4}$, i-butane; $\mathrm{n}-\mathrm{C}_{5}$, n-pentane; i- $\mathrm{C}_{5}$, $=$ i-pentane; CYC 5, cyclopentane; CYC 6, cyclohexane; Wet, gas wetness; Min, minimum; Max, maximum; Avg, arithmetic mean. Data are from Moore (1982)]

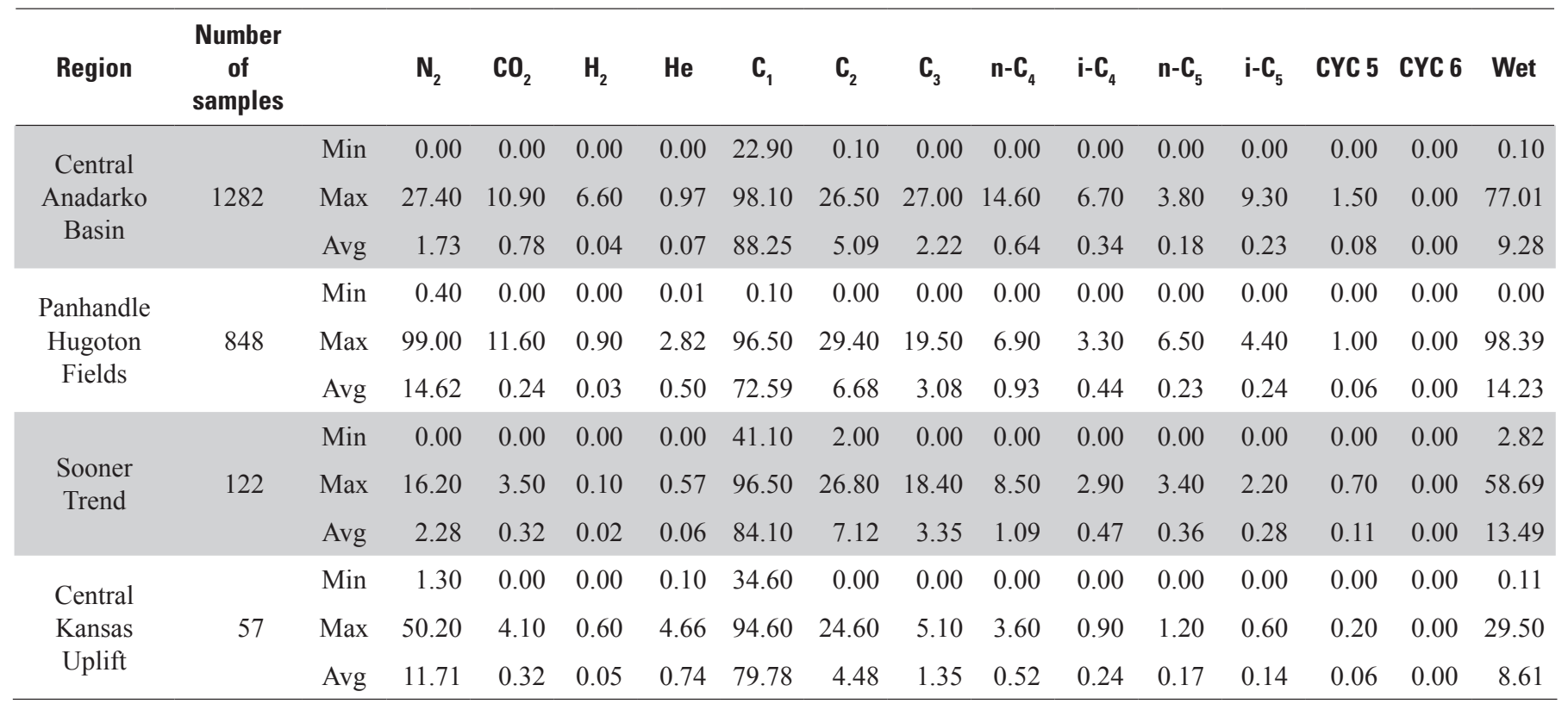


Table 2. Molecular and stable isotopic composition of gases from the central Anadarko Basin.

[Units for the compositional data are in mole percent, and the $\delta^{13} \mathrm{C}$ and $\delta^{2} \mathrm{H}$ values are in per mil relative to the Vienna Pee Dee Belemnite and Vienna Standard Mean Ocean Water scales, respectively. Gas wetness is calculated as $\Sigma \mathrm{C}_{2+} / \Sigma \mathrm{C}_{1}+\mathrm{C}_{2+}$. $\mathrm{C}_{1}$, methane; $\mathrm{C}_{2}$, ethane; $\mathrm{C}_{3}$, propane; $\mathrm{n}-\mathrm{C}_{4}$, n-butane; i- $\mathrm{C}_{4}$, i-butane; $\mathrm{n}-\mathrm{C}_{5}$, n-pentane; i- $\mathrm{C}_{5}$, = i-pentane; $\mathrm{C}_{6+}$, six carbon hydrocarbons and higher; $\mathrm{CO}_{2}$, carbon dioxide; $\mathrm{N}_{2}$, nitrogen; Wet, gas wetness in percent and $\delta^{13} \mathrm{C}_{1}, \delta^{13} \mathrm{C}_{2}$, and $\delta^{13} \mathrm{C}_{3}$ are the stable carbon isotopic composition of methane, ethane, and propane, respectively. $\delta^{2} \mathrm{HC} \mathrm{C}_{1}$ is the stable hydrogen isotopic composition of methane. ND, not determined. S indicates the source of the data and the numbers refer to the following publications: (1) Jenden and Kaplan (1989a), (2) Jenden and others (1988), (3) Rice and others (1988a), and (4) this study]

\begin{tabular}{|c|c|c|c|c|c|c|c|c|c|c|c|c|c|c|c|c|c|}
\hline $\begin{array}{l}\text { Well } \\
\text { name }\end{array}$ & $\begin{array}{c}\text { Formation } \\
\text { name }\end{array}$ & $C_{1}$ & $C_{2}$ & $\mathrm{C}_{3}$ & $\mathrm{n}-\mathrm{C}_{4}$ & $\mathrm{i}-\mathrm{C}_{4}$ & $n-C_{5}$ & $\mathrm{i}-\mathrm{C}_{5}$ & $\mathbf{C}_{6+}$ & $\mathrm{CO}_{2}$ & $\mathbf{N}_{2}$ & Wet & $\delta^{13} \mathbf{C}_{1}$ & $\delta^{13} \mathbf{C}_{2}$ & $\delta^{13} \mathbf{C}_{3}$ & $\delta^{2} \mathrm{HC}_{1}$ & $\mathbf{S}$ \\
\hline Cattle \#1-34 & Atoka & 95.80 & 1.64 & 0.32 & 0.12 & 0.14 & ND & ND & ND & 1.24 & 1.08 & $2.26 \%$ & -42.90 & -32.20 & ND & -139 & 1 \\
\hline Welks \#1-17 & Red Fork & 87.80 & 3.04 & 0.59 & 0.07 & 0.14 & ND & ND & ND & 1.07 & 1.69 & $4.18 \%$ & -43.20 & -32.50 & ND & -136 & 1 \\
\hline Allison \#1-3 & L. Atoka & 95.00 & 1.98 & 0.26 & 0.03 & 0.04 & ND & ND & 0.00 & 0.88 & 1.07 & $2.38 \%$ & -42.80 & -34.30 & ND & -147 & 1 \\
\hline Allison \#1-3 & L. Atoka & 95.00 & 2.01 & 0.32 & 0.04 & 0.05 & ND & ND & 0.00 & 1.70 & 0.90 & $2.48 \%$ & -42.70 & -34.40 & ND & ND & 1 \\
\hline Allison \#1-3 & L. Atoka & 96.30 & 2.10 & 0.98 & ND & ND & ND & ND & 0.00 & 0.86 & 0.42 & $3.10 \%$ & ND & ND & ND & ND & 1 \\
\hline Ashby \#1-36 & L \& M Atoka & 78.00 & 8.80 & 3.60 & 0.64 & 0.46 & ND & ND & 0.00 & 0.66 & 1.97 & $14.75 \%$ & -48.10 & -34.30 & ND & -162 & 1 \\
\hline Baker \#1-20 & Red Fork & 91.00 & 4.19 & 0.63 & 0.08 & 0.13 & ND & ND & 0.00 & 1.06 & 1.34 & $5.24 \%$ & -42.30 & -32.90 & ND & -133 & 1 \\
\hline Barham \#1-32 & Morrow & 94.00 & 0.46 & 0.04 & 0.00 & ND & ND & ND & 0.00 & 1.41 & 1.76 & $0.53 \%$ & -38.50 & ND & ND & -135 & 1 \\
\hline Berry \#1-8 & U. Morrow & 93.00 & 0.56 & 0.56 & ND & ND & ND & ND & 0.00 & 1.58 & 1.32 & $1.19 \%$ & -38.30 & ND & ND & -139 & 1 \\
\hline Bull Elk \#1 & L. Springer & 94.10 & 0.95 & 0.12 & 0.01 & 0.00 & ND & ND & 0.00 & 0.96 & 1.76 & $1.14 \%$ & -41.40 & -35.90 & ND & -145 & 1 \\
\hline Canyon Camp \#1 & Red Fork & 84.50 & 8.30 & 2.80 & 0.49 & 0.39 & ND & ND & 0.00 & 0.82 & 1.15 & $12.42 \%$ & -46.70 & -33.30 & ND & -152 & 1 \\
\hline Clark \#1-33 & Morrow & 95.90 & 0.45 & 0.04 & 0.01 & 0.00 & ND & ND & 0.00 & 1.41 & 1.34 & $0.51 \%$ & -38.20 & -36.10 & ND & -147 & 1 \\
\hline Cornstalk \#1 & Springer & 87.10 & 2.67 & 0.75 & 0.10 & 0.12 & ND & ND & 0.00 & 1.04 & 1.94 & $4.01 \%$ & -41.90 & -34.60 & ND & -142 & 1 \\
\hline Coulson \#5-1 & L. \& M. Atoka & 91.10 & 2.30 & 0.45 & 0.04 & 0.08 & ND & ND & 0.00 & 1.12 & 2.44 & $3.06 \%$ & -43.00 & -31.30 & ND & -151 & 1 \\
\hline Dugger \#1-18 & Morrow & 89.90 & 0.30 & 0.01 & ND & ND & ND & ND & 0.00 & 2.45 & 1.71 & $0.35 \%$ & -38.10 & ND & ND & -140 & 1 \\
\hline Farrar \#2-22 & Puryear & 93.20 & 0.50 & 0.04 & 0.00 & ND & ND & ND & 0.00 & 1.25 & 1.86 & $0.58 \%$ & -38.20 & ND & ND & -133 & 1 \\
\hline Flaming \#1-20 & Springer & 88.90 & 2.17 & 0.97 & 0.18 & 0.13 & ND & ND & 0.00 & 1.29 & 1.83 & $3.73 \%$ & -40.70 & -36.50 & ND & -149 & 1 \\
\hline Flaming \#1-20 & Springer & 92.90 & 0.62 & 0.08 & 0.01 & 0.01 & ND & ND & 0.00 & 1.63 & 0.92 & $0.76 \%$ & -38.90 & -35.70 & ND & -148 & 1 \\
\hline Foundation \#1-16 & Springer & 89.00 & 0.52 & 0.07 & ND & 0.00 & ND & ND & 0.00 & 1.25 & 0.83 & $0.66 \%$ & -39.40 & ND & ND & -139 & 1 \\
\hline Gregory \#1-12 & L. \& M. Atoka & 93.00 & 1.59 & 0.35 & 0.08 & 0.18 & ND & ND & 0.00 & 1.13 & 1.00 & $2.31 \%$ & -43.90 & -31.20 & ND & -145 & 1 \\
\hline Gregory \#2-27 & Morrow & 91.20 & 0.52 & 0.04 & 0.00 & 0.00 & ND & ND & 0.00 & 1.49 & 1.52 & $0.62 \%$ & -38.20 & -36.60 & ND & -133 & 1 \\
\hline Hamberger \#1-9 & Springer & 96.60 & 0.76 & 0.12 & ND & 0.01 & ND & ND & 0.00 & 1.38 & 1.65 & $0.91 \%$ & -39.00 & -35.50 & ND & -142 & 1 \\
\hline Harris \#2 & Bromide 3 & 83.60 & 8.50 & 3.81 & 0.87 & 0.56 & ND & ND & 0.00 & 0.67 & 1.86 & $14.11 \%$ & -46.90 & -34.70 & ND & -161 & 1 \\
\hline Hazlett \#1-21 & Red Fork & 82.00 & 9.13 & 3.20 & 0.59 & 0.46 & ND & ND & 0.00 & 0.71 & 1.46 & $14.03 \%$ & -46.90 & -32.80 & ND & -158 & 1 \\
\hline Hintchel \#1 & Springer & 87.50 & 2.34 & 0.57 & 0.06 & 0.06 & ND & ND & 0.00 & 1.03 & 2.24 & $3.35 \%$ & -40.30 & -34.50 & ND & -146 & 1 \\
\hline Holder \#1A & Bromide A & 82.00 & 9.13 & 3.20 & 0.66 & 0.46 & ND & ND & 0.00 & 0.44 & 0.89 & $14.09 \%$ & -47.00 & -34.80 & ND & -158 & 1 \\
\hline Holder \#1A & Arbuckle & 85.60 & 4.13 & 1.90 & 0.67 & 0.31 & ND & ND & 0.00 & 0.33 & 1.02 & $7.57 \%$ & -45.40 & -34.90 & ND & -156 & 1 \\
\hline
\end{tabular}


[Units for the compositional data are in mole percent, and the $\delta^{13} \mathrm{C}$ and $\delta^{2} \mathrm{H}$ values are in per mil relative to the Vienna Pee Dee Belemnite and Vienna Standard Mean Ocean Water scales, respectively. Gas wetness is calculated as $\Sigma \mathrm{C}_{2+} / \Sigma \mathrm{C}_{1}+\mathrm{C}_{2+}$. $\mathrm{C}_{1}$, methane; $\mathrm{C}_{2}$, ethane; $\mathrm{C}_{3}$, propane; $\mathrm{n}-\mathrm{C}_{4}$, n-butane; i- $\mathrm{C}_{4}$, i-butane; $\mathrm{n}-\mathrm{C}_{5}, \mathrm{n}$-pentane; i- $\mathrm{C}_{5}$, $=\mathrm{i}$-pentane; $\mathrm{C}_{6+}$, six carbon hydrocarbons and higher; $\mathrm{CO}_{2}$, carbon dioxide; $\mathrm{N}_{2}$, nitrogen; Wet, gas wetness in percent and $\delta^{13} \mathrm{C}_{1}, \delta^{13} \mathrm{C}_{2}$, and $\delta^{13} \mathrm{C}_{3}$ are the stable carbon isotopic composition of methane, ethane, and propane, respectively. $\delta^{2} \mathrm{HC}$ is the stable hydrogen isotopic composition of methane. ND, not determined. S indicates the source of the data and the numbers refer to the following publications: (1) Jenden and Kaplan (1989a), (2) Jenden and others (1988), (3) Rice and others (1988a), and (4) this study]

\begin{tabular}{|c|c|c|c|c|c|c|c|c|c|c|c|c|c|c|c|c|c|}
\hline $\begin{array}{l}\text { Well } \\
\text { name }\end{array}$ & $\begin{array}{c}\text { Formation } \\
\text { name }\end{array}$ & $C_{1}$ & $\mathrm{C}_{2}$ & $\mathrm{C}_{3}$ & $\mathrm{n}-\mathrm{C}_{4}$ & $\mathrm{i}-\mathrm{C}_{4}$ & $n-C_{5}$ & $\mathrm{i}-\mathrm{C}_{5}$ & $C_{6+}$ & $\mathrm{CO}_{2}$ & $\mathbf{N}_{2}$ & Wet & $\delta^{13} \mathbf{C}_{1}$ & $\delta^{13} \mathbf{C}_{2}$ & $\delta^{13} \mathbf{C}_{3}$ & $\delta^{2} \mathrm{HC}_{1}$ & S \\
\hline Jones \#1-17 & Boatwright & 92.00 & 2.20 & 0.77 & 0.13 & 0.11 & ND & ND & 0.00 & 0.87 & 1.25 & $3.37 \%$ & -39.50 & -34.30 & ND & -143 & 1 \\
\hline Kardokys \#10-1 & Morrow & 95.10 & 0.71 & 0.14 & 0.02 & 0.01 & ND & ND & 0.00 & 1.68 & 0.84 & $0.92 \%$ & -38.70 & -35.10 & ND & -148 & 1 \\
\hline Kardokys \#10-1 & Permian Lm & 96.50 & 0.69 & 0.13 & 0.02 & 0.01 & ND & ND & 0.00 & 1.52 & 0.88 & $0.87 \%$ & -38.80 & -34.90 & ND & -147 & 1 \\
\hline Marik \#1-11 & L. Desmoinesian & 84.70 & 5.90 & 2.10 & 0.41 & 0.34 & ND & ND & 0.00 & 0.93 & 4.35 & $9.36 \%$ & -46.40 & -34.80 & ND & -150 & 1 \\
\hline McConnel \#1-34 & L. \& M. Atoka & 92.00 & 1.55 & 0.27 & 0.08 & 0.04 & ND & ND & 0.00 & 0.95 & 0.87 & $2.07 \%$ & -42.30 & -34.30 & ND & -149 & 1 \\
\hline McKay \#1 & Red Fork & 83.00 & 9.48 & 2.29 & 0.42 & 0.34 & ND & ND & 0.00 & 1.77 & 1.33 & $13.12 \%$ & -46.70 & -32.80 & ND & -159 & 1 \\
\hline Palmer \#1-17 & Red Fork & 83.50 & 6.70 & 2.90 & 0.57 & 0.43 & ND & ND & 0.00 & 0.76 & 1.35 & $11.26 \%$ & -45.90 & -35.10 & ND & -153 & 1 \\
\hline Parker \#1 & Springer & 89.90 & 1.08 & 0.16 & 0.01 & 0.01 & ND & ND & 0.00 & 1.02 & 2.11 & $1.37 \%$ & -43.00 & -36.30 & ND & -136 & 1 \\
\hline Partain \#1-6 & Red Fork & 82.10 & 4.56 & 0.96 & 0.13 & 0.20 & ND & ND & 0.00 & 1.35 & 7.10 & $6.66 \%$ & -45.00 & -30.90 & ND & -152 & 1 \\
\hline Ranch \#1-15 & Morrow & 85.50 & 6.50 & 2.80 & 0.57 & 0.36 & ND & ND & 0.00 & 0.68 & 1.64 & $10.69 \%$ & -49.20 & -38.30 & ND & -154 & 1 \\
\hline Rayner \#1 & Springer & 93.00 & 1.61 & 0.57 & 0.10 & 0.08 & ND & ND & 0.00 & 1.05 & 1.34 & $2.47 \%$ & -39.90 & -34.90 & ND & -144 & 1 \\
\hline Rice \#1-35 & Springer & 94.00 & 0.17 & 0.01 & ND & ND & ND & ND & 0.00 & 2.89 & 1.90 & $0.19 \%$ & -33.20 & ND & ND & -154 & 1 \\
\hline Rice \#1-35 & Morrow & 93.80 & 1.87 & 0.01 & ND & ND & ND & ND & 0.00 & 3.50 & 1.00 & $1.96 \%$ & -33.20 & ND & ND & ND & 1 \\
\hline Rymer \#1 & Morrow & 87.20 & 5.04 & 1.32 & 0.24 & 0.29 & ND & ND & 0.00 & 0.78 & 1.17 & $7.32 \%$ & -43.00 & -32.70 & ND & -149 & 1 \\
\hline Ten Bears \#1 & Springer & 95.10 & 0.91 & 0.11 & 0.01 & ND & ND & ND & 0.00 & 1.04 & 0.71 & $1.07 \%$ & -40.00 & -34.40 & ND & -144 & 1 \\
\hline Thornton \#2-30 & Morrow & 93.00 & 0.43 & 0.04 & ND & ND & ND & ND & 0.00 & 1.52 & 0.88 & $0.50 \%$ & -37.80 & ND & ND & -131 & 1 \\
\hline Thurmond \#1-27 & Atoka & 91.40 & 1.67 & 0.32 & 0.07 & 0.13 & ND & ND & 0.00 & 1.14 & 1.26 & $2.33 \%$ & -43.10 & -31.90 & ND & -137 & 1 \\
\hline Thurmond \#1-32 & Red Fork & 86.90 & 4.90 & 1.50 & 0.22 & 0.25 & ND & ND & 0.00 & 0.94 & 1.76 & $7.33 \%$ & -44.50 & -34.10 & ND & -145 & 1 \\
\hline Washita \#1 & Springer & 94.20 & 1.12 & 0.10 & 0.01 & ND & ND & ND & 0.00 & 1.24 & 1.16 & $1.28 \%$ & -39.00 & -34.00 & ND & -140 & 1 \\
\hline Watkins \#2-21 & Red Fork & 92.00 & 5.10 & 0.73 & 0.09 & 0.14 & ND & ND & 0.00 & 0.96 & 1.09 & $6.18 \%$ & -44.10 & -32.30 & ND & -145 & 1 \\
\hline Webb \#2-1 & L. \& M. Atoka & 87.90 & 2.68 & 0.41 & 0.03 & 0.04 & ND & ND & 0.00 & 1.01 & 1.01 & $3.47 \%$ & -43.70 & -30.70 & ND & -150 & 1 \\
\hline West \#1-7 & Morrow & 90.00 & 2.66 & 0.64 & 0.07 & 0.08 & ND & ND & 0.00 & 0.87 & 1.10 & $3.69 \%$ & -41.40 & -34.00 & ND & -150 & 1 \\
\hline Seacat $1,2,3-19$ & Chat & 81.4 & 7.19 & 3.00 & 1.200 & 0.700 & 0.385 & 0.486 & ND & 0.217 & 4.63 & $13.74 \%$ & -41.0 & -34.9 & -29.4 & -158 & 2 \\
\hline Harden 7-17 & Viola & 59.0 & 8.25 & 8.04 & 3.570 & 1.440 & 1.020 & 0.761 & ND & 0.117 & 12.30 & $28.12 \%$ & -39.4 & -37.6 & -31.2 & -179 & 2 \\
\hline McAninch Gregg 4 & Chat & 92.2 & 3.34 & 1.06 & 0.267 & 0.139 & 0.072 & 0.073 & ND & 0.088 & 1.12 & $5.10 \%$ & -40.9 & -34.6 & -30.8 & -148 & 2 \\
\hline Barby 5-23 & Morrow & 91.7 & 3.41 & 1.41 & 0.170 & 0.385 & 0.121 & 0.107 & ND & 0.193 & 3.27 & $5.76 \%$ & -39.3 & -33.3 & -28.6 & -179 & 2 \\
\hline Barby-Harper 1-22A & Topeka & 56.7 & 13.50 & 13.70 & 4.440 & 2.420 & 1.190 & 1.540 & ND & 0.309 & 6.71 & $39.35 \%$ & -47.9 & -37.3 & -32.3 & -192 & 2 \\
\hline
\end{tabular}


Table 2. Molecular and stable isotopic composition of gases from the central Anadarko Basin.-Continued

[Units for the compositional data are in mole percent, and the $\delta^{13} \mathrm{C}$ and $\delta^{2} \mathrm{H}$ values are in per mil relative to the Vienna Pee Dee Belemnite and Vienna Standard Mean Ocean Water scales, respectively. Gas wetness is calculated as $\Sigma \mathrm{C}_{2+} / \Sigma \mathrm{C}_{1}+\mathrm{C}_{2+} \mathrm{C}_{1}$, methane; $\mathrm{C}_{2}$, ethane; $\mathrm{C}_{3}$, propane; $\mathrm{n}-\mathrm{C}_{4}$, n-butane; i- $\mathrm{C}_{4}$, i-butane; $\mathrm{n}-\mathrm{C}_{5}$, n-pentane; i- $\mathrm{C}_{5}$, $=\mathrm{i}$-pentane; $\mathrm{C}_{6+}$, six carbon hydrocarbons and higher; $\mathrm{CO}_{2}$, carbon dioxide; $\mathrm{N}_{2}$, nitrogen; Wet, gas wetness in percent and $\delta^{13} \mathrm{C}_{1}, \delta^{13} \mathrm{C}_{2}$, and $\delta^{13} \mathrm{C}_{3}$ are the stable carbon isotopic composition of methane, ethane, and propane, respectively. $\delta^{2} \mathrm{HC} \mathrm{C}_{1}$ is the stable hydrogen isotopic composition of methane. ND, not determined. S indicates the source of the data and the numbers refer to the following publications: (1) Jenden and Kaplan (1989a), (2) Jenden and others (1988), (3) Rice and others (1988a), and (4) this study]

\begin{tabular}{|c|c|c|c|c|c|c|c|c|c|c|c|c|c|c|c|c|c|}
\hline $\begin{array}{l}\text { Well } \\
\text { name }\end{array}$ & $\begin{array}{c}\text { Formation } \\
\text { name }\end{array}$ & $C_{1}$ & $\mathrm{C}_{2}$ & $\mathrm{C}_{3}$ & $n-C_{4}$ & i-C 4 & $n-C_{5}$ & $\mathrm{i}-\mathrm{C}_{5}$ & $\mathrm{C}_{6+}$ & $\mathrm{CO}_{2}$ & $\mathbf{N}_{2}$ & Wet & $\delta^{13} \mathbf{C}_{1}$ & $\delta^{13} \mathbf{C}_{2}$ & $\delta^{13} \mathbf{C}_{3}$ & $\delta^{2} \mathrm{HC}_{1}$ & $\mathbf{S}$ \\
\hline McMoran 2 & Kansas City & 90.2 & 3.72 & 1.59 & 0.034 & 0.058 & 0.007 & 0.010 & ND & 0.007 & 2.00 & $5.67 \%$ & -41.8 & -36.7 & -33.3 & -150 & 2 \\
\hline Coldwater No. 213 & Council Grove & 76.93 & 3.41 & 0.71 & 0.00 & 0.08 & 0.00 & 0.00 & 0.00 & 0.00 & 18.90 & $5.18 \%$ & -42.60 & ND & $\mathrm{ND}$ & $\mathrm{ND}$ & 3 \\
\hline Angleton No. B-2 & Council Grove & 82.39 & 4.84 & 1.50 & 0.16 & 0.15 & 0.00 & 0.00 & 0.00 & 0.00 & 10.96 & $7.47 \%$ & -43.40 & ND & ND & ND & 3 \\
\hline Barby No. A-1 & Council Grove & 81.32 & 4.59 & 1.62 & 0.20 & 0.20 & 0.00 & 0.00 & 0.00 & 0.00 & 12.07 & $7.52 \%$ & -44.10 & ND & ND & ND & 3 \\
\hline Follett Beard No. A-1 & Council Grove & 81.54 & 5.47 & 2.93 & 0.30 & 0.39 & 0.00 & 0.00 & 0.00 & 0.00 & 9.37 & $10.03 \%$ & -41.90 & -29.60 & ND & ND & 3 \\
\hline O’Neill Barby No. 1 & Virgilian & 81.52 & 5.33 & 3.15 & 0.47 & 0.41 & 0.00 & 0.08 & 0.00 & 0.28 & 8.76 & $10.38 \%$ & -42.40 & ND & ND & ND & 3 \\
\hline $\begin{array}{l}\text { O'Neil Barby Estate } \\
\text { No. } 1\end{array}$ & Council Grove & 74.74 & 4.98 & 1.92 & 0.16 & 0.19 & 0.00 & 0.00 & 0.00 & 0.14 & 17.87 & $8.84 \%$ & -45.40 & ND & ND & ND & 3 \\
\hline Barby No. 1-35 & Chester B & 62.80 & 12.52 & 8.33 & 0.62 & 0.72 & 0.00 & 0.00 & 0.00 & 0.00 & 15.00 & $26.11 \%$ & -48.60 & -36.40 & ND & ND & 3 \\
\hline Muehlebach No. 1 & Tonkawa & 82.90 & 5.62 & 1.79 & 0.20 & 0.17 & 0.00 & 0.00 & 0.00 & 0.19 & 9.06 & $8.58 \%$ & -44.30 & ND & ND & ND & 3 \\
\hline Verna Roberts & Tonkawa & 80.01 & 7.30 & 2.62 & 0.22 & 0.31 & 0.00 & 0.00 & 0.00 & 0.31 & 9.21 & $11.55 \%$ & -47.30 & -28.60 & ND & ND & 3 \\
\hline $\begin{array}{l}\text { Ira E. Northrup } \\
\text { No. 1-21 }\end{array}$ & Tonkawa & 79.84 & 7.88 & 2.66 & 0.20 & 0.31 & 0.00 & 0.00 & 0.00 & 0.36 & 8.73 & $12.16 \%$ & -47.30 & -30.00 & ND & ND & 3 \\
\hline Berryman No. 1 & Tonkawa & 81.41 & 5.60 & 1.82 & 0.20 & 0.20 & 0.00 & 0.00 & 0.00 & 0.11 & 10.74 & $8.76 \%$ & -46.80 & ND & ND & ND & 3 \\
\hline Nuttall No.1-32 & Tonkawa & 80.42 & 5.63 & 1.99 & 0.29 & 0.34 & 0.00 & 0.00 & 0.00 & 0.18 & 11.16 & $9.30 \%$ & -47.00 & ND & ND & ND & 3 \\
\hline Dunaway No.2-4 & Hoover & 86.19 & 5.51 & 2.28 & 0.47 & 0.31 & 0.09 & 0.13 & 0.00 & 0.19 & 4.83 & $9.25 \%$ & -44.20 & ND & ND & ND & 3 \\
\hline Shaller No. 1 & Douglas & 79.37 & 7.59 & 4.27 & 1.17 & 0.62 & 0.31 & 0.35 & 0.00 & 0.52 & 5.80 & $15.28 \%$ & -49.80 & ND & ND & ND & 3 \\
\hline $\begin{array}{l}\text { Texas Wildlife and } \\
\text { Parks No. } 2\end{array}$ & Douglas & 78.05 & 7.36 & 4.46 & 1.21 & 0.64 & 0.33 & 0.38 & 0.00 & 0.30 & 7.27 & $15.56 \%$ & -49.60 & ND & ND & ND & 3 \\
\hline Baker No. 1-20 & Cherokee & 92.49 & 4.01 & 0.67 & 0.09 & 0.14 & ND & ND & 0.00 & 1.20 & 1.30 & $5.04 \%$ & -42.30 & -32.90 & ND & -133 & 3 \\
\hline Watkins No. 2-21 & Red Fork & 92.30 & 4.26 & 0.82 & 0.10 & 0.16 & $\mathrm{ND}$ & ND & 0.00 & 1.10 & 1.20 & $5.47 \%$ & -44.10 & -32.30 & ND & -145 & 3 \\
\hline Wilks No. 1-17 & Red Fork & 93.36 & 3.40 & 0.66 & 0.08 & 0.16 & ND & ND & 0.00 & 1.12 & 1.20 & $4.40 \%$ & -43.20 & -32.50 & ND & -136 & 3 \\
\hline Thurmond No. 1-32 & Red Fork & 89.58 & 5.57 & 1.56 & 0.22 & 0.26 & ND & ND & 0.00 & 0.90 & 1.90 & $7.83 \%$ & -44.50 & -34.10 & ND & -145 & 3 \\
\hline Marik No. 1-11 & L. Desmoinesian & 83.09 & 7.20 & 2.36 & 0.43 & 0.38 & ND & ND & 0.00 & 1.00 & 5.60 & $11.10 \%$ & -46.40 & -43.80 & ND & -150 & 3 \\
\hline Partain No. 1-6 & Red Fork & 90.57 & 5.52 & 1.04 & 0.14 & 0.22 & ND & ND & 0.00 & 1.30 & 1.20 & $7.10 \%$ & -45.00 & -31.00 & ND & -152 & 3 \\
\hline Flaming No. 1-20 & Springer & 94.28 & 1.80 & 1.06 & 0.20 & 0.14 & ND & ND & 0.00 & 1.43 & 1.09 & $3.28 \%$ & -40.70 & -36.50 & ND & -149 & 3 \\
\hline Palmer No. 1-17 & Red Fork & 85.79 & 6.95 & 3.76 & 0.70 & 0.55 & ND & ND & 0.00 & 0.82 & 1.43 & $12.24 \%$ & -45.90 & -35.10 & ND & -153 & 3 \\
\hline $\begin{array}{l}\text { Canyon Camp Unit } \\
\text { No. I }\end{array}$ & Red Fork & 86.51 & 7.36 & 2.94 & 0.50 & 0.41 & ND & ND & 0.00 & 0.92 & 1.37 & $11.47 \%$ & -46.70 & -33.30 & ND & -152 & 3 \\
\hline
\end{tabular}


[Units for the compositional data are in mole percent, and the $\delta^{13} \mathrm{C}$ and $\delta^{2} \mathrm{H}$ values are in per mil relative to the Vienna Pee Dee Belemnite and Vienna Standard Mean Ocean Water scales, respectively. Gas wetness is calculated as $\Sigma \mathrm{C}_{2+} / \Sigma \mathrm{C}_{1}+\mathrm{C}_{2+} \cdot \mathrm{C}_{1}$, methane; $\mathrm{C}_{2}$, ethane; $\mathrm{C}_{3}$, propane; $\mathrm{n}-\mathrm{C}_{4}, \mathrm{n}$-butane; i- $\mathrm{C}_{4}$, i-butane; $\mathrm{n}-\mathrm{C}_{5}, \mathrm{n}-$ pentane; $\mathrm{i}-\mathrm{C}_{5}$, $=\mathrm{i}$-pentane; $\mathrm{C}_{6+}$, six carbon hydrocarbons and higher; $\mathrm{CO}_{2}$, carbon dioxide; $\mathrm{N}_{2}$, nitrogen; Wet, gas wetness in percent and $\delta^{13} \mathrm{C}_{1}, \delta^{13} \mathrm{C}_{2}$, and $\delta^{13} \mathrm{C}_{3}$ are the stable carbon isotopic composition of methane, ethane, and propane, respectively. $\delta^{2} \mathrm{HC}$ is the stable hydrogen isotopic composition of methane. ND, not determined. S indicates the source of the data and the numbers refer to the following publications: (1) Jenden and Kaplan (1989a), (2) Jenden and others (1988), (3) Rice and others (1988a), and (4) this study]

\begin{tabular}{|c|c|c|c|c|c|c|c|c|c|c|c|c|c|c|c|c|c|}
\hline $\begin{array}{l}\text { Well } \\
\text { name }\end{array}$ & $\begin{array}{c}\text { Formation } \\
\text { name }\end{array}$ & $C_{1}$ & $\mathrm{C}_{2}$ & $\mathrm{C}_{3}$ & $n-C_{4}$ & $\mathrm{i}-\mathrm{C}_{4}$ & $n-C_{5}$ & i-C $C_{5}$ & $\mathrm{C}_{6+}$ & $\mathrm{CO}_{2}$ & $\mathbf{N}_{2}$ & Wet & $\delta^{13} \mathbf{C}_{1}$ & $\delta^{13} \mathbf{C}_{2}$ & $\delta^{13} \mathbf{C}_{3}$ & $\delta^{2} \mathrm{HC}_{1}$ & $\mathbf{S}$ \\
\hline $\begin{array}{l}\text { Rickenberg McKay } \\
\text { No. } 1\end{array}$ & Red Fork & 86.57 & 6.26 & 2.67 & 0.48 & 0.40 & ND & ND & 0.00 & 2.10 & 1.51 & $10.18 \%$ & -46.70 & -32.80 & ND & -159 & 3 \\
\hline Hazlett No. 1 & Red Fork & 80.19 & 11.51 & 4.47 & 0.75 & 0.64 & ND & ND & 0.00 & 0.77 & 1.65 & $17.80 \%$ & -46.90 & -32.80 & ND & -158 & 3 \\
\hline Worley Unit No. I & Cherokee & 83.74 & 4.19 & 1.61 & 0.36 & 0.20 & 0.05 & 0.09 & 0.00 & 0.47 & 9.10 & $7.20 \%$ & -42.60 & ND & ND & ND & 3 \\
\hline Brown No. $1 \mathrm{H}$ & Red Fork & 75.11 & 2.65 & 0.66 & 0.09 & 0.06 & 0.01 & 0.02 & 0.00 & 0.33 & 21.07 & $4.44 \%$ & -41.60 & ND & ND & ND & 3 \\
\hline Outhier No. I & Big Lime & 78.08 & 2.96 & 0.96 & 0.10 & 0.11 & 0.02 & 0.02 & 0.00 & 0.20 & 17.55 & $5.07 \%$ & -40.70 & ND & ND & ND & 3 \\
\hline Carl No. 1-35 & Red Fork & 73.82 & 6.64 & 1.99 & 0.15 & 0.24 & 0.00 & 0.02 & 0.00 & 0.41 & 16.73 & $10.91 \%$ & -43.70 & ND & ND & ND & 3 \\
\hline Thurmond No. 1-27 & Atoka & 94.65 & 2.04 & 0.36 & 0.07 & 0.15 & ND & ND & 0.00 & 1.20 & 1.50 & $2.69 \%$ & -43.10 & -32.00 & ND & -137 & 3 \\
\hline McConnell & L. \& M. Atoka & 95.72 & 1.87 & 0.29 & 0.04 & 0.04 & ND & ND & 0.00 & 1.10 & 1.00 & $2.29 \%$ & -42.30 & -34.30 & ND & -149 & 3 \\
\hline K.C. Cattle No. 1-34 & Atoka & 95.20 & 1.48 & 0.35 & 0.08 & 0.17 & ND & ND & 0.00 & 1.30 & 1.40 & $2.14 \%$ & -42.90 & -32.20 & ND & -139 & 3 \\
\hline Gregory No. 1-12 & L. \& M. Atoka & 95.20 & 1.74 & 0.38 & 0.09 & 0.13 & ND & ND & 0.00 & 1.20 & 1.20 & $2.40 \%$ & -43.90 & -31.20 & ND & -145 & 3 \\
\hline Ashby No. 1-36 & L. \& M. Atoka & 82.00 & 9.64 & 4.20 & 0.73 & 0.61 & ND & ND & 0.00 & 0.07 & 2.10 & $15.62 \%$ & -48.10 & -34.30 & ND & -162 & 3 \\
\hline Coulson No. 5-1 & L. \& M. Atoka & 95.06 & 1.91 & 0.48 & 0.04 & 0.09 & ND & ND & 0.00 & 1.10 & 1.40 & $2.58 \%$ & -43.00 & -31.30 & ND & -151 & 3 \\
\hline Webb No. 2-1 & L. \& M. Atoka & 95.23 & 2.08 & 0.44 & 0.03 & 0.04 & ND & ND & 0.00 & 1.10 & 1.10 & $2.65 \%$ & -43.70 & -30.70 & ND & -150 & 3 \\
\hline Allison No. 103 & L. Atoka & 95.72 & 1.77 & 0.28 & 0.03 & 0.04 & ND & ND & 0.00 & 1.01 & 1.20 & $2.17 \%$ & -42.80 & -34.30 & ND & -147 & 3 \\
\hline Risley No. 4 & Granite Wash & 81.84 & 8.67 & 3.65 & 0.90 & 0.47 & 0.25 & 0.28 & 0.00 & 0.77 & 3.16 & $14.80 \%$ & -45.60 & ND & ND & ND & 3 \\
\hline Risley No. 3 & Granite Wash & 79.00 & 8.56 & 3.70 & 0.97 & 0.50 & 0.30 & 0.30 & 0.00 & 0.68 & 5.99 & $15.35 \%$ & -45.70 & ND & ND & ND & 3 \\
\hline Ross No. 2-71 & Granite Wash & 70.75 & 8.06 & 3.59 & 0.87 & 0.47 & 0.22 & 0.24 & 0.00 & 0.32 & 15.47 & $15.97 \%$ & -46.20 & ND & ND & ND & 3 \\
\hline Hobart No. 8-70 & Granite Wash & 78.02 & 10.45 & 4.83 & 1.28 & 0.69 & 0.30 & 0.34 & 0.00 & 0.69 & 3.42 & $18.65 \%$ & -48.30 & ND & ND & ND & 3 \\
\hline Long “A” No. 2 & Morrow & 79.61 & 5.39 & 2.81 & 0.26 & 0.27 & 0.00 & 0.00 & 0.00 & 0.25 & 11.34 & $9.88 \%$ & -43.10 & ND & ND & ND & 3 \\
\hline Barby No. 1-36 & Morrow & 79.39 & 5.53 & 3.45 & 0.30 & 0.23 & 0.00 & 0.00 & 0.00 & 0.32 & 10.76 & $10.70 \%$ & -44.40 & -33.80 & ND & ND & 3 \\
\hline Barby No. 1-29 & Chester & 83.48 & 3.81 & 1.64 & 0.15 & 0.16 & 0.00 & 0.00 & 0.00 & 0.20 & 10.49 & $6.45 \%$ & -42.10 & ND & ND & ND & 3 \\
\hline Boatman No. 1-23 & Morrow & 83.30 & 4.12 & 1.18 & 0.08 & 0.11 & 0.00 & 0.00 & 0.00 & 0.46 & 10.73 & $6.18 \%$ & -40.20 & ND & ND & ND & 3 \\
\hline Elliot No. 1-24 & Morrow & 77.98 & 3.58 & 1.13 & 0.08 & 0.13 & 0.00 & 0.00 & 0.00 & 0.10 & 17.00 & $5.93 \%$ & -40.40 & ND & ND & ND & 3 \\
\hline Frisby No. 1-29 & Morrow & 82.55 & 4.47 & 1.53 & 0.10 & 8.18 & 0.00 & 0.00 & 0.00 & 0.26 & 10.91 & $14.75 \%$ & -37.70 & ND & ND & ND & 3 \\
\hline Harrison No. 1-19 & Chester & 86.34 & 2.13 & 0.40 & 0.00 & 0.00 & 0.00 & 0.00 & 0.00 & 0.32 & 10.78 & $2.85 \%$ & -38.00 & ND & ND & ND & 3 \\
\hline Hudson No. 1-35 & Morrow & 86.94 & 5.12 & 2.30 & 0.50 & 0.33 & 0.08 & 0.14 & 0.00 & 0.29 & 4.20 & $8.88 \%$ & -41.90 & ND & ND & ND & 3 \\
\hline Conley No. 1 & L. Morrow & 80.62 & 2.61 & 0.67 & 0.06 & 0.07 & 0.01 & 0.02 & 0.00 & 0.28 & 15.66 & $4.09 \%$ & -39.60 & ND & ND & ND & 3 \\
\hline
\end{tabular}


Table 2. Molecular and stable isotopic composition of gases from the central Anadarko Basin._Continued

[Units for the compositional data are in mole percent, and the $\delta^{13} \mathrm{C}$ and $\delta^{2} \mathrm{H}$ values are in per mil relative to the Vienna Pee Dee Belemnite and Vienna Standard Mean Ocean Water scales, respectively. Gas wetness is calculated as $\Sigma \mathrm{C}_{2+} / \Sigma \mathrm{C}_{1}+\mathrm{C}_{2+}$. $\mathrm{C}_{1}$, methane; $\mathrm{C}_{2}$, ethane; $\mathrm{C}_{3}$, propane; $\mathrm{n}-\mathrm{C}_{4}$, n-butane; i- $\mathrm{C}_{4}$, i-butane; $\mathrm{n}-\mathrm{C}_{5}$, n-pentane; i- $\mathrm{C}_{5}$, = i-pentane; $\mathrm{C}_{6+}$, six carbon hydrocarbons and higher; $\mathrm{CO}_{2}$, carbon dioxide; $\mathrm{N}_{2}$, nitrogen; Wet, gas wetness in percent and $\delta^{13} \mathrm{C}_{1}, \delta^{13} \mathrm{C}_{2}$, and $\delta^{13} \mathrm{C}_{3}$ are the stable carbon isotopic composition of methane, ethane, and propane, respectively. $\delta^{2} \mathrm{HC} \mathrm{C}_{1}$ is the stable hydrogen isotopic composition of methane. ND, not determined. S indicates the source of the data and the numbers refer to the following publications: (1) Jenden and Kaplan (1989a), (2) Jenden and others (1988), (3) Rice and others (1988a), and (4) this study]

\begin{tabular}{|c|c|c|c|c|c|c|c|c|c|c|c|c|c|c|c|c|c|}
\hline $\begin{array}{l}\text { Well } \\
\text { name }\end{array}$ & $\begin{array}{c}\text { Formation } \\
\text { name }\end{array}$ & $C_{1}$ & $\mathrm{C}_{2}$ & $\mathrm{C}_{3}$ & $n-C_{4}$ & $\mathrm{i}-\mathrm{C}_{4}$ & $n-C_{5}$ & $\mathrm{i}-\mathrm{C}_{5}$ & $\mathrm{C}_{6+}$ & $\mathrm{CO}_{2}$ & $\mathbf{N}_{2}$ & Wet & $\delta^{13} \mathbf{C}_{1}$ & $\delta^{13} \mathbf{C}_{2}$ & $\delta^{13} \mathbf{C}_{3}$ & $\delta^{2} \mathrm{HC}_{1}$ & S \\
\hline Bradford No. 1-18 & Morrow & 78.25 & 6.24 & 3.02 & 0.38 & 0.36 & 0.01 & 0.08 & 0.00 & 0.45 & 11.21 & $11.42 \%$ & -42.40 & ND & ND & ND & 3 \\
\hline Tomkinson No. 17-1 & L. Morrow & 78.41 & 3.52 & 0.01 & 0.08 & 0.19 & 0.00 & 0.02 & 0.00 & 0.52 & 17.30 & $4.65 \%$ & -39.90 & ND & ND & ND & 3 \\
\hline Youngheim No. I & Morrow & 84.12 & 1.09 & 0.38 & 0.01 & 0.01 & 0.00 & 0.00 & 0.00 & 0.22 & 14.17 & $1.74 \%$ & -37.80 & ND & ND & ND & 3 \\
\hline Barnum No. 1-32 & Morrow & 96.59 & 0.95 & 0.04 & 0.00 & 0.00 & ND & ND & 0.00 & 1.40 & 0.90 & $1.01 \%$ & -38.50 & ND & ND & -135 & 3 \\
\hline Clark No. 1-33 & Puryear & 95.81 & 1.06 & 0.04 & 0.00 & 0.00 & ND & ND & 0.00 & 1.50 & 1.50 & $1.14 \%$ & -38.20 & -36.10 & ND & -147 & 3 \\
\hline Gregory No. 2-27 & Morrow & 96.41 & 1.08 & 0.04 & 0.00 & 0.00 & ND & ND & 0.00 & 1.60 & 0.80 & $1.15 \%$ & -38.20 & -36.60 & ND & -133 & 3 \\
\hline Farrar No. 1-22 & Morrow & 96.18 & 0.26 & 0.04 & 0.00 & 0.00 & ND & ND & 0.00 & 1.40 & 1.90 & $0.31 \%$ & -38.20 & ND & ND & -133 & 3 \\
\hline Watkins No. 1-21 & Morrow & 96.80 & 0.41 & 0.03 & 0.00 & 0.00 & ND & ND & 0.00 & 1.50 & 1.20 & $0.45 \%$ & -38.20 & ND & ND & -135 & 3 \\
\hline Thornton No.2-30 & Morrow & 96.75 & 0.46 & 0.03 & 0.00 & 0.00 & ND & ND & 0.00 & 1.70 & 1.00 & $0.50 \%$ & -37.80 & ND & ND & -131 & 3 \\
\hline Berry No. 1-8 & Morrow & 96.48 & 0.52 & 0.00 & 0.00 & 0.00 & ND & ND & 0.00 & 1.70 & 1.30 & $0.54 \%$ & -38.30 & ND & ND & -139 & 3 \\
\hline Dugger No. 1-18 & Morrow & 95.45 & 0.30 & 0.01 & 0.00 & 0.00 & $\mathrm{ND}$ & ND & 0.00 & 2.80 & 1.60 & $0.32 \%$ & -38.10 & ND & ND & -140 & 3 \\
\hline Rymer No. 1 & Morrow & 92.28 & 3.65 & 1.40 & 0.25 & 0.31 & $\mathrm{ND}$ & ND & 0.00 & 0.90 & 1.30 & $5.73 \%$ & -43.00 & -32.70 & ND & -149 & 3 \\
\hline West No. 1-7 & Morrow & 94.61 & 2.36 & 0.69 & 0.08 & 0.08 & ND & ND & 0.00 & 0.98 & 1.19 & $3.28 \%$ & -41.40 & -34.00 & ND & -150 & 3 \\
\hline Ranch No. 1-15 & Morrow & 87.08 & 6.69 & 2.90 & 0.59 & 0.37 & $\mathrm{ND}$ & $\mathrm{ND}$ & 0.00 & 0.07 & 1.65 & $10.81 \%$ & -49.20 & -38.30 & ND & -154 & 3 \\
\hline Kardokus No. 10-1 & Morrow & 96.24 & 1.07 & 0.14 & 0.03 & 0.02 & ND & ND & 0.00 & 1.56 & 0.93 & $1.29 \%$ & -38.80 & -34.90 & ND & -170 & 3 \\
\hline Lesperance No. 1-35 & Springer & 85.05 & 0.82 & 0.02 & 0.00 & 0.01 & 0.00 & 0.01 & 0.00 & 0.75 & 13.16 & $1.00 \%$ & -36.60 & ND & ND & ND & 3 \\
\hline Rice No. 1-35 & Morrow & 94.01 & 0.66 & 0.00 & 0.00 & 0.00 & ND & ND & 0.00 & 3.10 & 2.10 & $0.70 \%$ & -33.20 & ND & ND & -154 & 3 \\
\hline Cornstalk Unit No. 1-8 & Springer & ND & 2.29 & 0.81 & 0.11 & 0.13 & ND & ND & 0.00 & 1.10 & 1.20 & $100.00 \%$ & -41.90 & -34.60 & ND & -142 & 3 \\
\hline Rayner No. 2 & Springer & 95.60 & 1.20 & 0.50 & 0.09 & 0.08 & ND & ND & 0.00 & 1.10 & 1.30 & $1.92 \%$ & -39.90 & -34.90 & ND & -144 & 3 \\
\hline Hamburger No 1-9 & Springer & 96.46 & 0.00 & 0.13 & 0.00 & 0.00 & ND & ND & 0.00 & 1.54 & 1.86 & $0.13 \%$ & -39.00 & -35.50 & ND & -142 & 3 \\
\hline $\begin{array}{l}\text { Brown Foundation } \\
\text { No. 1-16 }\end{array}$ & Springer & 97.23 & 0.16 & 0.07 & 0.00 & 0.00 & ND & ND & 0.00 & 1.45 & 1.00 & $0.24 \%$ & -39.40 & ND & ND & -139 & 3 \\
\hline Flaming No. 1-20 & Springer & 96.30 & 0.98 & 0.08 & 0.01 & 0.01 & ND & ND & 0.00 & 1.67 & 0.94 & $1.11 \%$ & -38.90 & -35.70 & ND & -148 & 3 \\
\hline Hintchel Unit No. 1 & Springer & 94.99 & 2.17 & 0.62 & 0.07 & 0.07 & ND & $\mathrm{ND}$ & 0.00 & 0.98 & 1.09 & $2.99 \%$ & -40.30 & -34.50 & ND & -146 & 3 \\
\hline Jones No. 1-17 & Boatwright & 93.92 & 2.19 & 1.03 & 0.18 & 0.16 & ND & ND & 0.00 & 1.03 & 0.48 & $3.65 \%$ & -39.50 & -34.30 & ND & -143 & 3 \\
\hline Bull Elk No. 1 & L. Springer & 97.02 & 0.55 & 0.14 & 0.00 & 0.00 & ND & ND & 0.00 & 0.96 & 1.32 & $0.71 \%$ & -41.40 & -35.90 & ND & -145 & 3 \\
\hline Ten Bears No.1 & Springer & 96.36 & 1.50 & 0.12 & 0.00 & 0.00 & ND & $\mathrm{ND}$ & 0.00 & 1.18 & 0.82 & $1.65 \%$ & -40.00 & -34.40 & ND & -144 & 3 \\
\hline Little Washita No. 1 & Springer & 96.21 & 1.24 & 0.10 & 0.00 & 0.00 & ND & ND & 0.00 & 1.22 & 1.22 & $1.37 \%$ & -38.90 & -34.00 & ND & -140 & 3 \\
\hline
\end{tabular}


[Units for the compositional data are in mole percent, and the $\delta^{13} \mathrm{C}$ and $\delta^{2} \mathrm{H}$ values are in per mil relative to the Vienna Pee Dee Belemnite and Vienna Standard Mean Ocean Water scales, respectively. Gas wetness is calculated as $\Sigma \mathrm{C}_{2+} / \Sigma \mathrm{C}_{1}+\mathrm{C}_{2+} \cdot \mathrm{C}_{1}$, methane; $\mathrm{C}_{2}$, ethane; $\mathrm{C}_{3}$, propane; $\mathrm{n}-\mathrm{C}_{4}, \mathrm{n}$-butane; i- $\mathrm{C}_{4}$, i-butane; $\mathrm{n}-\mathrm{C}_{5}, \mathrm{n}-$ pentane; $\mathrm{i}-\mathrm{C}_{5}$, $=\mathrm{i}$-pentane; $\mathrm{C}_{6+}$, six carbon hydrocarbons and higher; $\mathrm{CO}_{2}$, carbon dioxide; $\mathrm{N}_{2}$, nitrogen; Wet, gas wetness in percent and $\delta^{13} \mathrm{C}_{1}, \delta^{13} \mathrm{C}_{2}$, and $\delta^{13} \mathrm{C}_{3}$ are the stable carbon isotopic composition of methane, ethane, and propane, respectively. $\delta^{2} \mathrm{HC}$ is the stable hydrogen isotopic composition of methane. ND, not determined. S indicates the source of the data and the numbers refer to the following publications: (1) Jenden and Kaplan (1989a), (2) Jenden and others (1988), (3) Rice and others (1988a), and (4) this study]

\begin{tabular}{|c|c|c|c|c|c|c|c|c|c|c|c|c|c|c|c|c|c|}
\hline $\begin{array}{l}\text { Well } \\
\text { name }\end{array}$ & $\begin{array}{c}\text { Formation } \\
\text { name }\end{array}$ & $C_{1}$ & $\mathrm{C}_{2}$ & $\mathrm{C}_{3}$ & $n-C_{4}$ & $\mathrm{i}-\mathrm{C}_{4}$ & $n-C_{5}$ & $\mathrm{i}-\mathrm{C}_{5}$ & $\mathrm{C}_{6+}$ & $\mathrm{CO}_{2}$ & $\mathbf{N}_{2}$ & Wet & $\delta^{13} \mathbf{C}_{1}$ & $\delta^{13} \mathbf{C}_{2}$ & $\delta^{13} \mathbf{C}_{3}$ & $\delta^{2} \mathrm{HC}_{1}$ & S \\
\hline Quanah Parker No. 1 & Springer & 95.62 & 1.47 & 0.16 & 0.01 & 0.01 & ND & ND & 0.00 & 1.01 & 1.71 & $1.70 \%$ & -43.00 & -36.30 & ND & -136 & 3 \\
\hline Kardokus No. 10-1 & Springer & 96.02 & 1.14 & 0.15 & 0.02 & 0.01 & ND & ND & 0.00 & 1.80 & 0.85 & $1.36 \%$ & -38.70 & ND & ND & -148 & 3 \\
\hline APC Long No. A-1 & Chester & 86.67 & 2.91 & 0.97 & 0.13 & 0.12 & 0.00 & 0.00 & 0.00 & 0.19 & 9.00 & $4.55 \%$ & -40.20 & ND & ND & ND & 3 \\
\hline McFarland No. 1-32 & Chester & 87.54 & 1.99 & 0.45 & 0.00 & 0.00 & 0.00 & 0.00 & 0.00 & 0.32 & 9.69 & $2.71 \%$ & -39.20 & ND & ND & ND & 3 \\
\hline Barby No. 1-10 & Chester & 86.61 & 3.04 & 0.74 & 0.00 & 0.09 & 0.00 & 0.00 & 0.00 & 0.53 & 8.99 & $4.28 \%$ & -40.20 & ND & ND & ND & 3 \\
\hline Barby No. 1-25 & Morrow & 86.46 & 3.45 & 0.96 & 0.08 & 0.12 & 0.00 & 0.00 & 0.00 & 0.80 & 8.75 & $5.06 \%$ & -40.50 & ND & ND & ND & 3 \\
\hline Barby No. 1-24 & Chester & 86.14 & 3.75 & 1.16 & 0.13 & 0.17 & 0.00 & 0.00 & 0.00 & 0.47 & 8.19 & $5.70 \%$ & -40.70 & -30.70 & ND & ND & 3 \\
\hline Brown No. 1-H & Red Fork & 78.45 & 3.30 & 1.00 & 0.10 & 0.11 & 0.00 & 0.02 & 0.00 & 0.35 & 16.68 & $5.46 \%$ & -40.50 & ND & ND & ND & 3 \\
\hline Holden No. 1A & Simpson & 80.52 & 9.40 & 5.90 & 1.81 & 1.02 & ND & ND & 0.00 & 0.41 & 0.93 & $18.38 \%$ & -47.00 & -34.80 & ND & -158 & 3 \\
\hline Harris No. 2 & Bromide 4 & 85.17 & 7.97 & 3.93 & 0.90 & 0.58 & ND & ND & 0.00 & 0.52 & 0.93 & $13.58 \%$ & -46.90 & -34.70 & ND & -161 & 3 \\
\hline Holden No. 1A & Arbuckle & 89.51 & 5.31 & 2.41 & 0.88 & 0.46 & ND & ND & 0.00 & 0.33 & 1.09 & $9.19 \%$ & -45.40 & -34.90 & ND & -156 & 3 \\
\hline Alexander 1-30 & Desmoinesian & 81.69 & 9.49 & 3.91 & 1.14 & 0.67 & 0.36 & 0.41 & 0.68 & 0.54 & 0.81 & $16.95 \%$ & ND & ND & ND & ND & 4 \\
\hline Alice 1-31 & Granite Wash & 89.74 & 5.49 & 2.19 & 0.56 & 0.29 & 0.12 & 0.14 & 0.21 & 0.53 & 0.61 & $9.12 \%$ & -40.73 & -37.12 & -32.73 & -135 & 4 \\
\hline Armstrong a 1-30 & Desmoinesian & 73.69 & 12.28 & 6.79 & 2.09 & 0.94 & 0.52 & 0.48 & 0.40 & 0.74 & 1.27 & $24.19 \%$ & -48.71 & -34.49 & -29.75 & ND & 4 \\
\hline Davis $1-20$ & Desmoinesian & 84.31 & 8.59 & 3.27 & 0.89 & 0.54 & 0.27 & 0.31 & 0.47 & 0.59 & 0.58 & $14.54 \%$ & -47.84 & -32.26 & -28.47 & ND & 4 \\
\hline Davis $1-29$ & Desmoinesian & 82.88 & 9.09 & 3.69 & 1.00 & 0.62 & 0.28 & 0.34 & 0.47 & 0.58 & 0.83 & $15.74 \%$ & -48.31 & -32.51 & -28.81 & -154 & 4 \\
\hline Davis 2-30 & Desmoinesian & 83.02 & 9.24 & 3.45 & 0.85 & 0.54 & 0.27 & 0.26 & 0.34 & 0.55 & 1.20 & $15.26 \%$ & -47.87 & -32.47 & -28.81 & ND & 4 \\
\hline Davis 3-20 ST & Desmoinesian & 82.19 & 9.37 & 3.89 & 1.07 & 0.95 & 0.27 & 0.33 & 0.45 & 0.56 & 0.71 & $16.58 \%$ & -48.49 & -32.73 & -28.98 & -151 & 4 \\
\hline Davis $3-30$ & Desmoinesian & 82.11 & 9.75 & 3.87 & 1.05 & 0.64 & 0.30 & 0.35 & 0.13 & 0.66 & 0.77 & $16.38 \%$ & -48.03 & -32.72 & -29.09 & ND & 4 \\
\hline Davis 4-30 & Desmoinesian & 83.31 & 9.23 & 3.72 & 1.02 & 0.62 & 0.28 & 0.34 & 0.10 & 0.54 & 0.67 & $15.53 \%$ & -48.08 & -32.44 & -28.64 & ND & 4 \\
\hline Fowler 1-29 & Desmoinesian & 82.99 & 9.00 & 3.49 & 0.93 & 0.59 & 0.26 & 0.32 & 0.38 & 0.58 & 0.71 & $15.28 \%$ & -48.18 & -32.46 & -28.84 & -150 & 4 \\
\hline Haley 2-31 & Desmoinesian & 78.10 & 10.53 & 5.51 & 1.75 & 0.84 & 0.43 & 0.49 & 0.34 & 0.77 & 1.06 & $20.30 \%$ & -48.11 & -34.70 & -31.83 & ND & 4 \\
\hline Haley 4-31 & Desmoinesian & 87.65 & 6.26 & 2.23 & 0.58 & 0.37 & 0.15 & 0.18 & 0.19 & 0.51 & 1.22 & $10.20 \%$ & -45.52 & ND & ND & ND & 4 \\
\hline Haley 5-31 & Desmoinesian & 82.65 & 9.18 & 3.81 & 1.07 & 0.59 & 0.30 & 0.34 & 0.35 & 0.81 & 0.73 & $15.92 \%$ & -47.23 & -33.67 & -30.58 & ND & 4 \\
\hline Hart 1-31 & Desmoinesian & 80.95 & 9.76 & 4.74 & 1.31 & 0.71 & 0.29 & 0.34 & 0.26 & 0.72 & 0.82 & $17.69 \%$ & -47.52 & -34.40 & -31.16 & ND & 4 \\
\hline Hubert 1-4 & Desmoinesian & 75.54 & 9.86 & 6.09 & 2.29 & 1.11 & 0.67 & 0.69 & 0.26 & 0.98 & 1.84 & $21.72 \%$ & -48.13 & -36.55 & -32.41 & ND & 4 \\
\hline Johnson 1-22 & Desmoinesian & 76.05 & 11.13 & 5.95 & 2.08 & 0.99 & 0.58 & 0.01 & 0.49 & 0.97 & 1.02 & $21.82 \%$ & -47.48 & -32.91 & -29.87 & -159 & 4 \\
\hline
\end{tabular}


Table 2. Molecular and stable isotopic composition of gases from the central Anadarko Basin._-Continued

[Units for the compositional data are in mole percent, and the $\delta^{13} \mathrm{C}$ and $\delta^{2} \mathrm{H}$ values are in per mil relative to the Vienna Pee Dee Belemnite and Vienna Standard Mean Ocean Water scales, respectively. Gas wetness is calculated as $\Sigma \mathrm{C}_{2+} / \Sigma \mathrm{C}_{1}+\mathrm{C}_{2+}$. $\mathrm{C}_{1}$, methane; $\mathrm{C}_{2}$, ethane; $\mathrm{C}_{3}$, propane; $\mathrm{n}-\mathrm{C}_{4}$, n-butane; i- $\mathrm{C}_{4}$, i-butane; $\mathrm{n}-\mathrm{C}_{5}$, n-pentane; i- $\mathrm{C}_{5}$, = i-pentane; $\mathrm{C}_{6+}$, six carbon hydrocarbons and higher; $\mathrm{CO}_{2}$, carbon dioxide; $\mathrm{N}_{2}$, nitrogen; Wet, gas wetness in percent and $\delta^{13} \mathrm{C}_{1}, \delta^{13} \mathrm{C}_{2}$, and $\delta^{13} \mathrm{C}_{3}$ are the stable carbon isotopic composition of methane, ethane, and propane, respectively. $\delta^{2} \mathrm{HC} \mathrm{C}_{1}$ is the stable hydrogen isotopic composition of methane. ND, not determined. S indicates the source of the data and the numbers refer to the following publications: (1) Jenden and Kaplan (1989a), (2) Jenden and others (1988), (3) Rice and others (1988a), and (4) this study]

\begin{tabular}{|c|c|c|c|c|c|c|c|c|c|c|c|c|c|c|c|c|c|}
\hline $\begin{array}{l}\text { Well } \\
\text { name }\end{array}$ & $\begin{array}{l}\text { Formation } \\
\text { name }\end{array}$ & $C_{1}$ & $C_{2}$ & $\mathrm{C}_{3}$ & $\mathrm{n}-\mathrm{C}_{4}$ & $\mathrm{i}-\mathrm{C}_{4}$ & $n-C_{5}$ & $\mathrm{i}-\mathrm{C}_{5}$ & $C_{6+}$ & $\mathrm{CO}_{2}$ & $\mathbf{N}_{2}$ & Wet & $\delta^{13} C_{1}$ & $\delta^{13} \mathbf{C}_{2}$ & $\delta^{13} \mathbf{C}_{3}$ & $\delta^{2} \mathrm{HC}_{1}$ & $\mathbf{S}$ \\
\hline Marriot 1-36 & Morrow & 95.33 & 0.90 & 0.16 & 0.08 & 0.04 & 0.01 & 0.02 & 0.04 & 1.15 & 1.79 & $1.29 \%$ & -38.77 & -34.13 & -29.93 & ND & 4 \\
\hline Megan 2-5 & Atoka & 96.84 & 1.38 & 0.24 & 0.11 & 0.08 & 0.02 & 0.03 & 0.03 & 0.93 & 0.29 & $1.90 \%$ & -39.77 & -31.03 & -26.24 & -130 & 4 \\
\hline Merit 2-5 & Atoka & 96.62 & 1.41 & 0.27 & 0.12 & 0.09 & 0.03 & 0.04 & 0.07 & 0.96 & 0.29 & $2.07 \%$ & -39.81 & -31.09 & -25.96 & ND & 4 \\
\hline Mulberry 1-20 & Desmoinesian & 83.46 & 9.07 & 3.58 & 0.99 & 0.60 & 0.31 & 0.35 & 0.04 & 0.54 & 0.78 & $15.18 \%$ & -48.21 & -32.31 & -28.52 & ND & 4 \\
\hline Mulberry 2-20 & Desmoinesian & 84.60 & 8.59 & 3.14 & 0.79 & 0.50 & 0.22 & 0.25 & 0.47 & 0.51 & 0.71 & $14.17 \%$ & -48.03 & -32.36 & -28.48 & ND & 4 \\
\hline Seymour 1-12 & Cherokee & 94.41 & 3.14 & 0.60 & 0.14 & 0.15 & 0.02 & 0.05 & 0.03 & 0.69 & 0.46 & $4.18 \%$ & -43.90 & -31.85 & -27.89 & ND & 4 \\
\hline Tipton 1-32 & Desmoinesian & 80.81 & 10.14 & 4.38 & 1.20 & 0.76 & 0.30 & 0.38 & 0.41 & 0.56 & 0.87 & $17.87 \%$ & -48.48 & -32.27 & -28.69 & ND & 4 \\
\hline Tucker 2-17 & Desmoinesian & 86.24 & 8.34 & 2.87 & 0.65 & 0.44 & 0.12 & 0.17 & 0.04 & 0.49 & 0.59 & $12.78 \%$ & -47.65 & -32.06 & -28.39 & -149 & 4 \\
\hline Smith 1-11 & Cherokee & 95.23 & 2.78 & 0.52 & 0.14 & 0.14 & 0.02 & 0.05 & 0.09 & 0.66 & 0.32 & $3.77 \%$ & -42.85 & -32.08 & -28.07 & $\mathrm{ND}$ & 4 \\
\hline A \& C 6 & Desmoinesian & 86.47 & 7.75 & 2.55 & 0.52 & 0.43 & 0.11 & 0.18 & 0.30 & 1.20 & 0.36 & $12.05 \%$ & -44.88 & -33.03 & -29.41 & ND & 4 \\
\hline Apatite Federal 2 & Desmoinesian & 86.21 & 7.57 & 2.48 & 0.51 & 0.43 & 0.11 & 0.18 & 0.28 & 1.14 & 0.35 & $11.81 \%$ & -44.50 & -32.91 & -29.20 & ND & 4 \\
\hline Beals 1 & Desmoinesian & 79.21 & 10.83 & 4.96 & 1.34 & 0.72 & 0.38 & 0.43 & 0.33 & 0.98 & 0.42 & $19.33 \%$ & -45.83 & -34.18 & -30.23 & ND & 4 \\
\hline Beulah 2 & U. Desmoinesian & 78.23 & 10.84 & 5.45 & 1.41 & 0.78 & 0.41 & 0.48 & 0.43 & 1.03 & 0.44 & $20.18 \%$ & -45.27 & -33.68 & -30.02 & ND & 4 \\
\hline Beulah 3 & Desmoinesian & 81.96 & 9.58 & 4.27 & 1.12 & 0.67 & 0.27 & 0.34 & 0.25 & 0.97 & 0.38 & $16.76 \%$ & -44.92 & -33.72 & -30.01 & -158 & 4 \\
\hline Beulah 9 & Desmoinesian & 83.52 & 8.69 & 3.63 & 0.81 & 0.63 & 0.20 & 0.30 & 0.36 & 1.16 & 0.32 & $14.91 \%$ & -43.53 & -32.10 & -28.20 & ND & 4 \\
\hline Bottom 3 & Desmoinesian & 85.91 & 7.82 & 2.76 & 0.55 & 0.52 & 0.13 & 0.22 & 0.44 & 1.14 & 0.34 & $12.66 \%$ & -43.67 & -32.35 & -28.06 & -150 & 4 \\
\hline Clayton 6 & Desmoinesian & 81.69 & 9.89 & 4.20 & 0.99 & 0.63 & 0.21 & 0.30 & 0.47 & 0.96 & 0.49 & $16.97 \%$ & -46.08 & -32.91 & -29.24 & ND & 4 \\
\hline Earl B-3 & Desmoinesian & 82.58 & 8.88 & 3.79 & 1.01 & 0.65 & 0.31 & 0.40 & 0.35 & 1.15 & 0.38 & $15.71 \%$ & -44.20 & -32.57 & -28.76 & ND & 4 \\
\hline Hohbein 1 & Marmaton & 82.54 & 9.54 & 3.99 & 0.94 & 0.69 & 0.21 & 0.03 & 0.47 & 1.02 & 0.39 & $16.14 \%$ & -45.45 & -32.60 & -28.81 & -156 & 4 \\
\hline Hohbein 7 & Desmoinesian & 84.90 & 8.03 & 3.00 & 0.63 & 0.61 & 0.15 & 0.26 & 0.34 & 1.26 & 0.32 & $13.31 \%$ & -44.01 & -32.40 & -28.12 & ND & 4 \\
\hline Kimzey 8-5 & Desmoinesian & 89.42 & 6.10 & 1.60 & 0.26 & 0.34 & 0.07 & 0.13 & 0.15 & 1.33 & 0.35 & $8.81 \%$ & -42.70 & -31.21 & -27.21 & ND & 4 \\
\hline Lamb 2 & Red Fork & 87.24 & 6.85 & 2.28 & 0.46 & 0.45 & 0.10 & 0.18 & 0.19 & 1.33 & 0.69 & $10.77 \%$ & -43.93 & -33.13 & -28.91 & -142 & 4 \\
\hline Lovett 3 & Desmoinesian & 86.02 & 5.90 & 2.01 & 0.39 & 0.36 & 0.10 & 0.16 & 0.18 & 0.86 & 3.23 & $9.57 \%$ & -36.72 & -23.97 & -21.64 & ND & 4 \\
\hline Lucas 5 & Granite Wash & 89.26 & 5.33 & 2.31 & 0.64 & 0.32 & 0.18 & 0.18 & 0.34 & 0.43 & 0.81 & $9.44 \%$ & -41.00 & -38.00 & -33.69 & -134 & 4 \\
\hline Mooney 2 & Desmoinesian & 84.82 & 7.96 & 2.87 & 0.59 & 0.53 & 0.14 & 0.23 & 0.46 & 1.16 & 0.31 & $13.10 \%$ & -43.92 & -32.45 & -28.48 & -149 & 4 \\
\hline Olivia 1 & Desmoinesian & 82.02 & 9.53 & 3.95 & 0.92 & 0.69 & 0.22 & 0.35 & 0.55 & 0.98 & 0.57 & $16.49 \%$ & -45.37 & -32.51 & -28.83 & $\mathrm{ND}$ & 4 \\
\hline Patton B-3 & Desmoinesian & 89.24 & 5.88 & 1.98 & 0.37 & 0.42 & 0.07 & 0.15 & 0.26 & 1.26 & 0.26 & $9.28 \%$ & -42.19 & -31.90 & -27.60 & -140 & 4 \\
\hline
\end{tabular}


[Units for the compositional data are in mole percent, and the $\delta^{13} \mathrm{C}$ and $\delta^{2} \mathrm{H}$ values are in per mil relative to the Vienna Pee Dee Belemnite and Vienna Standard Mean Ocean Water scales, respectively. Gas wetness is calculated as $\Sigma \mathrm{C}_{2+} / \Sigma \mathrm{C}_{1}+\mathrm{C}_{2+} \mathrm{C}_{1}$, methane; $\mathrm{C}_{2}$, ethane; $\mathrm{C}_{3}$, propane; $\mathrm{n}_{4} \mathrm{C}_{4}$, n-butane; i- $\mathrm{C}_{4}$, i-butane; $\mathrm{n}-\mathrm{C}_{5}, \mathrm{n}$-pentane; i- $\mathrm{C}_{5}$, $=\mathrm{i}$-pentane; $\mathrm{C}_{6+}$, six carbon hydrocarbons and higher; $\mathrm{CO}_{2}$, carbon dioxide; $\mathrm{N}_{2}$, nitrogen; Wet, gas wetness in percent and $\delta^{13} \mathrm{C}_{1}, \delta^{13} \mathrm{C}_{2}$, and $\delta^{13} \mathrm{C}_{3}$ are the stable carbon isotopic composition of methane, ethane, and propane, respectively. $\delta^{2} \mathrm{HC}$ is the stable hydrogen isotopic composition of methane. ND, not determined. S indicates the source of the data and the numbers refer to the following publications: (1) Jenden and Kaplan (1989a), (2) Jenden and others (1988), (3) Rice and others (1988a), and (4) this study]

\begin{tabular}{|c|c|c|c|c|c|c|c|c|c|c|c|c|c|c|c|c|c|}
\hline $\begin{array}{l}\text { Well } \\
\text { name }\end{array}$ & $\begin{array}{c}\text { Formation } \\
\text { name }\end{array}$ & $C_{1}$ & $C_{2}$ & $\mathrm{C}_{3}$ & $\mathrm{n}-\mathrm{C}_{4}$ & $\mathrm{i}-\mathrm{C}_{4}$ & $n-C_{5}$ & $\mathrm{i}-\mathrm{C}_{5}$ & $\mathrm{C}_{6+}$ & $\mathrm{CO}_{2}$ & $\mathbf{N}_{2}$ & Wet & $\delta^{13} \mathbf{C}_{1}$ & $\delta^{13} \mathbf{C}_{2}$ & $\delta^{13} \mathbf{C}_{3}$ & $\delta^{2} \mathrm{HC}_{1}$ & $\mathbf{S}$ \\
\hline Phillips 3 & Desmoinesian & 86.45 & 7.48 & 2.55 & 0.50 & 0.48 & 0.11 & 0.20 & 0.34 & 1.32 & 0.40 & $11.89 \%$ & -43.35 & -32.20 & -28.08 & -146 & 4 \\
\hline Rounds 6-3 & Desmoinesian & 85.34 & 8.25 & 3.00 & 0.52 & 0.38 & 0.13 & 0.17 & 0.29 & 1.19 & 0.38 & $12.99 \%$ & -43.87 & -32.32 & -28.30 & ND & 4 \\
\hline Sam 1 & Desmoinesian & 86.32 & 8.08 & 2.93 & 0.61 & 0.58 & 0.16 & 0.26 & 0.56 & 0.02 & 0.29 & $13.25 \%$ & -43.90 & -32.23 & -27.91 & -151 & 4 \\
\hline Welty 3 & Desmoinesian & 87.52 & 7.34 & 2.28 & 0.42 & 0.11 & 0.10 & 0.17 & 0.27 & 1.24 & 0.32 & $10.89 \%$ & -43.76 & -32.79 & -28.96 & ND & 4 \\
\hline Banks 3-20 & Cleveland & 81.08 & 7.80 & 3.50 & 0.83 & 0.61 & 0.19 & 0.28 & 0.37 & 1.04 & 3.47 & $14.35 \%$ & -45.12 & -34.14 & -30.01 & -154 & 4 \\
\hline Bloch 3-34 & Morrow & 85.41 & 6.95 & 3.65 & 1.16 & 0.47 & 0.38 & 0.32 & 0.47 & 0.38 & 0.57 & $13.56 \%$ & -45.83 & -37.36 & -34.58 & -151 & 4 \\
\hline Bloch 6-34 & Morrow & 88.51 & 6.15 & 2.64 & 0.75 & 0.32 & 0.24 & 0.21 & 0.19 & 0.20 & 0.47 & $10.59 \%$ & -45.44 & -38.29 & -35.29 & ND & 4 \\
\hline Bobbie 1-21 & Morrow & 85.94 & 5.99 & 3.29 & 1.13 & 0.46 & 0.42 & 0.35 & 0.68 & 0.35 & 0.84 & $12.53 \%$ & -47.01 & -38.04 & -35.38 & -148 & 4 \\
\hline Flick Trust 1-3 & Cherokee & 83.26 & 7.45 & 2.95 & 0.63 & 0.50 & 0.11 & 0.20 & 0.12 & 1.00 & 3.10 & $12.56 \%$ & -44.88 & -34.42 & -30.48 & -150 & 4 \\
\hline Hamby 2-4 & Morrow & 93.52 & 1.40 & 0.17 & 0.01 & 0.01 & ND & 0.01 & ND & 1.06 & 3.03 & $1.69 \%$ & ND & ND & ND & -146 & 4 \\
\hline Hershy 1-4 & Granite Wash & 84.50 & 4.79 & 3.15 & 1.09 & 0.53 & 0.27 & 0.28 & 0.38 & 0.27 & 4.56 & $11.05 \%$ & -39.44 & -41.80 & -37.86 & -143 & 4 \\
\hline Indian School 2-32 & Cherokee & 86.43 & 7.33 & 2.58 & 0.50 & 0.44 & 0.11 & 0.19 & 0.16 & 1.14 & 0.76 & $11.58 \%$ & -44.63 & -34.59 & -30.46 & ND & 4 \\
\hline Mackey 1-3 & L. Cherokee & 95.93 & 2.43 & 0.44 & 0.09 & 0.10 & 0.02 & 0.03 & 0.06 & 0.60 & 0.27 & $3.20 \%$ & -42.75 & -33.06 & -29.40 & -139 & 4 \\
\hline Mosley 1-29 & Cherokee & 82.40 & 7.41 & 3.07 & 0.69 & 0.58 & 0.18 & 0.26 & 0.43 & 1.12 & 3.07 & $13.28 \%$ & -44.00 & -32.99 & -29.02 & ND & 4 \\
\hline Puckett 1-16 & Desmoinesian & 84.52 & 7.30 & 3.74 & 1.39 & 0.50 & 0.45 & 0.36 & 0.28 & 0.51 & 0.78 & $14.23 \%$ & -44.10 & -38.93 & -33.76 & ND & 4 \\
\hline Red Moon 7-29 & Cherokee & 82.04 & 7.69 & 3.13 & 0.67 & 0.56 & 0.17 & 0.26 & 0.44 & 1.26 & 2.90 & $13.61 \%$ & ND & ND & ND & ND & 4 \\
\hline Slusher 1-28 & Morrow & 85.64 & 6.34 & 3.48 & 1.22 & 0.48 & 0.48 & 0.40 & 0.68 & 0.36 & 0.65 & $13.23 \%$ & -46.29 & -37.98 & -35.14 & ND & 4 \\
\hline Strack Trust 1-1 & Morrow & 85.31 & 5.08 & 3.13 & 0.88 & 0.53 & 0.16 & 0.27 & 0.35 & 0.53 & 3.03 & $10.87 \%$ & -42.15 & -36.07 & -32.09 & -154 & 4 \\
\hline Thunder 1-13 & Morrow & 83.79 & 5.89 & 3.17 & 0.80 & 0.61 & 0.21 & 0.30 & 0.46 & 0.80 & 3.17 & $12.01 \%$ & ND & ND & ND & ND & 4 \\
\hline Viersen 2-20 & Morrow & 90.62 & 3.41 & 1.04 & 0.23 & 0.13 & 0.04 & 0.06 & 0.11 & 0.49 & 3.17 & $5.25 \%$ & ND & ND & ND & ND & 4 \\
\hline Viersen 4-20 & Morrow & 89.07 & 3.76 & 1.08 & 0.19 & 0.12 & 0.04 & 0.06 & 0.10 & 0.57 & 4.02 & $5.68 \%$ & ND & ND & ND & ND & 4 \\
\hline Whitledge 1-2 & L. Cherokee & 95.21 & 2.64 & 0.50 & 0.14 & 0.13 & 0.03 & 0.05 & 0.07 & 0.70 & 0.28 & $3.61 \%$ & -42.84 & -32.99 & -29.27 & ND & 4 \\
\hline Wigington 1-31 & Morrow & 88.99 & 2.65 & 0.66 & 0.11 & 0.08 & 0.03 & 0.03 & 0.06 & 0.62 & 5.73 & $3.91 \%$ & ND & ND & ND & ND & 4 \\
\hline Boeckman D-1-20 & Morrow & 90.50 & 4.96 & 2.04 & 0.50 & 0.23 & 0.13 & 0.13 & 0.40 & 0.42 & 0.39 & $8.50 \%$ & -42.04 & -35.64 & -32.73 & ND & 4 \\
\hline Carl 1-2 & Morrow & 83.49 & 6.62 & 3.32 & 1.12 & 0.44 & 0.40 & 0.32 & 0.58 & 0.37 & 2.55 & $13.29 \%$ & -45.62 & -36.97 & -34.15 & ND & 4 \\
\hline Chiles C-2-20 & Morrow & 93.04 & 3.09 & 1.15 & 0.26 & 0.14 & 0.06 & 0.08 & 0.33 & 1.47 & 0.28 & $5.20 \%$ & -40.31 & -36.22 & -33.13 & ND & 4 \\
\hline Ford 1-7 & Deese 1 & 82.47 & 9.80 & 4.25 & 1.12 & 0.32 & 0.27 & 0.17 & 0.18 & 0.30 & 0.96 & $16.35 \%$ & -46.75 & -39.84 & -36.61 & -164 & 4 \\
\hline
\end{tabular}


Table 2. Molecular and stable isotopic composition of gases from the central Anadarko Basin._Continued

[Units for the compositional data are in mole percent, and the $\delta^{13} \mathrm{C}$ and $\delta^{2} \mathrm{H}$ values are in per mil relative to the Vienna Pee Dee Belemnite and Vienna Standard Mean Ocean Water scales, respectively. Gas wetness is calculated as $\Sigma \mathrm{C}_{2+} / \Sigma \mathrm{C}_{1}+\mathrm{C}_{2+}$. $\mathrm{C}_{1}$, methane; $\mathrm{C}_{2}$, ethane; $\mathrm{C}_{3}$, propane; $\mathrm{n}-\mathrm{C}_{4}$, n-butane; i- $\mathrm{C}_{4}$, i-butane; $\mathrm{n}-\mathrm{C}_{5}, \mathrm{n}$-pentane; i- $\mathrm{C}_{5}$, = i-pentane; $\mathrm{C}_{6+}$, six carbon hydrocarbons and higher; $\mathrm{CO}_{2}$, carbon dioxide; $\mathrm{N}_{2}$, nitrogen; Wet, gas wetness in percent and $\delta^{13} \mathrm{C}_{1}, \delta^{13} \mathrm{C}_{2}$, and $\delta^{13} \mathrm{C}_{3}$ are the stable carbon isotopic composition of methane, ethane, and propane, respectively. $\delta^{2} \mathrm{HC}$ is the stable hydrogen isotopic composition of methane. ND, not determined. S indicates the source of the data and the numbers refer to the following publications: (1) Jenden and Kaplan (1989a), (2) Jenden and others (1988), (3) Rice and others (1988a), and (4) this study]

\begin{tabular}{|c|c|c|c|c|c|c|c|c|c|c|c|c|c|c|c|c|c|}
\hline $\begin{array}{l}\text { Well } \\
\text { name }\end{array}$ & $\begin{array}{l}\text { Formation } \\
\text { name }\end{array}$ & $C_{1}$ & $C_{2}$ & $C_{3}$ & $\mathrm{n}-\mathrm{C}_{4}$ & $\mathrm{i}-\mathrm{C}_{4}$ & $n-C_{5}$ & $\mathrm{i}-\mathrm{C}_{5}$ & $\mathrm{C}_{6+}$ & $\mathrm{CO}_{2}$ & $\mathbf{N}_{2}$ & Wet & $\delta^{13} \mathbf{C}_{1}$ & $\delta^{13} \mathbf{C}_{2}$ & $\delta^{13} \mathbf{C}_{3}$ & $\delta^{2} \mathrm{HC}_{1}$ & $\mathbf{S}$ \\
\hline Ford 4-7 & $\begin{array}{l}\text { Woodford, } \\
\text { Sycamore }\end{array}$ & 81.50 & 10.28 & 4.39 & 1.23 & 0.34 & 0.35 & 0.20 & 0.28 & 0.23 & 0.99 & $17.32 \%$ & -46.29 & -39.38 & -36.01 & -162 & 4 \\
\hline Haigler Gas Unit 2-28 & Morrow & 83.81 & 6.86 & 4.21 & 1.35 & 0.55 & 0.35 & 0.36 & 0.57 & 0.33 & 1.21 & $14.53 \%$ & -44.49 & -36.13 & -33.35 & ND & 4 \\
\hline Harmon Heirs 4-8 & Woodford & 83.13 & 9.53 & 3.94 & 1.03 & 0.30 & 0.26 & 0.16 & 0.18 & 0.30 & 1.01 & $15.63 \%$ & -47.12 & -39.54 & -36.38 & -164 & 4 \\
\hline Harrell 2-7 & Bromide & 80.32 & 10.87 & 4.79 & 1.32 & 0.36 & 0.34 & 0.20 & 0.27 & 0.23 & 0.85 & $18.43 \%$ & -46.39 & -40.37 & -36.93 & -163 & 4 \\
\hline Harris 2-8 & $\begin{array}{l}\text { Woodford, } \\
\text { Sycamore }\end{array}$ & 84.04 & 8.82 & 3.75 & 1.05 & 0.30 & 0.24 & 0.14 & 0.13 & 0.19 & 1.20 & $14.66 \%$ & -46.39 & -39.18 & -35.84 & ND & 4 \\
\hline Heath Cattle 1-31 & Morrow & 83.60 & 6.72 & 4.01 & 1.22 & 0.50 & 0.28 & 0.31 & 0.51 & 0.40 & 1.92 & $13.94 \%$ & -44.69 & -36.50 & -33.72 & -148 & 4 \\
\hline Lyda May 1-2 & Morrow & 84.07 & 7.56 & 3.87 & 1.30 & 0.51 & 0.46 & 0.36 & 0.31 & 0.41 & 0.67 & $14.60 \%$ & ND & ND & ND & ND & 4 \\
\hline Mays 1-8 & $\begin{array}{c}\text { Hunton, Viola \& } \\
\text { Sycamore }\end{array}$ & 75.77 & 12.15 & 6.19 & 1.73 & 0.50 & 0.48 & 0.30 & 0.35 & 0.27 & 1.76 & $22.27 \%$ & -46.88 & -39.57 & -36.11 & ND & 4 \\
\hline Myia 1-11 & Morrow & 84.74 & 6.58 & 3.38 & 1.15 & 0.46 & 0.41 & 0.33 & 0.53 & 0.24 & 0.92 & $13.15 \%$ & -45.10 & -35.76 & -33.09 & -146 & 4 \\
\hline Myia 2-11 & Morrow & 86.79 & 6.30 & 3.12 & 0.96 & 0.40 & 0.31 & 0.25 & 0.42 & 0.17 & 1.04 & $11.93 \%$ & ND & ND & ND & ND & 4 \\
\hline Royle 1-11 & Morrow & 92.52 & 4.13 & 1.36 & 0.27 & 0.15 & 0.06 & 0.08 & 0.12 & 0.69 & 0.37 & $6.25 \%$ & -45.20 & -36.56 & -33.91 & ND & 4 \\
\hline Stewart Blackburn 1-30 & Morrow & 92.52 & 4.13 & 1.36 & 0.27 & 0.15 & 0.06 & 0.08 & 0.12 & 0.69 & 0.37 & $6.25 \%$ & -41.50 & -36.08 & -33.32 & ND & 4 \\
\hline Vera $1-11$ & Morrow & 83.76 & 6.30 & 3.16 & 1.07 & 0.42 & 0.39 & 0.31 & 0.59 & 0.18 & 2.28 & $12.75 \%$ & -45.70 & -36.70 & -34.02 & ND & 4 \\
\hline Cassell C-3-7H & Bromide & 80.57 & 9.87 & 5.26 & 1.78 & 0.71 & 0.50 & 0.38 & 0.26 & 0.33 & 0.20 & $18.89 \%$ & -48.16 & -33.98 & -32.58 & -162 & 4 \\
\hline
\end{tabular}


[Units for the compositional data are in mole percent, and the $\delta^{13} \mathrm{C}$ and $\delta^{2} \mathrm{H}$ values are in per mil relative to the Vienna Pee Dee Belemnite and Vienna Standard Mean Ocean Water scales, respectively. Gas wetness is calculated as $\Sigma \mathrm{C}_{2+} / \Sigma \mathrm{C}_{1}+\mathrm{C}_{2} . \mathrm{C}_{1}$, methane; $\mathrm{C}_{2}$, ethane; $\mathrm{C}_{3}$, propane; $\mathrm{n}_{4}, \mathrm{n}$-butane; i- $\mathrm{C}_{4}$, i-butane; $\mathrm{n}-\mathrm{C}_{5}, \mathrm{n}$-pentane; i- $\mathrm{C}_{5}$, = i-pentane; $\mathrm{CO}_{2}$, carbon dioxide; $\mathrm{N}_{2}$, nitrogen; Wet, gas wetness in percent and $\delta^{13} \mathrm{C}_{1}, \delta^{13} \mathrm{C}_{2}$, and $\delta^{13} \mathrm{C}_{3}$ are the stable carbon isotopic composition of methane, ethane, and propane, respectively. $\delta^{2} \mathrm{HC}$ is the stable hydrogen isotopic composition of methane. ND, not determined. $\mathrm{S}$ indicates the source of the data and the numbers refer to the following publications: (1) Ballentine and Sherwood Lollar (2002), (2) Jenden and Kaplan (1989), (3) Jenden and others (1988), and (4) Rice and others (1988a)]

\begin{tabular}{|c|c|c|c|c|c|c|c|c|c|c|c|c|c|c|c|c|}
\hline $\begin{array}{l}\text { Well } \\
\text { name }\end{array}$ & $\begin{array}{c}\text { Formation } \\
\text { name }\end{array}$ & $C_{1}$ & $\mathrm{C}_{2}$ & $\mathrm{C}_{3}$ & $\mathrm{n}-\mathrm{C}_{4}$ & $\mathrm{i}-\mathrm{C}_{4}$ & $n-C_{5}$ & $\mathrm{i}-\mathrm{C}_{5}$ & $\mathrm{CO}_{2}$ & $\mathbf{N}_{2}$ & Wet & $\delta^{13} \mathbf{C}_{1}$ & $\delta^{13} \mathbf{C}_{2}$ & $\delta^{13} \mathbf{C}_{13}$ & $\delta^{2} \mathrm{HC}_{1}$ & $\mathbf{S}$ \\
\hline Ratzlaff D “A” \#1 & Herington & 66.20 & 6.00 & 4.80 & ND & ND & ND & ND & ND & 0.19 & $14.03 \%$ & -43.5 & -35.6 & ND & ND & 1 \\
\hline Hefner Gas Unit \#1 & Fort Riley & 68.00 & 6.20 & 4.90 & ND & ND & ND & ND & ND & 0.18 & $14.03 \%$ & -43.4 & -35.2 & ND & ND & 1 \\
\hline Guldner Unit \#1 & Chase & 66.10 & 5.80 & 4.40 & ND & ND & ND & ND & ND & 0.21 & $13.37 \%$ & -43.5 & -35.7 & ND & ND & 1 \\
\hline Guldner Unit \#2 & Council Grove & 65.90 & 5.80 & 4.40 & ND & ND & ND & ND & ND & 0.20 & $13.40 \%$ & -43.6 & -35.7 & ND & ND & 1 \\
\hline Campbell, R.W. \#2 & Council Grove & 69.80 & 6.50 & 5.30 & ND & ND & ND & ND & ND & 0.15 & $14.46 \%$ & -43.6 & -35.9 & ND & ND & 1 \\
\hline Keller, Ernest \#2 & Council Grove & 69.50 & 6.10 & 4.60 & ND & ND & ND & ND & ND & 0.15 & $13.34 \%$ & -43.0 & -34.9 & ND & ND & 1 \\
\hline Jarvis Unit \#2 & Council Grove & 69.50 & 6.20 & 4.70 & ND & ND & ND & ND & ND & 0.15 & $13.56 \%$ & -43.3 & -35.1 & ND & ND & 1 \\
\hline Ball, Clyde H. \#2 & Council Grove & 70.00 & 6.30 & 5.10 & ND & ND & ND & ND & ND & 0.15 & $14.00 \%$ & -43.5 & -36.1 & ND & ND & 1 \\
\hline Wright "C" Unit \#1 & Chase & 66.90 & 6.20 & 4.90 & ND & ND & ND & ND & ND & 0.16 & $14.23 \%$ & -43.9 & -35.7 & ND & ND & 1 \\
\hline Baughman H-2 & Chase & 70.20 & 4.00 & 2.40 & ND & ND & ND & ND & ND & 0.18 & $8.36 \%$ & -45.1 & -35.3 & ND & ND & 1 \\
\hline Crayton A-1 & Chase & 68.20 & 6.40 & 5.20 & ND & ND & ND & ND & ND & 0.17 & $14.54 \%$ & -43.5 & -36.0 & ND & ND & 1 \\
\hline Mills C-1 & Herington & 71.50 & 5.70 & 4.20 & ND & ND & ND & ND & ND & 0.13 & $12.16 \%$ & -43.4 & -36.0 & ND & ND & 1 \\
\hline Parsely A-1 & Herington & 71.30 & 4.30 & 3.00 & ND & ND & ND & ND & ND & 0.15 & $9.29 \%$ & -44.9 & -35.5 & ND & ND & 1 \\
\hline Oberly A-1 & Topeka & 64.60 & 6.30 & 5.80 & ND & ND & ND & ND & ND & 0.21 & $15.78 \%$ & -44.5 & -36.4 & ND & ND & 1 \\
\hline Tucker B-1 & Chase & 68.40 & 5.80 & 4.80 & ND & ND & ND & ND & ND & 0.15 & $13.42 \%$ & -43.3 & -35.2 & ND & ND & 1 \\
\hline Barnes A-1 & Topeka & 59.20 & 8.20 & 10.30 & ND & ND & ND & ND & ND & 0.20 & $23.81 \%$ & -44.9 & -37.0 & ND & ND & 1 \\
\hline Hill A-1 & Chase & 68.40 & 6.00 & 4.60 & ND & ND & ND & ND & ND & 0.15 & $13.42 \%$ & -43.1 & -35.1 & ND & ND & 1 \\
\hline Buzzard D-1 & Permian Lm. & 65.20 & 5.60 & 4.70 & ND & ND & ND & ND & ND & 0.18 & $13.64 \%$ & -43.2 & -34.9 & ND & ND & 1 \\
\hline Stonebraker A-69 & Permian Lm. & 62.60 & 5.30 & 4.90 & ND & ND & ND & ND & ND & 0.21 & $14.01 \%$ & -43.0 & -34.9 & ND & ND & 1 \\
\hline Coffee Estate \#1 & Brown Dolomite & 69.10 & 6.60 & 4.90 & ND & ND & ND & ND & ND & 0.14 & $14.27 \%$ & -43.5 & -35.5 & ND & ND & 1 \\
\hline Blake Trust Estate \#2 & Brown Dolomite & 65.10 & 6.00 & 3.60 & ND & ND & ND & ND & ND & 0.21 & $12.85 \%$ & -43.3 & -35.8 & ND & ND & 1 \\
\hline Mary A Long \#1 & Brown Dolomite & 68.50 & 6.10 & 5.20 & ND & ND & ND & ND & ND & 0.17 & $14.16 \%$ & -44.0 & -35.7 & ND & ND & 1 \\
\hline Donelson et al \#1 & Brown Dolomite & 56.10 & 5.00 & 4.80 & ND & ND & ND & ND & ND & 0.26 & $14.87 \%$ & -43.2 & -35.0 & ND & ND & 1 \\
\hline Sarah Claybaugh \#1 & Brown Dolomite & 60.30 & 5.40 & 5.30 & ND & ND & ND & ND & ND & 0.25 & $15.07 \%$ & -42.4 & -35.1 & ND & ND & 1 \\
\hline Cameron Walls \#1 & Brown Dolomite & 62.80 & 5.70 & 5.60 & ND & ND & ND & ND & ND & 0.18 & $15.25 \%$ & -43.1 & -36.4 & ND & ND & 1 \\
\hline Hormer \#1 & Brown Dolomite & 64.90 & 6.20 & 5.30 & ND & ND & ND & ND & ND & 0.22 & $15.05 \%$ & -42.1 & -35.0 & ND & ND & 1 \\
\hline Whitherbee \#2 & Brown Dolomite & 61.70 & 9.30 & 11.50 & ND & ND & ND & ND & ND & 0.08 & $25.21 \%$ & -44.1 & -36.2 & ND & ND & 1 \\
\hline
\end{tabular}


Table 3. Molecular and stable isotopic composition of gases from the Panhandle Hugoton region of the Anadarko Basin Province.-Continued

[Units for the compositional data are in mole percent, and the $\delta^{13} \mathrm{C}$ and $\delta^{2} \mathrm{H}$ values are in per mil relative to the Vienna Pee Dee Belemnite and Vienna Standard Mean Ocean Water scales, respectively. Gas wetness is calculated as $\Sigma \mathrm{C}_{2+} / \Sigma \mathrm{C}_{1}+\mathrm{C}_{2+}$. $\mathrm{C}_{1}$, methane; $\mathrm{C}_{2}$, ethane; $\mathrm{C}_{3}$, propane; $\mathrm{n}-\mathrm{C}_{4}$, n-butane; i- $\mathrm{C}_{4}$, i-butane; $\mathrm{n}-\mathrm{C}_{5}$, n-pentane; i- $\mathrm{C}_{5}$, = i-pentane; $\mathrm{CO}_{2}$, carbon dioxide; $\mathrm{N}_{2}$, nitrogen; Wet, gas wetness in percent and $\delta^{13} \mathrm{C}_{1}, \delta^{13} \mathrm{C}_{2}$, and $\delta^{13} \mathrm{C}_{3}$ are the stable carbon isotopic composition of methane, ethane, and propane, respectively. $\delta^{2} \mathrm{HC}_{1}$ is the stable hydrogen isotopic composition of methane. ND, not determined. $\mathrm{S}$ indicates the source of the data and the numbers refer to the following publications: (1) Ballentine and Sherwood Lollar (2002), (2) Jenden and Kaplan (1989), (3) Jenden and others (1988), and (4) Rice and others (1988a)]

\begin{tabular}{|c|c|c|c|c|c|c|c|c|c|c|c|c|c|c|c|c|}
\hline $\begin{array}{c}\text { Well } \\
\text { name }\end{array}$ & $\begin{array}{c}\text { Formation } \\
\text { name }\end{array}$ & $C_{1}$ & $\mathrm{C}_{2}$ & $\mathrm{C}_{3}$ & $\mathrm{n}-\mathrm{C}_{4}$ & $\mathrm{i}-\mathrm{C}_{4}$ & $n-C_{5}$ & $\mathrm{i}-\mathrm{C}_{5}$ & $\mathrm{CO}_{2}$ & $\mathbf{N}_{2}$ & Wet & $\delta^{13} \mathbf{C}_{1}$ & $\delta^{13} \mathbf{C}_{2}$ & $\delta^{13} \mathbf{C}_{13}$ & $\delta^{2} \mathrm{HC}_{1}$ & S \\
\hline Flores 23 & Brown Dolomite & 69.20 & 6.50 & 5.60 & ND & ND & ND & ND & ND & 0.14 & $14.88 \%$ & -42.6 & -35.2 & ND & ND & 1 \\
\hline Nisbett \#1 & Brown Dolomite & 68.80 & 7.20 & 7.30 & ND & ND & ND & ND & ND & 0.14 & $17.41 \%$ & -42.7 & -35.1 & ND & ND & 1 \\
\hline McDade \#2+\#5 & Brown Dolomite & 59.40 & 10.50 & 17.20 & ND & ND & ND & ND & ND & 0.07 & $31.80 \%$ & -42.4 & -35.0 & ND & ND & 1 \\
\hline Brumley A\#1 & Brown Dolomite & 60.00 & 7.30 & 12.30 & ND & ND & ND & ND & ND & 0.09 & $24.62 \%$ & -42.6 & -35.2 & ND & ND & 1 \\
\hline Helm \#1 & Glorieta & 0.01 & & 0.00 & 0.00 & 0.00 & 0.01 & 0.00 & 0.06 & 93.50 & $55.17 \%$ & ND & ND & ND & ND & 2 \\
\hline Arnold \#1-1 & Chase & 49.60 & 3.18 & 2.19 & 0.72 & 0.29 & 0.18 & 0.15 & 0.00 & 43.50 & $11.92 \%$ & -42.1 & -34.8 & -30.2 & -145 & 2 \\
\hline Atkins B\#5 & Chase & 70.00 & 5.36 & 2.90 & 0.70 & 0.36 & 0.13 & 0.14 & 0.02 & 17.00 & $12.04 \%$ & -43.9 & -35.0 & ND & -163 & 2 \\
\hline Beaver \#1 & Brown Dolomite & 74.60 & 6.13 & 3.27 & 0.73 & 0.36 & 0.13 & 0.13 & 0.03 & 13.20 & $12.60 \%$ & -43.3 & -34.5 & ND & -162 & 2 \\
\hline Benedict A\#1 & Brown Dolomite & 75.40 & 7.68 & 5.60 & 2.35 & 1.09 & 0.64 & 0.61 & 0.02 & 4.49 & $19.25 \%$ & -41.9 & -36.6 & -31.4 & -149 & 2 \\
\hline Bivins A-111 & Brown Dolomite & 67.10 & 4.63 & 2.82 & 0.96 & 0.42 & 0.25 & 0.25 & 0.98 & 18.80 & $12.21 \%$ & -42.3 & -34.2 & ND & -165 & 2 \\
\hline Bivins A\#21 & Red Cave & 67.00 & 8.04 & 7.18 & 2.63 & 1.13 & 0.59 & 0.59 & 0.20 & 10.90 & $23.13 \%$ & -42.6 & -34.0 & -30.1 & -166 & 2 \\
\hline Bivins A\#64 & Dolomite & 71.90 & 5.82 & 3.69 & 1.09 & 0.51 & 0.25 & 0.27 & 1.13 & 13.70 & $13.93 \%$ & -42.6 & -34.3 & -30.3 & -161 & 2 \\
\hline Bivins A\#9 & Dolomite & 65.90 & 4.02 & 1.88 & 0.44 & 0.22 & 0.08 & 0.09 & 0.94 & 24.10 & $9.27 \%$ & -43.0 & -35.1 & ND & -167 & 2 \\
\hline Burton No. 1 & Herington & 70.90 & 6.45 & 3.67 & 0.98 & 0.43 & 0.20 & 0.19 & 0.03 & 16.50 & $14.39 \%$ & -43.7 & -34.5 & ND & -163 & 2 \\
\hline Cherokee No. 1B & Morrow & 84.10 & 4.36 & 2.53 & 0.75 & 0.40 & 0.25 & 0.23 & 0.36 & 6.79 & $9.20 \%$ & -43.0 & -33.5 & ND & -161 & 2 \\
\hline Crawford No. 1R & Red Cave & 75.10 & 9.13 & 4.78 & 1.23 & 0.58 & 0.24 & 0.25 & 0.02 & 5.94 & $17.75 \%$ & -44.7 & -32.8 & -28.8 & -173 & 2 \\
\hline Cubine \#1 & Brown Dolomite & 66.60 & 11.30 & 11.20 & 3.87 & 1.95 & 0.78 & 0.86 & 0.06 & 3.46 & $31.02 \%$ & -41.6 & -36.5 & -31.4 & -150 & 2 \\
\hline Dear \#1 & Brown Dolomite & 68.70 & 6.58 & 4.52 & 1.47 & 0.63 & 0.34 & 0.31 & 0.34 & 14.80 & $16.77 \%$ & -42.8 & -34.4 & ND & -157 & 2 \\
\hline Dunaway \#1R & Red Cave & 80.00 & 6.08 & 2.57 & 0.60 & 0.27 & 0.12 & 0.12 & 0.03 & 8.55 & $10.88 \%$ & -43.9 & -32.1 & -27.4 & -165 & 2 \\
\hline Eagley A\#1 & Wabaunsee & 66.40 & 4.80 & 2.02 & 0.36 & 0.18 & 0.33 & 0.15 & 0.05 & 25.20 & $10.56 \%$ & -43.5 & -35.1 & -30.3 & -160 & 2 \\
\hline Esta \#1 & L. Krider & 48.40 & 4.42 & 4.13 & 1.48 & 0.70 & 0.32 & 0.31 & 0.05 & 36.60 & $19.01 \%$ & -42.6 & -33.8 & ND & -155 & 2 \\
\hline Evers A\#1 & Chase & 60.90 & 4.84 & 2.83 & 0.81 & 0.31 & 0.18 & 0.17 & 0.02 & 29.30 & $13.05 \%$ & -44.0 & -35.0 & ND & -166 & 2 \\
\hline Fecht \#7 & Chase & 52.30 & 4.39 & 2.89 & 0.89 & 0.36 & 0.24 & 0.21 & 0.00 & 37.70 & $14.65 \%$ & -43.2 & -34.9 & -30.4 & -188 & 2 \\
\hline Fee A\#3 & Dolomite & 71.50 & 8.05 & 6.71 & 2.33 & 1.00 & 0.53 & 0.50 & 0.61 & 7.85 & $21.10 \%$ & -42.3 & -34.8 & -30.3 & -157 & 2 \\
\hline Fee \#3R & Red Cave & 81.50 & 6.52 & 2.82 & 0.60 & 0.32 & 0.10 & 0.11 & 0.00 & 7.23 & $11.38 \%$ & -44.1 & -31.7 & -27.0 & -163 & 2 \\
\hline Finley Dolomite \#11 & Brown Dolomite & 16.10 & 6.08 & 11.70 & 5.48 & 2.29 & 1.12 & 1.14 & 0.33 & 50.50 & $63.33 \%$ & -41.1 & -34.5 & -30.2 & -152 & 2 \\
\hline First National Bank B\#1 & Chase & 66.30 & 5.20 & 2.94 & 0.80 & 0.31 & 0.17 & 0.16 & 0.06 & 23.80 & $12.63 \%$ & -44.3 & -35.2 & ND & -168 & 2 \\
\hline
\end{tabular}


[Units for the compositional data are in mole percent, and the $\delta^{13} \mathrm{C}$ and $\delta^{2} \mathrm{H}$ values are in per mil relative to the Vienna Pee Dee Belemnite and Vienna Standard Mean Ocean Water scales, respectively. Gas wetness is calculated as $\Sigma \mathrm{C}_{2+} / \Sigma \mathrm{C}_{1}+\mathrm{C}_{2+} \mathrm{C}_{1}$, methane; $\mathrm{C}_{2}$, ethane; $\mathrm{C}_{3}$, propane; $\mathrm{n}_{4}, \mathrm{n}$-butane; i- $\mathrm{C}_{4}$, i-butane; $\mathrm{n}-\mathrm{C}_{5}, \mathrm{n}$-pentane; i- $\mathrm{C}_{5}$, = i-pentane; $\mathrm{CO}_{2}$, carbon dioxide; $\mathrm{N}_{2}$, nitrogen; Wet, gas wetness in percent and $\delta^{13} \mathrm{C}_{1}, \delta^{13} \mathrm{C}_{2}$, and $\delta^{13} \mathrm{C}_{3}$ are the stable carbon isotopic composition of methane, ethane, and propane, respectively. $\delta^{2} \mathrm{HC}$ is the stable hydrogen isotopic composition of methane. ND, not determined. $\mathrm{S}$ indicates the source of the data and the numbers refer to the following publications: (1) Ballentine and Sherwood Lollar (2002), (2) Jenden and Kaplan (1989), (3) Jenden and others (1988), and (4) Rice and others (1988a)]

\begin{tabular}{|c|c|c|c|c|c|c|c|c|c|c|c|c|c|c|c|c|}
\hline $\begin{array}{c}\text { Well } \\
\text { name }\end{array}$ & $\begin{array}{c}\text { Formation } \\
\text { name }\end{array}$ & $C_{1}$ & $\mathrm{C}_{2}$ & $\mathrm{C}_{3}$ & $n-C_{4}$ & $\mathrm{i}-\mathrm{C}_{4}$ & $n-C_{5}$ & $\mathrm{i}-\mathrm{C}_{5}$ & $\mathrm{CO}_{2}$ & $\mathbf{N}_{2}$ & Wet & $\delta^{13} \mathbf{C}_{1}$ & $\delta^{13} \mathbf{C}_{2}$ & $\delta^{13} \boldsymbol{C}_{13}$ & $\delta^{2} \mathbf{H C}_{1}$ & $\mathbf{S}$ \\
\hline Fox \#8 Composite) & Granite Wash & 30.60 & 16.70 & 23.60 & 6.11 & 2.96 & 0.79 & 0.87 & 1.07 & 17.60 & $62.51 \%$ & -42.1 & -34.6 & -30.3 & -152 & 2 \\
\hline Gracey \#1 & Chase & 71.20 & 6.21 & 3.51 & 0.84 & 0.41 & 0.13 & 0.15 & 0.02 & 12.40 & $13.64 \%$ & -43.1 & -34.3 & ND & -162 & 2 \\
\hline Hobson \#1A & Brown Dolomite & 67.30 & 6.00 & 3.90 & 1.10 & 0.51 & 0.21 & 0.21 & 0.10 & 18.50 & $15.05 \%$ & -42.3 & -34.3 & -30.1 & -156 & 2 \\
\hline Hyer A\#3 & Chase & 51.50 & 4.05 & 2.38 & 0.71 & 0.28 & 0.15 & 0.14 & 0.02 & 39.10 & $13.03 \%$ & -43.6 & -34.8 & -30.4 & -165 & 2 \\
\hline Kaser No. 1 & Permian Lm. & 73.60 & 6.44 & 3.95 & 0.12 & 0.53 & 0.31 & 0.28 & 0.05 & 11.40 & $13.65 \%$ & -43.2 & -34.4 & ND & -161 & 2 \\
\hline Kaser No. 2 & U. Morrow & 84.30 & 5.86 & 2.60 & 0.79 & 0.25 & 0.21 & 0.15 & 0.27 & 1.86 & $10.47 \%$ & -43.6 & -36.1 & ND & -158 & 2 \\
\hline Kaser No. 2 & Oswego & 77.30 & 7.38 & 5.25 & 1.79 & 0.71 & 0.55 & 0.43 & 0.30 & 4.73 & $17.25 \%$ & -45.3 & -34.3 & ND & -164 & 2 \\
\hline Lela (Composite) & Granite Wash & 27.60 & 19.50 & 22.30 & 6.49 & 3.34 & 1.10 & 1.23 & 1.21 & 19.60 & $66.16 \%$ & -42.3 & -36.1 & -31.0 & -147 & 2 \\
\hline Lemon C\#1 & Chase & 72.00 & 4.39 & 2.21 & 0.53 & 0.26 & 0.11 & 0.11 & 0.02 & 20.80 & $9.57 \%$ & -44.4 & -35.4 & ND & -167 & 2 \\
\hline Long A\#1 & Chase & 71.00 & 6.42 & 3.81 & 1.08 & 0.42 & 0.26 & 0.22 & 0.03 & 16.30 & $14.68 \%$ & -43.8 & -35.1 & -30.4 & -164 & 2 \\
\hline Masterson A\#35R & Red Cave & 76.40 & 6.66 & 3.19 & 0.71 & 0.35 & 0.12 & 0.13 & 0.02 & 11.50 & $12.74 \%$ & -44.1 & -32.6 & -28.8 & -166 & 2 \\
\hline Masterson B\#14 & Brown Dolomite & 67.40 & 6.86 & 6.21 & 2.92 & 1.13 & 0.88 & 0.80 & 0.29 & 10.80 & $21.81 \%$ & -42.4 & -34.3 & -30.1 & -156 & 2 \\
\hline Masterson B\#37 & Dolomite & 75.10 & 6.03 & 3.11 & 0.84 & 0.38 & 0.16 & 0.17 & 0.15 & 12.60 & $12.46 \%$ & -43.3 & -33.5 & -29.2 & -166 & 2 \\
\hline Masterson G\#3 & Dolomite & 68.40 & 7.87 & 7.38 & 3.21 & 1.27 & 0.88 & 0.82 & 0.28 & 8.16 & $23.85 \%$ & -42.4 & -34.3 & -30.2 & -157 & 2 \\
\hline Masterson \#16R & Red Cave & 69.30 & 8.75 & 6.76 & 2.15 & 0.93 & 0.40 & 0.44 & 0.06 & 9.11 & $21.89 \%$ & -43.3 & -33.1 & -28.9 & -165 & 2 \\
\hline Masterson \#31R & Red Cave & 74.70 & 8.75 & 5.02 & 1.30 & 0.62 & 0.23 & 0.25 & 0.04 & 8.12 & $17.79 \%$ & -44.1 & -32.7 & -28.5 & -166 & 2 \\
\hline Masterson \#97R & Red Cave & 17.00 & 2.71 & 2.28 & 0.83 & 0.28 & 0.18 & 0.18 & 0.01 & 73.00 & $27.52 \%$ & -42.7 & -30.5 & -27.3 & -159 & 2 \\
\hline McCall \#18 & Permian Lm. & 55.60 & 5.02 & 3.95 & 1.24 & 0.57 & 0.26 & 0.25 & 0.06 & 31.00 & $16.88 \%$ & -42.5 & -34.1 & ND & -158 & 2 \\
\hline Morris B\#1 & Chase & 55.70 & 3.13 & 1.64 & 0.44 & 0.20 & 0.01 & 0.10 & 0.02 & 37.10 & $9.01 \%$ & -44.8 & -35.1 & ND & -168 & 2 \\
\hline Morris \#1-33 & Chase & 74.00 & 6.44 & 3.77 & 1.08 & 0.47 & 0.23 & 0.21 & 0.03 & 12.50 & $14.15 \%$ & -43.4 & -34.4 & -30.2 & -162 & 2 \\
\hline Osborne \#10 & Brown Dolomite & 76.80 & 7.42 & 5.77 & 2.34 & 1.08 & 0.64 & 0.62 & 0.02 & 4.73 & $18.88 \%$ & -41.8 & -36.3 & -31.2 & -150 & 2 \\
\hline Osborne \#4 (Composite) & Brown Dolomite & 60.00 & 16.40 & 11.30 & 2.64 & 1.34 & 0.55 & 0.53 & 0.02 & 6.90 & $35.31 \%$ & -41.9 & -33.4 & -29.6 & -162 & 2 \\
\hline Parmele \#1 & Dolomite & 70.90 & 6.00 & 3.68 & 1.05 & 0.46 & 0.24 & 0.22 & 0.00 & 15.70 & $14.11 \%$ & -44.5 & -35.3 & -30.9 & -165 & 2 \\
\hline Porter A\#1 & Chase & 73.10 & 6.58 & 3.80 & 1.06 & 0.42 & 0.24 & 0.21 & 0.02 & 14.00 & $14.41 \%$ & -43.6 & -34.9 & ND & -166 & 2 \\
\hline Posey A\#1 & Topeka & 68.00 & 6.67 & 4.23 & 1.33 & 0.47 & 0.38 & 0.30 & 0.09 & 18.20 & $16.44 \%$ & -45.1 & -35.9 & -30.9 & -173 & 2 \\
\hline Posey A\#2A & Morrow, Upper & 70.40 & 6.42 & 5.22 & 1.08 & 0.61 & 0.23 & 0.21 & 0.14 & 14.70 & $16.37 \%$ & -46.1 & -34.0 & -27.8 & -187 & 2 \\
\hline Posey A\#2B & Marmaton & 64.40 & 5.77 & 3.18 & 0.96 & 0.39 & 0.25 & 0.24 & 0.05 & 25.00 & $14.36 \%$ & -44.3 & -34.7 & -30.1 & -172 & 2 \\
\hline
\end{tabular}


Table 3. Molecular and stable isotopic composition of gases from the Panhandle Hugoton region of the Anadarko Basin Province.-Continued

[Units for the compositional data are in mole percent, and the $\delta^{13} \mathrm{C}$ and $\delta^{2} \mathrm{H}$ values are in per mil relative to the Vienna Pee Dee Belemnite and Vienna Standard Mean Ocean Water scales, respectively. Gas wetness is calculated as $\Sigma \mathrm{C}_{2+} / \Sigma \mathrm{C}_{1}+\mathrm{C}_{2+}$. $\mathrm{C}_{1}$, methane; $\mathrm{C}_{2}$, ethane; $\mathrm{C}_{3}$, propane; $\mathrm{n}-\mathrm{C}_{4}$, n-butane; i- $\mathrm{C}_{4}$, i-butane; $\mathrm{n}-\mathrm{C}_{5}$, n-pentane; i- $\mathrm{C}_{5}$, =i-pentane; $\mathrm{CO}_{2}$, carbon dioxide; $\mathrm{N}_{2}$, nitrogen; Wet, gas wetness in percent and $\delta^{13} \mathrm{C}_{1}, \delta^{13} \mathrm{C}_{2}$, and $\delta^{13} \mathrm{C}_{3}$ are the stable carbon isotopic composition of methane, ethane, and propane, respectively. $\delta^{2} \mathrm{HC}_{1}$ is the stable hydrogen isotopic composition of methane. ND, not determined. $\mathrm{S}$ indicates the source of the data and the numbers refer to the following publications: (1) Ballentine and Sherwood Lollar (2002), (2) Jenden and Kaplan (1989), (3) Jenden and others (1988), and (4) Rice and others (1988a)]

\begin{tabular}{|c|c|c|c|c|c|c|c|c|c|c|c|c|c|c|c|c|}
\hline $\begin{array}{l}\text { Well } \\
\text { name }\end{array}$ & $\begin{array}{c}\text { Formation } \\
\text { name }\end{array}$ & $C_{1}$ & $\mathrm{C}_{2}$ & $\mathrm{C}_{3}$ & $\mathrm{n}-\mathrm{C}_{4}$ & $\mathrm{i}-\mathrm{C}_{4}$ & $n-C_{5}$ & $\mathrm{i}-\mathrm{C}_{5}$ & $\mathrm{CO}_{2}$ & $\mathbf{N}_{2}$ & Wet & $\delta^{13} \mathbf{C}_{1}$ & $\delta^{13} \mathbf{C}_{2}$ & $\delta^{13} \mathbf{C}_{13}$ & $\delta^{2} \mathrm{HC}_{1}$ & $\mathbf{S}$ \\
\hline Ree \#1 & Chase & 73.00 & 6.25 & 3.67 & 1.02 & 0.47 & 0.23 & 0.22 & 0.04 & 11.90 & $13.97 \%$ & -43.1 & -34.4 & ND & -161 & 2 \\
\hline Rees A\#1 & Chase & 79.70 & 5.88 & 3.77 & 1.40 & 0.52 & 0.52 & 0.40 & 0.00 & 5.44 & $13.56 \%$ & -44.8 & -34.8 & ND & -163 & 2 \\
\hline Ross \#1 & Chase & 61.80 & 5.24 & 3.28 & 1.04 & 0.37 & 0.28 & 0.22 & 0.02 & 27.20 & $14.44 \%$ & -43.9 & -35.1 & -30.6 & -169 & 2 \\
\hline Schaaf \#1 & Chase & 66.60 & 5.89 & 3.45 & 1.00 & 0.37 & 0.23 & 0.20 & 0.01 & 23.00 & $14.32 \%$ & -44.0 & -35.0 & ND & -166 & 2 \\
\hline Shores A\#1 & Chase & 58.00 & 5.30 & 4.00 & 1.20 & 0.55 & 0.25 & 0.25 & 0.09 & 28.20 & $16.61 \%$ & -42.7 & -34.2 & ND & -158 & 2 \\
\hline Stonebraker \#1AA & Permian Lm. & 65.00 & 5.61 & 3.87 & 1.18 & 0.51 & 0.27 & 0.25 & 0.06 & 22.00 & $15.24 \%$ & -42.2 & -33.7 & ND & -159 & 2 \\
\hline Stonebraker A\#12 & Permian Lm. & 60.20 & 5.40 & 3.29 & 0.84 & 0.37 & 0.14 & 0.15 & 0.02 & 23.00 & $14.48 \%$ & -41.9 & -34.0 & ND & -162 & 2 \\
\hline Stonebraker A\#50 & Herington & 72.20 & 6.11 & 3.71 & 1.04 & 0.45 & 0.21 & 0.20 & 0.06 & 14.90 & $13.97 \%$ & -43.0 & -34.3 & ND & -160 & 2 \\
\hline Stonebraker A\#62 & Permian Lm. & 64.20 & 5.48 & 3.61 & 1.01 & 0.47 & 0.20 & 0.20 & 0.06 & 20.00 & $14.60 \%$ & -43.1 & -34.2 & ND & -158 & 2 \\
\hline Stoops A\#1 & Chase & 70.50 & 2.50 & 0.99 & 0.25 & 0.14 & 0.06 & 0.07 & 0.02 & 24.50 & $5.38 \%$ & -45.4 & -35.8 & -30.3 & -167 & 2 \\
\hline Stubbs A\#1 & Chase & 73.50 & 6.59 & 3.76 & 1.01 & 0.43 & 0.22 & 0.19 & 0.02 & 14.40 & $14.24 \%$ & -43.5 & -34.6 & -30.3 & -162 & 2 \\
\hline Tate B\#1 & Chase & 72.40 & 6.14 & 3.44 & 0.87 & 0.38 & 0.18 & 0.16 & 0.02 & 14.30 & $13.36 \%$ & -43.5 & -34.6 & -30.3 & -164 & 2 \\
\hline Thompson B\#4 & Dolomite & 69.90 & 6.86 & 5.37 & 2.25 & 0.88 & 0.65 & 0.59 & 0.56 & 10.90 & $19.19 \%$ & -42.6 & -34.3 & ND & -161 & 2 \\
\hline Towler \#1-21 & Chase & 70.50 & 6.31 & 3.59 & 0.95 & 0.42 & 0.19 & 0.19 & 0.02 & 14.50 & $14.17 \%$ & -43.6 & -34.7 & ND & -163 & 2 \\
\hline Tucker \#1-12 & Chase & 68.80 & 5.70 & 3.46 & 1.01 & 0.37 & 0.25 & 0.21 & 0.03 & 19.20 & $13.78 \%$ & -43.6 & -35.1 & -30.7 & -160 & 2 \\
\hline United \#1 & Dolomite & 69.90 & 6.97 & 5.28 & 1.84 & 0.74 & 0.45 & 0.40 & 0.41 & 11.20 & $18.33 \%$ & -42.1 & -34.4 & -30.5 & -158 & 2 \\
\hline Vent A\#1 & Dolomite & 72.20 & 6.74 & 5.19 & 2.15 & 0.84 & 0.64 & 0.55 & 0.23 & 8.31 & $18.25 \%$ & -42.5 & -34.6 & -30.3 & -157 & 2 \\
\hline Wheat (Composite) & Brown Dolomite & 44.40 & 10.50 & 16.80 & 4.02 & 2.25 & 0.68 & 0.76 & 0.07 & 20.30 & $44.09 \%$ & -42.4 & -33.8 & -29.5 & -169 & 2 \\
\hline Whitman \#1 & Chase & 70.70 & 6.28 & 3.63 & 1.01 & 0.39 & 0.21 & 0.20 & 0.02 & 17.10 & $14.22 \%$ & -43.8 & -35.0 & -30.5 & -164 & 2 \\
\hline Wilson \#1-4 & Chase & 74.30 & 6.45 & 3.71 & 0.97 & 0.43 & 0.20 & 0.19 & 0.03 & 11.80 & $13.86 \%$ & -43.2 & -34.4 & $\mathrm{ND}$ & -161 & 2 \\
\hline Wolff C\#1 & Chase & 70.70 & 6.33 & 3.60 & 0.95 & 0.43 & 0.18 & 0.18 & 0.06 & 14.90 & $14.17 \%$ & -43.6 & -34.6 & ND & -161 & 2 \\
\hline Zimmerman No. 1-35 & Chase & 73.20 & 6.65 & 3.81 & 0.99 & 0.44 & 0.20 & 0.19 & 0.02 & 14.20 & $14.35 \%$ & -43.7 & -34.6 & -30.2 & -161 & 2 \\
\hline Fecht No. 7 & Chase & 52.4 & 4.39 & 2.89 & 0.888 & 0.356 & 0.239 & 0.214 & 0.001 & 37.70 & $14.63 \%$ & -43.2 & -34.9 & -30.4 & -188 & 3 \\
\hline Eagley A 1 & Wabaunsee & 65.6 & 4.80 & 2.02 & 0.364 & 0.182 & 0.325 & 0.150 & 0.046 & 25.20 & $10.68 \%$ & -43.5 & -35.1 & -30.3 & -160 & 3 \\
\hline Posey A 1 & Topeka & 67.4 & 6.67 & 4.23 & 1.330 & 0.468 & 0.382 & 0.300 & 0.089 & 18.20 & $16.56 \%$ & -45.1 & -35.9 & -30.9 & -173 & 3 \\
\hline Arnold 1-1 & Chase & 49.6 & 3.18 & 2.19 & 0.719 & 0.291 & 0.180 & 0.151 & 0.001 & 43.50 & $11.92 \%$ & -42.1 & -34.8 & -30.2 & -145 & 3 \\
\hline Burton 1 & Chase & 70.9 & 6.45 & 3.67 & 0.980 & 0.429 & 0.199 & 0.189 & 0.030 & 16.50 & $14.39 \%$ & -43.7 & -34.5 & -30.2 & -163 & 3 \\
\hline
\end{tabular}


[Units for the compositional data are in mole percent, and the $\delta^{13} \mathrm{C}$ and $\delta^{2} \mathrm{H}$ values are in per mil relative to the Vienna Pee Dee Belemnite and Vienna Standard Mean Ocean Water scales, respectively. Gas wetness is calculated as $\Sigma \mathrm{C}_{2} / \Sigma \mathrm{C}_{1}+\mathrm{C}_{2}$. $\mathrm{C}_{1}$, methane; $\mathrm{C}_{2}$, ethane; $\mathrm{C}_{3}$, propane; $\mathrm{n}-\mathrm{C}_{4}, \mathrm{n}$-butane; i- $\mathrm{C}_{4}$, i-butane; $\mathrm{n}-\mathrm{C}_{5}, \mathrm{n}$-pentane; i- $\mathrm{C}_{5}$, = i-pentane; $\mathrm{CO}_{2}$, carbon dioxide; $\mathrm{N}_{2}$, nitrogen; Wet, gas wetness in percen and $\delta^{13} \mathrm{C}_{1}, \delta^{13} \mathrm{C}_{2}$, and $\delta^{13} \mathrm{C}_{3}$ are the stable carbon isotopic composition of methane, ethane, and propane, respectively. $\delta^{2} \mathrm{HC}$ is the stable hydrogen isotopic composition of methane. ND, not determined. $\mathrm{S}$ indicates the source of the data and the numbers refer to the following publications: (1) Ballentine and Sherwood Lollar (2002), (2) Jenden and Kaplan (1989), (3) Jenden and others (1988), and (4) Rice and others (1988a)]

\begin{tabular}{|c|c|c|c|c|c|c|c|c|c|c|c|c|c|c|c|c|}
\hline $\begin{array}{l}\text { Well } \\
\text { name }\end{array}$ & $\begin{array}{c}\text { Formation } \\
\text { name }\end{array}$ & $C_{1}$ & $\mathrm{C}_{2}$ & $\mathrm{C}_{3}$ & $n-C_{4}$ & i-C 4 & $n-C_{5}$ & $\mathrm{i}-\mathrm{C}_{5}$ & $\mathrm{CO}_{2}$ & $\mathbf{N}_{2}$ & Wet & $\delta^{13} \mathbf{C}_{1}$ & $\delta^{13} \mathbf{C}_{2}$ & $\delta^{13} \mathbf{C}_{13}$ & $\delta^{2} \mathrm{HC}_{1}$ & S \\
\hline Evers A 1 & Chase & 61.0 & 4.84 & 2.83 & 0.805 & 0.313 & 0.183 & 0.169 & 0.015 & 29.30 & $13.03 \%$ & -44.0 & -35.0 & ND & -166 & 3 \\
\hline First National Bank B 1 & Chase & 66.1 & 5.20 & 2.94 & 0.801 & 0.314 & 0.168 & 0.158 & 0.058 & 23.80 & $12.66 \%$ & -44.3 & -35.2 & ND & -168 & 3 \\
\hline Gracey 1 & Chase & 72.6 & 6.21 & 3.51 & 0.842 & 0.408 & 0.129 & 0.147 & 0.020 & 12.40 & $13.41 \%$ & -43.1 & -34.3 & ND & -162 & 3 \\
\hline Hyer A 3 & Chase & 51.5 & 4.05 & 2.38 & 0.709 & 0.282 & 0.153 & 0.140 & 0.021 & 39.10 & $13.03 \%$ & -43.6 & -34.8 & -30.4 & -165 & 3 \\
\hline Lemon C 1 & Chase & 71.1 & 4.39 & 2.21 & 0.532 & 0.261 & 0.110 & 0.114 & 0.023 & 20.80 & $9.68 \%$ & -44.4 & -35.4 & ND & -167 & 3 \\
\hline Long A 1 & Chase & 70.7 & 6.42 & 3.81 & 1.080 & 0.422 & 0.259 & 0.221 & 0.025 & 16.30 & $14.73 \%$ & -43.8 & -35.2 & -30.5 & -166 & 3 \\
\hline Morris B 1 & Chase & 55.8 & 3.13 & 1.64 & 0.435 & 0.200 & 0.010 & 0.099 & 0.016 & 37.10 & $8.99 \%$ & -44.8 & -35.1 & ND & -168 & 3 \\
\hline Morris 1-33 & Chase & 74.1 & 6.44 & 3.77 & 1.080 & 0.469 & 0.228 & 0.211 & 0.031 & 12.50 & $14.13 \%$ & -43.4 & -34.4 & -30.2 & -162 & 3 \\
\hline Porter A 1 & Chase & 72.8 & 6.58 & 3.80 & 1.060 & 0.420 & 0.241 & 0.209 & 0.019 & 14.00 & $14.46 \%$ & -43.6 & -34.9 & ND & -166 & 3 \\
\hline Ross 1 & Chase & 61.6 & 5.24 & 3.28 & 1.040 & 0.372 & 0.275 & 0.222 & 0.024 & 27.20 & $14.48 \%$ & -43.9 & -35.1 & -30.6 & -169 & 3 \\
\hline Schaaf 1 & Chase & 65.4 & 5.89 & 3.45 & 0.998 & 0.365 & 0.233 & 0.199 & 0.011 & 23.00 & $14.55 \%$ & -44.0 & -35.0 & ND & -166 & 3 \\
\hline Stoops A 1 & Chase & 70.4 & 2.50 & 0.99 & 0.246 & 0.138 & 0.064 & 0.066 & 0.017 & 24.50 & $5.39 \%$ & -45.4 & -35.8 & -30.3 & -167 & 3 \\
\hline Stubbs A 1 & Chase & 73.0 & 6.59 & 3.76 & 1.010 & 0.426 & 0.224 & 0.194 & 0.021 & 14.40 & $14.32 \%$ & -43.5 & -34.6 & -30.3 & -162 & 3 \\
\hline Tate B 1 & Chase & 72.6 & 6.14 & 3.44 & 0.874 & 0.375 & 0.176 & 0.158 & 0.017 & 14.30 & $13.33 \%$ & -43.5 & -34.6 & -30.3 & -164 & 3 \\
\hline Towler 1-21 & Chase & 70.8 & 6.31 & 3.59 & 0.949 & 0.416 & 0.185 & 0.185 & 0.018 & 14.50 & $14.11 \%$ & -43.6 & -34.7 & ND & -163 & 3 \\
\hline Tucker 1-12 & Chase & 68.7 & 5.70 & 3.46 & 1.010 & 0.366 & 0.248 & 0.211 & 0.033 & 19.20 & $13.80 \%$ & -43.6 & -35.1 & -30.7 & -160 & 3 \\
\hline Whitman 1 & Chase & 70.3 & 6.28 & 3.63 & 1.010 & 0.394 & 0.213 & 0.196 & 0.018 & 17.10 & $14.29 \%$ & -43.8 & -35.0 & -30.5 & -164 & 3 \\
\hline Wolff C 1 & Chase & 70.8 & 6.33 & 3.60 & 0.952 & 0.425 & 0.180 & 0.184 & 0.061 & 14.90 & $14.15 \%$ & -43.6 & -34.6 & ND & -161 & 3 \\
\hline Zimmerman 1-35 & Chase & 73.8 & 6.65 & 3.81 & 0.987 & 0.438 & 0.196 & 0.187 & 0.020 & 14.20 & $14.25 \%$ & -43.4 & -34.6 & -30.2 & -161 & 3 \\
\hline Cherokee 1B & Morrow & 84.1 & 4.36 & 2.53 & 0.752 & 0.402 & 0.251 & 0.230 & 0.361 & 6.79 & $9.20 \%$ & -43.0 & -33.5 & -28.1 & -161 & 3 \\
\hline Posey A-2A & U. Morrow & 70.0 & 6.42 & 5.22 & 1.080 & 0.611 & 0.233 & 0.212 & 0.140 & 14.70 & $16.44 \%$ & -46.1 & -34.2 & -27.8 & -189 & 3 \\
\hline Posey A-2B & Marmaton & 64.1 & 5.77 & 3.18 & 0.963 & 0.392 & 0.254 & 0.236 & 0.047 & 25.00 & $14.41 \%$ & -44.3 & -34.7 & -30.1 & -172 & 3 \\
\hline G.W. Deahl No. 2R & Red Cave & 75.78 & 7.15 & 4.12 & 1.17 & 0.48 & 0.30 & 0.30 & 0.32 & 10.38 & $15.14 \%$ & -44.0 & -32.2 & ND & -164 & 4 \\
\hline Burnett No. 3R & Leonardlan & 74.17 & 6.97 & 3.88 & 1.14 & 0.45 & 0.28 & 0.28 & 0.09 & 12.72 & $14.91 \%$ & -43.9 & ND & ND & ND & 4 \\
\hline Interstate No. C-6 & Red Cave & 50.46 & 1.61 & 0.91 & 0.30 & 0.19 & 0.00 & 0.10 & 0.00 & 46.43 & $5.81 \%$ & -46.4 & ND & ND & ND & 4 \\
\hline G.W. Deahl No. 2 & Red Cave & 63.00 & 10.63 & 10.12 & 3.69 & 1.69 & 0.92 & 0.88 & 0.60 & 8.44 & $30.72 \%$ & -42.0 & ND & ND & ND & 4 \\
\hline Burnett No. 5G & Chase & 72.72 & 6.48 & 5.20 & 2.19 & 0.92 & 0.77 & 0.67 & 0.69 & 10.32 & $18.25 \%$ & -42.4 & ND & ND & ND & 4 \\
\hline
\end{tabular}


Table 3. Molecular and stable isotopic composition of gases from the Panhandle Hugoton region of the Anadarko Basin Province.-Continued

[Units for the compositional data are in mole percent, and the $\delta^{13} \mathrm{C}$ and $\delta^{2} \mathrm{H}$ values are in per mil relative to the Vienna Pee Dee Belemnite and Vienna Standard Mean Ocean Water scales, respectively. Gas wetness is calculated as $\Sigma \mathrm{C}_{2+} / \Sigma \mathrm{C}_{1}+\mathrm{C}_{2+}$. $\mathrm{C}_{1}$, methane; $\mathrm{C}_{2}$, ethane; $\mathrm{C}_{3}$, propane; $\mathrm{n}-\mathrm{C}_{4}$, n-butane; i- $\mathrm{C}_{4}$, i-butane; $\mathrm{n}-\mathrm{C}_{5}$, n-pentane; i- $\mathrm{C}_{5}$, =i-pentane; $\mathrm{CO}_{2}$, carbon dioxide; $\mathrm{N}_{2}$, nitrogen; Wet, gas wetness in percent and $\delta^{13} \mathrm{C}_{1}, \delta^{13} \mathrm{C}_{2}$, and $\delta^{13} \mathrm{C}_{3}$ are the stable carbon isotopic composition of methane, ethane, and propane, respectively. $\delta^{2} \mathrm{HC}_{1}$ is the stable hydrogen isotopic composition of methane. ND, not determined. $\mathrm{S}$ indicates the source of the data and the numbers refer to the following publications: (1) Ballentine and Sherwood Lollar (2002), (2) Jenden and Kaplan (1989), (3) Jenden and others (1988), and (4) Rice and others (1988a)]

\begin{tabular}{|c|c|c|c|c|c|c|c|c|c|c|c|c|c|c|c|c|}
\hline $\begin{array}{l}\text { Well } \\
\text { name }\end{array}$ & $\begin{array}{c}\text { Formation } \\
\text { name }\end{array}$ & $C_{1}$ & $\mathrm{C}_{2}$ & $\mathrm{C}_{3}$ & $\mathrm{n}-\mathrm{C}_{4}$ & $\mathrm{i}-\mathrm{C}_{4}$ & $n-C_{5}$ & $\mathrm{i}-\mathrm{C}_{5}$ & $\mathrm{CO}_{2}$ & $\mathbf{N}_{2}$ & Wet & $\delta^{13} \mathbf{C}_{1}$ & $\delta^{13} \mathbf{C}_{2}$ & $\delta^{13} \mathbf{C}_{13}$ & $\delta^{2} \mathrm{HC}_{1}$ & $\mathbf{S}$ \\
\hline Burnett No. 1-12 & Chase & 75.62 & 6.25 & 4.88 & 1.93 & 0.87 & 0.70 & 0.61 & 0.37 & 8.73 & $16.77 \%$ & -41.8 & -34.3 & ND & -154 & 4 \\
\hline Burnett No. 7 & Chase & 73.52 & 6.36 & 4.77 & 1.85 & 0.86 & 0.64 & 0.55 & 0.31 & 11.10 & $16.97 \%$ & -42.0 & ND & ND & ND & 4 \\
\hline Burnett No. 24 & Chase & 76.99 & 6.30 & 4.33 & 1.71 & 0.73 & 0.01 & 0.02 & 0.14 & 9.75 & $14.54 \%$ & -42.1 & -34.0 & ND & -157 & 4 \\
\hline State No. 1 & Herington & 54.97 & 4.90 & 4.00 & 1.23 & 0.58 & 0.28 & 0.28 & 0.18 & 33.57 & $17.01 \%$ & -42.8 & ND & ND & ND & 4 \\
\hline L.C. Christian No. 1 & Chase & 76.76 & 7.80 & 4.77 & 1.36 & 0.44 & 0.37 & 0.24 & 0.30 & 7.96 & $16.33 \%$ & -46.1 & ND & ND & ND & 4 \\
\hline Sharpe No. 1 & Krider & 59.20 & 5.38 & 3.90 & 1.19 & 0.53 & 0.30 & 0.98 & 0.15 & 29.07 & $17.18 \%$ & -42.5 & ND & ND & ND & 4 \\
\hline Myers No.1 & Permian Lm. & 69.85 & 6.07 & 3.66 & 0.97 & 0.43 & 0.21 & 0.19 & 0.13 & 18.49 & $14.17 \%$ & -42.8 & ND & ND & $\mathrm{ND}$ & 4 \\
\hline Mackay No. 2 & Council Grove & 72.39 & 6.08 & 3.51 & 0.84 & 0.42 & 0.15 & 0.16 & 0.16 & 16.27 & $13.36 \%$ & -42.5 & -33.4 & ND & -163 & 4 \\
\hline Mackay No. 1 & Permian Lm. & 72.76 & 6.25 & 3.64 & 0.83 & 0.40 & 0.17 & 0.17 & 0.12 & 15.66 & $13.61 \%$ & -42.8 & ND & ND & ND & 4 \\
\hline Cargill No. 11 & Chase & 67.97 & 5.88 & 3.66 & 1.02 & 0.39 & 0.27 & 0.26 & 0.15 & 20.41 & $14.45 \%$ & -43.5 & ND & ND & ND & 4 \\
\hline Cargill No. 2 & Council Grove & 67.42 & 5.88 & 3.57 & 0.98 & 0.38 & 0.24 & 0.21 & 0.22 & 21.10 & $14.31 \%$ & -43.3 & -32.2 & ND & -164 & 4 \\
\hline Kinsler No. 1 & Krider & 68.53 & 5.91 & 3.70 & 0.98 & 0.43 & 0.24 & 0.21 & 0.14 & 19.85 & $14.34 \%$ & -43.2 & ND & ND & ND & 4 \\
\hline Conan No. A-1 & Morrow & 70.24 & 6.43 & 3.95 & 1.11 & 0.41 & 0.28 & 0.24 & 0.15 & 17.18 & $15.03 \%$ & -43.5 & ND & ND & ND & 4 \\
\hline Conan No. A-2 & Council Grove & 68.93 & 6.33 & 3.78 & 1.06 & 0.41 & 0.26 & 0.22 & 0.20 & 18.88 & $14.89 \%$ & -43.5 & ND & ND & ND & 4 \\
\hline Bond No. 1 & Chase & 67.67 & 6.31 & 4.05 & 1.12 & 0.45 & 0.30 & 0.25 & 0.16 & 19.70 & $15.57 \%$ & -43.8 & ND & ND & ND & 4 \\
\hline Bond No. 2 & Council Grove & 68.26 & 6.44 & 4.07 & 1.10 & 0.45 & 0.29 & 0.24 & 0.15 & 18.94 & $15.57 \%$ & -43.8 & ND & ND & ND & 4 \\
\hline Collingwood No. 1 & Herington & 67.45 & 6.30 & 3.79 & 1.09 & 0.42 & 0.27 & 0.23 & 0.12 & 20.50 & $15.21 \%$ & -44.0 & ND & ND & $\mathrm{ND}$ & 4 \\
\hline Julian No. 3 & Chase & 69.80 & 6.44 & 3.81 & 1.02 & 0.41 & 0.25 & 0.21 & 0.11 & 17.95 & $14.82 \%$ & -43.7 & -33.7 & ND & -165 & 4 \\
\hline Claypool & Chase & 62.28 & 5.46 & 3.54 & 1.04 & 0.39 & 0.28 & 0.22 & 0.11 & 26.69 & $14.93 \%$ & -43.1 & -33.7 & ND & -169 & 4 \\
\hline Bentley & Chase & 68.19 & 6.27 & 3.83 & 1.06 & 0.41 & 0.28 & 0.22 & 0.13 & 19.62 & $15.04 \%$ & -43.4 & ND & ND & ND & 4 \\
\hline Kenny & Hollenberg & 65.65 & 5.60 & 3.40 & 0.90 & 0.36 & 0.21 & 0.19 & 0.15 & 23.52 & $13.97 \%$ & -43.3 & ND & ND & ND & 4 \\
\hline Rohlman No. A-1 & Chase & 64.64 & 5.79 & 3.60 & 0.37 & 0.29 & 0.25 & 0.21 & 0.11 & 24.75 & $13.99 \%$ & -43.3 & -33.6 & ND & -161 & 4 \\
\hline Rohlman No. A-2 & Council Grove & 65.19 & 5.80 & 3.57 & 0.91 & 0.39 & 0.20 & 0.18 & 0.10 & 23.65 & $14.49 \%$ & -43.3 & ND & ND & ND & 4 \\
\hline Berryman No. B-1 & Winfield & 64.38 & 4.79 & 2.37 & 0.44 & 0.25 & 0.06 & 0.08 & 0.00 & 27.65 & $11.04 \%$ & -42.8 & -33.6 & ND & ND & 4 \\
\hline Webster No. C-4 & Wabaunsee & 61.11 & 5.28 & 3.10 & 0.49 & 0.33 & 0.00 & 0.10 & 0.11 & 29.48 & $13.21 \%$ & -42.6 & -34.9 & ND & ND & 4 \\
\hline Roseberry No. 2 & Cherokee & 74.89 & 5.03 & 3.35 & 1.05 & 0.57 & 0.08 & 0.22 & 0.32 & 14.48 & $12.09 \%$ & -45.2 & ND & ND & ND & 4 \\
\hline L.C. Christian No. 2 & U. Morrow & 65.45 & 5.85 & 3.76 & 1.03 & 0.47 & 0.24 & 0.22 & 0.21 & 22.76 & $15.02 \%$ & -42.7 & -35.9 & ND & -172 & 4 \\
\hline
\end{tabular}


[Units for the compositional data are in mole percent, and the $\delta^{13} \mathrm{C}$ and $\delta^{2} \mathrm{H}$ values are in per mil relative to the Vienna Pee Dee Belemnite and Vienna Standard Mean Ocean Water scales, respectively. Gas wetness is calculated as $\Sigma \mathrm{C}_{2+} / \Sigma \mathrm{C}_{1}+\mathrm{C}_{2}$. $\mathrm{C}_{1}$, methane; $\mathrm{C}_{2}$, ethane; $\mathrm{C}_{3}$, propane; $\mathrm{n}-\mathrm{C}_{4}$, n-butane; i- $\mathrm{C}_{4}$, i-butane; $\mathrm{n}-\mathrm{C}_{5}$, n-pentane; i- $\mathrm{C}_{5}$, $=\mathrm{i}$-pentane; $\mathrm{CO}_{2}$, carbon dioxide; $\mathrm{N}_{2}$, nitrogen; Wet, gas wetness in percent and $\delta^{13} \mathrm{C}_{1}, \delta^{13} \mathrm{C}_{2}$, and $\delta^{13} \mathrm{C}_{3}$ are the stable carbon isotopic composition of methane, ethane, and propane, respectively. $\delta^{2} \mathrm{HC}$ is the stable hydrogen isotopic composition of methane. ND, not determined. $\mathrm{S}$ indicates the source of the data and the numbers refer to the following publications: (1) Ballentine and Sherwood Lollar (2002), (2) Jenden and Kaplan (1989), (3) Jenden and others (1988), and (4) Rice and others (1988a)]

\begin{tabular}{|c|c|c|c|c|c|c|c|c|c|c|c|c|c|c|c|c|}
\hline $\begin{array}{c}\text { Well } \\
\text { name }\end{array}$ & $\begin{array}{c}\text { Formation } \\
\text { name }\end{array}$ & $C_{1}$ & $\mathrm{C}_{2}$ & $\mathrm{C}_{3}$ & $n-C_{4}$ & $\mathrm{i}-\mathrm{C}_{4}$ & $n-C_{5}$ & $\mathrm{i}-\mathrm{C}_{5}$ & $\mathrm{CO}_{2}$ & $\mathbf{N}_{2}$ & Wet & $\delta^{13} \mathbf{C}_{1}$ & $\delta^{13} \mathbf{C}_{2}$ & $\delta^{13} \mathbf{C}_{13}$ & $\delta^{2} \mathrm{HC}_{1}$ & $\mathbf{S}$ \\
\hline Gardner No.1 & Chase & 71.79 & 5.22 & 3.11 & 0.87 & 0.39 & 0.22 & 0.20 & 0.43 & 17.75 & $12.24 \%$ & -43.1 & ND & ND & ND & 4 \\
\hline Wiggins No. 1 & Morrow & 75.83 & 6.00 & 3.75 & 1.19 & 0.47 & 0.38 & 0.30 & 0.25 & 11.82 & $13.75 \%$ & -43.8 & ND & ND & ND & 4 \\
\hline Wiggins No. 1 & Morrow & 77.33 & 5.99 & 3.67 & 1.18 & 0.46 & 0.40 & 0.30 & 0.41 & 10.27 & $13.43 \%$ & -43.7 & ND & ND & ND & 4 \\
\hline Carpenter No. 6-8 & L. Morrow & 90.36 & 2.48 & 1.06 & 0.28 & 0.14 & 0.10 & 0.09 & 0.40 & 5.08 & $4.39 \%$ & -39.9 & ND & ND & ND & 4 \\
\hline
\end{tabular}

Table 4. Molecular and stable isotopic composition of gases from the Sooner trend region of the Anadarko Basin Province.

[Units for the compositional data are in mole percent, and the $\delta^{13} \mathrm{C}$ and $\delta^{2} \mathrm{H}$ values are in per mil relative to the Vienna Pee Dee Belemnite and Vienna Standard Mean Ocean Water scales, respectively. Gas wetness is calculated as $\Sigma \mathrm{C}_{2} / \Sigma \mathrm{C}_{1}+\mathrm{C}_{2}, \mathrm{C}_{1}$, methane; $\mathrm{C}_{2}$, ethane; $\mathrm{C}_{3}$, propane; $\mathrm{n}-\mathrm{C}_{4}$, n-butane; $\mathrm{i}-\mathrm{C}_{4}$, i-butane; $\mathrm{n}-\mathrm{C}_{5}, \mathrm{n}$-pentane; i- $\mathrm{C}_{5},=\mathrm{i}$-pentane; $\mathrm{CO}_{2}$, carbon dioxide; $\mathrm{N}_{2}$, nitrogen; Wet, gas wetness in percent and $\delta^{13} \mathrm{C}_{1}, \delta^{13} \mathrm{C}_{2}$, and $\delta^{13} \mathrm{C}_{3}$ are the stable carbon isotopic composition of methane, ethane, and propane, respectively. $\delta^{2} \mathrm{HC}$ is the stable hydrogen isotopic composition of methane. ND, not determined. Data are from Rice and others (1988a)]

\begin{tabular}{|c|c|c|c|c|c|c|c|c|c|c|c|c|c|c|c|c|}
\hline $\begin{array}{c}\text { Well } \\
\text { name }\end{array}$ & $\begin{array}{c}\text { Formation } \\
\text { name }\end{array}$ & $C_{1}$ & $\mathrm{C}_{2}$ & $\mathrm{C}_{3}$ & $\mathrm{n}-\mathrm{C}_{4}$ & $\mathrm{i}-\mathrm{C}_{4}$ & $n-C_{5}$ & $\mathrm{i}-\mathrm{C}_{5}$ & $\mathrm{CO}_{2}$ & $\mathbf{N}_{2}$ & Wet & $\delta^{13} \mathbf{C}_{1}$ & $\delta^{13} \mathbf{C}_{2}$ & $\delta^{13} \mathbf{C}_{3}$ & $\delta^{2} \mathrm{HC}_{1}$ & Source \\
\hline Little Charlie No. 1 & Desmoinesian & 64.75 & 9.22 & 3.69 & 0.60 & 0.30 & 0.04 & 0.09 & 0.21 & 21.11 & $17.72 \%$ & -46.10 & -35.60 & ND & ND & Rice and others, 1988 \\
\hline Jarvis No. 1 & Bartlesville & 51.00 & 13.09 & 9.42 & 1.51 & 1.26 & 0.04 & 0.21 & 0.26 & 23.22 & $33.36 \%$ & -47.30 & ND & ND & -149 & Rice and others, 1988 \\
\hline Walker No. 1-35U & Manning & 82.84 & 4.27 & 1.72 & 0.28 & 0.26 & 0.02 & 0.07 & 0.12 & 10.42 & $7.40 \%$ & -42.30 & -31.20 & ND & ND & Rice and others, 1988 \\
\hline Dietz No. 1 & Manning & 74.39 & 5.48 & 2.52 & 0.51 & 0.31 & 0.06 & 0.11 & 0.07 & 16.54 & $10.78 \%$ & -42.10 & ND & ND & -146 & Rice and others, 1988 \\
\hline Gilger No.1 & Mississippian & 64.88 & 4.43 & 1.45 & 0.32 & 0.20 & 0.03 & 0.07 & 0.32 & 28.31 & $9.11 \%$ & -43.10 & ND & ND & ND & Rice and others, 1988 \\
\hline Schneider No. 1 & Middle Mississippian & 66.40 & 5.26 & 1.84 & 0.30 & 0.20 & 0.02 & 0.05 & 0.27 & 25.67 & $10.36 \%$ & -41.80 & $\mathrm{ND}$ & ND & ND & Rice and others, 1988 \\
\hline Meadowlake No. 1 & Middle Mississippian & 70.81 & 5.63 & 1.92 & 0.21 & 0.24 & 0.00 & 0.02 & 0.21 & 20.95 & $10.17 \%$ & -43.80 & -31.70 & ND & ND & Rice and others, 1988 \\
\hline Quintle No. 1 & Chester & 85.86 & 0.57 & 0.23 & 0.12 & 0.12 & 0.01 & 0.04 & 0.02 & 13.03 & $1.25 \%$ & -42.80 & ND & ND & -142 & Rice and others, 1988 \\
\hline Hill No. 1 & Mississippian & 67.93 & 10.69 & 4.64 & 0.70 & 0.35 & 0.02 & 0.07 & 0.41 & 15.19 & $19.51 \%$ & -46.00 & -38.10 & ND & ND & Rice and others, 1988 \\
\hline Simmons No. 1 & Hunton & 66.08 & 8.92 & 3.71 & 0.49 & 0.42 & 0.01 & 0.07 & 0.21 & 20.09 & $17.09 \%$ & -46.70 & -31.00 & ND & -157 & Rice and others, 1988 \\
\hline Killough No. 1-21 & Hunton & 67.61 & 5.80 & 2.46 & 0.32 & 0.16 & 0.02 & 0.04 & 0.08 & 23.51 & $11.52 \%$ & -45.30 & ND & ND & -150 & Rice and others, 1988 \\
\hline Rigdon Unit No. 2 & Cleveland & 67.43 & 8.70 & 3.45 & 0.60 & 0.45 & 0.02 & 0.08 & 0.19 & 19.08 & $16.47 \%$ & -42.90 & ND & ND & ND & Rice and others, 1988 \\
\hline Blaney Nos. 2,3,5 & Cleveland & 66.91 & 7.90 & 3.74 & 0.57 & 0.46 & 0.02 & 0.08 & 0.20 & 20.12 & $16.03 \%$ & -43.20 & ND & ND & ND & Rice and others, 1988 \\
\hline Waswo No. 1 & $\begin{array}{l}\text { Desmoinesian, } \\
\text { Mississippian, and } \\
\text { Hunton Group }\end{array}$ & 71.16 & 7.46 & 2.30 & 0.27 & 0.25 & 0.02 & 0.02 & 0.19 & 18.33 & $12.67 \%$ & -43.50 & ND & ND & ND & Rice and others, 1988 \\
\hline
\end{tabular}


Table 4. Molecular and stable isotopic composition of gases from the Sooner trend region of the Anadarko Basin Province.-Continued

[Units for the compositional data are in mole percent, and the $\delta^{13} \mathrm{C}$ and $\delta^{2} \mathrm{H}$ values are in per mil relative to the Vienna Pee Dee Belemnite and Vienna Standard Mean Ocean Water scales, respectively. Gas wetness is calculated as $\Sigma \mathrm{C}_{2+} / \Sigma \mathrm{C}_{1}+\mathrm{C}_{2+}$. $\mathrm{C}_{1}$, methane; $\mathrm{C}_{2}$, ethane; $\mathrm{C}_{3}$, propane; $\mathrm{n}-\mathrm{C}_{4}$, n-butane; i- $\mathrm{C}_{4}$, i-butane; n- $\mathrm{C}_{5}$, n-pentane; i- $\mathrm{C}_{5}$, = i-pentane; $\mathrm{CO}_{2}$, carbon dioxide; $\mathrm{N}_{2}$, nitrogen; Wet, gas wetness in percent and $\delta^{13} \mathrm{C}_{1}, \delta^{13} \mathrm{C}_{2}$, and $\delta^{13} \mathrm{C}_{3}$ are the stable carbon isotopic composition of methane, ethane, and propane, respectively. $\delta^{2} \mathrm{HC}_{1}$ is the stable hydrogen isotopic composition of methane. ND, not determined. Data are from Rice and others (1988a)]

\begin{tabular}{|c|c|c|c|c|c|c|c|c|c|c|c|c|c|c|c|c|}
\hline $\begin{array}{l}\text { Well } \\
\text { name }\end{array}$ & $\begin{array}{c}\text { Formation } \\
\text { name }\end{array}$ & $C_{1}$ & $C_{2}$ & $C_{3}$ & $n-C_{4}$ & $\mathrm{i}-\mathrm{C}_{4}$ & $n-C_{5}$ & $\mathrm{i}-\mathrm{C}_{5}$ & $\mathrm{CO}_{2}$ & $\mathbf{N}_{2}$ & Wet & $\delta^{13} \mathbf{C}_{1}$ & $\delta^{13} \mathbf{C}_{2}$ & $\delta^{13} \mathbf{C}_{3}$ & $\delta^{2} \mathrm{HC}_{1}$ & Source \\
\hline Mach No. 1 & Skinner & 72.41 & 8.81 & 3.36 & 0.48 & 0.27 & 0.03 & 0.07 & 0.45 & 14.12 & $15.24 \%$ & -44.50 & -34.90 & ND & ND & Rice and others, 1988 \\
\hline Schein Nos. 1,2,3 & Mississippian & 71.79 & 8.75 & 3.34 & 0.09 & 0.56 & 0.04 & 0.09 & 0.41 & 14.73 & $15.20 \%$ & -45.20 & ND & ND & -164 & Rice and others, 1988 \\
\hline FIFI No. 1 & Hunton & 71.50 & 8.17 & 2.89 & 0.47 & 0.28 & 0.03 & 0.07 & 0.25 & 16.33 & $14.28 \%$ & -43.60 & ND & ND & ND & Rice and others, 1988 \\
\hline Omega No. 19-1 & Chester & 76.31 & 6.37 & 2.07 & 0.38 & 0.28 & 0.09 & 0.08 & 0.14 & 14.28 & $10.83 \%$ & -43.10 & ND & ND & ND & Rice and others, 1988 \\
\hline
\end{tabular}

Table 5. Molecular and stable isotopic composition of gases from the central Kansas uplift region of the Anadarko Basin Province.

[Units for the compositional data are in mole percent, and the $\delta^{13} \mathrm{C}$ and $d^{2} \mathrm{H}$ values are in per mil relative to the Vienna Pee Dee Belemnite and Vienna Standard Mean Ocean Water scales, respectively. Gas wetness is calculated as $\Sigma \mathrm{C}_{2+} / \Sigma \mathrm{C}_{1}+\mathrm{C}_{2+}$. $\mathrm{C}_{1}$, methane; $\mathrm{C}_{2}$, ethane; $\mathrm{C}_{3}$, propane; $\mathrm{n}-\mathrm{C}_{4}$, n-butane; i- $\mathrm{C}_{4}$, i-butane; $\mathrm{n}-\mathrm{C}_{5}, \mathrm{n}$-pentane; i- $\mathrm{C}_{5}$, $=\mathrm{i}$-pentane; $\mathrm{CO}_{2}$, carbon dioxide; $\mathrm{N}_{2}$, nitrogen; Wet, gas wetness in percent and $\delta^{13} \mathrm{C}_{1}, \delta^{13} \mathrm{C}_{2}$, and $\delta^{13} \mathrm{C}_{3}$ are the stable carbon isotopic composition of methane, ethane, and propane, respectively. $\delta^{2} \mathrm{HC}$ is the stable hydrogen isotopic composition of methane. ND, not determined. Data are from Jenden and others (1988)]

\begin{tabular}{|c|c|c|c|c|c|c|c|c|c|c|c|c|c|c|c|c|}
\hline $\begin{array}{l}\text { Well } \\
\text { name }\end{array}$ & $\begin{array}{c}\text { Formation } \\
\text { name }\end{array}$ & $C_{1}$ & $C_{2}$ & $\mathrm{C}_{3}$ & $\mathrm{n}-\mathrm{C}_{4}$ & $\mathrm{i}-\mathrm{C}_{4}$ & $n-C_{5}$ & i- $C_{5}$ & $\mathrm{CO}_{2}$ & $\mathbf{N}_{2}$ & Wet & $\delta^{13} \mathbf{C}_{1}$ & $\delta^{13} \mathbf{C}_{2}$ & $\delta^{13} \mathbf{C}_{3}$ & $\delta^{2} \mathrm{HC}_{1}$ & Source \\
\hline Dutton I-17 & Kinderhook & 85.8 & 3.92 & 1.62 & 0.56 & 0.25 & 0.19 & 0.16 & 0.12 & 3.89 & $7.24 \%$ & -40.0 & -34.6 & -30.9 & -150 & Jenden and others, 1988 \\
\hline Embry Lease & Kansas City & 63.8 & 8.64 & 8.35 & 3.72 & 1.56 & 1.20 & 0.97 & 0.20 & 7.98 & $27.70 \%$ & -41.9 & -34.5 & -31.2 & -164 & Jenden and others, 1988 \\
\hline Chalk A 1-18 & Cherokee & 83.6 & 4.29 & 1.77 & 0.50 & 0.26 & 0.16 & 0.11 & 0.13 & 7.88 & $7.81 \%$ & -39.7 & -34.4 & -30.5 & -150 & Jenden and others, 1988 \\
\hline Enlow-Miller \#1 & Limestone & 85.5 & 4.88 & 2.36 & 0.93 & 0.42 & 0.33 & 0.26 & 0.20 & 4.51 & $9.70 \%$ & -39.6 & -35.1 & -30.8 & -151 & Jenden and others, 1988 \\
\hline Thurow \#1 & Foraker Limestone & 84.0 & 2.36 & 0.68 & 0.15 & 0.08 & 0.05 & 0.04 & 0.02 & 11.60 & $3.85 \%$ & -40.8 & -35.1 & -30.5 & -154 & Jenden and others, 1988 \\
\hline Isern \#1 & Arbuckle & 79.2 & 3.81 & 1.22 & 0.29 & 0.23 & 0.05 & 0.10 & 0.04 & 12.70 & $6.72 \%$ & -42.2 & -34.2 & -30.4 & -148 & Jenden and others, 1988 \\
\hline Miller \#1-34 & Indian Cave & 87.4 & 2.30 & 1.47 & 0.43 & 0.22 & 0.13 & 0.12 & 0.11 & 5.11 & $5.06 \%$ & -41.2 & -35.9 & -31.4 & -152 & Jenden and others, 1988 \\
\hline Urban Q3 & Lansing-Kansas City & 54.0 & 1.97 & 1.56 & 1.12 & 0.53 & 0.38 & 0.31 & 0.19 & 36.40 & $9.81 \%$ & -39.2 & -35.6 & -31.2 & -148 & Jenden and others, 1988 \\
\hline Doran 2-14 & Arbuckle & 74.9 & 6.59 & 3.49 & 1.02 & 0.62 & 0.24 & 0.24 & 0.67 & 9.69 & $14.00 \%$ & -41.1 & -34.3 & -30.4 & -149 & Jenden and others, 1988 \\
\hline Wessler A-1 & Fractured Quartzite & 43.9 & 13.00 & 16.60 & 8.58 & 3.51 & 1.79 & 2.06 & 2.18 & 7.53 & $50.92 \%$ & -40.9 & -37.4 & -33.3 & -162 & Jenden and others, 1988 \\
\hline Apple 2 & Topeka & 65.9 & 3.07 & 1.30 & 0.50 & 0.35 & 0.22 & 0.22 & 0.12 & 27.10 & $7.91 \%$ & -41.5 & -34.3 & -30.2 & -148 & Jenden and others, 1988 \\
\hline Honderick "D" 1 & Granite Wash & 77.5 & 3.75 & 1.48 & 0.50 & 0.32 & 0.15 & 0.16 & 0.04 & 14.70 & $7.57 \%$ & -41.7 & -34.1 & -29.9 & -147 & Jenden and others, 1988 \\
\hline Muth 1 & Chase & 63.6 & 2.18 & 0.92 & 0.30 & 0.22 & 0.11 & 0.10 & 0.01 & 30.50 & $5.67 \%$ & -40.0 & -34.5 & -30.4 & -139 & Jenden and others, 1988 \\
\hline Wellman 2 & Simpson & 77.7 & 6.29 & 2.68 & 1.01 & 0.38 & 0.28 & 0.19 & 0.14 & 12.10 & $12.24 \%$ & -44.1 & -35.0 & -32.2 & -174 & Jenden and others, 1988 \\
\hline Colglazier 1 & Viola & 68.1 & 4.15 & 1.68 & 0.45 & 0.24 & 0.16 & 0.11 & 0.21 & 22.00 & $9.06 \%$ & -39.6 & -34.4 & -30.7 & -149 & Jenden and others, 1988 \\
\hline
\end{tabular}


for stable isotopic analysis; so only the $\delta^{13} \mathrm{C}$ of methane was determined. After the stable carbon isotopic analyses were performed, 31 samples contained sufficient amounts of methane for stable hydrogen isotopic analysis. One sample was analyzed for the stable hydrogen isotopic composition of methane prior to stable carbon isotopic analysis, and the residual gas was not sufficient for further isotopic analyses.

The results of the stable isotopic analyses of the 75 gas samples that were analyzed are presented in table 2. For the samples collected for this study, the $\delta^{13} \mathrm{C}$ composition of methane ranges from $-48.7 \%$ to $-36.7 \%$ and averages $-44.9 \%$ $(1 \sigma=2.6 \%)$; the $\delta^{13} \mathrm{C}$ composition of ethane ranges from $-41.8 \%$ to $-24.0 \%$ and averages $-34.4 \%$ ( $(1 \sigma=2.9 \%)$; the $\delta^{13} \mathrm{C}$ composition of propane ranges from $-37.9 \%$ to $-21.6 \%$ and averages $-30.8 \%$ o $(1 \sigma=3.1 \%)$; and the $\delta^{2} \mathrm{H}$ composition of methane ranges from $-164 \%$ to $-130 \%$ and averages $-150 \%(1 \sigma=9 \%)$. In addition to the data generated from the samples collected for this study, stable isotopic data from producing gas wells in the central Anadarko Basin published by Jenden and others (1988), Rice and others (1988a), and Jenden and Kaplan (1989a) are included in table 2. Additional published stable isotopic data from the Panhandle-Hugoton field, the Sooner trend, and the central Kansas uplift are included in tables 3, 4, and 5, respectively, and these values are taken from the works of Jenden and others (1988), Rice and others (1988a), Jenden and Kaplan (1989a), and Ballentine and Sherwood Lollar (2002).

\section{Discussion}

A plot of the stable carbon isotopic composition of methane, ethane, and propane can be used to distinguish among abiogenic, thermogenic, and microbial sources of natural gases (Schoell, 1983a; Chung and others, 1988; Sherwood Lollar and others, 2002). Figure 3 shows the average $\delta^{13} \mathrm{C}$ composition of methane, ethane, and propane for the four regions within the study area; these data clearly indicate that the gases of the Anadarko Basin Province are from organic sources (that is, there is no significant contribution of natural gases from abiogenic sources). These data also indicate that the majority of the gases in the province are from thermogenic sources, which is consistent with the findings of previous studies of the gas geochemistry of the province (Jenden and others, 1988; Rice and others, 1988b, 1989). Although the average stable carbon isotopic values for methane, ethane, and propane for the four regions within the Anadarko Basin Province are consistent with a single thermogenic source (fig. 3), individual wells in parts of the study area do contain methane that is isotopically lighter than expected, given the $\delta^{13} \mathrm{C}$ composition of the associated ethane and propane. A significant contribution of methane from microbial sources is expected to result in methane depleted in ${ }^{13} \mathrm{C}$ relative to the stable carbon isotopic composition of ethane and propane (Schoell, 1980; Rice and Claypool, 1981; Schoell, 1983a; Whiticar, 1994). Consequently, microbial methane contributions in restricted portions of the province

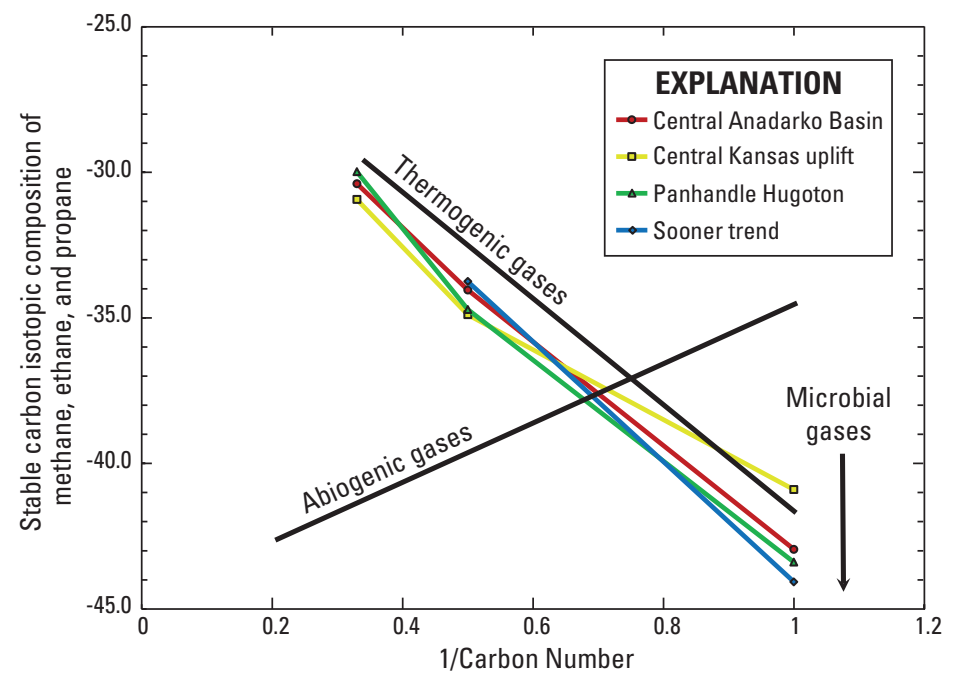

Figure 3. Plot of the stable carbon isotopic composition of methane, ethane, and propane versus the reciprocal of the carbon number of the hydrocarbon species. Data are average values for each of the interpretive regions of the study area. Heavy black lines indicate the expected trends for thermogenic and abiogenic sources of hydrocarbon gases. Microbial gases have isotopically depleted methane compositions as shown by the arrow. 
cannot be completely discounted. An alternative explanation for the occurrence of isotopically light methane is that it is the product of thermal cracking of oil (Tang and Schoell, 2005; Fusetti and others, 2010).

Plots of the $\delta^{13} \mathrm{C}$ composition of methane versus the $\delta^{2} \mathrm{H}$ of methane and versus the gas wetness (that is, the sum of the $\mathrm{C}_{2+}$ hydrocarbon gases) are widely used to identify the thermal regime of natural gas generation (Schoell, 1980; Schoell, 1983a, 1983b; Jenden and others, 1988; Rice and others, 1988b). For the Anadarko Basin Province data, the $\delta^{13} \mathrm{C}$ composition of methane plotted against the $\delta^{2} \mathrm{H}$ of methane (fig. 4) and against the gas wetness (fig. 5) indicate mature (oil window) to post-mature (dry gas window) sources for gas generation consistent with the findings of previous studies (Jenden and others, 1988; Rice and others, 1988b). The variation in thermal maturity of the generated gases is undoubtedly the result of differential burial of the source rocks in the basin. To address this point, Rice and others (1988b) attempted to correlate the $\delta^{13} \mathrm{C}$ of methane with the depth of the reservoir from which it was produced. They observed a wide scatter in the data and concluded that a significant portion of the gases had migrated variable distances from different sources into a single reservoir resulting in a lack of correlation between reservoir depth and gas maturity.

Given the size (approximately 58,000 $\mathrm{mi}^{2}$ ) and complexity of the Anadarko Basin Province, the study area has been broken down into four separate regions to facilitate a more detailed discussion of the natural gas geochemistry. Following the approach of Rice and others (1988b) and Jenden and others (1988), these four regions are defined as the central Anadarko Basin, the Panhandle-Hugoton field, the Sooner trend, and the central Kansas uplift (fig. 1). Details of the gas geochemistry of each of these regions are given in the following four sections.

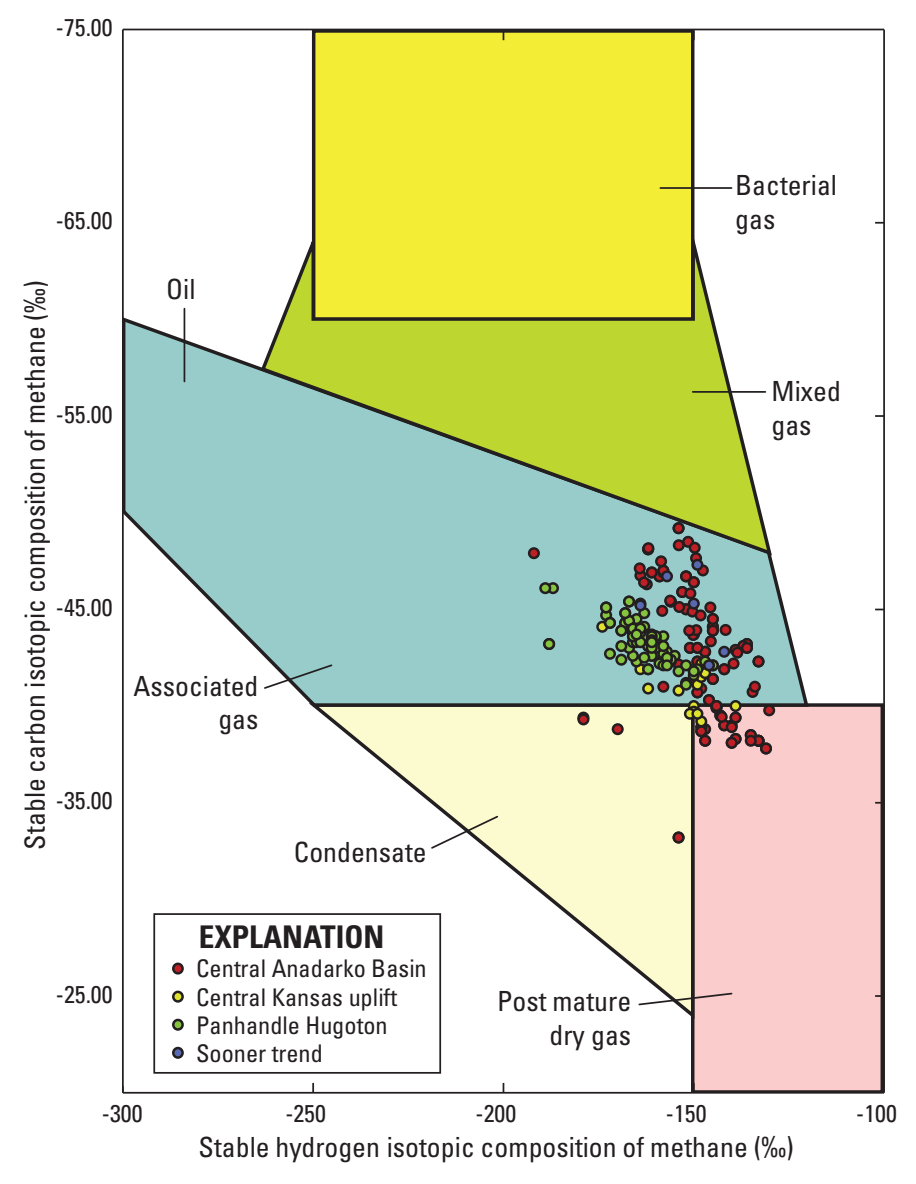

Figure 4. Plot of the stable carbon versus the stable hydrogen isotopic composition of methane. Note that stable hydrogen isotopic data for methane are not available for all of the gases in this study. Assoc. Gas, oil associated natural gas. 


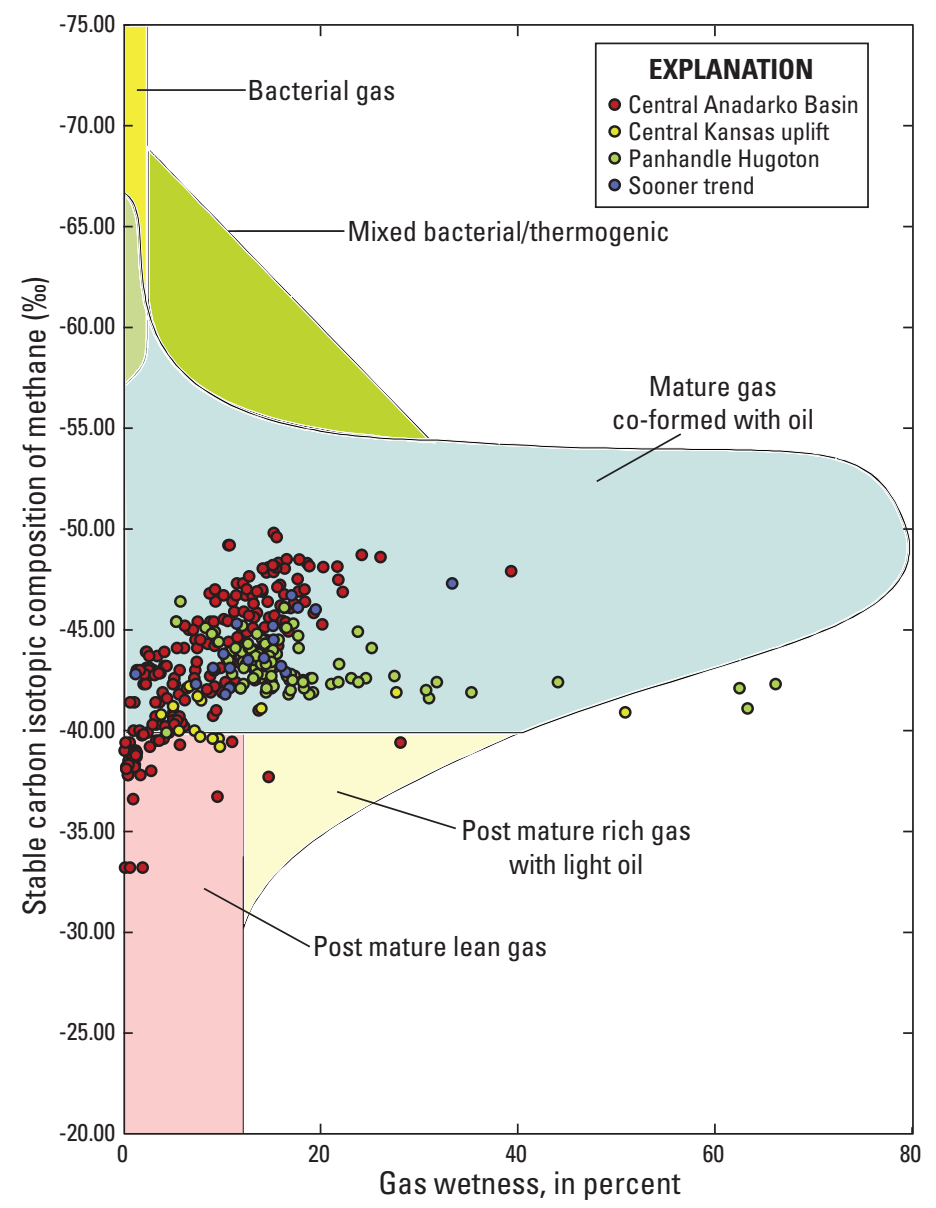

Figure 5. Plot of the gas wetness $\left(\Sigma C_{2+} / \Sigma C_{1}+C_{2+}\right)$ versus the stable carbon isotopic composition of methane.

\section{Central Anadarko Basin}

Rice and others (1988b) reported that gases in Upper Mississippian and Pennsylvanian reservoirs in the central Anadarko Basin become chemically drier (that is, lower $\mathrm{C}_{2+}$ content) and isotopically heavier (that is, increased ${ }^{13} \mathrm{C}$ content) with increasing age of the producing formation. They noted that (1) for gases derived from the Virgilian, the average $\delta^{13} \mathrm{C}$ of methane is $-46.6 \%$ and the mean $\mathrm{C}_{2+}$ value is 12 percent; and (2) gases produced from the Atokan and Desmoinesian contain methane with a mean $\delta^{13} \mathrm{C}$ value of $-43.9 \%$ and an average $\mathrm{C}_{2+}$ content of 7 percent. The authors concluded that these gases were most likely generated during the mature stage of hydrocarbon generation, which is consistent with the genetic classification scheme proposed by Schoell (1983a). Furthermore, the data presented by Rice and others (1988b) showed that gases produced from Springer Formation and Morrowan reservoirs contain methane with a mean $\delta^{13} \mathrm{C}$ value of $-39.9 \%$ and an average $\mathrm{C}_{2+}$ content of 3 percent, which likely indicates a mature to post-mature source for these gases.
The authors proposed two explanations for these observations. First, the Springer and Morrowan reservoirs are older and more deeply buried than the Virgilian, Atokan, and Desmoinesian reservoirs and are sourced from older, deeper source rocks that have reached a higher level of thermal maturity. Second, potential source rocks in the Springer and Morrowan intervals contain predominately Type III kerogen, whereas the Atokan and Desmoinesian lithologies contain mixed Type II and III and in the Virgilian the kerogen is mainly Type II (Rice and others, 1988b). Numerous field observations from around the world have shown that, in general, Type III kerogens generate gases that are drier and have enriched ${ }^{13} \mathrm{C}$ compositions relative to Type II kerogen-sourced gases of equivalent thermal maturity (Whiticar, 1994).

Rice and others (1988b) further noted that the geochemistry of gases in lower Paleozoic reservoirs in the central Anadarko Basin does not follow the trend observed in the Upper Mississippian and Pennsylvanian reservoirs. Whereas these deeper reservoirs would be expected to contain drier and heavier gases than those encountered in the shallower Springer 
and Morrowan lithologies, in fact the lower Paleozoic gases are, on average, the lightest $\left(\delta^{13} \mathrm{C}_{1}=-46.4 \%\right)$ and wettest $\left(\mathrm{C}_{2+}\right.$ $=14 \%$ ) gases in the central basin (Rice and others, 1988b). The authors explained this finding as the result of the source of the gases being a Type II kerogen and the fact that the gases are coproduced with oil. Most of the gases from the younger reservoirs examined by Rice and others (1988b), however, were from nonassociated accumulations. They did not consider the possibility that some gas generation resulted from oil cracking, but given that the average depth for the lower Paleozoic reservoirs is greater than 16,000 feet, it is reasonable to expect that some oil cracking has occurred and contributed to the associated gas pool. Gases derived from the thermal cracking of oil are expected to contain isotopically light methane and to have a greater $\mathrm{C}_{2+}$ content than primary gases generated at an equivalent thermal maturity (Tang and Schoell, 2005; Fusetti and others, 2010).

Data from other published studies of the geochemistry of gases from the central Anadarko Basin (Jenden and others, 1988; Jenden and Kaplan, 1989a) combined with the results of this study generally support the findings of Rice and others (1988b) that for Upper Mississippian and Pennsylvanian reservoirs, gas composition becomes chemically drier and isotopically heavier with increasing age. This expanded dataset (table 2) shows that gases derived from Virgilian reservoirs have an average $\delta^{13} \mathrm{C}$ composition for methane of $-45.5 \%$ and a mean $\mathrm{C}_{2+}$ value of 14 percent, and gases produced from Atokan and Desmoinesian units contain methane with a mean $\delta^{13} \mathrm{C}$ value of $-44.7 \%$ and an average $\mathrm{C}_{2+}$ content of 10 percent. Additionally, gases produced from Springer and Morrowan reservoirs contain methane with a mean $\delta^{13} \mathrm{C}$ value of $-40.6 \%$ and an average $\mathrm{C}_{2+}$ content of 6 percent. The mean $\delta^{13} \mathrm{C}$ composition for methane from lower Paleozoic reservoirs is $-46.1 \%$ and the average wetness is 16 percent. These values are consistent with the numbers reported by Rice and others (1988b) and support their interpretation of the geochemistry of gases from the central Anadarko Basin.

\section{Panhandle Hugoton Field}

The molecular and stable isotopic composition of the gases produced from the Panhandle Hugoton field region is remarkably consistent given the size of these giant fields (table 3). Plots of the $\delta^{13} \mathrm{C}$ composition of methane versus both the $\delta^{2} \mathrm{H}$ of methane (fig. 4) and gas wetness (fig. 5) for this region indicate that the gases are derived from a thermally mature source. Previous studies have noted the general absence of thermally mature source rocks in this region (Cardott and Lambert, 1985), and this has led to the inference that the Panhandle Hugoton gases are not locally sourced (Jenden and others, 1988; Rice and others, 1988b). Based on similarities among the gas wetness and the $\delta^{13} \mathrm{C}$ composition of methane of Panhandle Hugoton gases and those from Desmoinesian and Atokan reservoirs in the central Anadarko Basin, Rice and others (1988b) suggested that Pennsylvanian and (or) older source rocks in the central basin are the source of the gases in the Panhandle and Hugoton fields. Consequently, the gases produced from reservoirs in the Texas and Oklahoma Panhandles and in western Kansas are thought to have migrated several hundred kilometers from source kitchen areas in central Oklahoma. Sorenson (2005) proposed that hydrocarbons from the central Anadarko Basin initially charged structural traps in the pre-Laramide Panhandle field, and that early Tertiary Laramide orogenic events resulted in a remigration of fluids that charged the Hugoton field with gas. However, Jenden and others (1988) pointed out that, for a given level of thermal maturity, gases sourced from Type III (humic) kerogen tend to be enriched in ${ }^{13} \mathrm{C}$ relative to Type II (sapropelic) sourced gases. Consequently, early thermogenic gases derived from humic organic matter may be difficult to distinguish from mature gases generated from sapropelic organic matter, and Jenden and others (1988) proposed that the amount of natural gas derived from local source rocks in the Panhandle Hugoton region may be underestimated.

Examination of all of the published gas geochemistry data available for the Panhandle Hugoton region (table 3 ) shows that the average gas wetness is 16.0 percent $(1 \sigma=8.2$ percent $)$ and the mean $\delta^{13} \mathrm{C}$ composition of methane is $-43.4 \%$ o $(1 \sigma=1.0 \%)$. These values are comparable to the mean values reported for gases produced from Atokan and Desmoinesian reservoirs in the central Anadarko Basin. However, given the significant migration distance (hundreds of miles) required for gases generated in the central Anadarko Basin to charge reservoirs in the Panhandle Hugoton region, it seems likely that the long-distance-migrated gases might represent an earlier stage of gas generation compared to the more locally reservoired gases. This implies that the source of the Panhandle Hugoton gases is pre-Pennsylvanian, with the Woodford Shale being the most likely candidate.

An interesting feature of the geochemistry of the gases of the Panhandle Hugoton region is the elevated concentration of nitrogen. Based on more than 12,000 gas samples collected and analyzed by the U.S. Bureau of Mines, the median nitrogen concentration in produced natural gases throughout the United States is 3 percent (Jenden and Kaplan, 1989b). By contrast, for the gases examined in this study the average concentration of nitrogen from the Panhandle Hugoton region is 15.3 percent $(1 \sigma=12.0$ percent) (table 3$)$, which is in agreement with the approximate 15 percent value reported by Ballentine and Sherwood Lollar (2002). Nitrogen in natural gas reservoirs is generally thought to be derived from one or more of the following sources: (1) atmospheric nitrogen dissolved in groundwater, (2) thermogenic degradation of nitrogen containing sedimentary organic matter, (3) release of mineral bound nitrogen during metamorphism, and (4) igneous or deep mantle sources (Ballentine and Sherwood Lollar, 2002, and references therein). On the basis of the consistent association of helium with nitrogen throughout the Panhandle and Hugoton fields, Gold and Held (1987) suggested that all of the nitrogen must be derived from a source deeper than the total sedimentary accumulation of the Anadarko Basin (that is, a crustal and (or) mantle source). Jenden and others (1988) 
noted that in the Hugoton field higher concentrations of $\mathrm{N}_{2}$ are associated with enriched ${ }^{13} \mathrm{C}$ compositions in methane and that the $\mathrm{N}_{2} / \mathrm{Ar}$ ratio is nearly five times that of the atmospheric ratio indicating that an immature sedimentary origin for the $\mathrm{N}_{2}$ is unlikely. Citing the work of Oxburgh and others (1986), Jenden and others (1988) reported that more than 98 percent of the helium in the Panhandle and Hugoton fields is derived, at least in part, from crustal sources and that the nitrogen can be inferred to have a similar source. A more recent study of the noble gases $\left({ }^{3} \mathrm{He} /{ }^{4} \mathrm{He},{ }^{21} \mathrm{Ne} /{ }^{22} \mathrm{Ne}\right.$, and $\left.{ }^{40} \mathrm{Ar} /{ }^{36} \mathrm{Ar}\right)$ and the stable isotopic composition of the hydrocarbon and nonhydrocarbon gases $\left(\delta^{13} \mathrm{C}\right.$ of methane, ethane and propane; and $\delta^{15} \mathrm{~N}$ of $\left.\mathrm{N}_{2}\right)$ excludes the possibility of significant atmospheric and magmatic sources, and concludes that the nitrogen in the Panhandle Hugoton region is derived from a mixture of crustal rocks and thermally mature sedimentary organic matter (Ballentine and Sherwood Lollar, 2002).

\section{Sooner Trend}

All of the data for gases from the Sooner trend presented in this study are taken from Rice and others (1988a) (table 4). Gas production in this region is generally associated with oil, and the oils have been geochemically correlated with a Woodford Shale source (Rice and others, 1989). Hydrocarbon production in the Sooner trend is from relatively shallow (less than 3,000 meters) Silurian, Devonian, and Mississippian carbonate reservoirs (Rice and others, 1988b). Plots of the $\delta^{13} \mathrm{C}$ of methane versus both the $\delta^{2} \mathrm{H}$ of methane (fig. 4) and gas wetness (fig. 5) for this region indicate that the gases are derived from a thermally mature source rock containing Type II kerogen. The Woodford Shale is the most likely source for these gases given that the associated oils are derived from this formation. Within the Sooner trend region the Woodford Shale is only marginally mature; therefore, it is most likely that the gases have migrated from deeper portions of the central Anadarko Basin (Rice and others, 1988b).

\section{Central Kansas Uplift}

The central Kansas uplift gas geochemistry data presented in table 5 are from Jenden and others (1988). The gases appear to be derived primarily from a thermally mature Type II kerogen source, as shown in the plots of the $\delta^{13} \mathrm{C}$ of methane versus both the $\delta^{2} \mathrm{H}$ of methane (fig. 4) and gas wetness (fig. 5). The limited data available for thermal maturity in the central Kansas uplift region indicate that the local source rocks are marginally mature; consequently the shallow reservoirs were most likely charged by gases that had migrated hundreds of miles from the deeper central Anadarko Basin (Jenden and others, 1988). The average wetness for produced gases from the central Kansas uplift is 12.4 percent $(1 \sigma=12.0$ percent $)$ and the mean $\delta^{13} \mathrm{C}$ composition of the methane is $-40.9 \%(1 \sigma=1.3 \%)$, which are comparable to the mean values reported for gases produced from Springer and Morrowan reservoirs in the central Anadarko Basin (6.0 percent and $-40.6 \%$ ). However, as noted for the gases of the Panhandle Hugoton region, the long-distance migrated gases likely represent an earlier stage of gas generation compared to the more locally reservoired gases in the central Anadarko Basin. Consequently, the deep source of the central Kansas uplift gases is probably Early Mississippian or older, and may be the Woodford Shale.

Importantly, there is some evidence to suggest that gases in the central Kansas uplift region may contain a contribution of locally sourced early thermogenic gas. Figure 3 shows that the average $\delta^{13} \mathrm{C}$ composition of methane is slightly heavier than the expected value if the methane, ethane, and propane were derived from a single source of similar thermal maturity. The best explanation for this observation is that a dry mature gas mixed with a wetter lower maturity gas. Moreover, as discussed for the Panhandle Hugoton gases, gases sourced from Type III kerogen tend to be enriched in ${ }^{13} \mathrm{C}$ relative to Type II-sourced gases at an equivalent level of thermal maturity. Therefore, early Type II thermogenic gases may be difficult to distinguish from mature Type III gases, and Jenden and others (1988) suggested that the amount of natural gas derived from local source rocks in the central Kansas uplift region may be underestimated.

\section{Conclusions}

Approximately 100 produced gases were collected from the Anadarko Basin Province and analyzed for their molecular and stable isotopic composition. The study of these gases is intended to provide an interpretation of the significance of these new geochemical data in the context of the previous studies that have been conducted in the province. Given the extensive area encompassed by the province, natural gas production was considered in four separate regions within the province: the central Anadarko Basin, the Panhandle-Hugoton field, the Sooner trend, and the central Kansas uplift. These regions were largely defined on the basis of the age of the reservoir rocks, type of trap, and the composition and origin of the gases following the distinctions made in previous studies (Jenden and others, 1988; Rice and others, 1988b).

As noted in previous studies of the geochemistry of gases from the Anadarko Basin Province (Jenden and others, 1988; Rice and others, 1988b, 1989; Ballentine and Sherwood Lollar, 2002), the average $\delta^{13} \mathrm{C}$ composition of methane, ethane, and propane for the four regions within the study area indicate that (1) the majority of the natural gases are from organic sources with no significant contribution of gas from abiogenic sources (fig. 3), and that (2) most of the gases in the province were generated by thermogenic processes; although, limited contributions from microbial sources may be possible in localized areas. The molecular and stable carbon and hydrogen isotopic compositions of the hydrocarbon gases indicate mature (oil window) to post-mature (dry gas window) sources for gas generation (figs. 4 and 5), consistent with the findings of previous studies (Jenden and others, 1988; Rice and others, 1988b). 
In the central Anadarko Basin region, gas composition becomes chemically drier (lower $\mathrm{C}_{2+}$ content) and isotopically heavier (increased ${ }^{13} \mathrm{C}$ content) with increasing age of the producing formation within the Upper Mississippian and Pennsylvanian reservoirs. This is apparently the result of two factors. First, the older and more deeply buried reservoirs (Springer and Morrowan) are sourced from older, deeper, and more thermally mature source rocks (compared to the younger Virgilian, Atokan, and Desmoinesian reservoirs). Second, potential source rocks in the older intervals (Springer and Morrowan) contain predominately Type III kerogen, whereas the intermediate age lithologies (Atokan and Desmoinesian) contain mixed Type II and III, and in the youngest rocks (Virgilian) the kerogen is mainly Type II (Rice and others, 1988b). The geochemistry of gases in lower Paleozoic reservoirs in the central Anadarko Basin does not follow the trend observed in the Upper Mississippian and Pennsylvanian reservoirs; in fact, the gases in these deeper reservoirs are, on average, the wettest and isotopically lightest gases in the central basin (table 2). This is most likely the result of some contribution of gas from oil cracking because of the significant burial depth of these reservoirs (averaging more than 16,000 feet).

The geochemical composition of the gases produced from the giant Panhandle Hugoton field region is notably homogeneous and indicative of a thermally mature source. However, the general lack of thermally mature source rocks in this region (Cardott and Lambert, 1985) has led to the interpretation that these gases are not locally sourced (Jenden and others, 1988; Rice and others, 1988b). Based on similarities in the geochemistry of Panhandle Hugoton gases and those from Desmoinesian and Atokan reservoirs in the central Anadarko Basin, Rice and others (1988b) suggested that Pennsylvanian and (or) older source rocks in the central basin are the source of the gases in the Panhandle and Hugoton fields. However, long-distance-migrated gases likely represent an earlier stage of gas generation compared to the more locally reservoired gases in the central Anadarko Basin. This indicates a probable Early Mississippian or older source (possibly Woodford Shale) for the Panhandle Hugoton field gases.

The Panhandle Hugoton gases are also remarkable for their elevated nitrogen content (greater than five times the median concentration for United States gases) (Jenden and Kaplan, 1989b). Studies of the molecular and stable isotopic composition of the hydrocarbon and nonhydrocarbon gases (including noble gases) of the Panhandle Hugoton fields have shown that the nitrogen is derived from a mixture of crustal sources and thermally mature sedimentary organic matter (Oxburgh and others, 1986; Jenden and others, 1988; Rice and others, 1988b; Ballentine and Sherwood Lollar, 2002).

Gas production in the Sooner trend is associated with Woodford Shale-sourced oil, and the geochemistry of the gases indicates that they are derived from a thermally mature Type II kerogen source (most likely the Woodford Shale). Locally the Woodford Shale is only marginally mature; therefore, the gases must have migrated from deeper portions of the central basin (Rice and others, 1988b).
The central Kansas uplift gases appear to be primarily derived from a thermally mature Type II kerogen source. Local source rocks in the region are thought to be marginally mature, and consequently the source of the gas is most likely hundreds of miles away in the deeper central Anadarko Basin (Jenden and others, 1988). Comparison of the molecular and stable isotopic composition of the central Kansas uplift gases with those of the central Anadarko Basin gases indicates that the source may be Early Mississippian or older (possibly the Woodford Shale). However, it should be noted that there is some evidence to suggest that gases in the region may contain a contribution of locally sourced early thermogenic gas.

\section{Acknowledgments}

Access to producing gas wells for sample collection was kindly provided by Chesapeake Energy, Cimarex Energy, and Marathon Oil Corporation. The logistics for sample collection was provided by the staff of the U.S. Geological Survey Oklahoma Water Science Center, and this study would not have been possible without the contributions of Marvin Abbott and Stan Paxton. Molecular and stable isotopic analyses of the collected gases were performed in the U.S. Geological Survey, Central Energy Resources Science Center, Organic Geochemistry Laboratory by Augusta Warden and Mark Dreier. Constructive reviews of this manuscript were provided by Michael Lewan, Tongwei Zhang, and W.R. Keefer.

\section{References Cited}

Adler, F.J., 1971, Anadarko Basin and Central Oklahoma Area: Memoir-American Association of Petroleum Geologists, v. 15, p. 1061-1070.

Ballentine, C.J., and Sherwood Lollar, B., 2002, Regional groundwater focusing of nitrogen and noble gases into the Hugoton-Panhandle giant gas field, USA: Geochimica et Cosmochimica Acta, v. 66, no. 14, p. 2483-2497.

Bartram, J.G., Imbt, W.C., and Shea, E.F., 1950, Oil and gas in Arbuckle and Ellenburger formations, Mid-Continent region: American Association of Petroleum Geologists Bulletin, v. 34, no. 4, p. 682-700.

Baylis, S.A., Hall, K., and Jumeau, E.J., 1994, The analysis of the $\mathrm{C} 1-\mathrm{C} 5$ components of natural-gas samples using gaschromatography combustion isotope ratio mass-spectrometry: Organic Geochemistry, v. 21, no. 6-7, p. 777-785.

Bebout, D.G., White, W.A., Hentz, T.F., and Grasmick, M.K., eds., 1993, Atlas of major mid-continent gas reservoirs, Bureau of Economic Geology. 
Burgøyne, T.W., and Hayes, J.M., 1998, Quantitative production of $\mathrm{H}_{2}$ by pyrolysis of gas chromatographic effluents: Analytical Chemistry, v. 70, no. 24, p. 5136-5141.

Burke, K., 1977, Aulacogens and continental breakup: Annual Review of Earth and Planetary Sciences, v. 5, no. 00846597, p. 371-396.

Burruss, R.C., and Hatch, J.R., 1989, Geochemistry of oils and hydrocarbon source rocks, greater Anadarko Basin: Evidence for multiple sources of oils and long-distance oil migration, in Johnson, K.S., ed., Anadarko Basin symposium, 1988: Oklahoma Geological Survey Circular 90, p. 53-64.

Cardott, B.J., and Lambert, M.W., 1985, Thermal maturation by vitrinite reflectance of Woodford Shale, Anadarko Basin, Oklahoma: American Association of Petroleum Geologists Bulletin, v. 69, no. 11, p. 1982-1998.

Cardwell, L., 1985, Petroleum source rock potential of Arbuckle and Ellenburger groups, Oklahoma and North Texas: Bulletin of the American Association of Petroleum Geologists, v. 69, no. 1, p. 142.

Chung, H.M., Gormly, J.R., and Squires, R.M., 1988, Origin of gaseous hydrocarbons in subsurface environments: Theoretical considerations of carbon isotope distribution: Chemical Geology, v. 71, no. 1-3, p. 97-103.

Comer, J.B., and Hinch, H.H., 1987, Recognizing and quantifying expulsion of oil from the Woodford Formation and age-equivalent rocks in Oklahoma and Arkansas: Bulletin of the American Association of Petroleum Geologists, v. 71, no. 7, p. $844-858$.

Craig, L.C., and Varnes, K.L., 1979, History of the Mississippian System-An interpretive summary, in Craig, L.C., Connor, C.W., and others, eds., Paleotectonic investigations of the Mississippian System in the United States-Part II: Interpretive summary and special features of the Mississippian System: Washington, D.C., U.S. Geological Survey Professional Paper 1010-R, p. 371-406.

Energy Information Administration, 2010, Summary: U.S. Crude Oil, Natural Gas, and Natural Gas Liquids Proved Reserves, 2009: U.S. Department of Energy, 28 p.

Frezon, S.E., and Jordan, L., 1979, Introduction and regional analysis of the Mississippian System, in Craig, L.C., Connor, C.W., and others, a., eds., Part I: Paleotectonic investigations of the Mississippian System in the United States: Washington, D.C., U.S. Geological Survey Professional Paper 1010-I, p. 147-159.

Fusetti, L., Behar, F., Lorant, F., Grice, K., and Derenne, S., 2010, New insights into secondary gas generation from the thermal cracking of oil: Methylated monoaromatics. A kinetic approach using 1,2,4-trimethylbenzene. Part III: An isotopic fractionation model: Organic Geochemistry, v. 41, no. 5, p. 431-436.
Gold, T., and Held, M., 1987, Helium- nitrogen- methane systematics in natural gases of Texas and Kansas: Journal of Petroleum Geology, v. 10, no. 4, p. 415-424.

Ham, W.E., Denison, R.E., and Merrit, C.A., 1964, Basement rocks and structural evolution of southern Oklahoma: Oklahoma Geological Survey Bulletin 95, 302 p.

Ham, W.E., and Wilson, J.L., 1967, Paleozoic epeirogeny and orogeny in the central United States: American Journal of Science, v. 265, no. 5, p. 332-407.

Hatch, J.R., Rice, D.D., Burruss, R.C., Schmoker, J.W., and Clayton, J.L., 1986, Thermal maturity modeling and geochemical characterization of hydrocarbon source rocks, oils, and natural gases of the Anadarko Basin, in Carter, L.M.H., ed., U.S. Geological Survey Circular, Report: C 0974: Reston, U.S. Geological Survey, p. 21-23.

Higley, D.K., 2014, Thermal maturation of petroleum source rocks in the Anadarko Basin Province, Colorado, Kansas, Oklahoma, and Texas, Chap. 3, in Higley, D.K., compiler, Petroleum systems and assessment of undiscovered oil and gas in the Anadarko Basin Province, Colorado, Kansas, Oklahoma, and Texas-USGS Province 58: U.S. Geological Survey, Digital Data Series DDS-69-EE, 53 p.

Higley, D.K., Gaswirth, S.B., Abbott, M.M., Charpentier, R.R., Cook, T.A., Ellis, G.S., Gianoutsos, N.J., Hatch, J.R., Klett, T.R., Nelson, P.H., Pawlewicz, M.J., Pearson, O.N., Pollastro, R.M., and Schenk, C.J., 2011, Assessment of undiscovered oil and gas resources of the Anadarko Basin Province of Oklahoma, Kansas, Texas, and Colorado, 2010: U. S. Geological Survey Fact Sheet, p. 2.

Hoffman, P.F., Bally, A.W., and Palmer, A.R., 1989, Precambrian geology and tectonic history of North America: Boulder, CO, USA, Geological Society of America, 447-512 p.

IHS Energy, 2011, Energy Production Data, in IHS Energy Production Data on CD-ROM: Englewood, CO 80112.

Jenden, P.D., and Kaplan, I.R., 1989a, Analysis of Gases in the Earth's crust: Gas Research Institute Final Report GRI$88 / 0262,580 \mathrm{p}$.

Jenden, P.D., and Kaplan, I.R., 1989b, Origin of natural gas in Sacramento basin, California: American Association of Petroleum Geologists Bulletin, v. 73, no. 4, p. 431-453.

Jenden, P.D., Newell, K.D., Kaplan, I.R., and Watney, W.L., 1988, Composition and stable-isotope geochemistry of natural gases from Kansas, Midcontinent, USA: Chemical Geology, v. 71, no. 1-3, p. 117-147.

Johnson, K.S., 1989, Geologic evolution of the Anadarko Basin, in Johnson, K.S., ed., Circular - Oklahoma Geological Survey, vol. 90: Norman, University of Oklahoma, p. 3-12. 
Johnson, K.S., Amsden, T.W., Denison, R.E., Dutton, S.P., Goldstein, A.G., Rascoe, B., Jr., Sutherland, P.K., and Thompson, D.M., 1988, Southern mid-continent region, in Sloss, L.L., ed., Sedimentary cover-North American Craton; U.S.: Denver, CO, Geological Society of America, p. 307-359.

Johnson, K.S., and Cardott, B.J., 1992, Geologic framework and hydrocarbon source rocks of Oklahoma: CircularOklahoma Geological Survey, p. 21-37.

Jones, P.J., and Philp, R.P., 1990, Oils and source rocks from Pauls Valley, Anadarko Basin, Oklahoma, U.S.A: Applied Geochemistry, v. 5, no. 4, p. 429-448.

Jordan, L., and Vosburg, D.L., 1963, Permian salt and associated evaporites in the Anadarko basin of the western Oklahoma-Texas Panhandle region: Oklahoma Geological Survey Bulletin 102, 76 p.

Lewan, M.D., 1983, Effects of thermal maturation on stable organic carbon isotopes as determined by hydrous pyrolysis of Woodford Shale: Geochimica et Cosmochimica Acta, v. 47 , no. 8 , p. $1471-1479$.

McKee, E.D., Oriel, S.S., and others, 1967, Paleotectonic investigations of the Permian system in the United States: U.S. Geological Survey Professional Paper 515, 271 p.

Moore, B.J., 1982, Analysis of natural gases, 1917-1980: U.S. Bureau of Mines Information Circular 8870, 1,055 p.

Oxburgh, E.R., O’Nions, R.K., and Hill, R.I., 1986, Helium isotopes in sedimentary basins: Nature, v. 324, no. 6098, p. 632-635.

Petroleum Information Corporation, 1982, The Deep Anadarko Basin: Denver, CO Baker and Taylor (BTCP), 359 p.

Price, L.C., 1995, Origins, characteristics, controls, and economic viabilities of deep-basin gas resources: Chemical Geology, v. 126, no. 3-4, p. 335-349.

Rice, D.D., and Claypool, G.E., 1981, Generation, accumulation, and resource potential of biogenic gas: American Association of Petroleum Geologists Bulletin, v. 65, no. 1, p. 5-25.

Rice, D.D., Threlkeld, C.N., and Vuletich, A.K., 1988a, Analyses of natural gases from Anadarko Basin, southwestern Kansas, western Oklahoma, and Texas Panhandle: U.S. Geological Survey OF 88-0391, 5 p. [Open-File Report U.S. Geological Survey].

Rice, D.D., Threlkeld, C.N., and Vuletich, A.K., 1988b, Character, origin, and occurrence of natural gases in the Anadarko Basin, Southwestern Kansas, Western Oklahoma and Texas Panhandle, USA: Chemical Geology, v. 71, no. $1-3$, p. $149-157$.
Rice, D.D., Threlkeld, C.N., and Vuletich, A.K., 1989, Characterization and origin of natural gases of the Anadarko Basin, in Johnson, K.S., ed., Circular - Oklahoma Geological Survey, vol. 90: Norman, University of Oklahoma, p. 47-52.

Schoell, M., 1980, The hydrogen and carbon isotopic composition of methane from natural gases of various origins: Geochimica et Cosmochimica Acta, v. 44, no. 5, p. 649-662.

Schoell, M., 1983a, Genetic characterization of natural gases: Bulletin of the American Association of Petroleum Geologists, v. 67, no. 12, p. 2225-2238.

Schoell, M., 1983b, Isotope techniques for tracing migration of gases in sedimentary basins, in Brooks, J., ed., Journal of the Geological Society of London: London, Geological Society of London, p. 415-422.

Shatski, N.S., 1946, The Great Donets basin and the Wichita System; comparative tectonics of ancient platforms: Akademiia Nauk SSSR Doklady, Geology Series, v. 6, p. 57-90.

Sherwood Lollar, B., Westgate, T.D., Ward, J.A., Slater, G.F., and Lacrampe-Couloume, G., 2002, Abiogenic formation of alkanes in the Earth's crust as a minor source for global hydrocarbon reservoirs: Nature, v. 416, no. 6880, p. $522-524$.

Sorenson, R.P., 2005, A dynamic model for the Permian Panhandle and Hugoton fields, western Anadarko basin: Bulletin of the American Association of Petroleum Geologists, v. 89 , no. 7, p. 921-938.

Tang, Y., and Schoell, M., 2005, A new genetic scheme for natural gas formation and isotopic evidence for oil cracking: Geochimica et Cosmochimica Acta, v. 69, no. 10, p. A501.

Wang, H.D., and Philp, R.P., 1997, Geochemical study of potential source rocks and crude oils in the Anadarko Basin, Oklahoma: Bulletin of the American Association of Petroleum Geologists, v. 81, no. 2, p. 249-275.

Webb, G.W., 1976, Oklahoma City oil; second crop from preserved subunconformity source rocks: Bulletin of the American Association of Petroleum Geologists, v. 60, no. 1, p. 115-122.

Whiticar, M.J., 1994, Correlation of natural gases with their sources: Memoir - American Association of Petroleum Geologists, v. 60, p. 261-283. 

Chapter 5

Geologic Assessment of Undiscovered

Oil and Gas Resources in the Cambrian-

Devonian Stratigraphy of the Anadarko

Basin, Oklahoma, Kansas, Texas, and

Click here to return to

Volume Title Page Colorado

By Stephanie B. Gaswirth and Debra K. Higley

Chapter 5 of 13

Petroleum Systems and Assessment of Undiscovered Oil and Gas in the Anadarko Basin Province, Colorado, Kansas, Oklahoma, and Texas-USGS Province 58

Compiled by Debra K. Higley

U.S. Geological Survey Digital Data Series DDS-69-EE

U.S. Department of the Interior

U.S. Geological Survey 


\title{
U.S. Department of the Interior SALLY JEWELL, Secretary
}

\section{U.S. Geological Survey \\ Suzette M. Kimball, Acting Director}

\author{
U.S. Geological Survey, Reston, Virginia: 2014
}

For more information on the USGS - the Federal source for science about the Earth, its natural and living resources, natural hazards, and the environment, visit http://WWW.usgs.gov or call 1-888-ASK-USGS.

For an overview of USGS information products, including maps, imagery, and publications, visit http://WwW.usgs.gov/pubprod

To order this and other USGS information products, visit http://store.usgs.gov

Any use of trade, firm, or product names is for descriptive purposes only and does not imply endorsement by the U.S. Government.

Although this information product, for the most part, is in the public domain, it also may contain copyrighted materials as noted in the text. Permission to reproduce copyrighted items must be secured from the copyright owner.

Suggested citation:

Gaswirth, S.B. and Higley, D.K., 2014, Geologic assessment of undiscovered oil and gas resources in the CambrianDevonian stratigraphy of the Anadarko Basin, Oklahoma, Kansas, Texas, and Colorado, chap. 5, in Higley, D.K., compiler, Petroleum systems and assessment of undiscovered oil and gas in the Anadarko Basin Province, Colorado, Kansas, Oklahoma, and Texas-USGS Province 58: U.S. Geological Survey Digital Data Series DDS-69-EE, 42 p., http://dx.doi.org/10.3133/ds69EE. 


\section{Contents}

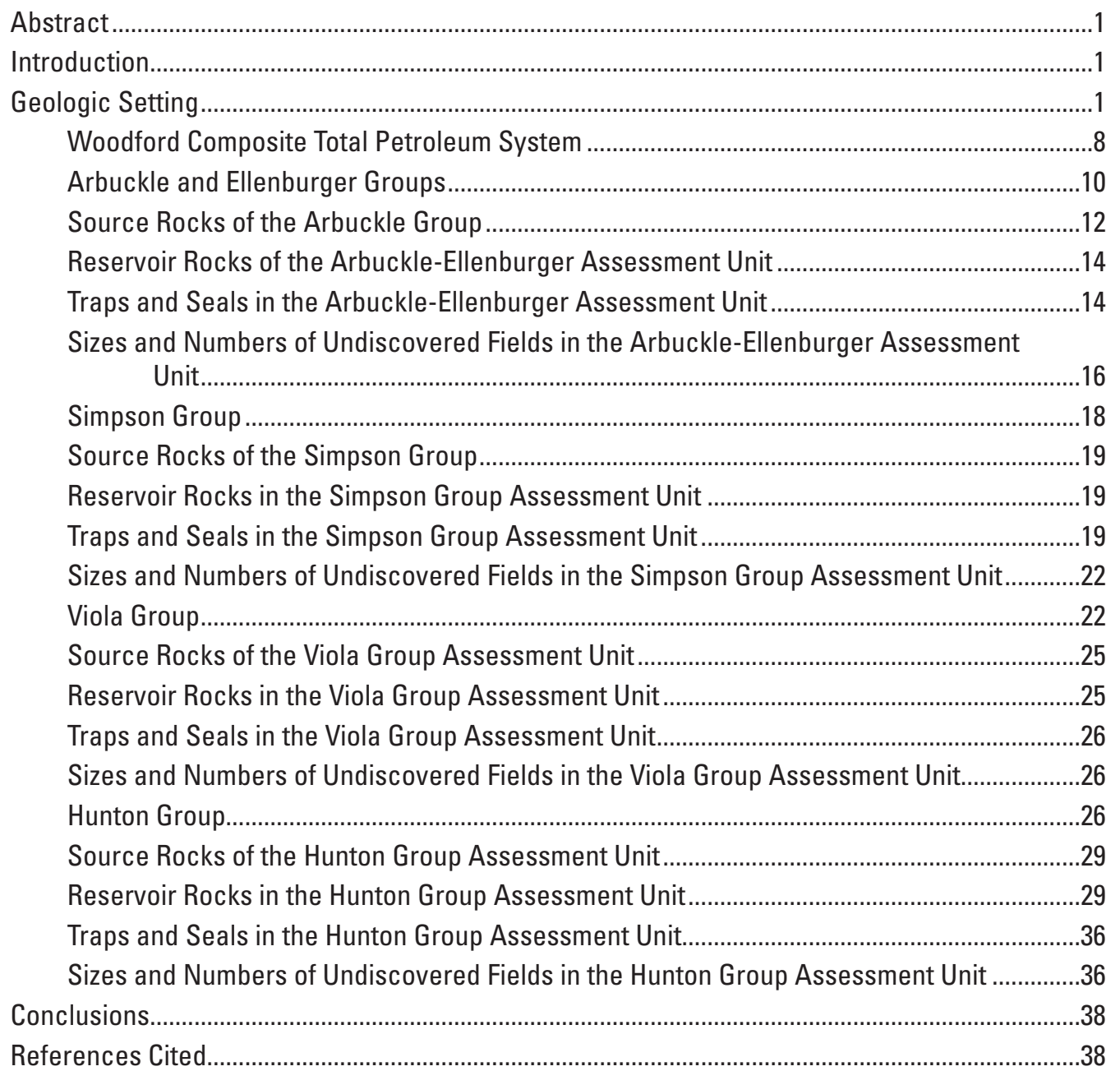

\section{Figures}

1. Anadarko Basin Province (red line) and boundary of the Woodford Composite Total Petroleum Systems (TPS; brown line) and assessment units (AU) discussed in this chapter.....

2. Generalized surface and subsurface stratigraphic columns for the Anadarko Basin and the Southern Oklahoma Fold Belt Provinces for the Precambrian to Mississippian. Assessment units (AU) are included in the Woodford Composite Total Petroleum System. Italics (blue text) indicate informal names. Formal formation- and member-rank units are not necessarily differentiated (as used by Bebout and others, 1993). Modified from Bebout and others (1993) and Henry and Hester (1996). Ages in millions of years (Ma) from Haq and Van Eysinga (1998), and Gradstein and others, (2004) (red text). Fm., Formation; Mbr., Member.

3. Map of the midcontinent of the United States, showing the approximate outline of the Oklahoma basin, southern Oklahoma aulacogen and other major features that existed in early and middle Paleozoic time. Modified from Johnson (1991)... 
4. Schematic cross sections showing restored thickness of Upper Cambrian and Ordovician strata in Oklahoma (modified from Johnson, 1991). S. OK. A., Southern Oklahoma Aulacogen. Scale is the same for both cross sections.

5. View to the southeast of elevation on the top of the Arbuckle Group. Major structures are labeled. Precambrian faults (red) are from Adler and others (1971). Wichita Mountain uplift faults are vertical for four-dimensional model purposes only, and surface in this area is unknown because of insufficient data. Data sources for this surface include Arbuckle picks from well logs, Rottmann (2000a, 2000b), and edited IHS Energy Group (2009) formation tops

6. Map showing production from the Arbuckle and Ellenburger Groups and Arbuckle Group thickness; contour interval is $\mathbf{5 0 0}$ feet.

7. Generalized stratigraphic column for the Anadarko Basin province with oil and gas source rocks (red text). Increases in source rock potential are indicated by larger numbers. Modified from Hatch and others (1986). Hatched vertical lines represent an unconformity (Bebout and others, 1993). Gp., Group; Fm., Formation; HC, Hydrocarbon.

8. Map showing vitrinite reflectance $\left(\% \mathrm{R}_{0}\right)$ data for the Woodford Shale. Contour interval is $0.4 \% \mathrm{R}_{0}$. Blue triangles and white dots indicate locations of $\% \mathrm{R}_{0}$ data from Cardott (1989), Price (1997), and Chesapeake Energy Corporation (2009, written commun.). White dot well locations are, from left to right, Bertha Rogers 1 and West Edmond SWD 1-24. Brown lines are faults in the underlying Hunton Group (Rottmann, 2000a)

9. Burial history curves for the Bertha Rogers 1 and West Edmond SWD 1-24 wells. Modeled vitrinite reflectance through time includes heat flow of 70 milliwatts per square meter $\left(\mathrm{mW} / \mathrm{m}^{2}\right)$ to 260 million years ago (Ma), followed by $40 \mathrm{~mW} / \mathrm{m}^{2}$ for Bertha Rogers 1 and $50 \mathrm{~mW} / \mathrm{m}^{2}$ for West Edmond SWD 1-24. White and pink lines follow the upper part of the Arbuckle Group and Woodford Shale, respectively. Ord., Ordovician; Sil., Silurian; Neo., Neogene; Gp., Group; \% R $\mathrm{o}^{\prime}$ percent vitrinite reflectance.

10. Depositional model of the Arbuckle Group in southern Oklahoma, showing a shallow-marine setting passing landward into a near-shore to shoreline setting, and finally into a tidal-flat setting. Sea-level transgressions and regressions resulted in the deposition of thin carbonate sequences (modified from Lindsay and Koskelin, 1991).

11. Arbuckle-Ellenburger Asessment Unit events chart showing the timing of source rock deposition and trap formation, and the age of overburden, reservoir, and seal rocks with different color bars (black, source rock; gray, overburden rock; blue, reservoir rock; pink, seal rock; purple, trap formation; dark green, generation; light green, migration accumulation). The chart also depicts the timing of oil generation, migration and accumulation as modeled for various wells in different parts of the Anadarko Basin. Ma, mega-annum; Plio, Pliocene; Mio, Miocene; Olig, Oligocene; Eoc, Eocene; Pal, Paleocene; L, Late; M, Middle; E, Early.

12. Lower Paleozoic stratigraphy in the southern Oklahoma aulacogen, with a burial-time curve illustrating the position of the Signal Mountain Formation and other hydrocarbon source rocks present in the Anadarko Basin. Modified from Donovan and Critchfield (2001). Ma, millions years before the present.

13. Image showing vitrinite reflectance $\left(\% R_{0}\right)$ contours on the Arbuckle Group, extracted from the four-dimensional model of the Anadarko Basin. The burial history model illustrates decompaction through subtle increases in layer thickness backward through time. The vitrinite reflectance profile was calculated using Sweeney and Burnham (1990) kinetics. 
14. Map showing oil and gas production and dry hole penetrations for the Arbuckle and Ellenburger Groups in the Anadarko Basin Province (IHS Energy Group, 2010). Structure contours are drawn on the top of the Arbuckle Group; contour interval is 2,000 feet. The Anadarko Basin Province boundary is the black line, the Woodford Composite Total Petroleum System (TPS) boundary the red line, and the Arbuckle-Ellenburger Assessment Unit (AU) boundary the blue line..

15. Map showing accumulations for the Arbuckle-Ellenburger Assessment Unit (AU) (Nehring Associates, Inc., 2007). Accumulations without numbers are in purple. TPS, Total Petroleum System.

16. Stratigraphic section of Simpson Group rocks (modified from Flores and Keighin, 1989)

17. Image showing major lithologies of Ordovician Simpson Group strata in the southern midcontinent (modified from Northcutt and Johnson, 1997).

18. Map showing production from and thickness of the Simpson Group; contour interval is 500 feet.

19. Image showing approximate distribution of the Joins sea during Ordovician time (modified from Denison, 1997)

20. Simpson Goup Assessment Unit events chart showing the timing of source rock deposition and trap formation, and the age of overburden, reservoir, and seal rocks with different color bars (black, source rock; gray, overburden rock; blue, reservoir rock; pink, seal rock; purple, trap formation; dark green, generation; light green, migration accumulation). The chart also depicts the timing of oil generation, migration and accumulation as modeled for various wells in different parts of the Anadarko Basin. Plio, Pliocene; Mio, Miocene; Olig, Oligocene; Eoc, Eocene; Pal, Paleocene; L, Late; M, Middle; E, Early...

21. Map showing oil and gas production and dry hole penetrations from the Simpson Group in the Anadarko Basin Province (IHS Energy Group, 2010). The structure contours are drawn on the top of the Simpson Group; contour interval is 2,000 feet. The Anadarko Basin Province boundary is the black line, the Woodford Composite Total Petroleum System (TPS) boundary the red line, and the Simpson Group Assessment Unit (AU) Boundary the blue line.

22. Map showing accumulations for the Simpson Group Assessment Unit (AU) (Nehring Associates, Inc., 2007). Nehring accumulations with grown reserve accumulation numbers are shown in light blue. Accumulations without numbers are in purple. TPS, Total Petroleum System

23. Image showing major lithologies of the Ordovician Viola Group strata in the southern midcontinent (modified from Northcutt and Johnson, 1997).

24. Map showing production from and thickness of the Viola Group; contour interval is variable...

25. Map showing production from and thickness of the Sylvan Shale; contour interval is 100 feet.

26. Viola Group Assessment Unit events chart showing the timing of source rock deposition and trap formation, and the ages of overburden, reservoir, and seal rocks with different color bars (black, source rock; gray, overburden rock; blue, reservoir rock; pink, seal rock; purple, trap formation; dark green, generation; light green, migration accumulation). The chart also depicts the timing of oil generation, migration and accumulation as modeled for various wells in different parts of the Anadarko Basin. Ma, mega-annum; Plio, Pliocene; Mio, Miocene; Olig, Oligocene; Eoc, Eocene; Pal, Paleocene; L, Late; M, Middle; E, Early.. 
27. Map showing oil and gas production and dry hole penetrations for the Viola Group in the Anadarko Basin Province (IHS Energy Group, 2010). Structure contours are drawn on the top of the Viola Group; contour interval is 2,000 feet. The Anadarko Basin Province boundary is the black line, the Woodford Composite Total Petroleum System (TPS) boundary the red line, and the Viola Group Assessment Unit (AU) boundary the blue line.

28. Map showing accumulations for the Viola Group Assessment Unit (AU) (Nehring Associates, Inc., 2007). Nehring accumulations with grown reserve accumulation numbers are shown in light blue. Accumulations without numbers are in purple. TPS, Total Petroleum System.

29. Image showing depositional setting during Hunton time in the midcontinent (modified from Fritz and Medlock, 1994)

30. Depositional model for Chimneyhill subgroup through Bois d'Arc Formation strata of the Hunton Group (modified from Fritz and Medlock, 1994)...

31. Type log of the Hunton Group in central Oklahoma; gray are shales, pink are calcareous shales, blue are limestones, and purple represents dolomite (modifed from Fritz and Medlock, 1994). GR, gamma-ray; R, resisitivity; Miss., Mississippian.......31

32. Pre-Woodford Shale subcrop map for Oklahoma and the northeast corner of the Texas panhandle (modified from Amsden, 1989). Fm, Formation...

33. Image showing approximate distribution of the informal Misener sandstone of Woodford Shale and its relation to the Simpson Group subcrop (modifed from Kuykendall and Fritz, 1993)

34. Map showing production from the Hunton Group and informal Misener sandstone of the Woodford Shale, and Hunton Group thickness; contour interval is 250 feet. Black oval is the West Edmond field. TPS, Total Petroleum System; AU, Assessment Unit

35. Hunton Group Assessment Unit events chart showing the timing of source rock deposition and trap formation, and the age of overburden, reservoir, and seal rocks with different color bars (black, source rock; gray, overburden rock; blue, reservoir rock; pink, seal rock; purple, trap formation; dark green, generation; light green, migration accumulation). The chart also depicts the timing of oil generation, migration and accumulation as modeled for various wells in different parts of the Anadarko Basin. Ma, mega-annum; Plio, Pliocene; Mio, Miocene; Olig, Oligocene; Eoc, Eocene; Pal, Paleocene; L, Late; M, Middle; E, Early.

36. Map showing oil and gas production and dry hole penetrations for the Hunton Group and informal Misener sandstone of the Woodford Shale in the Anadarko Basin Province (IHS Energy Group, 2010). Structure contours are drawn on the top of the Hunton Group; contour interval is 2,000 feet. The Anadarko Basin Province boundary is the black line, the Woodford Composite Total Petroleum System (TPS) boundary the red line, and the Hunton Group Assessment Unit (AU) Boundary the blue line

37. Map showing production from informal Misener sandstone of the Woodford Shale in the Anadarko Basin Province (IHS Energy Group, 2010). The structure contours are on the top of the Misener sandstone; contour interval is 500 feet. TPS, Total Petroleum System; Au, Assessment Unit

38. Map showing accumulations for the Hunton Group Assessment Unit (AU) (Nehring Associates, Inc., 2007). Nehring accumulations with grown reserve accumulation numbers are shown in light blue. Accumulations without numbers are in purple. TPS, Total Petroleum System. 


\title{
Geologic Assessment of Undiscovered Oil and Gas Resources in the Cambrian-Devonian Stratigraphy of the Anadarko Basin, Oklahoma, Kansas, Texas, and Colorado
}

\author{
By Stephanie B. Gaswirth and Debra K. Higley
}

\begin{abstract}
The Woodford Composite Total Petroleum System contains four Cambrian through Devonian conventional assessment units (AU) in the Anadarko Basin: (1) the ArbuckleEllenburger Assessment Unit; (2) the Simpson Group Assessment Unit; (3) the Viola Group Assessment Unit; (4) the Hunton Group Assessment Unit. These assessment units have collectively produced more than 900 million barrels of oil (MMBO) and 7 trillion cubic feet of gas (TCFG) from the Anadarko Basin, which covers central and western Oklahoma, south-central Kansas, southeast Colorado, and the Texas Panhandle. Based on available geologic and production data, the undiscovered oil resources for these conventional assessment units are estimated at a mean of 25 million barrels of oil and 646 billion cubic feet of gas (BCFG).
\end{abstract}

\section{Introduction}

The U.S. Geological Survey (USGS) completed a geologic-based assessment of the undiscovered oil and gas resources of the Anadarko Basin Province in western Oklahoma and Kansas, northern Texas, and southeastern Colorado in 2010 (Higley and others, 2011; fig. 1). The assessment was based on geologic elements and processes within a total petroleum system (TPS) that include: (1) source-rock distribution, thickness, organic richness, and history of generation, maturation and migration; (2) reservoir-rock type (conventional or continuous), distribution and quality; and (3) character of traps and timing of formation with respect to petroleum generation, accumulation and migration. Each TPS contains multiple assessment units (AU), the basic geologic unit of the oil and gas assessment, and undiscovered oil and gas resources were quantitatively estimated within each AU.

Detailed stratigraphic and structural framework studies and petroleum system modeling, combined with historical exploration records and production analyses, were used to estimate the undiscovered, technically recoverable resources. Using this geologic framework, the USGS defined two TPSs (the Woodford Composite and Pennsylvanian Composite) in the Anadarko Basin that contain nine conventional AUs and two continuous (unconventional) AUs. The Woodford Composite TPS includes the AUs that are the focus of this chapter: the Arbuckle-Ellenburger AU, the Simpson Group AU, the Viola Group AU, and the Hunton Group AU (fig. 1). These AUs comprise the strata in the Anadarko Basin from the Cambrian Reagan Sandstone through the OrdovicianDevonian Hunton Group (fig. 2). The conventional Mississippian AU and the continuous Woodford Shale Oil AU and Woodford Shale Gas AU (included in table 1) are also part of the Woodford Composite TPS, but documented in another chapter of this report. The Pennsylvanian Composite TPS includes the conventional Morrowan-Atokan AU, the Desmoinesian AU, the Missourian-Permian AU, the Greater Granite Wash Composite AU, and the continuous Thirteen Finger Limestone-Atoka Shale Gas AU, which are also included in chapter 7 of the DDS.

\section{Geologic Setting}

The Anadarko Basin is an asymmetrical, south-dipping sedimentary basin that began during the Precambrian as the southern Oklahoma aulacogen (Rascoe and Adler, 1983; fig. 3). The northwest-trending southern Oklahoma aulacogen is the deepest sedimentary trough in North America and was formed by rift-related igneous intrusive and extrusive occurrences in the late Early Cambrian though the Middle Cambrian (Lindsay and Koskelin, 1991). Following emplacement of Early and Middle Cambrian basement rocks during an episode of igneous activity, a basal transgressive sandstone, the Reagan Sandstone (fig. 4), was deposited across a moderately mature erosion surface of low relief (Johnson, 1989). The sandstone grades upward into a succession of shallow-water marine limestones and dolomites of the Arbuckle Group, which were deposited almost continuously until the Middle Ordovician.

The overlying Simpson Group sandstones and limestones were derived from northeastern and eastern sources. They are overlain by limestones of the Viola Group, followed by the gray and green-gray shales of the Sylvan Shale, 


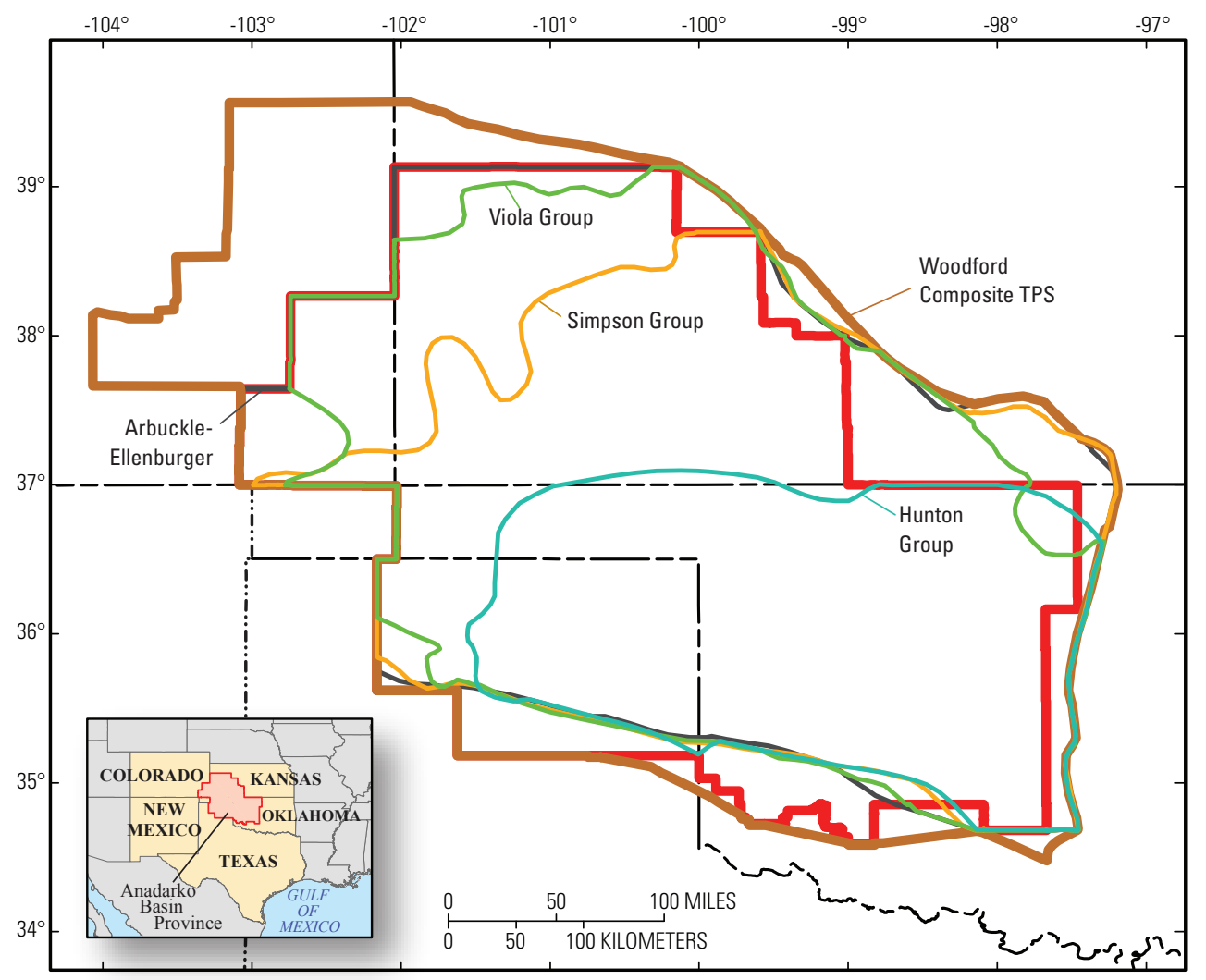

Figure 1. Anadarko Basin Province (red line) and boundary of the Woodford Composite Total Petroleum Systems (TPS; brown line) and assessment units (AU) discussed in this chapter.

and finally the Upper Ordovician to Devonian carbonates of the Hunton Group (fig. 4). The Devonian-Mississippian Woodford Shale unconformably overlies the Hunton Group. The Arbuckle through Viola strata are still present in most parts of the Anadarko Basin, though there is a pre-Woodford unconformity that is widespread in the Midcontinent (Johnson, 1989). The Sylvan Shale, Hunton Group and Woodford Shale are only present in Oklahoma, Texas, and southernmost Kansas.

The present-day basin configuration is largely controlled by movement of the late Paleozoic Wichita fault zone and Ouachita thrust plate (Amsden, 1989). The southern margin of the basin is defined by the Amarillo-Wichita Mountains. The eastern basin boundary is defined by the Nemaha uplift, the north by the Central Kansas uplift, and the west by the Sierra Grande uplift (figs. 5 and 6). The deepest part of the basin is parallel to the southern margin, where petroleum production extends below 20,000 feet (ft).

Basinward dip of strata results in a wide range of production depths for many of the Ordovician through Devonian units in the basin (Smith and Woods, 2000). As much as $15,000 \mathrm{ft}$ of Upper Cambrian through Mississippian shallow marine carbonates and clastics were deposited across the vast Oklahoma basin during an early epeirogenic phase of the Anadarko Basin (Johnson, 1989). Increase in formation thickness towards the basin depocenter was because of an increased rate of subsidence during the Paleozoic.

The majority of hydrocarbons produced in the Anadarko Basin are from Permian-Carboniferous reservoirs (Rascoe and Adler, 1983). However, the pre-Mississippian carbonates and clastics of the Arbuckle, Simpson, Viola and Hunton Groups contribute a substantial volume of gas and oil production in the basin. The Hunton Group (including production from the overlying informal Misener sandstone of the Woodford Shale) has produced approximately 290 million barrels of oil (MMBO) and 5 trillion cubic feet as gas (TCFG) (IHS Energy, 2010). The Arbuckle Group has produced $52 \mathrm{MMBO}$ and 285 BCFG, the Simpson Group $471 \mathrm{MMBO}$ and 1.2 TCFG, and the Viola Group $112 \mathrm{MMBO}$ and $505 \mathrm{BCFG}$ (IHS Energy Group, 2010). 


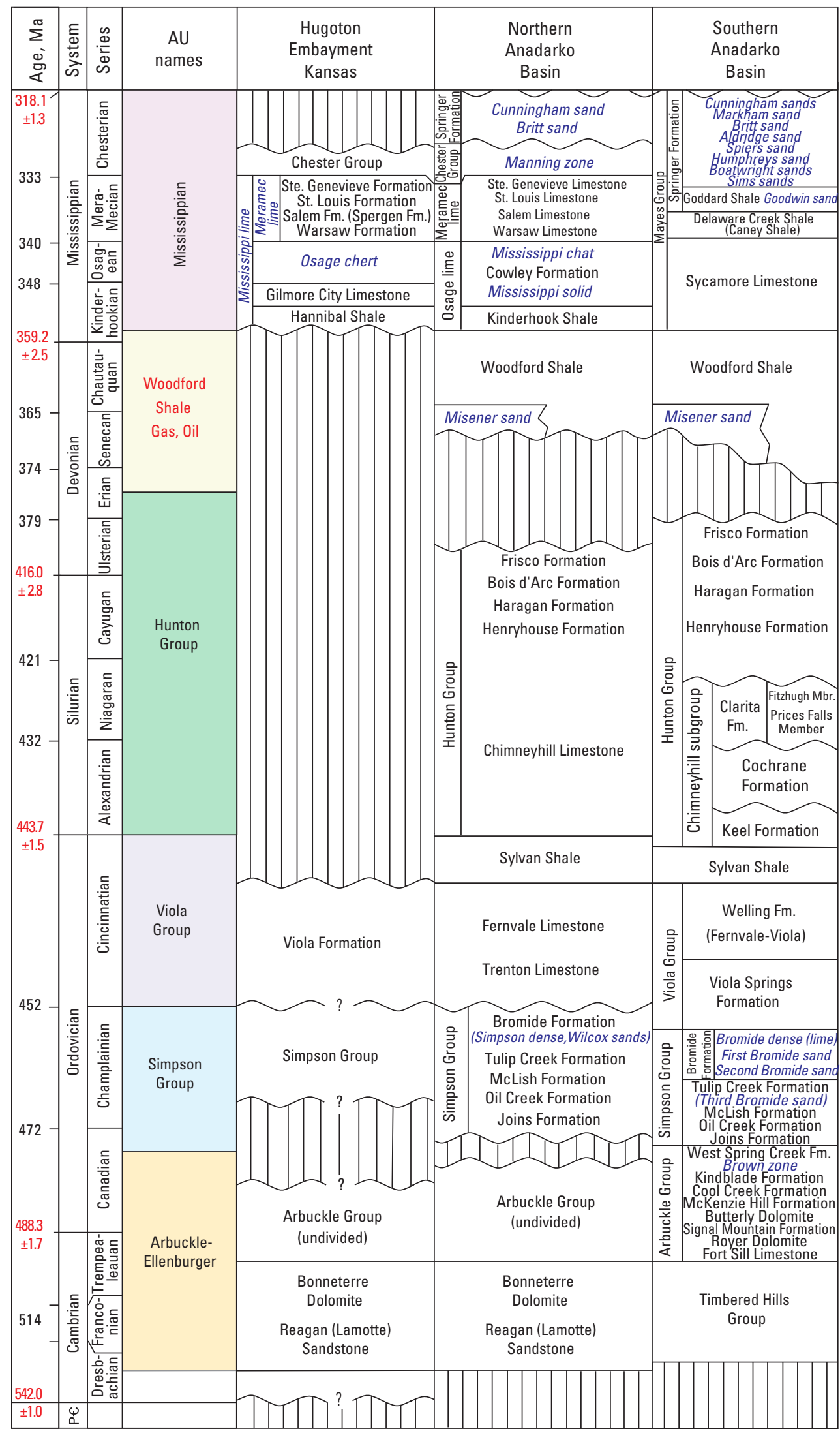

Figure 2. Generalized surface and subsurface stratigraphic columns for the Anadarko Basin and the Southern Oklahoma Fold Belt Provinces for the Precambrian to Mississippian. Assessment units (AU) are included in the Woodford Composite Total Petroleum System. Italics (blue text) indicate informal names. Formal formationand member-rank units are not necessarily differentiated (as used by Bebout and others, 1993). Modified from Bebout and others (1993) and Henry and Hester (1996). Ages in millions of years (Ma) from Haq and Van Eysinga (1998), and Gradstein and others, (2004) (red text). Fm., Formation; Mbr., Member. 
Table 1. Anadarko Basin assessment results for all of the Woodford Composite total petroleum system.

[MMBO, million barrels of oil. BCFG, billion cubic feet of gas. MMBNGL, million barrels of natural gas liquids. Results shown are fully risked estimates. For gas accumulations, all liquids are included as NGL (natural gas liquids). F95 represents a 95 percent chance of at least the amount tabulated; other fractiles are defined similarly. Fractiles are additive under the assumption of perfect positive correlation. TPS, Total Petroleum System; AU, Assessment Unit. Gray shading indicates not applicable]

\begin{tabular}{|c|c|c|c|c|c|c|c|c|c|c|c|c|c|}
\hline \multirow{3}{*}{$\begin{array}{l}\text { Total Petroleum Systems } \\
\text { (TPS) } \\
\text { and Assessment Units (AU) }\end{array}$} & \multirow{3}{*}{$\begin{array}{l}\text { Field } \\
\text { Type }\end{array}$} & \multicolumn{12}{|c|}{ Total Undiscovered Resources } \\
\hline & & \multicolumn{4}{|c|}{ Oil (MMBO) } & \multicolumn{4}{|c|}{ Gas (BCFG) } & \multicolumn{4}{|c|}{ NGL (MMBNGL) } \\
\hline & & F95 & $\mathrm{F} 50$ & F5 & Mean & F95 & F50 & F5 & Mean & F95 & $\mathrm{F} 50$ & $\mathrm{F5}$ & Mean \\
\hline \multicolumn{14}{|c|}{ Woodford Composite TPS } \\
\hline \multirow{2}{*}{ Arbuckle-Ellenburger AU } & Oil & 2 & 5 & 12 & 6 & 7 & 24 & 61 & 28 & 0 & 1 & 2 & 1 \\
\hline & Gas & & & & & 43 & 164 & 371 & 181 & 0 & 1 & 2 & 1 \\
\hline \multirow{2}{*}{ Simpson Group AU } & Oil & 2 & 4 & 9 & 5 & 6 & 17 & 39 & 19 & 0 & 0 & 1 & 1 \\
\hline & Gas & & & & & 33 & 114 & 252 & 125 & 2 & 9 & 21 & 10 \\
\hline \multirow{2}{*}{ Viola Group AU } & Oil & 2 & 5 & 10 & 5 & 3 & 9 & 20 & 10 & 0 & 1 & 2 & 1 \\
\hline & Gas & & & & & 10 & 27 & 58 & 30 & 0 & 0 & 0 & 0 \\
\hline \multirow{2}{*}{ Hunton Group AU } & Oil & 2 & 8 & 21 & 9 & 8 & 32 & 87 & 38 & 0 & 1 & 3 & 1 \\
\hline & Gas & & & & & 71 & 281 & 641 & 310 & 0 & 2 & 4 & 2 \\
\hline \multirow{2}{*}{ Mississippian AU } & Oil & 5 & 16 & 31 & 17 & 15 & 46 & 99 & 50 & 0 & 2 & 4 & 2 \\
\hline & Gas & & & & & 125 & 350 & 663 & 367 & 3 & 8 & 17 & 9 \\
\hline \multicolumn{14}{|c|}{ Pennsylvanian Composite TPS } \\
\hline \multirow{2}{*}{ Morrowan-Atokan AU } & Oil & 6 & 14 & 29 & 15 & 21 & 55 & 121 & 61 & 1 & 2 & 5 & 2 \\
\hline & Gas & & & & & 101 & 261 & 469 & 271 & 2 & 5 & 10 & 5 \\
\hline \multirow{2}{*}{ Desmoinesian AU } & Oil & 2 & 6 & 12 & 6 & 8 & 23 & 52 & 26 & 0 & 1 & 2 & 1 \\
\hline & Gas & & & & & 29 & 87 & 167 & 92 & 1 & 3 & 5 & 3 \\
\hline \multirow{2}{*}{ Missourian-Permian AU } & Oil & 10 & 22 & 38 & 23 & 49 & 114 & 223 & 122 & 2 & 4 & 8 & 4 \\
\hline & Gas & & & & & 61 & 130 & 231 & 136 & 2 & 4 & 7 & 4 \\
\hline \multirow{2}{*}{$\begin{array}{l}\text { Greater Granite Wash } \\
\text { Composite AU }\end{array}$} & Oil & 4 & 14 & 34 & 16 & 22 & 78 & 198 & 90 & 1 & 2 & 7 & 3 \\
\hline & Gas & & & & & 192 & 646 & 1,496 & 719 & 7 & 24 & 60 & 27 \\
\hline $\begin{array}{l}\text { Total Conventional } \\
\text { Resources }\end{array}$ & & 35 & 94 & 196 & 102 & 804 & 2,458 & 5,248 & 2,675 & 21 & 70 & 160 & 77 \\
\hline \multicolumn{14}{|c|}{ Woodford Composite TPS } \\
\hline \multirow{2}{*}{$\begin{array}{l}\text { Woodford Shale Oil AU } \\
\text { Woodford Shale Gas AU }\end{array}$} & Oil & 175 & 357 & 730 & 393 & 795 & 1,750 & 3,851 & 1,963 & 22 & 51 & 121 & 59 \\
\hline & Gas & & & & & 8,806 & 15,131 & 25,998 & 15,973 & 94 & 178 & 336 & 192 \\
\hline \multicolumn{14}{|c|}{ Pennsylvanian Composite TPS } \\
\hline \multirow{2}{*}{$\begin{array}{l}\text { Thirteen Finger Limestone- } \\
\text { Atoka Shale Gas AU }\end{array}$} & Oil & & & & & & & & & & & & \\
\hline & Gas & & & & & 3,040 & 6,229 & 12,763 & 6,850 & 33 & 73 & 161 & 82 \\
\hline $\begin{array}{l}\text { Total Continuous } \\
\text { Resources }\end{array}$ & & 175 & 357 & 730 & 393 & 12,641 & 23,110 & 42,612 & 24,786 & 149 & 302 & 618 & 333 \\
\hline Total Resources & & 210 & 451 & 926 & 495 & 13,445 & 25,568 & 47,860 & 27,461 & 170 & 372 & 778 & 410 \\
\hline
\end{tabular}




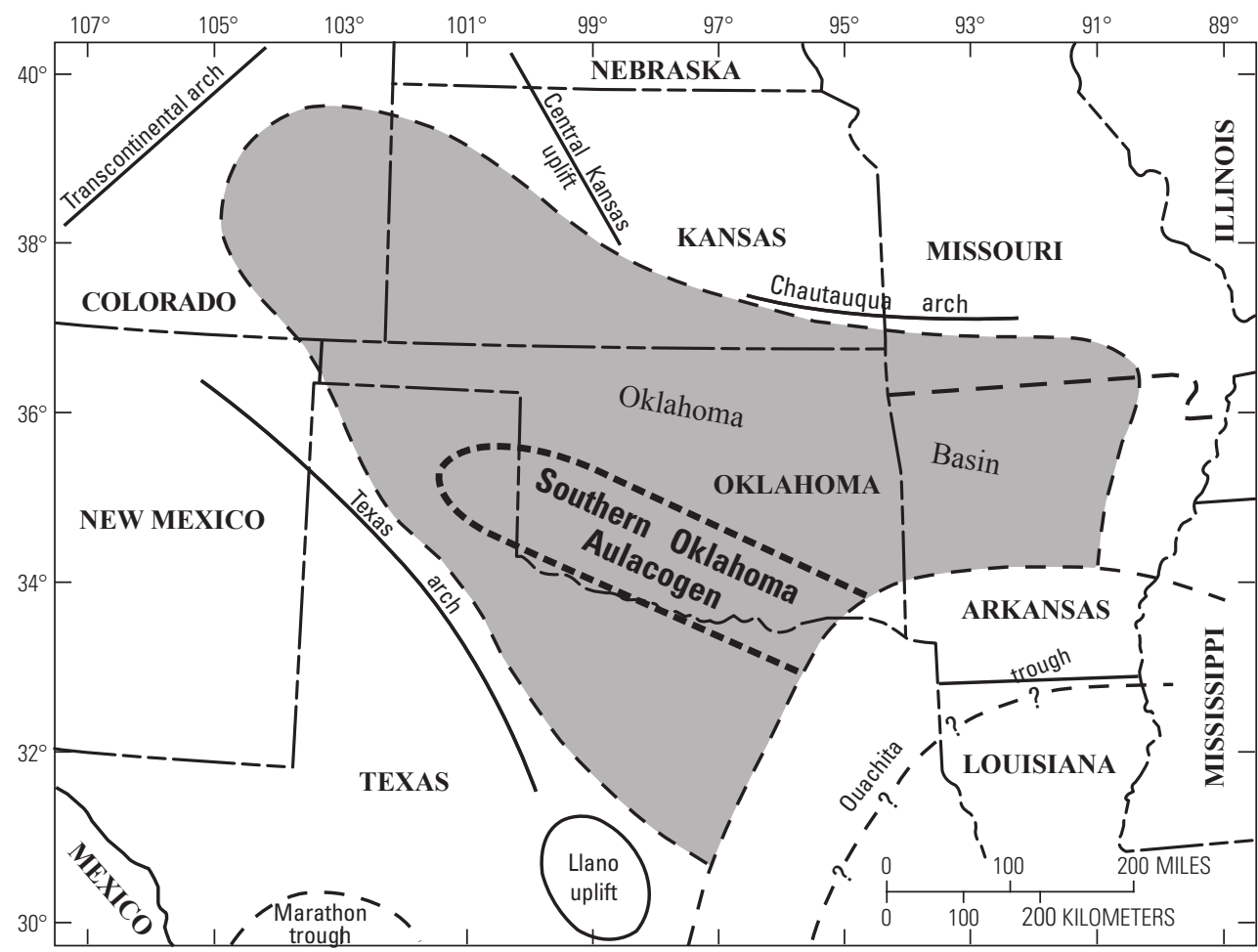

Figure 3. Map of the midcontinent of the United States, showing the approximate outline of the Oklahoma basin, southern Oklahoma aulacogen and other major features that existed in early and middle Paleozoic time. Modified from Johnson (1991). 

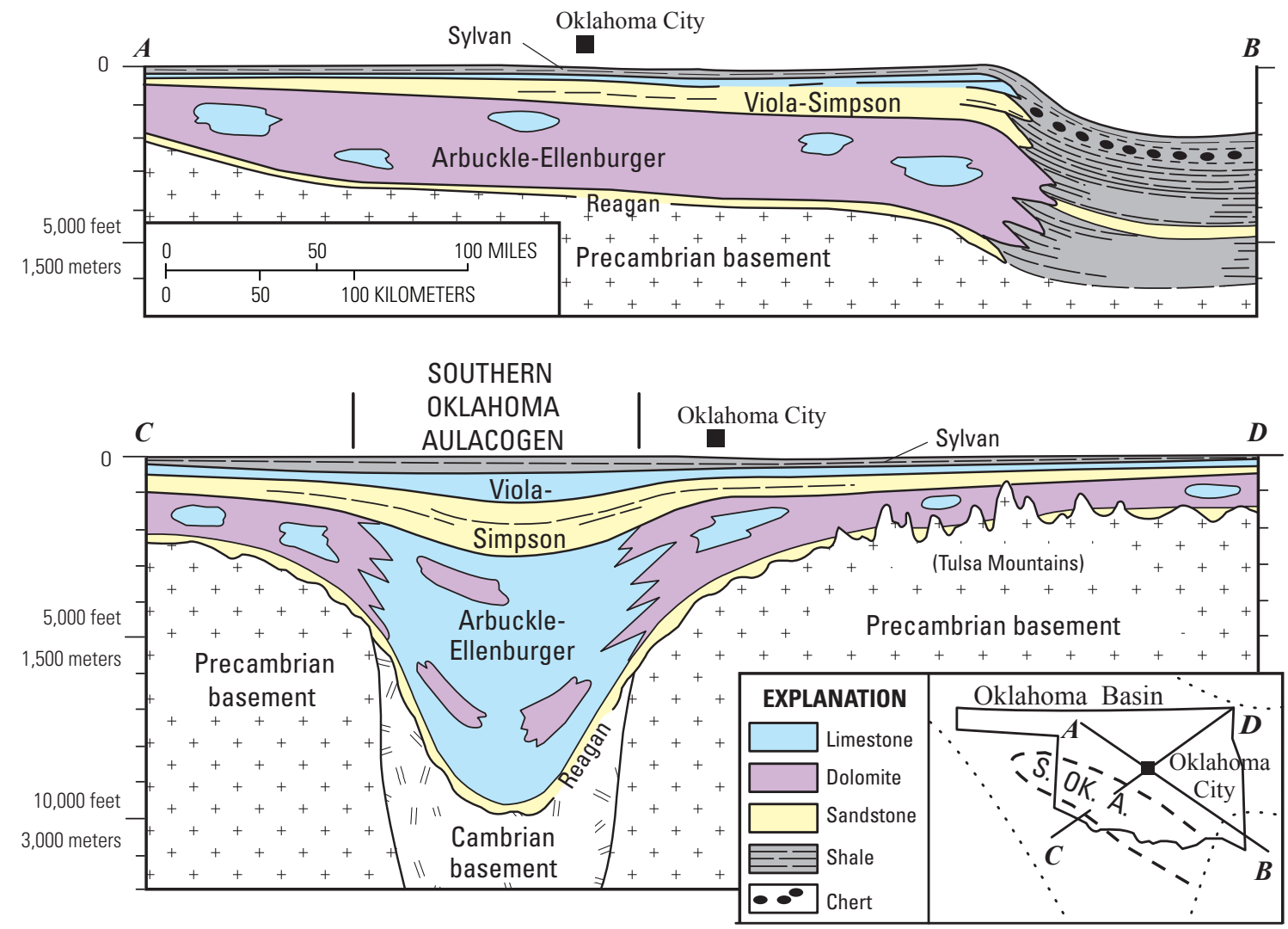

Figure 4. Schematic cross sections showing restored thickness of Upper Cambrian and Ordovician strata in Oklahoma (modified from Johnson, 1991). S. OK. A., Southern Oklahoma Aulacogen. Scale is the same for both cross sections. 


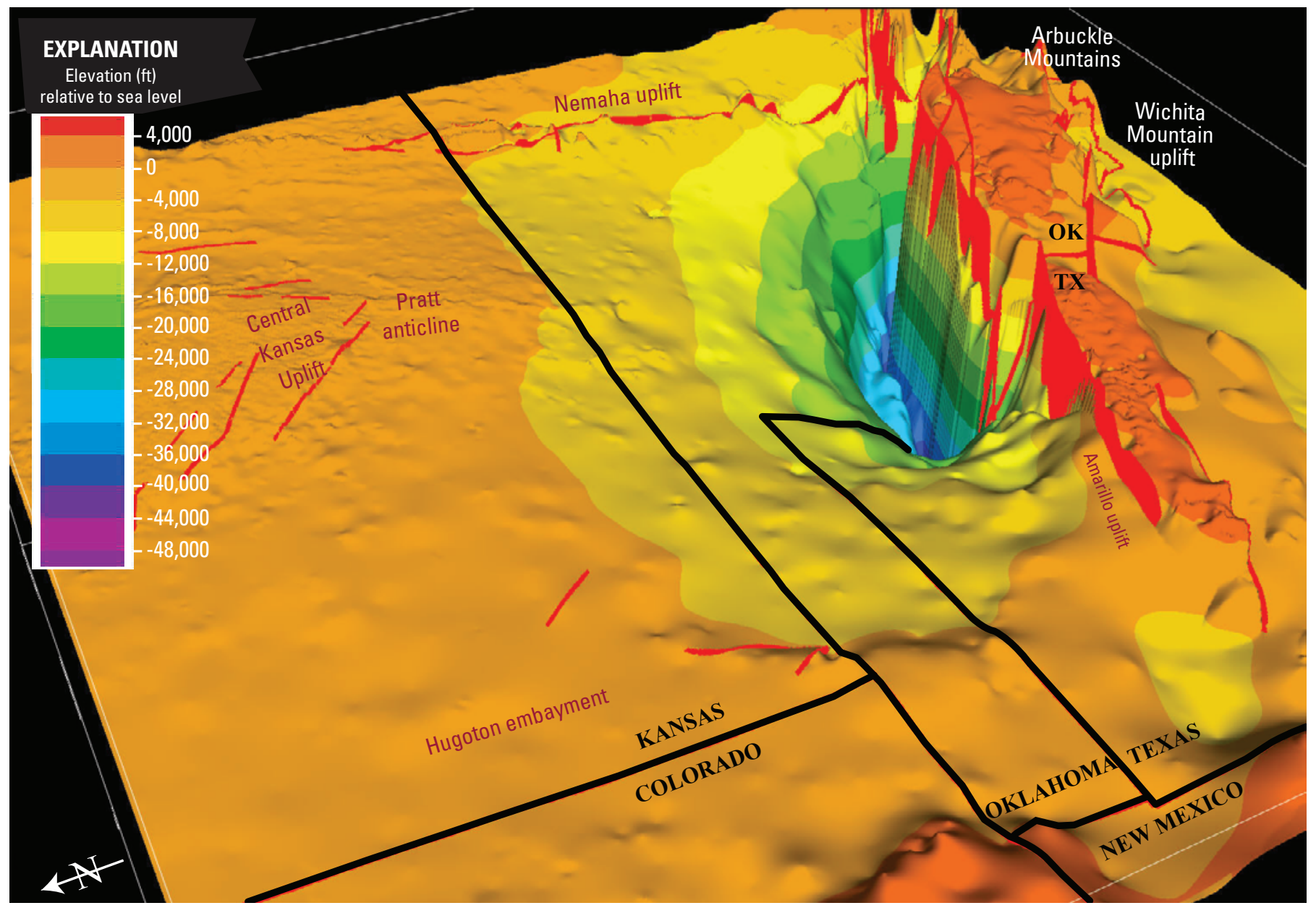

Figure 5. View to the southeast of elevation on the top of the Arbuckle Group. Major structures are labeled. Precambrian faults (red) are from Adler and others (1971). Wichita Mountain uplift faults are vertical for four-dimensional model purposes only, and surface in this area is unknown because of insufficient data. Data sources for this surface include Arbuckle picks from well logs, Rottmann (2000a, 2000b), and edited IHS Energy Group (2009) formation tops. 


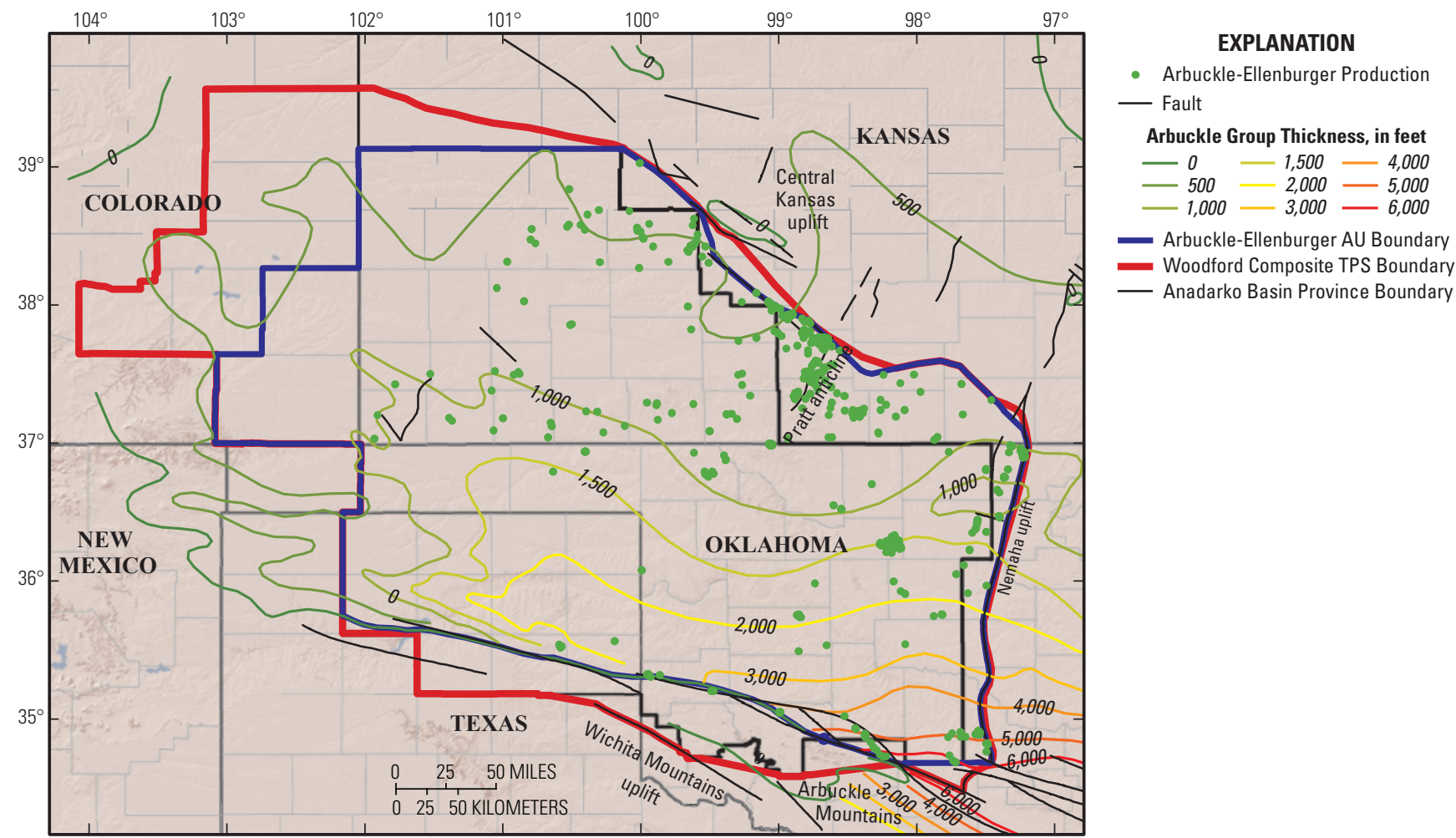

Figure 6. Map showing production from the Arbuckle and Ellenburger Groups and Arbuckle Group thickness; contour interval is 500 feet.

\section{Woodford Composite Total Petroleum System}

The Woodford Composite TPS boundary is defined where hydrocarbons generated from the Upper DevonianLower Mississippian Woodford Shale and other potential Ordovician source rocks (Arbuckle, Simpson and Viola Groups) have accumulated in Cambrian through Mississippian-age reservoirs (fig. 6). The Woodford Composite TPS is defined by the Nemaha uplift to the east, the border with the Central Kansas uplift to the northeast, the extent of maximum fluid distribution in the Mississippian AU to the north and northwest, the Anadarko Basin province boundary to the west, and the generalized province boundary to the south, mainly because of absence of data in the Wichita-Amarillo uplift areas (fig. 6).

The Woodford Shale was deposited under anoxic conditions in a shallow epicontinental sea over a regional unconformable erosional surface of the Silurian-Devonian Hunton Group (von Almen, 1970; Walper, 1977; Sullivan, 1985). The thickness ranges up to $375 \mathrm{ft}$ across the province, and the Woodford Shale is absent or thin in some areas because of erosion or non-deposition. It is thickest in the southern basin of Oklahoma and where it fills erosional channels of the Hunton Group. The Woodford Shale averages 40-ft thick based on Rottmann (2000b), well-log picks from about 100 wells across the basin, and edited formation tops from IHS Energy Group (2009).

The Woodford Shale in the Anadarko Basin is a dark gray to black carbonaceous, siliceous, pyritic shale (Cardott, 1989). It is considered one of the most prolific hydrocarbon source rocks in the Anadarko Basin (fig. 7), with predominantly Type II kerogen and total organic content (TOC) ranging from 1 to 14 percent (Burruss and Hatch, 1989). Vitrinite reflectance (Ro) data from the Woodford Shale (Cardot, 1989; Price, 1997) is contoured in figure 8 . Values range from 4.9 $\left(\% \mathrm{R}_{\mathrm{o}}\right)$ in the deepest basin to $0.5 \% \mathrm{R}_{\mathrm{o}}$ on the shelf, and indicate that the Woodford Shale is overmature for gas generation to marginally mature for oil generation in the deep basin and on the shelf, respectively. Vitrinite reflectance values increase along the Nemaha uplift on the eastern edge of the Anadarko Basin (fig. 8). Onset of petroleum generation from Woodford source rocks in the deep basin was at $335 \mathrm{Ma}$ based on onedimensional burial history modeling from the Bertha Rogers 1 well using variable heat flow through time, and a transformation ratio at $0.1 \% \mathrm{R}_{\mathrm{o}}$ and $0.55 \% \mathrm{R}_{\mathrm{o}}$ (fig. 9). The same parameters for the West Edmond SWD 1-24 well indicate that the onset of generation occurred at $225 \mathrm{Ma}$ for the Woodford Shale. Completion of generation for the Bertha Rogers 1 well occurred at about $310 \mathrm{Ma}$; the West Edmond SWD 1-24 well remains in the main oil generation window. 


\begin{tabular}{|c|c|c|c|c|c|}
\hline 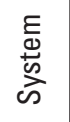 & Series & \multicolumn{2}{|c|}{$\begin{array}{l}\text { Lithostratigraphic Unit } \\
\text { (HC Source Rocks in Red) }\end{array}$} & $\begin{array}{c}\text { Relative HC } \\
\text { Source Rock } \\
\text { Potential (1-5) }\end{array}$ & $\begin{array}{l}\text { Expected } \\
\text { Hydrocarbons }\end{array}$ \\
\hline \multirow{2}{*}{ 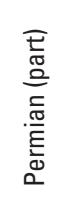 } & Leonardian & \multicolumn{2}{|c|}{ Sumner Gp; Enid Gp.; Hennessey Gp. } & & \\
\hline & Wolfcampian & $\begin{array}{l}\text { Chase Group } \\
\text { Council Grove Group } \\
\text { Admire Group }\end{array}$ & Pontotoc Group & & \\
\hline \multirow{6}{*}{ 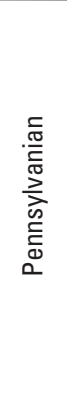 } & \multirow[t]{2}{*}{ Virgilian } & $\begin{array}{l}\text { Wabaunsee Group } \\
\text { Shawnee Group }\end{array}$ & Ada Group & & \\
\hline & & \multicolumn{2}{|c|}{ Douglas Group } & & \\
\hline & Missourian & $\begin{array}{l}\text { Lansing Group } \\
\text { Kansas City Group }\end{array}$ & Hoxbar Group & $1-2$ & - Oil \\
\hline & Desmoinesian & $\begin{array}{l}\text { Marmaton Group } \\
\text { Cherokee Group }\end{array}$ & Deese Group & $1-2$ & - Gas - Oil \\
\hline & Atokan & \multicolumn{2}{|c|}{ Atoka Gp.; Thirteen Finger limestone } & $1-2$ & - \\
\hline & Morrowan & \multicolumn{2}{|c|}{$\overbrace{}^{\text {Morrow Gp./Fm.; lower Dornick Hills Gp. }}$} & $2-3$ & - Gas \\
\hline \multirow{4}{*}{ 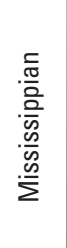 } & Chesterian & $\begin{array}{l}\text { Springer Formation } \\
\text { Chester Group }\end{array}$ & \multirow{4}{*}{ Mayes Group } & $1-2$ & - Gas - \\
\hline & Meramecian & Meramec lime & & & \\
\hline & Osagean & Osage lime & & 2 & - Gas Oil \\
\hline & Kinderhookian & Kinderhook Shale & & & \\
\hline \multirow{4}{*}{ 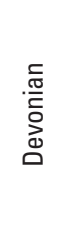 } & Chautauquan & \multirow{2}{*}{\multicolumn{2}{|c|}{ Woodford Shale, Chattanooga Shale }} & \multirow{3}{*}{$5+$} & \multirow{3}{*}{$-\alpha_{\text {Gas }} \quad-\phi_{-1 \mathrm{l}}$} \\
\hline & \multirow{3}{*}{$\begin{array}{l}\text { Senecan } \\
\text { Erian } \\
\text { Ulsterian }\end{array}$} & & & & \\
\hline & & & & & \\
\hline & & \multirow{2}{*}{\multicolumn{2}{|c|}{ Hunton Group }} & & \\
\hline 竞 & $\begin{array}{c}\text { Cayugan } \\
\text { Niagaran } \\
\text { Alexandrian }\end{array}$ & & & & \\
\hline \multirow{4}{*}{$\begin{array}{l}\frac{.}{.0} \\
\frac{0}{0} \\
\frac{0}{0} \\
\text { 힘 }\end{array}$} & \multirow{2}{*}{ Cincinnatian } & \multicolumn{2}{|c|}{ Sylvan Shale; Maquoketa Shale } & & \\
\hline & & \multicolumn{2}{|c|}{ Viola Group/Formation } & 2 & - Gas \\
\hline & Champlainian & \multicolumn{2}{|c|}{ Simpson Group } & $1-2$ & - \\
\hline & Canadian & \multicolumn{2}{|c|}{ Arbuckle Group } & $?$ & $? ?$ \\
\hline \multirow{2}{*}{ 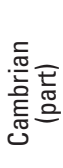 } & Trempealeauan & \multirow{2}{*}{\multicolumn{2}{|c|}{ Reagan Sandstone }} & & \\
\hline & Franconian & & & & \\
\hline
\end{tabular}

Figure 7. Generalized stratigraphic column for the Anadarko Basin province with oil and gas source rocks (red text). Increases in source rock potential are indicated by larger numbers. Modified from Hatch and others (1986). Hatched vertical lines represent an unconformity (Bebout and others, 1993). Gp., Group; Fm., Formation; HC, Hydrocarbon. 


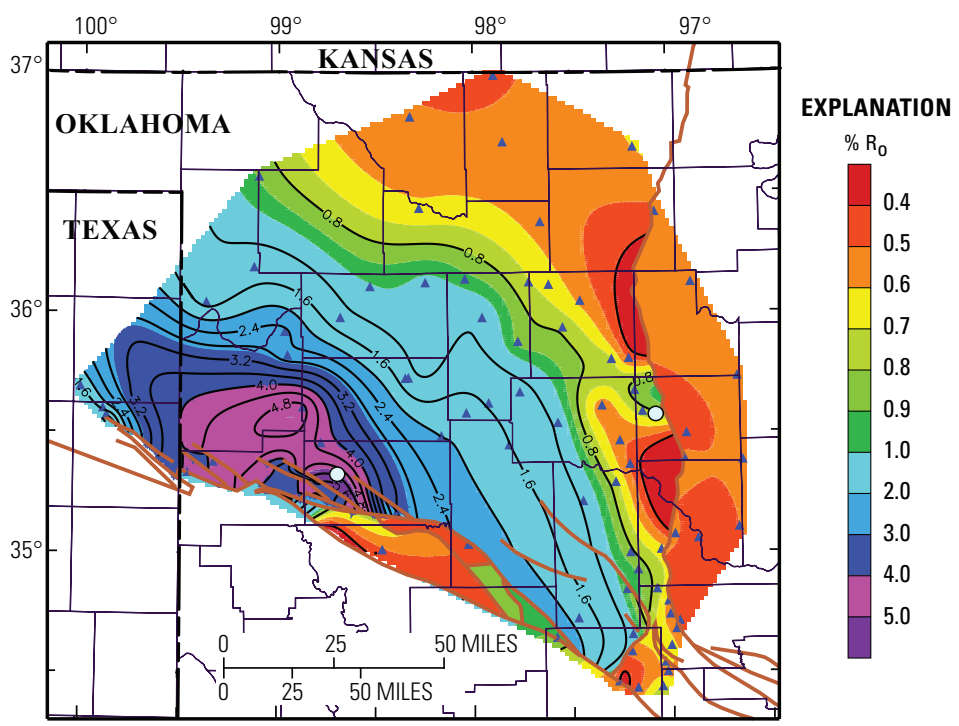

Figure 8. Map showing vitrinite reflectance $\left(\% \mathrm{R}_{0}\right)$ data for the Woodford Shale. Contour interval is $0.4 \% \mathrm{R}_{0}$. Blue triangles and white dots indicate locations of $\% \mathrm{R}_{0}$ data from Cardott (1989), Price (1997), and Chesapeake Energy Corporation (2009, written commun.). White dot well locations are, from left to right, Bertha Rogers 1 and West Edmond SWD 1-24. Brown lines are faults in the underlying Hunton Group (Rottmann, 2000a).

\section{Arbuckle and Ellenburger Groups}

The Arbuckle Group strata were deposited as a vast blanket of carbonate rock on a broad, nearly flat-lying carbonate ramp (commonly referred to as the "Great American Bank") that formed the southern margin of the North American craton during the Late Cambrian and Early Ordovician (Lindsay and Koskelin, 1991). The Arbuckle Group is age equivalent to the Ellenburger Group in Texas, and is the thickest sequence of lower Paleozoic strata in Oklahoma (Johnson and others, 2000). It overlies the Reagan Sandstone, and ranges from 400 to $8,000 \mathrm{ft}$ thick in the basin (fig. 6). It is thickest in the southern Oklahoma aulacogen (fig. 3), where it is limestone, thinning to approximately 4,000 $\mathrm{ft}$ of dolomite in the eastern Arbuckle Mountains, and 1,000 to 4,000 ft of dolomite on the shelf. The limestone to dolomite transition occurs near the boundary of the aulacogen (Johnson and others, 2000; fig. 4). Dolomitization was the result of water depth differences and restrictions in water circulation during deposition.

The base of the Arbuckle Group is lithologically defined by the abundance of lime mud that marked the beginning of platform deposition, associated with a rise in sea level (Donovan, 2000). The carbonates were deposited as a transgressive sequence on a shallow platform that was uplifted during the Paleozoic (Gao and others, 1992; Ayan and others, 2000).
Dolomite beds in these formations change laterally into limestone along the same stratigraphic interval. Lithologies include algal boundstones and stromatolites, mudstones, packstones, and grainstones, deposited in shallow marine, near-shore to shoreline, and tidal-flat settings (fig. 10). The Arbuckle Group is divided in the southern Anadarko Basin into six formations, and in ascending order are the Fort Sill Limestone, and Signal Mountain, McKenzie Hill, Cool Creek, Kindblade, and West Spring Creek Formations (fig. 2). The Royer Dolomite and Butterly Dolomite are also included in the Arbuckle stratigraphy in the Arbuckle Mountains, and to the north.

The Upper Cambrian Reagan Sandstone, which is part of the Timbered Hills Group in the Midcontinent, is included in the Arbuckle-Ellenburger AU (fig. 2). The Reagan Sandstone was deposited across a moderately mature erosional surface of low relief as the southern Oklahoma aulacogen began to subside during the Late Cambrian; it is a nonmarine, transgressive sandstone (Johnson, 1989; fig. 4). The Timbered Hills Group covers the province, with a thickness of approximately 35 to $120 \mathrm{ft}$. There is minimal hydrocarbon production from the Reagan Sandstone, with no accumulations reported in the Nehring database (Nehring Associates, Inc., 2009). Oil production from three wells in Kansas and Oklahoma totals approximately 130,000 barrels of oil from the Reagan Sandstone (IHS Energy, 2010). 


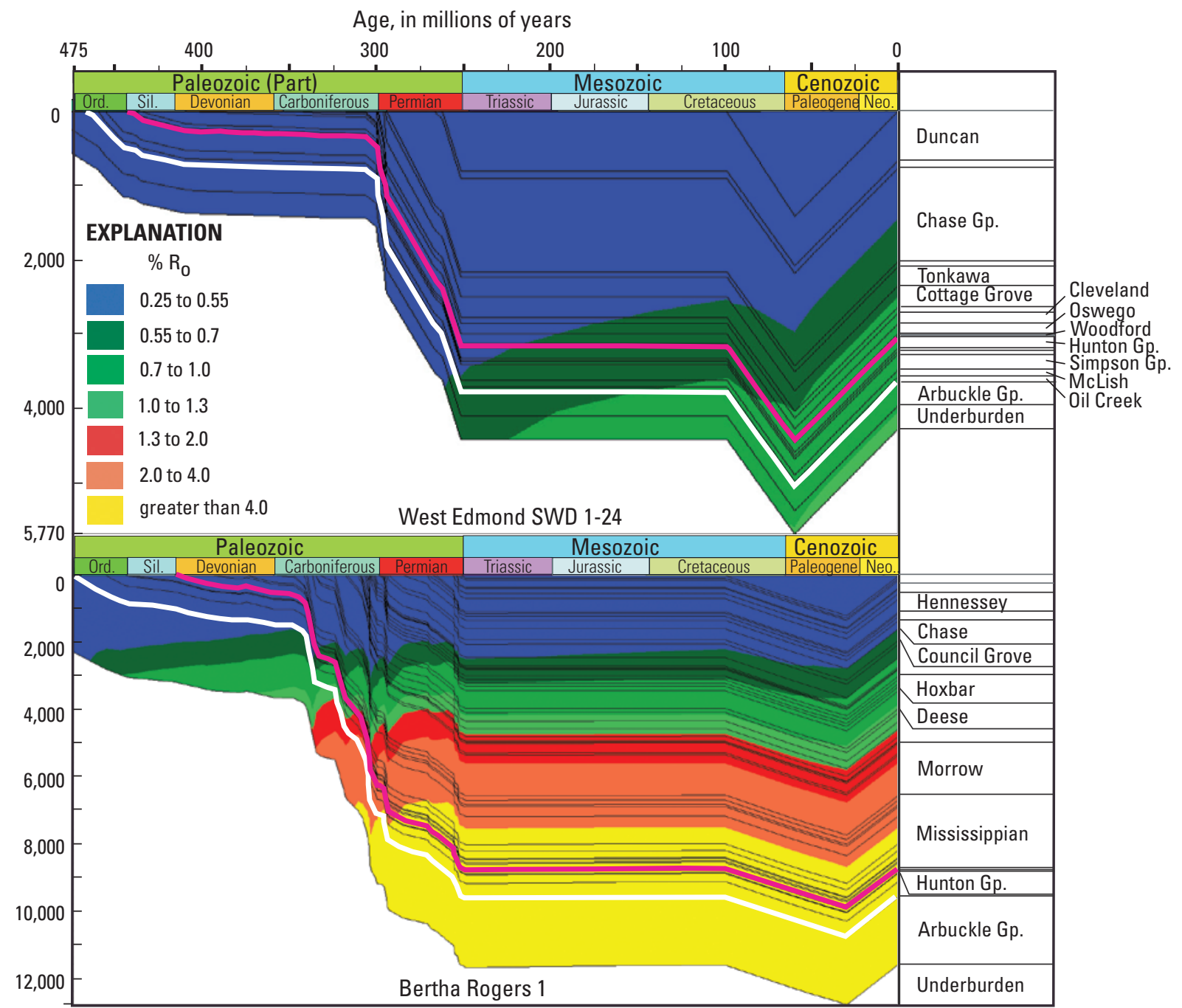

Figure 9. Burial history curves for the Bertha Rogers 1 and West Edmond SWD 1-24 wells. Modeled vitrinite reflectance through time includes heat flow of 70 milliwatts per square meter $\left(\mathrm{mW} / \mathrm{m}^{2}\right)$ to 260 million years ago (Ma), followed by $40 \mathrm{~mW} / \mathrm{m}^{2}$ for Bertha Rogers 1 and $50 \mathrm{~mW} / \mathrm{m}^{2}$ for West Edmond SWD 1-24. White and pink lines follow the upper part of the Arbuckle Group and Woodford Shale, respectively. Ord., Ordovician; Sil., Silurian; Neo., Neogene; Gp., Group; $\% R_{0^{\prime}}$ percent vitrinite reflectance. 


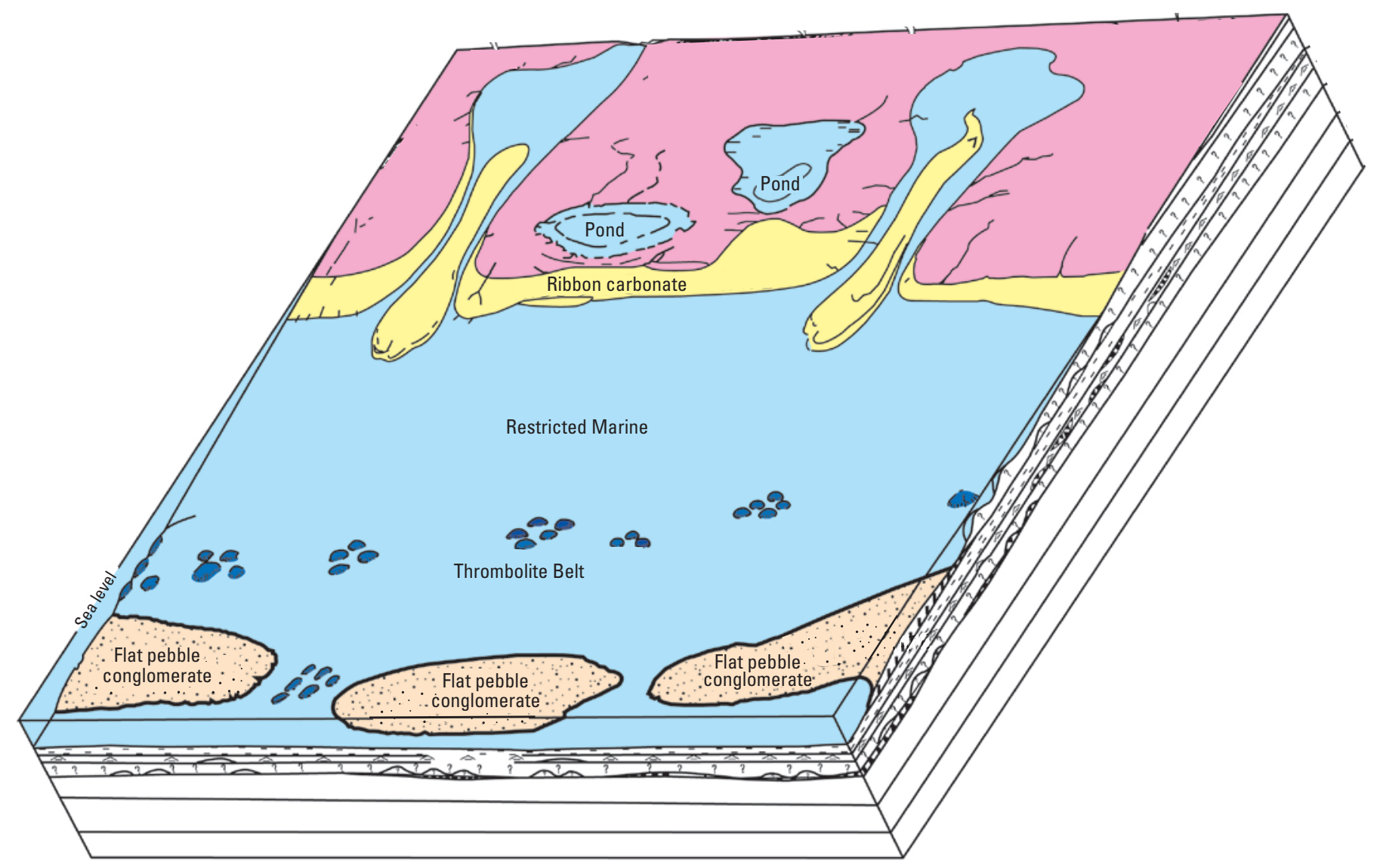

Figure 10. Depositional model of the Arbuckle Group in southern Oklahoma, showing a shallow-marine setting passing landward into a near-shore to shoreline setting, and finally into a tidal-flat setting. Sea-level transgressions and regressions resulted in the deposition of thin carbonate sequences (modified from Lindsay and Koskelin, 1991).

\section{Source Rocks of the Arbuckle Group}

Organic-rich rocks of the Woodford Shale and Simpson Group, and possibly the Viola Group, are considered the most likely sources of hydrocarbon for Arbuckle reservoirs (Burruss and Hatch, 1989; figs. 7 and 11). The Woodford Shale is likely the dominant source for the Arbuckle reservoirs, especially where faults juxtapose Woodford Shale source rock and Arbuckle carbonate reservoirs in the basin, or where migration occurred from the deep basin into Arbuckle reservoirs on the shelf.

The source rock potential of the Arbuckle Group has been long debated, as definitive source rocks have not yet been identified (Cardwell, 1977; Brown and Swetland, 1992; Williams, 1992; fig. 7). Bartram and others (1950) and Webb (1976) considered the Arbuckle hydrocarbons to be sourced from within the group. Possible hydrocarbon source rocks in the Cambrian and Ordovician Arbuckle Group were deposited in the rapidly subsiding aulacogen, and were buried to depths in the "oil window" (6,000-13,000 ft) from Middle Ordovician to Late Pennsylvanian time (Johnson and Cardott,
1992). Total organic carbon (TOC) data for the Arbuckle from Trask and Panode (1942) report 0.1-1.3 percent TOC in 81 Arbuckle samples from 16 wells, with lower values reported by Cardwell (1977). The Signal Mountain Formation, which was deposited in a deeper water setting than the rest of the Arbuckle, has shales with TOC values up to 1.26 percent and may have greater potential (Donovan and Critchfield, 2001; fig. 12). The Arbuckle, especially in the deep basin, has gone through the oil-generation window and has lost a substantial amount of the original organic matter that was converted to gas and oil (Johnson, 1992). Thus, Arbuckle rocks with low TOC could have had twice as much at the time of oil generation, according to arguments presented by Johnson (1992). It is also argued that organic-rich rocks in the Arbuckle Group may not yet have been located and (or) analyzed. Gatewood (1992) provided evidence of self-sourcing Arbuckle reservoirs, including production of large quantities of oil and gas 600$1,000 \mathrm{ft}$ or more below the top of the Arbuckle, oil produced beneath salt-water zones in some fields, organic remains in parts of the Arbuckle, and evaporites that serve as caps or seals for underlying dolomite reservoirs. 


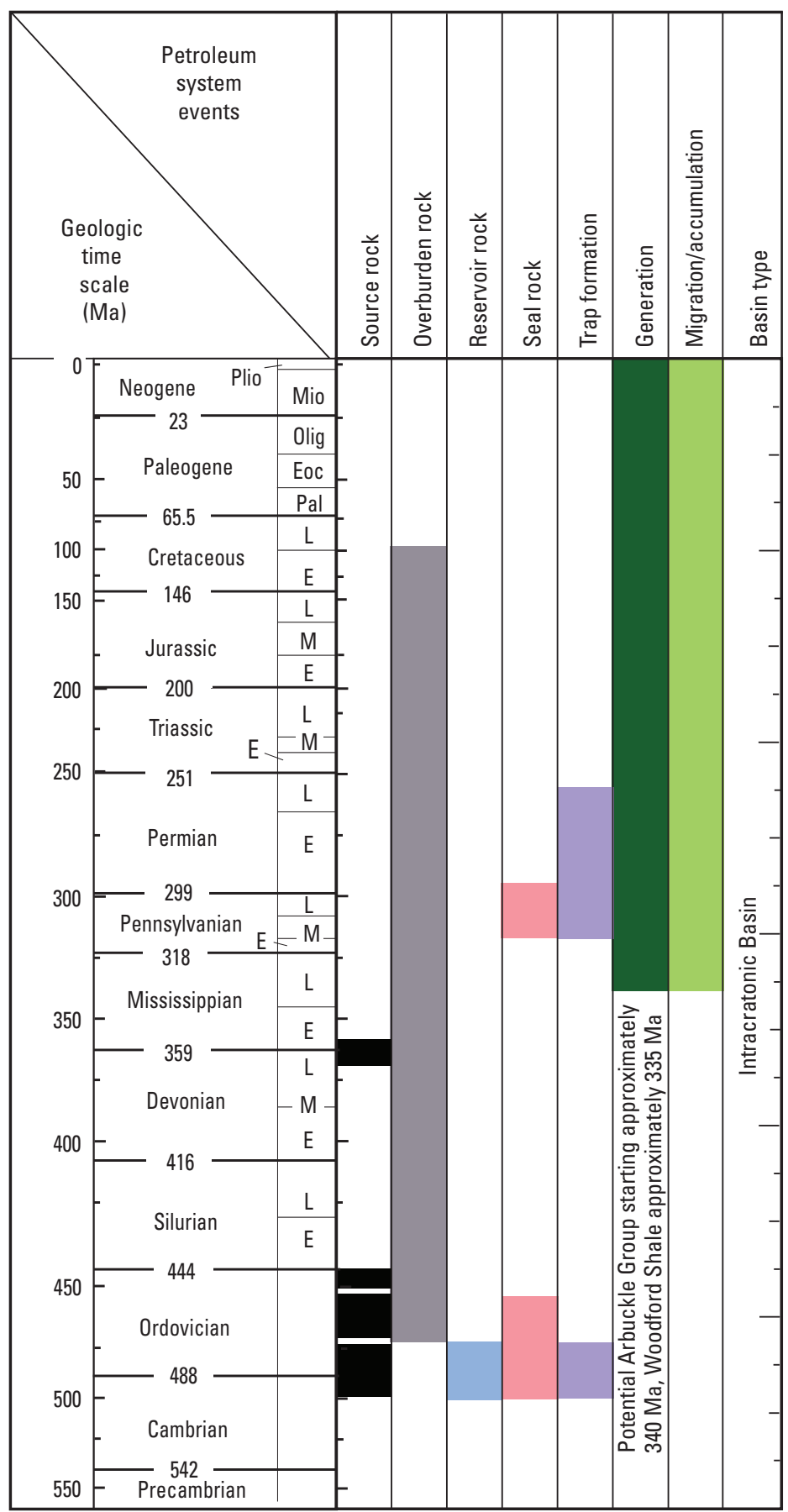

Figure 11. Arbuckle-Ellenburger Asessment Unit events chart showing the timing of source rock deposition and trap formation, and the age of overburden, reservoir, and seal rocks with different color bars (black, source rock; gray, overburden rock; blue, reservoir rock; pink, seal rock; purple, trap formation; dark green, generation; light green, migration accumulation). The chart also depicts the timing of oil generation, migration and accumulation as modeled for various wells in different parts of the Anadarko Basin. Ma, mega-annum; Plio, Pliocene; Mio, Miocene; Olig, Oligocene; Eoc, Eocene; Pal, Paleocene; L, Late; M, Middle; E, Early. 


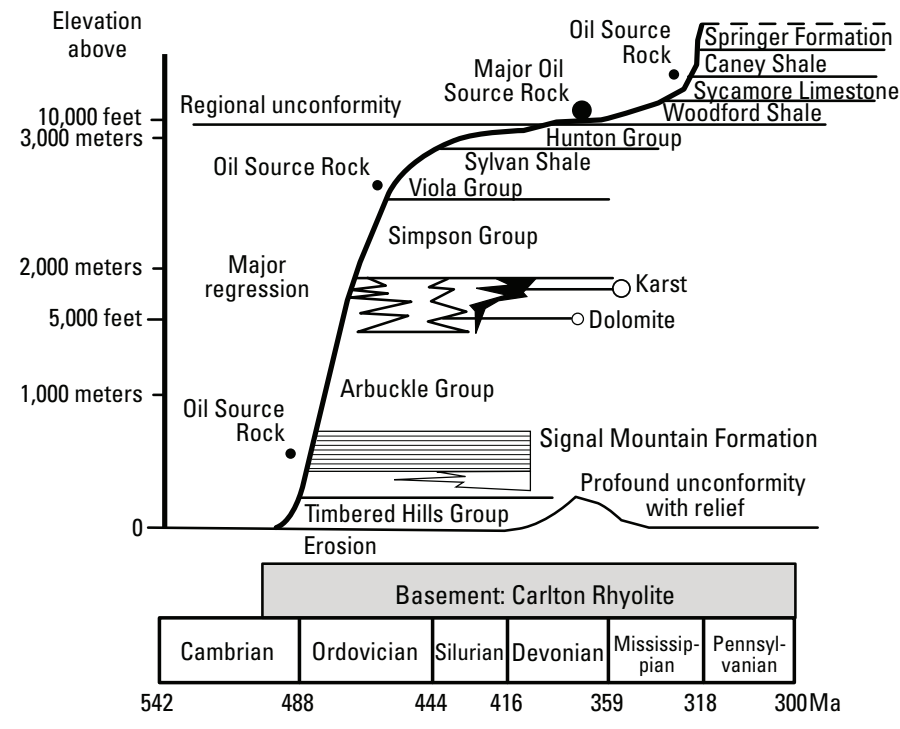

Figure 12. Lower Paleozoic stratigraphy in the southern Oklahoma aulacogen, with a burial-time curve illustrating the position of the Signal Mountain Formation and other hydrocarbon source rocks present in the Anadarko Basin. Modified from Donovan and Critchfield (2001). Ma, millions years before the present.

Potential Arbuckle source rocks reached the thermal zone of oil generation at approximately 340 million years ago (Ma) in the deep basin, according to the Bertha Rogers 1 one-dimensional (1D) burial history model, and $260 \mathrm{Ma}$ near the Nemaha uplift in the West Edmond SWD 1-24 well (fig. 9). This model used variable heat flow through time, a transformation ratio of 0.1 percent and 0.55 percent $R_{0}$. A map of present day Arbuckle percent $R_{0}$ extracted from the threedimensional (3D) model of the basin shows the Arbuckle as overmature in the deep basin and in the oil window along the Nemaha uplift and onto the shelf (fig. 13).

\section{Reservoir Rocks of the Arbuckle-Ellenburger Assessment Unit}

The Arbuckle Group is an important reservoir rock in the Anadarko Basin, with production from porous dolomite zones and limestones that were exposed to erosion on the crests of basement highs (Ball and others, 1991). Dolomites with high fracture intensity form better reservoirs as the late dolomization enhanced porosity and the dolomites did not undergo burial solution compaction or later cementation (Gao and others, 1992). Erosion of the Arbuckle appears to enhance its porosity and permeability, and fracture networks related to structure facilitate production and may have enhanced karstification (Ball and others, 1991). Known hydrocarbon accumulations are the result of the combined effects of diagenetic reservoir enhancement and proximity to major structural features. Porosity in the Arbuckle Group is variable and difficult to predict (Gao and others, 1992).
Production in the Arbuckle Group is scattered in Oklahoma and on the Kansas shelf and is almost exclusively from dolomitized facies (Johnson and others, 2000). The upper $250 \mathrm{ft}$ is more significantly explored than the lower, main body of the Arbuckle Group (Henry and Hester, 1996). Production in Kansas has largely been on and near the Central Kansas uplift and associated structures (fig.14).

The Ellenburger Group is the Arbuckle equivalent in the Texas Panhandle portion of the basin. They have common lithologic and physical characteristics, including: (1) the producing zone is dolomite, (2) it has secondary porosity, (3) the production is related to structure, and (4) fields have lateral and vertical heterogeneity.

The Arbuckle-Ellenburger AU boundary follows the Anadarko Basin province boundary on the north and west, the faults that define the southern end of the Central Kansas uplift in the northeast, the Nemaha uplift on the east, and the Precambrian fault system along the Arbuckle Mountains to the south (fig. 14). There is scattered production throughout the $\mathrm{AU}$, with exploration mainly focused along structures in the eastern, northeastern, and southern parts of the basin (fig. 14). There is the potential for oil migration from the deep basin onto the shelf, or towards the Texas Panhandle, as well as the possibility of self-sourcing in these areas.

\section{Traps and Seals in the Arbuckle-Ellenburger Assessment Unit}

Traps in the Arbuckle Group reservoirs are largely structural, or combination structure-stratigraphic, and most are near the eastern and southern fault systems. Seals for Arbuckle Group reservoirs are low porosity zones of Arbuckle evaporites, argillaceous carbonate, or shale, or overlying tight, impermeable shales of the Simpson Group (fig. 11). There are also areas in the basin where Pennsylvanian sands and shales unconformably overlie producing zones, creating the reservoir seal. Major Arbuckle fields that are producing from structurally controlled dolomite reservoirs are Cottonwood Creek and Haldton fields, as well as Oklahoma City field and Mayfield West field. Significant reserves are in Arbuckle reservoirs in Major County, Oklahoma, which is on the northern shelf of the Anadarko Basin, with oil and gas production since 1945. The primary trapping mechanism in the area is a horst and graben structural fabric, and within each horst block, structural reversals and stratigraphic pinchouts define reservoir geometry (Heyer, 1993). Reservoir lithology is dolomite, with textures ranging from bioclastic grainstones to mudstones. Intracrystalline and intercrystalline porosity is as high as 17 percent, with permeability up to 1.82 millidarcies (mD) (Heyer, 1993).

A substantial amount of gas has been produced from the Arbuckle in Mayfield field, in Beckham County, Oklahoma, and to the northwest in Wheeler County (fig. 14). Wells were drilled on faulted, domal structures with production from fractured Arbuckle carbonates which have undergone leaching, resulting in high porosity development at the unconformity level (Perry, 1990). 


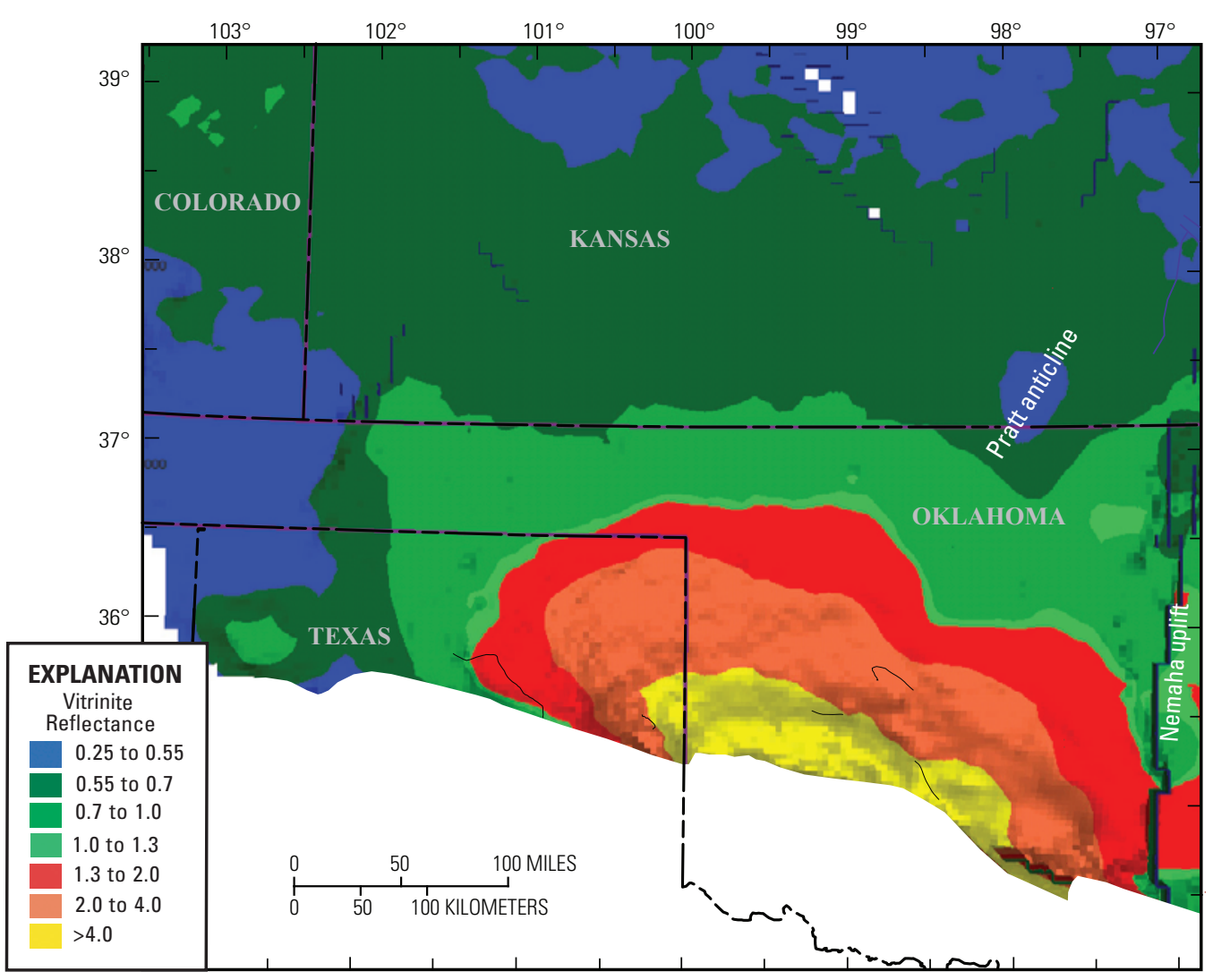

Figure 13. Image showing vitrinite reflectance $\left(\% R_{0}\right)$ contours on the Arbuckle Group, extracted from the four-dimensional model of the Anadarko Basin. The burial history model illustrates decompaction through subtle increases in layer thickness backward through time. The vitrinite reflectance profile was calculated using Sweeney and Burnham (1990) kinetics. 


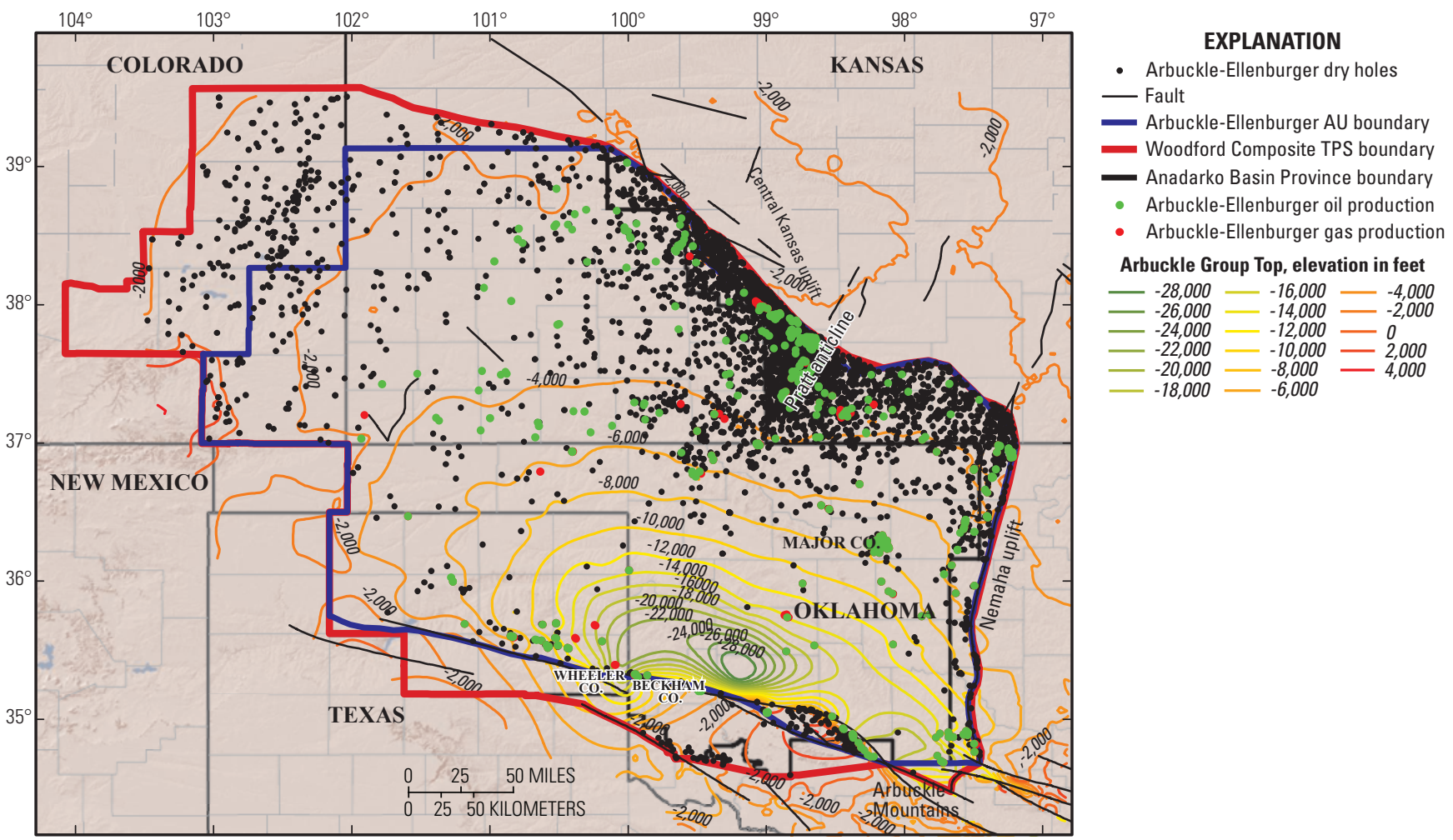

Figure 14. Map showing oil and gas production and dry hole penetrations for the Arbuckle and Ellenburger Groups in the Anadarko Basin Province (IHS Energy Group, 2010). Structure contours are drawn on the top of the Arbuckle Group; contour interval is 2,000 feet. The Anadarko Basin Province boundary is the black line, the Woodford Composite Total Petroleum System (TPS) boundary the red line, and the Arbuckle-Ellenburger Assessment Unit (AU) boundary the blue line.

\section{Sizes and Numbers of Undiscovered Fields in the Arbuckle-Ellenburger Assessment Unit}

The Arbuckle Group is not well explored, largely because of its depth, especially in the deep basin. There have been problems with seismic exploration of the Arbuckle because of (1) poor data quality beneath the complex overthrust zones that exist along the southern boundary of the Anadarko Basin (Brown and others, 1991) and (2) the relatively low amplitude of the Arbuckle reflections in the basin compared to the higher amplitude reflections of the overlying strata. Arbuckle wells are characterized by high initial potential, steep decline rates, production of large quantities of oil, and high water-oil ratios.

Cumulative oil production in the Arbuckle-Ellenburger $\mathrm{AU}$ is $52 \mathrm{MMBO}$, with $1.3 \mathrm{MMBO}$ from the Ellenburger Group in Texas (IHS Energy, 2010). Although there is oil production throughout the basin, gas production is limited to the deeper part of the basin, with 285 BCFG produced (IHS Energy, 2010; fig. 14). Production depths range from $3,000 \mathrm{ft}$ to $26,500 \mathrm{ft}$. The most recent reported discoveries are on the Anadarko shelf, but with low cumulative oil and gas numbers reported by IHS Energy (2010; fig. 15). In commingled fields, which are common in the basin, the Arbuckle composes a relatively small portion of the total production based on a comparison of field data versus Arbuckle accumulation data for a given field (Nehring Associates Inc., 2009).

Nehring Associates Inc. (2009) presented limited data for the Arbuckle-Ellenburger; only 4 of the 24 accumulations in the database have grown reserve accumulation numbers (fig. 15). This is likely because of a historical lack of reporting information from private operators to the state and other agencies for Midcontinent hydrocarbon production. Furthermore, there is a large amount of commingled production in the basin, as well as the incorrect reporting of the producing formation. Nehring field data were used to supplement the database, but are of marginal use because the production from all reservoirs is generally combined for a field, not just that of the ArbuckleEllenburger. In cases where the Arbuckle contribution can be determined by combining the field data with the grown data, the Arbuckle is a minor component of the total resource in the majority of the fields.

Using the USGS assessment methodology for undiscovered conventional resources (Klett and others, 2005; Schmoker and Klett, 2005), the mean undiscovered oil and 

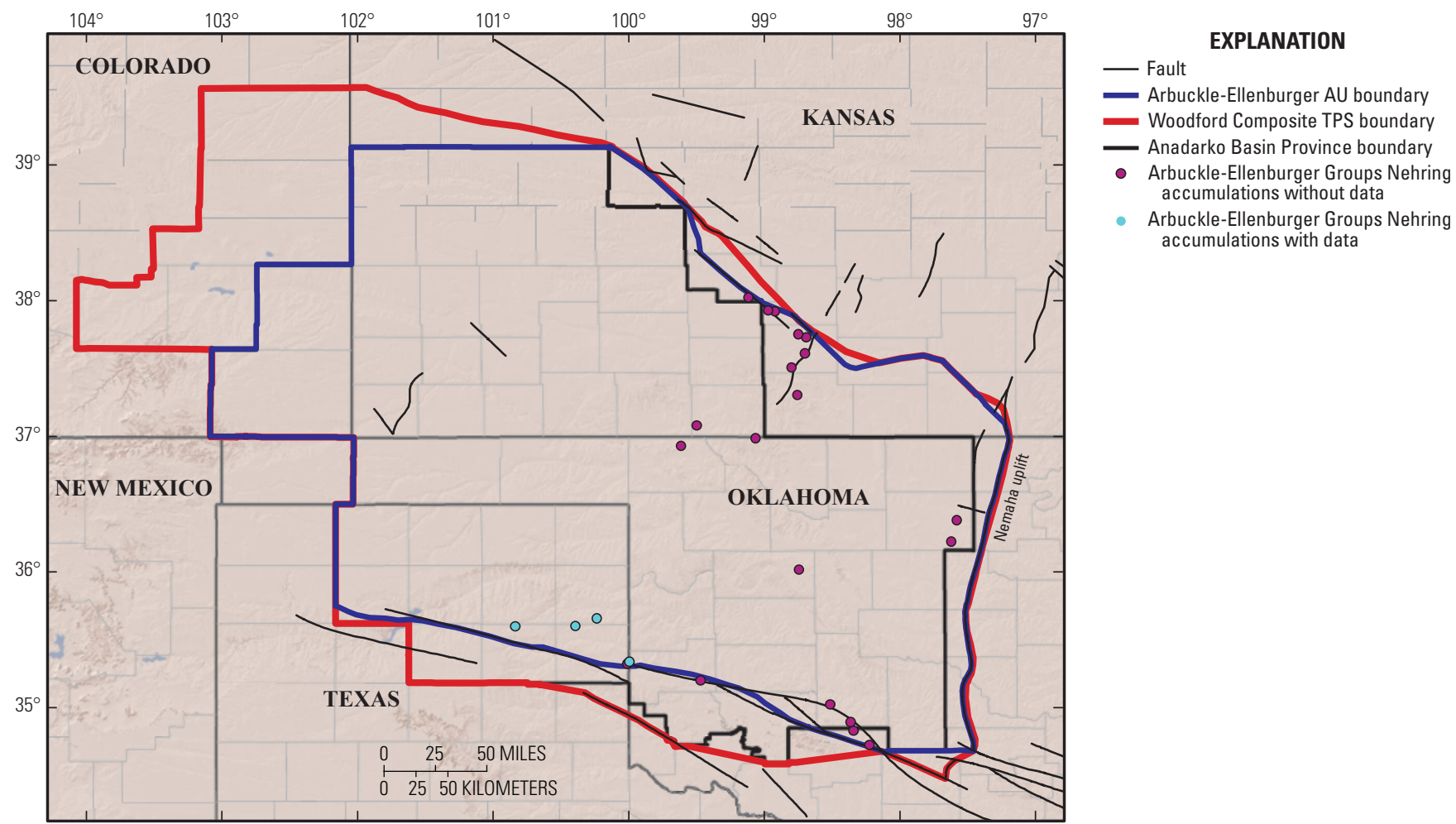

\section{EXPLANATION} accumulations with data

Figure 15. Map showing accumulations for the Arbuckle-Ellenburger Assessment Unit (AU) (Nehring Associates, Inc., 2007). Accumulations without numbers are in purple. TPS, Total Petroleum System.

gas resources for conventional reservoirs in the ArbuckleEllenburger AU are estimated at 5.5 MMBO and $181 \mathrm{BCFG}$ (table 1). Estimates of the minimum, median, and maximum numbers of undiscovered accumulations are 1,2, and 10 for oil, and 1, 10, and 60 for gas. The broad ranges reflect the uncertainty of future potential. The Arbuckle has been penetrated more extensively on the shelf of the basin, where production is predominantly oil (fig. 14). There is more undrilled area in the deep basin along structures; gas production is more likely in the deep basin based on gas versus oil production in the Arbuckle, and the fact that the deep basin is thermally mature to overmature for gas production (figs. 9 and 13). Future oil production will likely be as small accumulations on the shelf, or associated with deeper gas production. The most recent Arbuckle discoveries are on the shelf near the Pratt anticline, but oil numbers are low in these fields, whereas gas fields in the deeper basin are larger, based on field data (Nehring and Associates, Inc., 2009; fig. 15).

Large gas fields with Arbuckle production are Mayfield West (23 BCFG grown) and Bradbridge (7 BCFG grown). Production depth has a wide range (3,000 to $26,500 \mathrm{ft})$ depending on whether the Arbuckle is producing from the deep basin or the shelf (fig. 14).

Estimates of the minimum, median, and maximum sizes of undiscovered oil accumulations are $0.5,1.0$, and
10.0 MMBO. The 0.5 MMBO default signifies that there will be at least one field found greater than the minimum size, and the maximum reflects the uncertainty of Arbuckle accumulation sizes because of the lack of Nehring information. Production by field for the Arbuckle from the IHS database has been small (less than $4 \mathrm{MMBO}$ ). Estimates of the minimum, median, and maximum sizes of undiscovered gas accumulations are 3, 6, and 60 BCFG. The maximum reflects the uncertainty with deep gas production in the Arbuckle-Ellenburger, as it is sparsely drilled in the deep basin. The most recent accumulation reported by Nehring Associates, Inc. (2009) data was discovered in 2005. However, there are no Nehring accumulation data provided and none found for the Arbuckle in the IHS database (IHS Energy, 2010).

The future role of the Arbuckle Group is an important question in the development of hydrocarbon resources in Oklahoma, as there may be significant undiscovered resources, especially in the deepest part of the Anadarko Basin, where drilling into the Arbuckle is sparse. There is also potential for future production downdip from the central Kansas production areas, or along migration routes the hydrocarbons generated in the deep basin followed through Oklahoma. The Texas Panhandle is also a viable area for exploration, as the Arbuckle is deep enough there to have possibly self-sourced. 


\section{Simpson Group}

The Middle Ordovician Simpson Group overlies the Arbuckle Group in the Anadarko Basin, and the rocks represent a departure from carbonate deposition because of an influx of clastic sediments. Simpson Group strata in Oklahoma are clean quartzose sandstones interbedded with thick, shallow-water marine limestones and thin to moderately thick greenish-gray shales (fig. 16; Flores and Keighin, 1989; Johnson, 1991). In Kansas, the Simpson is a sequence of clastics and sandy carbonate rocks. Fine- to coarse-grained clastic sediments were derived from the uplifted Canadian Shield to the north and east when sea level was lowered during the Middle Ordovician; the windblown sediment was carried southward and covered the emergent carbonate shelf (Johnson, 1991). As sea level rose, sand was reworked into an extensive and sheet-like transgressive deposit in marine shoreface and tidal flat environments, which is overlain by marine shales and carbonates. Successive sea level fluctuations during the remainder of Simpson time produced a succession of sands, shales and limestones (Johnson, 1991). Clastic and carbonate rocks are dominant to the north, and offshore facies persist to the south (fig. 17).

The total thickness of Simpson Group rocks ranges from 100 to $300 \mathrm{ft}$ on the basin shelf, to $2,300 \mathrm{ft}$ in the basin depocenter (fig. 18). The Simpson is divided into five formations, in ascending order: (1) the Joins Formation, (2) the Oil Creek Formation, (3) the McLish Formation, (4) the Tulip Creek Formation, and (5) the Bromide Formation (figs. 2 and 16). Each has a basal sandstone facies and upper mudstone-siltstone-limestone facies (fig. 16). The basal sandstones of the Simpson are regionally extensive sheet-like bodies, ranging in thickness from 50 to $200 \mathrm{ft}$. Other sandstones are less continuous and cannot be traced for long distances laterally. In the subsurface of central Oklahoma, some of the sandstones are referred to as "Wilcox sands" (Johnson, 1991); the "Second Wilcox" is commonly correlated to the basal Bromide sand body (Rottmann, 1997).

The Joins Formation is the exception in that it lacks the sandstone facies present in the upper Simpson Group formations, and is mainly carbonate that was deposited in a shallow marine environment during the post-Arbuckle sea-level lowering in the remaining finger of the carbonate sea in Oklahoma (fig. 19; Denison, 1997). Over a large area of Oklahoma the Joins Formation was embayed by the basal Oil Creek Formation sandstones. The basal Oil Creek sandstone is overlain by shale and limestone, the latter of which is a reservoir in some areas of the basin. The basal McLish Formation sandstone is one of the most widespread and persistent quartz-rich sandstones of the Simpson Group. The McLish Formation grades into limestones, dolomites, and shales, and represents a continuation of clear-water shallow-marine sedimentation (Suhm, 1997). The Tulip Creek Formation has a basal sandstone body overlain by shale, and the uppermost Bromide Formation consists of varying amounts of sandstone, limestone, dolomite, and shale. The Bromide basal sandstone is the most areally

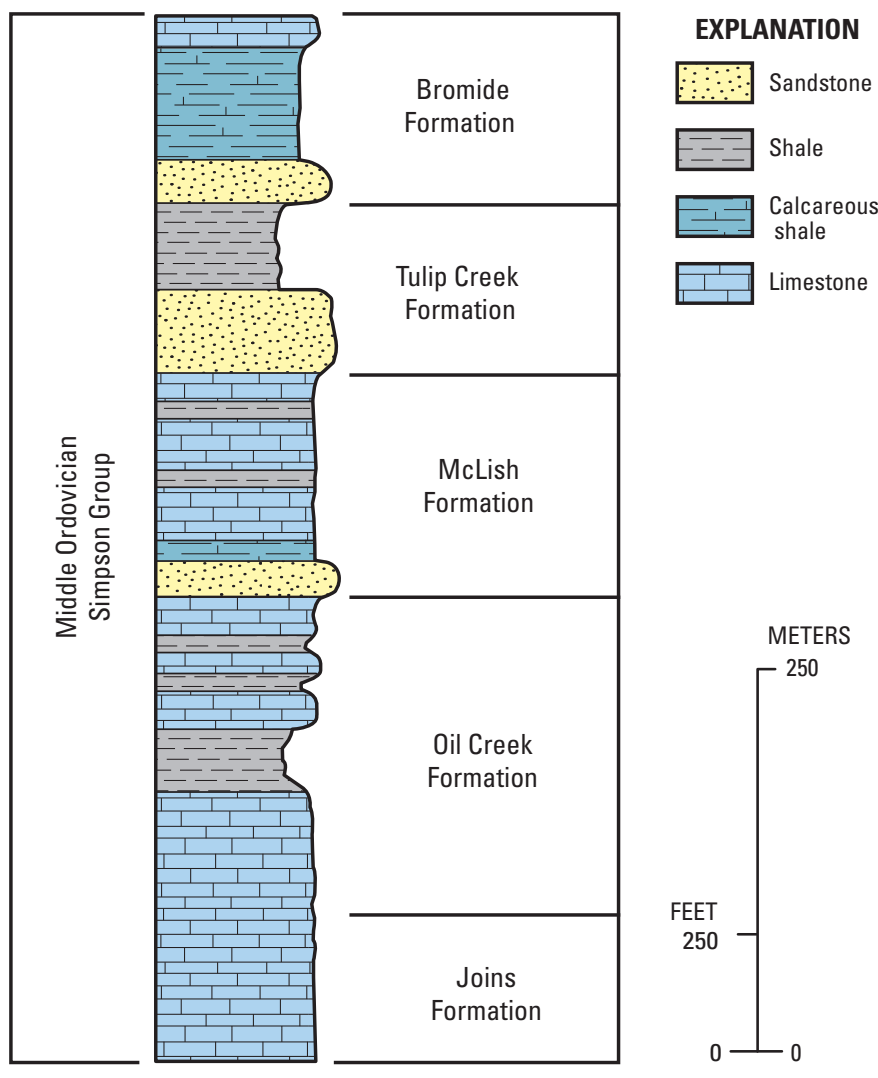

Figure 16. Stratigraphic section of Simpson Group rocks (modified from Flores and Keighin, 1989).

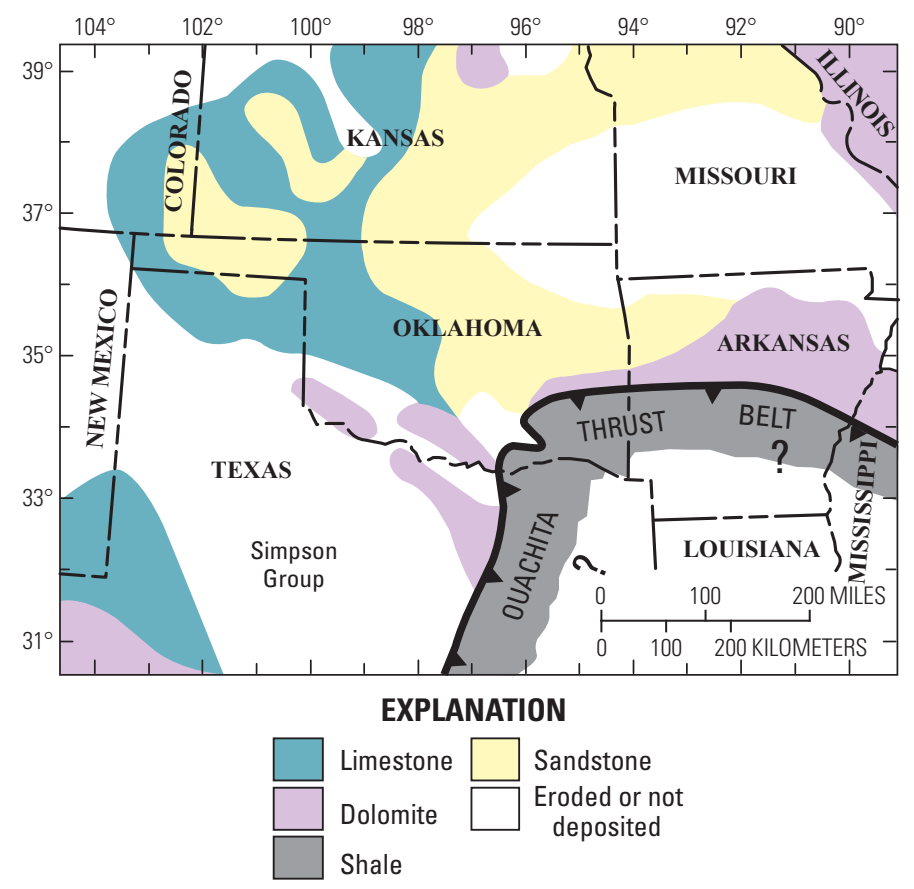

Figure 17. Image showing major lithologies of Ordovician Simpson Group strata in the southern midcontinent (modified from Northcutt and Johnson, 1997). 
extensive of the Simpson sandstones, and the contact with the overlying Viola Group is distinct based on the change to clean, crystalline limestone (Suhm, 1997).

In the Kansas portion of the Anadarko Basin, the Simpson is divided into six informal stratigraphic units that are correlated with the McLish and Bromide Formations. The McLish Formation corresponds to the two lower units and the Bromide Formation to the upper four units (Doveton and others, 1990; Charpentier and Doveton, 1991). The absence of the Joins, Tulip Creek, and Oil Creek Formations reflects the change from proximal shoreface facies in the deeper basin to offshore facies near the Pratt anticline (fig. 19; Charpentier and Doveton, 1991).

\section{Source Rocks of the Simpson Group}

Oil samples from the Middle Ordovician Simpson Group have the characteristics of Ordovician oils. The Woodford Shale is also a likely source rock for the Simpson Group reservoirs (figs. 7 and 20; Burruss and Hatch, 1989). Faults juxtapose the Woodford and Simpson in the basin, and migration from the Woodford Shale in the deep basin also charged Simpson reservoirs (Smith, 1997).

Simpson Group shales have been identified as a hydrocarbon source rock for the Oklahoma basin (fig. 3) by Adler and others (1971), Webster (1980), Hatch and others (1986), and Wavrek (1989). Philippie (1981) showed that shales of the Simpson Group acted as the source for oils in the stratigraphically older rocks of the Ellenburger Group in west Texas. Hatch and others (1986) also interpreted Simpson shales to be the probable source for Viola and Simpson Groups reservoirs in the Forest City Basin of Kansas and Nebraska.

Burruss and Hatch (1989) identified potential source rocks in the shales of the Simpson Group as containing Type I and II kerogen. Sparse sampling on the Kansas shelf indicates as much as 14 percent of the Simpson shales may be of source rock quality (greater than 1 percent TOC; Burruss and Hatch, 1989). Organic material in the Simpson Group is algal, and potentially improves in Kansas, where it is on the shelf. However, Simpson shales on the shelf are only marginally mature for oil and potential is considered poor to moderate (fig. 7; Burruss and Hatch, 1989). Quality is moderate in the deep basin where Simpson shales have reached maturity.

\section{Reservoir Rocks in the Simpson Group Assessment Unit}

The blanket-like sandstones with interbedded shales are the dominant reservoir facies within the Simpson Group. The Simpson Group is most productive from its sandstone units, but it also contains some carbonate reservoirs that are highly productive locally. Carbonate rocks within the lower Simpson yield oil and gas, especially in karsted reservoirs. All of the Simpson formations are productive to varying degrees. To the north in south-central Kansas, the Simpson increases in thickness west of the Pratt anticline and becomes dominated by sandy, cherty dolomite, which accounts for the loss of production in that area of the basin (Lynn Watney, written commun., 2010).

Reservoir porosity in producing sandstones ranges from 10 to 30 percent, with permeabilities ranging from 15 to 300 (mD) (Ball and others, 1991; Johnson, 1991). Reservoir quality is highest in well-sorted sandstones that are absent of clay, and which have had dissolution of intergranular carbonate cement during burial (Pollastro, 1989). The Second Wilcox (Bromide) is the principal producing unit of the Simpson on the central Oklahoma shelf, where overlying impermeable strata create stratigraphic traps when the sandstones are upthrown against impermeable strata and a fault seal occurs (Rottmann, 1997).

The Simpson AU boundary follows the Simpson subcrop on the northwest, the province boundary on the west, the faults that the define the southern end of the Central Kansas uplift in the northeast, the Nemaha uplift on the east, and the Precambrian fault system along the Arbuckle Mountains to the south (fig. 18). Production is concentrated on the eastern edge of the basin and to the north along the Central Kansas uplift. There is also production in the southeast corner of the basin along the Precambrian fault system.

\section{Traps and Seals in the Simpson Group Assessment Unit}

Simpson Group sandstones have produced substantial volumes of hydrocarbons from structural traps, especially along the Nemaha uplift on the eastern edge of the basin (fig. 18). On the Nemaha uplift, production is on the flanks of structural highs from erosionally truncated sandstone that is sealed by overlying Pennsylvanian shales. Multiple Simpson fields produce along these complex structures, such as the Oklahoma City, Golden Trend, and Sho-Vel-Turn fields. The Oklahoma City field produces from a large anticline near the south end of the Nemaha uplift, bounded on the east by a normal fault (Northcutt and Johnson, 1997). There is a large amount of commingled production in these fields.

Historically, Simpson exploration has been largely related to structural traps, with little exploration for possible stratigraphic traps related to unconformities, porosity development, and pinchouts of porous sandstone and carbonates. There may also be significant potential for stratigraphic traps that lie off structure and below seismic resolution (Candelaria and others, 1997).

Simpson reservoir seals are interbedded Simpson shales and tight sandstones or carbonates (fig. 20). Also, overlying Pennsylvanian shales on the flanks of structural highs form seals where the underlying strata have been removed by erosion. 


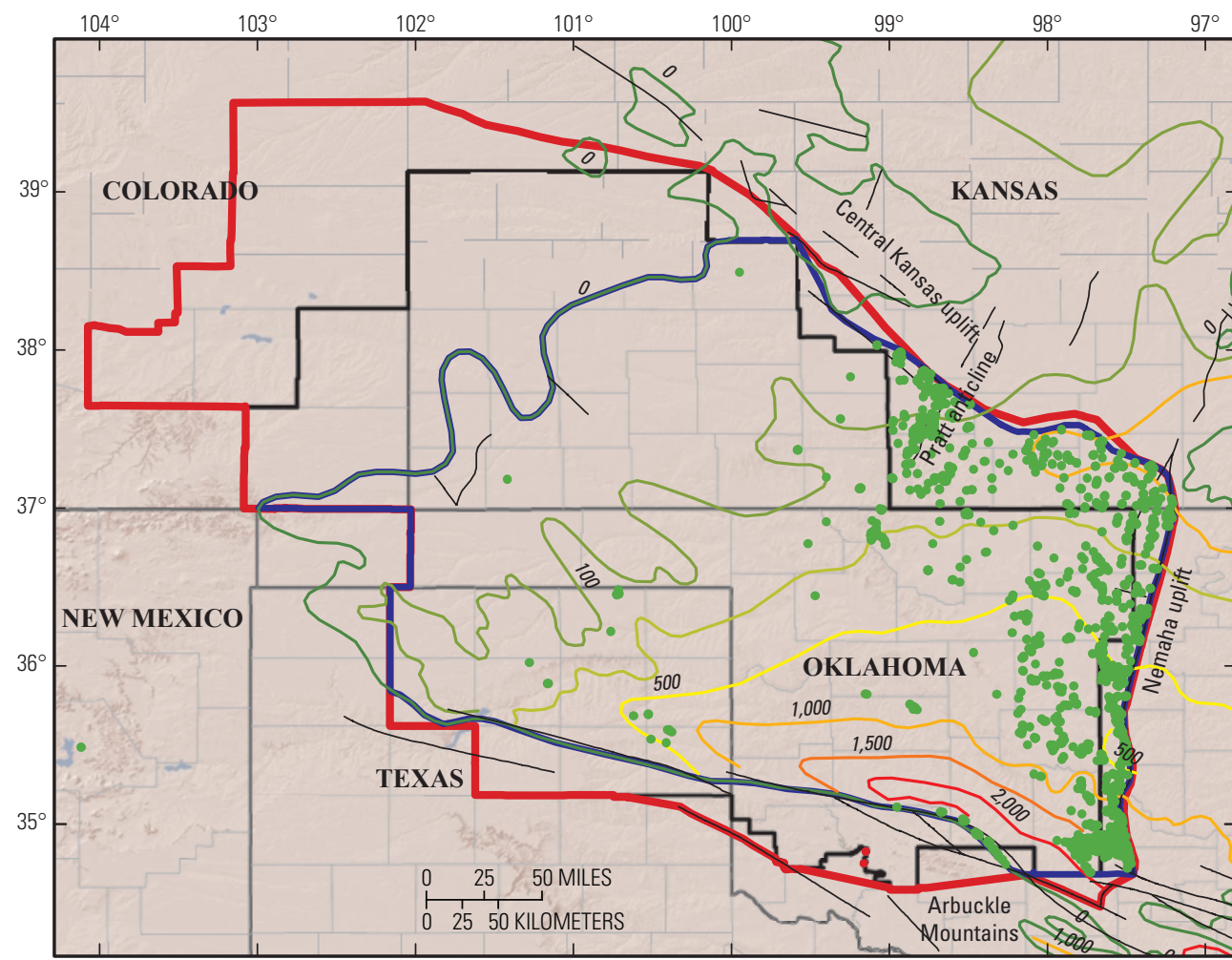

\section{EXPLANATION}

- Simpson Group Production

- Fault

Simpson Group Thickness, in feet

$-0=1,000$

$-100-1,500$

$-250-2,000$

Simpson Group AU boundary

Woodford Composite TPS boundary

- Anadarko Basin Province boundary

Figure 18. Map showing production from and thickness of the Simpson Group; contour interval is 500 feet.

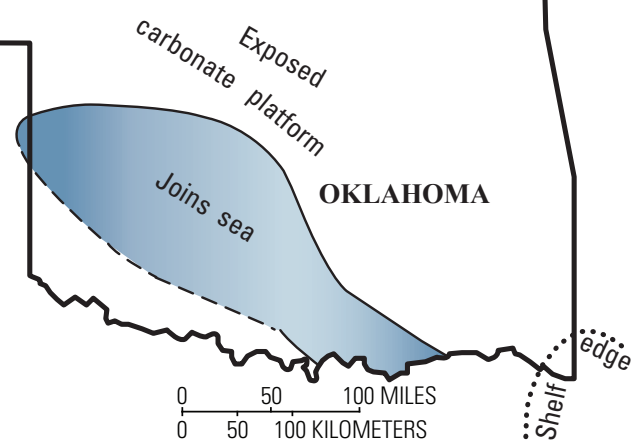

Figure 19. Image showing approximate distribution of the Joins sea during Ordovician time (modified from Denison, 1997). 


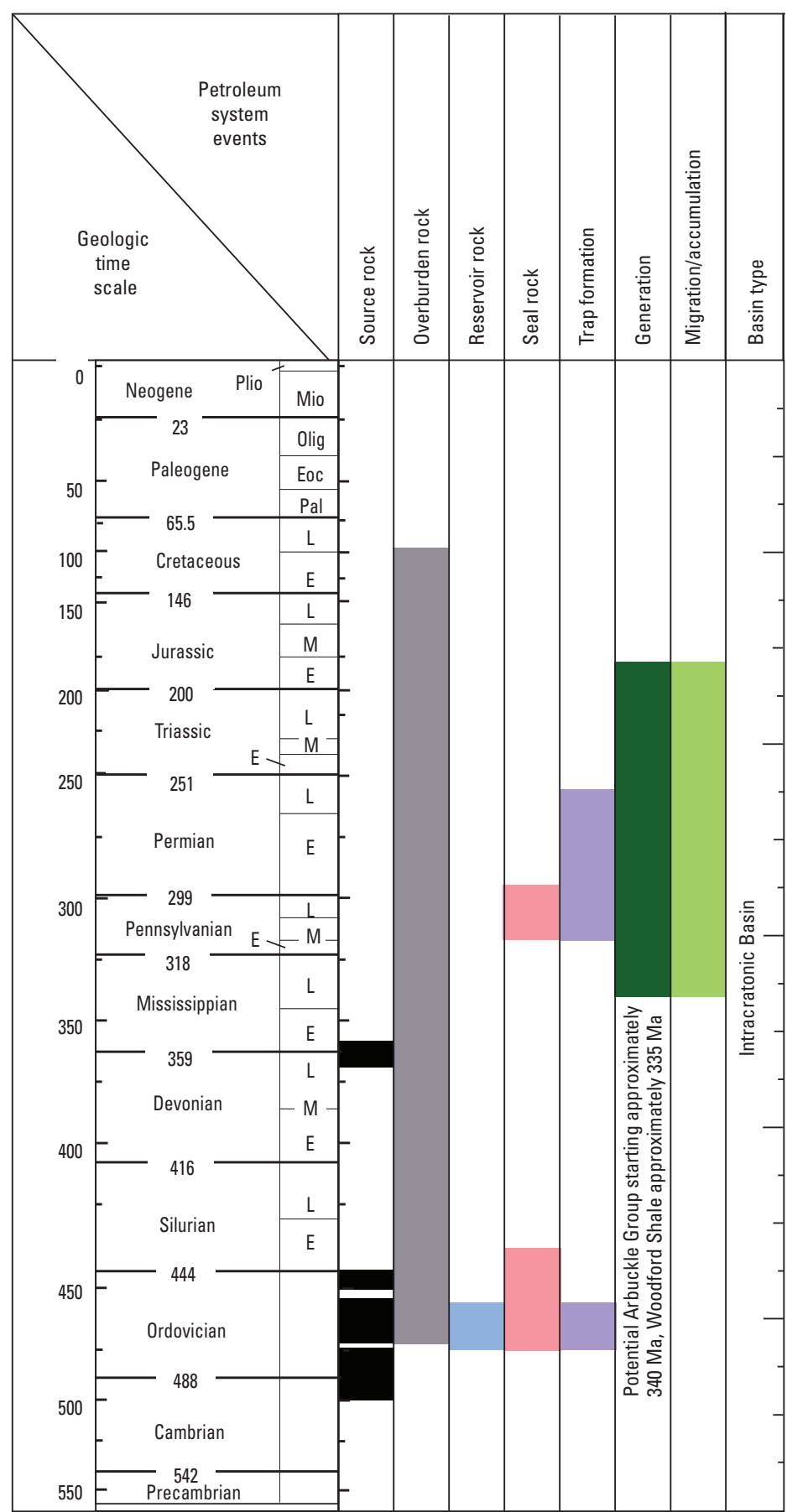

Figure 20. Simpson Goup Assessment Unit events chart showing the timing of source rock deposition and trap formation, and the age of overburden, reservoir, and seal rocks with different color bars (black, source rock; gray, overburden rock; blue, reservoir rock; pink, seal rock; purple, trap formation; dark green, generation; light green, migration accumulation). The chart also depicts the timing of oil generation, migration and accumulation as modeled for various wells in different parts of the Anadarko Basin. Plio, Pliocene; Mio, Miocene; Olig, Oligocene; Eoc, Eocene; Pal, Paleocene; L, Late; M, Middle; E, Early. 


\section{Sizes and Numbers of Undiscovered Fields in the Simpson Group Assessment Unit}

The Simpson reservoirs are of limited lateral and vertical extent, but important enough to enable individual wells to produce more than 1 MMBO (Northcutt and Johnson, 1997). Production is commonly commingled with other reservoirs, especially reservoirs in the Viola Group. Nehring accumulation data (Nehring Associates, Inc., 2009) indicates that individual fields in the Simpson produce approximately 0.5 to 8.0 MMBO in the basin. There is limited gas data available. There has been substantial penetration of the Simpson Group in south-central Kansas and near the Nemaha uplift, and scattered sporadic drilling throughout the remainder of the AU (fig. 21). There has been little drilling in the deep basin, near the basin depocenter.

Cumulative oil production in the Simpson AU is 470 MMBO (IHS Energy Group, 2010). There is oil production throughout the basin, whereas gas production is limited to the deeper part of the basin, with 1.2 TCFG produced (IHS Energy, 2010; fig. 21). Production depths range from 3,000 to $17,400 \mathrm{ft}$ in the structurally deepest part of the basin (fig. 21). There is an overlap in the oil and gas production, with production concentrated on the eastern and southern parts of the basin, and accumulations split between oil and gas.

The Nehring database (Nehring Associates, Inc., 2009) lists 105 Simpson accumulations, but only 18 have accumulation data (17 oil and 1 gas; fig. 22). As with the Arbuckle, this is likely because of the reporting of commingled production, or the erroneous reporting of the producing formation. Nehring field data (Nehring Associates, Inc., 2009) were used to supplement the database, but field data are of marginal use because it includes all producing reservoirs for a field, not just the Simpson Group.

Using the USGS assessment methodology for undiscovered conventional resources (Klett and others, 2005; Schmoker and Klett, 2005), we estimated the mean undiscovered oil resources for conventional reservoirs in the Simpson Group AU at $5 \mathrm{MMBO}$ and 125 BCFG (table 1). These estimates are based partly on data for 18 discovered accumulations that exceeded the minimum size of $0.5 \mathrm{MMBO}$ (Nehring Associates, Inc., 2009). The distribution of the number of undiscovered oil accumulations is estimated to have a minimum of 1 , a median of 3 , and a maximum of 10 . The estimated minimum, median, and maximum numbers of undiscovered gas accumulations exceeding the minimum size are 3,6, and 60, respectively. The Simpson has been penetrated extensively on the shelf near the Oklahoma-Kansas border, along the Nemaha uplift, and on other structures in the basin (fig. 21). There are also dry holes and wells with minor production in the Texas Panhandle and into southcentral Kansas, where the Simpson becomes dominated by chert (Doveton and others, 1990; Charpentier and Doveton, 1991). There is more undrilled area in the deep basin along structures in the same region where the Arbuckle is sparsely drilled. Future oil and gas production is likely limited on the shelf, as it has been extensively explored. The most recent Simpson discoveries were in the mid-1980s, suggesting that the interest and potential in the Simpson is waning.

The estimated minimum, median, and maximum sizes of undiscovered oil accumulations are 0.5, 0.8, and 8.0 MMBO. The $0.5 \mathrm{MMBO}$ default signifies that there will be at least one field discovered greater than the minimum size, and the maximum reflects the maximum size of a potential undiscovered oil accumulation, and the largest grown oil field reported (Nehring Associates, Inc., 2009). Oil production per field for the Simpson is generally less than $2 \mathrm{MMBO}$. Estimated minimum, median, and maximum sizes of undiscovered gas accumulations are 3 , 6 , and $60 \mathrm{BCFG}$. The maximum number reflects the uncertainty of deep gas production in the Simpson, similar to the ArbuckleEllenburger, as both are sparsely drilled in the deep basin.

\section{Viola Group}

Simpson Group sandstones and limestones are overlain by the Upper Ordovician limestones of the Viola Group. Following a brief marine withdrawal, Viola seas transgressed over the exposed upper Bromide carbonates of the Simpson Group, and deposition was in a broad, shallow, epicontinental sea (Denison, 1997). The Viola is a marine limestone sequence deposited on a carbonate platform (figs. 4 and 23), and is widespread in the Anadarko Basin. It contains chert at several stratigraphic levels and is highly fossiliferous (Johnson and others, 2000). There is terrigenous detritus in the lower Viola in the aulacogen, and the strata grade upward into clean, skeletal limestones (Johnson, 1991). The vertical change indicates an upward decrease in water depth and corresponding increase in energy level and aerobic activity (Johnson and others, 2000). Viola strata are 500-800 ft thick in the aulacogen, and thin to $50-500 \mathrm{ft}$ on the shelf (fig. 24). The rift margin played an important role in the thickness and facies of the Viola carbonates. The Viola Group is absent in the northern and western parts of the basin (fig. 24).

In the southern Anadarko Basin, the Viola Group consists of the lower Viola Springs Formation and the upper Welling Formation (fig. 2), and represents a shallowing-upward sequence (Denison, 1997). Within the aulacogen, the Viola Springs comprises organic-rich, finely laminated lime mudstones deposited in deeper water, and the Welling is pelmatozoan-rich grainstones deposited in shallow water (Denison, 1997). Outside the aulacogen, the basal Viola Springs lithology is packstone and wackestone.

Viola carbonate deposition ended abruptly as the system was flooded with clays of the overlying Sylvan Shale. The Sylvan is a widespread green to greenish-gray shale sequence that ranges from 300 to $400 \mathrm{ft}$ thick in the aulacogen and from 30 to $200 \mathrm{ft}$ thick in most shelf areas (Johnson and Cardott, 1992; fig. 25). The formation was included in the Viola Group AU for this assessment, although there is sparse, insignificant production despite numerous penetrations (fig. 25). Minor accumulations attributed to the Sylvan Shale by IHS Energy (2010) are likely erroneously reported and are from other units. 


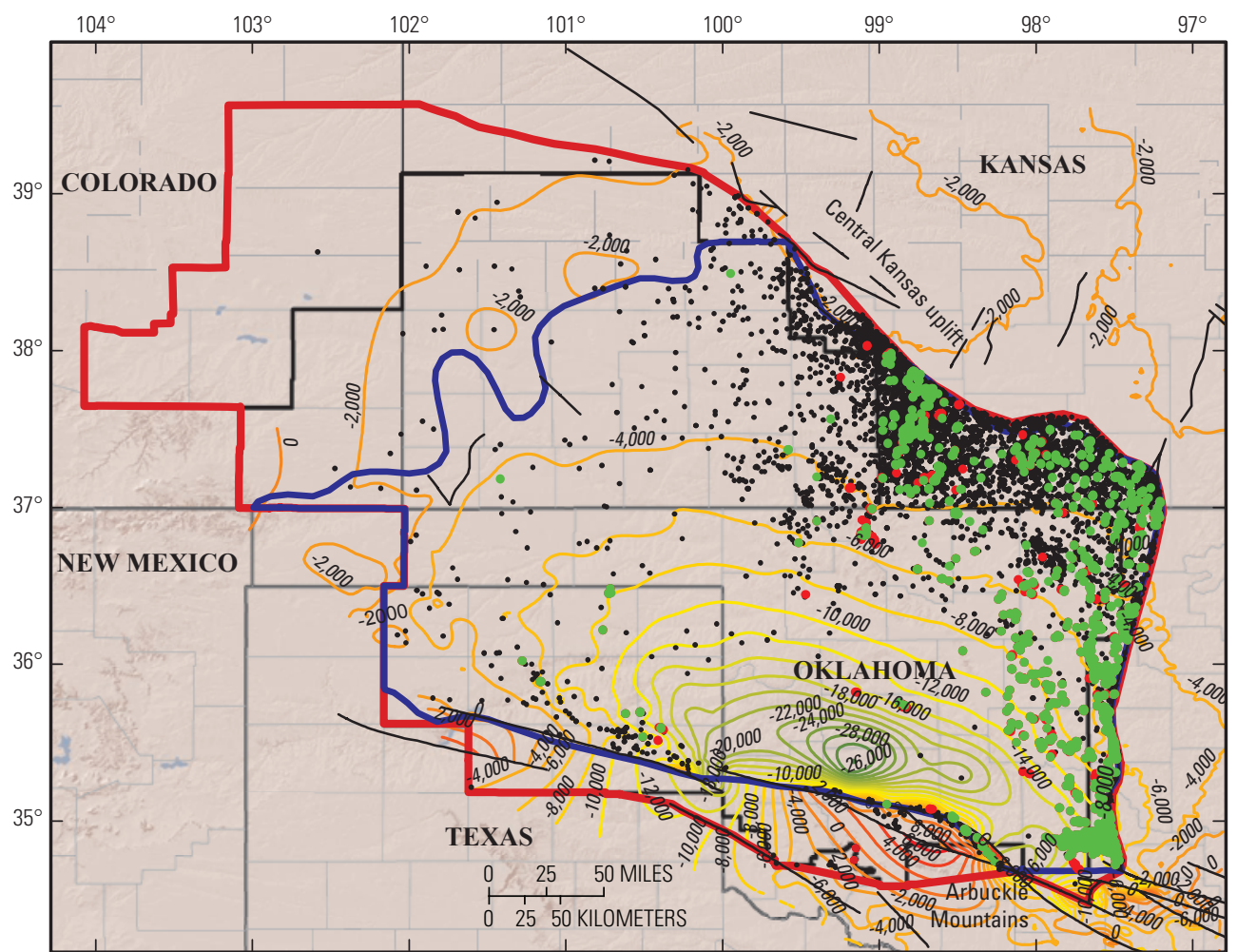

Figure 21. Map showing oil and gas production and dry hole penetrations from the Simpson Group in the Anadarko Basin Province (IHS Energy Group, 2010). The structure contours are drawn on the top of the Simpson Group; contour interval is 2,000 feet. The Anadarko Basin Province boundary is the black line, the Woodford Composite Total Petroleum System (TPS) boundary the red line, and the Simpson Group Assessment Unit (AU) Boundary the blue line.
EXPLANATION

- Simpson Group dry holes - Fault

Simpson Group AU boundary Woodford Composite TPS boundary

- Anadarko Basin Province boundary

- Simpson Group oil production

- Simpson Group gas production

Simpson Group Top, elevation in feet

$-28,000--14,000-0$

$-26,000-12,000-2,000$
$-2,000$

$-24,000--10,000-4,000$

$-22,000-8,000-6,000$

$-18,000+-4,000$

$-16,000--2,000$

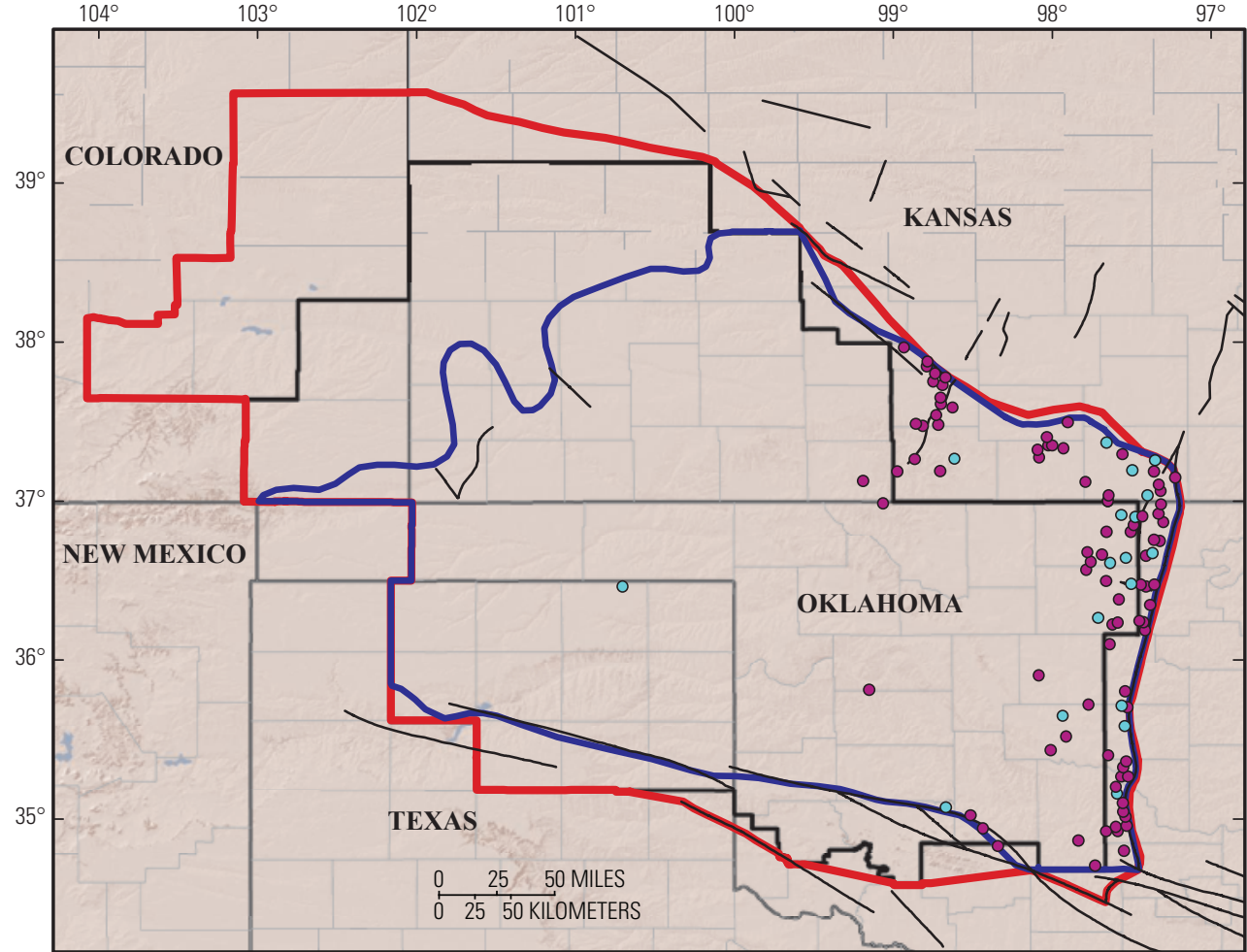

\section{EXPLANATION}

- Fault

- Simpson Group AU boundary

Woodford Composite TPS boundary

- Anadarko Basin Province boundary

- Simpson Group Nehring accumulations without data

- Simpson Group Nehring accumulations with data

Figure 22. Map showing accumulations for the Simpson Group Assessment Unit (AU) (Nehring

Associates, Inc., 2007).

Nehring accumulations with grown reserve accumulation numbers are shown in light blue. Accumulations without numbers are in purple. TPS, Total Petroleum System. 


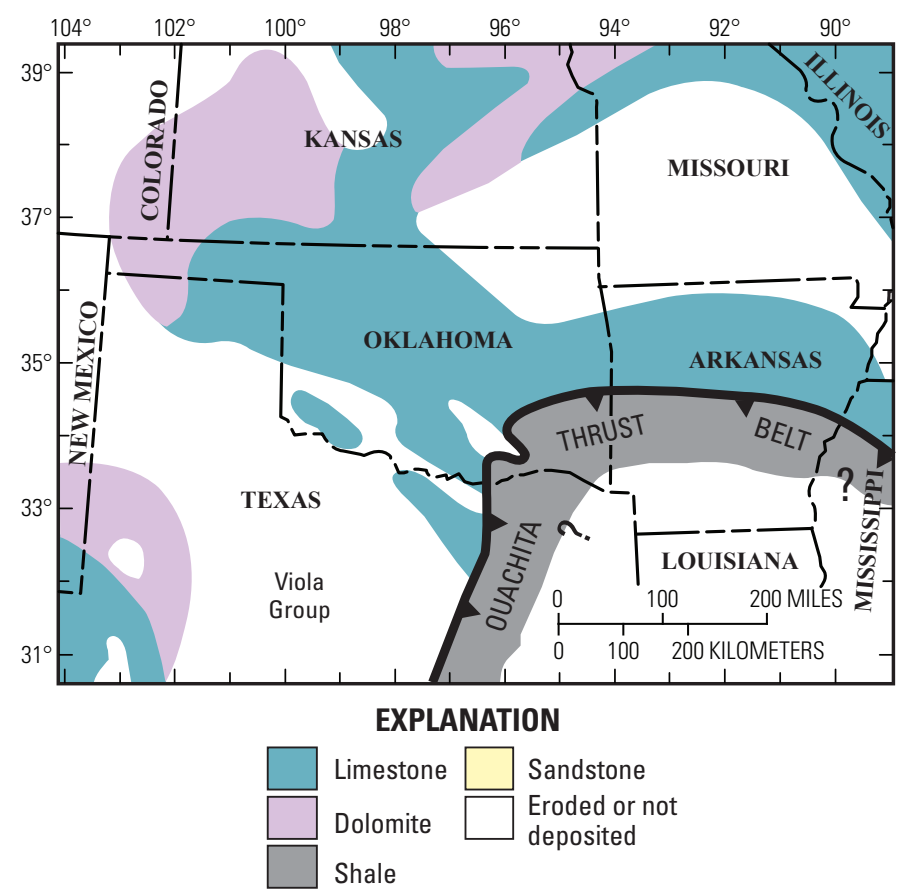

Figure 23. Image showing major lithologies of the Ordovician Viola Group strata in the southern midcontinent (modified from Northcutt and Johnson, 1997).

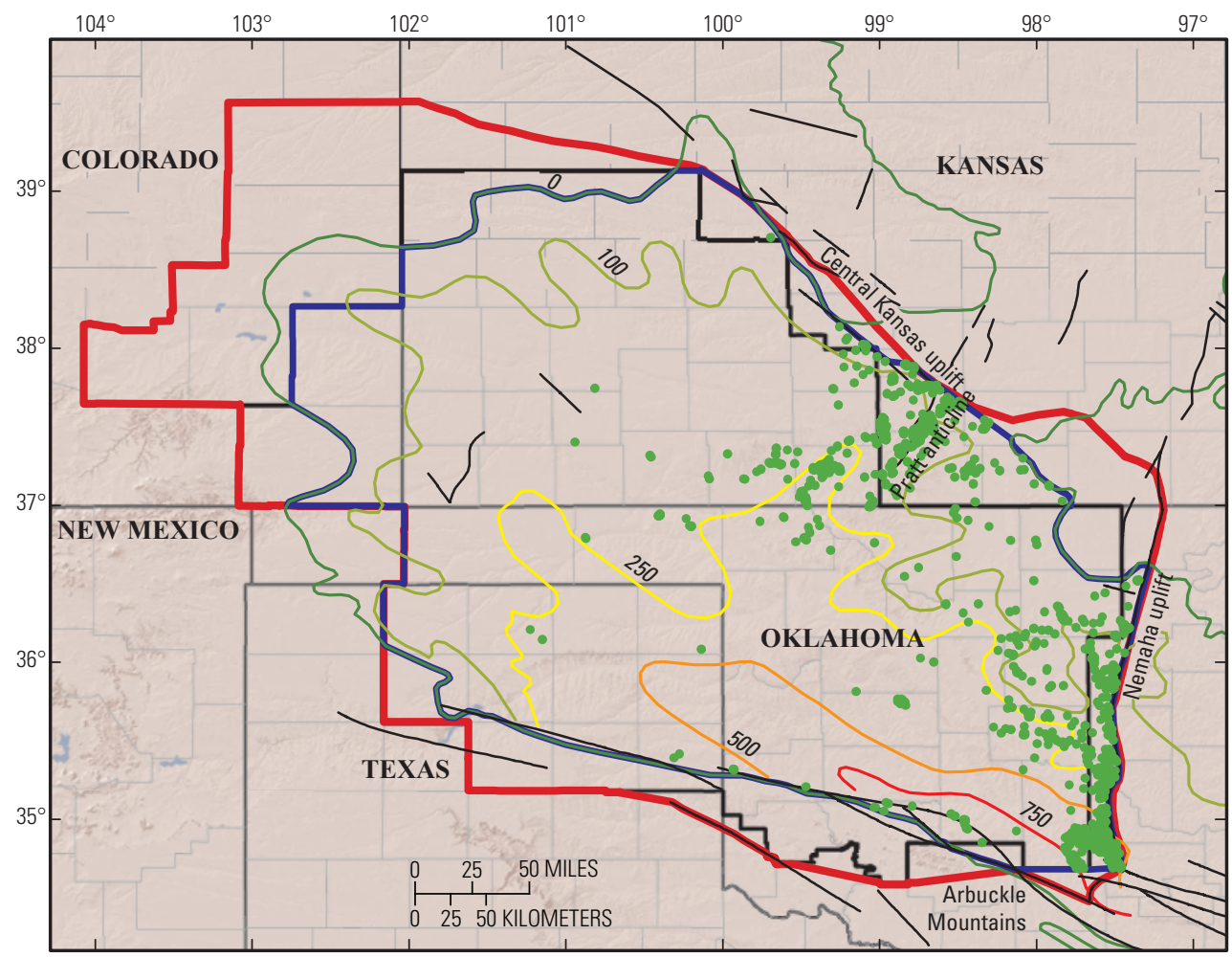

- Fault EXPLANATION

Viola Group AU boundary

Woodford Composite TPS boundary

- Anadarko Basin Province boundary

- Viola Group production

Viola Group thickness, in feet -0
$-100-500$
-250

Figure 24. Map showing production from and thickness of the Viola Group; contour interval is variable. 


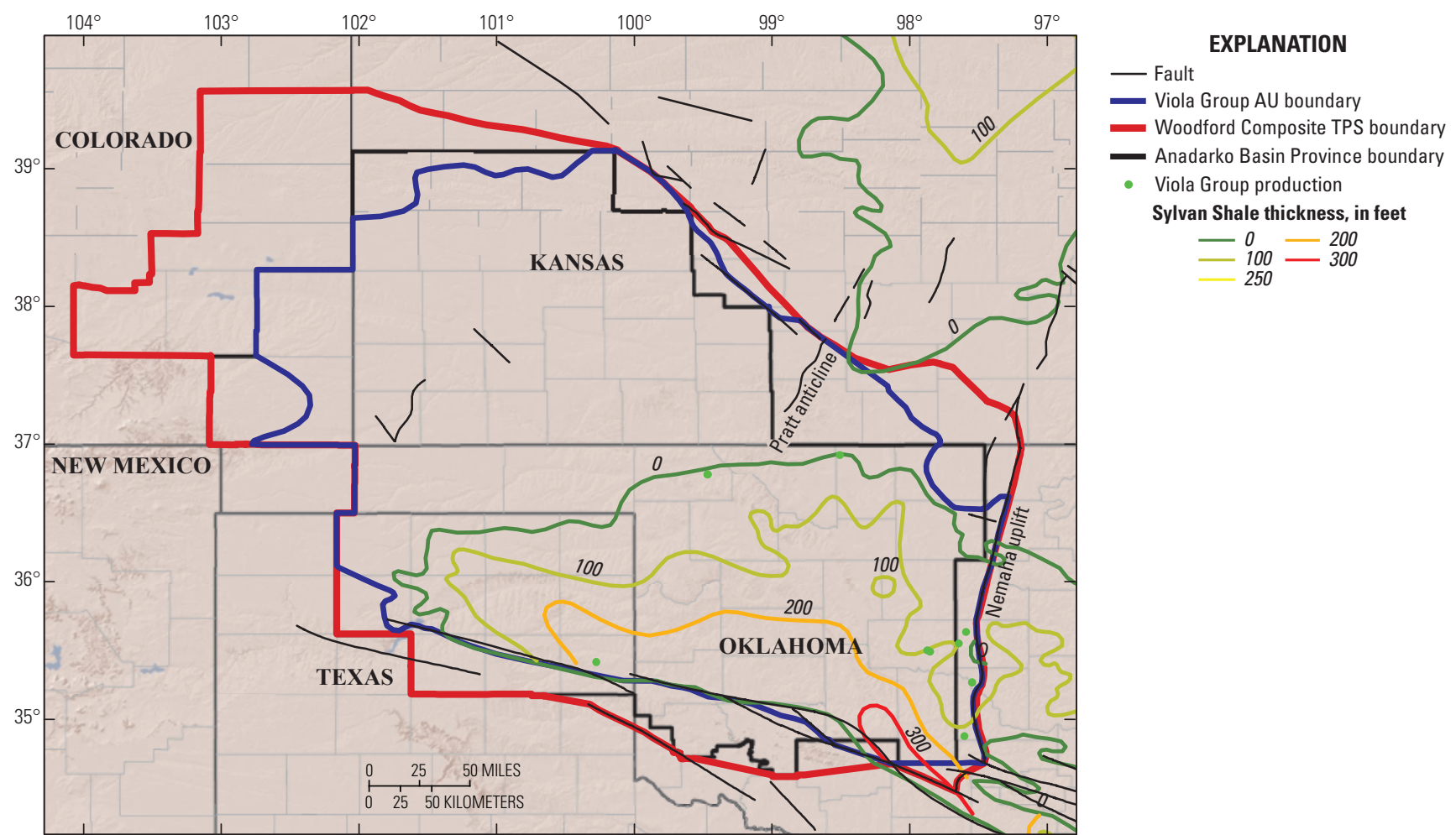

Figure 25. Map showing production from and thickness of the Sylvan Shale; contour interval is 100 feet.

\section{Source Rocks of the Viola Group Assessment Unit}

The Woodford Shale is the apparent source rock for Viola reservoirs, though there may be some self-sourcing as well as contributions from other Ordovician source rocks, such as the Simpson (figs. 7 and 26). Thin source beds that were sampled in the lowermost Viola Springs Formation have an average TOC of approximately 1 percent, with Type II kerogen (Denison, 1997; Wang and Philip, 1997). However, these data were disputed by Brown and Sentfle (1997) as a consequence of other studies indicating higher organic contents within the laminated marls of the Viola (average TOC of 2.4 percent) and basal chert (average TOC of 1.3 percent; see Wavrek and others, 1997). The Viola was reported as a source rock for oil in the Pauls Valley area of the Anadarko Basin by Jones and Philip (1990).

Data on the Sylvan Shale indicate that the source-rock potential is poor to moderate (Johnson and Cardott, 1992). The Sylvan contains Type II kerogen with low TOC (less than 1 percent; Burruss and Hatch, 1989).

\section{Reservoir Rocks in the Viola Group Assessment Unit}

Viola reservoirs are in thick sequences of limestones and dolomites that have been subjected to fracturing and dissolution, with karst features and zones of vuggy porosity. The Viola ranges from about 4,000 to $13,000 \mathrm{ft}$ in depth in the basin (fig. 27). Karstification occurred following Sylvan and Hunton deposition when the Viola was exhumed in southern Oklahoma (Sykes and others, 1997). Karst features include conduit-filling breccias, solution enlarged fractures, crackle breccias, vugs, and channels (Sykes and others, 1997). Surface karstic features are also common. Selective dolomitization also enhanced porosity and reservoir quality in the Viola.

Reservoirs of the Viola Group may have primary and secondary porosity, and fracture systems are developed as a function of the brittle nature of the carbonate. The highest porosity is in intercrystalline, moldic, and vuggy subtidal dolomitic mudstones and wackestones (Newell, 2000). Porosity averages 8-10 percent, but can be as high as 23 percent in dolomitized reservoirs in south-central Kansas (Newell, 2000). 
The Viola is absent in the northern and western Anadarko Basin, and the subcrop and province boundary define the assessment unit in those parts of the basin (fig. 24). The AU boundary is defined by the faults that delineate the southern end of the Central Kansas uplift in the northeast, the Nemaha uplift on the east, and the Precambrian fault system along the Arbuckle Mountains to the south (fig. 24).

\section{Traps and Seals in the Viola Group Assessment Unit}

Viola accumulations in the Anadarko Basin are commonly in structural or combination traps near the Central Kansas uplift and on the Nemaha uplift where the Viola is overlain by the Sylvan Shale or unconformably by Pennsylvanian shales (Carlson and Newell, 1997). The structural traps are in the same areas as the Simpson Group reservoirs. Production trends in south-central Kansas are in areas where dolomitized units are preserved on the crests of local anticlines (Newell, 2000). There is substantial Viola production in Fitts pool, to the southeast of the Anadarko Basin, on the western edge of the Arkoma Basin, where the structure is a large, faultbounded anticlinal fold. Stratigraphic variations resulting from localized movement during deposition can provide north-south closure for these structural traps. Stratigraphic traps may also be present where porous wedges of dolomitized Viola are enclosed by nonporous packstone and grainstone beds.

The primary seals in the Viola Group are the overlying Sylvan Shale, which has low porosity and permeability, or interbedded tight, impermeable limestone of the Viola (fig. 26). Oil and gas fields are also found where Viola reservoirs subcrop beneath the basal Pennsylvanian angular unconformity in south-central Kansas (Newell, 1997).

\section{Sizes and Numbers of Undiscovered Fields in the Viola Group Assessment Unit}

The Viola Group is not considered as significant a reservoir as the Simpson Group, though it yields oil and gas from thick sequences of carbonates that are fractured and have solution-enhanced porosity (Northcutt and Johnson, 1997). Production is from locally dolomitized sections on structures tested for Simpson and Arbuckle production. Most of the production is in the eastern basin, along the Nemaha uplift and near the Central Kansas uplift, where the Simpson Group produces (figs. 24 and 27).

The Viola has been extensively penetrated along the eastern and northern basin boundaries (fig. 27). Production is commonly commingled with other reservoirs, and formation water is produced with the oil. Hydraulic fracturing of the Viola reservoirs have been important to reservoir development (Johnson and others, 2000). The Viola has fair to good matrix porosity, but low permeability in many areas of the basin, requiring fracturing for production. The Viola is being pursued as a horizontal drilling candidate (Fritz and others, 1993).
There is little reservoir information reported for the Viola Group in the Nehring database (Nehring Associates, Inc., 2009); of the 29 accumulations only 1 has reported oil accumulation data (fig. 28). Cumulative oil production from the Viola Group AU is 112 MMBO (IHS Energy, 2010). Cumulative gas production is 505 BCF (IHS Energy, 2010). Production depths range from 4,000 to $18,000 \mathrm{ft}$ in the deepest basin. There is an overlap in the oil and gas production, with both concentrated on the eastern and northeastern parts of the basin (fig. 27).

Using the USGS assessment methodology for undiscovered conventional resources (Klett and others, 2005; Schmoker and Klett, 2005), it is estimated that the undiscovered oil and gas resources for conventional reservoirs in the Viola Group AU have a mean of $5 \mathrm{MMBO}$ and $30 \mathrm{BCFG}$ (table 1). The estimated minimum, median, and maximum numbers of (1) undiscovered oil accumulations exceeding the minimum size (0.5 MMBO) are 1, 2, and 10; and (2) undiscovered gas accumulations exceeding the minimum size ( 3 BCFG) are 1, 2, and 10. Like the Simpson, the Viola has been penetrated extensively on the shelf near the Oklahoma-Kansas border, along the Nemaha uplift, and on other structures in the basin (fig. 27). There is undrilled area in the deep basin along structures in the same region where the Arbuckle and Simpson are sparsely drilled. Future oil and gas production is likely limited on the shelf, as it has been extensively explored along the structures. The maximum number of oil and gas accumulations was raised to account for possible deep basin drilling. The most recent Viola discoveries were in 2000 , though no data were reported by Nehring Associates, Inc. (2009) for these fields.

The estimated minimum, median, and maximum sizes of undiscovered oil accumulations are 0.5, 1.0, and 5.0 MMBO. The $0.5 \mathrm{MMBO}$ default signifies that there will be at least one field found greater than the minimum size, and the maximum reflects the maximum size of a potential undiscovered oil accumulation, based on field data, as there is only one oil accumulation reported for the Viola Group AU (Nehring Associates, Inc., 2009). Oil production by field for the Viola is generally less than $3 \mathrm{MMBO}$. The distribution of sizes of undiscovered gas accumulations was estimated to have a minimum of 3 BCFG, a median of 6 BCFG, and a maximum of $30 \mathrm{BCFG}$. The maximum reflects the uncertainty with deep gas production in the Viola Group.

\section{Hunton Group}

The Silurian-Devonian was a time of widespread marine-carbonate deposition in the Midcontinent, and strata were deposited as a relatively thin veneer of limestones and dolomites in a ramp environment (fig. 29). Sea-level changes caused the migration of facies and generated the lateral distribution and vertical succession of carbonate strata (Al Shaieb and Puckette, 2002). The Hunton Group is a sequence of limestones, dolomites, and lesser clastic rocks of Late Ordovician to Early Devonian age (Al Shaieb and Puckette, 2002; fig. 2). Several distinct depositional environments are interpreted 


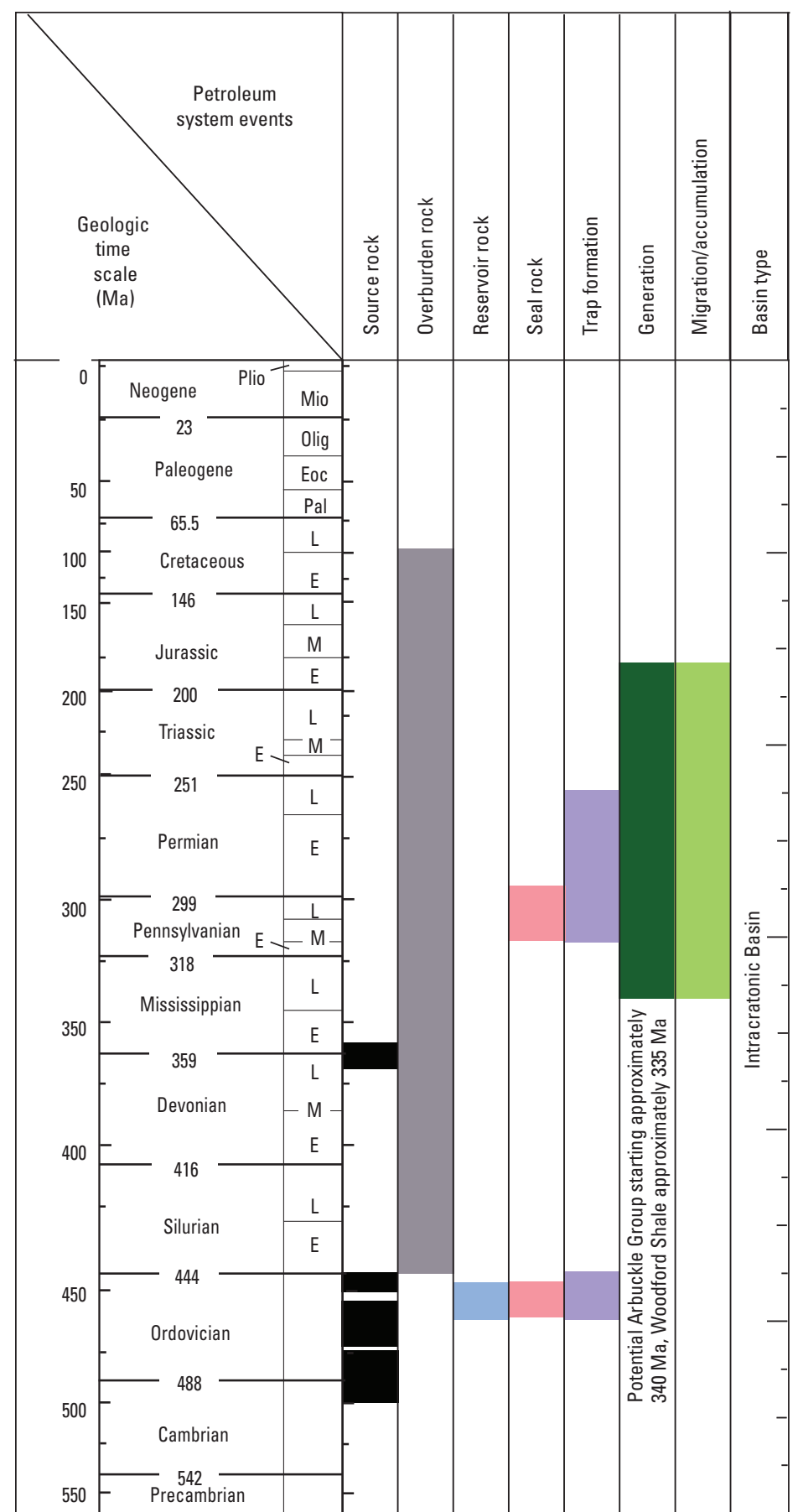

Figure 26. Viola Group Assessment Unit events chart showing the timing of source rock deposition and trap formation, and the ages of overburden, reservoir, and seal rocks with different color bars (black, source rock; gray, overburden rock; blue, reservoir rock; pink, seal rock; purple, trap formation; dark green, generation; light green, migration accumulation). The chart also depicts the timing of oil generation, migration and accumulation as modeled for various wells in different parts of the Anadarko Basin. Ma, mega-annum; Plio, Pliocene; Mio, Miocene; Olig, Oligocene; Eoc, Eocene; Pal, Paleocene; L, Late; M, Middle; E, Early. 


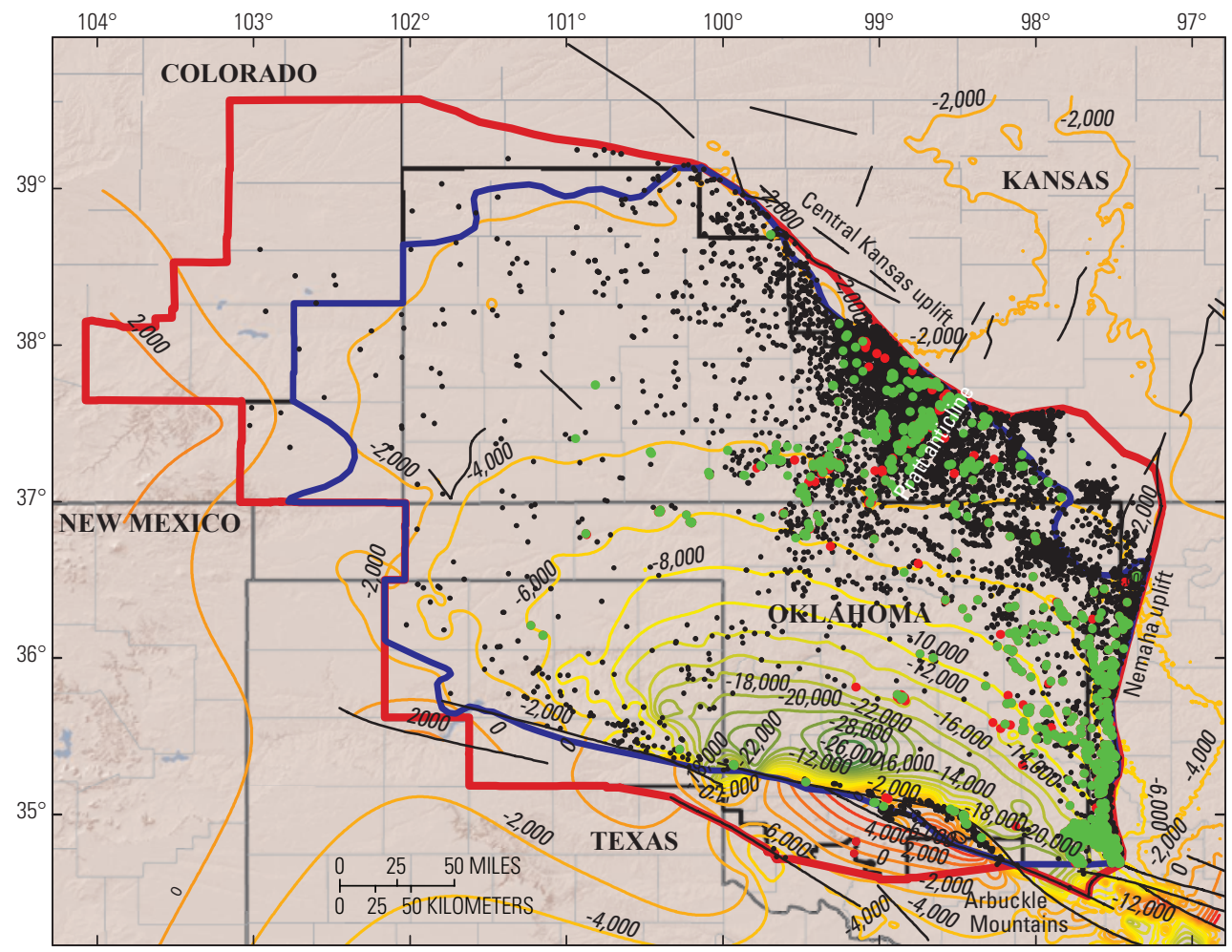

Figure 27. Map showing oil and gas production and dry hole penetrations for the Viola Group in the Anadarko Basin Province (IHS Energy Group, 2010). Structure contours are drawn on the top of the Viola Group; contour interval is 2,000 feet. The Anadarko Basin Province boundary is the black line, the Woodford Composite Total Petroleum System (TPS) boundary the red line, and the Viola Group Assessment Unit (AU) boundary the blue line.

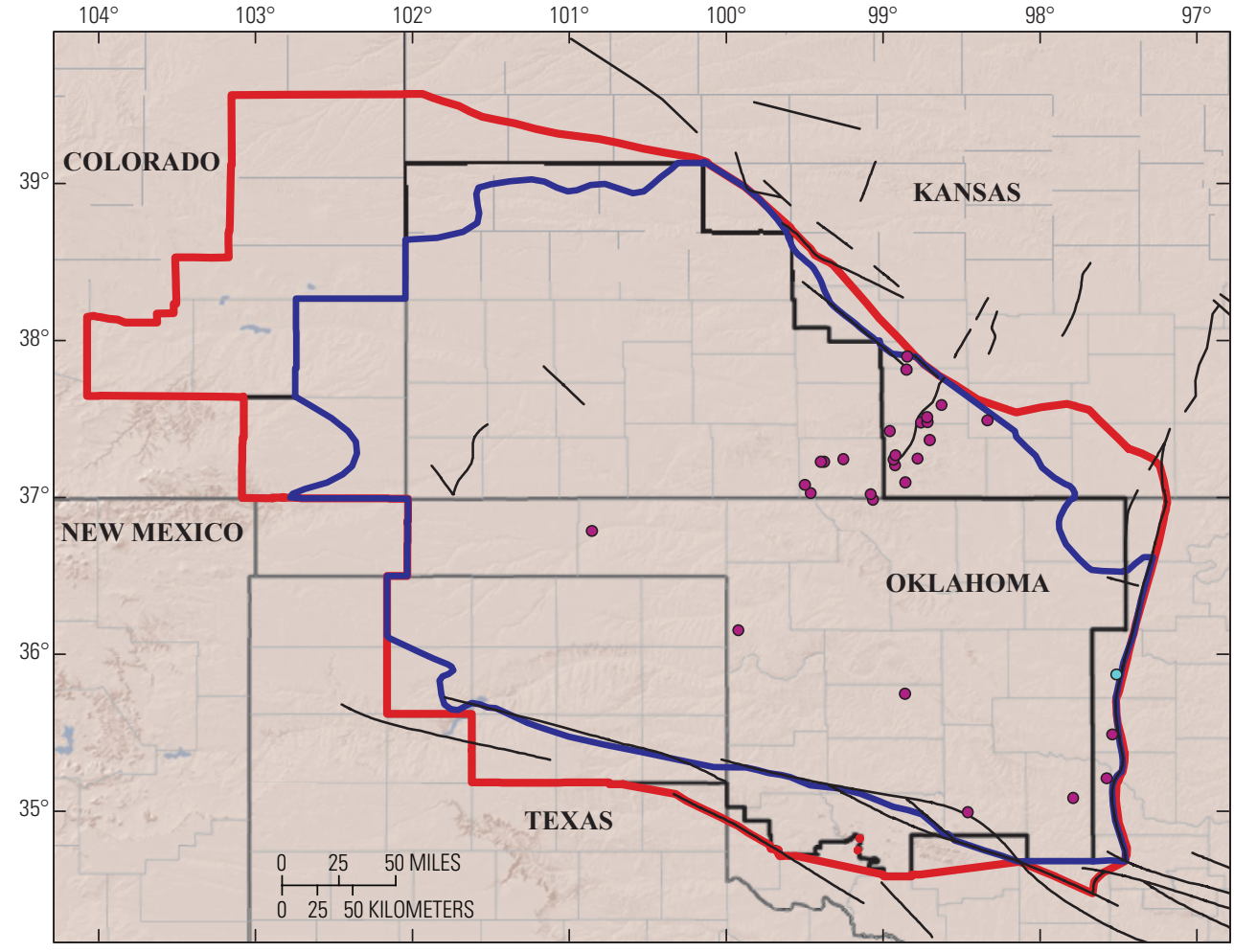

\section{EXPLANATION}

- Viola Group dry holes

- Precambrian fault

- Viola Group AU boundary

Woodford Composite TPS boundary

- Anadarko Basin Province boundary

- Viola Group oil production

- Viola Group gas production

Viola Group Top, elevation in feet above sea level

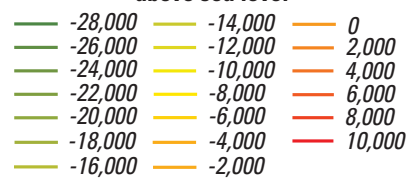

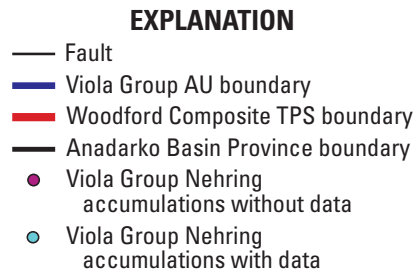

Figure 28. Map showing accumulations for the Viola Group Assessment Unit (AU) (Nehring Associates, Inc., 2007). Nehring accumulations with grown reserve accumulation numbers are shown in light blue. Accumulations without numbers are in purple. TPS, Total Petroleum System. 


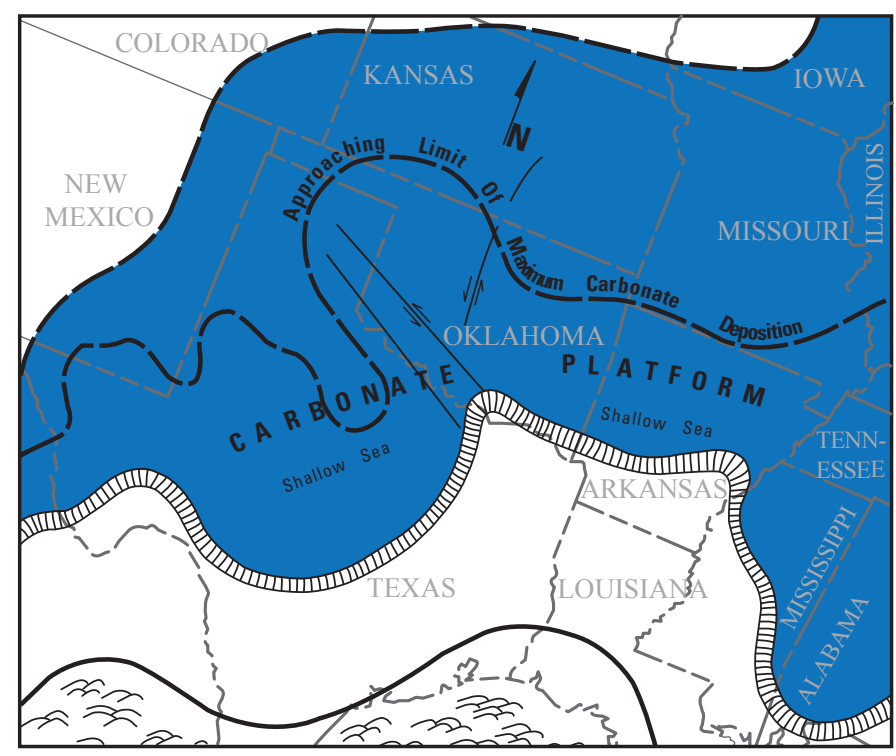

Figure 29. Image showing depositional setting during Hunton time in the midcontinent (modified from Fritz and Medlock, 1994).

from Hunton strata, including upper intertidal to supratidal, upper subtidal to lower intertidal, and subtidal facies (Fritz and Medlock, 1994; fig. 30). These facies are in shallowingupward cycles or parasequences. The Hunton comprises a series of progradational sequences that built generally southward across the carbonate ramp (Fritz and Medlock, 1994); predominantly subtidal rock, bioclastic, and oolitic facies that are less argillaceous and more bioturbated than the Arbuckle Group, the Hunton Group is divided into a number of formations (fig. 31). The basal unit in the southern Anadarko Basin of Oklahoma is the Ordovician Keel Formation, which resulted from eustatic shoaling of sea water with no significant terrigenous sedimentation, and has patchy distribution in Oklahoma (Johnson, 1991). The Keel is part of the informal Chimneyhill subgroup, which also contains the overlying Silurian Cochrane and Clarita Formations, which are dolomitic limestones and dolomite (fig. 31). The clean skeletal limestones and dolomites of the Chimneyhill are overlain by the argillaceous and silty limestones and dolomites of the Silurian Henryhouse and Silurian-Devonian Bois d'Arc Formations (Haragan Formation equivalent). The Henryhouse reservoirs are dolomitized intertidal facies (Al Shaieb and Puckette, 2002). In central and southern Oklahoma, they are overlain by the Devonian Frisco Formation (fig. 32). The Frisco Formation consists of skeletal packstone and grainstones, whose main components are pelmatozoans, brachiopods, and local corals (Morgan and Schneider, 1981). There are several intra-Hunton unconformities, including the Silurian-Devonian contact, and at the base of the Frisco Formation (fig. 31).

Following Hunton deposition, a pre-Woodford unconformity developed that is widespread in the Midcontinent (Johnson, 1989). This unconformity surface is one of the few in the Midcontinent in which the erosional geometry includes preserved incised channel features (Rottmann, 2000c).
The inundation of Devonian seas was initially confined to erosional channels, filling them with Woodford Shale and preserving them.

The Hunton conformably overlies the Sylvan Shale and is unconformably overlain by either the main body of the Woodford Shale or locally by the basal informal Misener sandstone of the Woodford, which is Late Devonian in age (fig. 2). The Misener was deposited on the post-Hunton unconformity in a marine environment, accumulating within preexisting fluvial channels (fig. 33). The sandstones are of the same source as the Simpson sandstones to the north and east, with the detritus being transported south as the early Woodford sea transgressed. The Misener is a fine-grained quartz sandstone with some dolomite, interbedded with Woodford-like shale.

The Hunton thickens from a wedge edge near the Kansas-Oklahoma border to more than 1,600 ft in Washita and Beckman Counties, Oklahoma, in the deepest part of the basin (fig. 34). The Hunton is typically 100 to $400 \mathrm{ft}$ thick on the northern shelf, where it is highly dolomitized (Johnson and others, 2000). The depositional extent of the Hunton has been modified by erosion (fig. 32).

\section{Source Rocks of the Hunton Group Assessment Unit}

The Hunton Group is not known to contain source rocks for hydrocarbons in the Anadarko Basin (Johnson and Cardott, 1992). However, the Woodford Shale, which lies unconformably on the Hunton (fig. 2) and has excellent source rock quality is considered to be the primary source for Hunton reservoirs throughout the basin (figs. 7 and 35). Vitrinite reflectance data indicates that the Woodford is mature for the generation of both oil and gas, especially in the deep basin (fig. 8).

Woodford hydrocarbons likely migrated up through the deep basin into Hunton reservoirs, although there is some indication from one-dimensional burial history models that there could be sourcing from the Woodford along the Nemaha uplift on the eastern edge of the basin (fig. 9). It is also likely that there is some contribution of Ordovician oil into the Hunton Group reservoirs as well, based on analyzed Hunton reservoir oils (J.R. Hatch, oral commun., 2010).

\section{Reservoir Rocks in the Hunton Group Assessment Unit}

The Hunton Group is a significant oil reservoir on the northern Anadarko Basin margin and a major gas producer in the deep (greater than 15,000 ft) basin (fig. 36). Reservoir development is largely facies dependent in the Hunton Group (Fritz and Medlock, 1994), though the wide range of depositional environments and diagenetic changes were also favorable for reservoir development. Typical facies for reservoir units are low-relief skeletal buildups and oolite shoals, both of which underwent some post-depositional dolomitization (Johnson and others, 2000). 

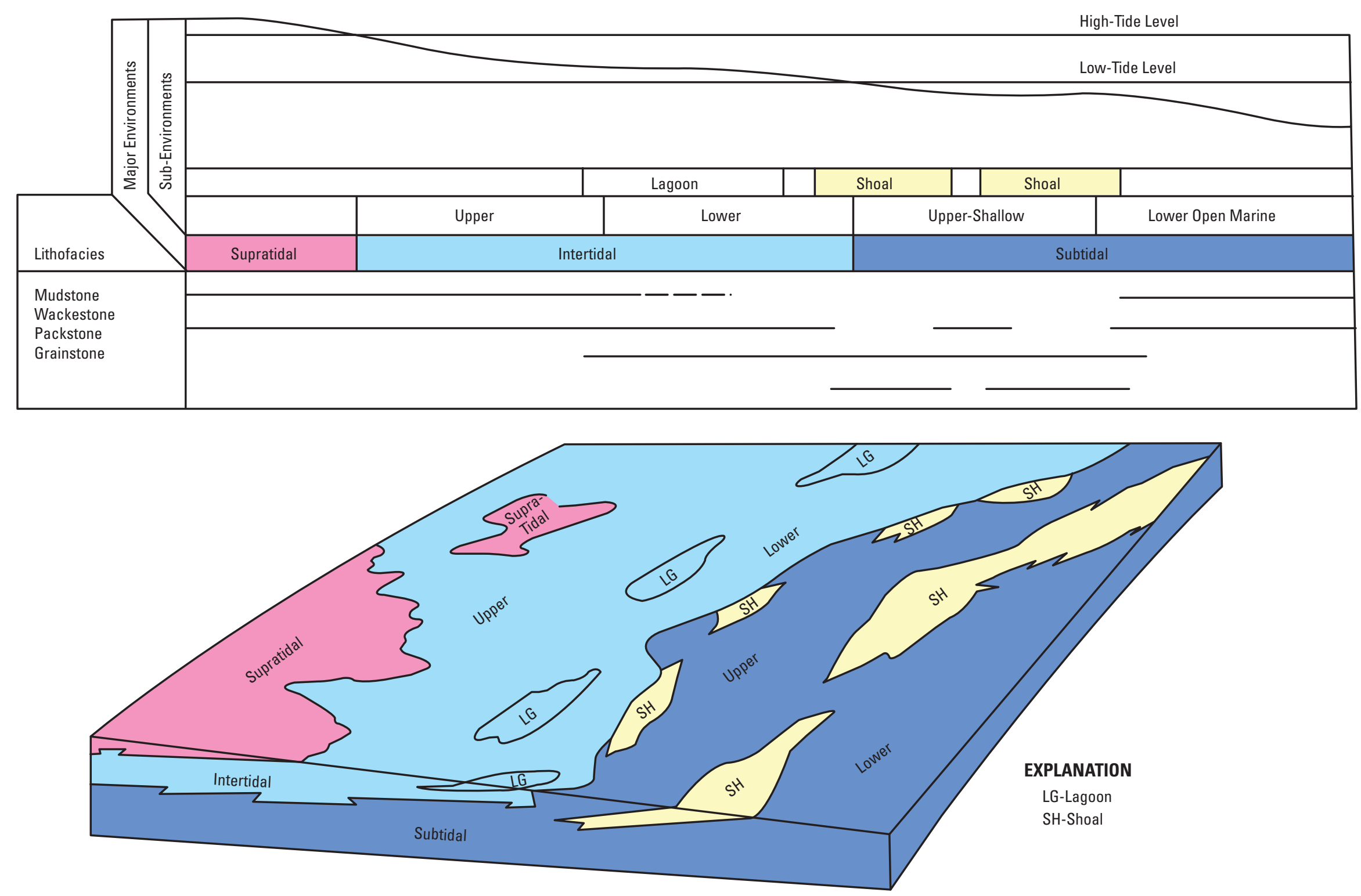

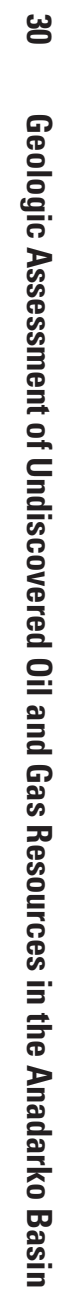

Figure 30. Depositional model for Chimneyhill subgroup through Bois d'Arc Formation strata of the Hunton Group (modified from Fritz and Medlock, 1994). 


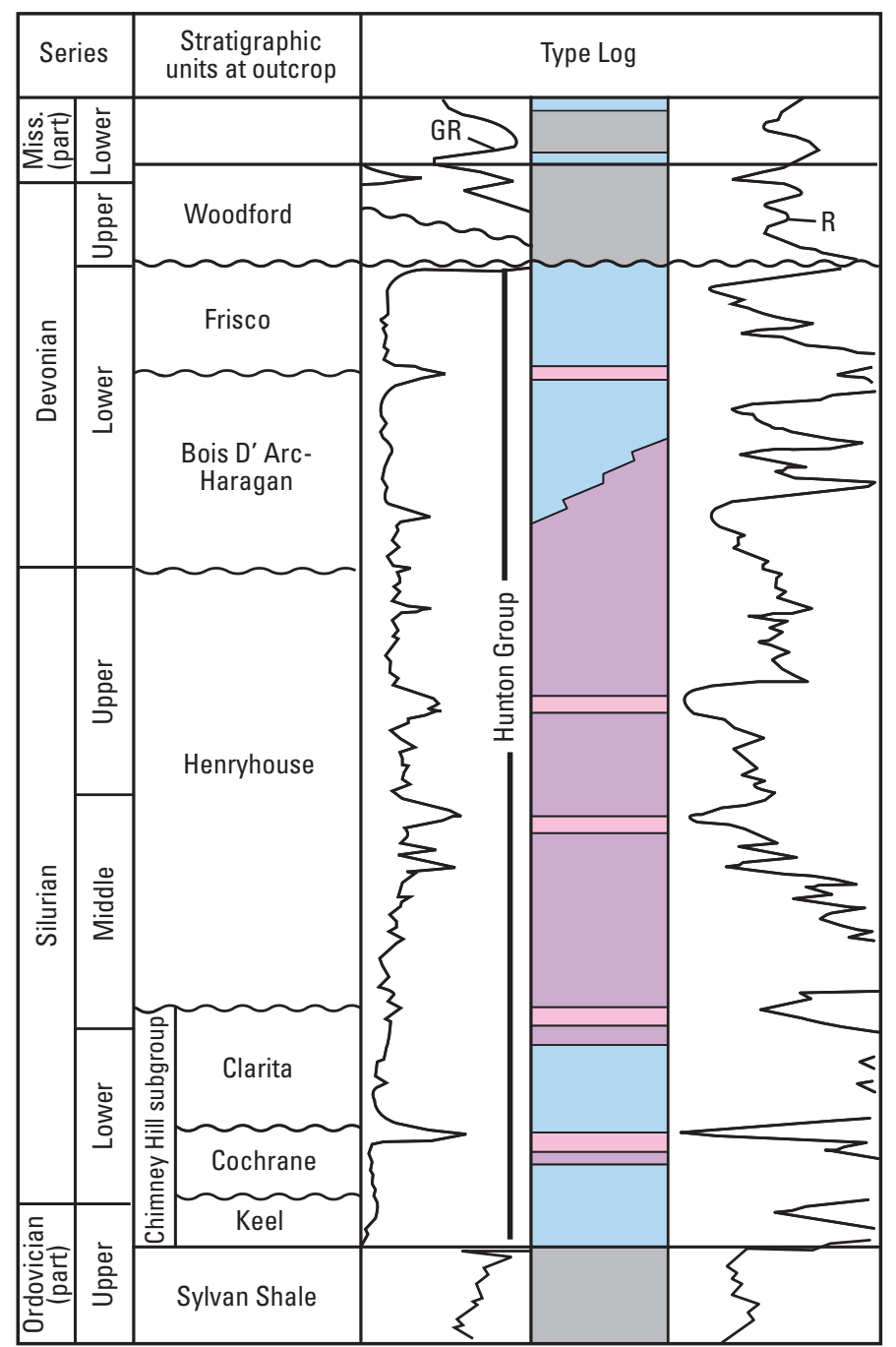

Figure 31. Type log of the Hunton Group in central Oklahoma; gray are shales, pink are calcareous shales, blue are limestones, and purple represents dolomite (modifed from Fritz and Medlock, 1994). GR, gamma-ray; R, resisitivity; Miss., Mississippian.

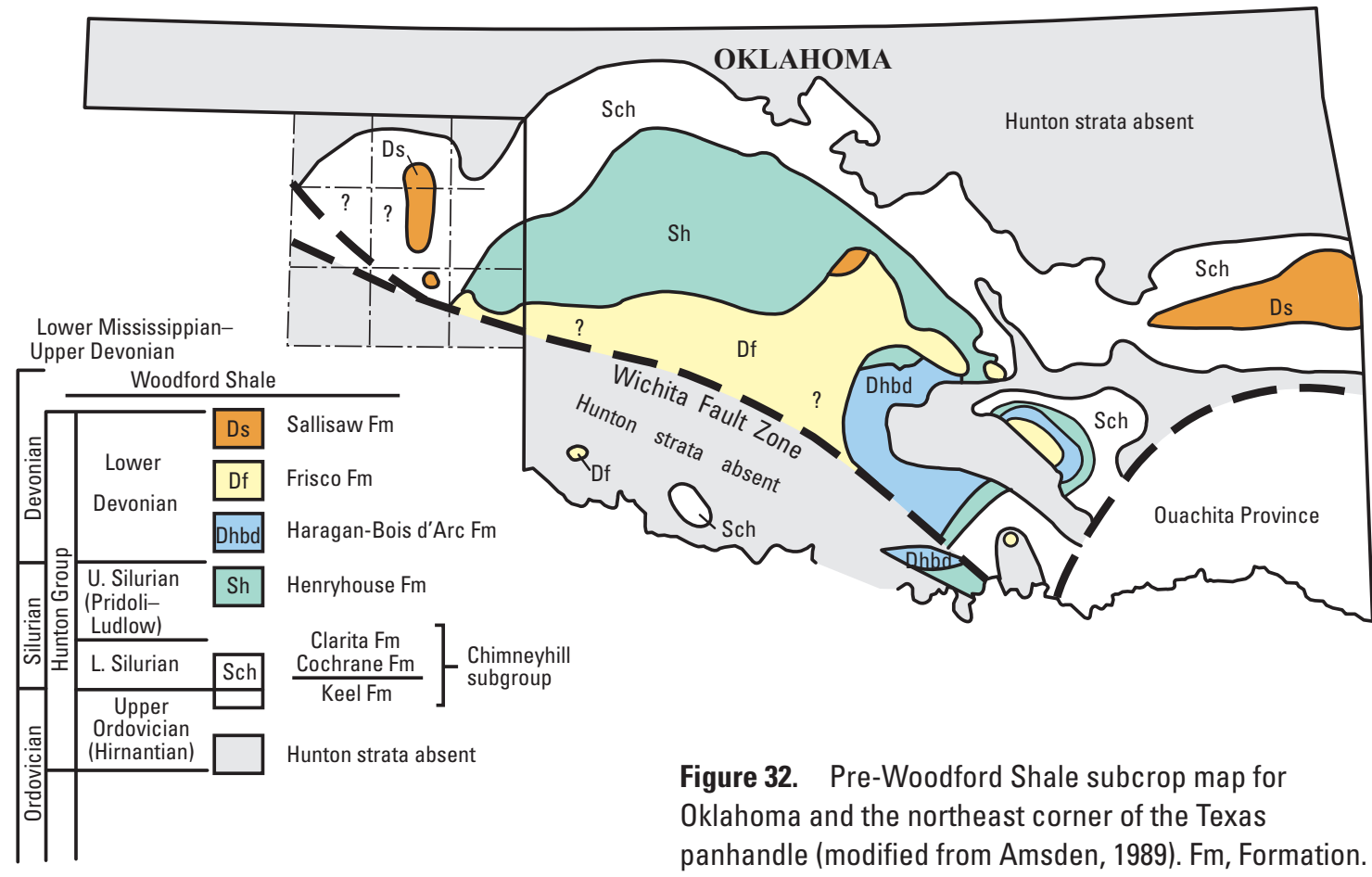




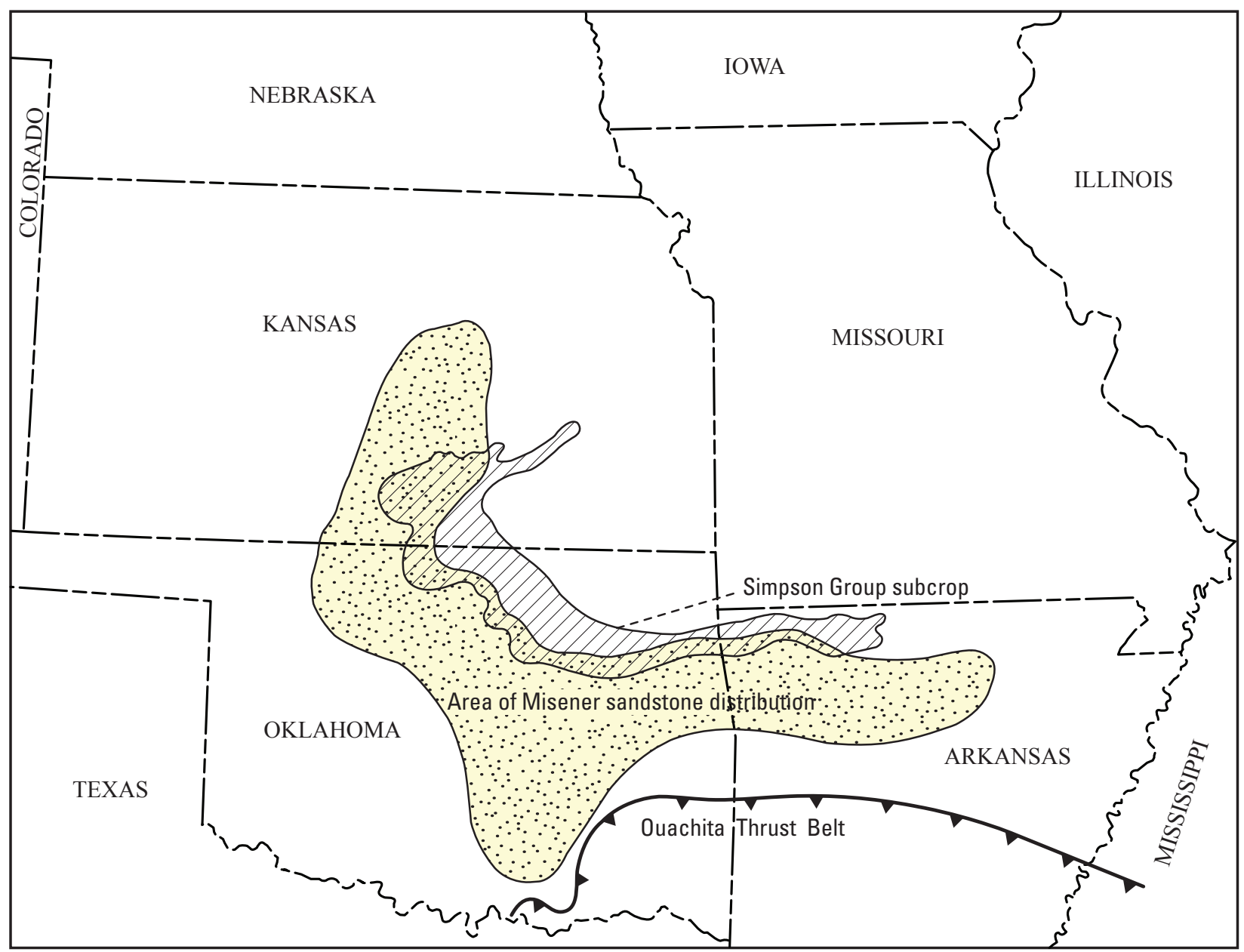

Figure 33. Image showing approximate distribution of the informal Misener sandstone of Woodford Shale and its relation to the Simpson Group subcrop (modifed from Kuykendall and Fritz, 1993). 


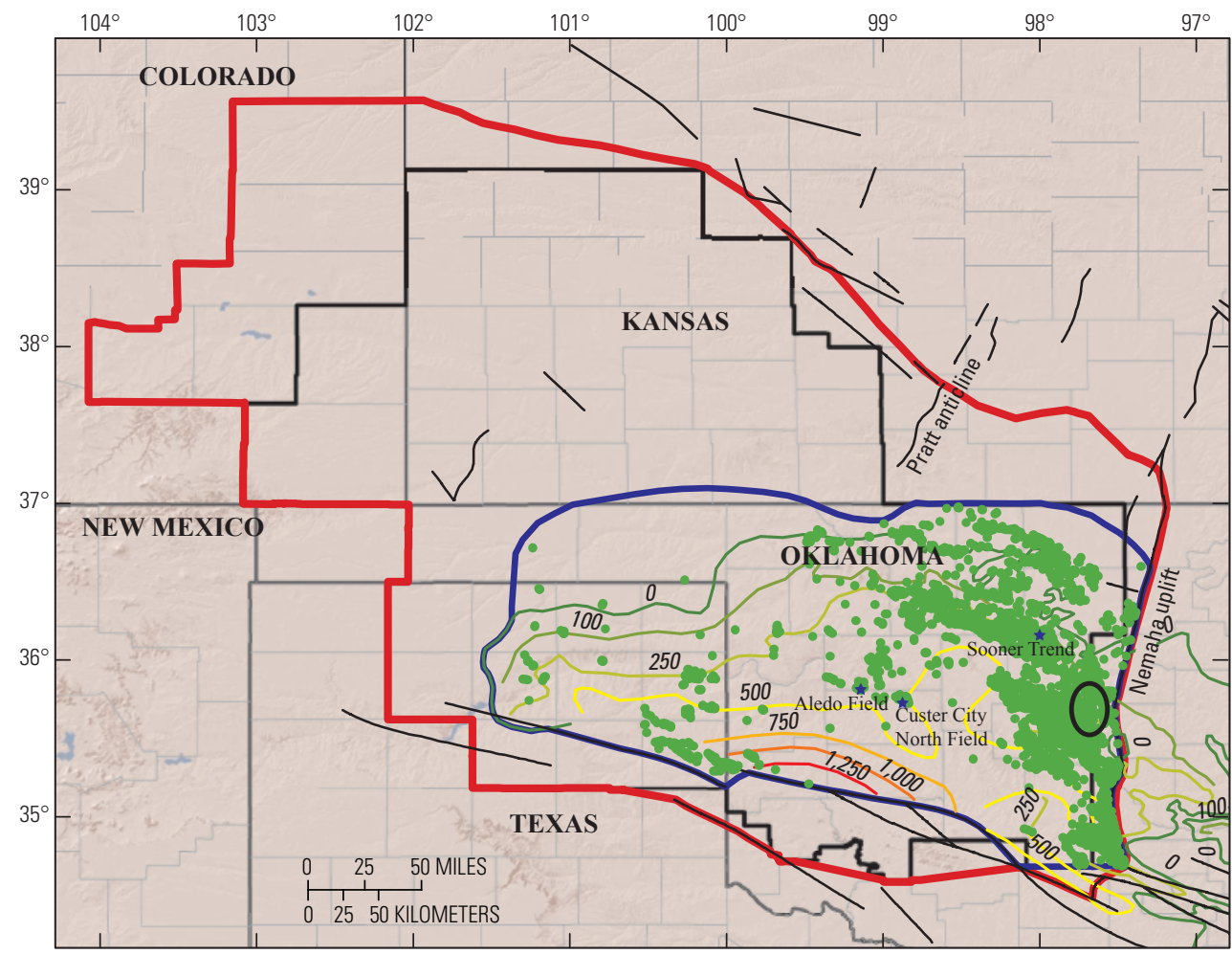

- Fault

EXPLANATION

Hunton Group AU boundary

Woodford Composite TPS boundary

- Anadarko Basin Province boundary

- Hunton Group and Misener sandstone production

Hunton Group thickness, in feet

-0
$-100-750$
$-1,000$

1,000
$-\quad 250-1,250$

$-500$

Figure 34. Map showing production from the Hunton Group and informal Misener sandstone of the Woodford Shale, and Hunton Group thickness; contour interval is 250 feet. Black oval is the West Edmond field. TPS, Total Petroleum System; AU, Assessment Unit. 


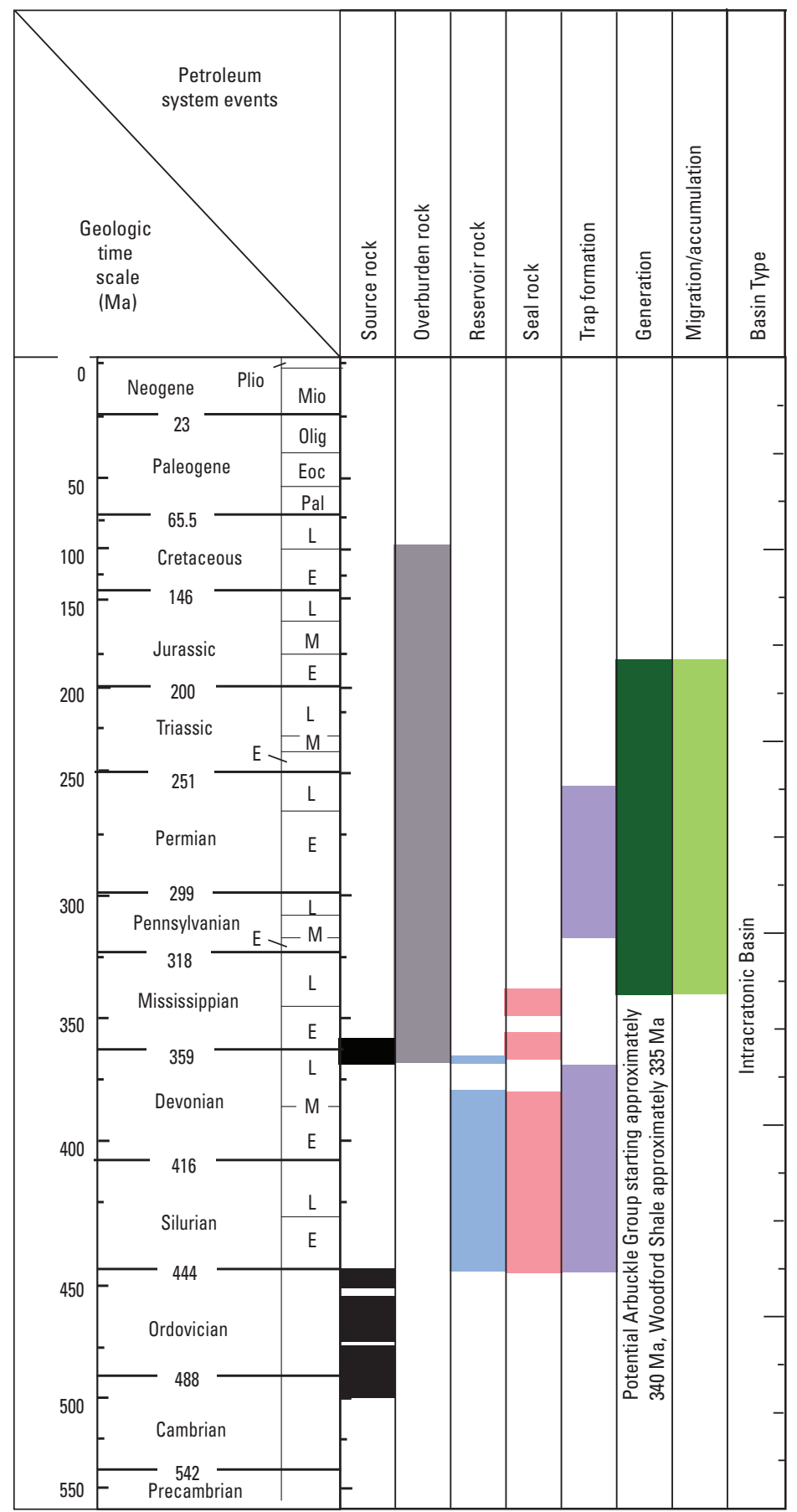

Figure 35. Hunton Group Assessment Unit events chart showing the timing of source rock deposition and trap formation, and the age of overburden, reservoir, and seal rocks with different color bars (black, source rock; gray, overburden rock; blue, reservoir rock; pink, seal rock; purple, trap formation; dark green, generation; light green, migration accumulation). The chart also depicts the timing of oil generation, migration and accumulation as modeled for various wells in different parts of the Anadarko Basin. Ma, mega-annum; Plio, Pliocene; Mio, Miocene; Olig, Oligocene; Eoc, Eocene; Pal, Paleocene; L, Late; M, Middle; E, Early. 


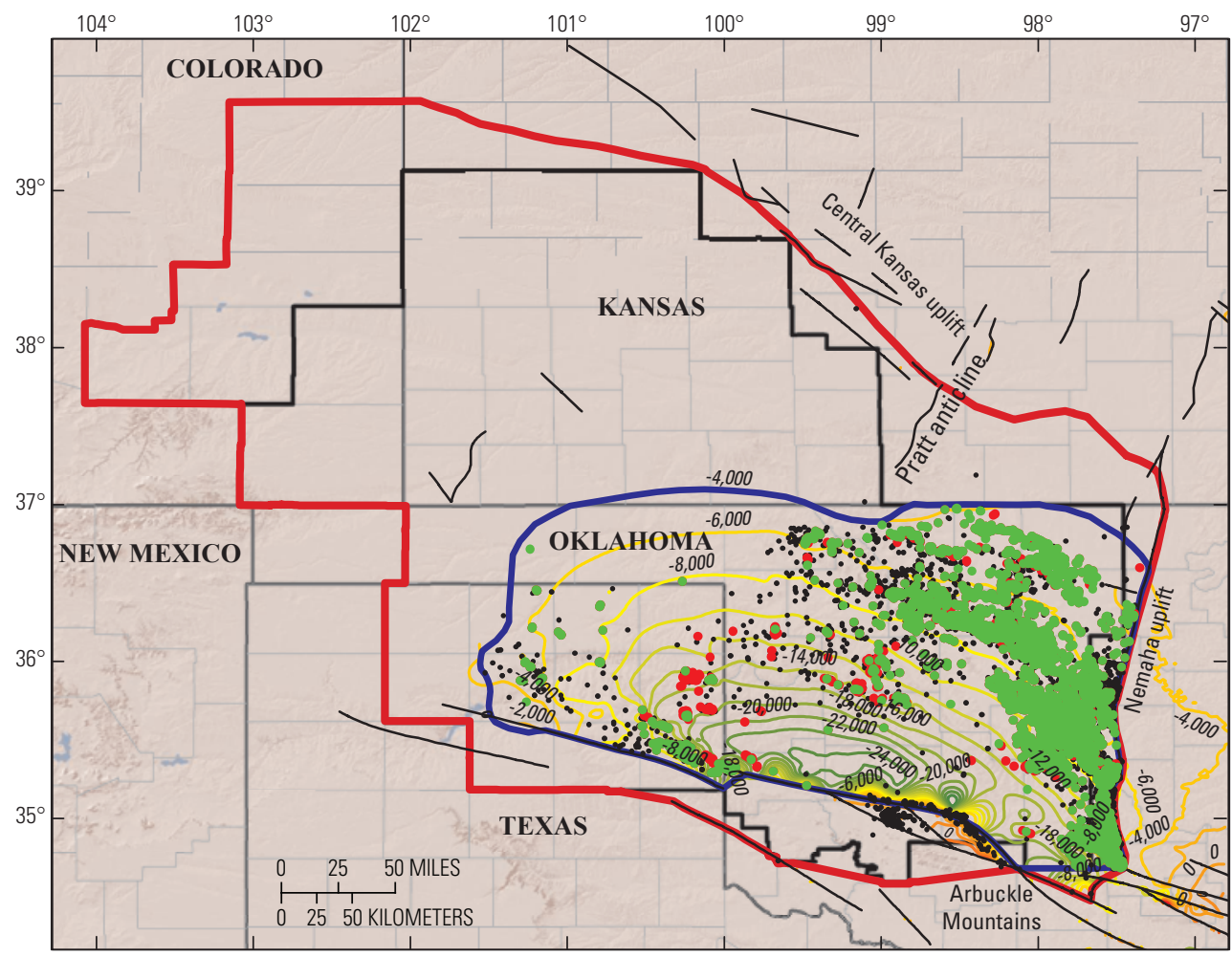

\section{EXPLANATION}

- Hunton Group dry holes Woodford TPS Fault

- Hunton Group AU boundary

Woodford Composite TPS boundary

- Anadarko Basin Province boundary

- Hunton Group oil production

- Hunton Group gas production

Hunton Group Top, elevation in feet above sea level

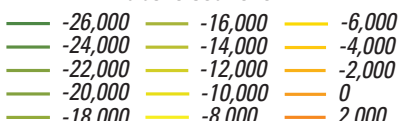

Figure 36. Map showing oil and gas production and dry hole penetrations for the Hunton Group and informal Misener sandstone of the Woodford Shale in the Anadarko Basin Province (IHS Energy Group, 2010). Structure contours are drawn on the top of the Hunton Group; contour interval is 2,000 feet. The Anadarko Basin Province boundary is the black line, the Woodford Composite Total Petroleum System (TPS) boundary the red line, and the Hunton Group Assessment Unit (AU) Boundary the blue line. 
Fracturing, dolomitization, and dissolution are important factors for porosity development in the Hunton. Most Hunton reservoirs are in dolomitized rock. Upper subtidal and lower intertidal facies are dolomitized and have higher porosity (Wilson and others, 1991). Porosity ranges from 3 to 12 percent with an average of 8.6 percent over a depth range of 9,000 to 19,500 ft (Ball and others, 1991). Significant porosity formation in the Hunton is commonly in oolitic dolomitized grainstones and dolomitized burrowed wackestones and packstones (Fritz and Medlock, 1994). The exception is the Frisco Formation (fig. 31), which is sparsely dolomitized and generally has interparticle and intraparticle porosity combined with moldic to vuggy porosity, as it has undergone multiple diagenesis phases (Fritz and Medlock, 1994; Medlock, 1984). Hydrocarbon accumulations in the Frisco are mainly in stratigraphic traps situated downdip of the areas where the formation has been severely truncated (Morgan and Schneider, 1981). Some giant fields that produce from the Devonian Frisco Formation include the Fitts and West Edmond fields. The West Edmond field in northwest Oklahoma County produces where the Hunton is truncated on the west flank of the Nemaha uplift.

Most Hunton fields are west of the Nemaha uplift on the northeastern shelf of the Anadarko Basin. Another major region of production is in central Oklahoma (Johnson and others, 2000; fig. 34), and on the north flank of the Anadarko Basin, including the Sooner Trend, Aledo, and Custer City North fields. Unlike the Arbuckle, Simpson, and Viola, where production is largely dependent on structures, the Hunton production is unique in that it extends away from structural features. There has also been a substantial amount of production near the Hunton erosional edge near the KansasOklahoma border.

Production from the Misener sandstone has been concentrated in the northeast corner of the AU, especially in Garfield, Grant, and Alfalfa Counties (fig. 37). Most of the production from the unit is reported commingled with that of the Hunton Group. It is a common target where present because of its shallow depth.

The Hunton Group AU boundary is defined by the Nemaha uplift on the east, the Precambrian fault system along the Arbuckle uplift to the south, and the Hunton subcrop and production to the east and north (fig. 34). The AU boundary was drawn slightly beyond the subcrop to account for remnant islands of Hunton carbonates that may have production potential.

\section{Traps and Seals in the Hunton Group Assessment Unit}

Most Hunton accumulations are in structure-stratigraphic combination traps, typically formed by the truncation of porous carbonate across structural noses (Fritz and Medlock, 1994). Most structures containing Hunton strata produce from the Hunton, but truncation traps are most common (Johnson and others, 2000). Another reservoir configuration in the Hunton Group is trapping by permeability barriers that are the result of facies changes along structural noses or faults. The upper surface of the Hunton is sculpted by the regional pre-Woodford unconformity, and these erosional structures also shape the traps on the basin margin (Ball and others, 1991). Weathering of the Hunton Group that occurred during pre-Woodford time and Pennsylvanian time also increased porosity and permeability.

The overlying Devonian-Mississippian Woodford Shale is a seal for the Hunton carbonates in the majority of the areas of the basin where the Hunton Group is present (fig. 35). However, the Woodford Shale is absent in some areas, and Mississippian strata form a seal for Hunton Group reservoirs in the Oklahoma and Texas Panhandles. There are also tight, lowporosity intervals within the Hunton Group that form intraformational seals for some reservoirs. Traps for the Misener sandstone are mainly stratigraphic, with some minor structural influence. Seals are likely interbedded shales.

\section{Sizes and Numbers of Undiscovered Fields in the Hunton Group Assessment Unit}

The Hunton Group is a prolific oil- and gas-producing unit in the Midcontinent. Some of the greatest potential for Hunton Group production is in the deepest parts of the Anadarko Basin, and some of the deepest gas fields in the world are located in the Hunton Group at depths below 20,000 ft along the Oklahoma-Texas border (Fritz and Medlock, 1994; fig. 36). Gas recovery in the basin increases with depth. Well spacing does not seem to have a significant effect on gas recovery, and most spacing on deep wells is 640-acre units (Smith and others, 2000).

The Hunton Group is a heterogenous reservoir, with a large amount of vertical and lateral variability. It is well explored and penetrated through most of the AU, but it is underexplored in the southwestern and north-central parts of the AU, where potential may exist, especially in stratigraphic plays (fig. 36).

There are 81 Nehring accumulations reported for the Hunton Group and Misener sandstone (fig. 38). Of these, 6 oil fields and 18 gas fields have accumulation data reported in the Nehring database (Nehring Associates, Inc., 2009). Cumulative oil production in the Hunton Group AU is 290 MMBO in the Hunton Group and $55 \mathrm{MMBO}$ from the Misener sandstone (IHS Energy, 2010). Cumulative gas production is 5 TCFG from the Hunton and 127 BCFG from the Misener (IHS Energy Group, 2010). Production depths range from 4,000 ft to over $25,000 \mathrm{ft}$ in the deepest part of the basin (fig. 36). There is an overlap in the oil and gas production, especially along the eastnortheast AU boundary and in the Texas Panhandle.

Using the USGS assessment methodology for undiscovered conventional resources (Klett and others, 2005; Schmoker and Klett, 2005), it is estimated that the undiscovered oil resources for conventional reservoirs in the Hunton Group AU have a mean of $9 \mathrm{MMBO}$ and $310 \mathrm{BCFG}$ (table 1). Estimates of the minimum, median, and maximum numbers of (1) undiscovered oil accumulations exceeding the minimum size are 1, 

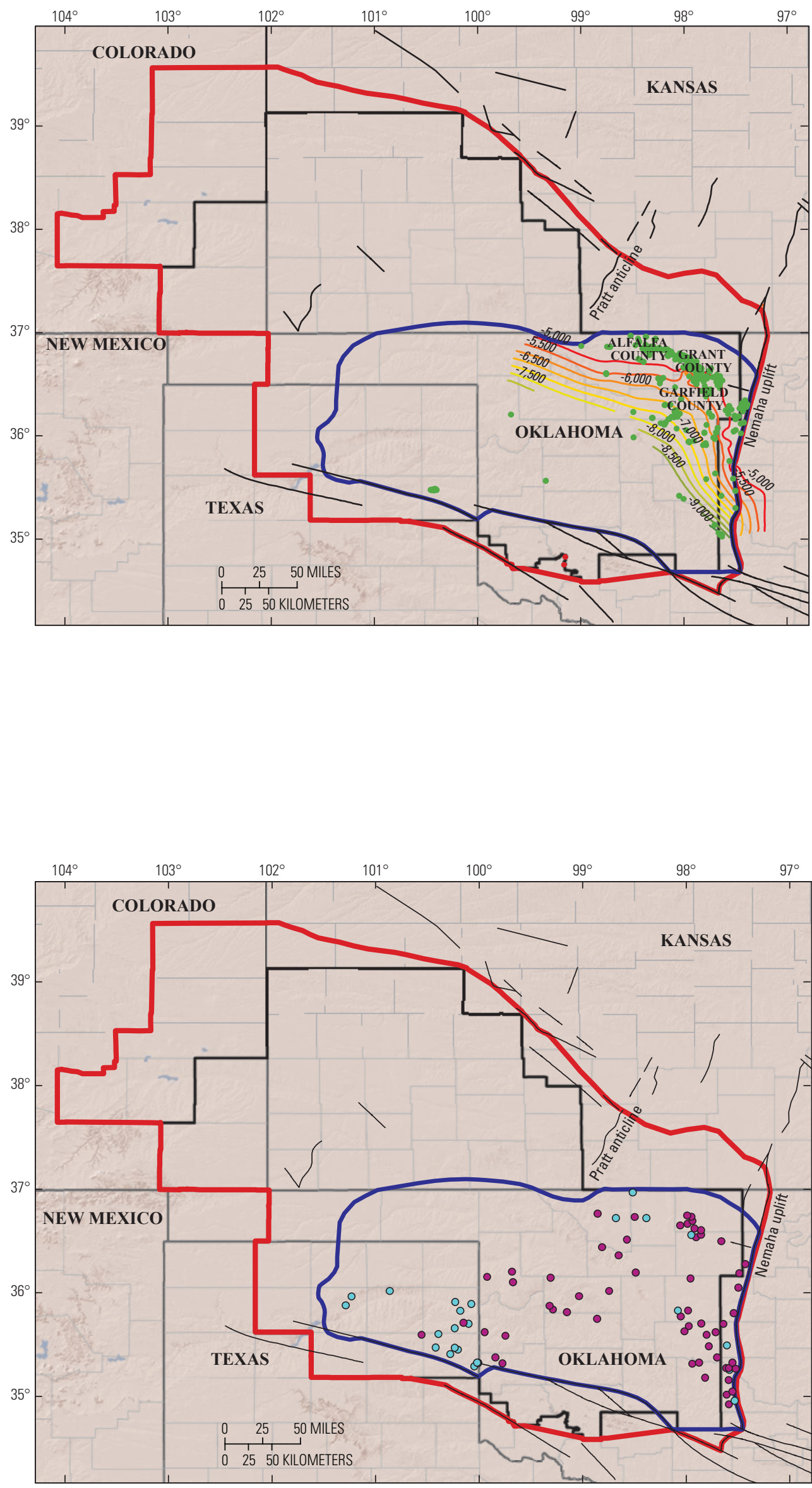

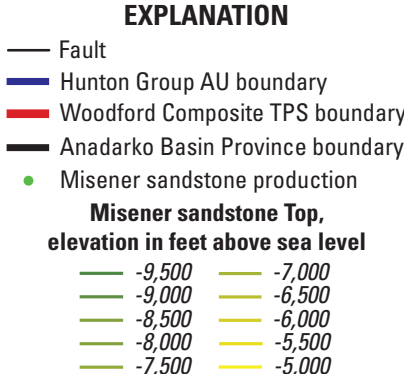

Figure 37. Map showing production from informal Misener sandstone of the Woodford Shale in the Anadarko Basin Province (IHS Energy Group, 2010). The structure contours are on the top of the Misener sandstone; contour interval is 500 feet. TPS, Total Petroleum System; Au, Assessment Unit.
Figure 38. Map showing accumulations for the Hunton Group Assessment Unit (AU) (Nehring Associates, Inc., 2007). Nehring accumulations with grown reserve accumulation numbers are shown in light blue. Accumulations without numbers are in purple. TPS, Total Petroleum System. 
3 , and 15 and (2) undiscovered gas accumulations exceeding the minimum size are 1, 10, and 50. The Hunton has been well-explored on the shelf and along the Nemaha uplift, and in the southwest part of the AU in the Texas Panhandle (fig. 36). There is undrilled area in the deep basin along structures in the same region where the Arbuckle, Simpson, and Viola are sparsely drilled. There is potential, especially for gas, in this region of the basin from karstification of the Hunton during channelization in pre-Woodford time. These channels were later filled in with Woodford Shale. In the deep basin, these potential gas reservoirs have not been explored.

Estimates of the minimum, median, and maximum numbers for the sizes of undiscovered oil accumulations are 0.5 , 1.0, and 20 MMBO. The 0.5 MMBO default signifies that there will be at least one field discovered greater than the minimum size, and the maximum reflects the large sizes of some of the historical Hunton oil accumulations, such as West Edmond field (100 MMBO), and along the Sooner trend (380 MMBO commingled; Nehring Associates, Inc., 2009). Estimates of the minimum, median, and maximum numbers for the sizes of undiscovered gas accumulations are 3, 10, and 200 BCFG. The maximum is raised to account for deep basin gas potential.

\section{Conclusions}

The Woodford Composite TPS includes the conventional Arbuckle-Ellenburger Group, Simpson Group, Viola Group and Hunton Group AUs. The Cambrian-Ordovician Arbuckle and Ellenburger Groups have produced $52 \mathrm{MMBO}$ and $285 \mathrm{BCFG}$ as of this assessment, with reservoirs concentrated in dolomitized Arbuckle strata on the shelf of the Anadarko Basin in Kansas and Oklahoma. The reservoirs are concentrated along structures and in combination structural-stratigraphic traps. Seals are low porosity zones of the Arbuckle Group or overlying, impermeable shales of the Simpson Group. The Arbuckle reservoirs are primarily sourced by the Woodford Shale, with contribution from Ordovician source rocks. The source rock potential of the Arbuckle Group has been debated, and it is suggested that the Arbuckle Group is self-sourcing. The results of $1 \mathrm{D}$ and $3 \mathrm{D}$ modeling indicate the Arbuckle Group is overmature in the deep basin and in the oil window on the basin shelf. Undiscovered potential exists in the undrilled areas in the deep basin along the flanks of structures. Gas production is likely in the deep basin, where the Arbuckle Group is mature to overmature for gas production.

The Simpson Group has produced $470 \mathrm{MMBO}$ and 1.2 TCFG, with reservoirs in blanket sandstones with high porosity and permeability, and minor contributions from carbonate reservoirs. Production is concentrated in structural traps along the eastern edge of the basin and up into Kansas south of the Central Kansas uplift. Seals are interbedded tight carbonates and shales. The Simpson reservoirs are sourced by Ordovician source rocks and the Woodford Shale. Source rock potential of the Simpson is considered moderate. Undiscovered potential exists in the undrilled deep basin.
The Viola Group has produced $112 \mathrm{MMBO}$ and $505 \mathrm{BCFG}$, with reservoirs in limestones and dolomites that have been subjected to fracturing and dissolution to create secondary porosity. Production is in areas similar to the Simpson, along the eastern part of the Anadarko Basin. Traps are structural and combination structural-stratigraphic, and seals are the overlying Sylvan Shale or interbedded tight carbonates of the Viola Group. The Viola reservoirs are sourced by Ordovician source rocks and the Woodford Shale. Undiscovered potential is in the deep basin, in areas similar to the Arbuckle and Simpson.

The Hunton Group has produced $290 \mathrm{MMBO}$ and 5 TCFG. Reservoirs are in carbonates of the Hunton Group and sandstones of the Misener. Misener sandstones are dominant reservoirs within the Hunton AU, producing $55 \mathrm{MMBO}$ and $127 \mathrm{BCFG}$. Production is in a more limited area because of erosion of the Hunton throughout much of the basin; it is concentrated along the northeast and east portion of the AU. Traps are structural and stratigraphic and seals are interbedded tight carbonates of the Hunton and the overlying Woodford Shale. Source rocks for the Hunton reservoirs are the Woodford Shale, with possible contributions from Ordovician rocks. Undiscovered potential is in the deep basin, especially for reservoirs within the Hunton Group.

\section{References Cited}

Adler, F.J., Caplan, W.M., Carlson, M.P., Goebel, E.D., Henslee H.T., Hick, I.C., Larson, T.G., McCracken, M.H., Parker, M.C., Rascoe, G., Jr., Schramm, M.W., and Wells, J.S., 1971, Future petroleum provinces of the mid-continent, in Cram, I.H., ed., Future petroleum provinces of the United States-Their geology and potential: American Association of Petroleum Geologists Memoir 15, v. 2, p. 985-1120.

Al Shaieb, Z., and Puckette, J., 2002, Sequence stratigraphy of Hunton Group ramp facies, Arbuckle Mountains and Anadarko Basin, Oklahoma, in Johnson, K.S., ed., Platform carbonates of the southern Midcontinent, 1996 symposium: Oklahoma Geological Survey Circular 101, p. 131-137.

Amsden, T.W., 1989, Depositional and post-depositional history of middle Paleozoic (late Ordovician through early Devonian) strata in the ancestral Anadarko Basin in Johnson, K.S., ed., Anadarko Basin Symposium: Oklahoma Geological Survey Circular 90, p. 143-146.

Ayan, D., Bucheit, A.K., Kulow, M.J., and Donovan, R.N., 2000, Preliminary cyclic stratigraphy of the Upper Arbuckle Group in the Richard's Spur Quarry, Slick Hills, Oklahoma in Johnson, K.S., ed., Platform carbonates in the southern Midcontinent, 1996 symposium: Oklahoma Geological Survey Circular 101, p. 97-102.

Ball, M.M., Henry, M.E., and Frezon, S.E., 1991, Petroleum geology of the Anadarko Basin region, Province (115), Kansas, Oklahoma, and Texas: U.S. Geological Survey Open-File Report 88-450W. 
Bartram, J.G., Imbt, W.C., and Shea, E.F., 1950, Oil and gas in Arbuckle and Ellenburger Formations, mid-continent region: American Association of Petroleum Geologists Bulletin, v. 34, p. 682-700.

Bebout, D.G., W.A. White, T.F. Hentz, and M.K. Grasmick, eds., 1993, Atlas of major mid-continent gas reservoirs: Bureau of Economic Geology, 85 p.

Brown, R.L., Everett, R., and Tadepalli, S., 1991, Special seismic exploration techniques for Arbuckle exploration: Oklahoma Geological Survey Circular 92, p. 183-184.

Brown, A.A., and Sentfle, J.T., 1997, Source potential of the Viola Springs Formation, southern limb of the Arbuckle anticline, Arbuckle Mountains, in Johnson, K.S., ed., Simpson and Viola Groups in the southern Midcontinent, 1994 symposium: Oklahoma Geological Survey Circular 99, p. 102.

Brown, S.W., and Swetland, P.J., 1992, Arbuckle oils-an overview in Johnson, K.S. and Cardott, B.J., eds., Source rocks in the southern Midcontinent: Oklahoma Geological Survey Circular 93, p. 175.

Burruss, R.C., and Hatch, J.R., 1989, Geochemistry of oils and hydrocarbon source rocks, greater Anadarko Basin: Evidence for multiple sources of oils and long-distance oil migration, in Johnson, Kenneth S., ed., Anadarko Basin symposium, 1988: Oklahoma Geological Survey Circular 90, p. 53-64.

Candelaria, M.P., Handford, C.R., and Reed, C.L., 1997, Sequence stratigraphic model for the Simpson Group of the southern Midcontinent: Key to a new stratigraphic play, in Johnson, K.S., ed., Simpson and Viola Groups in the southern Midcontinent, 1994 symposium: Oklahoma Survey Geological Circular 99, p. 218-223.

Cardott, B.J., 1989, Thermal maturation of the Woodford Shale in the Anadarko Basin, in Johnson, Kenneth S., ed., Anadarko Basin symposium, 1988: Oklahoma Geological Survey Circular 90, p. 32-46.

Cardwell, A.L., 1977, Petroleum source-rock potential of Arbuckle and Ellenburger Groups, southern mid-continent, United States: Quarterly of the Colorado School of Mines, v. 72 , no. 3,134 p.

Carlson, M.P., and Newell, K.D., 1997, Stratigraphy and petroleum production of the Simpson and Viola (Ordovician) in Kansas and Nebraska in Johnson, K.S., ed., Simpson and Viola Groups in the southern Midcontinent, 1994 symposium: Oklahoma Geological Survey Circular 99 , p. 58-64.

Charpentier, R.R., and Doveton, J.H., 1991, Stratigraphy and facies in the Middle Ordovician Simpson Group, southwestern Kansas and western Oklahoma, in, Johnson, K.S., ed.,
Late Cambrian-Ordovician geology of the southern Midcontinent, 1989 Symposium: Oklahoma Geological Survey Circular 92, p. 211.

Denison, R.E., 1997, Contrasting sedimentation inside and outside the southern Oklahoma aulacogen during the Middle and Late Ordovician, in Johnson, K.S., ed., Simpson and Viola Groups in the southern Midcontinent, 1994 symposium: Oklahoma Geological Survey Circular 99, p. 48-57.

Donovan, R.N., 2000, Initiation of the Arbuckle Platformview from the Slick Hills, Oklahoma: in Johnson, K.S., ed., Platform carbonates in the southern Midcontinent, 1996 symposium: Oklahoma Geological Survey Circular 101, p. $47-56$.

Donovan, R.N., and Critchfield, R., 2001, The Signal Mountain Formation-A source rock in hiding in Johnson, K.S. and Merriam, D.F., eds., Petroleum systems of sedimentary basins in the southern Midcontinent, 2000 Symposium: Oklahoma Geological Survey Circular 106, p. 71-80.

Doveton, J.H., Charpentier, R.R., and Metzger, E.P., 1990, Lithofacies analysis of the Simpson Group in south-central Kansas: Kansas Geological Survey, Petrophysical Series no. $5,34 \mathrm{p}$.

Flores, R.M., and Keighin, C.W., 1989, Petrology and depositional facies of siliciclastic rocks in the Middle Ordovician Simpson Group, Mazur well, southeastern Anadarko Basin, Oklahoma: U.S. Geological Survey Bulletin 1866-E, 45 p.

Fritz, R.D., and Medlock, P.L., 1994, Sequence stratigraphy of the Hunton Group as defined by core, outcrop, and log data: Bulletin Houston Geological Society, 11 p.

Fritz, R.D., Johnson, C.I., and Medlock, P.L., 1993, Application of horizontal drilling in fractured carbonates of Oklahoma, in Johnson, K.S. and Campbell, J.A., eds., Petroleum-reservoir geology in the southern Midcontinent, 1991 symposium: Oklahoma Geological Survey Circular 95, p. 66-68.

Gao, G., Land, L.S., and Folk, R.L. 1992, Meteoric modification of early dolomite and late dolomitization by basinal fluids, Upper Arbuckle Group, Slick Hills, southwestern Oklahoma: American Association of Petroleum Geologists Bulletin, v. 76, n. 11, p. 1649-1664.

Gatewood, L.E., 1992, Can carbonates be source rocks for commercial petroleum deposits? in Johnson, K.S. and Cardott, B.J., eds., Source rocks in the southern Midcontinent: Oklahoma Geological Survey Circular 93, p. 270-281.

Gradstein, F.M., Ogg, J.G., Smith, A.G., Bleeker, W., and Lourens, L.J., 2004, A new geologic time scale with special reference to Precambrian and Neogene: Episodes, v. 27, p. 83-100. 
Haq, B.U., and Van Eysinga, F.B., 1998, Geological time table: Elsevier Science, Amsterdam, New York, 1 chart.

Hatch, J.R., Rice, D.D., Burruss, R.C., Schmoker, J.W., and Clayton, J.L, 1986, Thermal maturity modeling and geochemical characterization of hydrocarbon source rocks, oils, and natural gases of the Anadarko Basin, in Carter, L.M.H., ed.: U.S. Geological Survey Circular 974, p. 21-23.

Henry, M.E., and Hester, T., 1996, Maps showing petroleum exploration intensity and production in major Cambrian to Ordovician reservoir rocks in the Anadarko Basin: U.S. Geological Survey Miscellaneous Field Studies Map MF-2313.

Heyer, J., 1993, Trapping mechanisms in Arbuckle sediments, eastern Major County, Oklahoma: Fort Worth Geological Society Transactions and Abstracts, p. 43-54.

Higley, D.K., Gaswirth, S.B, Abbott, M.M., Charpentier, R.R., Cook, T.A., Ellis, G.S., Gianoutos, N.J., Hatch, J.R., Klett, T.R., Nelson, P., Pawlewicz, M.J., Pearson, O.N., Pollastro, R.M., and Schenk, C.J., 2011, Assessment of undiscovered oil and gas resources of the Anadarko Basin Province of Oklahoma, Kansas, Texas and Colorado: U.S. Geological Survey Fact Sheet 2011-3003, 2 p.

IHS Energy Group, 2009, IHS energy well database: Unpublished database available from IHS Energy, 15 Inverness Way East, Englewood, CO 80112.

IHS Energy Group, 2010, Petroleum Information/Dwights petroROM Rocky Mountain Region production data on CDROM: IHS Energy Group, 15 Inverness Way East, D205, Englewood, CO, 80112.

Johnson, K.S., 1989, Geologic evolution of the Anadarko Basin in Johnson, K.S., ed., Anadarko Basin Symposium: Oklahoma Geological Survey Circular 90, p. 3-12.

Johnson, K.S., 1991, Geologic overview and economic importance, in Johnson, K.S., ed., Late Cambrian-Ordovician geology of the southern Midcontinent, 1989 symposium: Oklahoma Geological Survey Circular 92, p. 3-14.

Johnson, K.S., 1992, Discussion between audience and panel on the subject: can carbonates be source rocks for commercial petroleum deposits? in Johnson, K.S., and Cardott, B.J., eds., Source rocks in the southern Midcontinent: Oklahoma Geological Survey Circular 93, p. 283-286.

Johnson, K.S., and Cardott, B.J., 1992, Geologic framework and hydrocarbon source rocks of Oklahoma in Johnson, K.S., and Cardott, B.J., eds., Source rocks in the southern Midcontinent: Oklahoma Geological Survey Circular 93, p. 21-37.

Johnson, K.S., Hinshaw, G.C., and Northcutt, R.A., 2000, Petroleum production from platform carbonates of Oklahoma, in Johnson, K.S., ed., Platform carbonates in the southern Midcontinent, 1996 symposium: Oklahoma Geological Survey Circular 101, p. 1-11.
Jones, P.J., and Philip, R.P., 1990, Oils and source rocks from Pauls Valley, Anadarko basin, Oklahoma, U.S.A.: Applied Geochemistry, v. 5, p. 429-448.

Klett, T.R., Schmoker, J.W., and Charpentier, R.R., 2005, U.S. Geological Survey input-data form and operational procedure for the assessment of conventional petroleum accumulations: U.S. Geological Survey Digital Data Series 69-D, 7 p.

Kuykendall, M.D., and Fritz, R.D., 1993, Misener Sandstone: Distribution and relationship to late/post-Hunton unconformities, northern shelf, Anadarko Basin, in Johnson, K.S., ed., Hunton Group core workshop and field trip: Oklahoma Geological Survey Special Publication 93-4, p. 117-134.

Lindsay, R.F., and Koskelin, K.M., 1991, Arbuckle Group depositional parasequences in Late Cambrian-Ordovician geology of the southern Midcontinent, 1989 symposium: Oklahoma Geological Survey Circular 92, p. 71-84.

Medlock, P.L., 1984, Depositional environment and diagenetic history of the Frisco and Henryhouse Formations in central Oklahoma: Oklahoma State University, unpublished M.S. thesis, $146 \mathrm{p}$.

Morgan, W.A., and Schneider, R.E., 1981, Subtle porosity and traps within Frisco Formation (Devonian, Hunton Group): Geologic-seismic waveform approach, example from West El Reno field, Canadian County, Oklahoma: American Association of Petroleum Geologists Bulletin, v. 65, p. 960-961.

Nehring Associates, Inc., 2009, The significant oil and gas fields of the United States: Nehring Associates, Inc., P.O. Box 1655, Colorado Springs, CO, 80901.

Newell, K.D., 1997, Correlation and distribution of reservoir and sealing facies within the Viola Formation, south-central Kansas, in Johnson, K.S., ed., Simpson and Viola Groups in the southern Midcontinent, 1994 symposium: Oklahoma Geological Survey Circular 99, p. 247-259.

Newell, K.D., 2000, Depositional and diagenetic controls on porosity development in the Ordovician Viola Formation, south-central Kansas, in Johnson, K.S., ed., Platform carbonates in the southern Midcontinent, 1996 symposium: Oklahoma Geological Survey Circular 101, p. 107-115.

Northcutt, R.A., and Johnson, K.S., 1997, Major Simpson and Viola oil and gas reservoirs in Oklahoma in Johnson, K.S., ed., Simpson and Viola Groups in the southern Midcontinent, 1994 symposium: Oklahoma Geological Survey Circular 99, p. 48-57.

Perry, C.C., 1990, New Arbuckle frontier opens: Oil and Gas Journal, v. 88, p. 56.

Philippie, G.T., 1981, Correlation of crude oils with their oil source formations using high resolution GLD C6-C7 component analyses: Geochimica et Cosmochimica Acta, v. 45, p. 1495-1513. 
Pollastro, R.M., 1989, Mineralogic and textural relations in deeply buried rocks of the Simpson Group (Middle Ordovician)-Implications in diagenesis and petroleum geology, in Johnson, K.S., ed., Anadarko Basin symposium, 1988: Oklahoma Geological Survey Circular 90, p. 194-208.

Price, L.C., 1997, Minimum thermal stability levels and controlling parameters of methane, as determined by $\mathrm{C}_{15}{ }^{+}$ hydrocarbon thermal stabilities, in Dyman, T.S., Rice, D.D., and Westcott, P.A., eds., Geologic controls of deep natural gas resources in the United States: U. S. Geological Survey Bulletin 2146-K, p. 135-176.

Rascoe, B., and Adler, F.J., 1983, Permo-Carboniferous hydrocarbon accumulations, mid-continent, U.S.A.: American Association of Petroleum Geologists Bulletin, v. 67, p. $979-1001$.

Rottmann, K., 1997, Stratigraphy, paleogeomorphology, and structure of Simpson, Viola, and Mississippian strata and their integral relationships to "Second Wilcox" production in Lincoln and Logan counties, Oklahoma, in Johnson, K.S., ed., Simpson and Viola Groups in the southern Midcontinent, 1994 symposium: Oklahoma Geological Survey Circular 99, p. 137-154.

Rottmann, K., 2000a, Structure map of Hunton Group in Oklahoma and Texas Panhandle: Oklahoma Geologic Survey Special Publication 2000-2, pl. 3.

Rottmann, K., 2000b, Isopach map of Woodford Shale in Oklahoma and Texas Panhandle: Oklahoma Geologic Survey Special Publication 2000-2, pl. 2.

Rottmann, K., 2000c, Defining the role of Woodford-Hunton depositional relationships in Hunton stratigraphic traps of western Oklahoma, in Johnson, K.S., ed., Platform carbonates in the southern Midcontinent, 1996 symposium: Oklahoma Geological Survey Circular 101, p. 139-146.

Schmoker, J.W., and Klett, T.R., 2005, U.S. Geological Survey assessment concepts for conventional petroleum accumulations, chap. 19 of USGS Southwestern Wyoming Province Assessment Team, Petroleum systems and geologic assessment of oil and gas in the Southwestern Wyoming Province, Wyoming, Colorado, and Utah: U.S. Geological Survey Digital Data Series 69-D, 6 p.

Smith, P.W., 1997, Structural and Stratigraphic factors that influence Simpson Group production in central Oklahoma, in Johnson, K.S., ed., Simpson and Viola Groups in the southern Midcontinent, 1994 symposium: Oklahoma Geological Circular 99, p. 111-136.

Smith, P.W., and Woods, T.J., 2000, Effects of depth on quality of gas-well completions in carbonate reservoirs of the Anadarko Basin and shelf areas, in Johnson, K.S., ed., Platform carbonates in the southern Midcontinent, 1996 symposium: Oklahoma Geological Survey Circular 101, p. $25-45$.
Smith, P.W., Hendrickson, W.J., and Williams, C.M., 2000, Production and reservoir characteristics of selected Hunton Fields in the Anadarko Basin, in Johnson, K.S., ed., Platform carbonates in the southern Midcontinent, 1996 symposium: Oklahoma Geological Survey Circular 101, p. $147-156$.

Suhm, R.W., 1997, Simpson stratigraphy of the southern Midcontinent, in Johnson, K.S., ed., Simpson and Viola Groups in the southern Midcontinent, 1994 symposium: Oklahoma Geological Survey Circular 99, p. 3-38.

Sullivan, K.L., 1985, Organic facies variation of the Woodford Shale in western Oklahoma: Shale Shaker, v. 35, no. 4, p. $76-89$.

Sweeney, J.J., and Burnham, A.K., 1990, Evaluation of a simple model of vitrinite reflectance based on chemical kinetics: American Association of Petroleum Geologists Bulletin, v. 74, p. 1.559-1.570.

Sykes, M., Puckette, J., Abdalla, A., and Al-Shaieb, Z., 1997, Karst development in the Viola Limestone in southern Oklahoma, in Johnson, K.S., ed., Simpson and Viola Groups in the southern Midcontinent, 1994 symposium: Oklahoma Geological Survey Circular 99, p. 66-75.

Trask, P.D., and Patnode, H.W., 1942, Source beds of petroleum: American Association of Petroleum Geologists, Tulsa, $566 \mathrm{p}$.

von Almen, W.E., 1970, Palynomorphs of the Woodford Shale of south central Oklahoma with observations on their significance in zonation and paleoecology: East Lansing, Michigan, Michigan State University, Ph.D. dissertation, 179 p.

Walper, J.L., 1977, The geotectonic evolution of the Wichita aulacogen, Oklahoma, in Henry, G.E., ed., Basins of the Southwest, phase 2: North Texas Geological Society, p. 192-211.

Wang, H.D., and Philip, R.P., 1997, A geochemical study of Viola source rocks and associated crude oils in the Anadarko Basin, Oklahoma, in Johnson, K.S., ed., Simpson and Viola Groups in the southern Midcontinent, 1994 symposium: Oklahoma Geological Survey Circular 99, p. 81-101.

Wavrek, D.A., 1989, Characterization of oil types along Hewitt trend, Carter County, Oklahoma: Implications for future exploration (abs.): American Association of Petroleum Geologists, v. 73, p. 424.

Wavrek, D.A., Garcia, M.A., and Ferebee, C.D., 1997, The Viola Group as a petroleum system: Implications for horizontal-drilling prospects, in Johnson, K.S., ed., Simpson and Viola Groups in the southern Midcontinent, 1994 symposium: Oklahoma Geological Survey Circular 99, p. $78-86$. 
Webb, G.W., 1976, Oklahoma City oil-Second crop from preserved subunconformity source rocks: American Association of Petroleum Geologists Bulletin, v. 60, p. 115-122.

Webster, R.E., 1980, Evolution of the southern Oklahoma aulacogen: Oil and Gas Journal, v. 78, p. 150-172.

Williams, J.A., 1992, Can carbonates be source rocks for commercial petroleum deposits? in Johnson, K.S. and Cardott, B.J., eds., Source rocks in the southern Midcontinent: Oklahoma Geological Survey Circular 93, p. 267.

Wilson, J.L., Fritz, R.D., and Medlock, P.L., 1991, The Arbuckle Group - Relationship of core and outcrop analyses to cyclic stratigraphy and correlation in Johnson, K.S., ed., Late Cambrian-Ordovician geology of the southern Midcontinent, 1989 symposium: Oklahoma Geological Survey Circular 92, p. 61-63. 
Chapter 6

Petroleum Systems and Assessment of Undiscovered Oil and Gas in the Anadarko Basin Province, Colorado, Kansas, Oklahoma, and Texas-Woodford Shale Assessment Units

By Debra K. Higley, Troy A. Cook, and Mark J. Pawlewicz

Chapter 6 of 13

Petroleum Systems and Assessment of Undiscovered Oil and Gas in the Anadarko Basin Province, Colorado, Kansas, Oklahoma, and Texas-USGS Province 58

Compiled by Debra K. Higley

U.S. Geological Survey Digital Data Series DDS-69-EE

Version 1.1, June 2018 


\title{
U.S. Department of the Interior SALLY JEWELL, Secretary
}

\section{U.S. Geological Survey Suzette M. Kimball, Acting Director}

\author{
U.S. Geological Survey, Reston, Virginia \\ First release: 2014 \\ Revised: June 2018 (ver. 1.1)
}

For more information on the USGS —-the Federal source for science about the Earth, its natural and living resources, natural hazards, and the environment, visit http://Www.usgs.gov or call 1-888-ASK-USGS.

For an overview of USGS information products, including maps, imagery, and publications, visit $h t t p: / / W w w . u s g s . g o v / p u b p r o d$

To order this and other USGS information products, visit http://store.usgs.gov

Any use of trade, firm, or product names is for descriptive purposes only and does not imply endorsement by the U.S. Government.

Although this information product, for the most part, is in the public domain, it also may contain copyrighted materials as noted in the text. Permission to reproduce copyrighted items must be secured from the copyright owner.

Suggested citation:

Higley, D.K., T.A. Cook, M.J. Pawlewicz, 2018, Petroleum systems and assessment of undiscovered oil and gas in the Anadarko Basin Province, Colorado, Kansas, Oklahoma, and Texas-Woodford Shale Assessment Units, chap. 6, (ver 1.1, June 2018), in Higley, D.K., compiler, Petroleum systems and assessment of undiscovered oil and gas in the Anadarko Basin Province, Colorado, Kansas, Oklahoma, and Texas-USGS Province 58: U.S. Geological Survey Digital Data Series DDS-69-EE, 24 p., http://dx.doi.org/10.3133/ds69EE. 


\section{Contents}

Abstract
Introduction.
Geologic Setting of the Anadarko Basin Province
Petroleum Source and Reservoir Rocks
Thermal Maturation
Petroleum Production History
Refernowledgments

\section{Figures}

1. Map showing boundaries of the Anadarko Basin (red line) and the Woodford Composite Total Petroleum System (TPS). The contained Woodford Shale Oil and Woodford Shale Gas AUs are discussed in this chapter of the report. Green AU boundary approximately encloses all Woodford Shale within the province. The Henry House Creek quarry and State Route 77D outcrop locations are marked by the blue dot near the southeast corner of the figure. Background is surface elevation.

2. Generalized surface and subsurface stratigraphic columns for the Anadarko Basin Province. The horizontal red line divides assessment units (AUs) and total petroleum systems (TPSs) into those of the (above) Pennsylvanian Composite TPS and (below) Woodford Composite TPS. Italics (blue text) indicate informal names. Formal formation- and member-rank units are not necessarily differentiated (as used by Bebout and others, 1993). Modified from Bebout and others (1993) and Henry and Hester (1995). Ages in millions of years before present (Ma) from Haq and Van Eysinga (1998) and Gradstein and others (2004) (red text). Intervals with vertical bars and wavy horizontal lines represent time periods of nondeposition and (or) erosion. Formation, Fm.; Member, Mbr.

3. Map showing elevation on the top of the Woodford Shale in the 4D model layer. Extent of the Woodford Shale in the Anadarko Basin is approximated by that of the Woodford Shale Oil AU (pink line). Contours outside this boundary represent the highest elevation of underlying model layers. The Woodford Shale is also located east and northeast of the basin. The Woodford Shale Gas AU boundary (white line) corresponds to a thickness of about 75 feet ( 25 meters) and a transformation ratio of 0.99 percent, based on Woodford hydrous pyrolysis modeling. The black line corresponds to location of the southwest to northeast cross sections in figure 12. Precambrian faults (dark blue lines) and named structures are from Adler and others (1971) 
4. Generalized paleogeography at 360 million years before present showing marine influx (blue) near the end of Woodford Shale deposition (modified from Blakey, 2011). Ground surface is shades of brown. Superimposed Neoproterozoic and early Paleozoic rifts and continental margin (modified from Shatski, 1946; Perry, 1989) (purple line) shows possible Cambrian failed southern Oklahoma rift (SOA) along the southern boundary of and southeast of the Woodford Shale Gas assessment unit (AU) (pink line). The SOA approximates the northern border of the Wichita Mountain and Amarillo uplifts. Green line is the Woodford Shale Oil AU. Woodford extent is not shown outside of the Anadarko Basin Province.

5. Three-dimensional isopach image of the Woodford Shale in the Anadarko Basin. Vitrinite reflectance $\left(\% \mathrm{R}_{0}\right)$ contours (white lines) from figure 11 approximate the end of oil generation at about $1 \% R_{0^{\prime}}$ to onset of dry gas generation at $2 \% R_{0^{\prime}}$, and overmature for petroleum generation at $4 \% R_{0}$. Well locations (white dots) are, from left to right, Bertha Rogers 1, Petree 1, and Streeter 1. Woodford thickness is derived from Rottmann (2000b, pl. 2), analysis of more than 100 well logs, and edited formation tops from IHS Energy (2009a, 2009b). Faults (red) are from Rottmann (2000a, pl. 3). Image is tilted and 1,300 times vertical exaggeration

6. Generalized stratigraphic column for the Anadarko Basin Province, with petroleum source rocks identified with brown text. Increases in source rock potential are indicated by larger numbers. Petroleum source rocks may be more oil-prone (green) or gas-prone (red). Chart is modified from Burruss and Hatch (1989) and Hatch (oral commun., 2010). Italicized text indicates informal names. Vertical lines show a generalized time range of unconformity from Bebout and others (1993, fig. 5). Gp., Group; Fm., Formation

7. Photograph showing outcrop of Woodford Shale is as much as 231 feet. thick and is located on private property in the Henry House Falls quarry, sec. 6, T. 2 S., R. 1 E., on the south side of the Arbuckle uplift (Paxton, 2007) (fig. 1). Shown is a portion of the upper member of the Woodford Shale. It was described by Paxton (2007) as an organic-rich finely laminated fissile shale. The three resistant lighter-colored beds at Stan Paxton's thigh level are finely crystalline dolomite with hydrocarbon saturation. Spherical phosphate nodules are present above the upper dolomite layer. The upper member at this location is about 55 percent siliceous beds, 44 percent fissile beds, and 1 percent dolomite zones

8. Photographs showing outcrop of a slightly overturned lower member of Woodford Shale beds next to State Route 77D, in NE 1/4 sec. 30, T. 1 S., R 2 E., Murray County, Oklahoma (fig. 1). Stratigraphically up is to the left on the road-cut image. Red arrow on the upper right points to a fault and associated fault gouge, some of which is saturated with hydrocarbon. This section, as described in Paxton and Cardott (2008), strikes N. 70o W and dips 680 to the southwest, is highly siliceous, and was highly susceptible to fracturing during the structural deformation. Strata are generally less fissile than those in figure 7 ......

9. Photograph showing detail of fracture patterns in the lower member of the Woodford Shale at the State Route 77D outcrop photo from Paxton and Cardott (2008) (fig. 1). Spacing and orientation of fracture patterns in the dark gray siliceous shale differ from those in the underlying light-colored chert-rich facies. 
10. Map showing total organic carbon (TOC) for the Woodford Shale based on data from Burruss and Hatch (1989) and mean estimated values from Hester and others (1990). TOC ranges from 0.08 to $14.05 \mathrm{wt} \%$ and averages $3.7 \mathrm{wt} \%$ based on core and well log density calculations from 123 wells. There is considerable TOC variation, but almost the entire area that is thermally mature for petroleum generation contains greater than $2 \mathrm{wt} \%$ TOC. Decrease in TOC in the deep basin is partly the result of elevated levels of thermal maturation. Oil and gas generation depletes TOC and hydrogen indices (HI) — the fuels for petroleum—so original TOC and $\mathrm{HI}$ values would have been greater. Pink lines are Precambrian faults from Adler and others (1971)

11. Maps showing $A$, calculated vitrinite reflectance $\left(\% \mathrm{R}_{0}\right)$ (Sweeney and Burnham, 1990 ) and $B$, transformation ratios (\% TR) for the Woodford Shale from the four-dimensional petroleum system model. The three-dimensional rendering results in variable shading within contours. Pink contour line on the $\% \mathrm{R}_{0}$ map and the gas-oil ratio (GOR) inset corresponds to the $99 \%$ TR end of oil generation. The inset bubble symbols are GOR, with larger bubbles representing greater GOR. Greatest thermal maturation is in the deep basin of Oklahoma and Texas, whereas most of Colorado and Kansas is immature for oil generation, as characterized by an $R_{0}$ of about 6 percent and TR of 0.1 percent and greater. Blue lines are Precambrian faults from Adler and others (1971).

12. South to north thermal maturation cross sections showing transformation ratios $(\%$ TR) through time based on Woodford Shale hydrous pyrolysis kinetics from Lewan $(1983,1985)$, and Lewan and Ruble (2002). Pink and dark blue lines are the respective Woodford Shale and Thirteen Finger lime model layers. Cross section location is in figure 3. Modeled vitrinite reflectance $\left(R_{0}\right)$ lines mark the boundaries for generation and overmaturity for dry gas. Millions of year ago, Ma.

13. History of petroleum generation from the Oil Creek Formation, Woodford Shale, and Thirteen Finger lime (fig. 2) source layers in the four-dimensional petroleum system model. History is based on Woodford hydrous pyrolysis kinetics (Lewan and Ruble, 2002). Shaded area brackets the main period of peak oil generation from the Woodford Shale. Early oil generation from the Oil Creek layer, prior to about 70 million years ago, is in a narrow strip in the model that is proximal to the Wichita Mountain uplift. This strip exceeds maximum depth of the formation and is primarily the result of areas of poor data control in the area bordering the fault zone. The error could have been removed by clipping the four-dimensional model extent to the basin axis and northward, but less of the model would have been visible and usable.....

14. Events chart for the Woodford Shale Gas assessment unit (AU) and Woodford Shale Oil AU. Timing of oil and gas generation, migration, and accumulation is based primarily on vitrinite reflectance and transformation ratios through time from one-dimensional and four-dimensional (4D) models (Higley, 2014a). Timing of oil generation (gray polygon) is also based on (unpublished) volumes of petroleum generated through time using the 4D model; peak generation from the Woodford Shale (blue bars) is from about 310 to 230 million of years before present (Ma). A second phase of generation from about 80 to $50 \mathrm{Ma}$ is associated with deposition and erosion of strata associated with the Laramide orogeny. Lithofacies assignments in the "Formations" row are intended only to show periods of unconformities (wavy and horizontal lines) and deposition. Plio, Pliocene; Mio, Miocene; Eoc, Eocene; Pal, Paleocene; L, Late; M, Middle; E, Early... 
15. Map showing oil and gas wells that produce from the Devonian-Mississippian Woodford Shale Oil and Woodford Shale Gas assessment units (AUs) in the Anadarko Basin Province (code number 58) (IHS Energy, 2009a, 2010, 2011). Shown also are AU code numbers. Some wells include production from other units, primarily the Silurian-Devonian Hunton Group, Devonian informal Misener sand, and Mississippian strata. The Woodford Composite TPS code is 50580.

Precambrian faults (pink) are from Adler and others (1971)

16. Map showing oil wells (green dots) and gas wells (red dots) that produce from the Woodford Shale in the Anadarko Basin Province (IHS Energy, 2009a, 2010, 2011). White dots are wells that are productive only from the Woodford Shale based on a November 2011 retrieval of data from IHS Energy (2011). White dot wells that do not contain oil or gas wells were added to the IHS database since the January data retrieval. Extent of the Woodford Shale in the province is approximated by the Woodford Oil AU line. Thickness was derived from Rottmann (2000b, pl. 2), analysis of more than 100 well logs, and edited formation tops from IHS Energy (2009a, $2009 \mathrm{~b})$. The Kansas portion of the province contains variable thicknesses of Woodford Shale, and the Oklahoma and Texas portions have several small erosional remnants. The generalized location of the informal Misener sand (light blue line) in the lower part of the Woodford Shale (fig. 2) is modified from Amsden and Klapper (1972) and IHS Energy (2009a, 2009b). The Misener sand exhibits variable thickness and distribution, and is also present in isolated lows in the eroded, karst surface of the Hunton Group (Gaswirth and Higley, 2014). Pink lines are Precambrian faults from Adler and others (1971)

17. Discovery thirds for 74 wells with reported cumulative gas production from only the Woodford Shale from 1946 to September, 2009 (IHS Energy, 2010). The apparently poor production from the 3 rd third is misleading, and actual performance is probably greater, particularly with the present focus on horizontal wells. Reasons for the spread in data include that early production likely incorporates (unreported) oil and gas from the conventional basal Misener sand of the Woodford shale and (or) bounding reservoirs, and the 3rd third only represents 6 months of Woodford Shale gas from 24 wells. Average gas/oil ratio of the above data set is 237,000 cubic feet of gas per barrel of oil. Million cubic feet of gas, MMCFG.

18. Map showing Woodford Shale calculated gas estimated ultimate recoverable (EUR) ranges from about 0.1 million (MMCFG) to 11.9 billion cubic feet of gas for 76 wells based on production history from IHS Energy (2010). The dense cluster of wells has contoured values, whereas those outside are measured values. Inset map of contoured values is magnified 300 percent. Most gas production is located at the $1.2 \% R_{0}$ and greater Woodford Shale contours created using data from Cardott (1989), Price (1997), Chesapeake Energy Corporation (2009), and Mark Pawlewicz. Faults (brown lines) on the underlying Hunton Group (Rottmann, 2000a) were used to create the Woodford layer and to contour $\mathrm{R}_{0}$ values. 


\section{Tables}

1. Anadarko Basin Province assessment results are listed by name and code of total petroleum system (TPS) and assessment unit (AU).......................................................

2. Oil generation onset for the Anadarko Basin four-dimensional model, and onset and completion dates in millions of years before present (Ma) for the Bertha Rogers 1 and Petree Ranch 1 one-dimensional models based on Woodford Shale hydrous pyrolysis kinetics (Lewan and Ruble, 2002). The Douglas Group overlies the youngest potential petroleum source rocks in figure 6 . The HP kinetics-based onset of oil generation is $0.1 \%$ TR and completion is $99 \%$ TR. Oil generation histories in the four-dimensional model were recorded at 10 million year increments, so the plus symbol indicates onset was before the listed age 



\title{
Petroleum Systems and Assessment of Undiscovered Oil and Gas in the Anadarko Basin Province, Colorado, Kansas, Oklahoma, and Texas-Woodford Shale Assessment Units
}

\author{
By Debra K. Higley, Troy A. Cook, and Mark J. Pawlewicz
}

\section{Abstract}

The Woodford Composite Total Petroleum System in the Anadarko Basin Province includes assessment units of Cambrian through Lower Mississippian oil and gas reservoirs. Two of the assessment units are continuous (unconventional) - the Woodford Shale Gas assessment unit and The Woodford Shale Oil assessment unit of the Upper Devonian and Lower Mississippian Woodford Shale. Assessment unit boundaries were defined based on extent, thickness, and levels of thermal maturation of this reservoir/source unit. The assessment units are self-sourced from this rich petroleum source rock, with thermal maturity ranging from marginally mature for oil generation on the basin shelf to overmature for gas generation in the deep basin. The Woodford Shale Oil assessment unit boundary is approximated by the extent of the Woodford Shale in the basin; mean assessed resources are 393 million barrels of oil, 1.963 trillion cubic feet of gas (TCFG), and 59 million barrels of natural gas liquids. The Woodford Shale Gas assessment unit is located in the deep basin of Oklahoma and Texas. The assessment unit area includes a Woodford Shale thickness of approximately 75 feet ( 25 meters) and greater, and a transformation ratio of 0.99 percent based on Woodford hydrous pyrolysis kinetics modeling; this corresponds to a vitrinite reflectance of about 1.2 percent. Mean undiscovered assessed resources for the Woodford Shale Gas assessment unit are 15.973 trillion cubic feet of gas and 192 million barrels of natural gas liquids.

\section{Introduction}

The U.S. Geological Survey (USGS) completed an assessment of the undiscovered oil and gas resource potential of the Anadarko Basin Province of western Oklahoma and Kansas, northern Texas, and southeastern Colorado (fig. 1). The province area includes the Las Animas arch of southeastern Colorado, part of the Palo Duro Basin of Texas, and the Anadarko Basin. This assessment is hereafter referred to as the 2011 assessment, which corresponds to the publication release date of the results (Higley and others, 2011). Results of the geologic analysis and resource assessment are based on the geologic elements of each defined total petroleum system (TPS), including hydrocarbon source rocks (source-rock maturation, hydrocarbon generation and migration), reservoir rocks (sequence stratigraphic and petrophysical properties), hydrocarbon traps (trapping mechanisms and timing), and seals. Using this geologic framework, the USGS defined 2 TPS and 12 included Assessment Units (AUs), and quantitatively estimated the undiscovered oil and gas resources within these 9 conventional and 3 continuous AUs (table 1). TPSs and AUs are listed below.

Woodford Composite TPS 505801:

1. Arbuckle-Ellenburger, AU 50580101

2. Simpson Group, AU 50580102

3. Viola Group, AU 50580103

4. Hunton Group, AU 50580104

5. Mississippian, AU 50580105

6. Woodford Shale Gas, AU 50580161

7. Woodford Shale Oil, AU 50580162

Pennsylvanian Composite TPS 505802:

1. Morrowan-Atokan, AU 50580201

2. Desmoinesian, AU 50580202

3. Missourian-Permian, AU 50580203

4. Greater Granite Wash Composite, AU 50580204

5. Thirteen Finger Limestone-Atoka Shale Gas, AU 50580261

Continuous AUs are the Devonian and Mississippian (1) Woodford Shale Gas and (2) Woodford Shale Oil of the Woodford Composite TPS, and (3) the Pennsylvanian Thirteen Finger Limestone-Atoka Shale of the Pennsylvanian 


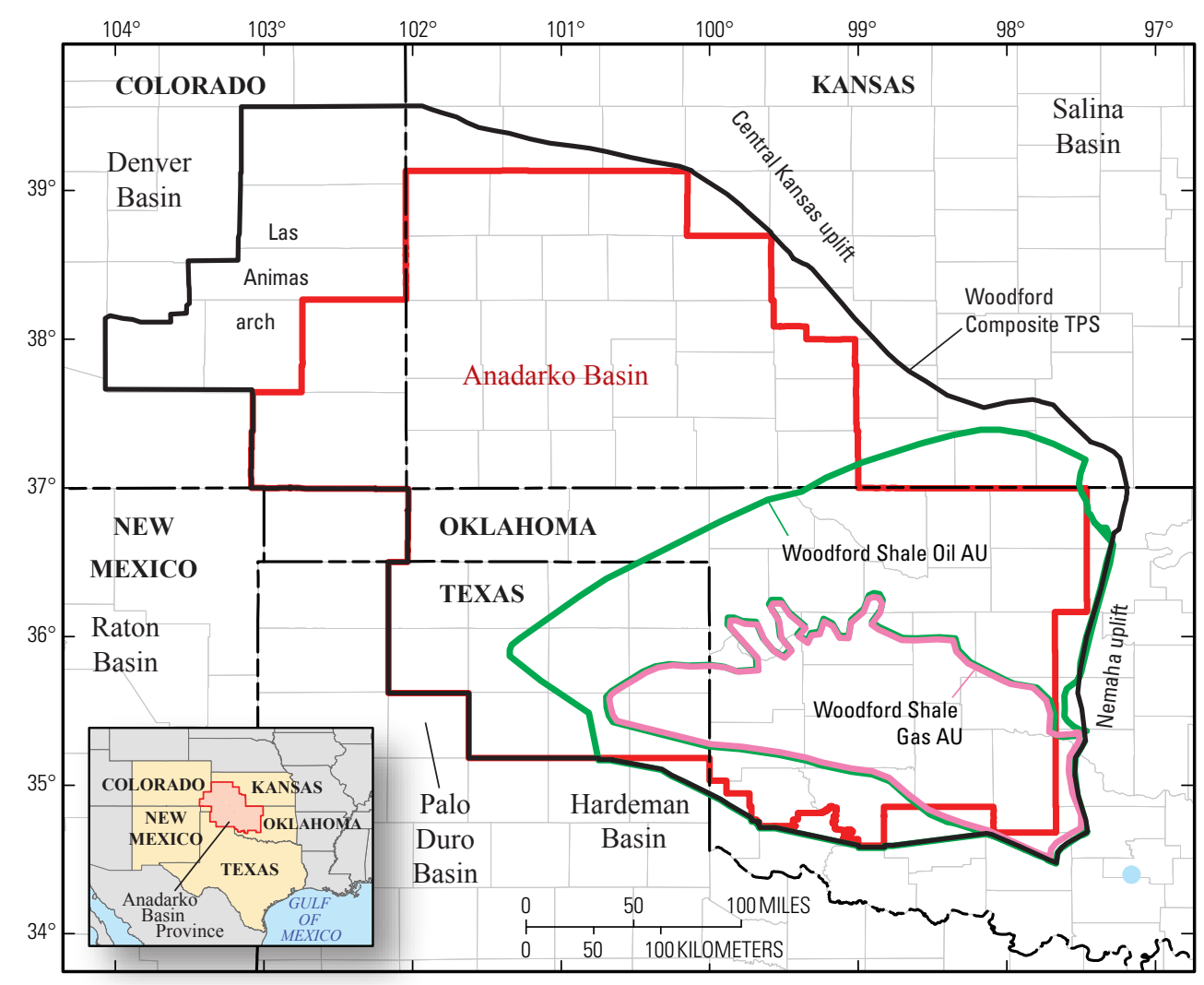

Figure 1. Map showing boundaries of the Anadarko Basin (red line) and the Woodford Composite Total Petroleum System (TPS). The contained Woodford Shale Oil and Woodford Shale Gas AUs are discussed in this chapter of the report. Green AU boundary approximately encloses all Woodford Shale within the province. The Henry House Creek quarry and State Route 77D outcrop locations are marked by the blue dot near the southeast corner of the figure. Background is surface elevation.

Composite TPS. The Woodford Shale Oil and Woodford Shale Gas AU assessment and geologic review are included in this chapter of the report. Description of the Thirteen Finger Limestone-Atoka Shale Gas AU is included in Higley (2014a, 2014b), and associated oil resources are assessed as conventional within the Morrowan-Atokan AU. The stratigraphic chart shows units within the Woodford Composite TPS (fig. 2).

The Woodford Composite TPS encompasses all included AU boundaries, although only the Woodford Shale Gas and Woodford Shale Oil AUs are shown in figure 1. Assignment of Woodford AU boundaries and assessment results were based primarily on (1) the lateral extent and thickness of the Woodford Shale, (2) timing and degree of thermal maturation of the Woodford Shale based on one-dimensional and four-dimensional (4D) petroleum system models of the basin (Higley, 2014a) and published vitrinite reflectance data, and (3) faults and other structures that would limit or enhance oil and gas accumulations. The "fingers" appearance of the Woodford Shale Gas AU boundary partly reflects Woodford deposition in eroded channels of the unconformably underlying
Silurian-Devonian Hunton Group. Results of the oil, gas, and natural gas liquids (NGL) assessment are in table 1 and Higley and others (2011).

The 4D petroleum system model consists of stacked grids of lithostratigraphic units that are referred to as layers. Each layer extends across the study area, a requirement of the PetroMod ${ }^{\circledR}$ petroleum system modeling software. This means that the highest elevation from the underlying layers is displayed if the modeled unit(s) has limited lateral extent. Model construction and data sources are discussed in Higley (2014a) and Higley and others (2014). Maps and models were generated using Dynamic Graphics ${ }^{\circledR}$ Earthvision ${ }^{\circledR}$ (Dynamic Graphics ${ }^{\circledR}$ and Earthvision ${ }^{\circledR}$ are registered trademarks of Dynamic Graphics, Inc.) and PetroMod ${ }^{\circledR}$ software (Schlumberger, 2011). PetroMod ${ }^{\circledR}$ software was used for 1D and 4D petroleum system models. Data sources for Woodford Shale isopach and structure surfaces include Rottmann (2000a, 2000b), well-log picks from about 100 wells across the basin, and edited formation tops from IHS Energy (2009a, 2009b). 
Table 1. Anadarko Basin Province assessment results are listed by name and code of total petroleum system (TPS) and assessment unit (AU).

[Resources are undiscovered oil, gas, and (or) natural gas liquids. MMBO, million barrels of oil; BCFG, billion cubic feet of gas; MMBNGL, million barrels of natural gas liquids. Type refers to mainly oil or gas accumulations in the assessment unit. Fractiles are fully risked estimates. F95 denotes a 95 -percent chance of at least the amount tabulated. Other fractiles are defined similarly. Fractiles are additive only under the assumption of perfect positive correlation]

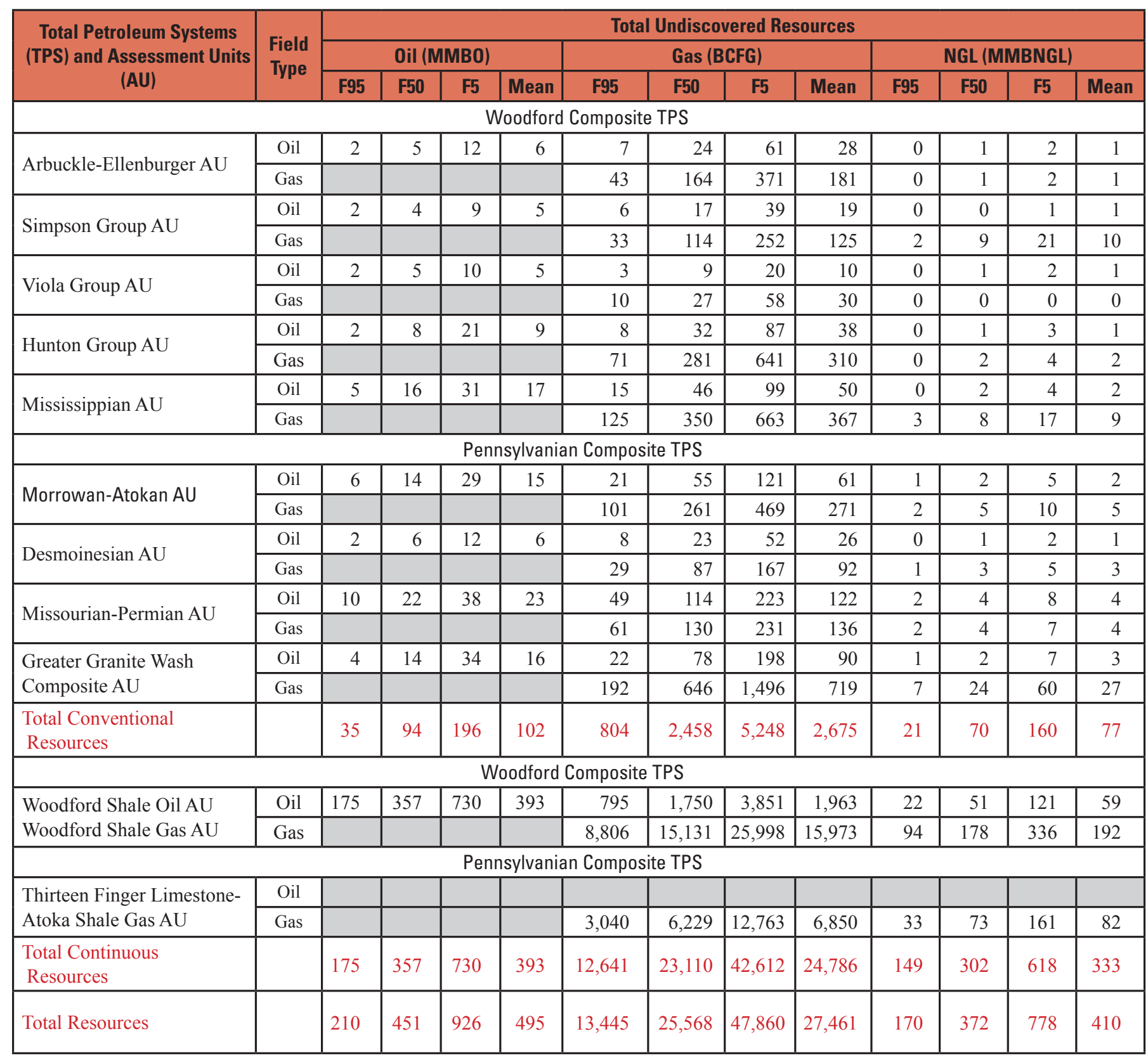




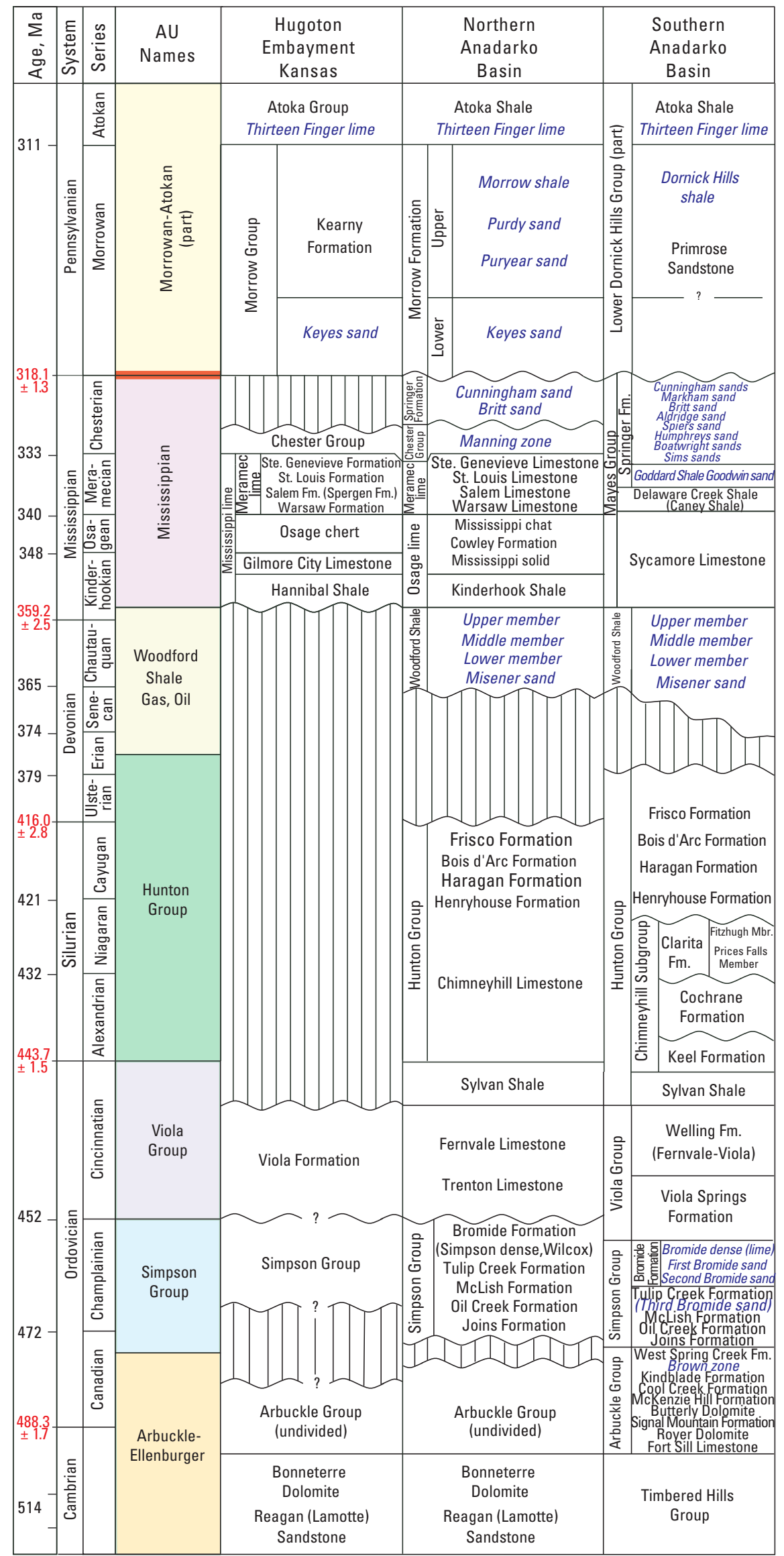

Figure 2. Generalized surface and subsurface stratigraphic columns for the Anadarko Basin Province. The horizontal red line divides assessment units (AUs) and total petroleum systems (TPSs) into those of the (above) Pennsylvanian Composite TPS and (below) Woodford Composite TPS. Italics (blue text) indicate informal names. Formal formation- and member-rank units are not necessarily differentiated (as used by Bebout and others, 1993). Modified from Bebout and others (1993) and Henry and Hester (1995). Ages in millions of years before present (Ma) from Haq and Van Eysinga (1998) and Gradstein and others (2004) (red text). Intervals with vertical bars and wavy horizontal lines represent time periods of nondeposition and (or) erosion. Formation, Fm.; Member, Mbr. 


\section{Geologic Setting of the Anadarko Basin Province}

The Anadarko Basin Province includes (1) the Anadarko Basin, a south-dipping asymmetric basin; (2) the southwestnortheast-trending Las Animas arch, and (3) part of the southeast-dipping Palo Duro Basin. The province is bounded on the southeast by the Arbuckle uplift and Marietta-Ardmore Basin, on the east by the Nemaha uplift and Salina Basin, on the north by the Central Kansas uplift, on the west by the Denver and Raton Basins, and on the south by the eroded Amarillo and Wichita Mountain uplifts (fig. 3). The Nemaha uplift consists of a discontinuous series of block-faulted uplifts, most of which are 5 to 20 miles long (north-south) and 3 to 5 miles wide, and forms the axis and western boundary of the broad, gently raised Central Oklahoma platform (Johnson, 1989) that separates the Anadarko and Ardmore Basins. Elevation on the top of the Woodford Shale in the province ranges from about $-2,500$ feet $(\mathrm{ft})[-760$ meters $(\mathrm{m})]$ on the southern Kansas shelf, to $-27,000 \mathrm{ft}(-8,000 \mathrm{~m})$ in the deep basin of southern Oklahoma. Extent of the Woodford Shale within the Anadarko Basin Province is approximated by the Woodford Shale Oil AU boundary in figure 3. The formation is mostly absent because of erosion in the Nemaha, Wichita Mountain and Amarillo uplifts, although the AU includes an area of Woodford in the Wichita Mountain uplift proximal to the fault zone. The Woodford Shale also is present northeast of the province in the Salina Basin of Kansas, and is commonly named the Chattanooga Shale. The Woodford Shale in the Arkoma Basin is also a petroleum source and reservoir rock. The Woodford Shale layer was contoured using the data and fault systems of Rottmann (2000a, 2000b), who showed the Woodford contact with the bounding fault of the Wichita Mountain uplift to be north of the Precambrian faults of Adler and others (1971). Because of this, Woodford contours terminate basinward of the fault system of Adler and others (1971) (fig. 3).

Southern Oklahoma was initially described by Shatski (1946) as a failed Cambrian aulacogen. The deepest part of the Anadarko Basin is along and north of this rift zone and is proximal to its southern margin along the Wichita Mountain uplift (fig. 3). This southern Oklahoma aulacogen (Rascoe and Adler, 1983) was emplaced on the Precambrian craton as described by Ham and others (1964) and is about 100 miles (mi) [160 kilometers $(\mathrm{km})]$ wide and $300 \mathrm{mi}(480 \mathrm{~km})$ long.

Most of Late Cambrian through Mississippian time was marked by a broad epicontinental sea (Johnson and others, 1988) that extended across most of the southern Midcontinent area, including the Anadarko Basin. Rates of basin subsidence were elevated in the Cambrian but decreased during the Silurian, Devonian, and Early Mississippian, one reason why Hunton and Woodford rocks are relatively thin (Feinstein, 1981; Johnson and others, 1988; Johnson, 1989) and of limited areal extent. Following Hunton Group deposition and prior to Woodford Shale deposition, the entire area was emergent and subjected to extensive erosion, and the Amarillo and Wichita
Mountain uplifts began to form as positive tectonic features (Eddleman, 1961). Early Mississippian seas advanced from the southeast and stable conditions again prevailed with deposition of mostly marine Mississippian carbonates (Eddleman, 1961). Ball and others (1991) indicated that the Mississippian Period closed with regional uplift and erosion that marked the onset of the Wichita orogeny; the associated erosional unconformity further removed Devonian strata on structural highs around the basin margin, and truncated formations as deeply as the Arbuckle Group. Figure 4 shows generalized paleogeography near the end of Woodford deposition. Euxinic seas of the Late Devonian-Early Mississippian were replaced by shallow, well-oxygenated marine waters in which fossiliferous (commonly crinoidal, and some oolitic) limestones were deposited, some of which were interbedded with shale and siltstone (Johnson, 1989). Depth of water during Woodford deposition is estimated at 160 to $1,300 \mathrm{ft}$ (50 to $400 \mathrm{~m}$ ) (Krystyniak and Paxton, 2006) to less than $500 \mathrm{ft}(150 \mathrm{~m})$ (Kirkland and others, 1992).

Various tectonic events strongly influenced rates of sediment deposition, sediment sources, depositional environments, erosion, and especially burial depths of reservoir, seal, and source rocks. During Late Mississippian to Pennsylvanian time, rapid rates of subsidence coincided with tectonic development of the Anadarko Basin and the Wichita Mountain uplift (Ball and others, 1991; Al-Shaieb and others, 1994).

Present basin configuration was impacted by movement of the late Paleozoic Wichita Mountain fault zone and the Ouachita thrust plate (Amsden, 1975, 1989). Depth of burial through time is the main control on thermal maturation of petroleum source rocks, although oil and gas generation is also influenced by factors such as basement heat flow and thermal conductivity of lithofacies.

\section{Petroleum Source and Reservoir Rocks}

Thickness of the Woodford Shale varies greatly across the province. It ranges up to $375 \mathrm{ft}$ thick and averages $40 \mathrm{ft}$ based on Rottmann (2000b), well-log picks from about 100 wells across the basin, and edited formation tops from IHS Energy (2009a, 2009b) (fig. 5). Increased thickness of the Woodford Shale and Hunton Group strata southward is probably related more to basin subsidence than sediment supply (Amsden, 1989). Greatest thickness of the Woodford is in the southern part of the basin in Oklahoma, largely as fill within eroded channels of the underlying Hunton Group. This is also the basin area that is most thermally mature for gas generation. Areas within the AUs (fig. 1) in which the Woodford is absent or thin resulted from erosion or nondeposition, and there are a few erosional remnants outside the Woodford Shale Oil AU boundary. Smooth contours near the southeastern corner of figure 5 reflect limited data and variable thicknesses because of extensive faulting. The Misener sand is an informal lower unit of the Woodford Shale (fig. 2) that is located mainly in the northeastern part of the basin in Oklahoma and 


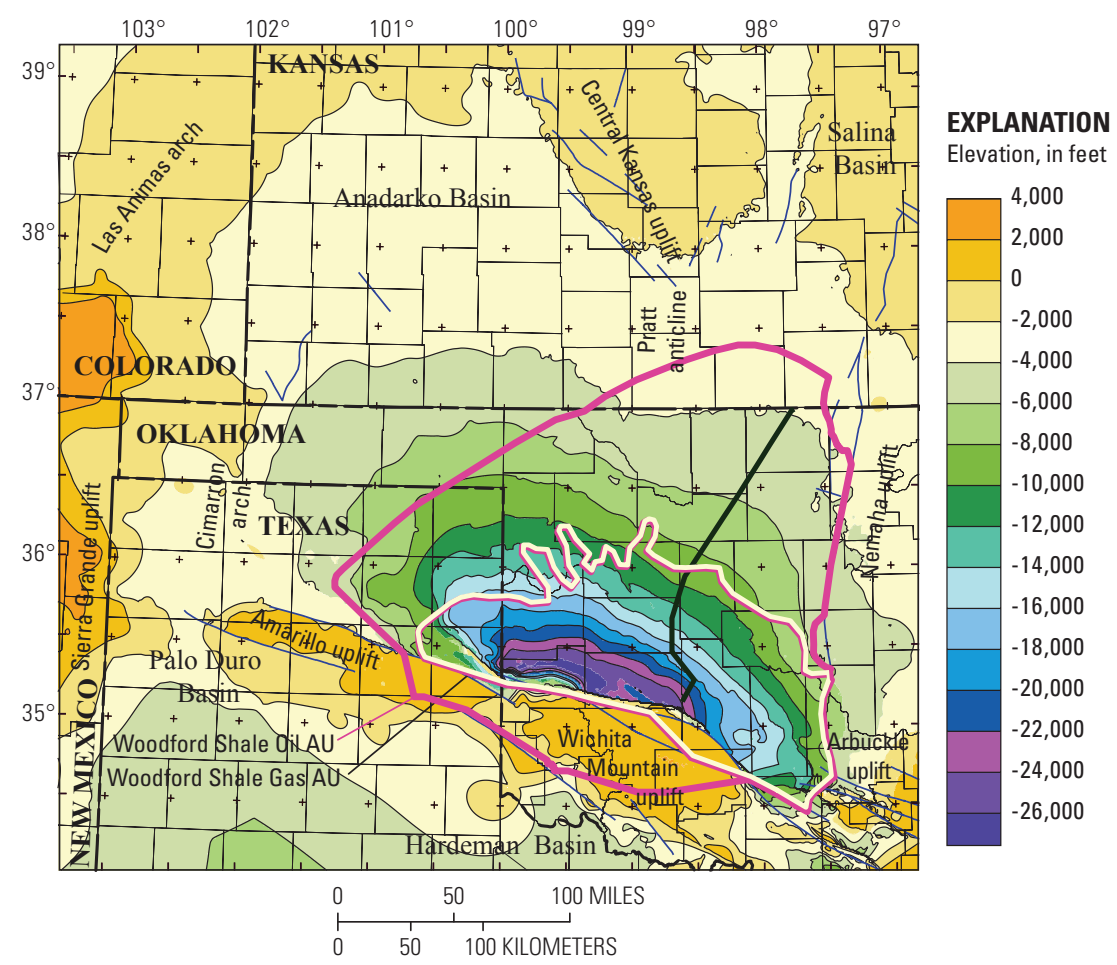

Figure 3. Map showing elevation on the top of the Woodford Shale in the $4 D$ model layer. Extent of the Woodford Shale in the Anadarko Basin is approximated by that of the Woodford Shale Oil AU (pink line). Contours outside this boundary represent the highest elevation of underlying model layers. The Woodford Shale is also located east and northeast of the basin. The Woodford Shale Gas AU boundary (white line) corresponds to a thickness of about 75 feet ( 25 meters) and a transformation ratio of 0.99 percent, based on Woodford hydrous pyrolysis modeling. The black line corresponds to location of the southwest to northeast cross sections in figure 12. Precambrian faults (dark blue lines) and named structures are from Adler and others (1971).
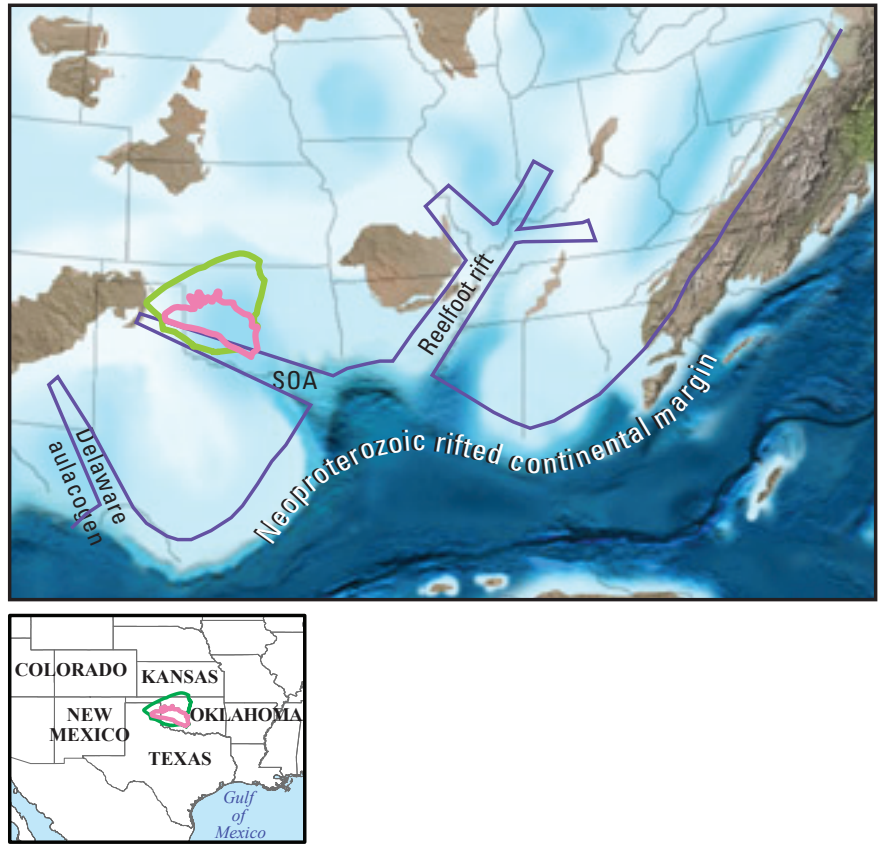

Figure 4. Generalized paleogeography at 360 million years before present showing marine influx (blue) near the end of Woodford Shale deposition (modified from Blakey, 2011). Ground surface is shades of brown. Superimposed Neoproterozoic and early Paleozoic rifts and continental margin (modified from Shatski, 1946; Perry, 1989) (purple line) shows possible Cambrian failed southern Oklahoma rift (SOA) along the southern boundary of and southeast of the Woodford Shale Gas assessment unit (AU) (pink line). The SOA approximates the northern border of the Wichita Mountain and Amarillo uplifts. Green line is the Woodford Shale Oil AU. Woodford extent is not shown outside of the Anadarko Basin Province. 


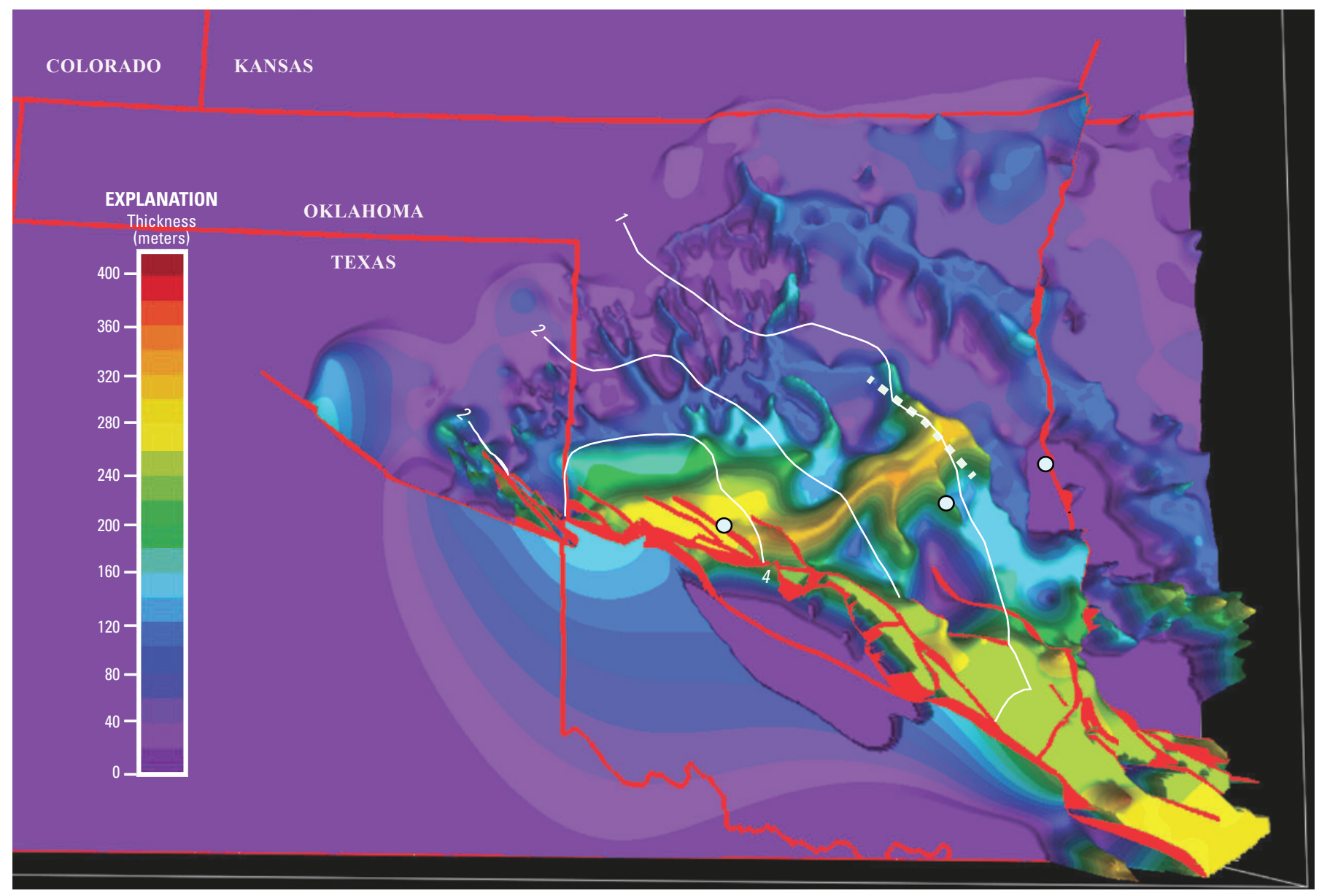

Figure 5. Three-dimensional isopach image of the Woodford Shale in the Anadarko Basin. Vitrinite reflectance $\left(\% \mathrm{R}_{0}\right)$ contours (white lines) from figure 11 approximate the end of oil generation at about $1 \% R_{0^{\prime}}$ to onset of dry gas generation at $2 \% R_{0^{\prime}}$ and overmature for petroleum generation at $4 \% R_{0}$. Well locations (white dots) are, from left to right, Bertha Rogers 1, Petree 1, and Streeter 1 . Woodford thickness is derived from Rottmann (2000b, pl. 2), analysis of more than 100 well logs, and edited formation tops from IHS Energy (2009a, 2009b). Faults (red) are from Rottmann (2000a, pl. 3). Image is tilted and 1,300 times vertical exaggeration.

Kansas; petroleum resources of this unit were evaluated with the Hunton Group AU (Gaswirth and Higley, 2014). Primary reasons for this assignment were that Misener sand reservoirs are conventional, and much of the petroleum production was reported as commingled with that of the Hunton Group.

The primary petroleum source rock for the Woodford Composite TPS is the Woodford Shale; additional sources may include limestones and (or) shales from the Mississippian Mayes Group, and the Ordovician Viola Formation (or Group) and Simpson Group (Hatch and others, 1987; Burruss and Hatch, 1989) (fig. 6). The Woodford Shale and other petroleum source rocks are discussed in more detail in Higley (2014a).

The Woodford Shale consists of upper, middle, and lower informal members of carbonaceous silica-rich fissile and non-fissile shales (von Almen, 1970; Sullivan, 1983, 1985; Hester and others, 1990; Lambert, 1993; Krystyniak and Paxton, 2006). Kirkland and others (1992, fig. 3) indicated
Woodford Shale lithofacies in northwest Kansas and southeast Nebraska are more carbonate rich than those of the deep Anadarko Basin. Based primarily on data from outcrops in the Arbuckle Mountains (fig. 3) and well core, Egenhoff and others (2011) determined that the upper shale member represents a downslope extension of a carbonate or carbonatesiliciclastic shallow marine system into deeper water siliciclastics. Egenhoff and others (2011) indicated that (1) the upper shale member is composed primarily of laminated silt-rich mudstones with lag deposits; and (2) these facies grade progressively basinward into finer-grained laminated mudstones and, (3) most distally, into quartz-rich radiolarian cherts that are intercalated with laminated mudstones. The non-fissile strata include phosphatic and siliceous shales, dolomite beds, phosphatic nodules, and rare chert beds (Krystyniak and Paxton, 2006). Fissile shale can also contain dolomite beds and phosphate nodules, such as in the upper member outcrop 


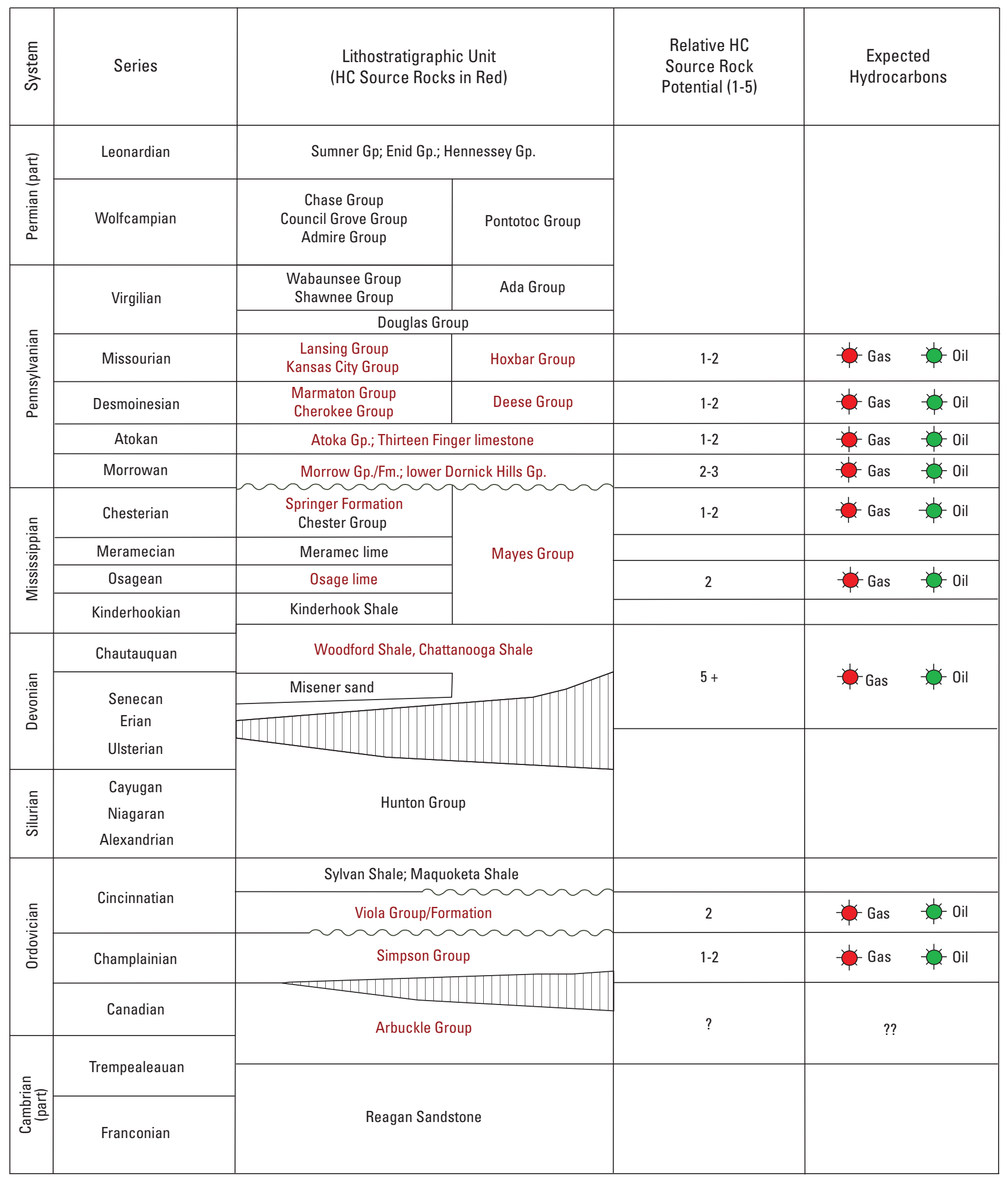

Figure 6. Generalized stratigraphic column for the Anadarko Basin Province, with petroleum source rocks identified with brown text. Increases in source rock potential are indicated by larger numbers. Petroleum source rocks may be more oil-prone (green) or gasprone (red). Chart is modified from Burruss and Hatch (1989) and Hatch (oral commun., 2010). Italicized text indicates informal names. Vertical lines show a generalized time range of unconformity from Bebout and others (1993, fig. 5). Gp., Group; Fm., Formation. 
at the Henry House Falls quarry (fig. 7). These fissile shales are thin-bedded and laterally continuous. The fissile and nonfissile shales exhibit different fracture behavior; shale from the upper member is thin-bedded and fairly friable (fig. 7), whereas the non-friable lower-member siliceous shale is more competent (figs. 8 and 9). Chert beds within the shales display different fracture patterns, competence, and orientations than the bounding shales (fig. 9).

Outcrop studies by Roberts and Mitterer (1992) and Krystyniak and Paxton (2006) determined that siliceous shale samples yield low weight percent total organic carbon (TOC) and fissile samples yield high TOC values. Outcrops and well cores were sampled at numerous locations across Oklahoma and Arkansas, including 11 in the Anadarko Basin Province, by Comer and Hinch (1987) in their analysis of source rock richness and hydrocarbon expulsion from the Woodford
Shale; their results showed that for interbedded black shale and chert, the chert beds contain relatively less TOC, but more bitumen and hydrocarbons than the shale. This suggests that, whereas both the shale and chert beds can be hydrocarbon sources, the shale was more efficient at expelling petroleum and the chert beds were better traps. Comer and Hinch (1987) described fractures that occur within the Woodford throughout their study area; these include fractures filled with bitumen, which are most common in the Arbuckle Mountain uplift and proximal Marietta-Ardmore Basin, the frontal zone of the Ouachita system, and in the southern part of the Anadarko Basin, where late Paleozoic orogenic activity was intense. Fractures filled with bitumen are also in central Oklahoma (along the Nemaha Ridge), where tectonic activity was of less certain timing and style (Comer and Hinch, 1987).

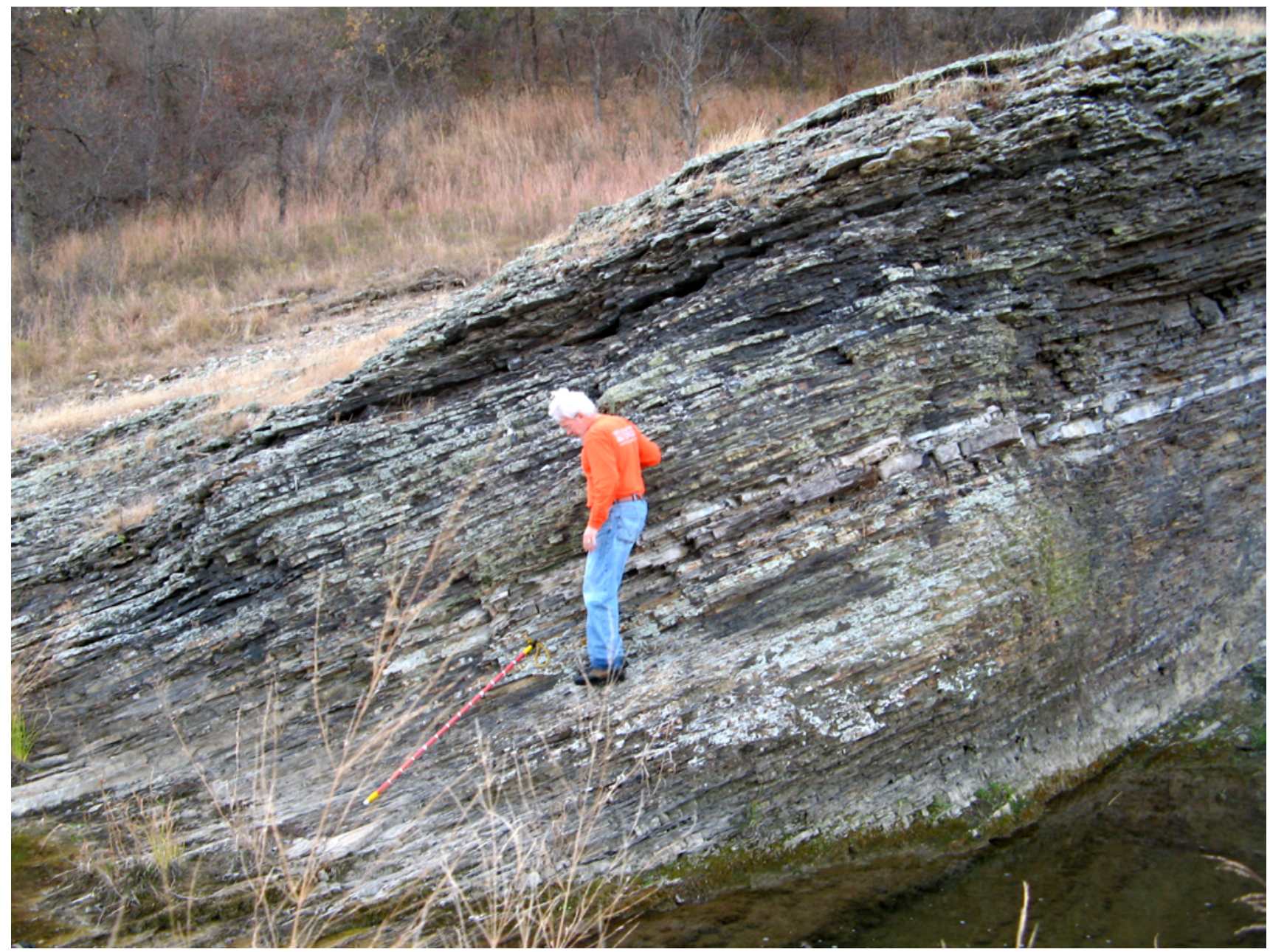

Figure 7. Photograph showing outcrop of Woodford Shale is as much as 231 feet. thick and is located on private property in the Henry House Falls quarry, sec. 6, T. 2 S., R. 1 E., on the south side of the Arbuckle uplift (Paxton, 2007) (fig. 1). Shown is a portion of the upper member of the Woodford Shale. It was described by Paxton (2007) as an organic-rich finely laminated fissile shale. The three resistant lighter-colored beds at Stan Paxton's thigh level are finely crystalline dolomite with hydrocarbon saturation. Spherical phosphate nodules are present above the upper dolomite layer. The upper member at this location is about 55 percent siliceous beds, 44 percent fissile beds, and 1 percent dolomite zones. 


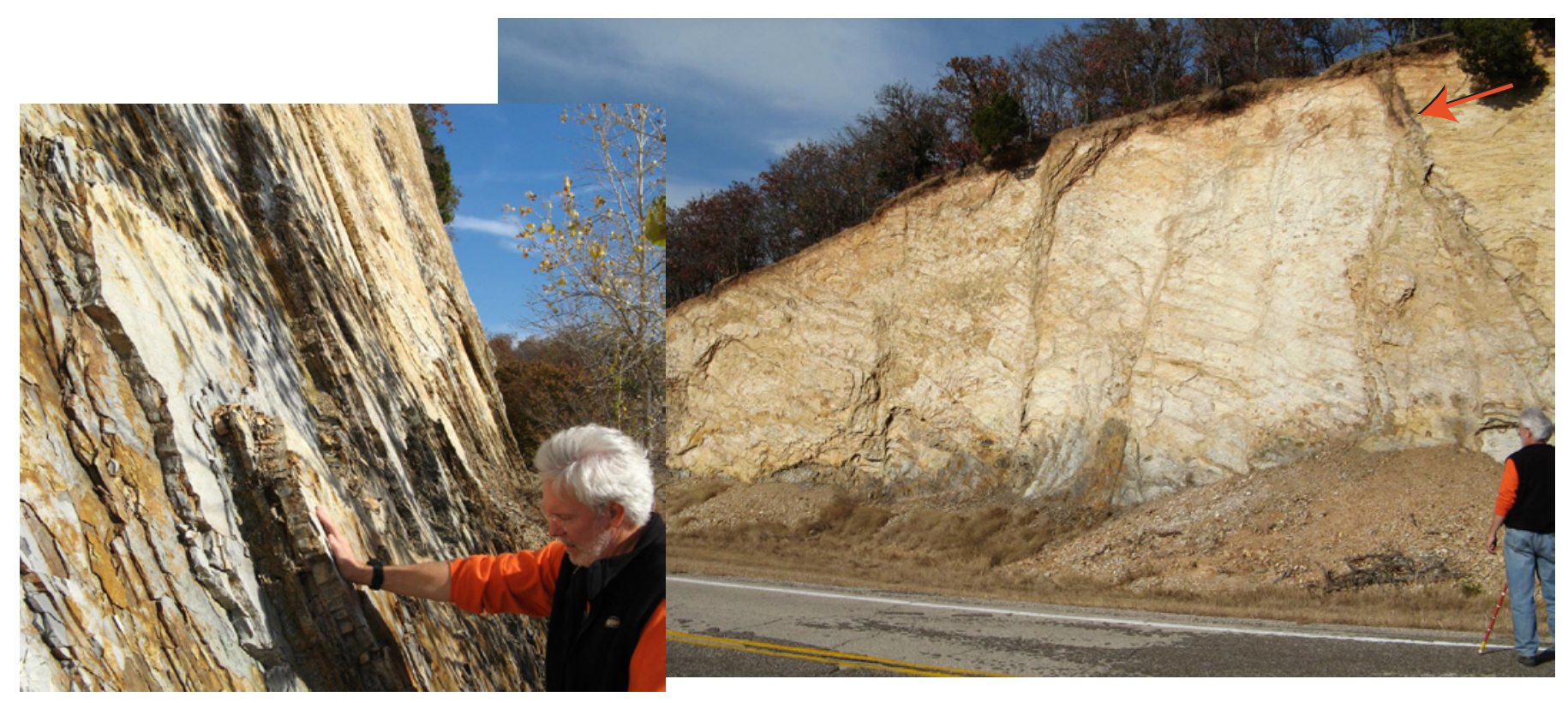

Figure 8. Photographs showing outcrop of a slightly overturned lower member of Woodford Shale beds next to State Route 77D, in NE 1/4 sec. 30, T. 1 S., R 2 E., Murray County, Oklahoma (fig. 1). Stratigraphically up is to the left on the road-cut image. Red arrow on the upper right points to a fault and associated fault gouge, some of which is saturated with hydrocarbon. This section, as described in Paxton and Cardott (2008), strikes N. 70o W and dips 680 to the southwest, is highly siliceous, and was highly susceptible to fracturing during the structural deformation. Strata are generally less fissile than those in figure 7.

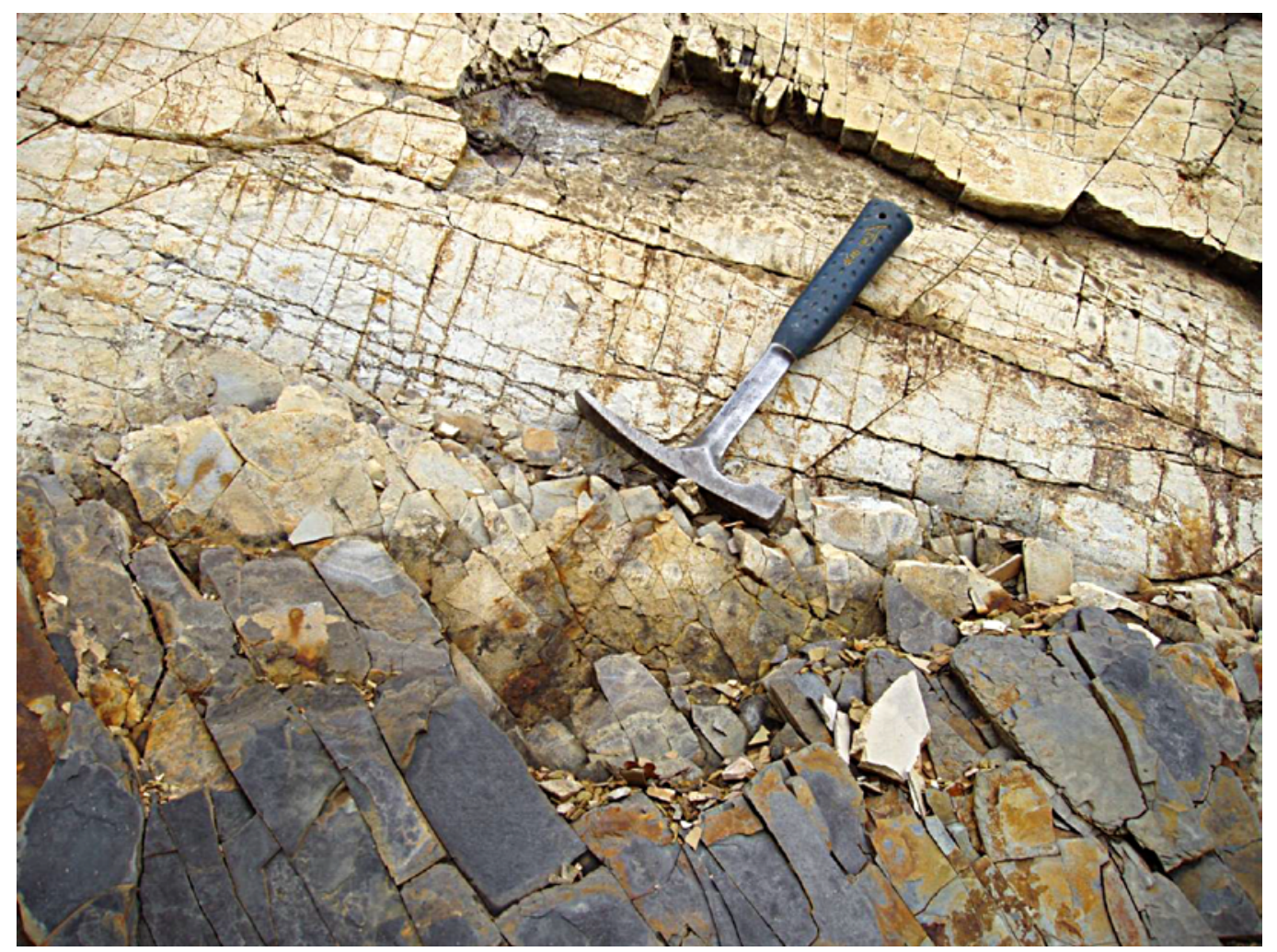

Figure 9. Photograph showing detail of fracture patterns in the lower member of the Woodford Shale at the State Route 77D outcrop photo from Paxton and Cardott (2008) (fig. 1). Spacing and orientation of fracture patterns in the dark gray siliceous shale differ from those in the underlying light-colored chert-rich facies. 
The Woodford Shale includes abundant type A amorphous type II kerogen (partially equivalent to the liptinite [exinite] maceral group) with the remaining primarily type III kerogen (vitrinite) and type I kerogen (Tasmanite alginite) (Lewan, 1983; Thompson and Dembicki, 1986; Crossey and others, 1986; Comer and Hinch, 1987; Burwood and others, 1988; Cardott, 1989, p. 35; Krystyniak and Paxton, 2006). A generalized composition of the Woodford Shale based on Abousleiman (2008) is 37 percent quartz, 26 percent illite, 15 percent kerogen, 17 percent carbonate, and 5 percent kaolinite. TOC content for the upper Woodford ranges from 0.8 weight percent (wt $\%$ ) up to $17 \mathrm{wt} \%$ and is highly variable vertically and laterally across the province based on data from Comer and Hinch (1987), Burruss and Hatch (1989), Hester and others (1990), Price (1997), Krystyniak and Paxton (2006), Joseph Hatch (oral commun., 2010), and data from the USGS Energy Geochemistry Data Base (2010, http://energy.cr.usgs.gov/ prov/og/) (fig. 10). Hester and others (1990) used log-derived formation density for 99 wells across the Anadarko Basin area to assign average TOC values of $3.2,5.5$, and $2.7 \mathrm{wt} \%$ for the respective lower, middle and upper members of the Woodford Shale; variation in TOC was not influenced by formation thickness, but by increased depth and thermal maturation with associated depletion by generation and expulsion of petroleum.

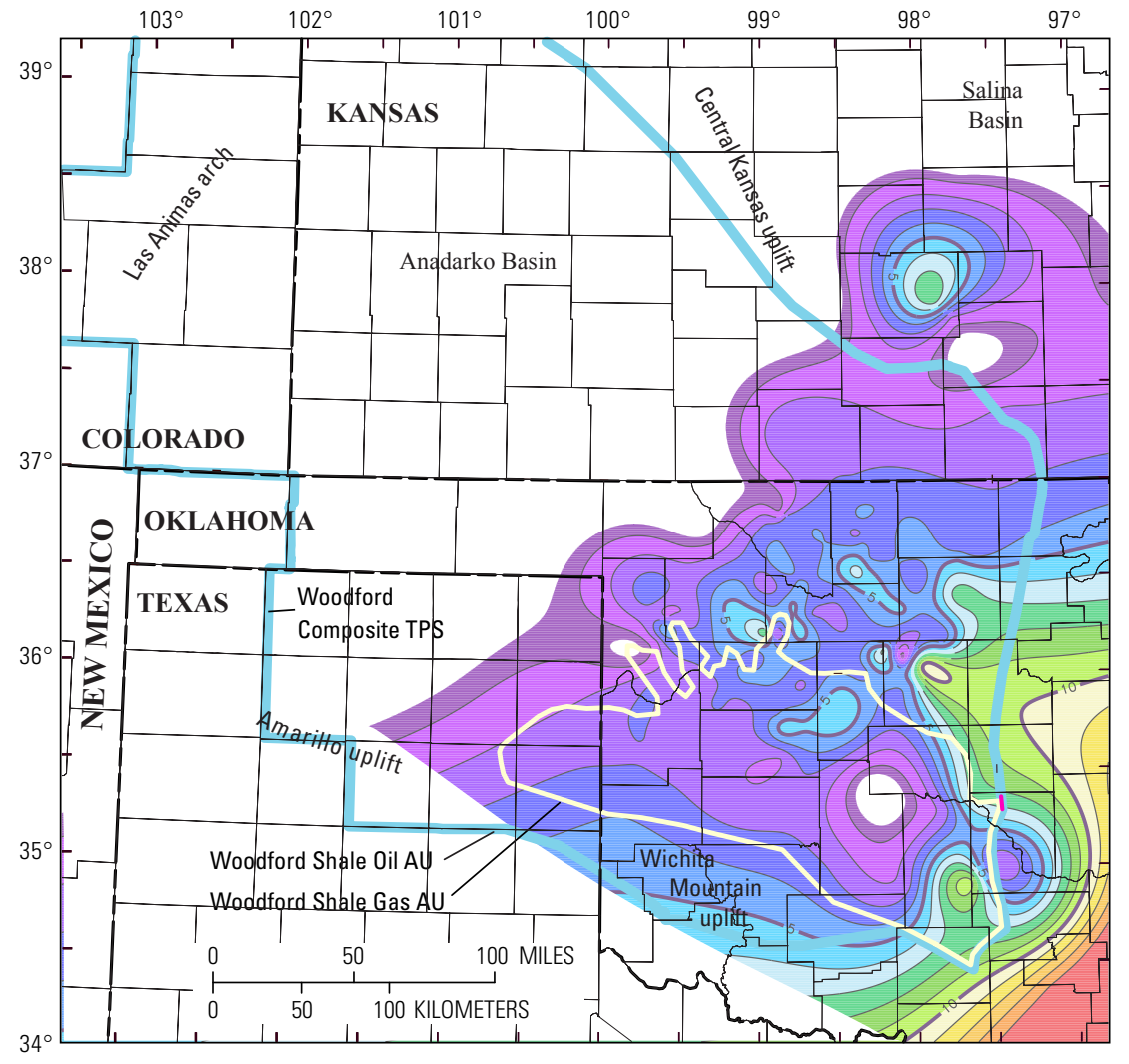

EXPLANATION

TOC

weight, in percent

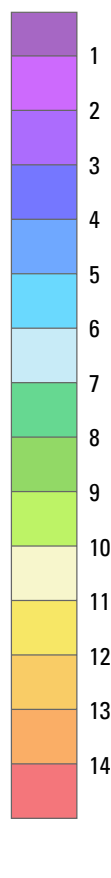

Figure 10. Map showing total organic carbon (TOC) for the Woodford Shale based on data from Burruss and Hatch (1989) and mean estimated values from Hester and others (1990). TOC ranges from 0.08 to $14.05 \mathrm{wt} \%$ and averages 3.7 $w t \%$ based on core and well log density calculations from 123 wells. There is considerable TOC variation, but almost the entire area that is thermally mature for petroleum generation contains greater than 2 wt\% TOC. Decrease in TOC in the deep basin is partly the result of elevated levels of thermal maturation. Oil and gas generation depletes TOC and hydrogen indices ( $\mathrm{HI}$ )- the fuels for petroleum - so original TOC and HI values would have been greater. Pink lines are Precambrian faults from Adler and others (1971). 


\section{Thermal Maturation}

The Woodford Shale is thermally mature for oil generation to overmature for gas generation over most of the Oklahoma and eastern Texas Panhandle portions of the Anadarko Basin Province (fig. 11). Levels of thermal maturation were based on $1 \mathrm{D}$ and $4 \mathrm{D}$ petroleum system models and contoured $\mathrm{R}_{\mathrm{o}}$ data from the Woodford Shale from Cardott (1989), Price (1997), and two wells in the Edmond West field provided by Chesapeake Energy and Mark Pawlewicz. Part of the area proximal to the Nemaha uplift is thermally immature for oil generation from the Woodford Shale based on Cardott (1989) $R_{o}$ data but marginally mature to mature based on $\mathrm{R}_{\mathrm{o}}$ measurements for the West Edmond SWD 1-24 (0.55 percent mean $\left.\mathrm{R}_{\mathrm{o}}\right)$ and Streeter 1 ( 0.75 percent mean $\mathrm{R}_{\mathrm{o}}$ ) wells from Brian Cardott and Mark Pawlewicz, respectively. The West Edmond SWD 1-24 sample was also analyzed by Chesapeake Energy on January 10, 2008, with an estimated mean 0.83 percent $R_{o}$ and range of 0.44 to 0.96 percent $\mathrm{R}_{\mathrm{o}}$ based on Rock Eval pyrolysis Tmax values for 19 samples of the Woodford Shale interval. The models were preferentially calibrated to measured $\mathrm{R}_{\mathrm{o}}$ data on the Woodford Shale. Extent of our modeled thermally mature source rocks is somewhat greater than that of Cardott (1989). The area of thermally mature Woodford Shale was also expanded slightly based on calibration of 1D and 1D extracted models (Gaswirth and Higley, 2014; Higley, 2014a) to incorporate areas in Oklahoma that are petroleum productive from the Woodford Shale, under the assumptions that the reservoir is self-sourced and internal lateral migration is minimal. The Woodford Shale may also be subject to $\mathrm{R}_{\mathrm{o}}$ suppression based on Price and Barker (1985) and Cardott (1989), which could result in higher values than those recorded and displayed by Cardott (1989). These factors are discussed in more detail in Higley (2014a).

Carter and others (1998) determined that oil generation from the Woodford Shale began as early as Late Mississippian in the western basin. Burial history models for two wells from the deep Anadarko Basin were used by Carter and others (1998) to determine that the Woodford Shales were thermally mature for oil generation by the end of the Permian; their burial history model for the northern shelf area of Oklahoma did indicate that the Woodford Shale has been in the early oil generation zone since Early Permian time.

The modeled onset of oil generation and expulsion from the Woodford Shale in the deep basin was about 330 million years ago (Ma), based on generated volumes of oil through time. Onset through completion of hydrocarbon generation is highly dependent on burial depth, which varies greatly across the basin. Figure 12 shows two-dimensional extractions from the 4D model that display thermal maturation through time for a south-north cross section. Displayed are transformation ratios (TR) that range from 0.1 percent at onset, 50 percent at peak, and 99 percent at completion of oil generation (Higley, 2014a, fig. 18). The 99-percent TR boundary corresponds to about 1.2 percent $\mathrm{R}_{\mathrm{o}}$ based on Lewan and Ruble (2002) Woodford Shale hydrous pyrolysis kinetics; this represents the transition from primarily oil to only gas generation. Modeled $\mathrm{R}_{\mathrm{o}}$ contours are also shown for the generalized onsets of dry gas (about 2 percent $\mathrm{R}_{\mathrm{o}}$ ), and overmaturity for gas generation (about 4 percent $\mathrm{R}_{\mathrm{o}}$ ) based on secondary cracking reactions (Tsuzuki and others, 1999; Higley, 2014a). Methane gas is stable in the Woodford Shale of the deep basin, based on thermodynamic calculations and constraints by Takach and others (1987) and Barker and Takach (1992), even considering the almost 28,000 ft current depth of the Woodford Shale in the Bertha Rogers 1 well (fig. 5) and greater than $30,000 \mathrm{ft}$ maximum burial depth of the unit in the basin. Although this indicates that methane is probably present in the deepest portion of the Woodford Shale Gas AU, it does not address the effects of elevated pressure and decreased pore size and permeability on the stored volumes of gas.

Oil generation began about $330 \mathrm{Ma}$ and slightly deeper in the basin than the 330 Ma-cross section image (fig. 12). By $300 \mathrm{Ma}$, the Woodford Shale on this section ranged from thermally immature to overmature for oil generation, and by $250 \mathrm{Ma}$ the range was marginally mature for oil to overmature for gas generation. Because $250 \mathrm{Ma}$ was approximately the time of maximum burial depth, changes in maturation from $250 \mathrm{Ma}$ to $0 \mathrm{Ma}$ were fairly minor. The present-day Woodford Shale and Thirteen Finger Limestone model layers range from marginally mature for oil to overmature for gas generation. As is the case with Carter and others' (1998) research, thermal maturation is strongly influenced by burial depth and temperature. Basement heat flow affects the wavy character of contours in the figure 12 thermal maturation cross sections, with areas of decreased heat flow exhibiting less thermal maturation. This relation is also displayed in Higley (2014a, figs. 39 and 40). Some of the "bumps" in the thermal maturation profiles were explained by Gallardo and Blackwell (1999) as associated with the thermal conductivity structure of the basin and heat generation properties of basement rocks. Cardott and Lambert (1985) and Cardott (1989) observed that the highest $\mathrm{R}_{\mathrm{o}}$ values in the Woodford Shale are about 19-25 mi $(30-40 \mathrm{~km})$ north of the deepest part of the basin and change rapidly over a small lateral distance. Heating history is also influenced by lithologies, for example, evaporites are thermally conductive and thick sections can "cool" underlying strata, whereas coals and shales are thermal insulators that increase heat of underlying rocks. Within the Anadarko Basin, coals are thin and sparse and evaporite sequences are relatively thin, so these lithologies would have minimal impact on heating history. Shales are more common, particularly in the Pennsylvanian section, and would have functioned as thermal blankets for underlying strata. Morrowan black shales compose as much as 60 percent of the rocks in the Oklahoma portion of the province (Wang and Philp, 1997), and the Morrowan section is as much as $4,000 \mathrm{ft}$ thick in the deep Anadarko Basin, thinning northward onto the shelf (Adler and others, 1971; Johnson, 1989).

Hydrocarbons from the Woodford AUs are self-sourced. Oil migration within the Woodford Shale is probably limited because of the low permeability; consequently, migration is predominantly expulsion outward to bounding carrier (reservoir) beds. Petroleum migration is preferentially from higher to 
A

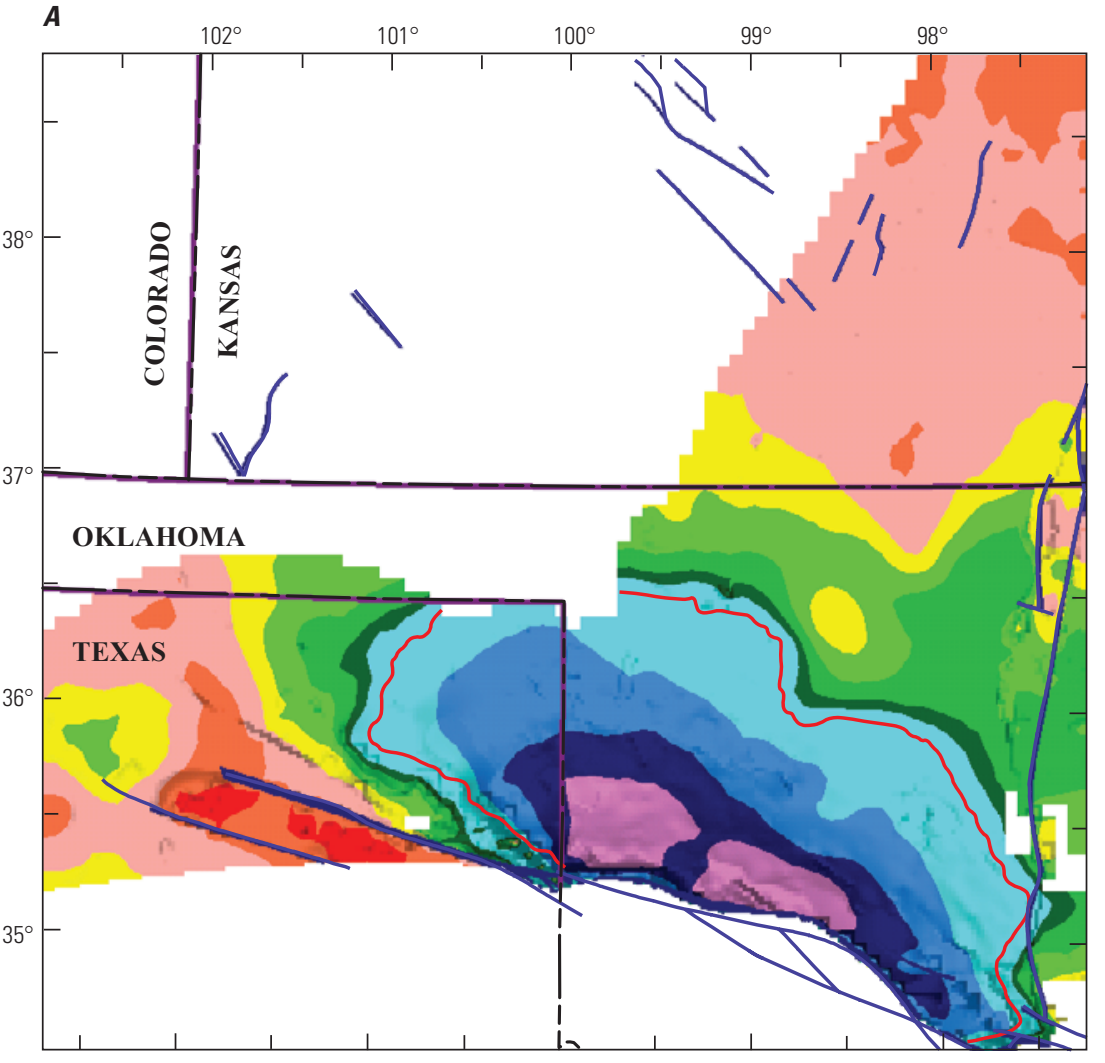

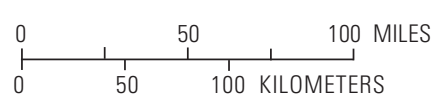

Figure 11. Maps showing $A$, calculated vitrinite reflectance $\left(\% \mathrm{R}_{\mathrm{o}}\right)$ (Sweeney and Burnham, 1990) and $B$, transformation ratios (\% TR) for the Woodford Shale from the four-dimensional petroleum system model. The three-dimensional rendering results in variable shading within contours. Pink contour line on the $\% \mathrm{R}_{0}$ map and the gas-oil ratio (GOR) inset corresponds to the $99 \%$ TR end of oil generation. The inset bubble symbols are GOR, with larger bubbles representing greater GOR. Greatest thermal maturation is in the deep basin of Oklahoma and Texas, whereas most of Colorado and Kansas is immature for oil generation, as characterized by an $R_{0}$ of about 6 percent and TR of 0.1 percent and greater. Blue lines are Precambrian faults from Adler and others (1971).
B

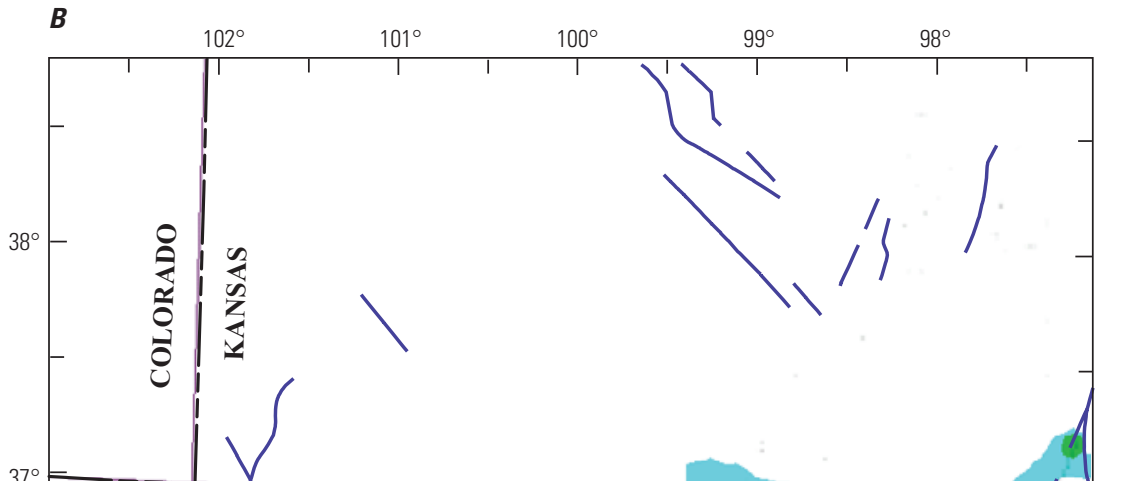




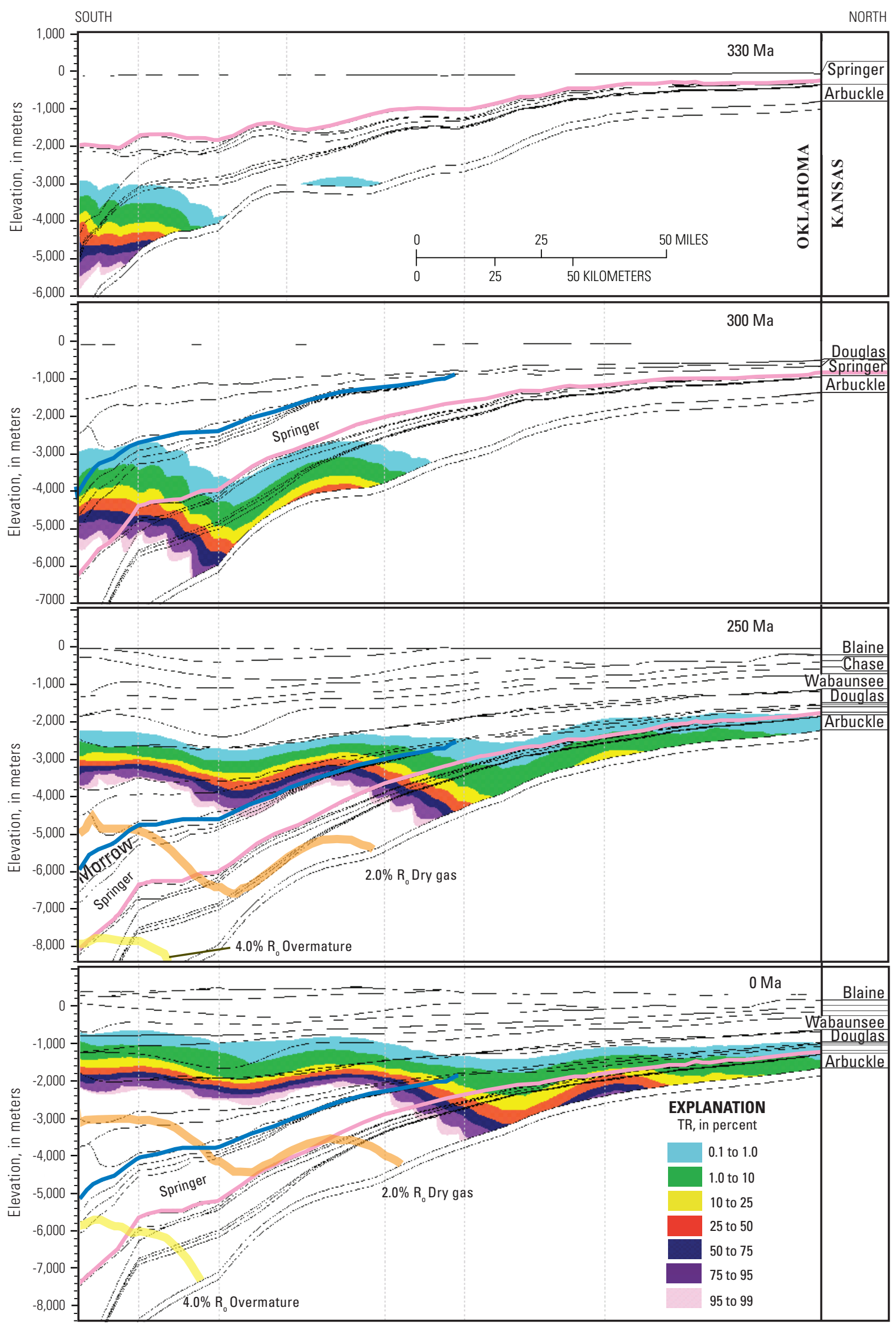


Figure 12. South to north thermal maturation cross sections showing transformation ratios (\% TR) through time based on Woodford Shale hydrous pyrolysis kinetics from Lewan (1983, 1985), and Lewan and Ruble (2002). Pink and dark blue lines are the respective Woodford Shale and Thirteen Finger lime model layers. Cross section location is in figure 3 . Modeled vitrinite reflectance $\left(R_{0}\right)$ lines mark the boundaries for generation and overmaturity for dry gas. Millions of year ago, Ma.-Previous page

lower pressure environments, regardless of whether those are underlying or overlying strata. Oil and gas within the Woodford Shale mainly represent saturation from nonexpelled hydrocarbons and that within faults and fractures. Because this is a reservoir, albeit with low permeability, there is also the potential of hydrocarbons migrating into the Woodford from deeper in the basin or from other source rocks, especially gas, because of its mobility. However, it is unlikely that other petroleum source rocks contributed petroleum to the Woodford Shale, because it is currently overpressured relative to underlying strata in the deep basin (Al-Shaieb and others, 1994) and has probably been overpressured since it was mature for hydrocarbon generation.

Comer and Hinch (1987) estimated the volume of expelled oil from their Woodford Shale study area by subtracting the TOC-normalized volume of hydrocarbons in black shale from the corresponding amount in chert. Their calculations also incorporated the Woodford Shale subcrop area and source rock total thickness, density and TOC concentration, as well as the density and amount of the expelled oil. Their calculated expulsion efficiency was between 27 and 50 percent. Estimated volume of expelled oil was 22 billion barrels from about (1) the eastern one-third of the Anadarko Basin, (2) the Marietta-Ardmore Basin, and (3) the western two thirds of the Central Oklahoma platform (Comer and Hinch, 1987, fig. 1). Figure 13 shows oil generation through time for the Oil Creek Formation, Woodford Shale, and Thirteen Finger lime petroleum source rocks (fig. 2). The curves are displayed as onset to completion, rather than volumes, partly because thickness of the Oil Creek layer is generalized and does not represent

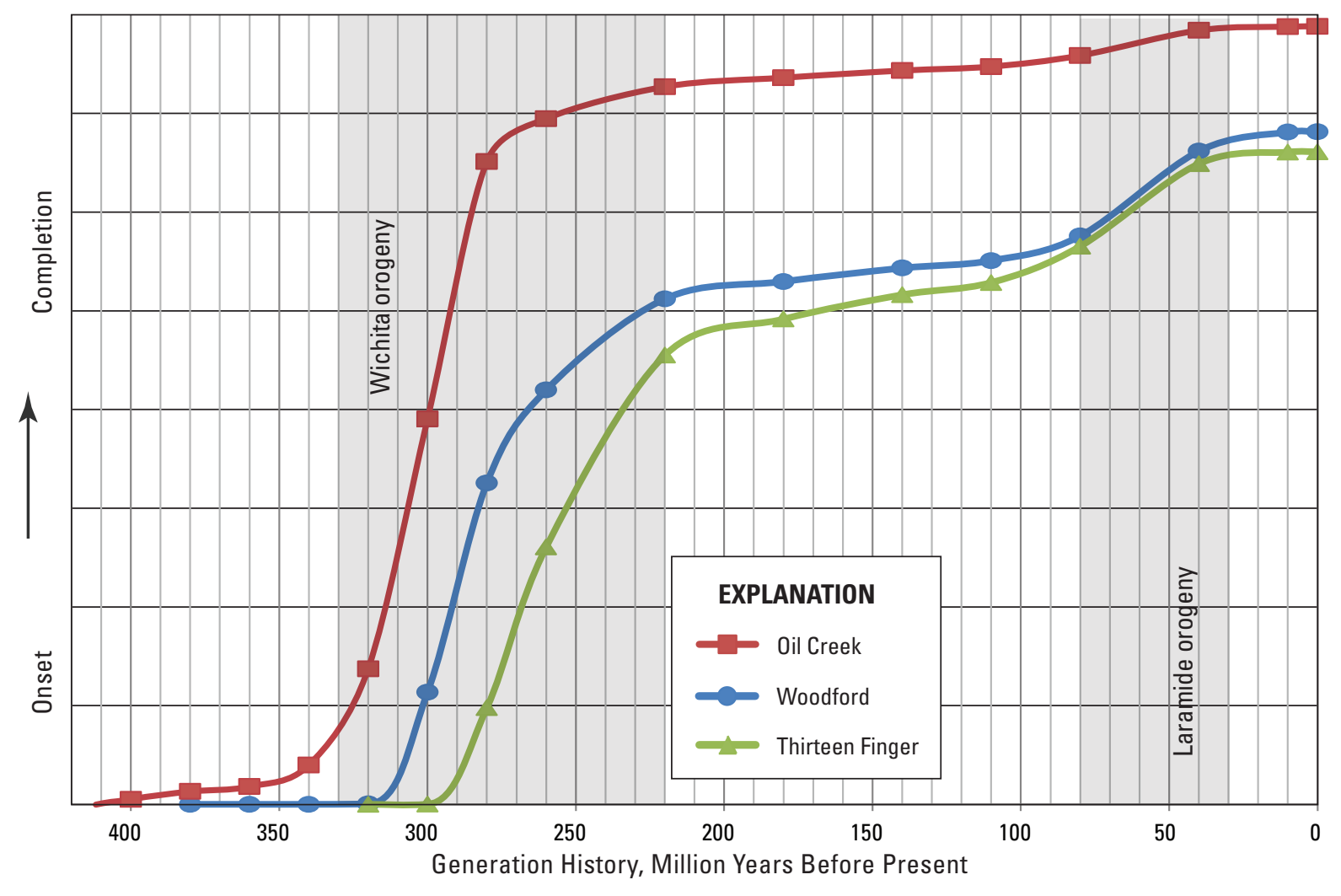

Figure 13. History of petroleum generation from the Oil Creek Formation, Woodford Shale, and Thirteen Finger lime (fig. 2) source layers in the four-dimensional petroleum system model. History is based on Woodford hydrous pyrolysis kinetics (Lewan and Ruble, 2002). Shaded area brackets the main period of peak oil generation from the Woodford Shale. Early oil generation from the Oil Creek layer, prior to about 70 million years ago, is in a narrow strip in the model that is proximal to the Wichita Mountain uplift. This strip exceeds maximum depth of the formation and is primarily the result of areas of poor data control in the area bordering the fault zone. The error could have been removed by clipping the four-dimensional model extent to the basin axis and northward, but less of the model would have been visible and usable. 


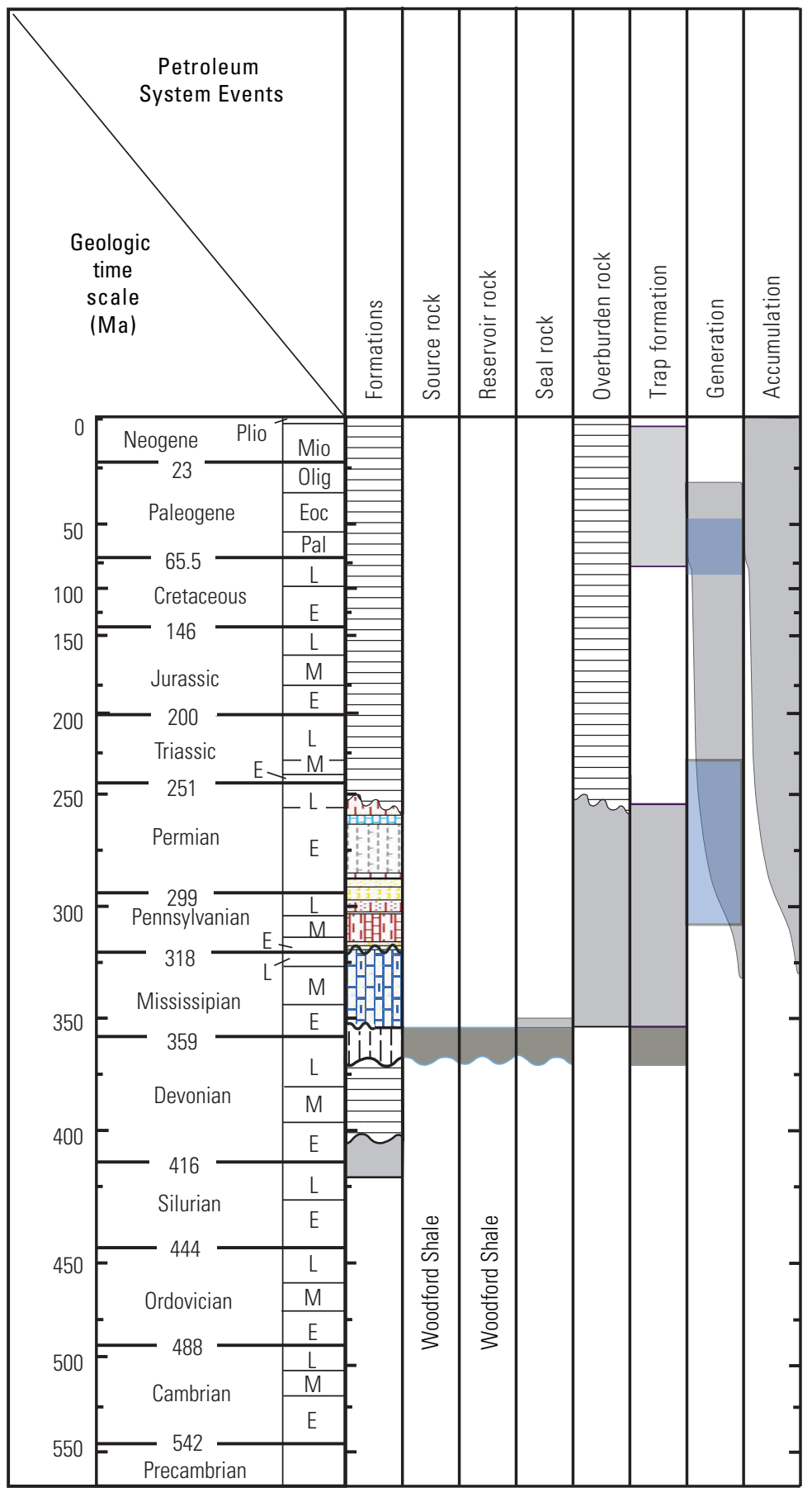

Figure 14. Events chart for the Woodford Shale Gas assessment unit (AU) and Woodford Shale Oil AU. Timing of oil and gas generation, migration, and accumulation is based primarily on vitrinite reflectance and transformation ratios through time from onedimensional and four-dimensional (4D) models (Higley, 2014a). Timing of oil generation (gray polygon) is also based on (unpublished) volumes of petroleum generated through time using the 4D model; peak generation from the Woodford Shale (blue bars) is from about 310 to 230 million of years before present (Ma). A second phase of generation from about 80 to $50 \mathrm{Ma}$ is associated with deposition and erosion of strata associated with the Laramide orogeny. Lithofacies assignments in the "Formations" row are intended only to show periods of unconformities (wavy and horizontal lines) and deposition. Plio, Pliocene; Mio, Miocene; Eoc, Eocene; Pal, Paleocene; L, Late; M, Middle; E, Early. the actual thickness of the source interval. Peak oil generation from the Woodford Shale was from about 310 to $230 \mathrm{Ma}$. Because Anadarko is a deep basin with a shallow shelf, age ranges for petroleum generation vary laterally as well as vertically, allowing for longer periods of oil and gas generation, migration, and trapping across the basin. A second period of Woodford oil generation from about 80 to $50 \mathrm{Ma}$ is associated with Laramide deposition, uplift, erosion, and subtle tilting of the basin. The petroleum system events chart for the Woodford Shale Oil and Woodford Shale Gas AUs (fig. 14) shows time periods of deposition of source, reservoir, seal, and overburden strata. Timing of hydrocarbon generation, migration, and accumulation are derived mainly from the Higley (2014a) petroleum system models (fig. 13, table 2). The curves are displayed as onset to completion, rather than volumes, partly because thickness of the Oil Creek layer is generalized and does not represent the actual thickness of the source interval. Peak oil generation from the Woodford Shale was from about 310 to $230 \mathrm{Ma}$. Because Anadarko is a deep basin with a shallow shelf, age ranges for petroleum generation vary laterally as well as vertically, allowing for longer periods of oil and gas generation, migration, and trapping across the basin. A second 
Table 2. Oil generation onset for the Anadarko Basin four-dimensional model, and onset and completion dates in millions of years before present (Ma) for the Bertha Rogers 1 and Petree Ranch 1 one-dimensional models based on Woodford Shale hydrous pyrolysis kinetics (Lewan and Ruble, 2002). The Douglas Group overlies the youngest potential petroleum source rocks in figure 6 . The HP kinetics-based onset of oil generation is $0.1 \%$ TR and completion is $99 \%$ TR. Oil generation histories in the four-dimensional model were recorded at 10 million year increments, so the plus symbol indicates onset was before the listed age.

\begin{tabular}{|c|c|c|c|c|c|}
\hline & \multirow{2}{*}{$\begin{array}{c}\text { Four Dimensional Model } \\
\text { Onset }\end{array}$} & \multicolumn{2}{|c|}{ Bertha Rogers 1} & \multicolumn{2}{|c|}{ Petree Ranch 1} \\
\hline & & Onset & End & Onset & End \\
\hline Virgilian (Douglas Group) & 270 & 100 & & & \\
\hline Thirteen Finger limestone & +300 & 290 & 265 & 270 & \\
\hline Woodford Shale & 330 & 330 & 310 & 290 & 200 \\
\hline Oil Creek Formation & +370 & 340 & 320 & & \\
\hline
\end{tabular}

period of Woodford oil generation from about 80 to $50 \mathrm{Ma}$ is associated with Laramide deposition, uplift, erosion, and subtle tilting of the basin. The petroleum system events chart for the Woodford Shale Oil and Woodford Shale Gas AUs (fig. 14) shows time periods of deposition of source, reservoir, seal, and overburden strata. Timing of hydrocarbon generation, migration, and accumulation are derived mainly from the Higley (2014a) petroleum system models (fig. 13, table 2).

\section{Traps and Seals}

The Woodford Shale Gas AU southern boundary includes a small portion of the overthrust belt that borders the Amarillo-Wichita Mountain uplift (fig. 3). Gas potential within wells in this area is influenced by complex faulting and fracturing that would likely enable leakage of gas from the Woodford Shale, adversely impacting production of gas. The area was included because Woodford intervals in this area are probably within the oil and gas generation windows, and some wells encounter repeated strata because of the faulting. For example, the Apache Gas Unit No. 1 vertical well penetrates the Woodford at least 4 times and at depths that range from about 1,900 to $19,000 \mathrm{ft}$.

The Woodford Shale AUs are within the Anadarko Basin area defined by Nelson and Gianoutsos (2014) as overpressured. Al-Shaieb and others (1994) outlined an overpressured megacompartment that is primarily in the Oklahoma and Texas portions of the basin; the diagenetically enhanced basal seal is stratigraphically controlled and seems to coincide with the Woodford Shale. Overpressuring occurs in all reservoirs between the top seal at depths of about 7,500 to $10,000 \mathrm{ft}$ $(2,290$ to $3,050 \mathrm{~m})$ to the base of the Woodford Shale, with return to normal pressure in the underlying Hunton Group (Al-Shaieb and others, 1994). Overpressure probably assists petroleum production from the Woodford Shale if natural and induced fractures are open. Effects of overpressure on lateral and bounding seals are dependent on their competence. Al-Shaieb and others (1994) indicated that the megacompartment complex is composed of sealed overpressured reservoirs with pressure gradients that exceed the normal gradient of 10.515 thousand Pascals per meter $(\mathrm{kPa} / \mathrm{m})[0.465$ pounds per square inch per foot (psi/ft)]; the megacompartment, including the Woodford Shale, is sealed to the south by a vertical cementation zone associated with the frontal fault zone of the Wichita Mountain uplift and by the convergence of the top and basal seals along its eastern, northern, and western boundaries. The overlying Kinderhook Shale probably provides a seal across its extent in the eastern basin.

\section{Petroleum Production History}

Distribution of oil and gas wells from the Woodford Shale is shown in figures 15 and 16. Gas production is located primarily within the Woodford Shale Gas AU and oil within the Woodford Shale Oil AU. Estimated mean undiscovered resources from the 2011 assessment are (1) 15.973 trillion cubic feet of gas (TCFG) and 192 million barrels of natural gas liquids (MMBNGL) for the Woodford Shale Gas AU; and (2) 393 million barrels of oil (MMBO), 1.963 TCFG, and 59 MMBNGL for the Woodford Shale Oil AU. The Woodford Shale was included in the 1995 play assessment of the Anadarko Basin as the Woodford/Chattanooga/Arkansas Novaculite play, which was described by J. Schmoker in Henry and Hester (1995) as a hypothetical continuous play with no production or assigned estimates of undiscovered resources. The Misener sand (fig. 16) of the basal Woodford Shale was separately assessed in 1995 as the Devonian Misener sandstone oil play with mean undiscovered accumulations of 4.1 MMBO (Henry and Hester, 1995). The Misener sand for the 2011 assessment was not assessed separately, but was included within the conventional Hunton Group AU (table 1) (Gaswirth and Higley, 2014). 


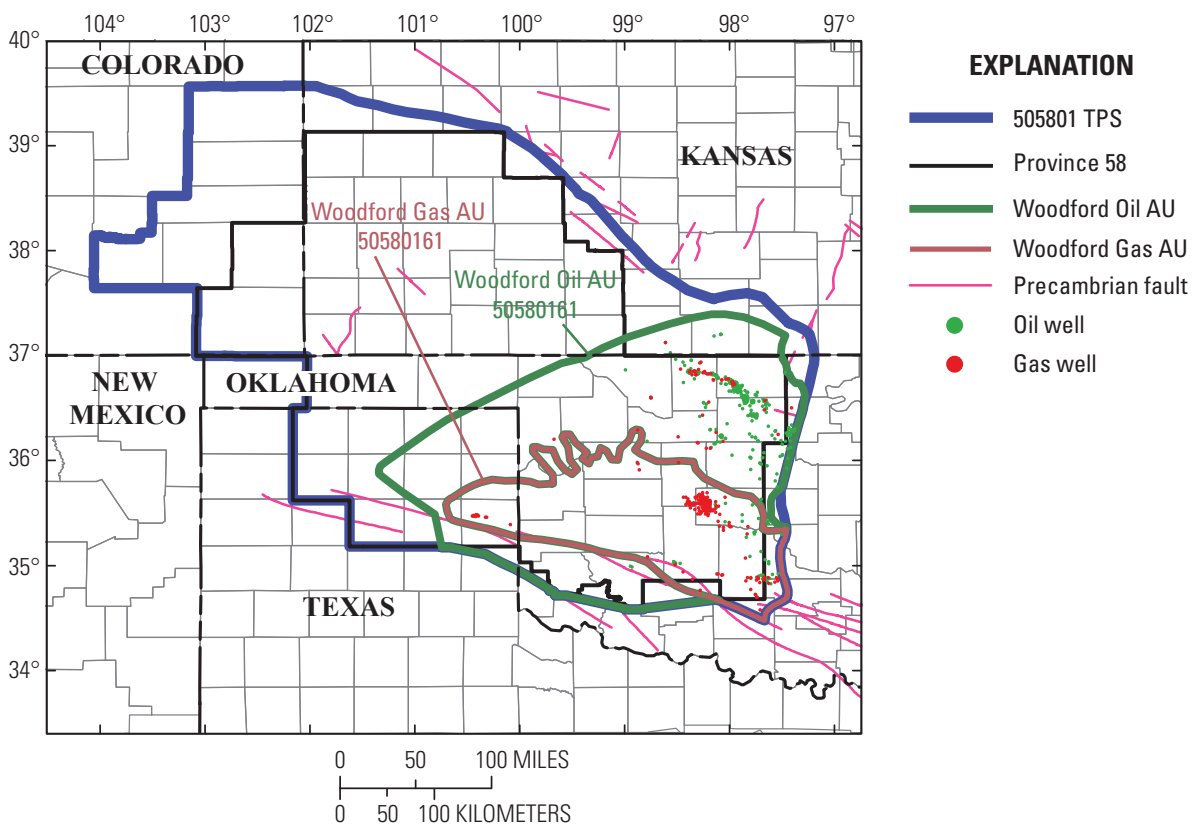

Figure 15. Map showing oil and gas wells that produce from the DevonianMississippian Woodford Shale Oil and Woodford Shale Gas assessment units (AUs) in the Anadarko Basin Province (code number 58) (IHS Energy, 2009a, 2010, 2011). Shown also are $\mathrm{AU}$ code numbers. Some wells include production from other units, primarily the Silurian-Devonian Hunton Group, Devonian informal Misener sand, and Mississippian strata. The Woodford Composite TPS code is 50580. Precambrian faults (pink) are from Adler and others (1971).

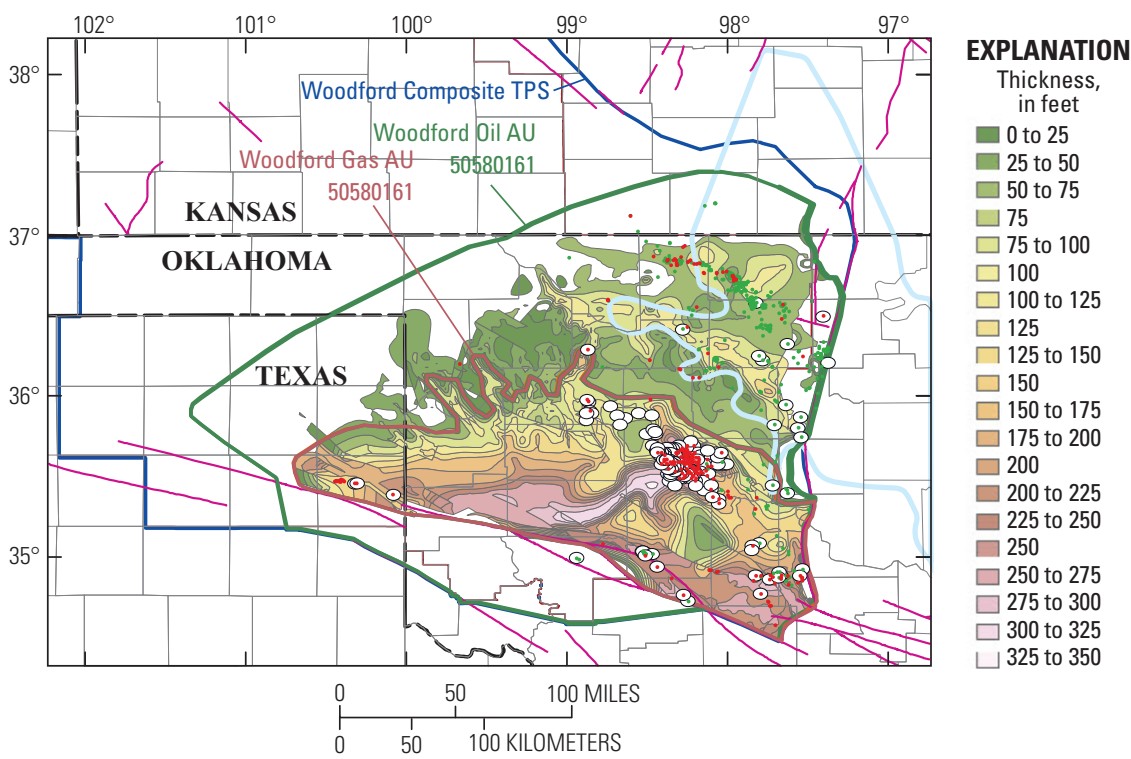

Figure 16. Map showing oil wells (green dots) and gas wells (red dots) that produce from the Woodford Shale in the Anadarko Basin Province (IHS Energy, 2009a, 2010, 2011). White dots are wells that are productive only from the Woodford Shale based on a November 2011 retrieval of data from IHS Energy (2011). White dot wells that do not contain oil or gas wells were added to the IHS database since the January data retrieval. Extent of the Woodford Shale in the province is approximated by the Woodford Oil AU line. Thickness was derived from Rottmann (2000b, pl. 2), analysis of more than 100 well logs, and edited formation tops from IHS Energy (2009a, 2009b). The Kansas portion of the province contains variable thicknesses of Woodford Shale, and the Oklahoma and Texas portions have several small erosional remnants. The generalized location of the informal Misener sand (light blue line) in the lower part of the Woodford Shale (fig. 2) is modified from Amsden and Klapper (1972) and IHS Energy (2009a, 2009b). The Misener sand exhibits variable thickness and distribution, and is also present in isolated lows in the eroded, karst surface of the Hunton Group (Gaswirth and Higley, 2014). Pink lines are Precambrian faults from Adler and others (1971). 


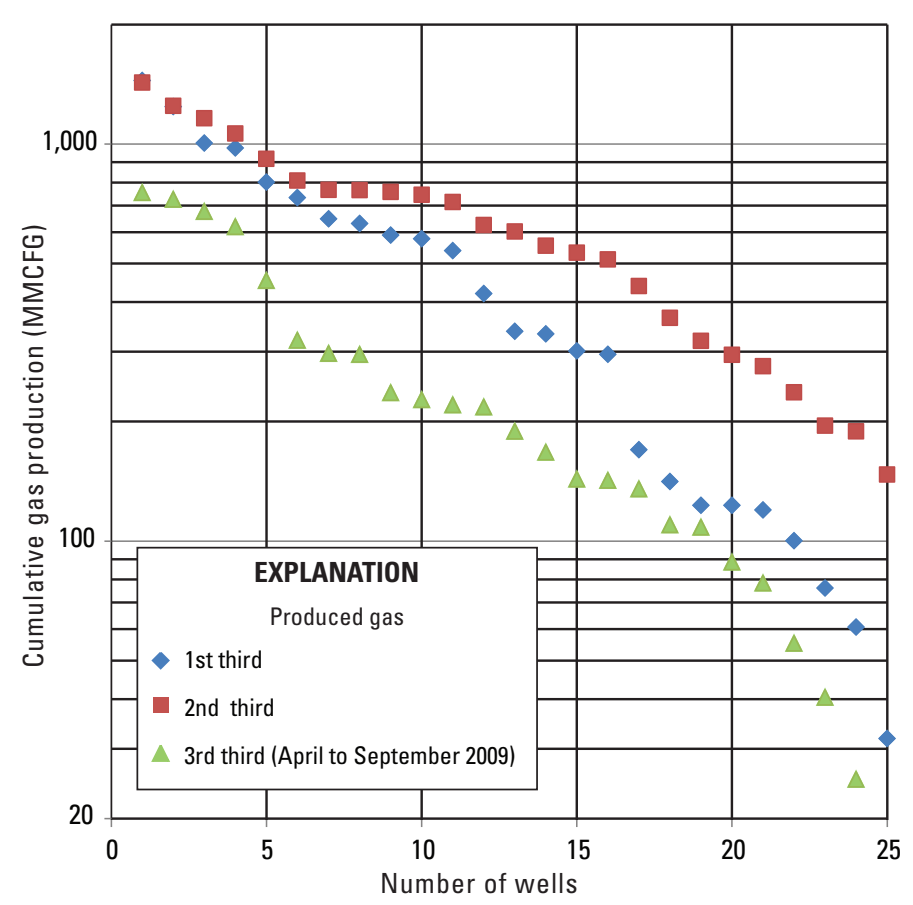

Figure 17. Discovery thirds for 74 wells with reported cumulative gas production from only the Woodford Shale from 1946 to September, 2009 (IHS Energy, 2010). The apparently poor production from the 3rd third is misleading, and actual performance is probably greater, particularly with the present focus on horizontal wells. Reasons for the spread in data include that early production likely incorporates (unreported) oil and gas from the conventional basal Misener sand of the Woodford shale and (or) bounding reservoirs, and the 3rd third only represents 6 months of Woodford Shale gas from 24 wells. Average gas/ oil ratio of the above data set is 237,000 cubic feet of gas per barrel of oil. Million cubic feet of gas, MMCFG.

The 2011 assessment incorporated multiple databases of well and field history and production that included IHS Energy (2009a, 2009b, 2010) and Nehring and Associates, Inc. (2009). Cumulative production from the Woodford Shale across the province was about $2 \mathrm{MMBO}$ and $34 \mathrm{BCFG}$, based mainly on data from IHS Energy (2010). Woodford Shale oil and gas has been mostly from vertical wells, with the production commonly reported commingled with the Hunton Group, Misener sand, Sycamore Limestone, and other units. Of the 130 total wells, there were about 49 horizontal Woodford Shale completions (IHS Energy, 2009a, 2010). Future Woodford Shale wells are expected to be mainly horizontal. Gas-oil ratio averaged about 237,000 cubic feet of gas/barrel of oil for 55 Woodford Shale wells in the province that did not report commingled production (IHS Energy, 2009b). Cumulative gas production for discovery thirds (fig. 17) shows largest gas production for the second third of production history. Largest decrease is for the last third of production history; however, the curves provide only limited information because of the short time range of production. Eighty-eight gas and 21 oil wells, respectively, with Woodford-only production were used to calculate estimated ultimate recovery (EUR) for the Woodford Shale Gas and Woodford Shale Oil AUs (IHS Energy, 2011). Contoured gas EURs are shown in figure 18. Present-day distribution of production for the Woodford Shale Gas AU is scattered across the formation extent, but gas production is concentrated in areas where $\mathrm{R}_{\mathrm{o}}$ values are greater than 1.2 percent (fig. 18). The isolated blue dot of greater than 5,000 MMCFG (fig. 18) appears to be in an area of lower thermal maturity, but there are no proximal $\mathrm{R}_{\mathrm{o}}$ samples, so the value here could be greater than indicated by the contours. The modeled TR through this point is 100 percent and $R_{o}$ is 1.3 percent. Methodology for determining EUR is in Cook (2004). 


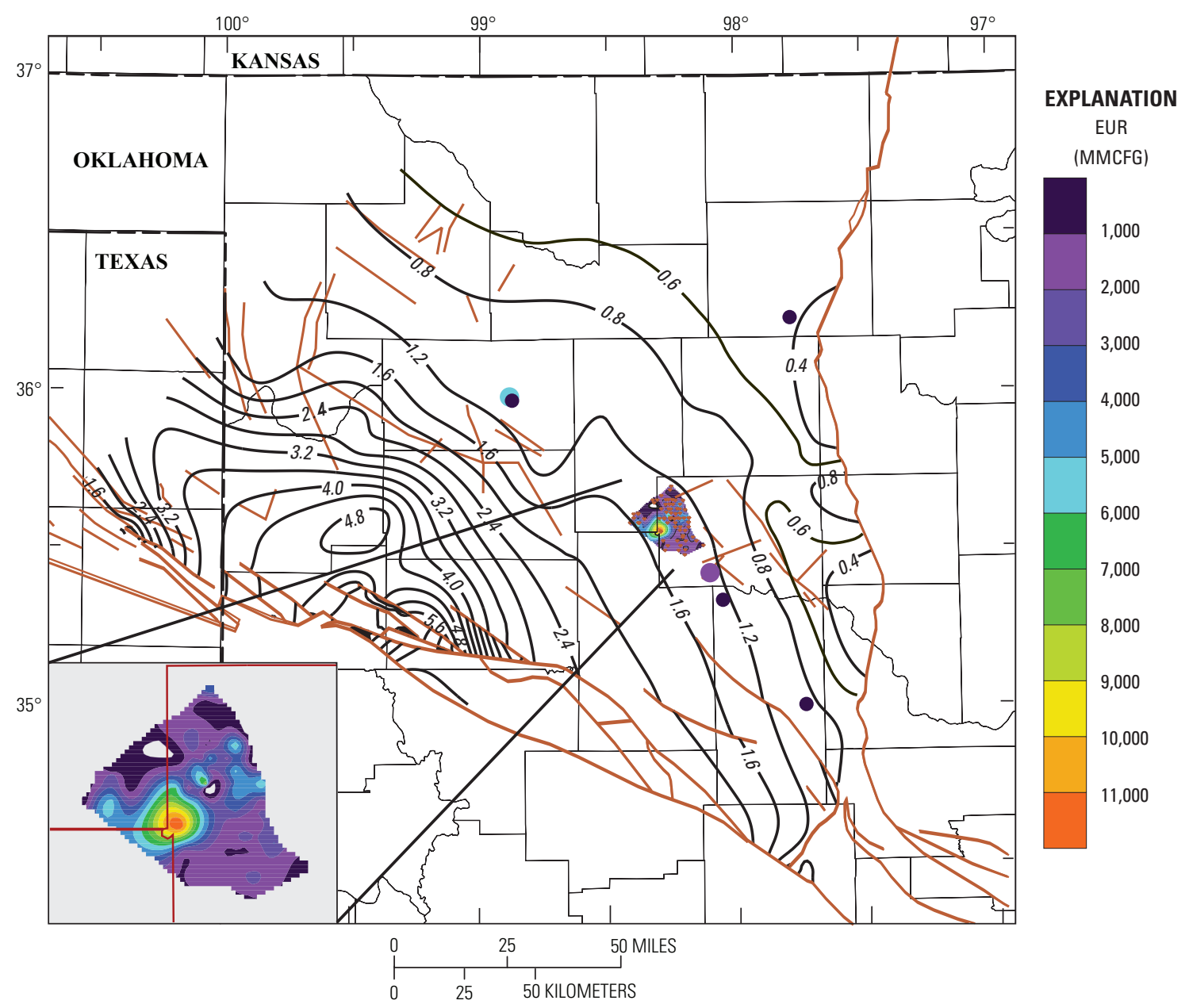

Figure 18. Map showing Woodford Shale calculated gas estimated ultimate recoverable (EUR) ranges from about 0.1 million (MMCFG) to 11.9 billion cubic feet of gas for 76 wells based on production history from IHS Energy (2010). The dense cluster of wells has contoured values, whereas those outside are measured values. Inset map of contoured values is magnified 300 percent. Most gas production is located at the $1.2 \% R_{0}$ and greater Woodford Shale contours created using data from Cardott (1989), Price (1997), Chesapeake Energy Corporation (2009), and Mark Pawlewicz. Faults (brown lines) on the underlying Hunton Group (Rottmann, 2000a) were used to create the Woodford layer and to contour $R_{0}$ values.

\section{Conclusions}

The Devonian-Mississippian Woodford Shale is an important petroleum source rock and reservoir in the Anadarko Basin Province. The formation consists of upper, middle, and lower informal members of carbonaceous silica-rich shales, and a lower informal Misener sand. The shale members contain fissile and non-fissile shale, including chert beds, all of which can exhibit different fracture behavior. Elevation on the top of the Woodford Shale ranges from about $-2,500 \mathrm{ft}(-760 \mathrm{~m})$ on the southern Kansas shelf, to $-27,000 \mathrm{ft}(-8,000 \mathrm{~m})$ in the deep basin of southern Oklahoma. Thickness of the Woodford Shale ranges up to $375 \mathrm{ft}$ and averages $40 \mathrm{ft}$. Greatest thickness is within eroded channels of the underlying Hunton Group. Present-day total organic carbon (TOC) content for the upper Woodford ranges from about $0.8 \mathrm{wt} \%$ to $17 \mathrm{wt} \%$.
Hydrocarbons in the Woodford Shale Gas and Woodford Shale Oil AUs are self-sourced. Gas production is concentrated in areas where $\mathrm{R}_{\mathrm{o}}$ values are greater than 1.2 percent, based mainly on the $4 \mathrm{D}$ model. Lateral migration distances are minor because of the low permeability within the source rock and primary expulsion is from this source outward to bounding carrier beds. Oil generation from the Woodford Shale began in the deep basin of southern Oklahoma about $330 \mathrm{Ma}$, based on 1D and 4D petroleum system models; peak oil generation was from about 310 to $230 \mathrm{Ma}$. Because Anadarko is a deep basin that has a shallow shelf, age ranges for generation vary laterally as well as vertically. These time ranges allowed for longer periods of oil and gas generation, migration, and trapping across the basin. A second period of Woodford oil generation from about 80 to $50 \mathrm{Ma}$ is associated with Laramide deposition, uplift, erosion, and subtle tilting of the basin. 


\section{Acknowledgments}

Tom Judkins, Kristen Marra, and Kate Whidden of the USGS and Brian Cardott of the Oklahoma Geological Survey provided excellent technical reviews of this chapter of the report. Brian has also been a wonderful resource for thermal maturation and petroleum source rock research in the basin. Chesapeake Energy has been very generous with their time, data, and expertise, for which we are grateful. I would like to thank Kathy Winner and IHS Energy for providing information for our mapping and modeling efforts.

\section{References Cited}

Abousleiman, Younane, Tran, M., Hoang, S., Bobko, C., Ortega, A., and Ulm, F.J., 2008, Study characterizes Woodford Shale: The American Oil and Gas Reporter, v. 51, no. 1, p. 106-115.

Adler, F.J., Caplan, W. M., Carlson, M.P., Goebel, E.D., Henslee H.T., Hick, I.C., Larson, T.G., McCracken, M.H., Parker, M.C., Rascoe, G., Jr., Schramm, M.W., and Wells, J.S.,1971, Future petroleum provinces of the mid-continent, in Cram, I.H., ed., Future petroleum provinces of the United States-Their geology and potential: American Association of Petroleum Geologist Memoir 15, v. 2, p. 985-1120.

Al-Shaieb, Zuhair, Puckett, J.O., Abdalla, A.A., and Ely, P.B., 1994, Megacompartment complex in the Anadarko Basin: A completely sealed overpressured phenomenon, in Ortoleva, P.J., ed., Basin compartments and seals: American Association of Petroleum Geologists Memoir 61, p. 55-68.

Amsden, T.W., 1975, Hunton Group (Late Ordovician, Silurian, and Early Devonian) in the Anadarko Basin of Oklahoma: Oklahoma Geological Survey Bulletin 129, 136 p.

Amsden, T.W., 1989, Depositional and post-depositional history of middle Paleozoic (late Ordovician through early Devonian) strata in the ancestral Anadarko Basin, in Johnson, K.S., ed., Anadarko Basin symposium, 1988: Oklahoma Geological Survey Circular 90, p. 143-146.

Amsden, T.W., and Klapper, G., 1972, Misener sandstone (Middle-Upper Devonian), north-central Oklahoma: American Association of Petroleum Geologists Bulletin, v. 56, no. 12 , p. 2323-2334.

Ball, M.M., Henry, M.E., and Frezon, S.E., 1991, Petroleum geology of the Anadarko Basin region, province (115), Kansas, Oklahoma, and Texas: U.S. Geological Survey OpenFile Report 88-450W, 36 p.

Barker, Colin, and Takach, N.E., 1992, Prediction of natural gas composition in ultradeep sandstone reservoirs: American Association of Petroleum Geologists Bulletin, v. 76, no. 12 , p. 1859-1873.
Bebout, D.G., White, W.A., Hentz, T.F., and Grasmick, M.K., eds., 1993, Atlas of major mid-continent gas reservoirs: Bureau of Economic Geology, 85 p.

Blakey, R.C., 2011, North American paleogeographic maps, Late Devonian (360Ma): accessed April 28, 2011, at http:// cpgeosystems.com/namD360.jpg.

Burruss, R.C., and Hatch, J.R., 1989, Geochemistry of oils and hydrocarbon source rocks, greater Anadarko Basin: Evidence for multiple sources of oils and long-distance oil migration, in Johnson, K.S., ed., Anadarko Basin symposium, 1988: Oklahoma Geological Survey Circular 90, p. 53-64.

Burwood, R., Drozd, R. J., Halpern, H.I., and Sedivy, R.R., 1988, Carbon isotopic variations of kerogen pyrolyzates: Organic Geochemistry, v. 12, p. 195-205.

Cardott, B.J., 1989, Thermal maturation of the Woodford Shale in the Anadarko Basin, in Johnson, K.S., ed., Anadarko Basin symposium, 1988: Oklahoma Geological Survey Circular 90, p. 32-46.

Cardott, B.J., and Lambert, M.W., 1985, Thermal maturation by vitrinite reflectance of Woodford Shale, Anadarko Basin, Oklahoma: American Association of Petroleum Geologists Bulletin, v. 69, no. 11, p. 1982-1998.

Carter, L.S., Kelly, S.A., Blackwell, D.D., and Naeser, N.D., 1998, Heat flow and thermal history of the Anadarko Basin, Oklahoma: American Association of Petroleum Geologists Bulletin, v. 82, no. 2, p. 291-316.

Comer, J.B., and Hinch, H.H., 1987, Recognizing and quantifying expulsion of oil from the Woodford Formation and age-equivalent rocks in Oklahoma and Arkansas: American Association of Petroleum Geologists Bulletin, v. 71, no. 7, p. 844-858.

Cook, Troy, 2004, Calculation of estimated ultimate recovery (EUR) for wells in assessment units of continuous hydrocarbon accumulations, chap. 7, in USGS Powder River Basin Province Assessment Team, compiler, Total petroleum system and assessment of coalbed gas in the Powder River Basin Province, Wyoming and Montana: U.S. Geological Survey Digital Data Series DDS-69C, 6 p., available online at http://pubs.usgs.gov/dds/dds-069/dds-069-c/REPORTS/ Chapter_7.pdf.

Crossey, L.J., Hagen, E.S., Surdam, R.C., and Papoint, T.W., 1986, Correlation of organic parameters derived from elemental analysis and programmed pyrolysis of kerogen, in Gautier, D.L., ed., Roles of organic matter in sediment diagenesis: Society of Economic Paleontologists and Mineralogists Special Publication 38, p. 35-45.

Eddleman, M.W., 1961, Tectonics and geologic history of the Texas and Oklahoma Panhandles, in Oil and gas fields of the Texas and Oklahoma Panhandles: Panhandle Geologic Society, p. 61-68. 
Egenhoff, S.O., Fishman, N.S., and Paxton, S.T., 2011, Cyclic nature of intracratonic versus passive margin mudstone systems - A reservoir perspective: American Association of Petroleum Geologists 2011 National Meeting, 1 p.

Feinstein, Shimon, 1981, Subsidence and thermal history of southern Oklahoma aulacogen: Implications for petroleum exploration: American Association of Petroleum Geologists Bulletin, v. 65 , no. 12 , p. $2521-2533$.

Gallardo, Jaquidon, and Blackwell, D.D., 1999, Thermal structure of the Anadarko Basin: Association of Petroleum Geologists Bulletin, v. 83, no. 2, p. 333-361.

Gaswirth, S.B., and Higley, D.K., 2014, Geologic assessment of undiscovered oil and gas resources in the CambrianDevonian stratigraphy of the Anadarko Basin, Oklahoma, Kansas, Texas, and Colorado, chap. 5, in Higley, D.K., compiler, Petroleum systems and assessment of undiscovered oil and gas in the Anadarko Basin Province, Colorado, Kansas, Oklahoma, and Texas-USGS Province 58: U.S. Geological Survey Digital Data Series 69-EE, 42 p.

Gradstein, F.M., Ogg, J.G., Smith, A.G., Bleeker, Wouter, Lourens, L.J., 2004, A new geologic time scale with special reference to Precambrian and Neogene: Episodes, v. 27, no. 2, p. 83-100.

Ham, W.E., Denison, R.E., and Merritt, C.H., 1964, Basement rocks and structural evolution of southern Oklahoma: Oklahoma Geological Survey Bulletin 95, 302 p.

Haq, B.U., and Van Eysinga, F.W.B., 1998, Geological time table: Elsevier Science, Amsterdam, New York, 1 chart.

Hatch, J.R., Jacobson, S.R., Witzke, B.J., Risatti, J.B., Anders, D.E., Watney, W.L., Newell, K.D., and Vuletich, A.K., 1987, Possible late Middle Ordovician organic carbon isotope excursion; evidence from Ordovician oils and hydrocarbon source rocks, mid-continent and east-central United States: American Association of Petroleum Geologists Bulletin v. 71, no. 11, p. 1342-1354.

Henry, M.E., and Hester, T.C., 1995, Anadarko Basin Province (058), in Gautier, D.L., Dolton, G.L., Takahashi, K.I., and Varnes, K.L., eds., 1995 National assessment of United States oil and gas resources on CD-ROM: U.S. Geological Survey Digital Data Series 30, 51 p., accessed April 20, 2011, at http://energy.cr.usgs.gov/oilgas/noga/1995.html.

Hester, T.C., Schmoker, J.W., and Sahl, H.L., 1990, Logderived regional source-rock characteristics of the Woodford Shale, Anadarko Basin, Oklahoma: U.S. Geological Survey Bulletin 1866-D, 38 p.

Higley, D.K., 2014a, Thermal maturation of petroleum source rocks in the Anadarko Basin Province, Colorado, Kansas, Oklahoma, and Texas, chap. 3, in Higley, D. K., compiler, Petroleum systems and assessment of undiscovered oil and gas in the Anadarko Basin Province, Colorado, Kansas,
Oklahoma, and Texas-USGS Province 58: U.S. Geological Survey Digital Data Series 69-EE, 53 p.

Higley, D.K., 2014b, Petroleum systems and assessment of undiscovered oil and gas in the Anadarko Basin Province, Colorado, Kansas, Oklahoma, and Texas-Mississippian through Permian assessment units, chap. 7, in Higley, D.K., compiler, Petroleum systems and assessment of undiscovered oil and gas in the Anadarko Basin Province, Colorado, Kansas, Oklahoma, and Texas-USGS Province 58: U.S. Geological Survey Digital Data Series 69-EE, 60 p.

Higley, D.K., Gaswirth, S.B., Abbott, M.M., Charpentier, R.R., Cook, T.A., Ellis, G.S., Gianoutsos, N.J., Hatch, J.R., Klett, T.R., Nelson, Philip, Pawlewicz, M.J., Pearson, O.N., Pollastro, R.M., and Schenk, C.J., 2011, Assessment of undiscovered oil and gas resources of the Anadarko Basin Province of Oklahoma, Kansas, Texas, and Colorado, 2010: U.S. Geological Survey Fact Sheet 2011-3003, 2 p., accessed February 2011, at http://pubs.usgs.gov/ $f_{s} / 2011 / 3003 /$.

Higley, D.K., Gianoutsos, N.J., Pantea, M.P., and Strickland, S.M., 2014, Precambrian to ground surface grid cell maps of the Anadarko Basin Province, chap. 13, in Higley, D.K., compiler, Petroleum systems and assessment of undiscovered oil and gas in the Anadarko Basin Province, Colorado, Kansas, Oklahoma, and Texas-USGS Province 58: U.S. Geological Survey Digital Data Series 69-EE, 7 p.

IHS Energy, 2009a, IHS energy well database: Unpublished database available from IHS Energy, 15 Inverness Way East, Englewood, CO 80112.

IHS Energy, 2009b, GDS database: Unpublished geological data services database available from IHS Energy, 15 Inverness Way East, Englewood, CO 80112.

IHS Energy, 2010, IHS energy production data on CD-ROM: Unpublished database available from IHS Energy, 15 Inverness Way East, Englewood, CO 80112.

IHS Energy, 2011, IHS Energy Production Data on CD-ROM: Unpublished database available from IHS Energy, 15 Inverness Way East, Englewood, CO 80112.

Johnson, K.S., 1989, Geologic evolution of the Anadarko Basin, in Johnson, K.S., ed., Anadarko Basin symposium, 1988: Oklahoma Geological Survey Circular 90, p. 3-12.

Johnson, K.S., Amsden, T.W., Denison, R.E., Dutton, S.P., Goldstein, A.G., Rascoe, B., Jr., Sutherland, P.K., and Thompson, D.M., 1988, Southern midcontinent region, in Sloss, L.L., ed., Sedimentary cover-North American craton, U.S., The Geology of North America: Boulder, Colo., Geological Society of America, v. D-2, p. 307-359.

Kirkland, D.W., Denison, R.E., Summers, D.M., and Gormly, J.R., 1992, Geology and organic geochemistry of the Woodford Shale in the Criner Hills and western Arbuckle 
Mountains, in Johnson, K.S., and Cardott, B.J., eds., Source rocks in the southern midcontinent, 1990 symposium: Oklahoma Geological Survey Circular 93, p. 38-69.

Krystyniak, A.M., and Paxton, S.T., 2006, Outcrop-based gamma-ray characterization of the Woodford Shale of south-central Oklahoma: Oklahoma Geological Survey, Coalbed methane and gas shales in the southern midcontinent conference, March 21, 2006, Oklahoma City, 16 p.

Lambert, M.W., 1993, Internal stratigraphy and organic facies of the Devonian-Mississippian Chattanooga (Woodford) Shale in Oklahoma and Kansas: Chapter 11, American Association of Petroleum Geology Studies in Geology 37: Source rocks in a sequence stratigraphic framework, p. $163-176$.

Lewan, M.D., 1983, Effect of thermal maturation on stable organic carbon isotopes as determined by hydrous pyrolysis of Woodford Shale: Geochemica et Cosmochimica Acta, v. 47, p. 1471-1479.

Lewan, M.D., 1985, Evaluation of petroleum generation by hydrous pyrolysis experimentation: Philosophical Transactions of the Royal Society, A 315, p. 123-134.

Lewan, M.D., and Ruble, T.E., 2002, Comparison of petroleum generation kinetics by isothermal hydrous and nonisothermal open-system pyrolysis: Organic Geochemistry, v. 33 , no. 12 , p. 1457-1475.

Nehring and Associates, Inc. (NRG), 2009, Significant oil and gas fields database: Colorado Springs, Colorado, NRG Associates, Inc. (Database available from NRG Associates, Inc., P.O. Box 1655, Colorado Springs, CO 80901).

Nelson, P.A., and Gianoutsos, N.J., 2014, Present-day overpressure and paleopressure indicators in the greater Anadarko Basin, Oklahoma, Texas, Kansas, and Colorado, chap. 8, in Higley, D.K., compiler, Petroleum systems and assessment of undiscovered oil and gas in the Anadarko Basin Province, Colorado, Kansas, Oklahoma, and TexasUSGS Province 58: U.S. Geological Survey Digital Data Series DDS-69-EE, 27 p.

Paxton, S.T., 2007, Stop 3. Complete section of Woodford Shale adjacent to the Henry House Falls quarry on the south side of the Arbuckle Mountains, in Cardott, B.J., Andrews, R.D., Miller, G.W., and Paxton, S.T., leaders, Woodford Shale gas field trip May 22 and 24, 2007: Oklahoma Geologic Survey Open File Report 1-2007, p. 42-47.

Paxton, S.T., and Cardott, B.J., 2008, Oklahoma gas shales field trip, October 21 and 23, 2008: Oklahoma Geological Survey Open File Report 2-2008, 61 p.

Perry, W.J., Jr., 1989, Tectonic evolution of the Anadarko Basin region, Oklahoma: U.S. Geological Survey Bulletin 1866-A, p. A1-A19.
Price, L.C., 1997, Minimum thermal stability levels and controlling parameters of methane, as determined by $\mathrm{C}_{15}+$ hydrocarbon thermal stabilities, in Dyman, T.S., Rice, D.D., and Westcott, P.A., eds., Geologic controls of deep natural gas resources in the United States: U.S. Geological Survey Bulletin 2146-K, p. 135-176.

Price L.C., and Barker, C.E., 1985, Suppression of vitrinite reflectance in amorphous rich kerogen-A major unrecognized problem: Journal of Petroleum Geology, v. 8, p. 59-84.

Rascoe, B., Jr., and Adler, F.J., 1983, Permo-Carboniferous hydrocarbon accumulations, Mid-continent USA: American Association of Petroleum Geologists Bulletin, v. 67, no. 6, p. 979-1001.

Roberts, C.T., and Mitterer, R.M. 1992, Laminated black shalebedded chert cyclicity in the Woodford Formation, southern Oklahoma, in Johnson, K.S., and Cardott, B.J., eds., Source rocks in the southern Midcontinent, 1990 symposium: Oklahoma Geological Survey Circular 93, p. 330-336.

Rottmann, Kurt, 2000a, Structure map of Hunton Group in Oklahoma and Texas Panhandle: Oklahoma Geological Survey Special Publication 2000-2, pl. 3.

Rottmann, Kurt, 2000b, Isopach map of Woodford Shale in Oklahoma and Texas Panhandle: Oklahoma Geological Survey Special Publication 2000-2, pl. 2.

Schlumberger, 2011, PetroMod Basin and Petroleum Systems Modeling Software: IES GmbH, Ritterstrasser, 23, 52072 Aachen, Germany, accessed January 2011, at http://www.ies.de.

Shatski, N.S., 1946, The Great Donetz Basin and the Wichita System, comparative tectonics of ancient platforms: Akad. Nauk SSSR Izv. Ser. Geol. 6, p. 57-90.

Sullivan, K.L., 1983, Organic facies variation of the Woodford Shale in western Oklahoma: University of Oklahoma, M.S. thesis, $100 \mathrm{p}$.

Sullivan, K.L., 1985, Organic facies variation of the Woodford Shale in western Oklahoma: Oklahoma City Geological Survey Shale Shaker, v. 35, no. 4, p. 76-89.

Sweeney, J.J., and A.K. Burnham, 1990, Evaluation of a simple model of vitrinite reflectance based on chemical kinetics: American Association of Petroleum Geologists Bulletin, v. 74, p. 1559-1570.

Takach, N.E., Barker, Colin, and Kemp, M.K., 1987, Stability of natural gas in the deep subsurface: Thermodynamic calculation of equilibrium compositions: American Association of Petroleum Geologists Bulletin, v. 71, no. 3, p. 322-333.

Thompson, C.L., and Dembicki, H., Jr., 1986, Optical characteristics of amorphous kerogens and the hydrocarbongenerating potential of source rocks: International Journal of Coal Geology, v. 6, p. 229-249. 
Tsuzuki, N., Takeda, N., Suzuki, M., and Yokoi, K., 1999, The kinetic modeling of oil cracking by hydrothermal pyrolysis experiments: International Journal of Coal Geology, v. 39, no. $1-3$, p. $227-250$.

von Almen, W.E., 1970, Palynomorphs of the Woodford Shale of south central Oklahoma with observations on their significance in zonation and paleoecology: East Lansing, Mich., Michigan State University, Ph.D. dissertation, 179 p.

Wang, H.D., and Philp, R.P., 1997, Geochemical study of potential source rocks and crude oils in the Anadarko Basin, Oklahoma: American Association of Petroleum Geologists Bulletin, v. 81, no. 2, p. 249-274. 
Chapter 7

Petroleum Systems and Assessment of Undiscovered Oil and Gas in the Anadarko Basin Province, Colorado, Kansas, Oklahoma, and Texas-Mississippian

\section{Through Permian Assessment Units}

By Debra K. Higley

Chapter 7 of 13

Petroleum Systems and Assessment of Undiscovered Oil and Gas in the Anadarko Basin Province, Colorado, Kansas, Oklahoma, and Texas-USGS Province 58

Compiled by Debra K. Higley

U.S. Geological Survey Digital Data Series DDS-69-EE 


\title{
U.S. Department of the Interior SALLY JEWELL, Secretary
}

\section{U.S. Geological Survey \\ Suzette M. Kimball, Acting Director}

\author{
U.S. Geological Survey, Reston, Virginia: 2014
}

For more information on the USGS - the Federal source for science about the Earth, its natural and living resources, natural hazards, and the environment, visit http://WWW.usgs.gov or call 1-888-ASK-USGS.

For an overview of USGS information products, including maps, imagery, and publications, visit http://WwW.usgs.gov/pubprod

To order this and other USGS information products, visit http://store.usgs.gov

Any use of trade, firm, or product names is for descriptive purposes only and does not imply endorsement by the U.S. Government.

Although this information product, for the most part, is in the public domain, it also may contain copyrighted materials as noted in the text. Permission to reproduce copyrighted items must be secured from the copyright owner.

Suggested citation:

Higley, D.K., 2014, Petroleum systems and assessment of undiscovered oil and gas in the Anadarko Basin Province, Colorado, Kansas, Oklahoma, and Texas-Mississippian through Permian Assessment Units, chap. 7, in Higley, D.K., compiler, Petroleum systems and assessment of undiscovered oil and gas in the Anadarko Basin Province, Colorado, Kansas, Oklahoma, and Texas_-USGS Province 58: U.S. Geological Survey Digital Data Series DDS-69-EE, 60 p., http://dx.doi.org/10.3133/ds69EE. 


\section{Contents}

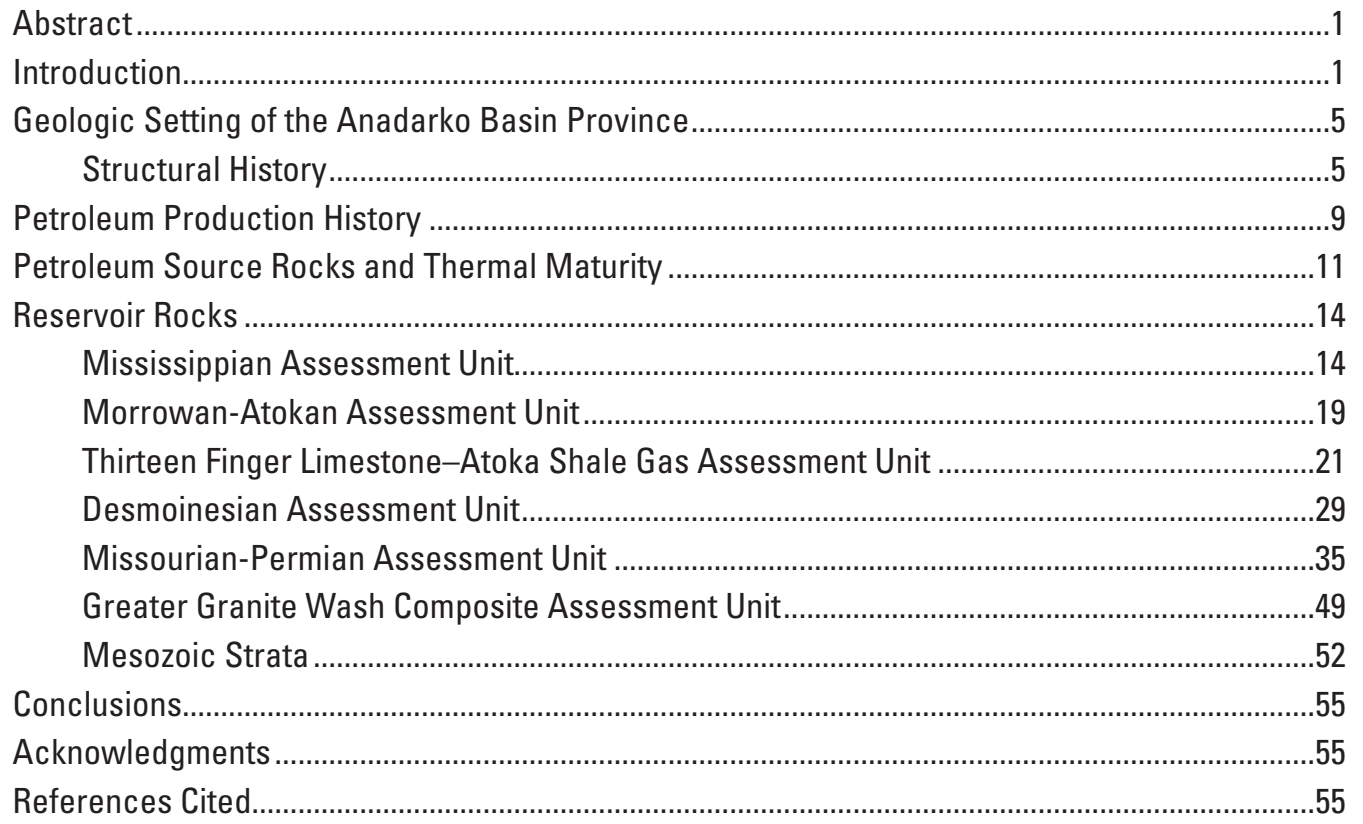

\section{Figures}

1. Map showing Anadarko Basin (red dashed line) and boundaries of total petroleum systems (TPS) and assessment units (AU) that are described in this chapter of the report. The Desmoinesian and Missourian-Permian AUs share a common boundary with the Pennsylvanian Composite TPS, with the exception that the AUs include a "donut hole" that is the Greater Granite Wash Composite AU. There are common boundaries for most AUs.

2. Generalized stratigraphic columns for Devonian through Permian strata in the Anadarko Basin Province. Assessment unit (AU) names and generalized divisions are split at the horizontal red line into (upper) Pennsylvanian Composite Total Petroleum System (TPS) and (lower) Woodford Composite TPS. Italics (blue text) and lowercase names indicate informal status. The Devonian Misener sand was evaluated as part of the Hunton Group AU (Gaswirth and Higley, 2014). Extension of the Morrowan-Atokan AU into the Desmoinesian reflects the unclear boundary of the Atoka Shale in the southern basin. Modified from Bebout and others (1993) and Henry and Hester (1995). Ages in millions of years from Haq and Van Eysinga (1998), and Gradstein and others, (2004) (red text). Fm.; Formation, Gp.; Group, Lst.; Limestone, Im.; lime, Sh., Shale, Ss.; Sandstone 
3. View to the southeast of elevation on the top of the informal Thirteen Finger limestone model layer (Higley and others, 2014b). Areal extent of the Thirteen Finger limestone is approximated by the light blue line. Major structures are labeled. CKU is Central Kansas uplift. The Las Animas arch (not shown) is north of the Sierra Grande uplift. The deep basin of Oklahoma and Texas is approximated by $-6,000$ feet elevation and deeper. Precambrian faults (red) are from Adler and others (1971). Faults bordering the Wichita Mountain uplift are vertical for 4D model purposes only, and subcropping strata on the uplift are unknown because of insufficient data. Data sources for this surface include formation picks from well logs and edited IHS Energy $(2009,2010 a)$ formation tops. Vertical exaggeration is 18 times.

4. South-north generalized cross sections showing stages of development of the southern Oklahoma aulacogen $(A, B)$ (modified from Gilbert, 1983; Perry, 1989), southern Oklahoma trough $(C)$, and Anadarko Basin and bounding Wichita Mountain uplift and fault zone $(D)$...

5. Map showing thickness of strata between the Hunton and Desmoinesian model layers ranges from less than 500 meters (1,640 feet) over most of the Kansas shelf to more than 5,000 meters $(16,400 \mathrm{feet})$ in the deep basin of 0 klahoma. This wedge of Woodford Shale and younger rocks is oriented northwest-southeast, similar to that of Woodford Shale thermal maturation contours. Precambrian faults (blue lines) and named structures are from Adler and others (1971)....

6. Maps showing $A$, Oil and $B$, gas wells that produce from Pennsylvanian and (or) Permian strata in the Anadarko Basin Province (IHS Energy, 2010a, 2010b). Generalized field locations are from IHS Energy (2010a) and Bebout and others (1993). Also shown are the Greater Granite Wash Composite Assessment Unit (AU) and Pennsylvanian Composite Total Petroleum System (TPS) boundaries. Precambrian faults are from Adler and others (1971)

7. Generalized stratigraphic column for the Anadarko Basin Province, with hydrocarbon source rocks highlighted (brown text). Increases in source rock potential are indicated by larger numbers. The expected hydrocarbons column heading indicate whether the source rock is more oil or gas prone. Blue highlights source intervals used in the four-dimensional petroleum system model. Vertical lines show a generalized time range of unconformity from Bebout and others (1993, fig. 5). Chart is from J. Hatch (oral commun., 2010, and modified from Burruss and Hatch (1989). Gp., Group; Fm., Formation.

8. Images showing present-day thermal maturation of Woodford to Douglas model layers using Woodford HP transformation ratio (\% TR) kinetics (Lewan and Ruble, 2002 ) and vitrinite reflectance $\left(\% R_{0}\right)$ (Sweeney and Burnham, 1990). Each layer extends across the study area, including units located outside of the petroleum source rocks. As an example, the Kansas portion of the Springer and Thirteen Finger layers are almost identical because these units are absent over much of this area and the thermal maturity reflects that of the underlying mostly Viola and Simpson Groups strata. The vitrinite reflectance contours in equivalent layers in chapter 3 (fig. 26) are limited to the extent of those strata, and also exhibit slightly different thermal maturation patterns, which largely represents a somewhat newer version of the model. Changes in thermal maturity on the shelf areas of Kansas and Colorado are also minor because of shallow burial relative to the deep basin of Oklahoma and Texas; source rocks are mostly immature for oil generation at less than $0.6 \% \mathrm{R}_{0}$. Thermal maturity for source rocks in the deep basin of Oklahoma and Texas ranges from mature for oil generation, to overmature for gas generation at about $4 \% R_{0}$ and greater. Red contour lines on the $R_{0}$ images mark the 99 percent transformation ratio that is approximately equivalent to $1.2 \% \mathrm{R}_{0}$. Precambrian faults (Adler and others, 1971) are shown as blue lines 
9. Isopach map of Mississippian to Woodford petroleum system model layers, which approximates the thickness of Mississippian rocks across the study area. Blank areas indicate missing strata or insufficient data. Extent of the Woodford Shale is approximated by the Woodford Shale Oil AU boundary in figure 1. Irregular contours are mainly because of subtraction of these unfiltered (unsmoothed) layer grids. Precambrian faults (green lines) are from Adler and others (1971)

10. Maps showing $A$, Oil and $B$, gas wells that produce from the Mississippian $A U$ in the Anadarko Basin Province (IHS Energy, 2010b). Also shown in figure 10A are 276 horizontal wells drilled into Mississippian strata; 166 of these were completed after 2010 (IHS Energy, 2012)

11. Cumulative number of producing wells by year for the Mississippian AU (IHS Energy, 2010a, 2010b). Some of the wells include production from units other than Mississippian. Onset of production from this AU for several major fields is also shown. Locations are in figure 6

12. Grown gas and oil accumulation sizes for discovery thirds of the Mississippian Assessment Unit of the Woodford Composite Total Petroleum System. Although this is a mature assessment unit, the oil accumulations only show a minor drop for the third of discoveries, and the gas accumulation history is stable. Data are from the Nehring and Associates, Inc. (2009) field database, which has limited information for the Mississippian fields. MMBO, million barrels of oil; BCFG, billion cubic feet of gas

13. Petroleum system events chart for the Mississippian Assessment Unit of the Woodford Composite Total Petroleum System. Timing of oil and gas generation, migration, and accumulation is based primarily on Higley (2014) petroleum system models. Potential petroleum source rocks (fig. 7; Hatch and others, 1986) are light gray rectangles. Primary petroleum source rock for the Mississippian AU is the Woodford Shale (Rice and others, 1988a, 1988b; Higley, 2014). The informal Osage lime and Springer Formation are potential petroleum sources for the AU. Times of peak generation from the Oil Creek Formation (blue), Woodford Shale (green) and Thirteen Finger limestone (tan) source rocks are within blue shaded rectangles. Paleogene trap formation, migration, and accumulation are based on Laramide generation and potential remigration associated with erosion and basin tilting. Unconformities, wavy and horizontal lines; Ist, limestone; sh., shale; Fm., Formation; Plio, Pliocene; Mio, Miocene; Eoc, Eocene; Pal, Paleocene; L, Late; M, Middle; E, Early

14. History of petroleum generation from the Oil Creek Formation, Woodford Shale, and informal Thirteen Finger limestone source layers in the four-dimensional (4D) petroleum system model. History is based on Woodford hydrous pyrolysis kinetics (Lewan and Ruble, 2002). Gray bars delineate periods of peak generation. Early oil generation from the 0il Creek layer, prior to about 370 million years ago, is in a narrow band that is proximal to the Wichita Mountain uplift. This band exceeds maximum depth of the formation and is because of areas of poor data control in the area bordering the fault zone. The error could have been removed by clipping the $4 \mathrm{D}$ model extent to the basin axis and northward, but less of the model would have been visible and usable

15. Map showing elevation on the top of the Atoka model layer. Extent of Morrowan and Atokan strata within the Pennsylvanian (Penn.) Composite Total Petroleum System (TPS) is approximated by the Morrowan-Atokan Assessment Unit (AU) boundary and in figure 3 elevation of the basal Thirteen Finger limestone. Elevation is relative to sea level. Precambrian faults are from Adler and others (1971)...... 
16. Maps showing $A$, Oil and $B$, gas wells that produce from Morrowan and Atokan reservoirs in the Anadarko Basin Province (IHS Energy, 2010a). The assessment unit (AU) boundary approximates the extent of Morrowan and Atokan strata. Precambrian faults are from Adler and others (1971). TPS, Total Petroleum System.....22

17. Cumulative number of wells by year that produce from the Morrowan and Atokan (IHS Energy, 2010a, 2010b). Several major fields are also shown by year of discovery; all are shown in figure 6

18. Discovery thirds of grown oil and gas accumulations for the Morrowan-Atokan Assessment Unit (AU) of the Pennsylvanian Composite Total Petroleum System (TPS). This is a mature AU, which is reflected by the generally larger field sizes early in the exploration history (first third) relative to later discoveries (second and third thirds). All thirds show similar trends in field sizes through time. Gas accumulation history is stable for fields less than 10 billion cubic feet of gas (BCFG), but exhibits progressive decline for fields greater than this. Data were constructed using the Nehring and Associates, Inc. (2009) field database, which has limited information for the Morrowan and Atokan fields. The database contains more information on Morrowan and Atokan gas fields than oil fields, as indicated by the data density on the plots. MMBO, million barrels of oil.

19. Petroleum system events chart for the Morrowan-Atokan AU of the Pennsylvanian Composite TPS. Woodford Shale (green) and Thirteen Finger limestone (tan) ranges of oil and gas generation, migration, and accumulation are based primarily on the petroleum system models. Potential petroleum source rocks from Hatch and others (1986) are light gray. Time ranges of peak generation (fig. 14) are within blue rectangles. Paleogene trap formation, migration, and accumulation are based on Laramide generation and potential remigration associated with basin tilting. Unconformities, wavy and horizontal lines; Ist, limestone; sh., shale; Fm., Formation; Plio, Pliocene; Mio, Miocene; Eoc, Eocene; Pal, Paleocene; L, Late; M, Middle; E, Early

20. Isopach map of the top of the Thirteen Finger limestone to the Morrow Formation, and the Thirteen Finger Limestone-Atoka Shale Gas AU (white line). Morrow and Thirteen Finger tops are based on Andrews (1999a, 1999b), examination of more than 120 well logs, and edited tops from IHS Energy (2009, 2010a). Morrow Formation faults (pink) are modified from Andrews (1999b). Color contour interval is $20 \mathrm{ft}$ and (black) line contours are 10 -feet intervals. Figure 21 cross sections are labeled $A-A^{\prime}$ and $B-B^{\prime}$. White polygons are Perryton (Texas) and Knowles Northwest (Oklahoma panhandle) oil fields, conventional oil reservoirs from the Thirteen Finger limestone.

21. A, Southwest to northeast and $B$, southeast to northwest cross sections of the Thirteen Finger limestone (TRFG) of the Pennsylvanian Atoka Group (ATOKA) in the Anadarko Basin. Lines of section are in figure 20. Vertical scale is in feet relative to the base of the Thirteen Finger limestone. MRMT is Marmaton Group, CHRK is Cherokee Group, MRRW is Morrow Formation, and MSSP is top of the Mississippian. Labeled well log traces are gamma (0 to 200 American Petroleum Institute [API]), resistivity, sonic density, and neutron density... 
22. Petroleum system events chart for the continuous Thirteen Finger Limestone-Atoka Shale Gas Assessment Unit (AU) of the Pennsylvanian Composite Total Petroleum System. Timing of basin oil and gas generation, migration, and accumulation is based primarily on the petroleum system models. Thirteen Finger limestone (tan) periods of peak oil and gas generation are shown by the blue rectangles. This $\mathrm{AU}$ is located in an overpressured zone of the deep basin (Al-Shaieb and others, 1994). Because of this, Laramide uplift and erosion probably had minimal effect on the pressure environment. Millions of years ago, $\mathrm{Ma}$; Unconformities, wavy and horizontal lines, Ist; limestone, sh; shale.

23. Photograph showing Desmoinesian outcrop along the San Juan River in the Paradox Basin, southeastern Utah, illustrates carbonate/siliclastic cyclicity and lateral continuity of beds. The informally named middle Desmoinesian "Old Yeller," Desert Creek, and Ismay zones are within the Paradox Formation and are roughly age-equivalent to the upper Cherokee and lower Marmaton Groups in the Anadarko Basin. This section is described in Grammer and others (1996) and is located in about sec. 26, T. 41 S., R. 19 E. Raft is for scale and this north view of the outcrop trends approximately west to east.

24. Modeled surface on the Desmoinesian layer shows a broad gentle slope in Colorado and Kansas that deepens near the basin axis, north of the Wichita Mountain uplift. Vertical yellow bars are Precambrian faults, and surface colors are generalized lithofacies from Adler and others (1971) that mainly record increased clastics basinward. White areas do not contain Desmoinesian or underlying Cherokee model layer lithofacies. Vertical exaggeration is 15 times.

25. Maps showing $A$, Oil and $B$, gas wells that produce from the Desmoinesian Assessment Unit (AU) Cherokee and Marmaton Groups in the Anadarko Basin Province (IHS Energy, 2010a, 2010b). Generalized field locations are from IHS Energy (2010a) and Bebout and others (1993). The Desmoinesian AU shares a common boundary with the Pennsylvanian Composite Total Petroleum System (TPS). Excluded from the AU is an oval area in the deep basin that is the Greater Granite Wash Composite AU and included wells. Precambrian faults (pink) are from Adler and others (1971).

26. Cumulative number of new-field wildcats by year that produce from the Desmoinesian AU (IHS Energy, 2010a, 2010b). Exploration and development was static until about 1950, after which activity increased and has been fairly steady through time. The displayed major fields by discovery dates produce oil and gas from multiple units and ages. Field locations are shown in figure 25.

27. Grown gas and oil accumulation sizes for discovery thirds for the Cherokee and Marmaton Groups reservoirs in the Desmoinesian Assessment Unit (AU) (50580202). This AU has a mature exploration and development status with production from about 1,400 fields in the province (IHS Energy, 2010a, 2010b); however, only a few of these fields are represented on the figure. This paucity of information from the Nehring and Associates, Inc. (2009) is primarily because of reporting of production, mainly as commingled production from multiple units and age ranges. The discovery thirds for oil accumulations show close agreement for fields less than $8 \mathrm{MMBO}$, and for gas accumulations the limited data suggest that the second third of discoveries was more successful than the first and third thirds.....33 
28. Petroleum system events chart for the Desmoinesian Assessment Unit (AU) of the Pennsylvanian Composite Total Petroleum system (TPS). Potential petroleum source rocks (fig. 7) from Hatch and others (1986) are light gray rectangles. The Thirteen Finger limestone (tan) range of oil and gas generation, migration, and accumulation represents Atokan and younger strata that are the primary petroleum source rocks; the 300-million years ago (Ma) onset of generation is based on the petroleum system one-dimensional and four-dimensional models. Potential oil and gas contributions from older source rocks is bracketed by the Woodford Shale (green) onset of generation of about $330 \mathrm{Ma}$. Time ranges of peak generation (fig. 14) are within blue rectangles. Paleogene trap formation, migration, and accumulation are based on Laramide generation and potential remigration associated with basin tilting. Unconformities, wavy and horizontal lines; Plio, Pliocene; Mio, Miocene; Eoc, Eocene; Pal, Paleocene; L, Late; M, Middle; E, Early ......34

29. Map showing elevation on the top of the Council Grove model layer for the Anadarko Basin province. Inset image on the top of the overlying Chase layer shows structures on this north-tilted view at 20 times vertical exaggeration. The primary basin axis is proximal to the Wichita Mountain uplift (fig. 24) in Oklahoma and Texas, the secondary axis trends northward along the west flank of the Central Kansas uplift (CKU), which is bounded approximately by displayed faults. White outlines the Panhandle (south) and Hugoton-Guymon (north) field areas (fig. 25). Precambrian faults (red) are from Adler and others (1971).

30. Oil (green) and gas (red) migration flow paths on the Douglas and Permian layers in the four-dimensional petroleum system model at present day. The image is tilted to the north and is at 15 times exaggeration to better show flow paths. Yellow outline is for the generalized locations of the Hugoton and Panhandle fields (fig. 25). In general, flow follows topography on the model layers. Petroleum is generated mostly in the deep basin and flows radially northward. Flow in northern Oklahoma and in Kansas is directed toward the Las Animas arch (LA), Central Kansas uplift (CKU), and Nemaha uplift. Gas flow paths at the western and eastern boundaries of the map and the CKU resulted from decrease in pressure-volume-temperature (PVT) conditions. Greenish-gold vertical bands are Precambrian faults (Adler and others, 1971). These are the only modeled Missourian-Permian Assessment Unit layers that have flow paths, mainly because (1) Heebner layer shale above the Douglas layer provided enough of a seal to prevent upward leakage, (2) the generalized lithologic assignments of other layers, and (3) coarse grid spacing resulted in most generated petroleum migrating out of the model.

31. Maps showing $A$, Oil and $B$, gas wells that produce from the Missourian-Permian Assessment Unit (AU) in the Anadarko Basin Province (IHS Energy, 2010a, 2010b). Also shown is the common boundary with the Greater Granite Wash Composite AU boundary and included wells. Generalized field locations are from IHS Energy (2010a) and Bebout and others (1993). Largest gas field is Hugoton-Guymon, which extends northward from the Panhandle field. Gold-colored line on $A$ is the southeast to northwest cross section and (red dot) burial history plot location for figure 39. Precambrian faults (pink) are from Adler and others (1971).

32. Graph showing production history of the Missourian-Permian Assessment Unit $(\mathrm{AU})$ in the Anadarko Basin Province with some of the major field discovery names and dates (IHS Energy, 2010a, 2010b; Oklahoma Geological Survey, 2011a, 2011b). Exploration and development was relatively slow until discovery of the Watonga-Chickasha field. Development after about 1950 shows the same general trends as those from other AUs 
33. Map showing generalized contours of estimated ultimate gas recovery (EUR) in million cubic feet of gas (MMCFG) from 1,024 leases in the Hugoton and Guymon fields (data from Troy Cook, written commun., 2010). Leases represent single-well production of gas from the Chase and (or) Council Grove Groups. Contours are based on $\log 10$ EUR values to better show variability across the area. Horizontal blue line is generalized location of cross sections shown in figure 35. Pink lines are Precambrian faults from Adler and others (1971).

34. Graph showing discovery thirds of estimated ultimate recovery of gas (EUR) in million cubic feet of gas (MMCFG) for 1,024 wells in the Hugoton and Guymon fields that produce gas from the Chase and (or) Council Grove Groups (T. Cook, written commun., 2010). Data represent leases that have one well per lease and are split into the first, second, and third thirds of onset of production to November 2009, based on data from IHS Energy (2010a, 2010b). The curves indicate that gas production has undergone a steady decline since onset of production. Decline rate is greatest for the more productive wells in the third of EUR

35. Lithofacies in west to east cross sections across the Hugoton field for the (B) Chase and (C) Council Grove Groups (modified from Dubois, 2007). These stratigraphic cross sections are hung on the top of the Chase (B) and the Council Grove $(C)$, and location is in figure 33. Orange through red layers are continental (Cont) sandstone (SS), siltstone (Silt) and shale. Other colors are marine lithofacies, including fractured (FxIn) and crystalline (CxIn) dolomite (Dolo). Both cross sections show lateral continuity of cyclical beds of marine and continental strata. Marine strata increase in thickness to the east and continental beds thicken westward. Dubois (2007) indicated that large-scale sedimentation patterns and distribution of resultant lithofacies (at the cycle scale) are largely a function of the position on the shelf and reflect the interaction of shelf geometry, sea level, and, possibly, the proximity to siliclastic sources. Lithofacies distribution and cycle-stacking patterns at larger scales may be a function of lower-order cyclicity and a shift from icehouse to greenhouse conditions (upward) during the Lower Permian

36. Graphs showing grown oil and gas accumulation sizes for discovery thirds of the Missourian-Permian Assessment Unit (AU) of the Pennsylvanian Composite Total Petroleum System. Only a fraction of the about 1,420 oil and gas fields that produce from this $A U$ are shown, largely because many fields produce from multiple units with reported commingled volumes. Discovery trends are similar of grown oil accumulations less than about 20 million barrels of oil (MMBO). At greater than this volume, the first third of discoveries are larger than those of the second and third thirds. The early development history was marked by the giant Hugoton-Guymon and Panoma gas fields. In general, the gas accumulation chart shows a general decrease through time in field sizes. Billion cubic feet of gas, BCFG

37. Model image showing oil and gas wells that produce from the Missourian-Permian and Greater Granite Wash Composite assessment units (AU) in the Anadarko Basin Province (IHS Energy, 2010a, 2010b) are superimposed on the image of Douglas plus Permian layer flow paths (fig. 30). In general, areas with less flow have fewer wells. Yellow outline is the Panhandle-Guymon-Hugoton field area, drilling is somewhat faint within the gas fields because of the wider drill spacing than for oil production. Absence of flow paths within the Panhandle field, with their appearance on the west side of the field suggests (invisible) Darcy flow through the reservoir because all potential source rocks are thermally immature in the western Amarillo uplift (fig. 8) (Higley, 2014). Sorenson (2005) also indicated that petroleum migrated southward from the basin into structural traps of the Panhandle field. Precambrian faults are from Adler and others (1971) 
38. Map showing basement heat flow contours across the Anadarko Basin based on data from Carter and others (1998), Blackwell and Richards (2004), and data downloads from the Southern Methodist University Web site ( $h t t p: / / s m u . e d u / g e o t h e r m a l /$. Shown are the cross sections (blue lines) in figures 39 and 40, location of the one-dimensional burial history model (red dot), and Panhandle-Guymon-Hugoton field outlines (pink lines). Values are milliwatts per square meter $\left(\mathrm{mW} / \mathrm{m}^{2}\right)$. The $A-A^{\prime}$ section crosses several zones of elevated heat flow and $B-B^{\prime}$ is mainly in areas of decreased heat flow. Basin areas north of the Wichita Mountain uplift and in the Amarillo uplift and northward exhibit generally lower heat flows than other basin areas. Highest measured heat flow is in the northwest, along the Las Animas arch. The northwest-trending Central Kansas uplift also exhibits elevated heat flow values. Red lines are faults on the top of the Hunton Group from Rottmann (2000a) that were used to contour Hunton and Woodford layers, and Precambrian faults (modified from Adler and others, 1971)

39. Southeast to northwest structural cross section with present-day transformation ratios (\% TR). The burial history plot of TR and vitrinite reflectance $\left(\% R_{0}\right)$ is near the red dot on the cross section, and both locations are in figure 38. Pink lines are the general location of the Woodford Shale layer. The two "bumps" of increased thermal maturation on the cross section correspond to areas of increased heat flow in figure 38. Lithologic variation has minor effect on these increases because of the generalized lithofacies assignments in the model. If basement heat flow was one value across the basin, then the TR contours would be approximately horizontal because of the strong influence of burial depth. The burial history plots show the Council Grove layer as marginally mature for oil generation at 0.1 percent TR and $0.6 \% R_{0^{\prime}}$ and the Woodford Shale layer is overmature for oil generation based on 100 percent TR and more than $1.2 \% R_{0}$. The one-dimensional model is about 2 kilometers southwest of the Woodford Shale measurement of Cardott (1989, p. 45) for the 1 Miami Cattle well (1.56\% mean $R_{0}$ at $-3,563$ m elevation). These images are extractions from the four-dimensional petroleum system model, and are displayed in elevation relative to sea level

40. Cross section showing vitrinite reflectance $\left(\% R_{0}\right)$ and transformation ratio $(\% \mathrm{TR})$ is an extraction from the four-dimensional model across the Panhandle field. Location is in figure 38. The Panhandle field is located on the Amarillo uplift and oil and gas are produced from Wolfcampian and Leonardian strata. The Stone Corral model layer is bounded by pink lines and includes units from the top of the Leonardian Wellington to the base of the Guadalupian Blaine Formations (fig. 2). It is underlain by Wolfcampian strata. The Amarillo uplift and areas to the northwest are thermally immature for oil generation, and potential petroleum source rocks are absent because of erosion or nondeposition. Oil and gas migration flow paths appear west of the Panhandle field in figures 30 and 36; this indicates that hydrocarbons did flow through this field but were not trapped in this leaky model, and were most likely sourced from the east and northeast from Pennsylvanian and older source rocks and through open fault systems. Vertical exaggeration is 33 times... 
41. Petroleum system events chart for the Missourian-Permian AU of the Pennsylvanian Composite TPS. Primary hydrocarbon source rocks are dark gray rectangles, and age ranges of potential source rocks from Hatch and others (1986) (fig. 7) are light gray rectangles. Onset and peak petroleum generation (fig. 14) are shown for the Oil Creek (blue), Woodford (green), and Thirteen Finger limestone (tan) source rocks. Age ranges of peak hydrocarbon generation are indicated by blue rectangles. Paleogene trap formation, migration, and accumulation are based on Laramide generation and potential remigration associated with basin tilting. Unconformities, wavy and horizontal lines; Fm(s)., Formation(s); Plio, Pliocene; Mio, Miocene; Eoc, Eocene; Pal, Paleocene; L, Late; M, Middle; E, Early.

42. Simplified southwest to northeast structural cross section from the Oklahoma deep basin to the shelf shows interfingering of granite wash lithofacies (blue) with those basinward (modified from Johnson, 1989). Greater Granite Wash Composite Assessment Unit (AU) boundary is gold line and cross section location is red line on inset map. Datum is sea level. Sil.-Dev., Silurian-Devonian.

43. Map showing thickness of the top of the Atoka to the Stone Corral model layers in the Greater Granite Wash Composite Assessment Unit (AU) ranges from about 0 to more than 14,000 feet ( $\mathrm{ft}$ ). Areas of smooth contours have more limited data, such as the Wichita Mountain uplift and Panhandle field areas; actual thicknesses here are generally less than $1,000 \mathrm{ft}$ ( 300 meters). Displayed wells produce oil and (or) gas from granite wash facies. Contour lines are 500-feet intervals. Precambrian faults (black) are from Adler and others (1971). Light gray lines are counties

44. Map showing oil and gas wells that produce from the Greater Granite Wash Composite Assessment Unit (AU) in the Pennsylvanian Composite Total Petroleum System (TPS), Anadarko Basin Province (IHS Energy, 2010a, 2010b). Smaller scale views of oil and of gas wells are in the Desmoinesian and Missourian-Permian oil and gas figures 24 and 31 . Generalized field locations are from IHS Energy (2010a), Nehring and Associates, Inc. (2009), and Bebout and others (1993). The largest field is Panhandle, which covers most of the Amarillo uplift and produces from granite wash and other lithofacies. Precambrian faults (blue) are from Adler and others (1971). Light gray lines are counties.

45. Map showing oil and (or) gas wells that produce from the granite wash within the Greater Granite Wash Composite Assessment Unit (AU). Some of the more than 3,600 Oklahoma Geological Survey (2011b) wells up to about 2008 are overlain by the more than 6,900 wells through September 2010 from IHS Energy (2010a, 2010b). Generalized field locations are from IHS Energy (2010a), Nehring and Associates, Inc. (2009), and Bebout and others (1993). Precambrian faults (blue) are from Adler and others (1971). Light gray lines are counties.

46. Discovery halves for oil and thirds for gas grown accumulations for the Greater Granite Wash Composite Assessment Unit (AU) in the Anadarko Basin Province. Only a fraction of the oil and gas fields that produce from this AU are shown, largely because most fields produce from multiple units with reported commingled volumes. These also include giant fields, such as Panhandle, which is not included in the discovery halves plot. Because these figures include some production from strata north of the granite wash lithofacies, the discovery plots are further compromised. The discovery halves of oil accumulations indicate that development has decreased through time. This "trend" probably reflects the paucity of data. The discovery thirds of gas accumulations indicates discovery has been fairly stable through time, although field sizes have decreased for larger fields as indicated by the third of accumulations greater than 20 billion cubic feet of gas (BCFG) 
47. Production history of leases in the Greater Granite Wash Composite Assessment Unit in the Anadarko Basin Province is shown relative to the more than 3,700 completed wells for the same time increments from Oklahoma Geological Survey (OGS, 2011b). Granite wash development started about 1950 and the Oklahoma Geological Survey (2011b) bars follow industry boom and bust cycles with an increase in completions from 1976 to 1985, followed by a lull until about the 2002 increase in exploration. Shown are some of the major field discovery names and year (Nehring and Associates, Inc., 2009; IHS Energy, 2010a, 2010b).

48. Petroleum system events chart for the Greater Granite Wash Composite Assessment Unit (AU) of the Pennsylvanian Composite Total Petroleum System (TPS). Dashed reservoir rock rectangle shows an interval of potential granite wash deposition that was included in the Morrowan-Atokan Assessment Unit (AU). Potential petroleum source rocks from Hatch and others (1986) (fig. 7) are light gray rectangles. Onset and peak petroleum generation (fig. 14) are shown for the 0il Creek Formation (blue), Woodford Shale (green), and Thirteen Finger limestone (tan) source rocks. Age ranges of peak generation are indicated by blue rectangles. Paleogene trap formation, migration, and accumulation are based on Laramide generation and potential remigration associated with basin tilting. Unconformities, wavy and horizontal lines; Fm., Formation; Plio, Pliocene; Mio, Miocene; Eoc, Eocene; Pal, Paleocene; L, Late; M, Middle; E, Early

\section{Tables}

1. Anadarko Basin Province assessment results are listed by name of Total Petroleum System (TPS) and Assessment Unit (AU)

2. Oil generation onset for the Anadarko Basin $4 \mathrm{D}$ model, and onset and completion dates in millions of years before the present (Ma) for the Bertha Rogers 1 and Petree Ranch 1 1D models based on Woodford Shale hydrous pyrolysis (HP) kinetics (Lewan and Ruble, 2002). The Douglas Group overlies the youngest potential petroleum source rocks in figure 7. The HP kinetics-based onset of oil generation is 0.1 percent transformation ratio (TR) and completion is 99 percent TR. Oil generation histories in the $4 \mathrm{D}$ model were recorded at 10-Ma increments; so the plus symbol indicates onset was before the listed age 


\title{
Petroleum Systems and Assessment of Undiscovered Oil and Gas in the Anadarko Basin Province, Colorado, Kansas, Oklahoma, and Texas-Mississippian Through Permian Assessment Units
}

\author{
By Debra K. Higley
}

\section{Abstract}

The Anadarko Basin Province includes the Anadarko Basin of western Oklahoma, western Kansas, and northern Texas, the Las Animas arch of southeastern Colorado, and the Palo Duro Basin of Texas. This is a mature petroleum province for conventional oil and gas reserves and resources. Two total petroleum systems were defined for the province. The Woodford Composite Total Petroleum System includes Cambrian through Mississippian strata, and the Pennsylvanian Composite Total Petroleum System comprises Pennsylvanian through Permian strata. This chapter of the report details assessment results and associated methodology for conventional and continuous resources for defined assessment units (AU) of Mississippian through Permian strata within the total petroleum systems. Mean undiscovered conventional resources for Mississippian through Permian assessment units in the Anadarko Basin Province total 77 million barrels of oil (MMBO), 1,934 billion cubic feet of gas (BCFG), and 60 million barrels of natural gas liquids (MMBNGL).

Total petroleum system assessment methods of analysis resulted in mean undiscovered resources of $17 \mathrm{MMBO}$, 417 BCFG, and 11 MMBNGL for the Mississippian Assessment Unit (AU) of the Woodford Composite Total Petroleum System (TPS). Mean undiscovered continuous gas resources for the Thirteen Finger Limestone-Atoka Shale Gas AU are 6.850 trillion cubic feet of gas (TCFG) and 82 MMBNGL. Undiscovered oil resources for the Thirteen Finger limestone and Atoka Shale are incorporated in the conventional Morrowan-Atokan AU. Mean undiscovered conventional resources for the Pennsylvanian Composite TPS include:

a. $15 \mathrm{MMBO}, 332 \mathrm{BCFG}$, and $7 \mathrm{MMBNGL}$ for the Morrowan-Atokan AU;

b. $6 \mathrm{MMBO}, 118 \mathrm{BCFG}$, and $4 \mathrm{MMBNGL}$ for the Desmoinesian AU; c. $23 \mathrm{MMBO}, 258 \mathrm{BCFG}$, and $8 \mathrm{MMBNGL}$ for the Missourian-Permian AU

d. $16 \mathrm{MMBO}, 809 \mathrm{BCFG}$, and $30 \mathrm{MMBNGL}$ for the Desmoinesian through Permian Greater Granite Wash Composite AU

\section{Introduction}

The U.S. Geological Survey (USGS) recently completed an assessment of the undiscovered oil and gas resource potential of the Anadarko Basin Province of western Oklahoma, western Kansas, northern Texas, and southeastern Colorado (fig. 1). The province area covers 59,861 square miles and includes the Anadarko Basin, Las Animas arch, and Palo Duro Basin. This is hereafter referred to as the 2011 assessment, which corresponds to the publication date of the assessment results by Higley and others (2011). Results of the assessment and geologic analysis (table 1) are based on the geologic elements that are used to define each total petroleum system (TPS), including hydrocarbon source rocks (source-rock maturation, hydrocarbon generation and migration), reservoir rocks (sequence stratigraphy and petrophysical properties), and hydrocarbon traps (trap formation and timing).

Using this geologic framework, the USGS defined the Woodford Composite TPS and Pennsylvanian Composite TPS and nine conventional and three continuous assessment units (AU), and quantitatively estimated the undiscovered oil and gas resources within each AU. The AU code numbers and assignments to TPSs are listed below. Those detailed within this chapter of the report are highlighted in bold text and AU boundaries are shown in figure 1 and the figure 2 stratigraphic column of Devonian through Permian units. The Woodford Shale Oil AU and Woodford Shale Gas AU are discussed in Higley and Cook (2014a); they are included 


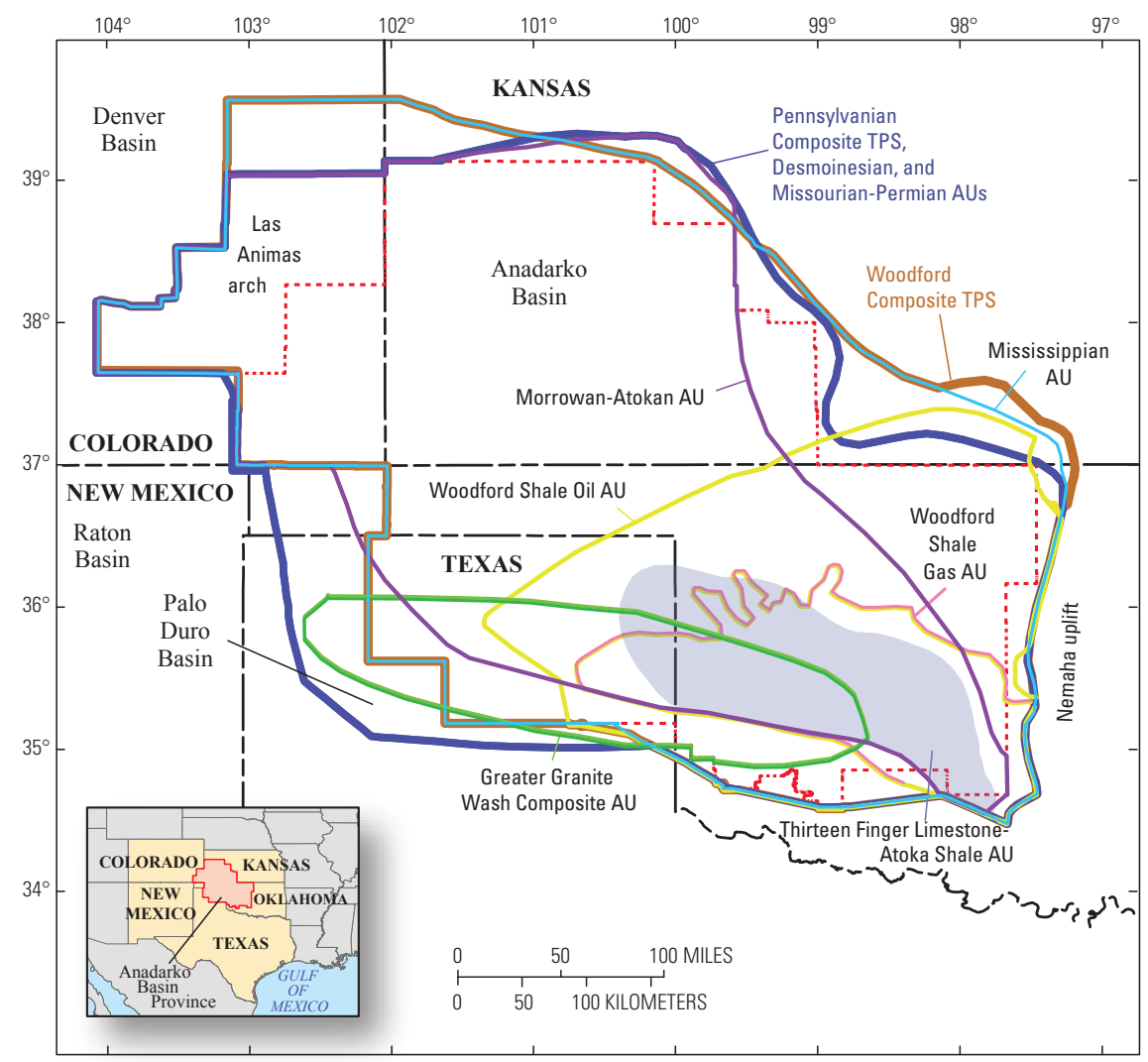

Figure 1. Map showing Anadarko Basin (red dashed line) and boundaries of total petroleum systems (TPS) and assessment units (AU) that are described in this chapter of the report. The Desmoinesian and Missourian-Permian AUs share a common boundary with the Pennsylvanian Composite TPS, with the exception that the AUs include a "donut hole" that is the Greater Granite Wash Composite AU. There are common boundaries for most AUs. 
Table 1. Anadarko Basin Province assessment results are listed by name of Total Petroleum System (TPS) and Assessment Unit (AU).

[Resources are undiscovered oil, gas, and (or) natural gas liquids. MMBO, million barrels of oil; BCFG, billion cubic feet of gas; MMBNGL, million barrels of natural gas liquids. Field type refers to mainly oil or gas accumulations in the assessment unit. Fractiles are fully risked estimates. F95 denotes a 95 -percent chance of at least the amount tabulated. Other fractiles are defined similarly. Fractiles are additive only under the assumption of perfect positive correlation]

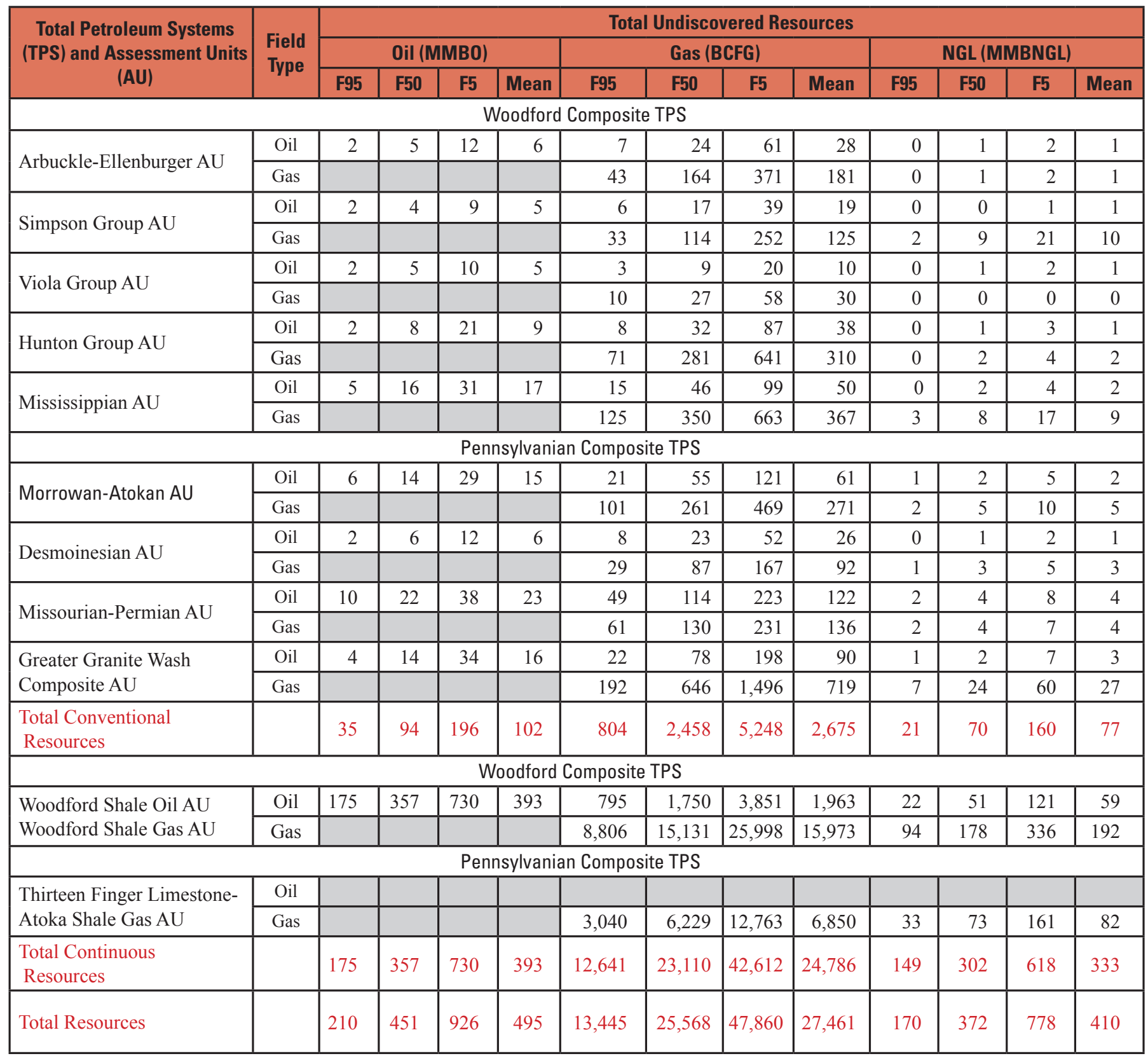




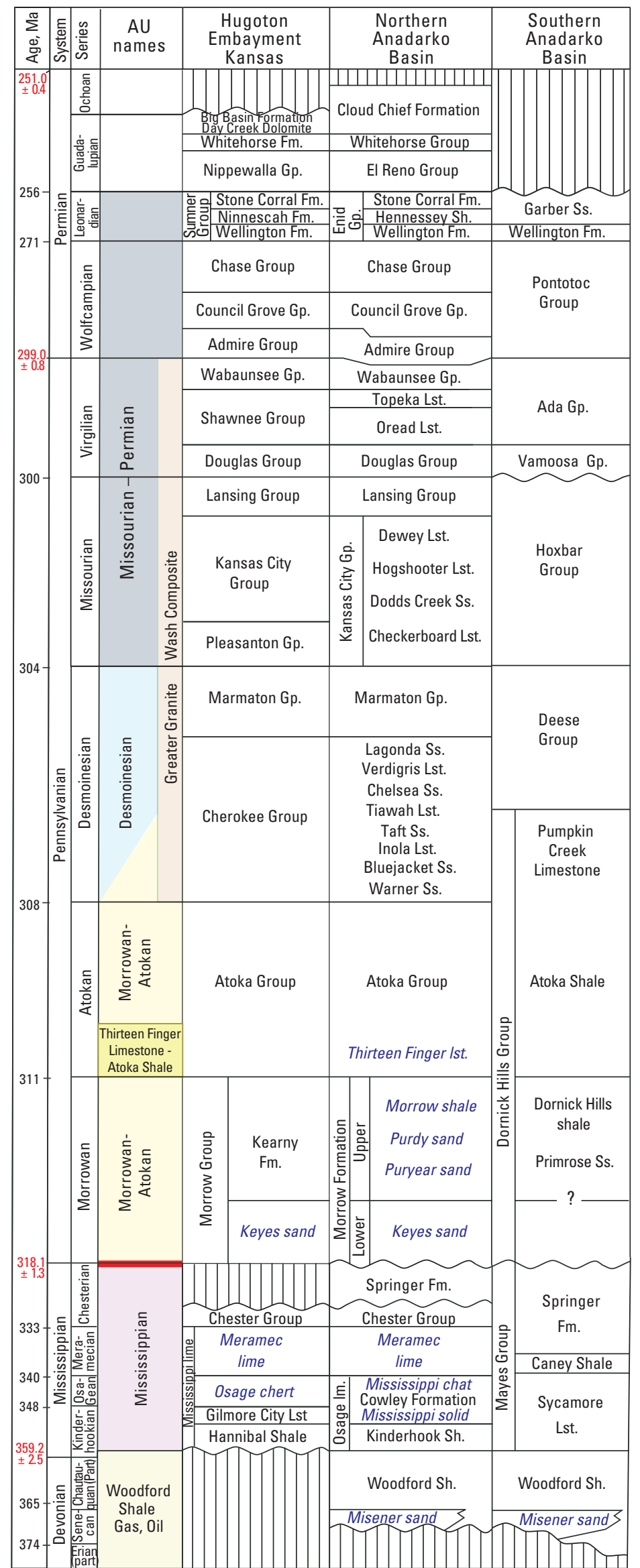

Figure 2. Generalized stratigraphic columns for Devonian through Permian strata in the Anadarko Basin Province.

Assessment unit (AU) names and generalized divisions are split at the horizontal red line into (upper) Pennsylvanian Composite Total Petroleum System (TPS) and (lower) Woodford Composite TPS. Italics (blue text) and lowercase names indicate informal status. The Devonian Misener sand was evaluated as part of the Hunton Group AU (Gaswirth and Higley, 2014). Extension of the Morrowan-Atokan AU into the Desmoinesian reflects the unclear boundary of the Atoka Shale in the southern basin. Modified from Bebout and others (1993) and Henry and Hester (1995). Ages in millions of years from Haq and Van Eysinga (1998), and Gradstein and others, (2004) (red text). Fm.; Formation, Gp.; Group, Lst.; Limestone, Im.; lime, Sh., Shale, Ss.; Sandstone. 
in figure 1 because they delineate major petroleum source rocks in the basin.

Woodford Composite TPS 505801:

1. Arbuckle-Ellenburger, AU 50580101

2. Simpson Group, AU 50580102

3. Viola Group, AU 50580103

4. Hunton Group, AU 50580104

5. Mississippian, AU 50580105

6. Woodford Shale Gas, AU 50580161

7. Woodford Shale Oil, AU 50580162

Pennsylvanian Composite TPS 505802:

1. Morrowan-Atokan, AU 50580201

2. Desmoinesian, AU 50580202

3. Missourian-Permian, AU 50580203

4. Greater Granite Wash Composite, AU 50580204

5. Thirteen Finger Limestone-Atoka Shale Gas, AU 50580261

\section{Geologic Setting of the Anadarko Basin Province}

Structural and isopach surfaces used in this chapter of the report and for building a four-dimensional (4D) petroleum system model (Higley, 2014; Higley and others, 2014) were constructed using edited formation tops data from (1) more than 220 wells across the province; (2) IHS Energy (2009, 2010a); (3) Kansas Geological Survey (2010, http://www. kgs.ku.edu/PRS/petroDB.html); and (4) maps and data from Fay (1964), Rascoe and Hyne (1987), Robbins and Keller (1992), Cederstrand, and Becker (1998), Andrews (1999a, 1999b, 2001), and Rottmann (2000a, 2000b). Rock unit ages and lithologies for surfaces and stratigraphic columns are commonly generalized; sources of information include Denison and others (1984), Ludvigon and others (2009), and the National Geologic Map Database (2011, http://ngmdb. usgs.gov/Geolex/). Figures within this chapter of the report may variously have feet or meters as vertical scales. Metric scales are mainly used for the one-dimensional (1D), two-dimensional, and 4D petroleum system models, largely because of ease in using the modeling software. Vertical scale of most contour maps and of well-log cross sections are in feet because that is traditionally used in the United States. Tilted three-dimensional (3D) images generally do not have map scales because edges would have different scales, with the associated clutter.

\section{Structural History}

The Anadarko Basin Province, which also incorporates the Las Animas arch and Palo Duro Basin, is located in western Kansas, western Oklahoma, northern Texas, and southeastern Colorado. The Anadarko Basin is the deepest in the onshore United States and markedly asymmetrical, with Precambrian basement ranging in depth from more than 40,000 feet ( $\mathrm{ft}$ ) at the basin axis in southern Oklahoma, to surface exposures on the broad shelf in the proximal Central Kansas uplift (CKU) of Kansas. The basin is bounded on the southeast by the Marietta-Ardmore Basin, on the east by the Nemaha uplift and Salina Basin, on the north by the CKU, and on the south by the eroded Amarillo-Wichita Mountain uplift (fig. 3). Western boundaries are the Denver and Raton Basins (fig. 1). The Nemaha uplift consists of a discontinuous series of block-faulted segments, each of which is about 5-20 mile long (north-south) and 3-5 mile wide, and forms the axis and western boundary of the broad, gently raised Central Oklahoma platform (Johnson, 1989) that separates the Anadarko Basin from the Ardmore Basin. The platform contains Precambrian and pre-Pennsylvanian rocks that are unconformably overlain by Desmoinesian and Missourian age strata (Rascoe and Adler, 1983).

Schatski (1946) stated that southern Oklahoma was initially an aulacogen. The latest Precambrian to earliest Cambrian structural setting of southern Oklahoma is considered to be that of a failed linear rift (aulacogen) of an inferred plate tectonic triple junction (Burke, 1977; Perry, 1989), the common name of which is the Southern Oklahoma aulacogen. Axis of the resulting southern Oklahoma trough extended northwest from the paleocontinental eastern margin through the area now occupied by the Ardmore Basin and Arbuckle Mountains, the Wichita Mountains and vicinity, and into the northern Panhandle of Texas (Perry, 1989). At the close of the rifting phase, the aulacogen began to cool and subside (Feinstein, 1981). From Cambrian to Mississippian time, the area of the Anadarko Basin was located near the equator and predominantly covered by warm, shallow seas with mostly carbonate deposition (Wang and Philp, 1997). A passive continental margin existed outward from the trough during this time period (Nicholas and Rozendal, 1975; Keller and others, 1983). More than 3.3 kilometers (km) [11,000 feet (ft)] of Cambrian through Lower Devonian rocks were deposited over the axis of the aulacogen in the region of the Wichita and southwestern Arbuckle Mountains (Ham, 1973).

The Late Mississippian (probably Chesterian) was marked by renewed tectonism (Perry, 1989). Major plate collision between the North American plate and either Gondwana or an intervening microplate resulted in onset of the Ouachita orogeny and formation of the ancestral Rocky Mountains (Kluth and Coney, 1981; Kluth, 1986). Ball and others (1991) indicated the Mississippian Period closed with regional uplift and erosion that marked the start of the Wichita orogeny. Near the end of Mississippian time, the seas retreated to the deeper areas of the geosyncline because of upwarping of the Wichita 


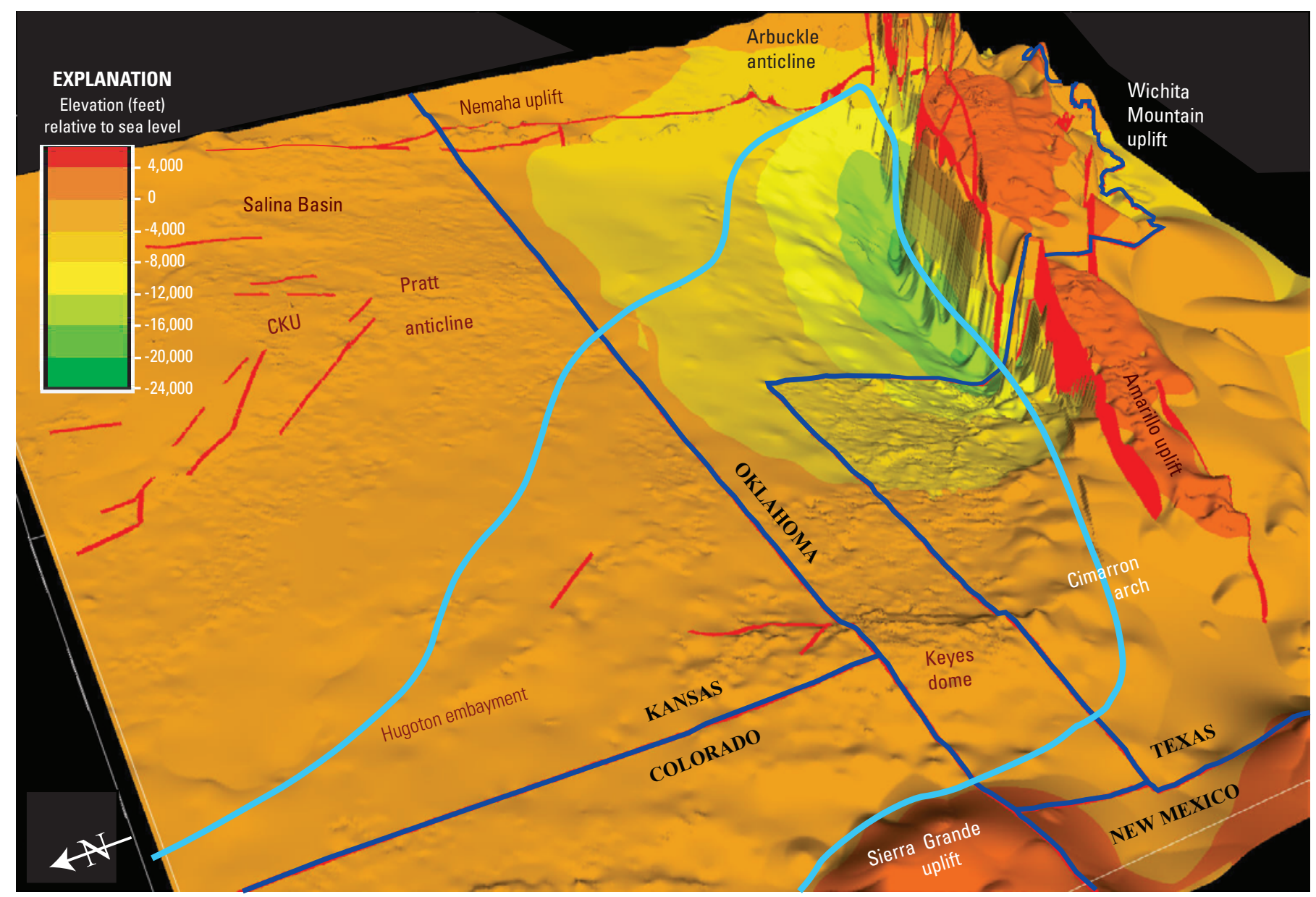

Figure 3. View to the southeast of elevation on the top of the informal Thirteen Finger limestone model layer (Higley and others, 2014b). Areal extent of the Thirteen Finger limestone is approximated by the light blue line. Major structures are labeled. CKU is Central Kansas uplift. The Las Animas arch (not shown) is north of the Sierra Grande uplift. The deep basin of Oklahoma and Texas is approximated by $-6,000$ feet elevation and deeper. Precambrian faults (red) are from Adler and others (1971). Faults bordering the Wichita Mountain uplift are vertical for 4D model purposes only, and subcropping strata on the uplift are unknown because of insufficient data. Data sources for this surface include formation picks from well logs and edited IHS Energy (2009, 2010a) formation tops. Vertical exaggeration is 18 times.

Mountain and Amarillo uplifts, elevation of the CKU, and subsequent emergence of the shelf areas, including the Oklahoma Panhandle and portions of the Texas Panhandle (Owen, 1975).

There is distinctive Precambrian layering on seismic profiles recorded south of the Wichita Mountains that is not visible under the Anadarko Basin. The Proterozoic basin containing that layering may have been bounded on its north side by a Precambrian fault that was probably twice reactivated during formation of the Southern Oklahoma aulacogen - once during the late Precambrian (?)-Early Cambrian extension, and again during Pennsylvanian compression (Brewer and others, 1983). Pennsylvanian compression resulted in crystalline basement rocks of the Wichita Mountains being thrusted northeastward over the basin along a series of deep faults (about 20- to $24-\mathrm{km}$ depths) with moderate (average $30^{\circ}$ to $40^{\circ}$ ) southwesterly dips (Brewer and others, 1983). Thrusting was northward toward the rapidly subsiding Anadarko Basin (Johnson, 1989). During the Early Pennsylvanian to Permian Ouachita and Wichita orogenies, Texas and Oklahoma moved northward and the Wichita Mountains and Amarillo arch were uplifted and thrust over the southern margins of the southern Oklahoma aulacogen and its superimposed basin, the southern Oklahoma trough (Ball and others, 1991). Figure 4 shows the generalized sequence of development from the Precambrian through Permian as the southern Oklahoma aulacogen proceeded from rifting into the Wichita Mountain uplift.

The Ouachita and Wichita orogenies were responsible for (1) folding and faulting of the Ouachita foldbelt in the Midcontinent, (2) subsidence of the Arkoma Basin, (3) emergence of the Amarillo, Wichita Mountain, and Nemaha uplifts; 

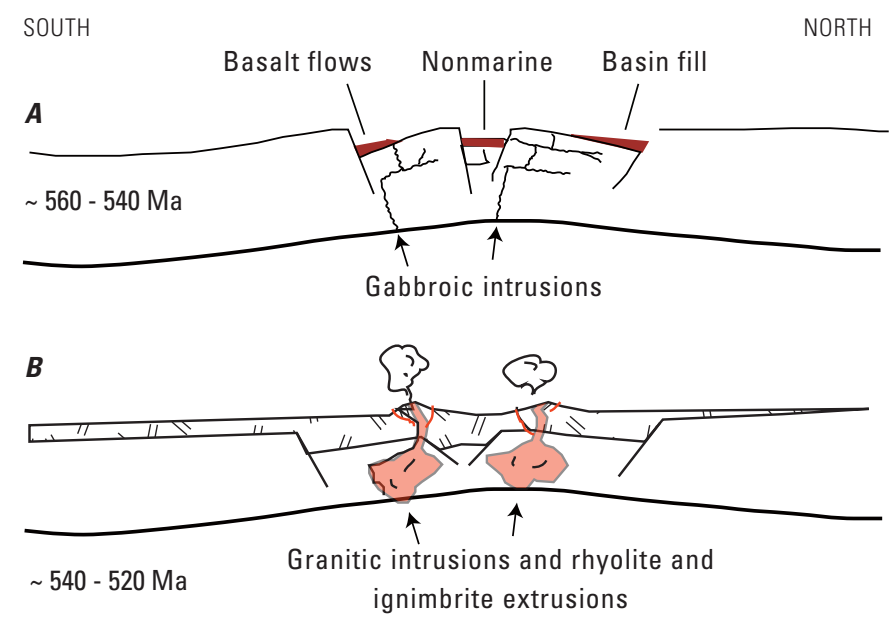

c
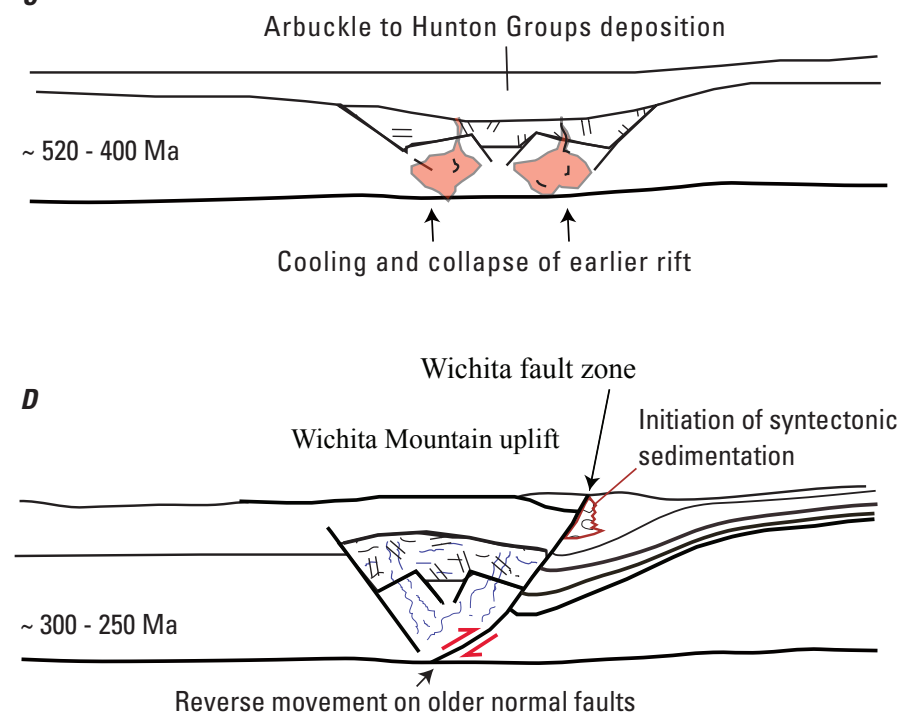

Figure 4. South-north generalized cross sections showing stages of development of the southern Oklahoma aulacogen $(A, B)$ (modified from Gilbert, 1983; Perry, 1989), southern Oklahoma trough $(C)$, and Anadarko Basin and bounding Wichita Mountain uplift and fault zone $(D)$. 
(4) uplift of the Cimarron arch and structures along the Las Animas arch; and (5) uplift of the Apishapa (Rascoe and Adler, 1983). The Anadarko Basin thus began to assume its present asymmetrical form (Owen, 1975). Associated faulting started at the southeast area of the basin in early Morrowan time (Ham and Wilson, 1967), whereas farther west it began in late Morrowan time; faulting persisted through the rest of the Pennsylvanian and probably died out during the Early Permian (Johnson, 1989). There is some evidence of left-lateral displacement associated with thrusting, but thrusting was most important in developing en echelon folds; strike-slip faulting was relatively minor and late, with most occurring during the Permian (Perry, 1989; Ball and others, 1991).

Pennsylvanian downwarping of the Anadarko Basin was at least partially the result of thrust loading, in contrast with interpretations of Pennsylvanian structures resulting from mainly vertical movements along high-angle faults (Brewer and others, 1983). Maximum structural displacement between the Wichita Mountain uplift and the basin floor exceeds 9,144 meters (m) (30,000 ft) (Al-Shaieb and others,
1994). Downwarping of the basin and compaction through time resulted in increased thickness of strata proximal to the Wichita Mountain and Amarillo uplifts. The Woodford Shale through Desmoinesian interval in particular has a pronounced wedge shape with a northwest-trending axis (fig. 5). Continued Permian filling of the Anadarko Basin following Pennsylvanian thrust loading may have resulted from compaction and dewatering of the thick Upper Mississippian and Pennsylvanian sediments (Perry, 1989). Compaction would have been greatest in the deep basin because these strata were thickest, with associated increased rate of deposition compared to shelf areas.

The current Rocky Mountains formed in the area of the eroded core of the Ancestral Rocky Mountains during the Laramide orogeny of Late Cretaceous and early Tertiary time. The orogeny raised and imparted an eastward and southeastward tilt to the entire region, with associated withdrawal of the Cretaceous epicontinental sea (Owen, 1975; Johnson, 1989) following deposition of the Upper Cretaceous Pierre Shale. Sorenson (2005) indicated that the Laramide orogeny may also

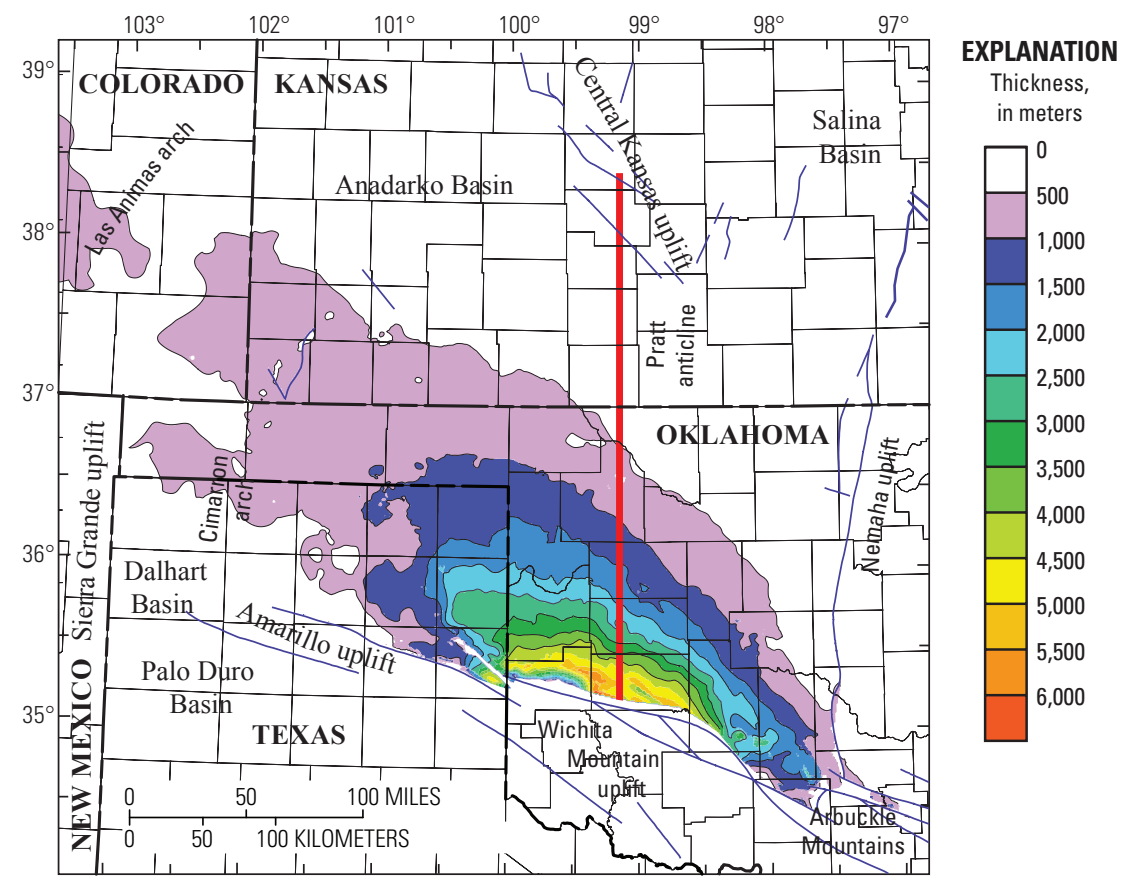

Figure 5. Map showing thickness of strata between the Hunton and Desmoinesian model layers ranges from less than 500 meters (1,640 feet) over most of the Kansas shelf to more than 5,000 meters (16,400 feet) in the deep basin of Oklahoma. This wedge of Woodford Shale and younger rocks is oriented northwest-southeast, similar to that of Woodford Shale thermal maturation contours. Precambrian faults (blue lines) and named structures are from Adler and others (1971). 
have influenced petroleum migration in the basin, specifically redistribution of Panhandle field fluid columns with northward spilling of gas into the Hugoton embayment.

Mostly alluvial sediments of the Miocene to Pliocene Ogallala Formation were deposited on an erosional surface of largely Permian, and Mesozoic strata in the western one-half of the basin (Seni, 1980; Johnson and others, 1988). Mesozoic units are present mostly in the Colorado and western Kansas portion of the province, with only scattered erosional remnants in Oklahoma and Texas.

\section{Petroleum Production History}

With more than 220,000 drill holes (IHS Energy, 2010a), the Anadarko Basin Province is in a mature state of exploration and development. Horizontal well completions for reported Oklahoma wells increased from less than 5 percent in 2002 to almost 30 percent in 2010 (Boyd, 2011, fig. 6). The Desmoinesian granite wash (Mitchell, 2011) and Devonian-Mississippian Woodford Shale are preferentially completed as horizontal wells. Anadarko is also an old exploration basin, dating back to the early 1900s for the still-productive panhandle, Cement, and numerous other fields (fig. 6). Historical exploration focus was for conventional oil and gas, and exploration is fairly recent for continuous (unconventional) accumulations.

Grouping of stratigraphic units into TPSs and AUs was affected by the quality and distribution of well and production data in the province. Production from most wells in the basin is reported as commingled from multiple units, and reservoir strata are sometimes misnamed. This commingling exists for small and large fields, and can extend across considerable formation ranges, such as for the Cement field of the deep basin in Oklahoma that has produced more than 186 million barrels of oil (MMBO) and 1.3 billion cubic feet of gas (BCFG) from Silurian through Permian strata (IHS Energy, 2010b). The Sooner trend of Oklahoma has yielded more than 334 MMBO, 2.3 trillion cubic feet of gas (TCFG), and no reported water from the Cambrian-Ordovician Arbuckle Group through Virgilian rock units (IHS Energy, 2010b). Produced water is almost exclusively reported for Colorado and Texas, and not for Kansas and Oklahoma. Commingling also influenced populating the Nehring and Associates, Inc. (2009) database on petroleum fields because of the difficulty in assigning production and associated data to rock units. Most Permian production in the Kansas portion of the basin is reported in the IHS Energy (2010b) database as Permian, as opposed to listing the lithostratigraphic unit name(s). As such, data are inadequate to assign production to, for example, the Chase or Council Grove Groups in the Hugoton and Guymon fields that have together produced more than $79 \mathrm{MMBO}$ and $26 \mathrm{TCFG}$ from Wolfcampian and Leonardian reservoirs. Figures $6 \mathrm{~A}$ and $6 B$ show oil and gas wells for Pennsylvanian through Permian production in the province; similar maps are presented for the Mississippian AU and other AUs. Although there is extensive well coverage, actual produced volumes and locations are adversely modified by reporting errors and commingling of production from multiple formations.

Mean undiscovered conventional resources that were assessed for Mississippian through Permian strata in the Anadarko Basin Province total 77 MMBO, 1,934 BCFG, and 60 million barrels of natural gas liquids (MMBNGL) (table 1). Oil and gas in the Anadarko Basin were assessed in 1995 (Henry and Hester, 1995) with mean undiscovered resources of 45.7 MMBO and 604.7 BCFG for their Mississippian through Permian plays (5812 through 5827). Primary reasons for the differences between these assessment results include: (1) technological advances have increased recovery of petroleum from existing fields and from undiscovered accumulations; (2) the increased contribution from unconventional (continuous) reserves and resources; and (3) assessment methodology and play/AU boundaries are different for the 1995 and 2011 assessments. For example, the Las Animas arch and Palo Duro Basin were assessed separately in 1995, whereas the Las Animas arch and most of the Palo Duro Basin were included in the Anadarko Basin Province for 2011. Well history and production data quality, although still variable, have improved since the 1995 assessment.

Application of the TPS assessment methodology resulted in mean undiscovered resources for the Mississippian AU of the Woodford Composite TPS of 17 MMBO, 417 BCFG, and 11 MMBNGL. Mean undiscovered oil and gas resources from the 1995 Anadarko Basin assessment totaled 14.0 MMBO and 133.2 BCFG for the Henry and Hester (1995) Lower Mississippian Stratigraphic Oil and Gas, Upper Mississippian Stratigraphic Oil and Gas, and Springer Stratigraphic Oil and Gas plays. Mean undiscovered resources were 4.4 MMBO and $0 \mathrm{BCFG}$ for the Mississippian Carbonate play in the Las Animas arch (Keighin, 1995). Ball and Henry (1995) assessed one play for the Palo Duro Basin, the Upper Paleozoic play that included all Mississippian through Permian strata for which mean undiscovered resources were $4.9 \mathrm{MMBO}$ and $0 \mathrm{BCFG}$.

Undiscovered oil resources for the Thirteen Finger limestone and Atoka Shale are included with the conventional Morrowan-Atokan AU. Mean undiscovered resources for the Thirteen Finger Limestone-Atoka Shale Gas continuous AU are assessed separately and are 6.850 TCFG and 82 MMBNGL. Undiscovered conventional resources for the Pennsylvanian Composite TPS include:

a. $15 \mathrm{MMBO}, 332 \mathrm{BCFG}$, and $7 \mathrm{MMBNGL}$ for the Morrowan-Atokan $\mathrm{AU}$;

b. 6 MMBO, 118 BCFG, and 4 MMBNGL for the Desmoinesian $\mathrm{AU}$;

c. $23 \mathrm{MMBO}, 258 \mathrm{BCFG}$, and $8 \mathrm{MMBNGL}$ for the Missourian-Permian AU;

d. $16 \mathrm{MMBO}, 809 \mathrm{BCFG}$, and $30 \mathrm{MMBNGL}$ for the Greater Granite Wash Composite AU. 


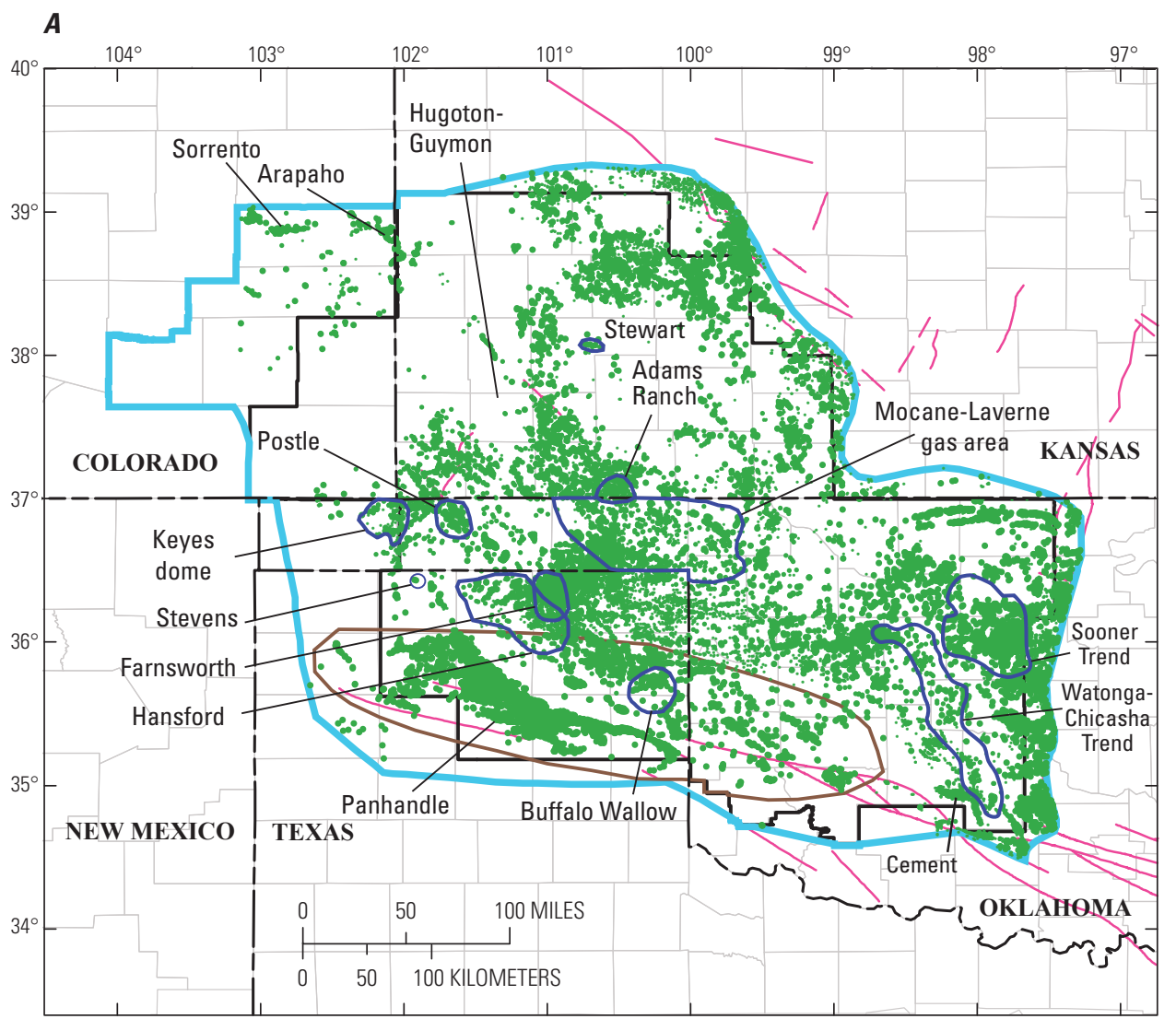

Figure 6. Maps showing $A$, Oil and $B$, gas wells that produce from Pennsylvanian and (or) Permian strata in the Anadarko Basin Province (IHS Energy, 2010a, 2010b). Generalized field locations are from IHS Energy (2010a) and Bebout and others (1993). Also shown are the Greater Granite Wash Composite Assessment Unit (AU) and Pennsylvanian Composite Total Petroleum System (TPS) boundaries. Precambrian faults are from Adler and others (1971).

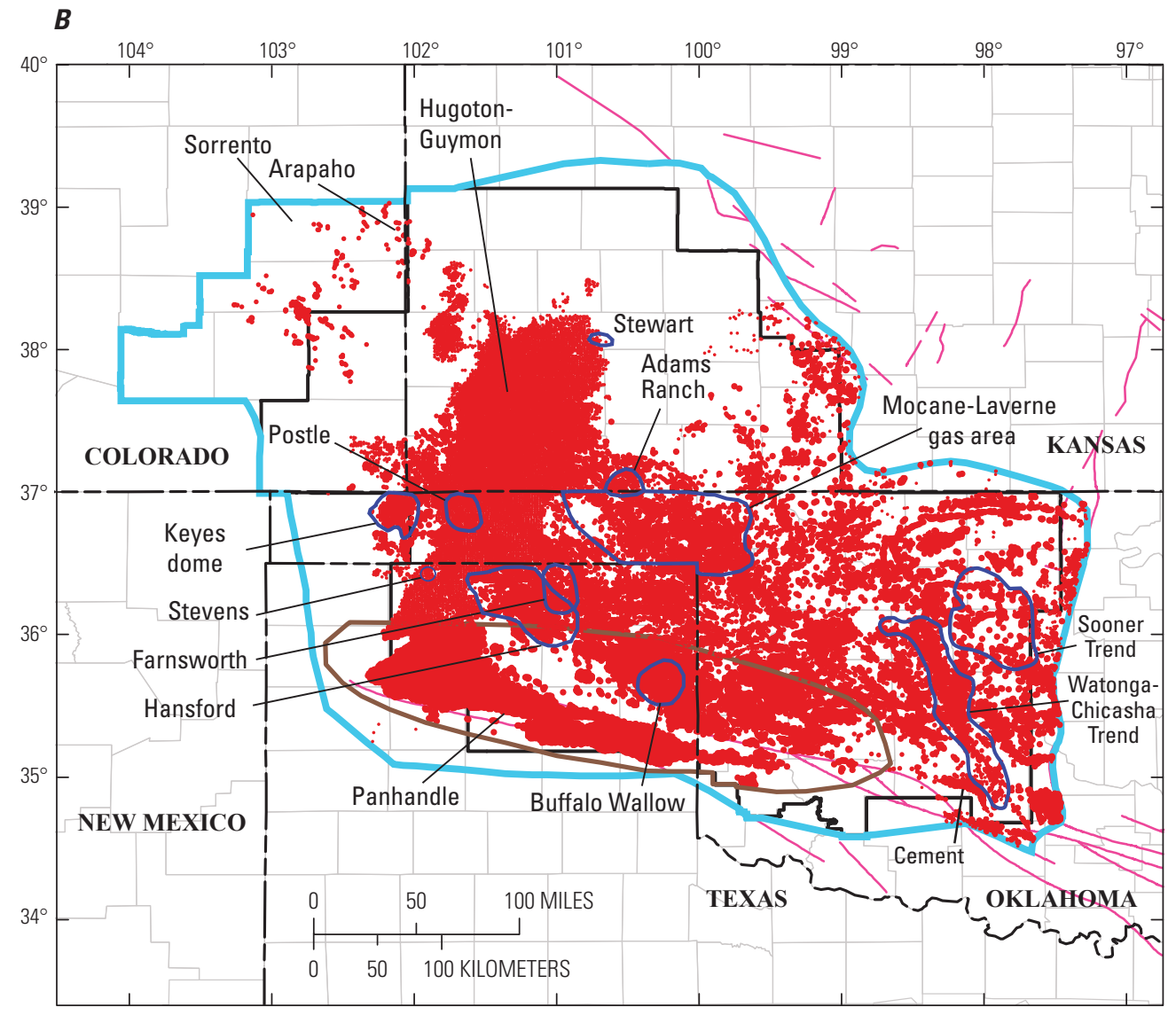

\section{EXPLANATION}

- Pennsylvanian Comp.TPS

- Province 58

- Greater Granite Wash Composite AU

- Precambrian fault

- Oil well

- Gas well 


\section{Petroleum Source Rocks and Thermal Maturity}

Petroleum source and potential source rocks in the Anadarko Basin include Ordovician through Missourian marine shale and limestone (fig. 7). Petroleum source rocks and thermal maturation history are discussed more fully in Higley (2014). The Woodford Composite TPS encompasses Ordovician through Mississippian petroleum source rocks and is named for the principal source rock in the province. The Pennsylvanian Composite TPS includes all Pennsylvanian petroleum source rocks. Primary reasons for this generalized TPS division are that (1) geochemical research in the basin has been focused on the Woodford Shale, with lesser information on other possible petroleum source rocks, and (2) the three source rocks with moderate to good hydrocarbon potential (Burruss and Hatch, 1989, p. 57) are the (a) Ordovician Simpson Group shale, (b) Devonian-Mississippian Woodford Shale, and (c) Pennsylvanian black shales. Organic-carbon values for thick shales in the Springer and Morrow Formations range from 0.5 to 3.4 percent; kerogen is Type III, indicating that it is mostly gas prone (Rice and others, 1989). Atokan, Desmoinesian, and Virgilian petroleum source rocks are mainly Types II and III kerogen (Rice and others, 1989). Figure 2 displays the Morrow as Morrow Group in western Kansas, and as Morrow Formation in the northern and southern Anadarko Basin; it is referred to as Morrow Formation in this report. The Atoka interval has group status in the Hugoton embayment and northern Anadarko Basin, and is the Atoka Shale and Pumpkin Creek Limestone of the Dornick Hills Group in the deep basin (fig. 2). It will be called Atoka Group in this chapter of the report. Discussions of Atoka shale refer to included shale intervals.

The three highlighted zones in figure 7 correspond to assigned hydrocarbon source rocks in the 4D petroleum system model. These generalized source intervals were chosen and based partly on the Burruss and Hatch (1989) determination of three oil types in the Anadarko Basin; their analyzed crude oils appeared to correlate with extracts from Ordovician, Devonian, and Pennsylvanian source rocks. The Atokan Thirteen Finger limestone was chosen to represent the Pennsylvanian hydrocarbon source rocks because the thickness and extent of this high gamma interval across the province. Morrowan black shales compose as much as 60 percent of the Morrow Formation in the Oklahoma portion of the province (Wang and Philp, 1997), but it would have been more difficult to define and map a specific petroleum source interval within the Morrow Formation across the basin.

Timing of generation, migration, and accumulation of oil and gas are derived from the Higley (2014) petroleum system models (fig. 8, table 2). Schlumberger (2011) Petro$\mathrm{Mod}^{\mathbb{B}}$ software was used for 1D and 4D petroleum system models. Within the Ordovician Simpson Group Oil Creek, Devonian-Mississippian Woodford, and Atokan Thirteen Finger petroleum source rocks in the 4D model, the Oil Creek Formation primarily sources Cambrian through Ordovician petroleum accumulations, the Woodford Shale is the major source for Devonian, Mississippian, and lower Morrowan accumulations, and the Thirteen Finger limestone is the main source for middle Morrowan through Permian accumulations (Higley, 2014). As is shown in figure 7, numerous other petroleum source rocks across the basin are potential contributors to reservoirs. The $4 \mathrm{D}$ petroleum system model consists of stacked grids of lithostratigraphic units that are referred to as layers. Each layer extends across the study area, a requirement of the PetroMod ${ }^{\circledR}$ software. This means that underlying layers are displayed if the modeled unit(s) has limited lateral extent. Model construction is discussed in Higley (2014) and Higley and others (2014b). Figure 8 shows modeled vitrinite reflectance $\left(\mathrm{R}_{\mathrm{o}}\right)$ and transformation ratio (TR) 3D images at present day for the Woodford through Douglas model layers that contain the source rocks that contributed oil and gas for Mississippian through Permian reservoirs. The TR maps record the beginning to the end of oil generation based on hydrous pyrolysis (HP) kinetics, and the $\mathrm{R}_{\mathrm{o}}$ images show assigned

Table 2. Oil generation onset for the Anadarko Basin $4 \mathrm{D}$ model, and onset and completion dates in millions of years before the present (Ma) for the Bertha Rogers 1 and Petree Ranch 1 1D models based on Woodford Shale hydrous pyrolysis (HP) kinetics (Lewan and Ruble, 2002). The Douglas Group overlies the youngest potential petroleum source rocks in figure 7. The HP kinetics-based onset of oil generation is 0.1 percent transformation ratio (TR) and completion is 99 percent TR. Oil generation histories in the $4 D$ model were recorded at 10-Ma increments; so the plus symbol indicates onset was before the listed age.

\begin{tabular}{lccccccc}
\hline & Four Dimensional Model & & \multicolumn{2}{c}{ Bertha Rogers 1 } & & \multicolumn{2}{c}{ Petree Ranch 1 } \\
\cline { 1 - 2 } & Onset & & Onset & End & & Onset & End \\
\hline Virgilian (Douglas Group) & 270 & & 100 & & & & \\
Thirteen Finger limestone & 300 & & 290 & 265 & & 270 & \\
Woodford Shale & 330 & & 330 & 310 & & 290 & 200 \\
Oil Creek Formation & 370 & & 340 & 320 & & & \\
\hline
\end{tabular}




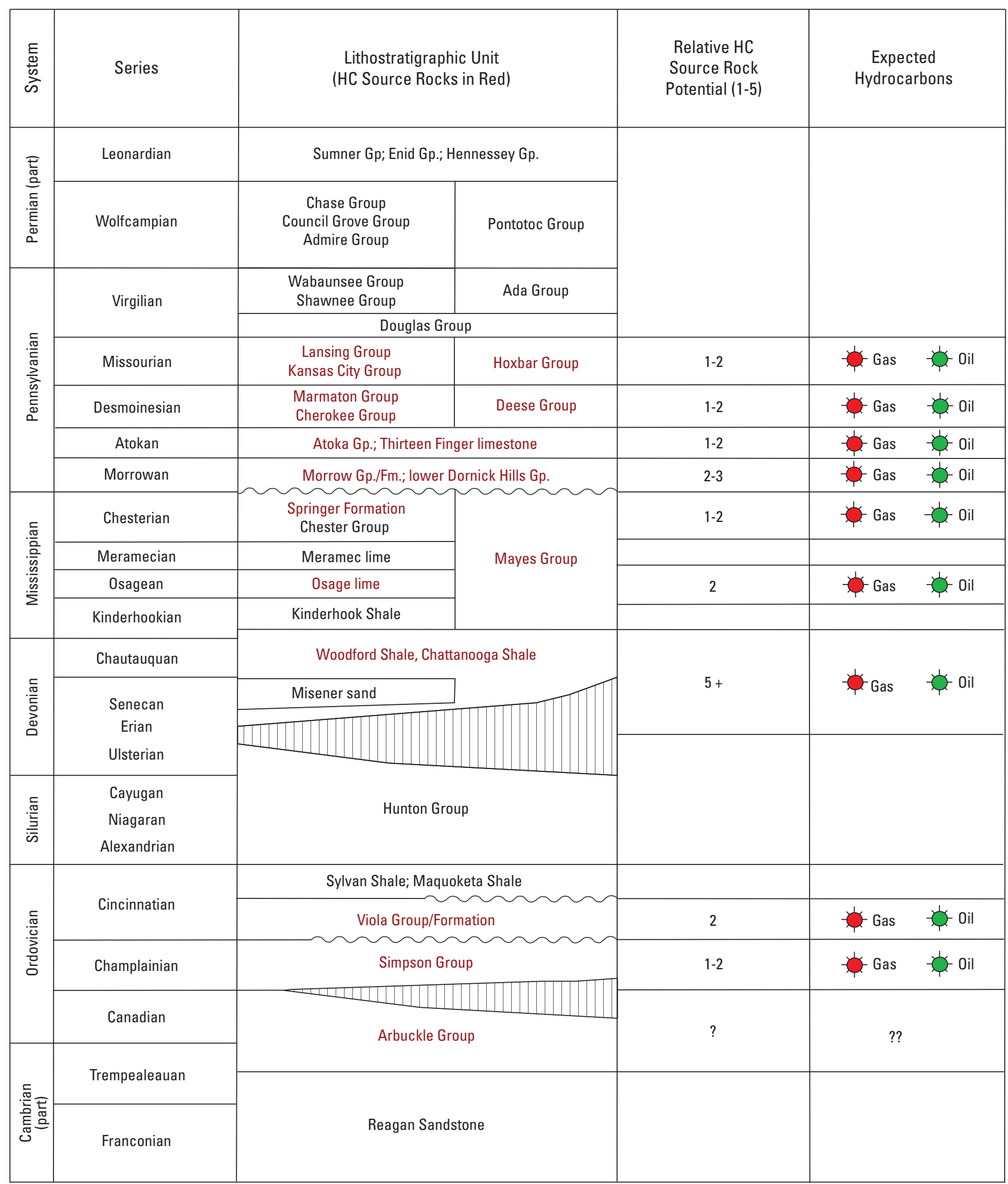

Figure 7. Generalized stratigraphic column for the Anadarko Basin Province, with hydrocarbon source rocks highlighted (brown text). Increases in source rock potential are indicated by larger numbers. The expected hydrocarbons column heading indicate whether the source rock is more oil or gas prone. Blue highlights source intervals used in the four-dimensional petroleum system model. Vertical lines show a generalized time range of unconformity from Bebout and others (1993, fig. 5). Chart is from J. Hatch (oral commun., 2010, and modified from Burruss and Hatch (1989). Gp., Group; Fm., Formation. 

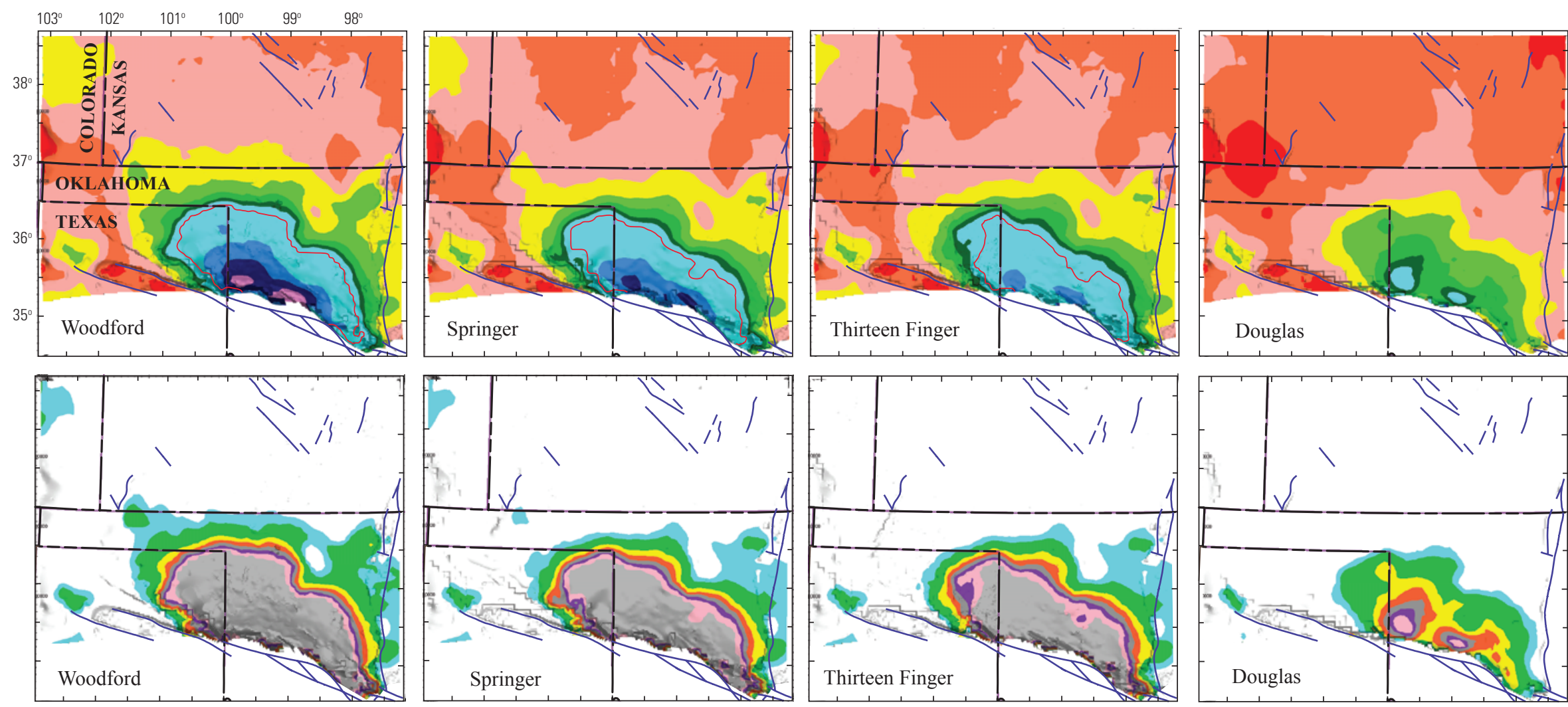

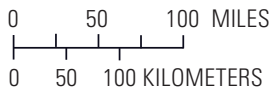

Figure 8. Images showing present-day thermal maturation of Woodford to Douglas model layers using Woodford HP transformation ratio (\% TR) kinetics (Lewan and Ruble, 2002) and vitrinite reflectance (\% $\mathrm{R}_{0}$ ) (Sweeney and Burnham, 1990). Each layer extends across the study area, including units located outside of the petroleum source rocks. As an example, the Kansas portion of the Springer and Thirteen Finger layers are almost identical because these units are absent over much of this area and the thermal maturity reflects that of the underlying mostly Viola and Simpson Groups strata. The vitrinite reflectance contours in equivalent layers in chapter 3 (fig. 26) are limited to the extent of those strata, and also exhibit slightly different thermal maturation patterns, which largely represents a somewhat newer version of the model. Changes in thermal maturity on the shelf areas of Kansas and Colorado are also minor because of shallow burial relative to the deep basin of Oklahoma and Texas; source rocks are mostly immature for oil generation at less than $0.6 \% \mathrm{R}$. Thermal maturity for source rocks in the deep basin of Oklahoma and Texas ranges from mature for oil generation, to overmature for gas generation at about $4 \% R_{0}$ and greater. Red contour lines on the $R_{0}$ images mark the 99 percent transformation ratio that is approximately equivalent to $1.2 \% R_{0}$. Precambrian faults (Adler and others, 1971) are shown as blue lines.

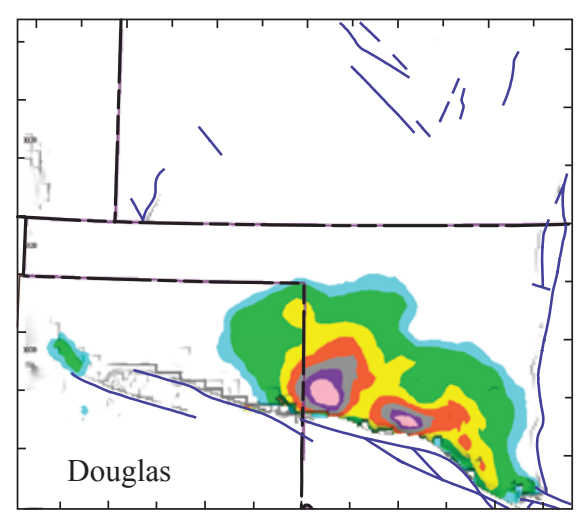

\section{EXPLANATION}

$\% R_{0} \quad T R$, in percent

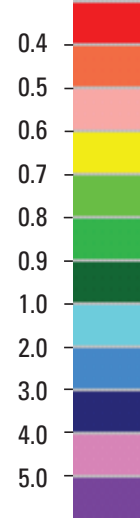

\begin{tabular}{|l|l|}
\hline 0.1 to 1.0 \\
\hline 1.0 to 10 \\
\hline 10 to 25 \\
\hline 25 to 50 \\
\hline 50 to 75 \\
\hline 75 to 95 \\
\hline 95 to 99 \\
\hline 99 to 100 \\
\hline
\end{tabular}


levels of maturation based on Sweeney and Burnham (1990) algorithms. The respective $\mathrm{R}_{\mathrm{o}}$ and TR values in figure 8 are not identical, but the good agreement of levels of thermal maturation is largely because of calibrating the model to available downhole temperature and thermal maturation data. Primary conclusions from the images are (1) thermal maturity of Oklahoma and Texas ranges from immature for oil generation to overmature for gas generation, although production of gas from the deep basin indicates all or most of the area is within the limit of gas preservation; (2) the Colorado and Kansas portions of the province are almost exclusively immature to marginally mature for oil generation. Elevated thermal maturation in Colorado is associated with elevated basement heat flow (Higley, 2014); (3) the Douglas layer represents lower Virgilian strata, that are younger than the petroleum source rocks in figure 7 , but if this layer contains source rocks in the deep basin they would mainly be immature to mature for oil generation; and (4) the primary factor that controls the degree of thermal maturation is depth of burial, which is mainly why the Woodford Shale is mature for oil and gas generation over a larger area than shallower units.

\section{Reservoir Rocks}

\section{Mississippian Assessment Unit}

A broad epicontinental sea extended across most of the southern Midcontinent from Late Cambrian through Mississippian time (Johnson and others, 1988). The Anadarko Basin Province area was a broad embayment that received a thick sequence of carbonates interbedded with thinner shales and sandstones (Johnson, 1989). Mississippian strata in the province are mainly limestones, cherty limestones, and shales that were deposited in shallow-marine environments (Craig and others, 1979; Frezon and Jordan, 1979; Mapel and others, 1979; Johnson and others, 1988). Thickness of the Mississippian is greatest in the deep basin of Oklahoma and Texas, where the Chesterian section can exceed 5,000 ft in thickness, and pre-Chesterian Mississippian rocks can be more than 2,000 ft thick (Adler and others, 1971). Craig and Connor (1979) indicated the total thickness of the Mississippian section along the northern shelf is about 1,000-2,000 ft. The Mississippian isopach (fig. 9) exhibits southeastward thickening from the Kansas and Colorado shelf to the deep basin. The irregular contours partly represent variations that resulted from subtracting the Mississippian and Woodford structure grids (layers) and (or) areas of poor data control.

Erosion of Springer Formation strata (fig. 2) over the Nemaha uplift resulted from pre-Pennsylvanian orogenic uplift (Johnson, 1989). The crest of the uplift has Precambrian and pre-Pennsylvanian rocks unconformably overlain by Desmoinesian and Missourian strata (Rascoe and Adler, 1983). Perry (1989) indicated that initial evidence of late Paleozoic tectonism is the abrupt pinchout of Springer strata in the adjacent western Ardmore Basin. This epeirogenic event caused gentle, widespread uplift and erosion over the Midcontinent; Mississippian and older Paleozoic rocks were eroded from the Cambridge arch, north of the province, and parts of the CKU, and from shelf areas bordering the Anadarko Basin and Arkoma Basin, to the east (Rascoe and Adler, 1983).

The angular unconformity that resulted from the Late Mississippian epeirogeny, separates Mississippian carbonate strata from the overlying Morrow Formation (Bowen and Weimer, 2003), except in the deep part of the basin where they conformably overlie lithologically similar Springer strata (Johnson, 1989). Because of the lithologic similarities, it can be difficult to determine the Springer-Morrow contact in the basin (Johnson, 1989). Keighin and Flores (1989) identified three depositional facies for the Springer and Morrow Formations that include (1) fluvial-influenced coastal (FIC), (2) tidalinfluenced nearshore (TINS), and (3) mixed tidal and non-tidal marine influence (MT/NTM).

The Mississippian AU extends across the province, with petroleum production from Kinderhookian through Chesterian strata. Reservoir rocks are mostly Lower Mississippian carbonates through Upper Mississippian sandstones and carbonates. Mississippian oil and gas wells are shown in figure 10. Springer Formation oil and gas are produced from thrust fault-bounded blocks on the northern flank of the Wichita Mountain uplift (Alder and others, 1971). Potential resources on the uplift should be predominantly oil because of shallower burial than the adjacent Anadarko Basin. There is also scattered petroleum production on and parallel to the Nemaha uplift. Horizontal drilling in Mississippian strata includes oil and gas wells (IHS Energy, 2012). Sixty percent of the 276 horizontal wells in the province were completed after 2010; these are scattered within existing fields and also include lightly explored areas close to the eastern Kansas/Oklahoma border in the province (fig. 10A). Horizontal wells completed in 2010 or later were not incorporated into the Higley and others (2011) assessment of undiscovered resources in the province; this is about 80 percent of the wells. Mississippian cumulative production across the basin is mainly oil with more than 1.16 BBO, 15.9 TCFG, and 277 million barrels of water (MMBW) from more than 29,000 leases in 2,357 fields (IHS Energy, 2010a, 2010b). Water production was reported only for Colorado and Texas. Exploration and development started early for Mississippian reservoirs (fig. 11). The earliest exploration for petroleum in the basin was the Cement field in 1917 (fig. 6), a faulted anticline in the deep basin that has produced oil and gas from Cambrian through Permian reservoirs (Davis and Northcutt, 1989). Exploration and development increased in the late 1950s with a continuing upward trend.

Discovery-thirds plots show the distribution of first, second, and third thirds of completion dates for Mississippian oil and gas fields. The division into thirds is based on early field discoveries frequently being the largest oil and gas accumulations, with later field sizes being smaller. Discovery thirds is also based on mathematically grown, as opposed to known, sizes of accumulations. This is incorporated because initial reserves of conventional accumulations are commonly 


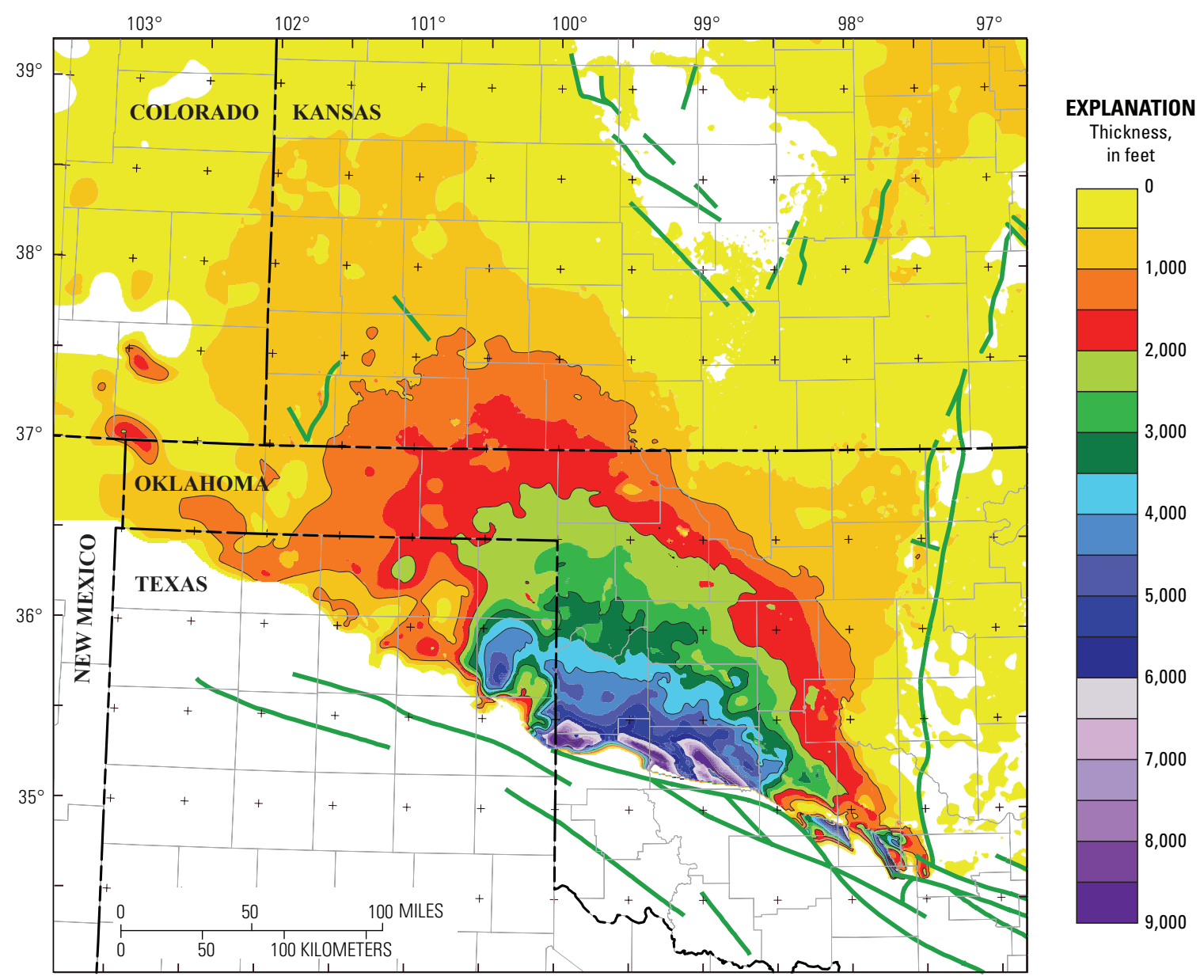

Figure 9. Isopach map of Mississippian to Woodford petroleum system model layers, which approximates the thickness of Mississippian rocks across the study area. Blank areas indicate missing strata or insufficient data. Extent of the Woodford Shale is approximated by the Woodford Shale Oil AU boundary in figure 1. Irregular contours are mainly because of subtraction of these unfiltered (unsmoothed) layer grids. Precambrian faults (green lines) are from Adler and others (1971). 


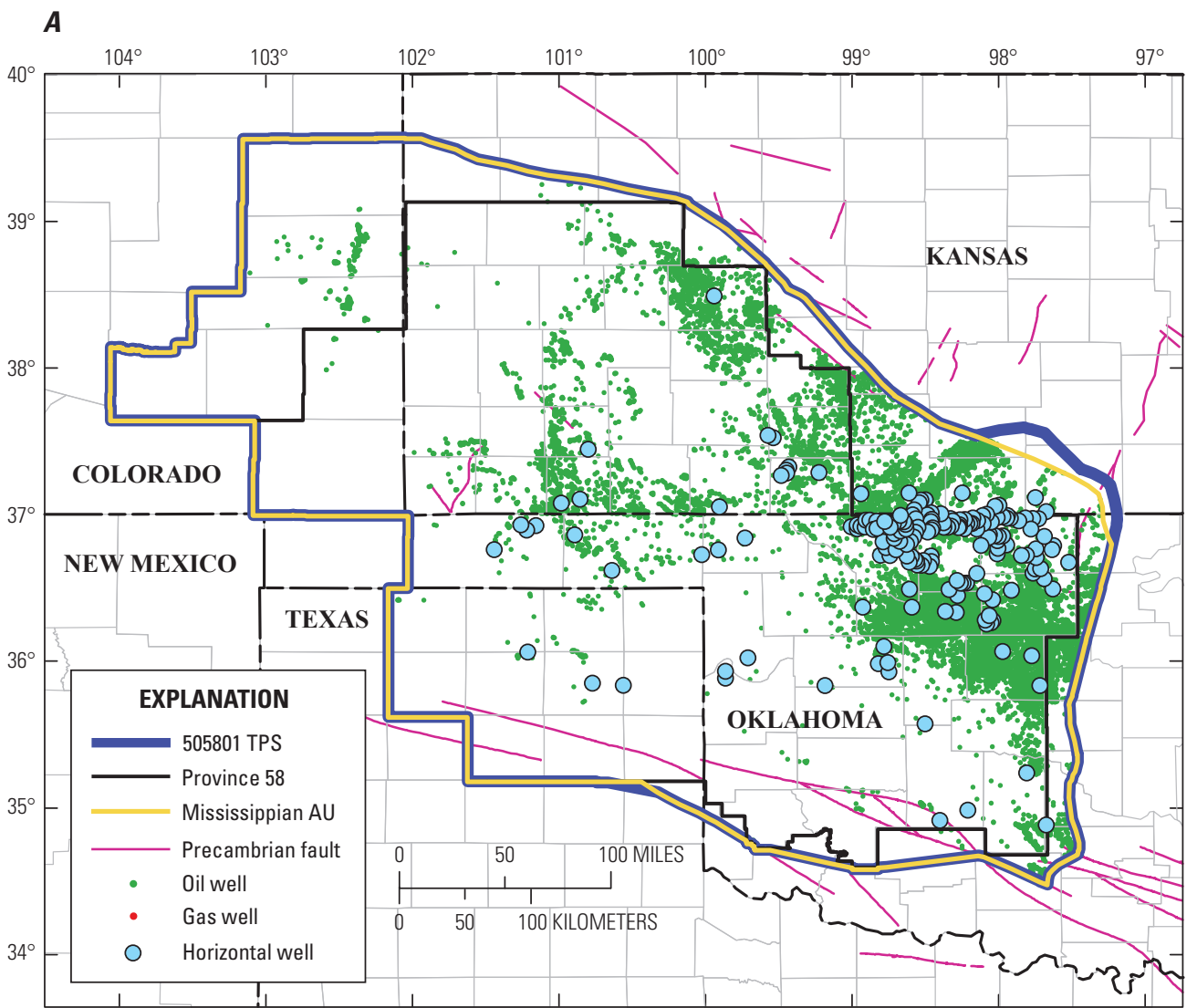

B

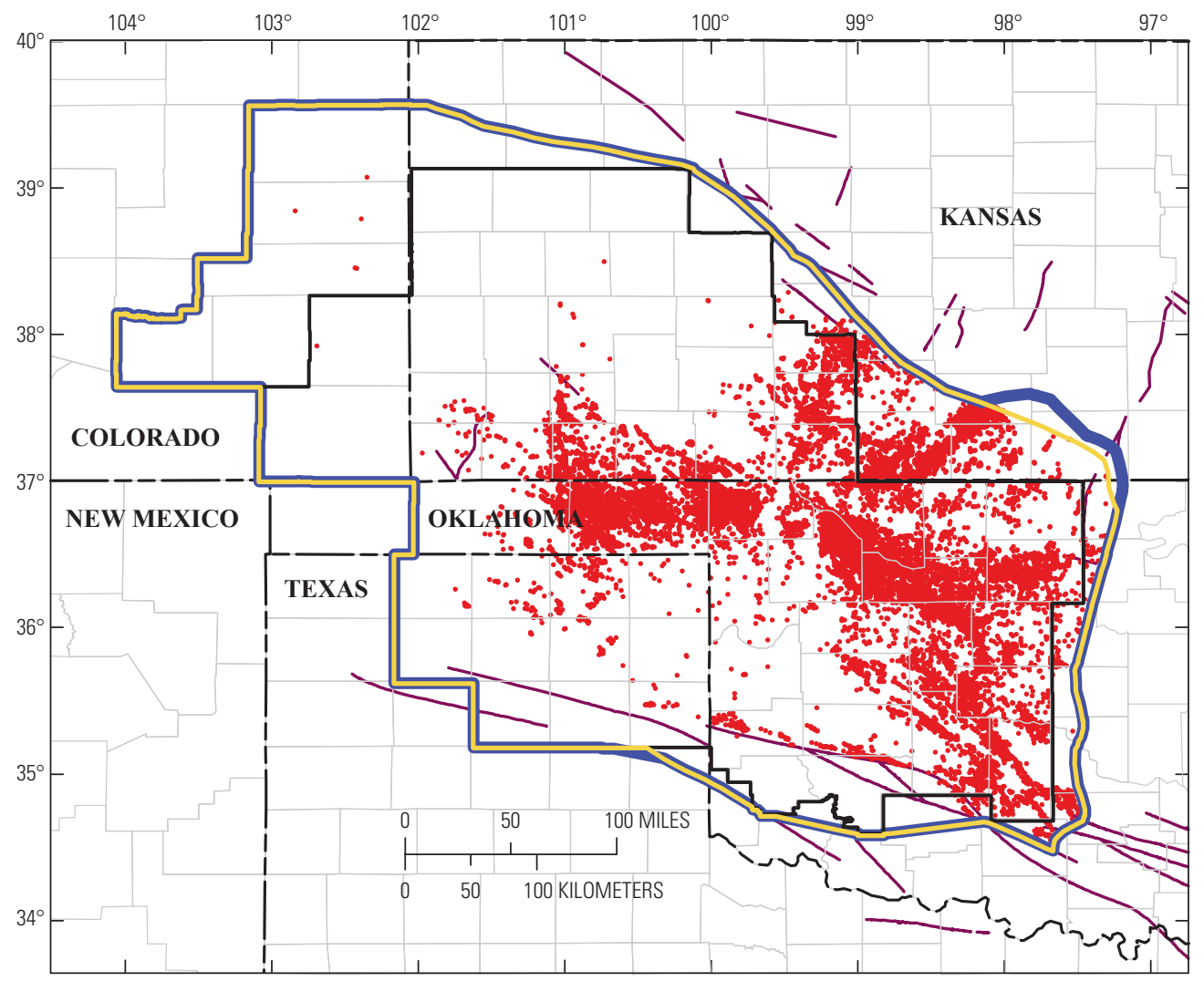

Figure 10. Maps showing $A$, Oil and $B$, gas wells that produce from the Mississippian AU in the Anadarko Basin Province (IHS Energy, 2010b). Also shown in figure $10 \mathrm{~A}$ are 276 horizontal wells drilled into Mississippian strata; 166 of these were completed after 2010 (IHS Energy, 2012). 


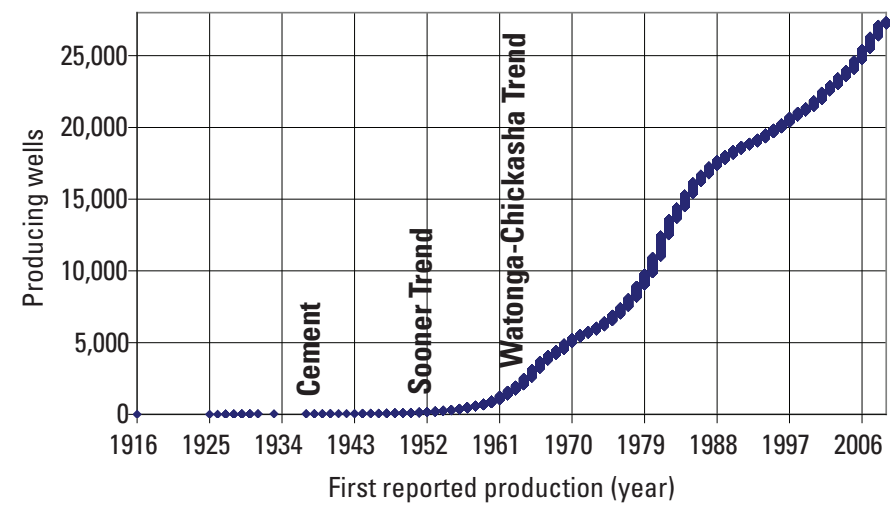

Figure 11. Cumulative number of producing wells by year for the Mississippian AU (IHS Energy, 2010a, 2010b). Some of the wells include production from units other than Mississippian. Onset of production from this AU for several major fields is also shown. Locations are in figure 6.

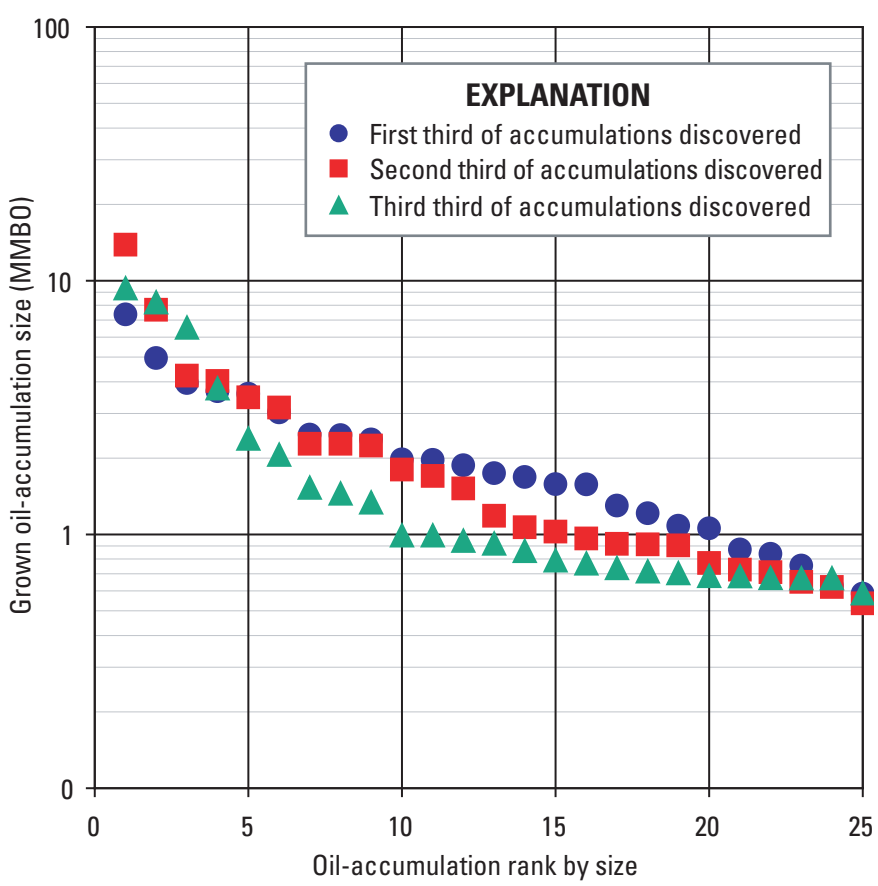

underestimated. The development success of an AU is measured by sizes of accumulations through time. The Mississippian AU discovery thirds oil and gas production plots show that, even with the mature status of exploration, that the latest discoveries exhibit similar grown accumulation sizes to the early and middle discoveries (fig. 12). This indicates that the AU has continuing potential for resources, even considering its fairly mature exploration status. The discovery trends also continue from the smallest to the largest accumulations. Discovery thirds for oil and gas accumulations (fig. 12) (Nehring and Associates, Inc., 2009) only show a few of the fields that produce from Mississippian units. Shown fields should produce oil and gas almost exclusively from the Mississippian, with lesser reported commingled production from other units.

Trapping mechanisms for the AU are structural, stratigraphic, and a combination of the two. Structural traps are evidenced by the linear trends of some of the oil and gas production in the deep basin, proximal to the greatest thickness of Mississippian strata. This is also the area that is overmature for oil generation, but is within the gas generation and preservation windows (Higley, 2014). Probable Mississippian petroleum source rocks include strata within the informal Osage lime and Springer Formation (fig. 7). Organic-carbon values for thick shales in the Mississippian Springer and Pennsylvanian Morrowan producing intervals range from 0.5 to $3.4 \mathrm{wt} \%$; however, the kerogen is mostly Type III indicating that it is primarily gas-prone (Hatch and others, 1986; Rice and others, 1989) (fig. 2). Mississippian oil generally

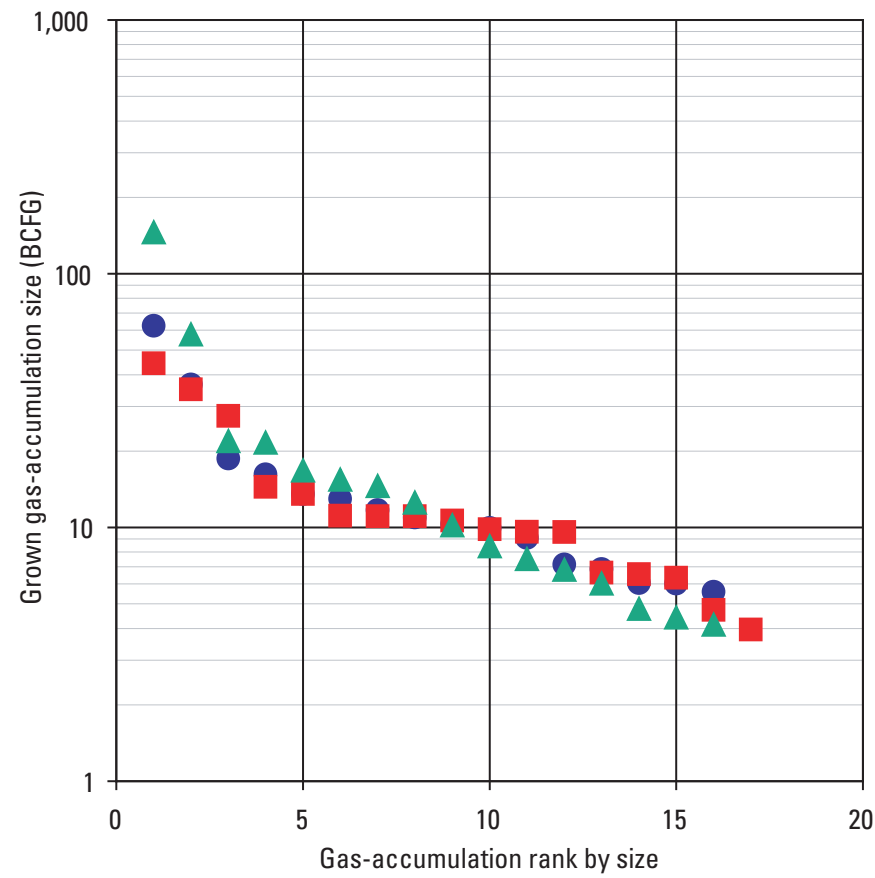

Figure 12. Grown gas and oil accumulation sizes for discovery thirds of the Mississippian Assessment Unit of the Woodford Composite Total Petroleum System. Although this is a mature assessment unit, the oil accumulations only show a minor drop for the third of discoveries, and the gas accumulation history is stable. Data are from the Nehring and Associates, Inc. (2009) field database, which has limited information for the Mississippian fields. MMBO, million barrels of oil; BCFG, billion cubic feet of gas. 


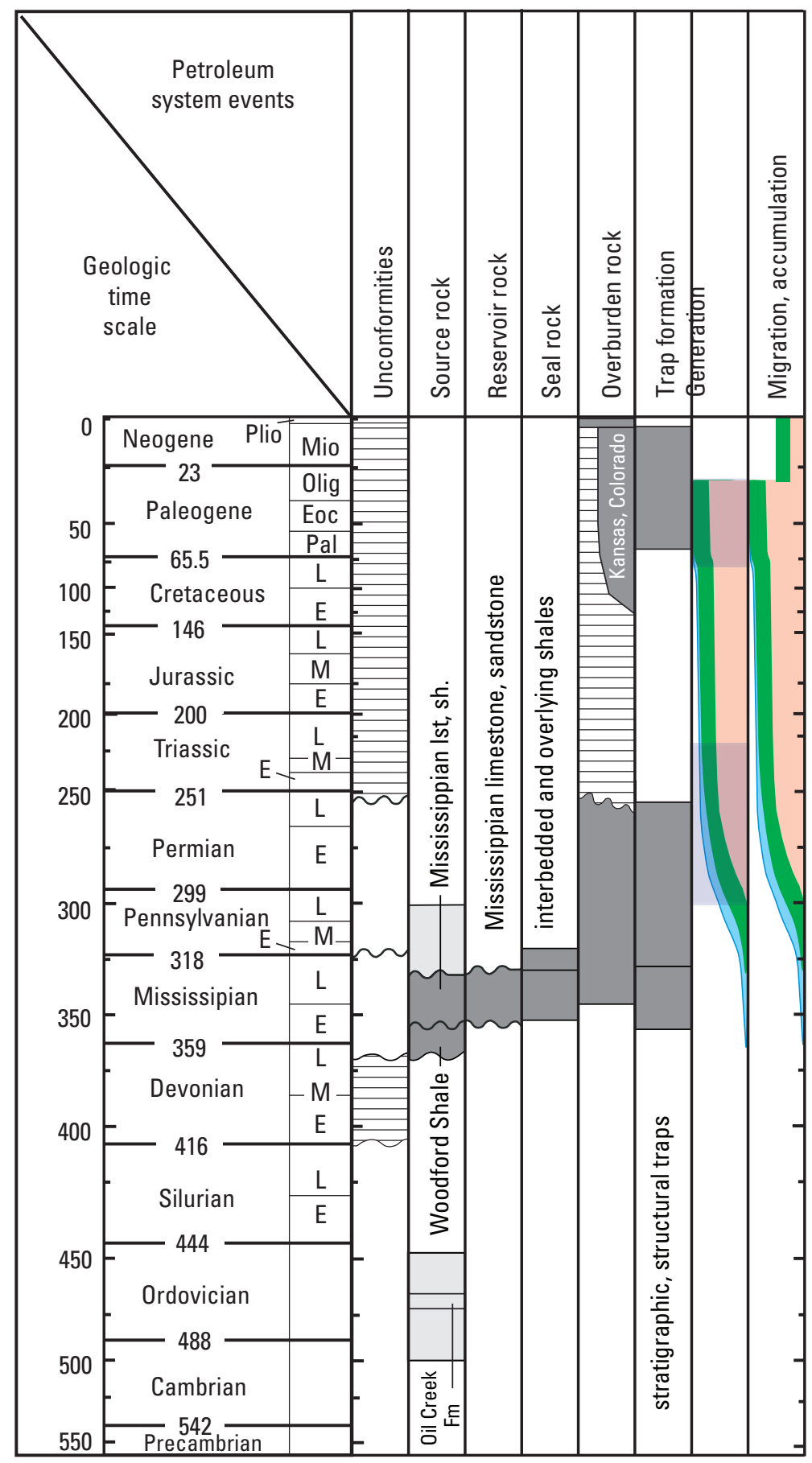

Figure 13. Petroleum system events chart for the Mississippian Assessment Unit of the Woodford Composite Total Petroleum System. Timing of oil and gas generation, migration, and accumulation is based primarily on Higley (2014) petroleum system models. Potential petroleum source rocks (fig. 7; Hatch and others, 1986) are light gray rectangles. Primary petroleum source rock for the Mississippian AU is the Woodford Shale (Rice and others, 1988a, 1988b; Higley, 2014). The informal Osage lime and Springer Formation are potential petroleum sources for the AU. Times of peak generation from the Oil Creek Formation (blue), Woodford Shale (green) and Thirteen Finger limestone (tan) source rocks are within blue shaded rectangles. Paleogene trap formation, migration, and accumulation are based on Laramide generation and potential remigration associated with erosion and basin tilting. Unconformities, wavy and horizontal lines; Ist, limestone; sh., shale; Fm., Formation; Plio, Pliocene; Mio, Miocene; Eoc, Eocene; Pal, Paleocene; L, Late; M, Middle; E, Early. correlates with extracts of the Woodford Shale (Rice and others, 1988a, 1988b). The underlying Woodford Shale is also the predominant petroleum source rock for the Mississippian AU based on petroleum system modeling (Higley, 2014). Associated gases from Silurian, Devonian, and Mississippian carbonates from the Sooner trend were from depths as great as 9,678 $\mathrm{ft}(2,950 \mathrm{~m})$ and were generated from Type II kerogen; gases are isotopically lighter (mean $\delta^{13} \mathrm{C}_{1}$ value is -43.9 percent) and chemically wetter (mean $\mathrm{C}_{2+}$ value is 14 percent) than those derived from Type III kerogen at equivalent levels of thermal maturity (Rice and others, 1988a, 1988b). The petroleum system events chart for the Mississippian AU (fig. 13) shows time periods of source, reservoir, seal, and overburden strata, and trap formation. Timing of generation, migration, and accumulation of oil and gas are derived from Higley (2014) petroleum system models (fig. 14, table 2). 


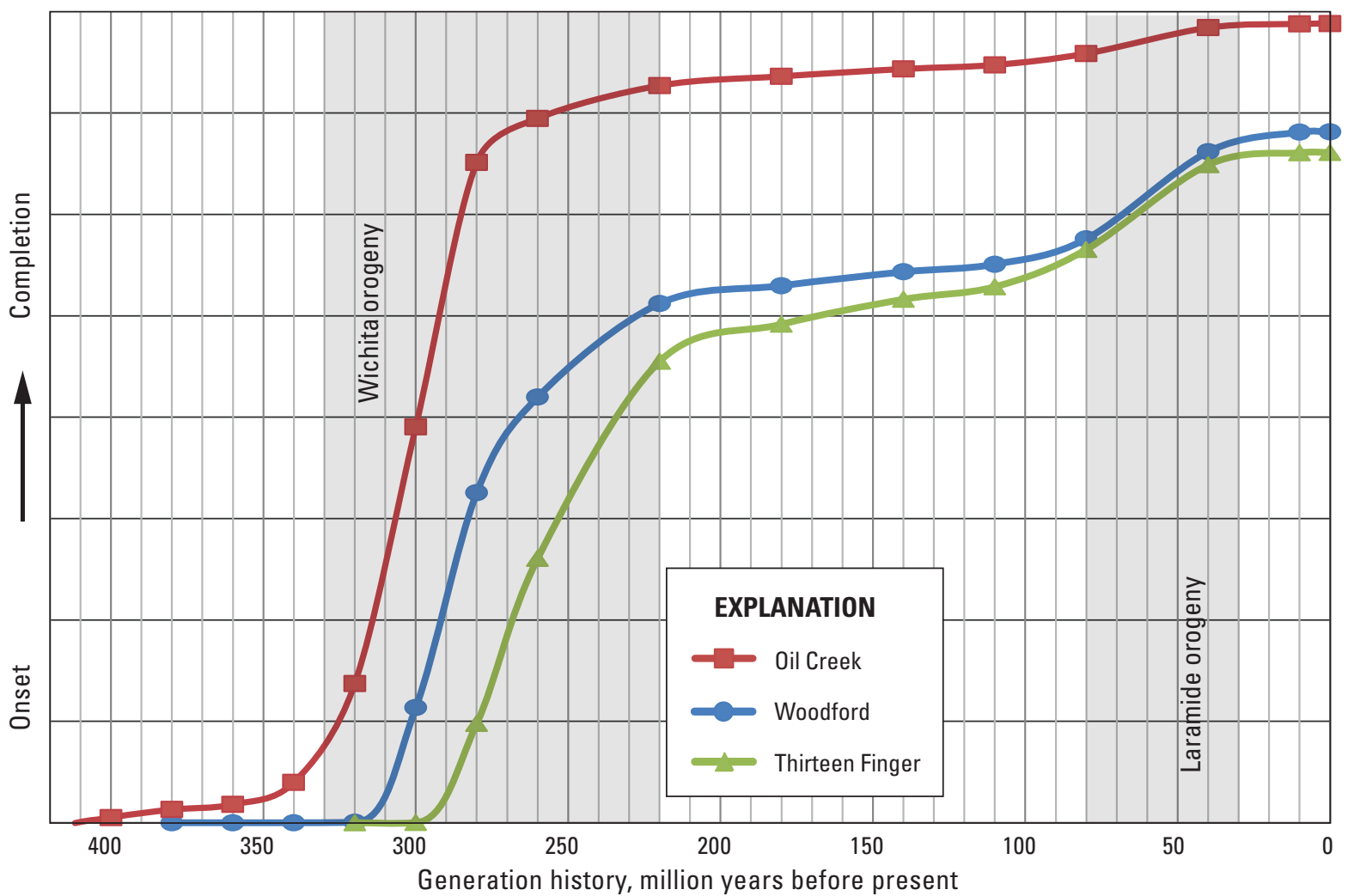

Figure 14. History of petroleum generation from the Oil Creek Formation, Woodford Shale, and informal Thirteen Finger limestone source layers in the four-dimensional (4D) petroleum system model. History is based on Woodford hydrous pyrolysis kinetics (Lewan and Ruble, 2002). Gray bars delineate periods of peak generation. Early oil generation from the Oil Creek layer, prior to about 370 million years ago, is in a narrow band that is proximal to the Wichita Mountain uplift. This band exceeds maximum depth of the formation and is because of areas of poor data control in the area bordering the fault zone. The error could have been removed by clipping the 4D model extent to the basin axis and northward, but less of the model would have been visible and usable.

\section{Morrowan-Atokan Assessment Unit}

The boundary of the Pennsylvanian Composite TPS (505802) and contained Morrowan-Atokan AU (50580201) are shown in figure 1. The AU approximates the extent of the contained assessed Morrow Formation and Atoka Group (fig. 2). The Colorado portion is limited by TPS and AU boundaries of the adjacent Denver Basin Province, for which Paleozoic AUs were assessed in 2002 (Higley and Cox, 2007; Higley and others, 2007).

By Early Pennsylvanian, as much as $10,000 \mathrm{ft}(3,050 \mathrm{~m})$ of Springer-Morrow and Atoka sediments were deposited in the basin (Rascoe and Adler, 1983; Al-Shaieb and others, 1994). The Morrow and Atoka Groups are each as much as 4,000 ft thick in the deep Anadarko Basin, thinning northward onto the shelf (Adler and others, 1971; Johnson, 1989). By the end of Permian time, more than 20,000 ft of Pennsylvanian and Permian sediments were deposited in the deep Anadarko
Basin (Jorgensen, 1989). Morrowan and Atokan strata were deposited on a broad low-relief shelf in Colorado and Kansas, and a subsiding deep basin in the Oklahoma and Texas portions of the province (figs. 3 and 15). The approximate Atokan onset of the Cimarron arch (fig. 5) is characterized by Morrowan rocks unconformably overlain on the crest by lower Desmoinesian strata (Rascoe and Adler, 1983). Lower Morrow strata pinch out along the flanks of the arch, but deposition of overlying Morrow sediments was associated with marine influx (Abels, 1959).

Morrow Formation and Atoka Group reservoirs are mostly marine and nonmarine sandstone and calcareous sandstone, with lesser limestone beds. Morrow Formation sediment supply was from the Sierra Grande uplift, Ancestral Rocky Mountains, Nebraska, and CKU (Swanson, 1979). The lower Morrow Formation consists of shallow-marine shales, sandstones, and limestones that were deposited under transgressive conditions and onlap the eroded Mississippian 


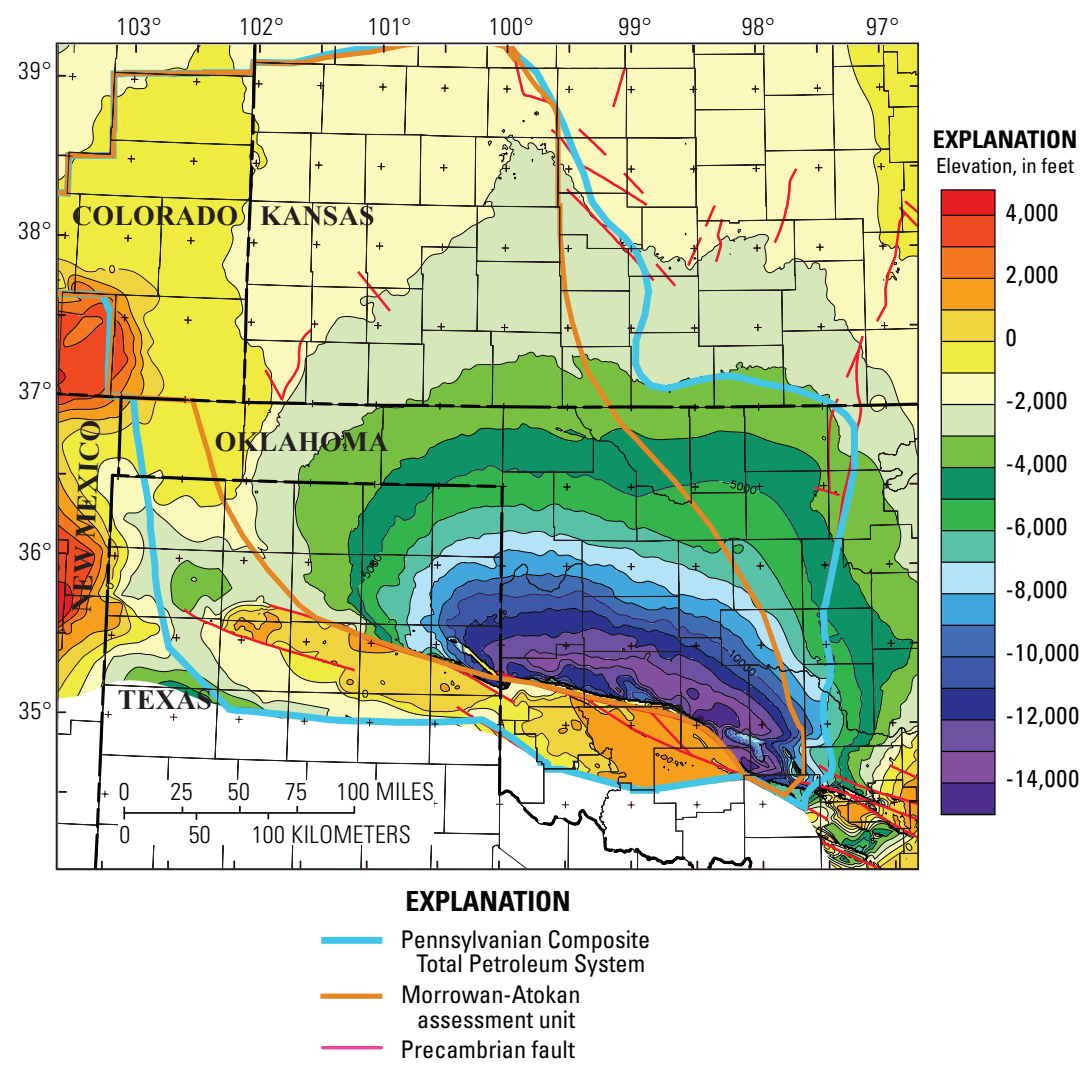

Figure 15. Map showing elevation on the top of the Atoka model layer. Extent of Morrowan and Atokan strata within the Pennsylvanian (Penn.) Composite Total Petroleum System (TPS) is approximated by the Morrowan-Atokan Assessment Unit (AU) boundary and in figure 3 elevation of the basal Thirteen Finger limestone. Elevation is relative to sea level. Precambrian faults are from Adler and others (1971). rocks toward the north and northeast (Johnson, 1979). Upper Morrow strata in the western part of the basin were primarily a deltaic sequence that prograded toward the southeast, resulting in deposition of mainly shales with lenticular, discontinuous sandstones, minor conglomerates, and thin coals and limestones (Swanson, 1979). Abels (1959) identified thin coal beds in the upper third of the Morrow Formation in the panhandle area of Texas and Oklahoma, and in southwestern Kansas. This area is mainly thermally immature for oil generation, so it is unlikely that the coals would have provided thermogenic natural gas.

The Morrowan on the shelf is characterized by marine to fluvial "clean, relatively well-sorted, non-glauconitic, and non-calcareous sandstone" (Adams, 1964), and the Hugoton field, Panhandle field, and Las Animas areas are mainly fluvial and shoreline sandstone reservoirs (Al-Shaieb and Walker, 1986). Bowen and Weimer (2003) described a compound incised-valley fill in western Kansas and eastern Colorado that contains three facies tracts with unique reservoir characteristics: (1) an updip facies tract of predominantly amalgamated fluvial channel sandstones overlies (2) a transitional facies tract of fluvial channel sandstones and interbedded finergrained estuarine sandstones, and (3) a downdip facies tract of fluvial channel sandstones isolated in estuarine shale. Traps in their study are combination, structural, and stratigraphic; the structural component is where valley-fill deposits cross anticlines, or the trend of the valley-fill deposits bends against structural strike. Reservoir compartmentalization can result from erosional truncation and subsequent deposition of strata, and lateral facies change from shale to sandstone in these elongate accumulations.

Morrowan strata in the deep basin were deposited in lowenergy environments and consist primarily of argillaceous, poorly sorted, glauconitic, calcareous sandstone (Rascoe and Adler, 1983). The lower permeability and depth of the deepbasin sandstones are associated with decreased exploration and development. These strata are also overpressured with gradients that exceed the normal $0.465 \mathrm{psi} / \mathrm{ft}$ gradient as part of the sealed megacompartment complex of Al-Shaieb and others (1994). Morrowan and Atokan intervals exhibit approximately normal pressure on the shelf.

Atokan strata consist of a series of southward-thickening marine shales, sandstones, and limestones that are about 50-200 ft thick in the northern and western parts of the Anadarko Basin (Johnson, 1989). Atokan strata tend to grade laterally into granite wash lithofacies close to the Wichita Mountain uplift, making well-log picks more difficult. The Morrow Formation is disconformably to gradationally overlain by Atokan Thirteen Finger limestone. Morrow and Atoka reservoir seals are primarily overlying and interbedded shales 
that help create the primarily stratigraphic traps in the province. Morrow Formation sandstones account for most of the pre-Permian oil and gas production in the basin (Davis and Northcutt, 1989). Morrowan plus Atokan production from almost 20,000 leases in 1,461 fields in the AU is approximately 850 MMBO, 26.08 TCFG, and 418.39 MMBW (IHS Energy, 2010a, 2010b).

Almost all reported water production in the province is from Colorado and Texas. Average production per well was about 51.8 thousand barrels of water (16,273 wells) and 1.5 BCFG (18,491 wells); average gas-oil ratio (GOR) for 12,865 wells is 3,530 MCFG/BO (IHS Energy, 2010a, 2010b). Morrow Formation reservoirs in western Kansas and eastern Colorado are largely valley-fill sequences that developed when extensive river systems incised the exposed marine shelf during relative lowstands, and have produced more than $100 \mathrm{MMBO}$ and 500 BCFG (Bowen and Weimer, 2003).

Best quality reservoirs are medium- to coarse-grained fluvial sandstones with porosities ranging from 18 to 28 percent and permeabilities of 0.5 to 2.0 Darcy; overlying estuarine reservoirs contain 8 to 18 percent porosity and 10 to 500 millidarcies $(\mathrm{mD})$ permeability (Bowen and Weimer, 2003). An area that also includes the Oklahoma Panhandle and northern Texas produced more than $200 \mathrm{MMBO}$ and 8 TCFG from updip fluvial to deep-water marine strata (Bowen and Weimer, 2004). Production from the Atoka Group (including the Thirteen Finger limestone) is $27 \mathrm{MMBO}$ and $1.7 \mathrm{TCFG}$ (IHS Energy, 2010a, 2010b).

Cumulative production numbers are approximate for the Morrow and Atoka, as well as most other reservoirs in the basin, because of extensive use of multiple completions and reporting of commingled production for multiple units. The first Morrow discovery was from Keyes dome (fig. 6) in 1943, which produced 1.7 MMBO and 500 BCFG through 1985 from the lower Morrow Formation Keyes sand (fig. 2) (Davis and Northcutt, 1989; Oklahoma Geological Survey (OGS) 2011a, fields discovery wells database (FDW). The WatongaChickasha trend (figs. 6 and 17), discovered in 1960 in Blaine County, Oklahoma, includes reservoirs of Atokan, Morrowan, and Springer age; production through 1985 was $44 \mathrm{MMBO}$ and 1.8 TCFG from sandstones in varied trap types (Davis and Northcutt, 1989). Since about 1955 trends in drilling and production have been relatively stable, even with the fairly extensive exploration and development of this AU. The discovery thirds of oil and gas fields are shown in figure 18. Discoveries in 1969 through 1978 of the (second thirds) Buffalo Wallow, Stiles Ranch, Allison-Parks, and Briscoe fields increased the associated discovery rate for $200 \mathrm{BCFG}$ discoveries.

The petroleum system events chart for the MorrowanAtokan AU (fig. 19) shows time periods of petroleum source, reservoir, seal, and overburden strata, and trapping. Timing of generation, migration, and accumulation of oil and gas are derived from Higley (2014) petroleum system models (table 2). Onset of oil generation from potential Morrowan source rocks (fig. 7) would be slightly older than the $300-\mathrm{Ma}$ age of the Thirteen Finger limestone on table 2.

\section{Thirteen Finger Limestone-Atoka Shale Gas Assessment Unit}

This is a continuous gas AU. Its boundary was determined based on the areal extent of the informally named Thirteen Finger limestone and overlying Atoka shale (fig. 2) of the Atoka Group (fig. 20), combined with the area that is thermally mature for gas generation based on petroleum system modeling by (fig. 20). Estimated oil resources for the Thirteen Finger limestone and Atoka shale were included as part of the Morrowan-Atokan AU assessment. Thirteen Finger limestone thickness contours that are outside the limits of the MorrowanAtokan AU shown in figure 20 are extrapolated outside areas of data control. Top, base, and extent of the Thirteen Finger limestone are based mainly on examination of 125 wells across the basin. Elevation on the top of the unit ranges from about $-4,000 \mathrm{ft}$ on the shelf areas of Colorado and Kansas to $-17,000 \mathrm{ft}$ in the deep basin area of Oklahoma and Texas (fig. 3 ). Average thickness is $95 \mathrm{ft}$, and the greatest thickness is in the deep basin and trending northwest toward Colorado (fig. 20). Also shown in figure 20 are locations of figure 21 cross sections.

The Thirteen Finger limestone across the Anadarko Basin Province comprises 75 to $100 \mathrm{ft}$ ( 23 to $30 \mathrm{~m}$ ) of cyclical thin marine limestones and shales (Rascoe and Adler, 1983). The "fingers" name is because of its stacked thin shale and carbonate beds with irregular high gamma kicks of 140 American Petroleum Institute (API) and greater on well logs over most of its extent (fig. 21). The Carr and Hentz (2009) described the unit as fissile, organic-rich shale interbedded with fossiliferous wackestones that represent a third-order transgressive systems tract; the thickest, most organic-rich shale in the Atoka, at the top of the unit, is inferred to represent a third-order marine condensed section. They indicated that a third-order highstand systems tract downlaps this condensed section and contains several progradational parasequences that are capped by "conventional" Atoka limestone and dolomite reservoirs. The thin carbonate beds in the basal Atokan Thirteen Finger limestone (fig. 2) are potential reservoirs that are overlain by Atoka Formation shales. The basal Atoka shales that exhibit gamma signatures of greater than 140 API in well logs in areas of the basin, suggest elevated levels of organic matter (fig. 21).

The Thirteen Finger limestone can be correlated across the extent of the underlying Morrow Formation in the province, except where proximal to the Wichita Mountain uplift. This is primarily the result of tilting of strata and increased contribution of clastic sediments relative to that of organic matter. Gamma response in wells in cross section $B-B$ ' are more pronounced than those in $A-A^{\prime}$, which suggests less organic matter in the deep basin. Source rocks in $A-A$ ' are thermally mature for oil and (or) gas generation, which would deplete the TOC and HI values. Gamma response of the overlying Atoka Group is unremarkable in the $A-A$ ' cross section but the $B-B$ ' response is similar to that of the Thirteen Finger limestone. The Atoka Group source interval differs in that it is primarily shale, rather than thin limestone and shale beds. 


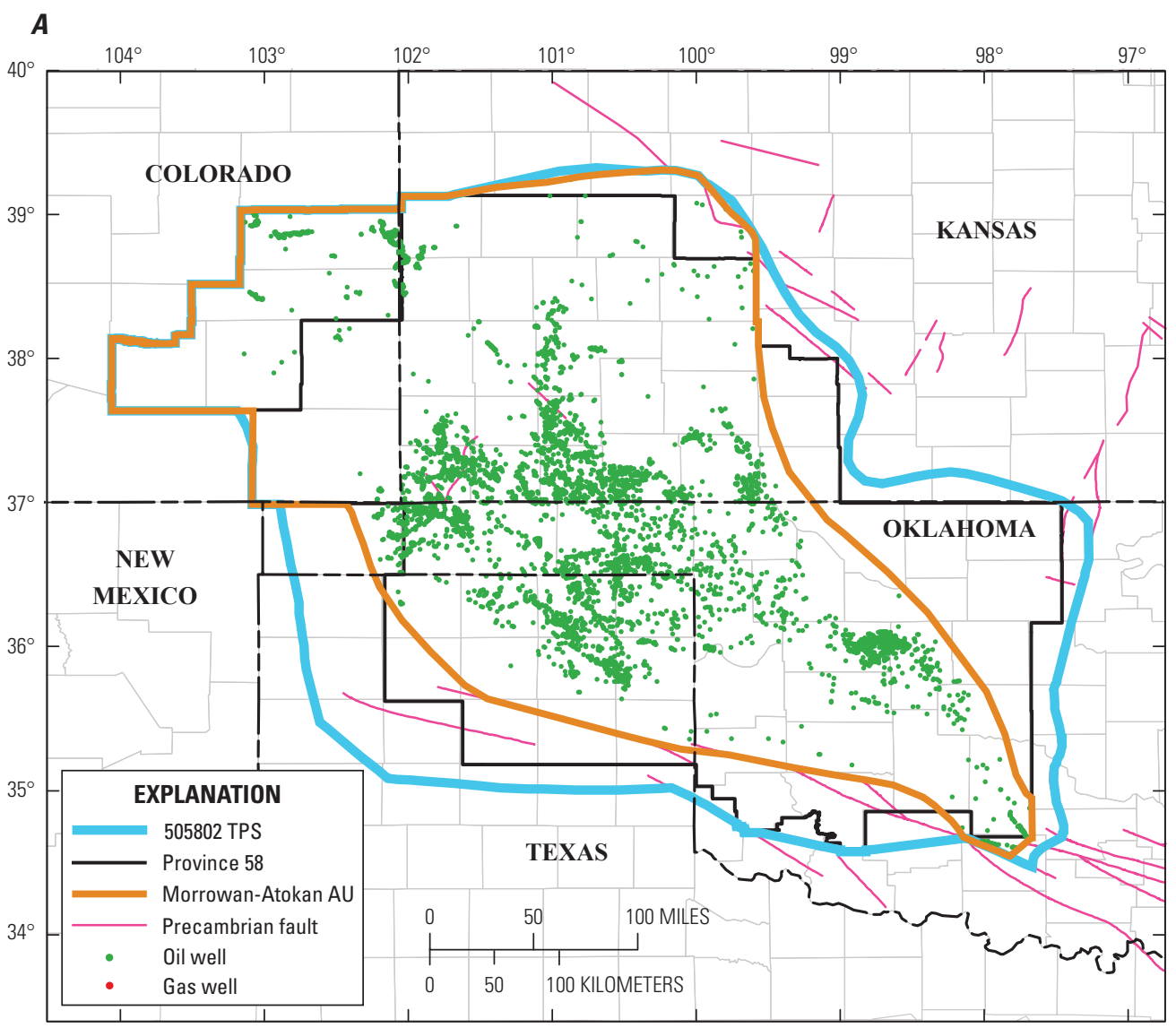

B

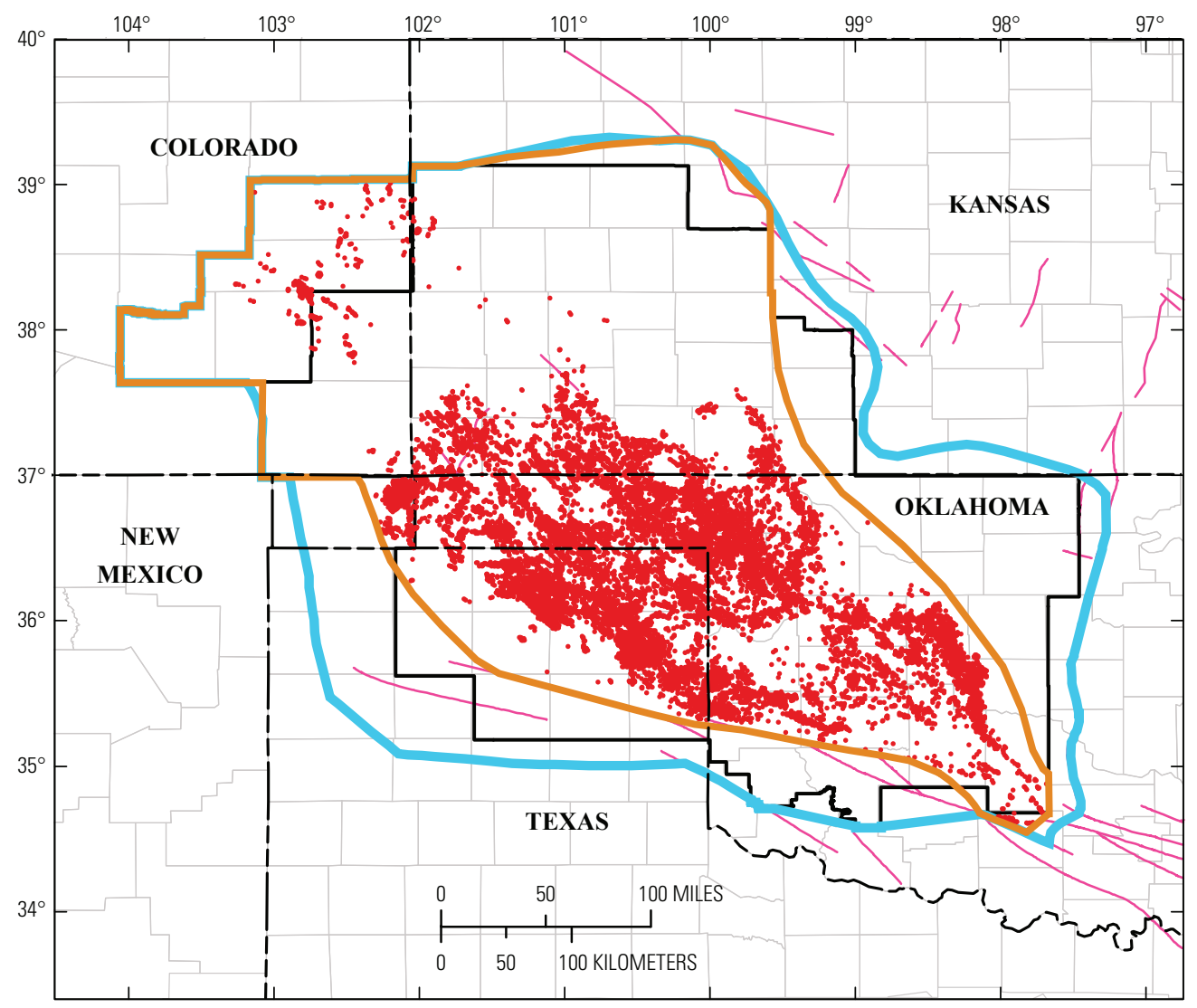

Figure 16. Maps showing $A$, Oil and $B$, gas wells that produce from Morrowan and Atokan reservoirs in the Anadarko Basin Province (IHS Energy, 2010a). The assessment unit (AU) boundary approximates the extent of Morrowan and Atokan strata. Precambrian faults are from Adler and others (1971). TPS, Total Petroleum System. 


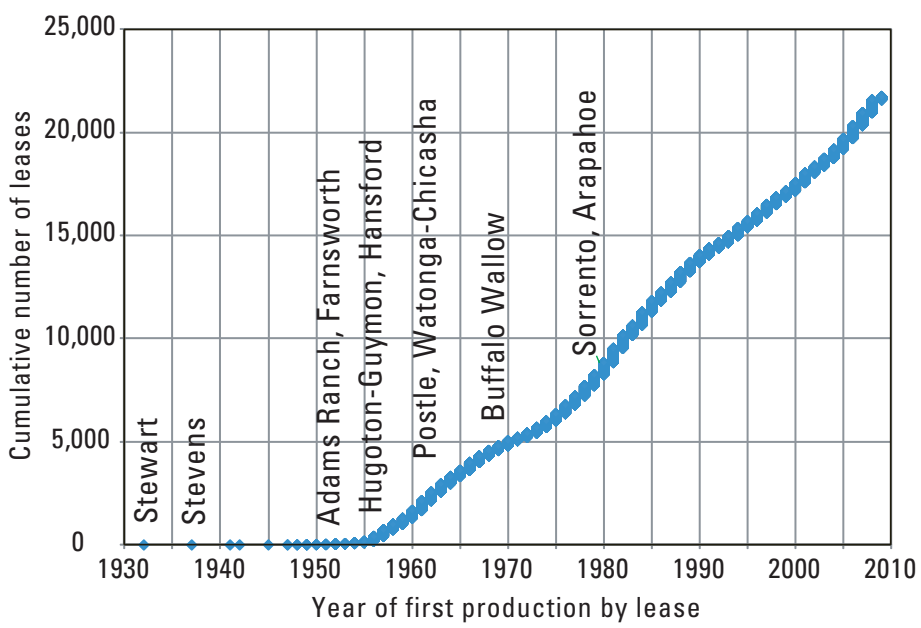

Figure 17. Cumulative number of wells by year that produce from the Morrowan and Atokan (IHS Energy, 2010a, 2010b). Several major fields are also shown by year of discovery; all are shown in figure 6 .
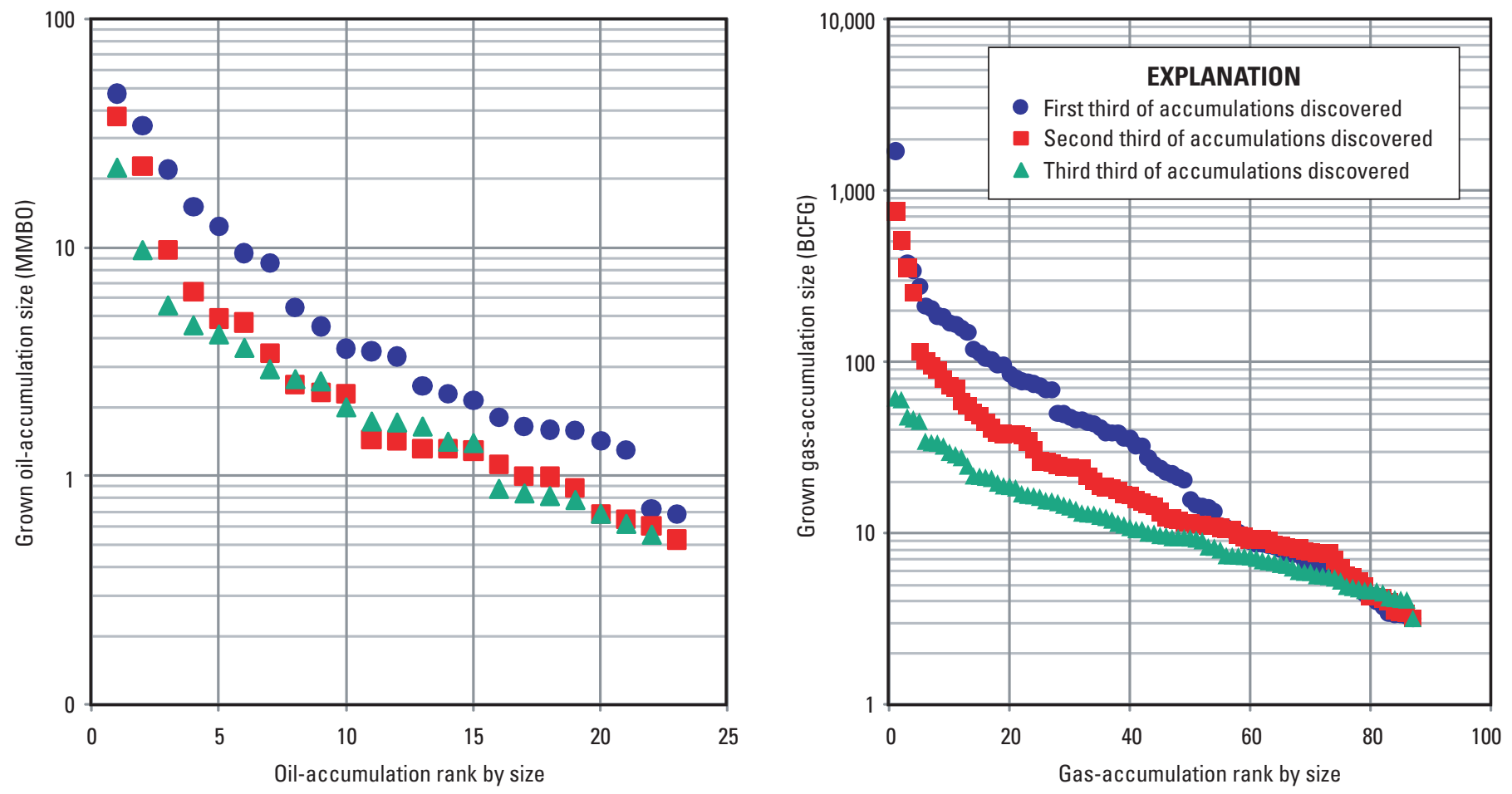

Figure 18. Discovery thirds of grown oil and gas accumulations for the Morrowan-Atokan Assessment Unit (AU) of the Pennsylvanian Composite Total Petroleum System (TPS). This is a mature AU, which is reflected by the generally larger field sizes early in the exploration history (first third) relative to later discoveries (second and third thirds). All thirds show similar trends in field sizes through time. Gas accumulation history is stable for fields less than 10 billion cubic feet of gas (BCFG), but exhibits progressive decline for fields greater than this. Data were constructed using the Nehring and Associates, Inc. (2009) field database, which has limited information for the Morrowan and Atokan fields. The database contains more information on Morrowan and Atokan gas fields than oil fields, as indicated by the data density on the plots. MMBO, million barrels of oil. 


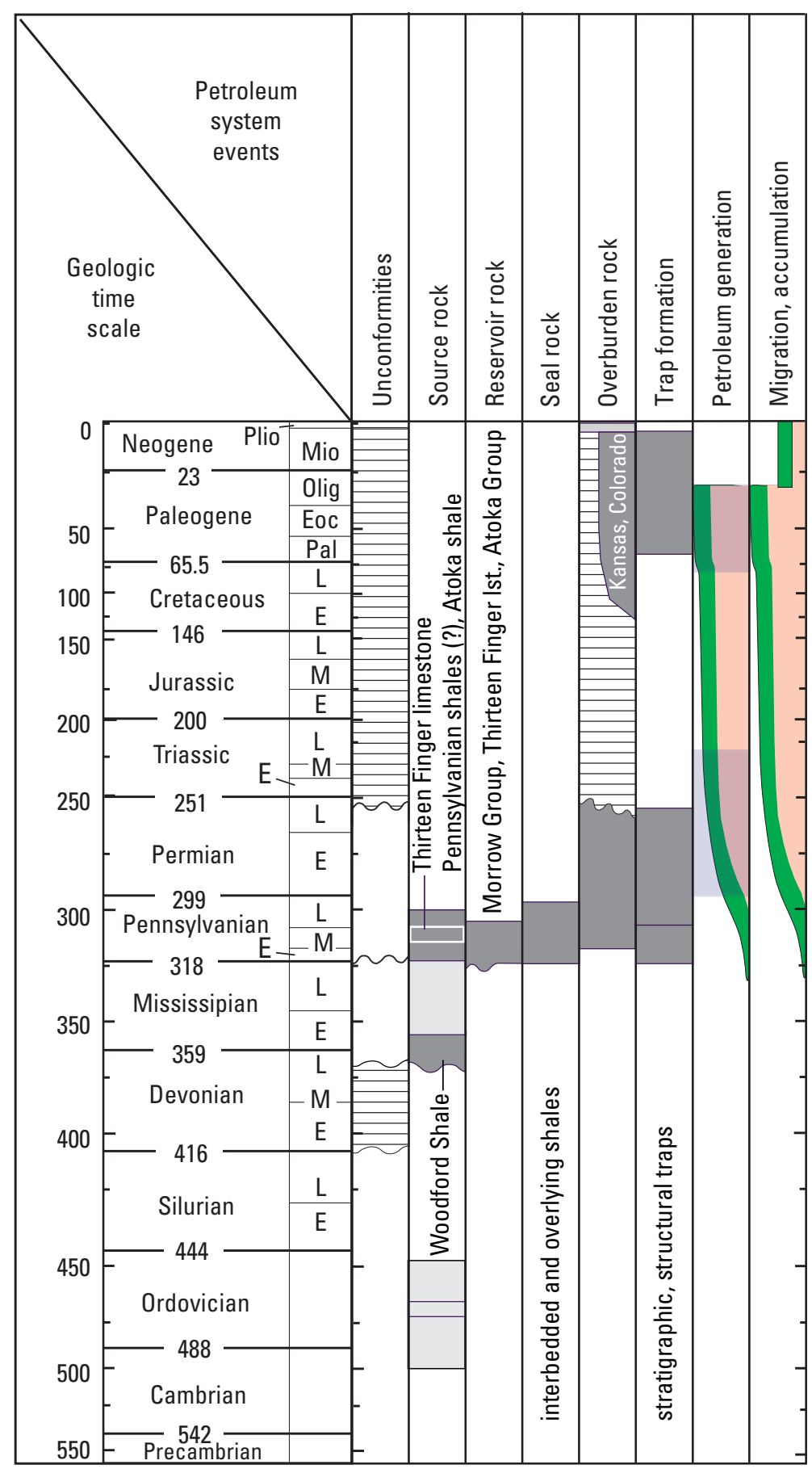

Figure 19. Petroleum system events chart for the Morrowan-Atokan AU of the Pennsylvanian Composite TPS. Woodford Shale (green) and Thirteen Finger limestone (tan) ranges of oil and gas generation, migration, and accumulation are based primarily on the petroleum system models. Potential petroleum source rocks from Hatch and others (1986) are light gray. Time ranges of peak generation (fig. 14) are within blue rectangles. Paleogene trap formation, migration, and accumulation are based on Laramide generation and potential remigration associated with basin tilting. Unconformities, wavy and horizontal lines; Ist, limestone; sh., shale; Fm., Formation; Plio, Pliocene; Mio, Miocene; Eoc, Eocene; Pal, Paleocene; L, Late; M, Middle; E, Early. 


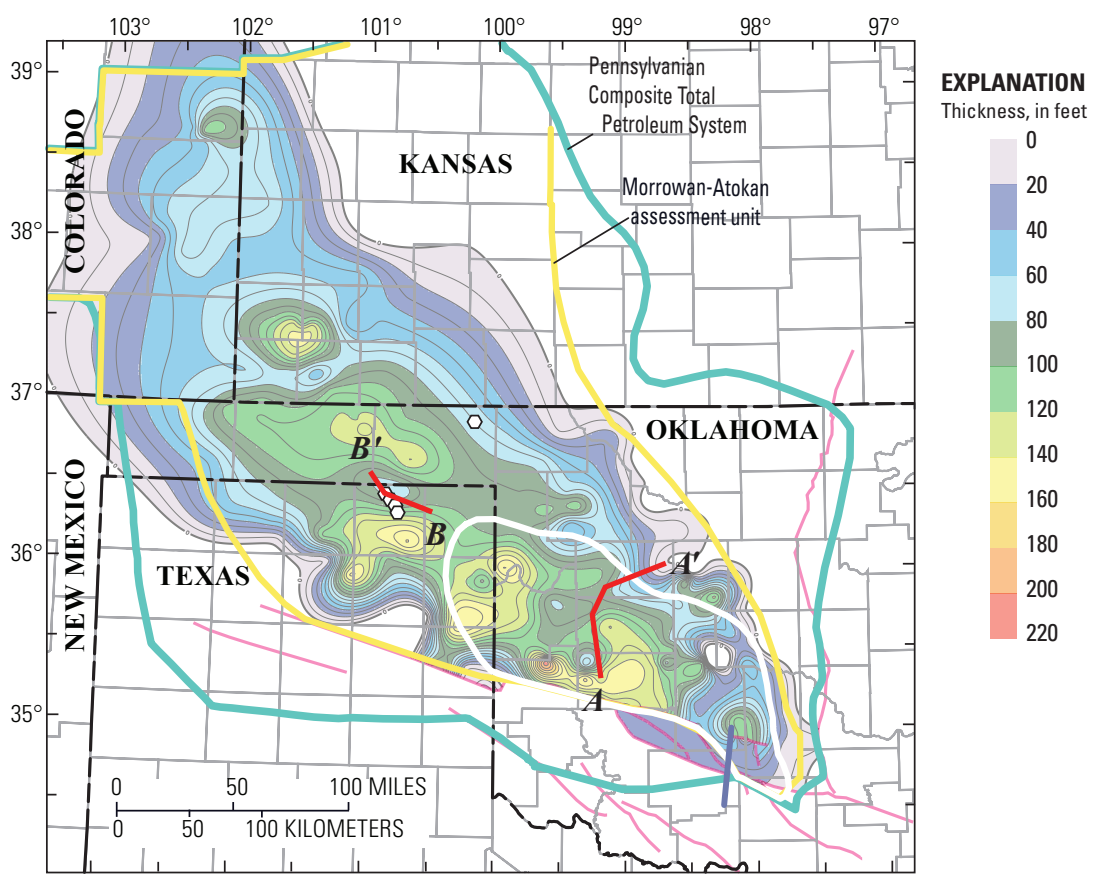

Figure 20. Isopach map of the top of the Thirteen Finger limestone to the Morrow Formation, and the Thirteen Finger Limestone-Atoka Shale Gas AU (white line). Morrow and Thirteen Finger tops are based on Andrews (1999a, 1999b), examination of more than 120 well logs, and edited tops from IHS Energy (2009, 2010a). Morrow Formation faults (pink) are modified from Andrews (1999b). Color contour interval is $20 \mathrm{ft}$ and (black) line contours are 10-feet intervals. Figure 21 cross sections are labeled $A-A^{\prime}$ and $B-B^{\prime}$. White polygons are Perryton (Texas) and Knowles Northwest (Oklahoma panhandle) oil fields, conventional oil reservoirs from the Thirteen Finger limestone.

There is no recorded continuous gas production in this AU. Within the Morrowan-Atokan AU there is minor conventional oil and associated gas production based on decline curves of petroleum and water production; the Thirteen Finger limestone in the Perryton and Knowles Northwest fields (fig. 20) have cumulative production of about 160,000 barrels of oil, 300 million cubic feet of gas (MMCFG), and 6,900 barrels of water (IHS Energy, 2010b). The fields are also located in an area marginally mature to mature for oil generation from the Thirteen Finger limestone, based on modeled TR and $\mathrm{R}_{\mathrm{o}}$ values (fig. 8). Carr and Hentz (2009) indicated that Atoka reservoirs are mainly limestone, and also sandstone, and have been productive since the 1940s in the northwestern part of the Anadarko Basin; operators have recently been drilling horizontally and applying hydraulic-fracture stimulations in the thin (6-27 ft, average $11 \mathrm{ft})$ carbonate reservoirs to tap adjacent organic-rich shales. Cumulative conventional petroleum production is $185 \mathrm{BCFG}$ and 6.7 MMBO in Ochiltree and Lipscomb Counties, northeast Texas (IHS Energy, 2010b).
The petroleum system events chart (fig. 22) shows the timing of generation, migration, and accumulation of petroleum that are based on petroleum system models of (table 2). Onset of gas generation from the Thirteen Finger limestone and Atoka shale (fig. 7) is probably soon after the 300-Ma onset of oil generation from these petroleum source rocks. The AU boundary (fig. 20) approximates the 0.99 percent TR boundary (fig. 8) that marks the transition from the end of oil generation to gas generation from the Thirteen Finger limestone. Although the Thirteen Finger limestone and Atoka shale are assumed to be the petroleum source rocks for this $\mathrm{AU}$, there could be potential contributions from shales of the underlying Morrow Formation. Also, timing of petroleum generation is based on Woodford hydrous pyrolysis (HP) kinetics. These boundaries would probably be slightly different, either larger or smaller, should HP kinetics values be available that is specific to the Thirteen Finger limestone and Atoka shale. 
Hohnke Leo Unit 1

Exxon Corporation

Arkla Exploration

Williams 1-18

Porter Willie 1-9

T 10 N, R 18 W, S. 6

T 14 N, R 19 W, S. 6

Company

Petroleum Co.

T 17 N, R 14 W, S. 9

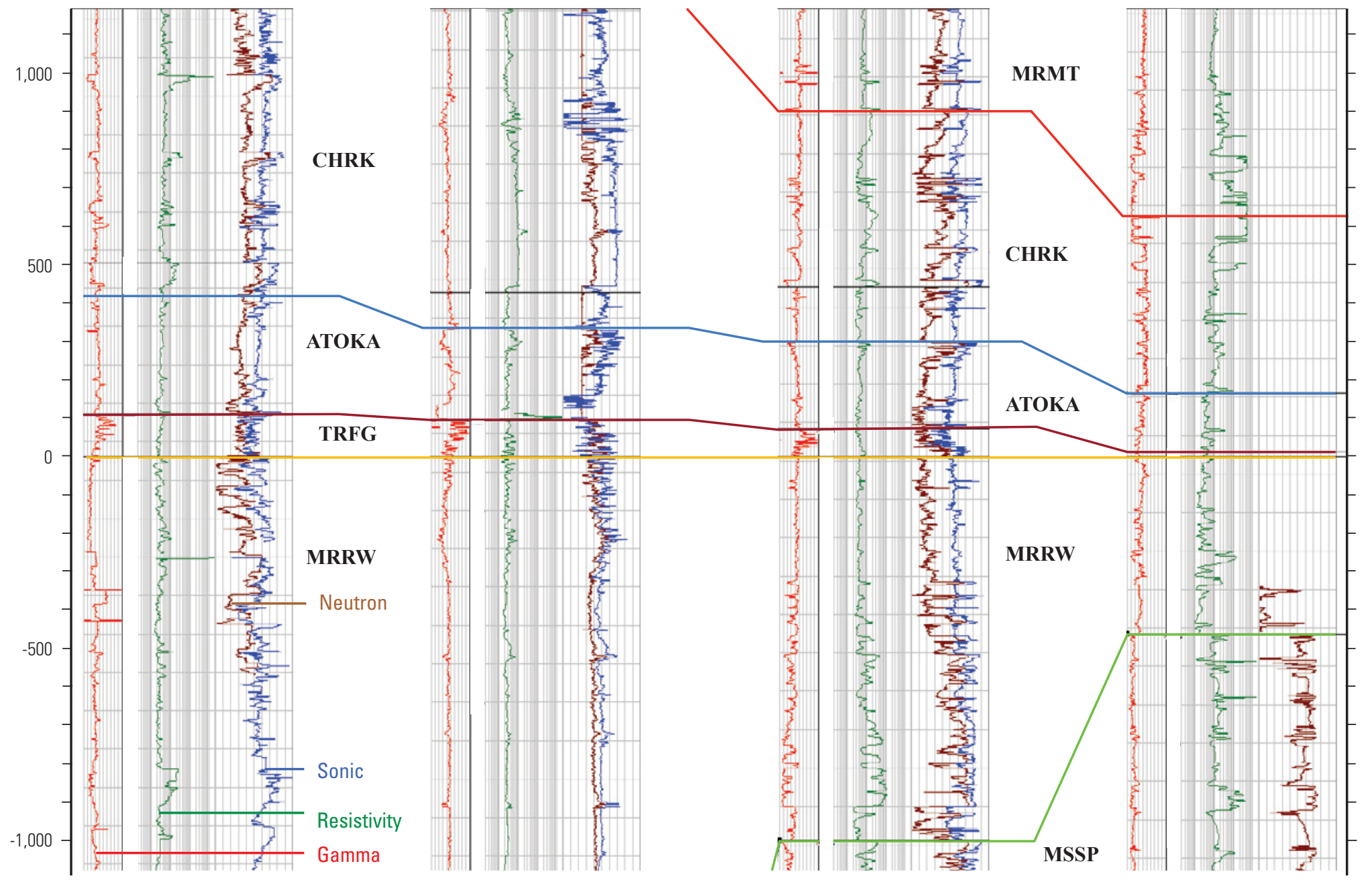

Figure 21. A, Southwest to northeast and $B$, southeast to northwest cross sections of the Thirteen Finger limestone (TRFG) of the

Pennsylvanian Atoka Group (ATOKA) in the Anadarko Basin. Lines of section are in figure 20. Vertical scale is in feet relative to the base of the Thirteen Finger limestone. MRMT is Marmaton Group, CHRK is Cherokee Group, MRRW is Morrow Formation, and MSSP is top of the

Mississippian. Labeled well log traces are gamma (0 to 200 American Petroleum Institute [API]), resistivity, sonic density, and neutron density. 


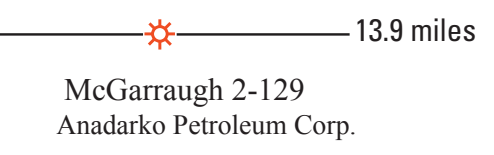

Anadarko Petroleum Corp.

Survey: SPRR Abs: 333, Blk. 10, S. 129

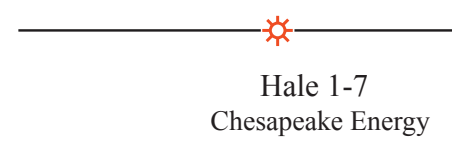

T. 1 N., R. 20 E., S. 7

Survey: H\&TC, Blk: 43

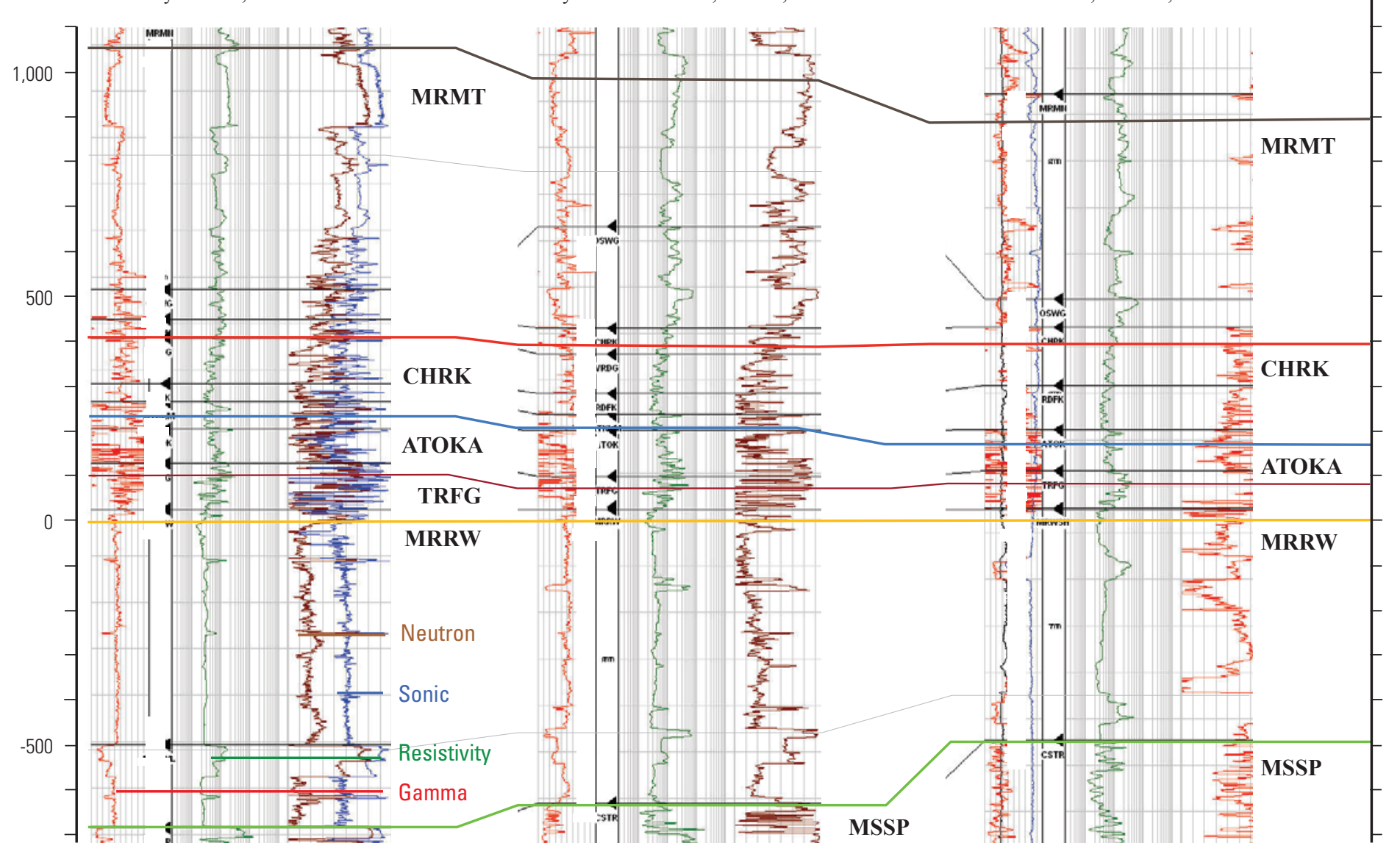

Figure 21. $A$, Southwest to northeast and $B$, southeast to northwest cross sections of the Thirteen Finger limestone (TRFG) of the Pennsylvanian Atoka Group (ATOKA) in the Anadarko Basin. Lines of section are in figure 20. Vertical scale is in feet relative to the base of the Thirteen Finger limestone. MRMT is Marmaton Group, CHRK is Cherokee Group, MRRW is Morrow Formation, and MSSP is top of the Mississippian. Labeled well log traces are gamma (0 to 200 American Petroleum Institute [API]), resistivity, sonic density, and neutron density.-Continued 


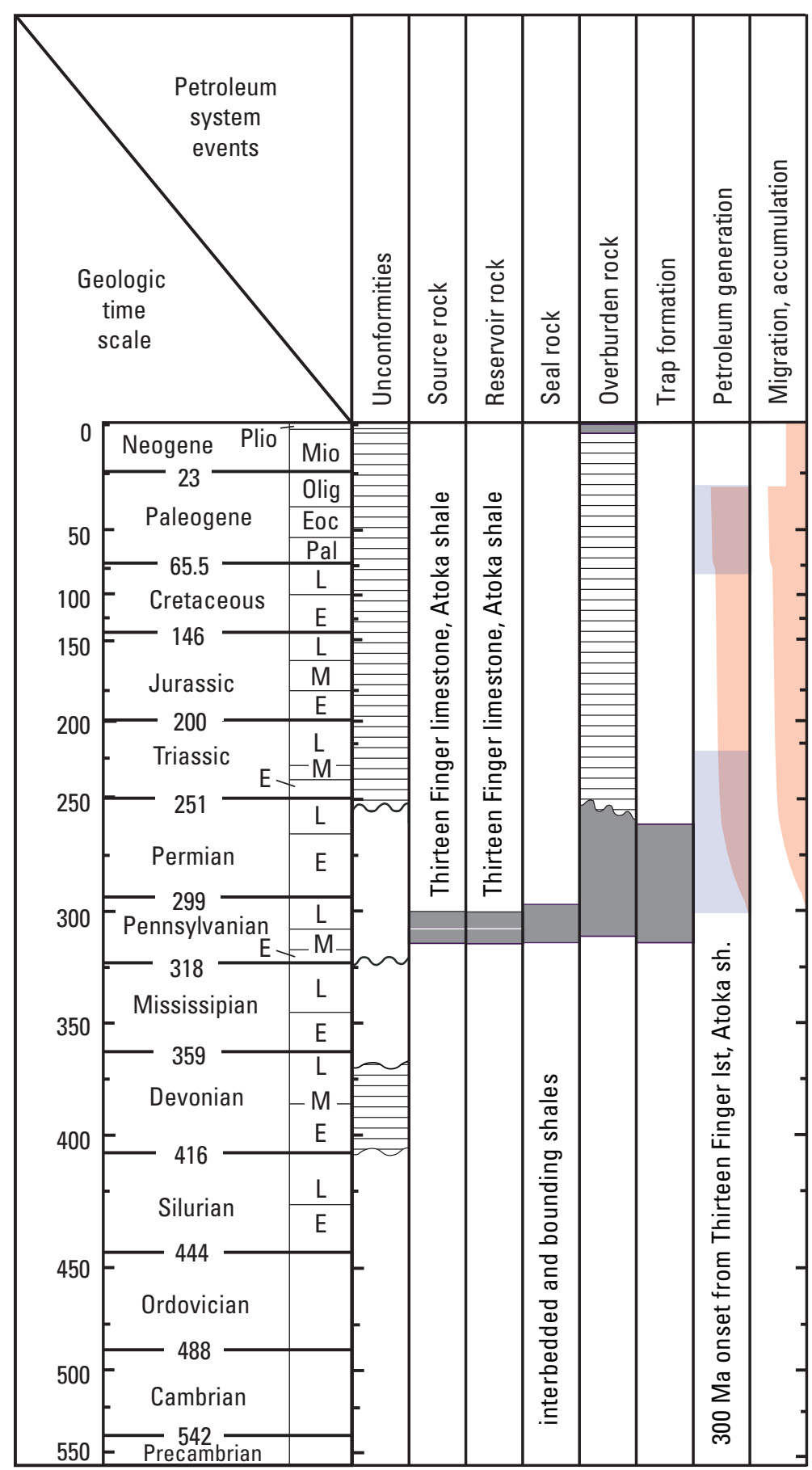

Figure 22. Petroleum system events chart for the continuous Thirteen Finger Limestone-Atoka Shale Gas Assessment Unit (AU) of the Pennsylvanian Composite Total Petroleum System. Timing of basin oil and gas generation, migration, and accumulation is based primarily on the petroleum system models. Thirteen Finger limestone (tan) periods of peak oil and gas generation are shown by the blue rectangles. This $\mathrm{AU}$ is located in an overpressured zone of the deep basin (Al-Shaieb and others, 1994). Because of this, Laramide uplift and erosion probably had minimal effect on the pressure environment. Millions of years ago, Ma; Unconformities, wavy and horizontal lines, Ist; limestone, sh; shale. 


\section{Desmoinesian Assessment Unit}

Desmoinesian, Missourian, and Virgilian strata were deposited in transgressive-regressive cycles that periodically inundated the province with alternating thin-bedded predominantly marine sandstones, limestones, and shales; these can be separated into stratigraphic packages of mostly sandstone or mostly limestone reservoirs (Moore, 1979; Rascoe and Adler, 1983). Desmoinesian strata, mainly of the Cherokee and Marmaton Groups (fig. 2) cover the province except where locally removed by erosion. They are absent over most of the Wichita Mountain uplift, in the CKU, Cimarron arch, and the Sierra Grande uplift (fig. 5). The lateral continuity of these strata are visible on well logs, but the Paradox Basin of southeastern Utah provides extensive outcrops to visualize the stacking symmetry and lateral continuity of beds (fig. 23).

Desmoinesian seas covered the entire central Oklahoma arch and the northern shelf area, extending into central Kansas (Johnson, 1989). The shallow sea was estimated at 50 to as much as $100 \mathrm{ft}$ (15 to $23 \mathrm{~m}$ ) deep (McCrone, 1964). Alluvial plain, deltaic, and incised channels flowed from the north and east southward and westward into the basin; during lowstands the shelf edge was exposed, the streams were incised during extension, and submarine fans were deposited on the basin floor (Anderson, 1991) with subsequent valley-fill deposition as the seal level rose. During temporary stillstands, deltaic conditions developed in the lower stretches of the streams, and relatively thin delta-fringe sands formed sheet-like units; further rise in sea level inundated the stream systems, and estuarine settings preceded widespread shallow marine conditions (Al-Shaieb and others, 1989). Source area for lower Desmoinesian clastics was from the north, whereas upper Desmoinesian strata were sourced from the south for the Arkoma and Anadarko Basins (Rascoe and Adler, 1983). West of the CKU, the lower Desmoinesian consists of an alternating sequence of thin limestones with lesser shale; sandstone is present but not common in this region, and was probably sourced from the Cambrian Reagan Sandstone from the northern CKU and Cambridge arch (Rascoe and Adler, 1983).

The lower Desmoinesian Cherokee Group and correlative strata are mainly marine shale with numerous thin limestones and some lenticular point-bar and channel-fill sandstones. The informal Red Fork sand is the most important reservoir of this group; its stacked channel-fill sandstones are generally coarser grained and thicker than the delta-fringe sandstone and other facies of the group (Al-Shaieb and others, 1994). The informal Prue sand, the uppermost unit of the Cherokee Group was deposited in fluvial, deltaic, and nearshore marine environments (Andrews and others, 1996; Boucher, 2009); it ranges in depth from $3,500 \mathrm{ft}$ on the northern shelf to about $12,000 \mathrm{ft}$ near the Wichita Mountain uplift (Henry and Hester, 1995). The upper Desmoinesian Marmaton Group across the northern Oklahoma shelf area consists almost entirely of limestones, but in the deep Anadarko Basin it is almost entirely clastic sediments that were derived from the Ouachita foldbelt to the south (Krumme, 1981; Rascoe and Adler, 1983).
Modeled thickness of the Desmoinesian in the deep basin ranges southward from about $500 \mathrm{ft}$ to more than 3,500 ft. The Marmaton Group comprises in ascending order, Fort Scott Limestone, Englevale Sandstone, and Oologah Limestone (Big lime) (Johnson, 1989) in the northern basin, and sandstones and shales of the Deese Group in the southern basin (fig. 2). Depths to the top of the Marmaton Group range from $3,500 \mathrm{ft}$ on the northern shelf, to about 13,000 ft proximal to the Wichita Mountain uplift (Henry and Hester, 1995). This marine sequence is $200-1,000 \mathrm{ft}$ thick in the north and grades southward in the deep Anadarko Basin into 4,000-5,000 ft of granite wash (Johnson, 1989). Granite wash lithofacies are generalized in the 4D model as the gray clastic sequence in figure 24. Desmoinesian strata in the deep basin were assessed as part of the Greater Granite Wash Composite AU.

The Desmoinesian AU boundary corresponds to that of the Pennsylvanian TPS, with the exception of an area in the deep basin that contains the Greater Granite Wash Composite AU (fig. 25). Oil and gas wells are distributed across the Desmoinesian AU, but are concentrated in the area of the Watonga-Chickasha and Sooner trends in the eastern half of the province, against the Nemaha uplift and, for oil wells, around the CKU. Trap types are structural and (or) stratigraphic. Fields close to the Wichita Mountain uplift are commonly structural, such as the large faulted anticline of the Cement field. Reservoirs are clastic and carbonate beds of the Cherokee and Marmaton Groups, and their lateral equivalents (fig. 2). Reservoirs range in depth from about 4,000 to 12,000 $\mathrm{ft}$ (Nehring and Associates, Inc., 2009). Cumulative production in the province is about $747 \mathrm{MMBO}, 8.29 \mathrm{TCFG}$, and 49.4 MMBW from about 14,235 wells in 1,640 fields (IHS Energy, 2010a, 2010b). Average GOR is 18,000 CFG/BO. Three of the larger fields are (1) Cement, for which 85 of 1,345 leases in the field record Desmoinesian production of 1.4 MMBO and 210 BCFG, (2) Postle, which has produced 5.4 MMBO and 9.5 BCFG from 25 Desmoinesian leases, and (3) the Watonga-Chickasha trend, which has produced 2.4 MMBO and $123 \mathrm{BCFG}$ from 180 of the 2,750 total leases (IHS Energy, 2010b) (fig. 25). Because much of the production in the province is reported as commingled from multiple units, volumes should be considered approximate. The discovery thirds plots of grown oil and gas accumulations contain only a few of the Desmoinesian reservoirs in the basin (fig. 26), largely because of the quality of well and production data, so trends in production are difficult to determine. The discovery thirds of oil accumulations appear to have a stable exploration and development history, although four of the five largest oil fields were discovered early in exploration. The gas discovery thirds has too few points to make valid conclusions.

Burruss and Hatch (1989) determined there are three oil types in the basin based on geochemical analysis of 104 crude oils and 190 shale core samples of Cambrian through Pennsylvanian rocks; these oil types are divided into (1) Pennsylvanian, (2) Silurian to Mississippian, and (3) Middle Ordovician Simpson Group reservoirs. This suggests that Pennsylvanian reservoirs are sourced from Pennsylvanian 


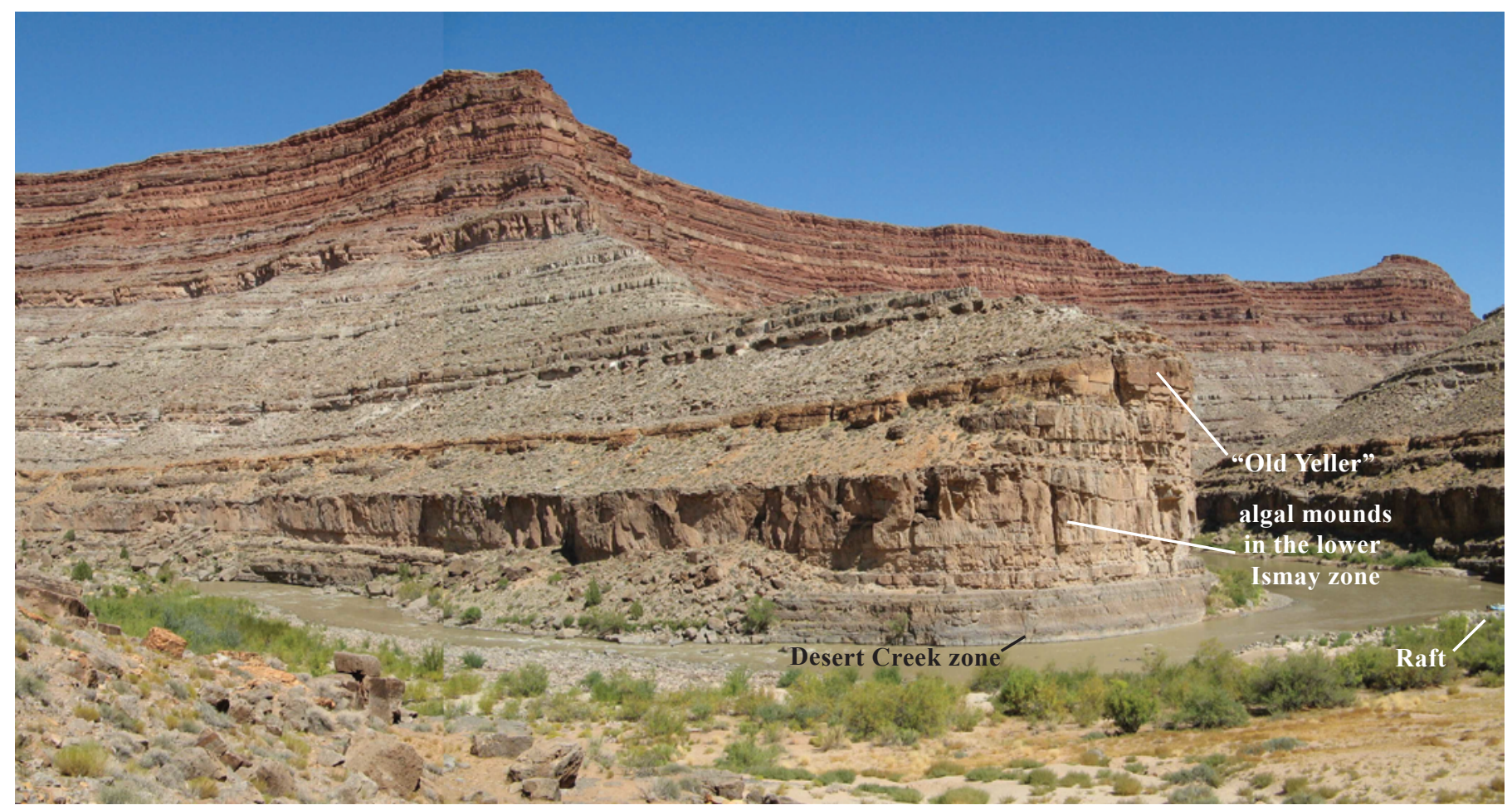

Figure 23. Photograph showing Desmoinesian outcrop along the San Juan River in the Paradox Basin, southeastern Utah, illustrates carbonate/siliclastic cyclicity and lateral continuity of beds. The informally named middle Desmoinesian "Old Yeller," Desert Creek, and Ismay zones are within the Paradox Formation and are roughly age-equivalent to the upper Cherokee and lower Marmaton Groups in the Anadarko Basin. This section is described in Grammer and others (1996) and is located in about sec. 26, T. 41 S., R. 19 E. Raft is for scale and this north view of the outcrop trends approximately west to east.

shales, although additional research may indicate more complexity. There are also potential petroleum contributions to Desmoinesian reservoirs from Mississippian strata in the northeastern Oklahoma portion of the basin because Morrowan and Atokan rocks are absent there. Oil and gas are concentrated along the Nemaha uplift and Central Kansas uplift, and a broad age range of petroleum source rocks could contribute in these areas because of migration along fault systems and thinning of strata in Kansas. Sampled shales within the Desmoinesian contain a mixture of Types II and III kerogen (Rice and others, 1989). Thermal maturation of potential Desmoinesian source rocks (fig. 7) is approximated by the Thirteen Finger images in figure 8 and shown in Higley
(2012, fig. 26). Cherokee and Marmaton Groups petroleum source rocks in the Texas and Oklahoma Panhandles would be slightly less mature than the Thirteen Finger image. Desmoinesian source rocks in the deep basin of Oklahoma and Texas are overmature for oil generation based on TR, and in the wet and dry gas generation windows using modeled vitrinite reflectance. The petroleum system events chart (fig. 28) displays the timing of generation, migration, and accumulation of petroleum based in part on petroleum system models (table 2) (Higley, 2014). Onset of oil generation from Pennsylvanian source rocks for the Desmoinesian AU (fig. 7) approximates the 300-Ma onset of oil generation from Thirteen Finger limestone petroleum source rocks. 


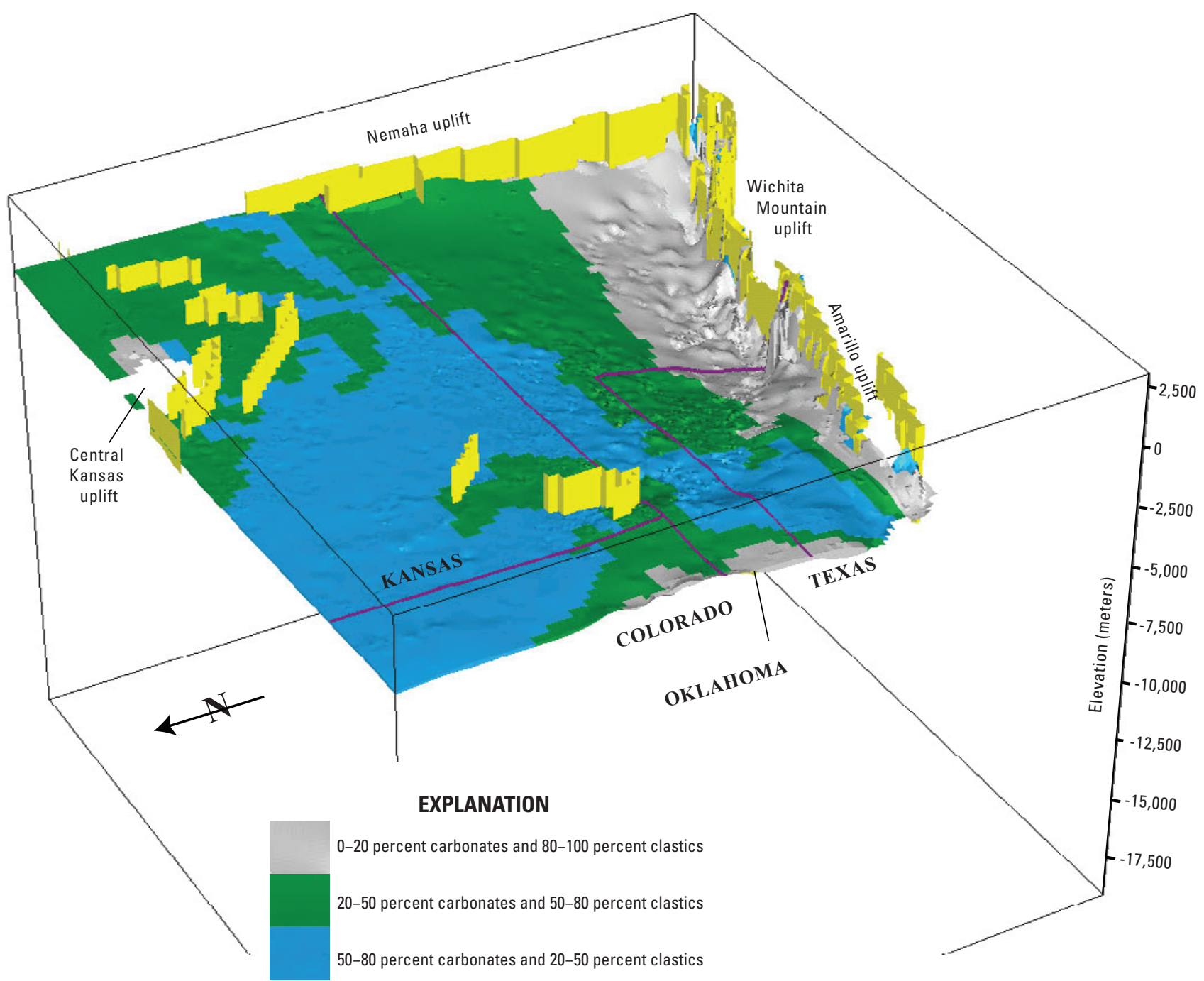

Figure 24. Modeled surface on the Desmoinesian layer shows a broad gentle slope in Colorado and Kansas that deepens near the basin axis, north of the Wichita Mountain uplift. Vertical yellow bars are Precambrian faults, and surface colors are generalized lithofacies from Adler and others (1971) that mainly record increased clastics basinward. White areas do not contain Desmoinesian or underlying Cherokee model layer lithofacies. Vertical exaggeration is 15 times. 
$\boldsymbol{A}$

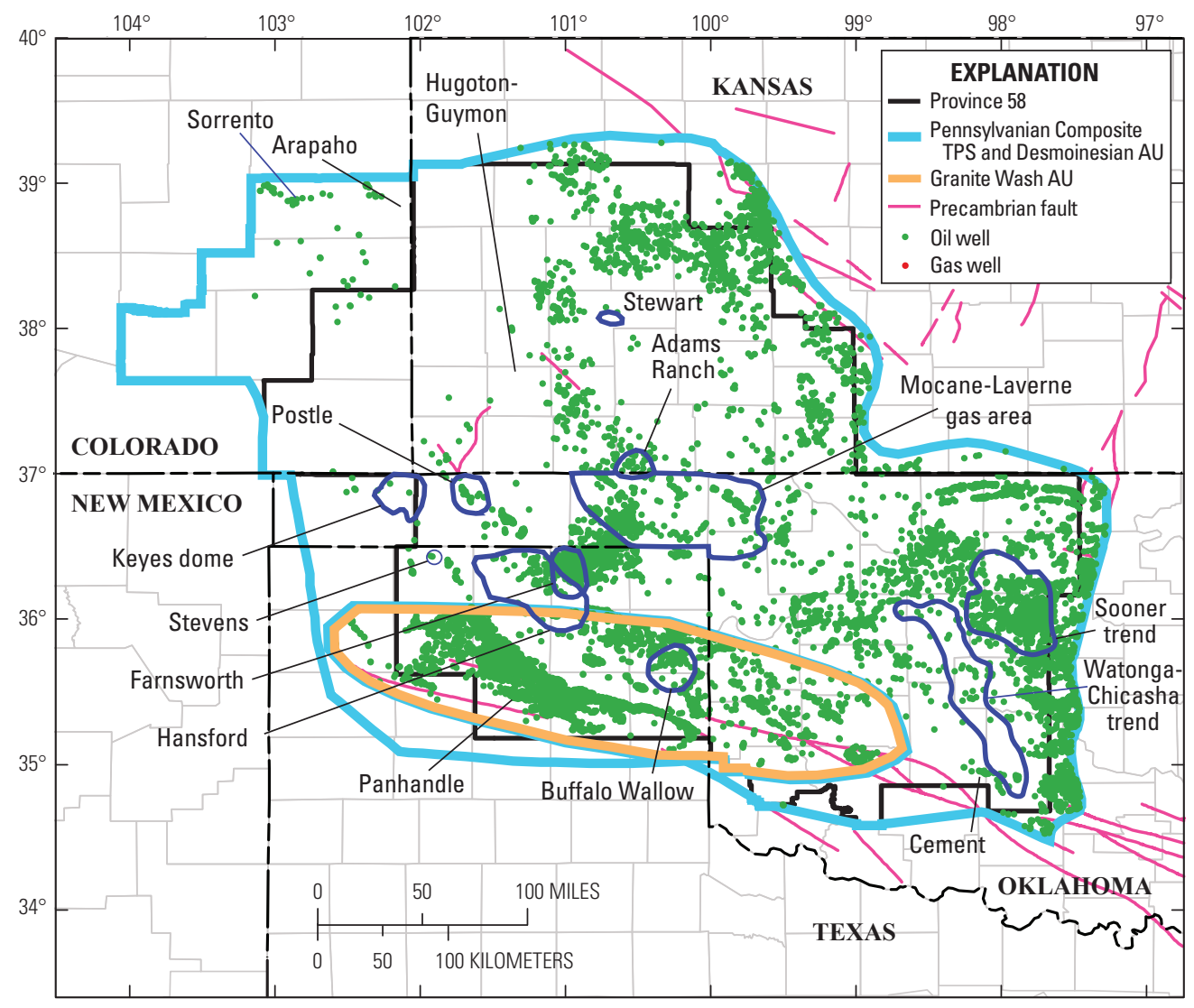

$\boldsymbol{B}$

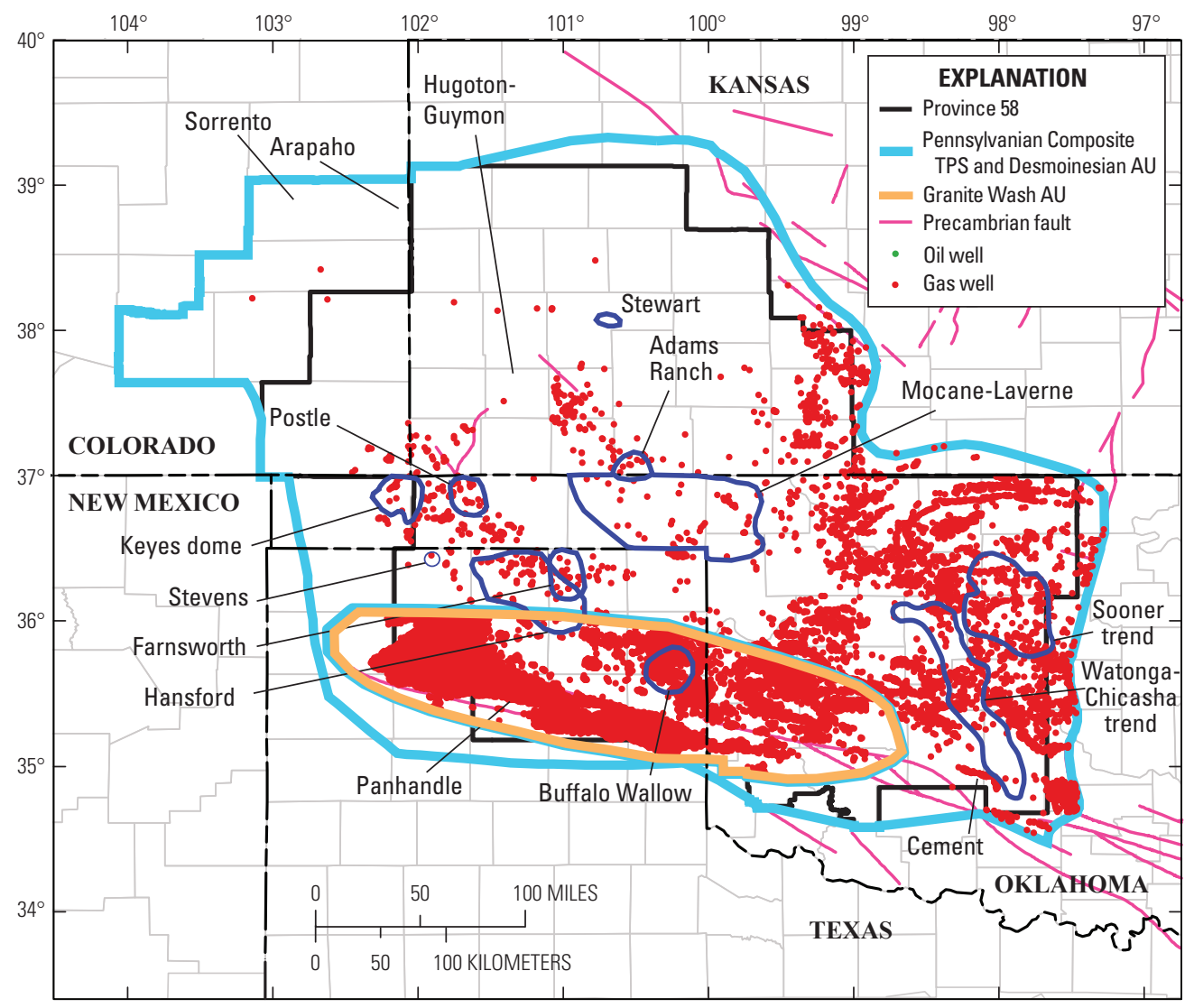

Figure 25. Maps showing $A$, Oil and $B$, gas wells that produce from the Desmoinesian Assessment Unit (AU) Cherokee and Marmaton Groups in the Anadarko Basin Province (IHS Energy, 2010a, 2010b). Generalized field locations are from IHS Energy (2010a) and Bebout and others (1993). The Desmoinesian AU shares a common boundary with the Pennsylvanian Composite Total Petroleum System (TPS). Excluded from the $A U$ is an oval area in the deep basin that is the Greater Granite Wash Composite AU and included wells. Precambrian faults (pink) are from Adler and others (1971). 


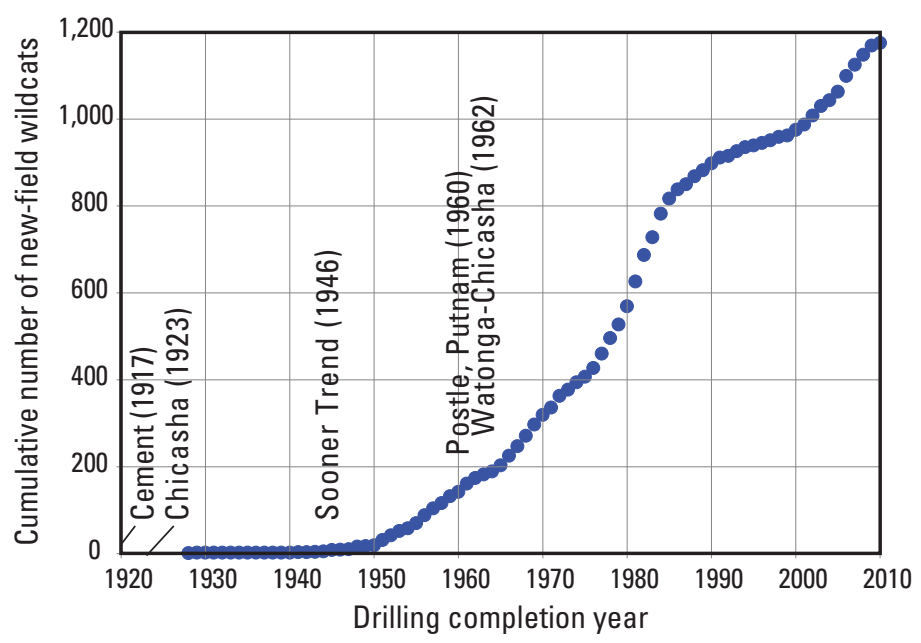

Figure 26. Cumulative number of new-field wildcats by year that produce from the Desmoinesian AU (IHS Energy, 2010a, 2010b). Exploration and development was static until about 1950, after which activity increased and has been fairly steady through time. The displayed major fields by discovery dates produce oil and gas from multiple units and ages. Field locations are shown in figure 25.
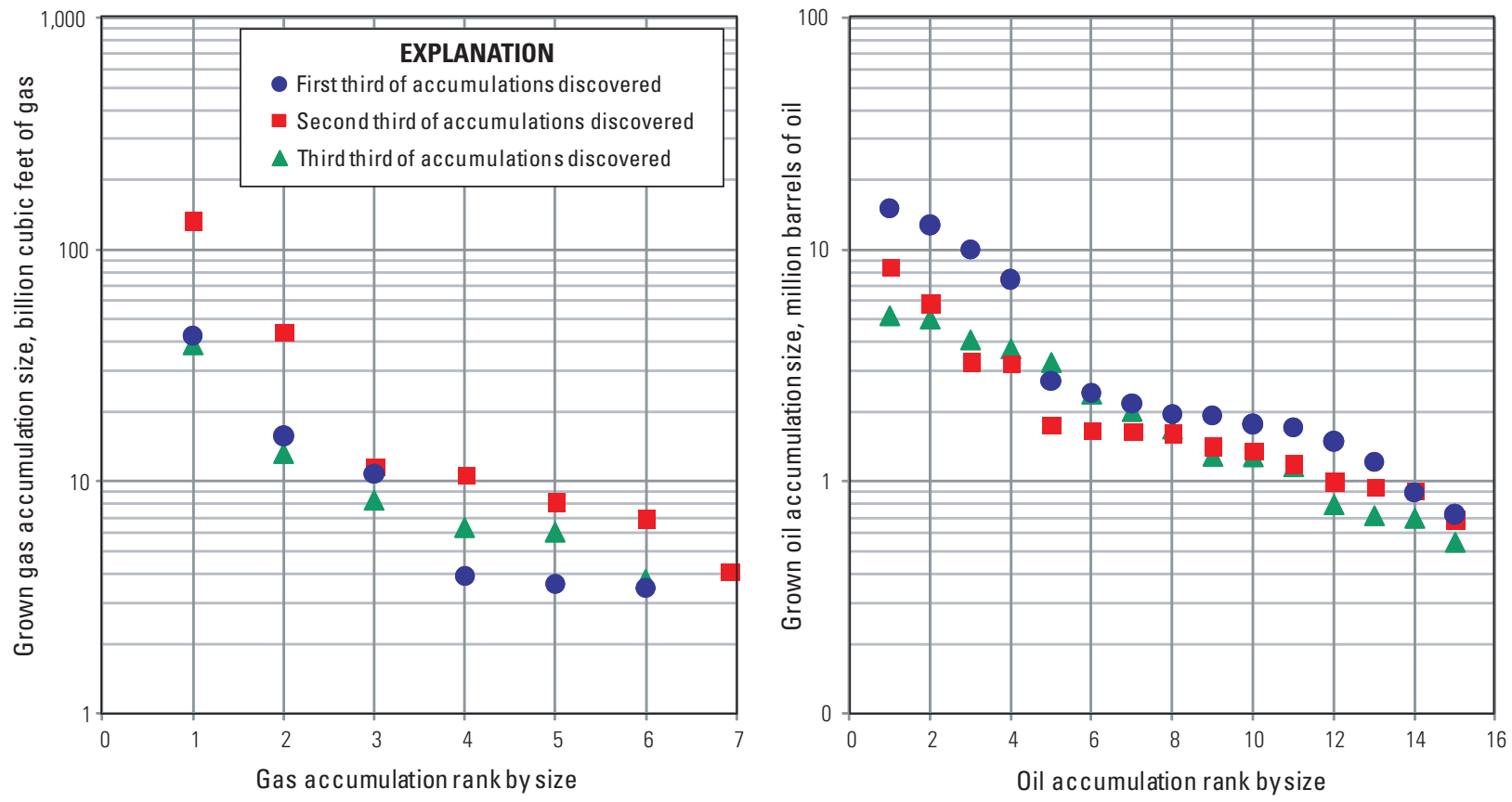

Figure 27. Grown gas and oil accumulation sizes for discovery thirds for the Cherokee and Marmaton Groups reservoirs in the Desmoinesian Assessment Unit (AU) (50580202). This AU has a mature exploration and development status with production from about 1,400 fields in the province (IHS Energy, 2010a, 2010b); however, only a few of these fields are represented on the figure. This paucity of information from the Nehring and Associates, Inc. (2009) is primarily because of reporting of production, mainly as commingled production from multiple units and age ranges. The discovery thirds for oil accumulations show close agreement for fields less than $8 \mathrm{MMBO}$, and for gas accumulations the limited data suggest that the second third of discoveries was more successful than the first and third thirds. 


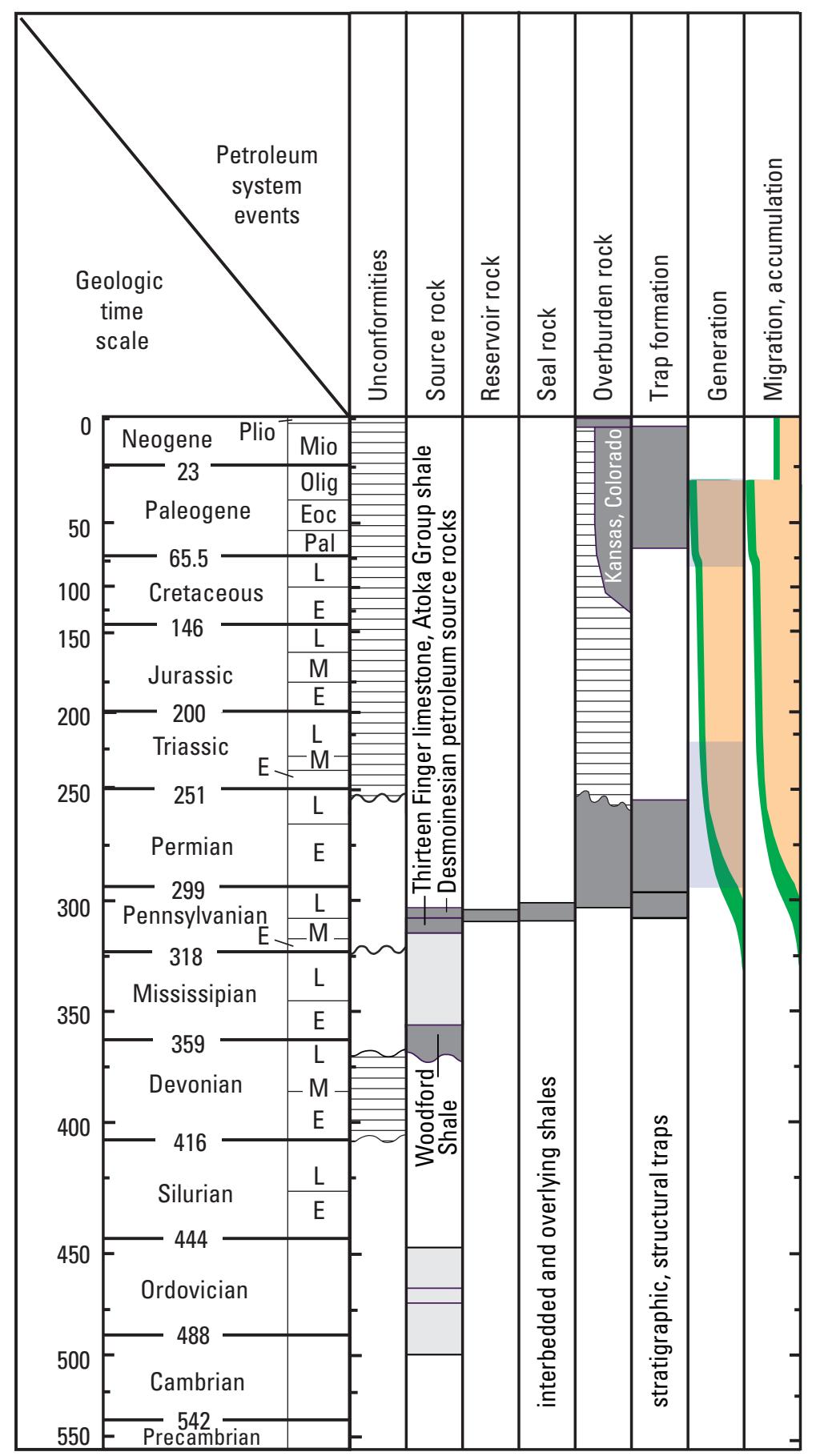

Figure 28. Petroleum system events chart for the Desmoinesian Assessment Unit (AU) of the Pennsylvanian Composite Total Petroleum system (TPS). Potential petroleum source rocks (fig. 7) from Hatch and others (1986) are light gray rectangles. The Thirteen Finger limestone (tan) range of oil and gas generation, migration, and accumulation represents Atokan and younger strata that are the primary petroleum source rocks; the 300-million years ago (Ma) onset of generation is based on the petroleum system one-dimensional and four-dimensional models. Potential oil and gas contributions from older source rocks is bracketed by the Woodford Shale (green) onset of generation of about $330 \mathrm{Ma}$. Time ranges of peak generation (fig. 14) are within blue rectangles. Paleogene trap formation, migration, and accumulation are based on Laramide generation and potential remigration associated with basin tilting. Unconformities, wavy and horizontal lines; Plio, Pliocene; Mio, Miocene; Eoc, Eocene; Pal, Paleocene; L, Late; M, Middle; E, Early. 


\section{Missourian-Permian Assessment Unit}

The Missourian-Permian AU shares a common boundary with the Pennsylvanian Composite TPS, but excludes the Greater Granite Wash Composite AU in the deep Anadarko Basin (fig. 1). The AU includes Missourian, Virgilian, Wolfcampian, and Leonardian carbonate and clastic reservoirs (fig. 2). Missourian and Virgilian strata were primarily deposited in transgressive-regressive cycles that periodically inundated the province with alternating thin-bedded predominantly marine sandstones, limestones, and shales; these can be separated into stratigraphic packages of mostly sandstone or mostly limestone reservoirs (Moore, 1979; Rascoe and Adler, 1983). Movement and erosion of the Wichita and Amarillo uplifts provided sediment for Missourian and Virgilian strata. Deformation and uplift of the Ouachitas, southeast of the Anadarko Basin, began during the Desmoinesian, and by late Missourian time several delta-front clastic wedges were deposited from streams that drained the Ouachitas and flowed into the eastern part of the Anadarko Basin (Johnson, 1989). Sandstone content increases from west to east in the Oklahoma portion of the basin, and the associated progression from marine to marginal marine indicates uplift with subsequent erosion of the Ouachita foldbelt was a sediment source (Rascoe and Adler, 1983). The Ouachitas continued to contribute clastics to the eastern basin during Virgilian time; a source of coarse detritus for the southeastern basin was provided by rapid rise of the Arbuckle Mountains uplift (Johnson, 1989).

The thickness of Missourian strata ranges from 500 to $1,000 \mathrm{ft}$ in most of the northern shelf and Hugoton embayment area, to more than $2,500 \mathrm{ft}$ at the depocenter in the southeastern Anadarko Basin (Johnson, 1989). The Missourian Series in southwest Kansas and the Oklahoma Panhandle are mostly marine limestones with some shale interbeds that grade to the south and southeast into shales and sandstones with minor carbonate beds; against the Wichita Mountain and Amarillo uplifts they become the thick arkosic and carbonate strata (Johnson, 1989) of the Greater Granite Wash Composite AU. Along the western margin of the Hugoton embayment, the Missourian Series consists of a clastic sequence of shales, siltstones, and sandstones, which are commonly red beds; Virgilian strata in the Hugoton embayment and proximal shelf area to the east are mainly marine limestones interbedded with shale (Johnson, 1989).

The Virgilian sequence ranges in thickness from about 500 to $1,500 \mathrm{ft}$ in the north and west, to more than $4,500 \mathrm{ft}$ in the southeastern corner of the province (Johnson, 1989). Virgilian and Wolfcampian marine carbonates and shales grade westward toward the Sierra Grande uplift into redbeds and sandstones that were sourced mainly from this uplift (Maher, 1953; Roth, 1955). An exception to the normal clastic/ carbonate sequences of the Missourian and Virgilian is the middle Virgilian Heebner Shale, which was deposited under deep marine conditions (Al-Shaieb and others, 1994). This laterally continuous interval provides a seal for underlying strata. Post-Heebner Virgilian sedimentation consisted of cyclic carbonates and shales on the Kansas shelf, and of cycles comprising carbonates and thick wedges of terrigenous deltaic strata on the eastern margin of the basin (Rascoe, 1978).

Lower Wolfcampian strata in-filled the sediment-starved Anadarko Basin at the end of the Pennsylvanian; estimated maximum water depth was about $700 \mathrm{ft}(213 \mathrm{~m})$ based on thickness of adjacent Virgilian deltaic wedges (Rascoe and Adler, 1983). The Wolfcampian Admire, Council Grove, and Chase Groups strata (fig. 2) cover most of the province, being absent over the Sierra Grande uplift and parts of the Amarillo, Central Kansas, and Wichita Mountain uplifts. The Chase Group in the basin averages about $200 \mathrm{ft}$ in thickness and consists of cyclical limestone, dolomitic limestone, dolomite, red shales, and siltstone lithofacies that become more shaly to the north and west (Ball and others, 1991). Modeled thickness of the Chase layer is about100 to $450 \mathrm{ft}$ thick in the Kansas portion of the basin, and thickens southward to about $800 \mathrm{ft}$ near the Amarillo and Wichita Mountain uplifts. The Council Grove model layer includes the underlying Admire Group; this interval also thickens southward in the basin to as much as $800 \mathrm{ft}$ in the Oklahoma and Texas portions of the basin. Unlike the Chase layer, which is thin in the Colorado portion of the model, the Council Grove and Admire interval has a northeasttrending increase in thickness that corresponds to the eastern flank of the Las Animas arch.

Owen (1975) ascribed evaporite deposition at the end of Wolfcampian time as resulting from landlocked seas. Continued basin subsidence in Guadalupian time resulted in deposition of as much as $1,500 \mathrm{ft}$ of red beds and evaporites in the deep basin; the Wichita Mountain uplift subsided at a somewhat slower rate and all but the highest parts were probably buried by fine clastics (Johnson, 1989). Thicknesses of 100-200 ft of Guadalupian Blaine Formation dolomite, anhydrite, and shale were deposited over almost the entire province during a marine transgression (Johnson, 1989). Leonardian and Guadalupian evaporites of the Wellington and Blaine Formations form important seals for underlying reservoir beds.

Figure 29 shows elevation on the top of the Wolfcampian Council Grove and Chase model layers. Data in the Palo Duro Basin area, south of the Panhandle field (figs. 1 and 29) were insufficient to model this surface. Shown are the basin axis proximal to the Wichita Mountain and eastern Amarillo uplifts, and the north-northwest trend of the flanks of the basin that are important to hydrocarbon migration. Also visible are the structural high of the Amarillo uplift in the Panhandle field area, a slight structural low between the Panhandle and Hugoton-Guymon fields, and the east-southeast tilt of strata in the Hugoton-Guymon fields.

Figure 30 is a tilted view of the present-day top of the Virgilian Douglas model layer with oil and gas migration flow paths. The process and methodology of modeled oil and gas generation, migration, and accumulation is explained in . Petroleum flow paths resemble hydrologic tributaries, with the exception that the flow is generally from deeper to shallower. Drilling depths to the top of the Douglas Group range from about $3,800 \mathrm{ft}$ in the northeastern part of the province 


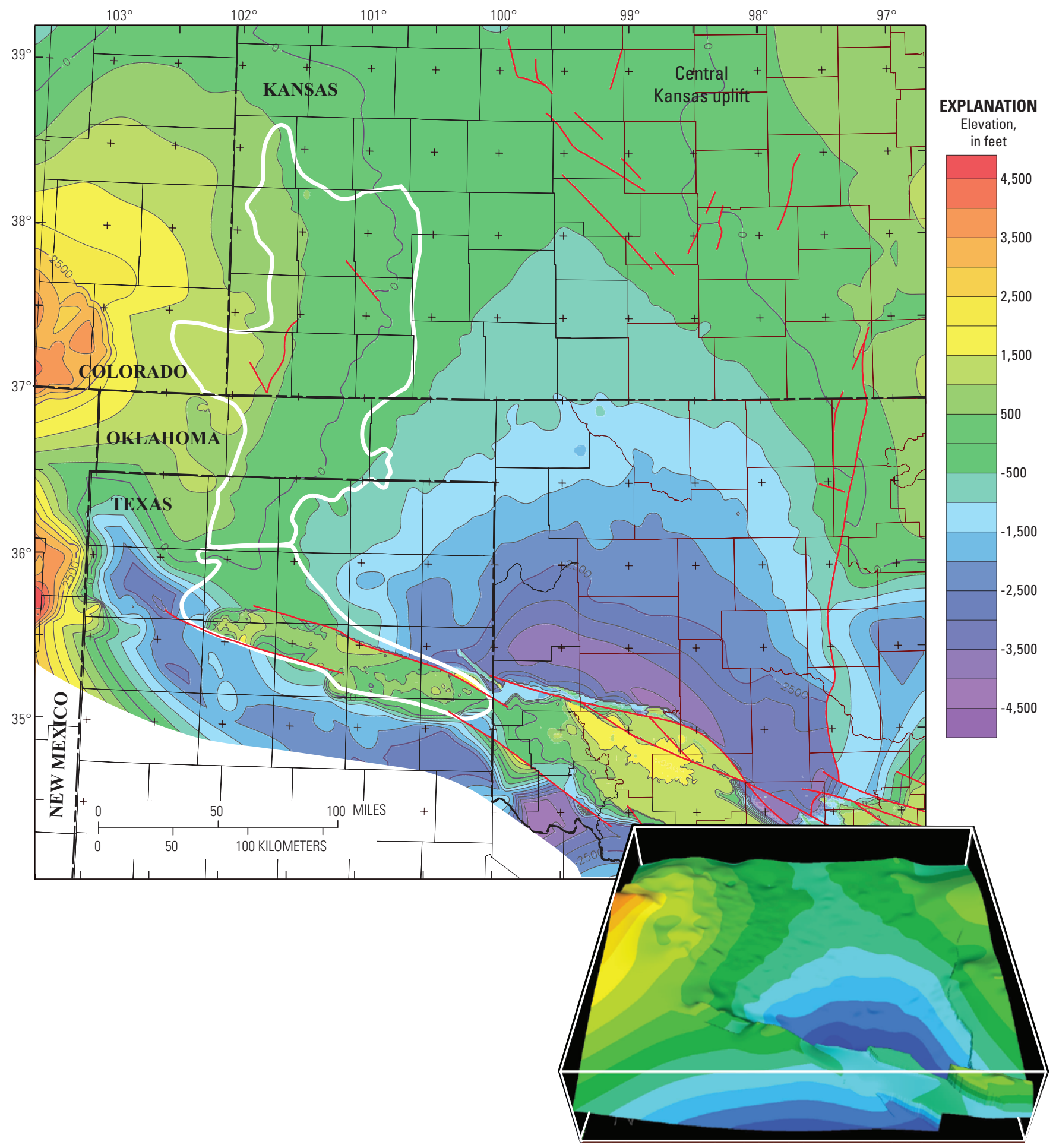

Figure 29. Map showing elevation on the top of the Council Grove model layer for the Anadarko Basin province. Inset image on the top of the overlying Chase layer shows structures on this north-tilted view at 20 times vertical exaggeration. The primary basin axis is proximal to the Wichita Mountain uplift (fig. 24) in Oklahoma and Texas, the secondary axis trends northward along the west flank of the Central Kansas uplift (CKU), which is bounded approximately by displayed faults. White outlines the Panhandle (south) and Hugoton-Guymon (north) field areas (fig. 25). Precambrian faults (red) are from Adler and others (1971). 
to about $11,000 \mathrm{ft}$ in the southern basin (Henry and Hester, 1995). Modeling indicates that migration on the Douglas layer is mainly from the deep basin radially outward to the Panhandle-Guymon-Hugoton fields, CKU, Salina Basin, and Nemaha uplift (fig. 30). Hydrocarbon flow follows topography on a layer, which is quite similar to structure on the Council Grove and Chase Groups (fig. 29), across the western and eastern flanks of the basin and up the secondary basin axis to the CKU. Gas flow paths are visible along the western and northwestern boundaries and CKU and mainly represent increase of gas volume because of pressure release proximal to outcrops and at major structures, such as the Sierra Grande uplift and Las Animas arch. Petroleum generation began in the deep basin (fig. 8) but then flowed up the flanks of the basin and toward bounding structures.

Potential Pennsylvanian petroleum source rocks that underlie the Douglas layer in Kansas and most of the Oklahoma and Texas panhandle area are thermally immature for oil generation (fig. 8), so long-distance migration factors into these reservoirs. Lateral migration can exceed $200 \mathrm{~km}$ (124 mi), which is the distance between the extent of thermally mature source rocks for the Oil Creek layer and the northern limit of the 4D model. Maximum migration is probably considerably greater because migration pathways tend to follow curved paths through permeable carrier lithofacies and along structures. Also, the wealth of oil and gas resources for the CKU and the Cambridge arch to the north are in areas that are thermally immature for petroleum generation. Because most oil and gas is not trapped in the 4D model, the flow paths are useful for visualizing migration on structural surfaces. The V-shaped areas of low flow to the right and left of the CKU correspond to areas of fewer oil and gas wells in figure 30, but flow also appears to be mostly absent in most of the Panhandle field area. That oil and gas migration flow paths appear west of the Panhandle field indicates that hydrocarbons did flow through this field but did not accumulate in the model.

Missourian-Permian AU oil and gas production is from more than 28,900 leases in 1,820 fields (fig. 31) and totals about $846 \mathrm{BBO}, 50 \mathrm{TCFG}$, and $263 \mathrm{MMBW}$ (IHS Energy, 2010a, 2010b). Because the reservoirs are subject to the same data reporting problems as for other strata in the basin, these numbers should be considered approximate. The Nehring and Associates, Inc. (2009) database contains information on 66 of the oil fields and 93 of the gas fields. This AU also includes oil and gas production from the Palo Duro Basin, and oil production from the Las Animas arch of southeastern Colorado (figs. 5 and 31). The Palo Duro Basin is bounded on the north by the Amarillo uplift (fig. 5), south of the Panhandle field. Oil and gas production, aside from the giant PanhandleGuymon-Hugoton field complex, is somewhat scattered across the province, particularly in regard to the number of wells that have been drilled. Oil and gas wells are concentrated in the Panhandle field, which is part of the Greater Granite Wash Composite AU (fig. 31); as such, some of the following discussion also applies to that AU. Within the MissourianPermian AU, oil wells are concentrated in northeast Texas, the
Cement, Watonga-Chickasha trend, and Sooner trend fields, and Nemaha uplift of Oklahoma, and near the CKU in Kansas. Gas wells are similarly distributed with the exception of the vast Panhandle-Guymon-Hugoton gas field complex (fig. 31), the largest in North America at 8,500 $\mathrm{mi}^{2}$; through 1985 it produced more than $48 \mathrm{TCFG}$, and accounted for about 58 percent of the gas and 26 percent of the oil production in the basin (Davis and Northcutt, 1989). This field complex will be referred to as the Panhandle-Guymon-Hugoton field, even though it includes other fields (fig. 31).

The Panhandle West field in the Panhandle-GuymonHugoton field was discovered in 1918, with production from Desmoinesian strata informally named "Big lime" (Mason, 1968) of the Marmaton Group (figs. 2 and 32). Galloway and others (1983) determined that porosity and permeability for the Panhandle field averages 13 percent and $25 \mathrm{mD}$, at $2,850 \mathrm{ft}$ depth. About 94 percent of the gas but less than 2 percent of the oil production from the Panhandle-Guymon-Hugoton fields is from the Missourian-Permian AU, almost all of it from the Permian, mainly Wolfcampian Council Grove and Chase Groups; most of the oil production is from granite wash in the Panhandle field, with other units producing minor amounts (IHS Energy, 2010a, 2010b). Hugoton-Guymon field production through September 2009 totaled about 1.4 BBO, 51.8 TCFG, and 864 MMBW for all reported Mississippian through Permian units (IHS Energy 2010a, 2010b).

Figure 33 shows estimated ultimate recovery (EUR) values for 1,024 wells across the Hugoton-Guymon portion of the field area. There is considerable variation in EURs, ranging mainly from about 400 to 7,000 MMCFG, and there are no clear trends in production. The EUR discovery thirds (fig. 34) show greatest production for the first third of discovered wells, followed by a progressive decline in EURs for the second and third of discovered wells. This is a complex set of reservoirs, and research by Dubois and others (2006) suggests that additional reserves may result from bypassed pay and by reservoir compartmentalization. Complex stacking, cyclicity, and lateral continuity of Council Grove and Chase Groups lithofacies are shown in figure 35. Average reservoir characteristics of the Chase Group in the field complex, north of about $35.5^{\circ}$ latitude, include about 5-mD permeability, 14 percent porosity, 25 percent water saturation, and 45 net feet of productive rock (Mason, 1968). Hugoton field initial reservoir pressure was 485 pounds per square inch (psia) in about 1928 and pressure declined to 360 psia by 1958 and 260 psia by 1968; average formation temperature and depth are $90{ }^{\circ} \mathrm{F}$ and $2,700 \mathrm{ft}$ (Mason, 1968).

Discovery thirds of grown oil accumulations for the Missourian-Permian AU (fig. 36) do not decline much through time, aside from effects on the curves of early fields from which $20 \mathrm{MMBO}$ and more was produced. The discovery thirds of gas fields do exhibit decline through time, particularly for larger fields.

Reservoir seals include interbedded shale lithofacies (Morse, 1963; Galloway and others, 1977). Missourian and Virgilian reservoir seals are mainly interbedded and overlying 


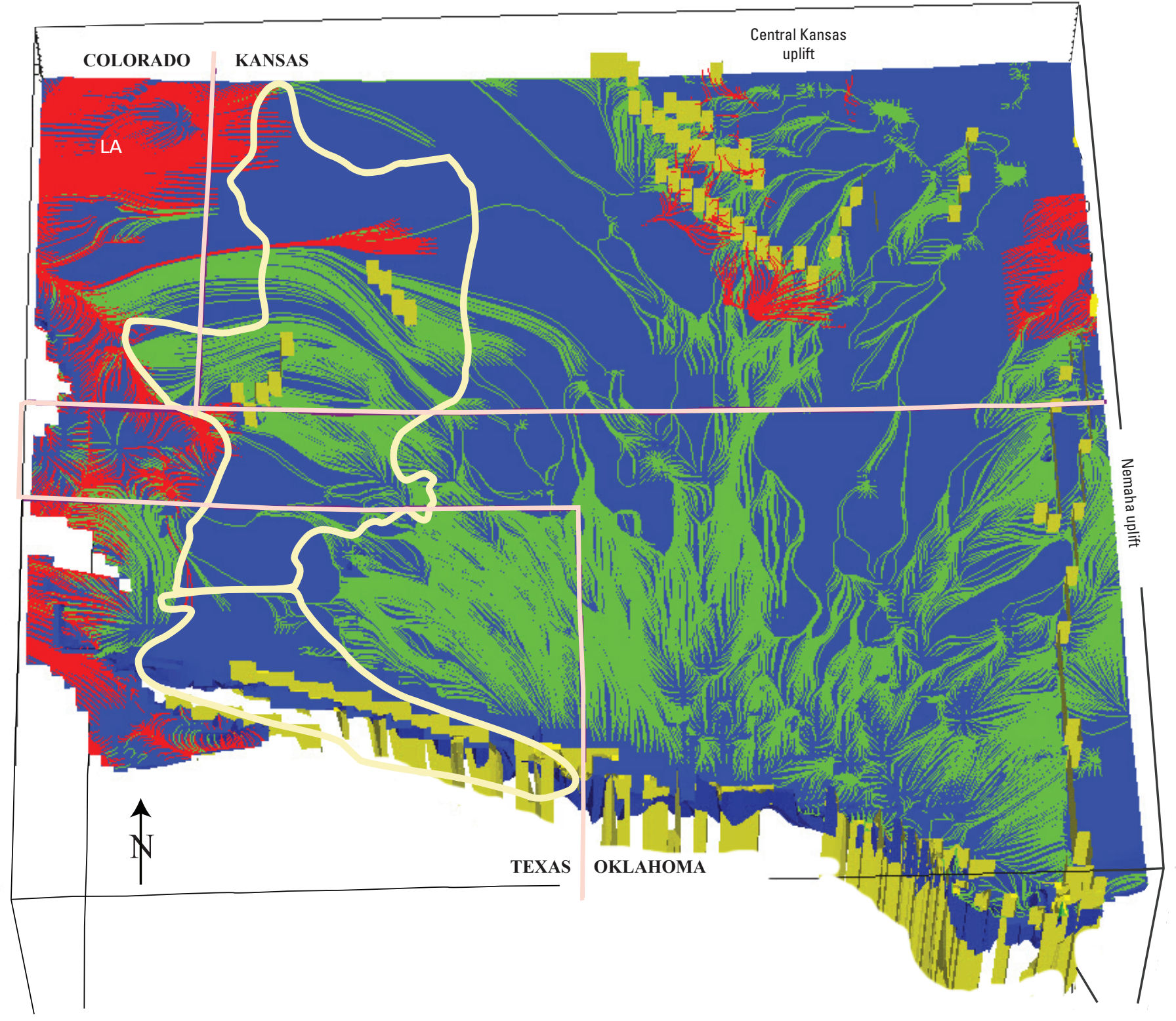

Figure 30. Oil (green) and gas (red) migration flow paths on the Douglas and Permian layers in the four-dimensional petroleum system model at present day. The image is tilted to the north and is at 15 times exaggeration to better show flow paths. Yellow outline is for the generalized locations of the Hugoton and Panhandle fields (fig. 25). In general, flow follows topography on the model layers. Petroleum is generated mostly in the deep basin and flows radially northward. Flow in northern Oklahoma and in Kansas is directed toward the Las Animas arch (LA), Central Kansas uplift (CKU), and Nemaha uplift. Gas flow paths at the western and eastern boundaries of the map and the CKU resulted from decrease in pressure-volume-temperature (PVT) conditions. Greenish-gold vertical bands are Precambrian faults (Adler and others, 1971). These are the only modeled Missourian-Permian Assessment Unit layers that have flow paths, mainly because (1) Heebner layer shale above the Douglas layer provided enough of a seal to prevent upward leakage, (2) the generalized lithologic assignments of other layers, and (3) coarse grid spacing resulted in most generated petroleum migrating out of the model. 
A

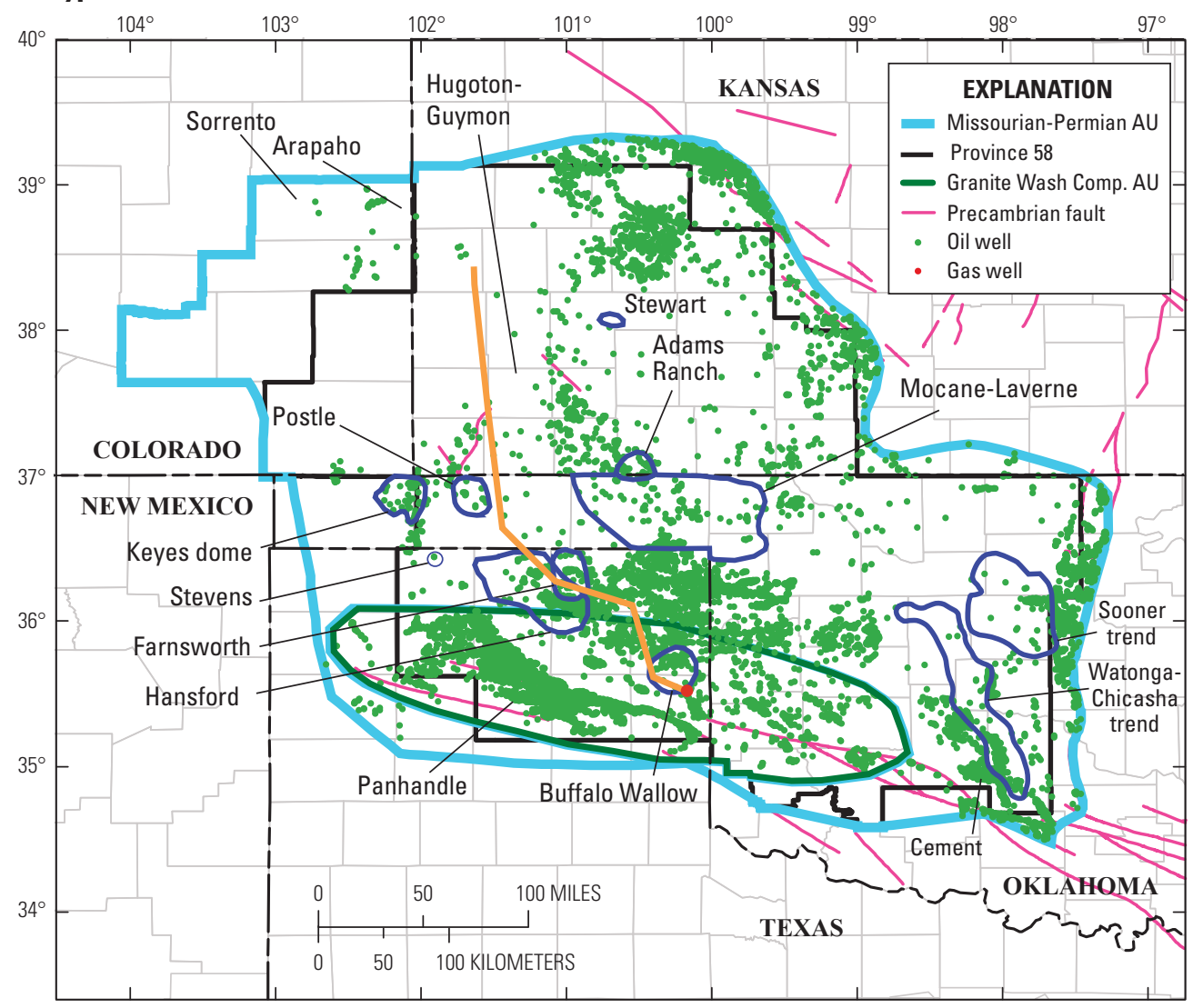

B

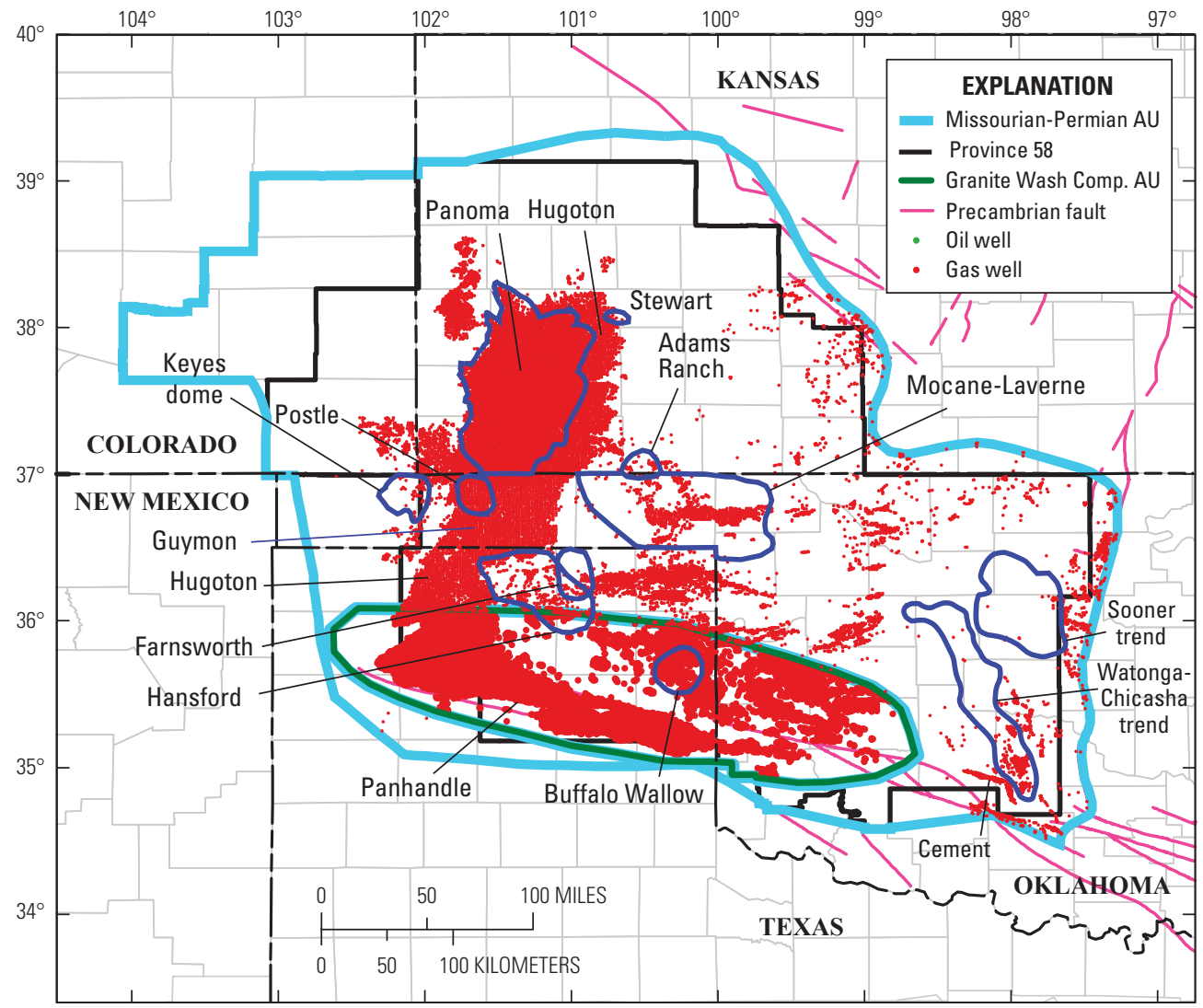

Figure 31. Maps showing $A$, Oil and $B$, gas wells that produce from the Missourian-Permian Assessment Unit (AU) in the Anadarko Basin Province (IHS Energy, 2010a, 2010b). Also shown is the common boundary with the Greater Granite Wash Composite AU boundary and included wells. Generalized field locations are from IHS Energy (2010a) and Bebout and others (1993). Largest gas field is Hugoton-Guymon, which extends northward from the Panhandle field. Gold-colored line on $A$ is the southeast to northwest cross section and (red dot) burial history plot location for figure 39. Precambrian faults (pink) are from Adler and others (1971). 


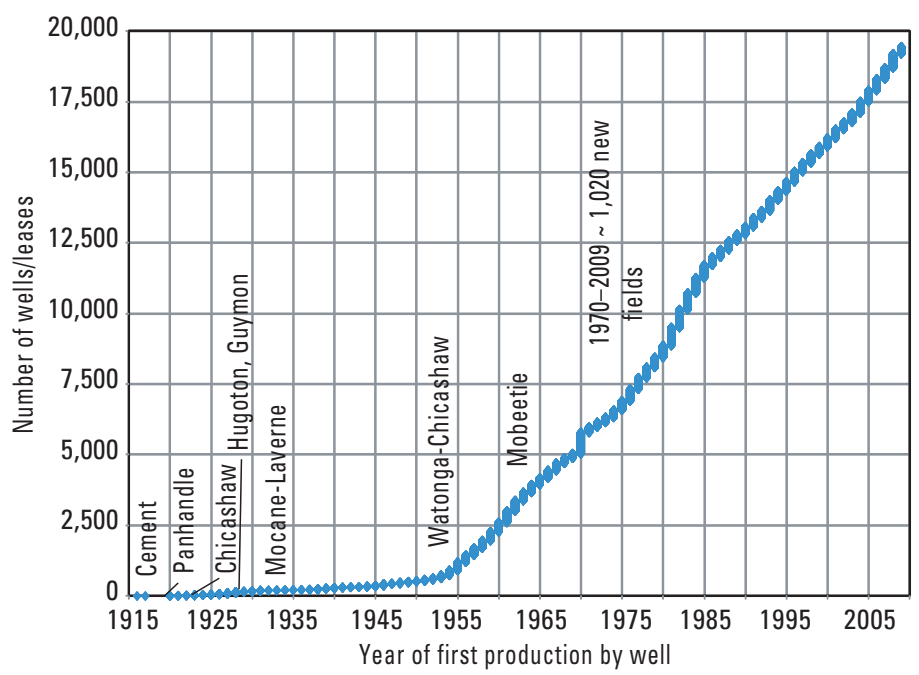

Figure 32. Graph showing production history of the Missourian-Permian Assessment Unit (AU) in the Anadarko Basin Province with some of the major field discovery names and dates (IHS Energy, 2010a, 2010b; Oklahoma Geological Survey, 2011a, 2011b). Exploration and development was relatively slow until discovery of the Watonga-Chickasha field. Development after about 1950 shows the same general trends as those from other AUs.

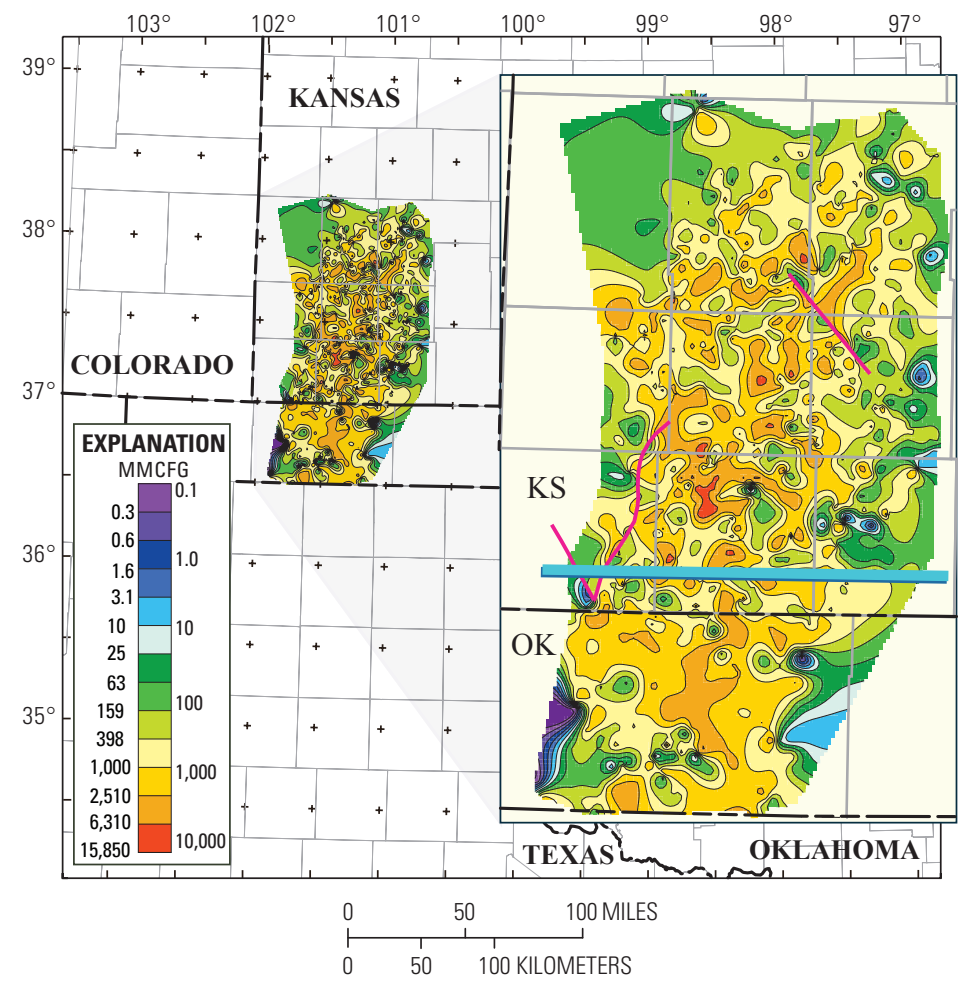

Figure 33. Map showing generalized contours of estimated ultimate gas recovery (EUR) in million cubic feet of gas (MMCFG) from 1,024 leases in the Hugoton and Guymon fields (data from Troy Cook, written commun., 2010). Leases represent single-well production of gas from the Chase and (or) Council Grove Groups. Contours are based on $\log 10$ EUR values to better show variability across the area. Horizontal blue line is generalized location of cross sections shown in figure 35 . Pink lines are Precambrian faults from Adler and others (1971). 


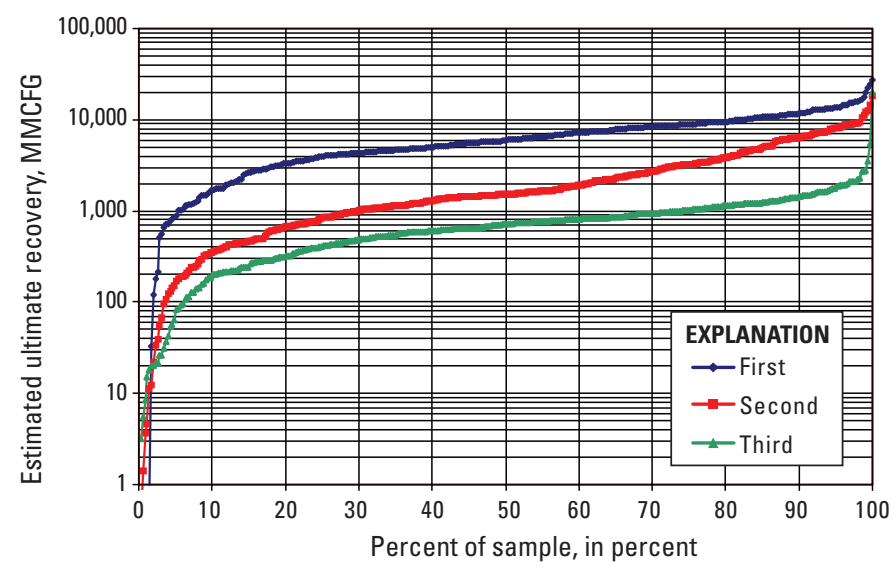

Figure 34. Graph showing discovery thirds of estimated ultimate recovery of gas (EUR) in million cubic feet of gas (MMCFG) for 1,024 wells in the Hugoton and Guymon fields that produce gas from the Chase and (or) Council Grove Groups (T. Cook, written commun., 2010). Data represent leases that have one well per lease and are split into the first, second, and third thirds of onset of production to November 2009, based on data from IHS Energy (2010a, 2010b). The curves indicate that gas production has undergone a steady decline since onset of production. Decline rate is greatest for the more productive wells in the third of EUR.

shales. Seals in Permian reservoirs include overlying shaleevaporite beds in the Leonardian strata, and seals for the Hugoton gas field may include capillary-pressure effects of bounding water. Trap types in this AU are mainly structural and (or) stratigraphic. Oil and gas fields that produce from Missourian rocks have a wide distribution, and are in structural, stratigraphic, and combination traps (Rascoe and Adler, 1983). The Cement field in the deep basin is a faulted anticline that produces from Silurian through Permian strata. The primary trapping mechanism is structural in the Panhandle field but stratigraphic in the Hugoton field, with a hydrodynamic component to both (Owen, 1975). Pippin (1970, p. 211) created a structure map on the granite underlying the Panhandle field that suggested the Amarillo uplift was a great horst block circumscribed almost completely by faults. The Hugoton field is an east-dipping homocline with the western edge being a somewhat steeply dipping monocline. Mason (1968) indicated that Chase and Council Grove Groups strata have a common gas-water contact in the Hugoton field, and that production in the west is limited by updip water and by a permeability decrease in the Chase Group, and on the east by an eastwarddipping gas-water interface.

Absence of an updip seal in the 4D model is indicated by flow of hydrocarbons westward through the Hugoton and Guymon fields (fig. 37); appearance of gas flow paths is largely the result of a decrease in pressure with shallower burial on the west and at the CKU. There were few accumulations with the 4D model, compared to overall production in the province, but production from this AU is generally focused along major Douglas layer flow paths. The overlying Heebner shale layer provided enough of a seal for the 4D petroleum system model to show migration of oil and gas on the underlying Douglas layer (fig. 30). The Heebner Shale is an exception to the normal clastic/carbonate sequences of the Missourian and Virgilian; its deep marine deposition was a period of uncommon Virgilian quiescence (Al-Shaieb and others, 1994). This shale generally is less than $100 \mathrm{ft}$ thick and can be mapped across most of the province; it thickens in the deep basin and proximal to the Nemaha and Arbuckle uplifts shown in figure 5. Rascoe and Adler (1983) indicated the shale is only about $3 \mathrm{ft}$ thick in the northern part of the basin, but increases to about $400 \mathrm{ft}$ in the deep basin.

Petroleum source rocks for the Missourian-Permian AU are probably Pennsylvanian shales and limestones. Rice and others (1988a, 1988b) indicated that Panhandle-Hugoton field nonassociated gases are mostly produced from Permian carbonates at depths less than $900 \mathrm{~m}(2,950 \mathrm{ft})$, and that Pennsylvanian and Permian gases display little compositional variation with a range of $\delta^{13} \mathrm{C}$ values from -46.4 to -39.9 percent (mean $\delta^{13} \mathrm{C}_{1}$ value is -43.2 percent, mean $\mathrm{C}_{2+}$ value is 14 percent). This isotopic composition is comparable to that of thermogenic gas from Atokan and Desmoinesian producing intervals in the central basin (Rice and others, 1989), which indicates Pennsylvanian sources of hydrocarbons. Shales in Virgilian-producing intervals have a mixture of Type II and III kerogen (Rice and others, 1989).

Permian oil and gas from the 4D model are mostly located in and west of the Panhandle through western Hugoton field areas (fig. 37); gas was sourced 56 percent from the Thirteen Finger limestone, 26 percent from the Oil Creek layer, and 18 percent from the Woodford layer. Because the 4D model has far fewer traps or seals than exist in the basin, modeled contributions from the Woodford layer source rocks may not be present with a more complex model. Model reasons for the Oil Creek contribution are that (1) Woodford Shale and Thirteen Finger limestone source rocks are thin to absent north of the Amarillo uplift area; (2) the Woodford through Desmoinesian isopach (fig. 5) is thin in the Texas Panhandle area; (3) Pennsylvanian strata in the Panhandle field area are underlain by up-tilted edges of lower Paleozoic rocks (Mason, 1968), which could allow for greater contributions from deeper source rocks; and (4) this leaky 4D model allows for more vertical and lateral migration than would a more complex model. The Panhandle field contains sweet and sour gases (Owen, 1975), which also suggests contributions from deeper sources than the mostly Type II and III kerogen of Pennsylvanian source rocks.

Measured $\mathrm{R}_{\mathrm{o}}$ values of the Upper Devonian-Lower Mississippian Woodford Formation are highest 30 to $40 \mathrm{~km}$ (20 to $40 \mathrm{mi}$ ) north-northwest of the deepest part of the Anadarko Basin, which is partly the result of the contrast in thermal conductivity between the high-conductivity granite wash strata adjacent to the Wichita uplift and the basinward low-conductivity shale section (Carter and others, 1998). The combination of decreasing heat flow toward the Wichita 

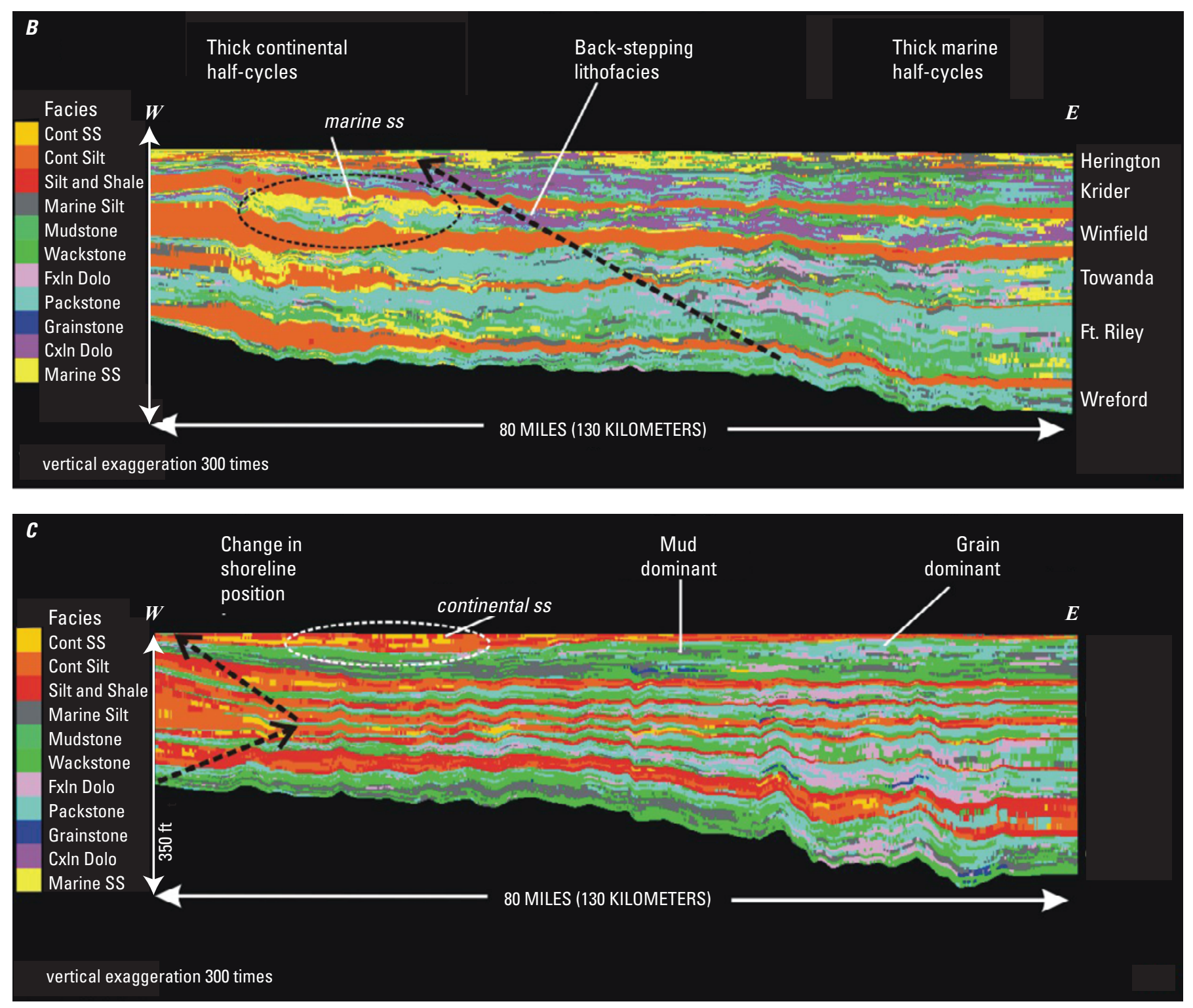

Figure 35. Lithofacies in west to east cross sections across the Hugoton field for the (B) Chase and (C) Council Grove Groups (modified from Dubois, 2007). These stratigraphic cross sections are hung on the top of the Chase (B) and the Council Grove (C), and location is in figure 33. Orange through red layers are continental (Cont) sandstone (SS), siltstone (Silt) and shale. Other colors are marine lithofacies, including fractured (FxIn) and crystalline (CxIn) dolomite (Dolo). Both cross sections show lateral continuity of cyclical beds of marine and continental strata. Marine strata increase in thickness to the east and continental beds thicken westward. Dubois (2007) indicated that large-scale sedimentation patterns and distribution of resultant lithofacies (at the cycle scale) are largely a function of the position on the shelf and reflect the interaction of shelf geometry, sea level, and, possibly, the proximity to siliclastic sources. Lithofacies distribution and cycle-stacking patterns at larger scales may be a function of lower-order cyclicity and a shift from icehouse to greenhouse conditions (upward) during the Lower Permian. 

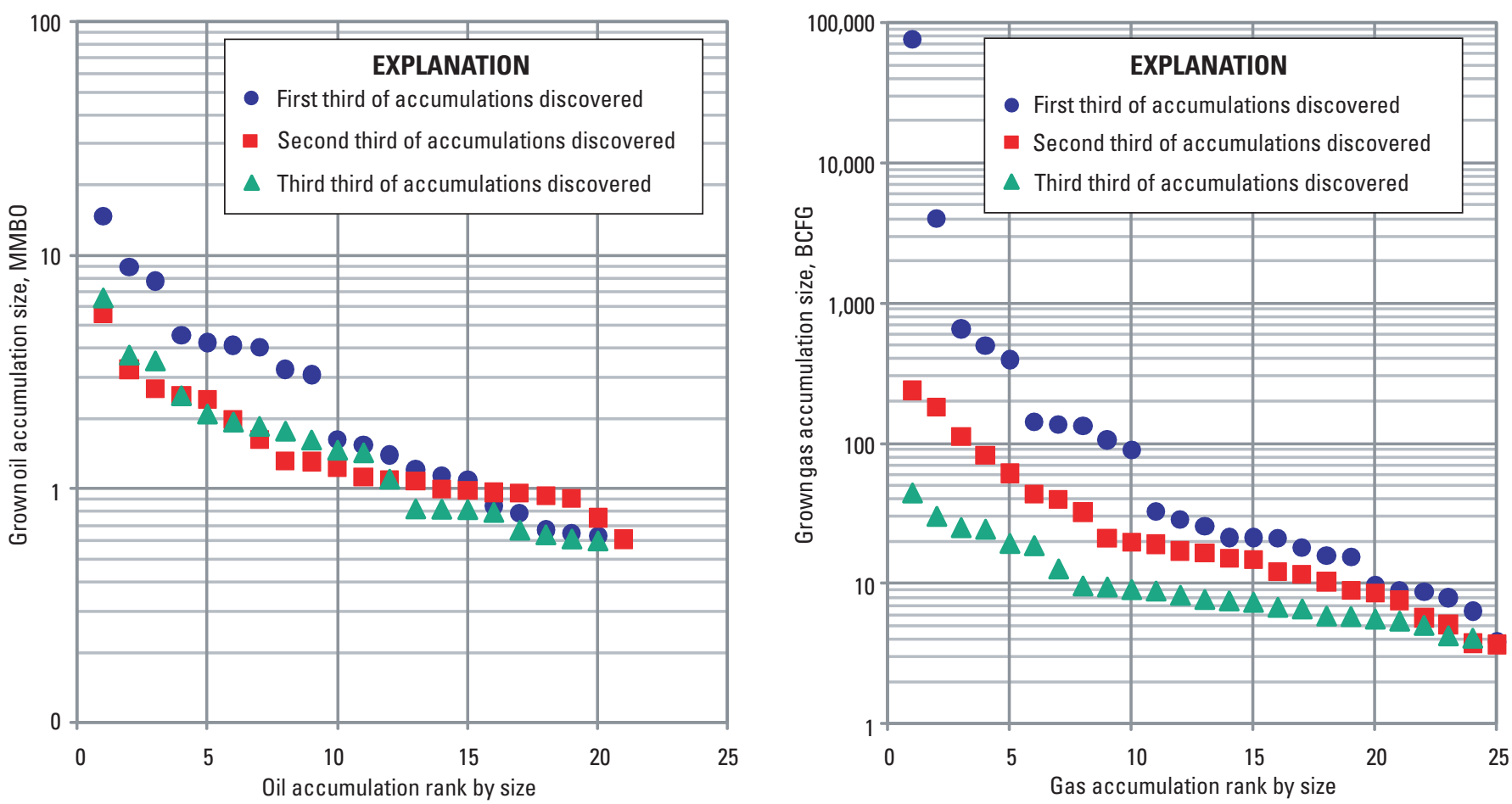

Figure 36. Graphs showing grown oil and gas accumulation sizes for discovery thirds of the Missourian-Permian Assessment Unit (AU) of the Pennsylvanian Composite Total Petroleum System. Only a fraction of the about 1,420 oil and gas fields that produce from this $\mathrm{AU}$ are shown, largely because many fields produce from multiple units with reported commingled volumes. Discovery trends are similar of grown oil accumulations less than about 20 million barrels of oil (MMBO). At greater than this volume, the first third of discoveries are larger than those of the second and third thirds. The early development history was marked by the giant Hugoton-Guymon and Panoma gas fields. In general, the gas accumulation chart shows a general decrease through time in field sizes. Billion cubic feet of gas, BCFG.

Mountains and the facies changes in the Pennsylvanian from marine shale to granite wash toward the uplift results in the highest formation temperatures being displaced about $50 \mathrm{~km}$ (31 mi) northward into the basin (Gallardo and Blackwell, 1999). Basement heat flow is greater basinward than it is close to the mountain front (fig. 38), which results in the pronounced thermal maturity "bump" in the figure 39 cross section from within the Greater Granite Wash Composite AU northward to Kansas. The associated burial history plot is in an area of low basement heat flow that contains granite wash arkosic strata. The Panhandle field is located on the Amarillo uplift, which exhibits low heat flow (fig. 38) and is thermally immature for hydrocarbon generation (fig. 40). The basin just north of the uplift deepens rapidly and thermal maturation of potential petroleum source rocks within $30 \mathrm{~km}(20 \mathrm{mi})$ ranges from mature for oil to gas generation. Some of the model layers are identified in figure 40 , but lateral continuity is variable mainly because of faulting and data quality of formation tops used to construct the layers.

The petroleum system events chart (fig. 41) shows the timing of hydrocarbon generation, migration, and accumulation based on petroleum system models (table 2) (Higley, 2014). Onset of oil generation from Pennsylvanian source rocks (fig. 7) approximates the 300-Ma beginning of oil generation from Thirteen Finger limestone petroleum source rocks based on 4D petroleum system modeling. This onset preceded deposition of reservoir rocks from this $\mathrm{AU}$; so the 270-Ma beginning of oil generation for this AU is based on the Higley (2012, table 3) modeled onset of generation from potential Virgilian source rocks. 


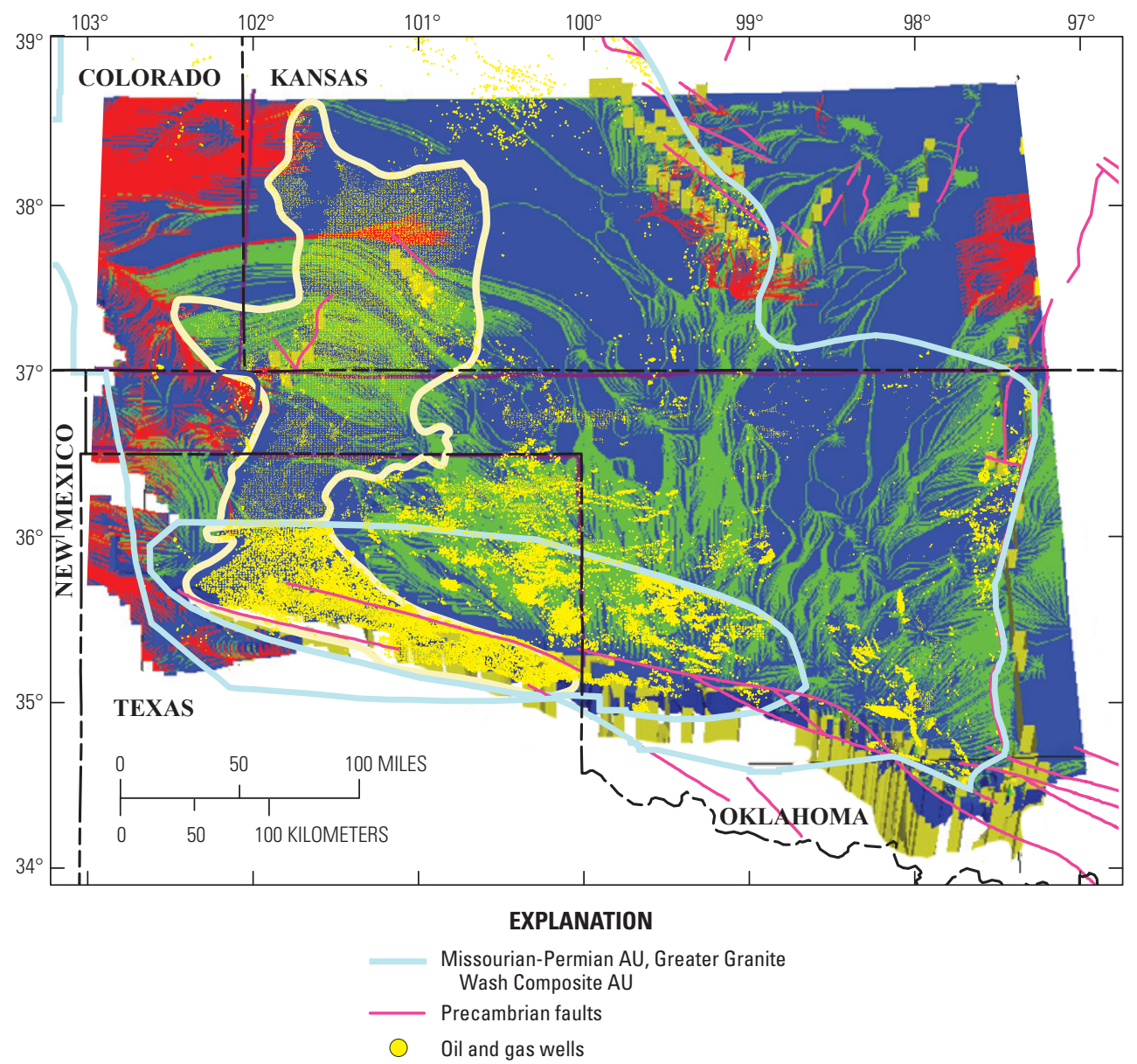

Figure 37. Model image showing oil and gas wells that produce from the MissourianPermian and Greater Granite Wash Composite assessment units (AU) in the Anadarko Basin Province (IHS Energy, 2010a, 2010b) are superimposed on the image of Douglas plus Permian layer flow paths (fig. 30). In general, areas with less flow have fewer wells. Yellow outline is the Panhandle-Guymon-Hugoton field area, drilling is somewhat faint within the gas fields because of the wider drill spacing than for oil production. Absence of flow paths within the Panhandle field, with their appearance on the west side of the field suggests (invisible) Darcy flow through the reservoir because all potential source rocks are thermally immature in the western Amarillo uplift (fig. 8) (Higley, 2014). Sorenson (2005) also indicated that petroleum migrated southward from the basin into structural traps of the Panhandle field. Precambrian faults are from Adler and others (1971). 


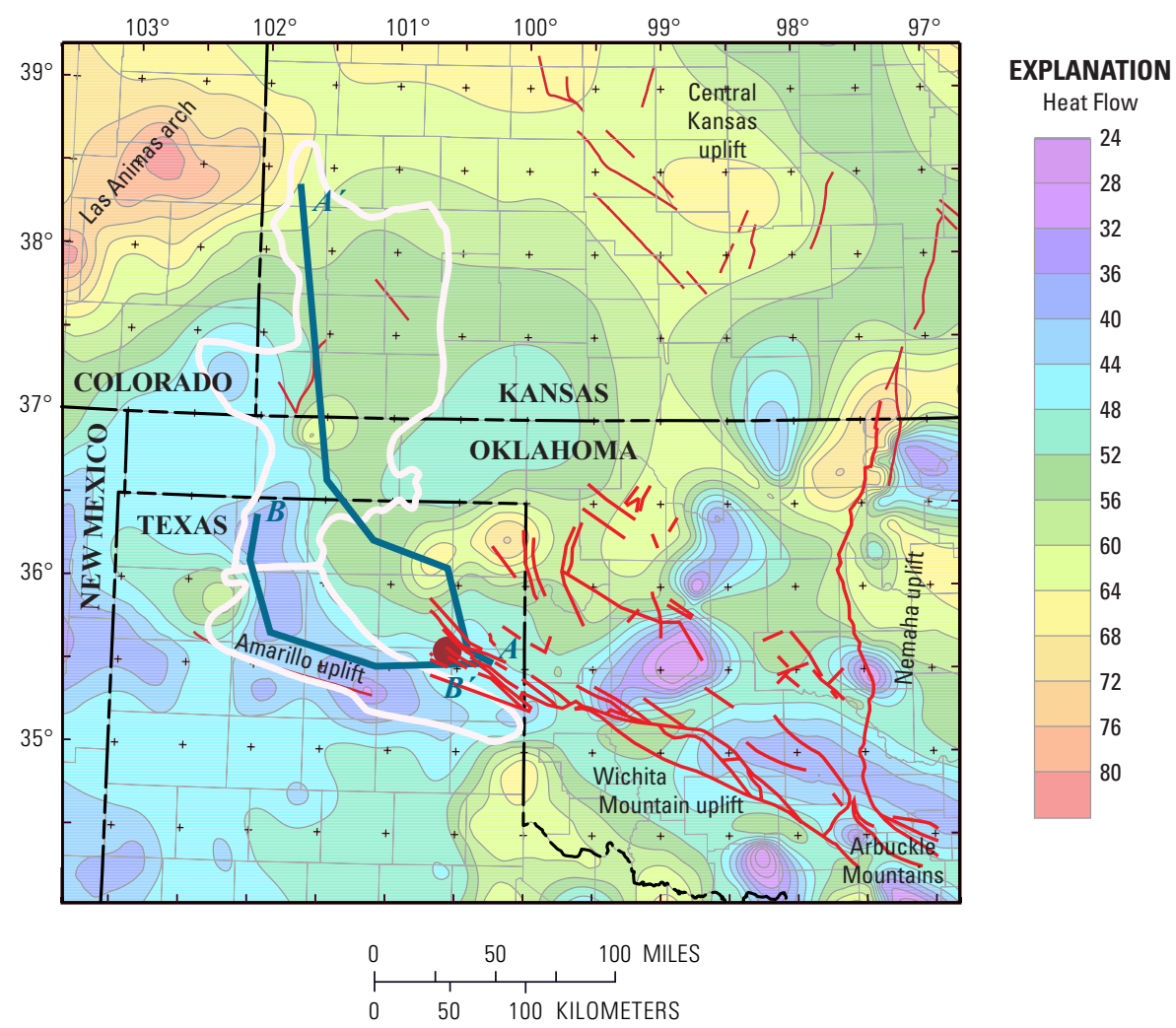

Figure 38. Map showing basement heat flow contours across the Anadarko Basin based on data from Carter and others (1998), Blackwell and Richards (2004), and data downloads from the Southern Methodist University Web site ( $h t t p: / / s m u . e d u$ / geothermal/. Shown are the cross sections (blue lines) in figures 39 and 40 , location of the one-dimensional burial history model (red dot), and Panhandle-Guymon-Hugoton field outlines (pink lines). Values are milliwatts per square meter $\left(\mathrm{mW} / \mathrm{m}^{2}\right)$. The $A-A^{\prime}$ section crosses several zones of elevated heat flow and $B-B^{\prime}$ is mainly in areas of decreased heat flow. Basin areas north of the Wichita Mountain uplift and in the Amarillo uplift and northward exhibit generally lower heat flows than other basin areas. Highest measured heat flow is in the northwest, along the Las Animas arch. The northwest-trending Central Kansas uplift also exhibits elevated heat flow values. Red lines are faults on the top of the Hunton Group from Rottmann (2000a) that were used to contour Hunton and Woodford layers, and Precambrian faults (modified from Adler and others, 1971). 

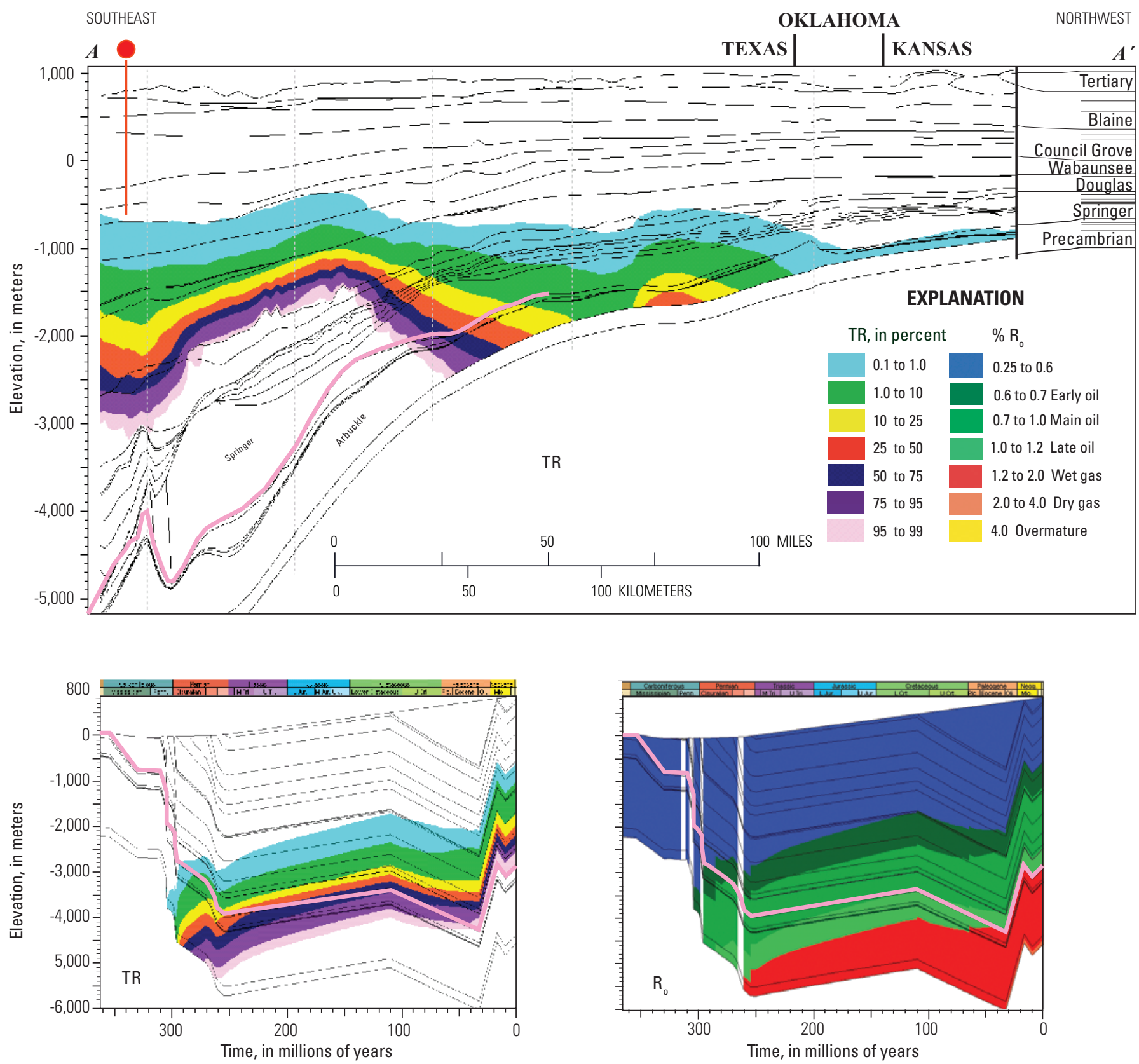

Figure 39. Southeast to northwest structural cross section with present-day transformation ratios (\% TR). The burial history plot of TR and vitrinite reflectance $\left(\% R_{0}\right)$ is near the red dot on the cross section, and both locations are in figure 38. Pink lines are the general location of the Woodford Shale layer. The two "bumps" of increased thermal maturation on the cross section correspond to areas of increased heat flow in figure 38. Lithologic variation has minor effect on these increases because of the generalized lithofacies assignments in the model. If basement heat flow was one value across the basin, then the TR contours would be approximately horizontal because of the strong influence of burial depth. The burial history plots show the Council Grove layer as marginally mature for oil generation at 0.1 percent $T R$ and $0.6 \% R_{0^{\prime}}$ and the Woodford Shale layer is overmature for oil generation based on 100 percent TR and more than $1.2 \% R_{0}$. The one-dimensional model is about 2 kilometers southwest of the Woodford Shale measurement of Cardott (1989, p. 45) for the 1 Miami Cattle well (1.56\% mean $R_{0}$ at $-3,563 \mathrm{~m}$ elevation). These images are extractions from the four-dimensional petroleum system model, and are displayed in elevation relative to sea level. 

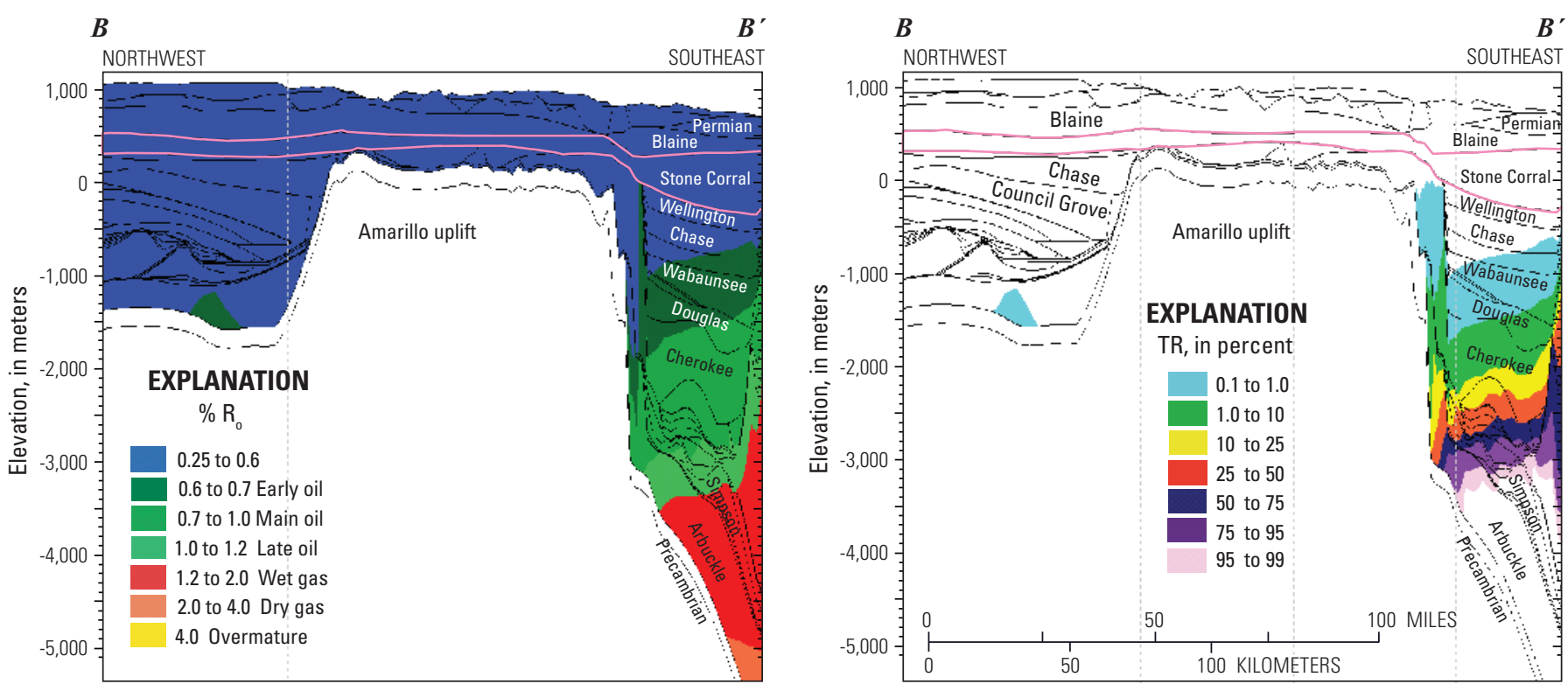

Figure 40. Cross section showing vitrinite reflectance $\left(\% R_{0}\right)$ and transformation ratio $(\% T R)$ is an extraction from the fourdimensional model across the Panhandle field. Location is in figure 38. The Panhandle field is located on the Amarillo uplift and oil and gas are produced from Wolfcampian and Leonardian strata. The Stone Corral model layer is bounded by pink lines and includes units from the top of the Leonardian Wellington to the base of the Guadalupian Blaine Formations (fig. 2). It is underlain by Wolfcampian strata. The Amarillo uplift and areas to the northwest are thermally immature for oil generation, and potential petroleum source rocks are absent because of erosion or nondeposition. Oil and gas migration flow paths appear west of the Panhandle field in figures 30 and 36 ; this indicates that hydrocarbons did flow through this field but were not trapped in this leaky model, and were most likely sourced from the east and northeast from Pennsylvanian and older source rocks and through open fault systems. Vertical exaggeration is 33 times. 


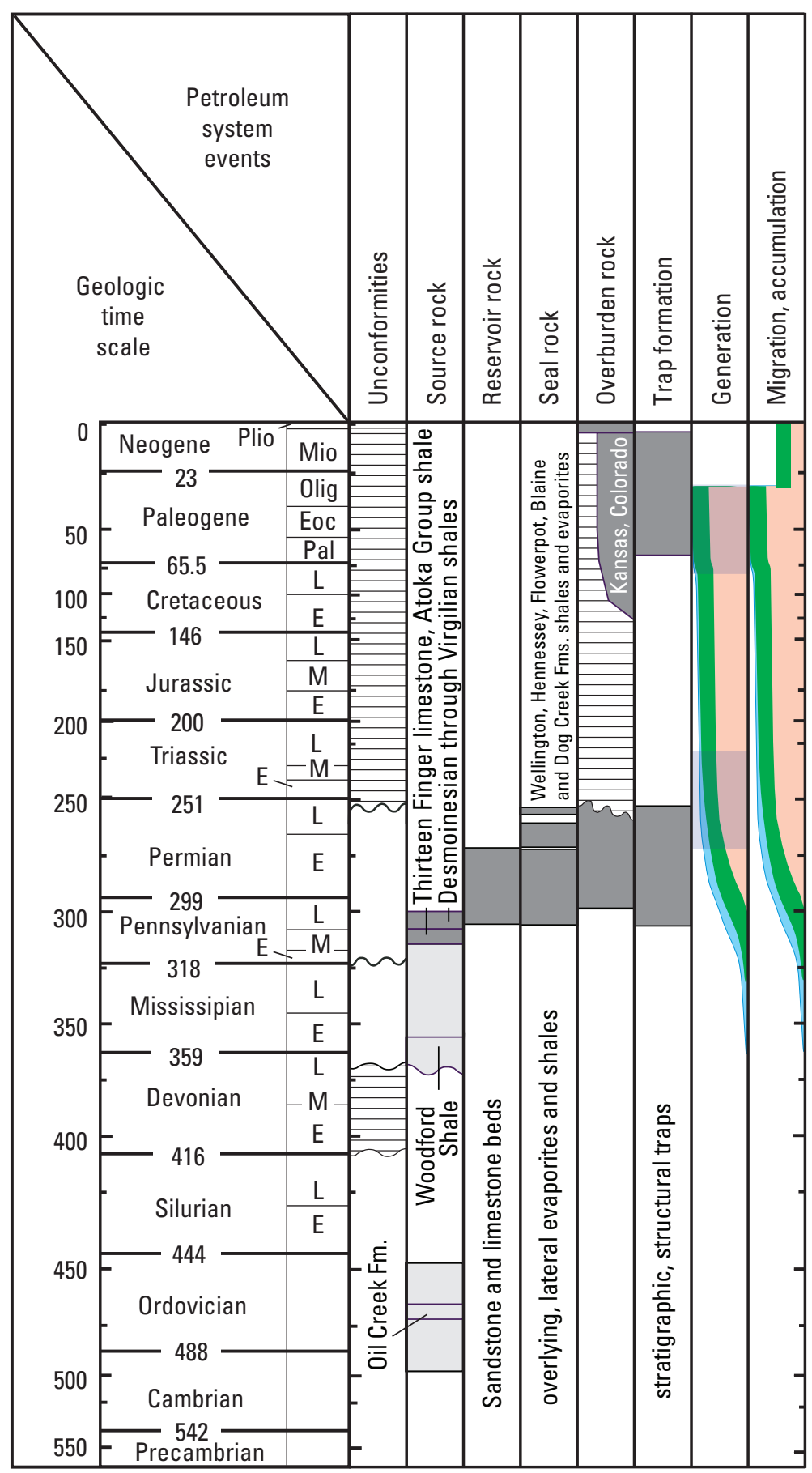

Figure 41. Petroleum system events chart for the Missourian-Permian AU of the Pennsylvanian Composite TPS. Primary hydrocarbon source rocks are dark gray rectangles, and age ranges of potential source rocks from Hatch and others (1986) (fig. 7) are light gray rectangles. Onset and peak petroleum generation (fig. 14) are shown for the Oil Creek (blue), Woodford (green), and Thirteen Finger limestone (tan) source rocks. Age ranges of peak hydrocarbon generation are indicated by blue rectangles. Paleogene trap formation, migration, and accumulation are based on Laramide generation and potential remigration associated with basin tilting. Unconformities, wavy and horizontal lines; Fm(s)., Formation(s); Plio, Pliocene; Mio, Miocene; Eoc, Eocene; Pal, Paleocene; L, Late; M, Middle; E, Early. 


\section{Greater Granite Wash Composite Assessment Unit}

The Greater Granite Wash Composite AU is defined as including Desmoinesian through Virgilian strata. The informally named granite wash strata consist mainly of arkosic shale to conglomerate. Every major stratigraphic unit from Morrow, and probably Springer, through Wolfcampian in the basin includes some granite wash facies near the mountains based on Dobervich and Parker (1958) and Edwards (1959).

The Wichita orogeny climaxed during the Atokan and slowed in Desmoinesian time; the orogeny was accompanied by uplift of tens of thousands of feet of the Wichita Mountain and Amarillo uplifts relative to the basin axis; associated erosion resulted in deposition of granite wash sediments in the subsiding basin north of the fault zones (Ball and others, 1991). Carbonate conglomerates were the first to form; these resulted from erosion of the thick sequence of early and middle Paleozoic carbonates that mantled the rising Wichita Mountain and Amarillo uplifts, subsequent deposition of conglomerates of arkosic lithofacies resulted from the exposure and erosion of granites and rhyolites in the core of the Wichita-Amarillo Mountains (Johnson, 1989; Gallardo and Blackwell, 1999). This heterogeneous mix of detritus in the granite wash became progressively finer northward of the source area.

The Anadarko Basin has been dormant since Early Permian time (Perry, 1989), as indicated by the relatively uniform thickness of Wolfcampian and Leonardian units across the basin, and that the Council Grove and Chase Groups, Hennessey Shale, and other strata can be correlated across the basin. Guadalupian and younger units exhibit more irregular distribution because of areas of nondeposition, and Laramide orogeny-associated uplift, tilting, and erosion but are present over much of the AU based on Adler and others (1971). The granite wash is generalized in figure 42 as the wedge-shaped

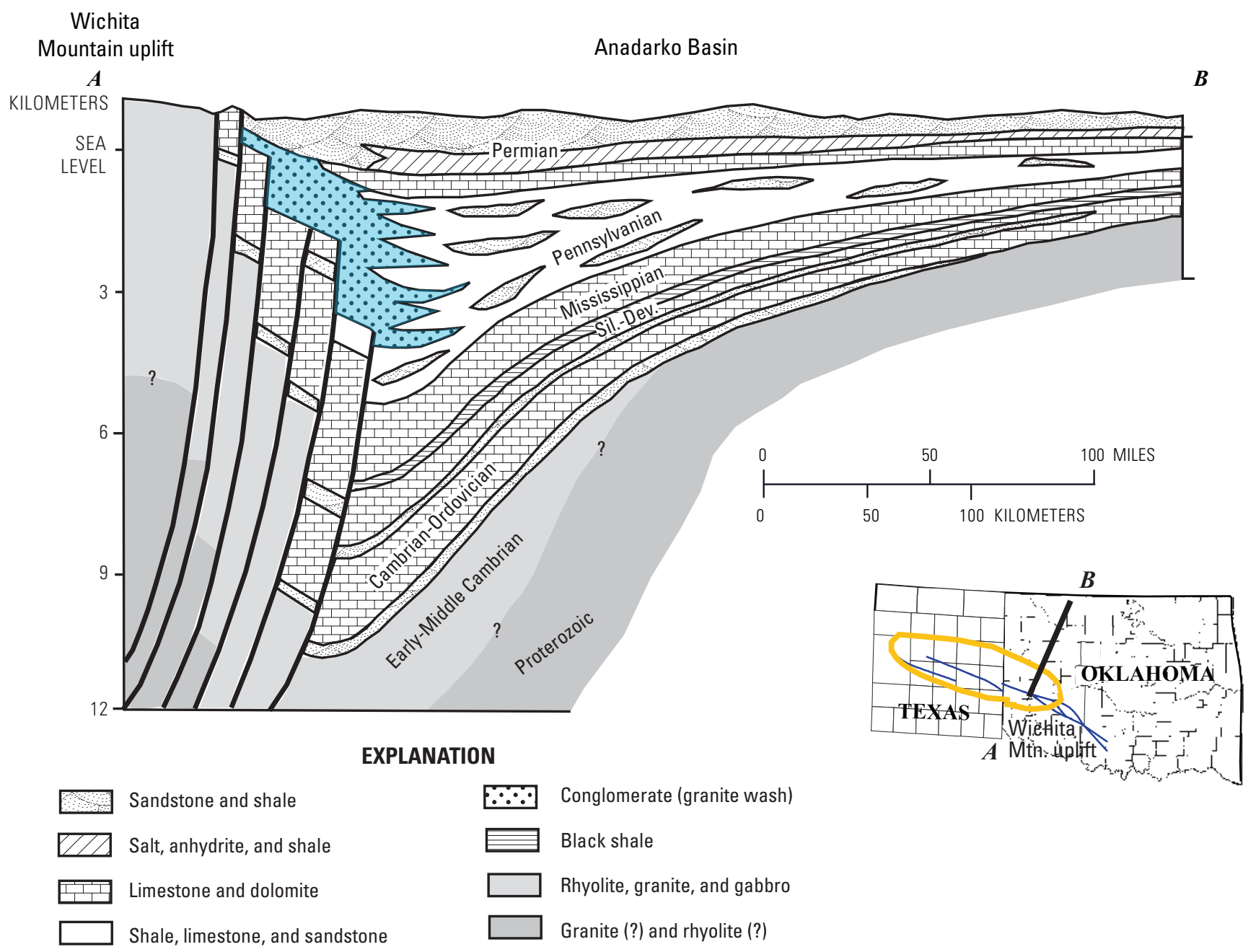

Figure 42. Simplified southwest to northeast structural cross section from the Oklahoma deep basin to the shelf shows interfingering of granite wash lithofacies (blue) with those basinward (modified from Johnson, 1989). Greater Granite Wash Composite Assessment Unit (AU) boundary is gold line and cross section location is red line on inset map. Datum is sea level. Sil.-Dev., Silurian-Devonian. 
granite wash interval; lithofacies grade basinward into mostly marine strata. The granite wash sequence is as much as $5,000 \mathrm{ft}$ thick and is located within about 30 miles north of the Wichita Mountain and Amarillo uplifts (Owen, 1975; Johnson, 1989). The extent of granite wash in Wolfcampian was less than 10 miles from the mountain front (Ball and others, 1991).

The greatest thickness of included strata in the AU is along the axis of the deep basin, with progressive northward and westward thinning. Thickness of the Morrowan through Wolfcampian section varies from 0 to more than $12,000 \mathrm{ft}$, and the Atoka Group to Stone Corral Formation interval has a similar thickness and distribution (fig. 43). Morrowan and Atokan strata, however, are absent over most of the Amarillo and Wichita Mountain uplifts. Determination of formation contacts from well logs south of the basin axis is complicated by interbedding of granite wash and marine lithofacies, and steeper dips proximal to the Wichita Mountain and Amarillo uplifts. Thicknesses in the figure 43 isopach map are not corrected to true stratigraphic thickness. More detailed descriptions and thickness maps of the various granite wash intervals are located in Mitchell (2011).

The Greater Granite Wash Composite AU has almost 20,000 wells, with cumulative production of about 1.6 BBO, 43.3 TCFG, and 1.1 BBW; about 11,000 of the wells are productive of oil and 19,000 have associated or nonassociated gas (IHS Energy, 2010a) (fig. 44). These totals include more than granite wash wells and production, mainly because the AU boundary includes strata that are not granite wash. This is because of the variable lateral extent and interfingering of these strata with other lithofacies, and some granite wash production is reported commingled with other units. Granite wash is listed as a producing interval from about 6,900 wells in the AU with cumulative production of about 1.44 BBO, 4.32 TCFG, and 1.03 BBW (IHS Energy, 2010b) (fig. 45). The Oklahoma Geological Survey (OGS, 2011b) Web site also includes information on granite wash and other plays, and reported granite wash production to 2008 as more than $61 \mathrm{MMBO}$ and $1.88 \mathrm{TCFG}$ from more than 4,200 wells. The apparent discrepancy between the IHS Energy and OGS numbers is partly the different reporting dates, but mainly because of the number and distribution of wells that were assigned to granite wash production (fig. 45). The OGS (2011b) included some wells just southeast of the TPS, and had fewer wells in the northwest Panhandle field area. Almost all wells in the western Panhandle field have completion dates after 2008, so the difference is likely how the OGS (2011b) evaluated the producing formations and associated production, compared to the reporting of IHS Energy (2011a, 2011b). Mitchell (2011) indicated that an average EUR from a vertical granite wash well is 1.5 BCFG and 21,000 BO. Many of the IHS Energy (2010a, 2010b) granite wash wells in the Panhandle field area correspond to oil wells in figure 44. Much of the Panhandle field gas production is identified as the informally named Wolfcampian Brown dolomite of the Chase Group and the Leonardian Red Cave of the Sumner Group, which are shallower than granite wash strata. The first Permian production

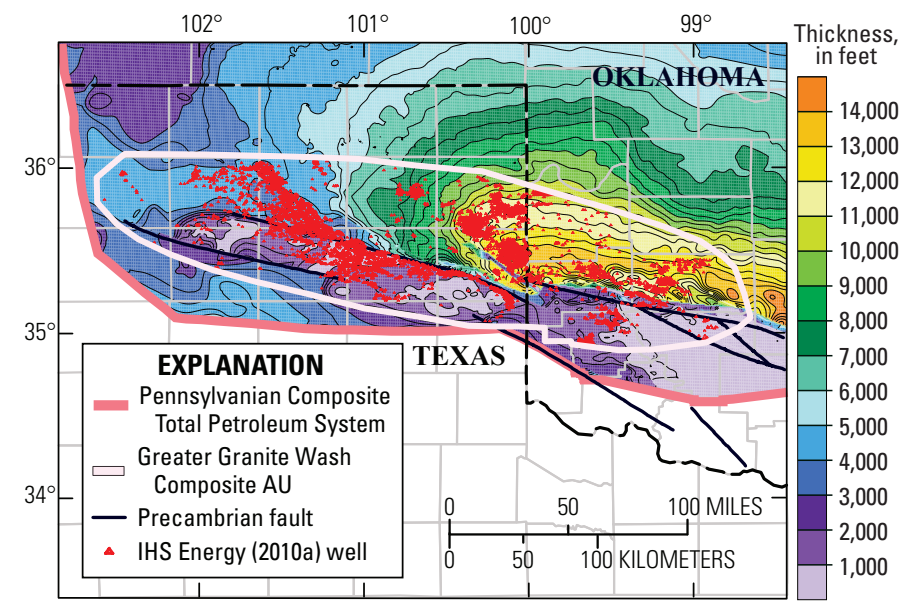

Figure 43. Map showing thickness of the top of the Atoka to the Stone Corral model layers in the Greater Granite Wash Composite Assessment Unit (AU) ranges from about 0 to more than 14,000 feet ( $\mathrm{ft}$ ). Areas of smooth contours have more limited data, such as the Wichita Mountain uplift and Panhandle field areas; actual thicknesses here are generally less than 1,000 ft (300 meters). Displayed wells produce oil and (or) gas from granite wash facies. Contour lines are 500-feet intervals. Precambrian faults (black) are from Adler and others (1971). Light gray lines are counties.

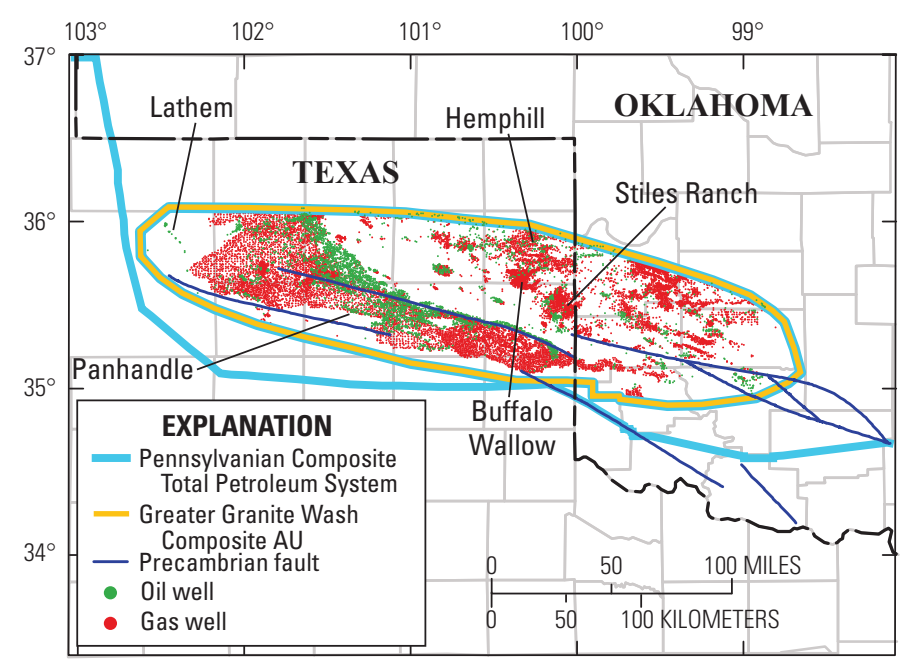

Figure 44. Map showing oil and gas wells that produce from the Greater Granite Wash Composite Assessment Unit (AU) in the Pennsylvanian Composite Total Petroleum System (TPS), Anadarko Basin Province (IHS Energy, 2010a, 2010b). Smaller scale views of oil and of gas wells are in the Desmoinesian and Missourian-Permian oil and gas figures 24 and 31 . Generalized field locations are from IHS Energy (2010a), Nehring and Associates, Inc. (2009), and Bebout and others (1993). The largest field is Panhandle, which covers most of the Amarillo uplift and produces from granite wash and other lithofacies. Precambrian faults (blue) are from Adler and others (1971). Light gray lines are counties. 
in the Greater Granite Wash Composite AU was from Red Cave interbedded redbeds and carbonates of the Panhandle field in 1919.

Cotner and Crum (1933) listed the range in GOR from 500 to $1,000,000 \mathrm{CFG} / \mathrm{BO}$ for the Panhandle field, which reflects that the Greater Granite Wash Composite AU includes oil and gas plays. Estimated gas/oil ratio (GOR) is 30,820 for the OGS (2011b) data. OGS (2011b) listed cumulative production of 99,400 BO, 1.0 BCFG, and more than 440,000 BW for a typical granite wash well. Smith and others (2001) indicated the average granite wash production for their 1,985 wells was $24 \mathrm{MBO}$ and $1.15 \mathrm{BCFG}$; associated mean GOR is 48,000 CFG/BO.

Discovery halves for oil and thirds for gas accumulations are shown in figure 46. Discovery halves data for oil fields are too limited to form conclusions. The first and second thirds of gas accumulations have been stable through time, and a decrease in discovery thirds more than 20 BCFG for gas accumulations may reflect the shorter production time for these fields, as well as the largest accumulations generally being discovered early in exploration. The curves were not skewed by the Panhandle field, which is not included on these charts. Exploration histories of Greater Granite Wash Composite AU leases and of wells within the granite wash (fig. 47) follow similar trends with those of other AUs in the province, with the exception of later onset of drilling.

Drilling depths in the AU range from less than $100 \mathrm{ft}$ to an estimated maximum of about $15,000 \mathrm{ft}$; petroleum production is as shallow as $300 \mathrm{ft}$, but most major reservoirs produce from depths of 4,700 to 16,500 feet based on Henry and Hester (1995) and Mitchell (2011). Permeability in the Pennsylvanian granite wash is variable, ranging from close to 0 to more than $300 \mathrm{mD}$, with about 20 percent average porosity (Mason, 1968) for these conglomeratic to arkosic strata. Published API gravity values of oil in the Panhandle field area range from 34 to $37^{\circ}$ (Owen, 1975). Mitchell (2011) listed API gravities of 45-60 for oil and condensate, and natural gas liquid yields are as high as 190 barrels per million cubic feet of gas (B/MMCFG); Virgilian and Missourian granite wash reservoirs are shallower and more oil-prone with API gravities of $45-50^{\circ}$.

Traps are structural and (or) stratigraphic. The northern fault of the Amarillo uplift brackets some oil wells, and wells north of the fault systems show a north-northwest trend that is oblique to Precambrian faults but fit well over Hunton fault systems of Rottman (2000a, 2000b) that are shown in figure 38. Panhandle field is located on the Amarillo uplift and is bracketed on the north and south by bounding faults (figs. 38 and 40). Seals include interbedded and overlying shales, change in updip and lateral facies, and fault offsets close to the Wichita and Amarillo uplifts. Differential compaction on the south side of the Amarillo uplift anticline also provided a seal (Mason, 1968). The Pennsylvanian in the Panhandle oil field area is underlain by up-tilted edges of older Paleozoic rocks (Mason, 1968), which probably resulted in contributions through time from multiple petroleum source rocks.

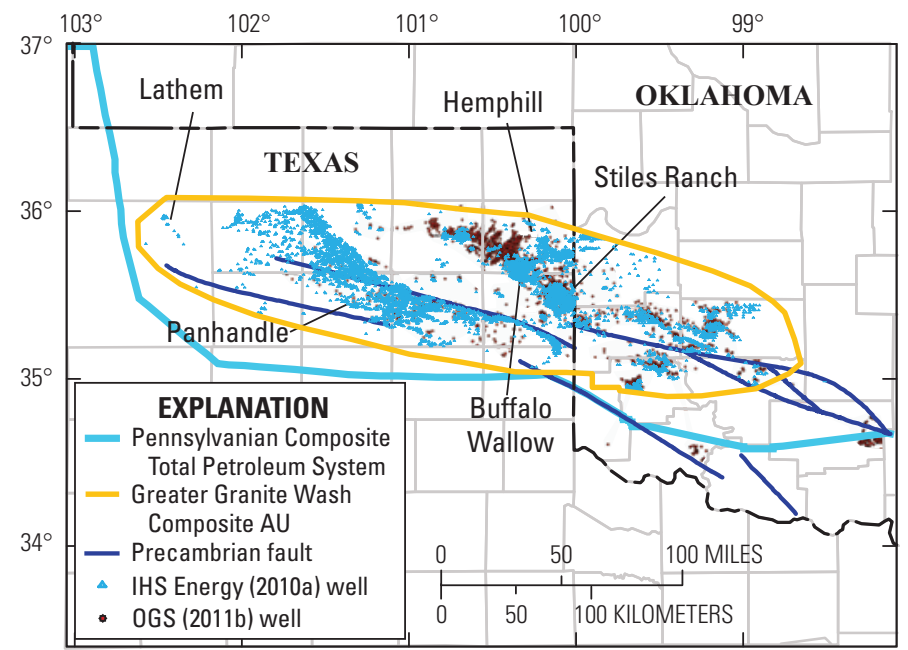

Figure 45. Map showing oil and (or) gas wells that produce from the granite wash within the Greater Granite Wash Composite Assessment Unit (AU). Some of the more than 3,600 Oklahoma Geological Survey (2011b) wells up to about 2008 are overlain by the more than 6,900 wells through September 2010 from IHS Energy (2010a, 2010b). Generalized field locations are from IHS Energy (2010a), Nehring and Associates, Inc. (2009), and Bebout and others (1993). Precambrian faults (blue) are from Adler and others (1971). Light gray lines are counties.

The western quarter of the $\mathrm{AU}$, including most of the Amarillo and Wichita Mountain uplifts, are thermally immature for oil generation because of shallow burial depths and decreased basement heat flow (figs. 8 and 38-40). Thermal maturation of Pennsylvanian petroleum source and potential source rocks in the rest of the AU ranges from immature for oil generation to mature for dry gas generation, as indicated by a modeled $R_{o}$ of less than 4 percent for the Thirteen Finger limestone layer (fig. 8). The Woodford Shale is absent over the western AU area, but deeper petroleum source rocks range in maturity from oil generation to overmature for gas generation. Hydrocarbon migration was primarily from the deep basin with flow to the west and northwest (fig. 30). The Greater Granite Wash Composite AU petroleum system events chart (fig. 48) shows the timing of hydrocarbon generation, migration, and accumulation based on petroleum system models (table 2) (Higley, 2014). Onset of oil generation from Pennsylvanian source rocks approximates the 300 -Ma beginning of oil generation from Thirteen Finger limestone petroleum source rocks, based on petroleum system modeling. This onset preceded deposition of reservoir rocks from this $\mathrm{AU}$, so the 270-Ma beginning of oil generation for this AU is based on the Higley (2012, table 3) modeled generation for potential Virgilian source rocks. 

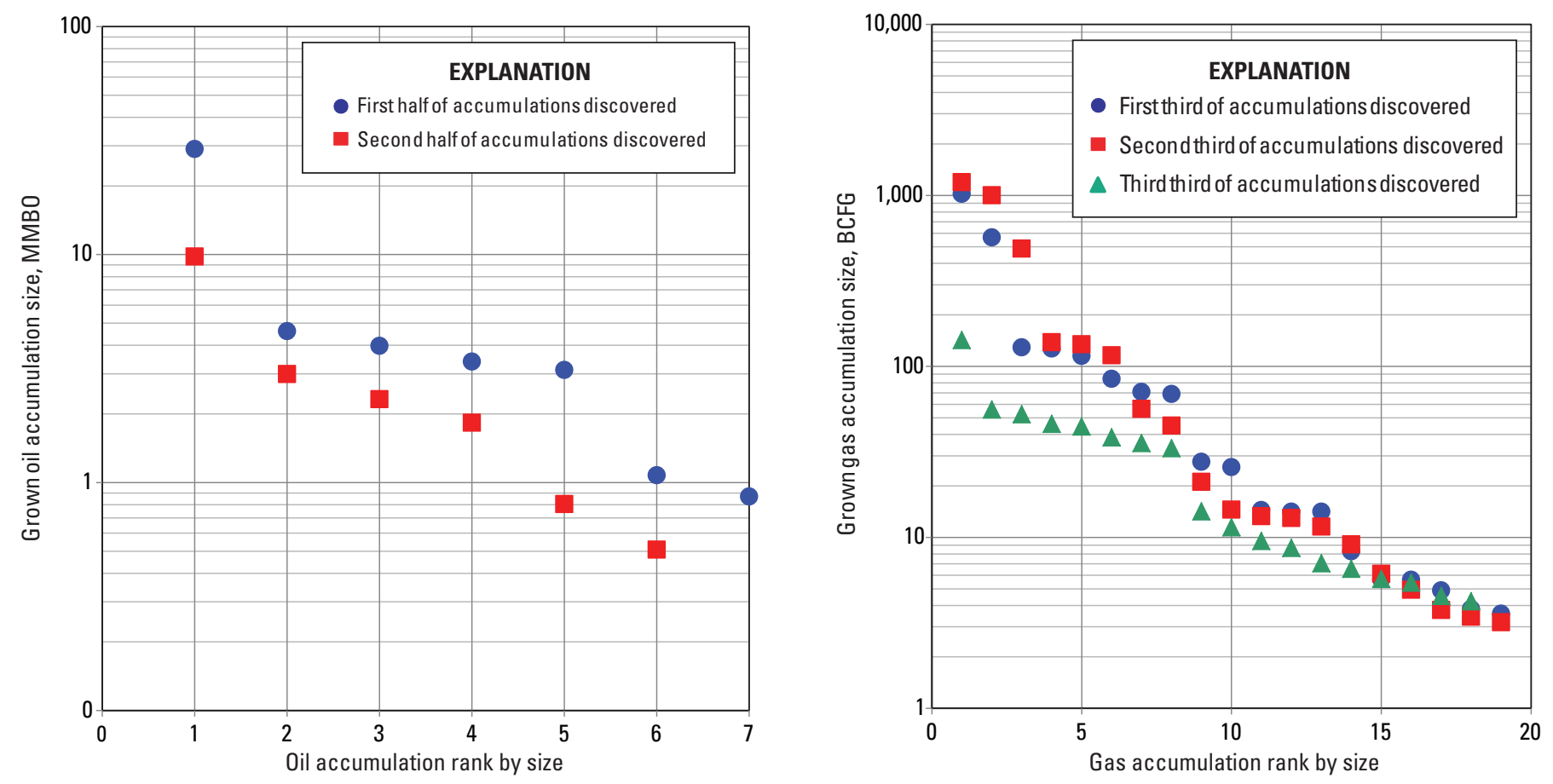

Figure 46. Discovery halves for oil and thirds for gas grown accumulations for the Greater Granite Wash Composite Assessment Unit $(\mathrm{AU})$ in the Anadarko Basin Province. Only a fraction of the oil and gas fields that produce from this AU are shown, largely because most fields produce from multiple units with reported commingled volumes. These also include giant fields, such as Panhandle, which is not included in the discovery halves plot. Because these figures include some production from strata north of the granite wash lithofacies, the discovery plots are further compromised. The discovery halves of oil accumulations indicate that development has decreased through time. This "trend" probably reflects the paucity of data. The discovery thirds of gas accumulations indicates discovery has been fairly stable through time, although field sizes have decreased for larger fields as indicated by the third of accumulations greater than 20 billion cubic feet of gas (BCFG).

\section{Mesozoic Strata}

Post-Permian strata in the basin include scattered remnants of rocks ranging in age from Triassic to Quaternary. Mostly alluvial sediments of the Miocene to Pliocene Ogallala Formation were deposited on an erosional surface of mostly Permian strata in the western half of the basin (Seni, 1980; Johnson and others, 1988). Underlying Mesozoic units are present mostly in the Colorado and western Kansas portions of the province, with only scattered erosional remnants in Oklahoma and Texas.

Johnson and others (1988) estimated that $500-1,000 \mathrm{ft}$ of Triassic and Jurassic strata were deposited in the Oklahoma and Texas portions of the basin but subsequent Early Cretaceous erosion removed all but the scattered 25-200 ft that remains in the far western part of the Anadarko Basin. Based on geologic maps of the States, outcrops of Mesozoic strata occur southeast of the province, in the Central Kansas uplift, along the western border in Colorado, Oklahoma, and Texas, the Amarillo uplift, and in scattered exposures along drainages in Kansas, New Mexico, and Oklahoma. The Lower Cretaceous Dakota Group sandstones and shales were deposited in the Colorado, western Kansas, and Oklahoma panhandle areas of the basin. Although the Muddy ("J") Sandstone of the Dakota Group is an important oil and gas reservoir in the Denver Basin, there is minimal potential for petroleum in the Anadarko Basin Province because of (1) thermally immature source rocks; (2) long vertical and lateral migration distances for Paleozoic source rocks; (3) depositional thinning and updip erosion of the strata; and (4) formation water in these porous and permeable sandstones. Other Mesozoic units have low potential for the same general reasons. An exception to this is the Upper Cretaceous Niobrara Shale in the northern Las Animas arch area of Colorado and western Kansas; however, this resource was included in the Denver Basin assessment (Higley and Cox, 2007; Higley and others, 2007) and will only be mentioned here. Because contained gas is biogenic, thermal maturity of source rocks and migration distances are not important considerations. Within the productive area, potential hydrocarbon source rocks are thermally immature for generation at about 0.3 to $0.4 \% \mathrm{R}_{\mathrm{o}}$ (Smagala and others, 1984 , Tainter, 1984). Biogenic gas is located within shallow, underpressured, fractured, relatively clean chalks (Longman and others, 1998) in fields that have low-relief structural closures. 


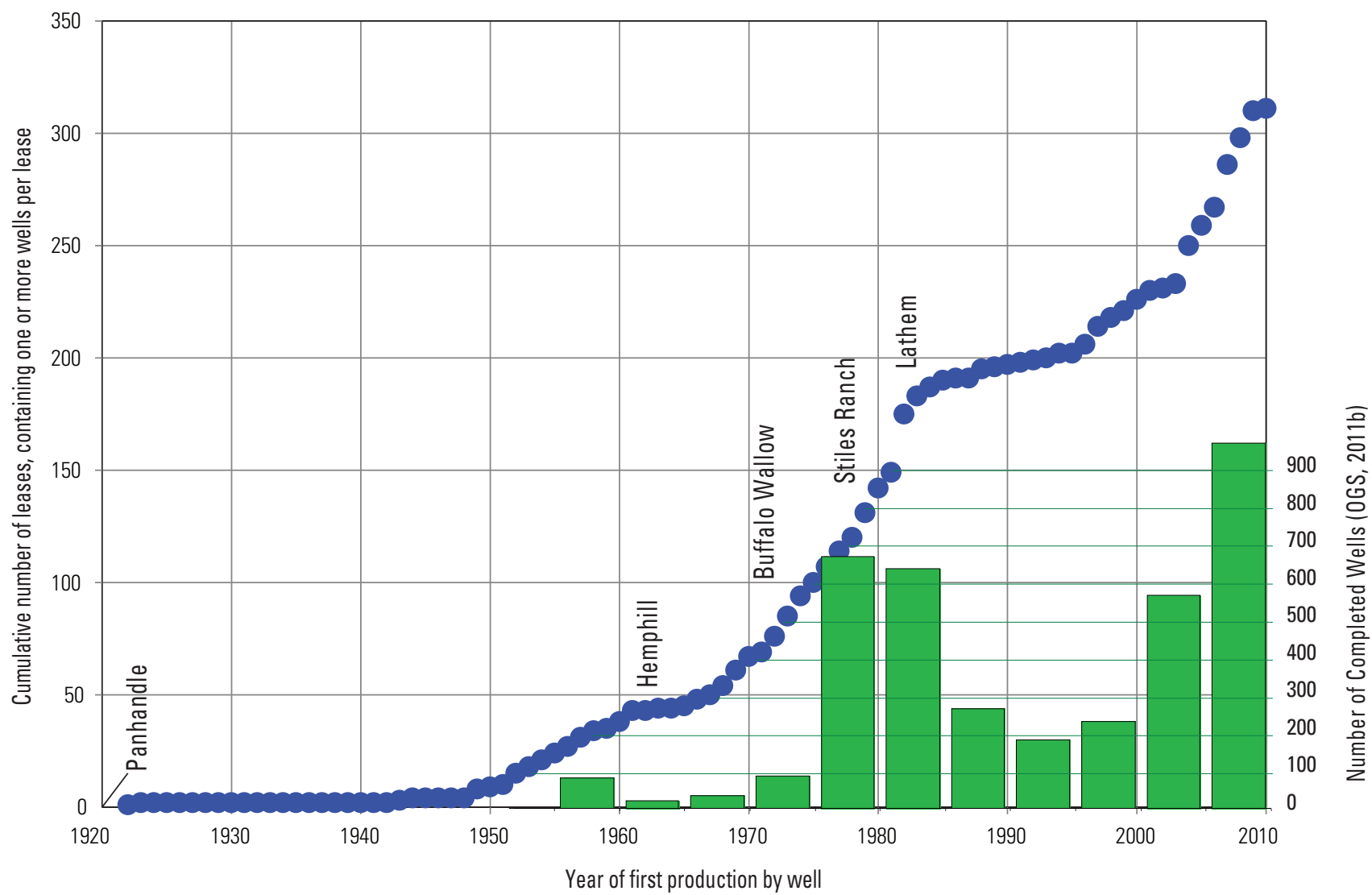

Figure 47. Production history of leases in the Greater Granite Wash Composite Assessment Unit in the Anadarko Basin Province is shown relative to the more than 3,700 completed wells for the same time increments from Oklahoma Geological Survey (OGS, 2011b). Granite wash development started about 1950 and the Oklahoma Geological Survey (2011b) bars follow industry boom and bust cycles with an increase in completions from 1976 to 1985, followed by a lull until about the 2002 increase in exploration. Shown are some of the major field discovery names and year (Nehring and Associates, Inc., 2009; IHS Energy, 2010a, 2010b). 


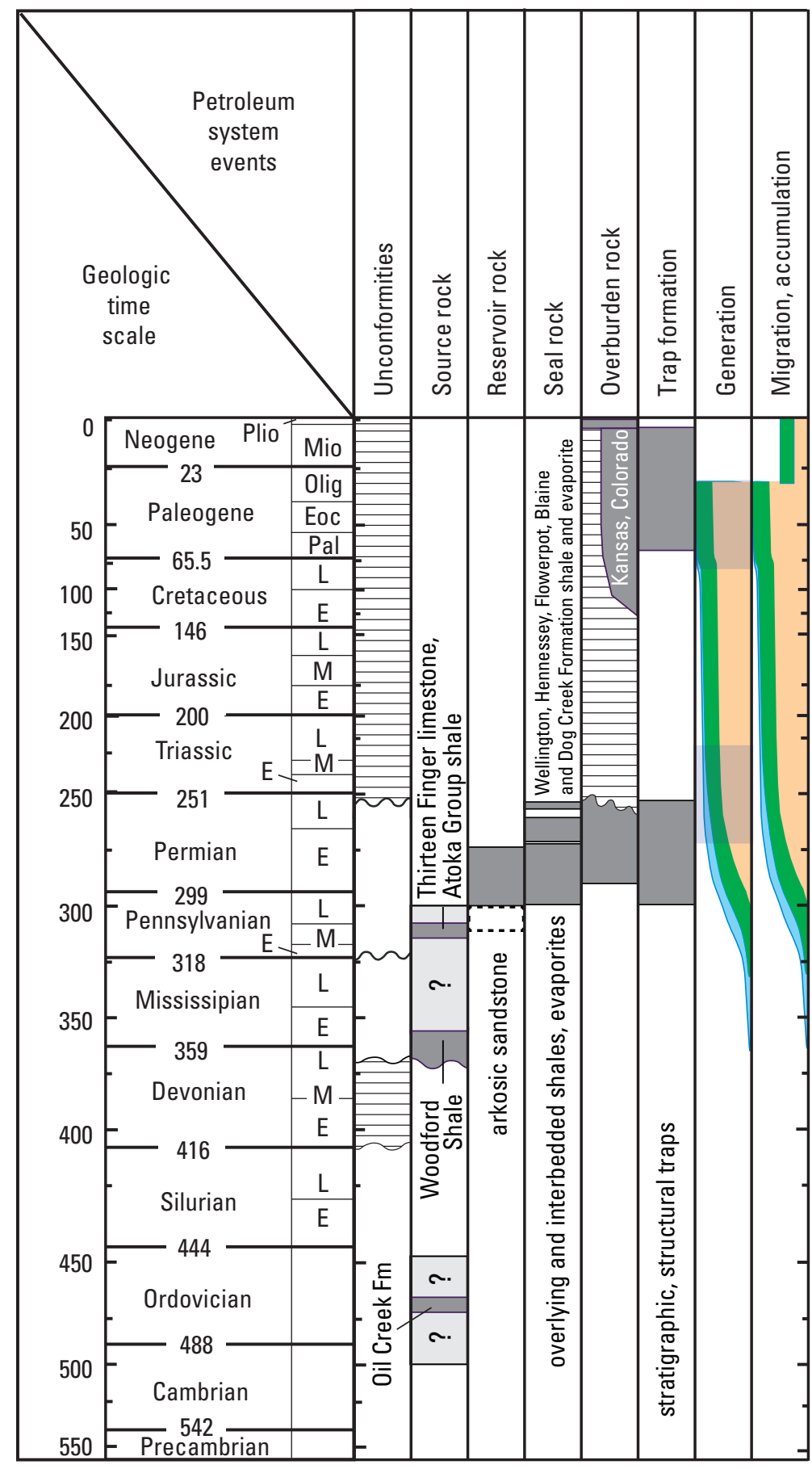

Figure 48. Petroleum system events chart for the Greater Granite Wash Composite Assessment Unit (AU) of the Pennsylvanian Composite Total Petroleum System (TPS). Dashed reservoir rock rectangle shows an interval of potential granite wash deposition that was included in the Morrowan-Atokan Assessment Unit (AU). Potential petroleum source rocks from Hatch and others (1986) (fig. 7) are light gray rectangles. Onset and peak petroleum generation (fig. 14) are shown for the Oil Creek Formation (blue), Woodford Shale (green), and Thirteen Finger limestone (tan) source rocks. Age ranges of peak generation are indicated by blue rectangles. Paleogene trap formation, migration, and accumulation are based on Laramide generation and potential remigration associated with basin tilting. Unconformities, wavy and horizontal lines; Fm., Formation; Plio, Pliocene; Mio, Miocene; Eoc, Eocene; Pal, Paleocene; L, Late; M, Middle; E, Early. 
Reservoir quality is controlled by burial diagenesis (Scholle, 1977) and influenced by fracturing on low-relief structures. Fracturing may also be associated with dissolution of underlying Permian evaporites. The fractured Niobrara chalks commonly have porosities of 40 percent and greater (Lockridge and Scholle, 1978) but low matrix permeability. Burial temperatures do not exceed $75^{\circ} \mathrm{C}$ in areas of active biogenic generation of gas (Rice and Claypool, 1981).

\section{Conclusions}

The Anadarko Basin Province is in a mature state of exploration and development, with more than 220,000 wells drilled. Oil and gas reserves and resources are distributed across the province and within Cambrian through Lower Permian strata. Much of the production is reported commingled from two or more formations, which complicates assigning oil and gas production and reserves to specific strata. This was a primary rationale for grouping assessment units (AU). The AUs discussed in this chapter of the report are conventional, with the exception of the continuous (unconventional) Thirteen Finger Limestone-Atoka Shale AU that has the same general extent as the vertically bounding Morrowan-Atokan AU.

The Mississippian AU extends across the province, with petroleum production from mostly Lower Mississippian carbonates through Upper Mississippian sandstones and carbonates. The Morrowan-Atokan AU outlines the extent of these strata. These Morrow and Atoka Group reservoirs are mostly marine and nonmarine sandstone and calcareous sandstone, with lesser limestone beds. The Thirteen Finger limestone and overlying Atoka shale intervals are the reservoir and petroleum source rocks for the Thirteen Finger Limestone-Atoka Shale AU. Cyclical thin limestone and shale beds are the potential reservoirs. The Greater Granite Wash Composite AU is located in the deep basin in Oklahoma and Texas. Informally named granite wash reservoirs consist mainly of Desmoinesian to Virgilian arkosic shale to conglomerate. Granite wash lithofacies grade northward and interfinger with mostly marine strata of other AUs. The surrounding Desmoinesian and MissourianPermian AUs extend to the province boundaries. Desmoinesian, Missourian, and Virgilian strata were deposited in transgressive-regressive cycles that periodically inundated the province with alternating thin-bedded mainly marine sandstones, limestones, and shales that are stratigraphic packages of mostly sandstone or mostly limestone reservoirs. Desmoinesian AU reservoirs are clastic and carbonate beds of the Cherokee and Marmaton Groups, and their lateral equivalents. The Missourian-Permian AU includes Missourian, Virgilian, Wolfcampian, and Leonardian carbonate and clastic reservoirs.

Assignment of petroleum source rocks to AUs was based mainly on previous research, analysis of well history and production data from proprietary databases, and 1D and 4D petroleum system models that were constructed for this project. The Devonian-Mississippian Woodford Shale was the primary petroleum source rock for Mississippian and lower Morrow
Formation units. Overlying Morrowan through Permian strata were mainly sourced from Pennsylvanian black shales, including the Atokan Thirteen Finger limestone.

The Anadarko Basin is the deepest basin in the onshore United States. It is markedly asymmetrical, with Precambrian basement ranging in depth from more than 40,000 $\mathrm{ft}$ at the basin axis in southern Oklahoma, to surface exposures on the broad shelf in the proximal Central Kansas uplift (CKU of Kansas). Ordovician through Pennsylvanian petroleum source rocks and potential source rocks in the shelf area are almost entirely thermally immature for oil generation. In the deep basin they range from marginally mature for oil generation to overmature for gas generation. Modeling indicates that oil generation began in the deep basin as early as $330 \mathrm{Ma}$ in the Woodford Shale, $300 \mathrm{Ma}$ in the Thirteen Finger limestone, and $270 \mathrm{Ma}$ in Virgilian strata, which may contain the youngest petroleum source rock(s). Oil and gas were generated mostly in the deep basin and flowed radially northward. Flow in northern Oklahoma and in Kansas was directed toward the Las Animas arch, CKU, and Nemaha uplift. The Laramide orogeny may also have influenced petroleum migration through uplift and by imparting a subtle southeastern tilt to the basin, specifically resulting in redistribution of Panhandle field fluid columns with northward spilling of gas into the Hugoton embayment. Migration distances range from self-sourced for the Thirteen Finger Limestone-Atoka Shale AU, to long range from thermally mature source rocks in Oklahoma and Texas to reservoirs in Kansas and Colorado.

\section{Acknowledgments}

Chesapeake Energy generously provided well logs for use in cross sections and correlating strata across the province. Troy Cook of the USGS contributed EUR data for the Hugoton-Guymon fields and his expertise for the Anadarko Basin project. Larry Anna, Tom Judkins, and Sarah Hawkins of the USGS and Neil Suneson, of the University of Oklahoma, gave excellent technical reviews of this paper.

\section{References Cited}

Abels, T.A., 1959, A subsurface study of the Morrowan Series in the northern Anadarko Basin: Oklahoma City Geological Society Shale Shaker, v. 9, p. 5-21.

Adams, W.L., 1964, Diagenetic aspects of lower Morrowan, Pennsylvanian, sandstones, northwestern Oklahoma: American Association of Petroleum Geologists Bulletin, v. 48 , p. $1568-1580$.

Adler, F.J., Caplan, W.M., Carlson, M.P., Goebel, E.D., Henslee H.T., Hick, I.C., Larson, T.G., McCracken, M.H., Parker, M.C., Rascoe, Jr., G., Schramm, M.W., and Wells, J.S.,1971, Future petroleum provinces of the mid-continent, 
in Cram, I.H., ed., Future petroleum provinces of the United States-Their geology and potential: American Association of Petroleum Geologist Memoir 15, v. 2, p. 985-1120.

Al-Shaieb, Z., and Walker, P., 1986, Evolution of secondary porosity in Pennsylvanian Morrow sandstones, Anadarko Basin, Oklahoma, in Spencer, C.W., and Mast, R.F., eds., 1986, Geology of tight gas reservoirs: American Association of Petroleum Geologists Studies in Geology, no. 24, p. 45-67.

Al-Shaieb, Z., Shelton, J.W., and Puckette, J.O., 1989, Sandstone reservoirs of the mid-continent syllabus for short course, Mid-continent section of the American Association of Petroleum Geologists 1989 meeting: Oklahoma City Geological Society, 125 p.

Al-Shaieb, Zuhair, Puckett, J.O., Abdalla, A.A., and Ely, P.B., 1994, Megacompartment complex in the Anadarko Basin: A completely sealed overpressured phenomenon, in Ortoleva, P.J., ed., Basin compartments and seals: American Association of Petroleum Geologists Memoir 61, p. 55-68.

Anderson, C.J., 1991, Distribution of submarine fan facies of the Upper Red Fork interval in the Anadarko Basin, western Oklahoma: Oklahoma State University, Unpublished M.S. thesis, $127 \mathrm{p}$.

Andrews, R.D., 1999a, Map showing regional (sic) structure at the top of the Morrow Formation in the Anadarko Basin and shelf of Oklahoma: Oklahoma Geological Survey Special Publication 99-4, pl. 3.

Andrews, R.D., 1999b, Morrow gas play in the Anadarko Basin and shelf of Oklahoma: Oklahoma Geological Survey Special Publication 99-4, 133 p., 7 pls.

Andrews, R.D., 2001, Map showing regional structure at the top of the Springer Group in the Ardmore Basin, and the Anadarko Basin and shelf of Oklahoma: Oklahoma Geological Survey Special Publication 2001-1, pl. 5.

Andrews, R.D., Campbell, J.A., and Northcutt, R.A., 1996, Fluvial-dominated deltaic (FDD) oil reservoirs in Oklahoma: The Skinner and Prue plays, Oklahoma Geological Survey Special Publication 96-2, 106 p.

Ball, M.M., and Henry, M.E., 1995, Palo Duro Basin Province (043), in Gautier, D.L., Dolton, G.L., Takahashi, K.I., and Varnes, K.L., eds., 1995 National assessment of United States oil and gas resources - Results, methodology, and supporting data: U.S. Geological Survey Digital Data Series 30, Release 2, one CD-ROM, 5 p., available online at $h t t p: / /$ pubs.usgs.gov/dds/dds-030/.

Ball, M.M., Henry, M.E., and Frezon, S.E., 1991, Petroleum geology of the Anadarko Basin region, province (115), Kansas, Oklahoma, and Texas: U.S. Geological Survey OpenFile Report 88-450W, 36 p.
Bebout, D.G., White, W.A., Hentz, T.F., and Grasmick, M.K., eds., 1993, Atlas of major mid-continent gas reservoirs: Bureau of Economic Geology, 85 p., 4 pls.

Blackwell, D.D., and Richards, M., 2004, Geothermal map of North America: American Association of Petroleum Geologists, 1 sheet, scale 1:6,500,000.

Boucher, Kurtis, 2009, Depositional setting, facies, and petroleum geology of the Cabaniss Group in portions of Washita, Custer, Blaine, Caddo, Canadian, and Grady Counties, OK, Part 2: Shale Shaker, the Journal of the Oklahoma City Geological Society, May-June 2009, p. 223-238.

Bowen, D.W., Weimer, Paul, 2003, Regional sequence stratigraphic setting and reservoir geology of Morrow incisedvalley sandstones (lower Pennsylvanian), eastern Colorado and western Kansas: American Association of Petroleum Geologists Bulletin, v. 87, no. 5, p. 781-815.

Bowen, D.W., Weimer, Paul, 2004, Reservoir geology of Nicholas and Liverpool Cemetery fields (lower Pennsylvanian), Stanton County, Kansas, and their significance to the regional interpretation of the Morrow Formation incisedvalley-fill systems in eastern Colorado and western Kansas: American Association of Petroleum Geologists Bulletin, v. 88 , no. 1 , p. $47-70$.

Boyd, D.T., 2011, Oklahoma 2010 drilling highlights: Shale Shaker, Oklahoma City Geological Society, March/April 2011, no. 5, p. 293-306, accessed August 2011, at http:// www.ogs.ou.edu/OilGas/pdf/2010DrillingHighlights.pdf.

Brewer, J.A., Good, R., Oliver, J.E., Brown, L.D., and Kaufman, S., 1983, COCORP profiling across the southern Oklahoma aulacogen: Overthrusting of the Wichita Mountains and compression within the Anadarko Basin: Geology, February 1983, v. 11, no. 2, p. 109-114.

Burke, K., 1977, Aulacogens and continental breakup: Annual Review of Earth and Planetary Sciences, v. 5, p. 371-396.

Burruss, R.C., and Hatch, J.R., 1989, Geochemistry of oils and hydrocarbon source rocks, greater Anadarko Basin: Evidence for multiple sources of oils and long-distance oil migration, in Johnson, K.S., ed., Anadarko Basin symposium, 1988: Oklahoma Geological Survey Circular 90, p. 53-64.

Carr, D.L., Hentz, Tucker F., 2009, Sequence stratigraphy and organic richness of the Lower Pennsylvanian Atoka series, northwest Anadarko Basin, Texas and Oklahoma Panhandle: American Association of Petroleum Geologists Annual Convention, June 7-10, 2009; Denver Colo., 1 p.

Carter, L.S., Kelly, S.A., Blackwell, D.D., and Naeser, N.D., 1998, Heat flow and thermal history of the Anadarko Basin, Oklahoma: American Association of Petroleum Geologists Bulletin, v. 82, no. 2, p. 291-316. 
Cederstrand, J.R., and Becker, M.F., 1998, Digital map of base of aquifer for the High Plains aquifer in parts of Colorado, Kansas, Nebraska, New Mexico, Oklahoma, South Dakota, Texas, and Wyoming: U.S. Geological Survey Open-File Report 98-393, digital data and metadata, accessed March 2011, at http://cohyst.dnr.ne.gov/metadata/ m001aqbs_99.html.

Cotner, Victor, and Crum, H.E., 1933, Geology and occurrence of natural gas in Amarillo district, Texas: American Association of Petroleum Geologists Bulletin, v. 17, no. 8, p. 877-906.

Craig, L.C., and Conner, C.W., 1979, Paleotectonic investigations of the Mississippian system in the United States, in Craig, L.C., and Varnes, K.L., comps., History of the Mississippian System, an interpretive summary: U.S. Geological Survey Professional Paper 101, p. 371-406.

Davis, H.G., and Northcutt, R.A., 1989, The greater Anadarko Basin: An overview of petroleum exploration and development, in Johnson, K.S., ed., Anadarko Basin symposium, 1988: Oklahoma Geological Survey Circular 90, p. 13-24.

Denison, R.E., Lidiak, E.G., Bickford, M.E., and Kisvarsanyi, E.B., 1984, Geology and geochronology of Precambrian rocks in the central interior region of the United States: U.S. Geological Survey Professional Paper 1241-C, 20 p.

Dobervich, George, and Parker, R.L., 1958, The Morrowan Series of the northern Texas Panhandle: Panhandle Geonews, v. 6 , no. 1 , p. 5-12.

Dubois, M.K., 2007, Chapter 3. Depositional model, in Dubois, M.K., Byrnes, A.P., Bhattacharya, Saibal, Bohline, G.C., Doveton, J.H., and Barba, R.E., comps., Hugoton asset management project (HAMP): Hugoton geomodel final report: Kansas Geological Survey Open-File Report 2007-06, 22 p., accessed August 2011, at http://www.kgs. ku.edu/PRS/publication/2007/OFR07_06/KGS_2007-06-3_ Chapter03_DepoModels.pdf.

Dubois, M.K., Byrnes, A.P., Bhattacharya, Saibal, Bohline, G.C., Doveton, J.H., and Barba, R.E., 2006, Hugoton asset management project (HAMP): Hugoton geomodel final report: Kansas Geological Survey Open-File Report 2007-6, 2 p., accessed August 2011, at http://www.kgs.ku.edu/PRS/ publication/2007/OFR07_06/.

Edwards, A.R., 1959, Facies changes in Pennsylvanian rocks along north flank of Wichita Mountains, in Petroleum geology of southern Oklahoma: American Association of Petroleum Geologists, v. 2, p. 142-153.

Fay, R.O., 1964, The Blaine and related formations of northwestern Oklahoma and southern Kansas: Oklahoma Geological Survey Bulletin B98, 238 p., 24 pls.

Feinstein, Shimon, 1981, Subsidence and thermal history of southern Oklahoma aulacogen: Implications for petroleum exploration: American Association of Petroleum Geologists Bulletin, v. 65, v. 12, p. 2521-2533.

Frezon, S.E., and Jordan, Louise, 1979, Oklahoma, in Craig, L.C., and Connor, C.W., coordinators, Paleotectonic investigations of the Mississippian Systems in the United States: U.S. Geological Survey Professional Paper 1010, p. $147-159$.

Gallardo, Jaquidon, and Blackwell, D.D., 1999, Thermal structure of the Anadarko Basin: Association of Petroleum Geologists Bulletin, v. 83, no. 2, p. 333-361.

Galloway, W.E., Ewing, T.E., Garrett, C.M., Tyler, N., and Bebout, D.G., 1983, Panhandle granite wash/dolomite, in Atlas of major Texas oil reservoirs: Bureau of Economic Geology, Austin, Tex., University of Texas, p. 135-136.

Galloway, W.E., Yancey, M.S., and Whipple, A.P., 1977, Seismic stratigraphic model of depositional platform margin, eastern Anadarko Basin, Oklahoma: American Association of Petroleum Geologists Bulletin, v. 61, no. 9, p. 1437-1447.

Gilbert, M.C., 1983, Timing and chemistry of igneous events associated with the southern Oklahoma aulacogen, in Morgan, P., and Baker, B.H., eds., Processes of continental rifting: Tectonophysics, v. 94, p. 439-455.

Gaswirth, S.B., and Higley, D.K., 2014, Geologic assessment of undiscovered oil and gas resources in the CambrianDevonian stratigraphy of the Anadarko Basin, Oklahoma, Kansas, Texas, and Colorado, chap. 5, in Higley, D.K., comp., Petroleum systems and assessment of undiscovered oil and gas in the Anadarko Basin Province, Colorado, Kansas, Oklahoma, and Texas-USGS Province 58: U.S. Geological Survey Digital Data Series 69-EE, 42 p.

Gradstein, F.M., Ogg, J.G., Smith, A.G., Bleeker, Wouter, Lourens, L.J., 2004, A new geologic time scale with special reference to Precambrian and Neogene: Episodes, June 2004 , v. 27 , no. 2 , p. $83-100$.

Grammer, G.M., Eberli, G.P., Van Buchem, F.S.P., Stevenson, G.M., and Homewood, Peter, 1996, Application of highresolution sequence stratigraphy to evaluate lateral variability in outcrop and subsurface-Desert Creek and Ismay intervals, in Longman, M.W., and Sonnenfeld, M.D., eds., Paradox Basin: Paleozoic Systems of the Rocky Mountain Region: Rocky Mountain Section of the Society for Sedimentary Geology (SEPM), p. 235-266.

Ham, W.E., 1973, Regional geology of the Arbuckle Mountains, Oklahoma: Oklahoma Geological Survey Special Publication 73-3, $56 \mathrm{p}$.

Ham, W.E., and Wilson, J.L., 1967, Paleozoic epiorogeny and orogeny in the central United States: American Journal of Science, v. 265, p. 332-407. 
Haq, B.U., and Van Eysinga, F.W.B., 1998, Geological time table: Amsterdam, New York, Elsevier Science, 1 chart.

Hatch, J.R., Rice, D.D., Burruss, R.C., Schmoker, J.W., and Clayton, J.L., 1986, Thermal maturity modeling and geochemical characterization of hydrocarbon source rocks, oils, and natural gases of the Anadarko Basin, in Carter, L.M.H., ed., U.S. Geological Survey research on energy resources-1986, program and abstracts: U.S. Geological Survey Circular 974, p. 21-23.

Henry, M.E., and Hester, T.C., 1995, Anadarko Basin Province (058), in Gautier, D.L., Dolton, G.L., Takahashi, K.I., and Varnes, K.L., eds., 1995 National assessment of United States oil and gas resources-Results, methodology, and supporting data: U.S. Geological Survey Digital Data Series 30, Release 2, one CD-ROM, accessed June 2011, at http:// certmapper.cr.usgs.gov/data/noga95/prov58/text/prov58.pdf.

Higley, D.K., 2014, Thermal maturation of petroleum source rocks in the Anadarko Basin Province, Colorado, Kansas, Oklahoma, and Texas, chap. 3, in Higley, D.K., comp., Petroleum systems and assessment of undiscovered oil and gas in the Anadarko Basin Province, Colorado, Kansas, Oklahoma, and Texas-USGS Province 58: U.S. Geological Survey Digital Data Series 69-EE, 53 p.

Higley, D.K., Charpentier, R.R., Cook, T.A., Klett, T.R., Pollastro, R.M., and Schmoker, J.W., 2007, Executive summary-2002 Assessment of undiscovered oil and gas in the Denver Basin Province, Colorado, Kansas, Nebraska, South Dakota, and Wyoming, in Higley, D.K., comp., Petroleum systems and assessment of undiscovered oil and gas in the Denver Basin Province, Colorado, Kansas, Nebraska, South Dakota, and Wyoming - USGS Province 39: U.S. Geological Survey Digital Data Series 69-P, chap. 1, 4 p.

Higley, D.K., and Cox, D.O., 2007, Oil and gas exploration and development along the Front Range in the Denver Basin of Colorado, Nebraska, and Wyoming, chap. 2, in Higley, D.K., comp., Petroleum systems and assessment of undiscovered oil and gas in the Denver Basin Province, Colorado, Kansas, Nebraska, South Dakota, and Wyoming-USGS Province 39: U.S. Geological Survey Digital Data Series DDS-69-P, 40 p., accessed June 2011, at http:// pubs.usgs.gov/dds/dds-069/dds-069-p/REPORTS/69_p_ CHAP_LIST.pdf.

Higley, D.K., Cook, T.A., and Pawlewicz, M.J., 2014a, Petroleum systems and assessment of undiscovered oil and Gas in the Anadarko Basin Province, Colorado, Kansas, Oklahoma, and Texas-Woodford Shale Assessment Units, chap. 6, in Higley, D.K., comp., Petroleum systems and assessment of undiscovered oil and gas in the Anadarko Basin Province, Colorado, Kansas, Oklahoma, and Texas-USGS Province 58: U.S. Geological Survey Digital Data Series DDS-69-EE, 24 .

Higley, D.K., Gaswirth, S.B., Abbott, M.M., Charpentier, R.R., Cook, T.A., Ellis, G.S., Gianoutsos, N.J., Hatch,
J.R., Klett, T.R., Nelson, Philip, Pawlewicz, M.J., Pearson, O.N., Pollastro, R.M., and Schenk, C.J., 2011, Assessment of undiscovered oil and gas resources of the Anadarko Basin Province of Oklahoma, Kansas, Texas, and Colorado, 2010: U.S. Geological Survey Fact Sheet 2011-3003, 2 p., accessed February 2011, at http://pubs.usgs.gov/ $f_{s} / 2011 / 3003 /$.

Higley, D.K., Gianoutsos, N.J., Pantea, M.P., and Strickland, S.M., 2014b, Precambrian to ground surface grid cell maps and 3D model of the Anadarko Basin Province, chap. 13, in Higley, D.K., comp., Petroleum systems and assessment of undiscovered oil and gas in the Anadarko Basin Province, Colorado, Kansas, Oklahoma, and Texas-USGS Province 58: U.S. Geological Survey Digital Data Series DDS-69EE, $7 \mathrm{p}$.

IHS Energy, 2009, GDS database: Unpublished geological data services database available from IHS Energy, 15 Inverness Way East, Englewood, CO 80112.

IHS Energy, 2010a, IHS energy well database: Unpublished database available from IHS Energy, 15 Inverness Way East, Englewood, CO 80112.

IHS Energy, 2010b, IHS energy production data on CD-ROM: Unpublished database available from IHS Energy, 15 Inverness Way East, Englewood, CO 80112.

IHS Energy, 2012, IHS energy production data on CD-ROM: Unpublished database available from IHS Energy, 15 Inverness Way East, Englewood, CO 80112.

Johnson, K.S., 1989, Geologic evolution of the Anadarko Basin, in Johnson, K.S., ed., Anadarko Basin symposium, 1988: Oklahoma Geological Survey Circular 90, p. 3-12.

Johnson, K.S., Amsden, T.W., Denison, R.E., Dutton, S.P., Goldstein, A.G., Rascoe, B., Jr., Sutherland, P.K., and Thompson, D.M., 1988, Southern midcontinent region, in Sloss, L.L., ed., Sedimentary cover-North American craton, U.S., The Geology of North America: Boulder, Colo., Geological Society of America, v. D-2, p. 307-359.

Jorgensen, D.G., 1989, Paleohydrology of the Anadarko Basin, central United States, in Johnson, K.S., ed., Anadarko Basin symposium, 1988: Oklahoma Geological Survey Circular 90, p. 176-193.

Kansas Geological Survey, 2010, downloadable formations tops and LAS well data: accessed February 2011, at http:// www.kgs.ku.edu/PRS/petroDB.html.

Keighin, C.W., 1995, Las Animas Arch Province (40), in Gautier, D.L., Dolton, G.L., Takahashi, K.I., and Varnes, K.L., eds., 1995 National assessment of United States oil and gas resources - Results, methodology, and supporting data: U.S. Geological Survey Digital Data Series 30, Release 2, one CD-ROM, 7 p. accessed August 2011, at http://pubs.usgs.gov/dds/dds-030/. 
Keighin, C.W., and Flores, R.M., 1989, Depositional facies, petrofacies, and diagenesis of siliclastics of Morrow and Springer rocks, Anadarko Basin, Oklahoma, in Johnson, K.S., ed., Anadarko Basin symposium, 1988: Oklahoma Geological Survey Circular 90, p. 147-159.

Keller, G.R., Lidiak, E.G., Hinze, W J., and Braile, L.W., 1983, The role of rifting in the tectonic development of the midcontinent, U.S.A., in Morgan, P., and Baker, B.H., eds., Processes of continental rifting: Tectonophysics, v. 95, p. 391-412.

Kluth, C.F., 1986, Plate tectonics of the Ancestral Rocky Mountains: in Peterson, J.A., ed., Paleotectonics and sedimentation in the Rocky Mountains region, United States: American Association of Petroleum Geologist Memoir 41, p. 353-369.

Kluth, C.F., and Coney, P.J., 1981, Plate tectonics of the Ancestral Rocky Mountains: Geology, v. 9, p. 10-15.

Krumme, G.W., 1981, Stratigraphic significance of limestones of the Marmaton Group (Pennsylvanian, Desmoinesian) in eastern Oklahoma: Oklahoma Geological survey Bulletin 131, $67 \mathrm{p}$.

Lewan, M.D., and Ruble, T.E., 2002, Comparison of petroleum generation kinetics by isothermal hydrous and nonisothermal open-system pyrolysis: Organic Geochemistry, v. 33 , no. 12 , p. 1457-1475.

Lockridge, J.P., and Scholle, P.A., 1978, Niobrara gas in eastern Colorado and northwestern Kansas, in Pruit, J.D., and Coffin, P.E., eds., Energy resources of the Denver Basin: Rocky Mountain Association of Geologists, p. 35-49.

Longman, M.W., Luneau, B.A., and Landon, S.M., 1998, Nature and distribution of Niobrara lithologies in the Cretaceous Western Interior Seaway of the Rocky Mountain region: The Mountain Geologist, v. 35, no. 4, p. 137-170.

Ludvigson, G.A., Sawin, R.S., Franseen, E.K., Watney, W.L., West, R.R., and Smith, J.J., 2009, A review of the stratigraphy of the Ogallala Formation and revision of Neogene ("Tertiary") nomenclature in Kansas: Kansas Geological Survey, Current Research in Earth Sciences, Bulletin 256, pt 2, 9 p., accessed February 2011, at http://www.kgs.ku.edu/ Current/2009/Ludvigson/index.html.

Maher, John C., 1953, Paleozoic history of southeastern Colorado: American Association of Petroleum Geologists Bulletin, v. 37, no. 11, p. 2475-2489.

Mapel, W.J., Johnson, R.B., Backman, G.O., and Varnes, K.L., 1979, Southern midcontinent and southern Rocky Mountains region, in Craig, L.C., Connor, C.W., coordinators, and Varnes, K.L., Mast, C.A., and Kitely, L.W., assistant coordinators, Introduction and regional analysis of the Mississippian System, part 1 of Paleotectonic investigations of the Mississippian Systems in the United States: U.S. Geological Survey Professional Paper 1010-J, p. 161-187.
Mason, J.W., 1968, Hugoton Panhandle field, Kansas, Oklahoma and Texas, in M 9: Natural gases of North America, volume two: American Association of Petroleum Geologists Volume A008, p. 1539-1547.

McCrone, A.W., 1964, Water depth and mid-continent cyclothems: Kansas Geological Survey Bulletin 169, v. 1, p. 275-281.

Mitchell, John, 2011, Horizontal drilling of deep granite wash reservoirs, Anadarko Basin, Oklahoma and Texas: Oklahoma City Geological Society Shale Shaker, SeptemberOklahoma, 2011, p. 118-167.

Moore, G.E., 1979, Pennsylvanian paleogeography of the southern mid-continent, in Hyne, N.J., ed., Pennsylvanian sandstones of the mid-continent: Tulsa Geological Society Special Publication 1, p. 2-12.

Morse, N.R., 1963, Northeast Waynoka, T25N, R's 15 and 16W, Woods County, in Cramer, R.D., Gatlin, Leroy, and Wessman, H.G., eds., Oil and gas fields of Oklahoma: Oklahoma City Geological Society Reference Report, v. 1, p. $118 \mathrm{~A}-121 \mathrm{~A}$.

National Geologic Map Database, 2011: U.S. Geological Survey, accessed September 2011, at http://ngmdb.usgs.gov/ Geolex/.

Nehring and Associates, Inc. ( NRG), 2009, Significant oil and gas fields database: Colorado Springs, Colorado, NRG Associates, Inc. (Database available from NRG Associates, Inc., P.O. Box 1655, Colorado Springs, CO 80901).

Nicholas, R.L., and Rozendal, R.A., 1975, Subsurface positive features within Wichita foldbelt in Texas and their relation to Paleozoic cratonic margin: American Association of Petroleum Geologists Bulletin, v. 59, no. 2, p. 193-216.

Oklahoma Geological Survey (OGS), 2011a, Databases: accessed September 2011, at http://www.ogs.ou.edu/level3databases.php.

Oklahoma Geological Survey (OGS) 2011b, Granite wash overview: accessed September 2011, at http://www.ogs. ou.edu/MEETINGS/.../GraniteWash08/GWOverview.ppt.

Owen, E.W., 1975, Part II. Panhandle-Hugoton gas and oil field, in Owen, E.W., ed., Trek of the oil finders: A history of exploration for petroleum: American Association of Petroleum Geologists Memoir 6, p. 939-959.

Perry, W.J., Jr., 1989, Tectonic evolution of the Anadarko Basin region, Oklahoma: U.S. Geological Survey Bulletin 1866-A, p. A1-A19.

Pippin, Lloyd, 1970, Panhandle-Hugoton field, TexasOklahoma-Kansas-The first fifty years, in Halbouty, M.T., ed., Geology of giant petroleum fields: American Association of Petroleum Geologists Memoir 14, p. 24-222. 
Rascoe, B., Jr., 1978, Sedimentary cycles in the Virgilian Stage (Upper Pennsylvanian) of the Anadarko Basin, parts 1 and 2: Oklahoma City Geological Society Shale Shaker, v. 28 , p. $123-131$, p. $144-149$.

Rascoe, B., Jr., and Adler, F.J., 1983, Permo-Carboniferous hydrocarbon accumulations, mid-continent USA: American Association of Petroleum Geologists Bulletin, v. 67, no. 6, p. 979-1001.

Rascoe, B., Jr., and Hyne, N.J., 1987, Petroleum geology of the midcontinent: Tulsa Geological Society Special Publication $3,162 \mathrm{p}$.

Rice, D.D., and Claypool, G.E., 1981, Generation, accumulation, and resource potential of biogenic gas: American Association of Petroleum Geologists Bulletin, v. 65, p. 5-25.

Rice, D.D., Threlkeld, C.N., and Vuletich, A.K., 1988a, Character, origin and occurrence of natural gases in the Anadarko Basin, western Kansas, western Oklahoma and Texas Panhandle, U.S.A.: Chemical Geology, v. 71, p. 149-157.

Rice, D.D., Threlkeld, C.N., and Vuletich, A.K., 1988b, Analysis of natural gases from the Anadarko Basin, southwestern Kansas, western Oklahoma and Texas Panhandle: U.S. Geological Survey Open-File Report 88-391, 6 p.

Rice, D.D., Threlkeld, C.N., and Vuletich, A.K 1989, Characterization and origin of natural gases of the Anadarko Basin, in Johnson, K.S., ed., Anadarko Basin symposium, 1988: Oklahoma Geological Survey Circular 90, p. 47-52.

Robbins, S.L., and Keller, G.R., 1992, Complete Bouguer and isostatic residual gravity maps of the Anadarko Basin, Wichita Mountains, and surrounding areas, Oklahoma, Kansas, Texas, and Colorado: U.S. Geological Survey Bulletin 1866-G, 11 p., 2 pls.

Roth, R.I., 1955, Paleogeology of Panhandle of Texas: American Association of Petroleum Geologists Bulletin, v. 39, no. 4, p. 422-443.

Rottmann, Kurt, 2000a, Structure map of Hunton Group in Oklahoma and Texas Panhandle: Oklahoma Geological Survey Special Publication 2000-2, pl. 3.

Rottmann, Kurt, 2000b, Isopach map of Woodford Shale in Oklahoma and Texas Panhandle: Oklahoma Geological Survey Special Publication 2000-2, pl. 2.

Schatski, N.S., 1946, The great Donets Basin and the Wichita system; comparative tectonics of ancient platforms: Akademiya Nauk SSSR Izvestiya, Seriya Geologicheskaya, v. 6, p. 57-90.
Schlumberger, 2011, PetroMod Basin and Petroleum Systems Modeling Software: IES GmbH, Ritterstrasser, 23, 52072 Aachen, Germany.

Scholle, P.A., 1977, Chalk diagenesis and its relation to petroleum exploration-Oil from chalks, a modern miracle: American Association of Petroleum Geologists Bulletin, v. 61, p. 982-1009.

Seni, S.J., 1980, Sand-body geometry and depositional systems, Ogallala Formation, Texas: Texas Bureau of Economic Geology Report of Investigations 105, 36 p.

Smagala, T.M., Brown, C.A., and Nydegger, G.L., 1984, Log-derived indicator of thermal maturity, Niobrara Formation, Denver Basin, Colorado, Nebraska, Wyoming, in Woodward, J., Meissner, F.F., and Clayton, J.C., eds., Hydrocarbon source rocks of the Greater Rocky Mountain area: Rocky Mountain Association of Geologists Guidebook, p. 355-363.

Smith, P.W., Hendrickson, W.J., and Woods, R.J., 2001, Comparison of production and reservoir characteristics in "granite-wash" fields in the Anadarko Basin: Oklahoma Geological Survey Circular 106, p. 19-27.

Sorenson, R.P., 2005, A dynamic model for the Permian Panhandle and Hugoton fields, western Anadarko Basin: American Association of Petroleum Geologists Bulletin, v. 89 , no. 7 , p. 921-938.

Swanson, D.C., 1979, Deltaic deposits in the Pennsylvanian upper Morrow Formation of the Anadarko Basin, in Hyne, N.J., ed., Pennsylvanian sandstone of the mid-continent: Tulsa Geological Society Special Publication 1, p. 115-168.

Sweeney, J.J., and Burnham, A.K., 1990, Evaluation of a simple model of vitrinite reflectance based on chemical kinetics: American Association of Petroleum Geologists Bulletin, v. 74, p. 1559-1570.

Tainter, P.A., 1984, Stratigraphic and paleostructural controls on hydrocarbon migration in Cretaceous D and $\mathrm{J}$ sandstone of the Denver Basin, in Woodward, J.F., Meissner, F., and Clayton, J., eds., Hydrocarbon source rocks of the greater Rocky Mountain region: Rocky Mountain Association of Geologists, p. 339-354.

Wang, H.D., and Philp, R.P., 1997, Geochemical study of potential source rocks and crude oils in the Anadarko Basin, Oklahoma: American Association of Petroleum Geologists Bulletin, v. 81, no. 2, p. 249-274. 


\section{Chapter 8 \\ Present-Day Overpressure and \\ Paleopressure Indicators in the Greater Anadarko Basin, Oklahoma, Texas, Kansas, and Colorado}

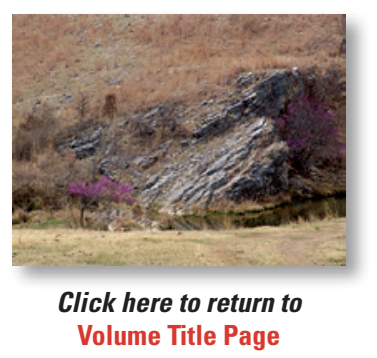

By Philip H. Nelson and Nicholas J. Gianoutsos

Chapter 8 of 13

Petroleum Systems and Assessment of Undiscovered Oil and Gas in the Anadarko Basin Province, Colorado, Kansas, Oklahoma, and Texas-USGS Province 58

Compiled by Debra K. Higley

U.S. Geological Survey Digital Data Series DDS-69-EE 


\title{
U.S. Department of the Interior SALLY JEWELL, Secretary
}

\section{U.S. Geological Survey \\ Suzette M. Kimball, Acting Director}

\author{
U.S. Geological Survey, Reston, Virginia: 2014
}

For more information on the USGS - the Federal source for science about the Earth, its natural and living resources, natural hazards, and the environment, visit http://www.usgs.gov or call 1-888-ASK-USGS.

For an overview of USGS information products, including maps, imagery, and publications, visit http://WwW.usgs.gov/pubprod

To order this and other USGS information products, visit http://store.usgs.gov

Any use of trade, firm, or product names is for descriptive purposes only and does not imply endorsement by the U.S. Government.

Although this information product, for the most part, is in the public domain, it also may contain copyrighted materials as noted in the text. Permission to reproduce copyrighted items must be secured from the copyright owner.

Suggested citation:

Nelson, P.H. and Gianoutsos, N.J., 2014, Present-day overpressure and paleopressure indicators in the greater Anadarko Basin, Oklahoma, Texas, Kansas, and Colorado, chap. 8, in Higley, D.K., comp., Petroleum systems and assessment of undiscovered oil and gas in the Anadarko Basin Province, Colorado, Kansas, Oklahoma, and TexasUSGS Province 58: U.S. Geological Survey Digital Data Series DDS-69-EE, 28 p., http://dx.doi.org/10.3133/ds69EE.

ISSN 2327-638X (online) 


\section{Contents}

Abstract
Introduction.
Overpressure in Rocks of Pennsylvanian and Mississippian Age
Indicators of Paleopressure from Previous Work
Indicators of Paleopressure from Reduction in Resistivity
Procedure
Semparison of Overpressured and Paleopressured Areas
References Cited

\section{Plates}

1. Pressure-depth plots for the Anadarko Basin in Oklahoma...................................... link

2. Resistivity logs in map position in the Anadarko Basin in Oklahoma ......................... link

3. Resistivity logs on west-east cross-section $A-A^{\prime}$.............................................. link

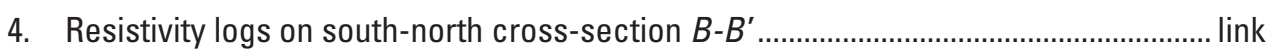

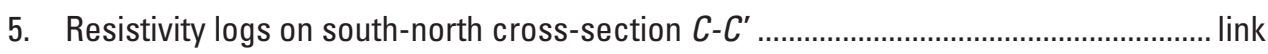

6. Resistivity logs on south-north cross-section $D-D^{\prime}$................................................ link

\section{Figures}

1. Map showing study area encompassing the greater Anadarko Basin in western Oklahoma, the Texas Panhandle, southwestern Kansas, and southeastern Colorado. Areas studied by Breeze (1970) and Al-Shaieb and others $(1994 a, b)$ are also shown. Basin axis coincides with deepest structural contours of the Woodford Shale.

2. Stratigraphic chart for the Anadarko Basin, from Higley (chapter 3 of this report). Wavy lines represent unconformities. Areas with vertical lines represent periods of non-deposition. (Camb., Cambrian; Miss., Mississippian).

3. Burial history for the Ferris 1-28 well, from Carter and others (1998). Well location is shown in figure 1. The times when overpressure and underpressure probably developed are highlighted. (pC, Precambrian; C, Cambrian; 0, Ordovician; S, Silurian; D, Devonian; M, Mississippian; IP, Pennsylvanian; P, Permian; $k$, Triassic; J, Jurassic; K, Cretaceous; $P$, Paleocene; E, Eocene; 0, Oligocene; M, Miocene; FMS., Formations).

4. Plots of pressure against depth for five areas $A$ through $E$ in the greater Anadarko Basin. Each plot contains pressure data from wells within a 30- by 30-mile area located in the index map. Faults in basement rocks bound the eastern and southern parts of the basin. Pressure data designated by green letters representing geologic periods are from Al-Shaieb and others (1994a). Pressure data from drillstem tests, bottom-hole pressures, and mud weights are taken from IHS Energy (2009) 
5. Map showing elevation of top of overpressure, based on data displayed in plate 1 .......7

6. Map showing outline of present-day overpressure in rocks of Desmoinesian age, based on data from Al-Shaieb and others (1994a) and augmented with pressure data from IHS Energy (2009). Contour lines and shading represent the ratio of pressure to depth in pounds per square inch per foot (psi/ft)

7. Map showing outline of present-day overpressure in rocks of Morrowan and Springer age, based on data from Al-Shaieb and others (1994a) and augmented by pressure data from IHS Energy (2009). Contour lines and shading represent the ratio of pressure to depth in pounds per square inch per foot (psi/ft).

8. A, Nine overpressured compartments in Morrowan and Springer age rocks, from Powley (1984). Elevation in feet (ft) of potentiometric surface $\mathrm{H}$ is given for each area. $B$, Pressure as a function of elevation for three overpressured areas in Morrow and Springer Formations, from Powley (1984). Pressure data are from oil and gas fields and individual wells. Each line has a slope of 0.465 pounds per square inch per foot (psi/ft).

9. Map showing contours of present-day pressure-depth ratios in the Morrow Formation, showing change from overpressure [greater than 0.5 pounds per square inch per $\mathrm{ft}$ (psi/ft)] to underpressure (less than $0.4 \mathrm{psi} / \mathrm{ft}$ ), based on data from Breeze (1970). Six named wells (small green circles) show well-log indicators of paleopressure, as interpreted by Breeze (1970). Water chemistry falls into three distinct types as shown by Stiff plots labeled X, Y, and Z, from Dickey and Soto (1974). Each brown circle is the locus of several wells from which water samples were taken.

10. Graphs showing indicators of paleopressure in mudrocks from resistivity, sonic, and self potential (SP) logs in six wells from Breeze (1970). Well locations are shown in figure 9 and in the inset map. Salinity of produced water from a nearby well is also from Breeze (1970). Pressure data from wells within a 3- to 10-mile distance from the designated well taken from Al-Shaieb and others (1994a). $A$, Neely 1 well; $B$, Cheyenne-Arapahoe Unit 1 well; $C$, Raymond Moss 1 well; $D$, Knabe 1 well; $E$, Kamp 1 well; and $F$, Fox 1 well.

11. Gamma-ray, resistivity, neutron, and density logs in the West Edmond SWD 1-24 well (location shown on plate 2). Resistivity trendline is based on lowest resistivity values in mudrocks, which are represented by gamma-ray values in excess of 70 American Petroleum Institute (API) units (gray shading) and neutron porosity in excess of 20 percent (brown shading). Low resistivity in sandstones (light yellow shading) is attributed to saline water in the pore space. In this well, resistivity and density in mudrocks increase steadily with depth and there is no indication of paleopressure.

12. Gamma-ray, resistivity, neutron, and sonic logs in the Bredy well. The resistivity trendline is based on the lowest resistivity values in mudrocks above 9,000 feet, which are represented by gamma-ray values in excess of 70 American Petroleum Institute (API) units (gray shading) and neutron porosity in excess of 20 percent (brown shading). In this well, the top of paleopressure is at 9,000 feet. Below the top of paleopressure, resistivity in mudrocks decreases with increasing depth and sonic slowness in mudrocks increases with depth.

13. Map of the elevation of top of paleopressure as determined from resistivity logs. Lines $A-A^{\prime}$ through $D-D^{\prime}$ show location of cross sections on plates $3-6$. A resistivity reversal was found in 107 wells (green circles) but was absent in 68 wells (red triangles)

14. Map of age of rock unit containing top of paleopressure, based on the depth where the resistivity log changes from increasing downwards to decreasing downwards. The green area includes rocks of either Morrowan or Springer age, or both. 
15. Map showing extents of paleopressure and overpressure in rocks of Morrowan and Springer age. The extent of the paleopressure indicator from resistivity logs is derived from figure 14 and the extent of present-day overpressure from pressure data is taken from figure 7. Modeled vitrinite reflectance contours of 0.8 and 1.2 percent for source rocks of Thirteen Finger lime of Atokan age taken from Higley (chapter 7 of this report).

16. Map showing extents of paleopressure and overpressure in rocks of Desmoinesian age. The extent of the paleopressure indicator from resistivity logs is derived from figure 14 and the extent of present-day overpressure from pressure data is taken from figure 6. Modeled vitrinite reflectance contours of 0.8 and 1.2 percent for source rocks of Desmoinesian age taken from Higley (chapter 7 of this report)

17. Map showing extents of paleopressure and overpressure in rocks of Missourian age. The extent of the paleopressure indicator from resistivity logs is derived from figure 14. Modeled vitrinite reflectance contours of 0.8 and 1.0 percent for source rocks of early Virgilian age taken from Higley (chapter 7 of this report)

\section{Appendix 1}

List of wells shown in plates 3-6..

\section{Conversion Factors}

Inch/Pound to SI

\begin{tabular}{|c|c|c|}
\hline Multiply & By & To obtain \\
\hline \multicolumn{3}{|c|}{ Length } \\
\hline foot $(\mathrm{ft})$ & 0.3048 & meter $(\mathrm{m})$ \\
\hline mile (mi) & 1.609 & kilometer (km) \\
\hline \multicolumn{3}{|c|}{ Volume } \\
\hline $\begin{array}{l}\text { barrel (bbl), (petroleum, } 1 \\
\quad \text { barrel=42 gal) }\end{array}$ & 0.1590 & cubic meter $\left(\mathrm{m}^{3}\right)$ \\
\hline cubic foot $\left(\mathrm{ft}^{3}\right)$ & 0.02832 & cubic meter $\left(\mathrm{m}^{3}\right)$ \\
\hline \multicolumn{3}{|c|}{ Flow rate } \\
\hline cubic foot per day $\left(\mathrm{ft}^{3} / \mathrm{d}\right)$ & 0.02832 & cubic meter per day $\left(\mathrm{m}^{3} / \mathrm{d}\right)$ \\
\hline \multicolumn{3}{|c|}{ Pressure } \\
\hline atmosphere, standard (atm) & 101.3 & kilopascal (kPa) \\
\hline bar & 100 & kilopascal $(\mathrm{kPa})$ \\
\hline pound per square inch $\left(\mathrm{lb} / \mathrm{in}^{2}\right)$ & 6.895 & kilopascal (kPa) \\
\hline \multicolumn{3}{|c|}{ Density } \\
\hline pound per cubic foot $\left(\mathrm{lb} / \mathrm{ft}^{3}\right)$ & 16.02 & kilogram per cubic meter $\left(\mathrm{kg} / \mathrm{m}^{3}\right)$ \\
\hline pound per cubic foot $\left(\mathrm{lb} / \mathrm{ft}^{3}\right)$ & 0.01602 & gram per cubic centimeter $\left(\mathrm{g} / \mathrm{cm}^{3}\right)$ \\
\hline
\end{tabular}

Temperature in degrees Celsius $\left({ }^{\circ} \mathrm{C}\right)$ may be converted to degrees Fahrenheit $\left({ }^{\circ} \mathrm{F}\right)$ as follows: ${ }^{\circ} \mathrm{F}=\left(1.8 x^{\circ} \mathrm{C}\right)+32$

Concentrations of chemical constituents in water are given either in milligrams per liter (mg/L) or micrograms per liter $(\mu \mathrm{g} / \mathrm{L})$. 



\title{
Present-Day Overpressure and Paleopressure Indicators in the Greater Anadarko Basin, Oklahoma, Texas, Kansas, and Colorado
}

\author{
By Philip H. Nelson and Nicholas J. Gianoutsos
}

\begin{abstract}
Formation fluids in Pennsylvanian strata of the Anadarko Basin are overpressured throughout the deep part of the basin, as known from previous studies. We take a closer look at the state of overpressuring, documenting the progressive deepening of the top of overpressure from north to south, the increasing vertical extent of overpressure from north to south as strata thicken, the compartmentalization of overpressure in strata of Desmoinesian and Morrowan age, and the areal extent of overpressure in these same strata. Resistivity logs distributed throughout the basin display trendline reversals, with resistivity decreasing with depth rather than continuing to increase along a normal compaction trend. These trendline reversals are interpreted as evidence of an overpressured state in which the pressure can either still be present or else has dissipated, a condition referred to herein as paleopressure. Areas where there are trendline reversals are called paleopressured areas. Paleopressured areas in rocks of Morrowan, Desmoinesian, and Missourian age are more than twice the size of present-day overpressured areas, indicating that the extent of present-day overpressured areas have contracted from paleopressured areas. We suggest that the contraction took place concurrently with the development of normal and subnormal pore pressures on the flanks of the basin when erosional processes exposed Permian and Pennsylvanian strata at the eastern edge of the basin.
\end{abstract}

\section{Introduction}

This study examines the distribution of present-day overpressure and the evidence that the extent of overpressure at one time occupied an area larger than the present-day distribution of overpressure within the greater Anadarko Basin. Chapter 9 of this report by Nelson and Gianoutsos, which also covers the study area of figure 1, examines the extent and cause of underpressure in the basin. Our two studies on formation fluid pressure were undertaken in support of and in parallel with the work reported by Higley (chapter 7 of this report) and Gaswirth (chapter 5 of this report) on the assessment of oil and gas in the Anadarko Basin. Previous studies dealing with fluid pressure in the basin from Breeze (1970) and Al-Shaieb and others $(1994 \mathrm{a}, \mathrm{b})$ are cited extensively in this chapter of the report; their study areas are shown in figure 1.

The elevation contours of the Woodford Shale illustrate the structure of the Anadarko Basin (fig. 1); a line drawn through the deepest part of the Woodford Shale serves to locate the deep basin in subsequent figures. Stratigraphic relations of Paleozoic rocks are shown in figure 2; further discussion of the geology of the basin is given by Higley (chapters 5 and 7 of this report). A representative burial history plot (fig. 3) shows that during times of rapid burial, overpressure developed in rocks of the Morrowan, Atokan, Desmoinesian, Missourian, and to a lesser degree in the Virgilian Series, all of Pennsylvanian age, as indicated schematically by the highlighting in figure 3 . The areal and stratigraphic distribution of overpressure and its contraction with time is the subject of this chapter of the report.

In this chapter of the report, the term "present-day overpressure" or "overpressure" refers to fluid pressure measurements for which the ratio of measured pressure to the depth of measurement is greater than that of saline formation water, nominally 0.465 pounds per square inch per foot (psi/ft). Moreover, these terms refer to the pressure prior to the production of oil or gas, because pore pressure decreases as hydrocarbons are produced. Measurements of post-production pressures are generally less than pre-production pressure and, although of great interest to reservoir engineers, are considered noise in our studies. The term "paleopressure" refers to overpressure inferred to have existed in the past, either where there is no present-day overpressure or where overpressure in the geologic past was possibly greater than present-day overpressure. In this chapter of the report, paleopressured rock volumes are delineated by the departure of resistivity logs from a normal mudrock compaction trend.

This chapter of the report comprises four parts. In the first part, we review the overpressured zone delineated by Al-Shaieb and others (1994a, b), which is notable because of its large extent and the compartmentalization of pressure within it. Access to the dataset used by Al-Shaieb and others 


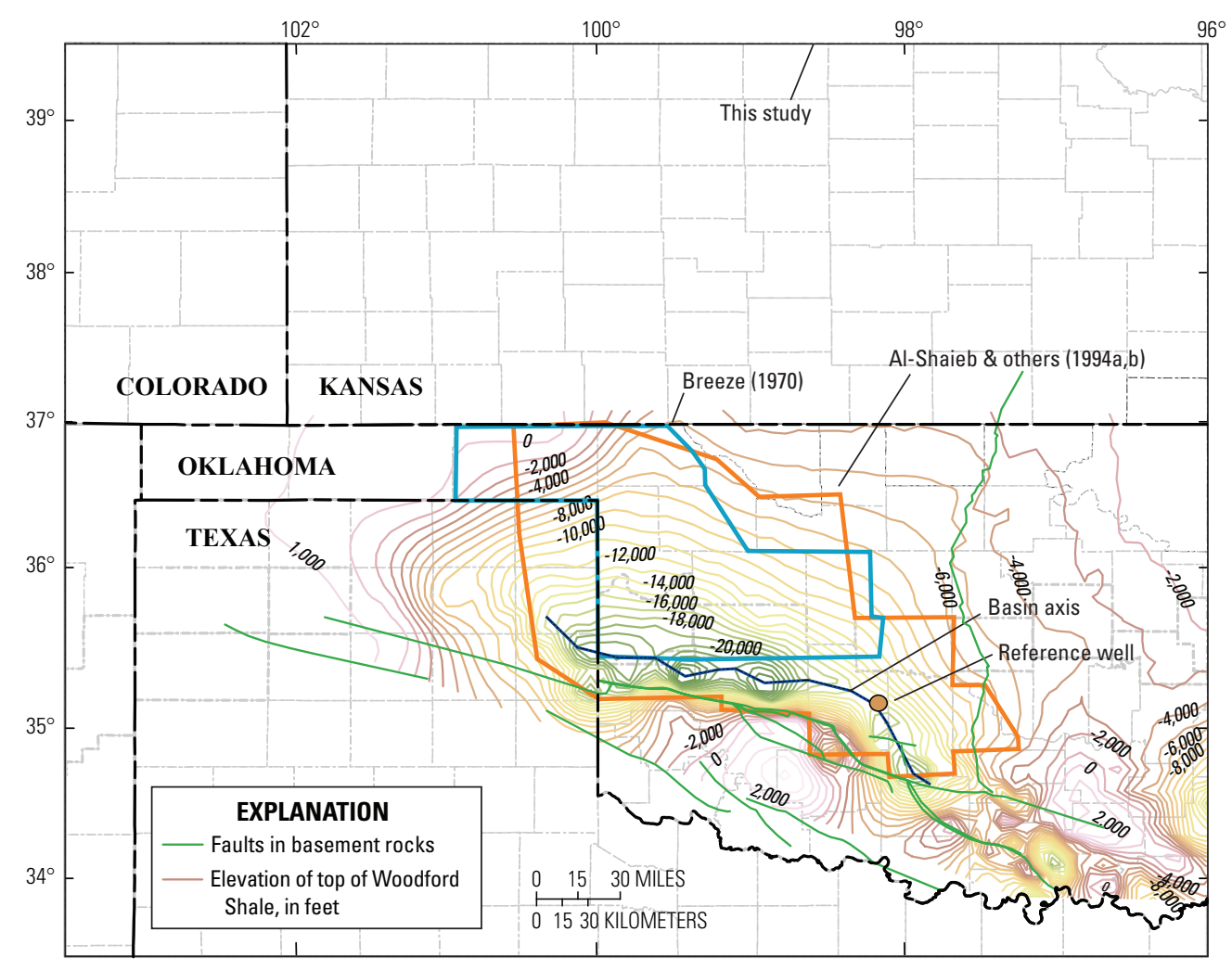

Figure 1. Map showing study area encompassing the greater Anadarko Basin in western Oklahoma, the Texas Panhandle, southwestern Kansas, and southeastern Colorado. Areas studied by Breeze (1970) and Al-Shaieb and others (1994a,b) are also shown. Basin axis coincides with deepest structural contours of the Woodford Shale.

(1994a) has made it possible to reformat the pressure-depth plots and produce maps of pressure as a function of geologic age. An important characteristic of the overpressured zone in the Anadarko basin is its preservation for the 250 million years that have elapsed since the presumed onset of overpressure during Permian time. We will return to this point when comparing the overpressured area with the paleopressured area in the third part of this chapter of the report.

In the second part of this chapter of the report, we review previously published work on pressure, salinity, and well log reversals and then, in the third part, describe our own examination of well-log evidence for high pressure in strata of Pennsylvanian and Mississippian age. A decrease of resistivity with increasing depth (rather than an increase along a normal mudrock compaction trend) is an indicator that rocks are not normally compacted (Bigelow, 1994). The lack of complete compaction can be attributed to either of two causes. (1) During burial, the rocks were partially prevented from compacting by pore pressure that remained higher than normal. This can happen if pore water is prevented from escaping from the pore space, so the pore water bears part of the vertical stress, a phenomenon referred to as disequilibrium compaction. (2) Excess pressure arises during the generation of oil and gas because of a volume increase in the amount of fluid in the pore space, causing microcracks to open within the rock. Microcracks that opened during generation of excess pressure remain partially open, with consequent reductions of resistivity and sonic velocity. Regardless of how the excess pressure was generated, if that pressure is maintained, either in whole or in part into the present day, then the excess pressure is measurable as overpressure. If the pressure from either of these two causes was not maintained but diminished either partly or entirely at some time in the past, then we refer to it as paleopressure. In this chapter of the report, the reversals in resistivity logs are called a "paleopressure indicator" and the rock volume thus affected is referred to as being paleopressured.

In the fourth part of this chapter of the report, we compare the overpressured and paleopressured areas with levels of thermal maturity obtained from basin modeling, finding that the highest levels of thermal maturity nearly coincide with the highest levels of present-day overpressure. We show that the area of the Anadarko Basin where resistivity reversals are present (the geographic extent of paleopressure) is greater than (and includes) the present-day overpressured zone delineated by Al-Shaieb and others (1994a, b). The main finding of this chapter of the report is the inferred contraction in horizontal 


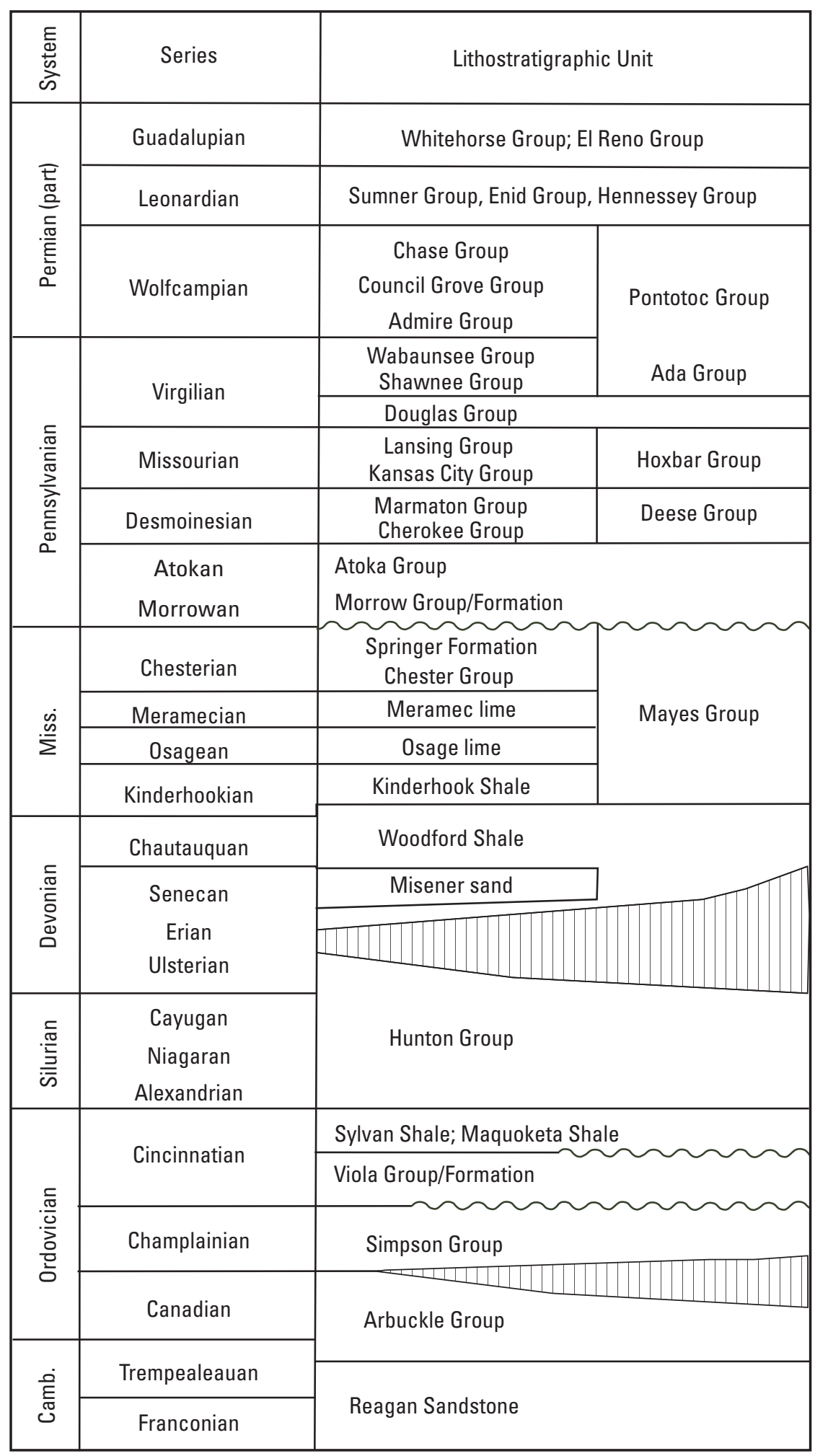

Figure 2. Stratigraphic chart for the Anadarko Basin, from Higley (chapter 3 of this report). Wavy lines represent unconformities. Areas with vertical lines represent periods of non-deposition. (Camb., Cambrian; Miss., Mississippian). 


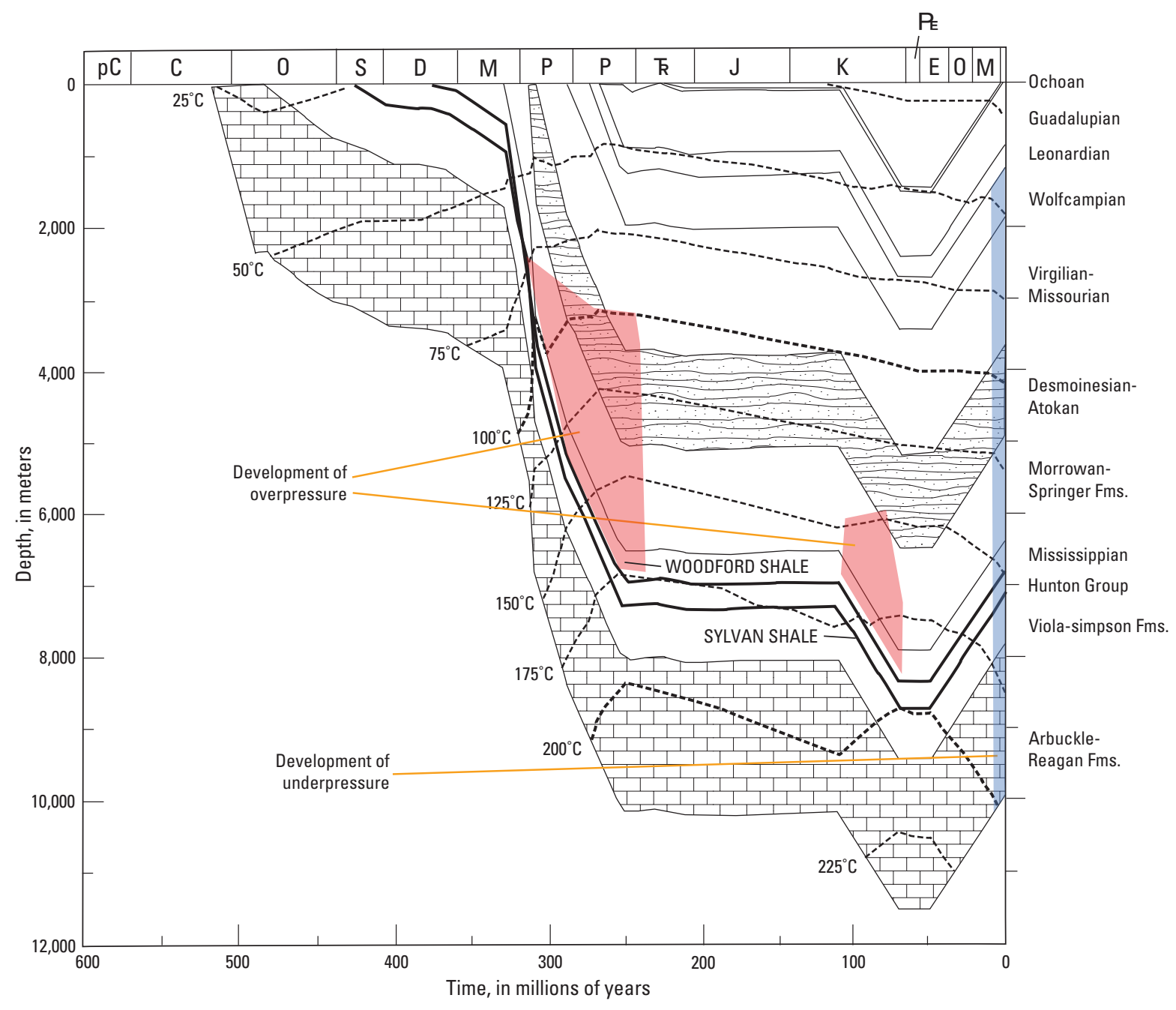

Figure 3. Burial history for the Ferris 1-28 well, from Carter and others (1998). Well location is shown in figure 1. The times when overpressure and underpressure probably developed are highlighted. (pC, Precambrian; C, Cambrian; O, Ordovician; S, Silurian; D, Devonian; M, Mississippian; IP, Pennsylvanian; P, Permian; K, Triassic; J, Jurassic; K, Cretaceous; $R$, Paleocene; E, Eocene; O, Oligocene; M, Miocene; FMS., Formations).

and vertical extent of the paleopressured rock volume to its smaller present-day overpressured volume. Some speculative ideas on the timing of the contraction are included in the fourth part of the chapter.

\section{Overpressure in Rocks of Pennsylvanian and Mississippian Age}

Al-Shaieb and others (1994a, b) used 4,439 reservoir-pressure data points to map the pressure regime in the Oklahoma and Texas portions of the Anadarko Basin. Sources were (1) pressures calculated from static initial wellhead shut-in pressures (2,579 points), (2) shut-in pressures from drillstem tests
(1,787 points), and (3) recorded bottom-hole pressures from $\mathrm{P} / \mathrm{Z}$ plots in production records (73 points). Their primary finding was the delineation of a large overpressured volume called the megacompartment complex (designated Level 1, with lateral dimensions of 100 miles) that contains sub-compartments within a single stratigraphic interval (Level 2 compartments, with lateral dimensions of tens of miles). Nested within Level 2 compartments are Level 3 compartments, with lateral dimensions of a few miles, that are linked to depositional facies within reservoirs. The megacompartment complex is bounded by the Woodford Shale at the base, and by major structural offsets on the south. The top of the overpressured zone lies between depths of 7,500 and 10,000 feet (ft) and crosses stratigraphic boundaries. The northern edge is formed by the convergence of the top and basal boundaries as strata thin to the north. 
We have combined data from Al-Shaieb and others (1994a) with data extracted from a commercial database (IHS Energy, 2009) to provide illustrative examples of pressuredepth plots from various parts of the basin (fig. 4). These examples demonstrate underpressure in the northwestern part of the basin ( $A$ and $B$ of fig. 4), normal pressure on the northern flank ( $C$ of fig. 4 ), and overpressure in the deep basin ( $D$ and $E$ of fig. 4). Underpressure is revealed by the slight gap between the mud-weight pressures, which track the $0.465 \mathrm{psi} / \mathrm{ft}$ line, and the drillstem-test pressures in $A$ and $B$ of figure 4. Underpressure in the Missourian-Virgilian and Morrowan rocks was mapped but not discussed by Al-Shaieb and others (1994a). The characterization and cause of underpressure in the greater Anadarko Basin is the subject of chapter 9 of this report by Nelson and Gianoutsos. Normal pressure is shown where the drillstem-test pressures coincide with the mud-weight pressures on the $0.465 \mathrm{psi} / \mathrm{ft}$ line as illustrated in figure $4 C$ and at depths shallower than $10,000 \mathrm{ft}$ in figure $4 D$. (Because many of the pressures from drillstem tests are lower than the actual pore pressure, because of either inadequate tests or to reservoir depletion, only the righthand edge of the drillstem-test data field should be considered as representative of pore pressure.)

Overpressure is present in rocks of Desmoinesian and Morrowan age as shown in figure $4 D$. Evidence of the compartmentalization cited by Al-Shaieb and others (1994a) is seen where the pressures in Morrowan rocks reach a maximum pressure-depth ratio of nearly $1.0 \mathrm{psi} / \mathrm{ft}$, whereas the pressures in Desmoinesian rocks reach a lesser maximum of nearly $0.9 \mathrm{psi} / \mathrm{ft}$. As pore pressure builds, drillers increase the mud weight to maintain control of the well while drilling. The mud-weight and drillstem-test pressures shown in $D$ and $E$ of figure 4 are consistent with the pressures compiled by Al-Shaieb and others (1994a), keeping in mind that the two datasets were not taken from the same wells but represent all wells with data in a 30- by 30-mile area.

The complete dataset for Oklahoma of Al-Shaieb and others (1994a) is displayed in 28 pressure-depth plots in plate 1. Each pressure-depth plot incorporates all data in a block of 8 to 15 townships. Many of the blocks (blocks B, C, F-O, R, and $\mathrm{S}$ on the index map in plate 1) are three townships (18 miles) in the south-north direction and four townships (24 miles) in the west-east direction; other blocks have comparable areas but different shapes. Grouping the data in this way permits inspection of the pressure-depth profiles and selection of the top of overpressure as a function of location; the pressure-depth trends cannot be examined in individual wells because a single well typically contributes only a few pressure measurements. Some observations regarding the relations among pressure, depth, and location within the basin are:

(1) The presence of megacompartments pointed out by Al-Shaieb and others (1994a, b) can be seen as a step increase in pressure with depth in Desmoinesian and Morrowan age rocks in blocks H, I, L, and M. Another step increase in pressure with depth takes place between Atokan/Desmoinesian and Morrowan rocks in block $\mathrm{S}$.
(2) Below the overpressured intervals in Desmoinesian and Morrowan rocks in blocks $\mathrm{H}, \mathrm{I}, \mathrm{J}$, and L, pressures return to normal in underlying rocks of Silurian age. The pressure regression supports the statement of Al-Shaieb and others (1994a, b) that the Devonian Woodford Shale is the base of the overpressured interval. In other blocks, the return to normal pressure at depth cannot be observed because rocks deeper than Morrowan were not penetrated. An exception occurs in the southeastern part of the study area in blocks $\mathrm{Z}$ and $\mathrm{ZZ}$ where Ordovician rocks are overpressured.

(3) Overpressured conditions prevail to a depth of 20,000 ft in rocks of Mississippian age in blocks X and Y.

(4) Normal to subnormal pressures prevail in blocks A-E on the northern flank of the area. The northernmost appearance of overpressure in Desmoinesian and Morrowan rocks is in blocks $\mathrm{F}$ and $\mathrm{G}$ in portions of T. 18-20 N. Normal pressures prevail in rocks of various ages on the east side of the basin in blocks Q, W, and YY.

(5) In general, the top of overpressure deepens from north to south. The top of overpressure is here defined as the depth below which several points have pressure-depth ratios exceeding $0.5 \mathrm{psi} / \mathrm{ft}$. Examining the north to south progression in R. 11-14 W., the top of overpressure is located at depths of approximately $6,500 \mathrm{ft}$ in block $\mathrm{G}, 8,100 \mathrm{ft}$ in block K, $10,100 \mathrm{ft}$ in block $\mathrm{O}$, and $13,000 \mathrm{ft}$ in block $\mathrm{U}$. A similar deepening of the top of overpressure can be observed in blocks F, J, $\mathrm{N}$, and T in R. 15-18 W.

The top of overpressure was determined for each of the 28 blocks shown in the index map on plate 1. Because the top of overpressure is determined by the depth of the shallowest overpressured measurements, and because there may be overpressured rocks above that depth for which no pressure was determined, it is likely that the top of overpressure is shallower in those blocks with sparse data, but it is unlikely that the top of overpressure is deeper than the selected depth. The depth of the top of overpressure and the average surface elevation was determined for each of the 28 blocks, and the elevation of the top of overpressure relative to sea level was then computed. The resulting contour map (fig. 5), based on 28 points and with a limited extension into the Texas Panhandle, shows the basinward drop in the top of overpressure from elevations above $-6,000 \mathrm{ft}$ to elevations deeper $-10,000 \mathrm{ft}$. No comparable map was presented by Al-Shaieb and others (1994a), although the map in figure 5 appears compatible with the top of overpressure illustrated on a cross section (Al-Shaieb and others 1994a, figure 11).

Al-Shaieb and others (1994a) presented pressure-contour maps of the (1) Missourian/Virgilian interval (showing mostly normal pressures), (2) Desmoinesian Red Fork Sandstone (showing pressure-depth ratios exceeding $0.8 \mathrm{psi} / \mathrm{ft}$ ), (3) Morrowan Series (showing pressure-depth ratios exceeding $0.9 \mathrm{psi} / \mathrm{ft}$ ), and (4) Hunton Group (showing a return to normal pressure below the base of the Woodford Shale, with the exception of one overpressured value of $0.74 \mathrm{psi} / \mathrm{ft}$ attributed to an isolated Hunton compartment). Using their digital dataset augmented by pressure data from IHS Energy (2009), we 

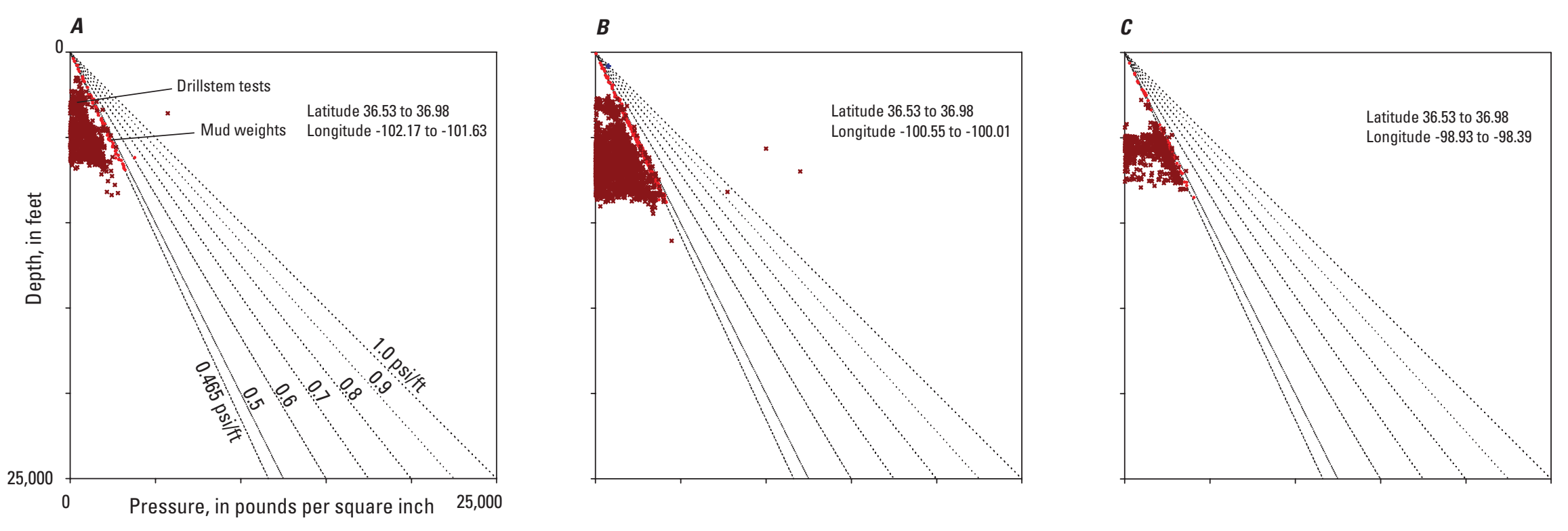

D
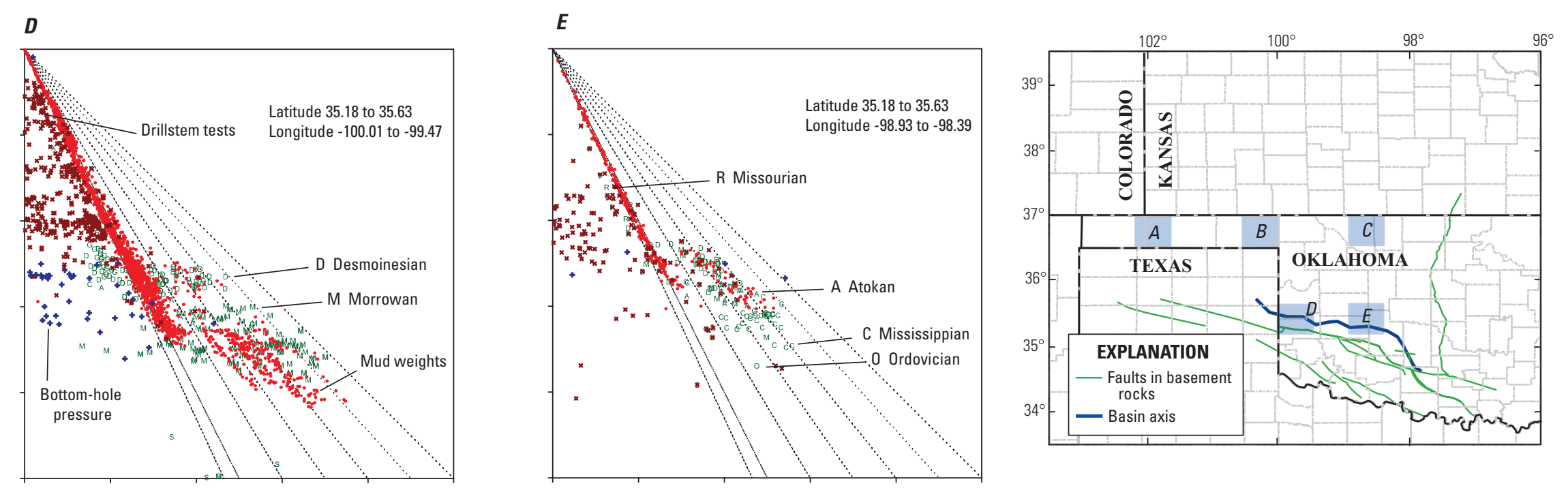

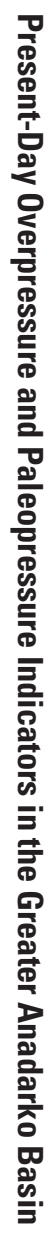

Figure 4. Plots of pressure against depth for five areas $A$ through $E$ in the greater Anadarko Basin. Each plot contains pressure data from wells within a 30 - by 30 -mile area located in the index map. Faults in basement rocks bound the eastern and southern parts of the basin. Pressure data designated by green letters representing geologic periods are from Al-Shaieb and others (1994a). Pressure data from drillstem tests, bottom-hole pressures, and mud weights are taken from IHS Energy (2009). 


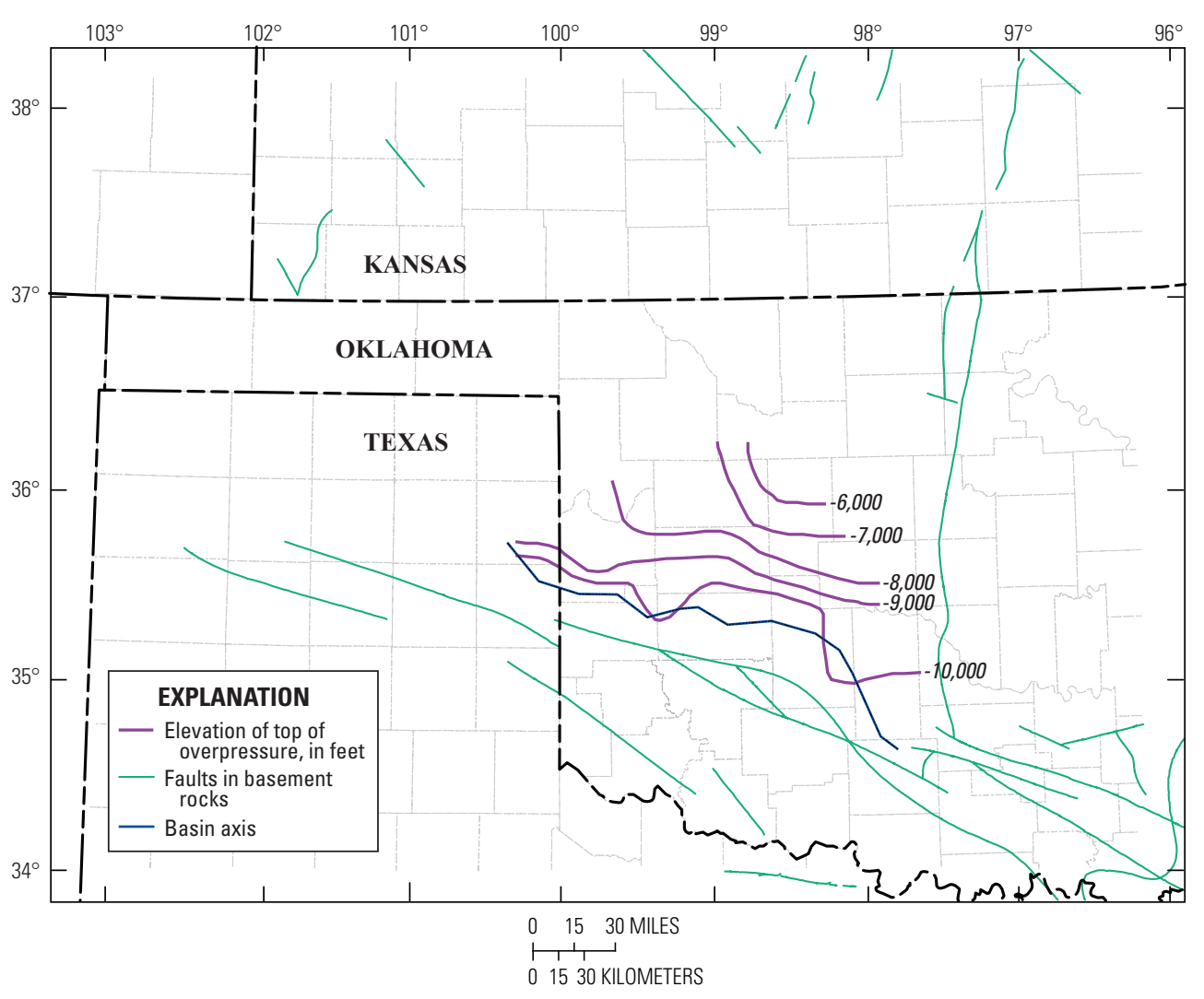

Figure 5. Map showing elevation of top of overpressure, based on data displayed in plate 1.

created outlines of present-day overpressure for the Morrowan and Desmoinesian series (figs. 6 and 7). The Desmoinesian outline (fig. 6), which includes pressures measured in rocks of Desmoinesian age, extends farther north and west than the area mapped by Al-Shaieb and others (1994a), which is restricted to pressures measured in the Desmoinesian Red Fork Sandstone. The outer boundary includes measurements with pressuredepth ratios greater than $0.5 \mathrm{psi} / \mathrm{ft}$ and the inner boundary shows the area of highest pressure with pressure-depth ratios greater than $0.7 \mathrm{psi} / \mathrm{ft}$. The southern edge of the outer boundary is drawn in close proximity to the bounding fault.

The Morrowan outline (fig. 7), which includes pressures measured in rocks of Morrowan and Springer age, follows the outline established by Al-Shaieb and others (1994a) except in Dewey County of Oklahoma, where the outline extends roughly 10 miles farther to the north. The eastern edge coincides with the truncation edge of Morrowan age rocks. The southern edge is drawn in close proximity to the bounding fault. Pressure compartments within the Morrow and Springer Formations (fig. $8 A$ ) were outlined by Powley (1984) in a study for Amoco. Amoco data were incorporated in the work of Al-Shaieb and others (1994a), and the boundaries of the two studies are similar. Moreover, Powley (1984) delineated nine pressure compartments within the Morrow and Springer Formations, each characterized by distinctive pressure-elevation relations with a unique potentiometric surface $H$ calculated from $H(\mathrm{ft})=Z(\mathrm{ft})+P(\mathrm{psi}) / 0.465$, where $Z$ is elevation and $P$ is pressure. Eight of the nine compartments are overpressured, as shown by values of $H$ that are greater than normal values of 2,000 to 3,500 ft (fig. 8A). Pressure-elevation plots for three of the nine areas demonstrate that differences in pressure between compartments is on the order of several thousand psi and that pressure gradients are around $0.465 \mathrm{psi} / \mathrm{ft}$ (fig. $8 B$ ). The determination of pressure-depth gradients of $0.465 \mathrm{psi} / \mathrm{ft}$, equivalent to the density of a moderately saline brine, shows that elevated hydrostatic gradients prevail over substantial areas within strata of Morrowan and Springer age. Thus, these overpressured compartments are water-dominated, and gas and oil accumulations within the compartments can be expected to be in buoyant equilibrium with water. 

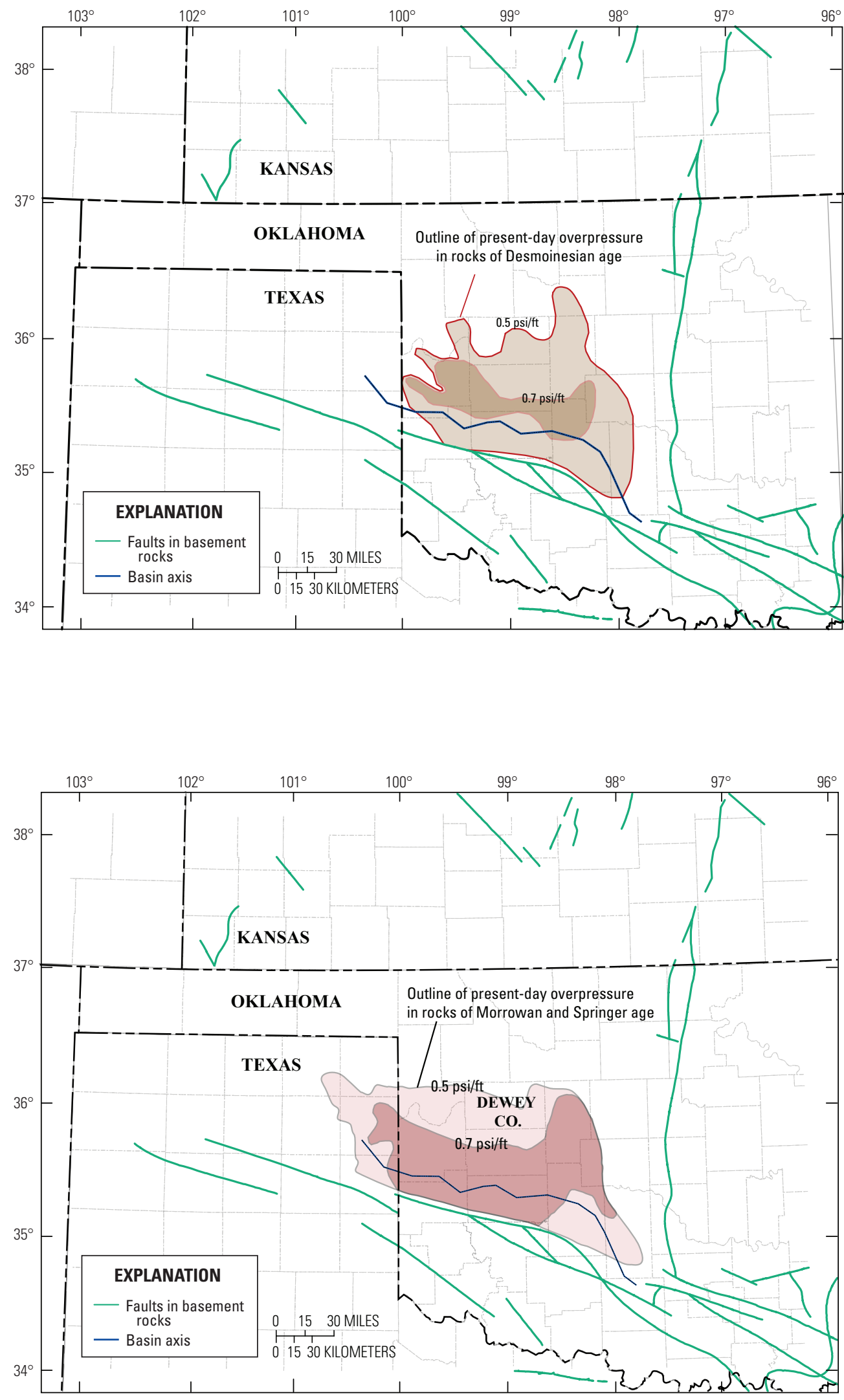

Figure 6. Map showing outline of present-day overpressure in rocks of Desmoinesian age, based on data from Al-Shaieb and others (1994a) and augmented with pressure data from IHS Energy (2009). Contour lines and shading represent the ratio of pressure to depth in pounds per square inch per foot (psi/ft).

Figure 7. Map showing outline of present-day overpressure in rocks of Morrowan and Springer age, based on data from Al-Shaieb and others (1994a) and augmented by pressure data from IHS Energy (2009). Contour lines and shading represent the ratio of pressure to depth in pounds per square inch per foot (psi/ft). 

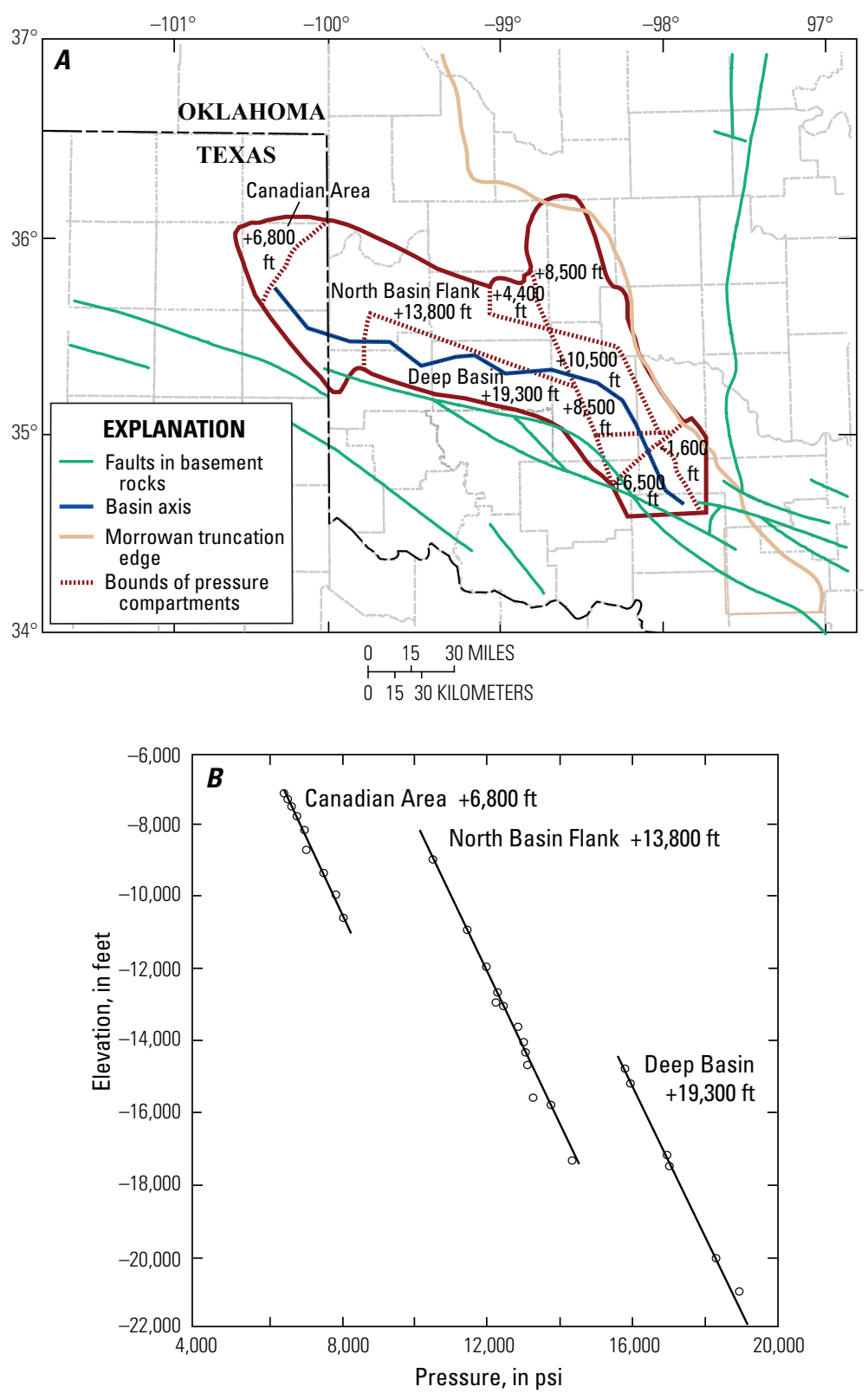

Figure 8. A, Nine overpressured compartments in Morrowan and Springer age rocks, from Powley (1984). Elevation in feet (ft) of potentiometric surface $\mathrm{H}$ is given for each area. $B$, Pressure as a function of elevation for three overpressured areas in Morrow and Springer Formations, from Powley (1984). Pressure data are from oil and gas fields and individual wells. Each line has a slope of 0.465 pounds per square inch per foot (psi/ft). 


\section{Indicators of Paleopressure from Previous Work}

In the course of investigating the cause of underpressure in the Morrowan sandstones of the northwestern Anadarko Basin, Breeze (1970) examined the characteristics of sonic logs, resistivity logs, and formation water salinity in six wells (fig. 9). The data from Breeze (1970), presented in the first three columns of figures $10 \mathrm{~A}-10 \mathrm{~F}$ of this chapter of the report, show increasing resistivity and decreasing sonic slowness ${ }^{1}$ of mudrocks in the upper part of each well, followed by reversals in these trends in the lower part of each well. The maximum resistivity at the top of the reversal in the six wells is about $10 \mathrm{ohm}-\mathrm{m}$, and the minimum sonic slowness is 70 to 75 $(\mu \mathrm{s} / \mathrm{ft})$. However, the density logs show a steady increase of density with depth with no reversals and hence do not show evidence of undercompaction (density logs shown by Breeze for the six wells of figures $10 A-10 F$ are not reproduced here).

${ }^{1}$ Sonic slowness, also called sonic travel time, is the inverse of sonic velocity. Slowness is measured by sonic logs and presented in units of microseconds per foot $(\mu \mathrm{s} / \mathrm{ft})$. The term "slowness" will be used throughout the remainder of this chapter of the report.
Pressure data, shown in the right-hand plot of figures $10 \mathrm{~A}-10 \mathrm{~F}$, behaves quite differently than the sonic and resistivity logs and formation water salinities. Present-day pressure varies from greater than hydrostatic (overpressured) in the Neely 1 well (fig. 10A), which is the southeasternmost of the six wells, to moderately overpressured in the CheyenneArapahoe Unit 1 well (fig. 10B), to normally pressured in the Raymond Moss and Knabe 1 wells (figs. 10C and 10D), to underpressured in the Kamp and Fox wells (figs. $10 E$ and $10 F)$. Thus, the present-day pressure ranges from overpressure to underpressure in a line of wells (fig. 9) in which the sonic and resistivity logs suggest overpressured conditions in mudrocks. Breeze (1970, p. 73) decided that the conditions producing the well log reversals differed from present-day conditions, "It is therefore concluded that the entire area was once subject to a similar history of deposition that left undercompacted shales as evidence." The thesis of this report follows the same line of reasoning.

The salinity of formation water, which is around $100,000 \mathrm{ppm}$ total dissolved solids in the upper part of each well, decreases in the lower part of each well to values about one-third of the values in the upper part (figs. 10A-10F). Water chemistry over a more extensive area was also reported by

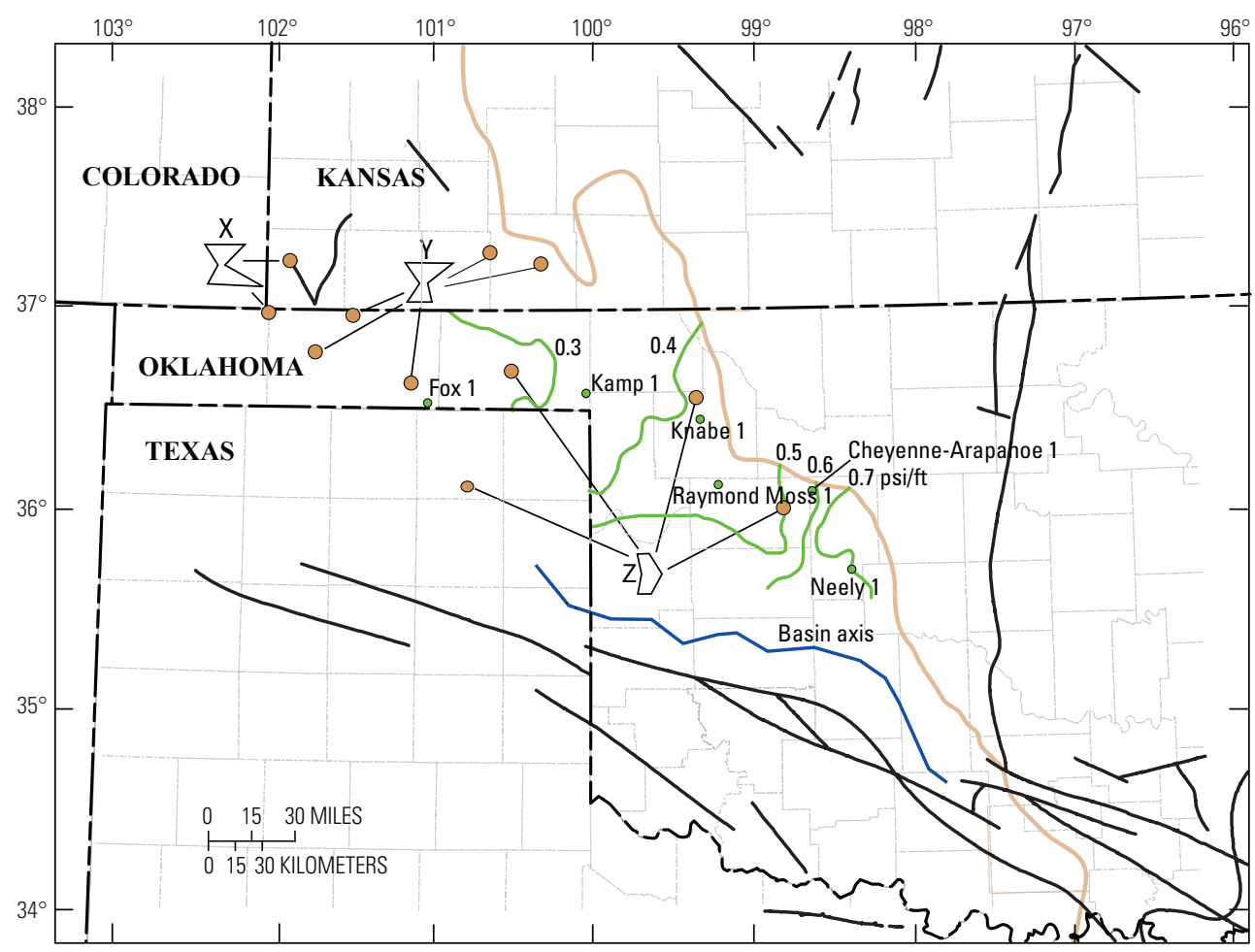

\footnotetext{
EXPLANATION

- Pressure-depth ratio in Morrow Formation in $\mathrm{psi} / \mathrm{ft}$

- Basin axix

Morrow truncation edge

- Locations of wells in figure 10

- Wells with water chemistry Stiff plot $\mathrm{Na} / 1,000>\mathrm{Cl} / 1,000$ $\left.\begin{array}{l}\mathrm{Ca} / 1,000 \\ \mathrm{Mg} / 100\end{array}\right\rangle\left\langle\begin{array}{l}\mathrm{CCO}_{3} / 10 \\ \mathrm{SO}_{4} / 10\end{array}\right.$
}

Figure 9. Map showing contours of present-day pressure-depth ratios in the Morrow Formation, showing change from overpressure [greater than 0.5 pounds per square inch per $\mathrm{ft}$ (psi/ft)] to underpressure (less than $0.4 \mathrm{psi} / \mathrm{ft}$ ), based on data from Breeze (1970). Six named wells (small green circles) show well-log indicators of paleopressure, as interpreted by Breeze (1970). Water chemistry falls into three distinct types as shown by Stiff plots labeled X, Y, and Z, from Dickey and Soto (1974). Each brown circle is the locus of several wells from which water samples were taken. 
A Well with overpressure and well log reversals

Hall-Jones Oil Corporation

Neely 1 sec. 9, T. 14 N., R. 11 W.

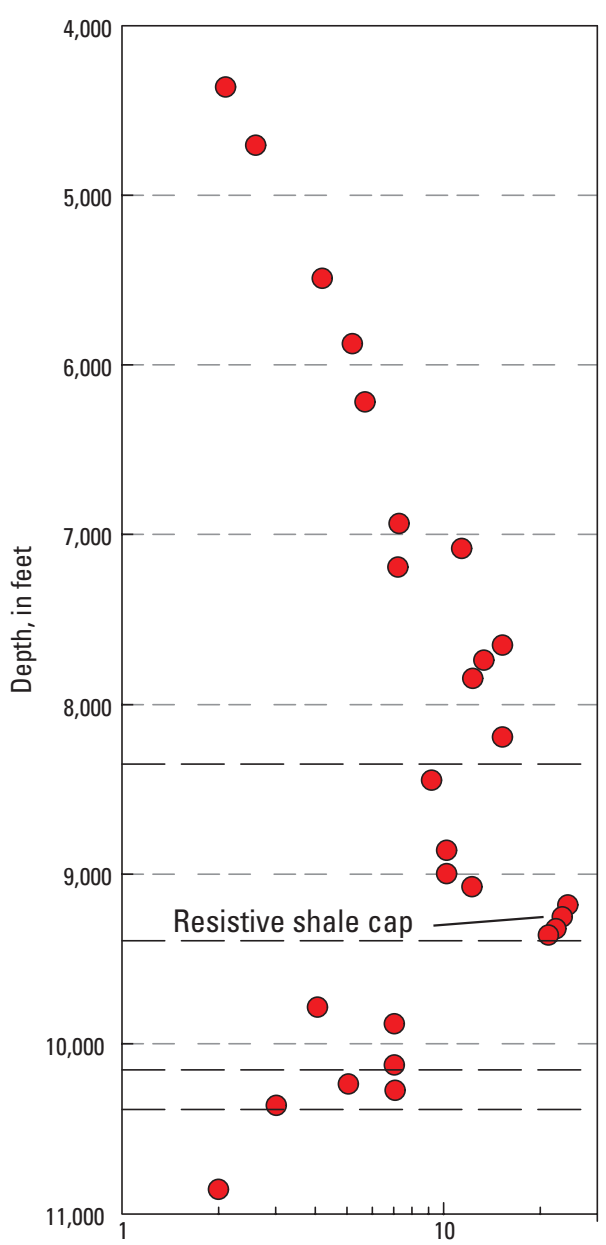

Resistivity, in ohm-meter

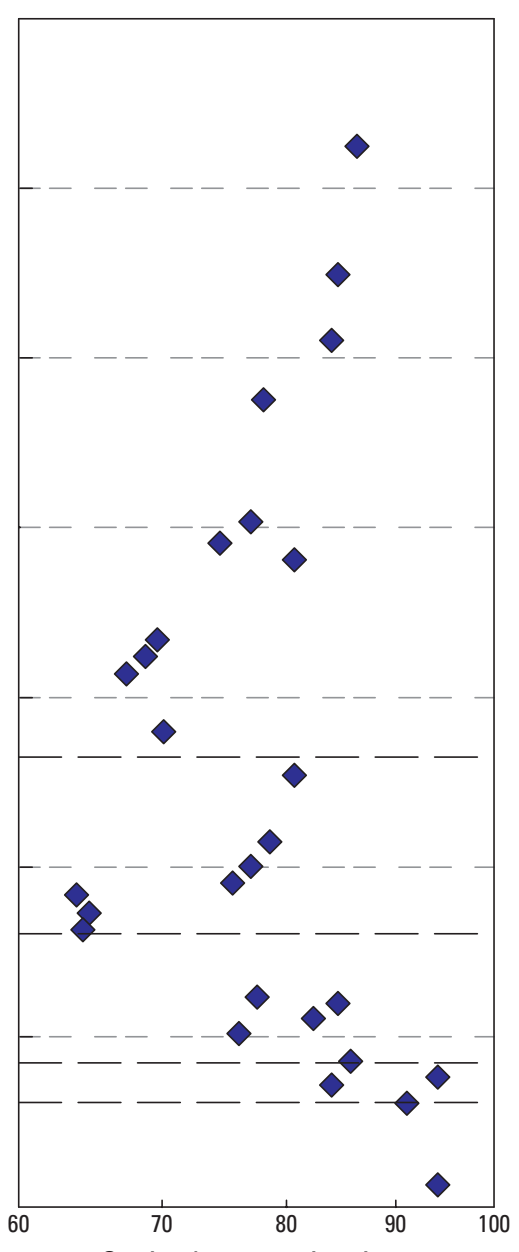

Sonic slowness, in micro seconds perfeet
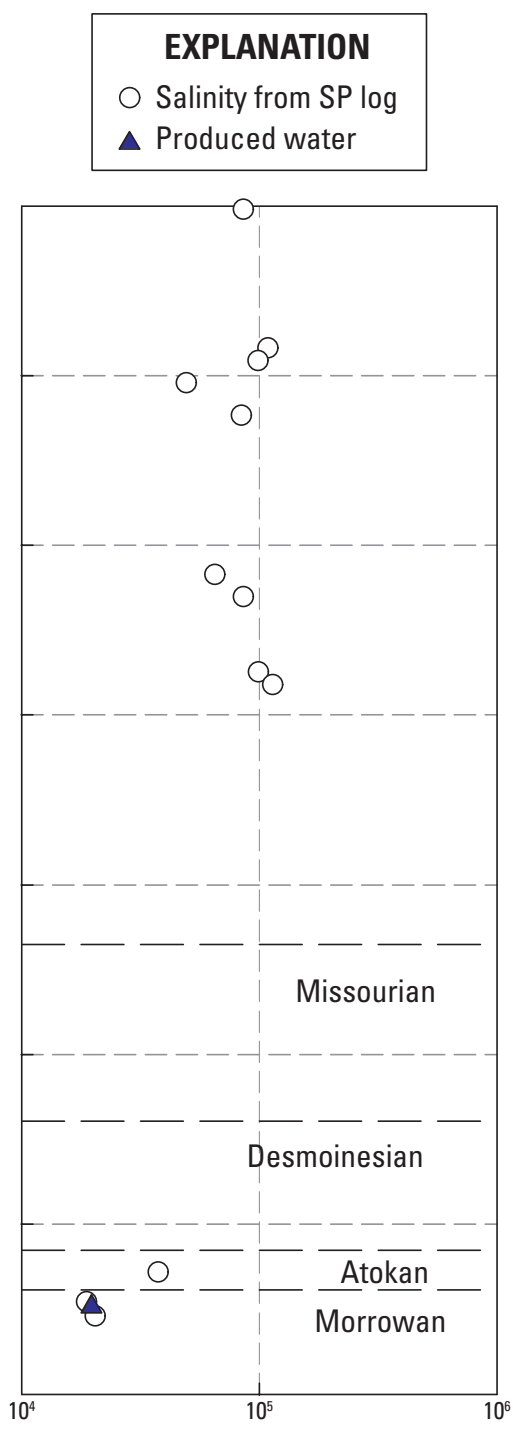

Water salinity, in parts per million of sodium chloride
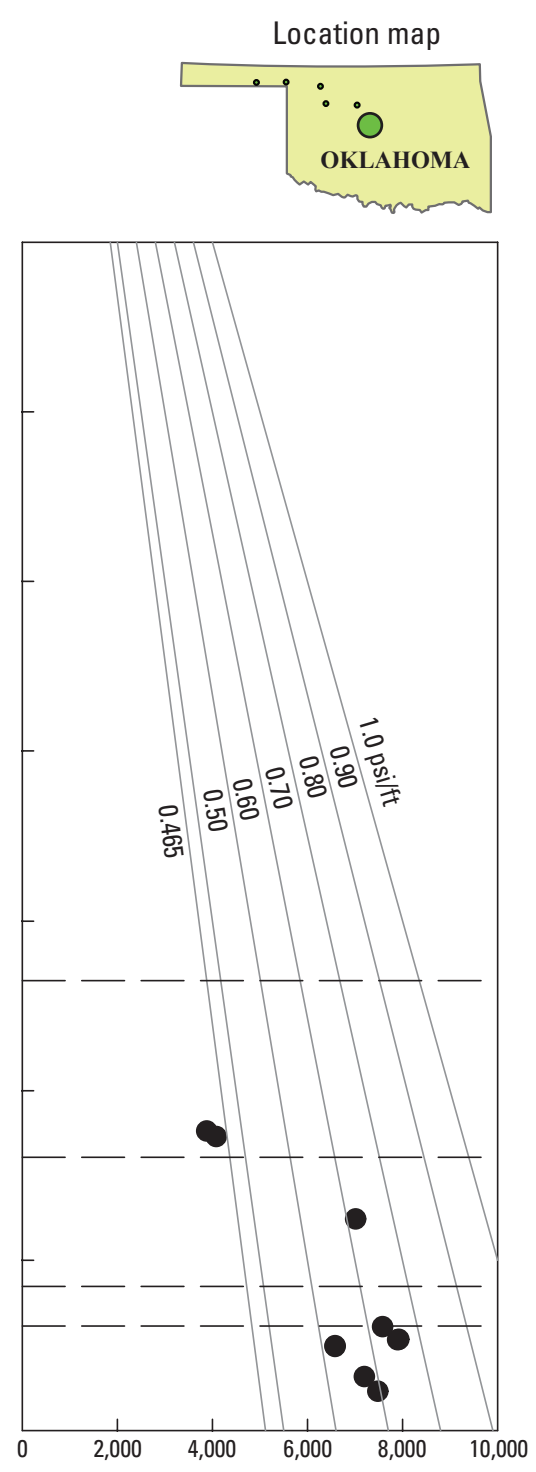

Pressure, in pounds per square inch

Figure 10. Graphs showing indicators of paleopressure in mudrocks from resistivity, sonic, and self potential (SP) logs in six wells from Breeze (1970). Well locations are shown in figure 9 and in the inset map. Salinity of produced water from a nearby well is also from Breeze (1970). Pressure data from wells within a 3to 10-mile distance from the designated well taken from Al-Shaieb and others (1994a). $A$, Neely 1 well; $B$, Cheyenne-Arapahoe Unit 1 well; $C$, Raymond Moss 1 well; $D$, Knabe 1 well; E, Kamp 1 well; and F, Fox 1 well. 
B Well with overpressure and well log reversals

Pan American Petroleum Corp.

Cheyenne-Arapaho Unit 1

Sec. 30 , T. 19 N., R. 13 W.

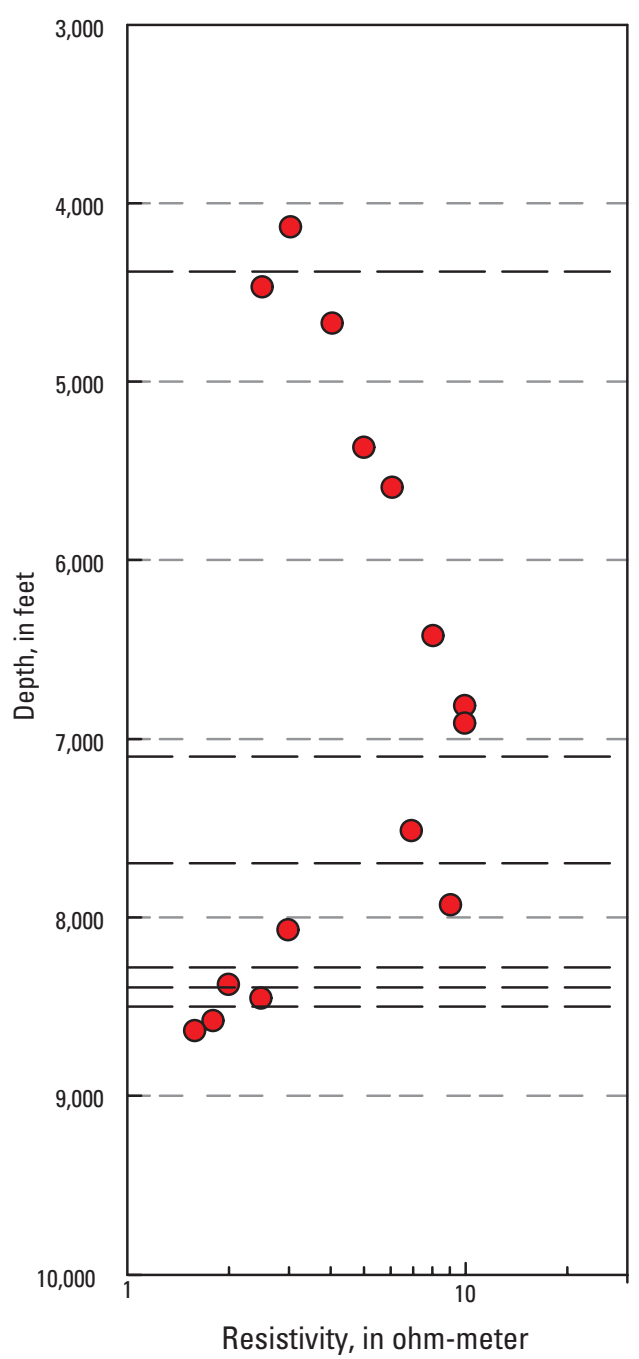

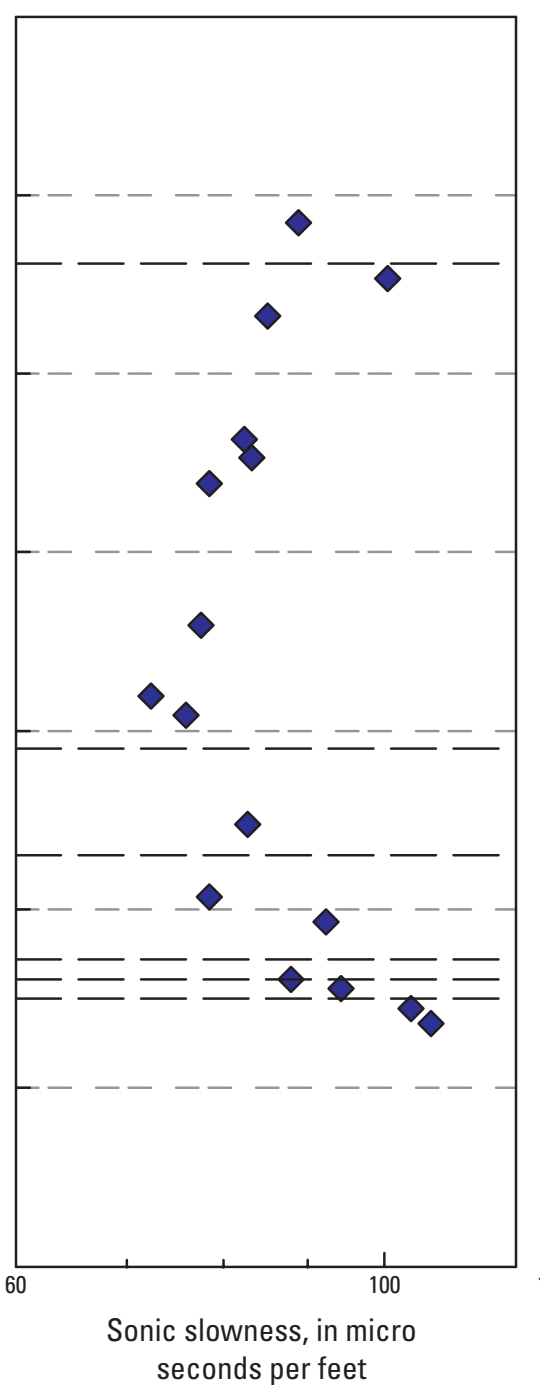

\section{EXPLANATION}

Salinity from SP log

$\triangle$ Produced water

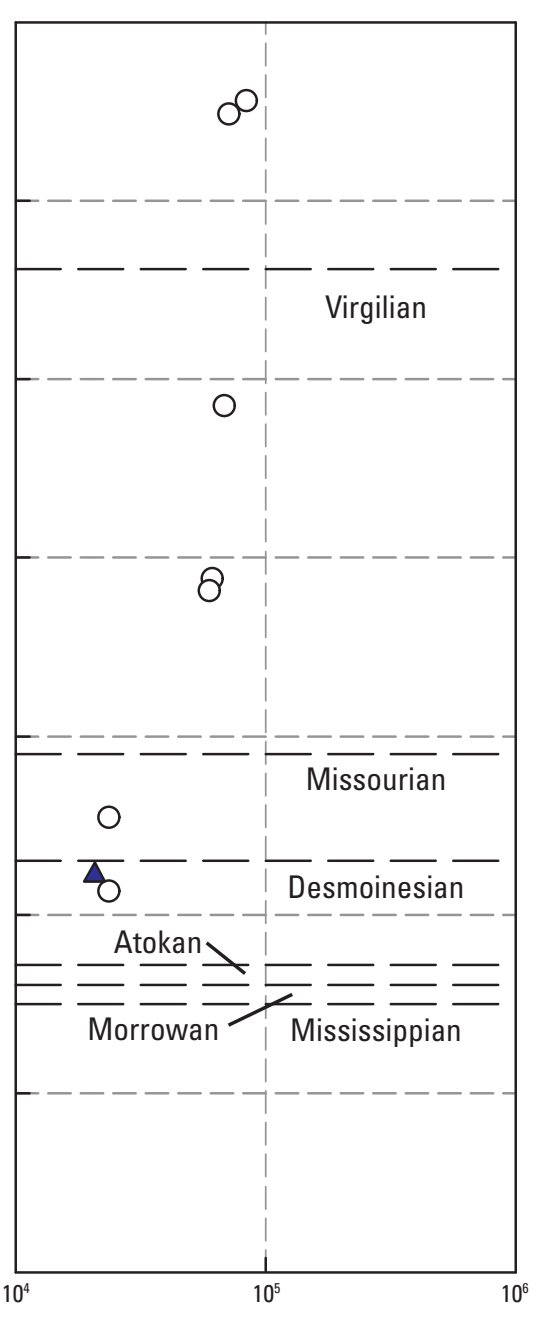

Water salinity, in parts per million of sodium chloride
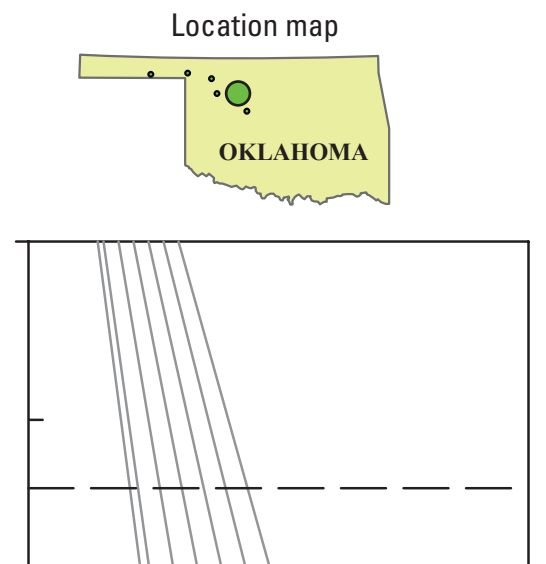

$10^{6}$

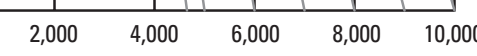

Pressure, in pounds per square inch

Figure 10. Graphs showing indicators of paleopressure in mudrocks from resistivity, sonic, and self potential (SP) logs in six wells from Breeze (1970). Well locations are shown in figure 9 and in the inset map. Salinity of produced water from a nearby well is also from Breeze (1970). Pressure data from wells within a 3- to 10-mile distance from the designated well taken from Al-Shaieb and others (1994a). A, Neely 1 well; $B$, Cheyenne-Arapahoe Unit 1 well; $C$, Raymond Moss 1 well; $D$, Knabe 1 well; $E$, Kamp 1 well; and $F$, Fox 1 well._-Continued 
C Well with normal pressure and well log reversals

Continental Oil Corporation

Raymond Moss 1

Sec. 16, T. 19 N., R. 19 W.
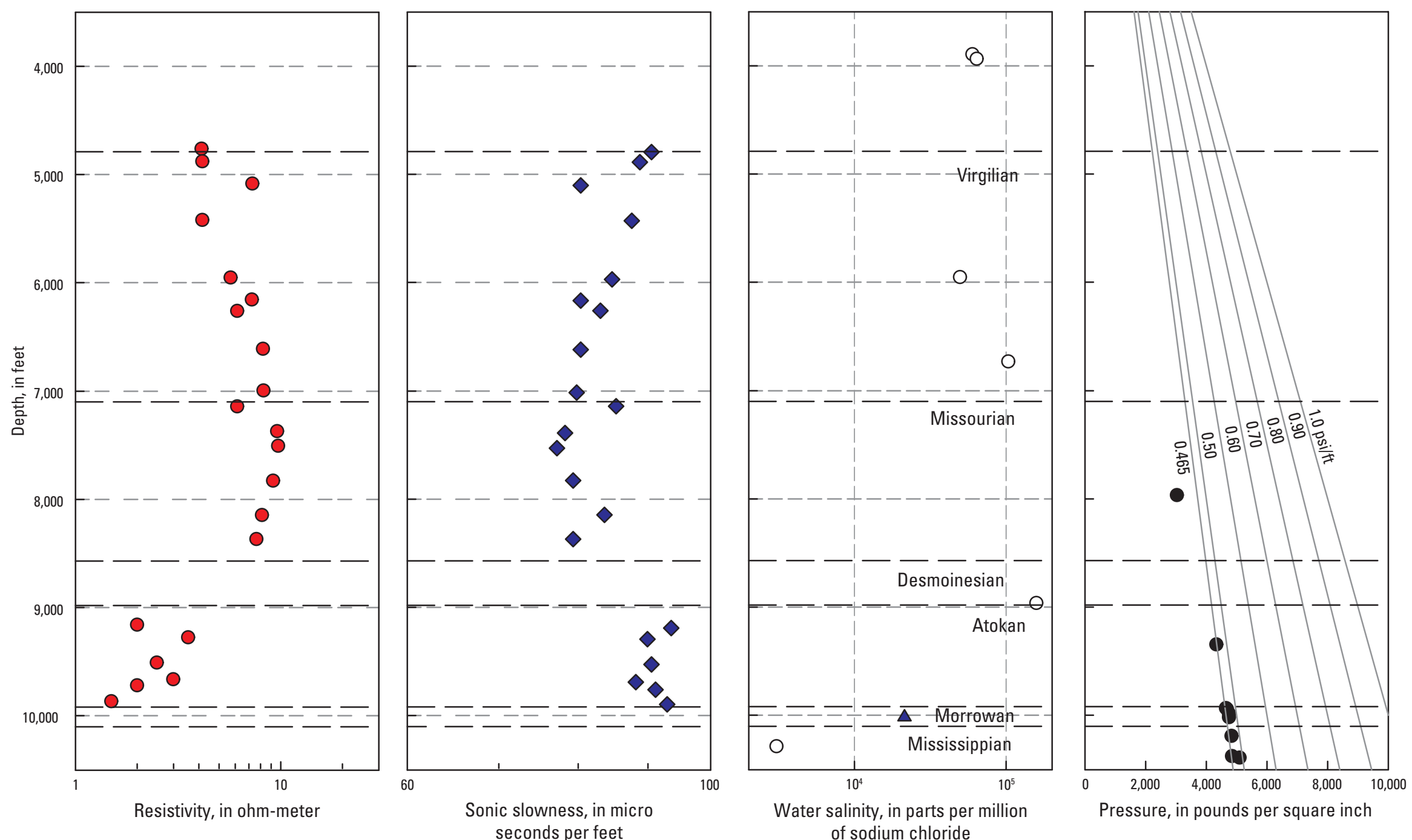

Figure 10. Graphs showing indicators of paleopressure in mudrocks from resistivity, sonic, and self potential (SP) logs in six wells from Breeze (1970). Well locations are shown in figure 9 and in the inset map. Salinity of produced water from a nearby well is also from Breeze (1970). Pressure data from wells within a 3- to 10-mile distance from the designated well taken from Al-Shaieb and others (1994a). $A$, Neely 1 well; $B$, Cheyenne-Arapahoe Unit 1 well; $C$, Raymond Moss 1 well; $D$, Knabe 1 well; E, Kamp 1 well; and F, Fox 1 well.-Continued 
D Well with normal pressure and well log reversals

W. G. Rogers Knabe 1

Sec. 22 , T. 23 N., R. 20 W.

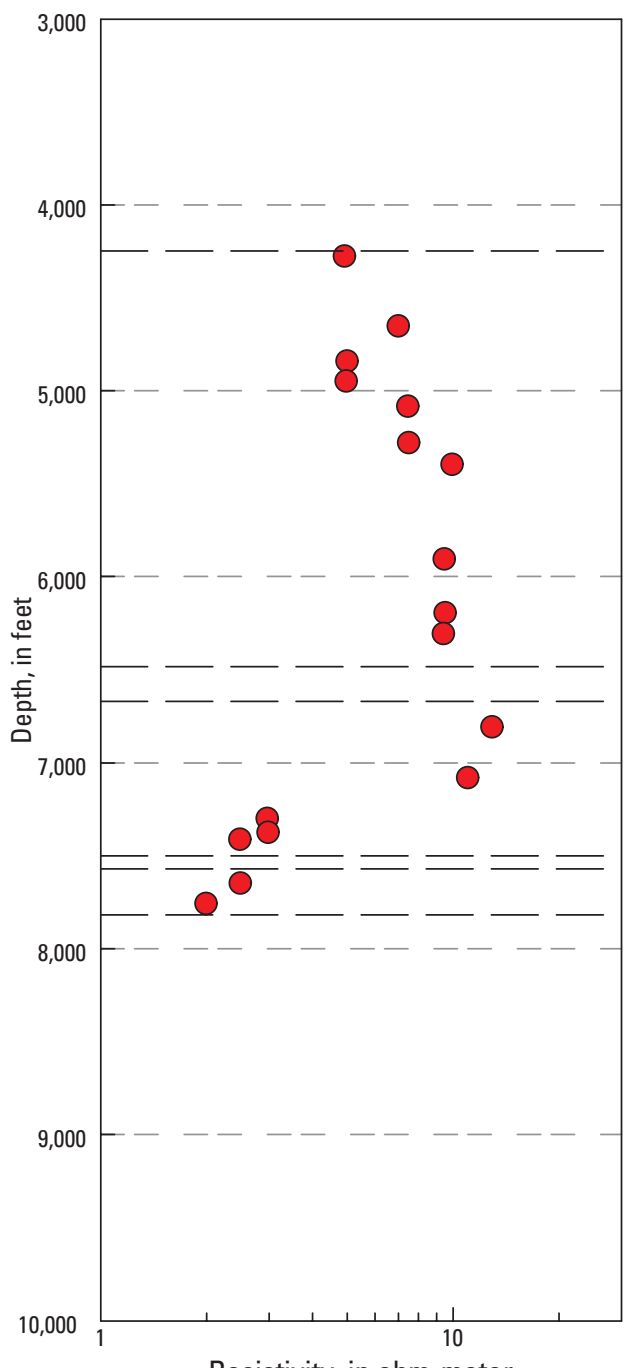

Resistivity, in ohm-meter

\section{EXPLANATION}

O Salinity from SP log

$\triangle$ Produced water

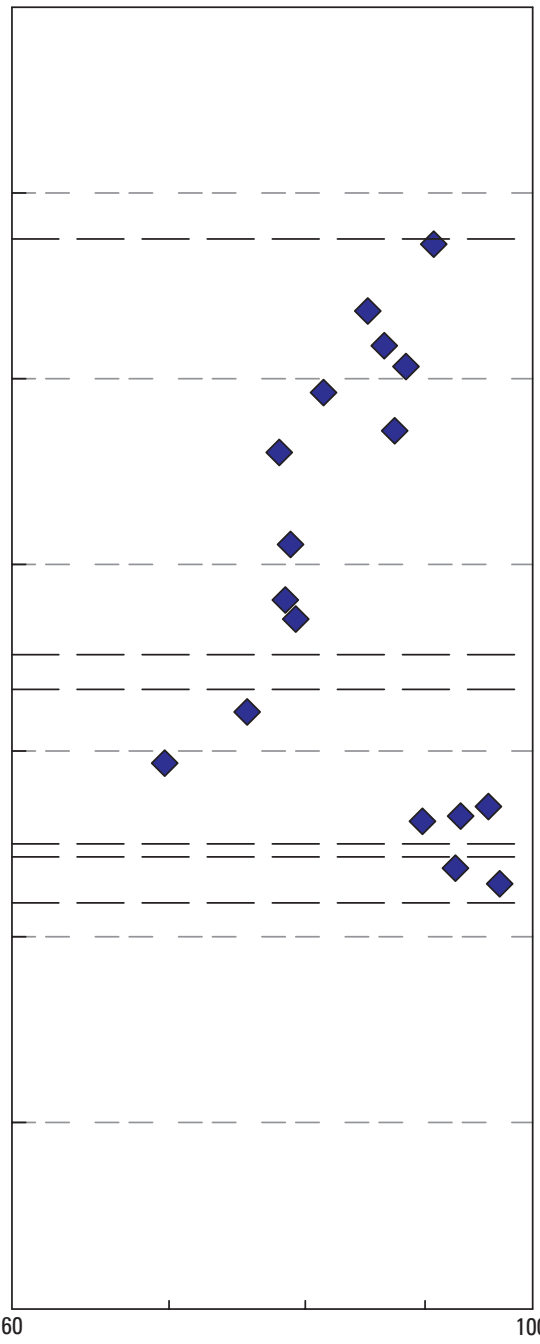

Sonic slowness, in micro seconds per feet
$10010^{4}$

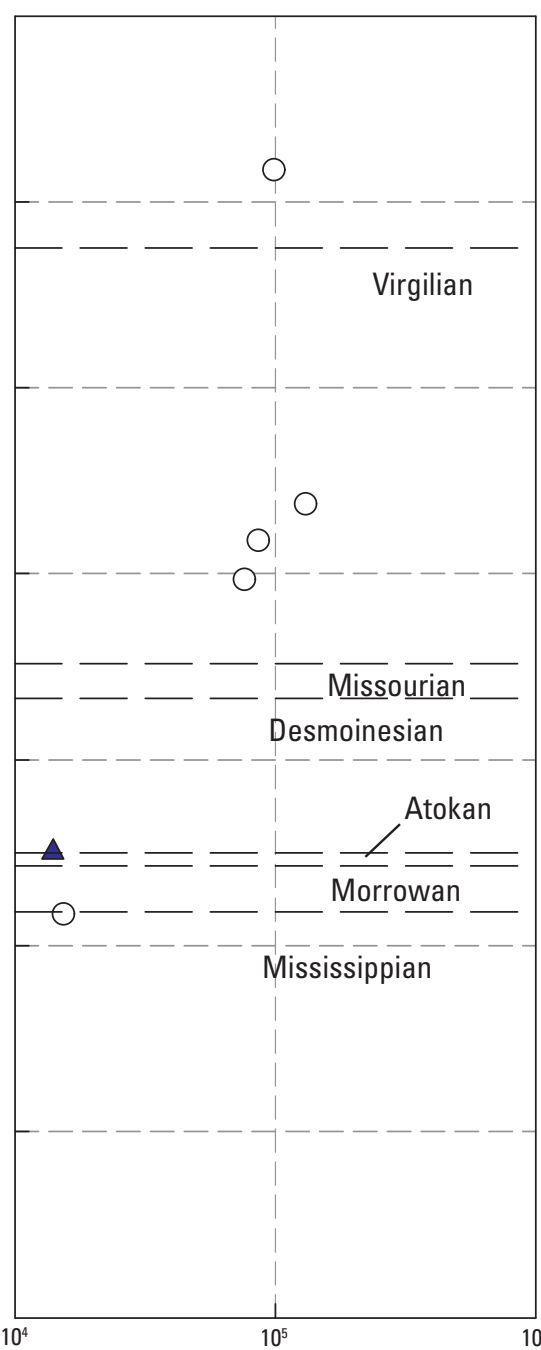

Water salinity, in parts per million of sodium chloride

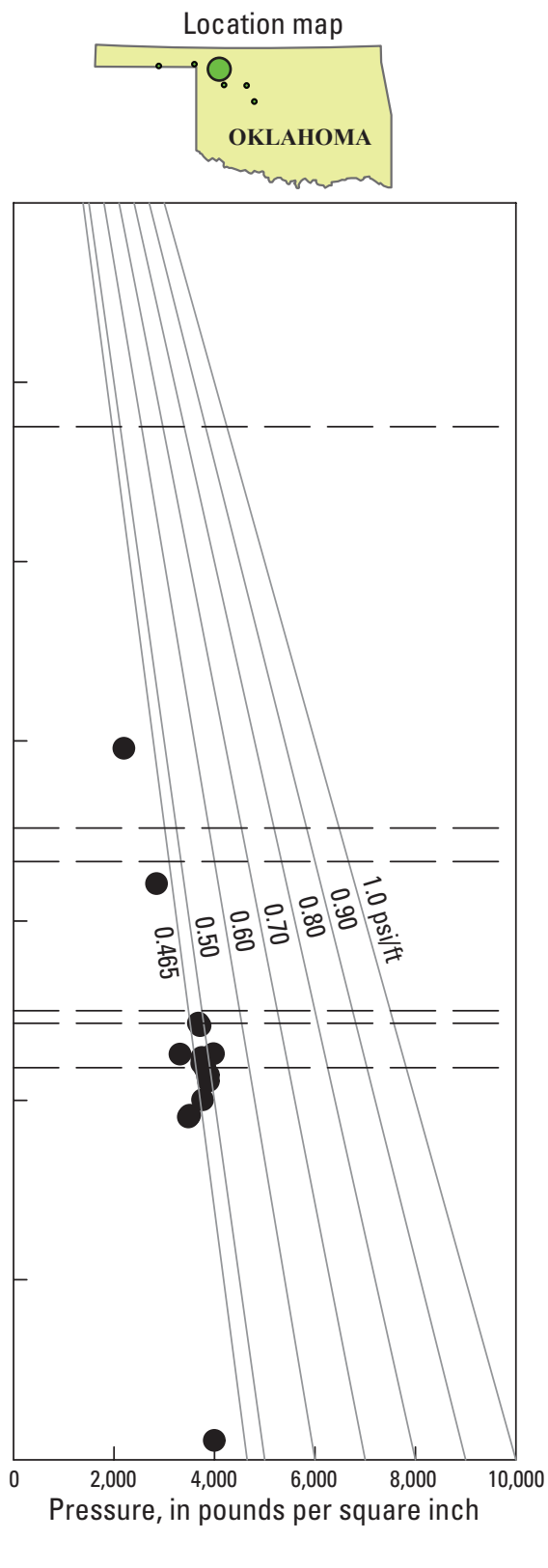

$\overrightarrow{+}$

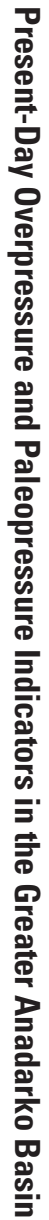

Figure 10. Graphs showing indicators of paleopressure in mudrocks from resistivity, sonic, and self potential (SP) logs in six wells from Breeze (1970). Well locations are shown in figure 9 and in the inset map. Salinity of produced water from a nearby well is also from Breeze (1970). Pressure data from wells within a 3- to 10-mile distance from the designated well taken from Al-Shaieb and others (1994a). $A$, Neely 1 well; $B$, Cheyenne-Arapahoe Unit 1 well; $C$, Raymond Moss 1 well; $D$, Knabe 1 well; $E$, Kamp 1 well; and F, Fox 1 well.-Continued 
$\boldsymbol{E}$ Well with underpressure and well log reversals

Phillips Petroleum Corp.

Kamp 1

Sec. 3, T. 1 N., R. 28 E.

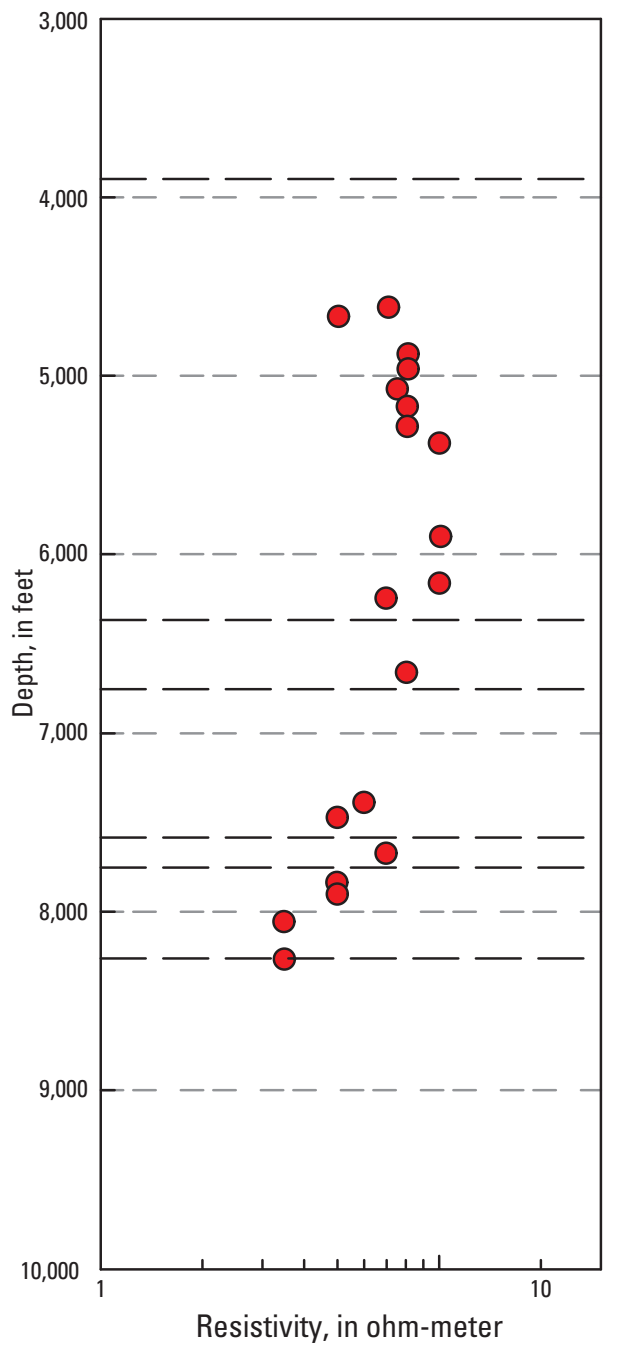

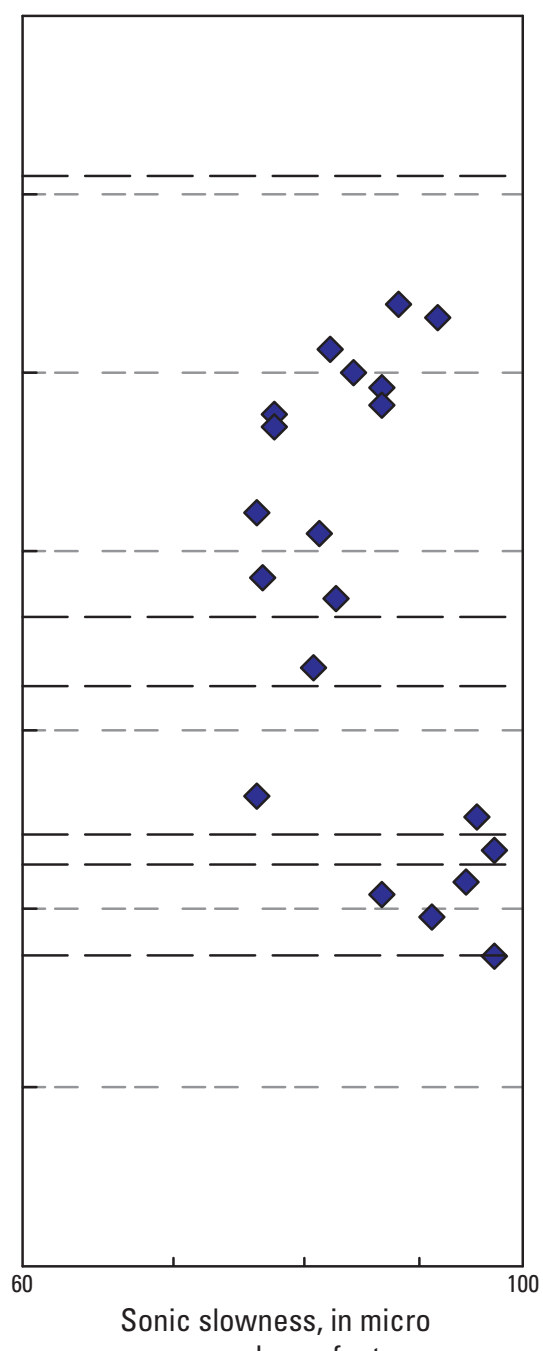

EXPLANATION

Salinity from SP log

- Produced water

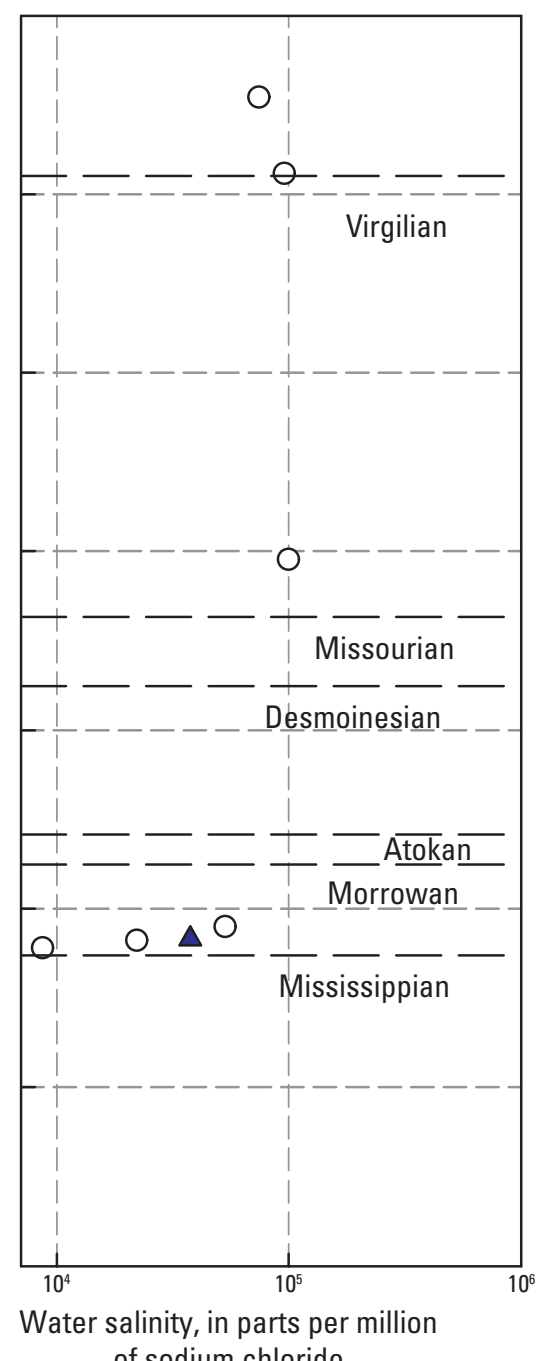

Location map
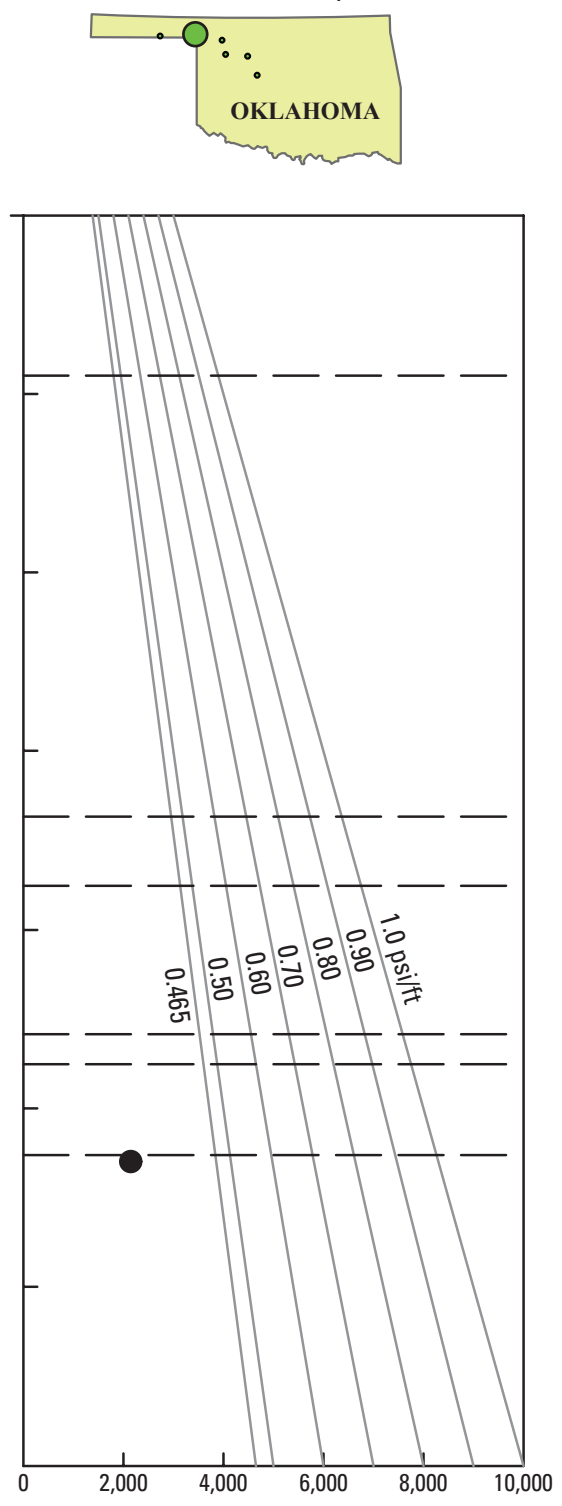

Pressure, in pounds per square inch

Figure 10. Graphs showing indicators of paleopressure in mudrocks from resistivity, sonic, and self potential (SP) logs in six wells from Breeze (1970). Well locations are shown in figure 9 and in the inset map. Salinity of produced water from a nearby well is also from Breeze (1970). Pressure data from wells within a 3- to 10-mile distance from the designated well taken from Al-Shaieb and others (1994a). $A$, Neely 1 well; $B$, Cheyenne-Arapahoe Unit 1 well; $C$, Raymond Moss 1 well; $D$, Knabe 1 well; E, Kamp 1 well; and F, Fox 1 well.-Continued 
$\boldsymbol{F}$ Well with underpressure and well log reversals

Horizon Oil and Gas Co.

Fox 1

Sec. 28, T. 1 N., R. 19 E.

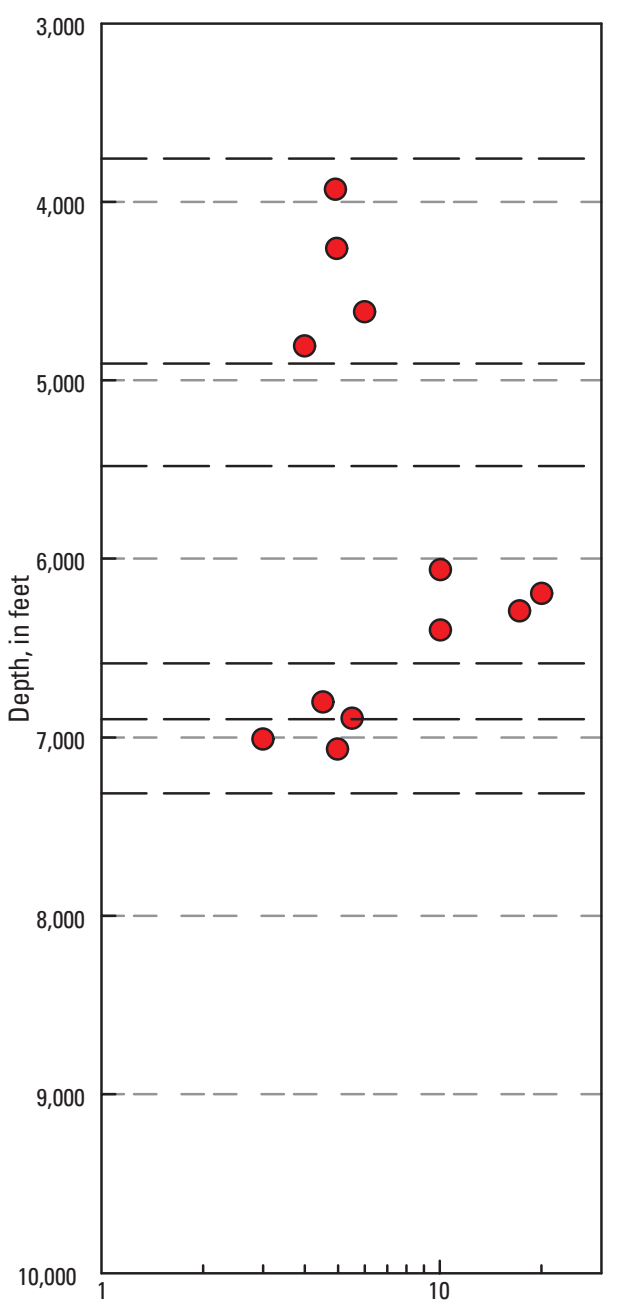

Resistivity, in ohm-meter

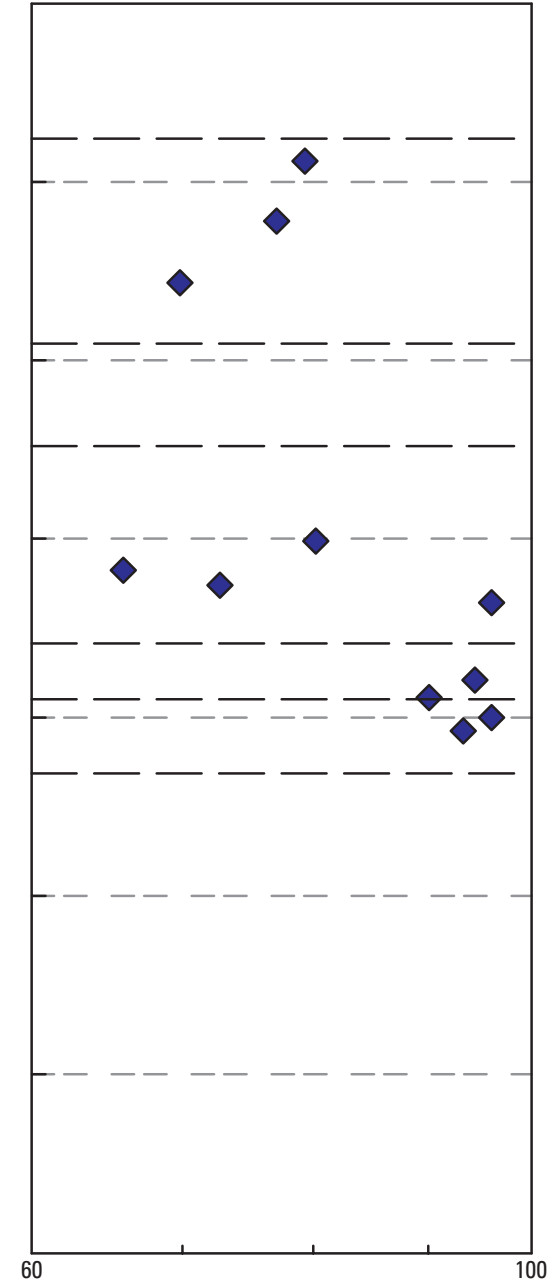

Sonic slowness, in micro seconds per feet
EXPLANATION

Salinity from SP log

$\Delta$ Produced water

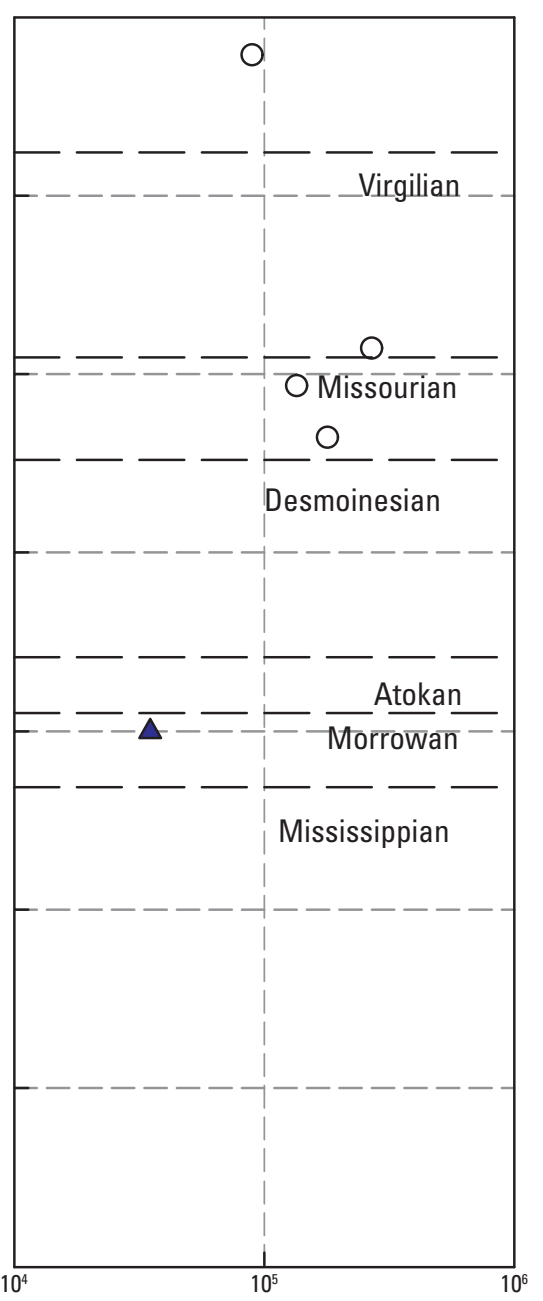

Water salinity, in parts per million of sodium chloride

Figure 10. Graphs showing indicators of paleopressure in mudrocks from resistivity, sonic, and self potential (SP) logs in six wells from Breeze (1970). Well locations are shown in figure 9 and in the inset map. Salinity of produced water from a nearby well is also from Breeze (1970). Pressure data from wells within a 3- to 10-mile distance from the designated well taken from Al-Shaieb and others (1994a). $A$, Neely 1 well; $B$, Cheyenne-Arapahoe Unit 1 well; $C$, Raymond Moss 1 well; $D$, Knabe 1 well; E, Kamp 1 well; and F, Fox 1 well.-Continued 
Dickey and Soto (1974), as shown by the Stiff diagrams in figure 9. Water types X, Y, and Z of figure 9 were defined by Dickey and Soto (1974) to distinguish three compositional groups. Water chemistry changes from moderate salinity $(30,000-80,000 \mathrm{mg} / \mathrm{L})$ sulfate waters of the chloride-calcium type $\mathrm{X}$ in the northwest, to typical connate high-salinity $(100,000-200,000 \mathrm{mg} / \mathrm{L})$ brines of the chloride-calcium type $\mathrm{Y}$, to a low-salinity $(10,000-30,000 \mathrm{mg} / \mathrm{L})$ high-bicarbonate type $Z$. The type $Z$ samples are located in the area studied by Breeze. Dickey and Soto (1974, p. 116) attempted to explain the presence of waters of a similar chemistry (type Z) throughout the transition from apparent overpressure to underpressure (fig. 9), first stating that "if the type $\mathrm{Z}$ water is meteoric in origin, it cannot be circulating now," and then concluding that "the abnormally high pressure in the Morrow sand and the presence of the dilute type $\mathrm{Z}$ water in that area indicate that they have been effectively isolated hydraulically. The relatively dilute waters, therefore, may not be of meteoric origin, but rather water somehow removed from the normal processes of concentration." In summary, both water chemistry and petrophysical properties reflected in the well logs indicate that the current pressure state of the Morrow Formation differs from an earlier condition.

\section{Indicators of Paleopressure from Reduction in Resistivity}

Following the finding by Breeze (1970), we examined resistivity logs from the Anadarko deep basin and shelf in Oklahoma, Texas, and Kansas. Our collection of sonic logs was not adequate for interpreting indicators of paleopressure, but the resistivity logs provided enough coverage and consistency that a shale trendline could be determined.

\section{Procedure}

Approximately 175 resistivity logs were inspected for indications of paleopressure. A resistivity trendline was established in each well based on the resistivity of mudrocks in strata below the evaporites and limestones of Permian age (figs. 11and 12). Mudrocks were identified by high gamma-ray and high neutron porosity readings, which were made distinguishable by shading the gamma-ray log where it exceeded 70 American Petroleum Institute (API) units and by shading the neutron log where it exceeded a neutron porosity of 20 percent - this rule of thumb was varied somewhat in wells where the gamma-ray or neutron log required shifting. The resulting trendline represents the normal resistivity compaction trend for that well. In many wells, the resistivity log follows the trendline without a reversal throughout the Pennsylvanian siliciclastic sequence, as in the example of the West Edmond well (fig. 11). The resistivity is less than the trendline in sandstones, as indicated by yellow shading in the resistivity curve, but not in the mudstones. Because no resistivity reversal is discernible in the West Edmond well, rocks in this area were either never overpressured or else the paleopressure signature was not retained. The density log also increases steadily with depth, also indicating a normal compaction trend.

In other wells, the resistivity trend in mudstones reverses and decreases with depth rather than continuing to increase along the trendline. The depth of the first clear separation between the resistivity log and the trendline is selected and recorded; this depth is considered to be the top of paleopressure. For example, in the Bredy well (fig. 12), the top of paleopressure is located at a depth of 9,000 ft. Resistivity then decreases further with depth below the top of paleopressure although the trendline indicates that resistivity would increase in the absence of the effect of paleopressure; the separation between the two is made visible by the red shading. Within the Springer Formation at $14,800 \mathrm{ft}$, resistivity in mudstones is around $2 \mathrm{ohm}-\mathrm{m}$ where the trendline value is greater than $10 \mathrm{ohm}-\mathrm{m}$. The sonic slowness in mudrocks tends to be higher within the paleopressured zone rather than lower as it would be under a normal compaction gradient. For example, at depths of 7,000 and 8,400 ft, sonic slowness is around $80(\mu \mathrm{s} / \mathrm{ft})$, but in deeper zones where resistivity is less than the resistivity trendline, as at 12,300 and $14,300 \mathrm{ft}$, the sonic slowness is around $90 \mu \mathrm{s} / \mathrm{ft}$.

After eliminating wells that were not suitable for our study because of incomplete well logs or insufficient depth of penetration, 175 wells were used in this study. Of these, 68 wells displayed no reversal in the resistivity log, that is, the resistivity of mudrocks increased steadily along the trendline determined for that well. Wells in which the resistivity reversal is absent establish the eastern, western, and northern limits of the area of paleopressure. The top of paleopressure was established in the remaining 107 wells and based on formation tops for each well, the approximate geologic age of the top of paleopressure was assigned in terms of Pennsylvanian provincial series (Morrowan, Atokan, Desmoinesian, Missourian, or Virgilian). Formation tops of Springer age were included within the Morrowan Series. Twenty-four wells in the northwestern part of the study area display reduced resistivity values in the Morrow Formation but not above or below the Morrow.

Selected resistivity logs are presented in map format on plate 2 to provide an overview of the resistivity variations throughout the Anadarko Basin. The geographic variation of resistivity and paleopressure indicators can also be examined on one west-east (pl. 3) and three south-north cross sections (pls. 4-6). The tops of one formation boundary and four provincial series boundaries are also shown on plates 3-6, along with a line labeled "top of paleopressure," that marks the shallowest depth where resistivity falls below the trendline value. The locations and elevations of the resistivity reversals for the 44 wells on the four cross sections in plates 3-6 are given in Appendix 1.

The top of the reversal in resistivity could be picked with a high level of certainty in some wells but with less certainty in other wells, depending on such factors as the thickness and abundance of mudrock units, the presence and quality of the neutron $\log$, and the slope of the trendline. For example, of the 13 wells in Line $C-C^{\prime}$ (pl. 5), inspection shows that the top of 
Well A - West Edmond SWD 1-24

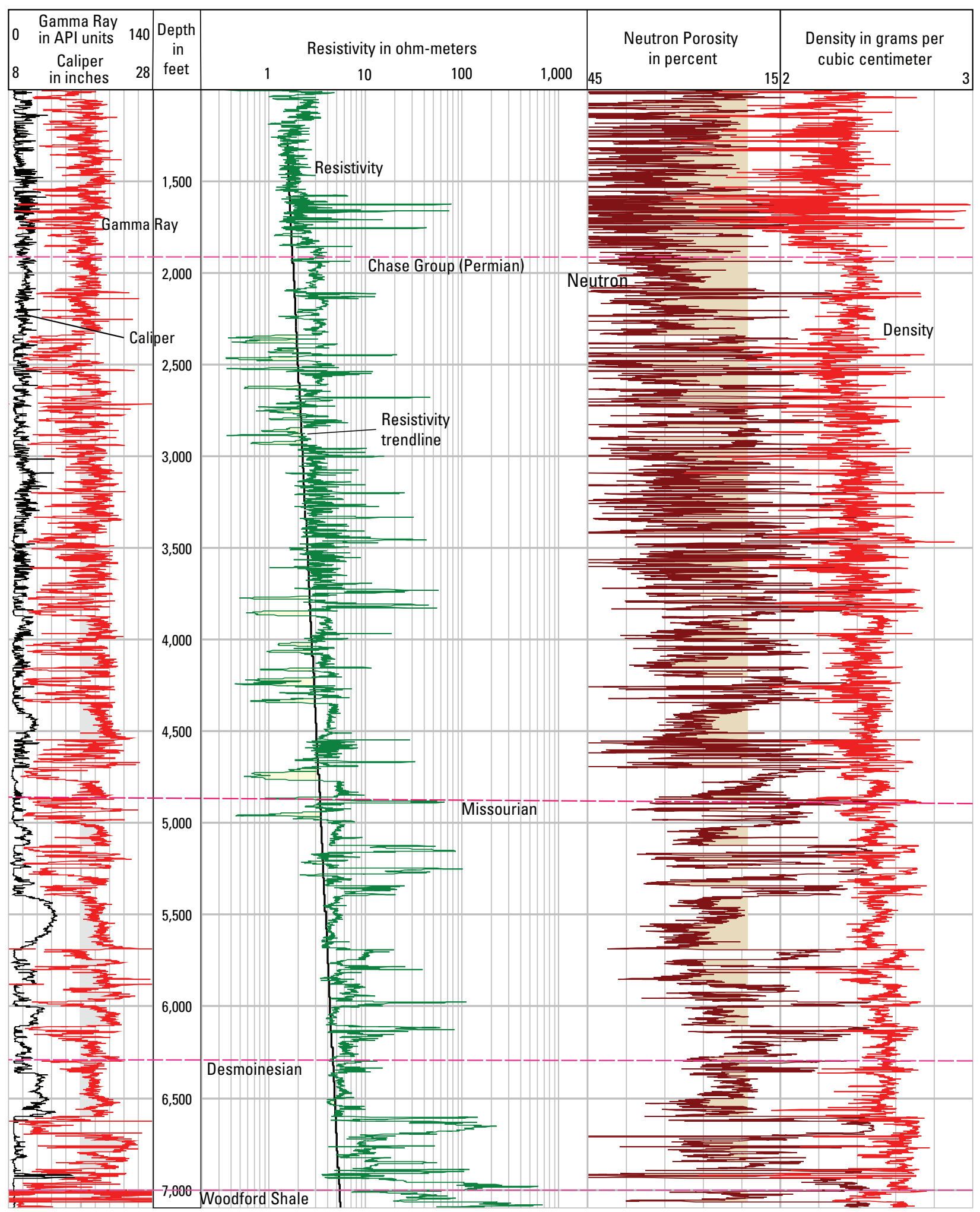

Figure 11. Gamma-ray, resistivity, neutron, and density logs in the West Edmond SWD 1-24 well (location shown on plate 2). Resistivity trendline is based on lowest resistivity values in mudrocks, which are represented by gamma-ray values in excess of 70 American Petroleum Institute (API) units (gray shading) and neutron porosity in excess of 20 percent (brown shading). Low resistivity in sandstones (light yellow shading) is attributed to saline water in the pore space. In this well, resistivity and density in mudrocks increase steadily with depth and there is no indication of paleopressure. 
Well B - Bredy 1-6

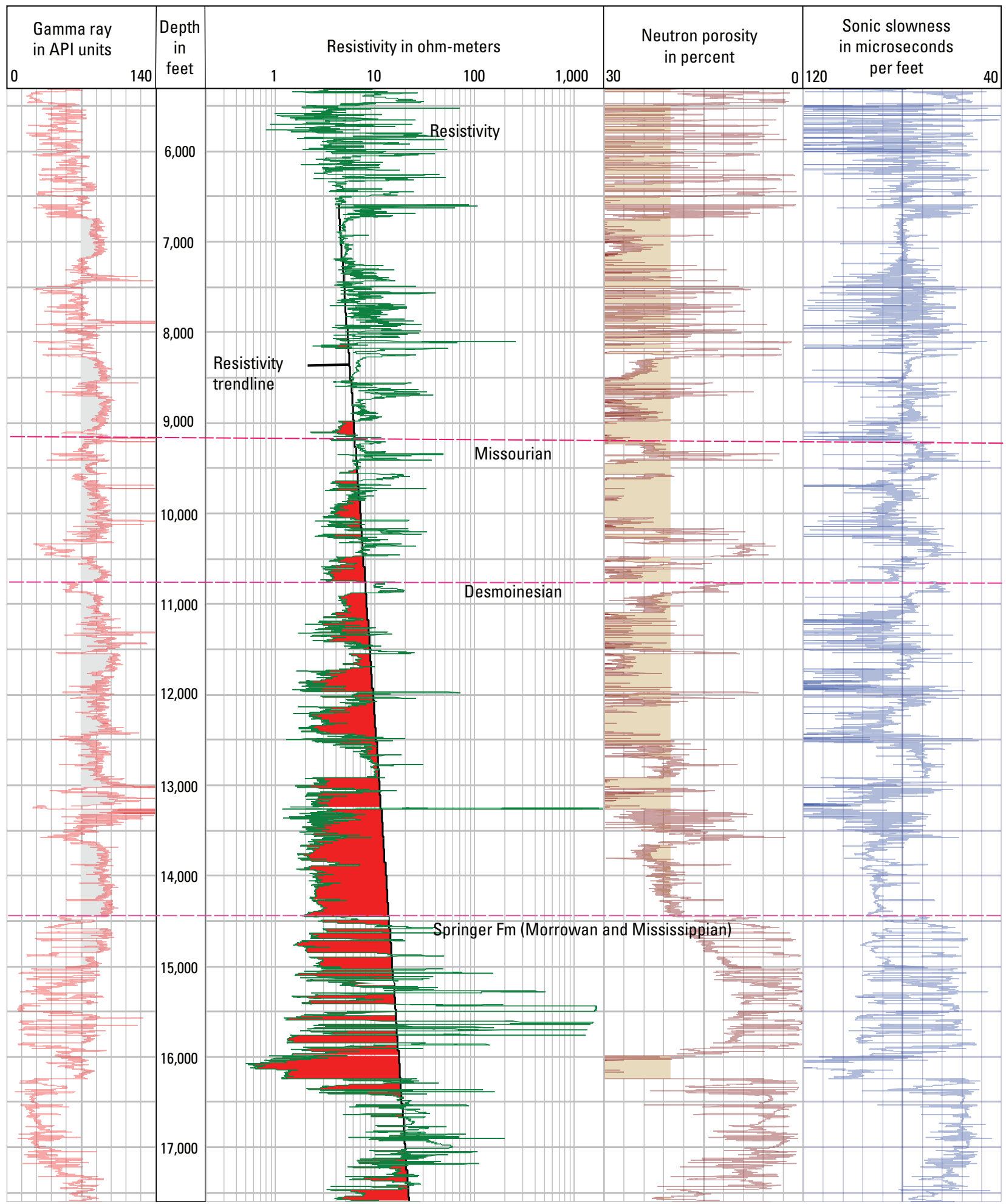

Figure 12. Gamma-ray, resistivity, neutron, and sonic logs in the Bredy well. The resistivity trendline is based on the lowest resistivity values in mudrocks above 9,000 feet, which are represented by gamma-ray values in excess of 70 American Petroleum Institute (API) units (gray shading) and neutron porosity in excess of 20 percent (brown shading). In this well, the top of paleopressure is at 9,000 feet. Below the top of paleopressure, resistivity in mudrocks decreases with increasing depth and sonic slowness in mudrocks increases with depth. 
the reversal could not have been picked at a shallower depth in 6 wells, but could have been picked at a shallower depth in the other 7 wells, with a range of 147 to $710 \mathrm{ft}$. Similarly, the top of the reversal could not have been picked at a deeper depth in 5 wells but could have been picked at a deeper depth in the other 8 wells, with a range of 64 to $297 \mathrm{ft}$. Because the errors are not normally distributed, it is difficult to estimate an overall error, but it does appear that a typical error is several hundred feet in about half of the wells.

\section{Results}

The geographic overview (pl. 2) shows how the paleopressure indicator varies with well position in the basin. The red shading between the trendline and the resistivity $\log$ indicates the presence of mudrocks, as determined by high gamma-ray (typically greater than 70 API units) or high neutron porosity (typically greater than 20 percent) readings. Resistivity is more reduced with respect to the trendline in the deeper part of the basin than on the flanks. Two maps show the elevation of the top of paleopressure (fig. 13) and the age of formations of the top of paleopressure (fig. 14).

The slope of the trendline is steepest on the flanks of the basin and not as steep in the deep basin, that is, resistivity increases with depth at a greater rate in the deep basin than on the flanks of the basin (pl. 2). This is not unexpected, as compaction determines the increase of resistivity with depth and compaction is greatest where burial depths are greatest. As a consequence, the width of the separation between the resistivity curve and the trendline is greatest in the deep basin, so the red shading is more obvious (fig. 12). Wells in which the shale resistivity decreases to less than $1 / 10$ of the trendline value are outlined with a red circle in figure 13; these wells are mostly in the southeastern part of the Anadarko Basin.

The zone of paleopressure is bounded on the west and east by wells with no resistivity reversal, indicating no evidence of paleopressure (see plate 2 and wells on the west and east end of line $A-A^{\prime}$ in plate 3). All strata are thinner on the shelf than in the deep basin, and as strata thin the zone of paleopressure also thins becoming more difficult to discern, as shown in the northern most wells on lines $B-B^{\prime}, C-C^{\prime}$, and $D-D^{\prime}$ (pls. 4-6). At the northern edge of the area, the paleopressure indicators reduce in number and thickness, until it is not possible to determine whether paleopressure is present or not. For this reason, no effort was made to trace the extent of paleopressure into southern Kansas, with the exception of the wells in the southwestern counties. In southwestern Kansas, paleopressure is only visible in strata of Morrowan age (pl. 2) with the exception of a single well in which the top of paleopressure is in Desmoinesian age rocks (fig. 14).

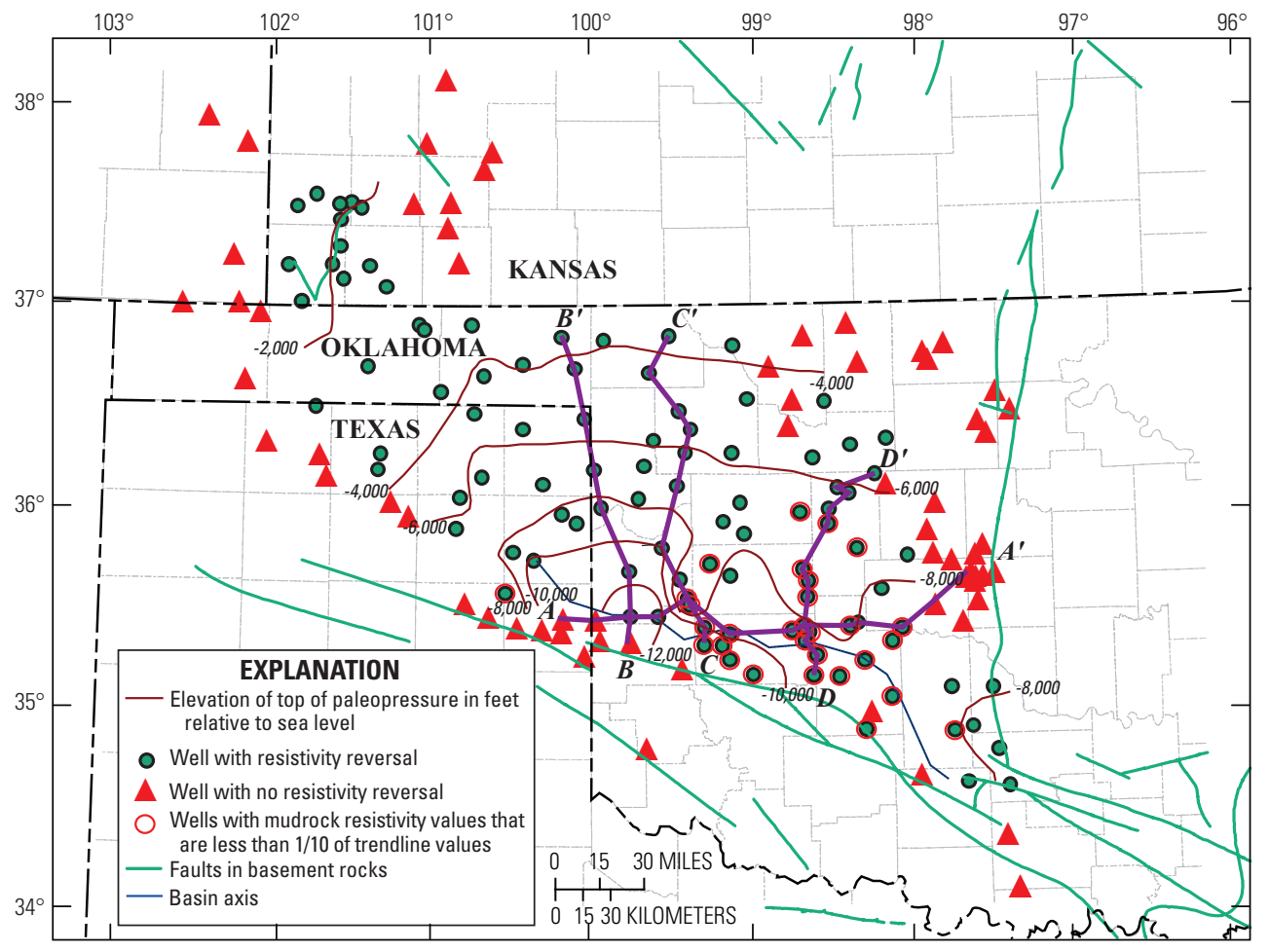

Figure 13. Map of the elevation of top of paleopressure as determined from resistivity logs. Lines $A-A^{\prime}$ through $D-D^{\prime}$ show location of cross sections on plates $3-6$. A resistivity reversal was found in 107 wells (green circles) but was absent in 68 wells (red triangles). 


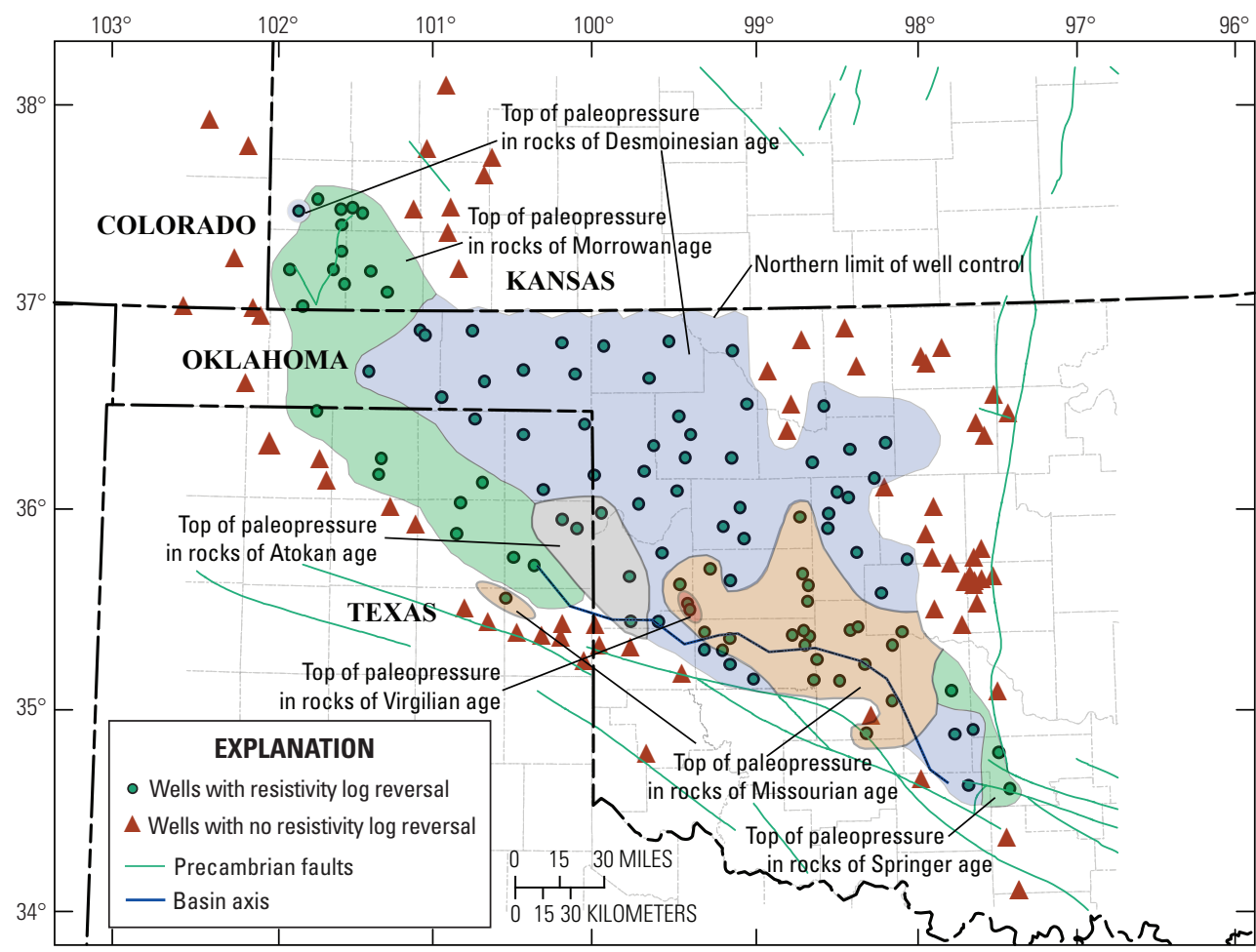

Figure 14. Map of age of rock unit containing top of paleopressure, based on the depth where the resistivity log changes from increasing downwards to decreasing downwards. The green area includes rocks of either Morrowan or Springer age, or both.

The changing relation between the top of paleopressure and rock unit age from west to east is seen by comparing the three north-south sections in plates 4-6. On the westernmost line $B-B^{\prime}$ (pl. 4), the top of paleopressure lies within the lower Desmoinesian or Atokan, within $500 \mathrm{ft}$ above the informal Thirteen Finger lime, which is considered to be a source rock (Higley, chapter 7 of this report). The same relation holds over the northern portion of line $C-C^{\prime}$ (pl. 5), but in the southern part of line $C-C^{\prime}$, paleopressure extends stratigraphically upwards into rocks of early Virgilian age in wells Bobby 1-14 and Weatherly 1 . The upward extension of paleopressure produces a large southward deflection of the $8,000-\mathrm{ft}$ contour in figure 13, encompassing a lobate area of approximately 15 by 30 miles where the top of paleopressure is higher in elevation (fig. 13) and higher stratigraphically (fig. 14) than immediately to the west and east. On the easternmost of the three southnorth cross sections, the top of paleopressure lies within rocks of Missourian age in the eight wells on the southern part of the line (pl. 6, fig. 14). The elevation of the top of paleopressure is fairly constant over a distance of about 50 miles, as can be seen in plate 6 and by the wide separation between the 6,000 and 8,000-ft contours of figure 13. Line $A-A^{\prime}$ (pl. 3) also shows the general west to east stratigraphic climb of the top of paleopressure, interrupted by the high point into rocks of Virgilian age that is reached in the Weatherly well where line $A-A^{\prime}$ intersects line $C-C^{\prime}$.
The top of paleopressure lies in rocks of Morrowan age along the west and northwest sides of the mapped area (fig. 14), with the exception of one outlying well in southwestern Kansas in which the paleopressure indicator extends into rocks of Desmoinesian age. The northwest-trending boundary between Morrowan and Desmoinesian age rocks is a transitional one because in some wells the resistivity reversals are in only a few thin units above the Thirteen Finger lime and cannot be unambiguously assigned to either Morrowan or Desmoinesian (Atokan units are absent or poorly defined in this area). The top of paleopressure does extend into recognizable Atokan units in five wells in an area straddling the Texas-Oklahoma border (fig. 14). Three wells in the southeastern edge of the basin show a clear indication of paleopressure in Springer mudrocks. Because there are no rocks older than Morrowan-Springer that show indications of paleopressure, areas marked Morrowan/ Springer in figure 14 do not show evidence of paleopressure in any other sequences. Paleopressure extends stratigraphically upward from Desmoinesian rocks into Missourian rocks in the southeastern part of the study area and also in a single isolated well in the Texas Panhandle. As previously mentioned, the top of paleopressure extends into rocks of Virgilian age in two wells, as shown in fig. 14.

As can be seen in plates 2-6, the greatest depth extent of the paleopressure indicator lies in the deep basin; that is, the depth range from top of paleopressure to either the base 
of paleopressure or the bottom of the well is greatest in the deep basin. Paleopressure extends downward through rocks of Morrowan age and into mudrocks of Mississippian age. Paleopressure does not register in Mississippian carbonates where mudrocks are absent. Somewhat surprisingly, the paleopressure indicator does not show up in the Devonian Woodford Shale (for example, Shirley 1-20 and Laubhan 35-1 wells of line $B-B^{\prime}$, pl. 4 ; Vierson and Coram A1 wells of line $C-C^{\prime}$, pl. 5). However, the Ordovician Sylvan Shale does produce a reduction in resistivity that shows up as a paleopressure indicator (Schneider 1 well, line $C-C^{\prime}$, pl. 5; Girard 1 well, line $A-A^{\prime}$, pl. 3).

\section{Comparison of Overpressured and Paleopressured Areas}

The area of paleopressure is much more extensive than that of present-day overpressure. The contraction of both areal and vertical extents are shown in figures 15-17. In these figures, the outlines of paleopressure are derived from figure 14, accounting for the fact that areas of paleopressure are stacked such that paleopressure exists in Morrowan (the oldest) through Virgilian (the youngest) rocks in the small area where the top of paleopressure extends to rocks of Virgilian age, paleopressure exists in Morrowan through Missourian rocks in the brown-shaded area where the top of paleopressure extends upward into rocks of Missourian age, and so on. The outlines of overpressured areas in Desmoinesian and Morrowan rocks are taken from figures 6 and 7 and the outline of overpressured areas in Missourian rocks, not previously displayed, is shown in figure 14. Figures 15-17 show that (1) the outline of present-day overpressure at $0.5 \mathrm{psi} / \mathrm{ft}$ in rocks of Morrowan and Springer age is less than half of its respective area of paleopressure (fig. 15), (2) the area of present-day overpressure for Desmoinesian age rocks is less than half the area of paleopressure for Desmoinesian-age rocks (fig. 16), and (3) for Missourian age rocks, the outline of present-day overpressure is less than one-fourth that of the area of paleopressure (fig. 17). (Based on evidence from mud logs and gas flares, the area of present-day overpressure in Missourian age rocks is greater than shown in figure 17 [John Mitchell, oral commun., 2011)].) In all three cases, the area of present-day overpressure is substantially less than the area of paleopressure.

The vertical extents of paleopressure and overpressure are more difficult to compare than the horizontal extents. North of the overpressured area, defined by the $0.5 \mathrm{psi} / \mathrm{ft}$ contours (figs. 15-17), overpressure is not present but paleopressure has a finite vertical extent. Within the overpressured areas, the situation is mixed. Where paleopressure extends upward into younger rocks of Virgilian age, as shown in figure 14 and in the southward bowing of the $-8,000$ - $\mathrm{ft}$ contour in figure 13 , the top of paleopressure is clearly at a higher elevation than the top of overpressure. In other areas, the two surfaces appear to be at comparable elevations (compare fig. 13 with fig. 5), suggesting that there has been little downward movement of the overpressured zone. In a few areas, the top of paleopressure is at a lower elevation than the top of overpressure. These lower-elevation areas are where well coverage for paleopressure is sparse, so the counter-intuitive relation of a paleopressure surface deeper than an overpressure surface is discounted as an artifact of inadequate coverage. In summary, it appears that the top of the original overpressured volume has moved downward a few thousand feet in some locations, but overall there has been little downward movement of the top of the original overpressured volume.

A somewhat analogous setting exists in the Green River Basin of Wyoming, where Jonah field, a fault-bounded, overpressured tight gas system lies within an extensive area of normally pressured strata. Resistivity and sonic logs display trend reversals at the top of the overpressured gas system in Jonah Field. The reversals are also at the same stratigraphic level in wells in non-productive, normally pressured Tertiary strata outside the field, where the well log signatures and present-day pressure are decoupled (Cluff and Cluff, 2004). Cluff and Cluff (2004) concluded that the well log reversals record pressure conditions at the time of maximum burial, and that the "signature was frozen into the rocks during subsequent exhumation" making Jonah Field "an anomalous remnant" of a former regional overpressured area (Cluff and Cluff, 2004, p. 143).

Thermal maturity maps based on petroleum system modeling (chapter 3 of this report) provide contours of vitrinite reflectance $\left(R_{0}\right)$ that can be compared with the outlines of paleopressure and present-day overpressure (figs. 15-17). In Morrowan age rocks, most of the overpressured area within the $0.7 \mathrm{psi} / \mathrm{ft}$ boundary lies within the $1.2 \% \mathrm{R}_{\mathrm{o}}$ contour, and almost all of the $0.5 \mathrm{psi} / \mathrm{ft}$ area lies within the $0.8 \% \mathrm{R}_{\mathrm{o}}$ contour (fig. 15). However, a substantial part of the paleopressured area lies outside the 0.8 percent contour. In Desmoinesian age rocks (fig. 16), the $1.2 \% \mathrm{R}_{\mathrm{o}}$ contour roughly coincides with the $0.7 \mathrm{psi} / \mathrm{ft}$ contour, showing that the highest present-day overpressures are in the same area as the levels of thermal maturity associated with gas generation. The $0.8 \% \mathrm{R}_{\mathrm{o}}$ contour includes most of the area within the $0.5 \mathrm{psi} / \mathrm{ft}$ contour but does not include all of the paleopressured area in rocks of Desmoinesian age. For comparison with paleopressured and presentday overpressured areas of Missourian age rocks (fig. 17), we display the contours of $0.8 \% \mathrm{R}_{\mathrm{o}}$ and $1.0 \% \mathrm{R}_{\mathrm{o}}$ from a model for the Douglas Group of early Virgilian age, which immediately overlies rocks of Missourian age. No $\mathrm{R}_{\mathrm{o}}$ values higher than 1.0 percent were computed for this model. The areas of paleopressure and present-day overpressure (fig. 17) coincide with part of the area circumscribed by a $0.8 \% \mathrm{R}_{\mathrm{o}}$ contour.

In summary, the comparisons among overpressured areas, paleopressured areas, and $\mathrm{R}_{\mathrm{o}}$ contours from petroleum system modeling (figs. 15-17) show that (1) the areas of high overpressure and high $\mathrm{R}_{\mathrm{o}}$ values are comparable in Morrowan and Desmoinesian rocks, (2) most of the overpressured areas lie within the $0.8 \% \mathrm{R}_{\mathrm{o}}$ contours, and (3) paleopressured areas generally extend beyond the $0.8 \% \mathrm{R}_{\mathrm{o}}$ contours. 


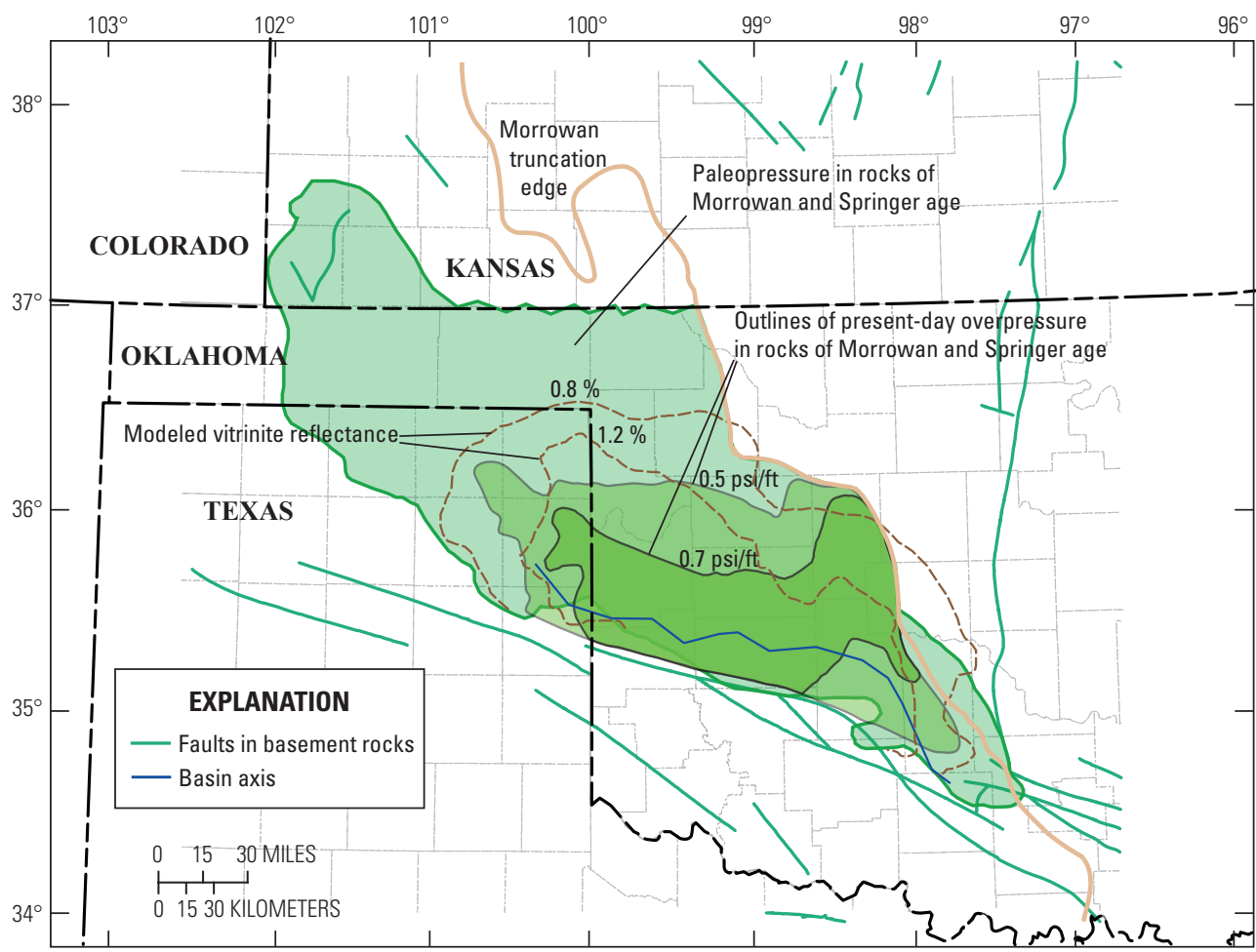

Figure 15. Map showing extents of paleopressure and overpressure in rocks of Morrowan and Springer age. The extent of the paleopressure indicator from resistivity logs is derived from figure 14 and the extent of present-day overpressure from pressure data is taken from figure 7. Modeled vitrinite reflectance contours of 0.8 and 1.2 percent for source rocks of Thirteen Finger lime of Atokan age taken from Higley (chapter 7 of this report).

The continued existence of an overpressured zone long after the cessation of burial and hydrocarbon generation has perplexed a number of investigators because containment of overpressure for long time periods requires extremely low values of permeability to fluid flow on the top, bottom, and sides of the overpressured volume. Using a one-dimensional model of pressure dissipation and parameters relevant to the Anadarko Basin, Lee and Deming (2002) observed that the retention of pressure from either compaction disequilibrium or hydrocarbon generation (with uplift) requires permeability in seals of $100-\mathrm{m}$ thickness to be so low as to be effectively zero. In a related report, Deming and others (2002) discuss the dilemma of long-lived overpressured volumes in the Anadarko Basin (and in sedimentary basins in general) and contribute a possible explanation for the retention of fluids within the basins. The explanation relies on laboratory experiments reported by Shosa and Cathles (2001), in which water flowing through a layered sand pack with flow rates and pressures governed by Darcy's law ceased flowing when gas was introduced into the system. Experimental checks verified that the cessation of flow was caused by gas bubbles immobilized at the interfaces between coarse and fine sand packs. Deming and others (2002) extrapolated the laboratory work to the basin scale, using well logs to show that the requisite number of fine-grain/coarse-grain interfaces are present in the Anadarko Basin.

The question of timing of the contraction of the overpressured area from its original (paleopressured) extent to its present configuration is another difficult question, and depends upon the chosen explanation for the retention of overpressure. The presence of underpressure in the northern and western parts of the basin is documented in a companion report by Nelson and Gianoutsos (chapter 9 of this report) and the timing of its development is conjectured to be quite recent in geological time. Assuming that the mechanism reported by Shosa and Cathles (2001) was (and is still) operative, then it is plausible that as erosion exposed Permian and Pennsylvanian strata, the pressure gradients changed direction in such a way as to render the gas-bubble lockup no longer effective on the northern flank of the basin. Moreover, the conversion from an overpressured system to a normal/underpressured system may be ongoing at the northern edge of the present-day overpressured area. 

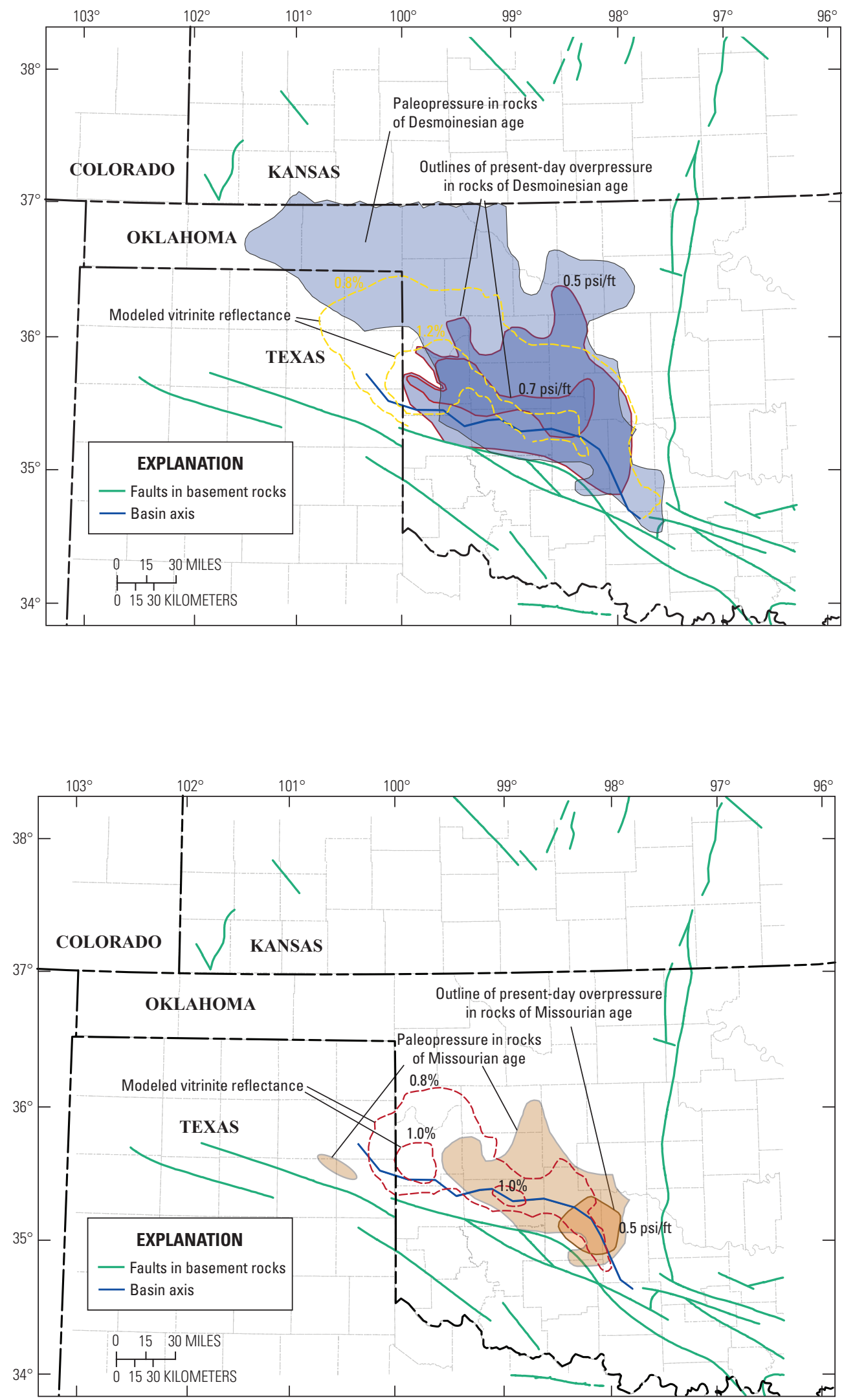

Figure 16. Map showing extents of paleopressure and overpressure in rocks of Desmoinesian age. The extent of the paleopressure indicator from resistivity logs is derived from figure 14 and the extent of present-day overpressure from pressure data is taken from figure 6. Modeled vitrinite reflectance contours of 0.8 and 1.2 percent for source rocks of Desmoinesian age taken from Higley (chapter 7 of this report).

Figure 17. Map showing extents of paleopressure and overpressure in rocks of Missourian age. The extent of the paleopressure indicator from resistivity logs is derived from figure 14. Modeled vitrinite reflectance contours of 0.8 and 1.0 percent for source rocks of early Virgilian age taken from Higley (chapter 7 of this report). 


\section{Summary and Conclusions}

Our review of the state of overpressure in the Anadarko Basin shows (1) the prevalence of normal to subnormal pressures on the northern flank of the basin (pl. 1, fig. 4), (2) the progressive deepening of the top of overpressure from north to south (pl. 1, fig. 5), (3) the increasing vertical extent of overpressure from north to south as strata thicken (pl. 1), and (4) the distinct compartmentalization of overpressure in rocks of Desmoinesian and Morrowan age (pl. 1).

The area of overpressure in rocks of Desmoinesian age is roughly 100 miles in the west-northwest direction and 50 miles in the east-northeast direction, based on the $0.5 \mathrm{psi} / \mathrm{ft}$ contour (fig. 6). The area of highest pressures within the $0.7 \mathrm{psi} / \mathrm{ft}$ contour nearly coincides with the $1.2 \% \mathrm{R}_{\mathrm{o}}$ contour (fig. 16), illustrating a close connection between high present-day overpressure and a thermal maturity level that is considered to be the end of oil generation for the Devonian-Mississippian Woodford Shale (chapter 3 of this report). The area of overpressure in rocks of Morrowan age is greater than 150 miles in the west-northwest direction and greater than 50 miles in the east-northeast direction, based on the $0.5 \mathrm{psi} / \mathrm{ft}$ contour (fig. 6). The area of highest pressures within the $0.7 \mathrm{psi} / \mathrm{ft}$ contour lies mostly within the $1.2 \% \mathrm{R}_{0}$ contour (fig. 15). Evaluation of a dataset from Powley (1984) strengthens the case for subcompartments within strata of Morrowan age and establishes the existence of pressure gradients of $0.465 \mathrm{psi} / \mathrm{ft}$ at elevated overall pressures in three of the subcompartments (fig. 8). These gradients show that water, not gas, is the continuous fluid within the subcompartments.

Reversals in resistivity logs from a normal mudrock compaction trend are most manifest in the deep basin and persist northward onto the shelf, but do not exist in wells east and west of the basin (pls. 2-5 and figs. 13-14). The top of the trend reversals lies in Virgilian and Missourian rocks in the deep basin and in Morrowan rocks on the western and southeastern fringes of the system (fig. 14), although the pattern with regard to rock age is an irregular one. The resistivity trend reversals in Desmoinesian rocks extend to the Oklahoma-Kansas border and may have extended farther to the north, but the top of the trend reversal cannot be selected with confidence where strata thin on the northern flank of the basin.

Equating the resistivity trend reversals with paleopressure allows comparisons between the areal and vertical extents of the paleopressured system with the extents of the present-day overpressured system. The areal extents of paleopressure are substantially larger, about twice as large, as the areal extents of present-day overpressure in rocks of Morrowan, Desmoinesian, and Missourian age (figs. 15-17). Changes in vertical extent, however, are more subtle and more difficult to determine. Although the high points of the original overpressured volume have moved downwards a few thousand feet in some locations, overall there has been little downward migration of the top of the original overpressured volume. The largest reductions in resistivity relative to trendlines, and correspondingly the most profound expressions of paleopressure, are in an area that extends for 100 miles in a northwesterly direction more or less aligned with the basin axis and extending 20 to 40 miles north from the basin axis (pls. 3-6, fig. 13). This area of large reductions in resistivity coincides with the area where paleopressures extended into rocks of Missourian age (fig 14).

The contraction of the paleopressured area to the area of present-day overpressure could conceivably have occurred over any span of geological time since the end of Permian deposition. However, it seems likely that the contraction occurred when the pressure reference for strata on the northern flank of the basin was established as erosional processes exposed strata of Permian and Pennsylvanian age at the eastern edge of the basin.

\section{Acknowledgments}

James Puckette, of the Geology Department at Oklahoma State University, provided access to files of pressure data from the publication by Al-Shaieb and others (1994a). Elena Hickman, U.S. Geological Survey (USGS) contractor, provided registered digital maps. Debra Higley, USGS, provided formation tops and other geological information. Many constructive review comments by Noel Osborne, USGS, led to revisions that have improved the clarity of the chapter of the report.

\section{References Cited}

Al-Shaieb, Z., Puckett, J.O., Abdalla, A.A., and Ely, P.B., 1994a, Megacompartment complex in the Anadarko Basin: A completely sealed overpressured phenomenon, in Ortoleva, Peter J., ed., Basin compartments and seals: American Association of Petroleum Geologists Memoir 61, p. 55-68.

Al-Shaieb, Z., Puckett, J.O., Abdalla, A.A., and Ely, P.B., 1994b, Three levels of compartmentation within the overpressured interval of the Anadarko Basin, in Ortoleva, Peter J., ed., Basin compartments and seals: American Association of Petroleum Geologists Memoir 61, p. 69-83.

Bigelow, E.L., 1994, Well logging methods to detect abnormal pressure, in Fertl, W.H., Chapman, R.E., and Hotz, R.F., eds., chap. 7, Studies in abnormal pressure: Developments in Petroleum Science 38, Amsterdam, Elsevier, 454 p.

Breeze, A.F., 1970, Abnormal-subnormal pressure relationships in the Morrow sands of northwestern Oklahoma: University of Oklahoma, M.S. thesis, 122 p.

Carter, L.S., Kelley, S.A., Blackwell, D.D., and Naeser, N.D., 1998, Heat flow and thermal history of the Anadarko Basin, Oklahoma: American Association of Petroleum Geologists Bulletin, v. 82, no. 2, p. 291-316. 
Cluff, R.M., and Cluff, S.G., 2004, The origin of Jonah Field, northern Green River Basin, Wyoming, chap. 8 in Robinson, J.W., and Shanley, K.W., eds.: Jonah Field: Case study of a tight-gas fluvial reservoir: AAPG Studies in Geology 52 and Rocky Mountain Association of Geologists 2004 Guidebook, p. 127-145.

Deming, D., Cranganu, C., and Lee, Y., 2002, Self-sealing in sedimentary basins: Journal of Geophysical Research, v. 107, n. B12, 2329, 9 p.

Dickey, P.A., and Soto, C., 1974, Chemical composition of deep subsurface waters on the western Anadarko Basin: Society of Petroleum Engineers Paper 5178, 17 p.

IHS Energy, 2009, U.S. production and well data: database available from IHS Energy, 15 Inverness Way East, D205, Englewood, CO 80112.

Lee, Y., and Deming, D., 2002, Overpressures in the Anadarko Basin, southwestern Oklahoma: Static or dynamic?: American Association of Petroleum Geologists Bulletin, v. 86, n. 1 , p. $145-160$.
Powley, D.E., 1984, Subsurface fluid compartments: American Association of Petroleum Geologists Search and Discovery Article 60006 (2006), adapted from Amoco Geological Research Report, 52 p. accessed December 14, 2010, at http://www.searchanddiscovery.com/ documents/2006/06015powley/images/powley01.pdf.

Shosa, J.D. and Cathles, L.M., 2001, Experimental investigation of capillary blockage of two phase flow in layered porous media, in Fillon, R.H. and others eds., Petroleum systems of deep-water basins: Global and Gulf of Mexico experience: 21st Annual Bob F. Perkins Research Conference [CD-ROM], Gulf Coast Section, Society of Economic Paleontological and Mineralogical Society, Houston, Texas.

U.S. Geological Survey, 2014, Colorado, Kansas, New Mexico, Oklahoma, and Texas: U.S. Geological Survey digital data, The National Map, accessed August 4, 2014, at: http://viewer.nationalmap.gov/viewer/. 


\section{Appendix 1}

List of wells shown on cross sections on plates 3-6 (Excel file format)

\begin{tabular}{|c|c|c|c|c|c|c|c|c|c|c|c|c|c|c|c|c|}
\hline $\begin{array}{c}\text { API } \\
\text { number }\end{array}$ & $\begin{array}{l}\text { Lease } \\
\text { name }\end{array}$ & No. & Operator & State & County & $\begin{array}{l}\text { Reference } \\
\text { elevation, } \\
\text { in feet }\end{array}$ & Reference & Latitude & Longitude & Section & Township & Range & $\begin{array}{l}\text { Rock unit } \\
\text { at top of } \\
\text { resistivity } \\
\text { reversal }\end{array}$ & $\begin{array}{l}\text { Depth } \\
\text { of top of } \\
\text { resistivity } \\
\text { reversal, } \\
\text { in feet }\end{array}$ & $\begin{array}{c}\text { Elevation } \\
\text { of top of } \\
\text { resistivity } \\
\text { reversal, } \\
\text { in feet }\end{array}$ & $\begin{array}{l}\text { Cross } \\
\text { section }\end{array}$ \\
\hline 3501723998 & $\begin{array}{l}\text { West Edmond } \\
\text { Swd }\end{array}$ & $1-24$ & Chesapeake Operating Inc & Oklahoma & Canadian & 1,159 & $\mathrm{DF}$ & 35.669 & -97.683 & 24 & $14 \mathrm{~N}$ & $05 \mathrm{~W}$ & Absent & -- & -- & A \\
\hline 4248330610 & Miles Gas Unit & 1 & Exxon Corporation & Texas & Wheeler & 2,509 & KB & -- & -- & $\begin{array}{c}\text { RRC } \\
\text { Dist 10, } \\
\text { Sec } 2\end{array}$ & Blk A-4 & $\begin{array}{l}\text { Survey: } \\
\text { H\&GN }\end{array}$ & Absent & -- & -- & A \\
\hline 4248331989 & Hagermann 21 & 1 & Chesapeake Operating Inc & Texas & Wheeler & 2,164 & KB & 35.433 & -100.00 & $\begin{array}{l}\text { RRC } \\
\text { Dist 10, } \\
\text { Sec } 21\end{array}$ & BLK OS2 & $\begin{array}{l}\text { Peter- } \\
\text { man CE } \\
\text { Survey }\end{array}$ & Absent & -- & -- & A \\
\hline 3512920675 & $\begin{array}{l}\text { Millington- } \\
\text { Shields }\end{array}$ & 1 & El Paso Natural Gas Company & Oklahoma & Roger Mills & 2,158 & GR & 35.454 & -99.589 & 1 & $11 \mathrm{~N}$ & $23 \mathrm{~W}$ IM & Desmoinesian & 14,600 & $-12,409$ & A \\
\hline 3501520950 & Tillman & 1 & Monsanto Company & Oklahoma & Caddo & 1,475 & $\mathrm{DF}$ & 35.418 & -98.355 & 22 & $11 \mathrm{~N}$ & $11 \mathrm{~W}$ IM & Missourian & 9,485 & $-8,008$ & A \\
\hline 3501522365 & Lindley & $3-30$ & Apache Corporation & Oklahoma & Caddo & 1,427 & GR & 35.402 & -98.401 & 30 & $11 \mathrm{~N}$ & $11 \mathrm{~W}$ IM & Missourian & 9,090 & $-7,639$ & A \\
\hline 3501721307 & Girard & 1 & Key Operating Company & Oklahoma & Canadian & 1,455 & DF & 35.388 & -98.083 & 31 & $11 \mathrm{~N}$ & 08W IM & Missourian & 9,550 & $-8,094$ & A \\
\hline 3514920050 & Richert & 1 & Conoco Inc & Oklahoma & Washita & 1,607 & KB & 35.382 & -98.760 & 35 & $11 \mathrm{~N}$ & $15 \mathrm{~W} \mathrm{IM}$ & Missourian & 9,665 & $-8,058$ & A \\
\hline 3514920342 & $\begin{array}{l}\text { Hohnke Leo } \\
\text { Unit }\end{array}$ & 1 & Exxon Corporation & Oklahoma & Washita & 1,849 & $\mathrm{DF}$ & 35.367 & -99.149 & 6 & $10 \mathrm{~N}$ & $18 \mathrm{~W}$ IM & Missourian & 11,600 & $-9,750$ & A \\
\hline 3501721288 & Royse & 1 & Samedan Oil Corporation & Oklahoma & Canadian & 1,342 & GR & 35.504 & -97.906 & 23 & $12 \mathrm{~N}$ & $7 \mathrm{~W} \mathrm{IM}$ & Absent & -- & -- & A \\
\hline 3512920639 & $\begin{array}{l}\text { A H Douglas } \\
\text { Estate }\end{array}$ & 1 & Exxon Corporation & Oklahoma & Roger Mills & 2,277 & $\mathrm{DF}$ & 35.456 & -99.759 & 5 & $11 \mathrm{~N}$ & $24 \mathrm{~W}$ & Atokan & 17,000 & $-14,753$ & $\mathrm{~A}, \mathrm{~B}$ \\
\hline 3512930019 & Weatherly & 1 & Petroleum Waste Recovery Inc & Oklahoma & Roger Mills & -- & -- & 35.543 & -99.406 & 3 & $12 \mathrm{~N}$ & $21 \mathrm{~W}$ IM & Virgilian & 8,730 & $-6,678$ & $\mathrm{~A}, \mathrm{C}$ \\
\hline 3514920232 & Davis & $28-1$ & Getty Oil Company & Oklahoma & Washita & 1,601 & $\mathrm{DF}$ & 35.403 & -98.689 & 28 & $11 \mathrm{~N}$ & $14 \mathrm{~W}$ IM & Missourian & 9,200 & $-7,598$ & A, D \\
\hline 3500921156 & Cat Creek & $1-19$ & Chesapeake Operating Inc & Oklahoma & Beckham & 2,045 & $\mathrm{DF}$ & 35.329 & -99.786 & 19 & $10 \mathrm{~N}$ & $24 \mathrm{~W}$ & Absent & -- & -- & B \\
\hline 3504520748 & Laubhan 35 & 1 & Marathon Oil Company & Oklahoma & Ellis & 2,370 & $\mathrm{DF}$ & 35.996 & -99.940 & 35 & $18 \mathrm{~N}$ & $26 \mathrm{~W}$ & Atokan & 11,090 & $-8,705$ & B \\
\hline 3500720640 & Dixon & $1-6$ & King Resources Company & Oklahoma & Beaver & 2,491 & DF & 36.839 & -100.188 & 6 & $04 \mathrm{~N}$ & 27E CM & Desmoinesian & 6,300 & $-3,807$ & B \\
\hline 3500721928 & $\begin{array}{c}\text { Homes Or } \\
\text { Holmes? }\end{array}$ & $36-2$ & Home Petroleum Corporation & Oklahoma & Beaver & -- & -- & 36.684 & -100.105 & 36 & $03 \mathrm{~N}$ & 27E CM & Desmoinesian & 6,745 & $-4,368$ & B \\
\hline 3504521097 & Schoenhals & 1 & Marathon Oil Company & Oklahoma & Ellis & 2,450 & $\mathrm{DF}$ & 36.183 & -99.984 & 28 & $20 \mathrm{~N}$ & 26W IM & Desmoinesian & 9,450 & $-7,000$ & B \\
\hline 3512922603 & Shirley & $1-20$ & The Ghk Company & Oklahoma & Roger Mills & 2,056 & $\mathrm{DF}$ & 35.679 & -99.768 & 20 & $14 \mathrm{~N}$ & $24 \mathrm{~W}$ & Desmoinesian & 13,580 & $-11,524$ & B \\
\hline 4229531218 & Gadberry & $1-174$ & Williford Energy Co & Texas & Lipscomb & 2,488 & KB & -- & -- & $\begin{array}{c}\text { RRC } \\
\text { Dist 10, } \\
\text { Sec 174 }\end{array}$ & Blk 10 & $\begin{array}{l}\text { Survey: } \\
\text { H\&GN }\end{array}$ & DesMonesian & 7,810 & $-5,322$ & B \\
\hline 3512922522 & Bobby & $1-14$ & Chesapeake Operating Inc & Oklahoma & Roger Mills & 2,007 & GR & 35.512 & -99.391 & 14 & $12 \mathrm{~N}$ & $21 \mathrm{~W}$ IM & Virgilian & 8,790 & $-6,763$ & $\mathrm{C}$ \\
\hline 3504520879 & Coram A & 1 & Cities Service Company & Oklahoma & Ellis & 2,268 & $\mathrm{DF}$ & 36.103 & -99.468 & 20 & $19 \mathrm{~N}$ & $21 \mathrm{~W}$ IM & Desmoinesian & 9,580 & $-7,293$ & $\mathrm{C}$ \\
\hline 3505921196 & Blasdel & 2 & Terra Resources Inc & Oklahoma & Harper & 1,759 & $\mathrm{DF}$ & 36.847 & -99.514 & 1 & $27 \mathrm{~N}$ & $22 \mathrm{~W}$ IM & Desmoinesian & 5,505 & $-3,743$ & $\mathrm{C}$ \\
\hline 3505922317 & Horton & $1-11$ & Chesapeake Operating Inc & Oklahoma & Harper & 2,125 & $\mathrm{DF}$ & 36.664 & -99.640 & 11 & $25 \mathrm{~N}$ & $23 \mathrm{~W} \mathrm{IM}$ & Desmoinesian & 6,535 & $-4,409$ & $\mathrm{C}$ \\
\hline 3512920125 & Viersen Unit & 1 & Pride Energy Company & Oklahoma & Roger Mills & -- & -- & 35.794 & -99.564 & 8 & $15 \mathrm{~N}$ & $22 \mathrm{~W}$ IM & Desmoinesian & 12,255 & $-10,095$ & $\mathrm{C}$ \\
\hline 3515320043 & Everett Johns & $1-18$ & A. R. Dillard Inc & Oklahoma & Woodward & 2,077 & GR & 36.383 & 99.379 & 18 & $22 \mathrm{~N}$ & 20W & Desmoinesian & 7,405 & $-5,316$ & C \\
\hline 3515321951 & Schneider & 1 & Plains Petroleum Operating Co & Oklahoma & Woodward & 1,896 & GR & 36.476 & -99.455 & 16 & $23 \mathrm{~N}$ & $21 \mathrm{~W}$ IM & Desmoinesian & 7,025 & $-5,111$ & $\mathrm{C}$ \\
\hline
\end{tabular}




\begin{tabular}{|c|c|c|c|c|c|c|c|c|c|c|c|c|c|c|c|c|}
\hline $\begin{array}{c}\text { API } \\
\text { number }\end{array}$ & $\begin{array}{l}\text { Lease } \\
\text { name }\end{array}$ & No. & Operator & State & County & $\begin{array}{l}\text { Reference } \\
\text { elevation, } \\
\text { in feet }\end{array}$ & Reference & Latitude & Longitude & Section & Township & Range & $\begin{array}{l}\text { Rock unit } \\
\text { at top of } \\
\text { resistivity } \\
\text { reversal }\end{array}$ & $\begin{array}{l}\text { Depth } \\
\text { of top of } \\
\text { resistivity } \\
\text { reversal, } \\
\text { in feet }\end{array}$ & $\begin{array}{c}\text { Elevation } \\
\text { of top of } \\
\text { resistivity } \\
\text { reversal, } \\
\text { in feet }\end{array}$ & $\begin{array}{l}\text { Cross } \\
\text { section }\end{array}$ \\
\hline 3515322725 & Grunewald & $1-26 \mathrm{~A}$ & Chesapeake Operating Inc & Oklahoma & Woodward & 2,165 & $\mathrm{DF}$ & 36.270 & -99.417 & 26 & $21 \mathrm{~N}$ & $21 \mathrm{~W}$ IM & Desmoinesian & 8,260 & $-6,093$ & $\mathrm{C}$ \\
\hline 3514920705 & Niece & $3-27$ & Meridian Oil Inc & Oklahoma & Washita & 1,828 & GR & 35.312 & -99.306 & 27 & $10 \mathrm{~N}$ & 20W IM & DesMonesian & 11,400 & $-9,535$ & $\mathrm{C}$ \\
\hline 3512923149 & Switzer & $4-32$ & Apache Corporation & Oklahoma & Roger Mills & 1,755 & DF & 35.640 & -99.453 & 32 & $14 \mathrm{~N}$ & $21 \mathrm{~W}$ IM & Missourian & 9,150 & $-7,395$ & $\mathrm{C}$ \\
\hline 3514920152 & Kilhoffer & $1-27$ & The Ghk Company & Oklahoma & Washita & 2,019 & $\mathrm{DF}$ & 35.400 & -99.303 & 27 & $11 \mathrm{~N}$ & 20W IM & Missourian & 12,100 & $-10,079$ & $\mathrm{C}$ \\
\hline 3514920020 & Bertha Rogers & 1 & Lone Star Producing Company & Oklahoma & Washita & 1,922 & $\mathrm{DF}$ & 35.309 & -99.193 & 27 & $10 \mathrm{~N}$ & 19W IM & Desmoinesian & 13,100 & $-11,178$ & $\mathrm{C}$ \\
\hline 3501121816 & Wisdom & $1-5$ & Sabine Production Company & Oklahoma & Blaine & 1,311 & $\mathrm{DF}$ & 36.062 & -98.401 & 5 & $18 \mathrm{~N}$ & $11 \mathrm{~W}$ IM & Desmoinesian & 7,540 & $-6,227$ & $\mathrm{D}$ \\
\hline 3501122895 & Gypsum U S & $1-27$ & Chesapeake Operating Inc & Oklahoma & Blaine & 1,595 & $\mathrm{DF}$ & 36.090 & -98.470 & 27 & $19 \mathrm{~N}$ & $12 \mathrm{~W}$ IM & Desmoinesian & 7,670 & $-6,073$ & $\mathrm{D}$ \\
\hline 3501122962 & Chain & $1-6$ & Chesapeake Operating Inc & Oklahoma & Blaine & 1,574 & $\mathrm{DF}$ & 35.982 & -98.525 & 6 & $17 \mathrm{~N}$ & $12 \mathrm{~W}$ IM & Desmoinesian & 8,250 & $-6,676$ & $\mathrm{D}$ \\
\hline 3501123150 & Christensen & $1-36$ & Chesapeake Operating Inc & Oklahoma & Blaine & 1,598 & DF & 35.910 & -98.532 & 36 & $17 \mathrm{~N}$ & $13 \mathrm{~W}$ IM & Desmoinesian & 8,580 & $-6,980$ & $\mathrm{D}$ \\
\hline 3501123174 & Gwyn & $1-2$ & Chesapeake Operating Inc & Oklahoma & Blaine & 1,166 & $\mathrm{DF}$ & 36.154 & -98.235 & 2 & $19 \mathrm{~N}$ & 10W IM & Desmoinesian & 6,960 & $-5,794$ & $\mathrm{D}$ \\
\hline 3501520860 & Allred & $18-1$ & L G Williams Oil Company & Oklahoma & Caddo & 1,462 & GR & 35.258 & -98.611 & 18 & $9 \mathrm{~N}$ & $13 \mathrm{~W}$ IM & Missourian & 9,800 & $-8,313$ & $\mathrm{D}$ \\
\hline 3503920435 & Swosu & 1 & Amoco Production Company & Oklahoma & Custer & 1,610 & DF & 35.548 & 98.661 & 3 & $12 \mathrm{~N}$ & $14 \mathrm{~W}$ IM & Missourian & 9,460 & $-7,848$ & $\mathrm{D}$ \\
\hline 3503921952 & Wingard & $1-16$ & Chesapeake Operating Inc & Oklahoma & Custer & 1,746 & $\mathrm{DF}$ & 35.685 & -98.689 & 16 & $14 \mathrm{~N}$ & $14 \mathrm{~W}$ IM & Missourian & 9,200 & $-7,453$ & $\mathrm{D}$ \\
\hline 3503922053 & Pitzer & $1-2$ & Zinke \& Trumbo Inc & Oklahoma & Custer & 1,699 & $\mathrm{DF}$ & 35.628 & -98.655 & 2 & $13 \mathrm{~N}$ & $14 \mathrm{~W}$ IM & Missourian & 9,360 & $-7,659$ & $\mathrm{D}$ \\
\hline 3514920141 & $\begin{array}{l}\text { Seger Indian } \\
\text { School }\end{array}$ & $1-21$ & $\begin{array}{l}\text { Hamilton Brothers Oil Com- } \\
\text { pany }\end{array}$ & Oklahoma & Washita & 1,601 & $\mathrm{DF}$ & 35.331 & -98.680 & 21 & $10 \mathrm{~N}$ & $14 \mathrm{~W}$ IM & Missourian & 9,610 & $-8,004$ & $\mathrm{D}$ \\
\hline 3514920153 & Aaron Unit & 1 & Amoco Production Company & Oklahoma & Washita & 1,558 & DF & 35.374 & -98.653 & 2 & $10 \mathrm{~N}$ & $14 \mathrm{~W}$ IM & Missourian & 9,440 & $-7,879$ & $\mathrm{D}$ \\
\hline 3514920202 & Arthur & $24-1$ & Towner Petroleum Company & Oklahoma & Washita & 1,429 & DF & 35.156 & -98.633 & 24 & $8 \mathrm{~N}$ & $14 \mathrm{~W}$ IM & Missourian & 10,390 & $-8,940$ & $\mathrm{D}$ \\
\hline
\end{tabular}

Explanation: IM, Indian Meridian; CM, Cimarron Meridian

Well names and locations from Oklahoma Corporation Commission (www.occeweb.com) and Railroad of Texas (http://www.rrc.state.tx.us) 
Chapter 9

Potentiometric Surfaces for Seven

Stratigraphic Units and an Explanation for Underpressure in the Greater Anadarko

Basin, Oklahoma, Texas, Kansas, and Colorado

By Philip H. Nelson and Nicholas J. Gianoutsos

Chapter 9 of 13

Petroleum Systems and Assessment of Undiscovered Oil and Gas in the Anadarko Basin Province, Colorado, Kansas, Oklahoma, and Texas-USGS Province 58

Compiled by Debra K. Higley

U.S. Geological Survey Digital Data Series DDS-69-EE 


\title{
U.S. Department of the Interior SALLY JEWELL, Secretary
}

\section{U.S. Geological Survey \\ Suzette M. Kimball, Acting Director}

\author{
U.S. Geological Survey, Reston, Virginia: 2014
}

For more information on the USGS - the Federal source for science about the Earth, its natural and living resources, natural hazards, and the environment, visit http://www.usgs.gov or call 1-888-ASK-USGS.

For an overview of USGS information products, including maps, imagery, and publications, visit http://WwW.usgs.gov/pubprod

To order this and other USGS information products, visit http://store.usgs.gov

Any use of trade, firm, or product names is for descriptive purposes only and does not imply endorsement by the U.S. Government.

Although this information product, for the most part, is in the public domain, it also may contain copyrighted materials as noted in the text. Permission to reproduce copyrighted items must be secured from the copyright owner.

Suggested citation:

Nelson, P.H., and Gianoutsos, N.J., 2014, Potentiometric surfaces for seven stratigraphic units and an explanation for underpressure in the Greater Anadarko Basin, Oklahoma, Texas, Kansas, and Colorado, chap. 9, in Higley, D.K., comp., Petroleum systems and assessment of undiscovered oil and gas in the Anadarko Basin Province, Colorado, Kansas, Oklahoma, and Texas_-USGS Province 58: U.S. Geological Survey Digital Data Series DDS-69-EE, 24 p., http://dx.doi.org/10.3133/ds69EE. 


\section{Contents}

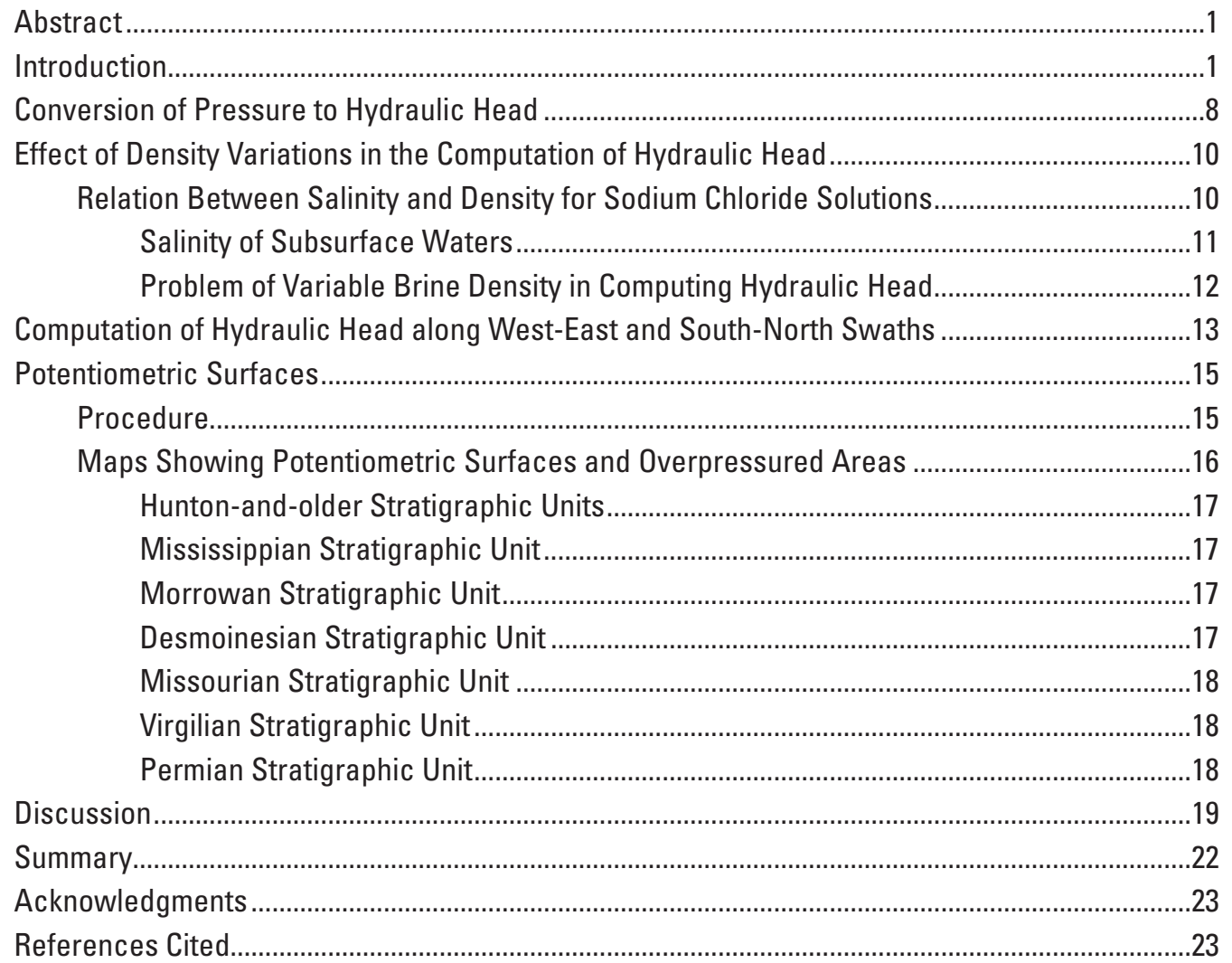

\section{Plates}

1. Maps of salinity in seven stratigraphic units in the Anadarko Basin ........................... link

2. Hydraulic head, surface elevation, and drillstem test elevation for four north-south swaths and seven stratigraphic units ....................................................................... link

3. Hydraulic head data and processing for 13 west-east lines and resulting potentiometric surfaces for rocks of Missourian age ................................................... link

4. Potentiometric surfaces for seven stratigraphic units .............................................. link

5. Separation between potentiometric and land surfaces for seven stratigraphic

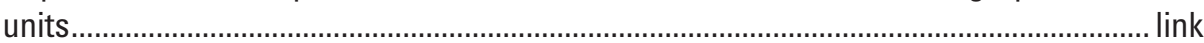

\section{Figures}

1. Map showing areas of this and previous studies of overpressure and underpressure. Contours for the top of the Woodford Shale show the structure of the Anadarko Basin; basin axis appears in subsequent figures as a reference line. Reference well is the Ferris $1-28$ well. The study area for this chapter extends from latitude $33.5^{\circ}$ to $39.5^{\circ} \mathrm{N}$. and longitude $96^{\circ}$ to $103.5^{\circ} \mathrm{W}$ 
2. Stratigraphic chart of the Anadarko Basin, with stratigraphic units (purple shading) as defined in this study to examine pressure data. The lithostratigraphic units are modified from Higley (chapter 3 of this report). Geohydrologic units were defined by Jorgensen and others (1996) for a regional study; light blue shading indicates the presence of rock units with well defined patterns of hydraulic head that can be considered to be aquifers. Cretaceous rocks are not present in the study area of figure 1 but are present in the regional study area of Jorgensen and others (1996). Wavy lines represent unconformities. Areas with vertical lines represent periods of non-deposition. PC, Precambrian; Camb., Cambrian; Miss., Mississippian.

3. Map of Anadarko Basin showing pressure-depth ratios for reservoirs of Desmoinesian age, based on data from NRG Associates (2009). psi/ft, pounds per square inch per foot.

4. Map of greater Anadarko Basin showing surface elevation contours in 400-foot intervals, basin axis, Precambrian faults, and outcrops of selected formations. Precambrian faults from Adler and others (1971). Thickness of Permian evaporites from Gianoutsos and others (chapter 10 of this report). Outcrops of Garber Sandstone and three Virgilian formations, and base of Wellington Formation in Kansas and Oklahoma from Stoesser and others (2005). East-west oriented Line F shows location of drillstem test data shown in figure $10 .$.

5. Burial history for the Ferris 1-28 well, from Carter and others (1998). Overpressure developed during rapid burial during Pennsylvanian time with a possible secondary development around $100 \mathrm{Ma}$. Underpressure development is believed to be recent, commensurate with exposure of strata in central Oklahoma. The Permian cap provides a low permeability seal over the area. $\mathrm{pC}$, Precambrian; $€$, Cambrian; 0 , Ordovician; S, Silurian; D, Devonian; M, Mississippian; $P$, Pennsylvanian; $P$, Permian, T, Triassic, J, Jurassic; $K$, Cretaceous; $P_{E^{\prime}}$ Paleocene; E, Eocene; 0 , Oligocene, M, Miocene; Fms, Formations; Gp, Group; Ss, Sandstone, ${ }^{\circ} \mathrm{C}$, degrees Celsius

6. Structural cross section showing Permian and younger rocks in the Texas Panhandle (see inset for location). Mudstone, anhydrite, and salt form impermeable layers in Permian rocks. An interpreted salt dissolution feature is near the center of the section. From McGookey and others (1988). Co., County; Quat., Quaternary; Tert., Tertiary; Fm., Formation; Gp., Group.

7. Graph showing pressure in relation to elevation from wells in a small area in the Oklahoma Panhandle. The average surface elevation of 2,542 feet is represented by the $y$-intercept of the hydrostatic gradient. The dashed lines are reference lines for the pressure data, most of which correspond to underpressured conditions.

8. Example demonstrating the calculation of hydraulic head and pressure-depth ratio for two wells testing the same formation. The pressure-depth ratio is the same in the two wells, but the hydraulic head is much greater in Well A than in Well B. $\mathrm{ft}$, foot; psi, pounds per square inch; psi/ft, pounds per square inch per foot

9. Box-and-whiskers plot of salinity in seven stratigraphic units with number of samples in each group. Each whisker and each box represents 25 percent of the data. Salinity data taken from a compilation by Breit (2002), which is based on waters produced from oil and gas wells

10. Data from drillstem tests in Missourian rocks from west-east swath Line $\mathrm{F}$ close to and parallel with the Kansas-Oklahoma border (location in figure 4). $A$, Surface elevation (black plus symbols), elevation of drillstem tests (triangular symbols), and computed hydraulic head (red dash symbols); $B$, pressure measurements; $C$, computed pressure-depth ratio indicating underpressure where less than 0.465 pounds per square inch per foot (psi/ft) 
11. Hydraulic head $\mathrm{H}$ computed for one-dimensional flow $\mathrm{Q}$ in a medium of low hydraulic conductivity in the west, $\mathrm{K}_{\mathrm{W}^{\prime}}$ and high conductivity in the east, $\mathrm{K}_{\mathrm{E}^{\prime}}$ with $K_{W} / K_{E}=1 / 30$. The length of the low-conductivity section, $X_{W^{\prime}}$ is $1 / 4$ the length of the high-conductivity section, $X_{E}$. Values of $\mathrm{H}$ range from 1,250 to 3,000 feet, with colors between 250-foot contours of $\mathrm{H}$ selected to match the colors in plate 4. Concept for one-dimensional model taken from Belitz and Bredehoeft (1988)...

12. West-east structural cross section showing features influencing the creation and maintenance of underpressure. Permian and Pennsylvanian strata crop out along and east of the Nemaha uplift. Brown shading indicates the impermeable evaporite-bearing strata of Permian age. Pinchout and thinning of strata on the Sierra Grande arch provide limited entry for recharge. East of longitude $102^{\circ} \mathrm{W}$. the cross section is based largely on well data. West of long $102^{\circ} \mathrm{W}$. the cross section is based on information from Roberts and others (1976), Weeks and Gutentag (1981), and McGookey and others (1988)

13. Comparison between a separation map from plate $5 \mathrm{~F}$ and degree of underpressuring for the Virgilian unit. Contours on the separation map give the Virgilian potentiometric surface minus the surface elevation. Dots give the location of individual oil and gas fields for which pressure-depth ratios were computed from data provided by NRG Associates (2009)

14. Map with contours showing separation between the potentiometric surface in Permian rocks and land surface elevation, in feet. For example, the yellow areas show where the potentiometric surface lies $-1,500$ to $-1,800 \mathrm{ft}$ below surface elevation. The underpressured Hugoton-Panhandle gas field lies in a trough of maximum separation between the potentiometric surface and surface elevation

\section{Tables}

1. Increments of density used for salinity maps of plate 1 ...................................................11

2. Specific volume of water as a function of pressure and temperature.............................11 


\section{Conversion Factors}

\begin{tabular}{|c|c|c|}
\hline Multiply & By & To obtain \\
\hline \multicolumn{3}{|c|}{ Length } \\
\hline foot $(\mathrm{ft})$ & 0.3048 & meter $(\mathrm{m})$ \\
\hline mile (mi) & 1.609 & kilometer (km) \\
\hline \multicolumn{3}{|c|}{ Volume } \\
\hline barrel (bbl), (petroleum, 1 barrel=42 gal) & 0.1590 & cubic meter $\left(\mathrm{m}^{3}\right)$ \\
\hline cubic foot $\left(\mathrm{ft}^{3}\right)$ & 0.02832 & cubic meter $\left(\mathrm{m}^{3}\right)$ \\
\hline \multicolumn{3}{|c|}{ Pressure } \\
\hline atmosphere, standard (atm) & 101.3 & kilopascal (kPa) \\
\hline bar & 100 & kilopascal (kPa) \\
\hline pound per square inch $\left(\mathrm{lb} / \mathrm{in}^{2}\right)$ & 6.895 & kilopascal (kPa) \\
\hline \multicolumn{3}{|c|}{ Density } \\
\hline pound per cubic foot $\left(\mathrm{lb} / \mathrm{ft}^{3}\right)$ & 16.02 & kilogram per cubic meter $\left(\mathrm{kg} / \mathrm{m}^{3}\right)$ \\
\hline pound per cubic foot $\left(\mathrm{lb} / \mathrm{ft}^{3}\right)$ & 0.01602 & gram per cubic centimeter $\left(\mathrm{g} / \mathrm{cm}^{3}\right)$ \\
\hline
\end{tabular}




\title{
Potentiometric Surface Maps for Seven Stratigraphic Units and an Explanation for Underpressure in the Greater Anadarko Basin, Oklahoma, Texas, Kansas, and Colorado
}

\author{
By Philip H. Nelson and Nicholas J. Gianoutsos
}

\section{Abstract}

The controls on subsurface pressure across the northern part of the Anadarko Basin are examined with the aid of potentiometric maps constructed for seven stratigraphic units ranging in age from Cambrian to Early Permian. Pressure data from drillstem tests are converted to hydraulic head, a step that is complicated by variations in brine density within the basin, as shown by maps of salinity for each of the seven stratigraphic units considered in this study. Seven potentiometric maps were constructed using a series of filtering and mapping steps to drape a surface over the highest values of hydraulic head. The maps show that, for rocks of Desmoinesian, Missourian, Virgilian, and Lower Permian age, hydraulic head values approach surface elevation values along the Nemaha uplift in central Oklahoma where Lower Permian and Pennsylvanian strata are exposed. From the Nemaha uplift westward to southwestern Kansas and the panhandle areas of Oklahoma and Texas, hydraulic head increases by several hundred feet in each rock unit, whereas surface elevation increases by thousands of feet. The underpressuring of the aquifer-supported oil and gas fields, which increases from east to west, is a consequence of the westward-increasing vertical separation between surface elevation and hydraulic potential. Recharge to the deep confined rock units is limited. The thick cap of Permian evaporites and shales restricts recharge to the underlying strata, preventing reestablishment of a normal hydrostatic gradient. Recharge from the west is restricted because of onlap of Pennsylvanian and older strata onto the eastern flank of the Sierra Grande arch. Present-day underpressuring of oil and gas reservoirs on the northwest flank of the basin is the result of uplift and exposure of Lower Permian and Pennsylvanian strata along the Nemaha uplift and restricted recharge to the basin.

\section{Introduction}

This study explores the cause of widespread underpressure in the western and northern parts of the greater Anadarko Basin, an area that extends from the southeast corner of
Colorado eastward to central Kansas and Oklahoma and includes the Oklahoma Panhandle and a portion of the Texas Panhandle (fig. 1). The structure of the Anadarko Basin is defined by the elevation contours of the Woodford Shale (fig. 1); a line drawn through the deepest part of the Woodford Shale serves to locate the deep basin in subsequent figures. Further discussion of the geology of the Anadarko Basin is given by Higley (chapters 5 and 7 of this report). Underpressure exists in rock units that are Paleozoic in age (fig. 2); rock units younger than Permian form unconfined aquifers above the deeper confined rock units where both underpressure and overpressured conditions exist. Chapter 8 of this report (Nelson and Gianoutsos), which also covers the study area of figure 1, examines the extent of overpressure which presently is confined to the deep basin but is hypothesized to have had a greater areal extent in the geologic past. The terms overpressure and underpressure refer to preproduction fluid pressures that are respectively greater than and less than hydrostatic pressure. The two studies on formation fluid pressure were undertaken in conjunction with the work reported by Higley (chapter 7 of this report) and Gaswirth and Higley (chapter 5 of this report) on the assessment of oil and gas in the Anadarko Basin.

The existence of underpressured oil and gas reservoirs in parts of the Anadarko Basin has been reported by a number of authors. Breeze (1970) documented the transition in the Morrow Formation in northwestern Oklahoma from overpressure in the deep basin to underpressure on the flank of the basin; his area of study is outlined in figure 1. Blubaugh (1999a, 1999b) mapped the widespread extent of underpressuring in the Hunton Group of Silurian and Devonian age. In the course of documenting the overpressured megacompartments in the deep Anadarko Basin, Al-Shaieb and others (1994) noted the existence of underpressured areas but did not investigate their cause; their study area is outlined in figure 1 and their data set is discussed in a companion report (Nelson and Gianoutsos, chapter 8 of this report). Original reservoir pressure in the extensive Permian age Panhandle-Hugoton gas field (fig. 1) was 435 pounds per square inch (psi) at a depth range of 2,500 to 3,000 feet for a pressure-depth ratio of less than 0.18 pounds per square inch per foot (psi/ft), as described by Pippin (1970) and Sorenson (2005). In 14 fields of 6 counties 


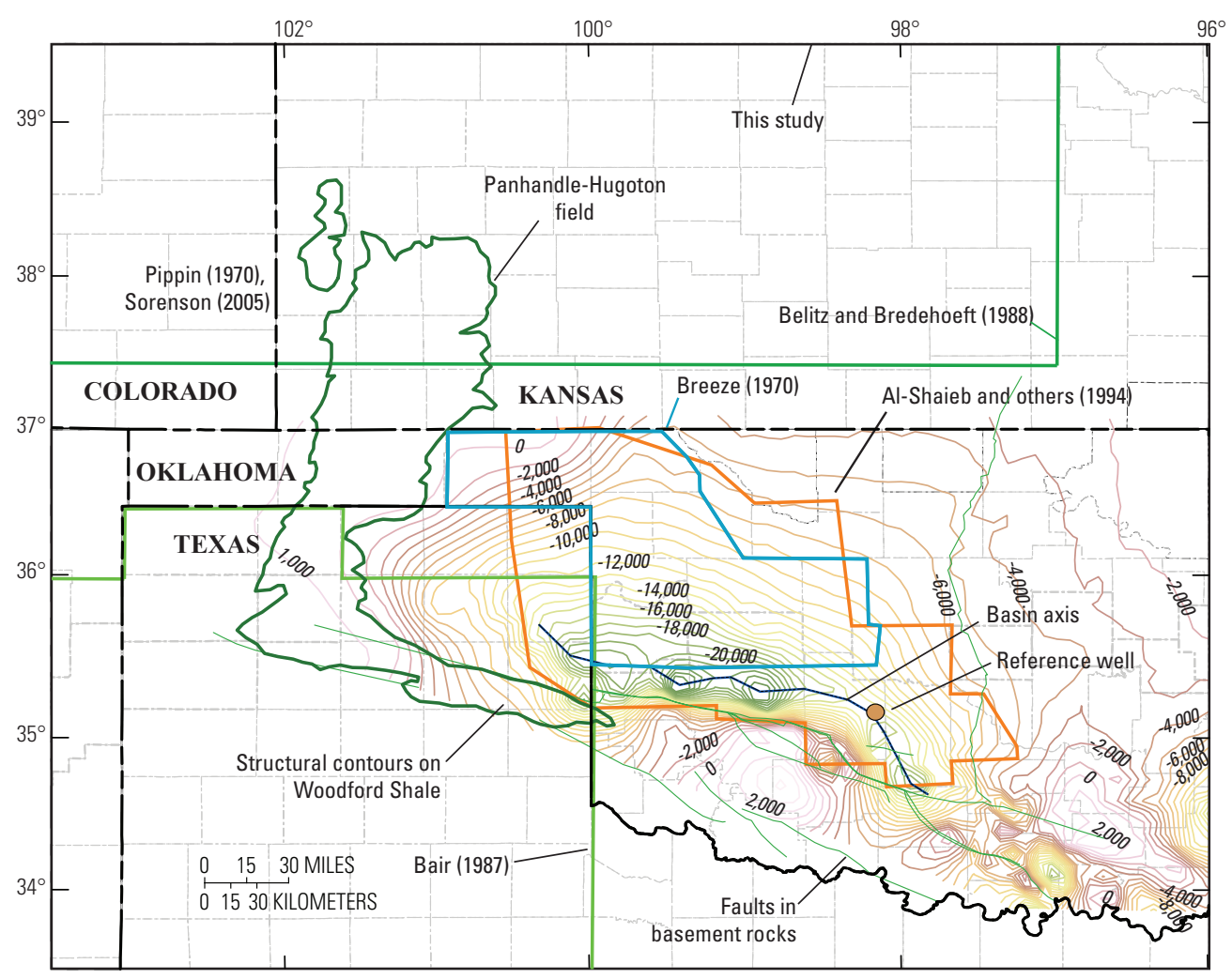

Figure 1. Map showing areas of this and previous studies of overpressure and underpressure. Contours for the top of the Woodford Shale show the structure of the Anadarko Basin; basin axis appears in subsequent figures as a reference line. Reference well is the Ferris $1-28$ well. The study area for this chapter extends from latitude $33.5^{\circ}$ to $39.5^{\circ} \mathrm{N}$. and longitude $96^{\circ}$ to $103.5^{\circ} \mathrm{W}$.

of the eastern part of the Texas Panhandle, the pressure-depth ratio ranges from 0.21 to $0.40 \mathrm{psi} / \mathrm{ft}$ with a median value of $0.29 \mathrm{psi} / \mathrm{ft}$ (Taylor and others, 1977) — rocks in these 14 fields range from Ordovician to Late Pennsylvanian in age, representing 6 of the 7 stratigraphic units considered in this report. Thus, many authors have either noted the presence of underpressure or have reported pore pressure values significantly less than a nominal hydrostatic pressure-depth ratio of 0.465 $\mathrm{psi} / \mathrm{ft}$. From the broad geographic and stratigraphic ranges reported, it is clear that underpressuring in the Anadarko Basin is a widespread phenomenon.

In addition to the aforementioned studies, pressure and depth to the top of reservoir are available for many reservoirs in the Anadarko Basin (NRG Associates, 2009). The ratios of those two values are posted on a map for reservoirs of Desmoinesian age (fig. 3). For purposes of discussion, pressuredepth ratios between 0.43 and $0.50 \mathrm{psi} / \mathrm{ft}$ can be considered to be normally pressured. Only 10 values fall within the normal pressure range and these are scattered throughout much of the mapped area. A few values exceed $0.50 \mathrm{psi} / \mathrm{ft} ; 3$ of these are along or immediately north of the basin axis where overpressuring is known to exist. The rest of the pressure-depth ratios are less than $0.43 \mathrm{psi} / \mathrm{ft}$, showing that underpressuring is widespread throughout much of the area. The lowest values, which are less than $0.30 \mathrm{psi} / \mathrm{ft}$, are mostly clustered in the Texas and Oklahoma Panhandles. The map (fig. 3) substantiates that much of the area is underpressured, most notably the northern and northwestern flanks of the basin. It is also apparent that pressure-depth ratios for reservoirs do not follow any trends in relation to geographic position. The same can be said of pressure data from individual wells, a condition which has provided a challenge in determining potentiometric levels in this study.

Three studies concerned with the distribution of subsurface pressure were helpful to us in developing our hypotheses on the nature of underpressure in the Anadarko Basin. Sorenson (2005) explained the underpressuring of the HugotonPanhandle gas field in terms of aquifer communication with outcrop in eastern Kansas. This report builds on Sorenson's findings by examining the potentiometric distribution of the entire stratigraphic section throughout the basin, finding that the tie to outcrop lies along the Nemaha uplift in central Oklahoma. A hydrodynamic study of the Palo Duro Basin (Bair, 1987) used water level and drillstem measurements extending beyond the limits of the basin itself; the northern 


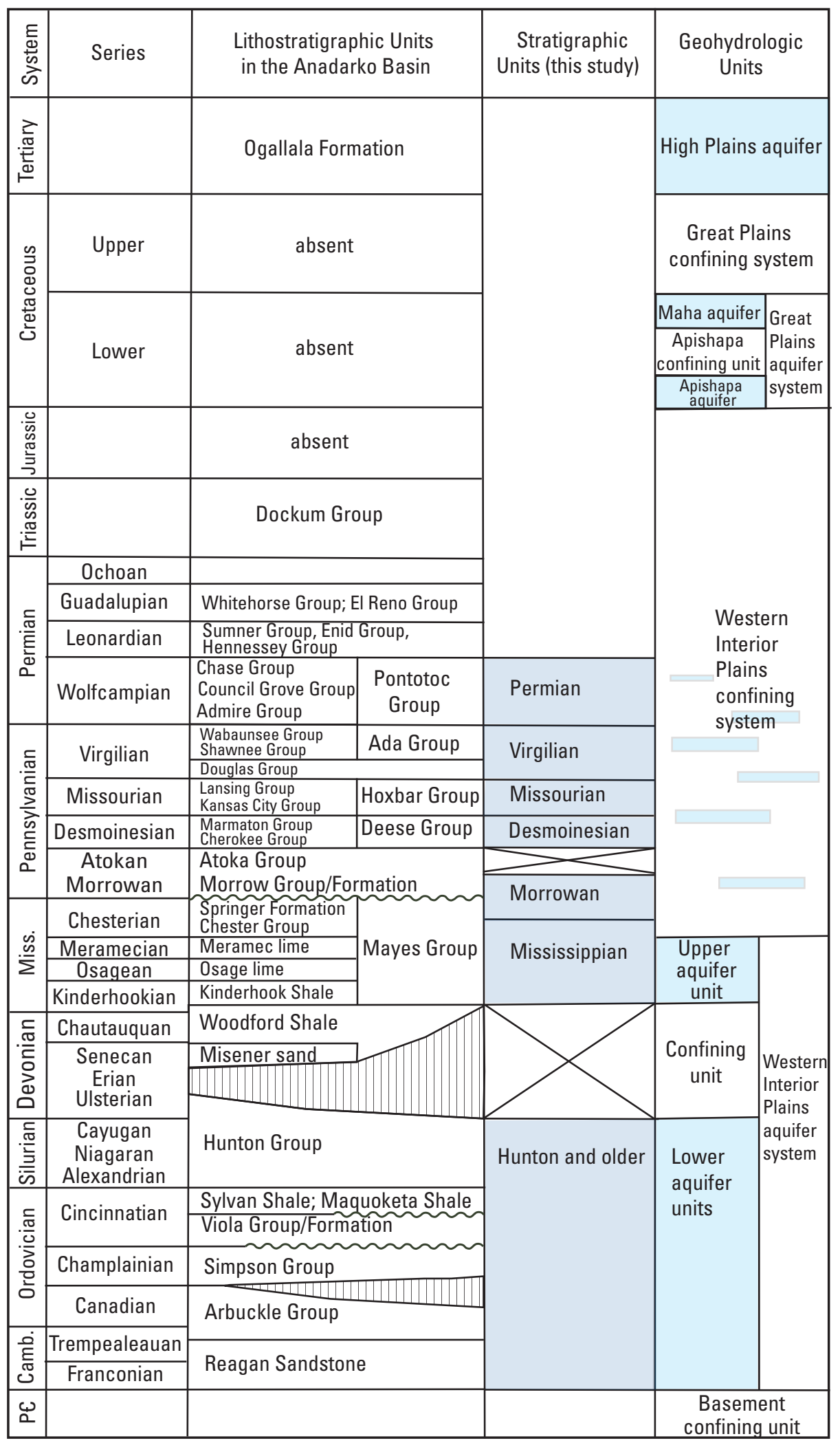

Figure 2. Stratigraphic chart of the Anadarko Basin, with stratigraphic units (purple shading) as defined in this study to examine pressure data. The lithostratigraphic units are modified from Higley (chapter 3 of this report). Geohydrologic units were defined by Jorgensen and others (1996) for a regional study; light blue shading indicates the presence of rock units with well defined patterns of hydraulic head that can be considered to be aquifers. Cretaceous rocks are not present in the study area of figure 1 but are present in the regional study area of Jorgensen and others (1996). Wavy lines represent unconformities. Areas with vertical lines represent periods of non-deposition. PC, Precambrian; Camb., Cambrian; Miss., Mississippian. 


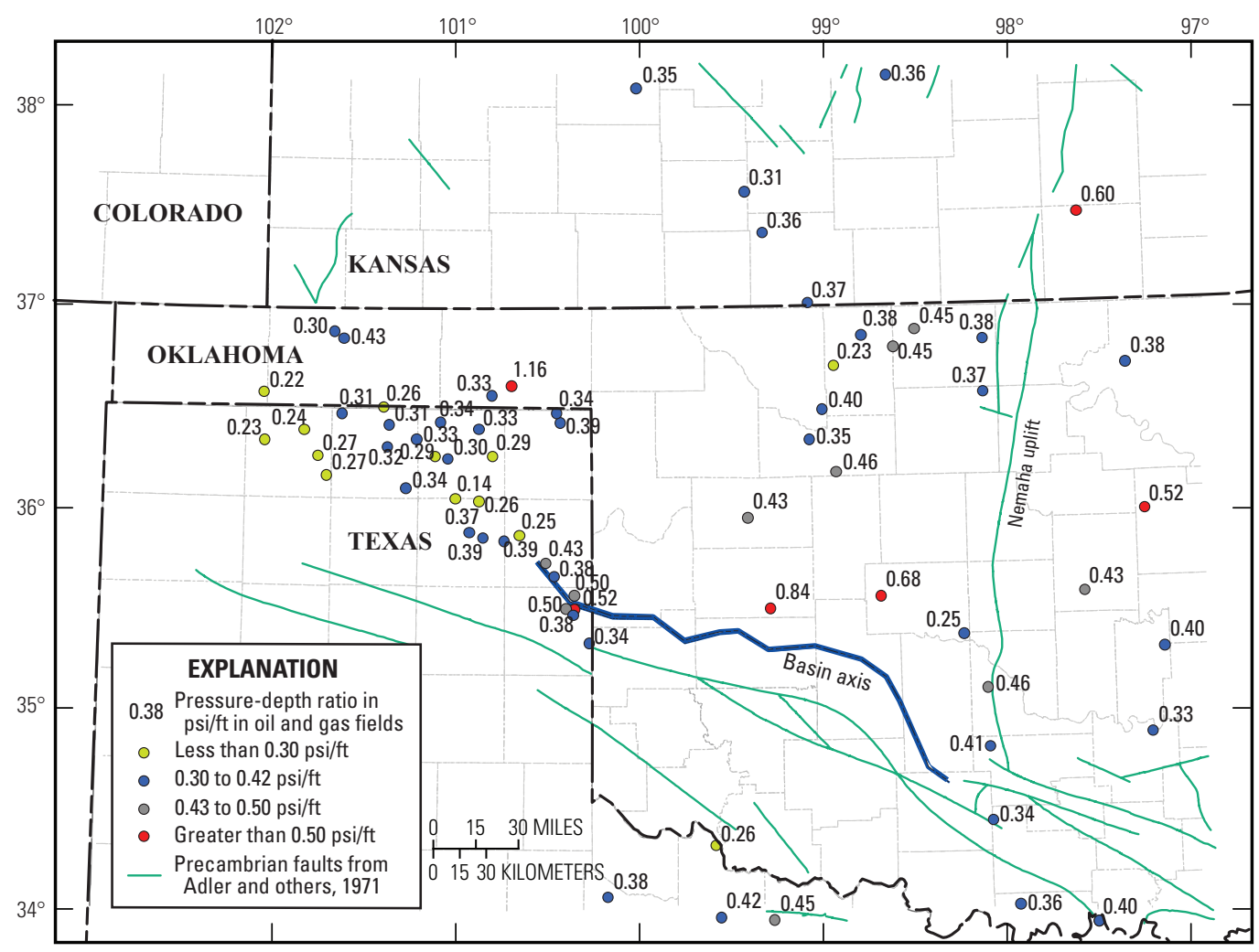

Figure 3. Map of Anadarko Basin showing pressure-depth ratios for reservoirs of Desmoinesian age, based on data from NRG Associates (2009). psi/ft, pounds per square inch per foot.

and eastern boundaries of Bair's study are shown in figure 1. The strata in the deepest of three hydrostratigraphic flow units, which includes Cambrian to Wolfcampian rocks, are underpressured. The underpressured condition is partly attributed to the "greater depth from the ground surface to potentiometric levels in the deep-basin strata beneath the High Plains compared to the Rolling Plains" and that "the regional flow pattern is controlled by the elevation and location of outcrops serving as regional recharge and discharge areas" (Bair, 1987, p. 169). Belitz and Bredehoeft (1988) examined the cause of underpressure in the Denver Basin and developed a hydrologic flow model extending from eastern Colorado into southern South Dakota, Nebraska, and Kansas; the southwestern corner of their study area is indicated in figure 1. The general cause of an underpressured or subnormal condition was stated by Belitz and Bredehoeft (1988, p. 1356):

"Generally, subnormal fluid pressures might be found in any subaerial, topographically tilted, structural basin capped by a thick sequence of lowpermeability rocks (i.e., shale or evaporites). The tilt can provide the topographic driving force for the fluid flow. The low-permeability cap can provide insulation from the elevation head of the water table, and the structure can provide the mechanism for reducing permeability in the basin deep that allows for better hydrologic connection to low-elevation outcrops than to high-elevation outcrops."

As indicated by the findings of Bair (1987), Belitz and Bredehoeft (1988), and Sorenson (2005), surface topography and outcrop elevation are critical considerations in diagnosing an underpressured condition. Surface elevation is greater than 4,400 $\mathrm{ft}$ above sea level in the northwestern part of the study area, and declines to less than $800 \mathrm{ft}$ in the southeastern part (fig. 4). Contours of surface elevation trend north-south in most of the study area, with westward swings where river valleys are present. As a consequence of the general north-south elevation contours, outcrops in central Oklahoma and Kansas also trend north-south, as shown by outcrops of Lower Permian Garber Sandstone and Virgilian formations, and the base of the Permian Wellington Formation (fig 4). These north-south 


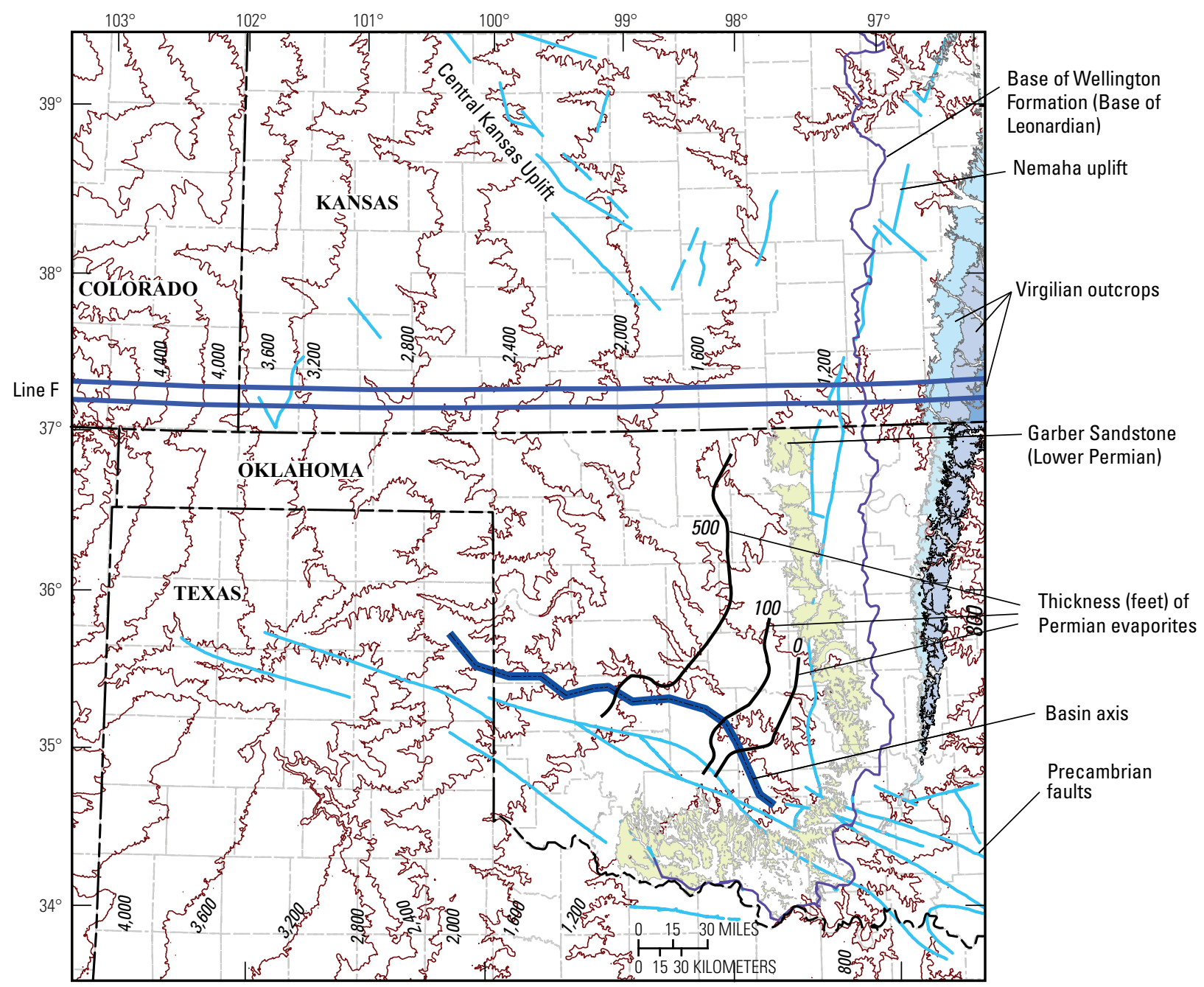

Figure 4. Map of greater Anadarko Basin showing surface elevation contours in 400-foot intervals, basin axis, Precambrian faults, and outcrops of selected formations. Precambrian faults from Adler and others (1971). Thickness of Permian evaporites from Gianoutsos and others (chapter 10 of this report). Outcrops of Garber Sandstone and three Virgilian formations, and base of Wellington Formation in Kansas and Oklahoma from Stoesser and others (2005). East-west oriented Line $\mathrm{F}$ shows location of drillstem test data shown in figure 10.

trending outcrops of Early Permian and Late Pennsylvanian age, in close proximity to the Nemaha uplift, play an important role in our interpretation of the hydrologic regime of the basin.

The burial history chart (fig. 5) reveals rapid deposition and burial during Late Mississippian and Pennsylvanian time. During times of rapid burial, overpressure developed in rocks of the Morrowan, Atokan, Desmoinesian, Missourian, and to a lesser degree in the Virgilian Series, all or in part of Pennsylvanian age, as indicated schematically by the red highlighting in figure 5; overpressure development is the subject of a companion report (Nelson and Gianoutsos, chapter 8 of this report). Uplift and erosion occurred in recent geologic time as indicated by the linear decrease in depth for all rock units from 45 to $0 \mathrm{Ma}$. As uplift and erosion took place, hydrologic conditions were altered, causing the development of underpressure, as symbolized by the vertical blue bar at the right hand side of figure 5. The areal and stratigraphic distribution of underpressure, as expressed in potentiometric and derivative maps, is the subject of this chapter of the report. The burial history chart demonstrates that the creation of overpressure and underpressure are widely separated in geologic time.

Mudstones and evaporites of Permian age form a low permeability cap (fig. 5) that covers most of the study area. The cross section of figure 6 shows that a sequence of mudstones and evaporites is 1,800-ft thick at the Texas-Oklahoma border. Anhydrites and gypsum of the Blaine Formation (fig. 6) extend into Oklahoma where they are mapped in outcrop (Fay, 1964). Based on mud log data from oil and 


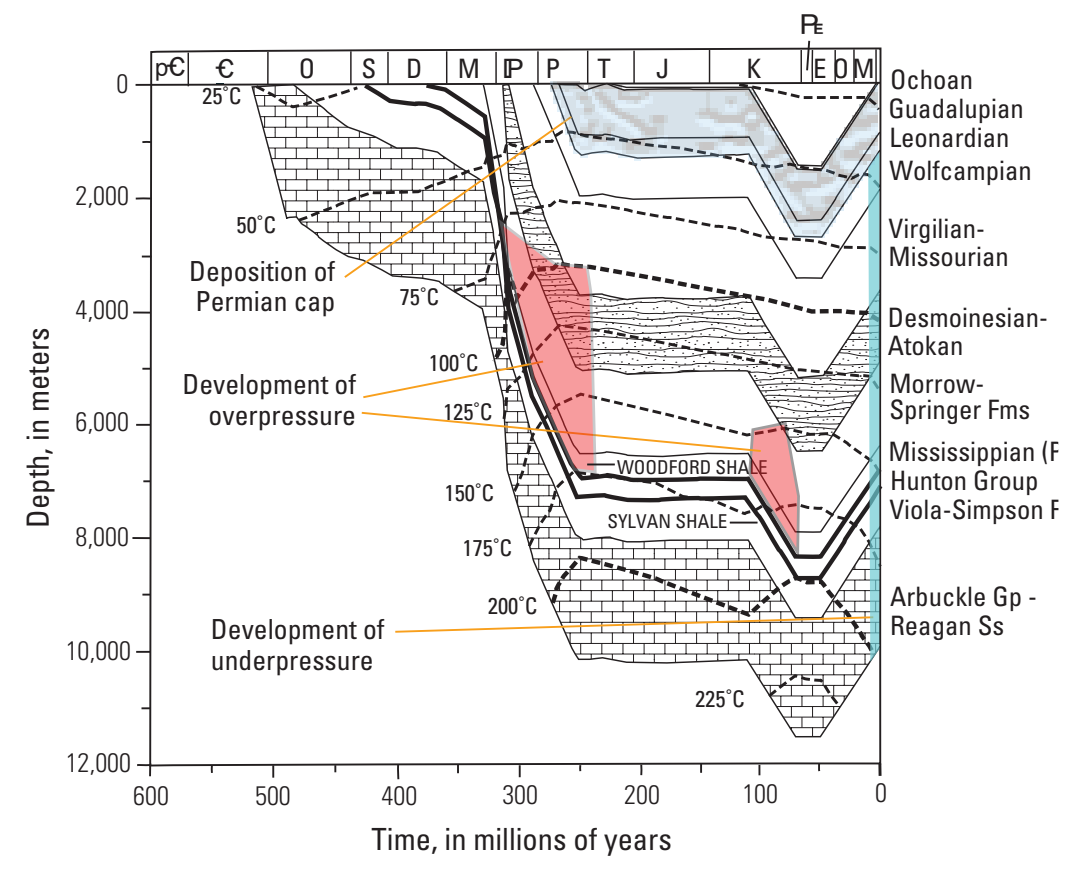

Figure 5. Burial history for the Ferris 1-28 well, from Carter and others (1998). Overpressure developed during rapid burial during Pennsylvanian time with a possible secondary development around $100 \mathrm{Ma}$. Underpressure development is believed to be recent, commensurate with exposure of strata in central Oklahoma. The Permian cap provides a low permeability seal over the area. $\mathrm{pC}$, Precambrian; $C$, Cambrian; 0, Ordovician; S, Silurian; D, Devonian; M, Mississippian; P, Pennsylvanian; P, Permian, T, Triassic, J, Jurassic; K, Cretaceous; $P_{E^{\prime}}$ Paleocene; $E$, Eocene; 0, Oligocene, M, Miocene; Fms, Formations; Gp, Group; Ss, Sandstone, ${ }^{\circ} \mathrm{C}$, degrees Celsius.

gas wells, evaporites are nearly ubiquitous in Upper Permian strata throughout the Anadarko Basin (Gianoutsos and others, chapter 10 of this report), thinning to zero thickness immediately west of the Nemaha uplift (fig. 4). The eastward extent of Leonardian strata is demarcated by the base of the Wellington Formation of the Enid Group in the vicinity of the Nemaha uplift (fig. 4). Although no direct measurements of permeability were conducted for this study, the permeability of evaporites below $1,500 \mathrm{ft}$ of burial is reduced to levels so low that the beds are considered impermeable to groundwater flow, based on geological evidence and laboratory measurements (Ingebritsen and others, 2008, p. 340-342). Thus an impermeable cap of Permian age separates shallow aquifers from deeper strata that are Pennsylvanian and older.

These four geologic elements - basin structure, surface topography, uplift and erosion, and an impermeable cap (figs. $1,4,5$, and 6) - compose the essential elements resulting in the present-day hydrologic regime. The main contribution of this report is documentation of the potentiometric surfaces at the basin scale for seven stratigraphic units-Hunton Groupand-older, Mississippian, Morrowan, Desmoinesian, Missourian, Virgilian, and Permian. Inspection of the potentiometric surfaces, and of a second set of surfaces showing the separation between each potentiometric surface and surface elevation, provide an explanation for the underpressured conditions that exist in the northern and northwestern portions of the greater Anadarko Basin.

The present study lies in the southwestern part of a regional examination of major hydrologic units in the central Midwest, an area encompassing all of Kansas and Nebraska and parts of adjoining states (Jorgensen and others, 1993). The relation between the aquifers and confining units of the regional study and the stratigraphic units of this chapter of the report can be examined in figure 2. Of the units defined in the regional study, the Western Interior Plains aquifer and confining systems are most relevant to the present study. The lower and upper aquifer units of the Western Interior Plains aquifer are nearly identical to the Hunton-and-older and Mississippian 
ELEVATION

METERS FEET

$1,300-4,200$

$1,200-4,000$

$-3,800$

$1,100-3,600$

$1,000-3,400$

$900-3,000$

$-2,800$

$800-2,600$

$-2,400$

$-2,200$

$600-2,000$

$-1,800$

$500-1,600$

$400=-1,400$

$300-1,000$

$-800$

$200-600$

$00-\begin{array}{r}-400 \\ -200\end{array}$

$-200$

Sea ${ }^{\text {Sevel }}$

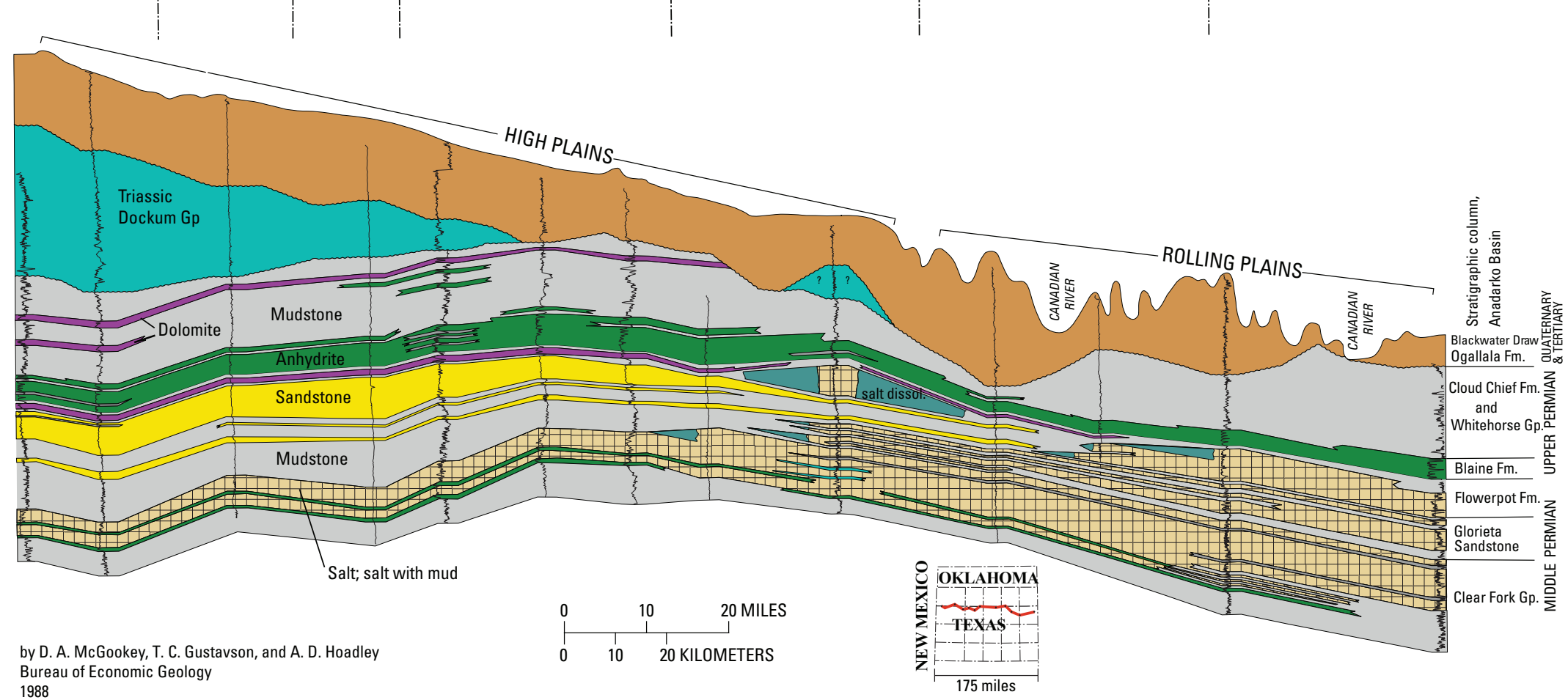

VERTICAL EXAGGERATION = 106

Dalhart Basin

Cimarron Arch

Anadarko Basin

Figure 6. Structural cross section showing Permian and younger rocks in the Texas Panhandle (see inset for location). Mudstone, anhydrite, and salt form impermeable layers in Permian rocks. An interpreted salt dissolution feature is near the center of the section. From McGookey and others (1988). Co., County; Quat., Quaternary; Tert., Tertiary; Fm., Formation; Gp., Group. 
stratigraphic units of the present study. The Western Interior Plains confining system includes permeable sandstone and limestone beds within thick shale units. Because the sandstone and limestone beds do not extend across the entire area of the confining system, they are not considered a regional hydraulic system (Jorgensen and others, 1993, p. B50). However, as demonstrated in this paper, maps of hydraulic head values for the Morrowan, Desmoinesian, Missourian, Virgilian, and Permian stratigraphic units suggest good hydraulic continuity over our study area. Corresponding lithostratigraphic units for the Cretaceous age Great Plains aquifer and confining systems are not shown in figure 2, because Cretaceous age rocks are only marginally present in our study area and were not considered as part of this study. The High Plains aquifer, which consists of the Ogallala Formation and unconsolidated deposits, will receive only brief consideration in this chapter of the report.

To develop potentiometric surfaces, we used pressure measurements in oil and gas wells that were obtained from drillstem tests over a time period of more than 50 years. Two steps are required to construct a potentiometric surface from pressure data. The first step, the conversion of a pressure measurement to hydraulic head, seemingly a straightforward computation, is complicated by the presence of gas and by variations in the density of water, the latter primarily because of salinity variations; these complications are discussed in the following sections, "Conversion of pressure to hydraulic head" and "Effect of density variations on the computation of hydraulic head." The second step, the conversion of individual hydraulic head values to form a potentiometric surface, requires examination of a large number of values distributed unevenly within the study area. For several reasons, only a fraction of these values are valid and can be used to define a potentiometric surface. The useful fraction is determined on a set of 13 west-east swaths on which the data are inspected and filtered, and from which a potentiometric level is defined as a continuous curve along the length of the swath. The potentiometric levels from the swaths are then combined using mapping algorithms to construct a potentiometric surface. These procedures are described in detail in the section "Potentiometric surfaces."

\section{Conversion of Pressure to Hydraulic Head}

In oil and gas exploration, formation pressure $P$ is routinely measured and plotted as a primary parameter of interest. As an example, pressure data from the Oklahoma Panhandle are plotted as a function of the elevation of the test interval (fig. 7). A solid blue line represents a hydrostatic gradient of $0.465 \mathrm{psi} / \mathrm{ft}$ and an intercept of 2,542 ft on the vertical axis, which is the average surface elevation for this dataset. Virtually all the pressure data fall to the left of the hydrostatic line, indicating a state of underpressure. Four dashed lines, each with a gradient of $0.465 \mathrm{psi} / \mathrm{ft}$, intercept the elevation axis at $0,500,1,000$, and 1,500 ft. Each intercept represents the value of hydraulic head $H$ for a point lying on its respective line. For example, a point lying on the upper dashed line has a value of $H$ equal to $1,500 \mathrm{ft}$.

In hydrological work, hydraulic head $H$ (also referred to as potentiometric elevation) is a primary parameter of interest. In order to map potentiometric surfaces within a stratigraphic unit, we need to convert measurements of $P$ to $H$. The relation between the two, which is discussed in textbooks (for example, Fetter, 1988; Toth, 2009), is straightforward if three assumptions can be made regarding the fluids in the formation. First, it is assumed that flow velocity is negligible, so that kinetic energy is unimportant compared to potential energy. Second, it is assumed that fluid density does not vary much within the area under study. This second assumption is violated because the density of water varies with pressure, temperature, and salinity; the resulting limitations on the conversion of $P$ to $H$ and the resulting potentiometric surfaces are discussed in the section "Effect of density variations in the computation of hydraulic head." Third, if gas instead of water is the continuous phase, then the simple relation that follows cannot be applied, as discussed by Nelson and Condon (2008). Consequently, we chose not to compute $H$ in the deep overpressured basin, because we suspect that gas is the continuous phase in parts of the deep basin.

Hydraulic head, $H$, is the sum of two components,

$$
H=Z+P / \rho g
$$

where

$$
\begin{aligned}
& Z \quad \text { is the elevation of the perforated interval, } P \text { is } \\
& \text { the formation pressure, } \rho \text { is the density of } \\
& \text { water, and } g \text { is the acceleration of gravity. } \\
& \text { In oilfield units } Z \text { is in feet, } P \text { is in psi, } \rho g \\
& \text { is in psi/ft, and consequently } H \text { is in feet. } \\
& \text { Equation } 1 \text { a can be rearranged, }
\end{aligned}
$$

$$
Z=H-P / \rho g
$$

which is the equation for the lines plotted in figure 7 , with $Z$ the variable on the vertical axis, $H$ the intercept on the vertical axis, $P$ the variable on the horizontal axis, and $1 / \rho g$ the slope of the line. For any pressure measurement $P$ at an elevation $Z$, the equivalent $H$ can be determined graphically (fig. 7) or computationally using equation $1 \mathrm{a}$. It should be mentioned that a pressure-depth plot is not compatible with equation $1 \mathrm{~b}$. Compatibility with equation $1 \mathrm{~b}$ requires that pressure be plotted as a function of elevation, as it is in figure 7.

Following the convention of other investigators in the Anadarko Basin, we set $\rho g=(\rho g)_{0}=0.465 \mathrm{psi} / \mathrm{ft}$, which corresponds to a brine density of $1.07 \mathrm{~g} / \mathrm{cm}^{3}$. As already mentioned, density varies as a function of salinity, temperature and pressure, so adoption of a fixed value of $0.465 \mathrm{psi} / \mathrm{ft}$ introduces errors that are discussed in the section "Effect of density variations in the computation of hydraulic head." The reference 


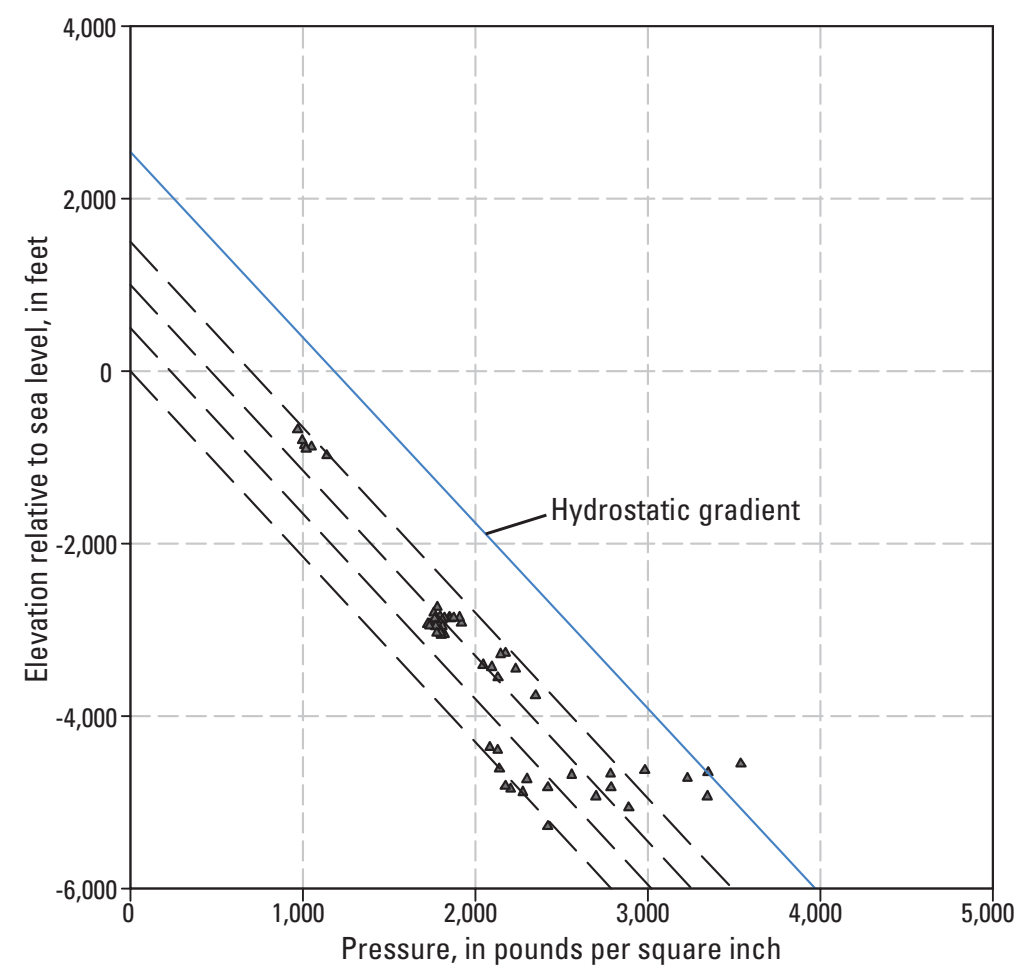

Figure 7. Graph showing pressure in relation to elevation from wells in a small area in the Oklahoma Panhandle. The average surface elevation of 2,542 feet is represented by the $y$-intercept of the hydrostatic gradient. The dashed lines are reference lines for the pressure data, most of which correspond to underpressured conditions.

elevation for $Z$ is taken to be sea level, which means that $H$ is determined relative to sea level. Elevations above sea level are positive and elevations below sea level are negative.

As an example, consider the case where the pressure $P$ determined from a drillstem test is $700 \mathrm{psi}$, the mean perforated depth $D$ for the drillstem test is $2,000 \mathrm{ft}$, and the Kelly Bushing elevation (KBE), is 3,500 ft (Well $A$ in fig. 8). (Depth in a well is measured from the Kelly bushing on the drill rig rather than from ground surface; consequently KBE must be used to determine the elevation of a point in the well corresponding to a particular depth.)

The mean elevation for the drillstem test is

$$
Z=-(D-K B E)=-(2,000-3,500)=1,500 \mathrm{ft} .
$$

The hydraulic head is

$$
H=Z+P /(\rho \mathrm{g})_{o}=1,500+700 / 0.465=3,000 \mathrm{ft} .
$$

And the pressure/depth ratio for this example is

$$
P / D=700 / 2,000=0.35 \mathrm{psi} / \mathrm{ft} .
$$

Hydraulic head is computed for each stratigraphic unit and displayed as a function of latitude or longitude. Pressures from drillstem tests, depths of measurement, and KBEs were taken from IHS Energy (2009). The pressure value is the largest of either the initial shut-in value or the final shut-in value of a drillstem test. A very low pressure will result in a hydraulic head not much greater than $Z$, the measurement elevation, and is considered invalid. A potentiometric surface is determined by mapping and contouring the high values of hydraulic head of each stratigraphic unit.

The pressure-depth ratio can be expressed in terms of $H$, $Z$, and $D$ by rearranging equation $1 \mathrm{a}$ and dividing by $D$,

$$
P / D=0.465^{*}(H-Z) / D
$$

which shows that the pressure-depth ratio is proportional to $(H-Z) / D$. In figure $8,(H-Z) / D=(3,000-1,500) / 2,000=$ $0.75 \mathrm{ft}$ in Well A and $(H-Z) / D=[1,000-(-2,000)] / 4,000=0.75$ in Well $\mathrm{B}$, showing that the $\mathrm{P} / \mathrm{D}$ ratio is 0.75 that of normal in both wells, and is equal to $0.75 \times 0.465=0.35 \mathrm{psi} / \mathrm{ft}$. Equation 2a has a simple geometrical interpretation: if the cross formed by the vertical brown line (length $D$ ) and the horizontal blue 


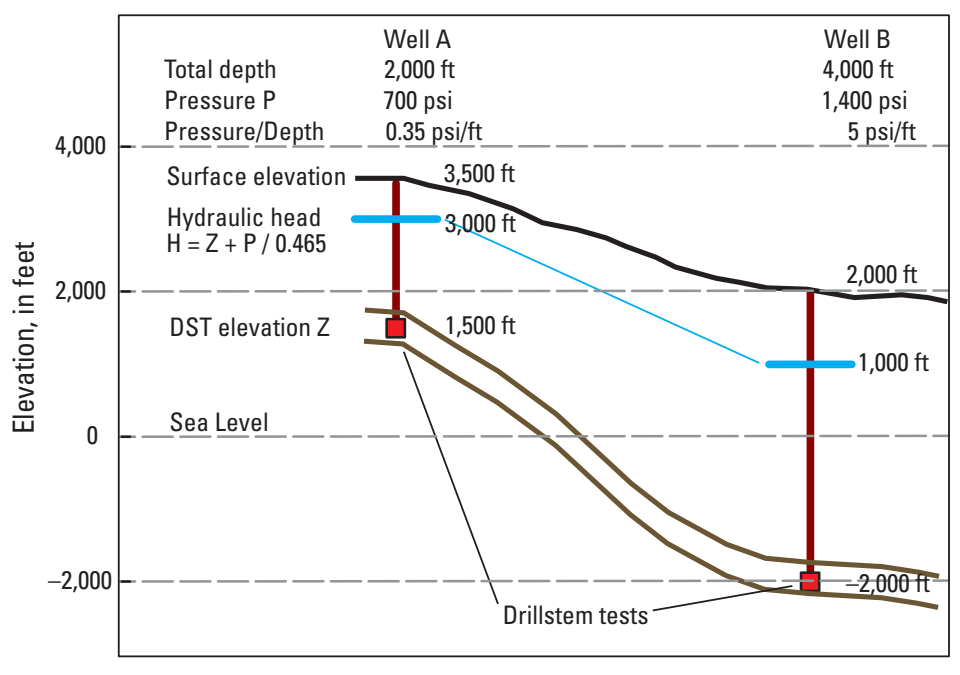

Horizontal distance, many miles

Figure 8. Example demonstrating the calculation of hydraulic head and pressure-depth ratio for two wells testing the same formation. The pressure-depth ratio is the same in the two wells, but the hydraulic head is much greater in Well A than in Well B. ft, foot; psi, pounds per square inch; psi/ft, pounds per square inch per foot.

line (elevation $H$ ) retains the same proportion from one well to the next, then the pressure-depth ratio is constant.

Equation 2a can also be written in terms of the vertical separation between the surface elevation $K B E$ and $H$,

$$
P / D=0.465^{*}\{1-(K B E-H) / D\}
$$

The main products of the present work are maps of hydraulic head $H$, referred to as potentiometric surfaces, and of $H-K B E$, the separation between $H$ and the Earth's surface. The separation $H-K B E$ is used as an indicator of underpressure rather than the ratio $P / D$.

\section{Effect of Density Variations in the Computation of Hydraulic Head}

The conversion of pressure $P$ to hydraulic head $H$ in equation 1a requires a choice of density $\rho g$. We used a value $(\rho g)_{o}=0.465 \mathrm{psi} / \mathrm{ft}$, a value that corresponds to a sodium chloride brine with a specific gravity of 1.074 and a salinity of approximately 101,700 parts per million (ppm) at standard conditions of pressure and temperature. Because density varies with salinity, pressure, and temperature, the effect of this assumption on $H$ at different locations in the basin requires evaluation.

\section{Relation Between Salinity and Density for Sodium Chloride Solutions}

Salinity is measured by weighing a salt solution, then weighing the solids that remain after evaporating the solution. Salinity is referred to as "total dissolved solids" (TDS) and expressed as weight percent or as ppm. For example, 1,000 grams ( $\mathrm{g}$ ) of solution containing $30 \mathrm{~g}$ of solids would have TDS $=(30 / 1,000) * 100=3$ weight percent or $(30 / 1,000)^{*} 10^{6}=30,000 \mathrm{ppm}$. The TDS of sea water is around $35,000 \mathrm{ppm}$.

The volume of brine is not a simple function of solute weight and water weight. Consequently, the density of brine (weight divided by volume) cannot be computed simply from TDS, it must be measured. Measurements for sodium chloride brine are tabulated in terms of specific gravity (SG) as a function of TDS (Wolf and others, 1973). (The specific gravity of brine is equivalent to the weight of brine divided by the weight of an equivalent volume of distilled water. Specific gravity is a dimensionless quantity.) The density of brine in field units 
such as pounds per gallon (ppg), or pounds per square inch per foot (psi/ft) are related to SG by

$$
\mathrm{SG}=\rho \mathrm{g}(\mathrm{ppg}) / 8.33=\rho \mathrm{g}(\mathrm{psi} / \mathrm{ft}) / 0.433
$$

where $8.33 \mathrm{ppg}$ is the density of water in pounds per gallon and $0.433 \mathrm{psi} / \mathrm{ft}$ is the density of water in $\mathrm{psi} / \mathrm{ft}$. In the petroleum industry, the unit of $\mathrm{psi} / \mathrm{ft}$ is referred to as pressure gradient. However, psi/ft is also a unit of density, being the weight in pounds of water contained in 12 cubic inches (one square inch in area by one foot in height). The relations among density, specific gravity, and salinity for sodium chloride solutions are given in table 1.

The density of water increases with an increase in pressure and decreases with an increase in temperature. Because both pressure and temperature increase with increasing depth, the two effects tend to cancel over restricted ranges of pressure and temperature. For example, the volume factor for water is $1.00 \pm 0.01$ for a temperature of $100^{\circ} \mathrm{F}$ and pressures of 1,000 , 2,000 , and $3,000 \mathrm{psi}$, and also for a temperature of $150^{\circ} \mathrm{F}$ and pressures of 3,000, 4,000, and 5,000 psi (table 2). However, at $200^{\circ} \mathrm{F}$, a pressure greater than $5,000 \mathrm{psi}$ is required to maintain a volume factor of $1.00 \pm 0.01$. Temperatures of $200^{\circ} \mathrm{F}$ and greater are attained in the deep basin, but not on the flanks of the basin that are the focus of this study. Thus, most of the variation in density will be because of salinity variations, which are examined next.

\section{Salinity of Subsurface Waters}

In the Anadarko Basin, the salinity of produced waters takes a wide range of values, from less than $10,000 \mathrm{ppm}$ to greater than $300,000 \mathrm{ppm}$ (fig. 9, pl. 1). The 8 salinity increments of approximately $30,000 \mathrm{ppm}$ chosen for the maps of plate 1 correspond to equal increments of $0.01 \mathrm{psi} / \mathrm{ft}$ for density, as shown in table 1 . High salinity values are shown in the brightly colored symbols and low salinities are in dull colors. Unfortunately, the salinity data are not distributed evenly across the basin, but are clustered along and east of the Nemaha uplift in Oklahoma and within the Central Kansas uplift (pl. 1). Data within the deep Anadarko Basin are few in number, with the exception of data for the Morrow Formation (pl. 1C). Some observations are:

1. The highest values, greater than $220,300 \mathrm{ppm}$, lie along the south-north trending Nemaha uplift in the following units: Hunton-and-older, Mississippian (limited data), Desmoinesian, Missourian, and Virgilian. Salinities

Table 1. Increments of density used for salinity maps of plate 1, in units of pounds per square inch per foot. Specific gravity (dimensionless) is equivalent to density. Equivalent total dissolved solids (parts per million, ppm) for sodium chloride solutions taken from Wolf and others, 1973. $\Delta \rho / \rho$ (dimensionless) is the fractional change in density from the base value of $0.465 \mathrm{psi} / \mathrm{ft}$.

[psi/ft, pounds per square inch per foot; TDS, total dissolved solids]

\begin{tabular}{ccccccrrr}
\hline $\begin{array}{c}\text { Density } \\
\text { (psi/ft) }\end{array}$ & $\mathbf{0 . 4 3 3}$ & $\mathbf{0 . 4 4 5}$ & $\mathbf{0 . 4 5 5}$ & $\mathbf{0 . 4 6 5}$ & $\mathbf{0 . 4 7 5}$ & $\mathbf{0 . 4 8 5}$ & $\mathbf{0 . 4 9 5}$ & $\mathbf{0 . 5 0 5}$ \\
\hline Spec. gravity & 1 & 1.028 & 1.051 & 1.074 & 1.097 & 1.120 & 1.143 \\
TDS (ppm) & 0 & 35,754 & 70,552 & 101,737 & 132,349 & 162,377 & 191,723 & 220,284 \\
$\Delta \rho / \rho$ & -0.0688 & -0.0430 & -0.0215 & 0.0000 & 0.0215 & 0.0430 & 0.0645 & 0.0860 \\
\hline
\end{tabular}

Table 2. Specific volume of water as a function of pressure and temperature, from Amyx and others (1960).

\begin{tabular}{ccccc}
\hline $\begin{array}{c}\text { Pressure } \\
\text { (pounds per square inch) }\end{array}$ & \multicolumn{4}{c}{ Temperature $\left({ }^{\circ} \mathbf{F}\right)$} \\
\hline & $\mathbf{1 0 0}$ & $\mathbf{1 5 0}$ & $\mathbf{2 0 0}$ & $\mathbf{2 5 0}$ \\
\hline 1000 & 1.0025 & 1.0153 & 1.0335 & 1.0560 \\
2000 & 0.9995 & 1.0125 & 1.0304 & 1.0523 \\
3000 & 0.9966 & 1.0095 & 1.0271 & 1.0487 \\
4000 & 0.9938 & 1.0067 & 1.0240 & 1.0452 \\
5000 & 0.9910 & 1.0039 & 1.0210 & 1.0418 \\
\hline
\end{tabular}


decrease eastward from the Nemaha uplift in all five of these units, showing similar salinity trends in all units for which data are available for the Nemaha uplift.

2. Along the central Kansas uplift, in rocks of Missourian, Virgilian, and Permian age, TDS is greater than $220,300 \mathrm{ppm}$ at the southeast end of the uplift and decreases to the northwest, although values at the northwest end of the uplift generally remain greater than $100,000 \mathrm{ppm}$. In rocks that are Hunton and older in age, the highest values are 132,000 ppm at the southeast end of the central Kansas uplift, and decrease to the northwest where many values are less than 36,000 ppm. Thus, along the Central Kansas uplift, waters in rocks that are Hunton and older in age are considerably less saline than rocks of Missourian, Virgilian, and Permian age.

3. Waters in rocks of Morrowan age are less than $36,000 \mathrm{ppm}$ in the deep basin and onto the shelfal area of the northeastern Texas Panhandle and the eastern Oklahoma Panhandle (pl. 1C). Values are erratic north and west of the limit of the $36,000 \mathrm{ppm}$ values. Spatial variations in the chemistry of Morrowan waters are discussed in chapter 8 of this report on present-day overpressure and paleopressure indicators (Nelson and Gianoutsos, chapter 8 of this report).

4. In areas other than the Nemaha uplift and the Central Kansas uplift, and with the exception of the Morrowan unit, the salinity data are few in number and reveal no obvious trends.

\section{Problem of Variable Brine Density in Computing Hydraulic Head}

Variable brine density constitutes an important theoretical problem in basin-scale groundwater flow modeling (Bachu, 1995; Bachu and Michael, 2002). In dealing with aquifers of limited spatial extent, it is reasonable to assume constant brine density when computing hydraulic head in accordance with equation $1 \mathrm{a}$,

$$
H=Z+P / \rho g
$$

However, for the deep confined basin-scale aquifers that are considered in this chapter of this report, the brine density $\rho g$ varies throughout the basin because of variations in salinity, pressure, and temperature. As explained by Bachu (1995), because of the variations in density $\rho g$, the driving force on a unit quantity of brine cannot be written simply as the gradient of a potential, $H$. However, the horizontal component of flow in aquifers of finite dip can be represented in the following formulation of Darcy's law (Bachu and Michael, 2002, equation 5),

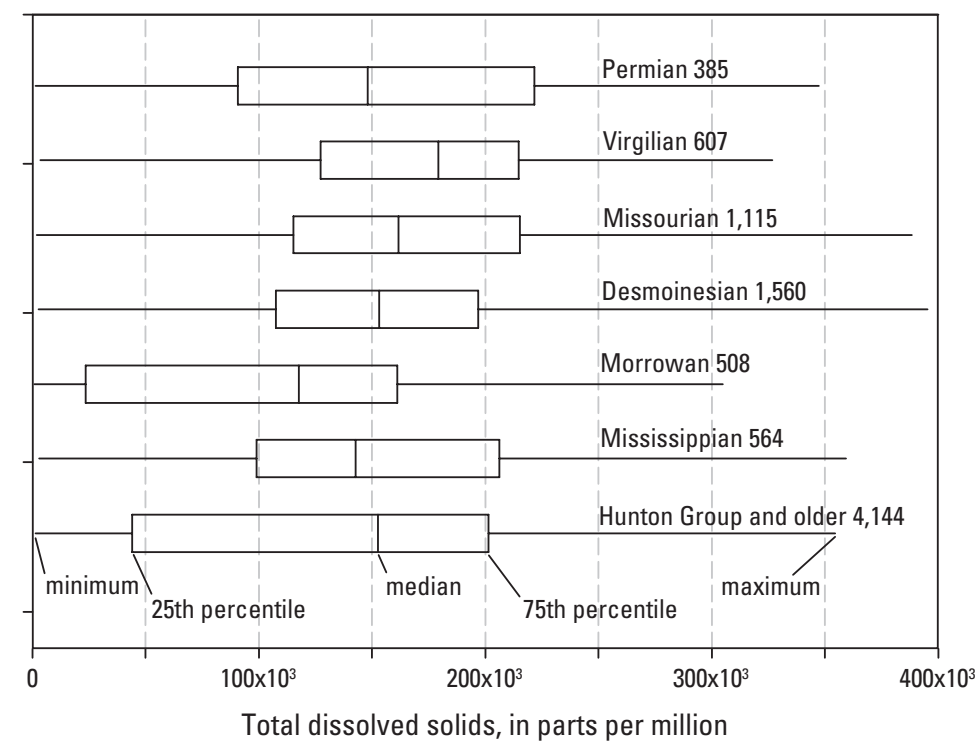

Figure 9. Box-and-whiskers plot of salinity in seven stratigraphic units with number of samples in each group. Each whisker and each box represents 25 percent of the data. Salinity data taken from a compilation by Breit (2002), which is based on waters produced from oil and gas wells. 


$$
q=-\frac{k \rho_{o} g}{\mu}\left(\nabla H_{o}+\frac{\Delta \rho}{\rho_{\mathrm{o}}} \nabla E\right)
$$

where

$k \quad$ is permeability, $\mu$ is viscosity, $H_{\mathrm{o}}$ is defined in equation 1a with $\rho$ set equal to $\rho_{o}, \Delta \rho=$ $\rho-\rho_{o}$, and $E$ is the elevation of the surface representing the aquifer. This formulation shows that the flow vector $\boldsymbol{q}$ is the vector sum of $\nabla H_{o}$, which is directed along the maximum hydraulic gradient, and $\nabla E$, which is directed along the maximum slope of the aquifer surface. The term $\nabla H_{o}$ is the force due to the combined gradients of pressure and measurement elevation and is directed perpendicular to the contours of $H_{\mathrm{o}}$. The term $(\Delta \rho / \rho) \nabla E$ is the buoyancy force because of variations in brine density and is directed downslope if $\rho>\rho_{o}$, and $\Delta \rho$ is therefore positive, or upslope if $\rho<\rho_{o}$. In areas where the leading term, is unchanging, the direction of flow vector $q$ is given by the vector sum of the two terms in equation 4.

The buoyancy term $(\Delta \rho / \rho) E$ in equation 4 is insignificant in areas where (1) density ( $\rho g)$ is equal to $(\rho g)$ or nearly so, and (or) (2) lateral changes in the elevation of the aquifer are small. In these two situations, the flow vector direction depends only on the gradient of hydraulic head. On the other hand, the $\nabla H_{o}$ term can be less than the $\nabla E$ term in equation 4 wherever the hydraulic head $H_{\mathrm{o}}$ has little lateral variation but density and aquifer elevation are changing. As a consequence of salinity and elevation changes, the flow vector $\boldsymbol{q}$ will undergo local variations in direction that cannot be derived from inspection of the potentiometric surface. This fact must be kept in mind when inspecting the potentiometric surfaces discussed in the subsection "Maps showing potentiometric surfaces and overpressured areas."

Numerical hydrologic models are a means of accounting for variations in density and permeability. As an example, along the Nemaha uplift and central Kansas uplift where salinity variations are greatest, a numerical model that accounts for variable density produced flow vectors that differ significantly from flow vectors produced by a constant-density model (Signor and others, 1996, p. C59-C75). However, at this point our purpose in constructing potentiometric maps and cross sections is to understand the nature of underpressuring in the Anadarko Basin and not to model groundwater flow. Equation 4 is not used to compute flow vectors but serves to explain the limitations of the potentiometric surfaces that are a product of this report.

The preceding discussion of density variations leads to two conclusions regarding the computation of hydraulic head in the Anadarko Basin. First, we cannot expect that local flow vectors can be accurately derived from the potentiometric surfaces. Second, the effect of salinity variations are likely to be greatest in those areas where salinity gradients are manifest, particularly along the Nemaha uplift and along the southern edge of the Central Kansas uplift.

\section{Computation of Hydraulic Head along West-East and South-North Swaths}

The computation of hydraulic head along a west-east profile is illustrated in figure 10, which shows drillstem tests within a swath that is six miles wide in the north-south direction. Each data point in parts $A, B$, and $C$ of figure 10 represents either data (surface elevation, elevation of drillstem tests, pressure) or a computed parameter (hydraulic head, pressuredepth ratio) from rocks of Missourian age. The surface elevations reveal the eastward drop from $4,200 \mathrm{ft}$ in the west to $1,000 \mathrm{ft}$ at the east end of the swath. The concave-upward pattern of the test elevations, which lie above sea level at the west and east ends of the swath, results from the intersection of the west-east line and the northward-shallowing basin geometry. The upper edge of the pressure data forms a convexupward pattern, which when summed with the test elevations in accordance with equation 1a, forms the upper edge of the hydraulic head pattern (fig. 10A) that is labeled "interpreted level of hydraulic head." Although the elevations and pressure measurements vary by thousands of feet, their values are complementary and tend to cancel when summed, leaving an interpreted level of hydraulic head that changes by less than $500 \mathrm{ft}$ from west to east.

Pressures falling below the convex-upward bound in figure $10 B$ did not sample the original formation pressure and result in hydraulic head values that fall below the upper edge in figure $10 \mathrm{~A}$. Thus, only a fraction of the pressure measurements convert to interpretable values of hydraulic head which are indicated by the horizontal lines at the upper edge of the red dash symbols. A few values of hydraulic head $H$ that are higher than the interpreted level of hydraulic head, such as those from longitude $101^{\circ}$ to $102^{\circ} \mathrm{W}$., could be because of small isolated compartments of high pressure or possibly because of pressure maintenance operations. The elevation of the Ogallala water table is considerably higher than the interpreted level of hydraulic head for the Missourian rocks (fig. 10A). The impermeable cap of Permian rocks (fig. 5) separates the shallow Ogallala aquifer from the deeper and older hydrological systems represented by Missourian rocks in figure 10 .

Dividing a pressure measurement by the depth of the measurement results in the pressure-depth ratio plotted in figure $10 C$. As was the case for hydraulic head, only the upper edge of the data swarm represents the true pressure-depth ratio at that location, because most of the pressure measurements were less than true formation pressure. The upper edge of the swarm of pressure-depth values reaches a maximum at long $98^{\circ} \mathrm{W}$., where it is somewhat less than the value of 

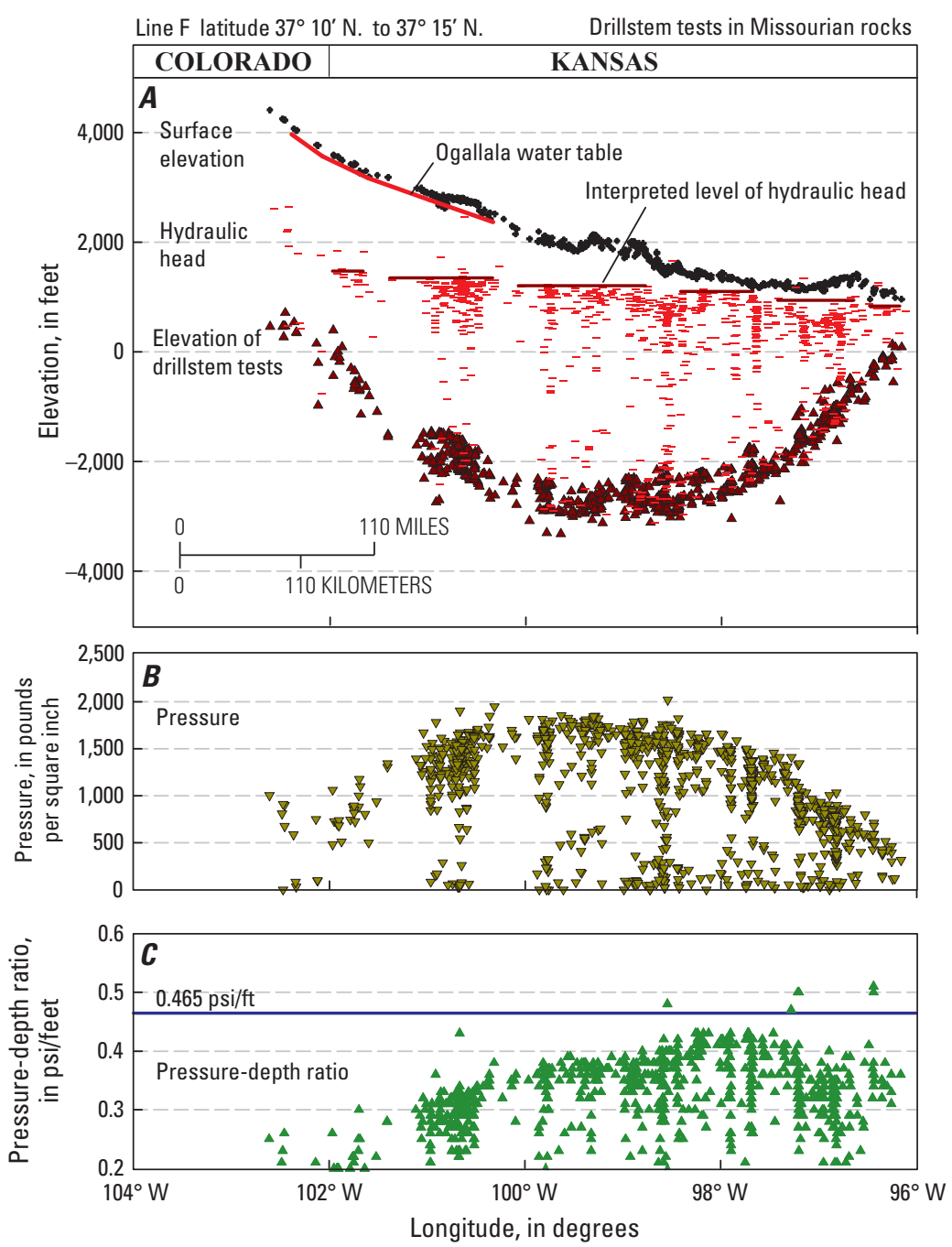

Figure 10. Data from drillstem tests in Missourian rocks from west-east swath Line $\mathrm{F}$ close to and parallel with the KansasOklahoma border (location in figure 4). A, Surface elevation (black plus symbols), elevation of drillstem tests (triangular symbols), and computed hydraulic head (red dash symbols); $B$, pressure measurements; $C$, computed pressure-depth ratio indicating underpressure where less than 0.465 pounds per square inch per foot (psi/ft). 
$0.465 \mathrm{psi} / \mathrm{ft}$ that was assumed for the conversion of pressure to hydraulic head (equation 1a). West of that point, the pressure-depth ratio declines to less than $0.3 \mathrm{psi} / \mathrm{ft}$, illustrating a westward increase in the degree of underpressure. As seen in figure $10 \mathrm{~A}$, the progressive westward increase in the degree of underpressure coincides with increasing separation between the surface elevation and the interpreted level of hydraulic head. In the following discussion, we will use the separation between surface elevation and the upper edge of hydraulic head as an indicator of underpressure, rather than the pressure-depth ratio.

The format of figure $10 \mathrm{~A}$ is repeated in plate 2 , which contains four south-north cross sections arranged in four columns. Seven stratigraphic units are displayed for each southnorth cross section; the stratigraphic units form the seven rows of plate 2. The geometry of the surface elevations and the drillstem test elevations on plate 2 have a different "look" than those of figure 10 . On each cross section of plate 2 , the surface elevation is relatively unvarying from south to north; however, the west-to-east drop in surface elevation is apparent on the progression from lines $\mathrm{H}$, where surface elevation is around $3,000 \mathrm{ft}$, to line $\mathrm{N}$, where surface elevation is around $1,000 \mathrm{ft}$. The drillstem test elevations, which display a U-shaped geometry on the west-east line in figure 10 , show the asymmetry of the Anadarko Basin on the south-north lines of plate 2. For example, the Desmoinesian test elevations on line $\mathrm{J}$ drop by $6,000 \mathrm{ft}$ from north to south. The major structure bounding the southern part of the basin is revealed by the upturn in test elevations at latitude $35^{\circ} 30^{\prime} \mathrm{N}$. on the Desmoinesian and the Hunton-and-older sections of line $\mathrm{J}$.

As previously discussed in connection with figure 10, only the uppermost values of hydraulic head are valid. The separation between those uppermost values and the surface elevation, which tracks the degree of apparent underpressuring, can be inspected as a function of longitude and stratigraphic unit. The separation feature is highlighted by yellow shading in the explanation on plate 2. Separation is greatest on the westernmost cross sections of Swath $\mathrm{H}$ and appears on all seven horizons, although data are sparse on the section for Hunton-and-older rocks. Separation appears to be greater in the deeper (Mississippian and Hunton-and-older) horizons than in the shallower (Permian and Virgilian) horizons. Separation diminishes from west to east in all of the seven stratigraphic units. For example, in rocks of Desmoinesian age at latitude $38^{\circ} \mathrm{N}$., the separation of $1,600 \mathrm{ft}$ on line $\mathrm{H}$ diminishes to $1,300 \mathrm{ft}$ on line $\mathrm{J}$, to $900 \mathrm{ft}$ on line $\mathrm{L}$, and to $500 \mathrm{ft}$ on line $\mathrm{N}$. The separation increases from south to north on line $\mathrm{N}$ as the surface elevation increases from south to north. A similar south-to-north increase in separation cannot be seen on lines $\mathrm{H}, \mathrm{J}$, or L because surface elevation is nearly constant along these three lines. In summary, the separation between the surface elevation and the upper edge of hydraulic head values (pl. 2) is fairly constant from south to north but diminishes from west to east.

\section{Potentiometric Surfaces}

A potentiometric surface is compiled by contouring the measurements of hydraulic head $H$ over the study area. The resulting surface represents the level to which water would rise in tightly cased wells that are in hydraulic connection with a confined aquifer. In this study, we compile potentiometric surfaces for each of the seven stratigraphic units.

\section{Procedure}

The problem of map representation of hydraulic head is analogous to fitting a rubber sheet downward onto a sea of data, in which only the sea surface is of interest. Points falling below the sea surface should not be represented. The problem is compounded by local spikes of unwanted high values that extend upwards from the sea surface. Ideally, the spikes should pass through the rubber sheet, leaving them extending upwards and unrepresented. To handle this problem, we used software designed to deal with potential field (gravity and magnetics) data. The data were divided into 13 west-east swaths (pl. 3), each displaying hydraulic head on the vertical axis and longitude on the $\mathrm{x}$-axis. Individual values of hydraulic head were either used in the filtering process (red points on lines 1-13 in pl. 3) or were deactivated if not used in the filtering process (green points in pl. 3).

Each swath was filtered independently of the others to establish 13 independent fits. A rolling statistic curve called ROLL was calculated by taking the maximum of 11 points, 5 to the left and 5 to the right of each hydraulic head value. The purpose of ROLL was to select points on the uppermost values of hydraulic head (values judged to be erroneously high were deactivated before the application of ROLL). The output of ROLL, shown as a green line in pl. 3, was input to a low-pass filter called LOWROLL. The 81-point low-pass filter utilized 40 points to the left and 40 points to the right of each output point. The smoothed output of the low-pass filter is shown as a series of blue dots in each swath of plate 3 .

Special problems that were encountered included areas of sparse data, areas of data with high scatter, and end effects. As already mentioned, each line for each stratigraphic unit was cleaned manually by removing points significantly below the tops of the values and removing high points that were considered outliers in the data set. In some cases, spaces were added in the database to manipulate the LOWROLL points to better follow the tops of the hydraulic head data. For example, if 40 blank spaces were added to the left of a point, the ROLL and LOWROLL would then only be calculated by the values to the right of that point. This process was used when the user clearly determined where the top of the hydraulic head values were but the output of LOWROLL was displayed in a slightly different location. Problems of this type were dealt with on a case-by-case basis as adjustments were made 
to improve the fit of the "rubber sheet" to the top of the surface on each swath.

The potentiometric surface for each stratigraphic unit was based upon the outputs of the low-pass filter for all of the swaths in a given stratigraphic unit. A grid was calculated using a minimum curvature algorithm with spacings of 0.1 and 0.01 (pl. 3). The 0.01-potentiometric surface, which clearly shows the relation between individual points on a swath and the resulting contour interval, was used for quality control and editing of the 0.1 -surface, which with additional notation is presented in plate 4 . In this way, potentiometric surfaces were constructed from computations of hydraulic head for each of the seven stratigraphic units.

In order to represent the height of the potentiometric surface with respect to the land surface, a second set of maps was constructed by computing the difference between the filtered (LOWROLL) values and the Kelly Bushing of each well. These data were then treated in a manner similar to that followed for the potentiometric surfaces to create maps of the separation be the Earth's surface and the potentiometric surface (pl. 5).

\section{Maps Showing Potentiometric Surfaces and Overpressured Areas}

For each stratigraphic unit (Permian, Virgilian, Missourian, Desmoinesian, Morrowan, Mississippian, and Huntonand-older), the potentiometric surface, in feet above sea level, is shown as a colored contour map (pl. 4). The Morrowan and Desmoinesian maps include an outline of their respective overpressured areas (red areas in parts $C$ and $D$ of pl. 4), drawn to include pressure-depth ratios greater than $0.5 \mathrm{psi} / \mathrm{ft}$, based on inspection of the data set from Al-Shaieb and others (1994) and the drillstem-test-derived map of potentiometric surface. Potentiometric contours are also interrupted in the Hunton-and-older, Missourian, and Virgilian units (gray areas in parts $A, E$, and $F$ of pl. 4), where the data in the central basin were insufficient to define a potentiometric surface. The overpressured areas in the deep basin are not considered further here, but are discussed in a companion paper by Nelson and Gianoutsos (chapter 8 of this report). Most importantly, the seven maps of plate 4 show that the overpressured zone in the deep basin has left an imprint in all rock units, except possibly those of Permian age.

As further introduction to plate 4 , consider the potentiometric surface for Permian rocks (pl. $4 G$ ), which has widely separated contours between 1,250 and 1,500 ft (light green area), demonstrating a low hydraulic gradient over more than one-half of the mapped area. The low hydraulic gradient is also shown in the west-east and south-north swaths of figure 10 and plate 2. In the western portion of the map (pl. $4 G$ ), contours are tightly grouped and the surface rises from 1,500 to $3,250 \mathrm{ft}$. The pattern of a broad area of low hydraulic gradient flanked on the west by a narrow area of high hydraulic gradient is common to all potentiometric surfaces except the Morrowan (pl. 4). The change in hydraulic gradient from high in the west to low in the east can be explained by changes in permeability or hydraulic conductivity. According to Darcy's law, an inverse relation exists between hydraulic gradient and hydraulic conductivity. One-dimensional flow can be expressed as $Q=-K(d H / d X)$, where $Q$ is the flow rate, $K$ is hydraulic conductivity, and $d H / d X$ is the hydraulic gradient expressed as the change in hydraulic head $H$ over a distance $X$. This concept is illustrated in a model (fig. 11), in which flow is constrained to a pipe. Hydraulic conductivity $K$ is low in the short, western segment of the pipe and high in the longer eastern segment. The tight clustering of increments of head $H$ in the western segment, which is comparable to the contours in plate $4 A$, was computed for the ratios of length and hydraulic conductivity indicated in the diagram: the length $X_{E}$ and hydraulic conductivity $K_{E}$ of the eastern segment are 4 and 30 times greater, respectively, than $X_{W}$ and $K_{W}$ of the western segment. Thus, areas with a low hydraulic gradient (wide spacing between contours in pl. 4) are likely to be areas with high $K$ relative to areas with a low hydraulic gradient (tightly spaced contours).

Because the pressure-depth ratio can be computed from the separation between potentiometric and land surfaces (eq. 2b), it follows that the separation between potentiometric surface and land surface is an indicator of whether pressure in a given stratigraphic unit is underpressured or normally pressured. The separation is mapped for the seven stratigraphic units (pl. 5) by subtracting the land surface represented in figure 4 from each of the potentiometric surfaces in parts $A-G$ of plate 4 . The zero contour, separating pink from grayblue areas, is the locus of intersection of the two surfaces.

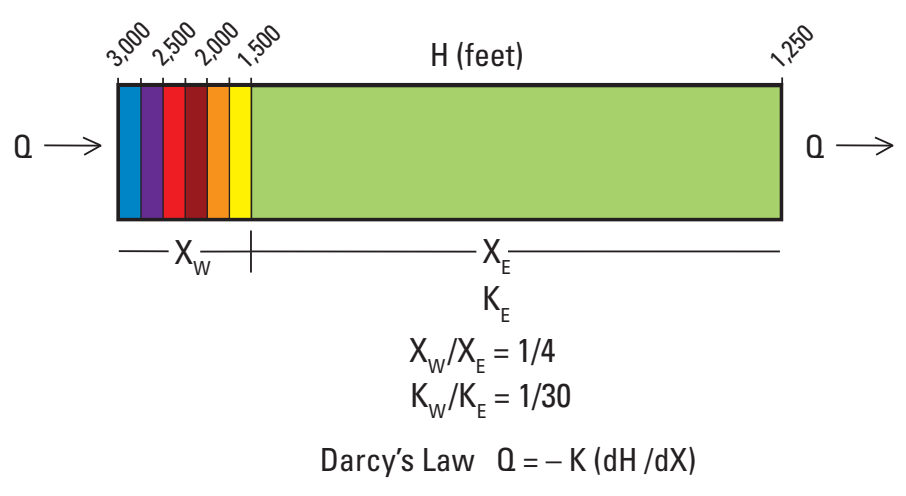

Figure 11. Hydraulic head $\mathrm{H}$ computed for one-dimensional flow $\mathrm{Q}$ in a medium of low hydraulic conductivity in the west, $\mathrm{K}_{\mathrm{w}}$, and high conductivity in the east, $\mathrm{K}_{\mathrm{E}^{\prime}}$ with $\mathrm{K}_{\mathrm{W}} / \mathrm{K}_{\mathrm{E}}=1 / 30$. The length of the low-conductivity section, $X_{w^{\prime}}$ is $1 / 4$ the length of the highconductivity section, $X_{E}$. Values of $H$ range from 1,250 to 3,000 feet, with colors between 250 -foot contours of $\mathrm{H}$ selected to match the colors in plate 4. Concept for one-dimensional model taken from Belitz and Bredehoeft (1988). 
Where the separation is positive (pink areas), groundwater has the potential to flow to the surface through tightly cased wells completed in the aquifer or through naturally occurring conduits in overlying geologic units. Under unconfined conditions, groundwater will discharge to springs and streams where the water table intersects land surface. The areas with wide spacing between contours that are present in plate 4 are not present in plate 5 because the land surface varies more than the potentiometric surfaces, especially in the western part of the study area. As a result, the land surface topography dominates the maps of plate 5 , resulting in the north-south banding over a large portion of the maps.

\section{Hunton-and-older Stratigraphic Units}

The potentiometric surface drops from around 1,500 ft in the western and southwestern portions of the study area to less than $500 \mathrm{ft}$ in the eastern part of the study area (pl. 4A). The potentiometric surface ranges between 750 and $1,000 \mathrm{ft}$ over much of central Kansas, including the Central Kansas uplift, showing a remarkably low gradient over an area that is more than 200 miles from west to east and more than 150 miles from south to north. The dense drilling pattern in Kansas is largely due to oil and gas production from the Arbuckle Group on the Central Kansas uplift. (The Cambrian-Ordovician Arbuckle Group may compose a separate hydrological system as the connection to the surface may lie along its surface exposures in central Missouri [L. Watney, Kansas Geological Survey, written commun., June 2011)] Groundwater, which flows from high head to low head, generally flows northnortheast across the northern flank of the Anadarko Basin, where the contour shading grades from yellow or light green to light blue in southern Kansas and northern Oklahoma. The 750 -foot contour lies immediately east of and parallel to the Nemaha uplift.

The separation between land surface and the potentiometric surface drops steadily from west to east ( $\mathrm{pl} .5 A$ ). Because the potentiometric surface is nearly flat (pl. $4 A)$, the separation between land surface and potentiometric surface is dominated by land surface topography. The $0 \pm 300$ contour areas occupy a large area of roughly 150 by 150 miles in the southeastern part of the map where the Nemaha and the Arbuckle fault systems intersect. Locally, the separation exceeds $+300 \mathrm{ft}$, suggesting artesian flow conditions for wells completed in the Huntonand-older stratigraphic unit. The Arbuckle-Simpson aquifer comprises the Simpson, Arbuckle, and Timbered Hills Groups (Christenson and others, 2011) and crops out in the southeast part of the map (pl. 5A) in a location that is consistent with the potentiometric surface.

\section{Mississippian Stratigraphic Unit}

This potentiometric surface (pl. 4B) is similar to the one for Hunton-and-older rocks (pl. 4A), with a broad low gradient area of values ranging from 750 to $1,000 \mathrm{ft}$ in Kansas, and a southeast-northwest trending 1,000-ft contour separating areas of higher hydraulic head in Oklahoma and Texas from lower values in Kansas. A west-east trend of values exceeding 1,750 ft in western Oklahoma and the northeast corner of the Texas Panhandle occupies an area that is overpressured in Desmoinesian and Morrowan rocks (figs. 4C and 4D); however this area is not overpressured in Mississippian rocks, although scattered measurement of high hydraulic head made it difficult to define a reliable potentiometric surface. The $750-\mathrm{ft}$ south-north contour lies east of the Nemaha uplift. As with the Hunton-and-older stratigraphic unit, groundwater flows generally north-northeastward from the northern flank of the basin, from northern Oklahoma into southern Kansas. A potentiometric surface for Mississippian rocks was not examined by Al Shaieb and others (1994), so no comparisons can be made.

The separation between land surface and the potentiometric surface for Mississippian rocks (pl. $5 B$ ) is similar to that for Hunton-and-older rocks (pl. 5A), as expected because the potentiometric surfaces are similar.

\section{Morrowan Stratigraphic Unit}

A northwest-southeast trending truncation edge bounds the Morrowan and Springer rock units (pl. 4C), which consequently occupy a smaller fraction of the study area than other rock units. The potentiometric surface rises from somewhat greater than $750 \mathrm{ft}$ at the truncation edge in Kansas to values greater than 2,000 ft in a four-county area of southwestern Kansas, southeastern Colorado, and the Oklahoma Panhandle. A west-east belt of values exceeding $1,750 \mathrm{ft}$ lies immediately north of the overpressured area.

Establishment and maintenance of a low (750-1,500 ft) potentiometric surface is presumed to be through hydraulic connectivity with either overlying Desmoinesian or underlying Mississippian strata, or both, because the Morrowan rocks do not extend far enough eastward to directly establish a discharge to surface. However, immediately north of the overpressured area, where the northeast corner of the Texas Panhandle meets the southeast corner of the Oklahoma Panhandle, lies an area of high hydraulic head (pl. 4C), as mentioned above, which creates a corresponding area where the potentiometric surface approaches the land surface ( $\mathrm{pl} .5 \mathrm{C}$ ). This area may be a relic of a once-overpressured part of the system, (see fig. 14 and relevant discussion in Nelson and Gianoutsos, chapter 8 of this report) as it lies well to the west of the zero contour on other difference maps.

\section{Desmoinesian Stratigraphic Unit}

The potentiometric surface drops from values in excess of 2,500 $\mathrm{ft}$ in southeastern Colorado to values ranging from 500 to $1,500 \mathrm{ft}$ in a broad low-gradient area in the eastern half of the mapped area (pl. $4 D$ ). A low-potential area approximately 30 by 50 miles in central Kansas includes values less than $500 \mathrm{ft}$. The southern edge of the overpressured area lies immediately south of the basin axis. Two salients projecting 
northward from the main overpressured area are mapped with both the data from this study (bright contours underlying the shaded overpressure) and the data set from Al-Shaieb and others (1994). A halo of hydraulic head values in the 1,000- to $1,500-\mathrm{ft}$ range surrounds the overpressured area. The overpressured area shown in plate $4 D$ is larger than that of the overpressured Red Fork Sandstone area shown by Al-Shaieb and others (1994), because the Desmoinesian pressure map includes formations and units of Desmoinesian age in addition to the Red Fork Sandstone.

The potentiometric surface approaches land surface $(-300 \mathrm{ft}$ line in pl. $5 D)$ along a line trending northeast in the eastern part of the mapped area. The $-300 \mathrm{ft}$ contour approaches and parallels the Nemaha uplift in northern Oklahoma and southern Kansas, suggesting that the Nemaha uplift is the discharge area for rocks of Desmoinesian age. The eastern edge of the overpressured area lies about 20 miles from the Nemaha uplift in the southeastern part of the Anadarko Basin. This relatively short distance from edge of overpressure to discharge point reflects the effectiveness of the pressure seal.

\section{Missourian Stratigraphic Unit}

The potentiometric surface ranges from 1,000 to $1,250 \mathrm{ft}$ along the Nemaha uplift and increases steadily westward to the western Kansas border where values exceed 1,750 ft (pl. 4E). As noted on the map, an area in north-central Kansas is well defined by the data, but sporadic measurements of higher head exist within the area. The potentiometric surface cannot be defined in a large southern area that encompasses the deep basin due to scatter in hydraulic head; numerous values of hydraulic head greater than 2,000 ft exist. Pressuredepth ratios of $0.5 \mathrm{psi} / \mathrm{ft}$ and greater suggest that this area is slightly overpressured, with pressure-depth ratios generally 0.5 to 0.6 in the informal lower Missourian sandstonesMedrano, Marchand, and Cleveland. This interpretation of overpressure in the lowermost Missourian sandstones differs from that of Al-Shaeib and others (1994), who categorized the Missourian unit as normally pressured.

As is the case with other maps of the separation between the potentiometric and land surfaces, the contour spacing and orientation of the Missourian stratigraphic unit (pl. $5 E$ ) is dominated by the land surface. The $-300-\mathrm{ft}$ contour lies 70 miles west of and parallel to the Nemaha uplift. The map indicates that an area east of the Nemaha uplift and another area south of the Arbuckle fault zone have potentials high enough for artesian flow (separation values greater than $0 \mathrm{ft}$ ).

\section{Virgilian Stratigraphic Unit}

The potentiometric surface increases from 1,000 to $1,250 \mathrm{ft}$ along the Nemaha uplift to more than $1,500 \mathrm{ft}$ in the northwestern part of the study area (pl. $4 F$ ). Between these two areas lies a broad low-gradient area with head values ranging from 1,500 to $1,250 \mathrm{ft}$. This low west-to-east decrease in the potential surface is interrupted in two areas. Values range up to 2,250 ft within the Central Kansas uplift, where the potentiometric surface is clearly defined despite a small fraction of measurements with higher hydraulic head. Higher head values are also located in the deep Anadarko Basin, but in this area there were insufficient data to determine the potentiometric surface.

The potentiometric surface approaches land surface $(-300 \mathrm{ft}$ line in pl. $5 F)$ along a line that is 70 miles west of and parallel to the Nemaha uplift. The broad discharge area for Virgilian strata extends from the $-300 \mathrm{ft}$ line to the eastern edge of the study area. West of the -300 line, the separation increases steadily to the western edge of the study area.

With the exception of the deep basin, the maps of potentiometric surface and separation between potentiometric and land surfaces are rather similar for Missourian and Virgilian rocks (compare pls. $4 E$ and $4 F$, and pls. $5 E$ and $5 F$ ). The similarity suggests that Missourian and Virgilian units are closely coupled and may act as one hydrologic system. The two systems were considered as one system by Al-Shaeib and others (1994).

\section{Permian Stratigraphic Unit}

The potentiometric surface increases from $750 \mathrm{ft}$ along the Arbuckle fault trend to $1,250 \mathrm{ft}$ along the Nemaha uplift and remains between 1,250 and 1,500 ft over an area that is roughly one-half of the study area (pl. $4 G$ ). In the westernmost counties of Kansas, an area which includes the western edge of the Hugoton gas field, values climb rapidly, exceeding $3,000 \mathrm{ft}$ at the western edge of the study area. The hydraulic gradient in western Kansas, Oklahoma, and Texas is the highest of any of the maps (pl. $4 A$ to $4 F$ ). The computed hydraulic head values that were used to define the map of plate $4 G$ are the most consistent of the seven mapped units, in that the surface is well defined on all 13 swaths with very few points above the surface and no areas, other than gaps shown as blank areas, where the surface could not be defined.

The map of the separation between the potentiometric surface and land surface for Permian rocks (pl. $5 G$ ) is similar to the maps for the Desmoinesian, Missourian, and Virgilian units (pls. $5 D, 5 E, 5 F$ ), the main difference being that, in the Permian stratigraphic unit, the separation is less in the westernmost part of Kansas and Oklahoma, due to the greater height of the Permian potentiometric surface in this area. The discharge area, represented by the $0 \pm 300$ contours, straddles the Nemaha uplift area and includes the high transmissibility Central Oklahoma aquifer (pl. $5 G$ ) where the water table lies just below surface elevation. The Central Oklahoma aquifer comprises the Garber Sandstone and Wellington Formation (fig. 4), as well as the Chase, Council Grove, and Admire Groups, all of Lower Permian age (Parkhurst and others, 1996). 


\section{Discussion}

The main feature of the potentiometric surfaces is the broad low-gradient area in central Kansas and northern Oklahoma, manifested as hydraulic head elevations between 1,250 and 1,500 $\mathrm{ft}$ in Permian, Virgilian, and Missourian units and as elevations between 750 and 1,000 ft in Desmoinesian, Mississippian, and Hunton-and-older units (pl. 4). The main feature in the maps showing separation between the Earth's surface and the potentiometric surfaces (pl. 5) is the north-south stripes formed as a result of subtracting the eastward-declining topography (fig. 4) from the broad low-gradient areas (pl. 4). Separation maps for Permian, Virgilian, Missourian, and Desmoinesian units show separation changing from $-2,400 \mathrm{ft}$ in the west to $0 \pm 300 \mathrm{ft}$ in the vicinity of the Nemaha uplift.

The position of the $0 \pm 300 \mathrm{ft}$ contour for Permian, Virgilian, Missourian, and Desmoinesian units is explained by the location of outcrops along and immediately east of the Nemaha uplift (fig. 12). The southern part of the Nemaha uplift also appears to be the control or partial control for the Mississippian and Hunton-and-older rock units. Along the Nemaha uplift, through exposure of strata to the surface or just below the surface and through connections with permeable faults, water pressure in the aquifers has equilibrated with atmospheric pressure causing the potentiometric elevation to be generally equal to surface elevation. The exact locations of equilibration are unknown and the areas where equilibration takes place is likely to be only a fraction of the area encompassed by the $0 \pm 300 \mathrm{ft}$ contours. Shales and evaporites of Permian age provide hydraulic isolation of deep strata from the surface so that the area around the Nemaha uplift is the dominant pressure equilibration point for these four units.

The separation maps reflect the degree of apparent underpressuring expected to exist in oil and gas wells in Permian and Pennsylvanian strata. To estimate the degree of underpressure at a desired location and depth, the separation $K B E-H$, which is expressed in feet, can be read from the appropriate map in plate 5 and converted to a pressure-depth ratio in $\mathrm{psi} / \mathrm{ft}$ using equation $2 \mathrm{~b}$. For example, consider a location on the Texas-Oklahoma border at the northeasternmost corner of the Texas Panhandle, where the separation contour on the Desmoinesian map (pl. 5D) is $H-K B E=-900 \mathrm{ft}$, or $K B E-H=+900$ $\mathrm{ft}$. For a Desmoinesian interval at a depth of $6,700 \mathrm{ft}$, the pressure/depth ratio from equation $2 \mathrm{~b}$ is $0.465 *(1-900 / 6700)=$ 0.403 , which is slightly underpressured. Note that underpressure cannot be read directly from the separation maps because equation $2 b$ requires that the depth be specified independently.

A comparison between the Virgilian separation map of plate $5 \mathrm{~F}$ and the pressure-depth ratio for a number of oil and gas reservoirs is shown in figure 13. The separation map was derived from well-based pressure measurements, not from reservoir-based pressure measurements, so the comparison of figure 13 is something of a check although not an independent check. The pressure-depth ratios are slightly subnormal $(0.42 \mathrm{psi} / \mathrm{ft})$ where the separation is close to $300 \mathrm{ft}$, are less than $0.30 \mathrm{psi} / \mathrm{ft}$ where the separation is greater than $1,500 \mathrm{ft}$, and decrease to values of $0.15 \mathrm{psi} / \mathrm{ft}$ where the separation is more than 1,800 ft. Between the eastern and western extremes, the pressure-depth ratio decreases more or less steadily as separation increases. Bearing in mind that the pressure-depth ratio is related to, but not directly proportional to separation, the general agreement between the trends in the pressure-depth ratios and the separation map gives confidence that underpressuring in oil and gas reservoirs and a low-gradient potentiometric surface are one and the same phenomenon.

A scenario for the origin of the Panhandle oil and gas field in Texas was presented by Sorenson (2005), in a pressure-depth plot and a series of five maps showing paleogeology from Late Permian to Quaternary. (Our only modification to Sorenson's scenario, which is summarized in this paragraph, is that aquifer pressure is equilibrated along the Nemaha uplift, in place of his proposed equilibration point in northeastern Kansas.) The Panhandle oil and gas field formed in Late Permian time and remained in place until early Tertiary when some spillage of gas northward into the Kansas Hugoton field may have begun. Aquifer pressure dropped in late Tertiary time as downcutting exposed Permian rocks. As aquifer pressure dropped, the Panhandle gas expanded, augmented by gas coming out of solution in oil. Reservoir pressure fell to its present-day value of $435 \mathrm{psi}$ at a depth range of 2,500-3,000

$\mathrm{ft}$, for a pressure-depth ratio of less than $0.18 \mathrm{psi} / \mathrm{ft}$ (Sorenson, 2005). In support of this scenario, the outline of the Hugoton field coincides with a maximum separation between surface elevation and potentiometric surface, as shown in figure 14. The maximum in separation corresponds to the western edge of the broad, low-gradient area where potentiometric elevation ranges between 1,250 and 1,500 $\mathrm{ft}(\mathrm{pl} .4 G)$, demonstrating that the location of the Hugoton gas field is bounded on the west by a sharp increase in potentiometric elevation.

Having established the discharge zone to be in the general vicinity of the Nemaha uplift, the question arises: where is the zone of recharge? Recharge is expected to take place where strata crop out at a high-elevation terminus of a groundwater system, in this case along the Rocky Mountain uplift in southeastern Colorado and northeastern New Mexico. However, during Pennsylvanian time the Sierra Grande arch in northeastern New Mexico was a positive feature. Pennsylvanian and pre-Pennsylvanian strata of the Anadarko Basin terminate on the eastern flank of the Sierra Grande arch, disrupting hydrological continuity with the Raton Basin and the Rocky Mountain uplift. Hydrological continuity to the west is limited to Lower Permian (Wolfcampian) continental deposits that drape the Sierra Grande arch in southeastern Colorado and a narrow west-east strip in New Mexico immediately south of the New Mexico-Colorado border (Wilson, 1977; Robson and Banta, 1987, Roberts and others, 1976).

Recharge is limited by what can best be described as a choke caused by onlap of Pennsylvanian and older strata on the Sierra Grande arch (fig. 12). Flow from west of the arch is restricted to permeable beds in Lower Permian (Wolfcampian and Leonardian) strata that are approximately 1,000 ft thick on top of the arch, as shown at longitude $104^{\circ} \mathrm{W}$. of figure 12 . 


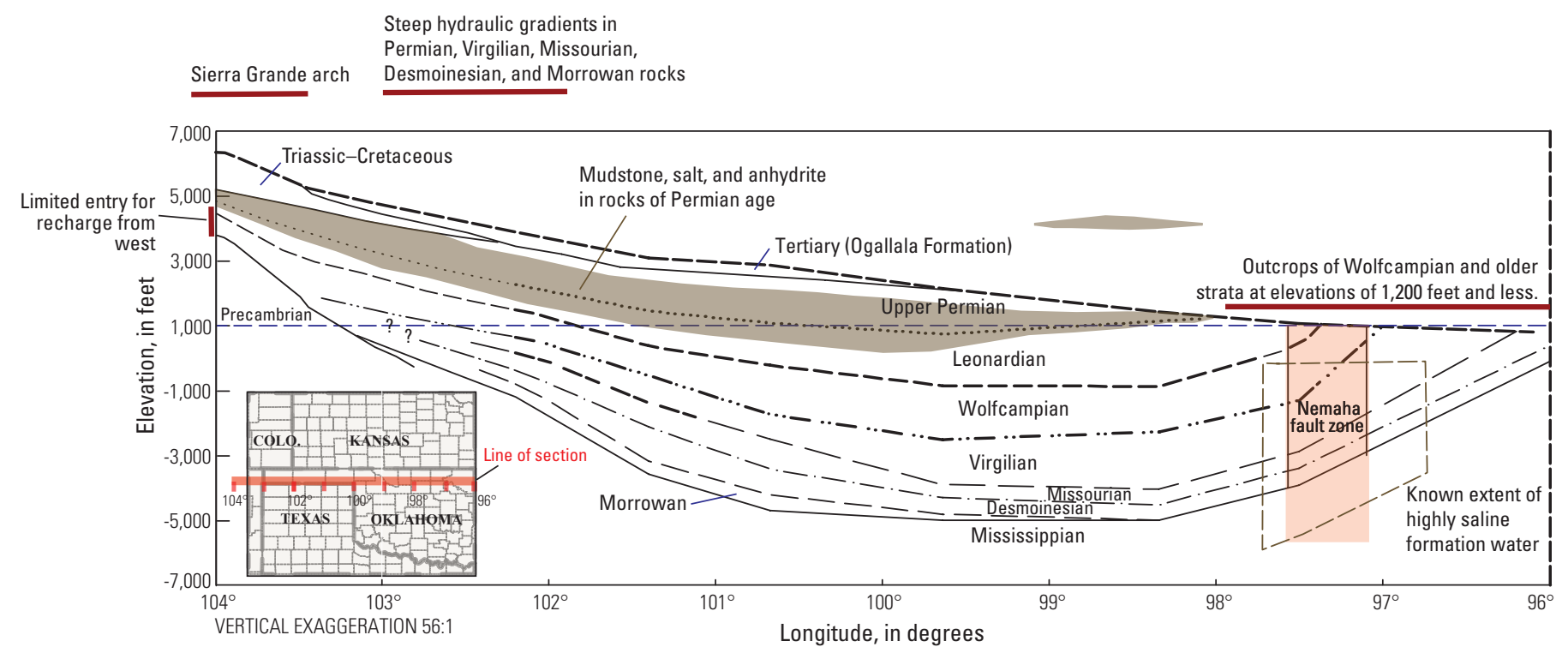

Figure 12. West-east structural cross section showing features influencing the creation and maintenance of underpressure. Permian and Pennsylvanian strata crop out along and east of the Nemaha uplift. Brown shading indicates the impermeable evaporite-bearing strata of Permian age. Pinchout and thinning of strata on the Sierra Grande arch provide limited entry for recharge. East of longitude $102^{\circ} \mathrm{W}$. the cross section is based largely on well data. West of long $102^{\circ} \mathrm{W}$. the cross section is based on information from Roberts and others (1976), Weeks and Gutentag (1981), and McGookey and others (1988).

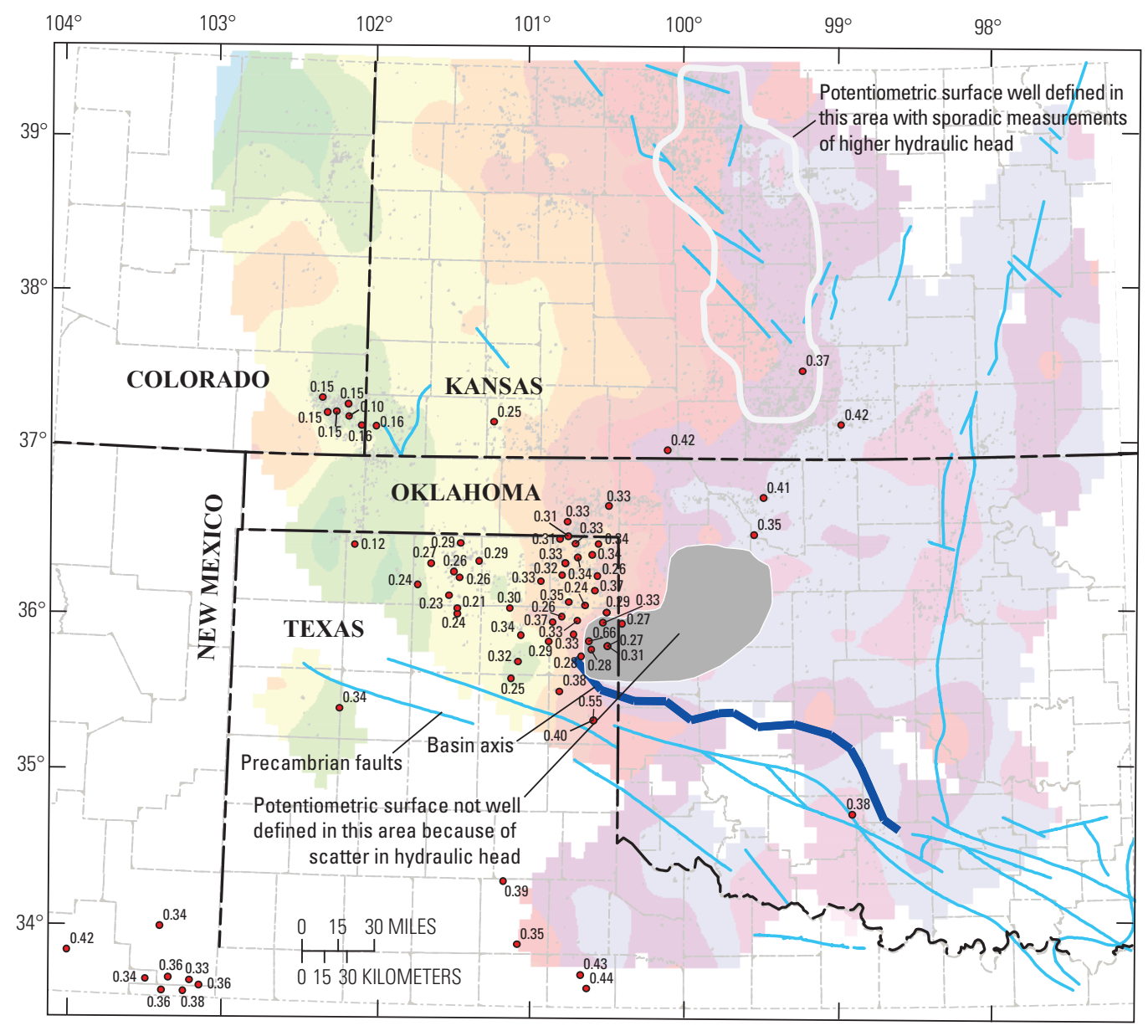

Figure 13. Comparison between a separation map from plate $5 \mathrm{~F}$ and degree of underpressuring for the Virgilian unit. Contours on the separation map give the Virgilian potentiometric surface minus the surface elevation. Dots give the location of individual oil and gas fields for which pressuredepth ratios were computed from data provided by NRG Associates (2009).

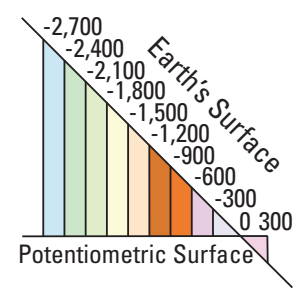




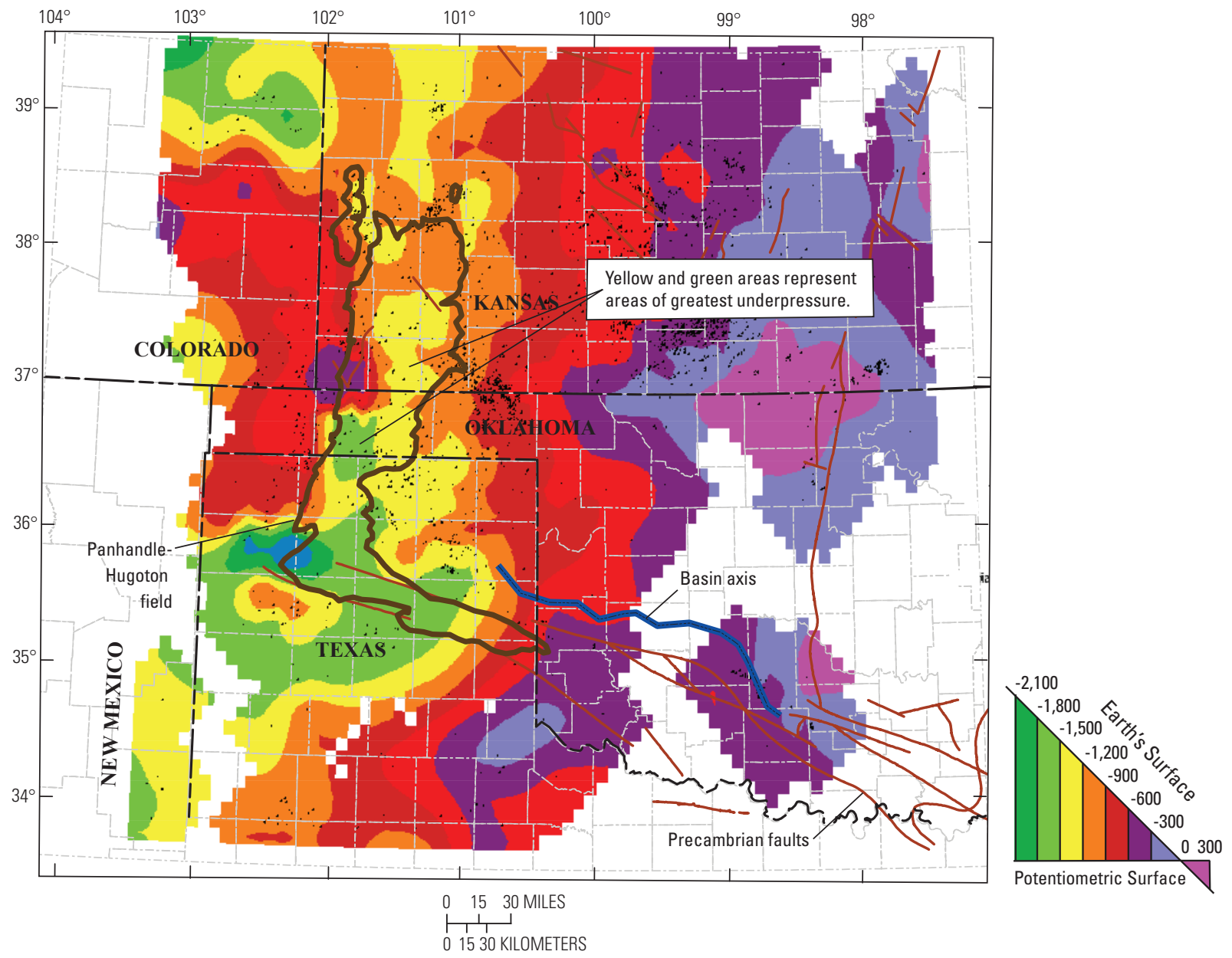

Figure 14. Map with contours showing separation between the potentiometric surface in Permian rocks and land surface elevation, in feet. For example, the yellow areas show where the potentiometric surface lies $-1,500$ to $-1,800$ $\mathrm{ft}$ below surface elevation. The underpressured Hugoton-Panhandle gas field lies in a trough of maximum separation between the potentiometric surface and surface elevation. 
East of the arch, groundwater flow must spread downward from Lower Permian strata into the eastward-thickening Pennsylvanian strata. Flow must also spread laterally, as the arch is not covered everywhere by rocks of Wolfcampian age (fig. 6 of Roberts and others, 1976). Consequently, the northsouth cross-sectional area open to flow over the arch is much smaller than the north-south cross-sectional area to be filled by eastward-migrating waters. In addition, discharge to the east may be inhibited by dense, high salinity waters in all seven stratigraphic units along the Nemaha uplift (pl. 1 and fig. 12). It is likely that these highly saline waters result from the dissolution of salt in Upper Permian strata. Buoyancy (negative buoyancy in this case) forces tend to cause the denser waters to descend westward and downward, counter to the overall west-to-east hydraulic gradients indicated in the potentiometric surfaces of plate 4 . These two effects-limited recharge from the west and density-induced counter-flow from the east - combine to set up a relatively stagnant groundwater system in the deep confined aquifers of the Anadarko Basin. The presence of overpressured strata in the deep basin (Nelson and Gianoutsos, chapter 8 of this report) further restricts groundwater circulation in deep confined aquifers. A supporting insight is provided by Gallardo and Blackwell (1999, p. 357) who studied the thermal regime of the basin: "We found no evidence for significant fluid flow in this basin at the present time ... the temperature structure can be explained adequately by normal conductive heat flow when the thermal conductivity of the rocks is taken into account in the modeling."

Our interpretation of a stagnant low-gradient groundwater system with limited hydraulic continuity to the west is counter to a scenario of a regional groundwater sysem recharged from the west and flowing from west to east in continuous strata that were tilted upward during Laramide uplift (Jorgensen, 1989).

\section{Summary}

Conversion of pressure from drillstem tests to hydraulic head, seemingly straightforward in a water-dominated system, is complicated by variations in salinity across the basin. Errors are not easily assessed because salinity is poorly documented in much of the basin. Nevertheless, the seven potentiometric surfaces and the seven derivative surfaces showing separation between land surface and potentiometric surface portray the state of hydraulic potential and the nature of underpressuring over the greater Anadarko Basin.

On the basis of their respective potentiometric and separation surfaces, the seven stratigraphic units fall into three groups.

1. In the units designated as Hunton-and-older and Mississippian, a large fraction of the potentiometric surface lies 750 to $1,000 \mathrm{ft}$ above sea level (pl. $4 A, B$ ). The separation maps indicate that the discharge area lies in the southeast part of the study area (pl. 5A, $B)$.
2. Northwest of the overpressured deep Anadarko Basin, the potentiometric and separation surfaces of the truncated Morrow age rocks are similar to the Mississippian surfaces. Hence, it appears that the Morrowan groundwater system is coupled to the groundwater system in Mississippian strata in this area.

3. The potentiometric surfaces of the four uppermost unitsDesmoinesian, Missourian, Virgilian, and Permian - share a general north-south to northeast-southwest trend in the contour patterns. The Nemaha uplift lies within the -300 to $+300 \mathrm{ft}$ contours of the separation surfaces in all four units (pl. $5 D-F)$, indicating that the discharge areas lie in the vicinity of the Nemaha uplift.

Outside the overpressured area in the deep basin, hydraulic potentials of Lower Permian and older strata are controlled by outcrops at elevations of 1,200 ft and less. Land surface rises to the west, so separation between land surface and potentiometric surface increases westward, and consequently, so does the degree of underpressuring. Underpressured reservoirs, including that of the Hugoton field, are the result of exposure of strata to atmospheric pressure in the vicinity of and east of the Nemaha uplift.

Four lines of evidence point to a low-flow to no-flow hydrologic system in the confined strata of the Anadarko Basin. Recharge of groundwater into Pennsylvanian and older strata on the northern flank of the Anadarko Basin is greatly limited by the presence of the low-permeability Permian cap. Recharge is further constricted by the pinchout of strata on the Sierra Grande uplift to the west (fig. 12). Highly saline formation water in strata along the Nemaha uplift imposes a fluid density barrier to west-to-east flow. And finally, overpressured strata in the deep basin further restrict fluid movement.

Our hypothesis of a markedly low-flow system warrants further investigation, possibly with detailed hydrological modeling. Features of the system awaiting elucidation are (1) the steep hydraulic gradient between longitude $102^{\circ} \mathrm{W}$. and $103^{\circ} \mathrm{W}$., (2) better mapping of salinity variations and the effect of high salinity waters along the Nemaha uplift, and (3) the details of pressure equilibration along the Nemaha uplift for the different stratigraphic units.

There are two practical ramifications of this work. First, the geologically recent drop in pressure brought on by the exposure of discharge zones along the Nemaha uplift has altered the original pressure distribution in the Anadarko Basin. The expansion of the Hugoton gas field is but one outcome. Other, smaller oil and gas fields have doubtless undergone gas expansion as a result of the pressure drop. The effects of this late-stage modification remain to be worked out for the distribution of oil and gas, particularly for gas. Second, the hydraulic potential of the deep confined strata is important when considering the injection and sequestration of carbon dioxide, if and when that takes place. A low-flow hydrologic regime, capped by impermeable strata, makes an attractive setting for carbon dioxide sequestration because the time durations for return flow to the surface are likely to be quite long. 


\section{Acknowledgments}

We benefitted from discussions with many people who visited our poster on this topic at annual meetings of the Geological Society of America and the American Association of Petroleum Geologists. We also benefitted from reviews by Noel Osborne, Sarah Hawkins, and Tom Judkins of the U.S. Geological Survey. Water salinity maps were originally compiled by Jessica Mager and Marvin Abbott of the U.S. Geological Survey. Rick Saltus of the U.S. Geological Survey introduced us to the Oasis Montaj software package and suggested the general filtering approach.

\section{References Cited}

Adler, F.J., Caplan, W.M., Carlson, M.P., Goebel, E.D., Henslee H.T., Hicks, I.C., Larson, T.G., McCracken, M.H., Parker, M.C., Rascoe, B., Jr., Schramm, Jr., M.W., and Wells, J.S., 1971, Future petroleum provinces of the midcontinent, Region 7, in Cram, I.H., ed., Future petroleum provinces of the United States-Their geology and potential: American Association of Petroleum Geologist Memoir 15, v. 2, p. 985-1120.

Al-Shaieb, Z., Puckett, J.O., Abdalla, A.A., and Ely, P.B., 1994, Megacompartment complex in the Anadarko Basin: A completely sealed overpressured phenomenon, in Ortoleva, P.J., ed., Basin compartments and seals: American Association of Petroleum Geologists Memoir 61, p. 55-68.

Amyx, J.W., Bass, D.M., Jr., and Whiting, R.L., 1960, Petroleum reservoir engineering, McGraw-Hill Book Company, $610 \mathrm{p}$.

Bair, E.S., 1987, Regional hydrodynamics of the proposed high-level nuclear-waste repository sites in the Texas Panhandle: Journal of Hydrology, v. 92, p. 149-172.

Bachu, S., 1995, Flow of variable-density formation water in deep sloping aquifers: Review of methods of representation with case studies: Journal of Hydrology, v. 164, p. 19-38.

Bachu, S., and Michael, K., 2002, Flow of variable-density formation water in deep sloping aquifers: Minimizing the error in representation and analysis when using hydraulic head distributions, Journal of Hydrology, v. 259, p. 49-65.

Belitz, K., and Bredehoeft, J.D., 1988, Hydrodynamics of Denver Basin: Explanation of subnormal fluid pressures: American Association of Petroleum Geologists Bulletin, v. 72 , no. 11, p. 1334-1359.

Blubaugh, P.E. Jr., 1999a, Hydrodynamics of the Hunton Group Anadarko Basin, Oklahoma and Texas Panhandle, pt. 1, Shale Shaker, Sept.-Oct., p. 61-80.
Blubaugh, P.E. Jr., 1999b, Hydrodynamics of the Hunton Group Anadarko Basin, Oklahoma and Texas Panhandle, pt. 2, Shale Shaker, Nov.-Dec., p. 90-105.

Breeze, A.F., 1970, Abnormal-subnormal pressure relationships in the Morrow sands of northwestern Oklahoma: M.S. thesis, University of Oklahoma, 122 p.

Breit, G.N., 2002, Produced waters database, U.S. Geological Survey, available online at http://energy.cr.usgs.gov/prov/ prodwat/index.htm.

Carter, L.S., Kelley, S.A., Blackwell, D.D., and Naeser, N.D., 1998, Heat flow and thermal history of the Anadarko Basin, Oklahoma: American Association of Petroleum Geologists Bulletin, v. 82, no. 2, p. 291-316.

Christenson, S.C., Osborn, N.I., Neel, C.R., Faith, J.R., Blome, C.D., Puckette, James, and Pantea, M.P., 2011, Hydrogeology and simulation of groundwater flow in the ArbuckleSimpson aquifer, south-central Oklahoma: U.S. Geological Survey Scientific Investigations Report 2011-5029, 104 p.

Fay, R.O., 1964, The Blaine and related formations of northwestern Oklahoma and southern Kansas: Oklahoma Geological Survey Bulletin B98, 238 p., 24 pls.

Fetter, C.W., 1988, Applied hydrogeology: Merrill Publishing Company, Columbus, Ohio, 592 p.

Gallardo, J., and Blackwell, D.D., 1999, Thermal structure of the Anadarko Basin: American Association of Petroleum Geologists Bulletin, v. 83, no. 2, p. 333-361.

IHS Energy, 2009, U.S. production and well data: database available from IHS Energy, 15 Inverness Way East, Englewood, CO 80112.

Ingebritsen, S., Sanford, W., and Neuzil, C., 2008, Groundwater in geologic processes: Cambridge University Press, New York, $536 \mathrm{p}$.

Jorgensen, D.G., 1989, Paleohydrology of the Anadarko Basin, central United States in Johnson, K.S., ed., Anadarko Basin Symposium, 1988: Oklahoma Geological Survey Circular 90, p. 176-193.

Jorgensen, D.G., Helgesen, J.O., and Imes, J.L., 1993, Regional aquifers in Kansas, Nebraska, and parts of Arkansas, Colorado, Missouri, New Mexico, Oklahoma, South Dakota, Texas, and Wyoming - Geohydrologic framework: U.S. Geological Survey Professional Paper 1414-B, 72 p.

Jorgensen, D.G., Helgesen, J.O., Signor, D.C., Leonard, R.B., Imes, J.L., and Christenson, S.C., 1996, Analysis of regional aquifers in the central Midwest of the United States in Kansas, Nebraska, and parts of Arkansas, Colorado, Missouri, New Mexico, Oklahoma, South Dakota, Texas, and Wyoming - Summary: U.S. Geological Survey Professional Paper 1414-A, 67 p. 
McGookey, D.A., Gustavson, T.C., and Hoadley, A.D., 1988, Regional structural cross sections, mid-Permian to Quaternary strata, Texas Panhandle and eastern New Mexico: Distribution of evaporites and areas of evaporite dissolution and collapse: Bureau of Economic Geology, University of Texas at Austin, $17 \mathrm{p}$.

NRG Associates, 2009, The significant oil and gas fields of the United States: NRG Associates Inc., P.O. Box 1655, Colorado Springs, CO 80901.

Nelson, P.H., and S.M., Condon, 2008, Attributes of a large underpressured gas system, San Juan Basin, New Mexico, Chap. 12 in Cumella, S.P, Shanley, K.W., and Camp, W.K., eds., Understanding, exploring, and developing tight-gas sands: American Association of Petroleum Geologists Hedberg Publication Series no. 3, p. 193-220.

Parkhurst, D.L., Christenson, S., and Breit, G.N., 1996, Ground-water-quality assessment of the central Oklahoma aquifer, Oklahoma-geochemical and geohydrologic investigations: U.S. Geological Survey Water-Supply Paper 2357-C, $101 \mathrm{p}$.

Pippin, L., 1970, Panhandle-Hugoton Field, Texas-OklahomaKansas-the first fifty years, in Halbouty, M.T., ed., Geology of giant petroleum fields,: American Association of Petroleum Geologists Memoir 14, p. 204-222.

Roberts, J.W., Barnes, J.J., and Wacker, H.J., 1976, Subsurface Paleozoic stratigraphy of the northeastern New Mexico basin and arch complex, in Ewing, R.C., and Kues, B.S., eds.: New Mexico Geological Society Guidebook of Vermejo Park, 27th Field Conference, p. 141-152.

Robson, S.G., and Banta, E.R., 1987, Geology and hydrology of the deep bedrock aquifers in eastern Colorado: U.S. Geological Survey Water Resources Investigations Report $85-4240,6$ sheets.

Signor, D.C., Helgesen, J.O., Jorgensen, D.G., and Leonard, R.B., 1996, Geohydrology and simulation of steady-state flow conditions in regional aquifer systems in Cretaceous and older rocks underlying Kansas, Nebraska, and parts of Arkansas, Colorado, Missouri, New Mexico, Oklahoma, South Dakota, Texas, and Wyoming: U.S. Geological Survey Professional Paper 1414-C, 105 p.
Sorenson, R.P., 2005, A dynamic model for the Permian Panhandle and Hugoton fields, western Anadarko Basin: American Association of Petroleum Geologists Bulletin, v. 89 , no. 7, p. 921-938.

Stoeser, D.B., Green, G.N., Morath, L.C., Heran, W.D., Wilson, A.B., Moore, D.W., and Van Gosen, B.S., 2005, Preliminary integrated geologic map databases for the United States: U.S. Geological Survey Open-File Report 2005-1351, available online at http://pubs.usgs.gov/ of/2005/1351/.

Taylor, I.D., Buckthal, W.P., Grant, W.D., and Pollock, M.E., 1977, Selected gas fields of the Texas Panhandle: Amarillo, Texas, Panhandle Geological Society, 83 p.

Toth, J., 2009, Gravitational systems of groundwater flow: Theory, evaluation, utilization: Cambridge University Press, $297 \mathrm{p}$.

U.S. Geological Survey, 2014, Colorado, Kansas, New Mexico, Oklahoma, and Texas: U.S. Geological Survey digital data, The National Map, accessed August 4, 2014, at: http://viewer.nationalmap.gov/viewer/.

Weeks, J.B., and Gutentag, E.D., 1981, Bedrock geology, altitude of base, and 1980 saturated thickness of the high plains aquifer in parts of Colorado, Kansas, Nebraska, Oklahoma, South Dakota, Texas, and Wyoming: Hydrological Atlas HA-648, two sheets.

Wilson, J.M., 1977, West-east subsurface correlation, Sierra Grande uplift to Kansas line, figure 22 in Irwin, D., ed., Subsurface cross-sections of Colorado: Denver, Colo., Rocky Mountain Association of Geologists Special Publication No. 2.

Wolf, A.V., Morden, G.B., and Prentiss, P.G., 1973, Concentrative properties of aqueous solutions: conversion tables, in Weast, R.C., ed., Handbook of Chemistry and Physics: CRC Press, p. D192-D234. 


\section{Chapter 10 Lithology of Paleozoic Rock Units in 62 Wells, Anadarko Basin, Oklahoma}

By Nicholas J. Gianoutsos, Jaquidon D. Kruger, Philip H. Nelson, and Debra K. Higley

Chapter 10 of 13

Petroleum Systems and Assessment of Undiscovered Oil and Gas in the Anadarko Basin Province, Colorado, Kansas, Oklahoma, and Texas-USGS Province 58

Compiled by Debra K. Higley

U.S. Geological Survey Digital Data Series DDS-69-EE 


\title{
U.S. Department of the Interior SALLY JEWELL, Secretary
}

\section{U.S. Geological Survey \\ Suzette M. Kimball, Acting Director}

\author{
U.S. Geological Survey, Reston, Virginia: 2014
}

For more information on the USGS - the Federal source for science about the Earth, its natural and living resources, natural hazards, and the environment, visit http://www.usgs.gov or call 1-888-ASK-USGS.

For an overview of USGS information products, including maps, imagery, and publications, visit http://WwW.usgs.gov/pubprod

To order this and other USGS information products, visit http://store.usgs.gov

Any use of trade, firm, or product names is for descriptive purposes only and does not imply endorsement by the U.S. Government.

Although this information product, for the most part, is in the public domain, it also may contain copyrighted materials as noted in the text. Permission to reproduce copyrighted items must be secured from the copyright owner.

Suggested citation:

Gianoutsos, N.J., Kruger, J.D., Nelson, P.H., and Higley, D.K., 2014, Lithology of Paleozoic rock units in 62 wells, Anadarko Basin, Oklahoma, chap. 10, in Higley, D.K., compiler, Petroleum systems and assessment of undiscovered oil and gas in the Anadarko Basin Province, Colorado, Kansas, Oklahoma, and Texas-USGS Province 58: U.S. Geological Survey Digital Data Series DDS-69-EE, 6 p., http://dx.doi.org/10.3133/ds69EE.

ISSN 2327-638X (online) 


\section{Contents}

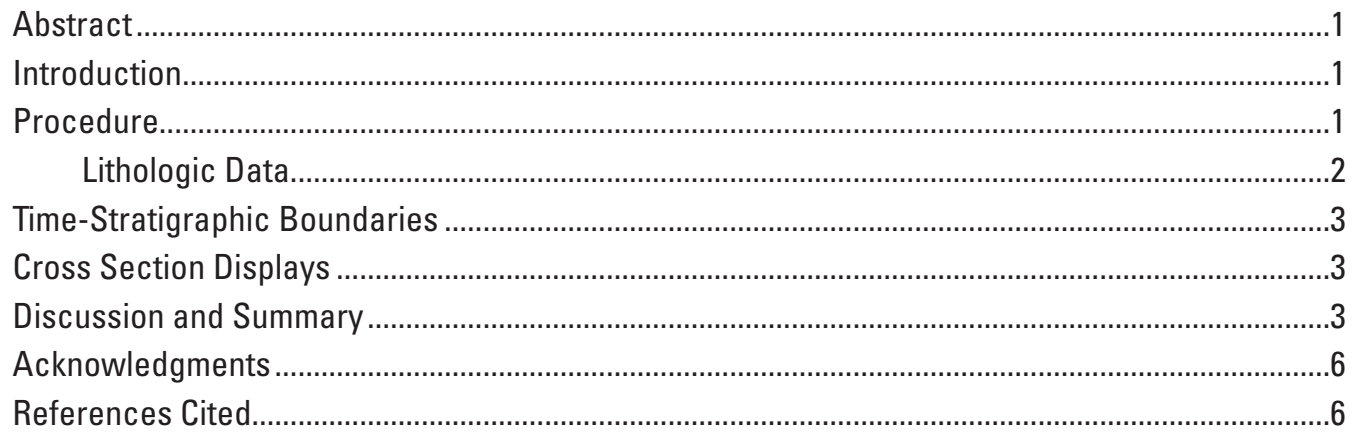

\section{Plates}

1. Cross section $A-A$, west to east.............................................................................. link

2. Cross section $B-B$, west to east.......................................................................... link

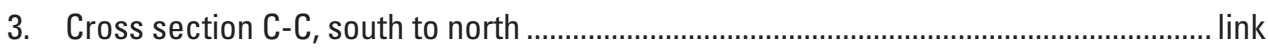

4. Cross section D-D, south to north................................................................... link

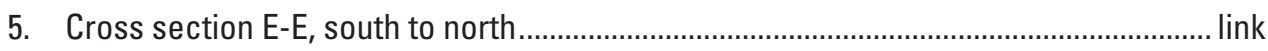

6. Cross section F-F, south to north....................................................................... link

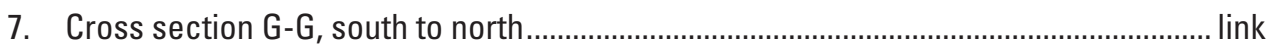

8. Cross section $\mathrm{H}-\mathrm{H}$, south to north...................................................................... link

\section{Figures}

1. Map of Anadarko Basin and shelf, Oklahoma showing locations of wells and cross sections .

2. Generalized stratigraphic section for the Anadarko Basin, listing prominent groups and formations, modified from Bebout and others (1993, fig. 5). The cross sections in this report used the tops of 5 horizons (red italicized print) to establish 5 time-stratigraphic boundaries. Granite wash is in the Upper Pennsylvanian and lowermost Permian strata. Red beds and evaporites are generally in rocks of Permian age. [Camb., Cambrian; Miss., Mississippian]...

3. Map of total thickness of evaporite in Permian rocks in the Anadarko Basin....................5 


\section{Appendixes (Digital Files)}

1. Spreadsheet with list of 62 wells used in this study .................................................... link

2. Spreadsheet with lithologic determinations from well information............................... link

\section{Conversion Factors}

Inch/Pound to SI

\begin{tabular}{|c|c|c|}
\hline Multiply & By & To obtain \\
\hline \multicolumn{3}{|c|}{ Length } \\
\hline foot (ft) & 0.3048 & meter $(\mathrm{m})$ \\
\hline mile (mi) & 1.609 & kilometer (km) \\
\hline
\end{tabular}




\title{
Lithology of Paleozoic Rock Units in 62 Wells, Anadarko Basin, Oklahoma
}

\author{
By Nicholas J. Gianoutsos, ${ }^{1}$ Jaquidon D. Kruger, ${ }^{2}$ Philip H. Nelson, ${ }^{1}$ and Debra K. Higley ${ }^{1}$
}

\begin{abstract}
Lithology derived from mud logs, sample logs, and well logs are presented on eight structural cross sections covering the Oklahoma portion of the Anadarko Basin. Seven lithologies are identified: dolomite, limestone, evaporite, sandstone, red shales/red beds, arkose (locally referred to as granite wash), and shale. All cross sections reveal three major lithologic groups: (1) the carbonate-dominated units of Mississippian age and older, (2) the siliciclastic units of Pennsylvanian age, and (3) the evaporites and red shales of Permian age. In addition to the cross section displays, the lithologic data are provided in spreadsheet form.
\end{abstract}

\section{Introduction}

This report focuses on lithologic determinations of Paleozoic strata in the Anadarko Basin, based primarily on interpretations of mud logs, sample logs, and well logs. It is part of a series of reports that includes (1) chapters by Higley (chapters 6 and 7 of this report) and Gaswirth and Higley (chapter 5 of this report) on the assessment of undiscovered oil and gas resources in the Anadarko basin, and (2) two chapters by Nelson and Gianoutsos (chapters 8 and 9 of this report) that examine the extent and cause of abnormal pressures in the greater Anadarko Basin.

The lithologies reported here were originally interpreted by Gallardo (1989) and Gallardo and Blackwell (1999) for the purpose of computing the temperature structure of the Anadarko Basin of western Oklahoma. Using mud logs, sample logs, and well logs, including spontaneous potential, gamma ray, density, and neutron porosity logs, they assigned 1 of 7 lithologies to each 10-ft interval in 63 wells distributed across the basin. A value of thermal conductivity was then assigned to each lithology, an independent estimate of heat flow was assigned to each well, and the temperature profile was computed for each of the 63 wells, 62 of which are used in the cross sections (pls. 1-8) contained in this chapter of the report.

A significant outcome of Gallardo and Blackwell's work was a map of present-day temperature contours at the top of the Woodford Shale that was compared with a previously published contour map of vitrinite reflectance (Gallardo and Blackwell, 1999, fig. 12). The maps show that the highest vitrinite reflectance (thermal maturity) values and highest present-day temperatures coincide but are offset from the deepest part of the basin. The location of the thermal maturity and temperature maxima was attributed to lateral lithologic variations and their impact on heat conduction (Gallardo and Blackwell, 1999).

The lithologic interpretations used to create the thermal profiles have previously gone unpublished. It is our belief that these gross lithologic interpretations, when viewed at the basin scale, are of sufficient value to warrant their presentation here on a series of structural cross sections showing the lithologic units across the basin, along with selected time stratigraphic boundaries. The 62 wells are arranged in 2 west-east and 6 south-north cross sections (fig. 1, pls. 1-8), providing a basinscale perspective of lithologic variations.

\section{Procedure}

The 62 wells used in this study are the same wells (with one exception) that were used in the thermal study by Gallardo and Blackwell (1999; Appendix 1); that list is repeated here with a few corrections and additions to the well locations and American Petroleum Institute (API) numbers (Appendix 1 of this chapter of the report) compiled from the Oklahoma Corporation Commission online database (2010). The Shell McDaniel 1 well (well number 8, Appendix 1) was not used in the cross sections because the well was not sufficiently deep. Our work flow consisted of preparation of the lithology files for plotting, selection of five time-stratigraphic boundaries for correlation, and the production of the cross-section displays.

${ }^{1}$ U.S. Geological Survey, Denver, Colorado.

${ }^{2}$ Raytheon Company, Dallas, Texas. 


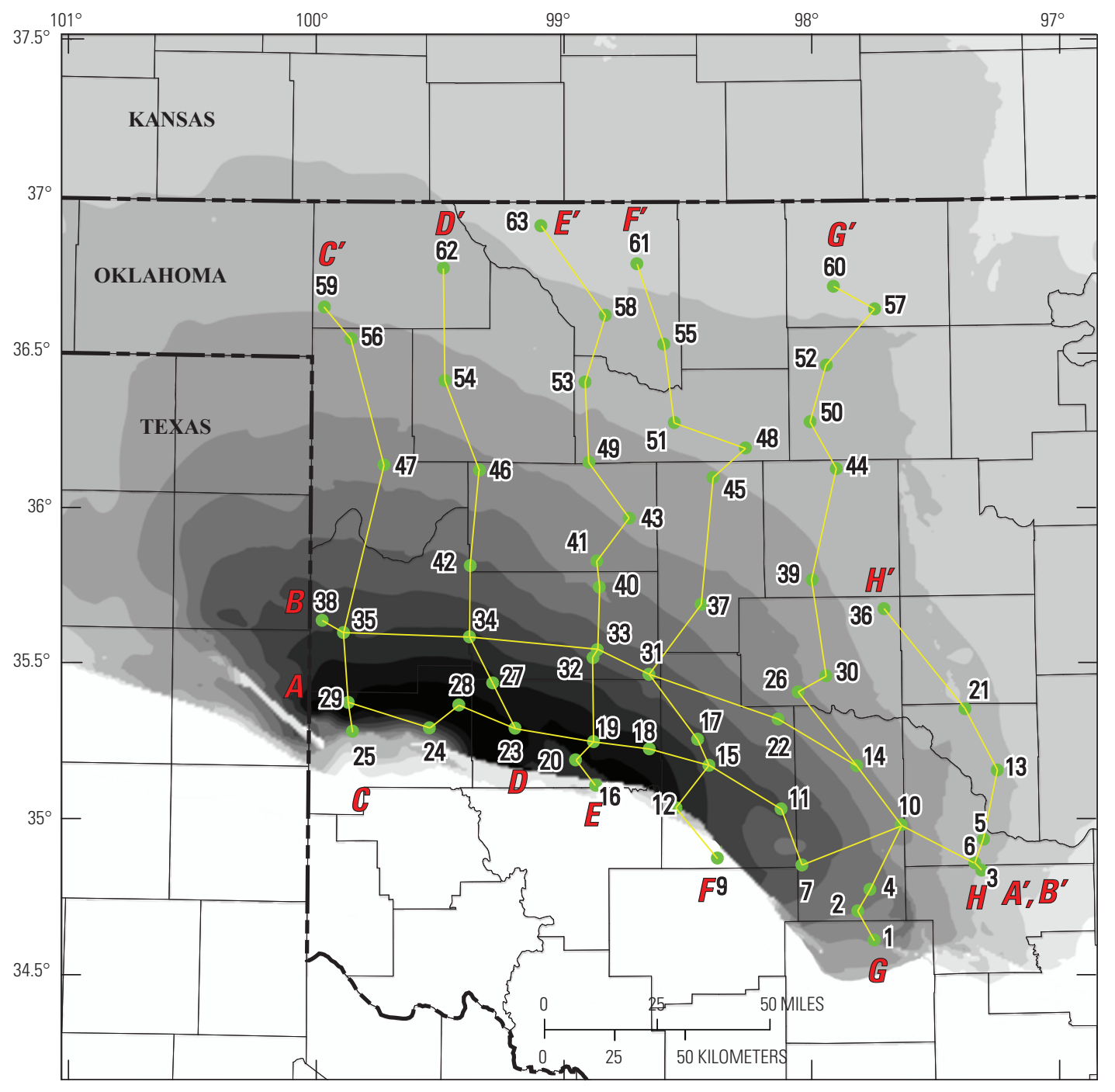

\section{EXPLANATION}

Elevation of the top of Woodford Shale relative to sea level, in feet.

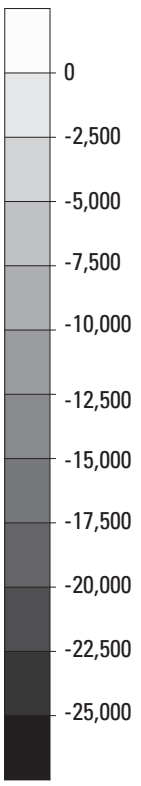

Note: Well 8 was not used in the cross sections and has been omitted from this map.

Figure 1. Map of Anadarko Basin and shelf, Oklahoma showing locations of wells and cross sections.

\section{Lithologic Data}

Seven lithologies are identified, based on well log response and lithologic information: (1) dolomite, (2) limestone, (3) evaporite, (4) sandstone, (5) red shales (referred to as red beds) (6) arkose (locally referred to as granite wash), and (7) shale. According to Gallardo and Blackwell (1999, p. 339), much of the Permian section is characterized by red shales with interbedded evaporates and other lithologies, commonly on a very fine vertical scale. As these types of deposits are typically referred to as red beds, the term red bed was assigned to all lithologies of Permian age that were not identifiable as another lithology. These seven lithologies are represented by colors on the cross sections shown on plates $1-8$.

Lithology was determined for each well on 10-foot (ft) intervals. In older wells from the shelf, sample logs were used in combination with well logs to assign lithologies. In wells from the deep basin, mud logs were the source of direct lithologic information along with the well logs. In cases where a mixed lithology was indicated (for example, dolomitic limestone), the dominant lithology (limestone) was assigned to that $10-\mathrm{ft}$ interval. Siltstone was not included as a category because siltstone could not be reliably interpreted from the available information. The "red bed" lithology was assigned to shales of Permian age whereas the "shale" lithology was assigned to shales (generally gray or black) older than Permian. This distinction, although somewhat arbitrary, was based on the interbedded red shales and evaporites that composed much of the Permian section and had a practical application in terms of the assignment of thermal conductivity values (Gallardo and Blackwell, 1999).

Spreadsheets originally compiled from well logs and other sources were modified to make them suitable as input files for LMKR Geographix plotting software. The spreadsheets gave the thickness but not the depth of each lithology, so the thicknesses were summed to provide a depth for each lithology interface. A single lithology can extend from a minimum of 10 feet to hundreds of feet. 
The original requirement for the lithology data was to assign thermal conductivity values from the surface to the base of the Paleozoic section, in order to model temperature in the basin (Gallardo and Blackwell, 1999). To produce continuous lithologies from the top to the bottom of each primary well, information from many primary wells was supplemented with information from nearby wells - lithologies obtained from nearby wells have been retained in this chapter of the report. In addition, where no information was available from either the primary or a nearby well, a lithology was assigned to fill the gap from the top of information to the surface; these lithologies have been deleted from this chapter of the report. Consequently, the lithology data presented in this chapter of the report are based mainly on the information available from each primary well, with supplemental information from nearby wells in many cases.

The lithologic data for each well are tabulated in Appendix 2, including the lithology type, the depth to the top of a given lithology, and the thickness of the lithology units.

\section{Time-Stratigraphic Boundaries}

To provide a stratigraphic framework for the lithology logs, five time-stratigraphic units were selected for portrayal on the cross sections: Devonian and older, Mississippian, Lower to Middle Pennsylvanian, Middle to Upper Pennsylvanian, Wolfcampian, and Leonardian to Guadalupian. The boundaries for these five time-stratigraphic units are based approximately on five lithostratigraphically defined tops (fig. 2). Three sources were used to establish the depth of a formation top: (1) mud log picks, which were available for only some of the formation tops; (2) transitions in lithology, which were judged to be definitive for two of the five boundaries (see below); and (3) maps representing stratigraphic units, as determined from mapping software (Dynamic Graphics ${ }^{\circledR}$ Earthvision $($ ). The maps were defined using a database consisting of stratigraphic unit picks from well logs, stratigraphic unit tops from various publications, and a database by IHS Energy (2010). Erroneously high values of a surface were eliminated by visual inspection, followed by spatial filtering to smooth the surfaces.

The five time-stratigraphic boundaries, located approximately using lithostratigraphic correlations (fig. 2), are:

1. Devonian and older. The boundary for the top of the Devonian is based on the top of the Woodford Shale, which is generally of Late Devonian age, although part of the formation appears to be of Early Mississippian age (Kirkland and others, 1992). There is high confidence in the location of the top of the Woodford Shale in most areas, so this boundary is generally shown as a solid line in the cross sections (pls. 1-8).

2. Mississippian. The boundary for the top of the Mississippian is based on the top of the Springer Group, although the uppermost part of the Springer Group is assigned a
Pennsylvanian age (Andrews, 2008). Given the uncertainty in the relation between the top of the Mississippian and the top of the Springer Group, this boundary is shown as a dashed line throughout the basin (pls. 1-8).

3. Lower to Middle Pennsylvanian. The boundary for the Middle Pennsylvanian is based on the top of the Cherokee Group of the Desmoinesian Series. As the location of the top of the Cherokee Group was uncertain in many areas, this boundary is shown as a dashed line throughout the basin (pls. 1-8).

4. Middle to Upper Pennsylvanian. The boundary between upper Pennsylvanian and Wolfcampian ages is based on the top of the Wabaunsee Group. In some locations, this boundary was adjusted to coincide with the base of the oldest red shale. Because of the uncertainty in the location of this boundary, it is shown as a dashed line throughout the basin (pls. 1-8).

5. Wolfcampian and Leonardian to Guadalupian. The boundary between Wolfcampian and Guadalupian ages is equivalent to the top of the Chase Group, which was adjusted to the base of the lowermost evaporite. Wherever the thick evaporite is present, confidence in the location of this boundary is high, as represented by the solid line on most of the cross sections.

\section{Cross Section Displays}

The structural cross sections (plates 1-8) are designed to be plotted and inspected at a scale of one inch per 2,000 vertical feet; elevations are displayed with respect to sea level rather than to a stratigraphic datum. Spacing between wells is proportional to actual distances. Correlations were not continued across major structural offsets in the southern part of the basin. At the scale of 1 inch to $2,000 \mathrm{ft}$, the resulting time-stratigraphic boundaries can only be approximated, with uncertainties ranging from a few feet to possibly several hundred feet in a few locations. Thus the results should not be used specifically for detailed correlations in certain cases, as noted on each plate.

\section{Discussion and Summary}

All cross sections reveal three major groups of lithologies in the Anadarko Basin: (1) the carbonate-dominated units of Mississippian age and older, (2) the siliciclastic units of Pennsylvanian age, and (3) the evaporites and red beds of Permian age. This association between lithologic groups and three major time-stratigraphic groups persists across the entire state of Oklahoma (Oklahoma City Geologic Society, 1971). These three major lithologic groups play an important role in:

- the thermal regime of the basin (Gallardo and Blackwell, 1999) 


\begin{tabular}{|c|c|c|c|}
\hline के & Series & \multicolumn{2}{|c|}{ Lithostratigraphic Unit } \\
\hline \multirow{3}{*}{ 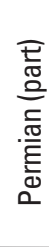 } & Guadalupian & \multicolumn{2}{|c|}{ Whitehorse Group; El Reno Group } \\
\hline & Leonardian & \multicolumn{2}{|c|}{ Sumner Group, Enid Group, Hennessey Group } \\
\hline & Wolfcampian & $\begin{array}{c}\text { Chase Group } \\
\text { Council Grove Group } \\
\text { Admire Group }\end{array}$ & Pontotoc Group \\
\hline \multirow{5}{*}{ 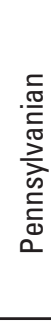 } & \multirow{2}{*}{ Virgilian } & $\begin{array}{l}\text { Wabaunsee Group } \\
\text { Shawnee Group }\end{array}$ & Ada Group \\
\hline & & \multicolumn{2}{|c|}{ Douglas Group } \\
\hline & Missourian & $\begin{array}{c}\text { Lansing Group } \\
\text { Kansas City Group }\end{array}$ & Hoxbar Group \\
\hline & Desmoinesian & $\begin{array}{l}\text { Marmaton Group } \\
\text { Cherokee Group }\end{array}$ & Deese Group \\
\hline & $\begin{array}{c}\text { Atokan } \\
\text { Morrowan }\end{array}$ & \multicolumn{2}{|c|}{ Atoka Group } \\
\hline \multirow{4}{*}{ 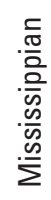 } & Chesterian & $\begin{array}{l}\text { Springer Formation } \\
\text { Chester Group }\end{array}$ & \multirow{4}{*}{ Mayes Group } \\
\hline & Meramecian & Meramec lime & \\
\hline & Osagean & Osage lime & \\
\hline & Kinderhookian & Kinderhook Shale & \\
\hline \multirow{3}{*}{. } & Chautauquan & \multicolumn{2}{|c|}{ Woodford Shale } \\
\hline & \multirow{2}{*}{$\begin{array}{l}\text { Senecan } \\
\text { Erian } \\
\text { Ulsterian }\end{array}$} & \multirow{2}{*}{\multicolumn{2}{|c|}{ Misener sand }} \\
\hline & & & \\
\hline 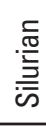 & $\begin{array}{c}\text { Cayugan } \\
\text { Niagaran } \\
\text { Alexandrian }\end{array}$ & \multicolumn{2}{|c|}{ Hunton Group } \\
\hline \multirow{4}{*}{$\begin{array}{l}\frac{.0}{0} \\
\frac{0}{3} \\
\frac{0}{0} \\
0\end{array}$} & Cincinnatian & \multicolumn{2}{|c|}{ Viola Group/Formation } \\
\hline & Champlainian & \multicolumn{2}{|c|}{ Simpson Group } \\
\hline & & 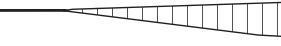 & \\
\hline & Canadian & \multicolumn{2}{|c|}{ Arbuckle Group } \\
\hline \multirow{2}{*}{ 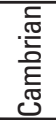 } & Trempealeauan & \multirow{2}{*}{\multicolumn{2}{|c|}{ Reagan Sandstone }} \\
\hline & Franconian & & \\
\hline
\end{tabular}

Figure 2. Generalized stratigraphic section for the Anadarko Basin, listing prominent groups and formations, modified from Bebout and others $(1993$, fig. 5). The cross sections in this report used the tops of 5 horizons (red italicized print) to establish 5 time-stratigraphic boundaries. Granite wash is in the Upper Pennsylvanian and lowermost Permian strata. Red beds and evaporites are generally in rocks of Permian age. [Camb., Cambrian; Miss., Mississippian] 
- the hydrologic regime of the basin (Nelson and Gianoutsos, chapter 9 of this report)

- the determination of oil and gas assessment units (Higley, chapters 1, 6 and 7 of this report; Gaswirth and Higley, chapter 5 of this report)

- the migration history of oil and gas within the basin (Higley, chapters 3, 5, 6 and 7 of this report)

In addition to these basin-scale features, other products can be derived from inspection of the plates and manipulation of the data in Appendix 2. For example, cross sections $A-A^{\prime}$ and
$B-B^{\prime}$ show that evaporite layers are present in the western part of each cross section but are absent in the eastern part of the cross sections. From the data in Appendix 2, the total thickness of evaporite was computed by summing all 10 -ft intervals listed for each well. These sums were used to construct a map showing total evaporite thickness (fig. 3), which shows that the total evaporite thickness exceeds $1,000 \mathrm{ft}$ in western Oklahoma and even exceeds 2,000 ft in three wells in the southwestern part of the Anadarko Basin. Evaporite thickness decreases steadily to the southeast and is absent in the southeastern part of the Anadarko Basin. Other mappable parameters and statistical results can be derived from the lithology data in Appendix 2.

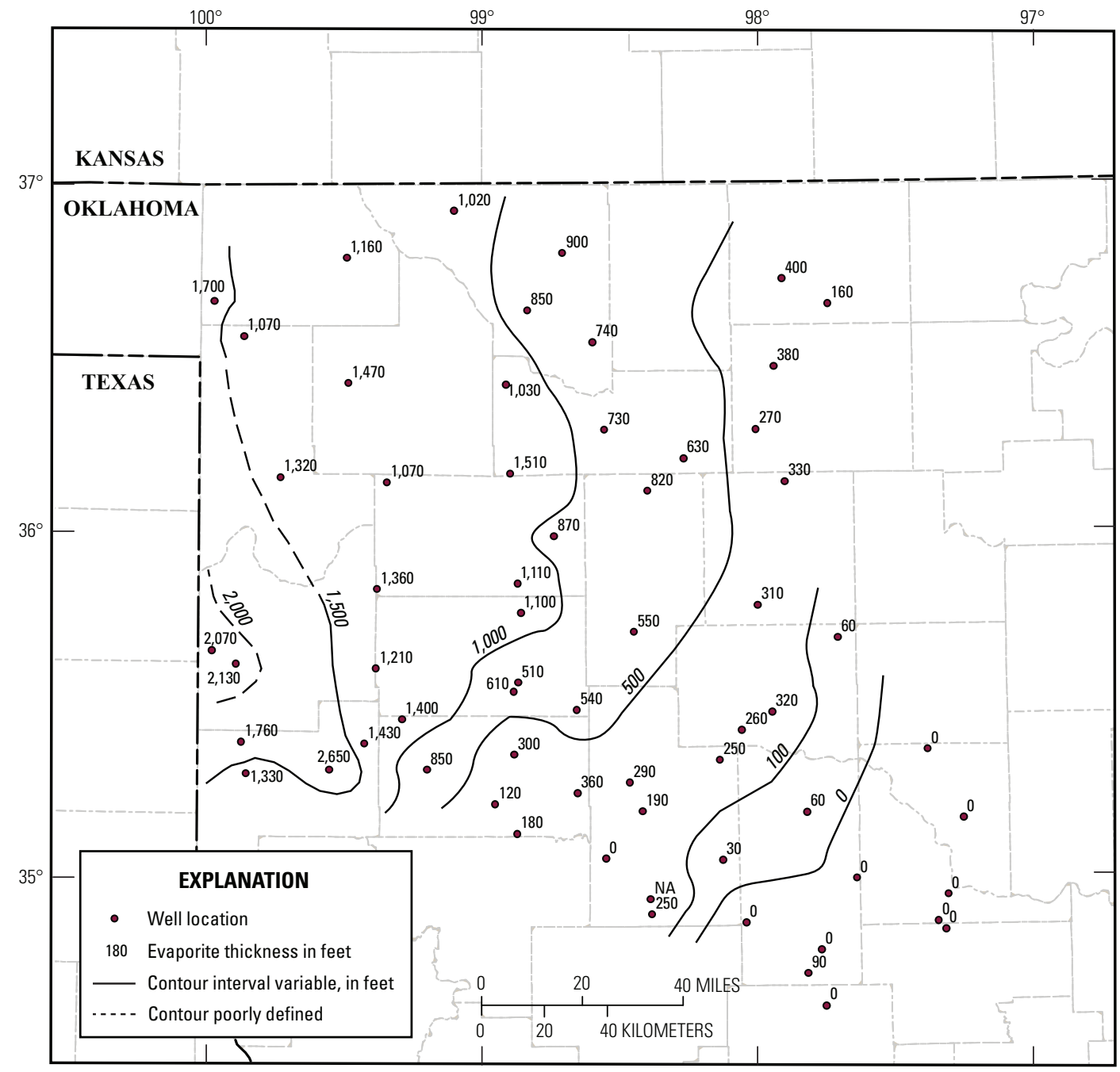

Figure 3. Map of total thickness of evaporite in Permian rocks in the Anadarko Basin. 


\section{Acknowledgments}

We thank David Blackwell of Southern Methodist University for his support and encouragement of this work. Reviews by Thomas Finn of the U.S. Geological Survey and Rick Andrews of the Oklahoma Geological Survey and guidance by Dick Keefer and Larry Anna of the U.S. Geological Survey led to improvements in the manuscript and cross sections.

\section{References Cited}

Andrews, R.D., 2008, Morrow and Springer strata in the southern midcontinent, in Andrews, R.D., ed., Morrow and Springer in the southern midcontinent, 2005 symposium: Oklahoma Geological Survey Circular 111, p. 81-97.

Bebout, D.G., White, W.A., Hentz, T.F., and Grasmick, M.K. eds., 1993, Atlas of major mid-continent gas reservoirs: Bureau of Economic Geology, 85 p.

Gallardo, J.D., 1989, Empirical model of temperature structure, Anadarko Basin, Oklahoma: Dallas, Tex., Southern Methodist University, Master's thesis, 186 p.
Gallardo, Jaquidon, and Blackwell, D.D., 1999, Thermal structure of the Anadarko Basin, American Association of Petroleum Geologists Bulletin, v. 83, no. 2, p. 333-361.

IHS Energy, 2010, U.S. Production and well data: database available from IHS Energy, 15 Inverness Way East, D205, Englewood, Colorado 80112.

Kirkland, D.W., Dension, R.E., Summers, D.M., and Gormly, J.R., 1992, Geology and organic geochemistry of the Woodford Shale in the Criner Hills and western Arbuckle Mountains, Oklahoma; Oklahoma Geological Survey Circular 93, p. 38-69.

Oklahoma City Geological Society, 1971, Cross section of Oklahoma from SW to NE corners of state, Witt, W.J., Chairman of Stratigraphic Committee, 1 pl.

Oklahoma Corporation Commission, 2010, Oil and gas database, http://www.occeweb.com/Orawebapps/OCCOraWebAppsone.html.

U.S. Geological Survey, 2014, Colorado, Kansas, New Mexico, Oklahoma, and Texas: U.S. Geological Survey digital data, The National Map, accessed August 4, 2014, at: http://viewer.nationalmap.gov/viewer/. 


\section{Chapter 11 \\ Tectonic and Structural Evolution of the Anadarko Basin and Structural Interpretation and Modeling of a Composite Regional 2D Seismic Line}

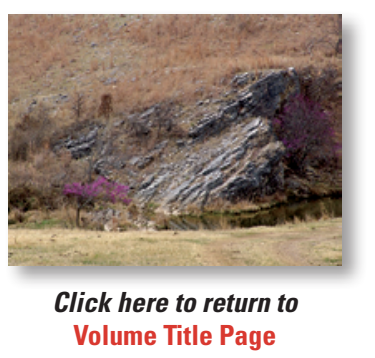

By Ofori N. Pearson and John J. Miller

Chapter 11 of 13

Petroleum Systems and Assessment of Undiscovered Oil and Gas in the Anadarko Basin Province, Colorado, Kansas, Oklahoma, and Texas-USGS Province 58

Compiled by Debra K. Higley

U.S. Geological Survey Digital Data Series DDS-69-EE 


\title{
U.S. Department of the Interior SALLY JEWELL, Secretary
}

\section{U.S. Geological Survey \\ Suzette M. Kimball, Acting Director}

\author{
U.S. Geological Survey, Reston, Virginia: 2014
}

For more information on the USGS - the Federal source for science about the Earth, its natural and living resources, natural hazards, and the environment, visit http://WWW.usgs.gov or call 1-888-ASK-USGS.

For an overview of USGS information products, including maps, imagery, and publications, visit http://WwW.usgs.gov/pubprod

To order this and other USGS information products, visit http://store.usgs.gov

Any use of trade, firm, or product names is for descriptive purposes only and does not imply endorsement by the U.S. Government.

Although this information product, for the most part, is in the public domain, it also may contain copyrighted materials as noted in the text. Permission to reproduce copyrighted items must be secured from the copyright owner.

Suggested citation:

Pearson, 0.N., and Miller, J.J., 2014, Tectonic and structural evolution of the Anadardo Basin and structural interpretation and modeling of a composite regional two-dimensional seismic line, chap. 11, in Higley, D.K., compiler, Petroleum systems and assessment of undiscovered oil and gas in the Anadarko Basin Province, Colorado, Kansas, Oklahoma, and Texas_-USGS Province 58: U.S. Geological Survey Digital Data Series DDS-69-EE, 23 p., http://dx.doi.org/10.3133/ds69EE. 


\section{Contents}

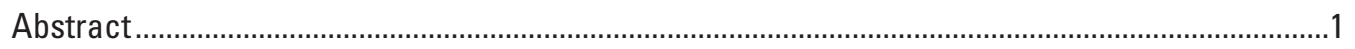

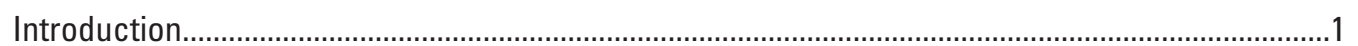

Tectonic, Structural, and Stratigraphic Overview.....................................................................

Precambrian Crustal Consolidation ....................................................................................

Late Precambrian to Middle Cambrian Development of the Southern Oklahoma

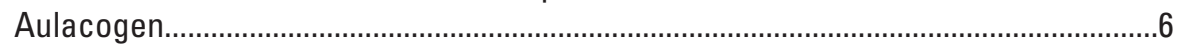

Late Cambrian to Early Mississippian Isostatic Subsidence .....................................................

Late Mississippian to Early Permian Development of the Anadarko Basin..............................8

Stratigraphic Overview ...................................................................................................

Cambrian and Ordovician Rocks .................................................................................

Silurian, Devonian, and Mississippian Rocks ..............................................................

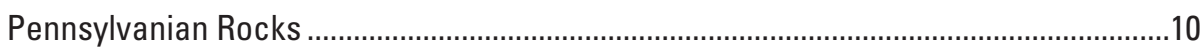

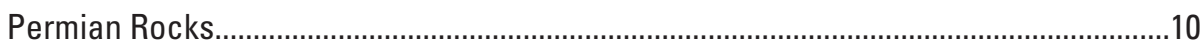

2D Reflection Seismic Datasets Available to the U.S. Geological Survey ......................................11

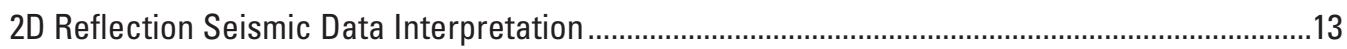

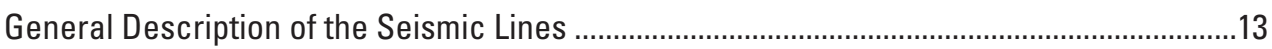

Seismic Interpretation of the Anadarko Composite Seismic Line ...................................................16

Structural Restoration of the Anadarko Composite Seismic Line ....................................................17

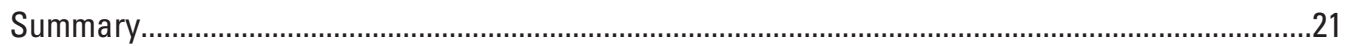

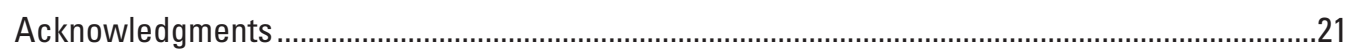

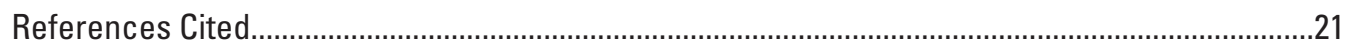

\section{Figures}

1. Map showing the location of the Anadarko Basin Province (red line). The general outline of the Anadarko Basin (blue line) is from Johnson and others (1988)......

2. Map showing the location of seismic lines (blue) licensed by the USGS within the Anadarko Basin Province (red line). Names by which the seismic lines are referred are also shown. Seismic data are owned or controlled by Seismic Exchange, Inc .........2

3. Map showing the major structural provinces of the Anadarko Basin and adjacent areas. Traces of major thrust faults are also shown in red, with $\mathrm{U}$ and $\mathrm{D}$ designating the upthrown and downthrown sides of selected faults. The fault system on the northern edge of the Wichita and Amarillo uplifts is known as the Wichita fault system (Harlton, 1963). Faults specifically referred to in the text are labeled. State boundaries are outlined in black; counties are outlined in gray. Modified from Luza and others (1987), Johnson (1989), and Luza (1989). Traces of seismic lines are shown in light blue

4. Simplified stratigraphic column for the northern Anadarko Basin (modified from Higley and Gaswirth, 2014, and references therein). Informal unit names are italicized. Names in red are the stratigraphic units used in the seismic interpretation and structural restoration. Wavy horizontal lines and vertical bars indicate unconformities. Ages are in millions of years before the present (Ma). Note the change of vertical scale between the Cambrian-Mississippian column and the Pennsylvanian-Permian column.... 
5. Maps showing the sequence of Proterozoic events that formed the complex suite of basement rocks beneath the Anadarko Basin Province. Modified from Whitmeyer and Karlstrom (2007). A, Yavapai orogeny; $B$, intrusion of the Yavapai Province by granitoids; $C$, Mazatzal orogeny; $D$, intrusion of Mazatzal granitoids; $E$, suturing of the Granite-Rhyolite Province; $F$, extensive granitoid intrusion of the Yavapai, Mazatzal, and Granite-Rhyolite Provinces; $G$, intrusion of Grenville-aged granitoids and development of the Midcontinent rift system; $H$, development of the southern Oklahoma aulacogen. Ga, billion years before the present.....

6. Map showing the geometry of the eastern Laurentian margin during the Cambrian in the southern and eastern United States. Locations and geometries of the southern Oklahoma aulacogen and the Reelfoot rift are shown. Paleogeographic map for the Middle Cambrian (510 Ma) is from Blakey (2011). Rifted margin geometry and location of the Reelfoot rift and southern Oklahoma aulacogen are from Keller and others (1983).

7. Map showing the location of the northwestern limits of the southern Oklahoma aulacogen relative to the present Anadarko Basin. The aulacogen is shown in light gray, thrust and reverse faults of the Wichita fault system are shown in red, and contour lines show approximate depths to basement in the Anadarko Basin, with depths in tens of thousands of feet below sea level. Modified from Perry (1989) and references therein. $\mathrm{Ga}$, billions of years before the present.

8. Root mean square velocity model used to migrate and convert seismic Line D. Velocities were determined using an automated velocity picking algorithm, and subsequently smoothed using a 201 common depth point horizontal, and 250-millisecond vertical, smoothing operator. A color bar illustrating the velocity scale is also shown. Seismic data are owned or controlled by Seismic Exchange, Inc.; interpretation is that of the U.S. Geological Survey

9. Uninterpreted seismic Line A. Location of line is shown in figure 2. Horizontal and vertical scales are equal. Black colors are amplitude peaks; red colors are amplitude troughs. Seismic data are owned or controlled by Seismic

Exchange, Inc

10. Uninterpreted seismic Line B. Location of line is shown in figure 2. Horizontal and vertical scales are equal. Dark black colors are amplitude peaks; dark red colors are amplitude troughs. Seismic data are owned or controlled by Seismic Exchange, Inc. Figure 10 is oversized. Click on the thumbnail to view the enlarged version

11. Uninterpreted seismic Line C. Location of line is shown in figure 2. Horizontal and vertical scales are equal. Dark black colors are amplitude peaks; dark red colors are amplitude troughs. Seismic data are owned or controlled by Seismic Exchange, Inc

12. Uninterpreted seismic Line D. Location of line is shown in figure 2. Horizontal and vertical scales are equal. Dark black colors are amplitude peaks; dark red colors are amplitude troughs. Seismic data are owned or controlled by Seismic Exchange, Inc. Figure 12 is oversized. Click on the thumbnail to view the enlarged version

13. Uninterpreted seismic Line E. Location of line is shown in figure 2. Horizontal and vertical scales are equal. Dark black colors are amplitude peaks; dark red colors are amplitude troughs. Seismic data are owned or controlled by Seismic Exchange, Inc. Figure 13 is oversized. Click on the thumbnail to view the enlarged version 
14. Map showing the location of wells used to aid in the interpretation of the composite seismic line. Oklahoma counties are shown in gray. The segments of the composite seismic line are shown in blue. American Petroleum Institute well numbers are given

15. Interpreted seismic Line C. Location of line is shown in figure 2. Horizontal and vertical scales are equal. Dark black colors are amplitude peaks; dark red colors are amplitude troughs. Well symbols are the same as those used in fig. 14. American Petroleum Institute well numbers are indicated. Lithology codes for well tops are given in table 1. Seismic data are owned or controlled by Seismic Exchange, Inc.; interpretation is that of the U.S. Geological Survey

16. Interpreted seismic Line D. Location of line is shown in figure 2. Horizontal and vertical scales are equal. Dark black colors are amplitude peaks; dark red colors are amplitude troughs. Well symbols are the same as those used in fig. 14. American Petroleum Institute well numbers are indicated. Lithology codes for well tops are given in table 1. Seismic data are owned or controlled by Seismic Exchange, Inc.; interpretation is that of the U.S. Geological Survey. Figure 16 is oversized. Click on the thumbnail to view the enlarged version .

17. Interpreted seismic Line E. Location of line is shown in figure 2. Horizontal and vertical scales are equal. Dark black colors are amplitude peaks; dark red colors are amplitude troughs. Well symbols are the same as those used in figure 14. American Petroleum Institute well numbers are indicated. Lithology codes for well tops are given in table 1. Seismic data are owned or controlled by Seismic Exchange, Inc.; interpretation is that of the U.S. Geological Survey. Figure 17 is oversized. Click on the thumbnail to view the enlarged version .

18. Structural restoration showing individual restoration stages. Depth in each stage is shown in thousands of feet below sea level; horizontal distance is given in miles from the southwestern edge of seismic Line $C$ (no vertical exaggeration). Seismic data are owned or controlled by Seismic Exchange, Inc.; interpretation is that of the U.S. Geological Survey. Figure 18 is oversized. Click on the thumbnail to view the enlarged version

19. Incremental shortening (blue line and round markers) and cumulative shortening (red line and star markers) is shown for all stages of the structural restoration shown in figure 18. Note that the vertical axis is time, rather than stratigraphic thickness.

\section{Tables}

1. Lithology codes and corresponding stratigraphic units for formation tops shown in figures 15,16 , and 17

2. Seismic line names, counties and states where seismic lines were recorded, the year the USGS purchased licenses for the data, and the lengths of each line. Seismic data are owned or controlled by Seismic Exchange, Inc.; interpretation is that of the U.S. Geological Survey

3. Data acquisition parameters for the five seismic lines used in this study. Seismic data are owned or controlled by Seismic Exchange, Inc.; interpretation is that of the U.S. Geological Survey... 



\title{
Tectonic and Structural Evolution of the Anadarko Basin and Structural Interpretation and Modeling of a Composite Regional 2D Seismic Line
}

\author{
By Ofori N. Pearson and John J. Miller
}

\begin{abstract}
The U.S. Geological Survey recently completed an assessment of undiscovered oil and gas resources in the Anadarko Basin Province of western Oklahoma and Kansas, northern Texas, and southeastern Colorado. The assessment methodology required a detailed look at the region's tectonic and structural evolution, which can be divided into the following four periods: (1) Precambrian crustal consolidation; (2) development of the southern Oklahoma aulacogen during late Precambrian to Middle Cambrian time; (3) thermally controlled isostatic subsidence of the failed rift from Late Cambrian to Early Mississippian time; and (4) development of the Ouachita-Marathon orogeny that caused the Wichita uplift and development of the asymmetric Anadarko Basin beginning in Late Mississippian time. Many of the basin's key structural elements are visible on two-dimensional reflection seismic lines, and interpretations of the data reveal the basin's subsurface structural geometry. A structural restoration based upon the seismic interpretation shows the possible geometric evolution for 22 of the Anadarko Basin's key stratigraphic units.
\end{abstract}

\section{Introduction}

The asymmetric Anadarko Basin (fig. 1) is the deepest interior cratonic basin in the conterminous United States, containing as much as $\approx 40,000$ feet of Upper Cambrian-Permian sedimentary rocks (Johnson and others, 1988). It has been one of the more productive U.S. basins in terms of production of oil and gas, with cumulative production in excess of 5.4 billion barrels of oil and 125 trillion cubic feet of gas (IHS Energy, 2010). In 2010, the U.S. Geological Survey (USGS) conducted an assessment of undiscovered oil and gas resources contained within the Anadarko Basin Province of western Oklahoma and Kansas, northern Texas, and southeastern Colorado (fig. 1), resulting in means of 495 million barrels of oil, 27,461 billion cubic feet of gas, and 410 million barrels of natural gas liquids (Higley and others, 2011).
As part of the 2010 assessment, an analysis of the Anadarko Basin's tectonic and structural evolution was conducted. A broad overview is presented in this chapter of the report, based on previously published studies and an interpretation and structural restoration of five twodimensional (2D) reflection seismic lines (fig. 2) acquired by the USGS within the basin. Published studies typically describe four main phases of tectonic and structural activity for the Anadarko Basin, and those divisions are adopted for this study. The acquisition and processing of the reflection seismic lines are also described. Three of those lines (fig. 2; Lines $\mathrm{C}, \mathrm{D}$, and $\mathrm{E}$ ) that lie in close proximity to one another were combined into a composite regional line, and the seismic interpretation of this line is discussed. A structural restoration is documented that was built from the seismic interpretation and key findings of the restoration are tied into elements of the broader tectonic and structural evolution of the Anadarko Basin.

\section{Tectonic, Structural, and Stratigraphic Overview}

Although the oldest sedimentary rocks in the Anadarko Basin are of Cambrian age, the basin's earlier tectonic and structural history plays an important role in the depositional history of the Paleozoic section and on the petroleum systems that are the subject of the 2010 USGS assessment. The eastern margin of North America has undergone two complete Wilson cycles (plate-tectonic spreading and convergence; Wilson, 1966) - the assembly and breakup of both the Rodinia and Pangaea land masses. Basement and sedimentary rocks of the Anadarko Basin Province were involved in the Proterozoic assembly and breakup of Rodinia, and the Paleozoic assembly of Pangaea.

Most workers describe the evolution of the Anadarko Basin in terms of four main tectonic and structural events, two of which occurred before deposition of the basin's oldest (Cambrian) sedimentary rocks. These four primary phases of tectonic and structural activity are: (1) Precambrian crustal 


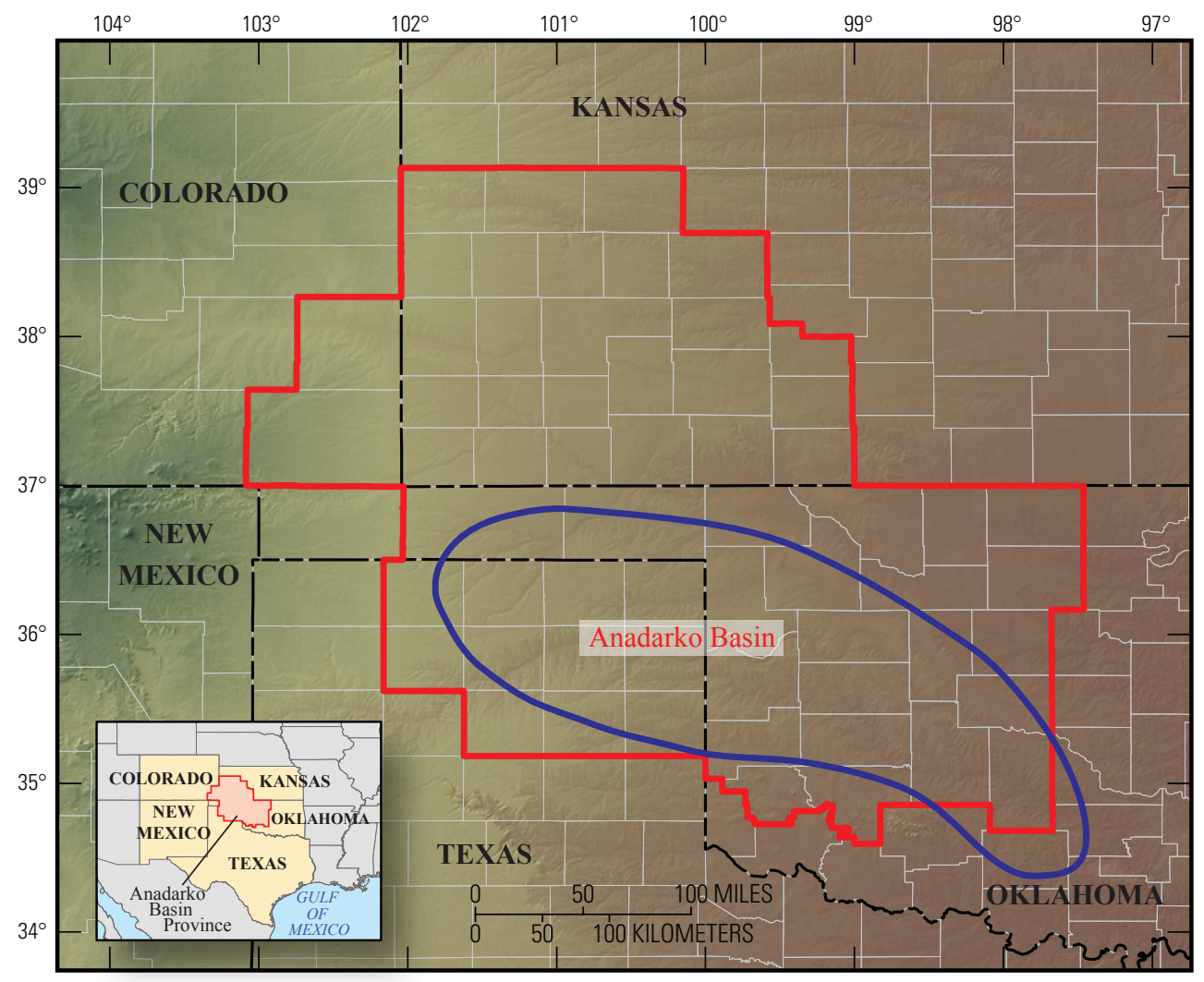

Figure 1. Map showing the location of the Anadarko Basin Province (red line). The general outline of the Anadarko Basin (blue line) is from Johnson and others (1988).

Figure 2. Map showing the location of seismic lines (blue) licensed by the USGS within the Anadarko Basin Province (red line). Names by which the seismic lines are referred are also shown. Seismic data are owned or controlled by Seismic Exchange, Inc. 
consolidation; (2) late Precambrian to Middle Cambrian development of the southern Oklahoma aulacogen; (3) Late Cambrian to Early Mississippian isostatic subsidence; and (4) development of the Anadarko Basin beginning in the Late Mississippian, because of the Ouachita orogeny. The following discussion gives a broad overview of each of these four phases, primarily as they relate to the Anadarko Basin proper. Although the Anadarko Basin Province also contains parts of other major structural elements (fig. 3) that make up the southern midcontinent region (for example, the Hugoton embayment, the Cimarron arch, the Palo Duro Basin, and the Nemaha uplift), these are discussed only in terms of how they relate to the Anadarko Basin. A simplified stratigraphic column for the basin is shown in figure 4 . A rationale for the simplification shown in the column and a description of the lithologies are provided in the stratigraphic summary portion of this report.

\section{Precambrian Crustal Consolidation}

Figure 5 (modified from Whitmeyer and Karlstrom, 2007) portrays the Proterozoic sequence of events that led to crustal consolidation of basement rock beneath the Anadarko Basin Province. Also shown in figure 5 are the ages of rocks that were sutured onto the southeastern margin of Laurentia and also the ages of intrusives that accompanied the various orogenies and stitched terranes together. The oldest basement rocks in the province (fig. $5 \mathrm{~A}$ ) were likely accreted onto the margin of Laurentia during the Yavapai orogeny $\approx 1.76-1.72$ billion years before the present $(\mathrm{Ga})$ (Whitmeyer and Karlstrom, 2007). During the orogeny, oceanic arc terranes (1.76-1.72 Ga) sutured onto Laurentia along the Cheyenne belt in Wyoming and the Spirit Lake tectonic zone at the southern margin of the western Superior and Penokean Provinces (Whitmeyer and Karlstrom, 2007). Following accretion, the Yavapai Province was intruded by granitoids (fig. $5 B$ ) dated at $\approx 1.72-1.68 \mathrm{Ga}$ (Whitmeyer and Karlstrom, 2007). The next major event to affect the southeastern margin of Laurentia was the Mazatzal orogeny, during which crust dated at 1.69-1.65 Ga formed in continental margin volcanic arcs and back-arc-related supracrustal successions were accreted along a bivergent suture zone (fig. $5 C$; Whitmeyer and Karlstrom, 2007). During later stages of the Mazatzal orogeny $(1.65-1.60 \mathrm{Ga})$, both the Yavapai and Mazatzal Provinces were intruded by granitoids (fig. $5 D$ ). The southernmost portion of the Anadarko Basin Province may be underlain by basement rocks of the 1.55-1.35 Ga Granite-Rhyolite Province (fig. 5E; Van Schmus and others, 1996). Following

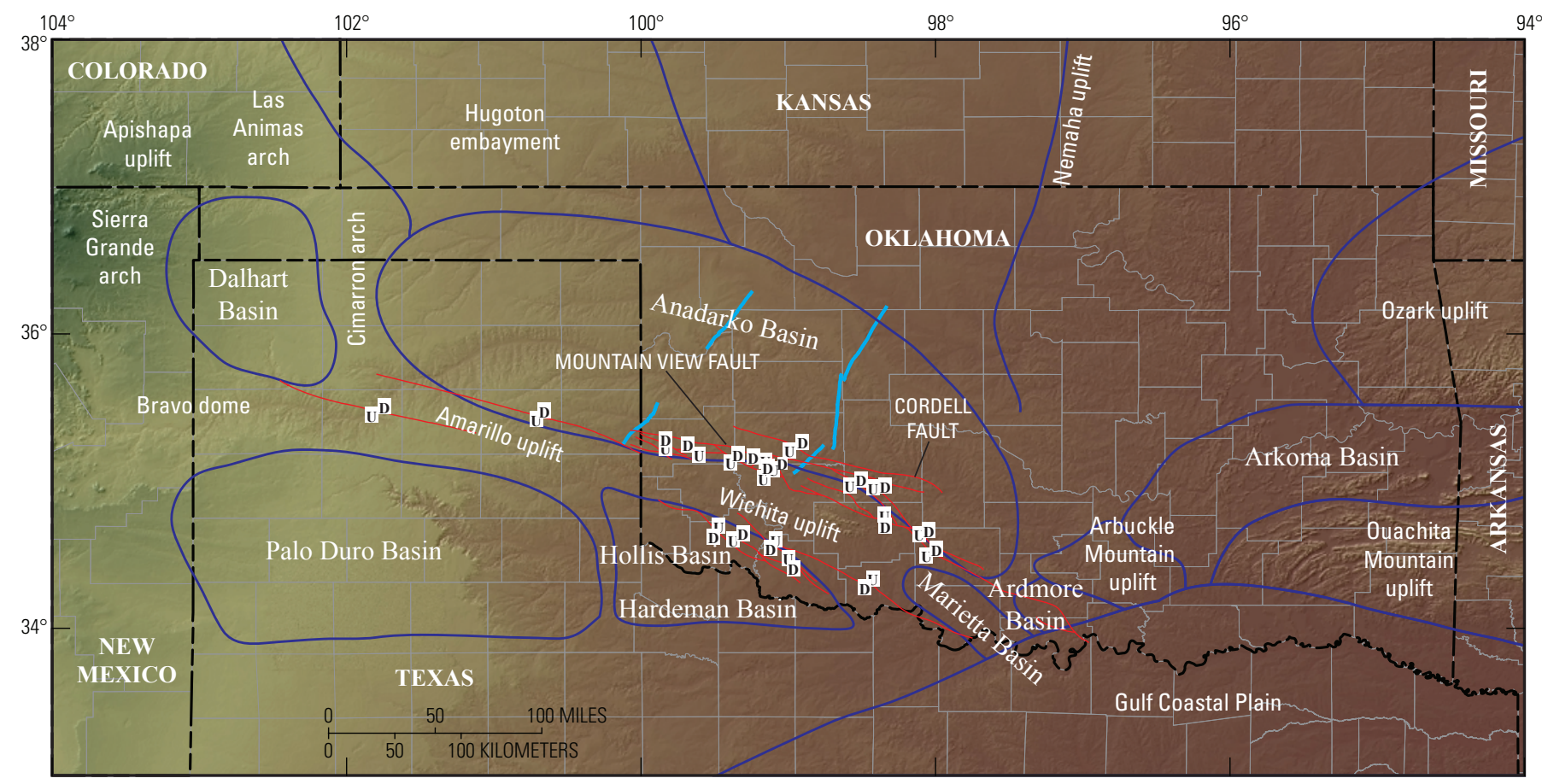

Figure 3. Map showing the major structural provinces of the Anadarko Basin and adjacent areas. Traces of major thrust faults are also shown in red, with $U$ and $D$ designating the upthrown and downthrown sides of selected faults. The fault system on the northern edge of the Wichita and Amarillo uplifts is known as the Wichita fault system (Harlton, 1963). Faults specifically referred to in the text are labeled. State boundaries are outlined in black; counties are outlined in gray. Modified from Luza and others (1987), Johnson (1989), and Luza (1989). Traces of seismic lines are shown in light blue. 


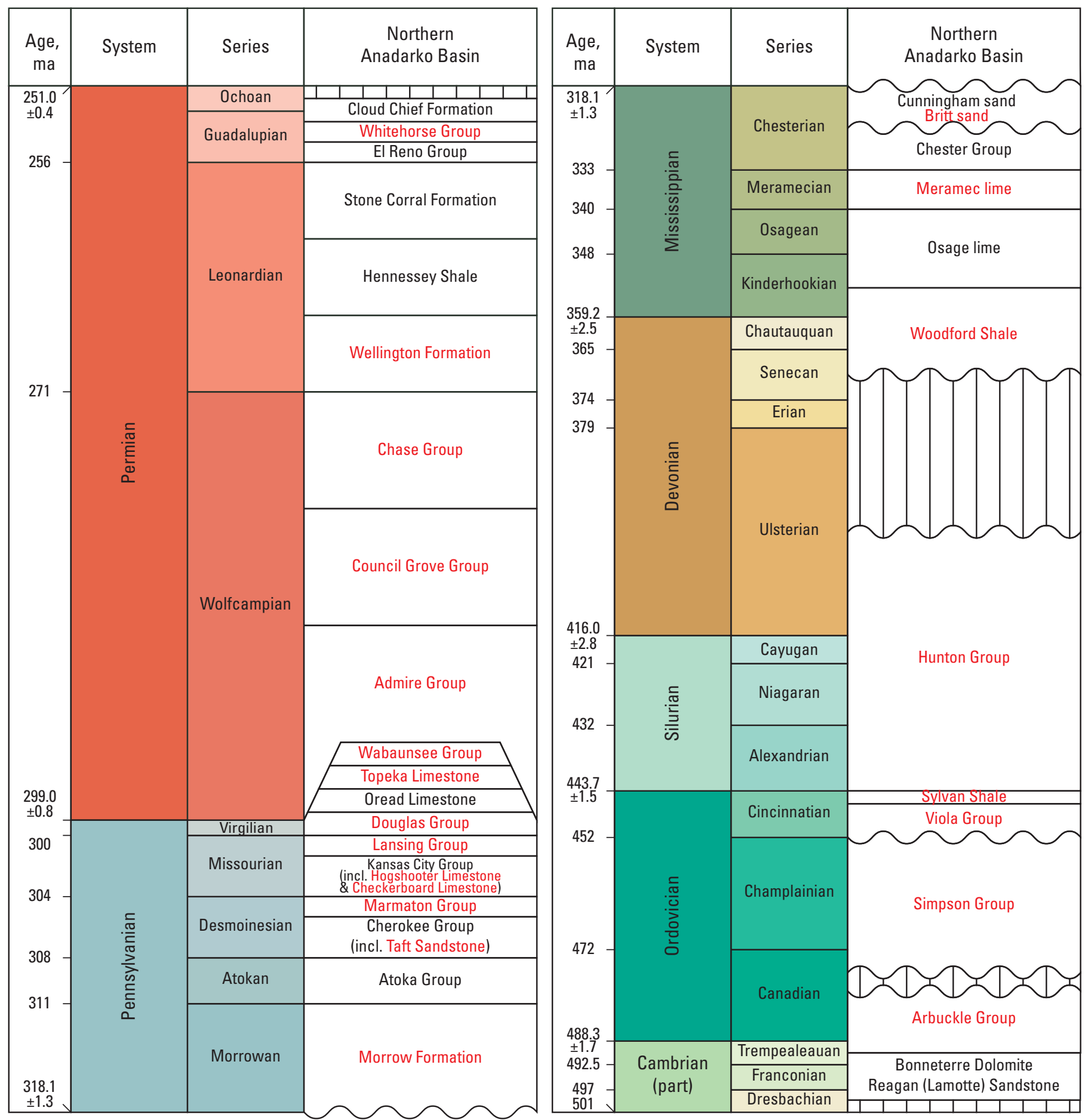

Figure 4. Simplified stratigraphic column for the northern Anadarko Basin (modified from Higley and Gaswirth, 2014, and references therein). Informal unit names are italicized. Names in red are the stratigraphic units used in the seismic interpretation and structural restoration. Wavy horizontal lines and vertical bars indicate unconformities. Ages are in millions of years before the present (Ma). Note the change of vertical scale between the Cambrian-Mississippian column and the Pennsylvanian-Permian column. 

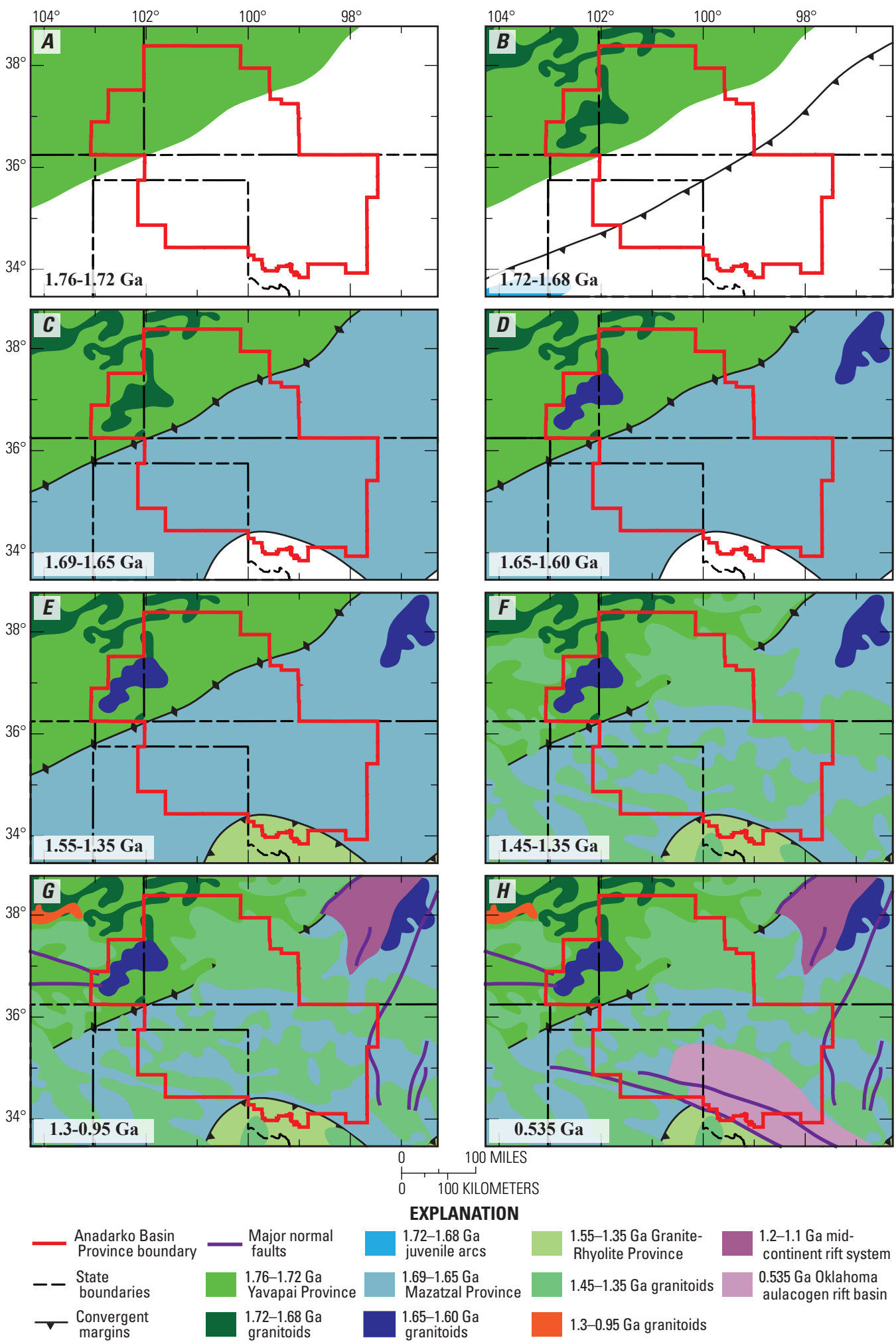

Figure 5. Maps showing the sequence of Proterozoic events that formed the complex suite of basement rocks beneath the Anadarko Basin Province. Modified from Whitmeyer and Karlstrom (2007). $A$, Yavapai orogeny; $B$, intrusion of the Yavapai Province by granitoids; $C$, Mazatzal orogeny; $D$, intrusion of Mazatzal granitoids; $E$, suturing of the Granite-Rhyolite Province; $F$, extensive granitoid intrusion of the Yavapai, Mazatzal, and Granite-Rhyolite Provinces; $G$, intrusion of Grenville-aged granitoids and development of the Midcontinent rift system; $H$, development of the southern Oklahoma aulacogen. Ga, billion years before the present. 
accretion of the Granite-Rhyolite Province to the southeastern margin of Laurentia, the Yavapai, Mazatzal, and Granite-Rhyolite Provinces were all intruded by extensive bimodal A-type granites (1.45-1.35 Ga, fig. $5 F$; Whitmeyer and Karlstrom, 2007) that stitched together the three provinces.

The Proterozoic assembly of Laurentia culminated during the 1.30-0.95 Ga Grenville orogeny (fig. 5G). The GrenvilleLlano deformation front (the Llano front is the likely southwestern continuation in Texas of the Grenville front) lay to the south of the Anadarko Basin Province in the Granite-Rhyolite Province (Thomas, 1991), thus probably did not affect basement rocks within the Anadarko Basin Province. Along with the Grenville deformation front, late Grenville granitoids intrusions in Colorado and the southernmost extent of the 1.2-1.1 Ga Midcontinent rift system in Kansas (fig. 5G) are likely the most proximal signatures of the Grenville orogeny. Following the amalgamation of Laurentia during the Grenville orogeny, a long process of diachronous rifting began. Early rifting (between 0.78 and $0.68 \mathrm{Ga}$ ) occurred to the west, and separated Australia, Antarctica, south China, and Siberia from Laurentia. Rifting on the eastern margin of Laurentia began between 0.62 and $0.55 \mathrm{Ga}$ (Whitmeyer and Karlstrom, 2007). The final rifting event that affected the midcontinent region was the separation of the Argentinian Precordillera terrane from the Ouachita embayment of eastern Oklahoma and Texas (Thomas and Astini, 1996). Although the mechanics behind the translation of the Argentinian Precordillera from the southeastern margin of Laurentia to the western margin of Gondwana are poorly understood, they likely involve the Reelfoot rift and Southern Oklahoma aulacogen (Whitmeyer and Karlstrom, 2007). Formation of the southern Oklahoma aulacogen (fig. $5 H)$ during the Cambrian $(\sim 0.535 \mathrm{Ga})$ was the next major tectonic and structural event to affect the Anadarko Basin Province.

\section{Late Precambrian to Middle Cambrian Development of the Southern Oklahoma Aulacogen}

The second half of the first complete Wilson cycle to affect the eastern margin of North America involved the breakup of Rodinia and the opening of the Iapetus Ocean. Toward the end of this rifting phase in the Early Cambrian, at least two triple junctions developed in what is now the southeastern United States (Whitmeyer and Karlstrom, 2007). Evidence for the presence of these triple junctions includes the Reelfoot rift and the southern Oklahoma aulacogen (fig. 6). Development of the southern Oklahoma aulacogen played a key role in the formation of the Anadarko Basin, as the aulacogen's geometry and thermal effect on the surrounding crust exhibited first-order controls on subsequent depositional and structural trends.

Shatsky (1946) was the first to suggest the presence of an aulacogen in southern Oklahoma. The primary lines of evidence that support the existence of a failed rift system are an

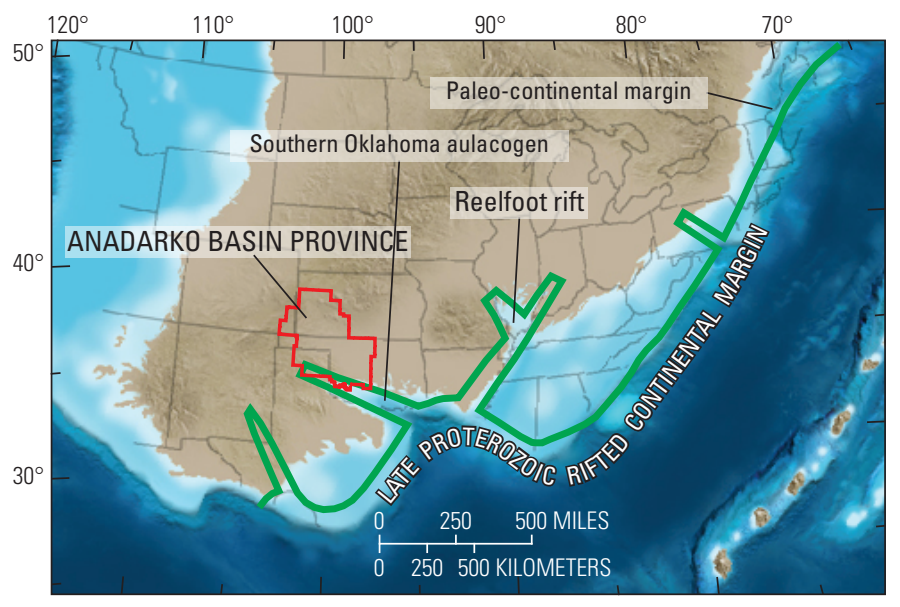

Figure 6. Map showing the geometry of the eastern Laurentian margin during the Cambrian in the southern and eastern United States. Locations and geometries of the southern Oklahoma aulacogen and the Reelfoot rift are shown. Paleogeographic map for the Middle Cambrian (510 Ma) is from Blakey (2011). Rifted margin geometry and location of the Reelfoot rift and southern Oklahoma aulacogen are from Keller and others (1983).

extensive suite of bimodal Cambrian igneous rocks and a linear trend of high Bouguer gravity anomaly values across southern Oklahoma. Bimodal igneous rocks of Cambrian age are wellexposed on the Wichita uplift (fig. 3) south of the Anadarko Basin. These rocks include: (1) the Glen Mountains Layered Complex, which is a layered mafic intrusion; (2) the 525-million years before the present (Ma) Carlton Rhyolite Group, which comprises over 40,000 cubic kilometers $\left(\mathrm{km}^{3}\right)$ of silicic intrusive and volcanic rocks (Gilbert, 1983); and (3) other basalts and granites (Keller and Stephenson, 2007). The other primary line of evidence that supports the existence of the southern Oklahoma aulacogen is the linear trend of high Bouguer gravity anomaly values extending from about long. $96^{\circ}$ to $101^{\circ} \mathrm{W}$. across southern Oklahoma (see fig. 3 of Keller and Stephenson, 2007). This gravity high has been linked to the presence of a deep and massive mafic intrusive (Keller and Baldridge, 1995). The three-armed pattern of Bouguer gravity highs at the southeastern edge of the aulacogen is likely tied to a rift-rift-rift triple junction (see fig. 3 of Keller and Stephenson, 2007).

Rift systems are typically bounded by extensive normal fault systems, and the presence of such faults around the southern Oklahoma aulacogen was inferred by Gilbert (1982, 1987). However, normal fault systems flanking the aulacogen that are clearly rift-related have not been recognized (Keller and Stephenson, 2007). This is likely because of a combination of poor exposures and extensive deformation during the latter parts of the Paleozoic that may have inverted or reactivated Cambrian extensional structures.

Although the axes of the southern Oklahoma aulacogen and the Anadarko Basin are subparallel, the two features do not completely overlap. The northern edge of the aulacogen probably lies beneath the southern edge of the Anadarko Basin 
(Perry, 1989; fig. 7). Some of the thrust and reverse faults that separate the basin from the Wichita uplift could be inverted normal faults associated with Cambrian rifting.

By the end of the Middle Cambrian, rifting along the incipient spreading center below southern Oklahoma ended, and the extensive suite of igneous rocks that had been incorporated into the crust in the vicinity of the aulacogen began to cool. This period of thermal subsidence lasted from the Late Cambrian through the Early Mississippian, and characterizes the next major phase in the region's tectonic, structural, and depositional evolution.

\section{Late Cambrian to Early Mississippian Isostatic Subsidence}

The oldest sedimentary rocks in the Anadarko Basin are the Upper Cambrian Reagan Sandstone (fig. 4). The deposition of these rocks marks the beginning of a lengthy phase dominated by basin subsidence, during which more than 40,000 ft of Upper Cambrian through Permian sediments accumulated. The initial phase of subsidence occurred from Late Cambrian through Early Mississippian time, and was dominated by thermally controlled isostatic subsidence caused primarily by cooling of the southern Oklahoma aulacogen (Feinstein, 1981).

This lengthy initial period of subsidence occurred during a time when the southern midcontinent region was isolated from tectonic events related to the start of the second Wilson cycle. In North America, the Appalachian-Ouachita orogenic belt records successive, diachronous orogenies that resulted in the closure of the Iapetus Ocean and the formation of Pangaea during the Permian (Thomas, 2006). These orogenies are the Taconic (Ordovician-Silurian), Acadian (DevonianMississippian), and the Alleghanian (Mississippian-Permian). During the Taconic and Acadian orogenies, which mainly affected more northern parts of the North American continent, a passive margin outboard of the southern midcontinent region persisted until Mississippian time (Thomas, 1989). The relative isolation of the southern midcontinent region from tectonic stresses that occurred farther to the north, suggests that unconformities within the Upper Cambrian through Lower

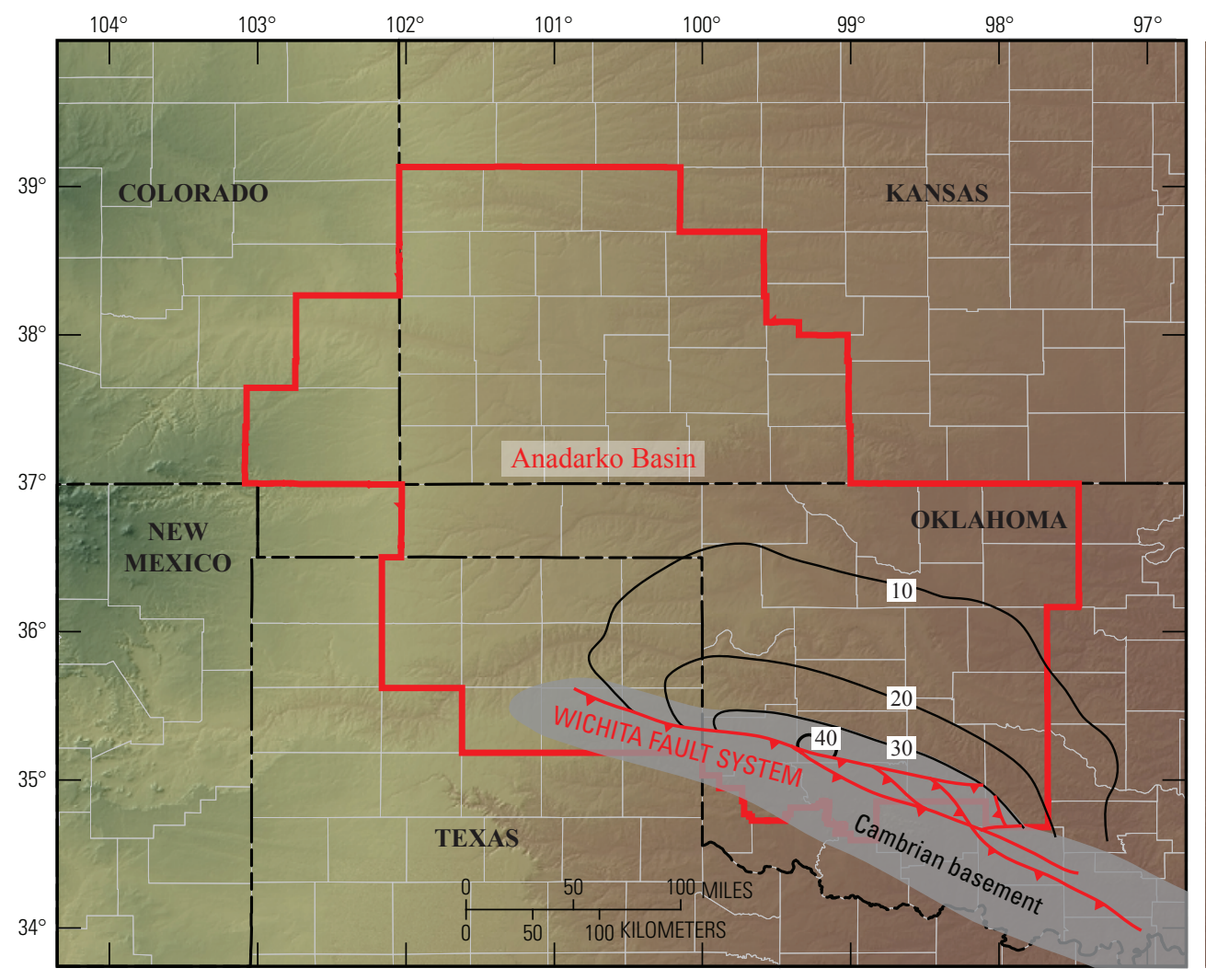

Figure 7. Map showing the location of the northwestern limits of the southern Oklahoma aulacogen relative to the present Anadarko Basin. The aulacogen is shown in light gray, thrust and reverse faults of the Wichita fault system are shown in red, and contour lines show approximate depths to basement in the Anadarko Basin, with depths in tens of thousands of feet below sea level. Modified from Perry (1989) and references therein. Ga, billions of years before the present. 
Tectonic and Structural Evolution of the Anadarko Basin, Structural Interpretation and Modeling of a Seismic Line

Mississippian stratigraphic section of the Anadarko Basin are more likely related to global sea level changes than to structural events.

The cooling southern Oklahoma aulacogen created accommodation space in what is known as the southern Oklahoma trough, which is generally coincident with the geometry of the aulacogen (Perry, 1989). More than 11,700 ft of Cambrian-Devonian rocks were deposited in this trough (Ham, 1973; Cardott and Lambert, 1985). Feinstein (1981) proposed a two-stage mechanism to explain the Late Cambrian through Early Mississippian subsidence of the Oklahoma trough. The earliest stage occurred during deposition of the basal Upper Cambrian Reagan Sandstone. The rate of subsidence at this stage was low, and can be compared to that of other basins where elastic flexure of the lithosphere in response to an increased load (rocks emplaced during the aulacogen) governed subsidence (Feinstein, 1981). Feinstein (1981) proposed that higher rates of subsidence that followed deposition of the Reagan Sandstone can be attributed to detachment of the aulacogen from the adjacent platform, possibly reactivating normal fault systems that bounded the aulacogen. After detaching from the adjacent platform, evolution of the Oklahoma trough generally followed an exponential curve of subsidence with time controlled by isostatic subsidence driven by cooling of rocks emplaced during formation of the aulacogen (Feinstein, 1981). This two-stage model supports observations that slow initial rates of Cambrian subsidence accelerated during the Early Ordovician before slowing again in the Late Ordovician as the aulacogen reached thermal equilibrium; slow gradual rates of subsidence continued during Silurian, Devonian, and Early Mississippian time (see figs. 2 and 3 in Feinstein, 1981).

\section{Late Mississippian to Early Permian Development of the Anadarko Basin}

Beginning in the Late Mississippian, the prolonged period of relative tectonic and structural quiescence in the southern midcontinent region ended abruptly as compressional forces associated with the Appalachian-Ouachita orogeny arrived. For the southern midcontinent region, this marked the onset of the second Wilson cycle, which included the assembly and subsequent breakup of Pangaea. For the remainder of this discussion, this orogenic period will be referred to as the Ouachita-Marathon orogeny, as this nomenclature is more commonly used in the literature to describe the collision between the North American and the South American-African plates. Compressional forces of the Ouachita-Marathon orogeny are generally thought to be responsible for the structural inversion of the southern Oklahoma aulacogen, and the subsequent formation of the Anadarko Basin (Kluth and Coney, 1981; Kluth, 1986).

Structures related to the Ouachita-Marathon orogeny in the Anadarko Basin Province have been extensively documented and described (see, for example, McConnell, 1989; Perry, 1989), as they have not been overprinted by subsequent deformation. Identification of many of these structures has been aided by the availability of extensive subsurface datasets produced during exploration for petroleum. Detailed descriptions of individual structural features are not provided in the following summary, but the tectonic forces that caused the formation of the Anadarko Basin and the resultant basin-scale structural features are discussed.

The earliest compressional stresses of the Ouachita-Marathon orogeny affected the Alabama promontory during Middle Mississippian time in what is now the Black Warrior Basin of Alabama (Thomas, 2006). The deformation front migrated toward the northwest along the Alabama-Oklahoma transform fault, and reached the Arkoma Basin (fig. 3) by the Early Pennsylvanian (Thomas, 1989). This migration of the deformation front is an excellent example of tectonic inheritance, as preexisting crustal weaknesses that developed during the rifting of Rodinia were exploited by the Ouachita-Marathon orogeny (Thomas, 2006).

Within the Anadarko Basin region, the earliest sign of the approaching deformation front may be the pinching-out of uppermost Mississippian-lowermost Pennsylvanian sandstones in the western Ardmore Basin (fig. 3; Perry, 1989). During Morrowan time, a broad uplift formed that extended from the Criner Hills southward into Texas and northwestward toward the Wichita Mountains. Tomlinson and McBee (1959) called this uplift the Wichita-Criner uplift, and it included the Wichita Mountains, which were rising during this time. To the northeast of the uplift, a narrow trough developed, into which greater thicknesses of Morrowan through Desmoinesian sediments were deposited relative to the shelf farther to the northeast (Johnson, 1989). Development of the Wichita-Criner uplift accelerated during the late Morrowan, and climaxed during Atokan time. On the northeast flank of the Wichita Mountains, synorogenic sediments derived from the weathering of both Mississippian limestones that mantled the Wichita Mountains and basement rocks were deposited in the immediate footwall of the developing Wichita fault system (fig. 3; Johnson, 1989). These synorogenic sediments suggest that the mountains had been unroofed by this time. The Wichita fault system may have reactivated normal faults that formed during emplacement of the southern Oklahoma aulacogen. By the beginning of Desmoinesian time, faulting along the margins of the Wichita Mountains had ended (Perry, 1989). However, the uplift remained a positive feature through the end of the Pennsylvanian, and continued to supply sediments into the deepening Anadarko Basin. During the Late Pennsylvanian, the deformation front appears to have migrated both to the east and to the southwest. The eastward migration is evidenced by structural activity in the Arbuckle Mountains (Perry, 1989) and in the Ouachita Mountains of southeastern Oklahoma and west-central Arkansas (Johnson, 1989). Westward migration is seen by deformation in the Marathon region of western Texas, which climaxed in the Virgilian or Wolfcampian; by Leonardian time, the Ouachita-Marathon orogeny had ended (Kluth and Coney, 1981). 
Major structural activity in the Anadarko Basin ended by the Early Permian (Perry, 1989). Deposition continued to the end of the Permian; accommodation space was likely created both by continued gradual subsidence of the basin and compaction of older rocks. The final act of the second Wilson cycle to affect the eastern margin of North America began in the Triassic with the onset of Atlantic rifting. By this time, however, the Anadarko Basin was located far inboard of the continental margin, and does not appear to have been affected. The most recent structural activity within the basin involves minor reactivation of normal faults that may have occurred during the Jurassic, Cretaceous, and Holocene (Perry, 1989).

As previously mentioned, the Wichita Mountains are bounded to the northeast by the Wichita fault system (fig. 3). Brewer and others (1983) reported that deep reflection seismic profiles across the Anadarko Basin and Wichita Mountains image a series of thrust faults that dip $30^{\circ}$ to $40^{\circ}$ toward the southeast, and accommodated $9.3 \pm 3.1$ miles of crustal shortening. This amount of shortening supports the idea that subsidence of the Anadarko Basin was at least partially related to hanging-wall crustal loading (Brewer and others, 1983). In addition to dip-slip motion along the Wichita fault system, there is considerable evidence based upon offset fold hinges and isochores that left-lateral strike slip motion may also have occurred. McConnell (1989) proposed that as much as 7.5 miles of left-lateral motion occurred along the Mountain View fault (fig. 3), and that this supports the arguments put forth by Kluth and Coney (1981) that intracratonic uplifts related to the Ouachita-Marathon orogeny were caused by reactivated slip on faults bounding basement-cored uplifts.

Geodynamic calculations discussed by Garner and Turcotte (1984) led to the suggestion that formation of the Anadarko Basin can best be modeled in terms of elastic flexure of the lithosphere. Under this scenario, compressional forces related to the Ouachita-Marathon orogeny did not cause basinal subsidence. Rather, Garner and Turcotte (1984) argued that compression was caused by flexure-related subsidence of the Anadarko Basin. Given the large amounts of compression accommodated by the Mountain View fault system, it seems likely that formation of the Anadarko Basin was caused by a combination of thrust-loading and flexurerelated subsidence.

\section{Stratigraphic Overview}

Although a detailed stratigraphic description of the rocks within the Anadarko Basin is beyond the scope of this report, it is important to summarize the key lithologic characteristics of the units shown in the interpretation of the composite seismic line and the structural model (discussed in subsequent sections). A simplified stratigraphic column for the northern Anadarko Basin is shown in figure 4 . This study adopts the commonly used stratigraphic nomenclature established for the northern part of the basin, as more well control was available for the northern parts of the composite seismic line. The Anadarko Basin contains too many stratigraphic units to incorporate all into a regional seismic interpretation and structural model. Therefore, stratigraphic picks on the seismic lines were made for key units based in part on available well control along the seismic lines. The units highlighted in red (fig. 4) were used in the seismic interpretation and structural model. For a more complete stratigraphic section for the Anadarko Basin, see Higley and Gaswirth (2014). Table 1 provides a list of codes for the formation tops identified in the selected wells; the names of some of these tops appear in the following stratigraphic summary. The subsequent paragraphs describe key lithologic features of only the units that are picked on the composite seismic line and incorporated into the structural restoration.

\section{Cambrian and Ordovician Rocks}

The oldest unit identified in the seismic interpretation is the Cambrian-Ordovician Arbuckle Group (fig. 4). It consists of shallow-water marine carbonate mudstones and secondary dolomites that reach thicknesses of $\approx 8,000 \mathrm{ft}$. (Ball and others, 1991), and forms a significant petroleum reservoir with production primarily from porous dolomite zones (Johnson, 1989). The unconformably overlying Middle Ordovician Simpson Group comprises shallow-water marine limestones, sandstones, and shales (Schramm, 1964). The next overlying Viola Group is a cherty, dolomitic limestone (Bornemann and Doveton, 1983), and the uppermost Ordovician unit is the Sylvan Shale, which is fissile dark green to brown silty marine shale that contains thin intervals of dolomitic sandstone (Huffman, 1953).

\section{Silurian, Devonian, and Mississippian Rocks}

Silurian and Lower Devonian rocks of the Anadarko Basin belong to the Hunton Group (fig. 4), which consists of a sequence of limestones and dolomites (Kopaska-Merkel and Friedman, 1989). A significant regional unconformity related to a major sea level low-stand separates the Hunton Group from the overlying Woodford Shale (Kuykendall and Fritz, 1993). The Woodford Shale, which includes the basal informal Misener sand, is one of the Anadarko Basin's major source rocks. The formation is a dark gray to black organic-rich siliceous shale (Kuykendall and Fritz, 1993). Overlying the Woodford Shale are Mississippian carbonates of the Kinderhookian, Osagean, and Meramecian Series. The informal Meramec lime is a thick unit of argillaceous to silty micritic limestone, with higher energy deposits increasing toward the north (Bokman, 1954; Harris, 1975). The Meramec lime is represented in the seismic interpretation by the Goddard Shale (table 1), which is a time equivalent unit (Higley and Gaswirth, 2014) from the southern Anadarko Basin. The youngest Mississippian unit identified in the seismic interpretation is the informal Britt sand (fig. 4), which is part of the Chesterian Series. The Britt sand is predominantly a quartzarenitic to subarkosic sandstone (Andrews and others, 2001). Unconformities within and at the top of the Chesterian Series are products of the onset of the Wichita orogeny. 
Table 1. Lithology codes and corresponding stratigraphic units for formation tops shown in figures 15,16 , and 17.

\begin{tabular}{cl}
\hline Lithology code & \multicolumn{1}{c}{ Corresponding stratigraphic unit (see fig. 4) } \\
\hline RSPG & Rush Springs Formation (upper part of the Whitehorse Group) (Higley and Gaswirth, 2014) \\
WLNG & Wellington Formation \\
CHSE & Chase Group \\
ADMR & Council Grove Group \\
WBNS & Admire Group \\
TOPK & Topeka Limestone \\
TNKW & Tonkawa sand (upper part of the Douglas Group) (Higley and Gaswirth, 2014) \\
CGGV & Cottage Grove Sandstone (lower part of the Lansing Group) \\
HGSR & Hogshooter Limestone \\
CCKB & Checkerboard Limestone \\
MRMN & Marmaton Group \\
RDFK & Taft Sandstone (also called the Red Fork sand) (Higley and Gaswirth, 2014) \\
MRRW & Morrow Formation \\
BRTT & Britt sand \\
GDRD & Goddard Shale (same age as the Meramec lime) (Higley and Gaswirth, 2014) \\
WDFD & Woodford Shale \\
HNTN & Hunton Group \\
SLVN & Sylvan Shale \\
VIOL & Viola Group \\
\hline
\end{tabular}

\section{Pennsylvanian Rocks}

The oldest Pennsylvanian unit identified in the seismic interpretation is the Morrow Formation (fig. 4). It consists mostly of shale deposited during a marine transgression; interbedded sandstones were deposited during brief regressions (Ball and others, 1991). Following the climax of the Wichita orogeny during Atokan time, rocks of the Cherokee Group, including the Taft Sandstone, were deposited. The Taft Sandstone is also known informally as the Red Fork sand (Higley and Gaswirth, 2014; table 1), which comprises mainly shale interbedded with thin limestone and calcareous siltstone (Mannhard and Busch, 1974). The overlying Marmaton Group consists of limestones interbedded with shales and a coarsening-upward sequence of mudstones and sandstones (Rascoe, 1962; Hentz, 1994). Missourian rocks of the Kansas City Group (including the Checkerboard and Hogshooter Limestones) and the Lansing Group (including the Cottage Grove Sandstone) are predominantly dark gray shales interbedded with a few sandstones and thin, dense limestones (Rascoe, 1962). The Douglas Group at the base of the Virgilian Series, which includes the informal Tonkawa sand (Higley and Gaswirth, 2014; table 1), is mostly red and gray sandy shales and thin, dense limestones (Rascoe, 1962). The Topeka Limestone is a massive shelfal carbonate that grades into a sequence of silty shales and thin limestones and sandstones in deeper parts of the Anadarko Basin (Rascoe, 1962). The Wabaunsee Group, which consists of shelfal limestones and calcareous shales in the deep basin (Rascoe, 1962), occupies the top of the Pennsylvanian section.

\section{Permian Rocks}

Rocks of the Wolfcampian Series (fig. 4) are primarily shallow marine limestones and shales. The basal Admire Group and overlying Council Grove Group are made up of massive marine limestones and shales; in the northern part of the basin, there are nonmarine red silty shales and siltstones (Rascoe and Adler, 1983). The Chase Group is mostly interbedded limestones and shales; in western parts of the basin, the group grades abruptly into red silty sandstones and shales (Rascoe, 1962). The only Leonardian unit identified in the seismic interpretation is the Wellington Formation, which consists of interbedded alluvial and deltaic sandstones and shales (Johnson and others, 1988). The youngest unit identified in the seismic interpretation is the Guadalupian Whitehorse Group (fig. 4), which includes the Rush Springs Formation (Higley and Gaswirth, 2014; table 1). The Whitehorse Group consists of red sandstones and thin anhydrites (Johnson and others, 1988). 


\section{D Reflection Seismic Datasets Available to the U.S. Geological Survey}

As part of the 2010 Anadarko Basin petroleum resource assessment, the USGS licensed five seismic lines from Seismic Exchange, Inc., that total approximately 137 line miles (220 km; fig. 2). The lines were acquired by four different companies between 1978 and 1985, using widely varying data acquisition (recording) parameters. The line names, locations where recorded (county names), year purchased by the USGS, and line lengths are given in table 2. Relevant recording parameters for each of the lines are given in table 3. Note that because of the proprietary nature of the seismic data, the lines shown in figure 2 are given general names (Line A, Line $\mathrm{B}$, Line $\mathrm{C}$, Line $\mathrm{D}$, and Line $\mathrm{E}$ ), rather than the original line names provided by Seismic Exchange, Inc.

The data were reprocessed by the USGS between 2008 and 2010 using Halliburton's ProMAX seismic data processing software. Lines B, D, and E were completely reprocessed by the USGS, beginning with the field data, through the point of migrated depth. Line A was reprocessed beginning with the field data through detailed velocity analysis, and the resulting velocity model was used to convert the industry-migrated data

Table 2. Seismic line names, counties and states where seismic lines were recorded, the year the USGS purchased licenses for the data, and the lengths of each line. Seismic data are owned or controlled by Seismic Exchange, Inc.; interpretation is that of the U.S. Geological Survey.

\begin{tabular}{|c|c|c|c|}
\hline Line name & Counties and States where recorded & Year licensed & Line lengths in miles/kilometers \\
\hline Line A & Beckham \& Roger Mills, OK; Wheeler, TX & 2009 & $17.0 / 27.4$ \\
\hline Line B & Woodward, Ellis, \& Dewey, OK & 2007 & $31.2 / 50.2$ \\
\hline Line $\mathrm{C}$ & Kiowa \& Washita, OK & 2009 & $16.8 / 27.0$ \\
\hline Line D & Washita \& Custer, OK & 2007 & $34.7 / 55.8$ \\
\hline Line $\mathrm{E}$ & Blaine, Caddo, Major, \& Washita, OK & 2007 & $36.9 / 59.3$ \\
\hline
\end{tabular}

Table 3. Data acquisition parameters for the five seismic lines used in this study. Seismic data are owned or controlled by Seismic Exchange, Inc.; interpretation is that of the U.S. Geological Survey.

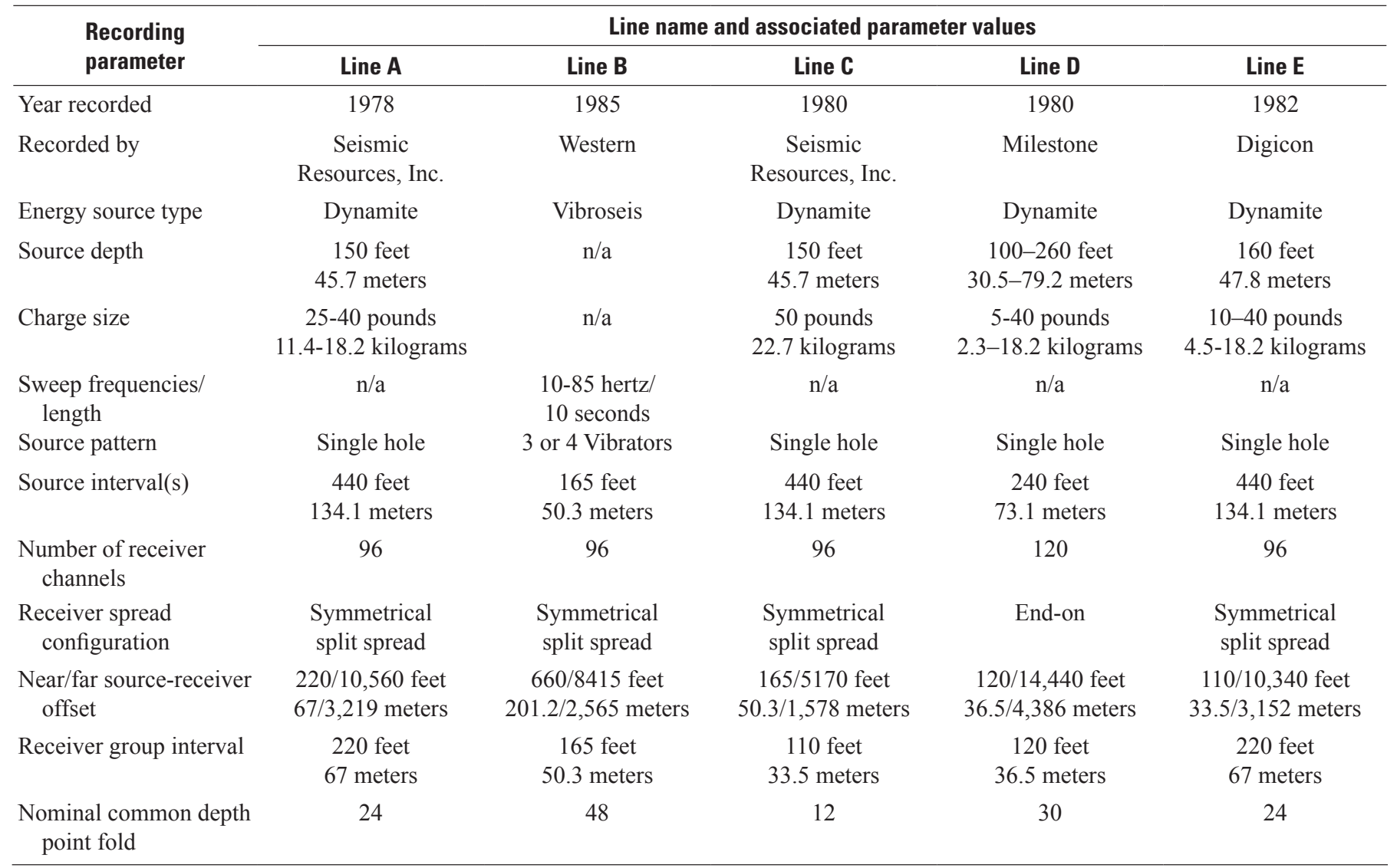


to depth. Line $\mathrm{C}$ was not reprocessed, but the stacking velocities were analyzed, edited, smoothed, and used to convert the industry-migrated data to depth. During reprocessing, the following generalized sequence of processing steps was used.

Before stacking: amplitude scaling, single or multiple window spiking deconvolution, datum statics using smoothed surface elevations, velocity analysis [root-mean-square (RMS) velocities determined directly from the seismic data], surfaceconsistent residual statics, a second velocity analysis, second pass of residual statics if necessary, normal moveout correction using the stacking velocities, and common depth point (CDP) stacking.

After stacking: shift both the seismic data and the velocity fields to a horizontal datum, automatic gain control scaling, bandpass filter, post-stack time migration using a smoothed version of the stacking velocity field, and depth conversion using the same smoothed velocity field. With the exception of Line $\mathrm{C}$ as explained below, the stacking velocities were determined directly from the seismic data using conventional velocity analysis applied at points along the lines where there was good signal-to-noise ratio. Two passes of velocity analyses were performed, one before and one after residual statics analysis. Velocity functions were picked manually from the interactive onscreen analysis display, except for the second pass on Line D, where an automated velocity picking routine was used. This method used the manually picked velocities from the first pass as a guide function. Velocity functions were picked at a 25-CDP interval, and were constrained to -5 percent to +10 percent about the guide function at the surface, and $\pm 10 \%$ about the guide function at 2,500 milliseconds (ms). This method was successful in producing a detailed velocity field for stacking. However, the stacked image produced using the automated velocity picks was only minimally improved over that of the manually picked velocity model. This velocity model was smoothed (201 CDPs horizontal; $250 \mathrm{~ms}$ vertical smoothing operator), and was used for migration and depth conversion (fig. 8). For Line $\mathrm{C}$, stacking velocities were provided with the industrymigrated data. These velocities were analyzed and edited to ensure that the interval velocities were geologically reasonable, and then a smoothed version of those velocities was used for migration and depth conversion.

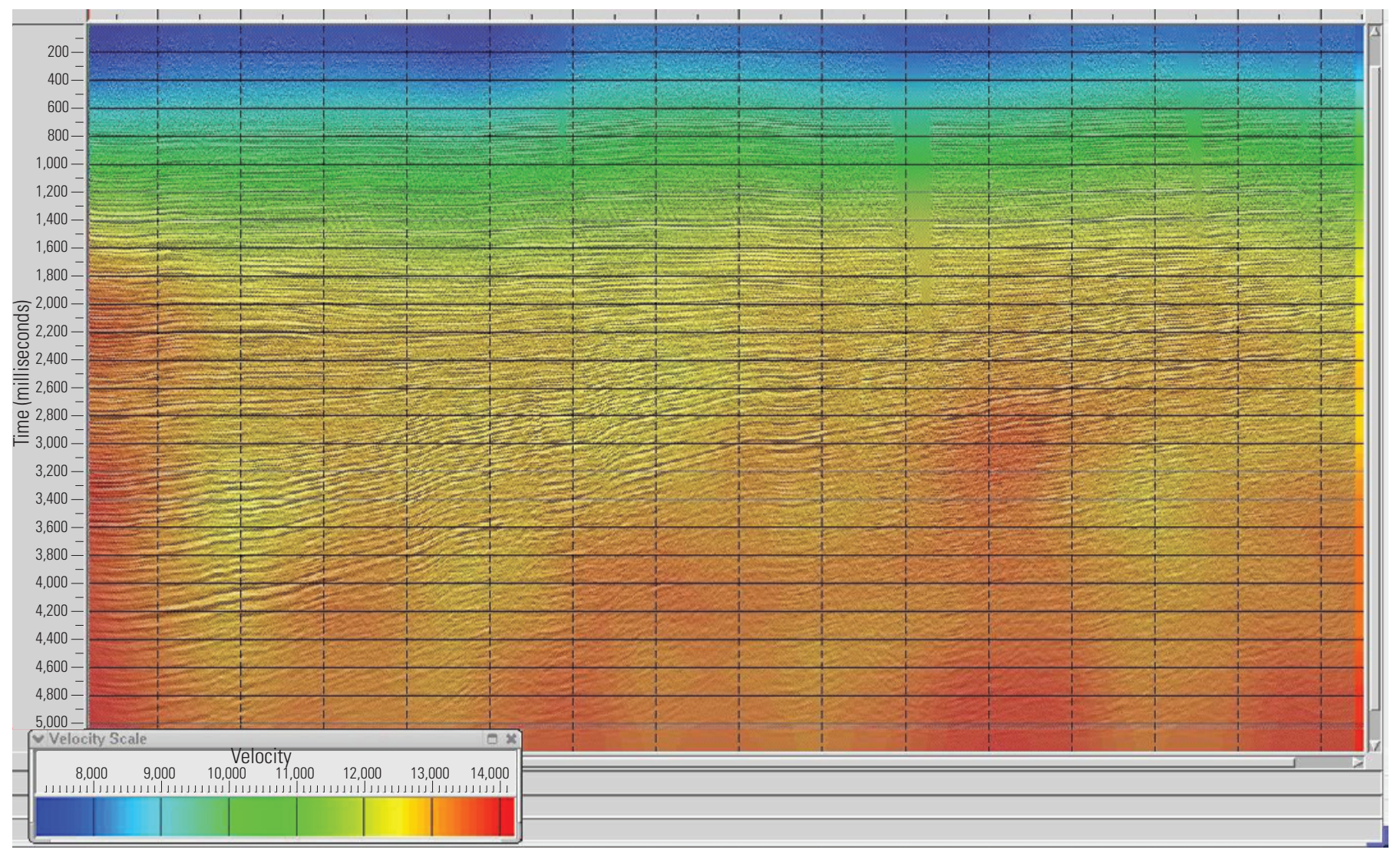

Figure 8. Root mean square velocity model used to migrate and convert seismic Line D. Velocities were determined using an automated velocity picking algorithm, and subsequently smoothed using a 201 common depth point horizontal, and 250-millisecond vertical, smoothing operator. A color bar illustrating the velocity scale is also shown. Seismic data are owned or controlled by Seismic Exchange, Inc.; interpretation is that of the U.S. Geological Survey. 


\section{D Reflection Seismic Data Interpretation}

\section{General Description of the Seismic Lines}

Although the seismic lines were all acquired almost 30 years ago, key stratigraphic and structural features of the Anadarko Basin can be seen in the images. Figures 9-13 show uninterpreted depth sections for Line A, Line B, Line C, Line $\mathrm{D}$, and Line E, respectively. All lines are shown at the same scale, with the vertical (depth) and horizontal (miles along the surface) axes equal. All lines are also shown with identical red to black color bars, where red colors represent amplitude troughs and black colors represent amplitude peaks. A brief qualitative description is given in the following paragraphs for each of the seismic lines.

Line A (fig. 9) crosses the southern boundary of the Anadarko Basin onto the Wichita uplift (fig. 3). Noncontinuous reflectors with apparent northeast dips can be seen at shallow depths along the southwestern portion of the line. The lack of continuous reflectors at the line's southwestern most edge likely shows the presence of Precambrian basement rocks of the Wichita uplift in the hanging-wall of the Mountain View fault system. The subparallel reflectors that reach depths of $\approx 30,000 \mathrm{ft}$ and extend across the northeastern two-thirds of the line are caused by Cambrian-Permian rocks of the Anadarko Basin. The axis of the basin lies between about three to five miles from the southwest edge of the seismic line, depending upon the stratigraphic level.

Line B (fig. 10) shows subparallel, largely continuous reflectors with apparent gentle southwest dips. It is possible to trace many of the shallow reflectors (above 15,000 ft at the southwest edge of the line) along the entire 31.2-mile length of the seismic line. The southwestern expansion of the Cambrian-Permian stratigraphic section can be clearly seen. For example, the prominent reflectors at depths of approximately $2,500 \mathrm{ft}$ and $7,500 \mathrm{ft}$ at the northeastern edge of the line reach depths of approximately $3,500 \mathrm{ft}$ and $12,000 \mathrm{ft}$ at the southwestern edge of the line.

Line C (fig. 11) is similar in many ways to Line A, in that it spans the boundary between the Anadarko Basin and the Wichita uplift. Although the basin-bounding thrust faults are not directly imaged, it is possible to infer the presence of at least two faults. The Mountain View fault system likely exists at the southwest edge of the line, and is responsible for elevating basement rock characterized by non-continuous reflectors. The obvious monoclinal flexure in the middle of the line may be caused by the Cordell fault (fig. 3). Although the southwestern third of the line is poorly imaged, the fold geometry is similar to that described for many Laramide basement-cored uplifts (Erslev, 1991; Brandenburg and others, 2012). Shallow

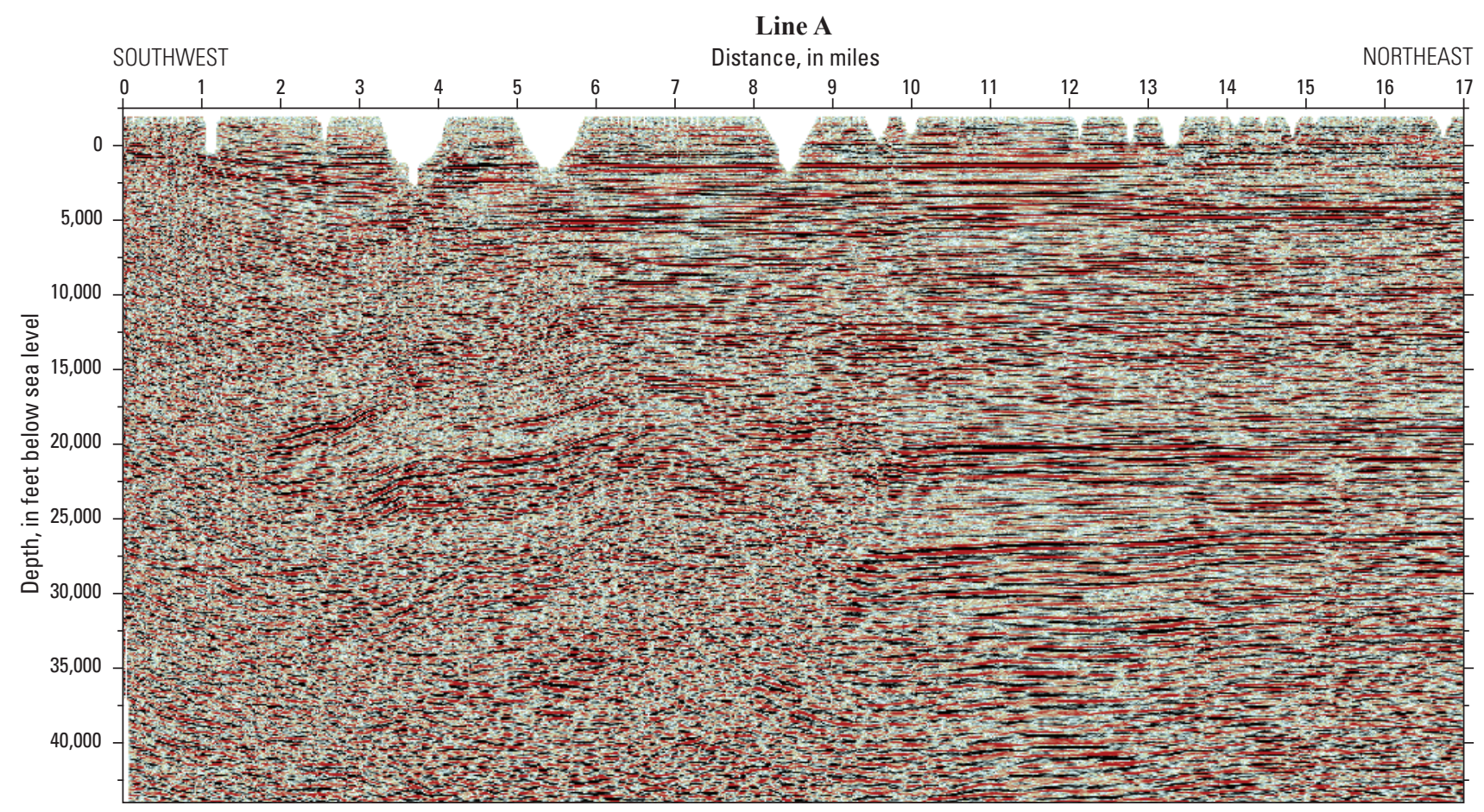

Figure 9. Uninterpreted seismic Line A. Location of line is shown in figure 2. Horizontal and vertical scales are equal. Black colors are amplitude peaks; red colors are amplitude troughs. Seismic data are owned or controlled by Seismic Exchange, Inc. 


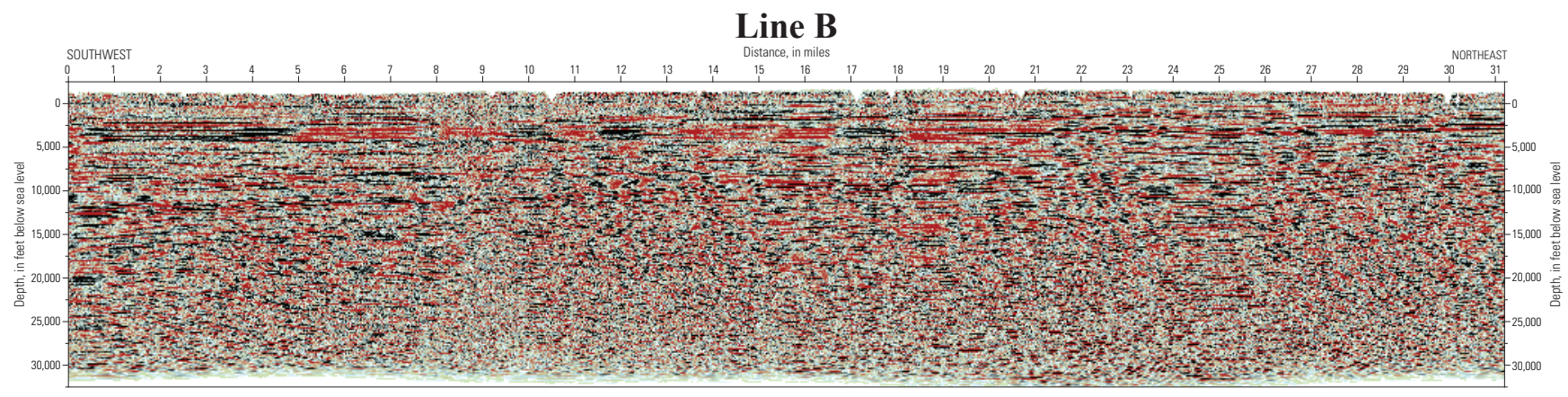

Figure 10. Uninterpreted seismic Line B. Location of line is shown in figure 2. Horizontal and vertical scales are equal. Dark black colors are amplitude peaks; dark red colors are amplitude troughs. Seismic data are owned or controlled by Seismic Exchange, Inc. Figure 10 is oversized. Click on the thumbnail to view the enlarged version.

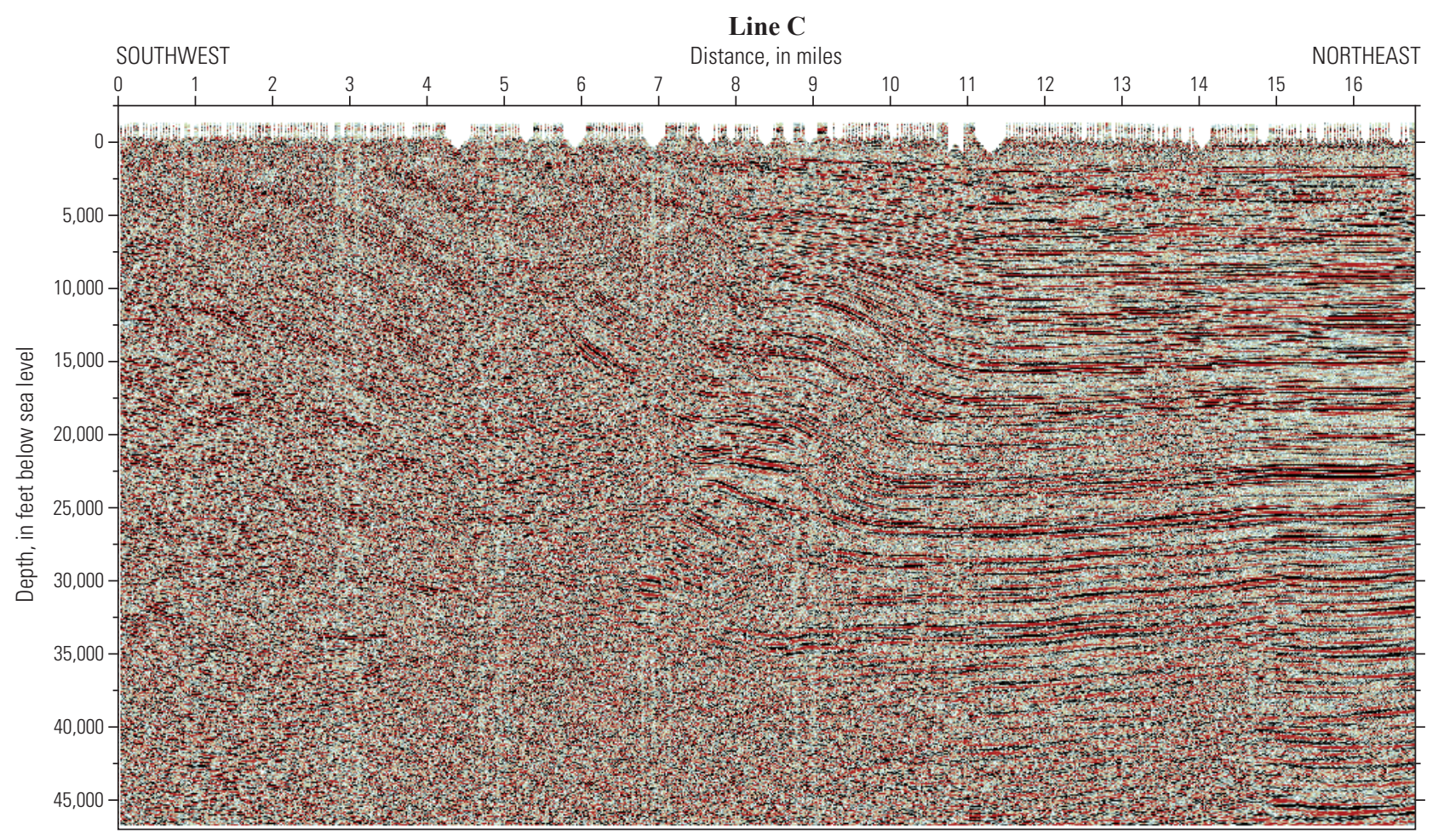

Figure 11. Uninterpreted seismic Line C. Location of line is shown in figure 2. Horizontal and vertical scales are equal. Dark black colors are amplitude peaks; dark red colors are amplitude troughs. Seismic data are owned or controlled by Seismic Exchange, Inc. 


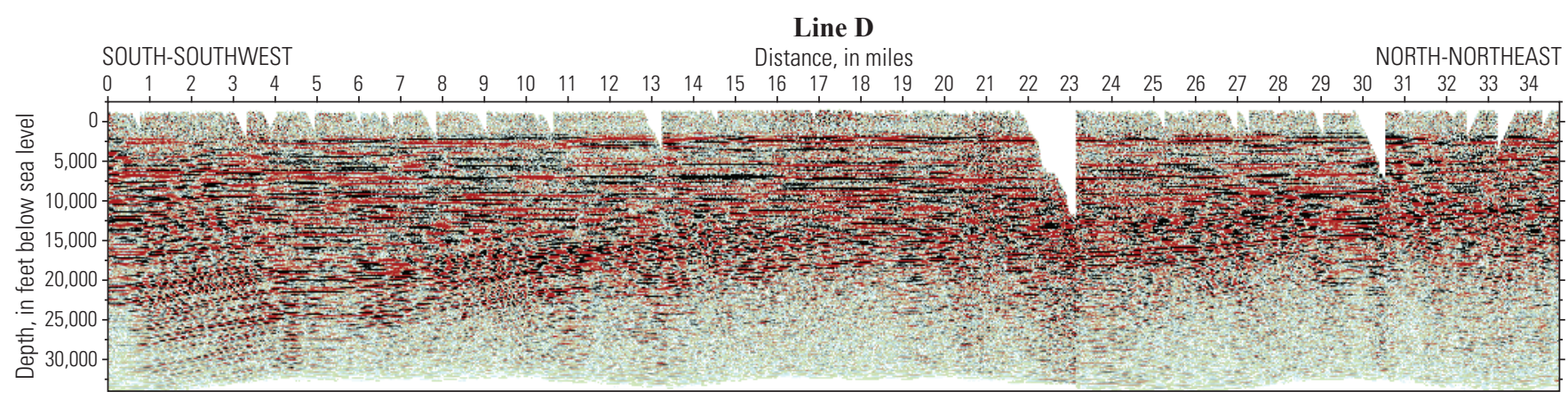

Figure 12. Uninterpreted seismic Line D. Location of line is shown in figure 2. Horizontal and vertical scales are equal. Dark black colors are amplitude peaks; dark red colors are amplitude troughs. Seismic data are owned or controlled by Seismic Exchange, Inc. Figure 12 is oversized. Click on the thumbnail to view the enlarged version.

\section{Line $\mathbf{E}$}

SOUTHWEST

Distance, in miles

NORTHEAST

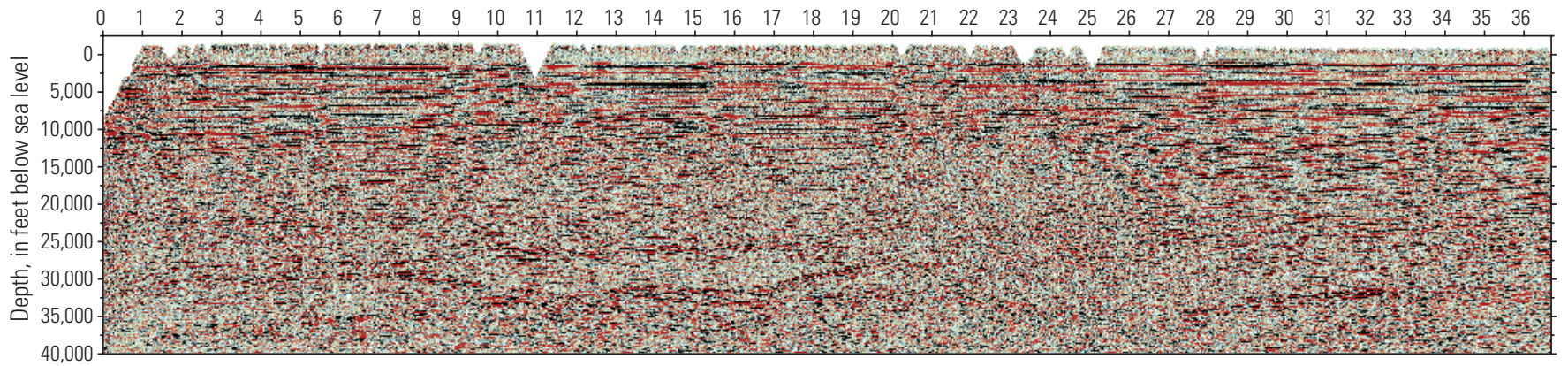

Figure 13. Uninterpreted seismic Line E. Location of line is shown in figure 2. Horizontal and vertical scales are equal. Dark black colors are amplitude peaks; dark red colors are amplitude troughs. Seismic data are owned or controlled by Seismic Exchange, Inc. Figure 13 is oversized. Click on the thumbnail to view the enlarged version. 
reflectors that have apparent dips toward the northeast along the southwestern third of the line are generally poorly imaged and are difficult to track. However, stratigraphic on-lap and angular truncation of reflectors can be seen at depths above $5,000 \mathrm{ft}$ from six to eight miles from the southwest edge of the seismic line. Depending upon the stratigraphic level, the axis of the basin is between approximately 8 and 12 miles from the southwestern edge of the seismic line. Northeast of the basin's axis, subparallel reflectors made by Cambrian-Permian rocks are well-imaged and are easy to track.

Line D (fig. 12) is characterized by well-imaged, subparallel reflectors with apparent south-southwest dips. As with Line $\mathrm{B}$, it is possible to track numerous reflectors across the entire 34.7-mile-long seismic line. At the resolution and scale of the image, reflectors (those above approximately $27,500 \mathrm{ft}$ at the south-southwest edge of the image) do not appear to be offset by faults. The south to southwestern expansion of the Cambrian-Permian section is well-imaged by this line;

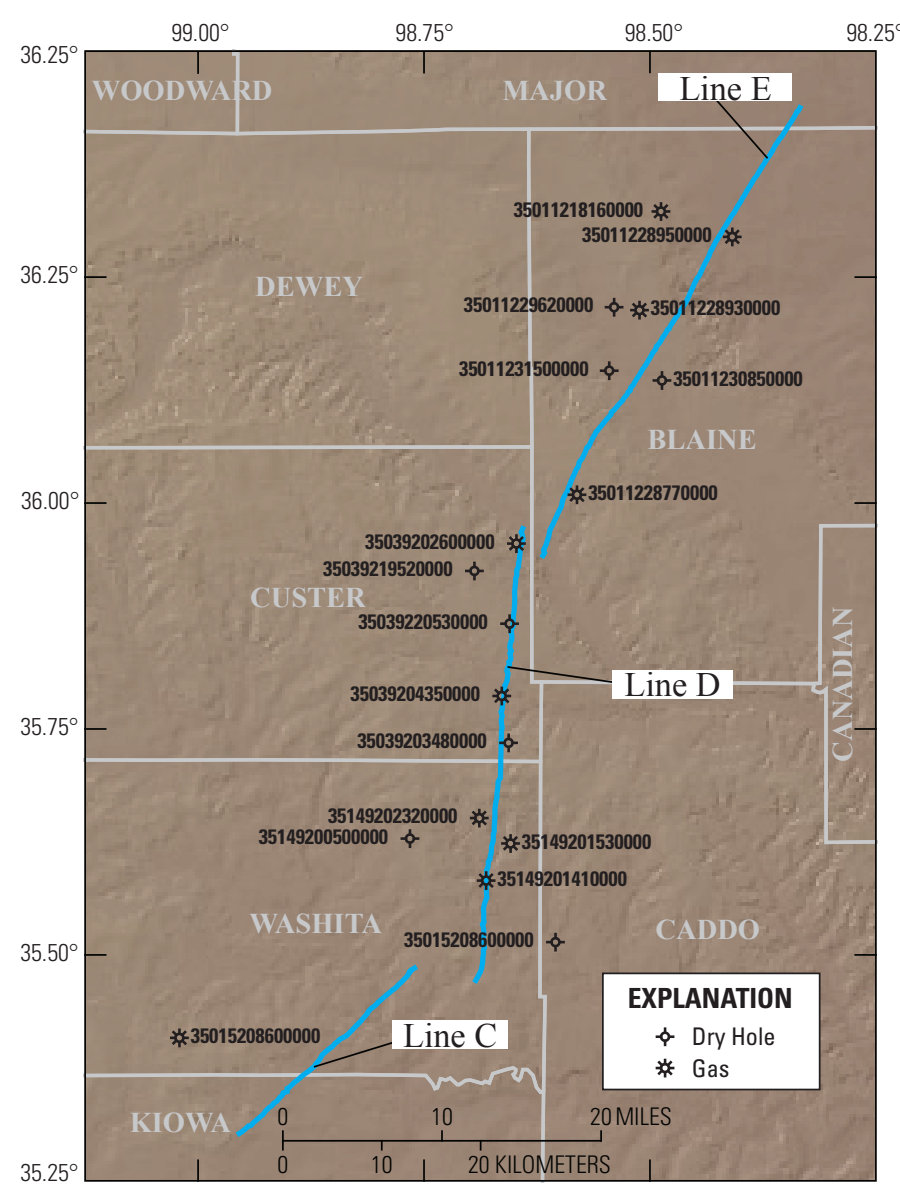

Figure 14. Map showing the location of wells used to aid in the interpretation of the composite seismic line. Oklahoma counties are shown in gray. The segments of the composite seismic line are shown in blue. American Petroleum Institute well numbers are given. reflectors at depths of approximately $12,500 \mathrm{ft}$ at the northnortheast edge of the line reach depths of approximately $27,500 \mathrm{ft}$ at the south-southwest edge of the line.

Line E (fig. 13) contains a well-imaged section down to approximately $15,000 \mathrm{ft}$ depth at the southwest edge of the line and 10,000 ft at the northeast edge. As with previously described lines, it is possible to trace numerous individual reflectors across the entire 36.9-mile length of the seismic image without encountering any offsets because of faulting. Reflectors have gentle apparent dips toward the southwest and show the thickening of the Cambrian-Permian section toward the basin's axis. Layered intrusives possibly related to the southern Oklahoma aulacogen may be visible between depths of 30,000 and $35,000 \mathrm{ft}$ along the southwestern half of the seismic line.

Because of their proximity to each other, Lines C, D, and $\mathrm{E}$ were selected for a more detailed seismic interpretation. These three lines are henceforth collectively referred to as the Anadarko "composite seismic line." Interpretation of the composite seismic line was guided by formation tops information from 18 selected wells (fig. 14) that were projected orthogonally onto the line of section from distances of up to 7.5 miles off the composite seismic line. These wells are a subset of more than 220 wells across the Anadarko Basin Province that contain edited formation tops. A detailed discussion of the numerous data sources and the methodology behind picking these formation tops is given in Higley and others (2014) and references therein.

\section{Seismic Interpretation of the Anadarko Composite Seismic Line}

Interpretations for Line $\mathrm{C}$, Line $\mathrm{D}$, and Line $\mathrm{E}$ are shown in figures 15,16 , and 17 , respectively. The lines are all shown at the same scale, and the vertical (depth) and horizontal (distance along the surface) axes are identical. The projected location of the wells and relevant formation tops (table 1) are also shown.

Interpretation of Lines $\mathrm{D}$ and $\mathrm{E}$ is facilitated by the ease with which numerous individual reflectors can be traced across the entire length of the lines. No faults were interpreted to be present on these two lines; however, if present, they were either not imaged by the seismic data or are of a subseismic scale. Formation tops from the 17 wells projected onto these two seismic lines permits identification of 20 horizons; the only two horizons for which well control is not available are the Simpson and Arbuckle Groups. On Line E (fig. 17), the top of the Simpson Group was placed at the next prominent reflector below the top of the Viola Group in well 35011230850000 , which gives an apparent thickness for the Viola Group at that location of $\approx 460 \mathrm{ft}$. The top of the Arbuckle Group was placed at the next prominent reflector below the top of the Simpson Group at the same location, which yields an apparent thickness for the Simpson Group of $\approx 750 \mathrm{ft}$. Although these two 
horizons probably do not represent the actual tops of the Simpson and Arbuckle Groups, they are likely to be close. Evidence in support of this comes from the combined thickness of the Viola and Simpson Groups at the southwestern edge of the interpretation on Line C. Johnson and others (1988) suggested that the maximum combined thickness of the two groups in southern Oklahoma is in excess of $\approx 2,500 \mathrm{ft}$; the maximum thickness of the two groups at the southwestern edge of the interpretation is $\approx 2,800 \mathrm{ft}$.

Interpretation was only done for the northeastern half of Line C (fig. 15), because of the poor quality of the seismic data combined with a lack of well control and the complex geometries of the frontal portion of the Wichita uplift in the southwestern part. McConnell (1989) provided examples of complex compressional geometries at the northern edge of the Wichita uplift. Although locations for thrust faults of the Mountain View fault system and the Cordell fault (fig. 3) could have been hypothesized, available data do not permit determination of the hanging-wall and footwall cutoff relations, and thus constrain either the magnitude or timing of displacement along the faults.

\section{Structural Restoration of the Anadarko Composite Seismic Line}

A structural restoration of the composite seismic line was built using Midland Valley's 2DMove software. The restoration shown in figure 18 details 22 stages corresponding to each of the interpreted stratigraphic units (fig. 4) described in the preceding sections. As with all other figures that show the seismic lines and interpretations, the horizontal and vertical scales in each panel of figure 18 are identical.

The restoration was pinned at the northeast edge of the composite seismic line, and all horizons were restored to a horizontal surface at an elevation of the present sea level. The primary restoration algorithm used within 2DMove was "Flexural Slip Unfolding." The flexural slip algorithm was used for the following reasons: (1) it maintains the line length of the template horizon (the template horizon is the horizon that is being restored to the horizontal surface) in the direction of unfolding; (2) it maintains the orthogonal bed thickness between the template horizon and all other passive horizons

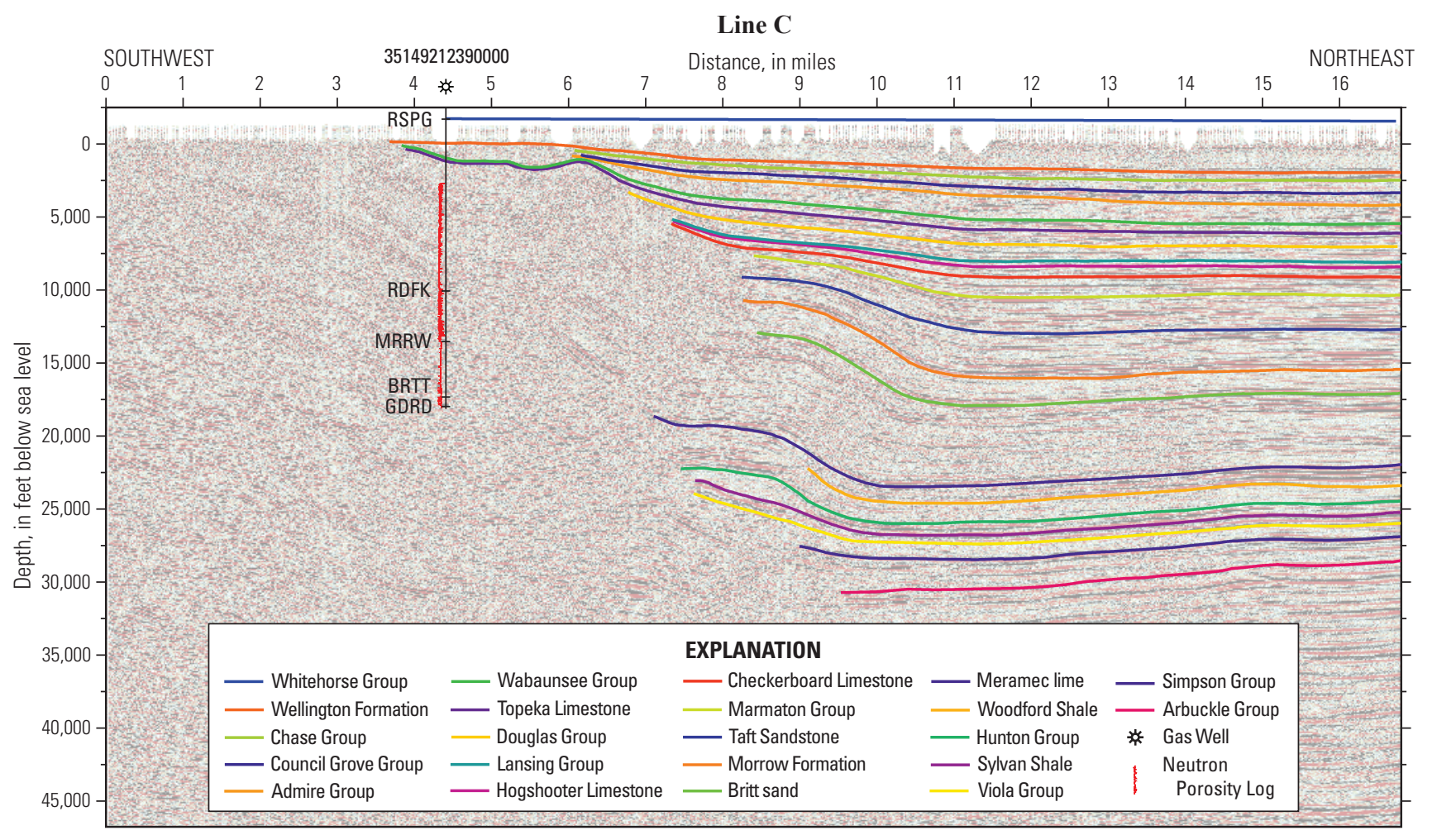

Figure 15. Interpreted seismic Line C. Location of line is shown in figure 2. Horizontal and vertical scales are equal. Dark black colors are amplitude peaks; dark red colors are amplitude troughs. Well symbols are the same as those used in fig. 14. American Petroleum Institute well numbers are indicated. Lithology codes for well tops are given in table 1. Seismic data are owned or controlled by Seismic Exchange, Inc.; interpretation is that of the U.S. Geological Survey. 


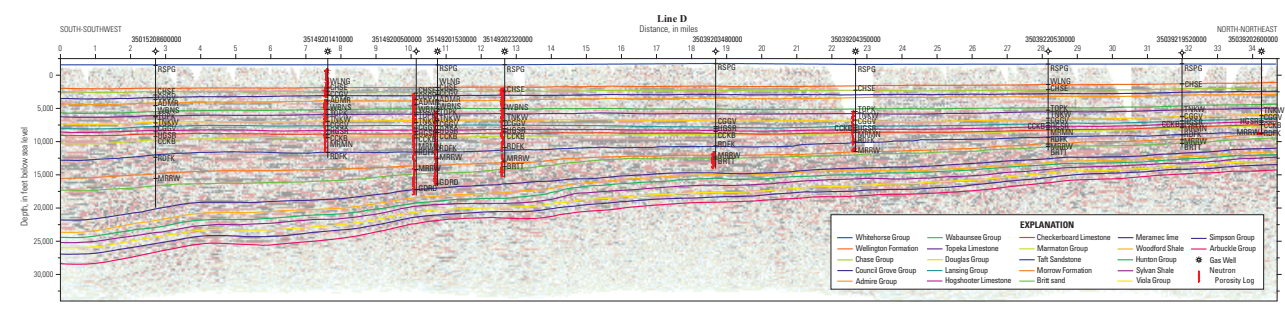

Figure 16. Interpreted seismic Line D. Location of line is shown in figure 2. Horizontal and vertical scales are equal. Dark black colors are amplitude peaks; dark red colors are amplitude troughs. Well symbols are the same as those used in fig. 14. American Petroleum Institute well numbers are indicated. Lithology codes for well tops are given in table 1. Seismic data are owned or controlled by Seismic Exchange, Inc.; interpretation is that of the U.S. Geological Survey. Figure 16 is oversized. Click on the thumbnail to view the enlarged version.

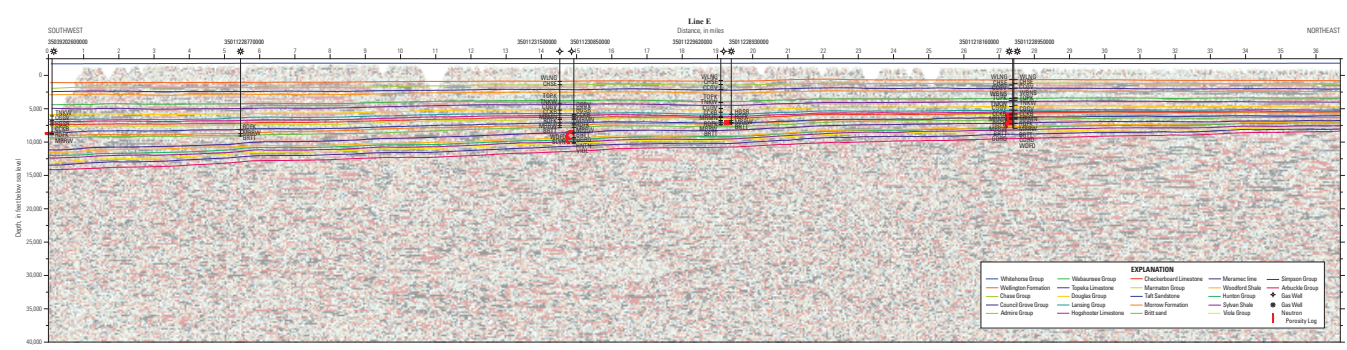

Figure 17. Interpreted seismic Line E. Location of line is shown in figure 2. Horizontal and vertical scales are equal. Dark black colors are amplitude peaks; dark red colors are amplitude troughs. Well symbols are the same as those used in figure 14. American Petroleum Institute well numbers are indicated. Lithology codes for well tops are given in table 1. Seismic data are owned or controlled by Seismic Exchange, Inc.; interpretation is that of the U.S. Geological Survey. Figure 17 is oversized. Click on the thumbnail to view the enlarged version.

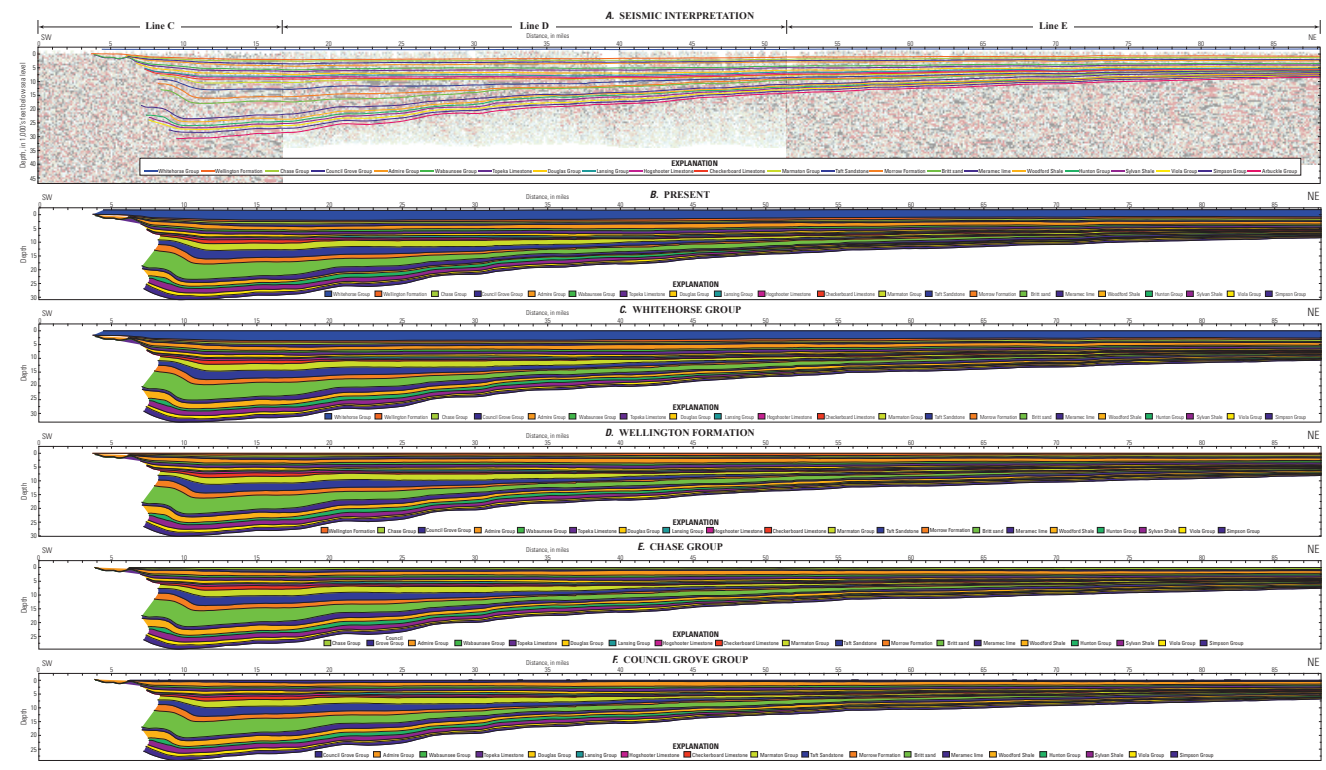

Figure 18. Structural restoration showing individual restoration stages. Depth in each stage is shown in thousands of feet below sea level; horizontal distance is given in miles from the southwestern edge of seismic Line $C$ (no vertical exaggeration). Seismic data are owned or controlled by Seismic Exchange, Inc.; interpretation is that of the U.S. Geological Survey. Figure 18 is oversized. Click on the thumbnail to view the enlarged version. 


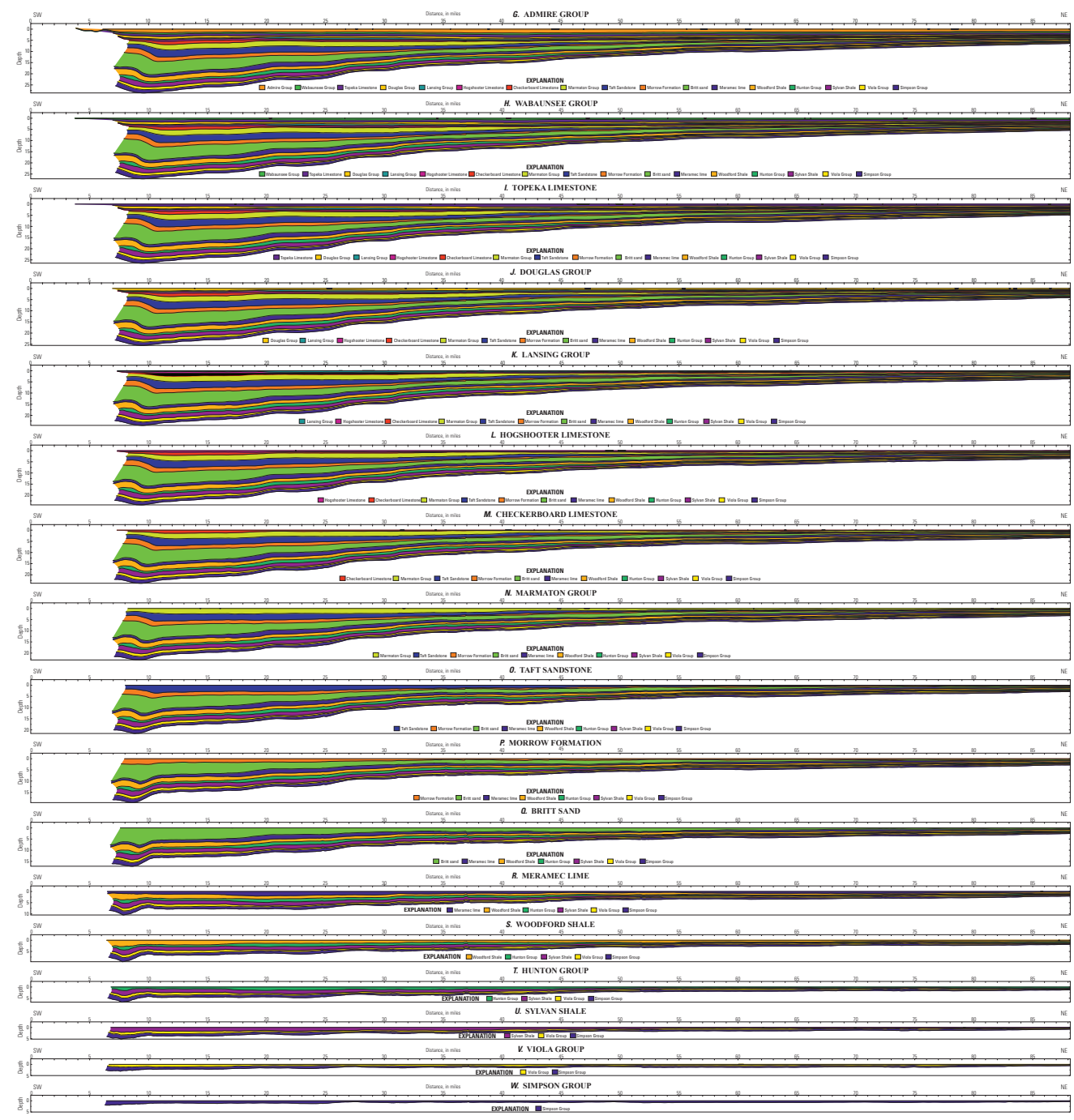

Figure 18. Structural restoration showing individual restoration stages. Depth in each stage is shown in thousands of feet below sea level; horizontal distance is given in miles from the southwestern edge of seismic Line $C$ (no vertical exaggeration). Seismic data are owned or controlled by Seismic Exchange, Inc.; interpretation is that of the U.S. Geological Survey. Figure 18 is oversized. Click on the thumbnail to view the enlarged version.-Continued 


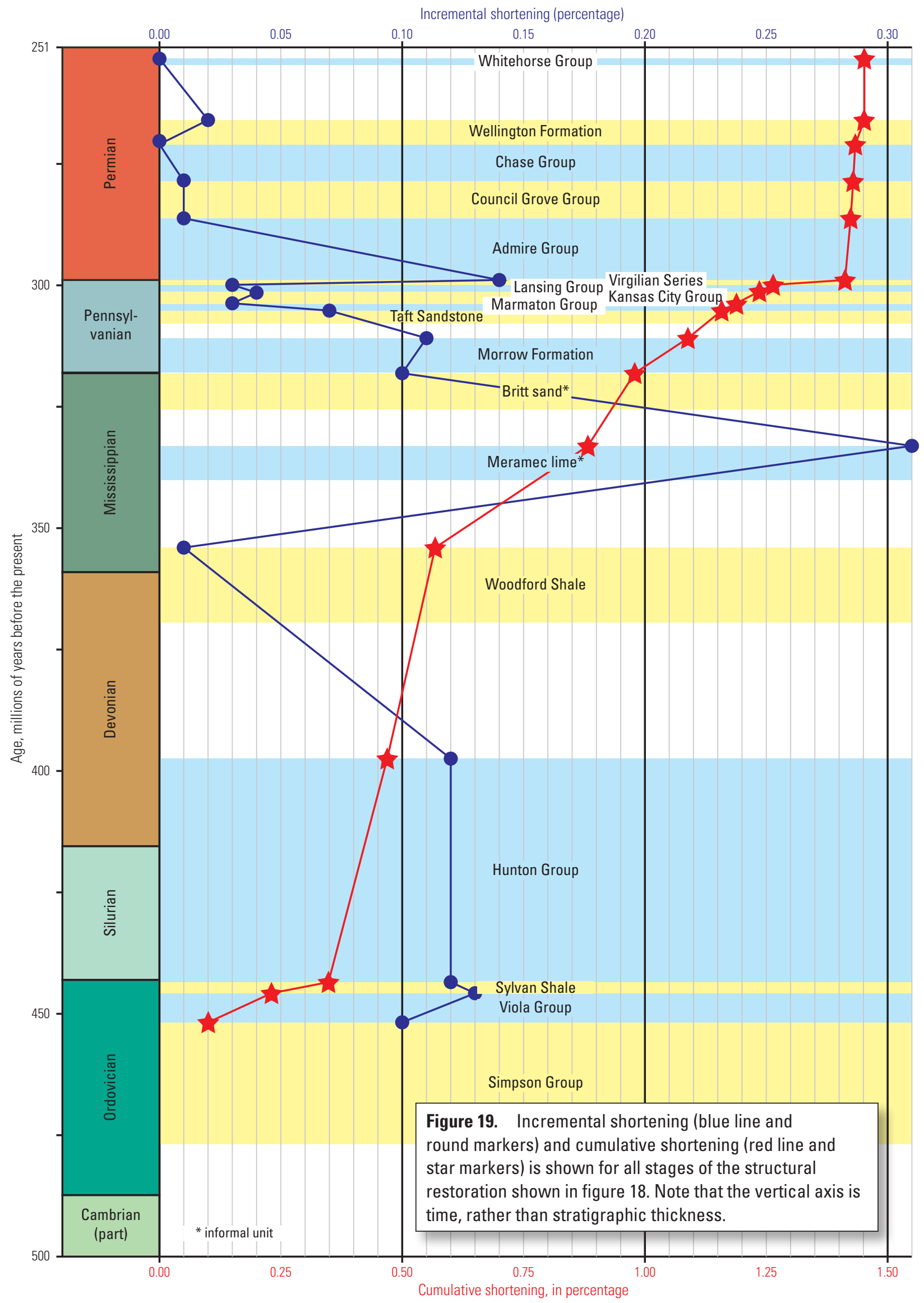


(passive horizons are the horizons below the template horizon); and (3) it maintains area of the fold and model. Additionally, the flexural slip algorithm is an appropriate choice inasmuch as the composite seismic line is roughly orthogonal to the northern flank of the Wichita uplift; the dominant stresses responsible for the creation of the Wichita uplift and the Anadarko Basin were therefore roughly aligned with the northeast-southwest orientation of the composite seismic line.

Each stage of the restoration (fig. $18 B-W$ ) shows the 2D geometry that may have existed at the end of deposition of each interpreted lithologic unit. The restoration shows that in deeper parts of the basin, between approximately 7 and 45 miles from the southwest end of the model, subtle structural relief developed along most of the horizons soon after the deposition of individual units. For example, a broad fold had developed in the Meramec lime about 20 miles from the southwestern end of the model (fig. $18 Q$ and $R$ ) by the end of deposition of the Britt sand. Some of the shorter wavelength features, such as the narrow fold at about 37 miles from the southwest end of the model (fig. $18 Q-W$ ), are likely artifacts of the restoration.

As previously mentioned, the restoration is pinned at the northeast edge of the composite seismic line. Relative to the margin of the Ouachita orogeny, this location is within the stable North American craton. Progressive shortening of the restoration can therefore be seen by individual horizons lengthening in a southwesterly direction, in reverse chronological order. During the time represented by the deposition of the Simpson Group through the Whitehorse Group, the cumulative amount of shortening along the cross-section is 1.45 percent. Although this is not a large amount of shortening, it is important to recognize that it took place entirely within the footwalls of the thrust faults that separate the Wichita uplift and the Anadarko Basin. McConnell (1989) estimated that approximately 6.7 miles of reverse-slip occurred on these frontal faults. The magnitude of Ordovician through Permian shortening is shown in figure 19, which displays both the incremental (blue line and round markers) and cumulative (red line and star markers) shortening percentages through time. For example, by the end of deposition of the Viola Group, the underlying Simpson Group rocks had been shortened by 0.10 percent; by the end of deposition of the Britt sand, the underlying Meramec lime had been shortened by 0.31 percent. The cumulative shortening line reveals that most of the shortening within the basin was during Mississippian and Pennsylvanian time. Since deposition of the Virgilian Series, almost no shortening has occurred. Significant tectonic shortening during the Permian is absent, so accommodation space for Permian rocks was likely created by isostatic subsidence of the basin. Calculation of the relatively minor amount of shortening experienced by rocks within the Anadarko Basin to the north of the Mountain View fault system supports the interpretation (figs. 15, 16, and 17) that no faults are present along the seismic lines, at least none that is visible at the scale of the seismic data available for this study. The key point is that shortening within the Anadarko Basin was primarily accommodated by minor folding, rather than faulting.

\section{Summary}

The structural evolution of the Anadarko Basin Province was affected by tectonic processes associated with two complete Wilson cycles that affected the eastern margin of North America. The first Wilson cycle, which involved the creation and subsequent breakup of the Rodinian supercontinent, accounts for the consolidation of basement rock beneath the Anadarko Basin Province and the emplacement of the southern Oklahoma aulacogen. The second Wilson cycle, which involves the formation and breakup of Pangaea, controlled the depositional environments of the Anadarko Basin's more than 40,000 feet of Cambrian through Permian sediments and the geometry of the basin's current geometry. The geometry of each of the major structural elements was partially inherited from prior tectonic and structural events.

The U.S. Geological Survey licensed and analyzed five two-dimensional reflection seismic lines within the Anadarko Basin. Three of the lines have endpoints that approximately overlap; these were combined into a regional composite seismic line that extends almost 90 miles in a northeast-southwest direction across the basin. A detailed seismic interpretation, based in part on well data, shows relatively undeformed Cambrian through Permian strata that dip toward the southwest into the trough of the Anadarko Basin. The Wichita Mountains structural high and disrupted strata associated with the Wichita fault system can also be seen on the southeastern edge of the composite seismic line. A structural restoration shows the sequential burial of 22 key stratigraphic horizons. At the scale of the available seismic data, there is little visible structural deformation within the Anadarko Basin northeast (in the footwall) of the Wichita fault system.

\section{Acknowledgments}

We would like to thank Kristen Marra, Sarah Hawkins, and Dick Keefer for constructive technical reviews of this paper. We also thank Seismic Exchange, Inc., for giving permission to publish the seismic data. Members of the USGS Anadarko Basin assessment team, in particular Debra Higley and Stephanie Gaswirth, provided insightful discussions about the Anadarko Basin, and were a valuable aid in our study.

\section{References Cited}

Andrews, R.D., Hendrickson, W.J., and Smith, P.W., 2001, Regional overview of the Springer gas play, Springer gas play in western Oklahoma: Oklahoma Geological Survey Special Publication 2001-1, p. 1-26.

Ball, M.M., Henry, M.E., and Frezon, S.E., 1991, Petroleum geology of the Anadarko Basin region, Province (115), Kansas, Oklahoma, and Texas: U.S. Geological Survey OpenFile Report 88-450W, 36 p. 
Blakey, R.C., 2011, North American paleogeographic maps, Middle Cambrian (510 Ma): accessed May 4, 2012, at http://cpgeosystems.com/namC510.jpg.

Bokman, J., 1954, Relative abundance of common sediments in Anadarko Basin of Oklahoma: American Association of Petroleum Geologists Bulletin, v. 38, p. 648-654.

Bornemann, E., and Doveton, J.H., 1983, Lithofacies mapping of Viola Limestone in south-central Kansas, based on wireline logs: American Association of Petroleum Geologists Bulletin, v. 67, p. 609-623.

Brandenburg, J.P., Alpak, F.O, Solum, J.G., and Naruk, S.J., 2012, A kinematic trishear model to predict deformation bands in a fault-propagation fold, East Kaibab monocline, Utah: American Association of Petroleum Geologists Bulletin, v. 96, p. 109-132.

Brewer, J.A., Good, R., Oliver, J.E., Brown, L.D., and Kaufman, L., 1983, COCORP profiling across the southern Oklahoma aulacogen: Overthrusting of the Wichita Mountains and compression within the Anadarko Basin: Geology, v. 11, p. 109-114.

Cardott, B.J., and Lambert, M.W., 1985, Thermal maturation by vitrinite reflectance of Woodford Shale, Anadarko Basin, Oklahoma: American Association of Petroleum Geologists Bulletin, v. 69, p. 1982-1998.

Erslev, E., 1991, Trishear fault-propagation folding: Geology, v. 19 , p. $617-620$.

Feinstein, S., 1981 Subsidence and thermal history of southern Oklahoma aulacogen: Implications for petroleum exploration: American Association of Petroleum Geologists Bulletin, v. 65, p. 2521-2533.

Garner, D.L, and Turcotte, D.L., 1984, The thermal and mechanical evolution of the Anadarko Basin: Tectonophysics, v. 107, p. 1-24.

Gilbert, M.C., 1982, Geologic setting of the eastern Wichita Mountains with a brief discussion of unresolved problems, in Gilbert, M.C. and Donovan, R.N., eds., Geology of the eastern Wichita Mountains: Oklahoma Geological Survey Guidebook 21, p. 1-30.

Gilbert, M.C., 1983, Timing and chemistry of igneous events associated with the southern Oklahoma aulacogen, in Morgan, P., and Baker, B.H., eds., Processes of continental rifting: Tectonophysics, v. 94, p. 439-455.

Gilbert, M.C., 1987, Petrographic and structural evidence from the igneous suite in the Wichita Mountains bearing on the Cambrian tectonic style of the southern Oklahoma aulacogen: Geological Society of America Abstracts with Programs, v. 19, p. 152.

Ham, W.E., 1973, Regional geology of the Arbuckle Mountains, Oklahoma: Oklahoma Geological Survey Special Publication 73-3, $56 \mathrm{p}$.
Harlton, B.H., 1963, Frontal Wichita fault system of southwestern Oklahoma: American Association of Petroleum Geologists Bulletin, v. 47, p. 1552-1580.

Harris, S.A., 1975, Hydrocarbon accumulation in "MeramecOsage" (Mississippian) rocks, Sooner trend, northwestcentral Oklahoma: American Association of Petroleum Geologists Bulletin, v. 59, p. 633-664.

Hentz, T.F., 1994, Sequence stratigraphy of the upper Pennsylvanian Cleveland Formation: A major tight-gas sandstone, western Anadarko Basin, Texas Panhandle: American Association of Petroleum Geologists Bulletin, v. 78, p. 569-595.

Higley, D.K. and Gaswirth, S.B., 2014, Overview of chapters within USGS DDS-69-EE, chap. 2, in Higley, D.K., comp., Petroleum systems and assessment of undiscovered oil and gas in the Anadarko Basin Province, Colorado, Kansas, Oklahoma, and Texas_-USGS Province 58: U.S. Geological Survey Digital Data Series DDS-69-EE, 5 p.

Higley, D.K., Gaswirth, S.B., Abbott, M.M., Charpentier, R.R., Cook, T.A., Ellis, G.S., Gianoutsos, N.J., Hatch, J.R., Klett, T.R., Nelson, P., Pawlewicz, M.J., Pearson, O.N., Pollastro, R.M., and Schenk, C.J., 2011, Assessment of undiscovered oil and gas resources of the Anadarko Basin Province of Oklahoma, Kansas, Texas, and Colorado, 2010: U.S. Geological Survey Fact Sheet 2011-3003, 2 p.

Higley, D.K., Gianoutsos, N.J., Pantea, M.P., and Strickland, S.M., 2014, Precambrian to ground surface grid cell maps of the Anadarko Basin Province, chap. 13, in Higley, D.K., compiler, Petroleum systems and assessment of undiscovered oil and gas in the Anadarko Basin Province, Colorado, Kansas, Oklahoma, and Texas-USGS Province 58: U.S. Geological Survey Digital Data Series DDS-69-EE, 7 p.

Huffman, G.G., 1953, Sylvan Shale in northeastern Oklahoma: Geological Notes, American Association of Petroleum Geologists Bulletin, v. 37, p. 447-450.

IHS Energy, 2010, IHS energy production data on CD-ROM: Unpublished database available from IHS Energy, 15 Inverness Way East, Englewood, CO 80112.

Johnson, K.S., 1989, Geologic evolution of the Anadarko Basin, in Johnson, K.S., ed., Anadarko Basin symposium, 1988: Oklahoma Geological Survey Circular 90, p. 3-12.

Johnson, K.S., Amsden, T.W., Denison, R.E., Dutton, S.P., Goldstein, A.G., Rascoe, B., Jr., Sutherland, P.K., and Thompson, D.M., 1988, in Sloss, L.L., ed., Sedimentary cover-North American craton, U.S.: The geology of North America: Boulder, Colo., Geological Society of America, v. D-2, p. 307-359.

Keller, G.R., and Baldridge, W.S., 1995, The southern Oklahoma aulacogen, in Olsen, K.H., ed., Continental rifts: Evolution, structure, tectonics: Amsterdam, Elsevier, p. 427-435. 
Keller, G.R., Lidiak, E.G., Hinze, W.J., and Braile, L.W., 1983, The role of rifting in the tectonic development of the midcontinent, U.S.A.: Tectonophysics, v. 94, p. 391-412.

Keller, G.R., and Stephenson, R.A., 2007, The southern Oklahoma and Dniepr-Donets aulacogens: A comparative analysis, in Hatcher, R.D., Jr., Carlson, M.P., McBride, J.H., and Martinez Catalán, J.R., eds., 4-D Framework of continental crust: Geological Society of America Memoir 200, p. $127-143$.

Kluth, C.F., 1986, Plate tectonics of the Ancestral Rocky Mountains: American Association of Petroleum Geologists Memoir 41, p. 353-369.

Kluth, C.F., and Coney, P.J., 1981, Plate tectonics of the Ancestral Rocky Mountains: Geology, v. 9, p. 10-15.

Kopaska-Merkel, D.C., and Friedman, G.M., 1989, Petrofacies analysis of carbonate rocks: Example from lower Paleozoic Hunton Group of Oklahoma and Texas: American Association of Petroleum Geologists Bulletin, v. 73, p. 1289-1306.

Kuykendall, M.D., and Fritz, R.D., 1993, Misener sandstone: Distribution and relationship to late/post-Hunton unconformities, northern shelf, Anadarko Basin, in Johnson, K.S. ed., Hunton Group core workshop and field trip: Oklahoma Geological Survey Special Publication 93-4, p. 117-134.

Luza, K.V., 1989, Neotectonics and seismicity of the Anadarko Basin, in Johnson, K.S., ed., Anadarko Basin symposium, 1988: Oklahoma Geological Survey Circular 90, p. 121-132.

Luza, K.V., Madole, R.F., and Crone, A.J., 1987, Investigation of the Meers Fault, southwestern Oklahoma: Oklahoma Geological Survey Special Publication 87-1, 75 p.

Mannhard, G.W., and Busch, D.A., 1974, Stratigraphic trap accumulations in southwestern Kansas and northwestern Oklahoma: American Association of Petroleum Geologists Bulletin, v. 58, p. 447-463.

McConnell, D.A., 1989, Constraints on magnitude and sense of slip across the northern margin of the Wichita uplift, southwestern Oklahoma, in Johnson, K.S., ed., Anadarko Basin symposium, 1988: Oklahoma Geological Survey Circular 90, p. 85-96.

Perry, W.J., Jr., 1989, Tectonic evolution of the Anadarko Basin region, Oklahoma: U.S. Geological Survey Bulletin 1866-A, p. A1-A19.

Rascoe, B., Jr., 1962, Regional stratigraphic analysis of Pennsylvanian and Permian rocks in western mid-continent, Colorado, Kansas, Oklahoma, Texas: American Association of Petroleum Geologists Bulletin, v. 46, p. 1345-1370.
Rascoe, B., Jr., and Adler, F.J., 1983, Permo-Carboniferous hydrocarbon accumulations, mid-continent, U.S.A.: American Association of Petroleum Geologists Bulletin, v. 67, p. $979-1001$.

Schramm, M.W., 1964, Paleogeologic and quantitative lithofacies analysis, Simpson Group, Oklahoma: Bulletin of the American Association of Petroleum Geologists, v. 48, p. 1164-1195.

Shatsky, N.S., 1946, The great Donets Basin and the Wichita system: Comparative tectonics of ancient platforms: Akademiya Nauk SSSR Izvestiya, Seriya Geologicheskaya, v. 6, p. 57-90.

Thomas, W.A., 1989, The Appalachian-Ouachita orogen beneath the Gulf Coastal Plain between the outcrops in the Appalachian and Ouachita Mountains, in Hatcher, R.D., Jr., Thomas, W.A., and Viele, G.W., eds., The AppalachianOuachita orogen in the United States: Geological Society of America, The Geology of North America, v. F-2, p. 537-553.

Thomas, W.A., 1991, The Appalachian-Ouachita rifted margin of southeastern North America: Geological Society of America Bulletin, v. 103, p. 415-431.

Thomas, W.A., 2006, Tectonic inheritance at a continental margin: GSA Today, v. 16, p. 4-11.

Thomas, W.A., and Astini, R.A., 1996, The Argentine Precordillera: A traveler from the Ouachita embayment of North American Laurentia: Science, v. 273, p. 752-757.

Tomlinson, C.W., and McBee, W., Jr., 1959, Pennsylvanian sediments and orogenies of Ardmore District, Oklahoma, in Petroleum geology of southern Oklahoma, volume 2: American Association of Petroleum Geologists, p. 3-52.

Van Schmus, W.R., Bickford, M.E., and Turek, E., 1996, Proterozoic geology of the east-central mid-continent basement, in van der Pluijm, B.A., and Catacosinos, P.A., eds., Basement and basins of eastern North America: Geological Society of America Special Paper 308, p. 7-32.

Van Schmus, W.R., Schneider, D.A., Holm, D.K., Dodson, S., and Nelson, B.K., 2007, New insights into the southern margin of the Archean-Proterozoic boundary in the northcentral United States based on U-Pb, Sm-Nd, and Ar-Ar geochronology: Precambrian Research, v. 157, p. 80-105.

Whitmeyer, S.J., and Karlstrom, K.E., 2007, Tectonic model for the Proterozoic growth of North America: Geosphere, v. 3, p. 220-259.

Wilson, J.T., 1966, Did the Atlantic close and then reopen?: Nature, v. 211, p. 676-681. 



\section{Chapter 12 \\ Tabular Data and Graphical Images in Support of the U.S. Geological Survey National Oil and Gas Assessment- Anadarko Basin Province (5058)}

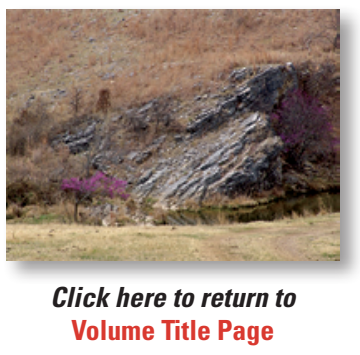

By T.R. Klett and P.A. Le

Chapter 12 of 13

Petroleum Systems and Assessment of Undiscovered Oil and Gas in the Anadarko Basin Province, Colorado, Kansas, Oklahoma, and Texas-USGS Province 58

Compiled by Debra K. Higley

U.S. Geological Survey Digital Data Series DDS-69-EE 


\title{
U.S. Department of the Interior SALLY JEWELL, Secretary
}

\section{U.S. Geological Survey \\ Suzette M. Kimball, Acting Director}

\author{
U.S. Geological Survey, Reston, Virginia: 2014
}

For more information on the USGS - the Federal source for science about the Earth, its natural and living resources, natural hazards, and the environment, visit http://WWW.usgs.gov or call 1-888-ASK-USGS.

For an overview of USGS information products, including maps, imagery, and publications, visit http://WwW.usgs.gov/pubprod

To order this and other USGS information products, visit http://store.usgs.gov

Any use of trade, firm, or product names is for descriptive purposes only and does not imply endorsement by the U.S. Government.

Although this information product, for the most part, is in the public domain, it also may contain copyrighted materials as noted in the text. Permission to reproduce copyrighted items must be secured from the copyright owner.

Suggested citation:

Klett T.R., and Le., P.A., 2014, Tabular data and graphical images in support of the U.S. Geological Survey national oil and gas assessment-Anadarko Basin Province (5058), chap. 12, in Higley, D.K., compiler, Petroleum systems and assessment of undiscovered oil and gas in the Anadarko Basin Province, Colorado, Kansas, Oklahoma, and TexasUSGS Province 58: U.S. Geological Survey Digital Data Series DDS-69-EE, 20 p., http://dx.doi.org/10.3133/ds69EE.

ISSN 2327-638X (online) 


\section{Contents}

Introduction
Disclaimers
Data Sources
Data Overview
Suta Tables
Summary Tables
Graphical Data.
File List
Tabular Data.
References Cited.





\title{
Tabular Data and Graphical Images in Support of the U.S. Geological Survey National Oil and Gas Assessment- Anadarko Basin Province (5058)
}

\author{
By T.R. Klett and P.A. Le
}

\section{Introduction}

This chapter describes data used in support of the U.S. Geological Survey (USGS) National Oil and Gas Assessment (NOGA) project. Digital tabular data used in this chapter of the report and archival data that permit the user to perform further analyses are available elsewhere with this report and online at: http://energy.usgs.gov/OilGas/AssessmentsData/ NationalOilGasAssessment.aspx.

Because of the number and variety of platforms and software available, graphical images are provided as Portable Document Format files (.pdf files) and tabular data are provided in a raw form as tab-delimited text files (.tab files).

\section{Disclaimers}

This publication was prepared by an agency of the U.S. Government. Neither the U.S. Government nor any agency thereof, nor any of its employees, make any warranty, expressed or implied, or assume any legal liability or responsibility for the accuracy, completeness, or usefulness of any information, apparatus, product, or process disclosed in this report, or represent that its use would not infringe upon privately owned rights. Although all data and software published with this report have been used by the USGS, no warranty, expressed or implied, is made by the USGS as to the accuracy of the data and related materials. The act of distribution shall not constitute any such warranty, and no responsibility is assumed by the USGS in the use of these data or related materials. Any use of trade, firm, or product names is for descriptive purposes only and does not imply endorsement by the U.S. Government.

\section{Data Sources}

Crude oil and natural gas production data (including all volumetric and descriptive data such as cumulative production, remaining reserves, known recoverable volumes, major producing reservoirs, and petroleum type) and historical data (including field-discovery dates, well-completion dates, exploration objectives, and well depths) for fields, reservoirs, and wells are derived from commercial databases leased and (or) purchased by the USGS, including (1) PI/ Dwights Plus US Production Data (IHS Energy Group, 2010a), (2) PI/Dwights Plus US Well Data (IHS Energy Group, 2010b), and (3) NRG Associates, Inc., "Significant Oil and Gas Fields of the United States" (NRG Associates, 2009), as well as older versions of these databases. Data from these databases are subject to proprietary constraints, but derivative representations in the form of graphs and summary statistics are allowed to be published and were prepared for each assessment unit. To supplement commercial databases, additional data are obtained, where available, from operators, other Federal and State agencies, or published geological reports.

The PI/Dwights Plus US Production database provides production data for wells, leases, or production units. The PI/ Dwights Plus US Well database provides individual well data (including data for dry holes), including well identifications, locations, initial and final well status classifications, completion dates, and information on penetrated formations. The NRG Associates, Inc., "Significant Oil and Gas Fields of the United States" database provides volumetric and production data for fields and reservoirs.

\section{Data Overview}

This report provides various data files supporting the NOGA project. The files contain data that are the sources for the various graphs, data tables, and summary tables used in the assessment process. Tabular data are provided as tabdelimited text files (.tab files), usable in spreadsheet and database software. Graphical and summary data are provided as Portable Document Format files (.pdf files). File name conventions are as follows: 


\section{Data Tables}

eco\#\#\#\#.tab

fed\#\#\#\#.tab

own\#\#\#\#.tab

sta\#\#\#\#.tab

vol\#\#\#\#.tab

inf\#\#\#\#.tab

ins\#\#\#\#.tab

kvol\#\#\#\#.tab

\section{Summary Tables}

c\#\#\#\#\#\#.pdf

d\#\#\#\#\#\#.pdf

u\#\#\#\#\#\#.pdf

\section{Graphs}

em\#\#\#\#\#\#.pdf

fp\#\#\#\#\#\#.pdf

g\#\#\#\#\#\#.pdf

k\#\#\#\#\#\#.pdf

Prefixes are defined as follows. The eco\#\#\#\#.tab, fed\#\#\#\#.tab, own\#\#\#\#.tab, and sta\#\#\#\#.tab files contain volume-percent data of undiscovered petroleum allocated to ecosystem regions, Federal lands, general land-ownership parcels, and States, respectively. The vol\#\#\#\#.tab (volumes of undiscovered resources) table contains estimates of undiscovered petroleum resources along with parameters that express uncertainty in these estimates. The inf\#\#\#\#.tab table (input FORSPAN) contains input data from the FORSPAN Assessment Model for Continuous Accumulations-Basic Input Data Form (Klett and Charpentier, 2003; Crovelli, 2005) used in this assessment and provided in the u\#\#\#\#\#\#. pdf (unconventional assessment units) files. The ins\#\#\#\#.tab (input Seventh) table contains input data from the Seventh Approximation Data Forms for Conventional Assessment Units (Klett and others, 2005; Schmoker and Klett, 2005) used in this assessment and provided in the c\#\#\#\#\#\#.pdf (conventional assessment units) files. Data in the kvol\#\#\#\#. tab (known volumes) table are recoverable volumes reported in a commercially purchased database. The d\#\#\#\#\#\#. pdf (discovery history) table contains known recoverable volumes divided into historic discovery segments (first- and second-half or first-, second-, and third-third of accumulations discovered). The em\#\#\#\#\#\#.pdf (output from the Monte Carlo program called EMC2; Charpentier and Klett, 2005) files contain graphs of input data and estimated petroleum resource volumes for conventional assessment units. The fp\#\#\#\#\#\#.pdf (FORSPAN probability distributions for input and output variables) files contain graphs of input data and estimated petroleum resource volumes for continuous assessment units. The g\#\#\#\#\#\#.pdf (grown recoverable volumes) and k\#\#\#\#\#\#.pdf (known recoverable volumes) files contain graphs of exploration and discovery data for conventional assessment units.

For identification purposes, numbers in the positions occupied by the four symbols "\#\#\#\#” represent the province code. Files with these numbers contain data for the entire province. Files having numbers in the positions occupied by the six symbols "\#\#\#\#\#\#" (which represent the last six digits of the assessment-unit code) contain data only for that assessment unit.

\section{Numeric Codes}

A hierarchical numeric code identifies each region, province, total petroleum system, and assessment unit. The criteria for assigning codes are uniform throughout the NOGA project and throughout all resulting publications. The numeric codes used in this study are listed below.

\begin{tabular}{lll}
\hline \multicolumn{1}{c}{ Unit } & \multicolumn{1}{c}{ Name } & \multicolumn{1}{c}{ Code } \\
\hline Region & North America & $\mathbf{5}$ \\
Province & Anadarko Basin & 5058 \\
Total Petroleum System & Woodford Composite & 505801 \\
& Pennsylvanian Composite & 505802 \\
Assessment Units & Arbuckle-Ellenburger & 50580101 \\
& Simpson Group & 50580102 \\
& Viola Group & 50580103 \\
& Hunton Group & 50580104 \\
& Mississippian & 50580105 \\
& Woodford Shale Gas & 50580161 \\
& Woodford Shale Oil & 50580162 \\
& Morrowan-Atokan & 50580201 \\
& Desmoinesian & 50580202 \\
& Missourian-Permian & 50580203 \\
& Greater Granite Wash & 50580204 \\
& Composite & \\
Thirteen Finger Lime- & 50580261 \\
& stone-Atoka Shale Gas & \\
\hline & &
\end{tabular}

The assessment-unit portion of the code (last two digits) defines the type of assessment unit. Numbers from 01 to 59 represent conventional assessment units: 61 to 79 represent continuous oil or gas assessment units, and 81 to 99 represent coalbed-gas assessment units. No total petroleum system or assessment-unit codes end with 0 , for example 505800, 50580160 or 50580180 .

To maintain the conventional labeling of files (8-character name and 3-character extension), some file names that contain assessment-unit numbers do not include the first two digits. For example, a summary table that contains input data for the Arbuckle-Ellenburger assessment unit is labeled "c580101.pdf." 


\section{Summary Tables}

The c\#\#\#\#\#.pdf and u\#\#\#\#\#.pdf files are copies of the completed input data forms used in the assessment process. The c\#\#\#\#\#.pdf files contain the Seventh Approximation Data Forms for Conventional Assessment Units. The u\#\#\#\#\#.pdf files contain the FORSPAN Assessment Model for Continuous Accumulations-Basic Input Data Form. These data are defined in the inf\#\#\#\#.tab and ins\#\#\#\#.tab sections of this report.

The d\#\#\#\#\#\#.pdf files contain three tables of known and grown petroleum volumes in an assessment unit as a whole and in terms of discovery history. Grown field sizes are defined as known accumulation sizes that were adjusted upward to account for estimated future reserve growth. NA means not applicable and is shown in place of volumes for which only one accumulation is present to protect the proprietary nature of the data.

\section{Graphical Data}

The fp\#\#\#\#\#\#.pdf files contain graphs of input data and estimated petroleum resource volumes for continuous assessment units. The data are defined in the inf\#\#\#\#.tab and vol\#\#\#\#.tab sections of this report. The graphs contained in these files are derived from a report generated by a commercial software package. The quality of these preformatted graphs, therefore, does not necessarily meet USGS editorial standards.

The em\#\#\#\#\#.pdf files contain graphs of input data and estimated petroleum resource volumes for conventional assessment units. The data are defined in the ins\#\#\#.tab and vol\#\#\#\#.tab sections of this report. The graphs contained in these files are derived from a report generated by a commercial software package. The quality of these preformatted graphs, therefore, does not necessarily meet USGS editorial standards.

The g\#\#\#\#\#.pdf and k\#\#\#\#\#\#.pdf files contain graphs of exploration and discovery data for conventional assessment units. The volumetric data are defined in the kvol\#\#\#\#.tab sections of this report. To protect the proprietary nature of the data, these files are not provided if the total number of accumulations in the assessment unit that are greater than or equal to the specified minimum size is less than four.

Two sets of exploration-activity and discovery-history graphs are provided for each of the assessment units, one set showing known field sizes (cumulative production plus remaining reserves) and the other showing field sizes that were adjusted to compensate for potential reserve growth that may occur in the next 30 years (labeled "grown"). Within each set of graphs, oil fields and gas fields are treated separately. The graphs include:
- Cumulative Number of New-Field Wildcat Wells vs. Drilling-Completion Year

- Number of New-Field Wildcat Wells vs. DrillingCompletion Year

- Oil- or Gas-Accumulation Size [million barrels of oil (MMBO) or billion cubic feet of gas (BCFG)] vs. Oilor Gas-Accumulation Rank by Size (With Respect to Discovery Halves or Thirds)

- Number of Oil or Gas Accumulations vs. Oil- or GasAccumulation Size Classes (MMBO or BCFG) (With Respect to Discovery Halves or Thirds)

- Volume of Oil or Gas (MMBO or BCFG) vs. Oil- or Gas-Accumulation Size Classes (MMBO or BCFG)

- Oil- or Gas-Accumulation Size (MMBO or BCFG) vs. Accumulation-Discovery Year

- Oil- or Gas-Accumulation Size (MMBO or BCFG) vs. Cumulative Number of New-Field Wildcat Wells

- Cumulative Oil or Gas Volume (MMBO or BCFG) vs. Accumulation-Discovery Year

- Cumulative Oil or Gas Volume (MMBO or BCFG) vs. Cumulative Number of New-Field Wildcat Wells

- Cumulative Number of Oil or Gas Accumulations vs. Accumulation-Discovery Year

- Cumulative Number of Oil or Gas Accumulations vs. Cumulative Number of New-Field Wildcat Wells

- Reservoir Depth, Oil or Gas Accumulations [feet (ft)] vs. Accumulation-Discovery Year

- Reservoir Depth, Oil or Gas Accumulations (ft) vs. Cumulative Number of New-Field Wildcat Wells

- Gas/Oil, Oil Accumulations [cubic feet of gas per barrel of oil, (CFG/BO)] vs. Mean Reservoir Depth (ft)

- NGL/Gas, Oil Accumulations [barrels of natural gas liquids per million cubic feet of gas (BNGL/MMCFG)] vs. Mean Reservoir Depth (ft)

- Liquids/Gas, Gas Accumulations [barrels of oil and natural gas liquids per million cubic feet of gas (BL/ MMCFG)] vs. Mean Reservoir Depth (ft)

- Number of Reservoirs in Oil Accumulations vs. American Petroleum Institute (API) Gravity (Degrees)

If data are insufficient or do not exist, graphs are not provided. Therefore, not all graphs are included in all files. 


\section{File List}

\begin{tabular}{|c|c|}
\hline \multicolumn{2}{|r|}{ Data tables } \\
\hline eco5058.tab & \\
\hline fed5058.tab & \\
\hline inf5058.tab & \\
\hline ins5058.tab & \\
\hline kvol5058.tab & \\
\hline own5058.tab & \\
\hline sta5058.tab & \\
\hline vol5058.tab & \\
\hline Summary tables & Assessment-unit name \\
\hline c580101.pdf & Arbuckle-Ellenburger \\
\hline c580102.pdf & Simpson Group \\
\hline c580103.pdf & Viola Group \\
\hline c580104.pdf & Hunton Group \\
\hline c580105.pdf & Mississippian \\
\hline c580201.pdf & Morrowan-Atokan \\
\hline c580202.pdf & Desmoinesian \\
\hline c580203.pdf & Missourian-Permian \\
\hline c580204.pdf & Greater Granite Wash Composite \\
\hline d580101.pdf & Arbuckle-Ellenburger \\
\hline d580102.pdf & Simpson Group \\
\hline d580103.pdf & Viola Group \\
\hline d580104.pdf & Hunton Group \\
\hline d580105.pdf & Mississippian \\
\hline d580201.pdf & Morrowan-Atokan \\
\hline d580202.pdf & Desmoinesian \\
\hline d580203.pdf & Missourian-Permian \\
\hline d580204.pdf & Greater Granite Wash Composite \\
\hline u580161.pdf & Woodford Shale Gas \\
\hline u580162.pdf & Woodford Shale Oil \\
\hline u580261.pdf & Thirteen Finger Limestone-Atoka Shale Gas \\
\hline Graphs & Assessment-unit name \\
\hline em580101.pdf & Arbuckle-Ellenburger \\
\hline em580102.pdf & Simpson Group \\
\hline em580103.pdf & Viola Group \\
\hline em580104.pdf & Hunton Group \\
\hline em580105.pdf & Mississippian \\
\hline em580201.pdf & Morrowan-Atokan \\
\hline em580202.pdf & Desmoinesian \\
\hline em580203.pdf & Missourian-Permian \\
\hline em580204.pdf & Greater Granite Wash Composite \\
\hline fp580161.pdf & Woodford Shale Gas \\
\hline fp580162.pdf & Woodford Shale Oil \\
\hline fp580261.pdf & Thirteen Finger Limestone-Atoka Shale Gas \\
\hline g580101.pdf & Arbuckle-Ellenburger \\
\hline g580102.pdf & Simpson Group \\
\hline
\end{tabular}

\begin{tabular}{ll}
\hline & \multicolumn{1}{c}{ Data tables } \\
\hline g580103.pdf & Viola Group \\
g580104.pdf & Hunton Group \\
g580105.pdf & Mississippian \\
g580201.pdf & Morrowan-Atokan \\
g580202.pdf & Desmoinesian \\
g580203.pdf & Missourian-Permian \\
g580204.pdf & Greater Granite Wash Composite \\
k580101.pdf & Arbuckle-Ellenburger \\
k580102.pdf & Simpson Group \\
k580103.pdf & Viola Group \\
k580104.pdf & Hunton Group \\
k580105.pdf & Mississippian \\
k580201.pdf & Morrowan-Atokan \\
k580202.pdf & Desmoinesian \\
k580203.pdf & Missourian-Permian \\
k580204.pdf & Greater Granite Wash Composite \\
\hline
\end{tabular}

\section{Tabular Data}

The eco\#\#\#\#.tab, fed\#\#\#\#.tab, own\#\#\#\#.tab, and sta\#\#\#\#. tab files contain volume-percent data of undiscovered petroleum allocated to ecosystem regions, Federal lands, general land-ownership parcels, and States. Cells are left blank if data are unavailable.

The eco\#\#\#\#.tab table contains 59 columns. The sum of the percentages should equal those for the total area (excluding offshore) in the own\#\#\#\#.tab file. Data columns for these files are as follows:

1. Code-assessment-unit code number.

2. Name-assessment-unit name.

3. Area $\mathrm{km}^{2}$ - area of the assessment unit in square kilometers.

4. Eco 1-name of first ecosystem that occupies all or part of the assessment-unit area.

5. E1 Area \%-percentage of assessment-unit area that is occupied by ecosystem 1 .

6. E1 Oil \%-estimated percentage of undiscovered oil volume allocated to ecosystem 1 .

7. E1 Gas \%-estimated percentage of undiscovered gas volume allocated to ecosystem 1 .

8. Eco 2-name of second ecosystem that occupies part of the assessment-unit area.

9. E2 Area \% - percentage of assessment-unit area that is occupied by ecosystem 2 . 
10. E2 Oil \%-estimated percentage of undiscovered oil volume allocated to ecosystem 2 .

11. E2 Gas \% — estimated percentage of undiscovered gas volume allocated to ecosystem 2 .

12. Eco 3-name of third ecosystem that occupies part of the assessment-unit area.

13. E3 Area $\%$ - percentage of assessment-unit area that is occupied by ecosystem 3 .

14. E3 Oil \% - estimated percentage of undiscovered oil volume allocated to ecosystem 3 .

15. E3 Gas \% - estimated percentage of undiscovered gas volume allocated to ecosystem 3 .

16. Eco 4-name of fourth ecosystem that occupies part of the assessment-unit area.

17. E4 Area \% - percentage of assessment-unit area that is occupied by ecosystem 4 .

18. E4 Oil \% — estimated percentage of undiscovered oil volume allocated to ecosystem 4 .

19. E4 Gas \% — estimated percentage of undiscovered gas volume allocated to ecosystem 4 .

20. Eco 5-name of fifth ecosystem that occupies part of the assessment-unit area.

21. E5 Area \%-percentage of assessment-unit area that is occupied by ecosystem 5 .

22. E5 Oil \% - estimated percentage of undiscovered oil volume allocated to ecosystem 5 .

23. E5 Gas \%-estimated percentage of undiscovered gas volume allocated to ecosystem 5 .

24. Eco 6-name of sixth ecosystem that occupies part of the assessment-unit area.

25. E6 Area $\%$ - percentage of assessment-unit area that is occupied by ecosystem 6 .

26. E6 Oil \%-estimated percentage of undiscovered oil volume allocated to ecosystem 6 .

27. E6 Gas \% - estimated percentage of undiscovered gas volume allocated to ecosystem 6 .

28. Eco 7-name of seventh ecosystem that occupies part of the assessment-unit area.

29. E7 Area \% - percentage of assessment-unit area that is occupied by ecosystem 7 .

30. E7 Oil \% - estimated percentage of undiscovered oil volume allocated to ecosystem 7.
31. E7 Gas \% - estimated percentage of undiscovered gas volume allocated to ecosystem 7.

32. Eco 8-name of eighth ecosystem that occupies part of the assessment-unit area.

33. E8 Area $\%$ - percentage of assessment-unit area that is occupied by ecosystem 8 .

34. E8 Oil \% - estimated percentage of undiscovered oil volume allocated to ecosystem 8 .

35. E8 Gas \% - estimated percentage of undiscovered gas volume allocated to ecosystem 8 .

36. Eco 9-name of ninth ecosystem that occupies part of the assessment-unit area.

37. E9 Area $\%$ - percentage of assessment-unit area that is occupied by ecosystem 9 .

38. E9 Oil \%-estimated percentage of undiscovered oil volume allocated to ecosystem 9.

39. E9 Gas \% - estimated percentage of undiscovered gas volume allocated to ecosystem 9.

40. Eco 10 - name of tenth ecosystem that occupies part of the assessment-unit area.

41. E10 Area \% - percentage of assessment-unit area that is occupied by ecosystem 10 .

42. E10 Oil \% - estimated percentage of undiscovered oil volume allocated to ecosystem 10 .

43. E10 Gas \% - estimated percentage of undiscovered gas volume allocated to ecosystem 10 .

44. Eco 11-name of eleventh ecosystem that occupies part of the assessment-unit area.

45. E11 Area \%-percentage of assessment-unit area that is occupied by ecosystem 11 .

46. E11 Oil \%-estimated percentage of undiscovered oil volume allocated to ecosystem 11 .

47. E11 Gas \%-estimated percentage of undiscovered gas volume allocated to ecosystem 11 .

48. Eco 12 - name of twelfth ecosystem that occupies part of the assessment-unit area.

49. E12 Area \% - percentage of assessment-unit area that is occupied by ecosystem 12 .

50. E12 Oil \% - estimated percentage of undiscovered oil volume allocated to ecosystem 12 .

51. E12 Gas \% - estimated percentage of undiscovered gas volume allocated to ecosystem 12 . 
52. Eco 13-name of thirteenth ecosystem that occupies part of the assessment-unit area.

53. E13 Area $\%$ - percentage of assessment-unit area that is occupied by ecosystem 13 .

54. E13 Oil \%-estimated percentage of undiscovered oil volume allocated to ecosystem 13.

55. E13 Gas \% - estimated percentage of undiscovered gas volume allocated to ecosystem 13 .

56. Eco 14-name of fourteenth ecosystem that occupies part of the assessment-unit area.

57. E14 Area \% - percentage of assessment-unit area that is occupied by ecosystem 14.

58. E14 Oil \%-estimated percentage of undiscovered oil volume allocated to ecosystem 14 .

59. E14 Gas \% - estimated percentage of undiscovered gas volume allocated to ecosystem 14 .

The fed\#\#\#\#.tab table contains 83 columns. The sum of the percentages should equal those for Federal lands in the own\#\#\#\#.tab file. Data columns for these files are:

1. Code-assessment-unit code number.

2. Name - assessment-unit name.

3. Area $\mathrm{km}^{2}$ - area of the assessment unit in square kilometers.

4. BLM — “Bureau of Land Management (BLM)," name of Federal land that occupies all or part of the assessmentunit area.

5. BLM Area \%-percentage of assessment-unit area that is occupied by Bureau of Land Management lands.

6. BLM Oil \% — estimated percentage of undiscovered oil volume allocated to Bureau of Land Management lands.

7. BLM Gas \% - estimated percentage of undiscovered gas volume allocated to Bureau of Land Management lands.

8. BLMW-“BLM Wilderness Areas (BLMW)," name of Federal land that occupies all or part of the assessmentunit area.

9. BLMW Area \%-percentage of assessment-unit area that is occupied by Bureau of Land Management Wilderness Areas.

10. BLMW Oil \%-estimated percentage of undiscovered oil volume allocated to Bureau of Land Management Wilderness Areas.

11. BLMW Gas \% - estimated percentage of undiscovered gas volume allocated to Bureau of Land Management Wilderness Areas.
12. BLMR — “BLM Roadless Areas (BLMR)," name of Federal land that occupies all or part of the assessment-unit area.

13. BLMR Area \% - percentage of assessment-unit area that is occupied by Bureau of Land Management Roadless Areas.

14. BLMR Oil \%-estimated percentage of undiscovered oil volume allocated to Bureau of Land Management Roadless Areas.

15. BLMR Gas \% - estimated percentage of undiscovered gas volume allocated to Bureau of Land Management Roadless Areas.

16. NPS - "National Park Service (NPS)," name of Federal land that occupies all or part of the assessment-unit area.

17. NPS Area $\%$ - percentage of assessment-unit area that is occupied by National Park Service lands.

18. NPS Oil \% - estimated percentage of undiscovered oil volume allocated to National Park Service lands.

19. NPS Gas \% - estimated percentage of undiscovered gas volume allocated to National Park Service lands.

20. NPSW- “NPS Wilderness Areas (NPSW)," name of Federal land that occupies all or part of the assessmentunit area.

21. NPSW Area \%-percentage of assessment-unit area that is occupied by National Park Service Wilderness Areas.

22. NPSW Oil \% - estimated percentage of undiscovered oil volume allocated to National Park Service Wilderness Areas.

23. NPSW Gas \% - estimated percentage of undiscovered gas volume allocated to National Park Service Wilderness Areas.

24. NPSP — "NPS Protected Withdrawals (NPSP)," name of Federal land that occupies all or part of the assessmentunit area.

25. NPSP Area $\%$ - percentage of assessment-unit area that is occupied by National Park Service Protected Withdrawals.

26. NPSP Oil \%-estimated percentage of undiscovered oil volume allocated to National Park Service Protected Withdrawals.

27. NPSP Gas \% - estimated percentage of undiscovered gas volume allocated to National Park Service Protected Withdrawals.

28. FS - "Forest Service (FS)," name of Federal land that occupies all or part of the assessment-unit area. 
29. FS Area \% - percentage of assessment-unit area that is occupied by USDA Forest Service lands.

30. FS Oil \% - estimated percentage of undiscovered oil volume allocated to USDA Forest Service lands.

31. FS Gas \%-estimated percentage of undiscovered gas volume allocated to USDA Forest Service lands.

32. FSW - "FS Wilderness Areas (FSW)," name of Federal land that occupies all or part of the assessment-unit area.

33. FSW Area \% - percentage of assessment-unit area that is occupied by USDA Forest Service Wilderness Areas.

34. FSW Oil \%-estimated percentage of undiscovered oil volume allocated to USDA Forest Service Wilderness Areas.

35. FSW Gas $\%$ - estimated percentage of undiscovered gas volume allocated to USDA Forest Service Wilderness Areas.

36. FSR - "FS Roadless Areas (FSR)," name of Federal land that occupies all or part of the assessment-unit area.

37. FSR Area $\%$ - percentage of assessment-unit area that is occupied by USDA Forest Service Roadless Areas.

38. FSR Oil \% — estimated percentage of undiscovered oil volume allocated to USDA Forest Service Roadless Areas.

39. FSR Gas $\%$ - estimated percentage of undiscovered gas volume allocated to USDA Forest Service Roadless Areas.

40. FSP — "FS Protected Withdrawals (FSP)," name of Federal land that occupies all or part of the assessment-unit area.

41. FSP Area $\%$ - percentage of assessment-unit area that is occupied by USDA Forest Service Protected Withdrawals.

42. FSP Oil $\%$ - estimated percentage of undiscovered oil volume allocated to USDA Forest Service Protected Withdrawals.

43. FSP Gas \% — estimated percentage of undiscovered gas volume allocated to USDA Forest Service Protected Withdrawals.

44. FWS - “U.S. Fish and Wildlife Service (USFWS)," name of Federal land that occupies all or part of the assessmentunit area.

45. FWS Area \%-percentage of assessment-unit area that is occupied by U.S. Fish and Wildlife Service lands.

46. FWS Oil \% — estimated percentage of undiscovered oil volume allocated to U.S. Fish and Wildlife Service lands.
47. FWS Gas \%-estimated percentage of undiscovered gas volume allocated to U.S. Fish and Wildlife Service lands.

48. FWSW-“USFWS Wilderness Areas (USFWSW)," name of Federal land that occupies all or part of the assessmentunit area.

49. FWSW Area $\%$ - percentage of assessment-unit area that is occupied by U.S. Fish and Wildlife Service Wilderness Areas.

50. FWSW Oil \%-estimated percentage of undiscovered oil volume allocated to U.S. Fish and Wildlife Service Wilderness Areas.

51. FWSW Gas \% - estimated percentage of undiscovered gas volume allocated to U.S. Fish and Wildlife Service Wilderness Areas.

52. FWSP - "USFWS Protected Withdrawals (USFWSP)," name of Federal land that occupies all or part of the assessment-unit area.

53. FWSP Area $\%$ - percentage of assessment-unit area that is occupied by U.S. Fish and Wildlife Service Protected Withdrawals.

54. FWSP Oil \% - estimated percentage of undiscovered oil volume allocated to U.S. Fish and Wildlife Service Protected Withdrawals.

55. FWSP Gas \%-estimated percentage of undiscovered gas volume allocated to U.S. Fish and Wildlife Service Protected Withdrawals.

56. WS - "Wilderness Study Areas (WS)," name of Federal land that occupies all or part of the assessment-unit area.

57. WS Area $\%$ - percentage of assessment-unit area that is occupied by Wilderness Study Areas.

58. WS Oil \% - estimated percentage of undiscovered oil volume allocated to Wilderness Study Areas.

59. WS Gas \% - estimated percentage of undiscovered gas volume allocated to Wilderness Study Areas.

60. DOE_ “Department of Energy (DOE)," name of Federal land that occupies all or part of the assessment-unit area.

61. DOE Area \%-percentage of assessment-unit area that is occupied by Department of Energy lands.

62. DOE Oil \% - estimated percentage of undiscovered oil volume allocated to Department of Energy lands.

63. DOE Gas \%-estimated percentage of undiscovered gas volume allocated to Department of Energy lands.

64. DOD — "Department of Defense (DOD)," name of Federal land that occupies all or part of the assessmentunit area. 
65. DOD Area \%-percentage of assessment-unit area that is occupied by Department of Defense lands.

66. DOD Oil \% - estimated percentage of undiscovered oil volume allocated to Department of Defense lands.

67. DOD Gas \% - estimated percentage of undiscovered gas volume allocated to Department of Defense lands.

68. BOR - "Bureau of Reclamation (BOR)," name of Federal land that occupies all or part of the assessment-unit area.

69. BOR Area \% - percentage of assessment-unit area that is occupied by Bureau of Reclamation lands.

70. BOR Oil \% - estimated percentage of undiscovered oil volume allocated to Bureau of Reclamation lands.

71. BOR Gas \% - estimated percentage of undiscovered gas volume allocated to Bureau of Reclamation lands.

72. TVA — “Tennessee Valley Authority (TVA)," name of Federal land that occupies all or part of the assessmentunit area.

73. TVA Area $\%$ - percentage of assessment-unit area that is occupied by Tennessee Valley Authority lands.

74. TVA Oil \% - estimated percentage of undiscovered oil volume allocated to Tennessee Valley Authority lands.

75. TVA Gas $\%$ - estimated percentage of undiscovered gas volume allocated to Tennessee Valley Authority lands.

76. Other- “'Other Federal," other unspecified Federal lands that occupy all part of the assessment-unit area.

77. Oth Area \% - percentage of assessment-unit area that is occupied by other unspecified Federal lands.

78. Oth Oil \% - estimated percentage of undiscovered oil volume allocated to other unspecified Federal lands.

79. Oth Gas \% - estimated percentage of undiscovered gas volume allocated to other unspecified Federal lands.

80. Fed 20 - name of additional specified Federal lands that occupy all or part of the assessment-unit area.

81. (81)F20 Area \% - percentage of assessment-unit area that is occupied by additional specified Federal lands.

82. F20 Oil \% - estimated percentage of undiscovered oil volume allocated to additional specified Federal lands.

83. F20 Gas \% - estimated percentage of undiscovered gas volume allocated to additional specified Federal lands.

The own\#\#\#\#.tab table contains 59 columns. The sum of the percentages should equal 100. Data columns for these files are as follows:

1. Code-assessment-unit code number.
2. Name-assessment-unit name.

3. Area $\mathrm{km}^{2}$ - area of the assessment unit in square kilometers.

4. Fed- "Federal Lands," all Federal lands that occupy all or part of the assessment-unit area.

5. Fed Area $\%$ - percentage of assessment-unit area that is occupied by Federal lands.

6. Fed Oil \%-estimated percentage of undiscovered oil volume allocated to Federal lands.

7. Fed Gas $\%$ - estimated percentage of undiscovered gas volume allocated to Federal lands.

8. Pri- "Private Lands," all private lands that occupy all or part of the assessment-unit area.

9. Pri Area \% - percentage of assessment-unit area that is occupied by private lands.

10. Pri Oil \% — estimated percentage of undiscovered oil volume allocated to private lands.

11. Pri Gas \% — estimated percentage of undiscovered gas volume allocated to private lands.

12. Tri- "Tribal Lands," all tribal lands that occupy all or part of the assessment-unit area.

13. Tri Area $\%$ - percentage of assessment-unit area that is occupied by tribal lands.

14. Tri Oil \% - estimated percentage of undiscovered oil volume allocated to tribal lands.

15. Tri Gas \%-estimated percentage of undiscovered gas volume allocated to tribal lands.

16. Oth-name of other unspecified lands or offshore areas that occupy all or part of the assessment-unit area.

17. Oth Area \% - percentage of assessment-unit area that is occupied by other unspecified lands or offshore areas.

18. Oth Oil \%-estimated percentage of undiscovered oil volume allocated to other unspecified lands or offshore areas.

19. Oth Gas \% - estimated percentage of undiscovered gas volume allocated to other unspecified lands or offshore areas.

20. State 1 -name of first State for which State-owned lands or waters occupy all or part of the assessment-unit area.

21. S1 Area \% - percentage of assessment-unit area that is occupied by State 1-owned lands or waters.

22. S1 Oil \% - estimated percentage of undiscovered oil volume allocated to State 1-owned lands or waters. 
23. S1 Gas \%-estimated percentage of undiscovered gas volume allocated to State 1-owned lands or waters.

24. State 2-name of second State for which State-owned lands or waters occupy all or part of the assessmentunit area.

25. S2 Area $\%$ - percentage of assessment-unit area that is occupied by State 2-owned lands or waters.

26. S2 Oil \%-estimated percentage of undiscovered oil volume allocated to State 2-owned lands or waters.

27. S2 Gas \%-estimated percentage of undiscovered gas volume allocated to State 2-owned lands or waters.

28. State 3-name of third State for which State-owned lands or waters occupy all or part of the assessment-unit area.

29. S3 Area \% - percentage of assessment-unit area that is occupied by State 3-owned lands or waters.

30. S3 Oil \% - estimated percentage of undiscovered oil volume allocated to State 3-owned lands or waters.

31. S3 Gas \% - estimated percentage of undiscovered gas volume allocated to State 3-owned lands or waters.

32. State 4 name of fourth State for which State-owned lands or waters occupy all or part of the assessment-unit area.

33. S4 Area \% - percentage of assessment-unit area that is occupied by State 4-owned lands or waters.

34. S4 Oil \% - estimated percentage of undiscovered oil volume allocated to State 4-owned lands or waters.

35. S4 Gas \%-estimated percentage of undiscovered gas volume allocated to State 4-owned lands or waters.

36. State 5-name of fifth State for which State-owned lands or waters occupy all or part of the assessment-unit area.

37. S5 Area \%-percentage of assessment-unit area that is occupied by State 5-owned lands or waters.

38. S5 Oil \%-estimated percentage of undiscovered oil volume allocated to State 5-owned lands or waters.

39. S5 Gas \% - estimated percentage of undiscovered gas volume allocated to State 5-owned lands or waters.

40. State 6-name of sixth State for which State-owned lands or waters occupy all or part of the assessment-unit area.

41. S6 Area $\%$ - percentage of assessment-unit area that is occupied by State 6-owned lands or waters.

42. S6 Oil \% - estimated percentage of undiscovered oil volume allocated to State 6-owned lands or waters.

43. S6 Gas \%-estimated percentage of undiscovered gas volume allocated to State 6-owned lands or waters.
44. State 7-name of seventh State for which State-owned lands or waters occupy all or part of the assessmentunit area.

45. S7 Area $\%$ - percentage of assessment-unit area that is occupied by State 7-owned lands or waters.

46. S7 Oil \% - estimated percentage of undiscovered oil volume allocated to State 7-owned lands or waters.

47. S7 Gas $\%$ - estimated percentage of undiscovered gas volume allocated to State 7-owned lands or waters.

48. State 8-name of eighth State for which State-owned lands or waters occupy all or part of the assessmentunit area.

49. S8 Area \% - percentage of assessment-unit area that is occupied by State 8-owned lands or waters.

50. S8 Oil \%-estimated percentage of undiscovered oil volume allocated to State 8-owned lands or waters.

51. S8 Gas \% - estimated percentage of undiscovered gas volume allocated to State 8-owned lands or waters.

52. State 9-name of ninth State for which State-owned lands or waters occupy all or part of the assessment-unit area.

53. S9 Area $\%$ - percentage of assessment-unit area that is occupied by State 9-owned lands or waters.

54. S9 Oil \% - estimated percentage of undiscovered oil volume allocated to State 9-owned lands or waters.

55. S9 Gas \% - estimated percentage of undiscovered gas volume allocated to State 9-owned lands or waters.

56. State 10 - name of tenth State for which State-owned lands or waters occupy all or part of the assessmentunit area.

57. S10 Area $\%$ - percentage of assessment-unit area that is occupied by State 10-owned lands or waters.

58. S10 Oil \% - estimated percentage of undiscovered oil volume allocated to State 10-owned lands or waters.

59. S10 Gas \% - estimated percentage of undiscovered gas volume allocated to State 10-owned lands or waters.

The sta\#\#\#\#.tab table contains 59 columns. The sum of the percentages should equal 100. Data columns for these files are as follows:

1. Code-assessment-unit code number.

2. Name-assessment-unit name.

3. Area $\mathrm{km}^{2}$ - area of the assessment unit in square kilometers.

4. State 1-name of first State (onshore and offshore) that occupies all or part of the assessment-unit area. 
5. S1 Area \%-percentage of assessment-unit area that is occupied by State 1.

6. S1 Oil \% - estimated percentage of undiscovered oil volume allocated to State 1.

7. S1 Gas \%-estimated percentage of undiscovered gas volume allocated to State 1.

8. State 2-name of second State (onshore and offshore) that occupies all or part of the assessment-unit area.

9. S2 Area \%-percentage of assessment-unit area that is occupied by State 2 .

10. S2 Oil \%-estimated percentage of undiscovered oil volume allocated to State 2.

11. S2 Gas \%-estimated percentage of undiscovered gas volume allocated to State 2.

12. State 3 - name of third State (onshore and offshore) that occupies all or part of the assessment-unit area.

13. S3 Area \%-percentage of assessment-unit area that is occupied by State 3 .

14. S3 Oil \%-estimated percentage of undiscovered oil volume allocated to State 3.

15. S3 Gas \%-estimated percentage of undiscovered gas volume allocated to State 3 .

16. State 4-name of fourth State (onshore and offshore) that occupies all or part of the assessment-unit area.

17. S4 Area \%-percentage of assessment-unit area that is occupied by State 4 .

18. S4 Oil \% - estimated percentage of undiscovered oil volume allocated to State 4.

19. S4 Gas \%-estimated percentage of undiscovered gas volume allocated to State 4.

20. State 5-name of fifth State (onshore and offshore) that occupies all or part of the assessment-unit area.

21. S5 Area \%-percentage of assessment-unit area that is occupied by State 5 .

22. S5 Oil \% - estimated percentage of undiscovered oil volume allocated to State 5 .

23. S5 Gas \%-estimated percentage of undiscovered gas volume allocated to State 5 .

24. State 6-name of sixth State (onshore and offshore) that occupies all or part of the assessment-unit area.

25. S6 Area \% - percentage of assessment-unit area that is occupied by State 6 .
26. S6 Oil \%-estimated percentage of undiscovered oil volume allocated to State 6 .

27. S6 Gas \% - estimated percentage of undiscovered gas volume allocated to State 6 .

28. State 7-name of seventh State (onshore and offshore) that occupies all or part of the assessment-unit area.

29. S7 Area \%-percentage of assessment-unit area that is occupied by State 7 .

30. S7 Oil \% - estimated percentage of undiscovered oil volume allocated to State 7 .

31. S7 Gas \%-estimated percentage of undiscovered gas volume allocated to State 7.

32. State 8-name of eighth State (onshore and offshore) that occupies all or part of the assessment-unit area.

33. S8 Gas \% - percentage of assessment-unit area that is occupied by State 8 .

34. S8 Oil \%-estimated percentage of undiscovered oil volume allocated to State 8 .

35. S8 Gas \%-estimated percentage of undiscovered gas volume allocated to State 8 .

36. State 9-name of ninth State (onshore and offshore) that occupies all or part of the assessment-unit area.

37. S9 Area \%-percentage of assessment-unit area that is occupied by State 9 .

38. S9 Oil \% - estimated percentage of undiscovered oil volume allocated to State 9.

39. S9 Gas \%-estimated percentage of undiscovered gas volume allocated to State 9 .

40. State 10 - name of tenth State (onshore and offshore) that occupies all or part of the assessment-unit area.

41. S10 Area \%-percentage of assessment-unit area that is occupied by State 10.

42. S10 Oil \% - estimated percentage of undiscovered oil volume allocated to State 10 .

43. S10 Gas \%-estimated percentage of undiscovered gas volume allocated to State 10.

44. State 11-name of eleventh State (onshore and offshore) that occupies all or part of the assessment-unit area.

45. S11 Area \%-percentage of assessment-unit area that is occupied by State 11.

46. S11 Oil \%-estimated percentage of undiscovered oil volume allocated to State 11. 
47. S11 Gas \%-estimated percentage of undiscovered gas volume allocated to State 11.

48. State 12 - name of twelfth State (onshore and offshore) that occupies all or part of the assessment-unit area.

49. S12 Area $\%$ - percentage of assessment-unit area that is occupied by State 12 .

50. S12 Oil \%-estimated percentage of undiscovered oil volume allocated to State 12 .

51. S12 Gas \%-estimated percentage of undiscovered gas volume allocated to State 12.

52. State 13 - name of thirteenth State (onshore and offshore) that occupies all or part of the assessment-unit area.

53. S13 Area $\%$ - percentage of assessment-unit area that is occupied by State 13 .

54. S13 Oil \%-estimated percentage of undiscovered oil volume allocated to State 13 .

55. S13 Gas \%-estimated percentage of undiscovered gas volume allocated to State 13 .

56. State 14 - name of fourteenth State (onshore and offshore) that occupies all or part of the assessment-unit area.

57. S14 Area \% - percentage of assessment-unit area that is occupied by State 14 .

58. S14 Oil \% - estimated percentage of undiscovered oil volume allocated to State 14.

59. S14 Gas \% - estimated percentage of undiscovered gas volume allocated to State 14.

The vol\#\#\#\#.tab table contains estimates of undiscovered petroleum resources along with parameters that express uncertainty in these estimates. The table contains 40 columns. Blank cells represent no data. Data columns for these files are as follows:

1. Code-assessment-unit code number.

2. Name - assessment-unit name.

3. Type - type of assessment unit, conventional, continuous oil, continuous gas, or coalbed gas.

4. Status - remarks indicating that assessment unit was not quantitatively assessed; otherwise blank.

5. Prob \%-geologic probability of the assessment unit.

6. OILF95 MMB - the estimated value of undiscovered oil (oil in oil accumulations) such that there is a 95-percent probability that this amount or more exists in the assessment unit. The volume is given in millions of barrels of oil (MMBO).
7. OILF50 MMB - the estimated value of undiscovered oil (oil in oil accumulations) such that there is a 50-percent probability that this amount or more exists in the assessment unit. This is the median value. The volume is given in millions of barrels of oil (MMBO).

8. OILF5 MMB - the estimated value of undiscovered oil (oil in oil accumulations) such that there is a 5-percent probability that this amount or more exists in the assessment unit. The volume is given in millions of barrels of oil (MMBO).

9. OILMN MMB - the estimated mean (average) value of undiscovered oil (oil in oil accumulations). The volume is given in millions of barrels of oil (MMBO).

10. OILSD MMB - the estimated standard deviation value of undiscovered oil (oil in oil accumulations). The volume is given in millions of barrels of oil (MMBO).

11. AGF95 BCF - the estimated value of undiscovered associated/dissolved gas (gas in oil accumulations) such that there is a 95-percent probability that this amount or more exists in the assessment unit. The volume is given in billions of cubic feet of gas (BCFG).

12. AGF50 BCF - the estimated value of undiscovered associated/dissolved gas (gas in oil accumulations) such that there is a 50-percent probability that this amount or more exists in the assessment unit. This is the median value. The volume is given in billions of cubic feet of gas (BCFG).

13. AGF5 BCF - the estimated value of undiscovered associated/dissolved gas (gas in oil accumulations) such that there is a 5-percent probability that this amount or more exists in the assessment unit. The volume is given in billions of cubic feet of gas (BCFG).

14. AGMN BCF - the estimated mean (average) value of undiscovered associated/dissolved gas (gas in oil accumulations). The volume is given in billions of cubic feet of gas (BCFG).

15. AGSD BCF - the estimated standard deviation value of undiscovered associated/dissolved gas (gas in oil accumulations). The volume is given in billions of cubic feet of gas (BCFG).

16. NGLF95 MMB - the estimated value of undiscovered natural gas liquids (NGL in oil accumulations) such that there is a 95-percent probability that this amount or more exists in the assessment unit. The volume is given in millions of barrels of NGL (MMBNGL).

17. NGLF50 MMB - the estimated value of undiscovered natural gas liquids (NGL in oil accumulations) such that there is a 50-percent probability that this amount or more exists in the assessment unit. This is the median value. The volume is given in millions of barrels of NGL (MMBNGL). 
18. NGLF5 MMB - the estimated value of undiscovered natural gas liquids (NGL in oil accumulations) such that there is a 5-percent probability that this amount or more exists in the assessment unit. The volume is given in millions of barrels of NGL (MMBNGL).

19. NGLMN MMB - the estimated mean (average) value of undiscovered natural gas liquids (NGL in oil accumulations). The volume is given in millions of barrels of NGL (MMBNGL).

20. NGLSD MMB - the estimated standard deviation value of undiscovered natural gas liquids (NGL in oil accumulations). The volume is given in millions of barrels of NGL (MMBNGL).

21. NGF95 BCF - the estimated value of undiscovered nonassociated gas (gas in gas accumulations) such that there is a 95-percent probability that this amount or more exists in the assessment unit. The volume is given in billions of cubic feet of gas (BCFG).

22. NGF50 BCF - the estimated value of undiscovered nonassociated gas (gas in gas accumulations) such that there is a 50-percent probability that this amount or more exists in the assessment unit. This is the median value. The volume is given in billions of cubic feet of gas (BCFG).

23. NGF5 BCF - the estimated value of undiscovered nonassociated gas (gas in gas accumulations) such that there is a 5-percent probability that this amount or more exists in the assessment unit. The volume is given in billions of cubic feet of gas (BCFG).

24. NGMN BCF - the estimated mean (average) value of undiscovered nonassociated gas (gas in gas accumulations). The volume is given in billions of cubic feet of gas (BCFG).

25. NGSD BCF - the estimated standard deviation value of undiscovered nonassociated gas (gas in gas accumulations). The volume is given in billions of cubic feet of gas (BCFG).

26. LIQF95 MMB - the estimated value of undiscovered liquids (oil and NGL in gas accumulations) such that there is a 95-percent probability that this amount or more exists in the assessment unit. The volume is given in millions of barrels of liquids (MMBL).

27. LIQF50 MMB - the estimated value of undiscovered liquids (oil and NGL in gas accumulations) such that there is a 50-percent probability that this amount or more exists in the assessment unit. This is the median value. The volume is given in millions of barrels of liquids (MMBL).

28. LIQF5 MMB - the estimated value of undiscovered liquids (oil and NGL in gas accumulations) such that there is a 5-percent probability that this amount or more exists in the assessment unit. The volume is given in millions of barrels of liquids (MMBL).

29. LIQMN MMB - the estimated mean (average) value of undiscovered liquids (oil and NGL in gas accumulations). The volume is given in millions of barrels of liquids (MMBL).

30. LIQSD MMB - the estimated standard deviation value of undiscovered liquids (oil and NGL in gas accumulations). The volume is given in millions of barrels of liquids (MMBL).

31. LOF95 MMB - the estimated size of the largest undiscovered conventional oil accumulation such that there is a 95-percent probability of that accumulation being this amount or larger. The volume is given in millions of barrels of oil (MMBO).

32. LOF50 MMB - the estimated size of the largest undiscovered conventional oil accumulation such that there is a 50-percent probability of that accumulation being this amount or larger. This is the median value. The volume is given in millions of barrels of oil (MMBO).

33. LOF5 MMB - the estimated size of the largest undiscovered conventional oil accumulation such that there is a 5-percent probability of that accumulation being this amount or larger. The volume is given in millions of barrels of oil (MMBO).

34. LOMN MMB - the estimated mean (average) value of the largest undiscovered conventional oil accumulation. The volume is given in millions of barrels of oil (MMBO).

35. LOSD MMB - the estimated standard deviation value of the largest undiscovered conventional oil accumulation. The volume is given in millions of barrels of oil (MMBO).

36. LGF95 BCF - the estimated size of the largest undiscovered conventional gas accumulation such that there is a 95-percent probability of that accumulation being this amount or larger. The volume is given in billions of cubic feet of gas (BCFG).

37. LGF50 BCF - the estimated size of the largest undiscovered conventional gas accumulation such that there is a 50-percent probability of that accumulation being this amount or larger. This is the median value. The volume is given in billions of cubic feet of gas (BCFG).

38. LGF5 BCF - the estimated size of the largest undiscovered conventional gas accumulation such that there is a 5-percent probability of that accumulation being this amount or larger. The volume is given in billions of cubic feet of gas (BCFG).

39. LGMN BCF - the estimated mean (average) value of the largest undiscovered conventional gas accumulation. The volume is given in billions of cubic feet of gas (BCFG). 
40. LGSD BCF - the estimated standard deviation value of the largest undiscovered conventional gas accumulation. The volume is given in billions of cubic feet of gas (BCFG).

The inf\#\#\#\#.tab table contains input data from the FORSPAN Assessment Model for Continuous Accumulations-Basic Input Data Form used in this assessment. Blank cells represent no data. This table contains 80 columns. Data columns are as follows:

1. Date - date of assessment.

2. Geol—assessing geologist's name.

3. Regno-region code number.

4. Reg-region name.

5. Provno-province code number.

6. Prov-province name.

7. TPSno-total petroleum system code number.

8. TPS - total petroleum system name.

9. AUno-assessment-unit code number.

10. AU—assessment-unit name.

11. Data1 — data sources used to aid in completing the datainput form.

12. Data2 - additional data sources used to aid in completing the data-input form.

13. Comm - assessment unit type, the primary commodity type in the assessment unit, based on the gas-to-oil ratio (GOR) of the petroleum endowment, which includes both the discovered and undiscovered petroleum. An assessment unit is characterized as being oil prone if the gas-tooil ratio (GOR) is less than 20,000 cubic feet of gas per barrel of oil; otherwise, it is gas prone.

14. RminMMBBCF - minimum total recovery per cell considered for assessment, in million barrels of oil (MMBO) for oil assessment units or billion cubic feet of gas $(\mathrm{BCFG})$ for gas assessment units.

15. Numtest $1-$ number of tested cells in the assessment unit.

16. Numtest2-number of tested cells in the assessment unit that have total recoveries equal to or larger than the specified minimum total recovery per cell.

17. AUM - assessment-unit maturity, the exploration maturity of the assessment unit. Assessment-unit maturity is classified as "established" if more than 24 cells exceed the minimum total recovery, "frontier" if 1 to 24 cells exceed the minimum total recovery, or "hypothetical" if no cells exceed the minimum total recovery.
18. Med1MMBBCF - median total recovery per cell of the set of tested cells equal to or greater than the minimum total recovery that constitute the first third of the total number of cells ranked according to date of discovery within the assessment unit, in million barrels of oil (MMBO) for oil assessment units or billion cubic feet of gas $(\mathrm{BCFG})$ for gas assessment units.

19. Med2MMBBCF - median total recovery per cell of the set of tested cells equal to or greater than the minimum total recovery that constitute the second third of the total number of cells ranked according to date of discovery within the assessment unit, in million barrels of oil (MMBO) for oil assessment units or billion cubic feet of gas (BCFG) for gas assessment units.

20. Med3MMBBCF - median total recovery per cell of the set of tested cells equal to or greater than the minimum total recovery that constitute the third third of the total number of cells ranked according to date of discovery within the assessment unit, in million barrels of oil (MMBO) for oil assessment units or billion cubic feet of gas (BCFG) for gas assessment units.

21. Prob $1 \%$ - charge probability, the probability for adequate petroleum charge for at least one untested cell equal to or larger than the minimum total recovery, somewhere in the assessment unit, having the potential to be added to reserves. Charge probability is given as a fractional value from 0 to 1.0 .

22. Prob $2 \%$-rocks probability, the probability for adequate reservoirs, traps, and seals for at least one untested cell equal to or larger than the minimum total recovery, somewhere in the assessment unit, having the potential to be added to reserves. Rocks probability is given as a fractional value from 0 to 1.0 .

23. Prob3 \% - timing probability, the probability for favorable geologic timing for at least one untested cell equal to or larger than the minimum total recovery, somewhere in the assessment unit, having the potential to be added to reserves. Timing probability is given as a fractional value from 0 to 1.0 .

24. Geoprob \% - geologic probability, the product of charge, rocks, and timing probabilities. Geologic probability is given as a fractional value from 0 to 1.0.

25. TAAmin a-estimated minimum (F100) area of the assessment unit. The area is given in acres.

26. TAAmod a - estimated mode of area of the assessment unit. The area is given in acres.

27. TAAmax a - estimated maximum (F0) area of the assessment unit. The area is given in acres.

28. TAAmean a-estimated mean area of the assessment unit. The area is given in acres. 
29. CAmin a-estimated minimum (F100) area per cell of untested cells having the potential for additions to reserves in the assessment unit. This area is equivalent to the drainage area of wells. This area is given in acres.

30. CAmod a — estimated mode of area per cell of untested cells having the potential for additions to reserves in the assessment unit. This area is equivalent to the drainage area of wells. This area is given in acres.

31. CAmax a - estimated maximum (F0) area per cell of untested cells having the potential for additions to reserves in the assessment unit. This area is equivalent to the drainage area of wells. This area is given in acres.

32. CAmean a — estimated mean area per cell of untested cells having the potential for additions to reserves in the assessment unit. This area is equivalent to the drainage area of wells. This area is given in acres.

33. CAminmn a-estimated minimum of the mean area per cell of untested cells having the potential for additions to reserves in the assessment unit. This area is equivalent to the drainage area of wells. This area is given in acres.

34. CAmaxmn a - estimated maximum of the mean area per cell of untested cells having the potential for additions to reserves in the assessment unit. This area is equivalent to the drainage area of wells. This area is given in acres.

35. TAAUmin \%-estimated minimum (F100) percentage of the total assessment-unit area that is untested.

36. TAAUmod \% - estimated mode of percentage of the total assessment-unit area that is untested.

37. TAAUmax \% — estimated maximum (F0) percentage of the total assessment-unit area that is untested.

38. (38)TAAUmean \% - estimated mean percentage of the total assessment-unit area that is untested.

39. UAAPmin \%-estimated minimum (F100) percentage of the untested area in the assessment unit having the potential for additions to reserves).

40. UAAPmod \% - estimated mode of percentage of the untested area in the assessment unit having the potential for additions to reserves.

41. UAAPmax \% — estimated maximum (F0) percentage of the untested area in the assessment unit having the potential for additions to reserves.

42. UAAPmean \%-estimated mean percentage of the untested area in the assessment unit having the potential for additions to reserves.

43. RminMMBBCF — estimated minimum (F100) total recovery per cell for untested cells in the assessment unit having the potential for additions to reserves. This volume is given as million barrels of oil (MMBO) for oil assessment units and billion cubic feet of gas (BCFG) for gas assessment units.

44. RmedMMBBCF — estimated median (F50) total recovery per cell for untested cells in the assessment unit having the potential for additions to reserves. This volume is given as million barrels of oil (MMBO) for oil assessment units and billion cubic feet of gas (BCFG) for gas assessment units.

45. RmaxMMBBCF — estimated maximum (F0) total recovery per cell for untested cells in the assessment unit having the potential for additions to reserves. This volume is given as million barrels of oil (MMBO) for oil assessment units and billion cubic feet of gas (BCFG) for gas assessment units.

46. RmeanMMBBCF - estimated mean total recovery per cell for untested cells in the assessment unit having the potential for additions to reserves. This volume is given as million barrels of oil (MMBO) for oil assessment units and billion cubic feet of gas (BCFG) for gas assessment units.

47. GminCF/B - estimated minimum (F100) gas to oil ratio (GOR), in cubic feet of gas per barrel of oil (CFG/BO), of untested cells equal to or larger than the minimum total recovery in oil assessment units.

48. GmodCF/B - estimated mode of gas to oil ratio (GOR), in cubic feet of gas per barrel of oil (CFG/BO), of untested cells equal to or larger than the minimum total recovery in oil assessment units.

49. GmaxCF/B - estimated maximum (F0) gas to oil ratio (GOR), in cubic feet of gas per barrel of oil (CFG/BO), of untested cells equal to or larger than the minimum total recovery in oil assessment units.

50. NminB/MMCF - estimated minimum (F100) natural gas liquids (NGL) to gas ratio, in barrels of natural gas liquids per million cubic feet of gas (BNGL/MMCFG), of untested cells equal to or larger than the minimum total recovery in oil assessment units.

51. NmodB/MMCF — estimated mode of natural gas liquids (NGL) to gas ratio, in barrels of natural gas liquids per million cubic feet of gas (BNGL/MMCFG), of untested cells equal to or larger than the minimum total recovery in oil assessment units.

52. NmaxB/MMCF — estimated maximum (F0) natural gas liquids (NGL) to gas ratio, in barrels of natural gas liquids per million cubic feet of gas (BNGL/MMCFG), of untested cells equal to or larger than the minimum total recovery in oil assessment units.

53. LminB/MMCF — estimated minimum (F100) liquids (oil plus natural gas liquids) to gas ratio (LGR), in barrels of liquids per million cubic feet of gas (BNGL/MMCFG), of 
untested cells equal to or larger than the minimum total recovery in gas assessment units.

54. LmodB/MMCF — estimated modal liquids (oil plus natural gas liquids) to gas ratio (LGR), in barrels of liquids per million cubic feet of gas (BNGL/MMCFG), of untested cells equal to or larger than the minimum total recovery in gas assessment units.

55. LmaxB/MMCF — estimated maximum (F0) liquids (oil plus natural gas liquids) to gas ratio (LGR), in barrels of liquids per million cubic feet of gas (BNGL/MMCFG), of untested cells equal to or larger than the minimum total recovery in gas assessment units.

56. APImin deg — estimated minimum (F100) American Petroleum Institute gravity, in degrees, of oil in untested cells in oil assessment units.

57. APImod deg — estimated modal American Petroleum Institute gravity, in degrees, of oil in untested cells in oil assessment units.

58. APImax deg - estimated maximum (F0) American Petroleum Institute gravity, in degrees, of oil in untested cells in oil assessment units.

59. Smin \%-estimated minimum (F100) sulfur (S) content, in percent, of oil in untested cells in oil assessment units.

60. Smod \% - estimated mode of sulfur (S) content, in percent, of oil in untested cells in oil assessment units.

61. Smax \%-estimated maximum (F0) sulfur (S) content, in percent, of oil in untested cells in oil assessment units.

62. ODmin $\mathrm{m}$ - estimated minimum (F100) drilling depth, in meters, of oil in untested cells in oil assessment units.

63. ODf75 $\mathrm{m}$ - estimated 75 th fractile (F75) of drilling depth, in meters, of oil in untested cells in oil assessment units.

64. ODmod $\mathrm{m}$ - estimated mode of drilling depth, in meters, of oil in untested cells in oil assessment units.

65. ODf $25 \mathrm{~m}$ - estimated 25 th fractile (F25) drilling depth, in meters, of oil in untested cells in oil assessment units.

66. ODmax m-estimated maximum (F0) drilling depth, in meters, of oil in untested cells in oil assessment units.

67. OWDmin $\mathrm{m}$ - estimated minimum (F100) water depth, in meters, of oil in untested cells in oil assessment units (ocean, bays, or lakes; if applicable).

68. OWDmod $\mathrm{m}$ - estimated mode of water depth, in meters, of oil in untested cells in oil assessment units (ocean, bays, or lakes; if applicable).

69. OWDmax m-estimated maximum (F0) water depth, in meters, of oil in untested cells in oil assessment units (ocean, bays, or lakes; if applicable).
70. IGmin \%-estimated minimum (F100) inert gas (IG) content, in percent, of gas in untested cells in gas assessment units (nitrogen and helium, and so forth).

71. IGmod \% - estimated mode of inert gas (IG) content, in percent, of gas in untested cells in gas assessment units (nitrogen and helium, and so forth).

72. IGmax \% - estimated maximum (F0) inert gas (IG) content, in percent, of gas in untested cells in gas assessment units (nitrogen and helium, and so forth).

73. $\mathrm{CO} 2 \mathrm{~min} \%$ - estimated minimum (F100) carbon dioxide $\left(\mathrm{CO}_{2}\right)$ content, in percent, of gas in untested cells in gas assessment units.

74. $\mathrm{CO} 2 \mathrm{med} \%$ - estimated mode of carbon dioxide $\left(\mathrm{CO}_{2}\right)$ content, in percent, of gas in untested cells in gas assessment units.

75. $\mathrm{CO} 2 \mathrm{max} \%$ - estimated maximum (F0) carbon dioxide $\left(\mathrm{CO}_{2}\right)$ content, in percent, of gas in untested cells in gas assessment units.

76. H2Smin \%-estimated minimum (F100) hydrogen sulfide $\left(\mathrm{H}_{2} \mathrm{~S}\right)$ content, in percent, of gas in untested cells in gas assessment units.

77. H2Smod $\%$ - estimated mode of hydrogen sulfide $\left(\mathrm{H}_{2} \mathrm{~S}\right)$ content, in percent, of gas in untested cells in gas assessment units.

78. H2Smax \%-estimated maximum (F0) hydrogen sulfide $\left(\mathrm{H}_{2} \mathrm{~S}\right)$ content, in percent, of gas in untested cells in gas assessment units.

79. HVmin btu — estimated minimum heating value, in British Thermal Units (btu), of gas in untested cells in gas assessment units.

80. HVmod btu - estimated mode of heating value, in British Thermal Units (btu), of gas in untested cells in gas assessment units.

81. HVmax btu - estimated maximum heating value, in British Thermal Units (btu), of gas in untested cells in gas assessment units.

82. GDmin $\mathrm{m}$ - estimated minimum (F100) drilling depth, in meters, of gas in untested cells in gas assessment units.

83. GDf25 $\mathrm{m}$ - estimated 25 th fractile (F25) drilling depth, in meters, of gas in untested cells in gas assessment units.

84. GDmod $\mathrm{m}$ - estimated mode of drilling depth, in meters, of gas in untested cells in gas assessment units.

85. GDf25 $\mathrm{m}$ - estimated 25th fractile (F25) drilling depth, in meters, of gas in untested cells in gas assessment units.

86. GDmax m-estimated maximum (F0) drilling depth, in meters, of gas in untested cells in gas assessment units. 
87. GWDmin m-estimated minimum (F100) water depth, in meters, of gas in untested cells in gas assessment units (ocean, bays, or lakes; if applicable).

88. GWDmod m- estimated mode of water depth, in meters, of gas in untested cells in gas assessment units (ocean, bays, or lakes; if applicable).

89. GWDmax m-estimated maximum (F0) water depth, in meters, of gas in untested cells in gas assessment units (ocean, bays, or lakes; if applicable).

90. FSRmean $\%$ - estimated mean future success ratio, as percent.

91. FSRmin \%-estimated minimum (F100) future success ratio, as percent.

92. FSRmod $\%$ - estimated mode of future success ratio, as percent.

93. FSRmax $\%$ - estimated maximum (F100) future success ratio, as percent.

94. HSR \%-historical success ratio, as percent.

95. TWCP-Typical well-completion practices (conventional, open hole, open cavity, other).

96. FWTS - Fraction of wells drilled that are typically stimulated.

97. FST - Predominant type of stimulation (none, frac, acid, other).

98. FHW-Fraction of wells drilled that are horizontal.

The ins\#\#\#\#.tab table contains input data from the Seventh Approximation Data Forms for Conventional Assessment Units used in this assessment and provided in the c\#\#\#\#\#. pdf files. Cells are left blank if data are unavailable. This table contains 78 columns. Data columns are as follows:

1. Date-date of assessment.

2. Geol_assessing geologist's name.

3. Regno-region code number.

4. Reg-region name.

5. Provno-province code number.

6. Prov-province name.

7. TPSno- - total petroleum system code number.

8. TPS — total petroleum system name.

9. AUno-assessment-unit code number.

10. AU—assessment-unit name.

11. Data - data sources used to aid in completing the datainput form.
12. Comm - primary commodity type in the assessment unit, based on the gas-to-oil ratio of the petroleum endowment, which includes both the discovered and undiscovered petroleum. An assessment unit is characterized as being oil prone if the gas-to-oil ratio is less than 20,000 cubic feet of gas per barrel of oil; otherwise, it is gas prone.

13. Minsize MMB - minimum accumulation size considered for assessment, in million barrels of oil equivalent (MMBOE).

14. Numoil-number of oil accumulations equal to or larger than the minimum accumulation size discovered in the assessment unit.

15. Numgas - number of gas accumulations equal to or larger than the minimum accumulation size discovered in the assessment unit.

16. AUM - assessment-unit maturity, the exploration maturity of the assessment unit. Assessment-unit maturity is classified as "established" if more than 13 accumulations exceeding minimum size have been discovered, "frontier" if 1 to 13 accumulations exceeding minimum size have been discovered, or "hypothetical" if no accumulations exceeding minimum size have been discovered.

17. MedO1MMB - median size of the set of discovered oil accumulations that constitute the first one-third or one-half of the total number of oil accumulations ranked according to date of discovery within the assessment unit, in million barrels of oil (MMBO). This size is derived from known oil volumes that were adjusted upward to account for estimated future reserve growth.

18. MedO2MMB - median size of the set of discovered oil accumulations that constitute the second one-third or one-half of the total number of oil accumulations ranked according to date of discovery within the assessment unit, in million barrels of oil (MMBO). This size is derived from known oil volumes that were adjusted upward to account for estimated future reserve growth.

19. MedO3MMB - median size of the set of discovered oil accumulations that constitute the third one-third of the total number of oil accumulations ranked according to date of discovery within the assessment unit, in million barrels of oil (MMBO). This size is derived from known oil volumes that were adjusted upward to account for estimated future reserve growth.

20. MedG1BCF - median size of the set of discovered gas accumulations that constitute the first one-third or onehalf of the total number of gas accumulations ranked according to date of discovery within the assessment unit, in billion cubic feet of gas (BCFG). This size is derived from known gas volumes that were adjusted upward to account for estimated future reserve growth. 
21. MedG2BCF - median size of the set of discovered gas accumulations that constitute the second one-third or one-half of the total number of gas accumulations ranked according to date of discovery within the assessment unit, in billion cubic feet of gas (BCFG). This size is derived from known gas volumes that were adjusted upward to account for estimated future reserve growth.

22. MedG3BCF - median size of the set of discovered gas accumulations that constitute the third one-third of the total number of gas accumulations ranked according to date of discovery within the assessment unit, in billion cubic feet of gas (BCFG). This size is derived from known gas volumes that were adjusted upward to account for estimated future reserve growth.

23. Prob $1 \%$ - charge probability, the probability for adequate petroleum charge for at least one undiscovered accumulation equal to or larger than the minimum accumulation size, somewhere in the assessment unit, having the potential to be added to reserves. Charge probability is given as a fractional value from 0 to 1.0 .

24. Prob $2 \%$-rocks probability, the probability for adequate reservoirs, traps, and seals for at least one undiscovered accumulation equal to or larger than the minimum accumulation size, somewhere in the assessment unit, having the potential to be added to reserves. Rocks probability is given as a fractional value from 0 to 1.0.

25. Prob3 \% - timing probability, the probability for favorable geologic timing for at least one undiscovered accumulation equal to or larger than the minimum accumulation size, somewhere in the assessment unit, having the potential to be added to reserves. Timing probability is given as a fractional value from 0 to 1.0 .

26. Geoprob \% - geologic probability, the product of charge, rocks, and timing probabilities. Geologic probability is given as a fractional value from 0 to 1.0 .

27. NOmin - estimated minimum (F100) number of undiscovered oil accumulations equal to or larger than the minimum accumulation size in the assessment unit.

28. NOmod - estimated mode of number of undiscovered oil accumulations equal to or larger than the minimum accumulation size in the assessment unit.

29. NOmax - estimated maximum (F0) number of undiscovered oil accumulations equal to or larger than the minimum accumulation size in the assessment unit.

30. NGmin - estimated minimum (F100) number of undiscovered gas accumulations equal to or larger than the minimum accumulation size in the assessment unit.

31. NGmod - estimated mode of number of undiscovered gas accumulations equal to or larger than the minimum accumulation size in the assessment unit.
32. NGmax - estimated maximum (F0) number of undiscovered gas accumulations equal to or larger than the minimum accumulation size in the assessment unit.

33. SOminMMB - estimated minimum (F100) size, in million barrels of oil (MMBO), of undiscovered oil accumulations in the assessment unit.

34. SOmedMMB — estimated median (F50) size, in million barrels of oil (MMBO), of undiscovered oil accumulations in the assessment unit.

35. SOmaxMMB - estimated maximum (F0) size, in million barrels of oil (MMBO), of undiscovered oil accumulations in the assessment unit.

36. SGminBCF - estimated minimum (F100) size, in billion cubic feet of gas (BCFG), of undiscovered gas accumulations in the assessment unit.

37. SGmedBCF - estimated median (F50) size, in billion cubic feet of gas (BCFG), of undiscovered gas accumulations in the assessment unit.

38. SGmaxBCF - estimated maximum (F0) size, in billion cubic feet of gas (BCFG), of undiscovered gas accumulations in the assessment unit.

39. GminCF/B - estimated minimum (F100) gas-to-oil ratio (GOR), in cubic feet of gas per barrel of oil (CFG/BO), of undiscovered oil accumulations equal to or larger than the minimum accumulation size in the assessment unit.

40. GmodCF/B - estimated mode of gas-to-oil ratio (GOR), in cubic feet of gas per barrel of oil (CFG/BO), of undiscovered oil accumulations equal to or larger than the minimum accumulation size in the assessment unit.

41. GmaxCF/B - estimated maximum (F0) gas-to-oil ratio (GOR), in cubic feet of gas per barrel of oil (CFG/BO), of undiscovered oil accumulations equal to or larger than the minimum accumulation size in the assessment unit.

42. NminB/MMCF - estimated minimum (F100) natural gas liquids (NGL) to gas ratio, in barrels of natural gas liquids per million cubic feet of gas (BNGL/MMCFG), of undiscovered oil accumulations equal to or larger than the minimum accumulation size in the assessment unit.

43. NmodB/MMCF — estimated mode of natural gas liquids (NGL) to gas ratio, in barrels of natural gas liquids per million cubic feet of gas (BNGL/MMCFG), of undiscovered oil accumulations equal to or larger than the minimum accumulation size in the assessment unit.

44. NmaxB/MMCF - estimated maximum (F0) natural gas liquids (NGL) to gas ratio, in barrels of natural gas liquids per million cubic feet of gas (BNGL/MMCFG), of undiscovered oil accumulations equal to or larger than the minimum accumulation size in the assessment unit. 
45. LminB/MMCF — estimated minimum (F100) liquids (oil plus natural gas liquids) to gas ratio (LGR), in barrels of liquids per million cubic feet of gas (BL/MMCFG), of undiscovered gas accumulations equal to or larger than the minimum accumulation size in the assessment unit.

46. LmodB/MMCF — estimated mode of liquids (oil plus natural gas liquids) to gas ratio (LGR), in barrels of liquids per million cubic feet of gas (BL/MMCFG), of undiscovered gas accumulations equal to or larger than the minimum accumulation size in the assessment unit.

47. LmaxB/MMCF — estimated maximum (F0) liquids (oil plus natural gas liquids) to gas ratio (LGR), in barrels of liquids per million cubic feet of gas (BL/MMCFG), of undiscovered gas accumulations equal to or larger than the minimum accumulation size in the assessment unit.

48. APImin deg-estimated minimum (F100) American Petroleum Institute gravity, in degrees, of oil in undiscovered oil accumulations in the assessment unit.

49. APImod deg — estimated mode of American Petroleum Institute gravity, in degrees, of oil in undiscovered oil accumulations in the assessment unit.

50. APImax deg - estimated maximum (F0) American Petroleum Institute gravity, in degrees, of oil in undiscovered oil accumulations in the assessment unit.

51. Smin \%-estimated minimum (F100) sulfur (S) content, in percent, of oil in undiscovered oil accumulations in the assessment unit.

52. Smod \%-estimated mode of sulfur (S) content, in percent, of oil in undiscovered oil accumulations in the assessment unit.

53. Smax \%-estimated maximum (F0) sulfur (S) content, in percent, of oil in undiscovered oil accumulations in the assessment unit.

54. ODmin $\mathrm{m}$ - estimated minimum (F100) drilling depth, in meters, of oil in untested cells in oil assessment units.

55. ODf75 m-estimated 75th fractile (F75) of drilling depth, in meters, of oil in untested cells in oil assessment units.

56. ODmod $\mathrm{m}$ - estimated mode of drilling depth, in meters, of oil in untested cells in oil assessment units.

57. ODf $25 \mathrm{~m}$ - estimated 25th fractile (F25) drilling depth, in meters, of oil in untested cells in oil assessment units.

58. ODmax $\mathrm{m}$ - estimated maximum (F0) drilling depth, in meters, of oil in untested cells in oil assessment units.

59. OWDmin $\mathrm{m}$ - estimated minimum (F100) water depth, in meters, of oil in untested cells in oil assessment units (ocean, bays, or lakes; if applicable).
60. OWDmod $\mathrm{m}$ - estimated mode of water depth, in meters, of oil in untested cells in oil assessment units (ocean, bays, or lakes; if applicable).

61. OWDmax m-estimated maximum (F0) water depth, in meters, of oil in untested cells in oil assessment units (ocean, bays, or lakes; if applicable).

62. IGmin \%-estimated minimum (F100) inert (IG) gas content, in percent, of gas in untested cells in gas assessment units (nitrogen and helium, and so forth).

63. IGmod \%-estimated mode of inert gas (IG) content, in percent, of gas in untested cells in gas assessment units (nitrogen and helium, and so forth).

64. IGmax \%-estimated maximum (F0) inert gas (IG) content, in percent, of gas in untested cells in gas assessment units (nitrogen and helium, and so forth).

65. CO2min \%-estimated minimum (F100) carbon dioxide $\left(\mathrm{CO}_{2}\right)$ content, in percent, of gas in untested cells in gas assessment units.

66. $\mathrm{CO} 2 \mathrm{mod} \%$ - estimated mode of carbon dioxide $\left(\mathrm{CO}_{2}\right)$ content, in percent, of gas in untested cells in gas assessment units.

67. $\mathrm{CO} 2 \mathrm{max} \%$ - estimated maximum (F0) carbon dioxide $\left(\mathrm{CO}_{2}\right)$ content, in percent, of gas in untested cells in gas assessment units.

68. H2Smin \%-estimated minimum (F100) hydrogen sulfide $\left(\mathrm{H}_{2} \mathrm{~S}\right)$ content, in percent, of gas in untested cells in gas assessment units.

69. H2Smod \%-estimated mode of hydrogen sulfide $\left(\mathrm{H}_{2} \mathrm{~S}\right)$ content, in percent, of gas in untested cells in gas assessment units.

70. H2Smax \% - estimated maximum (F0) hydrogen sulfide $\left(\mathrm{H}_{2} \mathrm{~S}\right)$ content, in percent, of gas in untested cells in gas assessment units.

71. GDmin $\mathrm{m}$ - estimated minimum (F100) drilling depth, in meters, of gas in untested cells in gas assessment units.

72. GDf25 m-estimated 25th fractile (F25) drilling depth, in meters, of gas in untested cells in gas assessment units.

73. GDmod $\mathrm{m}$ - estimated mode of drilling depth, in meters, of gas in untested cells in gas assessment units.

74. GDf25 $\mathrm{m}$ - estimated 25th fractile (F25) drilling depth, in meters, of gas in untested cells in gas assessment units.

75. GDmax m-estimated maximum (F0) drilling depth, in meters, of gas in untested cells in gas assessment units.

76. GWDmin m-estimated minimum (F100) water depth, in meters, of gas in untested cells in gas assessment units (ocean, bays, or lakes; if applicable). 
77. GWDmod m-estimated mode of water depth, in meters, of gas in untested cells in gas assessment units (ocean, bays, or lakes; if applicable).

78. GWDmax m-estimated maximum (F0) water depth, in meters, of gas in untested cells in gas assessment units (ocean, bays, or lakes; if applicable).

Data in the kvol\#\#\#\#.tab table are derived from a commercially purchased database (NRG Associates, 2009) and are current through 2007. This table contains 15 columns. Blank cells represent no data. Data columns for these files are as follows:

1. Code-assessment-unit code number.

2. Name-assessment-unit name.

3. Numres - number of reported reservoirs in the assessment unit.

4. CUMOIL MMB - cumulative production of oil from the reported reservoirs in the assessment unit. This volume is in million barrels of oil (MMBO).

5. REMOIL MMB - remaining oil reserves in the reported reservoirs in the assessment unit. This volume is in million barrels of oil (MMBO).

6. KROIL MMB - known recoverable oil volume (cumulative production plus remaining reserves) from the reported reservoirs in the assessment unit. This volume is in million barrels of oil (MMBO).

7. CUMGAS BCF - cumulative production of natural gas from the reported reservoirs in the assessment unit. This volume is in billion cubic feet of gas (BCFG).

8. REMGAS BCF-remaining natural gas reserves in the reported reservoirs in the assessment unit. This volume is in billion cubic feet of gas (BCFG).

9. KRGAS BCF — known recoverable natural gas volume (cumulative production plus remaining reserves) from the reported reservoirs in the assessment unit. This volume is in billion cubic feet of gas (BCFG).

10. CUMNGL MMB - cumulative production of natural gas liquids (NGL) from the reported reservoirs in the assessment unit. This volume is in million barrels of natural gas liquids (MMBNGL).

11. REMNGL MMB — remaining natural gas liquids (NGL) reserves in the reported reservoirs in the assessment unit. This volume is in million barrels of natural gas liquids (MMBNGL).

12. KRNGL MMB — known recoverable natural gas liquids (NGL) volume (cumulative production plus remaining reserves) from the reported reservoirs in the assessment unit. This volume is in million barrels of natural gas liquids (MMBNGL).

13. CUMPET MMB - cumulative production of petroleum (oil, gas, and NGL) from the reported reservoirs in the assessment unit. This volume is in million barrels of oil equivalent (MMBOE).

14. REMPET MMB - remaining petroleum (oil, gas, and NGL) reserves in the reported reservoirs in the assessment unit. This volume is in million barrels of oil equivalent (MMBOE).

15. KRPET MMB - known recoverable petroleum (oil, gas, and NGL) volume (cumulative production plus remaining reserves) from the reported reservoirs in the assessment unit. This volume is in million barrels of oil equivalent (MMBOE).

\section{References Cited}

Charpentier, R.R., and Klett, T.R., 2005, A Monte Carlo simulation method for the assessment of undiscovered, conventional oil and gas, chap. 21, in USGS Southwestern Wyoming Province Assessment Team, eds., Petroleum systems and geologic assessment of oil and gas in the Southwestern Wyoming Province, Wyoming, Colorado, and Utah: U.S. Geological Survey Digital Data Series DDS-69-D, 5 p. [available at http://energy.usgs.gov/OilGas/AssessmentsData/ NationalOilGasAssessment/Methodology.aspx].

Crovelli, R.A., 2005, Analytic resource assessment method for continuous petroleum accumulations - The ACCESS assessment method, chap. 22, in USGS Southwestern Wyoming Province Assessment Team, eds., Petroleum systems and geologic assessment of oil and gas in the Southwestern Wyoming Province, Wyoming, Colorado, and Utah: U.S. Geological Survey Digital Data Series DDS-69-D, 10 p. [available at http://energy.usgs.gov/OilGas/AssessmentsData/ NationalOilGasAssessment/Methodology.aspx].

IHS Energy Group, 2010a [includes data current as of December 2010], PI/Dwights Plus US production data: IHS Energy Group, database available from IHS Energy Group, 15 Inverness Way East, D205, Englewood, CO 80112.

IHS Energy Group, 2010b [includes data current as of December 2010], PI/Dwights Plus US well data: IHS Energy Group; database available from IHS Energy Group, 15 Inverness Way East, D205, Englewood, CO 80112.

Klett, T.R., and Charpentier, R.R., 2003, FORSPAN model users guide: U.S. Geological Survey Open-File Report 03-354, 30 p. [available at http://energy.usgs.gov/OilGas/AssessmentsData/ NationalOilGasAssessment/Methodology.aspx]. 
Klett, T.R., Schmoker, J.W., and Charpentier, R.R., 2005, U.S. Geological Survey input-data form and operational procedure for the assessment of conventional petroleum accumulations, chap. 20, in USGS Southwestern Wyoming Province Assessment Team, eds., Petroleum systems and geologic assessment of oil and gas in the Southwestern Wyoming Province, Wyoming, Colorado, and Utah: U.S. Geological Survey Digital Data Series DDS-69-D, 7 p. [available at http://energy.usgs.gov/OilGas/AssessmentsData/ NationalOilGasAssessment/Methodology.aspx].

NRG Associates, 2009 [includes data current as of December 2007], The significant oil and gas fields of the United States: NRG Associates, Inc., database available from NRG Associates, Inc., P.O. Box 1655, Colorado Springs, CO 80901.

Schmoker, J.W., and Klett, T.R., 2005, U.S. Geological Survey assessment concepts for conventional petroleum accumulations, chap. 19, in USGS Southwestern Wyoming Province Assessment Team, eds., Petroleum systems and geologic assessment of oil and gas in the Southwestern Wyoming Province, Wyoming, Colorado, and Utah: U.S. Geological Survey Digital Data Series DDS-69-D, 6 p. [available at http://energy.usgs.gov/OilGas/AssessmentsData/ NationalOilGasAssessment/Methodology.aspx]. 


\section{Chapter 13 \\ Precambrian to Ground Surface Grid Cell Maps and 3D Model of the Anadarko Basin Province}

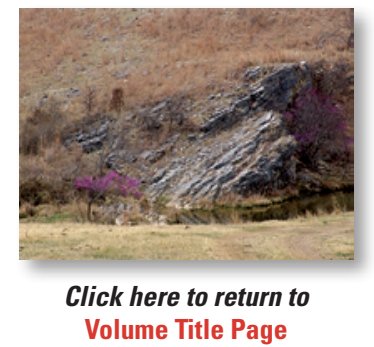

By Debra K. Higley, Nicholas J. Gianoutsos, Michael P. Pantea, and Sean M. Strickland

Chapter 13 of 13

Petroleum Systems and Assessment of Undiscovered Oil and Gas in the Anadarko Basin Province, Colorado, Kansas, Oklahoma, and Texas-USGS Province 58

Compiled by Debra K. Higley

U.S. Geological Survey Digital Data Series DDS-69-EE 


\title{
U.S. Department of the Interior SALLY JEWELL, Secretary
}

\section{U.S. Geological Survey \\ Suzette M. Kimball, Acting Director}

\author{
U.S. Geological Survey, Reston, Virginia: 2014
}

For more information on the USGS - the Federal source for science about the Earth, its natural and living resources, natural hazards, and the environment, visit http://WWW.usgs.gov or call 1-888-ASK-USGS.

For an overview of USGS information products, including maps, imagery, and publications, visit http://WwW.usgs.gov/pubprod

To order this and other USGS information products, visit http://store.usgs.gov

Any use of trade, firm, or product names is for descriptive purposes only and does not imply endorsement by the U.S. Government.

Although this information product, for the most part, is in the public domain, it also may contain copyrighted materials as noted in the text. Permission to reproduce copyrighted items must be secured from the copyright owner.

Suggested citation:

Higley, D.K., Gianoutsos, N.J., Pantea, M.P., and Strickland, S.M., 2014, Precambrian to ground surface grid cell maps and 3D model of the Anadarko Basin Province, chap. 13, in Higley, D.K., compiler, Petroleum systems and assessment of undiscovered oil and gas in the Anadarko Basin Province, Colorado, Kansas, Oklahoma, and Texas-USGS Province 58: U.S. Geological Survey Digital Data Series DDS-69-EE, 8 p., http://dx.doi.org/10.3133/ds69EE.

ISSN 2327-638X (online) 


\section{Contents}

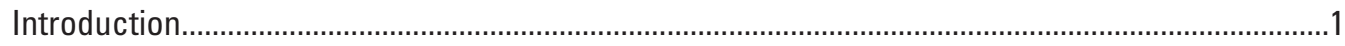

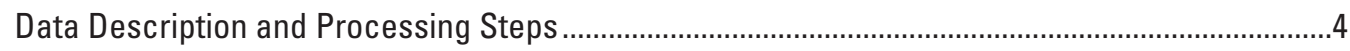

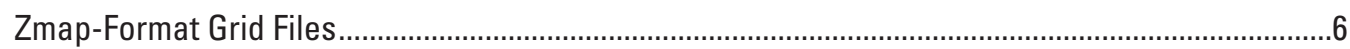

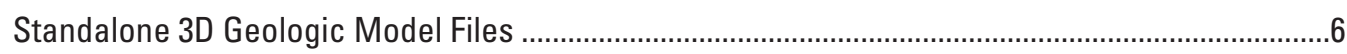

Computer Requirements to View the 3D EarthVision ${ }^{\circledR}$ Geologic Model ..........................................6

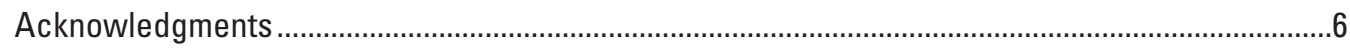

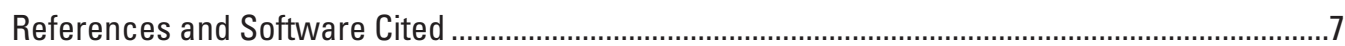

\section{Figures}

1. Geographic extent of grid files as displayed by basement heat flow contours across the Anadarko Basin Province based on data from Carter and others (1998), Blackwell and Richards (2004), and data downloads from the Southern Methodist University Web site (http://smu.edu/geothermal/). Basin areas north of the Wichita Mountain uplift and in the Amarillo uplift and northward exhibit generally lower heat flows than other basin areas. Highest measured heat flow is in the northwest, along the Las Animas uplift. The northwest-trending Central Kansas uplift (CKU) also exhibits elevated heat flow values. Heat flow units are milliwatts per square meter $\left(\mathrm{mW} / \mathrm{m}^{2}\right)$. Precambrian faults (red lines) are from Adler and others (1971)

2. View to the southeast showing the Woodford Shale layer and modeled lithofacies. Vertical exaggeration is 18 times. Extent of the Woodford Shale is shown in white. This PetroMod ${ }^{\circledR}$ image shows underlying and lateral formations and facies changes for the Woodford Shale layer. The southern half of the Kansas portion has almost 0 meter thickness and represents grid extrapolation from the Woodford (Chattanooga) Shale east of the Central Kansas uplift (CKU) to the Woodford Shale proximal to the Kansas-Oklahoma border. Lateral lithofacies are primarily limestone and dolomite of the Viola Group. Because the purpose of this image is to show lateral changes in formation and lithofacies assignments on a model layer, this information is generalized in the legend and not all listed formations are visible. Vertical yellow bars are Precambrian faults from Adler and others (1971) 


\section{Tables}

1. Two-dimensional grid file names, times intervals of deposition and erosion in millions of years ago (Ma), and lithofacies assignments. [Grids represent the highest elevation of the named unit relative to sea level. Lithofacies that were assigned in the PetroMod® v. 11.3 software are included for archival purposes and names are not defined, merely labeled with general terms. Lithology names that are similar to layer names are custom lithofacies based on published distributions of facies or compositions that are mainly derived from sources that include Adler and others (1971), Denison and others (1984), Howery (1993), Ludvigson and others (2009), and the National Geologic Map Database (2011, http://ngmdb.usgs.gov/Geolex/].

2. Geographic coordinate information for the zmap-format two-dimensional grid files is located in the ZmapFormatGridFiles folder. [All grid $\mathrm{x}$ and $\mathrm{y}$ dimensions are in meters and the grid spacings are 1 kilometer. Grid size refers to the total number of grid cells. Structure and erosional isopach grids z dimension is in feet relative to sea level. Contour values for total organic carbon (TOC) are weight percent carbon, and for basement heat flow are milliwatts per square meter $\left.\left(\mathrm{mW} / \mathrm{m}^{2}\right)\right]$ 


\title{
Precambrian to Ground Surface Grid Cell Maps and 3D Model of the Anadarko Basin Province
}

\author{
By Debra K. Higley, Nicholas J. Gianoutsos, Michael P. Pantea, and Sean M. Strickland
}

\section{Introduction}

The digital files listed in table 1 were compiled as part of the U.S. Geological Survey (USGS) 2010 assessment of the undiscovered oil and gas potential of the Anadarko Basin Province of western Oklahoma, western Kansas, northern Texas, and southeastern Colorado. This publication contains a three-dimensional (3D) geologic model that was constructed of two-dimensional (2D) structural surface grids across the province and Precambrian fault surfaces generated from Adler and others (1971). Also included are (1) 26 zmap-format structure grid files on Precambrian to present-day surfaces across the province; (2) estimated eroded thickness of strata following the Laramide orogeny and based on one-dimensional (1D) models and 1D extractions from the four-dimensional (4D) PetroMod ${ }^{\circledR}$ model (Schlumberger, 2011; Higley, 2014); (3) present-day weight percent total organic carbon (TOC) for the Woodford Shale based on TOC data from Burruss and Hatch (1989) and mean values from Hester and others (1990); and (4) basement heat flow contours (fig. 1) across the province based on data from Carter and others (1998), Blackwell and Richards (2004), and data downloads from the Southern Methodist University Web site (http://smu.edu/geothermal/).

The 3D geologic model and 2D grids were created using EarthVision $^{\circledR}$ software [Dynamic Graphics Inc. (DGI), 2010] and grids were saved in zmap format. Lateral scales of the 3D model and all grids are in meters, and vertical scales of the structure and eroded thickness grids and model are in feet. TOC grid values are weight percent (wt \%) and the heat flow grid is milliwatts per square meter $\left(\mathrm{mW} / \mathrm{m}^{2}\right)$ (fig. 1). The age range represented by the stratigraphic intervals composing the grid files is 1,600 million years ago $(\mathrm{Ma})$ to present day. File names and age ranges of deposition and erosion are listed in table 1 . These time period assignments are generalized because of the lack of precise information regarding formation ages; there are no time overlaps because of modeling software requirements.

Metadata associated with this publication are within the AnadarkoMetadata.xml, AnadarkoMetadata.doc, and AnadarkoMetadata.htm files. Included are information on the study area and the names of the zmap-format grid files, such as file name and type, geographic coordinates of the grids and 3D model (table 2), and background information on the files in this publication. 
Table 1. Two-dimensional grid file names, times intervals of deposition and erosion in millions of years ago (Ma), and lithofacies assignments.

[Grids represent the highest elevation of the named unit relative to sea level. Lithofacies that were assigned in the PetroMod ${ }^{\circledR}$ v. 11.3 software are included for archival purposes and names are not defined, merely labeled with general terms. Lithology names that are similar to layer names are custom lithofacies based on published distributions of facies or compositions that are mainly derived from sources that include Adler and others (1971), Denison and others (1984), Howery (1993), Ludvigson and others (2009), and the National Geologic Map Database (2011, http://ngmdb.usgs.gov/Geolex/)]

\begin{tabular}{|c|c|c|c|c|c|c|c|}
\hline \multirow{2}{*}{$\begin{array}{c}\text { Number } \\
1\end{array}$} & \multirow{2}{*}{$\begin{array}{c}\begin{array}{c}\text { Zmap } \\
\text { file name }\end{array} \\
\text { surfaceDEMft.DAT }\end{array}$} & \multicolumn{2}{|c|}{$\begin{array}{c}\text { Deposition age } \\
\text { from (Ma) to (Ma) }\end{array}$} & \multicolumn{2}{|c|}{$\begin{array}{c}\text { Erosion age } \\
\text { from (Ma) to (Ma) }\end{array}$} & \multirow{2}{*}{$\begin{array}{c}\text { Total petroleum system } \\
\text { assignment }\end{array}$} & \multirow[t]{2}{*}{$\begin{array}{c}\text { Lithofacies value } \\
\text { Assigned in PetroMod }{ }^{\circledR} \text { (Part) }\end{array}$} \\
\hline & & 1.83 & 1.8 & 1.8 & 0 & & \\
\hline 2 & OgallalaTft.DAT & 5.3 & 1.83 & 0 & 0 & Overburden rock & Sandstone (clay poor) \\
\hline 3 & CretaceousTft.DAT & 250 & 33 & 33 & 5.3 & Overburden rock & Sandstone (clay rich) \\
\hline 4 & PermianTft.DAT & 255 & 252 & 252 & 250 & Overburden rock & Sandstone (subarkose, quartz rich) \\
\hline 5 & BlaineTft.DAT & 261 & 255 & 0 & 0 & Seal rock & Blaine (custom lithologies) \\
\hline 6 & StoneCorralTft.DAT & 266 & 261 & 0 & 0 & Seal rock & Limestone (shaly) \\
\hline 7 & WellingtonTft.DAT & 271 & 266 & 0 & 0 & Seal rock & Wellington (custom lithologies) \\
\hline 8 & ChaseTft.DAT & 285 & 271 & 0 & 0 & Reservoir rock & Limestone (shaly) \\
\hline 9 & CouncilGroveTft.DAT & 296 & 285 & 0 & 0 & Reservoir rock & Limestone (shaly) \\
\hline 10 & WabaunseeTft.DAT & 298.2 & 296 & 0 & 0 & Reservoir rock & Siltstone (organic lean) \\
\hline 11 & HeebnerTTft.DAT & 298.4 & 298.2 & 0 & 0 & Seal rock & Shale (organic lean, typical) \\
\hline 12 & HeebnerBTft.DAT & 298.5 & 298.4 & 0 & 0 & Seal rock & Siltstone (organic lean) \\
\hline 13 & DouglasTft.DAT & 304 & 298.5 & 0 & 0 & Reservoir rock & Sandstone (typical) \\
\hline 14 & DesmoinesianTft.DAT & 305.3 & 304 & 0 & 0 & Reservoir rock & Limestone (shaly) \\
\hline 15 & CherokeeTft.DAT & 308 & 305.3 & 0 & 0 & Reservoir rock & Limestone (shaly) \\
\hline 16 & AtokanTft.DAT & 310 & 308 & 0 & 0 & Reservoir rock & SHALEcarb \\
\hline 17 & ThirteenFingerTft.DAT & 311 & 310 & 0 & 0 & Source rock & Limestone (shaly) \\
\hline 18 & MorrowTft.DAT & 324 & 311 & 0 & 0 & Reservoir, seal rock & SHALEsilt, Sandstone (clay poor) \\
\hline 19 & SpringerTft.DAT & 354 & 330 & 330 & 324 & Reservoir rock & Limestone (shaly) \\
\hline 20 & WoodfordTft.DAT & 369 & 354 & 0 & 0 & Source rock & Woodford (custom lithologies) \\
\hline 21 & HuntonTft.DAT & 442 & 379 & 379 & 369 & Reservoir rock & Limestone (shaly), Dolomite (typical) \\
\hline 22 & SylvanTft.DAT & 445 & 442 & 0 & 0 & Seal rock & Shale (black) \\
\hline 23 & ViolaTft.DAT & 456 & 445 & 0 & 0 & Reservoir rock & Limestone (organic rich - typical), Dolomite (typical), SHALEsilt \\
\hline 24 & SimpsonTft.DAT & 471 & 456 & 0 & 0 & Reservoir, source rock & SHALEsilt, Sandstone (quartzite, very quartz rich) \\
\hline 25 & ArbuckleTft.DAT & 520 & 476 & 0 & 0 & Reservoir rock & Limestone (organic rich - typical) \\
\hline 26 & PrecambrianTft.DAT & 1,600 & 544 & 544 & 520 & Underburden rock & Granite (greater than 1,000 Ma old), Rhyolite \\
\hline 27 & CreataceousErosionThickft.DAT & & 33 & 5.3 & & & \\
\hline 28 & HeatFlow_mWm2.DAT & & & & & & \\
\hline 29 & WoodfordTOC.DAT & & & & & & \\
\hline
\end{tabular}




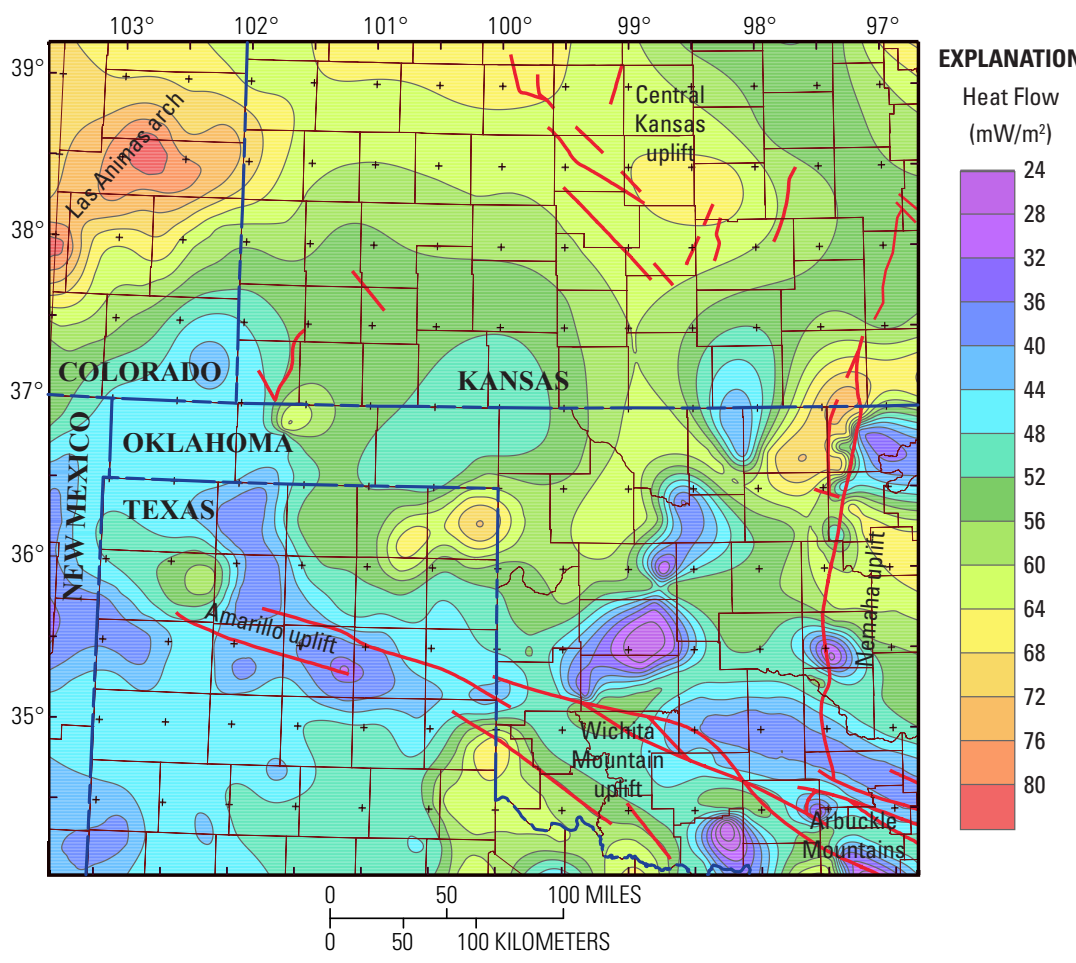

Figure 1. Geographic extent of grid files as displayed by basement heat flow contours across the Anadarko Basin Province based on data from Carter and others (1998), Blackwell and Richards (2004), and data downloads from the Southern Methodist University Web site (http://smu.edu/geothermal/). Basin areas north of the Wichita Mountain uplift and in the Amarillo uplift and northward exhibit generally lower heat flows than other basin areas. Highest measured heat flow is in the northwest, along the Las Animas uplift. The northwest-trending Central Kansas uplift (CKU) also exhibits elevated heat flow values. Heat flow units are milliwatts per square meter $\left(\mathrm{mW} / \mathrm{m}^{2}\right)$. Precambrian faults (red lines) are from Adler and others (1971).

Table 2. Geographic coordinate information for the zmap-format two-dimensional grid files is located in the ZmapFormatGridFiles folder.

[All grid $\mathrm{x}$ and $\mathrm{y}$ dimensions are in meters and the grid spacings are 1 kilometer. Grid size refers to the total number of grid cells. Structure and erosional isopach grids $\mathrm{z}$ dimension is in feet relative to sea level. Contour values for total organic carbon (TOC) are weight percent carbon, and for basement heat flow are milliwatts per square meter $\left.\left(\mathrm{mW} / \mathrm{m}^{2}\right)\right]$

Geographic coordinate system

World Geodetic System (WGS)

First and second standard parallels (degrees, north)

Central meridian (degrees, west)

Latitude of origin (degrees, north)

Semi-major axis, Semi-minor axis

False Northing, False easting

$\mathrm{X}$ and $\mathrm{Y}$ dimensions of grid files

Xminimum, Yminimum, grid cell size

Xmaximum, Ymaximum, grid cell size

\author{
Lambert Conformal Conic \\ WGS 84 ellipsoid \\ 35 \\ 99 \\ 35
}

6378137

0

6356752.3142

0

\begin{tabular}{rrr}
-400000.0000 & -100000.0000 & 601 \\
200000.0000 & 475000.0000 & 576 \\
\hline
\end{tabular}




\section{Data Description and Processing Steps}

1. Elevation, thickness, and fault data sources for the 2D grids and 3D model include formation tops from more than 220 wells across the province, edited formation tops from IHS Energy (2009a, 2009b) and the Kansas Geological Survey (2010, http://www.kgs.ku.edu/PRS/petroDB. html), and maps and data from Fay (1964), Adler and others (1971), Rascoe and Hyne (1987), Robbins and Keller (1992), Cederstrand and Becker (1998), Andrews (1999a, 1999b, 2001), and Rottmann (2000a, 2000b). Sources of ground elevations for 2D grids were well records and digital elevation model (DEM) data. Locations of formation outcrops/subcrops were derived primarily from surface geologic maps of the region and Rascoe and Hyne (1987). Formation ages and lithologies are commonly generalized; sources of information include Adler and others (1971), Denison and others (1984), Howery (1993), Ludvigson and others (2009), and the National Geologic Map Database (2011, http://ngmdb.usgs.gov/Geolex/).

2. Names and age ranges of formations change within and across the Anadarko Basin Province; consequently, data retrievals were based mainly on approximate age-equivalent units. Data files were edited using Environmental Systems Research Institute (ESRI) (2010) ArcMap ${ }^{\text {tm }}$ and Dynamic Graphics, Inc. (2010) EarthVision ${ }^{\circledR}$ software to remove anomalies, examples of which include location errors and incorrect formation-top elevations. Maps generated with EarthVision ${ }^{\circledR}$ software were compared to published cross sections and maps, and anomalous surfaces were corrected by editing the scattered data files and regridding the files.

3. This chapter of the report contains fault trace and volume views of a standalone 3D geologic model of the study area. The model can be viewed and manipulated, and .jpg or tiff images of user-defined views can be saved. Both a basic "getting started" and detailed help file were provided by Dynamic Graphics, Inc., and are in the 3Dviewer_HelpFiles folder as aids to understanding the included 3D viewer. The included 3D viewer is designed to work with the Microsoft Windows operating system. The USGS has licensed from Dynamic Graphics, Inc., the rights to provide an encrypted model that allows the viewers to use the enclosed data sets and interpreted model. The license allows the USGS the service and rights to provide unlimited distribution. We designed this product to function from the DVD-ROM media but recommend that the necessary files be copied to a local hard drive for better performance. No additional installation programs are needed to view the model and datasets using the 3D standalone viewer. Should there be error messages when starting the software that reference the Microsoft $\mathrm{C}++$ libraries; selecting "OK" several times will start the software. The folder "bug fix" includes a possible fix for this error message problem and is provided as a courtesy by Dynamic Graphics, Inc. More information about the viewing software and EarthVision ${ }^{\circledR}$ may be obtained from Dynamic Graphics, Inc., at http://www.dgi.com/.

4. The extent and elevation of model layers in highly faulted and deformed areas is not well documented or constrained. For that reason, surfaces on and south of the Wichita Mountain and Amarillo uplifts should be considered erroneous. The modeling software requires all grids to extend to the map boundaries, even if the modeled strata are only present within a portion of the layer. The shallowest elevation of the immediately underlying surface is included for strata with limited geographic range. For example, the Woodford Shale is only located in the deep part of the Anadarko Basin of Texas and Oklahoma and in a portion east of the Central Kansas uplift (fig. 2), but the structure grid of this surface also includes Precambrian through Silurian "subcrops."

5. 2D grids downloadable from this publication and used to build the standalone 3D models were generated using the Dynamic Graphics, Inc. EarthVision ${ }^{\circledR}$ Briggs Biharmonic Spline algorithm. Horizontal scales are in meters. Coordinate information is provided in table 2, grid file headers, and the metadata files. The $\mathrm{x}$ and $\mathrm{y}$ grid spacing are both 1,000 meters. As many as 15 data values were evaluated from each grid node and a scattered data feedback algorithm follows each biharmonic iteration. These modeling steps result in the curvature of the surface being distributed between data points rather than concentrated at individual data points. This generates a more natural appearing modeled surface of the modeled grid nodes that accurately reflect the scattered data. Grids generated for this publication were not smoothed or filtered. More information on this process and software are available from Dynamic Graphics, Inc., at http://www.dgi.com. Volumes of units are defined and shown as the space between (1) two geologic surfaces, (2) geologic surfaces and fault planes, or (3) geologic surfaces and model extents.

For the Earthvision ${ }^{\circledR}$ 3D model, faults were defined as extending from Precambrian basement to the ground surface. Due to modeling and time constraints, most intersecting faults were designated as vertical and thoroughgoing. Modeled faults were added sequentially as follows: (1) faults that cross the model, (2) faults that truncated other faults, and (3) faults to help show the basin geometry. Where data or details were missing, data points were extrapolated from known data points based on local thickness of modeled units or fault displacements. For example, if the only local data control for a surface was a contact on the geologic map, we used that $\mathrm{X}, \mathrm{Y}$, and $\mathrm{Z}$ value and calculated local overlying and (or) underlying $\mathrm{Z}$-surface-elevation values based on thickness. Some thickness and surface variations shown in the model may reflect additional small faulting or inherent uncertainties of defined picks from the data, but 


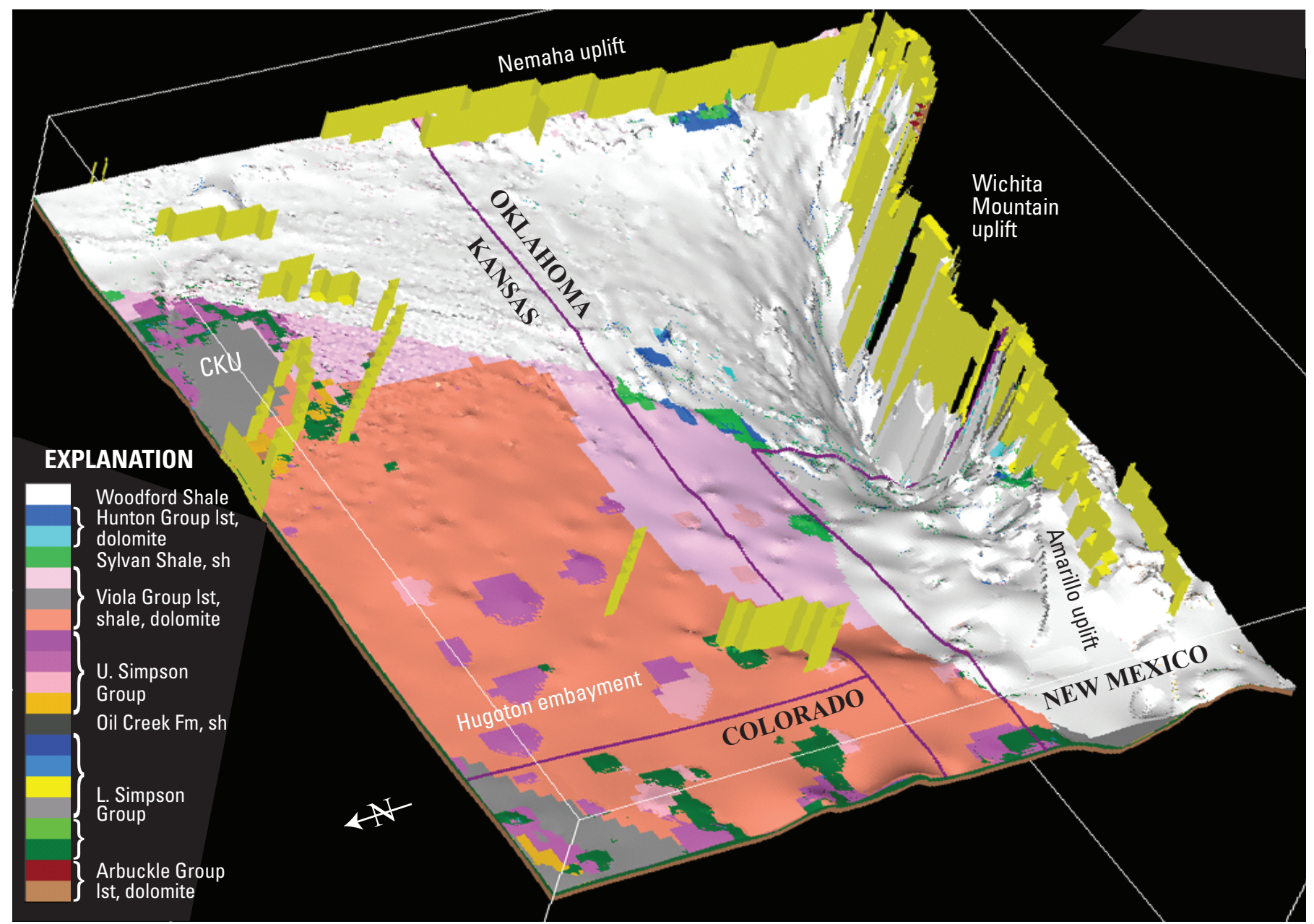

Figure 2. View to the southeast showing the Woodford Shale layer and modeled lithofacies. Vertical exaggeration is 18 times. Extent of the Woodford Shale is shown in white. This PetroMod ${ }^{\circledR}$ image shows underlying and lateral formations and facies changes for the Woodford Shale layer. The southern half of the Kansas portion has almost 0 meter thickness and represents grid extrapolation from the Woodford (Chattanooga) Shale east of the Central Kansas uplift (CKU) to the Woodford Shale proximal to the Kansas-Oklahoma border. Lateral lithofacies are primarily limestone and dolomite of the Viola Group. Because the purpose of this image is to show lateral changes in formation and lithofacies assignments on a model layer, this information is generalized in the legend and not all listed formations are visible. Vertical yellow bars are Precambrian faults from Adler and others (1971).

are considered to be reasonable interpretations based on objective criteria in surface maps, lithological descriptions, and geophysical interpretations.

The top of the Precambrian basement is the lowermost modeled unit and was used as a base for the deep fault structures in the 3D geologic model. This was necessary because the EarthVision ${ }^{\circledR}$ D modeling technique builds the geologic layers upward from the base, and fault displacement propagates vertically until other data are available or some model extent or boundary is reached. Geologic surface data were then edited proximal to the faults to generate clean fault scarps. This was necessary because converting grid files to $\mathrm{X}, \mathrm{Y}$, and $\mathrm{Z}$ data files commonly places data points on the fault scarps, which EarthVision $^{\circledR}$ tries to interpret as geologic surfaces. This process and the associated 3D EarthVision ${ }^{\circledR}$ model were created subsequent to the PetroMod ${ }^{\circledR}$ zmap-format grid files in this publication, most of which have different terminations against the southern fault system. The PetroMod ${ }^{\circledR} 4 \mathrm{D}$ petroleum system model of the Anadarko Basin includes a modeled sequence of northward-stepping vertical faults along the Amarillo-Wichita Mountains uplift that are connected laterally at the tops and bases. Vertically curved faults are not an option with PetroMod ${ }^{\circledR}$.

6. Negative isopach values can be present in grid files in areas where data are lacking, in which case negative thickness values were replaced by zero or 2 meter thickness because of requirements of the EarthVision ${ }^{\circledR}$ and Pet$\operatorname{roMod}^{\circledR}$ modeling software. Identical structural surfaces 
result in a mottled appearance in the EarthVison ${ }^{\circledR} 3 \mathrm{D}$ model because the software defines these intersections as a contact with a resulting black line. Grids were modified to exceed 1 foot in thickness in order to minimize identical surfaces. This process can result in the disappearance of units that are only a few feet thick.

\section{Zmap-Format Grid Files}

In table 1, names are listed for the 29 zmap-format grid files associated with this publication. Also included for archival purposes are PetroMod ${ }^{\circledR}$ model assignments of time periods of deposition and erosion, total petroleum system(s), and generalized lithofacies(s). Each PetroMod ${ }^{\circledR}$ structure grid contains at least one lithology but may have multiple assigned lithologies. These are represented by lateral changes in color within a model layer, such as is shown in figure 2 .

Zmap-format grids include file headers with (1) a comment section with original file names and locations, file creation date and time; and (2) original file name and folder, file type, grid spacing, and coordinate information. The file structure is a series of rows and columns with values listed for each grid cell. Included data and coordinates are incorporated in maps and models by using software that reads zmap-format files. Software programs are available to import and convert zmap-format files. These grid formats can be read by EarthVision ${ }^{\circledR}$, ArcMap $^{\circledR}$, and PetroMod ${ }^{\circledR}$, as well as other mapping and modeling software. Metadata are saved in text (.txt) and XML (.xml) formats, the latter of which is readable using ESRI ArcGIS ${ }^{\circledR}$ and some XML, WWW, and word-processing software.

\section{Standalone 3D Geologic Model Files}

There are three standalone EarthVision ${ }^{\circledR}$ 3D geologic models, which are opened by double-clicking on the open_viewer.bat file located within the 3DgeologicModel folder and then selecting one of the three ".faces" files below. Background information on PC requirements, loading, opening, viewing, and manipulating the models is located within the Demo3DViewer.pdf and QuickHelp.pdf files located in the 3Dviewer_HelpFiles. Because the standalone model uses considerable processing power, the 3DgeologicModel folder should be moved to a computer hard drive before opening the model.

1. 5_3_11_hor.sliced.encn.faces-Model comprises the 26 structural surfaces listed in table 1. Also displayed are vertical red bands that depict the Precambrian faults of Adler and others (1971).

2. 5_3_11_hor.sliced.fault.encn.faces_-Precambrian fault traces are treated as vertical faults, as opposed to incorporating the structural dips of these complex fault systems.
3. 5 3 11 hor.sliced.surf.encn.faces-Within this model, the Precambrian fault traces (gray) are vertical and extrapolated across the model to intersect other fault systems.

\section{Computer Requirements to View the 3D EarthVision ${ }^{\circledR}$ Geologic Model}

\section{Windows $®$ XP or Windows 7 Operating System Graphics Card Recommendations}

- An OpenGL capable graphics card with dedicated memory is required.

- We recommend the graphics card have at least 512MB of memory onboard.

- Some large monitors (30-inch or greater) require a dual-link DVI capable connector.

- DGI recommends graphics cards from the NVIDIA Quadro FX series (NVIDIA Quadro FX with at least $512 \mathrm{MB}$ of memory) for use with its software.

\section{Computer Processor Unit (CPU) Requirements}

- Time to open, view, and manipulate a model is partially dependent on the processor speed of the PC CPU.

- Although most of DGI's software does not currently take advantage of multiple CPUs/Cores, their presence will allow running more software simultaneously without impacting performance.

- CPUs designed for lower power solutions (UltraLow Voltage [ULV] or Consumer Ultra-Low Voltage [CULV]) are not recommended at this time as they are optimized for decreased power consumption, rather than performance.

\section{Memory Requirements}

- 4GB memory minimum

- For 32-bit systems, $4 \mathrm{~GB}$ is recommended. This is the maximum amount of memory supported on 32-bit Windows XP Professional system. However, depending on the BIOS (basic input output system) and operating system settings, the user may only see $3 \mathrm{~GB}$ or $3.5 \mathrm{~GB}$ available. 


\section{Acknowledgments}

This chapter of the report benefited from excellent technical reviews by Laura Biewick, Jennifer Eoff, and Gregory Gunther of the USGS. Gregory also generated the initial metadata associated with grid files in this chapter.

\section{References and Software Cited}

Adler, F.J., Caplan, W.M., Carlson, M.P., Goebel, E.D., Henslee H.T., Hick, I.C., Larson, T.G., McCracken, M.H., Parker, M.C., Rascoe, G., Jr., Schramm, M.W., and Wells, J.S., 1971, Future petroleum provinces of the mid-continent, in Cram, I.H., ed., Future petroleum provinces of the United States-Their geology and potential: American Association of Petroleum Geologist Memoir 15, v. 2, p. 985-1120.

Andrews, R.D., 1999a, Map showing regional structure at the top of the Morrow Formation in the Anadarko Basin and shelf of Oklahoma: Oklahoma Geological Survey Special Publication 99-4, pl. 3.

Andrews, R.D., 1999b, Morrow gas play in the Anadarko Basin and shelf of Oklahoma: Oklahoma Geological Survey Special Publication 99-4, 133 p., 7 pl.

Andrews, R.D., 2001, Map showing regionlal (sic) structure at the top of the Springer Group in the Ardmore Basin, and the Anadarko Basin and shelf of Oklahoma: Oklahoma Geological Survey Special Publication 2001-1, pl. 5.

Blackwell, D.D., and Richards, M., 2004, Geothermal map of North America: American Association of Petroleum Geologists, 1 sheet, scale 1:6,500,000.

Burruss, R.C., and Hatch, J.R., 1989, Geochemistry of oils and hydrocarbon source rocks, greater Anadarko Basin: Evidence for multiple sources of oils and long-distance oil migration, in Johnson, K.S., ed., Anadarko Basin symposium, 1988: Oklahoma Geological Survey Circular 90, p. 53-64.

Carter, L.S., Kelly, S.A., Blackwell, D.D., and Naeser, N.D., 1998, Heat flow and thermal history of the Anadarko Basin, Oklahoma: American Association of Petroleum Geologists Bulletin, v. 82, no. 2, p. 291-316.

Cederstrand, J.R., and Becker, M.F., 1998, Digital map of base of aquifer for the High Plains aquifer in parts of Colorado, Kansas, Nebraska, New Mexico, Oklahoma, South Dakota, Texas, and Wyoming: U.S. Geological Survey Open-File Report 98-393, digital data and metadata, accessed March 2011, at http://cohyst.dnr.ne.gov/ metadata/m001aqbs_99.html.
Dynamic Graphics, Inc., 2010, EarthVision software: Available from Dynamic Graphics, Inc., 1015 Atlantic Avenue, Alameda, CA 94501, accessed August 2010, at http://www. dgi.com.

Environmental Systems Research Institute, 2010, Geographic Information Systems software; accessed November 2011, at http:www.esri.com/.

Fay, R.O., 1964, The Blaine and related formations of northwestern Oklahoma and southern Kansas: Oklahoma Geological Survey Bulletin 98, 238 p., 24 pl.

Hester, T.C., Schmoker, J.W., and Sahl, H.L., 1990, Logderived regional source-rock characteristics of the Woodford Shale, Anadarko Basin, Oklahoma: U.S. Geological Survey Bulletin 1866-D, 38 p.

Higley, D.K., 2014, Thermal maturation of petroleum source rocks in the Anadarko Basin Province, Colorado, Kansas, Oklahoma, and Texas, chap. 3, in Higley, D.K., comp, Petroleum systems and assessment of undiscovered oil and gas in the Anadarko Basin Province, Colorado, Kansas, Oklahoma, and Texas-USGS Province 58: U.S. Geological Survey Digital Data Series 69-EE, 53 p.

Howery, S.D., 1993, A regional look at Hunton production in the Anadarko Basin, in Johnson, K.S., ed., Hunton Group core workshop and field trip: Oklahoma Geological Survey Special Publication 93-4, p. 77-81.

IHS Energy, 2009a, IHS energy well database: Unpublished database available from IHS Energy, 15 Inverness Way East, Englewood, CO 80112.

IHS Energy, 2009b, GDS database: Unpublished geological data services database available from IHS Energy, 15 Inverness Way East, Englewood, CO 80112.

Kansas Geological Survey, 2010, downloadable formations tops and LAS well data: accessed April 2012, at http://www. kgs.ku.edu/PRS/petroDB.html.

National Geologic Map Database, 2011, U.S. Geological Survey, accessed December 1, 2011, at http://ngmdb.usgs. gov/Geolex/.

Rascoe, B., Jr., and Hyne, N.J., 1987, Petroleum geology of the midcontinent: Tulsa Geological Society Special Publication $3,162 \mathrm{p}$.

Robbins, S.L., and Keller, G.R., 1992, Complete Bouguer and isostatic residual gravity maps of the Anadarko Basin, Wichita Mountains, and surrounding areas, Oklahoma, Kansas, Texas, and Colorado: U.S. Geological Survey Bulletin 1866-G, 11 p., 2 pls. 
Rottmann, Kurt, 2000a, Structure map of Hunton Group in Oklahoma and Texas Panhandle: Oklahoma Geological Survey Special Publication 2000-2, pl. 3.

Rottmann, Kurt, 2000b, Isopach map of Woodford Shale in Oklahoma and Texas Panhandle: Oklahoma Geological Survey Special Publication 2002-2, pl. 2.

Schlumberger, 2011, PetroMod Basin and Petroleum Systems Modeling Software: IES GmbH, Ritterstrasser, 23, 52072 Aachen, Germany, accessed January 2011, at http://www. ies.de. Southern Methodist University: accessed August 2011, at http://smu.edu/geothermal/. 
